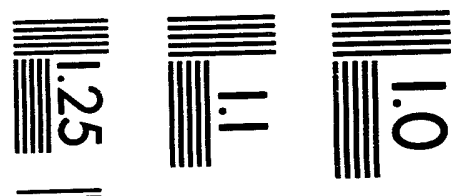

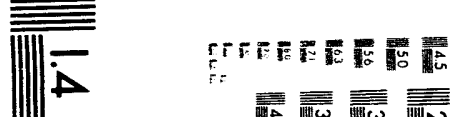

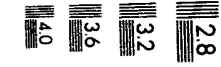

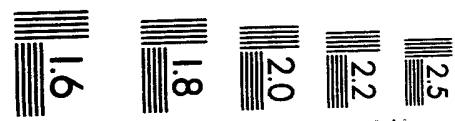



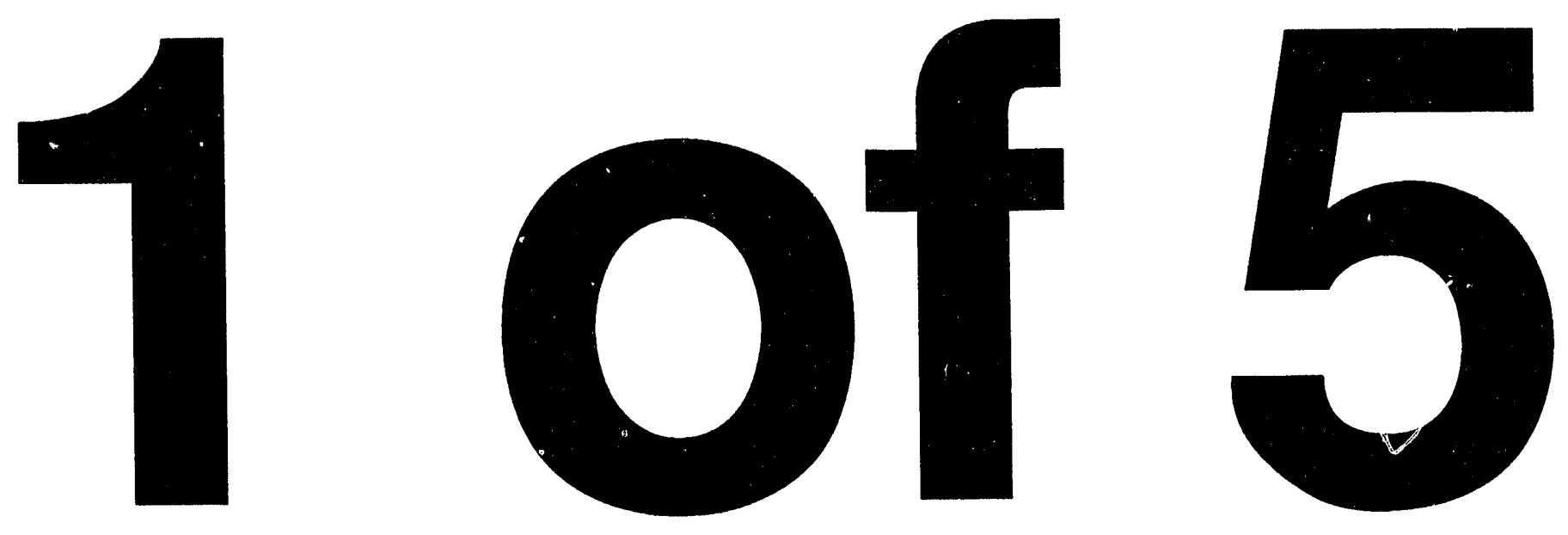
DOE/EIA-0370(93)

Distribution Category UC-950

\section{Oil and Gas Field Code Master List 1993}

December 1993

\section{Energy Information Administration \\ Office of Oil and Gas \\ U.S. Department of Energy \\ Washington, D.C. 20585}

This report was prepared by the Energy Information Administration, the independent statistical and analytical agency within the Department of Energy. The information contained herein should not be construed as advocating or reflecting any policy position of the Department of Energy or any other organization. 


\section{Preface}

The Oil and Gas Field Code Master List 1993 is the twelfth annual listing of all identified oil and gas fields in the United States. It is updated with field information collected through October 1993. The purpose of this publication is to provide codes for easy identification of domestic fields. Standardization on these field codes fosters consistency of field identification by government and industry.

The use of field names and codes listed in this publication is required on survey forms and other reports regarding field-specific data collected by the Energy Information Administration (EIA) and the Federal Energy Regulatory Commission (FERC). The surveys currently using these field codes are the following: Form EIA-23, "Annual Survey of Domestic Oil and Gas Reserves" and the Forms FERC 8 and EIA-191, both entitled "Underground Gas Storage Report".
A glossary of key terms is provided to assist readers in more fully understanding the information in this Field Code Master List.

EIA gratefully acknowledges the assistance provided by the various State organizations and trade associations in verifying the existence of fields and confirming their officially recognized names.

General information regarding this publication may be obtained from Craig $\mathrm{H}$. Cranston (202) 586-6083, Chief, Reserves and Production Branch, Reserves and Natural Gas Division, in the Energy Information Administration's Office of Oil and Gas. Detailed information regarding the preparation of this document may be obtained from Robert F. King (202) 586-4787, Reserves and Production Branch. 


\section{Contents}

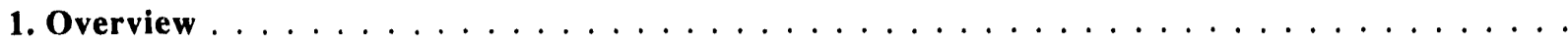

Introduction

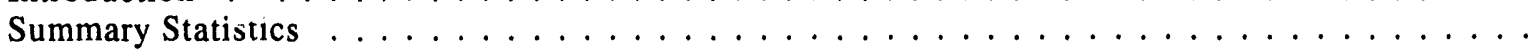

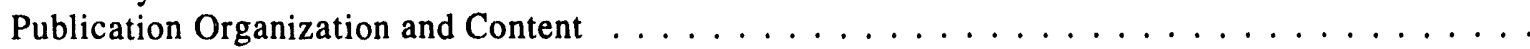

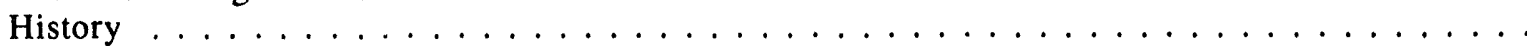

Definition of Field $\ldots \ldots \ldots \ldots \ldots \ldots \ldots \ldots \ldots \ldots \ldots \ldots \ldots \ldots$

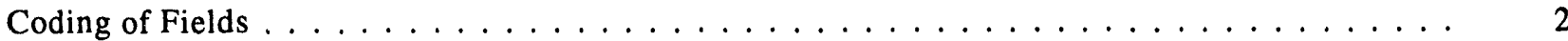

General Field Naming Conventions $\ldots \ldots \ldots \ldots \ldots \ldots \ldots \ldots \ldots \ldots$

2. Methodology for Field Code Assignments $\ldots \ldots \ldots \ldots \ldots \ldots \ldots \ldots$

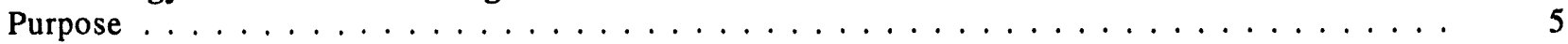

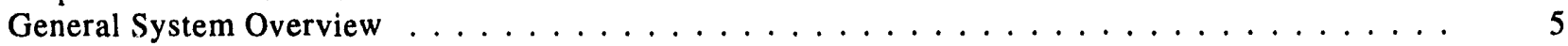

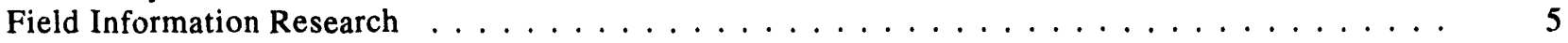

State Source Review Procedures $\ldots \ldots \ldots \ldots \ldots \ldots \ldots \ldots \ldots$

Assigning New Field Code Numbers $\ldots \ldots \ldots \ldots \ldots \ldots \ldots \ldots$

Field Alias Procedures $\ldots \ldots \ldots \ldots \ldots \ldots \ldots \ldots \ldots \ldots \ldots \ldots$

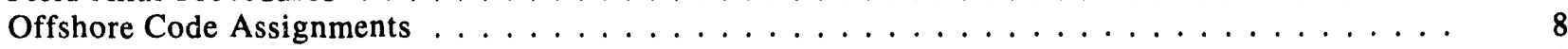

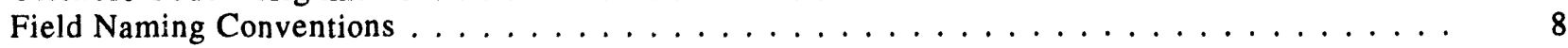

Invalid Field Record Procedures $\ldots \ldots \ldots \ldots \ldots \ldots \ldots \ldots$

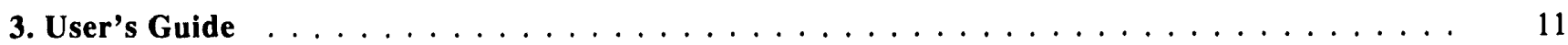

Field Code Master List $\ldots \ldots \ldots \ldots \ldots \ldots \ldots \ldots \ldots$

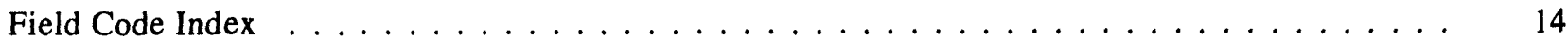

Coalbed Methane Field List $\ldots \ldots \ldots \ldots \ldots \ldots \ldots \ldots \ldots \ldots$

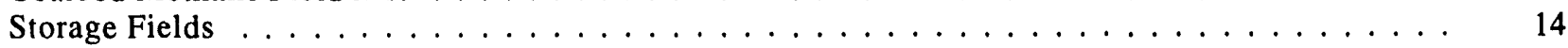

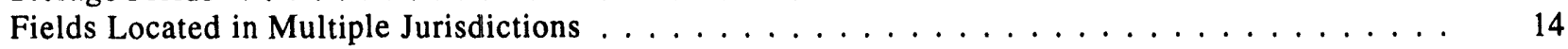

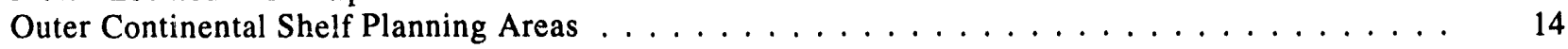

Glossary . . . . . . . . . . . . . . . . . . . . . . . . . . . . . . . 39

Oil and Gas Field Code Master List $\ldots \ldots \ldots \ldots \ldots \ldots \ldots \ldots \ldots$

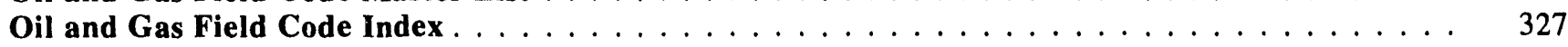

Oil and Gas Invalid Field Record List $\ldots \ldots \ldots \ldots \ldots \ldots \ldots$

\section{Tables}

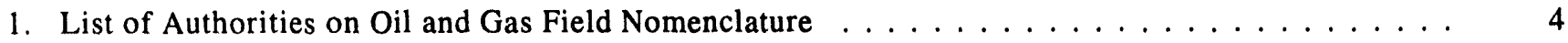

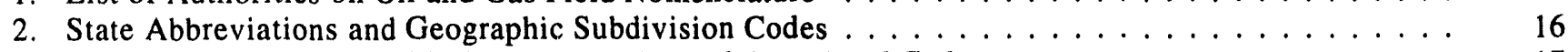

3. U.S. Geological Survey Alaska Quadrangles and Associated Codes . . . . . . . . . . . . . . . 17

4. Coalbed Methane Fields $\ldots \ldots \ldots \ldots \ldots \ldots \ldots \ldots$

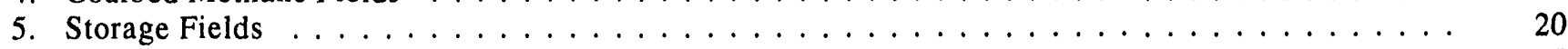

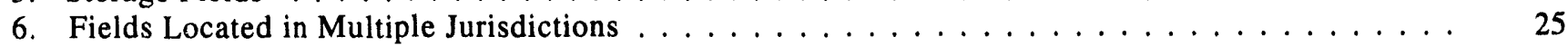




\section{Figures}

1. Field Code Processing Flow Chart $\ldots \ldots \ldots \ldots \ldots \ldots \ldots \ldots \ldots \ldots \ldots \ldots$

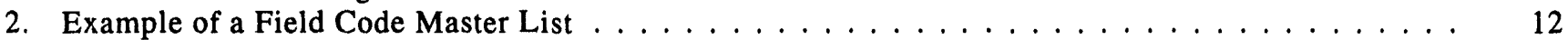

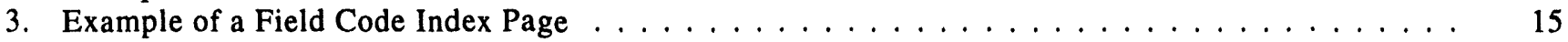

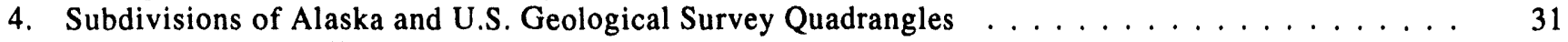

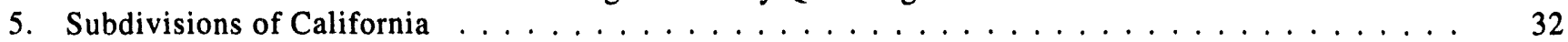

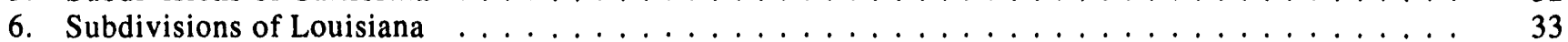

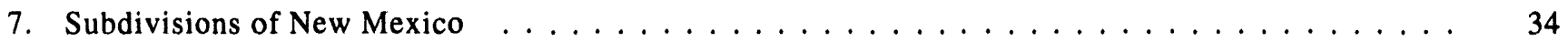

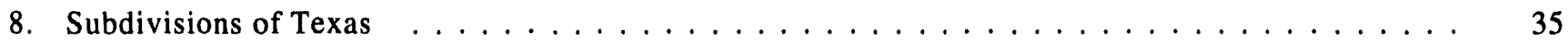

9. Western Planning Area, Gulf of Mexico Outer Continental Shelf Region . . . . . . . . . . . . . 36

10. Central Planning Area, Gulf of Mexico Outer Continental Shelf Region . . . . . . . . . . . . . . 37

11. Eastern Planning Area, Gulf of Mexico Outer Continental Shelf Region . . . . . . . . . . . . 38 


\section{Overview}

\section{Introduction}

This is the twelfth annual edition of the Energy Information Administration's (EIA) Oil and Gas Field Code Master List. It reflects data collected through October 1993 and provides standardized field name spellings and codes for all identified oil and/or gas fields in the United States. The master field name spellings and codes are to be used by respondents when filing the following Department of Energy (DOE) forms:

- Form EIA-23, "Annual Survey of Domestic Oil and Gas Reserves", filed by oil and gas well operators (field codes are required from larger operators only).

- Forms FERC 8 and EIA-191, "Underground Gas Storage Report", filed by natural gas producers and distributors who operate underground natural gas storage facilities.

Other Federal and State government agencies, as well as industry, use the EIA Oil and Gas Field Code Master List as the standard for field identification. A machinereadable version of the Oil and Gas Field Code Master List is available from the National Technical Information Service, 5285 Port Royal Road, Springfield, Virginia 22161, (703) 487-4650.

In order for the Master List to be useful, it must be accurate and remain current. To accomplish this, EIA constantly reviews and revises this list. The EIA welcomes all comments, corrections, and additions to the Master List. All such information should be given to the ElA Field Code Coordinator at (214) 953-1858.

EIA gratefully acknowledges the assistance provided by numerous State organizations and trade associations in verifying the existence of fields and their official nomenclature.

\section{Summary Statistics}

There are 56,423 field records in this year's Oil and Gas Field Code Master List (FCML). This amounts to 394 more than in last year's report. As it is maintained by EIA, the Master List includes the following:

- Field records for each State and county in which a field resides.

- Field records for each offshore area block in the Gulf of Mexico in which a field resides.
- Field records for each alias field name (see definition of alias below).

- Fields crossing State boundaries that may be assigned different names by the respective State naming authorities.

Taking into consideration the double-counting of fields under such circumstances, EIA can identify 45,743 distinct fields in the United States as of October 1993. This count includes fields that no longer produce oil or gas. Of this number, 382 are fields used in whole or in part for oil or gas storage. The following is a summary count by hydrocarbon type of distinct fields in the United States:

\begin{tabular}{|c|c|}
\hline Field Type & Number \\
\hline U.S. oil and gas distinct fields. . & 45,743 \\
\hline Fields with oil & 33,914 \\
\hline Fields with gas. . . . . . . . & 31,229 \\
\hline Fields with associated-dissolved gas. .... & 15,492 \\
\hline Fields with nonassociated gas $\ldots \ldots \ldots$ & 20,904 \\
\hline Fields with unspecified hydrocarbon & 150 \\
\hline
\end{tabular}

Note: The sum of these fields is greater than the total number of distinct fields, since many fields have more than one type of hydrocarbon present.

ElA has assigned a unique six-digit field code to each field name. A listing of all field names and the States in which they occur, ordered by field code, is presented in the Field Code Index. The number of entries in the Field Code Index, 51,142, represents the number of field names that have been recognized by State naming authorities. Of these, 44,737 are currently considered official, while 6,405 are no longer valid and have been designated as alias names by EIA.

In addition, this report contains an Invalid Field Record List of 42 records that have been removed from the FCML since last year's report. These records were found to be either technically incorrect or to represent field names which were never recognized by State naming authorities. 


\section{Publication Organization and Content}

Chapter 2 provides details on the methodology used to assign field codes and the procedures followed to standardize field names. Chapter 3, the User's Guide, and the Glossary provide users with the definitions and explanations needed to utilize this publication. The FCML follows the Glossary. It is organized by State, with fields sorted alphabetically by name within each individual State. Fields in the Federal Offshore Outer Continental Shelf are listed separately at the end after Wyoming. Each field name entry contains the field code, field name, geographical information, and other related information. The Field Code Index, which follows the Master List, is an abbreviated listing sorted by field code with field names and the State or States in which each field is located. This listing is intended for use as a quick reference. The Master List must be consulted for more detailed information. The Invalid Field Record List identifies those field records which were determined to be invalid since the previous report.

\section{History}

The EIA FCML evolved from the Federal Power Commission's Field/Plant Code List (FPC Field Code List). The FPC Field Code List, originally developed over 20 years ago, had a unique code assigned to each field on the list. That is, two fields having identical names in separate States had separate six-digit field codes. However, some respondents to Form FPC 15, "Interstate Pipeline's Annual Report of Gas Supply", began using the first code given in the list for a field name, regardless of the State involved. With few respondents utilizing computerized edits on their submissions at that time, miscoding of fields became a problem. The solution applied to this probiem was to recode the fields on the list so that any fields with identical names were assigned the same six-digit code (a field name code) but were differentiated by the State and county codes incorporated in the full field code. For example, $145385 \mathrm{KS} 101$ is the field code for the CLARK field in Kansas and 145385TX285 is the field code for the CLARK field in Texas, as 145385 is the field name code for CLARK.

The FPC Field Code List, originally designed to handle data relating to interstate gas fields, was expanded over the years, as resources permitted, to include the names of oil fields and intrastate gas fields. Six-digit codes were assigned in ascending order to alphabetically sorted field names. Codes from the FPC Field Code List were used in filing Form FPC 15 and Form FPC 8, "Underground Gas Storage Report".

After the establishment of the DOE in 1977, the requirement to gather annual, verifiable oil and gas reserves estimates led to the development of Form EIA-23, "Annual Survey of Domestic Oil and Gas Reserves". Form EIA-23 collects certain data by field, and the use of the FPC Field Code List aided the reporting and processing of these data. As use of the Master List expanded by way of the Form EIA-23 program, additional work to verify and update the code list was necessary to keep the list current. In 1981, the correlation between the code number sequence and the alphabetical field name sequence was dropped. This change precluded the necessity of periodically reassigning field codes in order to maintain the list in parallel numeric and alphabetic order.

A procedure was developed for the 1988 Field Code Master List publication which determined the actual count of distinct oil and gas fields in the United States. A part of this procedure was to aggregate the separate county portions of existing fields into their recognized entirety, even if the field crossed a State boundary. This procedure is time-consuming and does entail manual intervention, so that it is accomplished on a yearly cycle.

\section{Definition Of "Field"}

A field is defined as:

"An area consisting of a single reservoir or multiple reservoirs all grouped on, or related to, the same individual geological structural feature and/or stratigraphic condition. There may be two or more reservoirs in a field which are separated vertically by intervening impervious strata, or laterally by local geologic barriers, or by both."

This definition is not used by all States in their designation of fields; consequently, areas classified as individual fields by some States may be found combined in this Master List.

\section{Coding Of Fields}

As noted above, the six-digit field name code is common to a specific field name, regardless of whether one or several fields exist with that particular name. In order to uniquely identify a particular field, the field name code must be coupled with the corresponding State abbreviation and county code.

Most codes on the Master List remain in a numerically ascending order by alphabetized field name. However, field names added since 1981 have been assigned the first available (numerically lowest) unused code. Fields located in the Federal Offshore area and large State offshore blocks of the Gulf of Mexico will continue to be represented by codes above 800000 , according to their area, subarea, and block number.

Records that appear on the list with field name codes in light italics are alias records. The correct field name and field name code appear as part of the alias field name record after the label "Alias For:". Alias field 
names are not currently recognized by State naming authorities, and alias field names and their codes should not be used in DOE filings.

Records that were on the Field Code Master List last year but which were subsequently found to be invalid are entered on a separate Invalid Field Record List. These records were incorrectly placed on the FCML or when complete information was not available. Field names and codes on the Invalid Field Record List should not be used in DOE filings with the indicated State and county. Note, however, that the identified field name and field name code may still be valid for a different State/county combination.

\section{General Field Naming Conventions}

Field name spellings in the Master List reflect a number of conventions and conditions. In most instances, the 26-character name reflects the conventions imposed by the data block length in the DOE forms and by the State naming authority, usually the oil and gas regulatory agency. Field identification for a well, lease, block, unit, or section is the responsibility of the State naming authority. A listing of these agencies appears in Table 1. In the absence of a State authority, field names which have come into general acceptance in an area may be listed. In the Appalachian Region, field area names are often used. Detailed information is provided in Chapter 2, Methodology for Field Code Assignments, concerning the field naming conventions followed. 
Table 1. List of Authorities on Oil and Gas Field Nomenclature

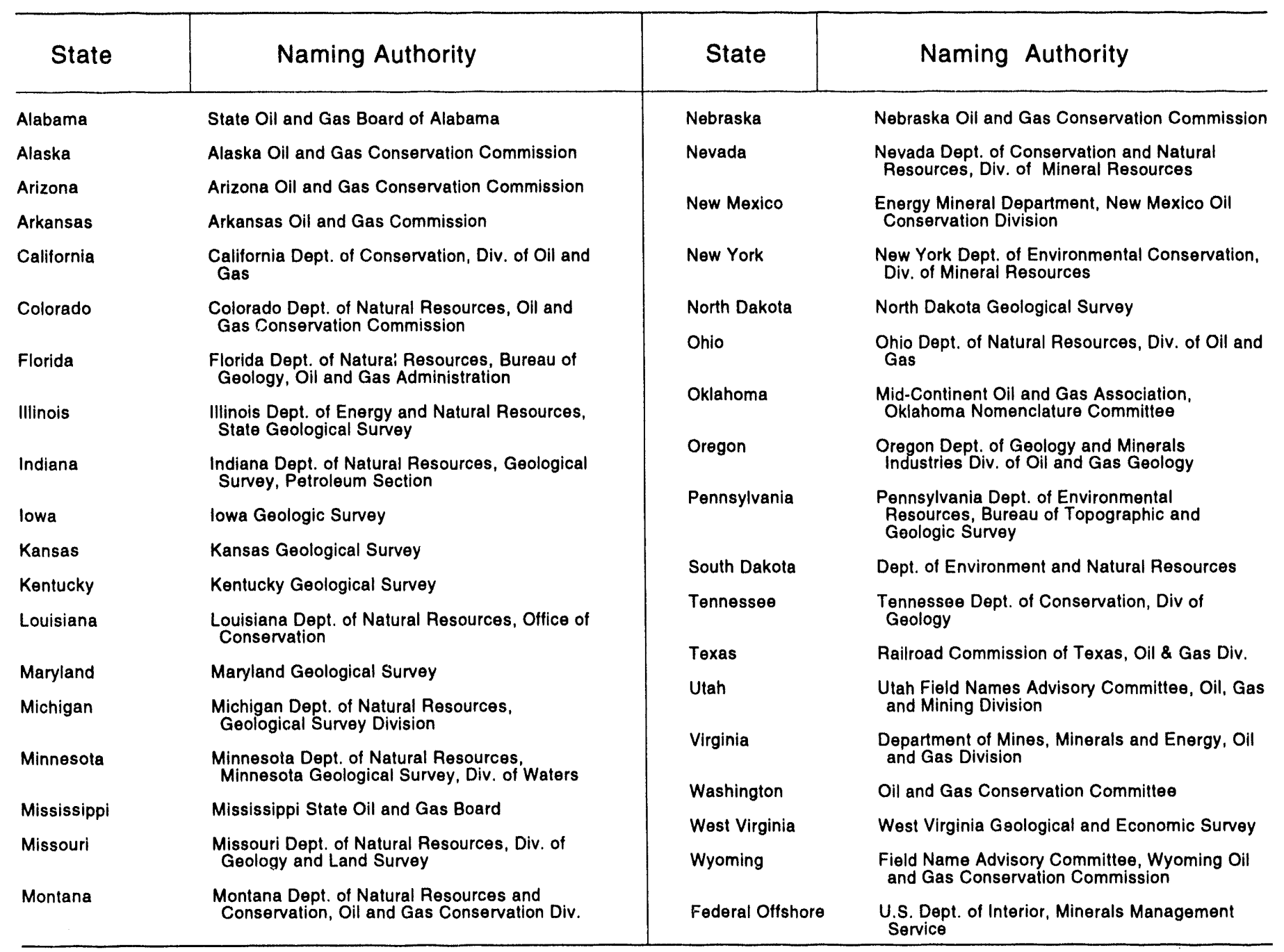

Source: Energy Information Administration, Office of Oil and Gas 


\section{Methodology for Field Code Assignments}

\section{Purpose}

The purpose of the Field Code Master List effort is to provide the Energy Information Administration (EIA) and others with standardized field name spellings and codes for all crude oil and natural gas fields throughout the United States. The codes are compiled on the computerized Field Code Master File (FCMF), which is updated on a quarterly cycle. The Oil and Gas Field Code Master List, derived from the FCMF, is published annually. The first edition was released by EIA in December 1982. Computer tapes containing the latest field code information are delivered to the National Technical Information Service in January of each year. Printouts of newly added fields and changes to existing fields become available in June of each year and may be requested in writing from Robert F. King, EI-443, Forrestal Bldg., U.S. Dept. of Energy, Washington, DC 20585 .

\section{General System Overview}

Figure 1 presents a flow chart of the activities necessary to process new field information. All new field information received by EIA goes through this cycle, which is designed to process field information received from respondents filing Forms EIA-23, and FERC 8 and EIA-191, as well as from other sources such as State publications.

The system incorporates both a Field Code Working File (FCWF) and the FCMF. The FCWF contains all new, or unverified, field information. It exists for two reasons:

- The large size of the FCMF makes it relatively difficult to manipulate.

- It is useful in tracking the progress made toward verifying the large number of in process fields, while at the same time assuring that redundant activities are not taking place.

The FCWF is updated as information about the field is obtained. Quarterly, and more often when warranted by the number of completed transactions, all fully resolved records are extracted from the working file and used to update the FCMF. Once the master file update is completed, the resolved records are deleted from the FCWF.

\section{Field Information Research}

Geologists and petroleum engineers are responsible for supervising research and final resolution of field information. Listings of the FCWF are produced by region for review. There are several possible explanations why field information under review is not already contained on the FCMF. That information could reflect the following:

- A relatively recent field discovery.

- A recently discovered extension of an old field into a new county or State.

- An alias used for the official name.

- An error exists in the reported information (e.g., a reporting error on the Form EIA-23).

The official recognition of a new field discovery by a State field naming authority is a prerequisite for the assignment of an official EIA field code. Table 1 on page 4 lists these naming authorities. Information regarding State recognition is obtained through official State publications and computer tapes, or through other contact with the State agencies.

If the field name in question has not been officially recugnized, several sources of information exist for further investigation into the third and fourth possibilities listed above. These include:

- Analysis of State data files (the Texas and New Mexico files, for example, contain detailed information for each field and operator.)

- Review of other oil and gas publications.

- Telephone contact with the source of the information (e.g., a respondent to Forms FERC 8 and EIA-191, or Form EIA-23).

\section{State Source Review Procedures}

State sources provide most of the field names. Field information is processed on a routine basis from State sources so that EIA field codes will hopefully be assigned as this information is needed. 
Figure 1. Field Code Processing Flow Chart

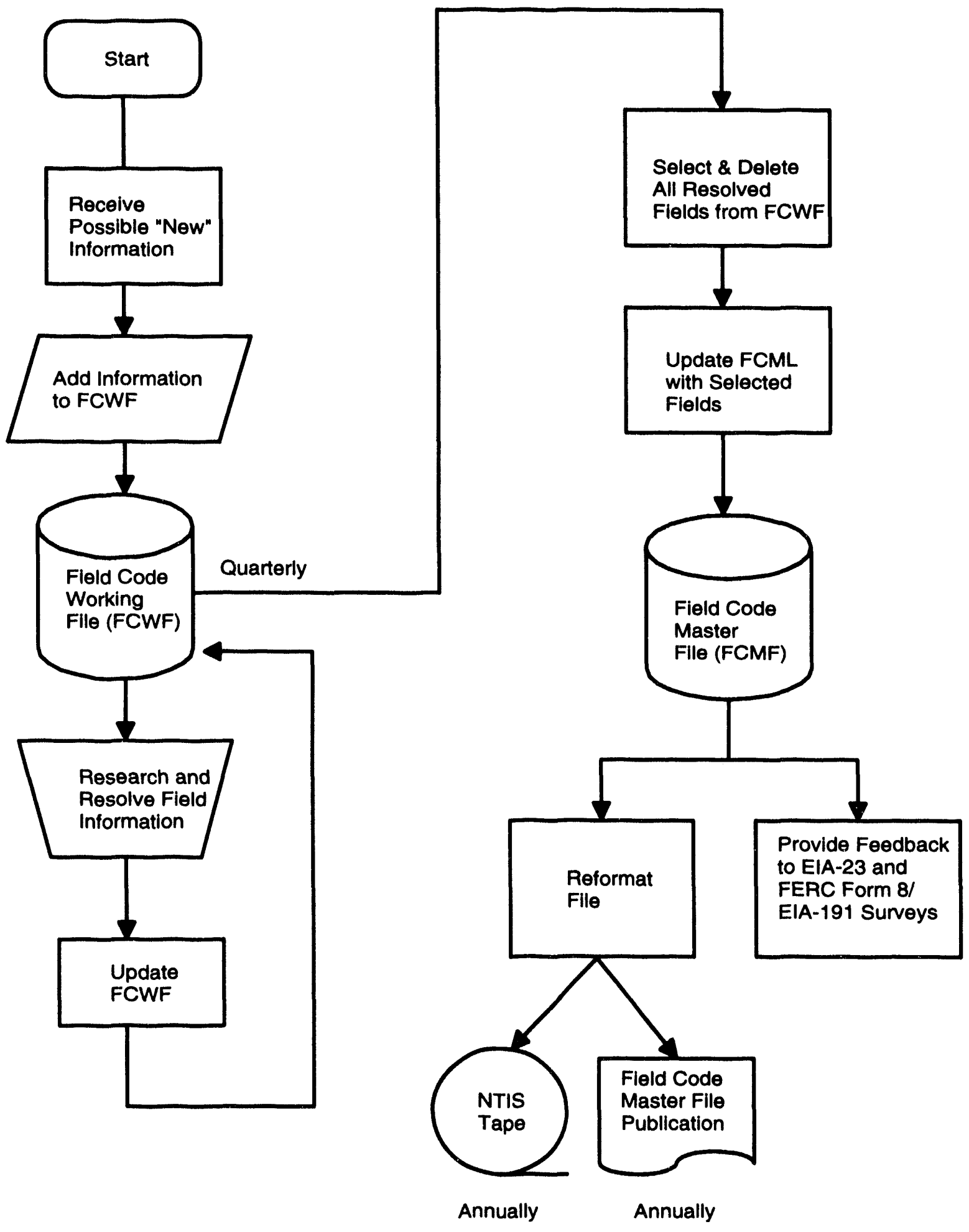

Source: Energy Information Administration, Office of Oll and Gas 
As State publications are released, they are routinely reviewed, and their information regarding new fields is compared to the information on the FCMF. If the FCMF does not contain the new field information, the working file is updated to reflect it. Prior to updating the working file, entries on it are reviewed in order to identify any research which might be in progress on the same field. Information added to the working file from State publications is added as resolved information, ready to be entered on the FCMF, since the States are the final authority for this information.

The quality and quantity of information available through the State publications varies. Some States publish new field information relatively frequently (e.g., monthly) in a format that is easily reviewed for incorporation into the FCMF. Routine processing of State sources begins with these publications. Additional State publications, such as State geological papers, are also included in these routine reviews.

Periodically, all FCMF records for a State are compared to the most recent State publication. Some States do not publish field information, or the field information is not carried in the latest publication but in a previous one. In an overall review of the FCMF, when a particular field is not found in any available State publication, the source NISP (not in State publication) is initially coded into the source section of the FCMF. When an earlier State source reports "No Production", "No Reports", or some similar remark for one or more years, and does not show the field in subsequent sources, the field is still considered to be official, whether it is producing at this time or not. When a field is found in an older State source in some sort of combination or in subordination with another field name, the State is contacted and asked to clarify the status of the field. This research resolves most of the NISP records, allowing them to be finally categorized as a master field record with a State naming authority source, as an alias field record, or as an invalid field record. Certain field records represented field names assigned by non-State Naming Authority publications which were coded prior to initiation of production of the field, which subsequently was abandoned without any recorded production. These records have been classified as invalid.

\section{Assigning New Field Code Numbers}

A sequential listing of available field code numbers starting with 000101 , has been produced for use by the Field Code Coordinator when new field codes are assigned. The first available code (numerically lowest) is used for a new field, regardless of name spelling, with the following exception: codes 800000-999998 are excluded from sequential code assignments, as they are reserved for offshore Gulf of Mexico fields.

\section{Field Alias Procedures}

A field alias is an unofficial name for an officially recognized field. Each name designated as an alias is cross-referenced to the official name. An official name is the current, State-sanctioned name. An unofficial name is one which no longer is, or never was, a State-sanctioned name.

A field alias may reflect the following possibilities:

- An alternate name for the official name.

- A field that has been renamed to something else.

- A field that has been combined, consolidated, or merged into another field.

- A field that has split into two or more new fields and the old name is no longer used.

For the combined or renamed types of aliases, both the official and alias names are on the FCMF.

For alternate name aliases, the official name must be on the FCMF in all cases. The alias name will be on the FCMF in the following cases:

- A representative from the State agency is aware of the alias name as an alternative for the official name.

- The alias name was submitted by a Form FERC 15 "Interstate Pipeline's Annual Report of Gas Supply" respondent.

- Two or more operators report the same alias.

- The alias is already on the FCMF for some reason.

An alternate name alias which does not meet one of these four criteria normally would not be added to the FCMF.

\section{Field Alias Records That Have Several Official Names}

Certain alias field records result from the splitting of a field into two or more fields, with none of the resulting fields using the original field name. For example, Field A was split into three fields: Field B, Field C, and Field D. Fields B, C, and D would be listed as field records on the Master List. Field $A$ would be listed as a field alias record referencing Field B, but not Fields $C$ and $D$ and would show SPLIT as its reason for alias. Because of space limitations, the appropriate State naming Authority or the Field Code Coordinator must be contacted to determine names of the other fields created out of Field A when it was split, as well as how many others were created. 


\section{Fields With More Than One} Name Change

The current official name may be the result of several name changes. For instance, in Oklahoma, RINGWOOD NE was combined with RINGWOOD NORTH in 1965, which was subsequently combined into RINGWOOD in 1966. The listing for RINGWOOD NE would show the correct field name as RINGWOOD. If multiple names were involved, the alias field name record would list only the current (correct) field name. (For exainple, in Oklahoma, HENNESSEY SE is an alias for HENNESSEY EAST which has since been changed first to DOVER-HENNESSEY and finally to SOONER TREND. The correct name for the alias HENNESSEY SE, HENNESSEY EAST and DOVER-HENNESSEY is SOONER TREND.) Information on the chain of successive field names may be obtained from the appropriate State naming authority or the Field Code Coordinator.

\section{Offshore Code Assignments}

Offshore fields on the Federal Gulf of Mexico Outer Continental Shelf receive a field code that is determined by the lease block (or blocks) for which they are named by the U.S. Minerals Management Service (MMS). The last three digits of the code are the block number. For instance, East Cameron (offshore area prefix code 824) Block 071 , receives the field code of 824071 . If several blocks are included in one field, the field code reflects the number of he block for which the field is named. The ther blocks included in the field are aliases to the offic al name. When the MMS has named two fields with the same basic block number i.e., Ship Shoal Blk 113 field and Ship Shoal Blk 113A field, for the second designated field the basic block number is combined with the prefix code of an adjacent area within which this block number would not occur.

There are cases where a particular OCS lease block contains portions of two or more fields. If one of the fields is named for the block concerned, there will be an entry in the REMARKS section indicating PBI, or Part of Block In, followed by the field code - $f$ the other included field. If the block concerned is oniy an alias to all the fields involved, it will be designated in MULTI to highlight that more than one field is included within the lease block.

Fields in large offshore State blocks in the Gulf of Mexico are assigned codes in the manner of offshore Federal fields. Special prefixes, generally unassigned prefixes for area surveys, are used for the small State block fields. A special prefix has been set aside for High Island-State. This prefix is used only with the small block fields. For State blocks that exceed 999 , an unused three-digit prefix ending in 1 is used, such as SOUTH PADRE IS BLK 1068 with code 951068.

Federal offshore fields in areas other than the Gulf of Mexico are given names in the same manner as for onshore fields, and are coded with the next sequential code.

\section{Field Naming Conventions}

\section{Use of Compass Directions in Field Names}

As a general rule, a compass direction used as part of a field name is placed at the end of the name. For example, the field named West Davenport by a State source bears the name DAVENPORT WEST on the FCMF. A field named after a known landmark, such as the town of East Davenport for example, bears the name EAST DAVENPORT on the FCMF.

If the field DAVENPORT WEST is then combined with other wells or fields and the word District, or some similar word, is added to the name, the name will appear on the FCMF as DAVENPORT WEST DISTRICT. This enables users to distinguish between two fields, one named as a result of forming a district with the DAVENPORT WEST field as its nucleus, e.g., DAVENPORT WEST DISTRICT, and the other which is a new district to the west of DAVENPORT DISTRICT, e.g., DAVENPORT DISTRICT WEST.

Abbreviations are not used in connection with onshore field names except for noncardinal compass points, such as NW for Northwest or SE for Southeast, and for names of combined fields in certain States which use all the individual names of the former fields to form the new field name. Cardinal compass points are spelled out in onshore field names.

Offshore field names in the Gulf of Mexico usually consist of an offshore area name and block number specified by the U.S. Minerals Management Service (MMS). Example: EAST CAMERON BLOCK 299. The Field Code Master List has retained the subarea identifiers such as EAST CAMERON SOUTH ADDITION BLOCK 299 field which yields a 37-character field name. Such lengthy offshore area names must be abbreviated to fit within the 26 characters available. When this is necessary, the following standard abbreviations are used:

$\begin{array}{lll}\text { North } & \ldots & \text { N } \\ \text { South } & \ldots & \text { S } \\ \text { East } \ldots . & \text { E } \\ \text { West } \ldots . & \text { W } \\ \text { Block } & \ldots & \text { BLK } \\ \text { Island } & \ldots & \text { IS }\end{array}$

$\begin{array}{ll}\text { North Addition .. } & \text { NA } \\ \text { South Addition .. } & \text { SA } \\ \text { East Addition ... } & \text { EA } \\ \text { West Addition . . . } & \text { WA } \\ \text { South Extension . . } & \text { SX } \\ \text { East Extension . . . } & \text { EX }\end{array}$

For example, High Island East Addition South Extension Block A376 is ahbreviated HIGH IS EA SX BLK A376. 


\section{Special Naming Conventions}

Some States regard reservoirs as fields and keep their records on that basis (e.g., Texas and New Mexico). The Master List does not follow the State conventions in these instances. For example, in Texas, PARKER (PENNSYLVANIAN) and PARKER (WOLFCAMP) are considered to be separate fields by the State. PARKER is actually the name of the field and PENNSYLVANIAN and WOLFCAMP are the names of reservoirs in the field. The Texas Railroad Commission requires that the reservoir name appear in parentheses after the field name in its publications. In the Master List, you will only find PARKER listed as the field.

The MMS quarterly publication, OCS Operations Field Names Master List, Gulf of Mexico OCS Region, (FNML) is the primary source for the Federal offshore Fields in the Gulf of Mexico. This publication, and the MMS computer file on which it is based, does not use the subarea identifiers, such as the SOUTH ADDITION, although maps of the region still carry these subareas. That is, where the MMS FNML carries the name WEST CAMERON BLOCK 617, most maps and the Field Code Master List call the field WEST CAMERON SA BLK 617 with SA the abbreviation for SOUTH ADDITION.

\section{Reused Field Names}

Some States occasionally reuse field names for areas other than the original field location. This problem is handled on the FCMF by indicating (OLD) and (NEW) after the field name, with each being assigned a differ- ent field code. This procedure facilitates computerized usage of the database.

\section{Invalid Field Record Procedures}

Field records are removed from the FCML when they are found to be incorrect for one of the following reasons:

- The field name as it appears was never approved by the State naming authority for that State and county; i.e., it could have been a misspelled field name or the name of a producing unit, well, lease or offshore platform.

- County or State location data are incorrect.

- Two separate field codes were assigned to the same field name.

When research on a NISP field record determines that one of those reasons applies to a field or field alias record, the record type is changed to INVALID. Invalid field records appear in the Invalid Field Record List section of the FCML publication for the year in which they have been designated INVALID. They will not appear in subsequent years. A cumulative list of INVALID records may be obtained by contacting the Field Code Coordinator. The National Technical Information Service machine-readable version of the EIA Oil and Gas Field Code Master List includes a separate and cumulative Invalid Field Record List. 


\section{User's Guide}

\section{Field Code Master List}

Fields in the Field Code Master List (FCML) are sorted alphabetically by State and alphabetically by field narie within a State. Fields in the Federal Offshore are listed separately. Fields that occur in multiple States are listec in each and are further sublisted by county.

The layout of the FCML is shown in Figure 2. Each field record is one line long except for records with a "REMARK", which is on a second line. Each alias field record is two lines long. A brief description of each data item follows.

\section{Master Field Record}

Item 1, FIELD CODE. The six-digit field code assigned to this field name.

Item 2, FIELD NAME. This is the field name (26-character limit).

Item 3, STATE POSTAL ABBREVIATION AND STATE SUBDIVISION CODE. This is a four-character code indicating the State and State subdivision. The first two positions are the State postal abbreviation. The last two positions are the two-digit subdivision code, used only in Alaska, California, Louisiana, New Mexico, and Texas, and in offshore areas to differentiate between State and Federal waters. Table 2 on page 16 is a listing of these codes. Figures 5 through 9, at the end of the Users' Guide, are subdivision maps for the aforementioned States.

Item 4, COUNTY CODE. This is a three-character code for the county or parish. For all States except Alaska this is the Gederal Information Processing Standards (FIPS) county code, as presented in FIPS publication 6-3 dated December 15, 1979 and its amendments. As Alaska has no counties, the FCML uses the U.S. Geological Survey $1^{0} \times 3^{\circ}$ quadrangles for Alaska and the three-digit pseudo-county codes assigned to them by the American Petroleum Institute. Codes used for fields located in State or Federal offshore areas are: Offshore-States, 990; Offshore-Federal, 995; OffshoreGeneral, 999.

Item 5, COUNTY NAME. This is the county or parish name (23-character limit) as defined in FIPS publication 6-3 for all State onshore areas except Alaska, For Alaska, the name associated with the USGS $1^{\circ} \times 3^{0}$ quadrangle is used. Table 3 on page 17 is a listing of the pseudo-county codes and names for Alaska. If the field is in an offshore area, see the list in Item 4 above.

Item 6, FIELD DISCOVERY YEAR. This is the fourdigit year of first discovery of oil or gas in this field, if it is known. In the case of combined fields, this is the earliest date among the formerly separate fields.

Item 7, FIELD TYPE. This is a three-character block giving the type of hydrocarbon found in the field using the symbols defined below.

\begin{tabular}{|c|c|}
\hline Symbol & Meaning of Symbol \\
\hline ONA & $\begin{array}{l}\text { Oil, nonassociated gas, and associated- } \\
\text { dissolved gas are present. }\end{array}$ \\
\hline ON & $\begin{array}{l}\text { Oii and nonassociated gas present; } \\
\text { associated-dissolved gas absent. }\end{array}$ \\
\hline $\mathbf{N}$ & $\begin{array}{l}\text { Nonassociated gas present; } \\
\text { oil and associated-dissolved gas absent. }\end{array}$ \\
\hline $\mathrm{O}$ & $\begin{array}{l}\text { Oil present; nonassociated gas and associated-dis } \\
\text { solved gas absent. }\end{array}$ \\
\hline OA & $\begin{array}{l}\text { Oil and associated-dissolved gas present; } \\
\text { nonassociated gas absent. }\end{array}$ \\
\hline Blank & Type of hydrocarbon is unknown. \\
\hline
\end{tabular}

Item 8, REMARKS. This is a 23-character entry specifying an attribute of the field. 
Figure 2. Example of a Field Code Master List

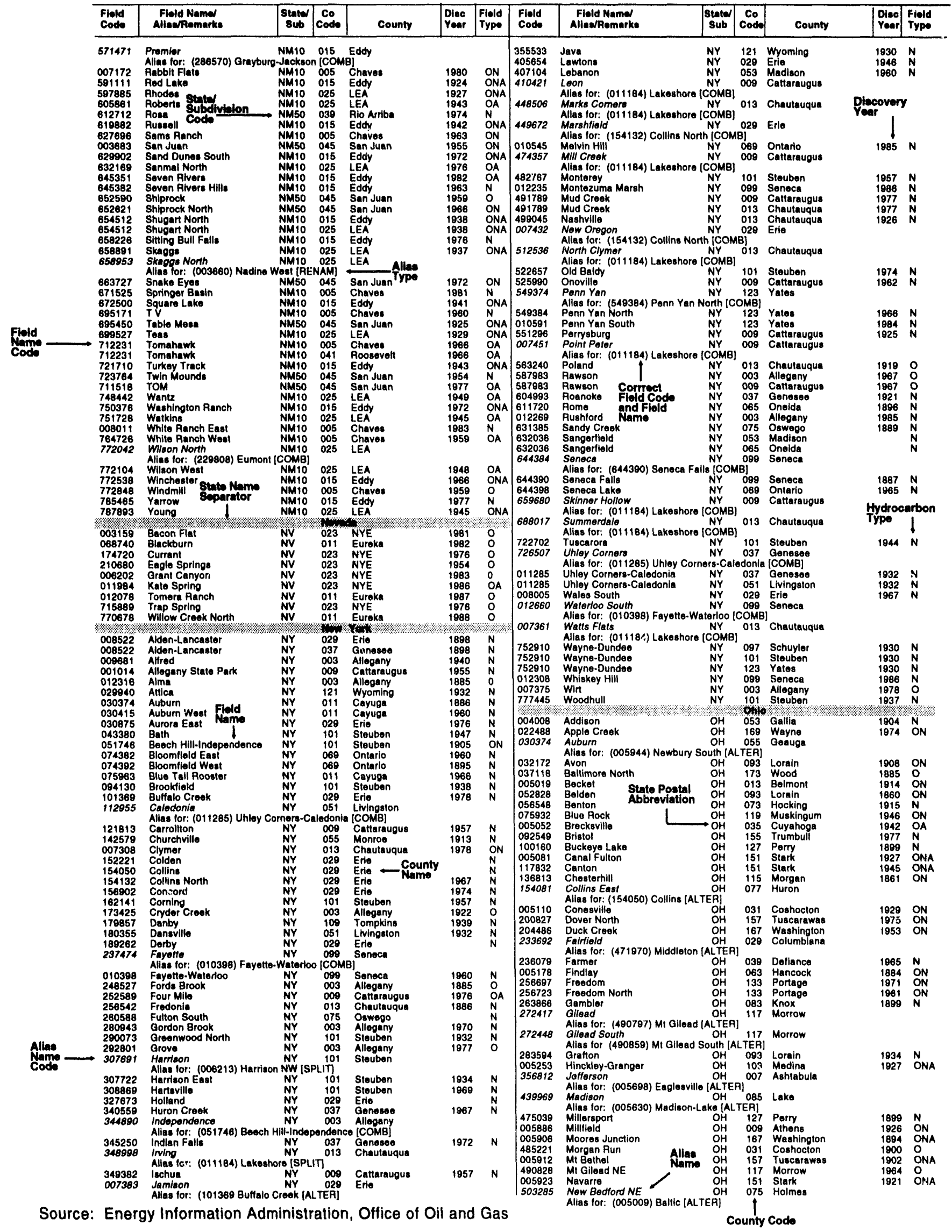


Common remarks are described below:

\begin{tabular}{ll}
\hline Remark & \multicolumn{1}{c}{ Description } \\
\hline AKA & $\begin{array}{l}\text { Field is also called by another name, } \\
\text { Also Known As. The year (YY) it be- } \\
\text { came known as this field also may be } \\
\text { shown. (AKA JOHN SMITH 71) }\end{array}$ \\
COMB & $\begin{array}{l}\text { Field combined with another field. The } \\
\text { year (YY) also may be shown after the } \\
\text { combined field code. (COMB 123456 78) }\end{array}$ \\
& $\begin{array}{l}\text { Although listed in State offshore, this } \\
\text { field and block are located Landward of } \\
\text { the Barrier Islands (see BRETON } \\
\text { SOUND BLK 18.) }\end{array}$ \\
& $\begin{array}{l}\text { Used when referring to offshore blocks. } \\
\text { Part of Block In will reference an- } \\
\text { other offshore field code (PBI 921300) in } \\
\text { which a portion of this block is included } \\
\text { (i.e., portions of this block occur in two } \\
\text { different fields). }\end{array}$
\end{tabular}

Remark

Description

STOR Field used to store natural gas or it is a DOE strategic oil reserve. The storage field will be gas unless the REMARKS section includes an entry such as DOE OIL. The year (YY) it was designated a storage field is shown in parentheses following the STOR label (STOR (69)

$A Q F)$. $A Q F$ is used in conjunction with STOR to signify aquifer gas storage field.

UNIT A unit is an area made up of a group of leases (either State or Federal). It may or may not include a field and it may be composed of one or more fields.

\section{Alias Field Record}

Item 1, ALIAS FIELD CODE. The six-digit field name code assigned to this alias field name, printed in light italics.

Item 2, ALIAS FIELD NAME. This is the alias field name (26-character limit), printed in italics.

Item 3, STATE POSTAL ABBREVIATION and STATE SUBDIVISION CODE. Same as Item 3 under Master Field Record.

Item 4, COUNTY CODE. Same as Item 4 under Master Field Record.

Item 5, COUNTY NAME. Same as Item 5 under Master Field Record.

Item 6, CROSS-REFERENCE. This is the identification of the master field code and field name which should be used in place of the alias name listed in Item 2 of this record. This item consists of the label "Alias For:" followed by the correct six-digit field name code and the correct (i.e., current) field name.

Item 7, ALIAS TYPE. This code (five-character limit) indicating type of alias is present only for alias fields. The types of alias codes are as follows:
ALTER This field name is an alternative for the official field name and should not be used in DOE forms. The correct name and code are listed after "Alias For:".

BLOCK This offshore field extends into more than one offshore block. This offshore block name is not the one for which the field is named. The block for which the field is named is given after "Alias For:".

COMB This field has been combined with another field which is listed after "Alias For:".

MULTI This offshore lease block has portions of two or more fields in it. In the Field Code Master List, the record is listed once for each field to which it is aliased so that appropriate cross reference is made.

RENAM This field has been renamed. The new name is listed after "Alias For:".

SPLIT This field has been split into two or more fields, none of which retains this name. One of the new fields is listed after Alias For:". Contact the Field Code Coordinator for the additional fields and information relating to this alias field.

Included on the following page is a listing of the State names and the page of the Oil and Gas Field Code Master List on which the first field in those States occurs. 


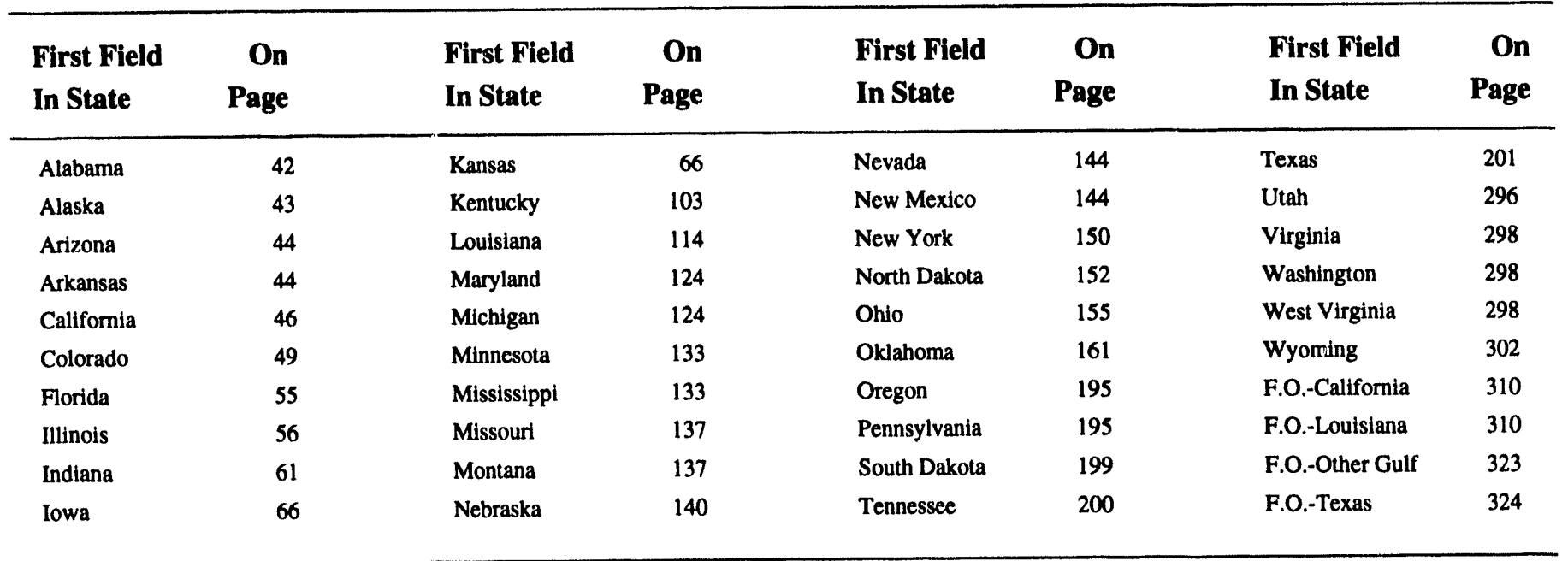

\section{Field Code Index}

In the Field Code Index, the field codes, State abbreviations, and associated field names are listed in ascending field code order. If a field name occurs in more than one State, the names are sorted in alphabetic State abbreviation order.

The field codes are arranged in four columns per page, as shown in Figure 3. As the six-digit field code relates directly to the particular field name, there is only one listing in this section per State/field code combination, regardless of the number of counties within that State in which it occurs.

The Index is intended for quick reference only. All detailed information is included on the Master List.

\section{Coalbed Methane Field List}

A dramatic rise in coalbed methane's share of natural gas production has heightened interest in those fields with coalbed methane potential. In Table 4, p. 18, Coalbed Methane Fields, the field name, field code, county code and name and state are given for those fields currently productive or with drilling activity.

\section{Storage Fields}

Storage fields have been developed in many States. Some were developed in existing, official oil and/or gas fields. All storage fields are listed in Table 5. In certain States, the storage portion of a field or pool has been given a different name. In this case, the storage field name is carried on the FCML as an alias to the official field name. The storage field names which are aliases are listed in both Table 5 and the FCML in italics. These alias field names and codes in italics should only be used in filing Forms FERC 8 and EIA-191, "Underground Gas Storage Report".

\section{Fields Located in Multiple Jurisdictions}

Table 6, Fields Located in Multiple Jurisdictions, is compiled to reflect those oil and/or gas fields which cross State boundaries. In developing the summary statistics on page 1 , a field is only counted once, no matter how many counties or States it is in.

\section{Outer Continental Shelf Planning Areas}

Gulf of Mexico Outer Continental Shelf statistics published by the Minerals Management Service (MMS) follow the boundary lines of their Western, Central and Eastern Planning areas, shown respectively in Figures 9, 10, and 11. In order that reports developed from the U.S. Crude Oil, Natural Gas, and Natural Gas Liquids Reserves 1992 track the reporting by MMS, fields currently found in the Garden Banks leasing area (GB), and those which may be found in GB or in the Keathley Canyon leasing area, will henceforth be listed in the FCML as Texas Federal Offshore rather than LouisianaSouth Federal Offshore. 
Figure 3. Example of a Field Code Index Page

\begin{tabular}{|c|c|c|c|c|c|c|c|c|c|c|c|}
\hline $\begin{array}{l}\text { Field } \\
\text { Codo }\end{array}$ & State & Field Name & $\begin{array}{l}\text { Field } \\
\text { Code }\end{array}$ & State & Fiold Name & $\begin{array}{l}\text { Fiold } \\
\text { Codo }\end{array}$ & State & Fiold Namo & $\begin{array}{l}\text { Fiold } \\
\text { Code }\end{array}$ & Stato & Fleld Name \\
\hline 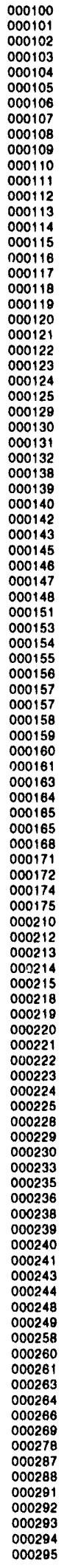 & 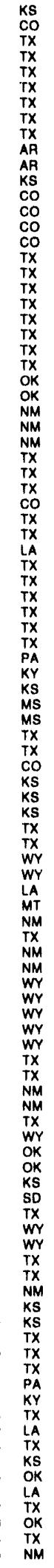 & 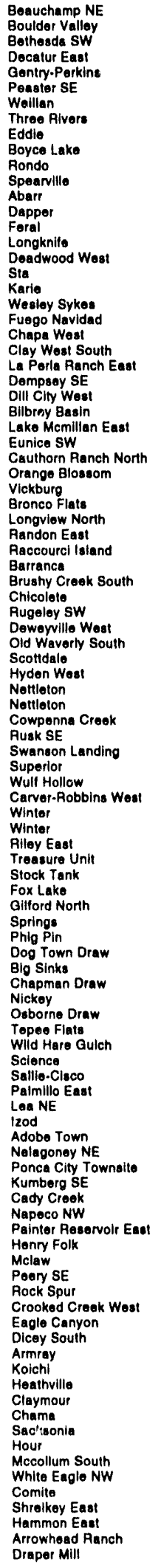 & 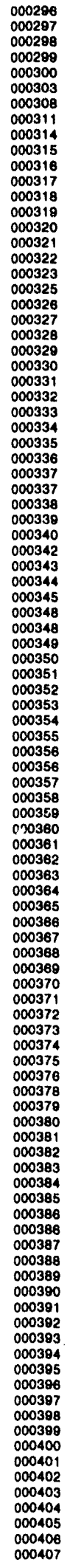 & 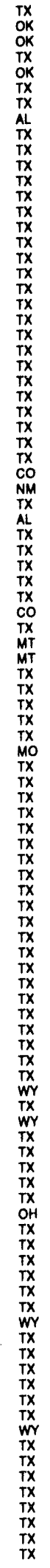 & 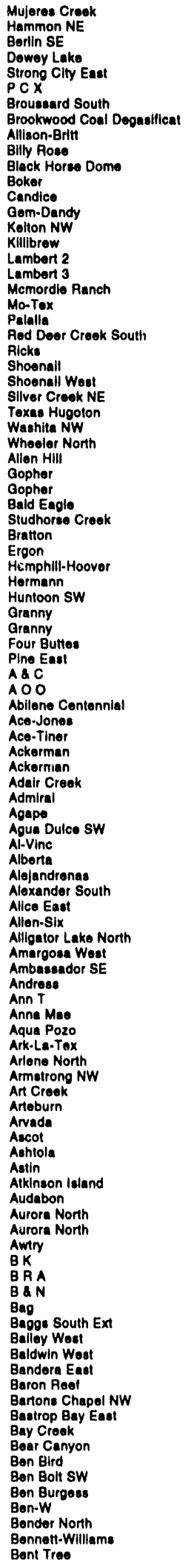 & 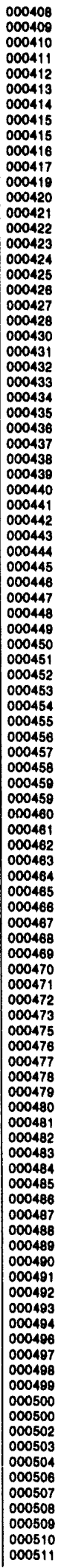 & 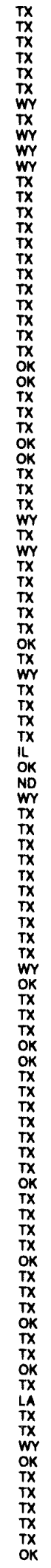 & 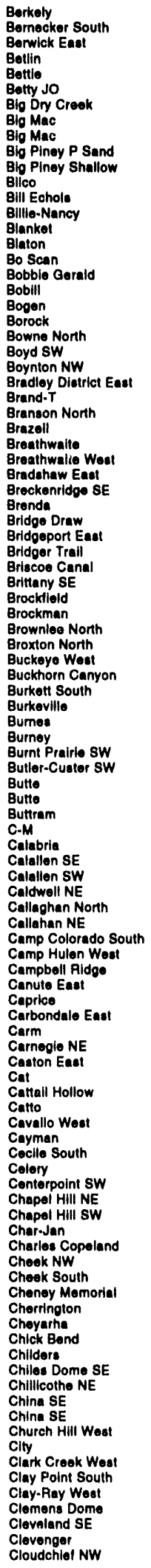 & 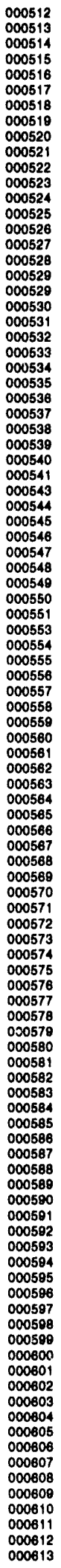 & 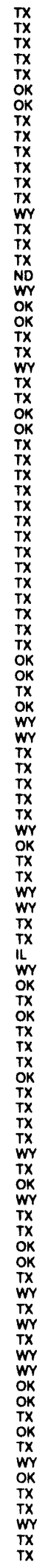 & 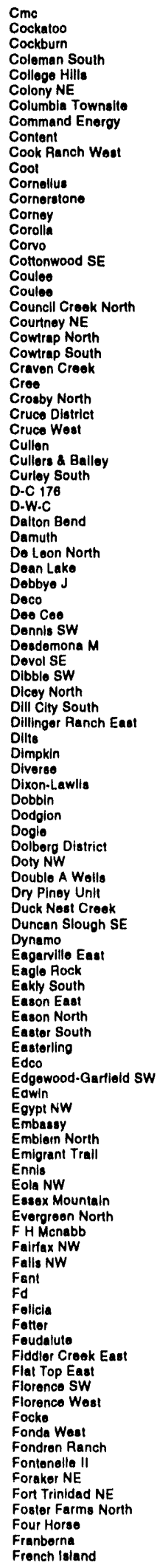 \\
\hline
\end{tabular}

Source: Energy Information Administration, Office of Oll and Gas 
Table 2. State Abbreviations and Geographic Subdivision Codes

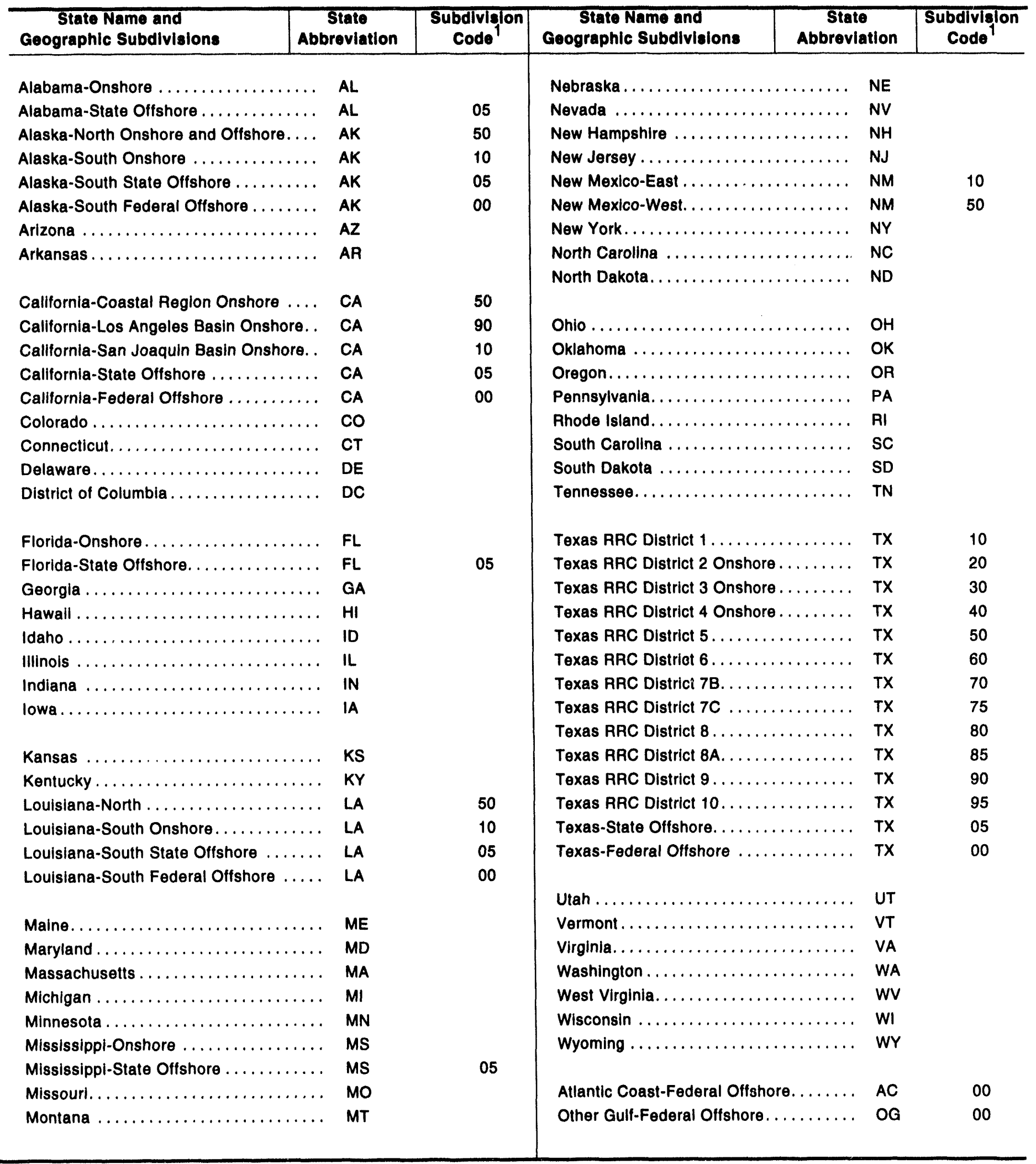

${ }^{1}$ If no subdivision code is specified, there is no subdivision.

Sources: National Bureau of Standards, U.S. Department of Commerce, FIPS PUB 6-3, Counties and County Equivalents of the States of the United States and the District of Columbia. Energy Information Administration, Office of Oil and Gas. 


\begin{tabular}{|c|c|c|c|c|c|c|c|}
\hline Quadrangle & $\begin{array}{l}\text { Quad } \\
\text { Code }\end{array}$ & Quadrangle & $\begin{array}{l}\text { Quad } \\
\text { Code }\end{array}$ & Quadrangle & $\begin{array}{l}\text { Quad } \\
\text { Cods }\end{array}$ & Quadrangle & $\begin{array}{l}\text { Quad } \\
\text { Code }\end{array}$ \\
\hline Adak. . . . . . . & 001 & Dillingham ......... & 077 & Marshall .. & 157 & Seward ....... & 233 \\
\hline Afognak ........... & 003 & Dixon Entrance..... & 079 & McCarthy ......... & 159 & Shishmaref ......... & 235 \\
\hline Ambler River........ & 005 & Eagle $\ldots \ldots \ldots \ldots$ & 081 & McGrath . ......... & 161 & Shungnak ......... & 237 \\
\hline Amukta $\ldots \ldots \ldots$ & 007 & Fairbanks ......... & 083 & Meade River. . . . . . . & 163 & Simeonof Island . .... & 239 \\
\hline Anchorage......... & 009 & False Pass. . . . . . . & 087 & Medfra........... & 165 & Sitka............ & 241 \\
\hline Arctic $\ldots \ldots \ldots \ldots$ & 011 & Flaxman Island ..... & 089 & Molozitna ......... & 167 & Skagway ........... & 243 \\
\hline Atka $\ldots \ldots \ldots \ldots$ & 013 & Fort Yukon......... & 091 & Middleton Island .... & 169 & Sleetmute ........ & 245 \\
\hline Atlin $\ldots \ldots \ldots \ldots$ & 015 & Cold Bay .......... & 093 & Misheguk Mtn. ..... & 171 & Solomon .......... & 247 \\
\hline Attu................ & 017 & Garelol island ...... & 095 & Mt Fairweather. ..... & 085 & St Lawrence ........ & 253 \\
\hline Baird Inlet .......... & 019 & Goodnews ......... & 097 & Mt Hayes . ........ & 173 & St Matthew ........ & 249 \\
\hline Baird Mts......... & 021 & Gulkana .......... & 099 & Mt Katmai ......... & 175 & St Michael. ......... & 255 \\
\hline Barrow ............ & 023 & Hagemeister Island . & 101 & Mt McKinley ........ & 177 & Stepovak Bay ....... & 251 \\
\hline Barter Island ........ & 025 & Harrison Bay ....... & 103 & Mt Michelson ....... & 179 & Sumdum .......... & 257 \\
\hline Beaver............ & 027 & Healy & 105 & Mt St Elias ........ & 181 & Survey Pass ........ & 259 \\
\hline Beechey Point . & 029 & Holy Cross . . . . . . . . & 107 & Nabesna.......... & 183 & Sutwik Island . ...... & 261 \\
\hline Bendeleben........ & 031 & Hooper Bay ........ & 109 & Naknek ........ & 185 & Table Min .......... & 263 \\
\hline Bering Glacier. ...... & 033 & Howard Pass....... & 111 & Noatak. ...... & 187 & Taku River. . . . . .... & 265 \\
\hline Bethel $\ldots \ldots \ldots \ldots$ & 035 & Hughes.......... & 113 & Nome............. & 189 & Talkeetna $\ldots \ldots \ldots$ & 267 \\
\hline Bettles ....... & 037 & Icy Bay ............ & 115 & Norton Bay......... & 191 & Talkeetna Mts. ...... & 269 \\
\hline Big Delta .......... & 039 & Iditarod........... & 117 & Nulato ........... & 193 & Tanacross ......... & 271 \\
\hline Black ............ & 041 & Ikpikpuk River . . . . . . & 119 & Nunivak Island...... & 195 & Tanana ........... & 273 \\
\hline Black River ......... & 043 & Illamna ........... & 121 & Nushagak Bay ...... & 197 & Taylor Mis ......... & 275 \\
\hline Blying Sound. ........ & 045 & Juneau . . . . . . . . . . & 123 & Ophir............. & 199 & Teller.............. & 277 \\
\hline Bradfleld Canal. ..... & 047 & Kaguyak........... & 125 & Petersburg ........ & 201 & Teshekpuk. ........ & 279 \\
\hline Bristol Bay. . . . . . . . & 049 & Kantishna River .... & 127 & Phillip Smith Mts.... & 203 & Trinity Islands. . . . . . & 281 \\
\hline 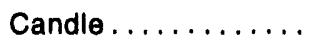 & 051 & Karluk........... & 129 & Point Hope ........ & 205 & Tyonek............ & 283 \\
\hline Cape Mendenhall. ... & 053 & Kateel River........ & 131 & Point Lay .......... & 207 & Ugashlk .......... & 285 \\
\hline Chandalar.......... & 055 & Kenal ............. & 133 & Port Alexander...... & 209 & Umiat ............ & 287 \\
\hline Chand'or Lake ..... . & 057 & Ketchikan..... & 135 & Port Moller .......... & 211 & Umnak............ & 289 \\
\hline Charley River . ...... & 059 & Killik River . . . . . . . & 137 & Pribilof Islands. . . . . & 213 & Unalakleet.......... & 291 \\
\hline Chignik ........... & 061 & Kiska............. & 139 & Prince Rupert. ..... . & 215 & Unalaska.......... & 293 \\
\hline Christian .......... & 063 & Kodiak .... & 141 & Rat Islands ......... & 217 & Unimak ........... & 295 \\
\hline Circle ............ & 065 & Kotzebue......... & 143 & Ruby $\ldots \ldots \ldots \ldots$ & 219 & Utukok River. ....... & 297 \\
\hline Cold Bay ...... & 093 & Kuskokwim Bay. .... & 145 & Russian Mission .... & 221 & Valdez ............ & 299 \\
\hline Coleen............. & 067 & Kwiguk ........... & 147 & Sagavanirktok ..... & 223 & Wainwright ........ & 301 \\
\hline Cordova............ & 069 & Lake Clark ......... & 149 & Samalga Island ..... & 225 & Wiseman ........... & 303 \\
\hline Craig ............. & 071 & Lime Hills. . . . . . . . . & 151 & Seguam........... & 227 & Yakutat .... & 305 \\
\hline De Long Mts ....... & 073 & Livengood $\ldots \ldots \ldots$ & 153 & Selawik... & 229 & & \\
\hline Demarcation Point ... & 075 & Lookout Ridge...... & 155 & Seldovia . & 231 & & \\
\hline
\end{tabular}

Note: The State of Alaska does not have counties that can be used for field identification. Areas identifled by U. S. Geological Survey quadrangles have been assigned codes by the American Petroleum Institute for use as pseudo-counties. These three-digit codes are analogous to the usual "county code".

Source: The API Well Number and Standard State and County Numeric Codes Including Offshore Waters. API Bulletin D12A, January 1979. American Petroleum Institute, Washington, DC. 
Table 4. Coalbed Methane Flelds

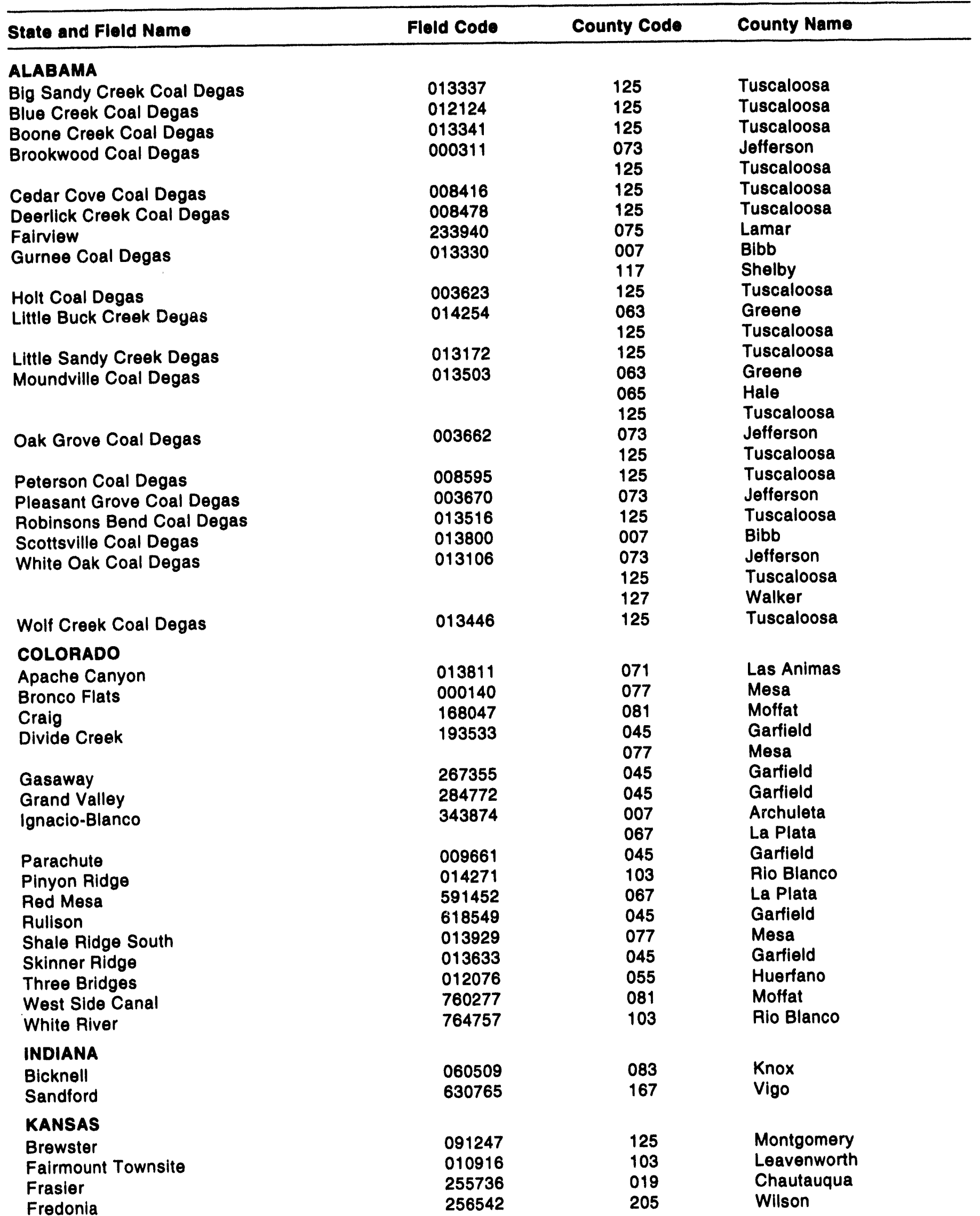


Table 4. Coalbed Methane Fields (continued)

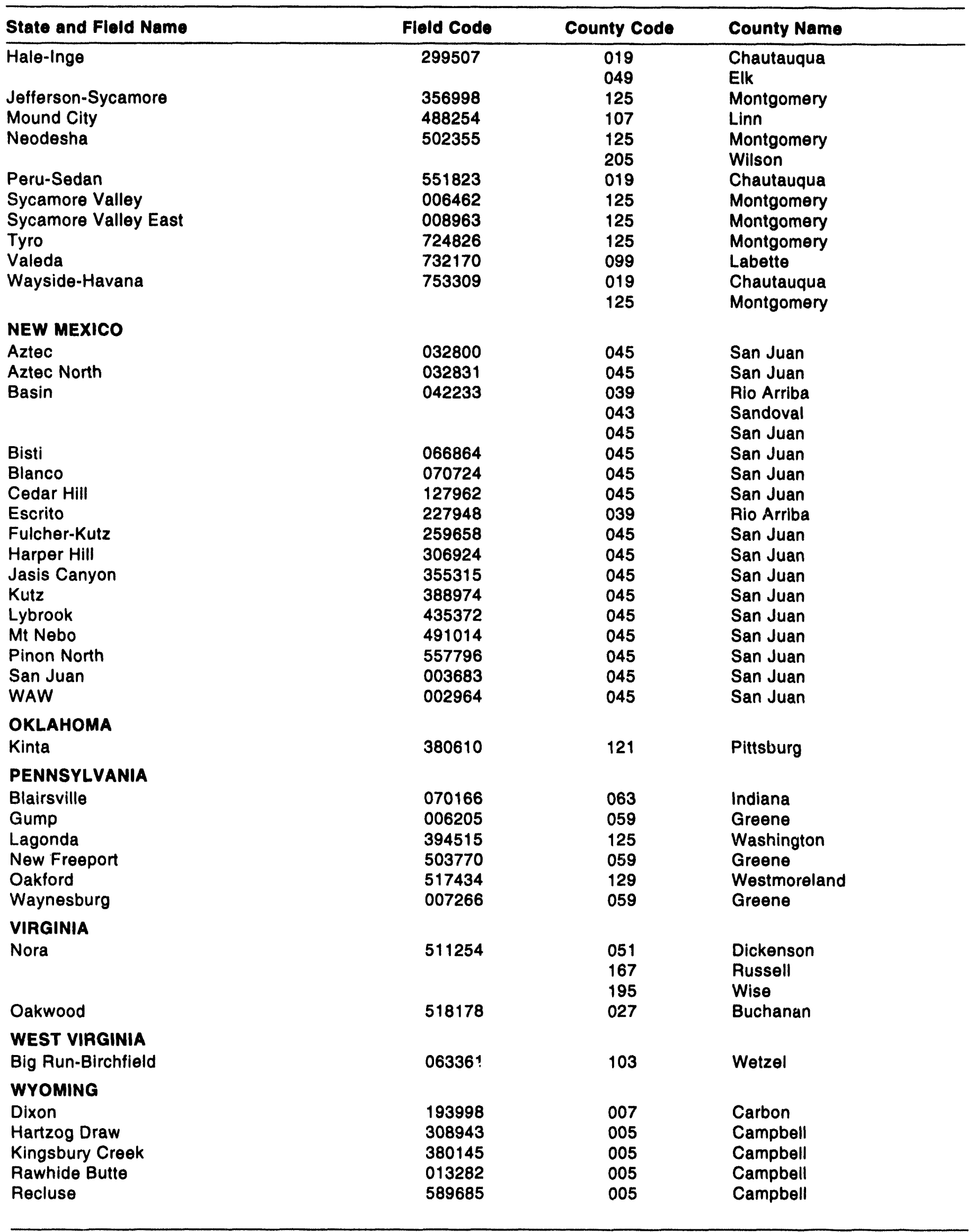

Source: Energy Information Administration, Office of Oil and Gas 
Table 5. Storage Fields*

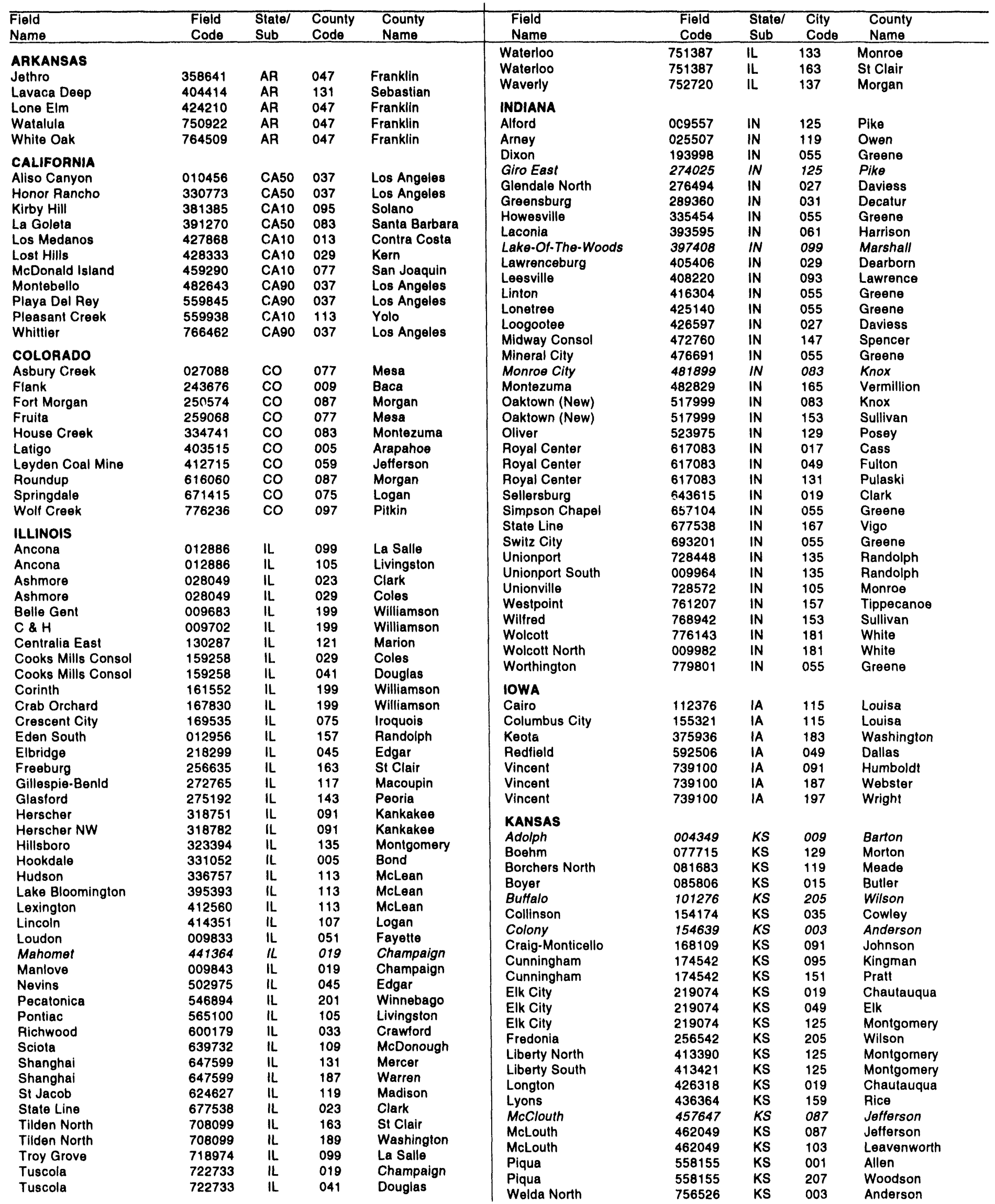

*Alias field names are in italics. 
Table 5. Storage Fields* (Continued)

\begin{tabular}{|c|c|c|c|c|c|c|c|c|c|}
\hline $\begin{array}{l}\text { Fiold } \\
\text { Name } \\
\end{array}$ & $\begin{array}{l}\text { Field } \\
\text { Code } \\
\end{array}$ & $\begin{array}{l}\text { State/ } \\
\text { Sub }\end{array}$ & $\begin{array}{l}\text { County } \\
\text { Code }\end{array}$ & $\begin{array}{l}\text { County } \\
\text { Name }\end{array}$ & $\begin{array}{l}\text { Field } \\
\text { Name } \\
\end{array}$ & $\begin{array}{l}\text { Field } \\
\text { Code } \\
\end{array}$ & $\begin{array}{l}\text { State/ } \\
\text { Sub } \\
\end{array}$ & $\begin{array}{l}\text { City } \\
\text { Code }\end{array}$ & $\begin{array}{l}\text { County } \\
\text { Name }\end{array}$ \\
\hline Welda South & 756557 & KS & 003 & Anderson & Columbus West & 155538 & MI & 147 & St Clair \\
\hline KENTUCKY & & & & & Cranberry Lake & 168233 & MI & 035 & Clare \\
\hline Adair North & 003171 & KY & 091 & Hancock & Cranberry Lake & 168233 & MI & 113 & Missaukee \\
\hline Anton & 021244 & KY & 107 & Hopkins & Croton & 172387 & MI & 123 & Newaygo \\
\hline Ballardsville & 036808 & KY & 185 & Oldham & Excelsior $06-27 \mathrm{~N}-6 \mathrm{~W}$ & 009735 & MI & 079 & Kalkaska \\
\hline Bon Harbor & 079544 & $K Y$ & 059 & Daviess & Four Corners & 252434 & MI & 147 & St Clair \\
\hline Canmer & 009704 & KY & 099 & Hart & Freeman-Lincoln & 256790 & MI & 035 & Clare \\
\hline Cocilia & 004841 & KY & 093 & Hardin & $\begin{array}{l}\text { Goodwell } \\
\text { Hamilton North }\end{array}$ & 280385 & MI & 123 & Newaygo \\
\hline Center & 129264 & KY & 009 & Barren & $\begin{array}{l}\text { Hamilton North } \\
\text { Hessen }\end{array}$ & 301584 & MI & 035 & Clare \\
\hline Center & 129264 & KY & 087 & Green & $\begin{array}{l}\text { Hessen } \\
\text { Howell }\end{array}$ & $\begin{array}{l}319123 \\
335361\end{array}$ & MI & 147 & St Clair \\
\hline Center & 129264 & KY & 169 & Metcalfe & Ira & $\begin{array}{l}335361 \\
347913\end{array}$ & MI & 093 & Livingston \\
\hline Crofton East & 009721 & KY & 047 & Christian & Kalkaska $01-27 \mathrm{~N}-7 \mathrm{~W}$ & $\begin{array}{l}347913 \\
009799\end{array}$ & MI & 147 & St Clair \\
\hline Dixio & 193812 & KY & 101 & Henderson & Lacey Station & $\begin{array}{l}009799 \\
303525\end{array}$ & MI & 079 & Kalkaska \\
\hline Doe Run & 194968 & KY & 163 & Meade & Lee 02-1S-5W & 393525 & MI & 015 & Barry \\
\hline Eagle Creek & 210463 & KY & 077 & Gallatin & Le日 04-1S-5W & 003265 & MI & 025 & Calhoun \\
\hline Eagle Crook & 210463 & KY & 081 & Grant & Lee $11.1 \mathrm{~S}-5 \mathrm{~W}$ & 009822 & MI & 025 & Calhoun \\
\hline Eagle Creok & 210463 & KY & 187 & Owen & Le日 $11 \cdot 15-5 \mathrm{~W}$ & 003273 & MI & 025 & Calhoun \\
\hline Flint Hill & 245385 & $K Y$ & 093 & Hardin & Le日 16 & 009823 & MI & 025 & Calhoun \\
\hline Flint Hill Consol & 012752 & KY & 093 & Hardin & Lenox & 410204 & MI & 099 & Macomb \\
\hline Graham Lake & 283842 & KY & 177 & Muhlenberg & Leonard & 410731 & MI & 125 & Oakland \\
\hline Grandview & 284927 & KY & 059 & Daviess & Marion(Winterfield) & 447874 & MI & 035 & Clare \\
\hline Greenville & 289577 & KY & 177 & Muhlenberg & Marion(Winterfield) & 447874 & MI & 133 & Osceola \\
\hline Hawesville NW & 011146 & KY & 091 & Hancock & Marsac Creek & 449207 & MI & 147 & St Clair \\
\hline Hickory School & 320852 & $K Y$ & 059 & Daviess & Marysville-Morton & 009849 & MI & 147 & St Clair \\
\hline Kettle Island & 005569 & KY & 013 & Bell & Muttonville & 495943 & MI & 099 & Macomb \\
\hline Kirkwood Springs & 381788 & KY & 107 & Hopkins & Northville & 513052 & MI & 125 & Oakland \\
\hline Magnolia & 440775 & KY & 087 & Green & Northville & 513052 & MI & 161 & Washtenaw \\
\hline Magnolia & 440775 & KY & 099 & Hart & Northville & 513052 & MI & 163 & Wayne \\
\hline Magnolia & 440775 & KY & 123 & LaRue & Orient & 527889 & MI & 035 & Clare \\
\hline Midland & 472280 & KY & 177 & Muhlenberg & Orient & 527889 & MI & 133 & Osceola \\
\hline Mt Olive Church & 009874 & KY & 227 & Warren & Overisel & 531485 & MI & 005 & Allegan \\
\hline Muldraugh & 493184 & KY & 163 & Meade & Partello & 541445 & MI & 025 & Calhoun \\
\hline Owensboro & 532074 & KY & 059 & Daviess & Puttygut & 575943 & MI & 147 & St Clair \\
\hline Owensboro West & 532167 & KY & 059 & Daviess & Rapid River $35-28 \mathrm{~N}-7 \mathrm{~W}$ & 003354 & MI & 079 & Kalkaska \\
\hline St Charles & 673074 & KY & 107 & Hopkins & $\begin{array}{l}\text { Ray } \\
\text { Reed City }\end{array}$ & $\begin{array}{l}588018 \\
593157\end{array}$ & $\begin{array}{l}\text { MI } \\
\text { MI }\end{array}$ & $\begin{array}{l}095 \\
085\end{array}$ & Macomb \\
\hline LOUISIANA & & & & & Reed City & 593157 & MI & 133 & Osceola \\
\hline Bayou Choctaw & 045581 & LA10 & 047 & Iberville & Riverside & 603992 & MI & 035 & Clare \\
\hline Bayou Choctaw & 045581 & LA10 & 121 & West Baton & Riverside & 603992 & MI & 113 & Missaukee \\
\hline & & & & Rouge & Rolland & $: 11379$ & MI & 073 & Isabella \\
\hline Bear Creok & 048643 & LA50 & 013 & Bienville & Salem & -25030 & MI & 005 & Allegan \\
\hline Epps & 226762 & LA50 & 035 & Eust Carroll & Salem & 625030 & MI & 139 & Ottawa \\
\hline Epps & 226762 & LA50 & 123 & West Carroll & Shaver & & & & \\
\hline Garden Island Bay & 265340 & LA10 & 075 & Plaquemines & (Sumner-New Haven) & 648622 & MI & 057 & Gratiot \\
\hline Hackberry West & 298329 & LA10 & 023 & Cameron & Shaver & & & & \\
\hline Hester & 319154 & LA10 & 093 & St James & (Sumner-New Haven) & 648622 & MI & 117 & Montcaim \\
\hline Holly & 328510 & LA50 & 031 & De Soto & Six Lakes & 658449 & MI & 073 & Isabella \\
\hline Lake Bistineau & 395331 & LA50 & 013 & Bienville & Six Lakes & 658449 & MI & 107 & Mecosta \\
\hline Lake Bistineau & 395331 & LA50 & 015 & Bossier & Six Lakes & 658449 & MI & 117 & Montcalm \\
\hline Lake Bistineau & 395331 & LA50 & 119 & Webster & Swan Creek & 691651 & MI & 147 & St Clair \\
\hline Ruston & 620502 & LA50 & 061 & Lincoln & W C Taggart(Six Lakes) & 743079 & $M I$ & 107 & Mecosta \\
\hline Sulphur Mines & 687737 & LA10 & 019 & Calcasieu & W C Taggart(Six Lakes) & 743079 & $M I$ & 117 & Montcalm \\
\hline Unionville & 728572 & LA50 & 061 & Lincoln & Winfield & 773189 & $M I$ & 107 & Mecosta \\
\hline Unionville & 728572 & LA50 & 111 & Union & Winfield & 773189 & MI & 117 & Montcalm \\
\hline Washington & 750240 & LA10 & 097 & St Landry & Woodville & 778406 & MI & 123 & Newaygo \\
\hline Weeks Island & 754914 & LA10 & 045 & Iberia & MINNESOTA & & & & \\
\hline Weeks Island & 754914 & LA10 & 101 & St Mary & Waterville-Waseca & 751666 & $M N$ & 079 & Le Sueur \\
\hline MARYLAND & & & & & Waterville-Waseca & 751666 & MN & 131 & Rice \\
\hline Accident & 002210 & MD & 023 & Garrett & Waterville-Waseca & 751666 & MN & 161 & Waseca \\
\hline MICHIGAN & & & & & MISSISSIPPI & & & & \\
\hline Austin & 030901 & MI & 107 & Mecosta & Amory & 016408 & MS & 095 & Monroe \\
\hline Belle River Mills & 053820 & MI & 147 & St Clair & Eminence & 223786 & MS & 031 & Covington \\
\hline Broomfield & 094843 & MI & 073 & Isabella & Jackson & 353006 & MS & 049 & Hinds \\
\hline Broomfield Deerfield & 074351 & MI & 073 & Isabella & Jackson & 353006 & MS & 121 & Rankin \\
\hline Capac & 118576 & MI & 147 & St Clair & Muldon & 492967 & MS & 095 & Monroe \\
\hline Charlton $01-30 \mathrm{~N}-1 \mathrm{~W}$ & 002860 & MI & 137 & Otsego & Petol Dome & 014074 & MS & 035 & Forrest \\
\hline Chester Sec 15 & 003494 & MI & 137 & Otsego & MISSOURI & & & & \\
\hline Clarence 19-1S-4W & 009710 & MI & 025 & Calhoun & Florissant & 002175 & MO & 183 & St Charles \\
\hline Cold Springs $12-28 \mathrm{~N}-6 \mathrm{~W}$ & 002922 & MI & 079 & Kalkaska & Florissant & 002175 & MO & 189 & St Louis \\
\hline $\begin{array}{l}\text { Cold Springs } 31-28 N-6 W \\
\text { Coldwater }\end{array}$ & $\begin{array}{l}009713 \\
152252\end{array}$ & $\begin{array}{l}\text { MI } \\
\text { MI }\end{array}$ & $\begin{array}{l}079 \\
073\end{array}$ & $\begin{array}{l}\text { Kalkaska } \\
\text { Isabella }\end{array}$ & MONTANA & & & & \\
\hline $\begin{array}{l}\text { Coldwater } \\
\text { Collin }\end{array}$ & $\begin{array}{l}152252 \\
153988\end{array}$ & $\mathrm{MI}$ & 147 & St Clair & Box Elder & 084938 & MT & 005 & Blaine \\
\hline Columbus & 155290 & MI & 147 & St Clair & Box Elder & 084938 & MT & 041 & Hill \\
\hline
\end{tabular}

*Alias field names are in italics. 
Table 5. Storage Flelds" (Continued)

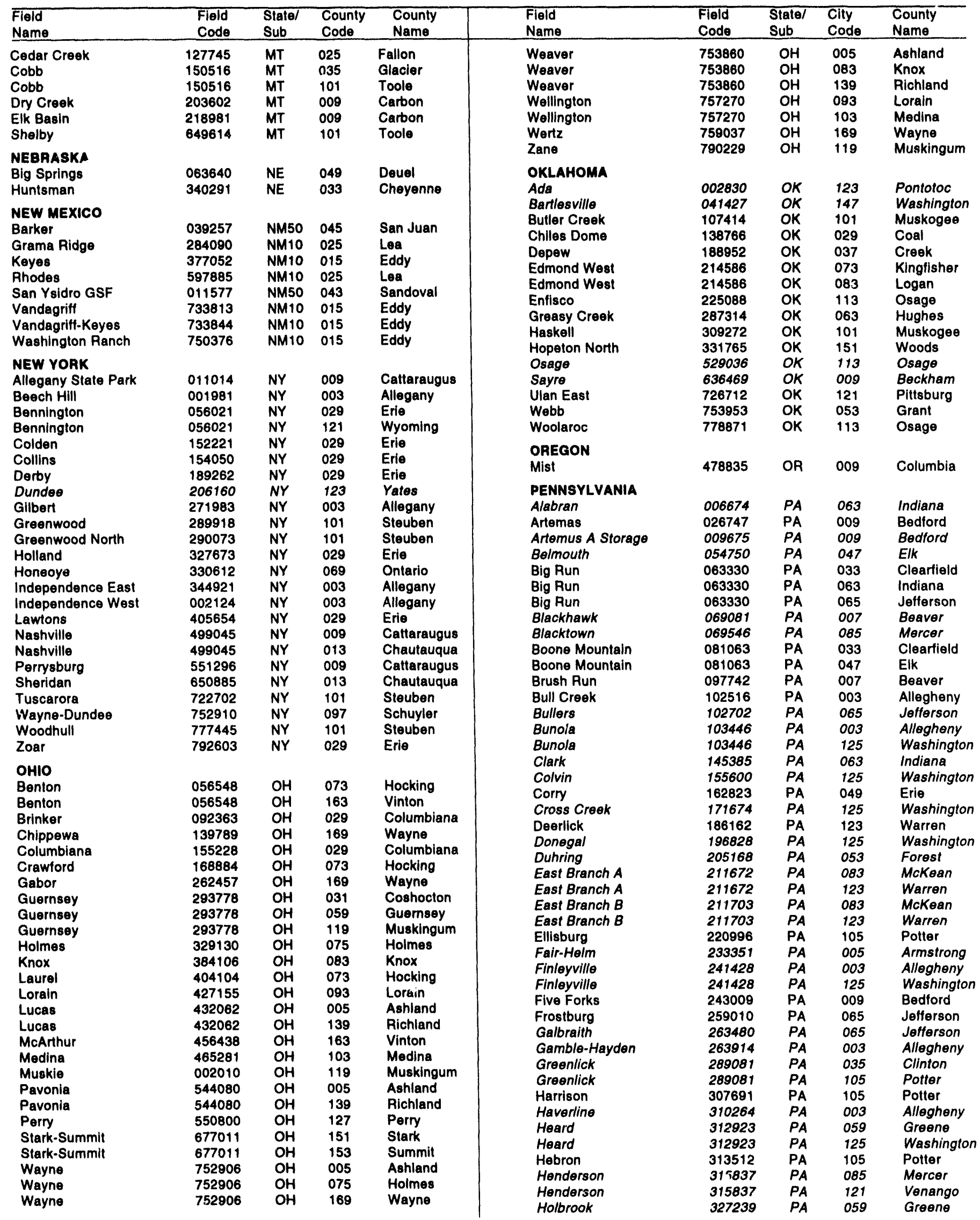

*Alias field names are in italics. 
Table 5. Storage Fields* (Continued)

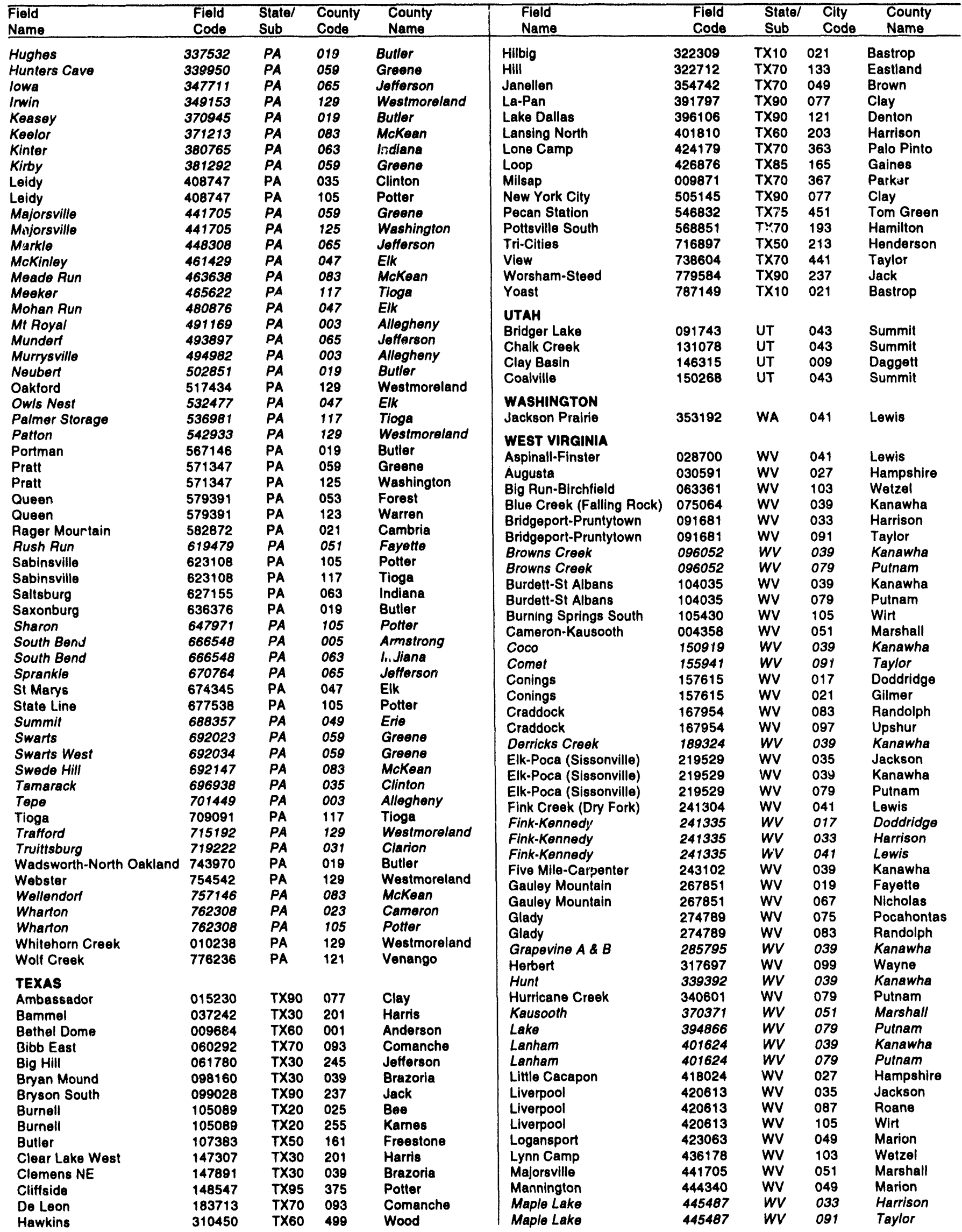

*Alias field names are in italics. 
Table 5. Storage Fields* (Continued)

\begin{tabular}{|c|c|c|c|c|}
\hline $\begin{array}{l}\text { Field } \\
\text { Name } \\
\end{array}$ & $\begin{array}{l}\text { Field } \\
\text { Code } \\
\end{array}$ & $\begin{array}{l}\text { State/ } \\
\text { Sub }\end{array}$ & $\begin{array}{l}\text { County } \\
\text { Code }\end{array}$ & $\begin{array}{l}\text { County } \\
\text { Name }\end{array}$ \\
\hline $\begin{array}{l}\text { Murphy Creek } \\
\text { (Freemansburg) } \\
\text { Murphy Creek } \\
\text { (Freemansburg) } \\
\text { Nowberne } \\
\text { Rockport } \\
\text { Rockport } \\
\text { Rockport } \\
\text { Silver HIII } \\
\text { Sinking Creek } \\
\text { Slab Fork } \\
\text { Terra Alta } \\
\text { Victory } \\
\text { Victory } \\
\text { Weston-Jane Lew } \\
\text { Weston-Jane Lew } \\
\text { Wilbur } \\
\text { Wilbur } \\
\text { X-1 }\end{array}$ & $\begin{array}{l}494610 \\
\\
494610 \\
505393 \\
608868 \\
608868 \\
608868 \\
656279 \\
657612 \\
659945 \\
701821 \\
738077 \\
738077 \\
760959 \\
760959 \\
767795 \\
767795 \\
782253\end{array}$ & $\begin{array}{l}W V \\
W V \\
W V \\
W V \\
W V \\
W V \\
W V \\
W V \\
W V \\
W V \\
W V \\
W V \\
W V \\
W V \\
W V \\
W V \\
W V \\
W V\end{array}$ & $\begin{array}{l}017 \\
041 \\
021 \\
035 \\
105 \\
107 \\
103 \\
021 \\
081 \\
077 \\
051 \\
103 \\
033 \\
041 \\
017 \\
095 \\
079\end{array}$ & $\begin{array}{l}\text { Doddridge } \\
\text { Lewls } \\
\text { Glimer } \\
\text { Jackson } \\
\text { Wirt } \\
\text { Wood } \\
\text { Wetzel } \\
\text { Gilmer } \\
\text { Raleigh } \\
\text { Preston } \\
\text { Marshall } \\
\text { Wetzel } \\
\text { Harrison } \\
\text { Lewis } \\
\text { Doddridge } \\
\text { Tyler } \\
\text { Putnam }\end{array}$ \\
\hline $\begin{array}{l}\text { WYOMING } \\
\text { Billy Creek } \\
\text { Brady } \\
\text { Bunker Hill } \\
\text { Chimney But'e } \\
\text { Chimney Butte } \\
\text { Elk Basin } \\
\text { Kirk } \\
\text { Oil Springs }\end{array}$ & $\begin{array}{l}064880 \\
087651 \\
103353 \\
139262 \\
139262 \\
218981 \\
381509 \\
520968\end{array}$ & $\begin{array}{l}\text { WY } \\
\text { WY } \\
\text { WY } \\
\text { WY } \\
\text { WY } \\
\text { WY } \\
\text { WY } \\
\text { WY }\end{array}$ & $\begin{array}{l}019 \\
037 \\
007 \\
023 \\
035 \\
029 \\
013 \\
007\end{array}$ & $\begin{array}{l}\text { Johnson } \\
\text { Sweetwater } \\
\text { Carbon } \\
\text { Lincoln } \\
\text { Sublette } \\
\text { Park } \\
\text { Fremont } \\
\text { Carbon }\end{array}$ \\
\hline
\end{tabular}

*Alias field names are in italics. 
Table 6. Fields Located in Multiple Jurisdictions

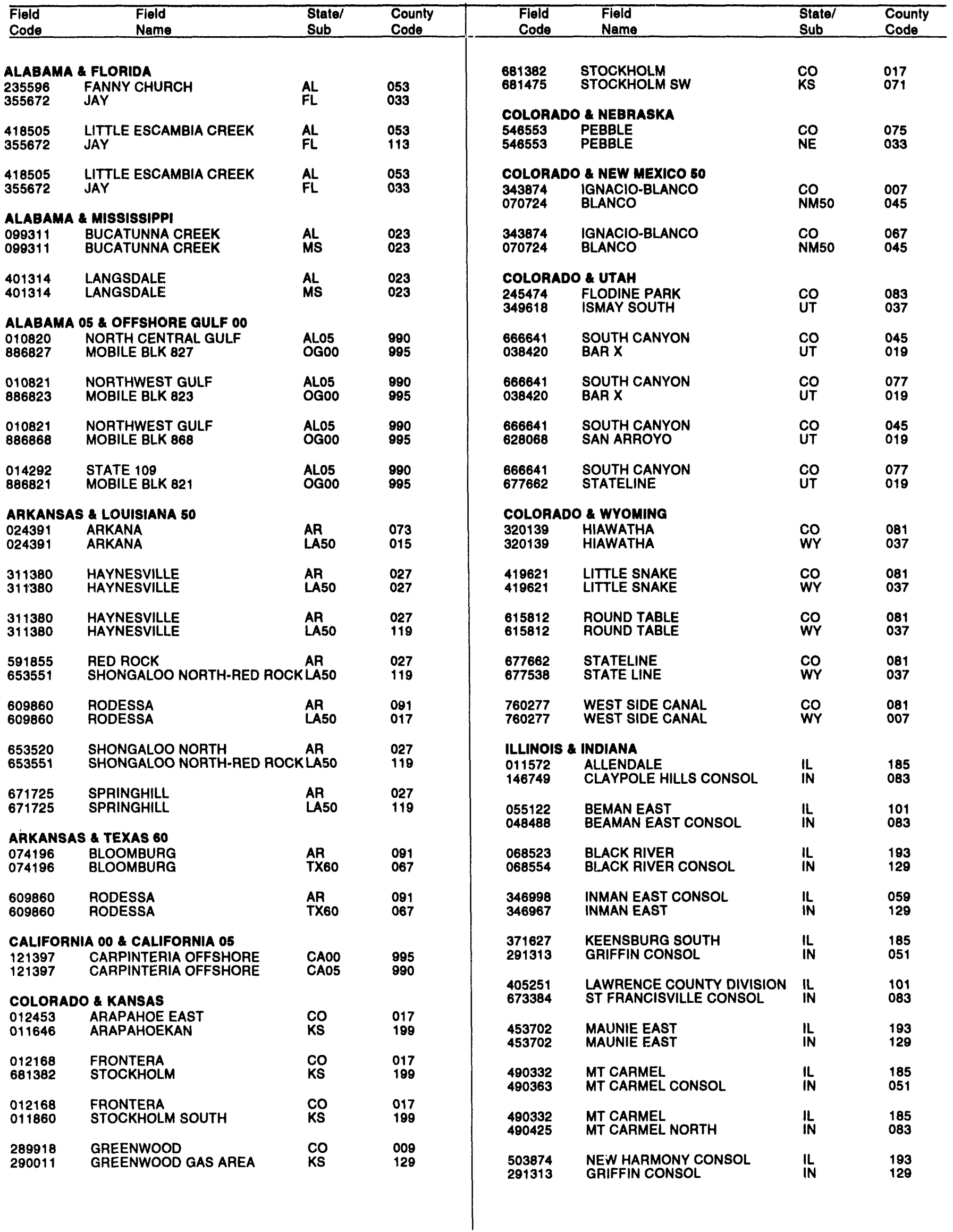


Table 6. Fields Located In Multiple Jurisdictions (Continued)

\begin{tabular}{|c|c|c|c|c|c|c|c|}
\hline $\begin{array}{l}\text { Figld } \\
\text { Code }\end{array}$ & $\begin{array}{l}\text { Flold } \\
\text { Name }\end{array}$ & $\begin{array}{l}\text { Statel } \\
\text { Sub }\end{array}$ & $\begin{array}{l}\text { County } \\
\text { Code } \\
\end{array}$ & $\begin{array}{l}\text { Field } \\
\text { Code } \\
\end{array}$ & $\begin{array}{l}\text { Field } \\
\text { Name } \\
\end{array}$ & $\begin{array}{l}\text { Statel } \\
\text { Sub }\end{array}$ & $\begin{array}{l}\text { County } \\
\text { Code }\end{array}$ \\
\hline $\begin{array}{l}503874 \\
291313\end{array}$ & $\begin{array}{l}\text { NEW HARMONY CONSOL } \\
\text { GRIFFIN CONSOL }\end{array}$ & IL & $\begin{array}{l}193 \\
051\end{array}$ & $\begin{array}{l}681041 \\
747512\end{array}$ & $\begin{array}{l}\text { STILLWELL } \\
\text { WALTON }\end{array}$ & $\begin{array}{l}\text { KS } \\
\text { MO }\end{array}$ & $\begin{array}{l}091 \\
037\end{array}$ \\
\hline $\begin{array}{l}503967 \\
756200\end{array}$ & $\begin{array}{l}\text { NEW HARMONY SOUTH } \\
\text { WELBORN NORTH CONSOL }\end{array}$ & IL & $\begin{array}{l}193 \\
129\end{array}$ & $\begin{array}{l}\text { KANSAS } \\
071468 \\
657643\end{array}$ & $\begin{array}{l}\text { NEBRASKA } \\
\text { BLEES } \\
\text { SINK }\end{array}$ & $\begin{array}{l}\mathrm{KS} \\
\mathrm{NE}\end{array}$ & $\begin{array}{l}039 \\
145\end{array}$ \\
\hline $\begin{array}{l}504029 \\
335051\end{array}$ & $\begin{array}{l}\text { NEW HAVEN CONSOL } \\
\text { HOVEY WEST }\end{array}$ & IL & $\begin{array}{l}193 \\
129\end{array}$ & $\begin{array}{l}332467 \\
332467\end{array}$ & $\begin{array}{l}\text { HORINEK } \\
\text { HORINEK }\end{array}$ & $\begin{array}{l}\text { KS } \\
\text { NE }\end{array}$ & $\begin{array}{l}153 \\
087\end{array}$ \\
\hline $\begin{array}{l}504029 \\
756185\end{array}$ & $\begin{array}{l}\text { NEW HAVEN CONSOL } \\
\text { WELBORN CONSOL } \\
\text { OAK GROVE }\end{array}$ & IN & $\begin{array}{l}193 \\
129 \\
059\end{array}$ & $\begin{array}{l}337377 \\
012564\end{array}$ & $\begin{array}{l}\text { HUFFSTUTTER } \\
\text { ALMA SOUTH }\end{array}$ & $\begin{array}{l}\text { KS } \\
\text { NE }\end{array}$ & $\begin{array}{l}147 \\
083\end{array}$ \\
\hline $\begin{array}{l}516721 \\
516721\end{array}$ & $\begin{array}{l}\text { OAK GROVE } \\
\text { OAK GROVE }\end{array}$ & IL & $\begin{array}{l}059 \\
129\end{array}$ & $\begin{array}{l}001551 \\
772166\end{array}$ & $\begin{array}{l}\text { LAWSON NE } \\
\text { WILSONVILLE SW }\end{array}$ & $\begin{array}{l}\mathrm{KS} \\
\mathrm{NE}\end{array}$ & $\begin{array}{l}039 \\
065\end{array}$ \\
\hline $\begin{array}{l}607411 \\
607411\end{array}$ & $\begin{array}{l}\text { ROCHESTER } \\
\text { ROCHESTER }\end{array}$ & IN & $\begin{array}{l}185 \\
051\end{array}$ & KANSAS & OKLAHOMA & & \\
\hline $\begin{array}{l}673415 \\
673384\end{array}$ & $\begin{array}{l}\text { ST FRANCISVILLE EAST } \\
\text { ST FRANCISVILLE CONSOL }\end{array}$ & IL & $\begin{array}{l}101 \\
083\end{array}$ & $\begin{array}{l}112872 \\
112872\end{array}$ & $\begin{array}{l}\text { CALDWELL } \\
\text { CALDWELL }\end{array}$ & $\begin{array}{l}\text { KS } \\
\text { OK }\end{array}$ & $\begin{array}{l}191 \\
053\end{array}$ \\
\hline $\begin{array}{l}677538 \\
677538\end{array}$ & $\begin{array}{l}\text { STATE LINE } \\
\text { STATE LINE }\end{array}$ & IL & $\begin{array}{l}023 \\
167\end{array}$ & $\begin{array}{l}116964 \\
116623\end{array}$ & $\begin{array}{l}\text { CANEY } \\
\text { CANARY }\end{array}$ & $\begin{array}{l}\text { KS } \\
\text { OK }\end{array}$ & $\begin{array}{l}125 \\
147\end{array}$ \\
\hline ILLINOIS & KENTUCKY & & & $\begin{array}{l}145447 \\
145447\end{array}$ & $\begin{array}{l}\text { CLARK CREEK } \\
\text { CLARK CREEK }\end{array}$ & $\begin{array}{l}\text { KS } \\
\text { OK }\end{array}$ & $\begin{array}{l}025 \\
059\end{array}$ \\
\hline $\begin{array}{l}649056 \\
649056 \\
\text { INDIANA }\end{array}$ & $\begin{array}{l}\text { SHAWNEETOWN EAST } \\
\text { SHAWNEETOWN EAST } \\
\text { KENTUCKY }\end{array}$ & $\mathbb{K}_{\mathrm{KY}}^{\mathrm{L}}$ & $\begin{array}{l}059 \\
225\end{array}$ & $\begin{array}{l}218702 \\
218733\end{array}$ & $\begin{array}{l}\text { ELGIN } \\
\text { ELGIN SOUTH }\end{array}$ & $\begin{array}{l}\text { KS } \\
\text { OK }\end{array}$ & $\begin{array}{l}019 \\
113\end{array}$ \\
\hline $\begin{array}{l}039257 \\
039257\end{array}$ & $\begin{array}{l}\text { BARKER } \\
\text { BARKER }\end{array}$ & $\underset{K Y}{I N}$ & $\begin{array}{l}163 \\
101\end{array}$ & $\begin{array}{l}219663 \\
122606\end{array}$ & $\begin{array}{l}\text { ELKHART } \\
\text { CARTHAGE DISTRICT NE }\end{array}$ & $\begin{array}{l}\text { KS } \\
\text { OK }\end{array}$ & $\begin{array}{l}129 \\
139\end{array}$ \\
\hline $\begin{array}{l}393595 \\
194968\end{array}$ & $\begin{array}{l}\text { LACONIA } \\
\text { DOE RUN }\end{array}$ & $\underset{K Y}{\mathbb{N}}$ & $\begin{array}{l}061 \\
163\end{array}$ & $\begin{array}{l}241118 \\
480070\end{array}$ & $\begin{array}{l}\text { FINCHAM } \\
\text { MOCANE-LAVERNE GAS AREA }\end{array}$ & $\begin{array}{l}\text { KS } \\
\text { OK }\end{array}$ & $\begin{array}{l}119 \\
007\end{array}$ \\
\hline $\begin{array}{l}569502 \\
569471\end{array}$ & $\begin{array}{l}\text { POWELLS LAKE CONSOL } \\
\text { POWELLS LAKE }\end{array}$ & $\underset{K Y}{\mathbb{N}}$ & $\begin{array}{l}129 \\
225\end{array}$ & $\begin{array}{l}290011 \\
122668\end{array}$ & $\begin{array}{l}\text { GREENWOOD GAS AREA } \\
\text { CARTHAGE GAS AREA }\end{array}$ & $\begin{array}{l}\text { KS } \\
\text { OK }\end{array}$ & $\begin{array}{l}129 \\
139\end{array}$ \\
\hline $\begin{array}{l}662394 \\
662332\end{array}$ & $\begin{array}{l}\text { SMITH MILLS NORTH } \\
\text { SMITH MILLS CONSOL }\end{array}$ & $\underset{K Y}{\mathbb{N}}$ & $\begin{array}{l}129 \\
101\end{array}$ & $\begin{array}{l}337780 \\
295545\end{array}$ & $\begin{array}{l}\text { HUGOTON GAS AREA } \\
\text { GUYMON-HUGOTON GAS AREA }\end{array}$ & $\begin{array}{l}\text { KS } \\
\text { OK }\end{array}$ & $\begin{array}{l}175 \\
139\end{array}$ \\
\hline $\begin{array}{l}\text { INDIANA } \\
219694 \\
215485\end{array}$ & $\begin{array}{l}\text { MICHIGAN } \\
\text { ELKHART NORTH } \\
\text { EDWARDSBURG }\end{array}$ & $\underset{\mathrm{MI}}{\mathbf{N}}$ & $\begin{array}{l}039 \\
027\end{array}$ & $\begin{array}{l}337780 \\
295545\end{array}$ & $\begin{array}{l}\text { HUGOTON GAS AREA } \\
\text { GUYMON-HUGOTON GAS AREA }\end{array}$ & $\begin{array}{l}\text { KS } \\
\text { OK }\end{array}$ & $\begin{array}{l}189 \\
139\end{array}$ \\
\hline INDIANA & OHIO & & & $\begin{array}{l}413049 \\
480070\end{array}$ & $\begin{array}{l}\text { LIBERAL-LIGHT } \\
\text { MOCANE-LAVERNE GAS AREA }\end{array}$ & $\begin{array}{l}\text { KS } \\
\text { OK }\end{array}$ & $\begin{array}{l}175 \\
007\end{array}$ \\
\hline $\begin{array}{l}716680 \\
005094\end{array}$ & $\begin{array}{l}\text { TRENTON } \\
\text { CHATTANOOGA }\end{array}$ & $\underset{\text { OH }}{\text { ON }}$ & $\begin{array}{l}001 \\
107\end{array}$ & $\begin{array}{l}461150 \\
139014\end{array}$ & $\begin{array}{l}\text { MCKAY } \\
\text { CHILOCCO EAST }\end{array}$ & $\begin{array}{l}\text { KS } \\
\text { OK }\end{array}$ & $\begin{array}{l}035 \\
071\end{array}$ \\
\hline $\begin{array}{l}716680 \\
005094\end{array}$ & $\begin{array}{l}\text { TRENTON } \\
\text { CHATTANOOGA }\end{array}$ & $\stackrel{\mathbb{N}}{\mathrm{OH}}$ & $\begin{array}{l}075 \\
107\end{array}$ & $\begin{array}{l}461491 \\
480070\end{array}$ & $\begin{array}{l}\text { MCKINNEY } \\
\text { MOCANE-LAVERNE GAS AREA }\end{array}$ & $\begin{array}{l}\text { KS } \\
\text { OK }\end{array}$ & $\begin{array}{l}025 \\
007\end{array}$ \\
\hline $\begin{array}{l}716680 \\
005777\end{array}$ & $\begin{array}{l}\text { TRENTON } \\
\text { WILLSSHIRE }\end{array}$ & $\stackrel{\mathbb{N}}{\mathrm{OH}}$ & $\begin{array}{l}001 \\
161\end{array}$ & $\begin{array}{l}461491 \\
480070\end{array}$ & $\begin{array}{l}\text { MCKINNEY } \\
\text { MOCANE-LAVERNE GAS AREA }\end{array}$ & $\begin{array}{l}\text { KS } \\
\text { OK }\end{array}$ & $\begin{array}{l}119 \\
007\end{array}$ \\
\hline $\begin{array}{l}\text { KANSAS } \\
178946 \\
411537\end{array}$ & $\begin{array}{l}\text { MISSOURI } \\
\text { DALLAS } \\
\text { LESTER }\end{array}$ & $\begin{array}{l}\text { KS } \\
\text { MO }\end{array}$ & $\begin{array}{l}081 \\
085\end{array}$ & $\begin{array}{l}494579 \\
192479\end{array}$ & $\begin{array}{l}\text { MURPHY } \\
\text { DILWORTH DISTRICT }\end{array}$ & $\begin{array}{l}\text { KS } \\
\text { OK }\end{array}$ & $\begin{array}{l}191 \\
071\end{array}$ \\
\hline $\begin{array}{l}265805 \\
002598\end{array}$ & $\begin{array}{l}\text { GARLAND } \\
\text { SWARTZ-GARLAND }\end{array}$ & $\begin{array}{l}\text { KS } \\
\text { MO }\end{array}$ & $\begin{array}{l}011 \\
217\end{array}$ & $\begin{array}{l}551823 \\
196518\end{array}$ & $\begin{array}{l}\text { PERU-SEDAN } \\
\text { DOMES-POND CREEK }\end{array}$ & $\begin{array}{l}\text { KS } \\
\text { OK }\end{array}$ & $\begin{array}{l}019 \\
113\end{array}$ \\
\hline $\begin{array}{l}429108 \\
002651\end{array}$ & $\begin{array}{l}\text { LOUISBURG } \\
\text { FREEMAN-WESTLINE }\end{array}$ & $\begin{array}{l}\text { KS } \\
\text { MO }\end{array}$ & $\begin{array}{l}121 \\
037\end{array}$ & $\begin{array}{l}551823 \\
721307\end{array}$ & $\begin{array}{l}\text { PERU-SEDAN } \\
\text { TURKEY CREEK }\end{array}$ & $\begin{array}{l}\text { KS } \\
\text { OK }\end{array}$ & $\begin{array}{l}019 \\
113\end{array}$ \\
\hline $\begin{array}{l}429108 \\
002602\end{array}$ & $\begin{array}{l}\text { LOUISBUAG } \\
\text { LIMPUS }\end{array}$ & $\begin{array}{l}\text { KS } \\
\text { MO }\end{array}$ & $\begin{array}{l}121 \\
037\end{array}$ & $\begin{array}{l}696659 \\
244482\end{array}$ & $\begin{array}{l}\text { TALOGA } \\
\text { FLATS NW }\end{array}$ & $\begin{array}{l}\text { KS } \\
\text { OK }\end{array}$ & $\begin{array}{l}129 \\
139\end{array}$ \\
\hline $\begin{array}{l}429108 \\
002603\end{array}$ & $\begin{array}{l}\text { LOUISBURG } \\
\text { LISLE }\end{array}$ & $\begin{array}{l}\text { KS } \\
\text { MO }\end{array}$ & $\begin{array}{l}121 \\
037\end{array}$ & $\begin{array}{l}723065 \\
429790\end{array}$ & $\begin{array}{l}\text { TUTTLE SE } \\
\text { LOVEDALE NW }\end{array}$ & $\begin{array}{l}\text { KS } \\
\text { OK }\end{array}$ & $\begin{array}{l}033 \\
059\end{array}$ \\
\hline
\end{tabular}


Table 6. Fields Located In Multiple Jurisdictions (Continued)

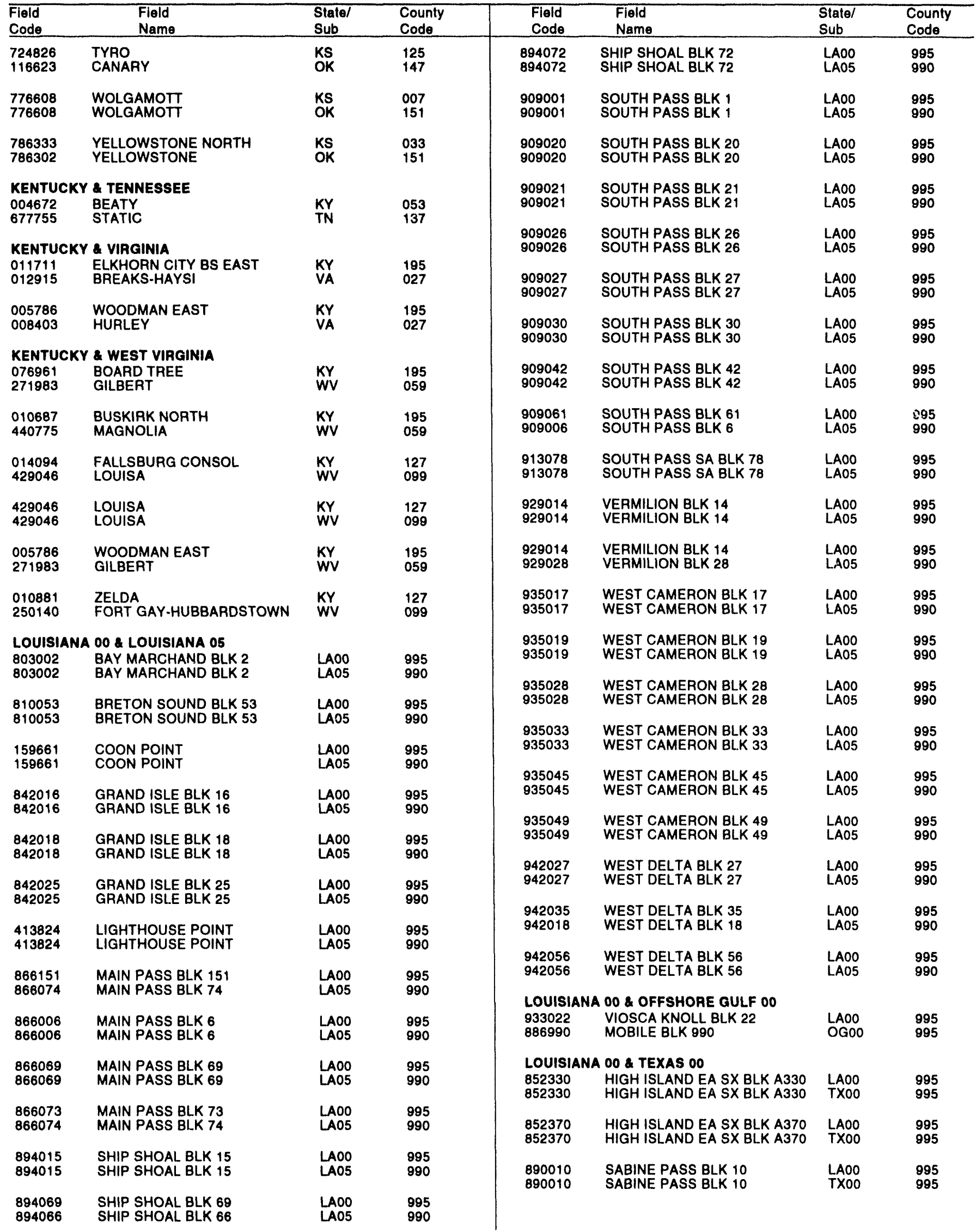


Table 6. Fields Located in Multiple Jurisdictions (Continued)

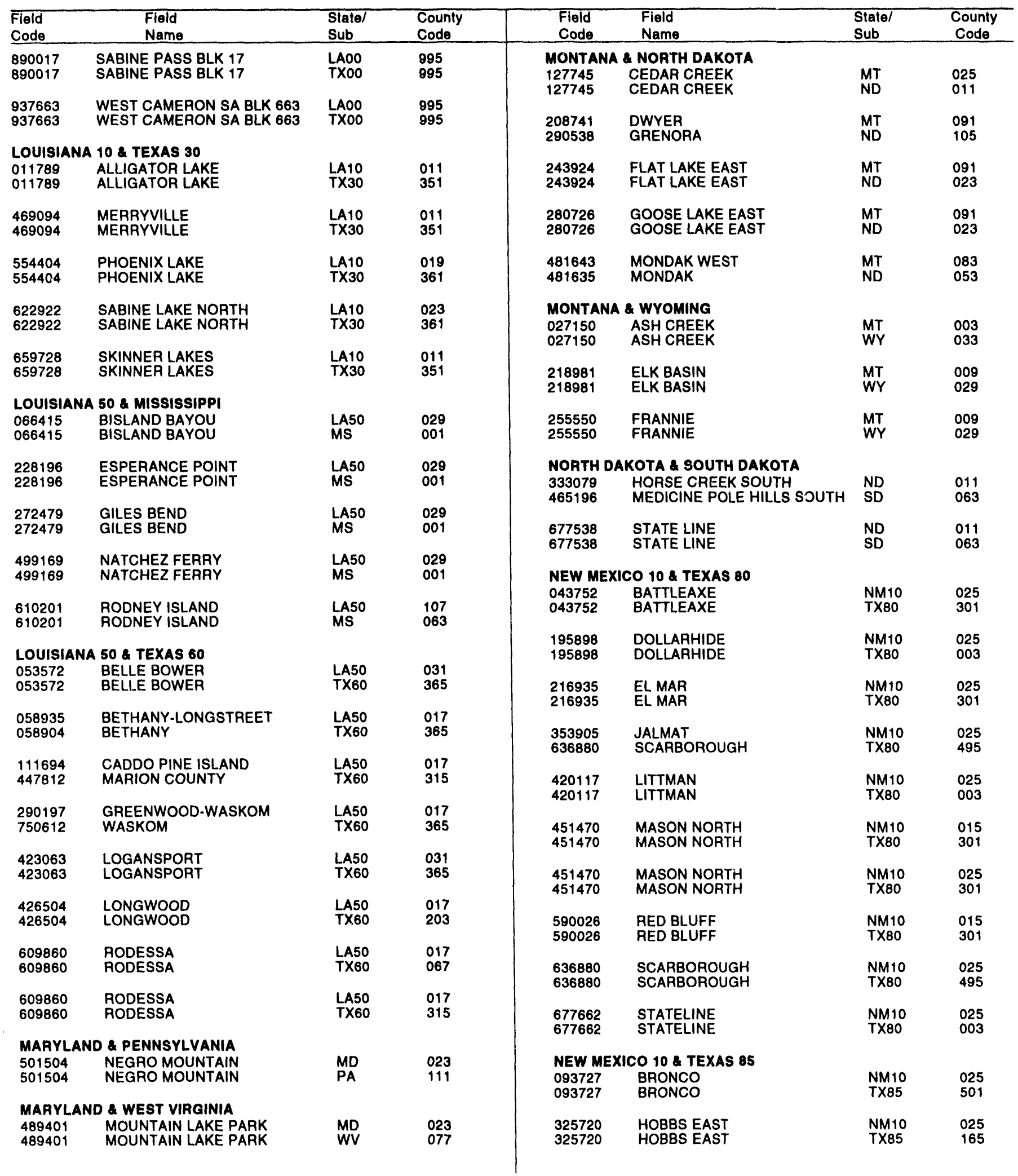


Table 6. Fields Located in Multiple Jurisdictions (Continued)

\begin{tabular}{|c|c|c|c|c|c|c|c|}
\hline $\begin{array}{l}\text { Field } \\
\text { Code } \\
\end{array}$ & $\begin{array}{l}\text { Fleld } \\
\text { Name }\end{array}$ & $\begin{array}{l}\text { Statel } \\
\text { Sub } \\
\end{array}$ & $\begin{array}{l}\text { County } \\
\text { Code } \\
\end{array}$ & $\begin{array}{l}\text { Fiold } \\
\text { Code } \\
\end{array}$ & $\begin{array}{l}\text { Fleld } \\
\text { Name } \\
\end{array}$ & $\begin{array}{l}\text { State/ } \\
\text { Sub }\end{array}$ & $\begin{array}{l}\text { County } \\
\text { Code }\end{array}$ \\
\hline $\begin{array}{l}420117 \\
420117\end{array}$ & $\begin{array}{l}\text { LITTMAN } \\
\text { LITTMAN }\end{array}$ & $\begin{array}{l}\text { NM10 } \\
\text { TX85 }\end{array}$ & $\begin{array}{l}025 \\
165\end{array}$ & $\begin{array}{l}407104 \\
502510\end{array}$ & $\begin{array}{l}\text { LEBANON } \\
\text { NEPTUNE }\end{array}$ & $\stackrel{\mathrm{OH}}{W V}$ & $\begin{array}{l}105 \\
035\end{array}$ \\
\hline $\begin{array}{l}086938 \\
086938\end{array}$ & $\begin{array}{l}\text { \& PENNSYLVANIA } \\
\text { BRADFORD } \\
\text { BRADFORD }\end{array}$ & $\begin{array}{l}\text { NY } \\
\text { PA }\end{array}$ & $\begin{array}{l}009 \\
083\end{array}$ & $\begin{array}{l}420644 \\
136565\end{array}$ & $\begin{array}{l}\text { LIVERPOOL EAST } \\
\text { CHESTER-PUGHTOWN }\end{array}$ & $\underset{W V}{\mathrm{OH}}$ & $\begin{array}{l}029 \\
029\end{array}$ \\
\hline $\begin{array}{l}094130 \\
094130\end{array}$ & $\begin{array}{l}\text { BROOKFIELD } \\
\text { BROOKFIELD }\end{array}$ & $\begin{array}{l}\text { NY } \\
\text { PA }\end{array}$ & $\begin{array}{l}101 \\
117\end{array}$ & $\begin{array}{l}430875 \\
077064\end{array}$ & $\begin{array}{l}\text { LOWER NEWPORT } \\
\text { BOAZ }\end{array}$ & $\underset{W V}{\mathrm{OH}}$ & $\begin{array}{l}167 \\
107\end{array}$ \\
\hline $\begin{array}{l}107176 \\
686683\end{array}$ & $\begin{array}{l}\text { BUSTI } \\
\text { SUGAR GROVE }\end{array}$ & $\begin{array}{l}\text { NY } \\
\text { PA }\end{array}$ & $\begin{array}{l}013 \\
123\end{array}$ & $\begin{array}{l}005882 \\
077064\end{array}$ & $\begin{array}{l}\text { MILE RUN } \\
\text { BOAZ }\end{array}$ & $\underset{W V}{\mathrm{OH}}$ & $\begin{array}{l}167 \\
107\end{array}$ \\
\hline $\begin{array}{l}307722 \\
307691\end{array}$ & $\begin{array}{l}\text { HARRISON EAST } \\
\text { HARRISON }\end{array}$ & $\begin{array}{l}\text { NY } \\
\text { PA }\end{array}$ & $\begin{array}{l}101 \\
105\end{array}$ & $\begin{array}{l}005906 \\
077064\end{array}$ & $\begin{array}{l}\text { MOORES JUNCTION } \\
\text { BOAZ }\end{array}$ & $\underset{W V}{\mathrm{OH}}$ & $\begin{array}{l}167 \\
107\end{array}$ \\
\hline $\begin{array}{l}442573 \\
759626\end{array}$ & $\begin{array}{l}\text { MALLORY } \\
\text { WFST BRANCH }\end{array}$ & $\begin{array}{l}\text { NY } \\
\text { PA }\end{array}$ & $\begin{array}{l}009 \\
083\end{array}$ & $\begin{array}{l}005945 \\
674345 \\
005968\end{array}$ & $\begin{array}{l}\text { NEWELL RUN } \\
\text { ST MARYS } \\
\text { OLIVE-MEIGS }\end{array}$ & $\underset{W V}{\mathrm{OH}}$ & $\begin{array}{l}167 \\
073 \\
105\end{array}$ \\
\hline $\begin{array}{l}599404 \\
652414\end{array}$ & $\begin{array}{l}\text { RICHBURG } \\
\text { SHINGLEHOUSE }\end{array}$ & $\begin{array}{l}\text { NY } \\
\text { PA }\end{array}$ & $\begin{array}{l}003 \\
083\end{array}$ & $\begin{array}{l}503688 \\
005980\end{array}$ & PATTON RUN & $\begin{array}{l}\text { WH } \\
\text { OH }\end{array}$ & $\begin{array}{l}105 \\
107 \\
013\end{array}$ \\
\hline $\begin{array}{l}599404 \\
652414\end{array}$ & $\begin{array}{l}\text { RICHBURG } \\
\text { SHINGLEHOUSE }\end{array}$ & $\begin{array}{l}\text { NY } \\
\text { PA }\end{array}$ & $\begin{array}{l}003 \\
105\end{array}$ & $\begin{array}{l}276711 \\
006036\end{array}$ & $\begin{array}{l}\text { GLENN RUN } \\
\text { PROCTORVILLE }\end{array}$ & $\begin{array}{l}\text { WV } \\
\mathrm{OH}\end{array}$ & $\begin{array}{l}069 \\
087\end{array}$ \\
\hline $\begin{array}{l}647971 \\
652414\end{array}$ & $\begin{array}{l}\text { SHARON } \\
\text { SHINGLEHOUSE }\end{array}$ & $\begin{array}{l}N Y \\
\text { PA }\end{array}$ & $\begin{array}{l}003 \\
105\end{array}$ & $\begin{array}{l}340105 \\
006036\end{array}$ & $\begin{array}{l}\text { HUNTINGTON } \\
\text { PROCTORVILLE }\end{array}$ & $\begin{array}{l}\text { WV } \\
\text { OH }\end{array}$ & $\begin{array}{r}011 \\
187\end{array}$ \\
\hline $\begin{array}{l}677538 \\
677538\end{array}$ & $\begin{array}{l}\text { STATE LINE } \\
\text { STATE LINE }\end{array}$ & $\begin{array}{l}\text { NY } \\
\text { PA }\end{array}$ & $\begin{array}{l}003 \\
105\end{array}$ & 340105 & HUNTINGTON & WV & 199 \\
\hline $\begin{array}{l}107011 \\
157832\end{array}$ & CST VIRGINIA & $\stackrel{\mathrm{OH}}{\mathrm{PA}}$ & $\begin{array}{l}007 \\
039\end{array}$ & $\begin{array}{l}006417 \\
056331\end{array}$ & $\begin{array}{l}\text { SHEETS RUN } \\
\text { BENS RUN }\end{array}$ & $\underset{W V}{\mathrm{OH}}$ & $\begin{array}{l}167 \\
095\end{array}$ \\
\hline $\begin{array}{l}\text { OHIO \& } \\
005001 \\
340105\end{array}$ & $\begin{array}{l}\text { ATHALIA } \\
\text { HUNTINGTON }\end{array}$ & $\underset{W V}{O H}$ & $\begin{array}{l}087 \\
011\end{array}$ & $\begin{array}{l}006417 \\
056331\end{array}$ & $\begin{array}{l}\text { SHEETS RUN } \\
\text { BENS RUN }\end{array}$ & $\underset{\mathrm{WV}}{\mathrm{OH}}$ & $\begin{array}{l}167 \\
073\end{array}$ \\
\hline $\begin{array}{l}005001 \\
340105\end{array}$ & $\begin{array}{l}\text { ATHALIA } \\
\text { HUNTINGTON }\end{array}$ & $\underset{W V}{\mathrm{OH}}$ & $\begin{array}{l}087 \\
099\end{array}$ & $\begin{array}{l}658139 \\
658139\end{array}$ & $\begin{array}{l}\text { SISTERSVILLE } \\
\text { SISTERSVILLE }\end{array}$ & $\underset{W V}{\mathrm{OH}}$ & $\begin{array}{l}111 \\
095\end{array}$ \\
\hline $\begin{array}{l}039505 \\
540174\end{array}$ & $\begin{array}{l}\text { BARLOW } \\
\text { PARKERSBURG }\end{array}$ & $\underset{\mathrm{WV}}{\mathrm{OH}}$ & $\begin{array}{l}167 \\
107\end{array}$ & $\begin{array}{l}691364 \\
503998\end{array}$ & $\begin{array}{l}\text { SUTTON } \\
\text { NEW HAVEN }\end{array}$ & $\underset{W V}{\mathrm{OH}}$ & $\begin{array}{l}105 \\
053\end{array}$ \\
\hline $\begin{array}{l}054409 \\
674345\end{array}$ & $\begin{array}{l}\text { BELLS RUN } \\
\text { ST MARYS }\end{array}$ & $\underset{W V}{\mathrm{OH}}$ & $\begin{array}{l}167 \\
073\end{array}$ & \multicolumn{2}{|c|}{$\begin{array}{l}\text { OKLAHOMA \& TEXAS } 90 \\
302824 \text { HANDY SE } \\
302824 \text { HANDY SE }\end{array}$} & $\begin{array}{l}\text { OK } \\
\text { TX90 }\end{array}$ & $\begin{array}{l}095 \\
181\end{array}$ \\
\hline $\begin{array}{l}082830 \\
674345\end{array}$ & $\begin{array}{l}\text { BOSWORTH } \\
\text { ST MARYS }\end{array}$ & $\underset{W V}{\mathrm{OH}}$ & $\begin{array}{l}167 \\
073\end{array}$ & $\begin{array}{l}641879 \\
482395\end{array}$ & $\begin{array}{l}\text { SEAY } \\
\text { MONTAGUE COUNTY REGULAR }\end{array}$ & $\begin{array}{l}\text { OK } \\
\text { TX90 }\end{array}$ & $\begin{array}{l}067 \\
337\end{array}$ \\
\hline $\begin{array}{l}005056 \\
686435\end{array}$ & $\begin{array}{l}\text { BRILLIANT } \\
\text { SUCKERROD }\end{array}$ & $\underset{\mathrm{WV}}{\mathrm{OH}}$ & $\begin{array}{l}081 \\
009\end{array}$ & $\begin{array}{l}658325 \\
658325\end{array}$ & $\begin{array}{l}\text { SIVELLS BEND } \\
\text { SIVELLS BEND }\end{array}$ & $\begin{array}{l}\text { OK } \\
\text { TX90 }\end{array}$ & $\begin{array}{l}085 \\
097\end{array}$ \\
\hline $\begin{array}{l}104872 \\
276711\end{array}$ & $\begin{array}{l}\text { BURLINGTON } \\
\text { GLENN RUN }\end{array}$ & $\underset{\text { WV }}{\mathrm{OH}}$ & $\begin{array}{l}013 \\
069\end{array}$ & \multicolumn{2}{|c|}{ OKLAHOMA TEXAS 95} & OK & 007 \\
\hline $\begin{array}{l}005096 \\
562837\end{array}$ & $\begin{array}{l}\text { CHESIRE } \\
\text { POINT PLEASANT }\end{array}$ & $\underset{W V}{\mathrm{OH}}$ & $\begin{array}{l}053 \\
053\end{array}$ & 011479 & ALLEN-PARKER & TX95 & 357 \\
\hline $\begin{array}{l}284927 \\
056331\end{array}$ & $\begin{array}{l}\text { GRANDVIEW } \\
\text { BENS RUN }\end{array}$ & $\stackrel{\mathrm{OH}}{\mathrm{WV}}$ & $\begin{array}{l}167 \\
073\end{array}$ & $\begin{array}{r}551389 \\
291499\end{array}$ & $\begin{array}{l}\text { CAMRICK DISTRICT } \\
\text { PERRYTON NORTH } \\
\text { GRIGGS SOUTH }\end{array}$ & TX95 & 357 \\
\hline $\begin{array}{l}284927 \\
056331\end{array}$ & $\begin{array}{l}\text { GRANDVIEW } \\
\text { BENS RUN }\end{array}$ & $\underset{\text { WH }}{\mathrm{OH}}$ & $\begin{array}{l}167 \\
095\end{array}$ & $\begin{array}{l}365433 \\
295669\end{array}$ & $\begin{array}{l}\text { JUDD } \\
\text { GUYMON SOUTH }\end{array}$ & $\begin{array}{l}\text { TX95 } \\
\text { OK }\end{array}$ & $\begin{array}{l}421 \\
139\end{array}$ \\
\hline $\begin{array}{l}292584 \\
292584\end{array}$ & $\begin{array}{l}\text { GROUNDHOG CREEK } \\
\text { GROUNDHOG CREEK }\end{array}$ & $\underset{W V}{\mathrm{OH}}$ & $\begin{array}{l}105 \\
035\end{array}$ & 324944 & HITCHLAND WEST & TX95 & 195 \\
\hline $\begin{array}{l}292584 \\
602752\end{array}$ & $\begin{array}{l}\text { GROUNDHOG CREEK } \\
\text { RIPLEY NORTH }\end{array}$ & $\stackrel{\text { OH }}{\text { WV }}$ & $\begin{array}{l}105 \\
035\end{array}$ & $\begin{array}{l}295545 \\
000333\end{array}$ & $\begin{array}{l}\text { GUYMON-HUGOTON GAS AREA } \\
\text { TEXAS HUGOTON }\end{array}$ & $\begin{array}{l}\text { OK } \\
\text { TX95 }\end{array}$ & $\begin{array}{l}139 \\
195\end{array}$ \\
\hline $\begin{array}{l}315775 \\
077064\end{array}$ & $\begin{array}{l}\text { HENDERSHOT } \\
\text { BOAZ }\end{array}$ & $\underset{W V}{\text { OH }}$ & $\begin{array}{l}167 \\
107\end{array}$ & $\begin{array}{l}295545 \\
000333\end{array}$ & $\begin{array}{l}\text { GUYMON-HUGOTON GAS AREA } \\
\text { TEXAS HUGOTON }\end{array}$ & $\begin{array}{l}\text { OK } \\
\text { TX95 }\end{array}$ & $\begin{array}{l}139 \\
421\end{array}$ \\
\hline
\end{tabular}


Table 6. Flelds Located In Multiple Jurisdictions (Continued)

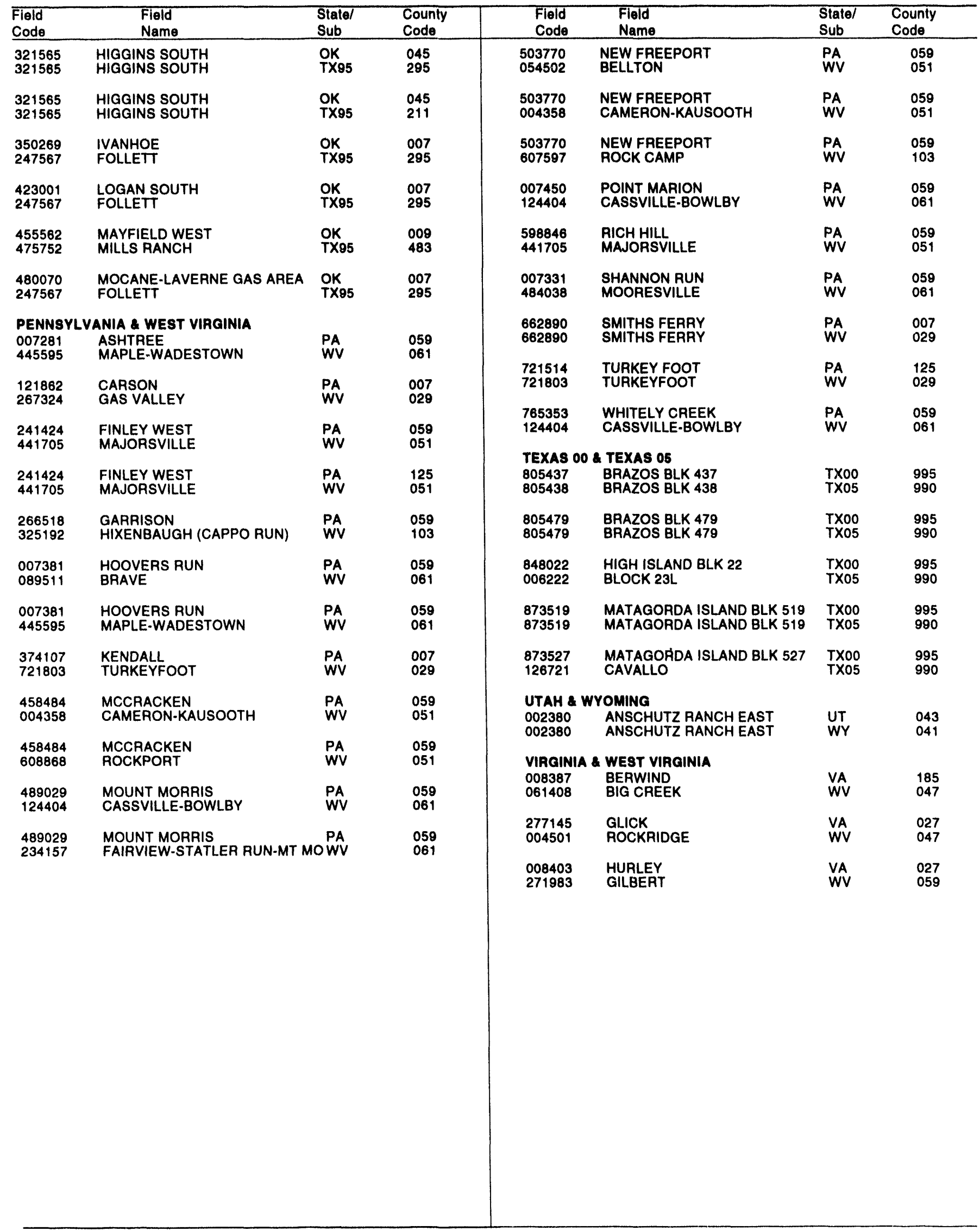


Figure 4. Subdivisions of Alaska and U.S. Geological Survey Quadrangles

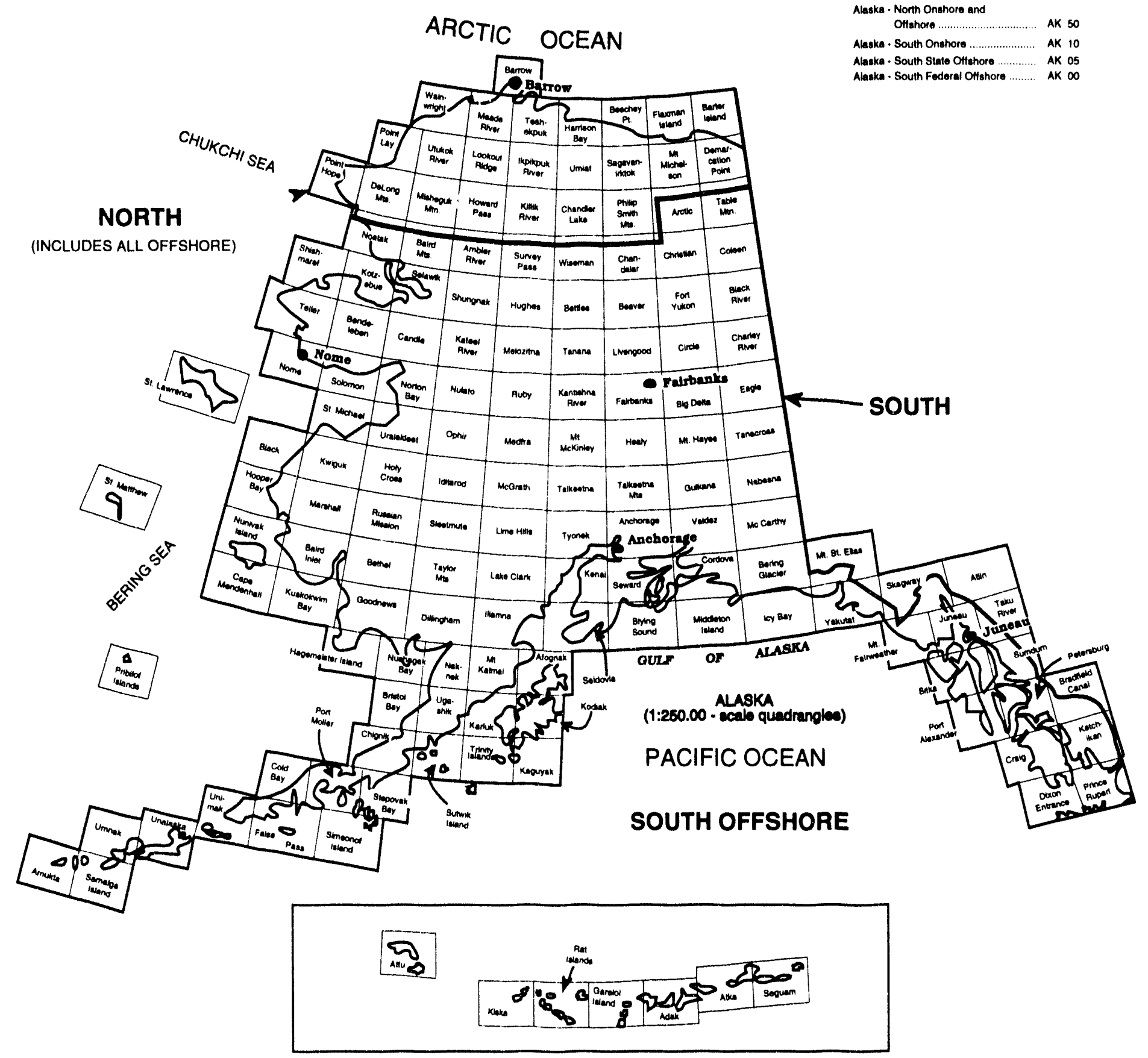

Sou,ce: After U.S. Geological Survey 
Figure 5. Subdivisions of California

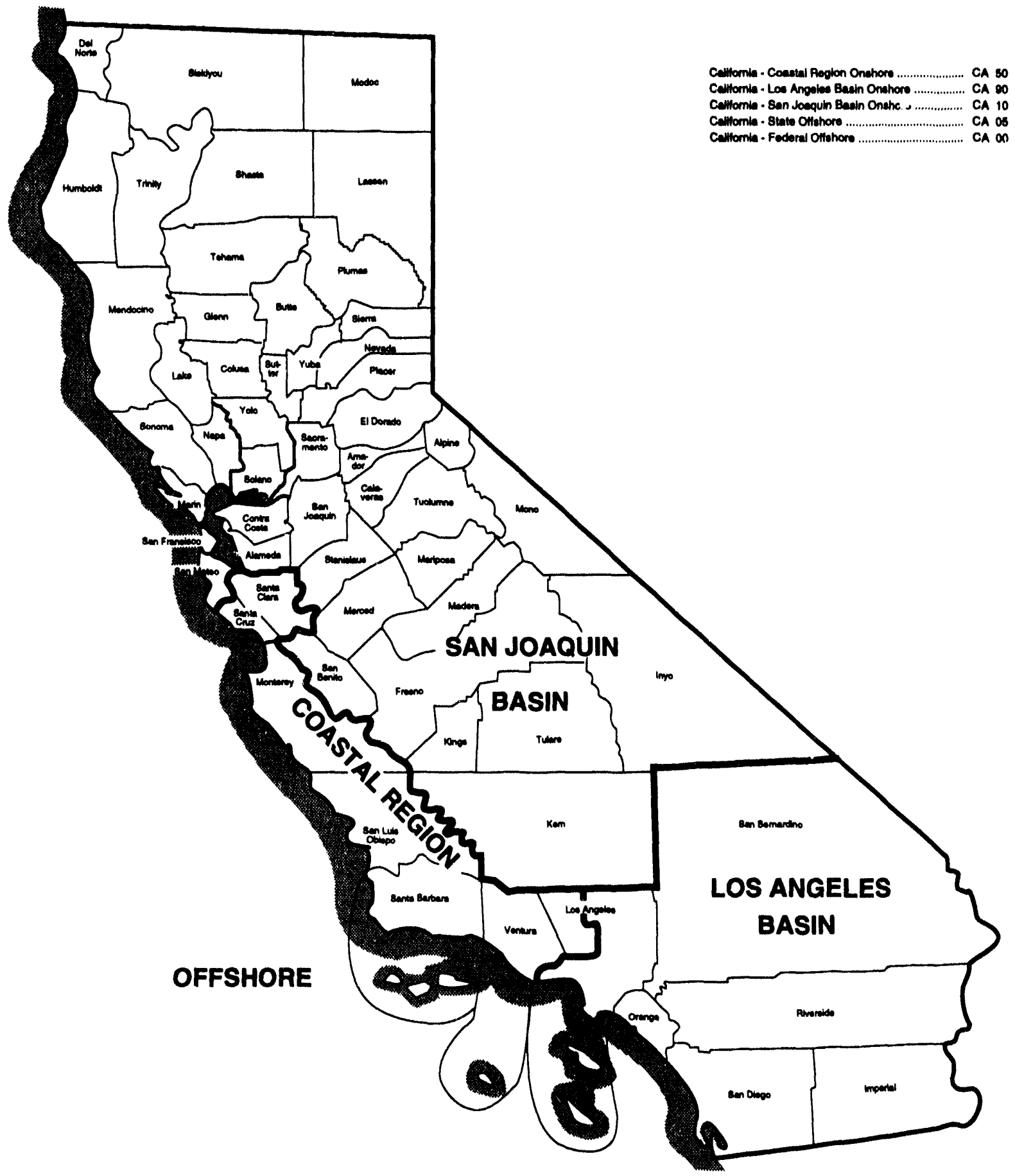

Source: Energy Information Administration, Office of Oll and Gas 
Figure 6. Subdivisions of Loulsiana

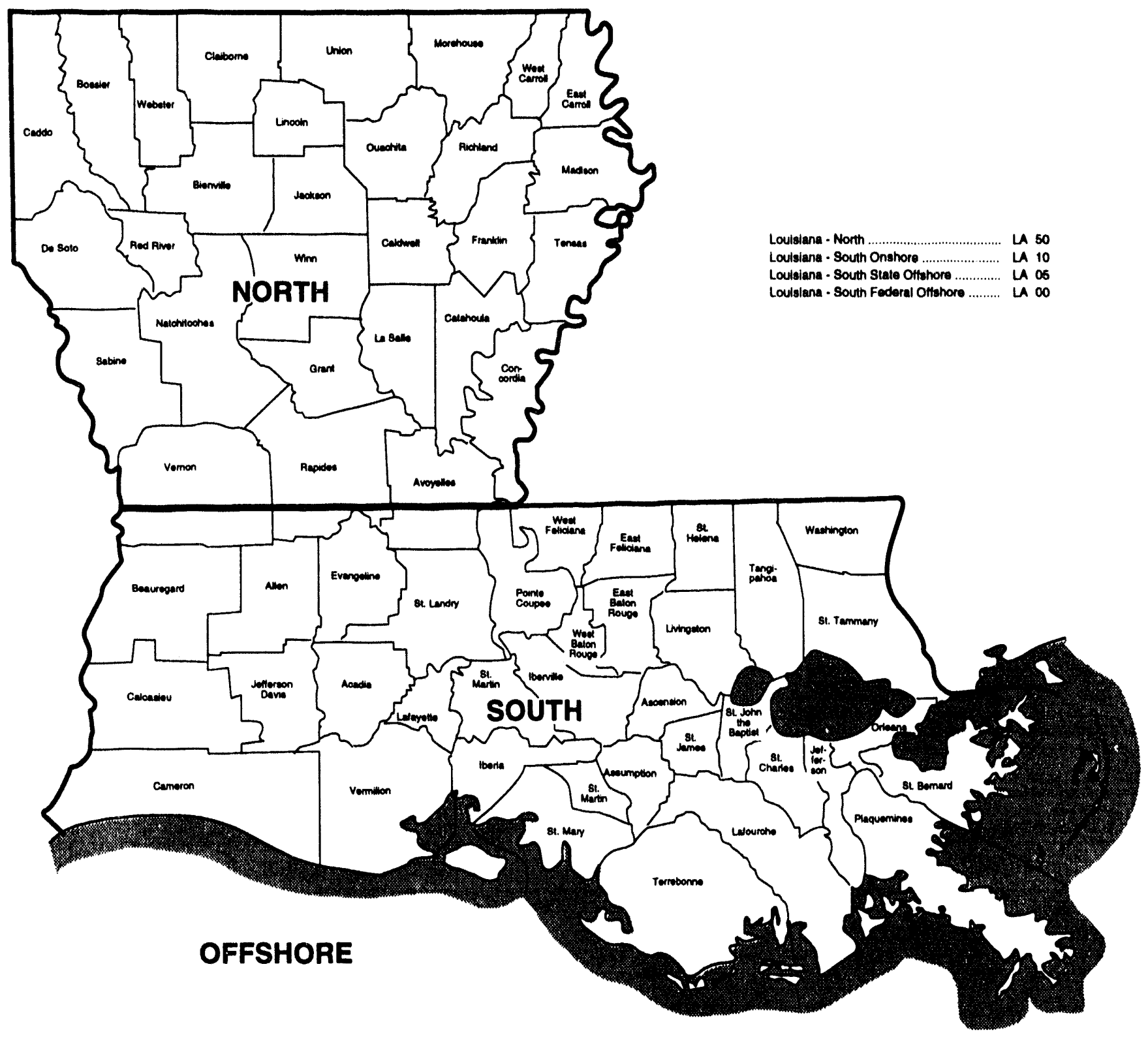

Source: Energy Information Administration, Office of Oll and Gas 
Figure 7. Subdivisions of New Mexico

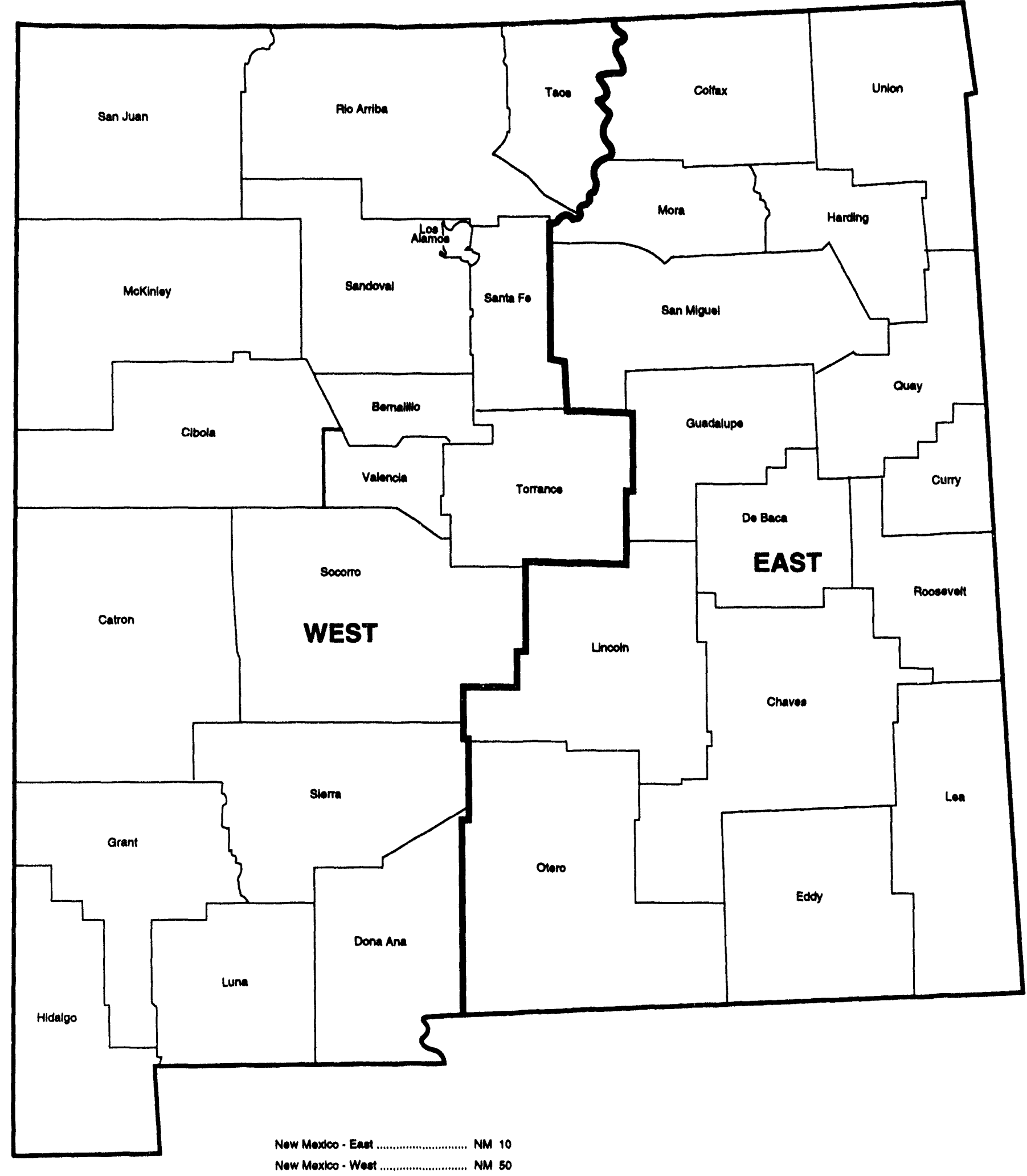

Source: Energy Information Administration, Office of Oll and Gas 


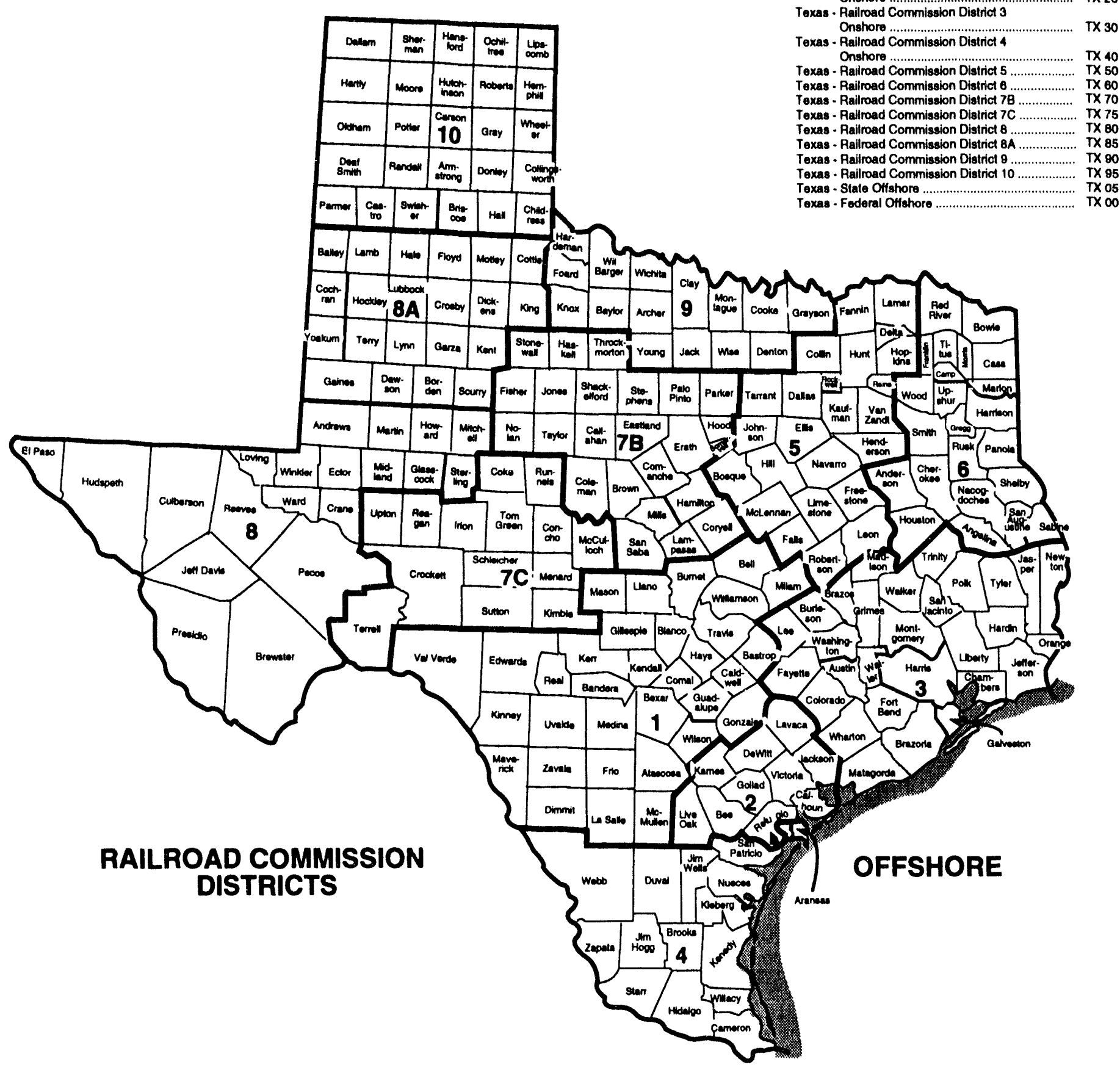

Source: Energy Information Administration, Office of Oll and Gas 
Figure 9. Western Planning Area, Gulf of Mexico Outer Continental Shelf Region

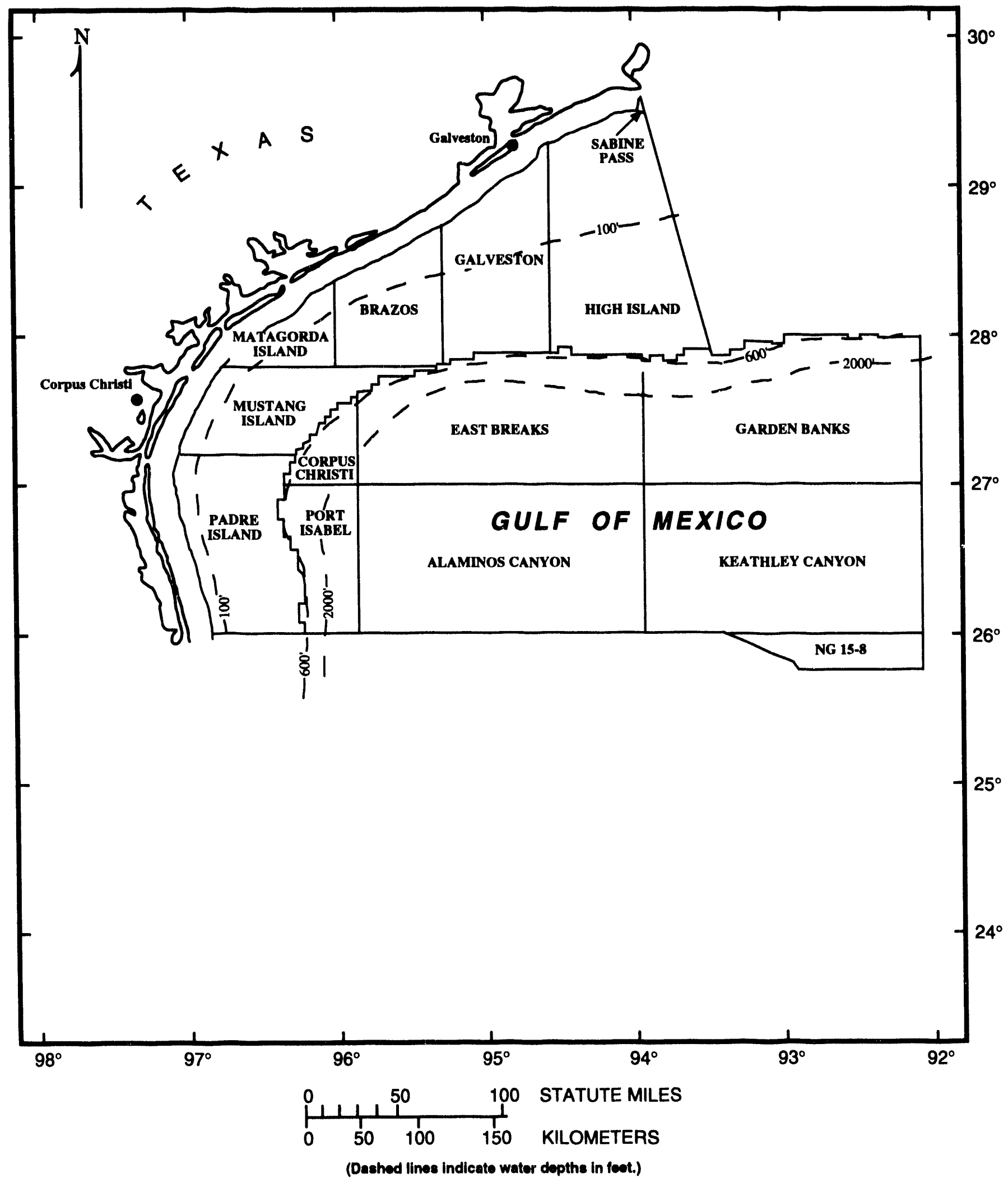

Source: After Minerals Management Service, U.S. Department of the Interior 
Figure 10. Central Planning Area, Gulf of Mexico Outer Continental Shelf Region

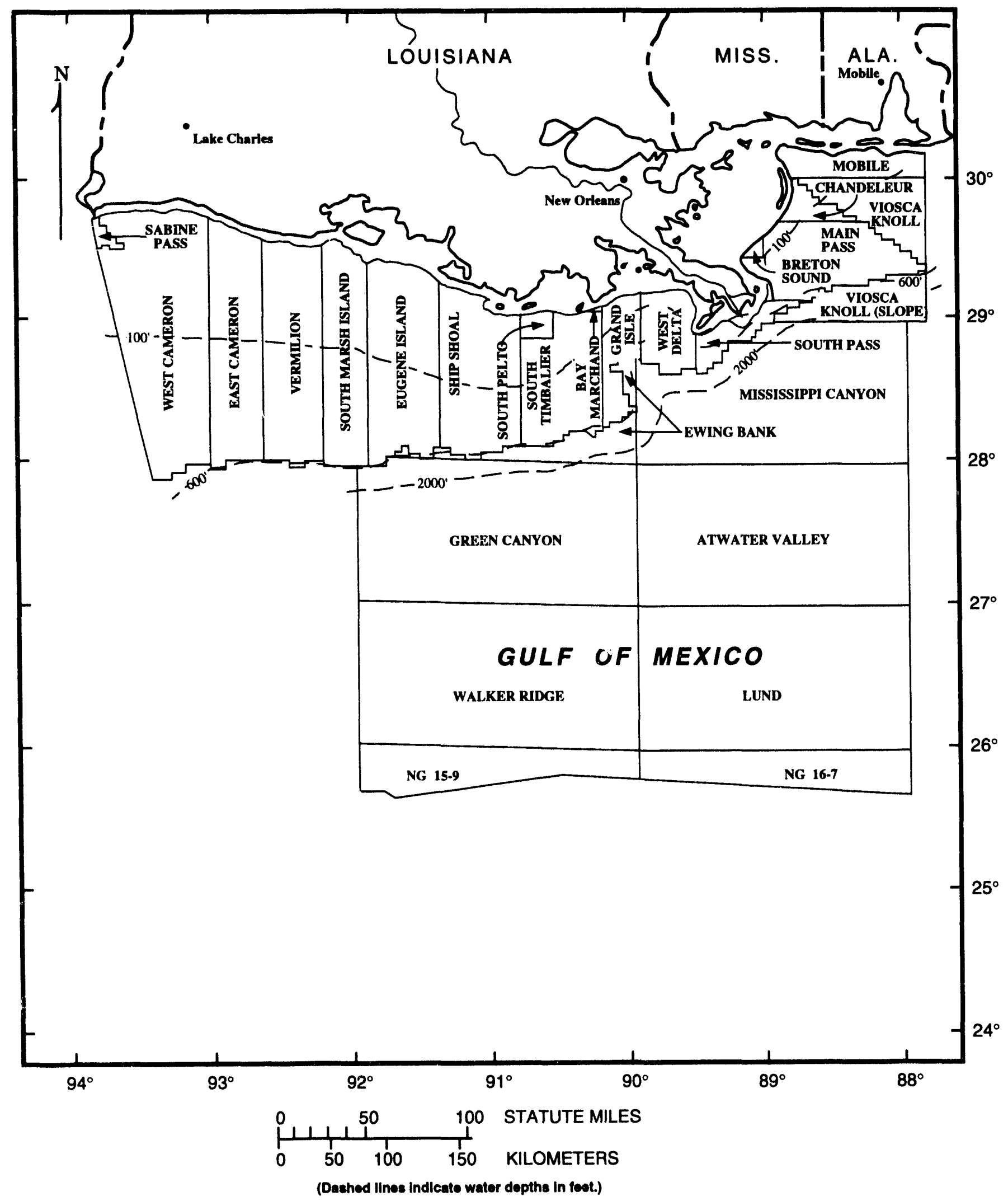

Source: After Minerals Management Service, U.S. Department of the Interior 
Figure 11. Eastern Planning Area, Gulf of Mexico Outer Continental Shelf Region

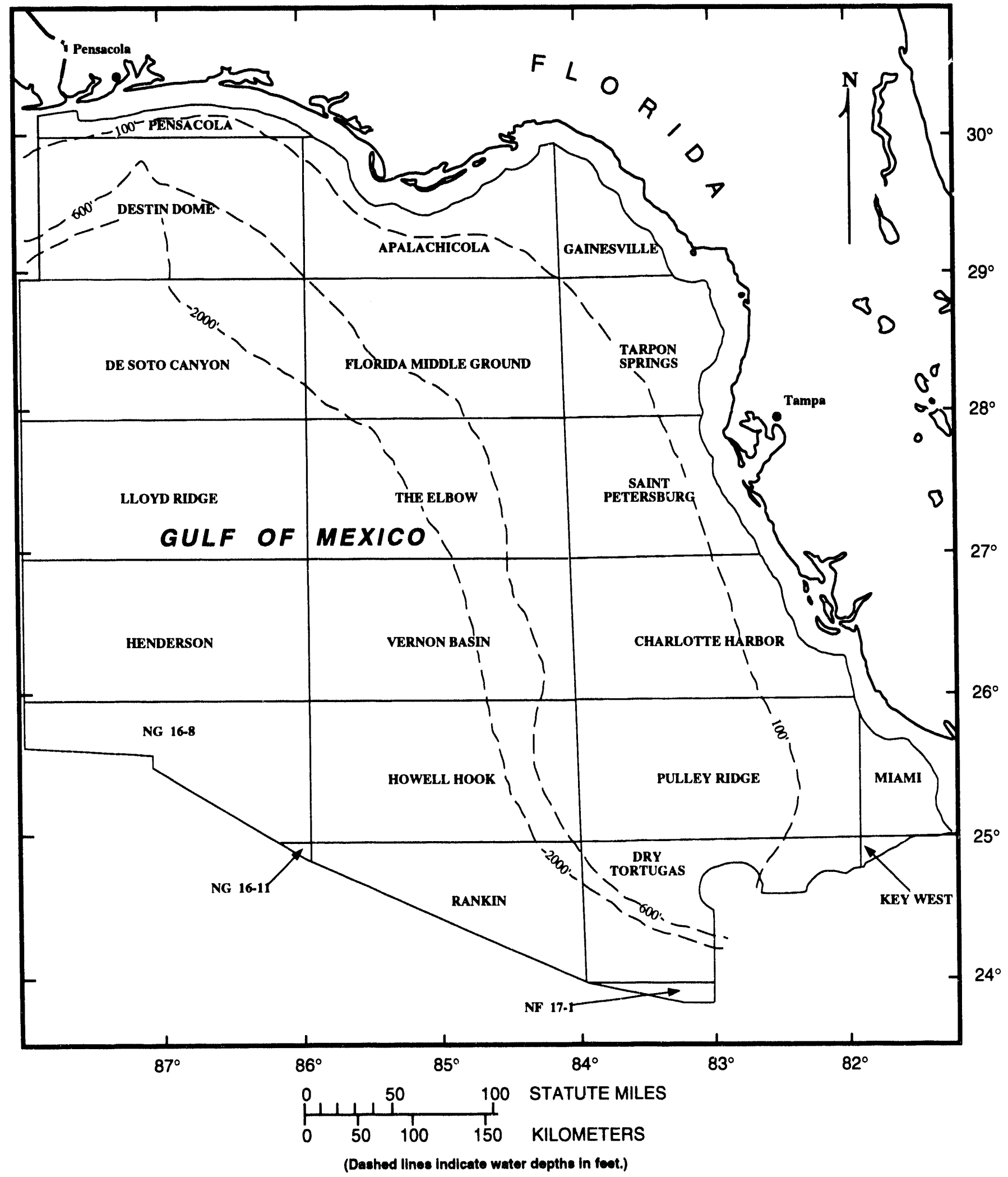

Source: After Minerals Management Service, U.S. Department of the Interior 


\section{Glossary}

This Glossary defines many of the technical terms used in this report.

Crude Oil: A mixture of hydrocarbons that exist in the liquid phase in natural underground reservoirs and remain liquid at atmospheric pressure after passing through surface separating facilities. Crude oil may also include:

- Small amounts of hydrocarbons that exist in the gaseous phase in natural underground reservoirs but are liquid at atmospheric pressure after being recovered from oil well (casinghead) gas in lease separators, and that subsequently are commingled with the crude stream without being separately measured.

- Small amounts of nonhydrocarbons produced with the oil.

Field: An area consisting of a single reservoir or multiple reservoirs all grouped on or related to the same individual geological structural feature and/or stratigraphic condition. There may be two or more reservoirs in a field which are separated vertically by intervening impervious strata, or laterally by local geologic barriers, or by both. (See Reservoir.)

Field Area: A geographic area encompassing two or more pools that have a common gathering and metering system, the reser "ns of which are reported as a single unit. This concept applies primarily to the Appalachian region. (See Pool.)
Field Discovery Year: The calendar year in which a field was first recognized as containing economically recoverable accumulations of oil and/or gas.

Natural Gas: A mixture of hydrocarbon compounds and small quantities of various nonhydrocarbons existing in the gaseous phase or in solution with crude oil in natural underground reservoirs at reservoir conditions. The principal hydrocarbons usually contained in the mixture are methane, ethane, propane, butane, and pentane. Typical nonhydrocarbon gases which may be present in reservoir natural gas are carbon dioxide, helium, hydrogen sulfide, and nitrogen. Under reservoir conditions, natural gas and the liquefiable portions occur either in a single gaseous phase in the reservoir or in solution with crude oil and are not distinguishable at the time as separate substances.

Pool: In general, a reservoir. In certain situations a pool may consist of more than one reservoir. (See Field Area.)

Reservoir: A porous and permeable underground formation containing an individual and separate natural accumulation of producible hydrocarbons (oil and/or gas) which is confined by impermeable rock or water barriers and is characterized by a single natural pressure system.

Subdivision: A prescribed portion of a given State of other geographical region defined in this publication for statistical reporting purposes. 


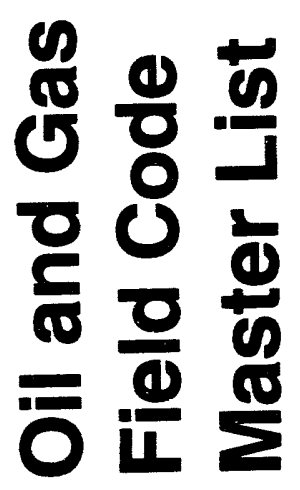


A L A B A M A

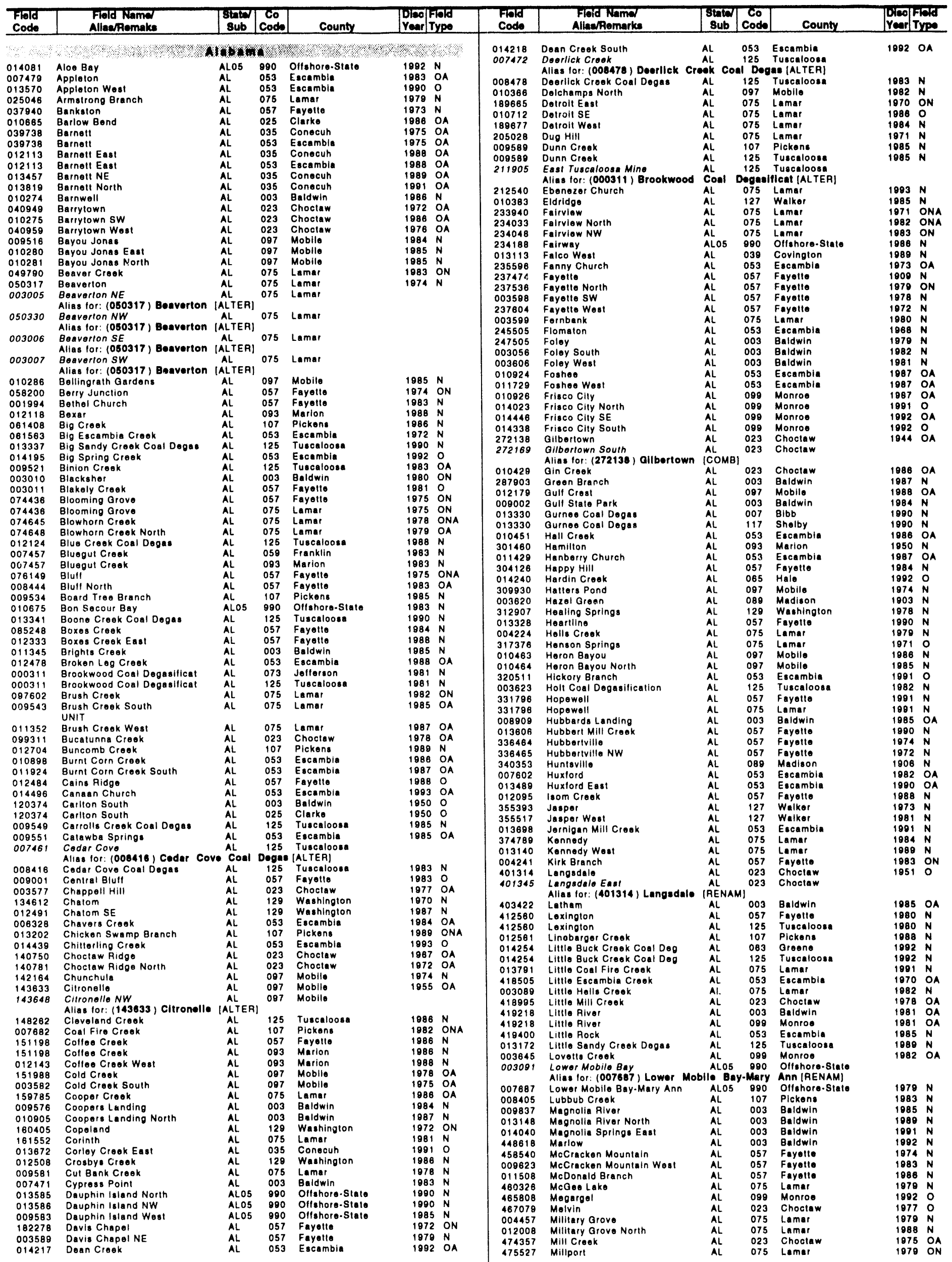


ALASKA

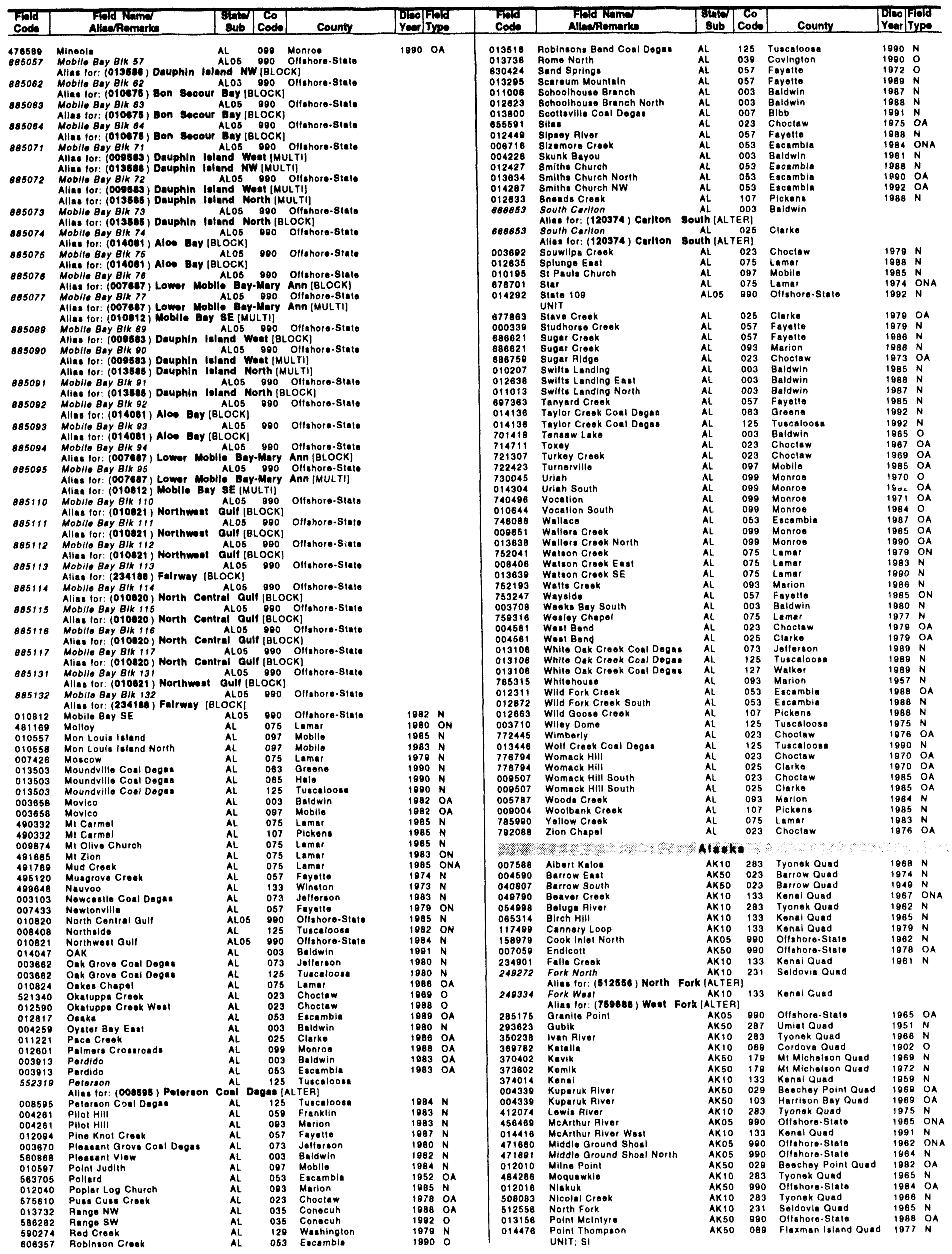


ALASKA

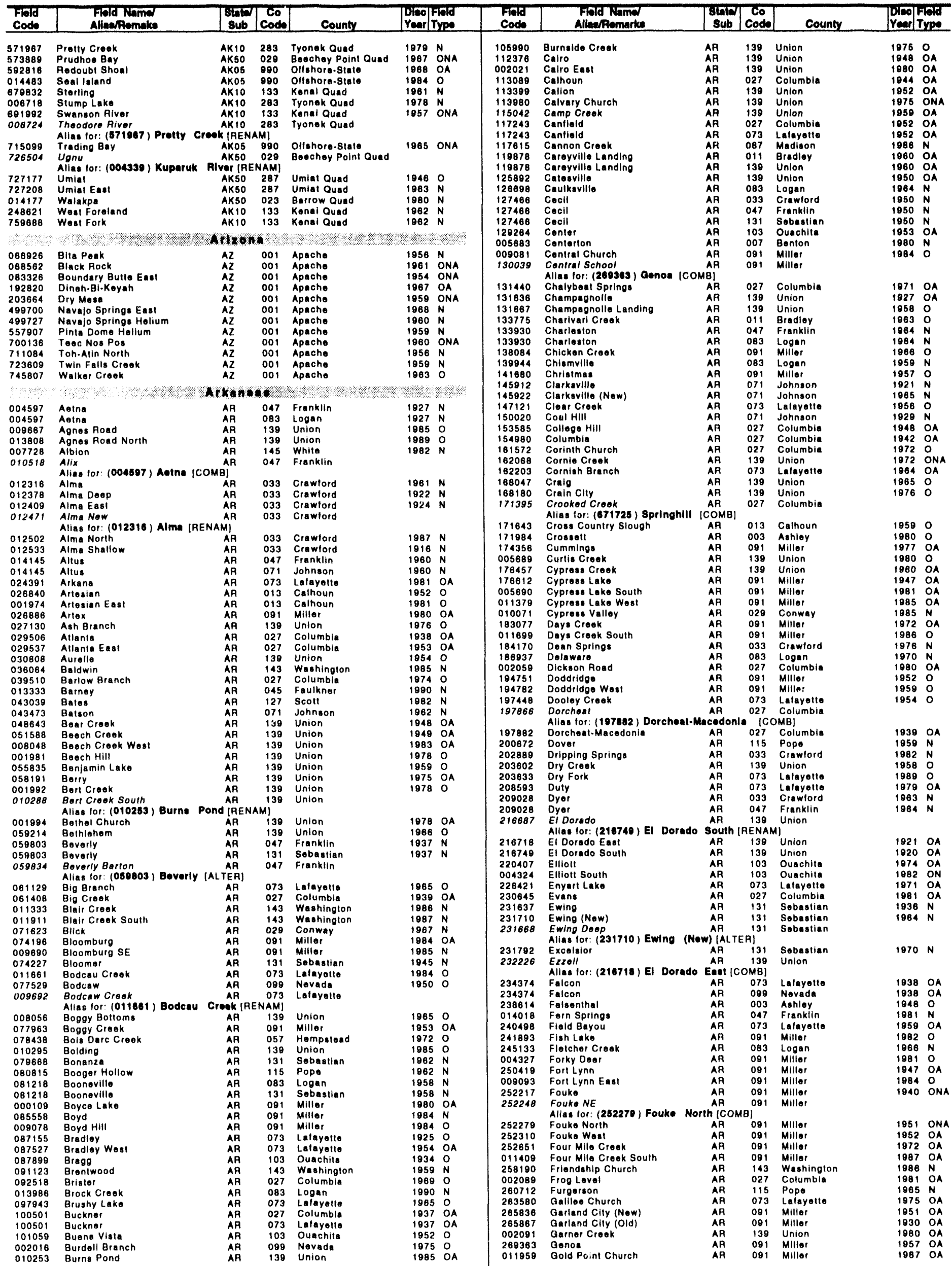


ARKANSAS

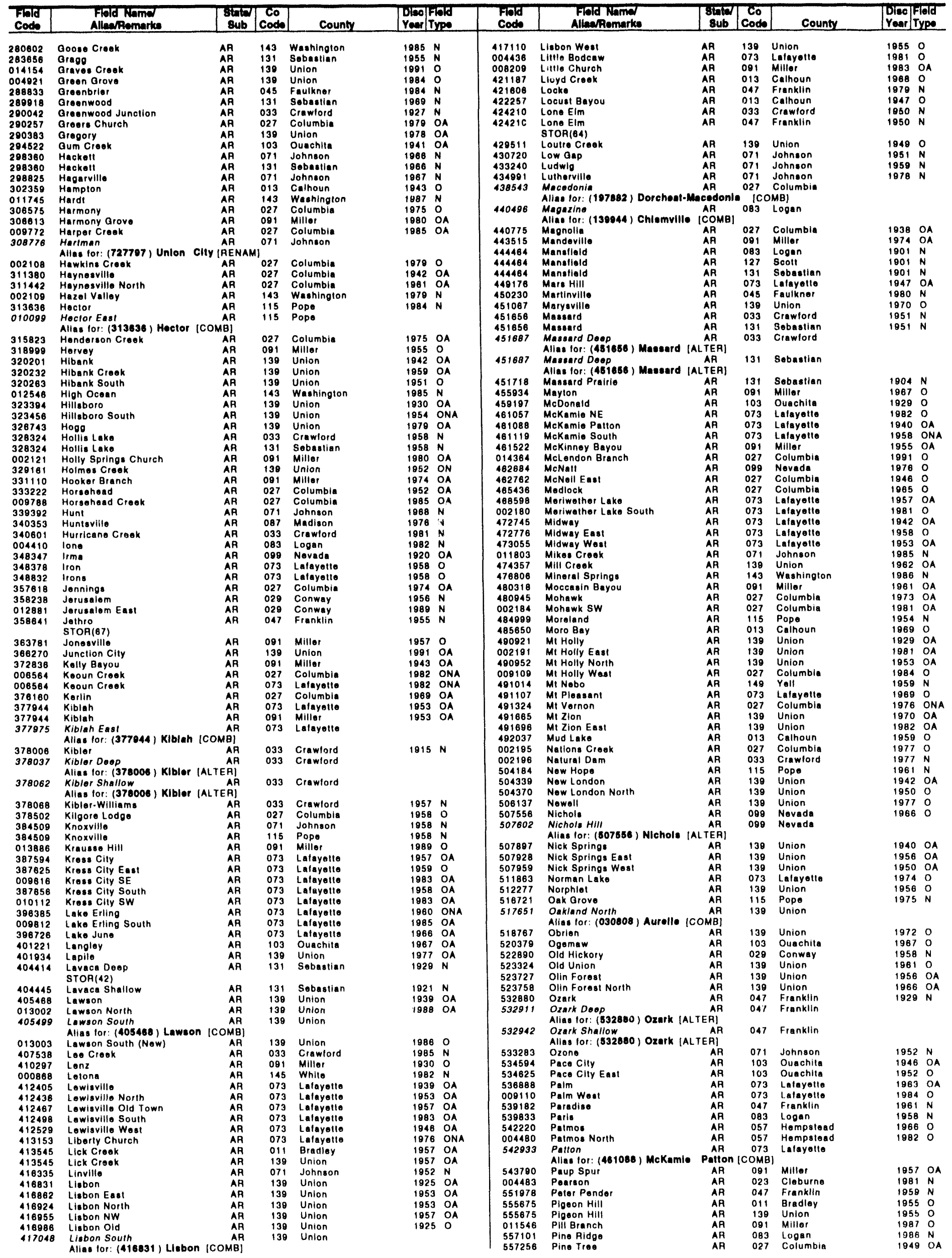


ARKANSAS

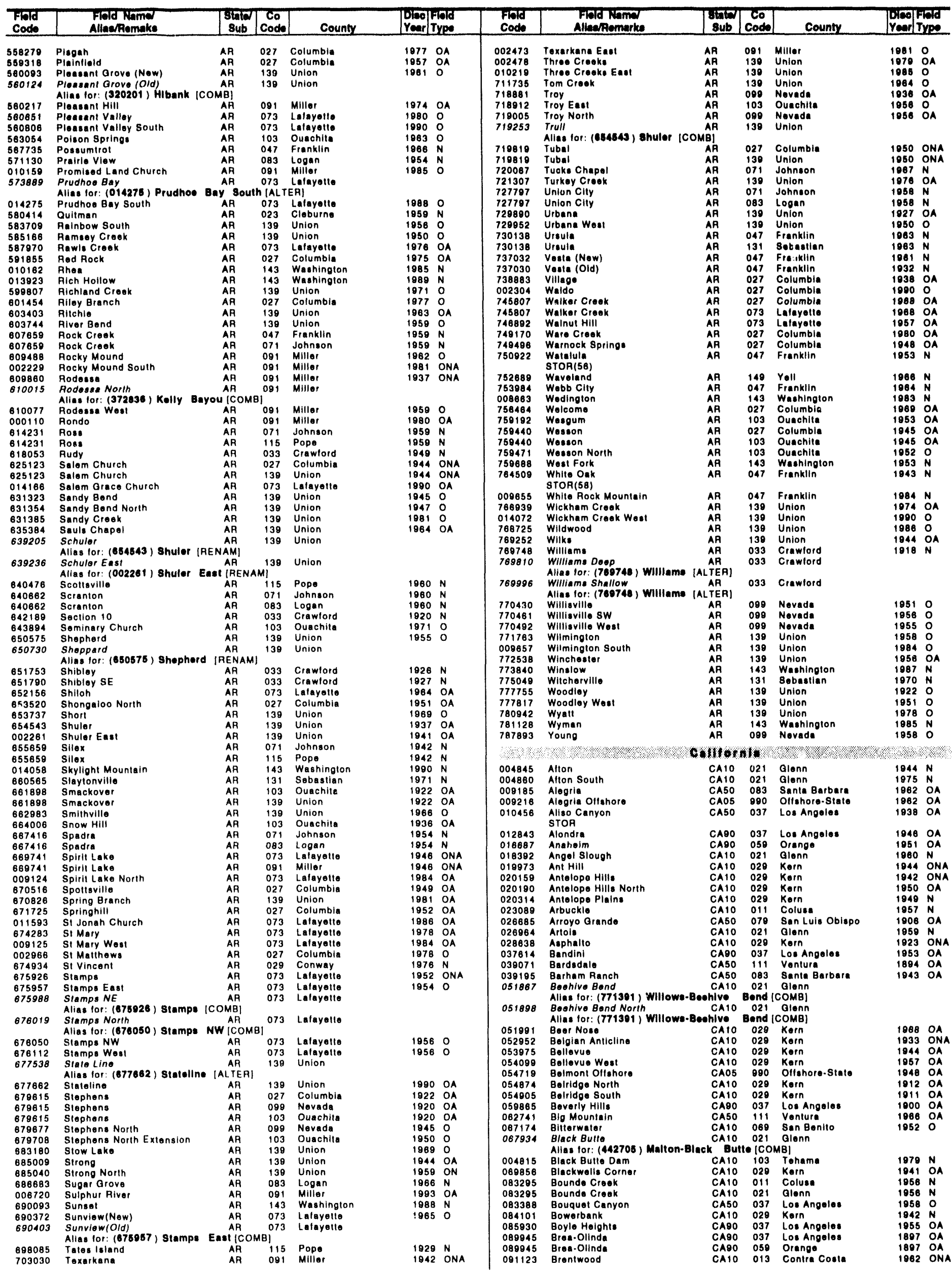


CALIFORNIA

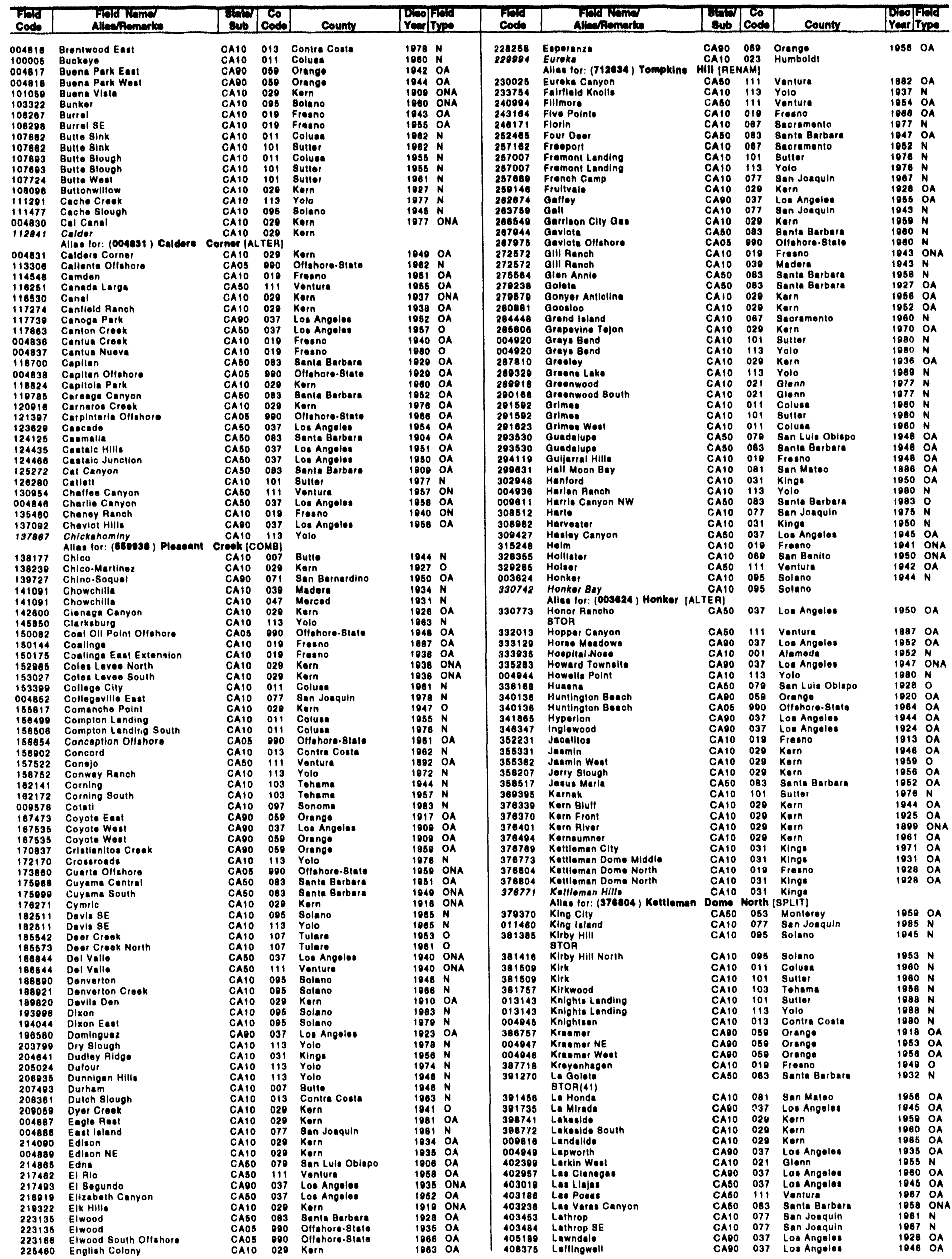


CALIFORNIA

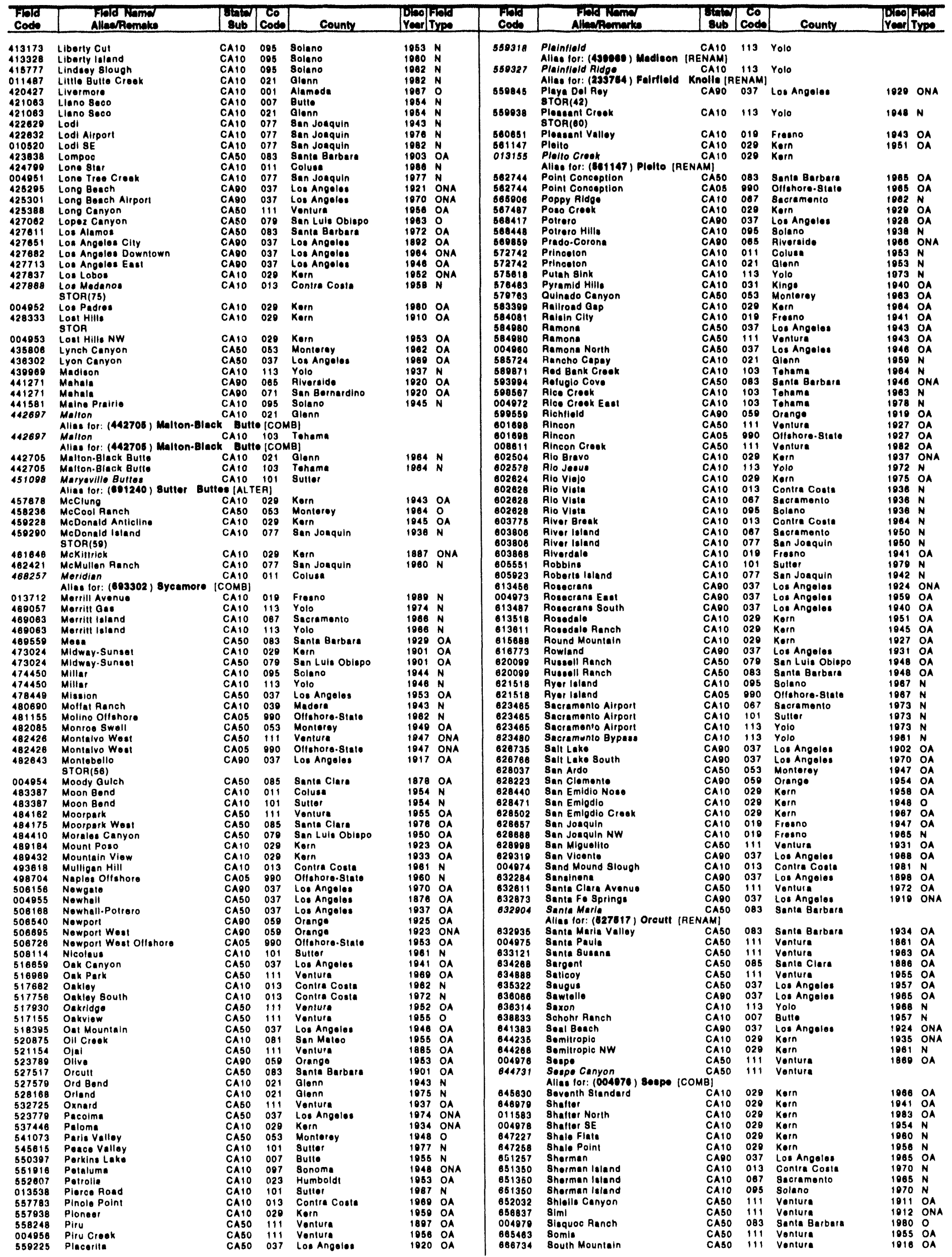


COLORADO

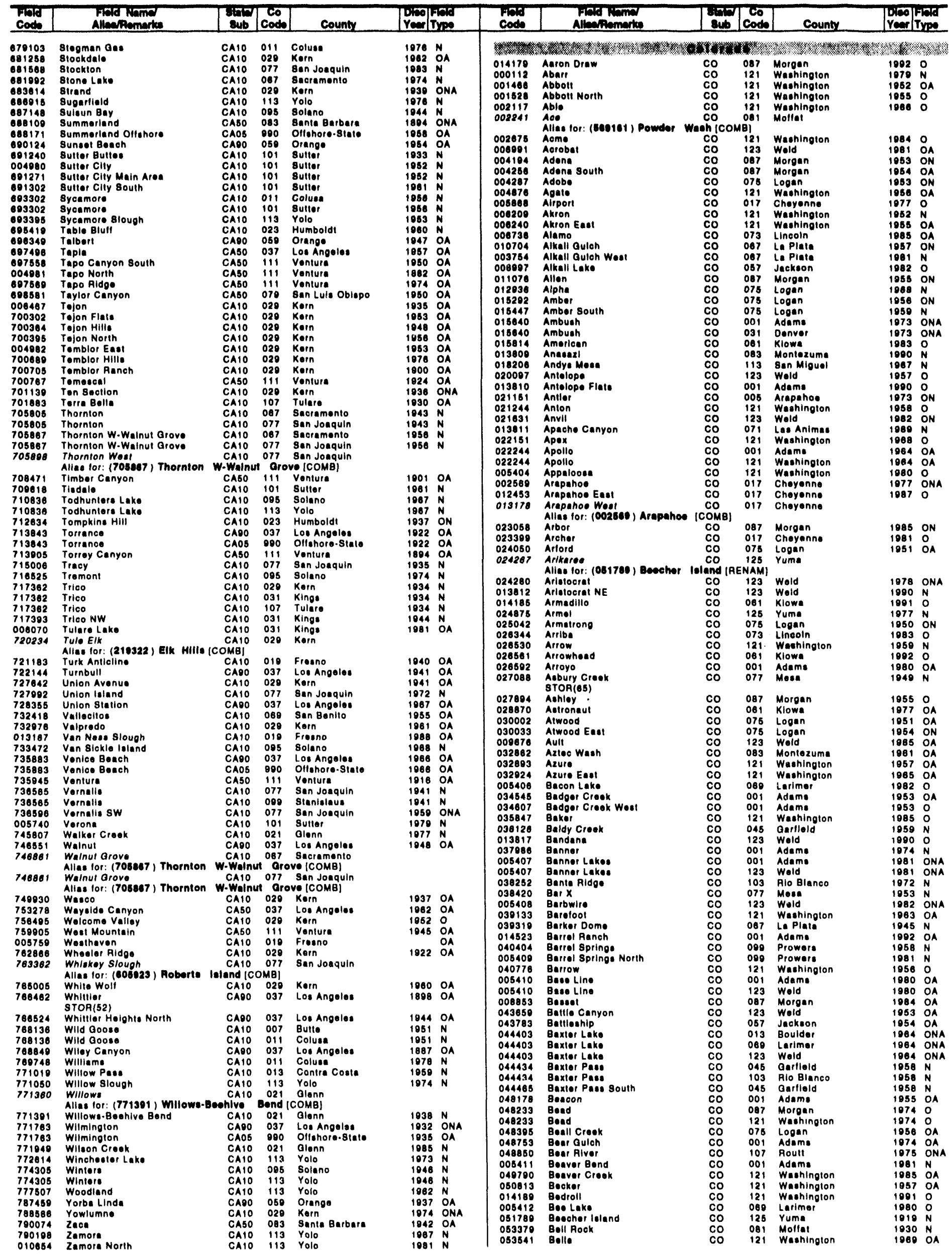




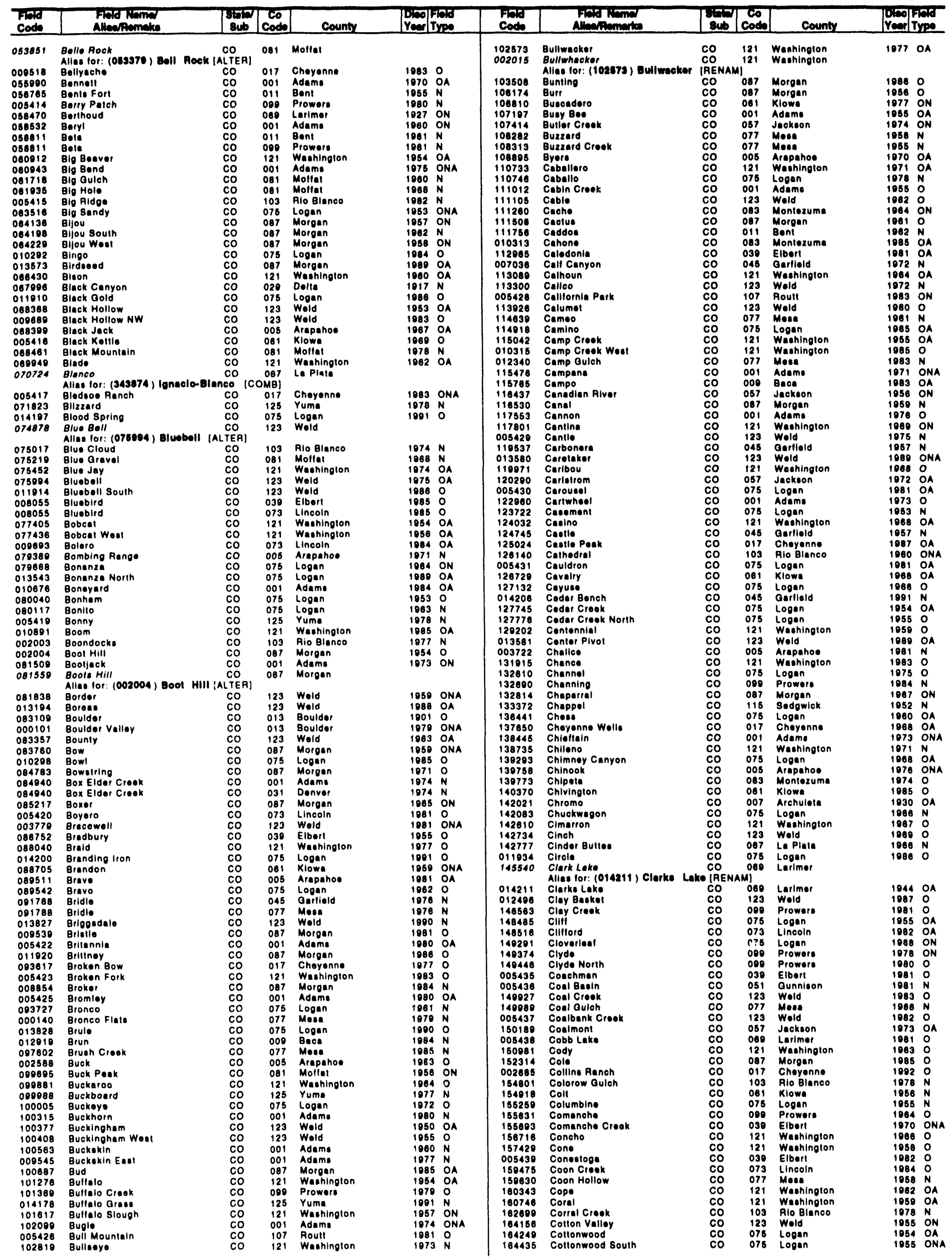


COLORADO

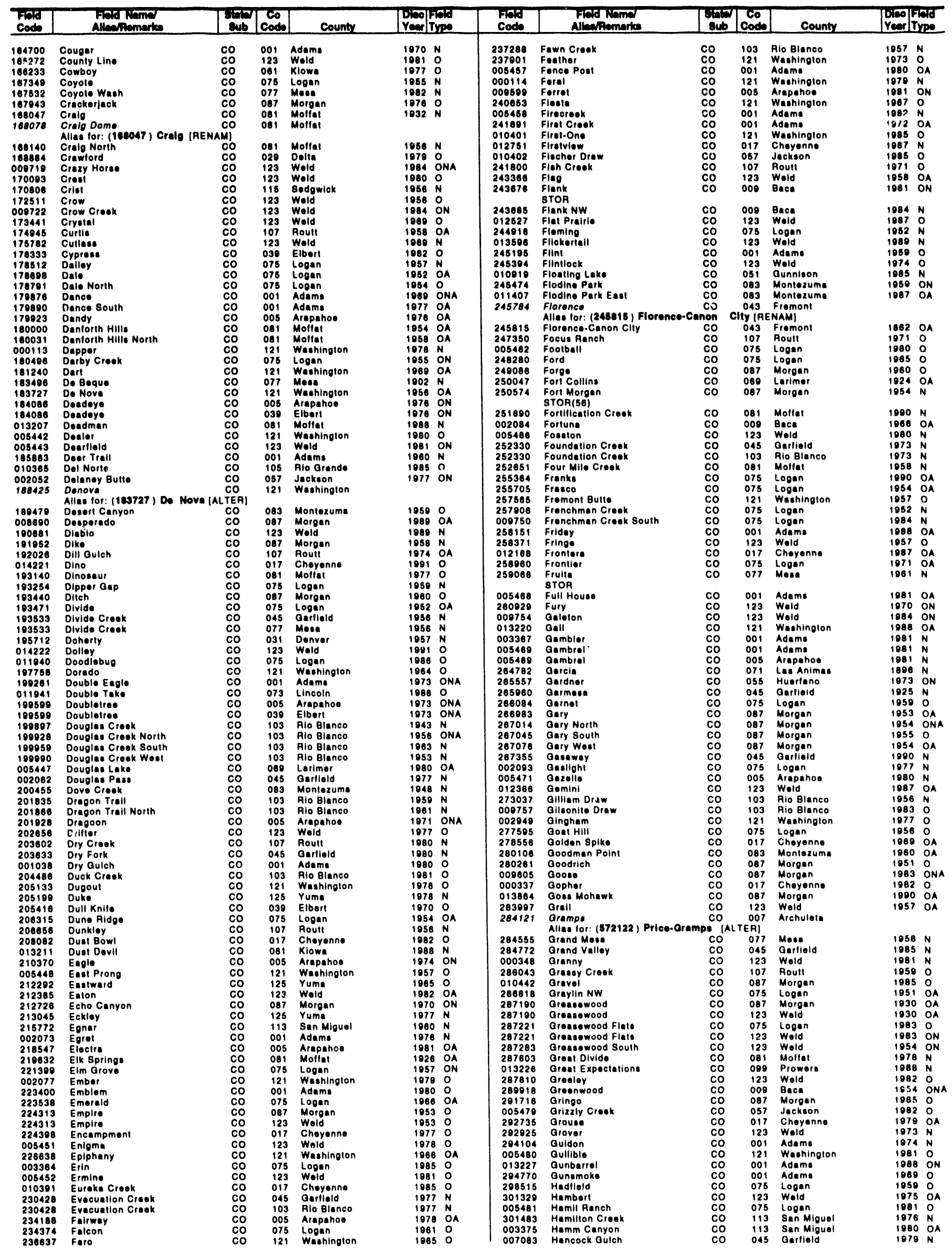




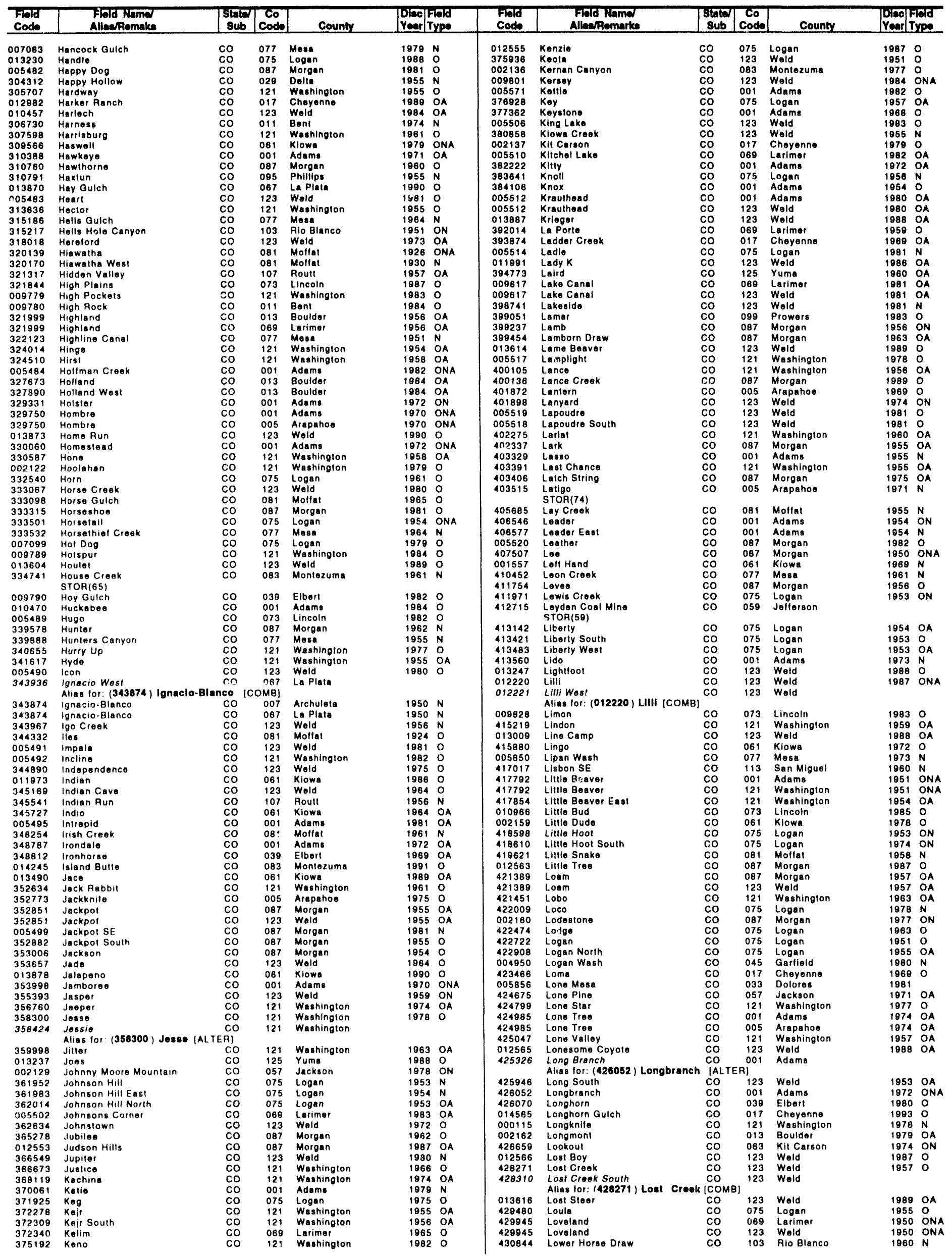


COLORADO

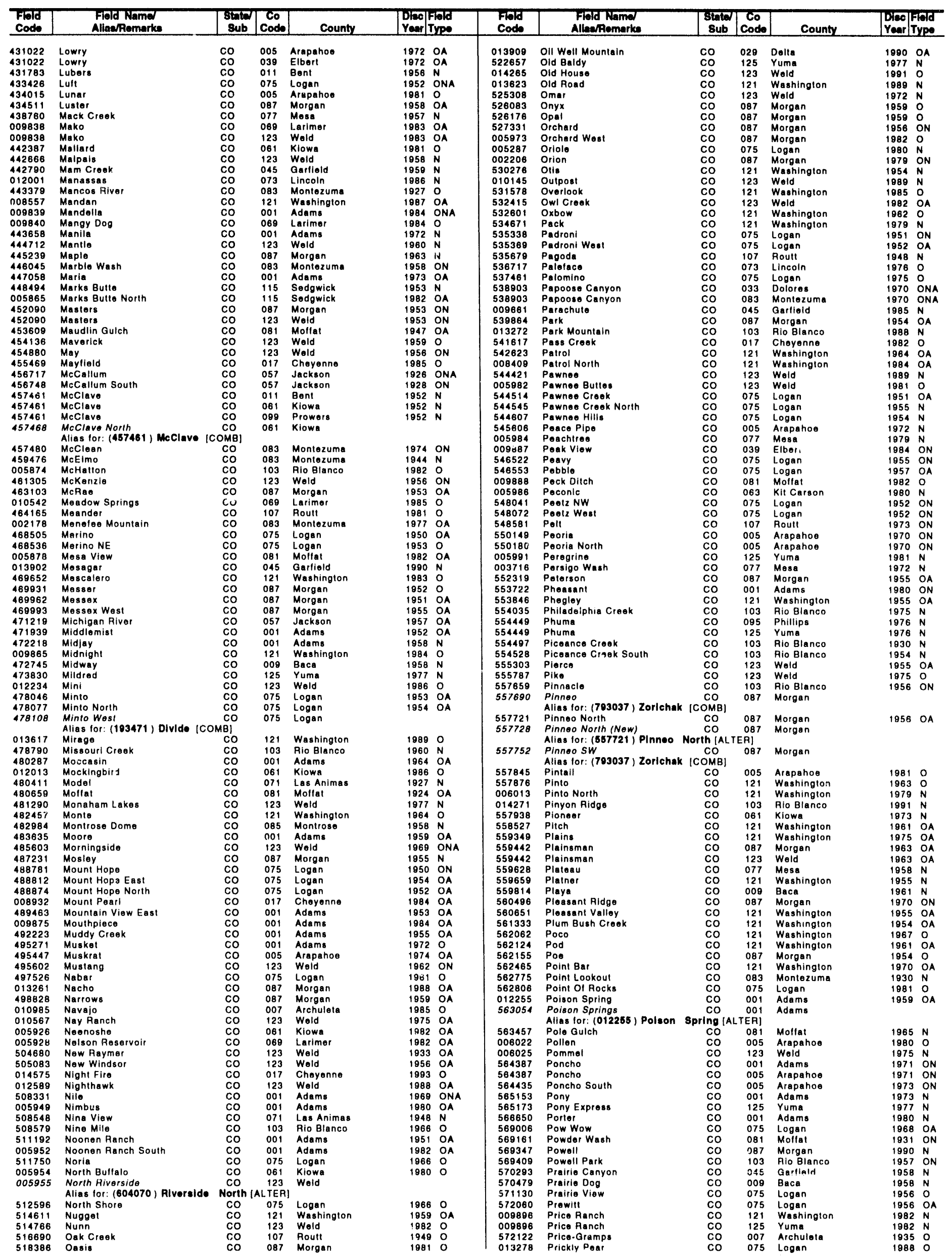


COLORADO

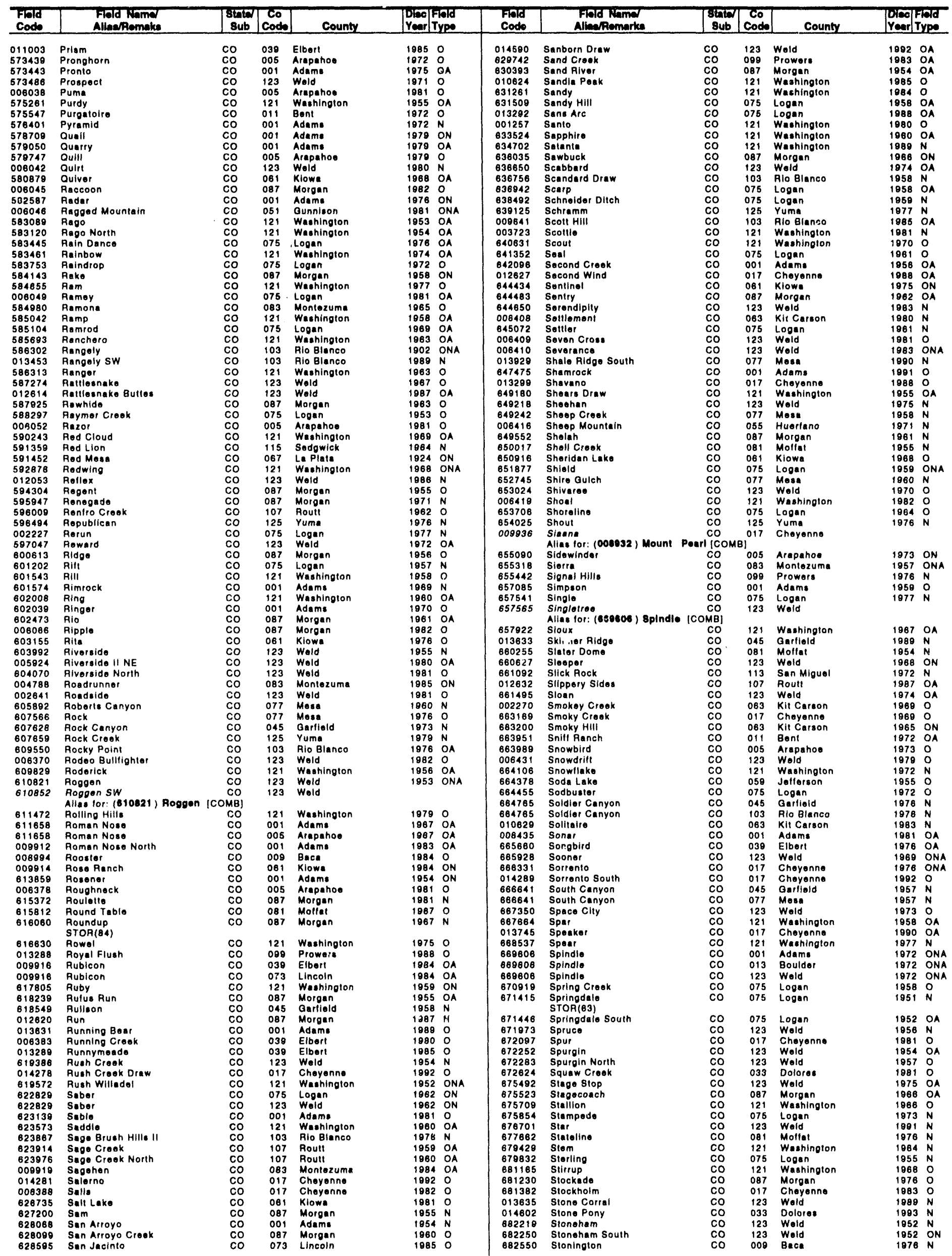


FLORIDA

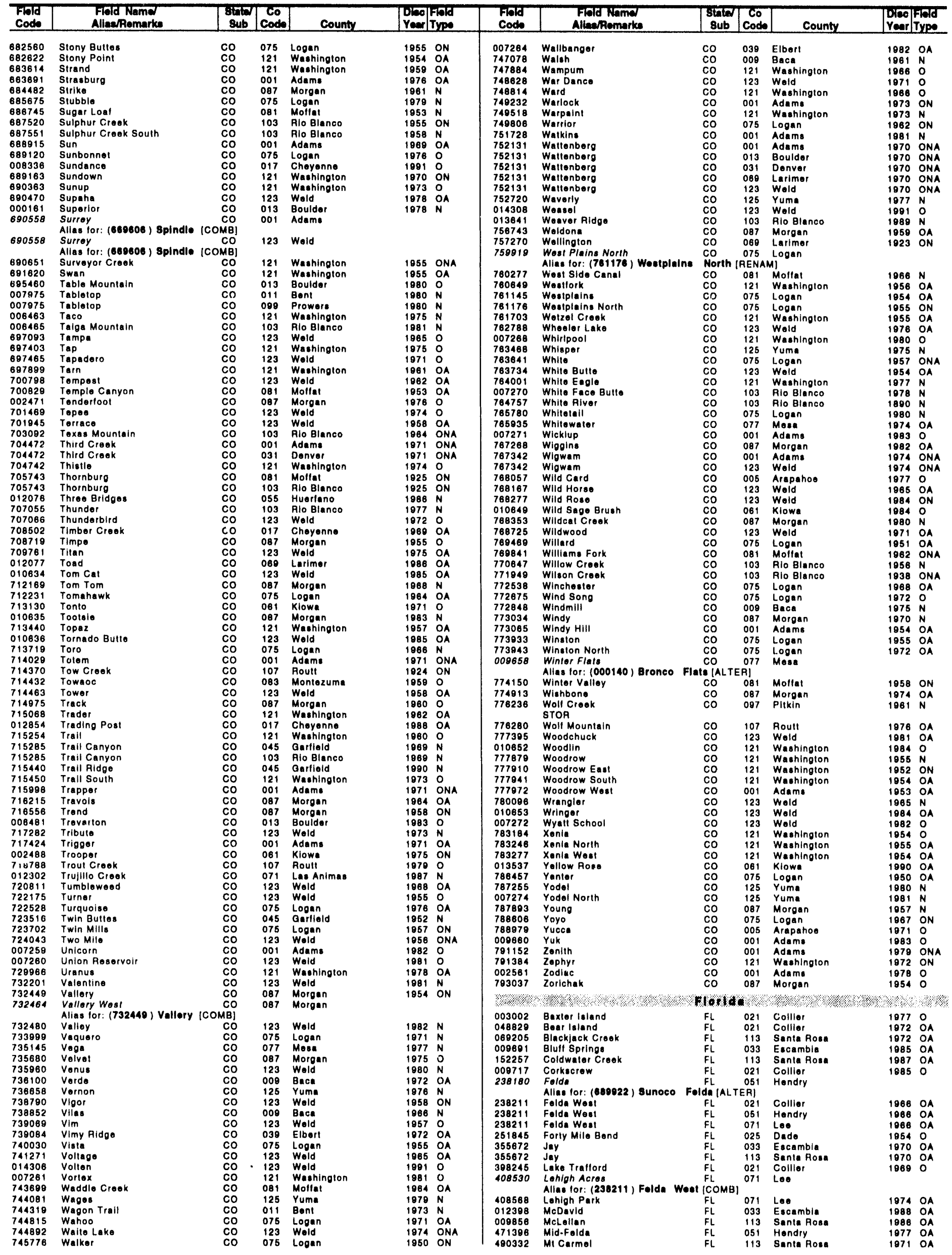




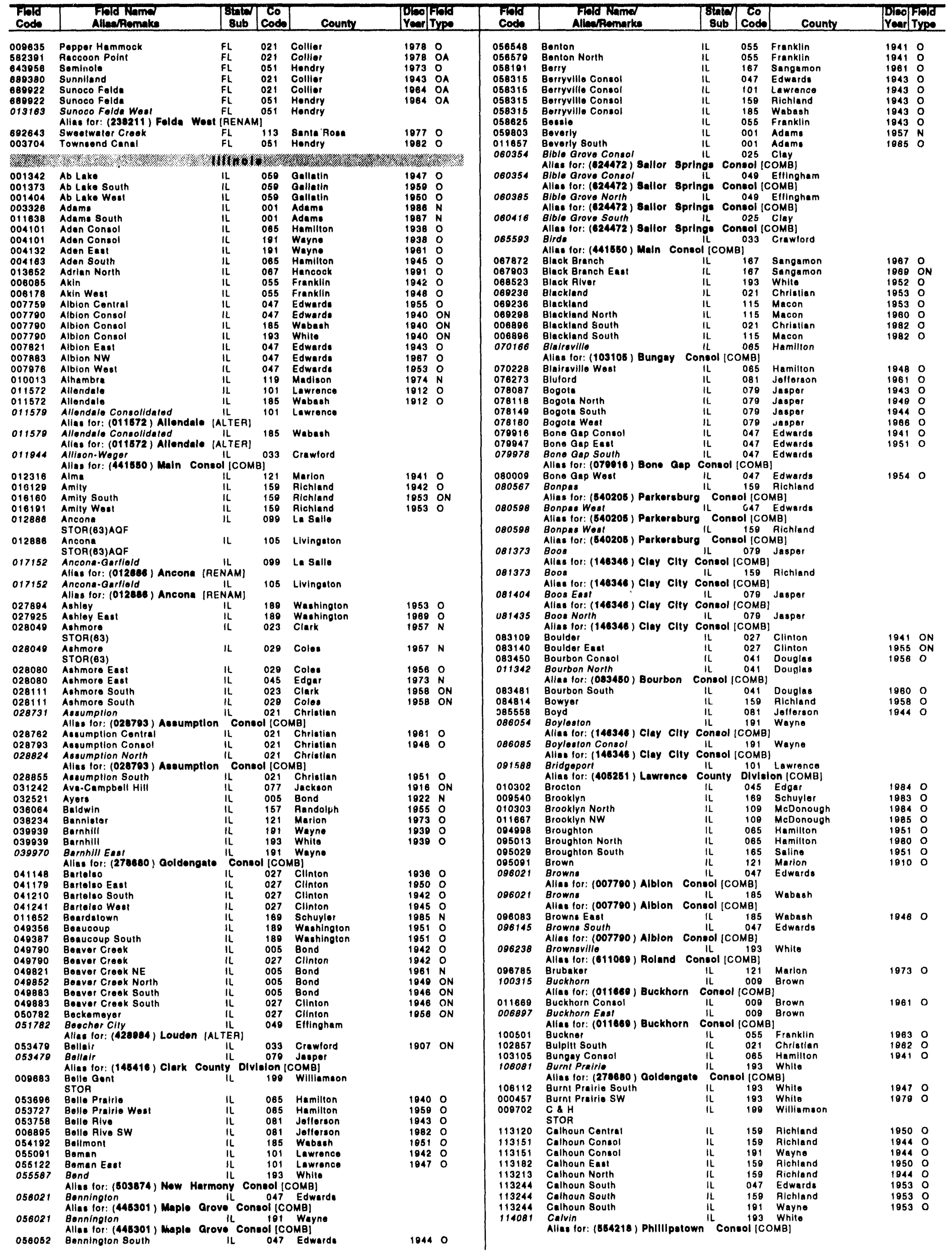


ILLINOIS

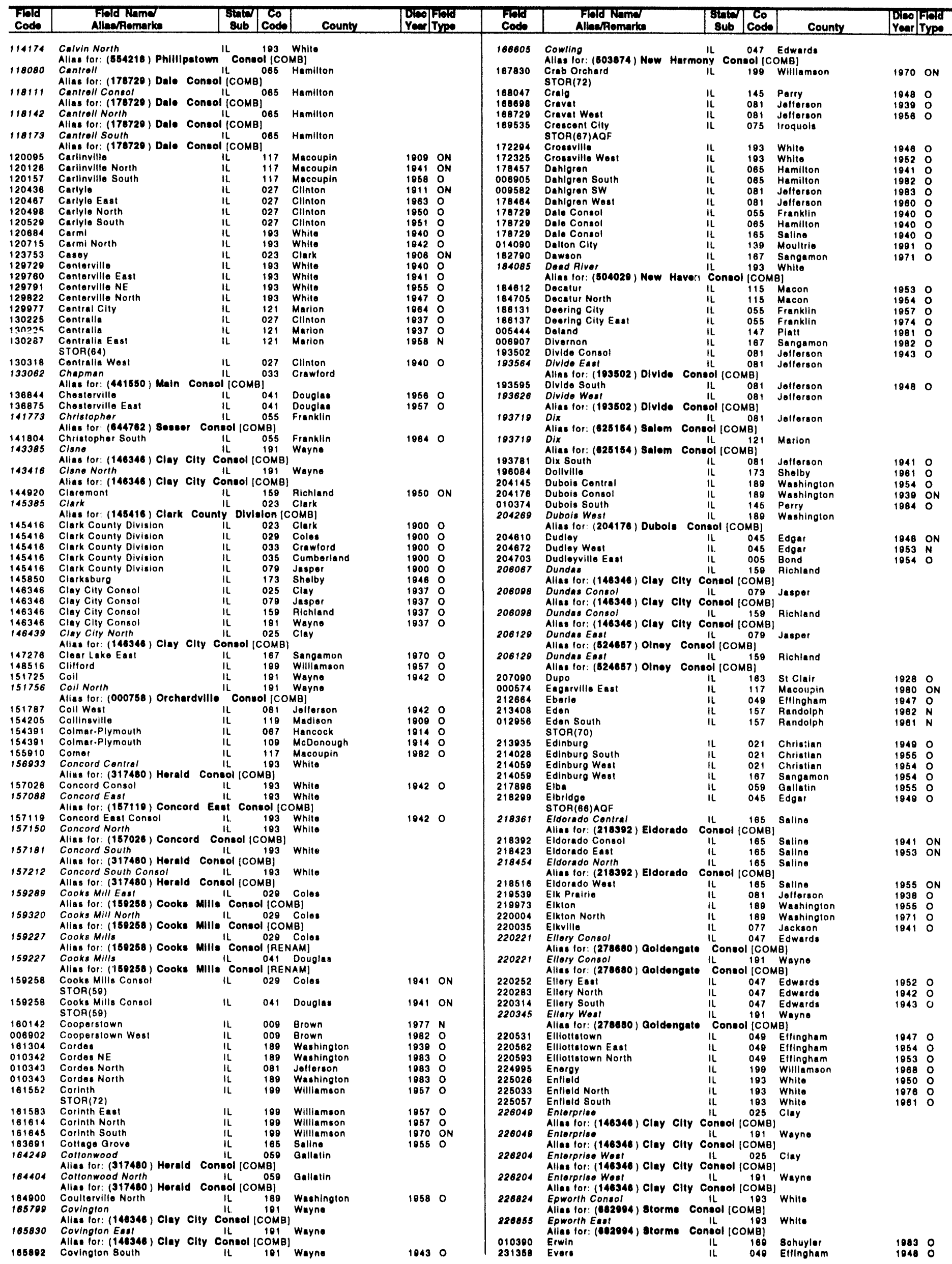




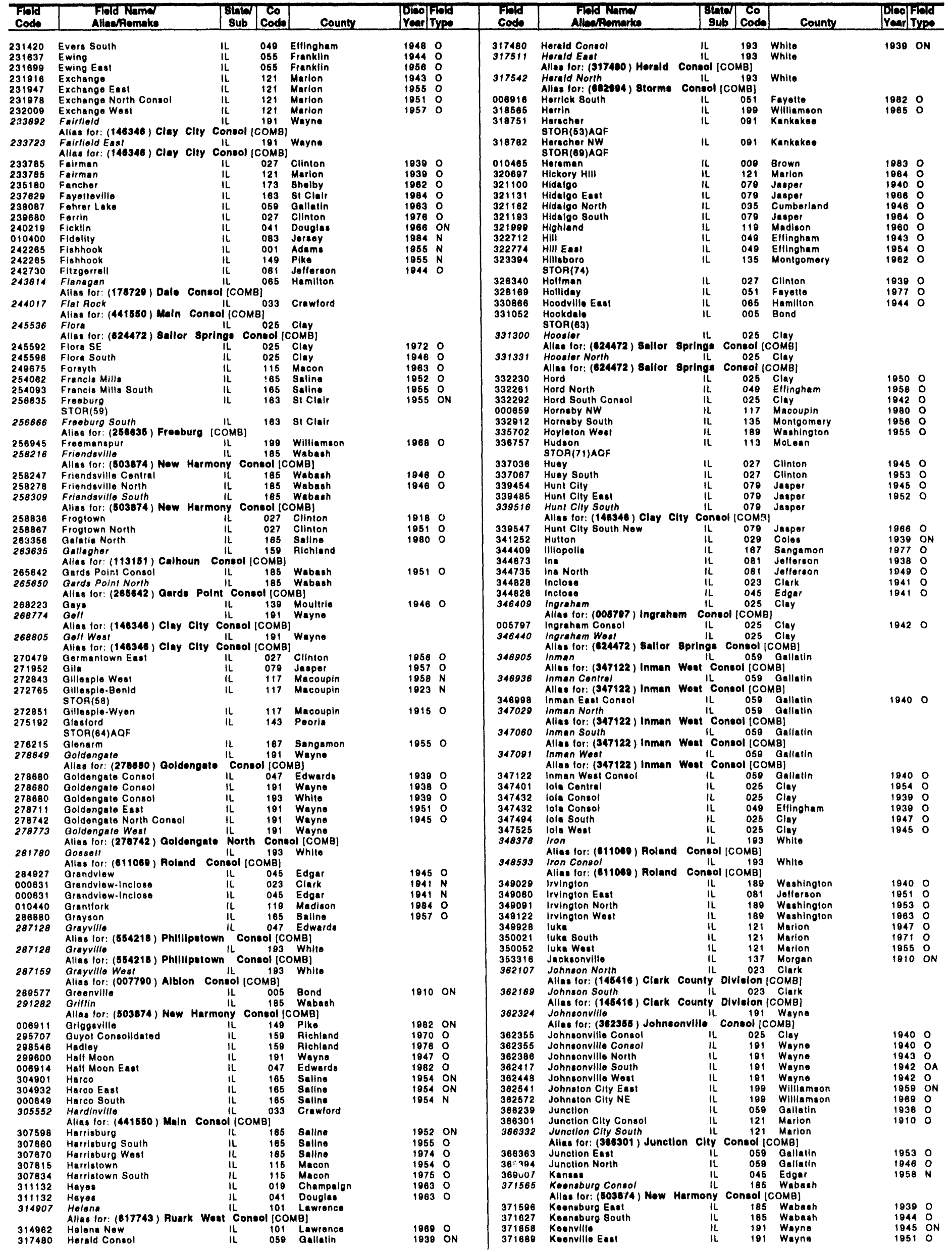


ILLINOIS

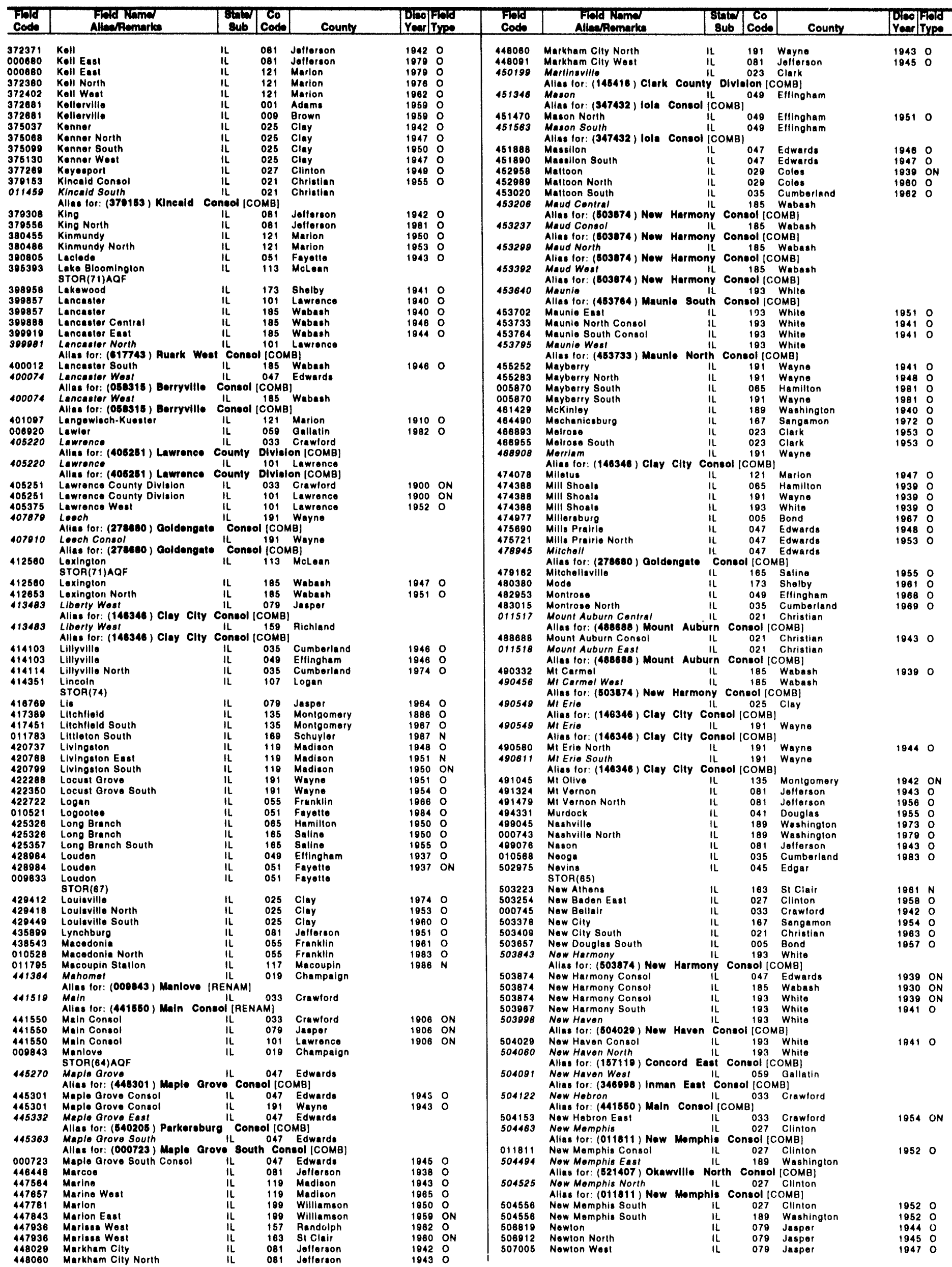




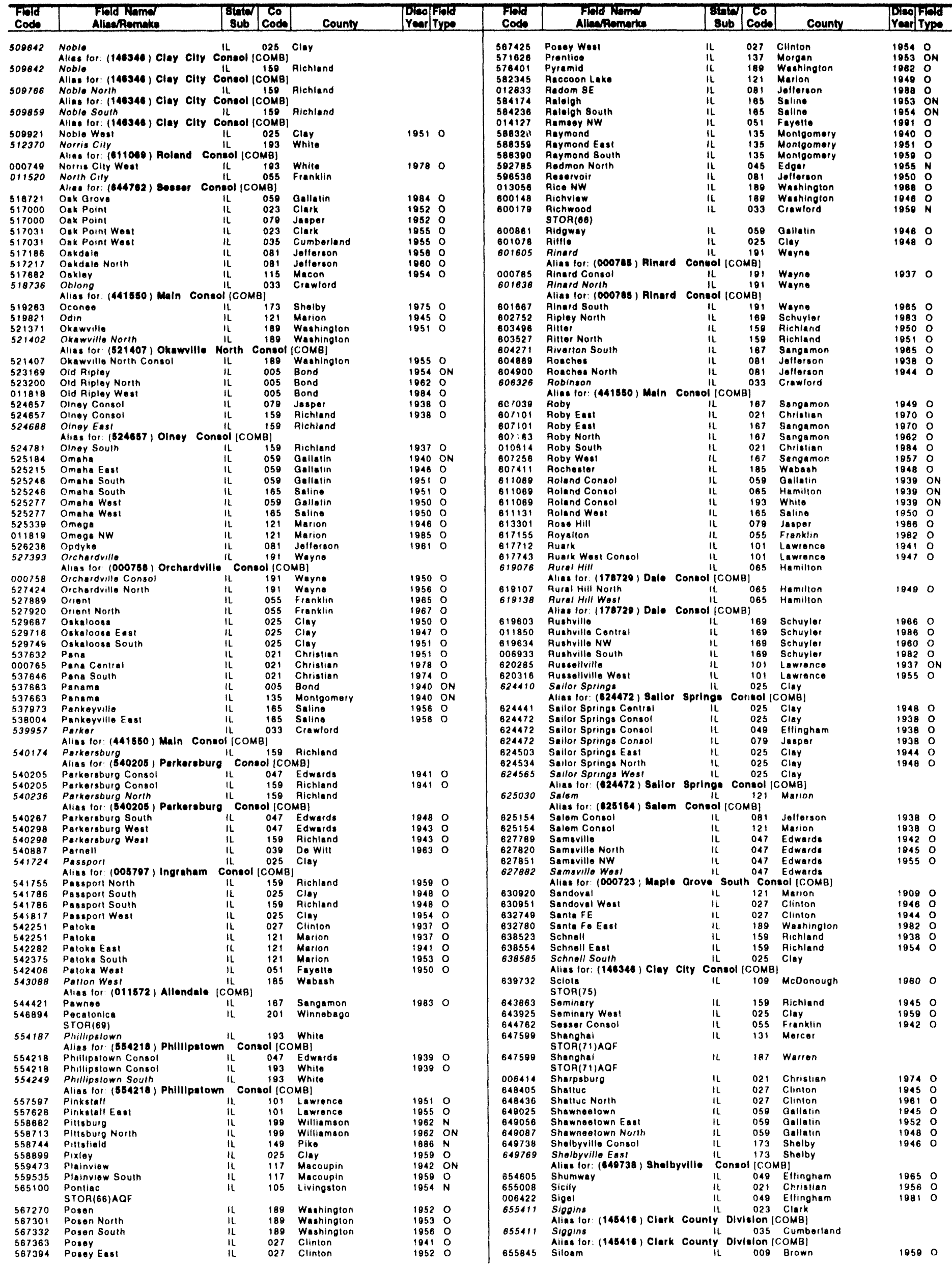


INDIANA

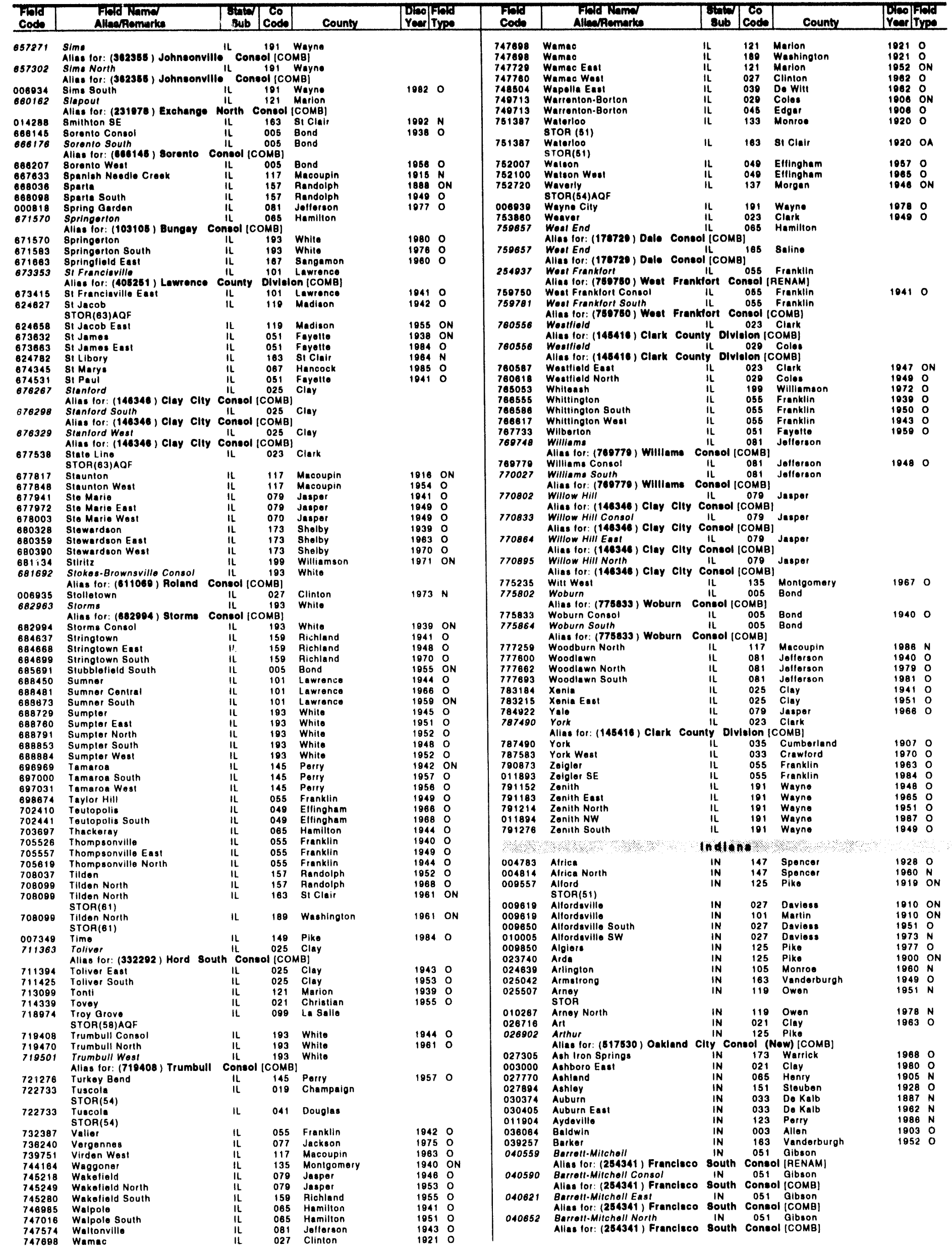


INDIANA

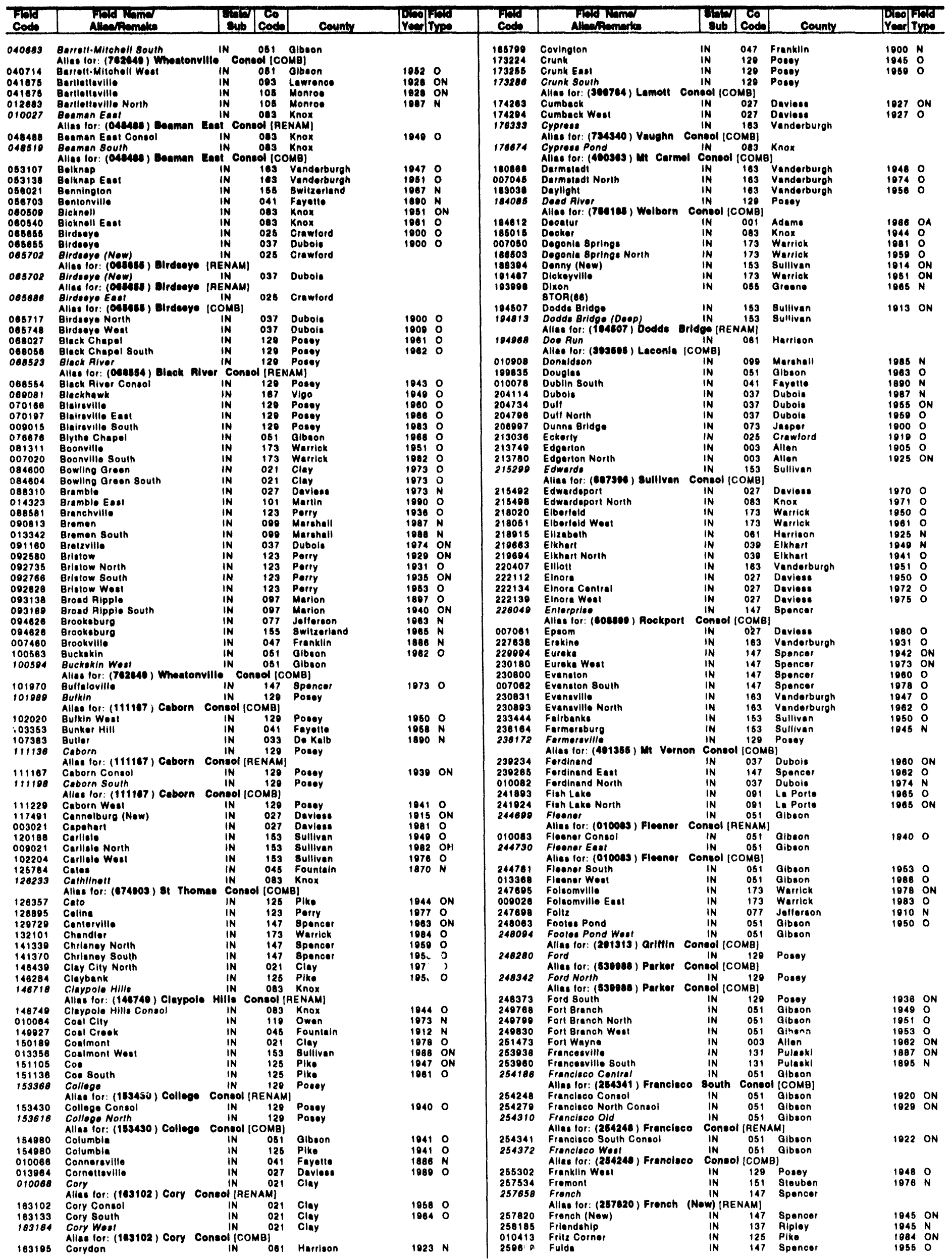


INDIANA

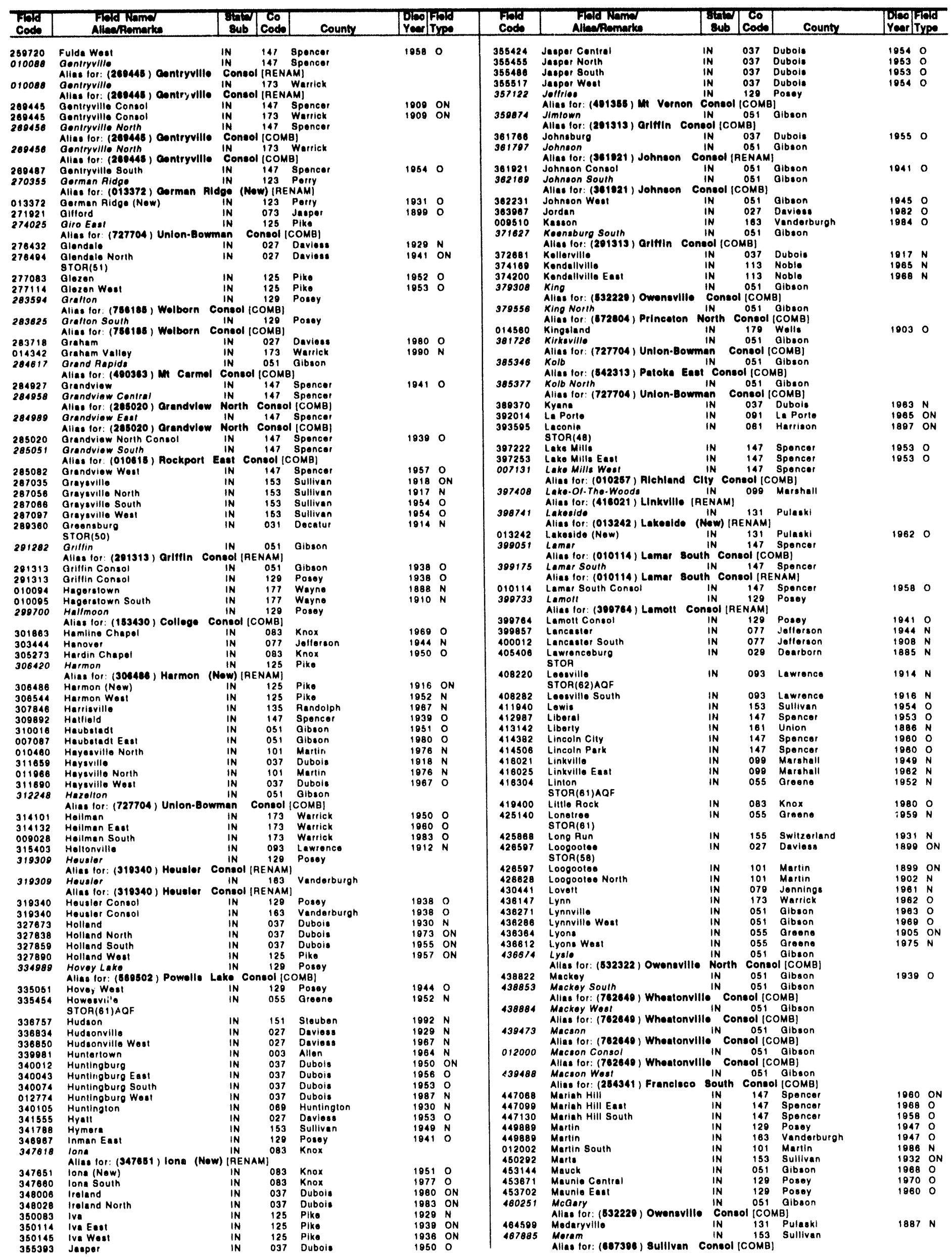


INDIANA

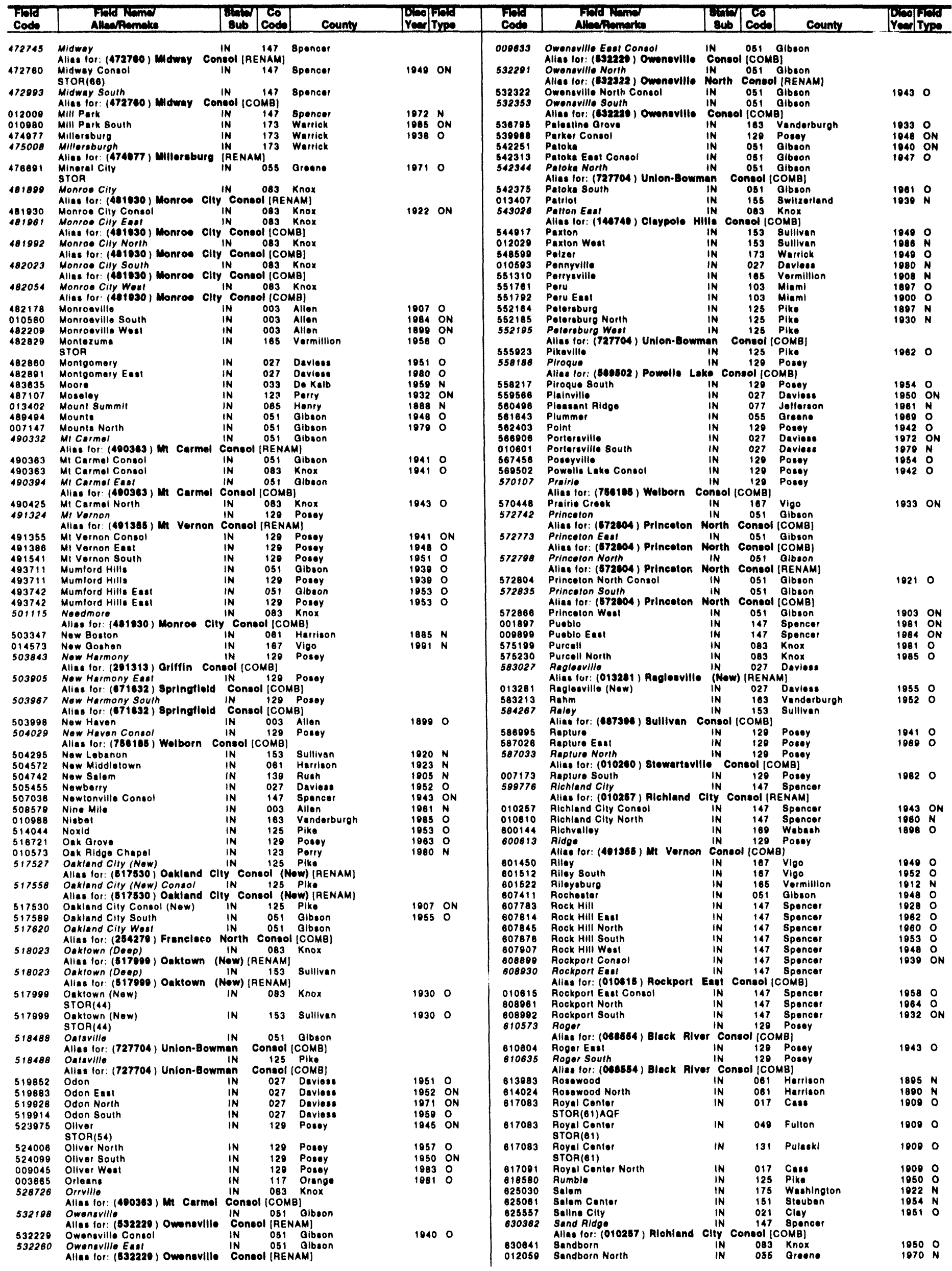


INDIANA

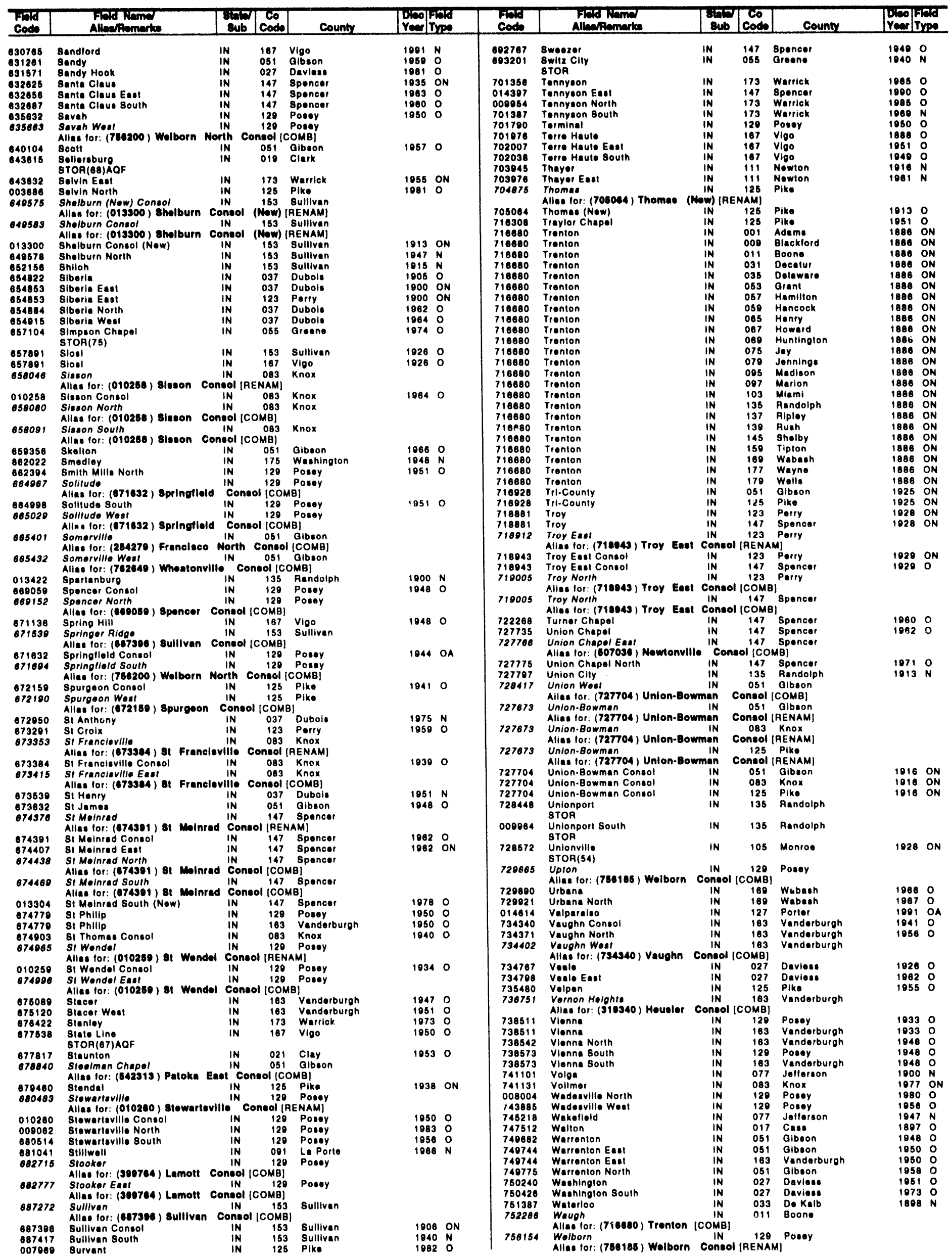


INDIANA

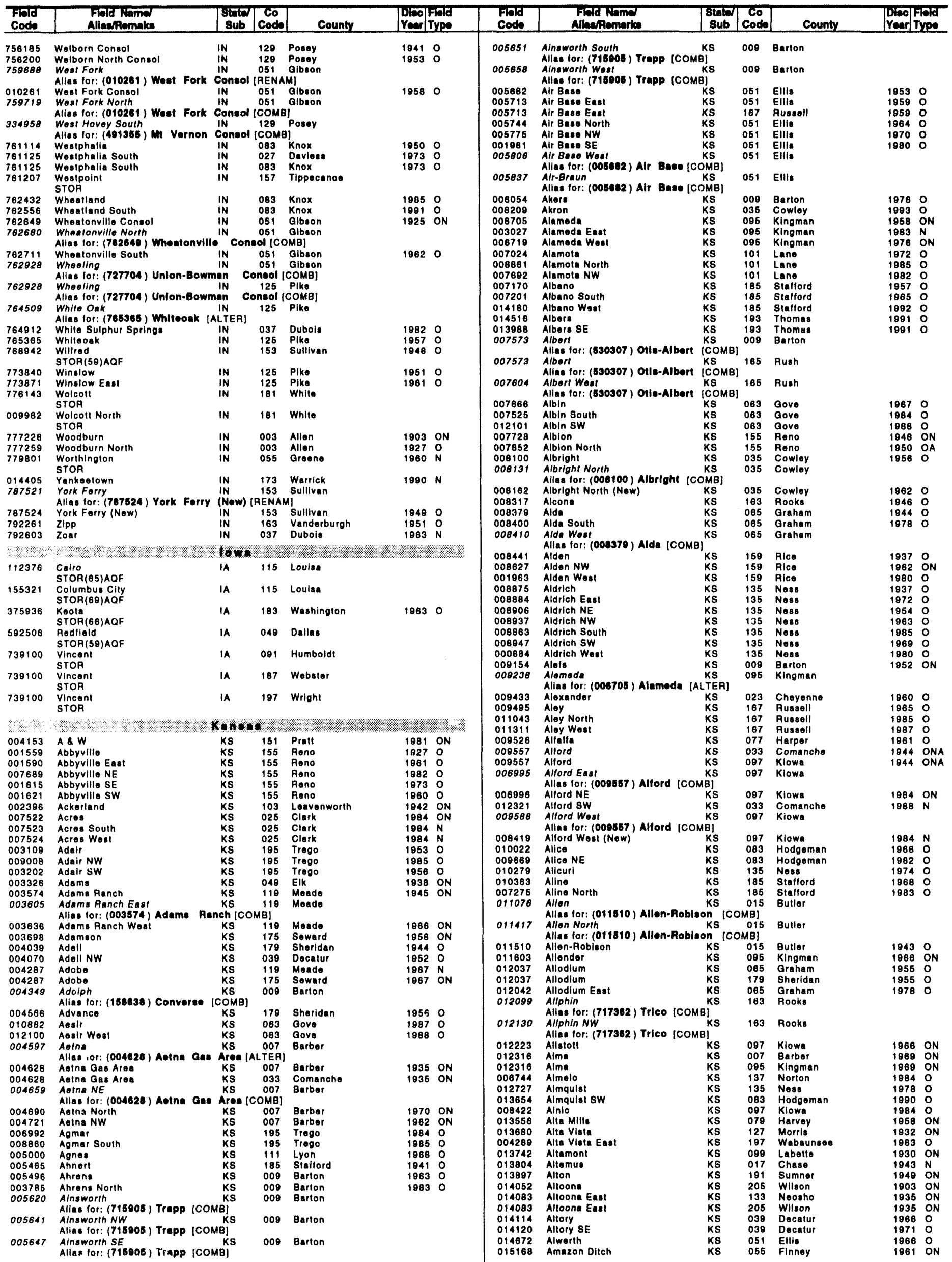


KANSAS

\begin{tabular}{|c|c|}
\hline $\begin{array}{l}\text { Find } \\
\text { Code } \\
\end{array}$ & $\begin{array}{l}\text { Fold Name } \\
\text { Aliear Remarke }\end{array}$ \\
\hline $\begin{array}{l}013957 \\
013988 \\
015323\end{array}$ & $\begin{array}{l}\text { Amazon Ditch East } \\
\text { Amazon Ditch NE } \\
\text { Amber Crook }\end{array}$ \\
\hline $\begin{array}{l}015323 \\
000070\end{array}$ & $\begin{array}{l}\text { Amber Crook } \\
\text { Amber Crook North }\end{array}$ \\
\hline 004071 & Ambor Crook South \\
\hline $\begin{array}{l}015385 \\
015394\end{array}$ & $\begin{array}{l}\text { Amber Mills } \\
\text { Amber Mills South }\end{array}$ \\
\hline $\begin{array}{l}015394 \\
015540\end{array}$ & $\begin{array}{l}\text { Amber Mills South } \\
\text { Amboy }\end{array}$ \\
\hline & Alias for: (446470) Marcotte ic \\
\hline 015571 & $\begin{array}{l}\text { Amboy SW } \\
\text { Alias for: }(446470) \text { Marcotte }\end{array}$ \\
\hline $\begin{array}{l}015633 \\
007526\end{array}$ & \\
\hline $\begin{array}{l}007526 \\
015637\end{array}$ & $\begin{array}{l}\text { Ambroso EAst } \\
\text { Ambrose SE }\end{array}$ \\
\hline $\begin{array}{l}015637 \\
007277\end{array}$ & $\begin{array}{l}\text { Ambrose SE } \\
\text { Ambrose South }\end{array}$ \\
\hline $\begin{array}{l}007277 \\
011313\end{array}$ & $\begin{array}{l}\text { Ambrose South } \\
\text { Ambrosia }\end{array}$ \\
\hline $\begin{array}{l}011313 \\
015664\end{array}$ & $\begin{array}{l}\text { Ambrosia } \\
\text { Ambh }\end{array}$ \\
\hline $\begin{array}{l}015664 \\
015788\end{array}$ & $\begin{array}{l}\text { Ameh } \\
\text { Amerest }\end{array}$ \\
\hline 007694 & $\begin{array}{l}\text { Amerest } \\
\text { Amerson }\end{array}$ \\
\hline 015912 & Amos \\
\hline 015974 & Amos NW \\
\hline 016036 & Amos Wost \\
\hline 016541 & Amstutz \\
\hline 016550 & Amstutz South \\
\hline 000888 & Amstutz sW \\
\hline 016552 & Amy \\
\hline 017690 & Andover South \\
\hline 017690 & Andover South \\
\hline 017772 & Andreson \\
\hline 017772 & Andreson \\
\hline 007527 & Androson South \\
\hline 017834 & $\begin{array}{l}\text { Androws } \\
\text { Alias for: (269208) Coneseo-Ed }\end{array}$ \\
\hline $\begin{array}{l}018268 \\
018298\end{array}$ & Ang Ang North \\
\hline 018547 & Angoll \\
\hline 007696 & Angell SE \\
\hline $\begin{array}{l}007278 \\
011900\end{array}$ & $\begin{array}{l}\text { Angell South } \\
\text { Angman }\end{array}$ \\
\hline $\begin{array}{l}011900 \\
014181\end{array}$ & $\begin{array}{l}\text { Angman } \\
\text { Angman South }\end{array}$ \\
\hline 018764 & Angola \\
\hline 018888 & Anita \\
\hline 019105 & Anna \\
\hline $\begin{array}{l}019229 \\
019260\end{array}$ & $\begin{array}{l}\text { Annabelle } \\
\text { Annamao }\end{array}$ \\
\hline $\begin{array}{l}018260 \\
018384\end{array}$ & $\begin{array}{l}\text { Annamae } \\
\text { Annelly }\end{array}$ \\
\hline 019415 & Annelly East \\
\hline 019446 & Annoliy North \\
\hline 018477 & Annelly South \\
\hline 019508 & Annelly West \\
\hline 019539 & Anness \\
\hline 009010 & Anness South \\
\hline 010545 & Anness West \\
\hline 019601 & $\begin{array}{l}\text { Annon } \\
\text { Alias for: (717362) Trico (COM }\end{array}$ \\
\hline 019632 & Annon South \\
\hline & \\
\hline 011047 & Annuschat \\
\hline $\begin{array}{l}019787 \\
019849\end{array}$ & $\begin{array}{l}\text { Anson } \\
\text { Anson SE }\end{array}$ \\
\hline $\begin{array}{l}019849 \\
019880\end{array}$ & $\begin{array}{l}\text { Anson SE } \\
\text { Anson South }\end{array}$ \\
\hline 019880 & Allas lor: (010787) Anson $1 C O$ \\
\hline 020097 & $\begin{array}{l}\text { Antolope } \\
\text { Alias for: (428581) Lost Sprln }\end{array}$ \\
\hline 020100 & Antelope Creok \\
\hline 020128 & Antolope East \\
\hline & Allas for: (428581) Lost Sprin \\
\hline 020252 & $\begin{array}{l}\text { Antelope North } \\
\text { Allas for: (428581) Loet Sprin }\end{array}$ \\
\hline 020376 & $\begin{array}{l}\text { Alias lor: (428581) Lost Sprln } \\
\text { Antolopo SE }\end{array}$ \\
\hline 然 & Aliss tor: (428581) Lost Sprin \\
\hline 020438 & Antolope South \\
\hline & Alias for: (428581) Lost Sprin \\
\hline 020562 & Anthony \\
\hline 021244 & Anton \\
\hline & Allas for: (220378) Ellinwood \\
\hline 021430 & Antone \\
\hline 021461 & $\begin{array}{l}\text { Antonino } \\
\text { Alies for: (387129) Kraus (COI }\end{array}$ \\
\hline 001985 & Antonino (Old) \\
\hline 003029 & Antonino NE \\
\hline $\begin{array}{l}021492 \\
021523\end{array}$ & $\begin{array}{l}\text { Antonino South } \\
\text { Antonino SW }\end{array}$ \\
\hline $\begin{array}{l}021523 \\
021554\end{array}$ & $\begin{array}{l}\text { Antonino SW } \\
\text { Antonino Townsite }\end{array}$ \\
\hline $\begin{array}{l}021554 \\
004854\end{array}$ & $\begin{array}{l}\text { Antonino Townsite } \\
\text { Antonino Townsite East }\end{array}$ \\
\hline 004654 & $\begin{array}{l}\text { Antonino Townsite East } \\
\text { Alles for: (184801) Dochant }\end{array}$ \\
\hline 021585 & Antonino Townsite West \\
\hline 021616 & Antrim \\
\hline 004994 & Antrim South \\
\hline 007699 & Applo Valloy \\
\hline $00747 \theta$ & Appleton \\
\hline 007700 & Apploton NW \\
\hline 022523 & Appling \\
\hline 011646 & Arapahookan \\
\hline 023058 & Arbor \\
\hline 023089 & Arbuckle \\
\hline 023399 & Archer \\
\hline 007701 & Alcher West \\
\hline 023905 & Ardroy \\
\hline 023915 & Ardrey West \\
\hline 024081 & Argo \\
\hline 009673 & Ark \\
\hline 014183 & Ark River \\
\hline 024298 & Arkalon \\
\hline 024329 & Arkalon East \\
\hline & Alias for: (024298) Arkalon \\
\hline & Arkalon NE \\
\hline & Arkalon North \\
\hline 009012 & Arkalon NW \\
\hline 024360 & Arkalon Wost \\
\hline 53 & $\begin{array}{l}\text { Alias for: (024208) Arkalon I } \\
\text { Arkansas Cliy Gas Area }\end{array}$ \\
\hline
\end{tabular}




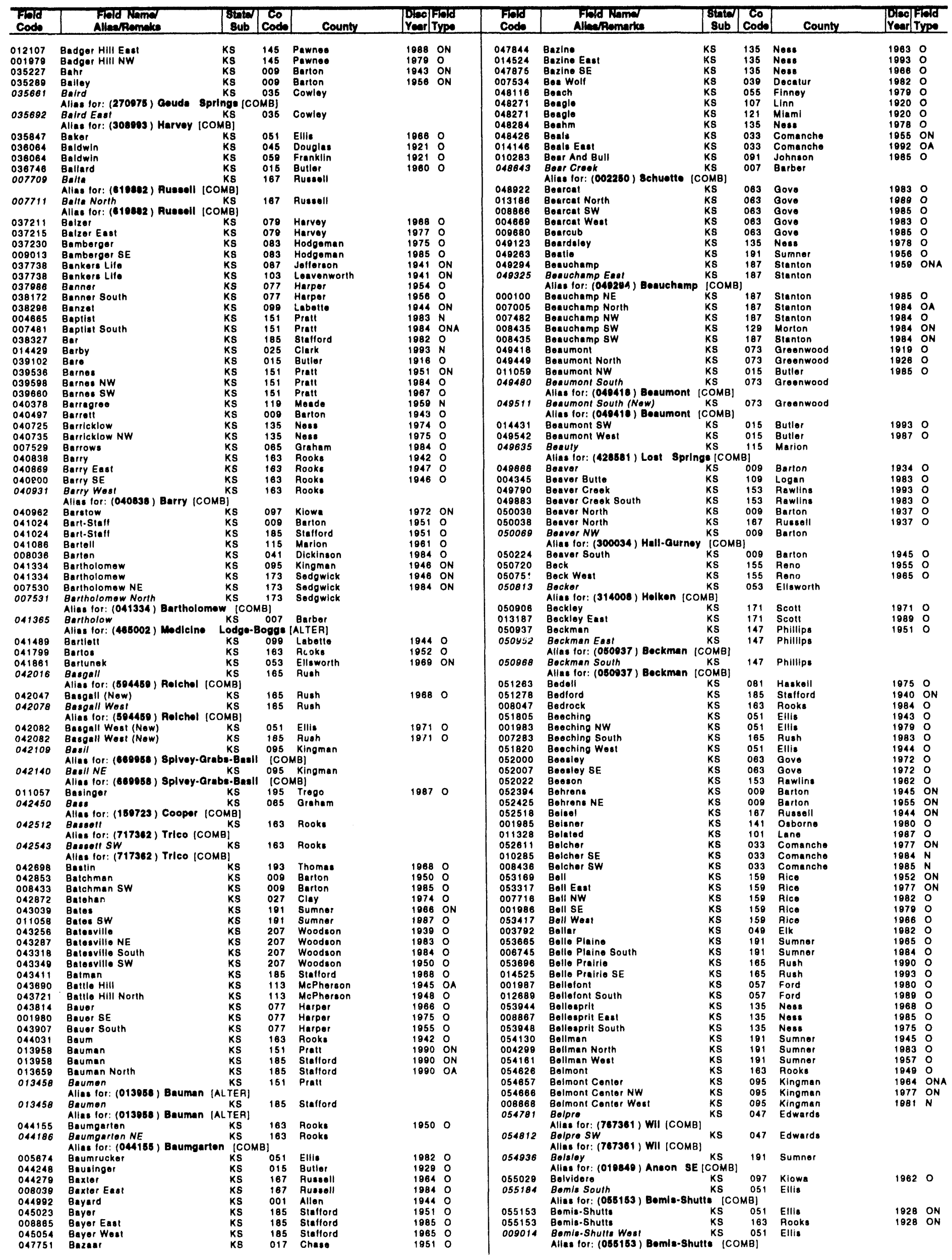


KANSAS

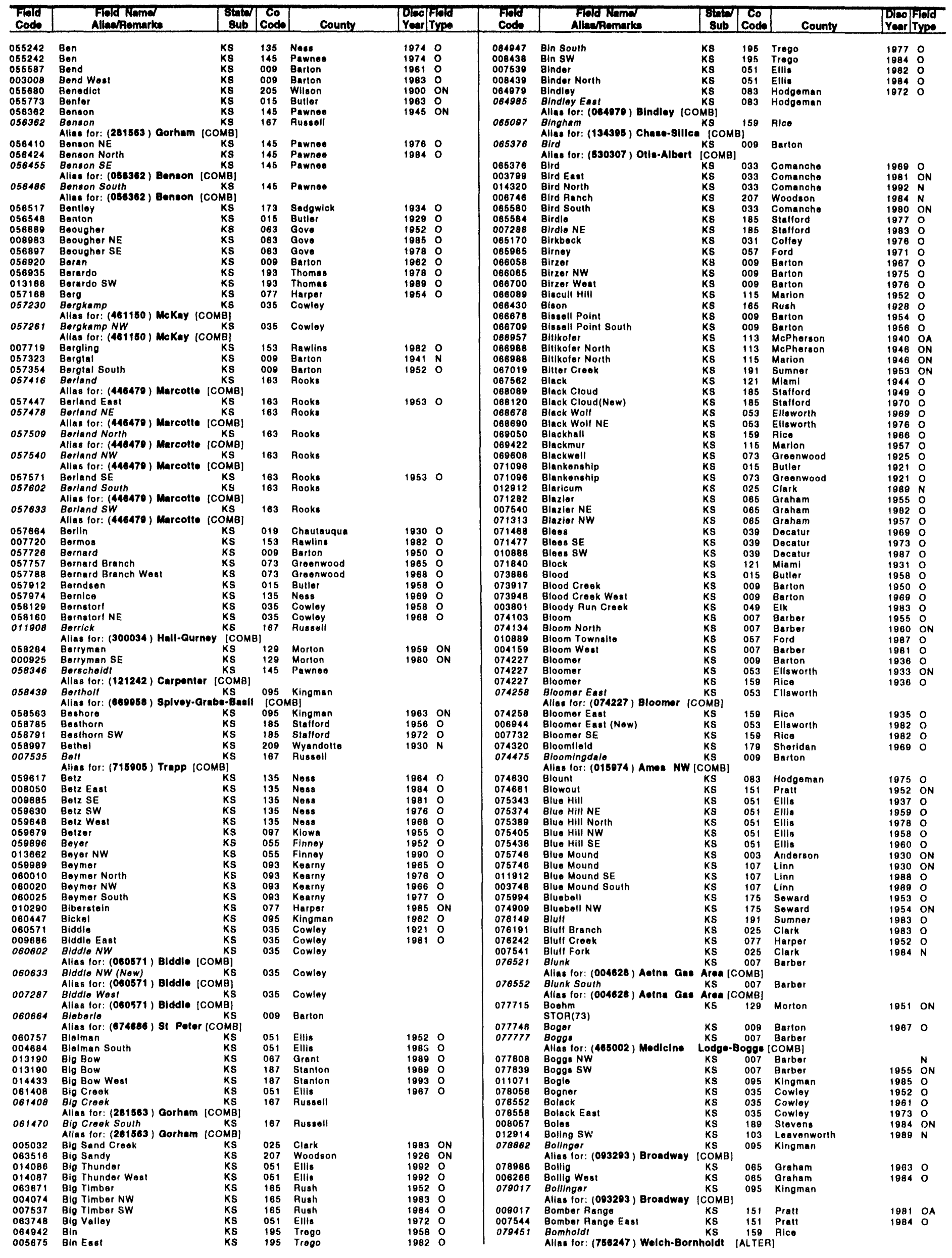


KANSAS

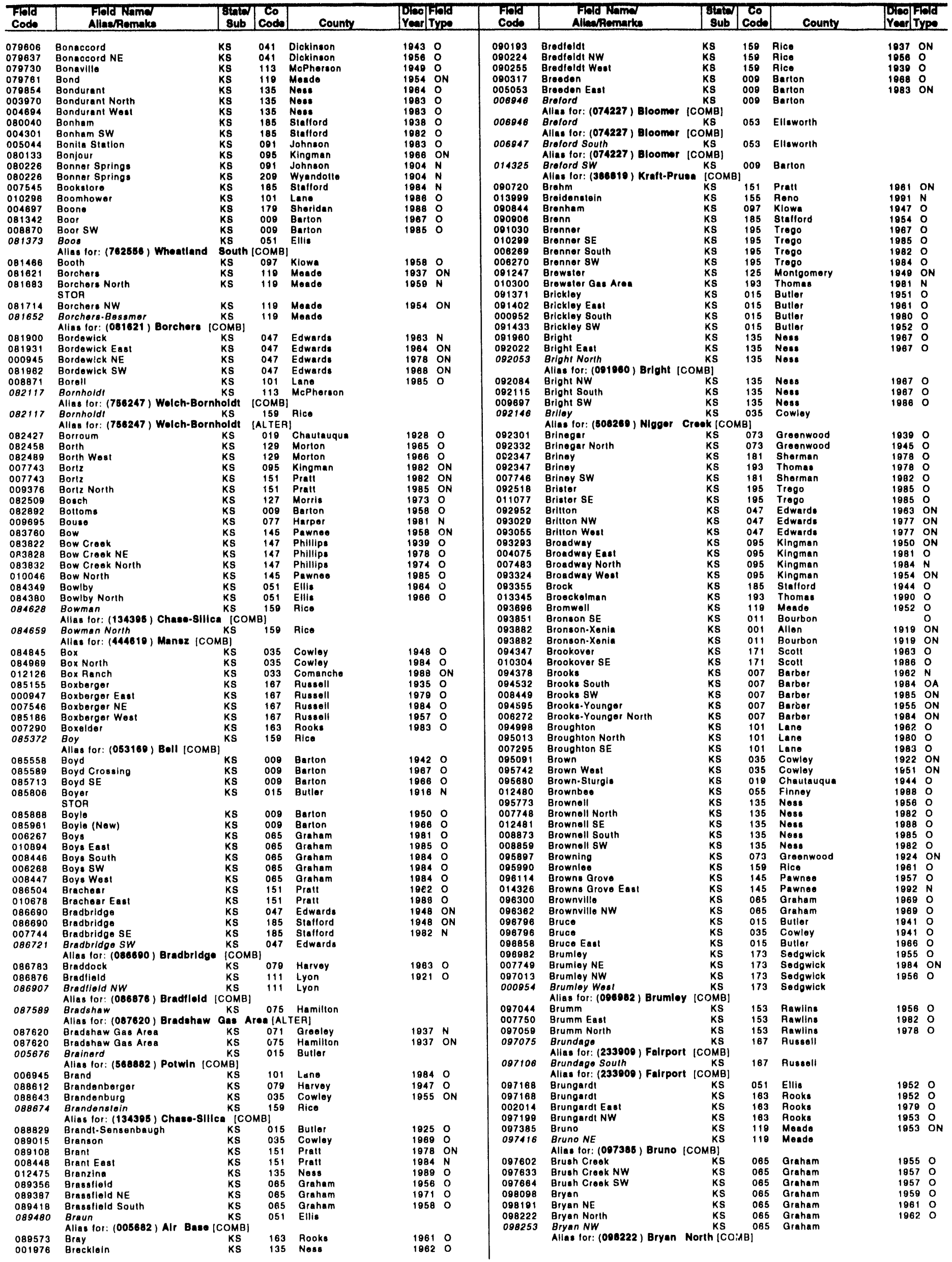


KANSAS

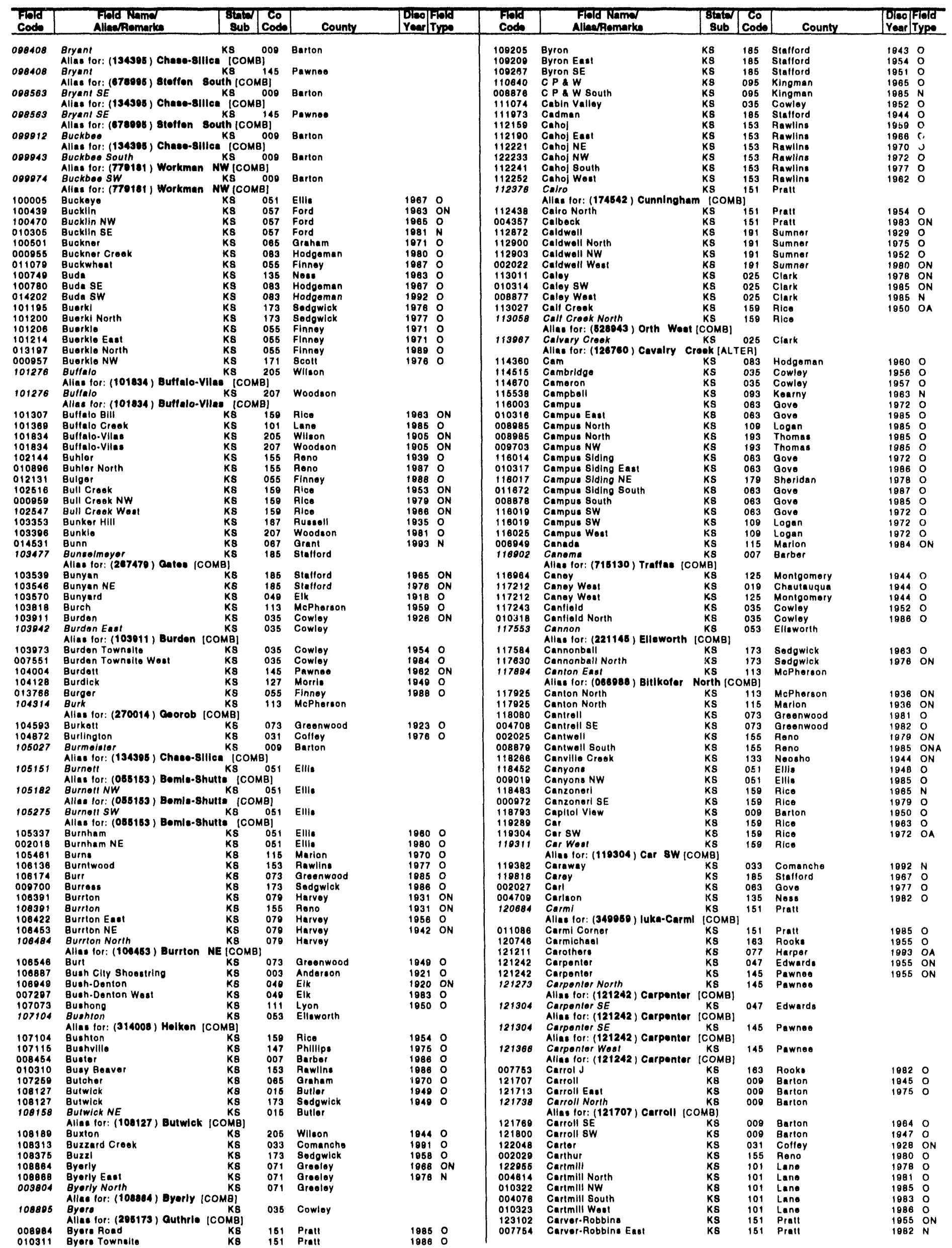


KANSAS

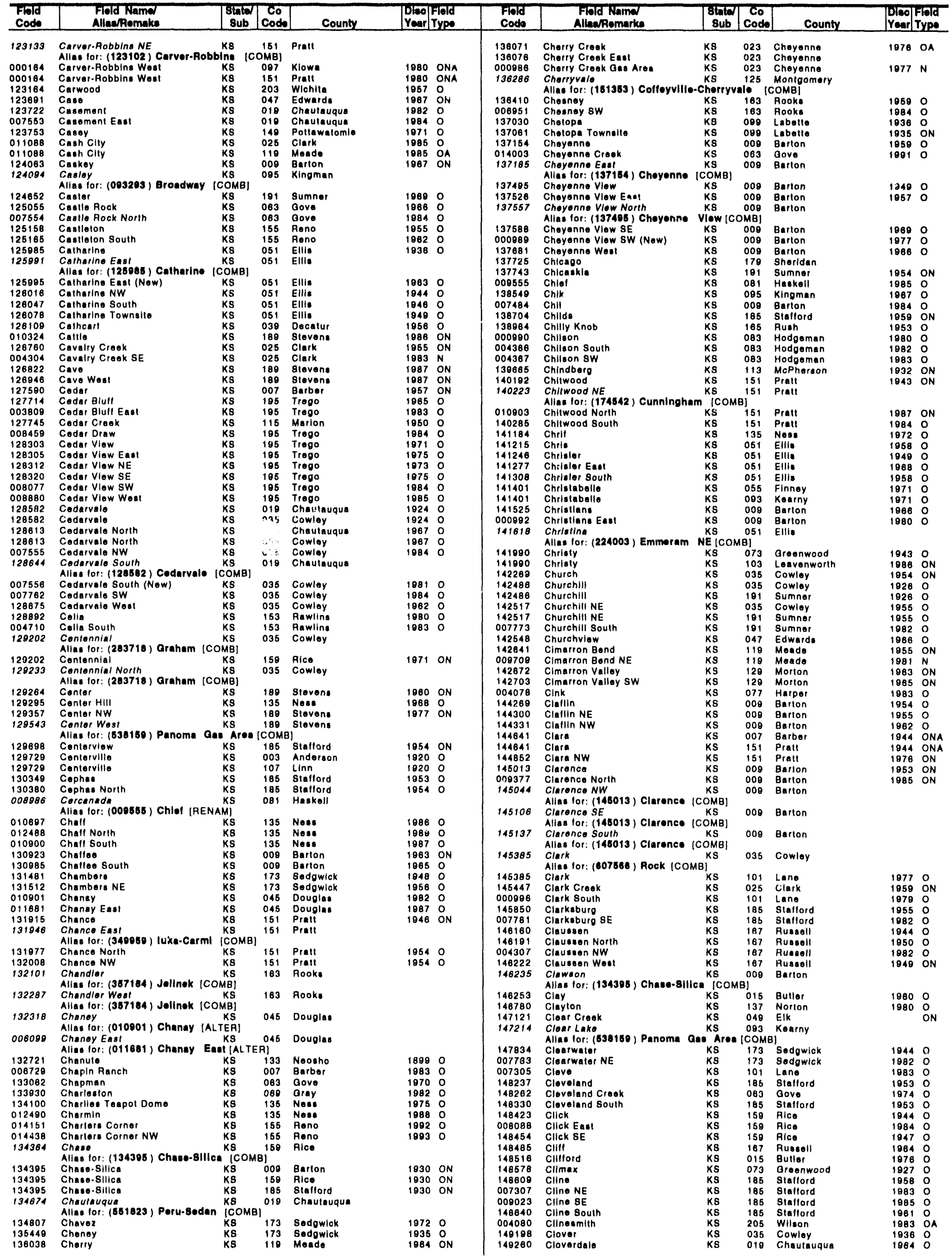


KANSAS

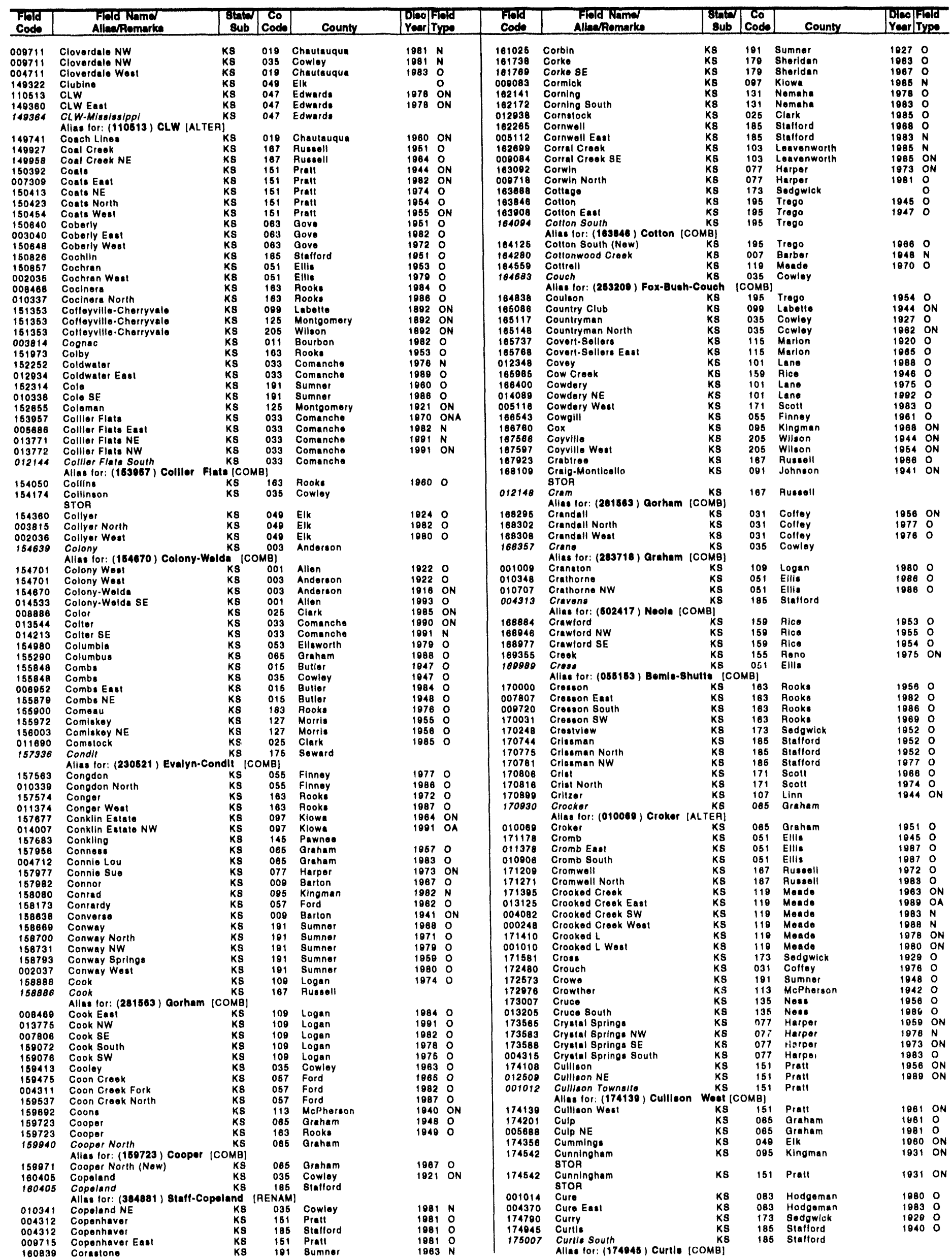


KANSAS

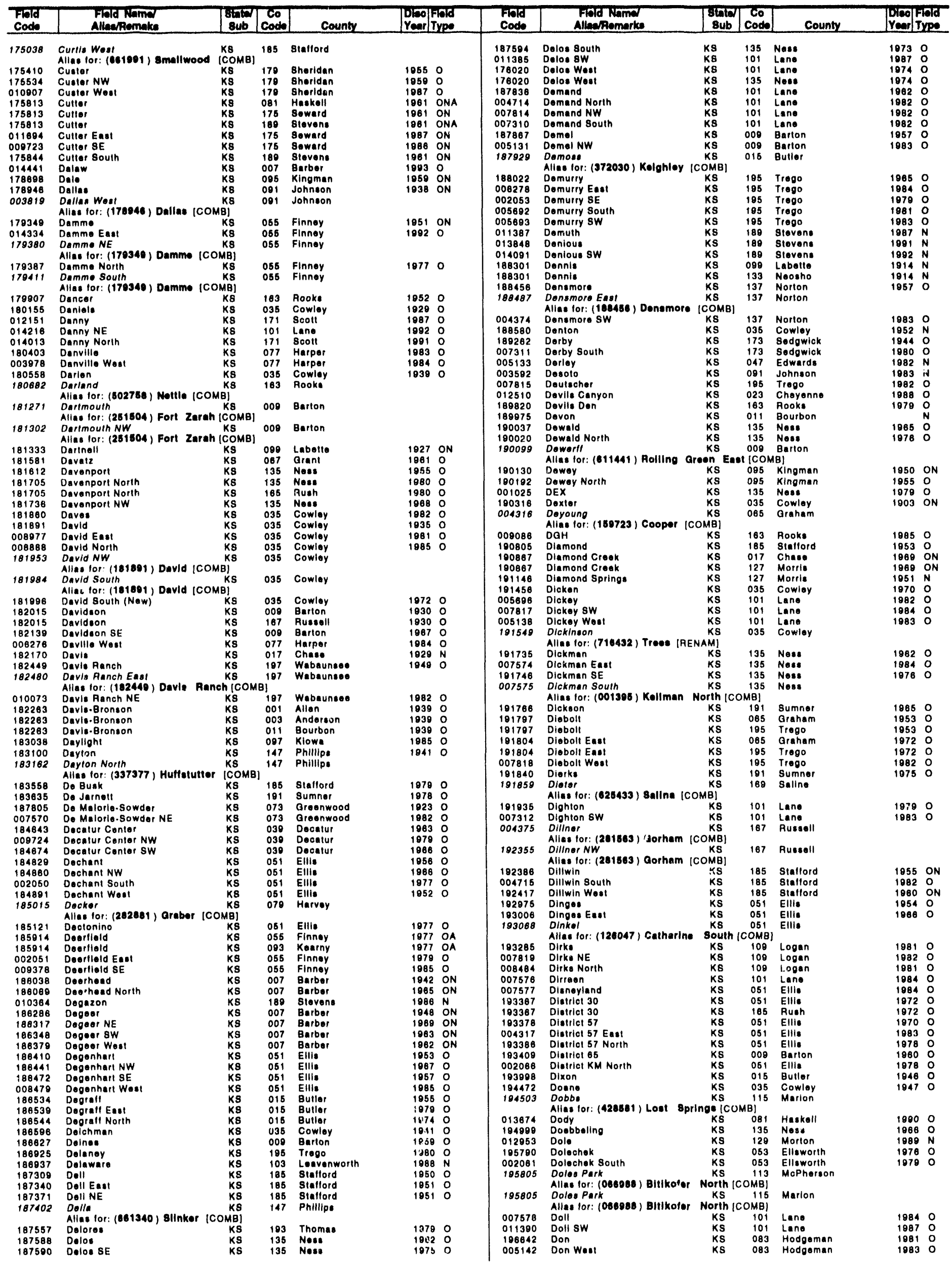


KANSAS

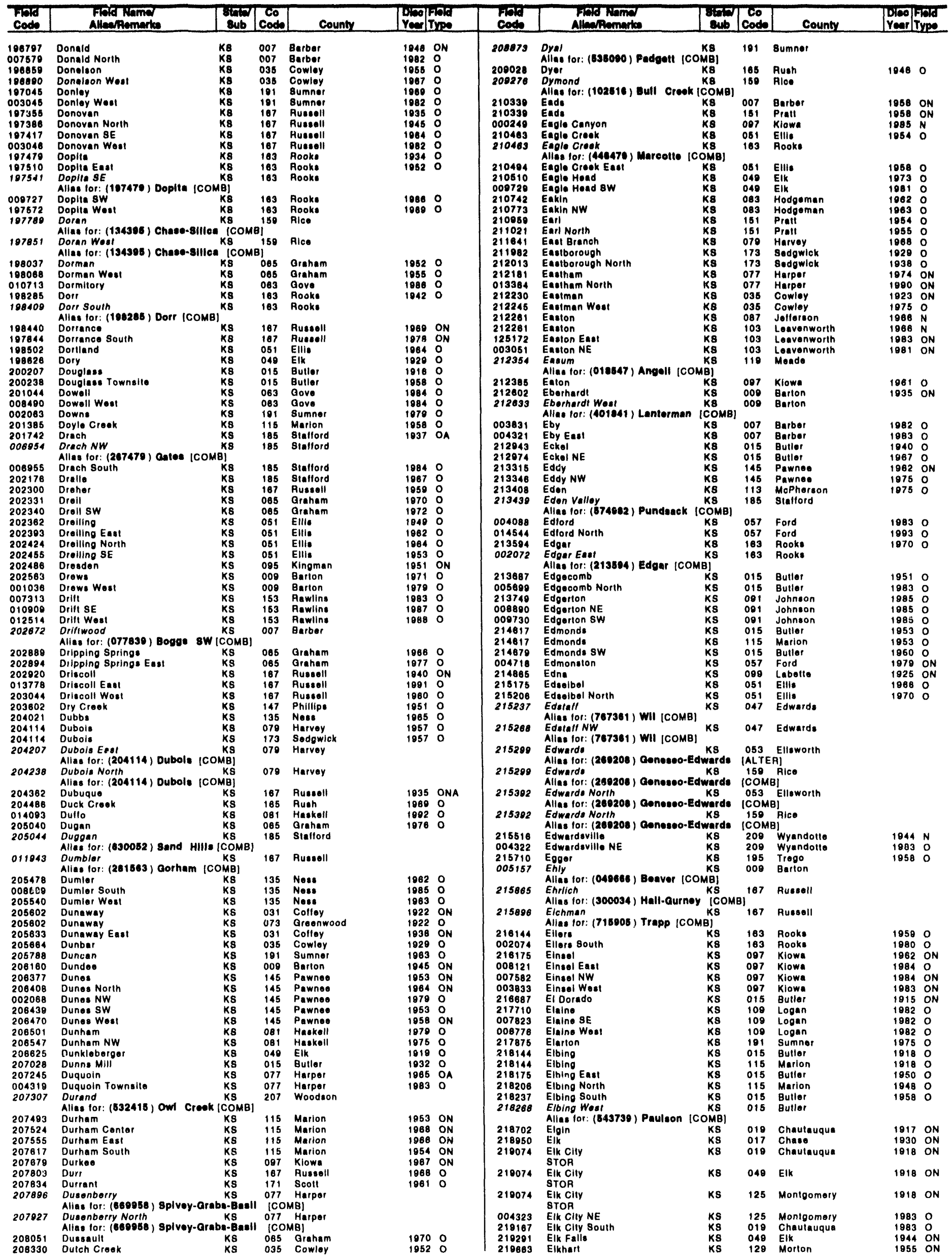


KANSAS

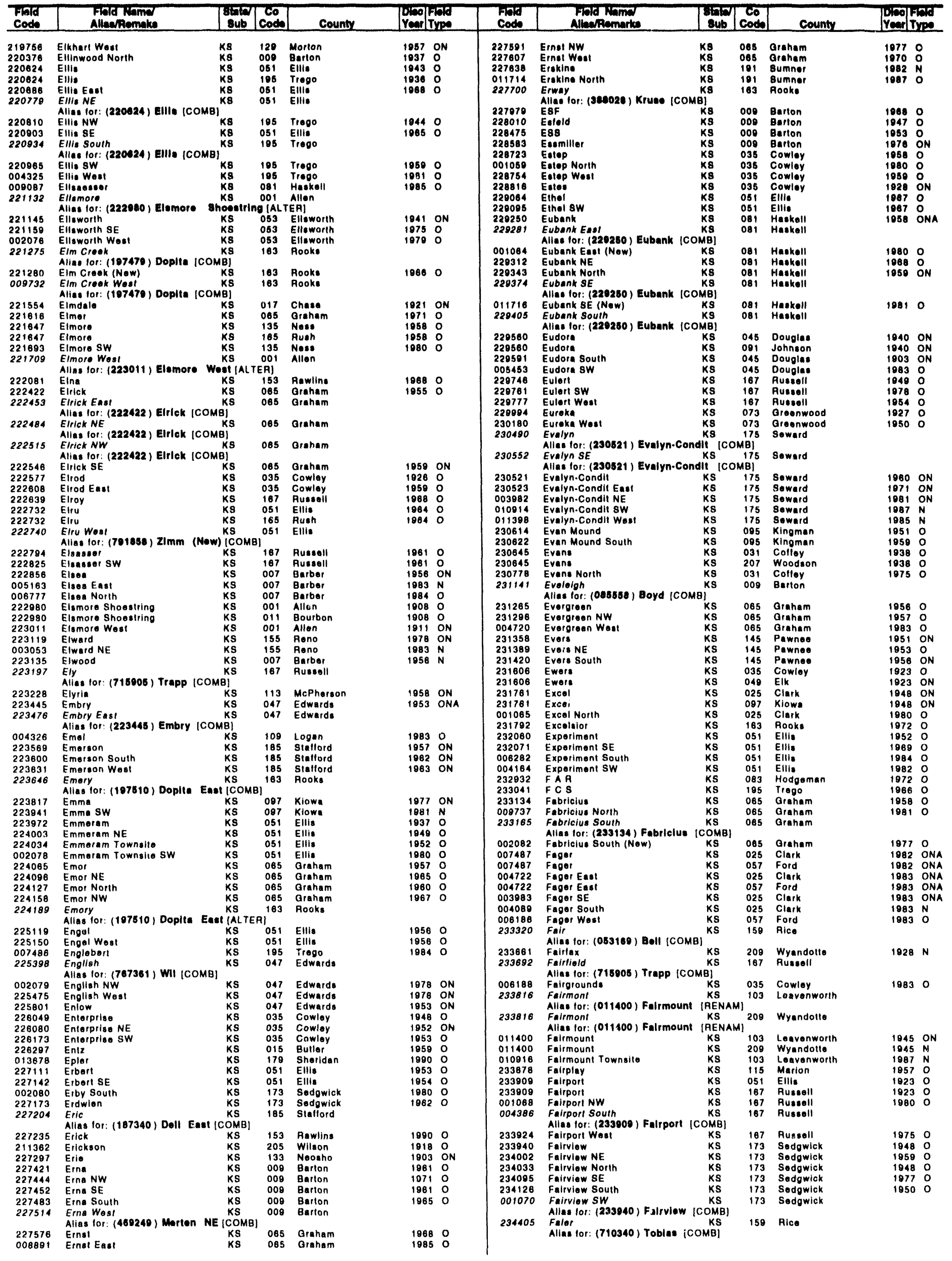


KANSAS

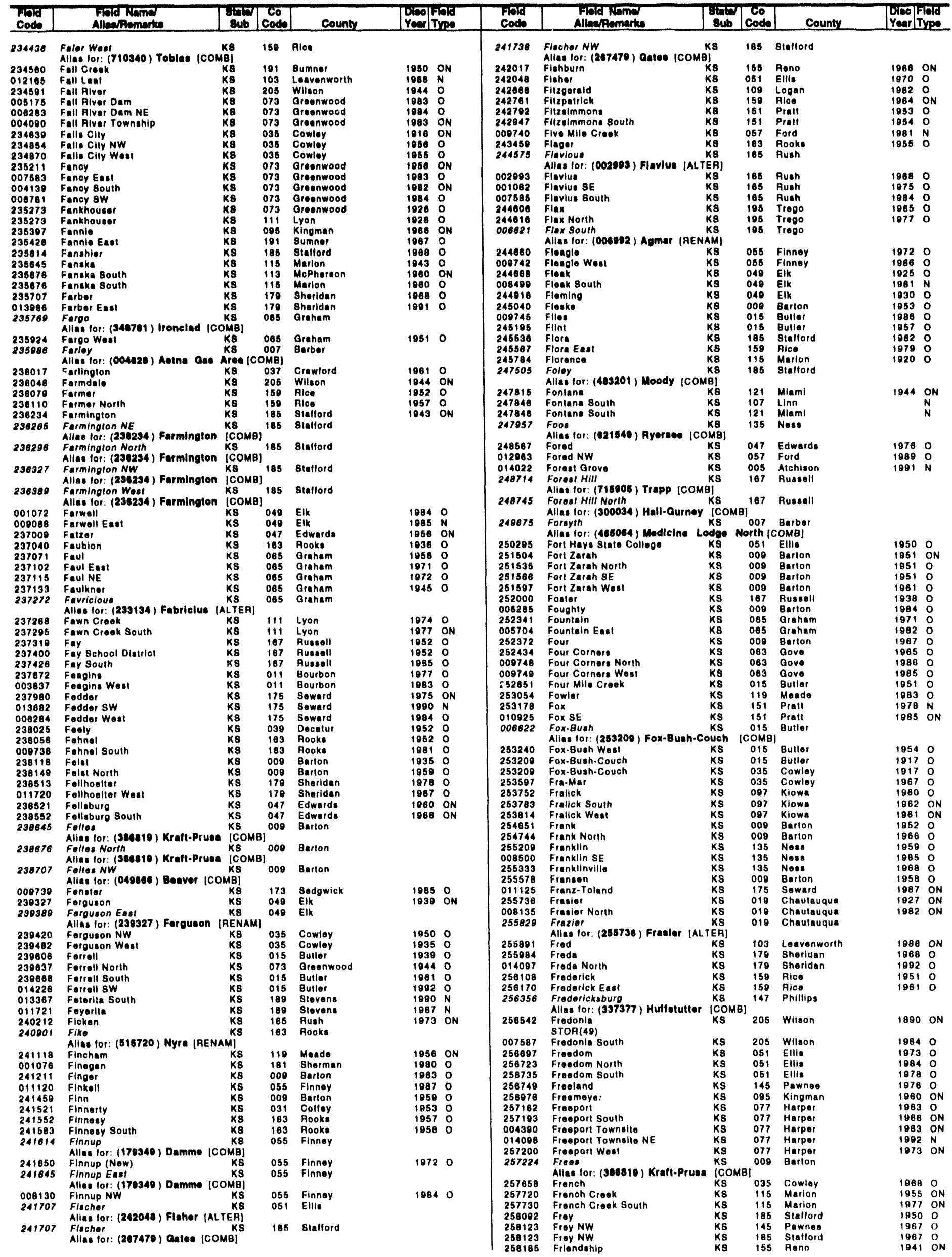


KANSAS

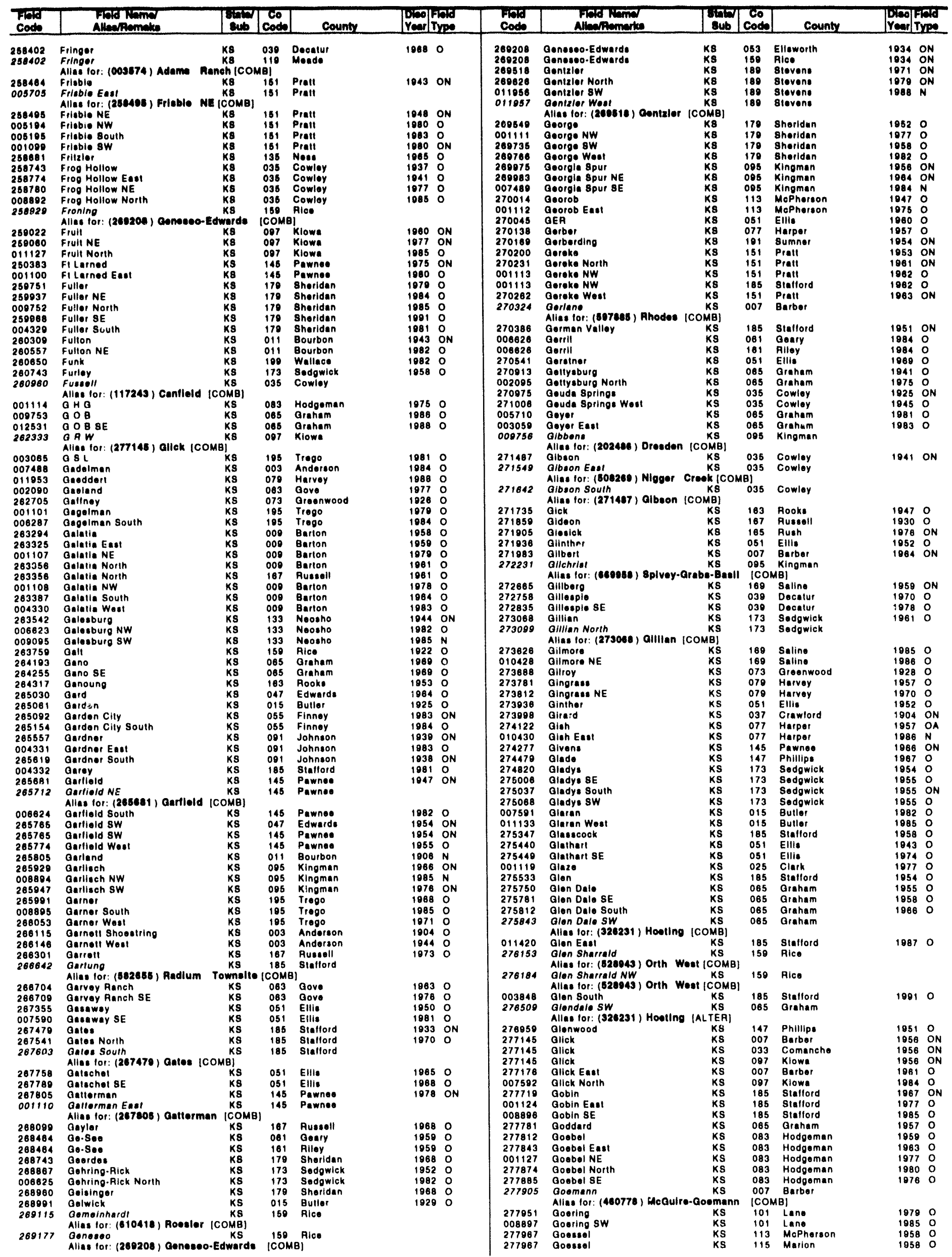


KANSAS

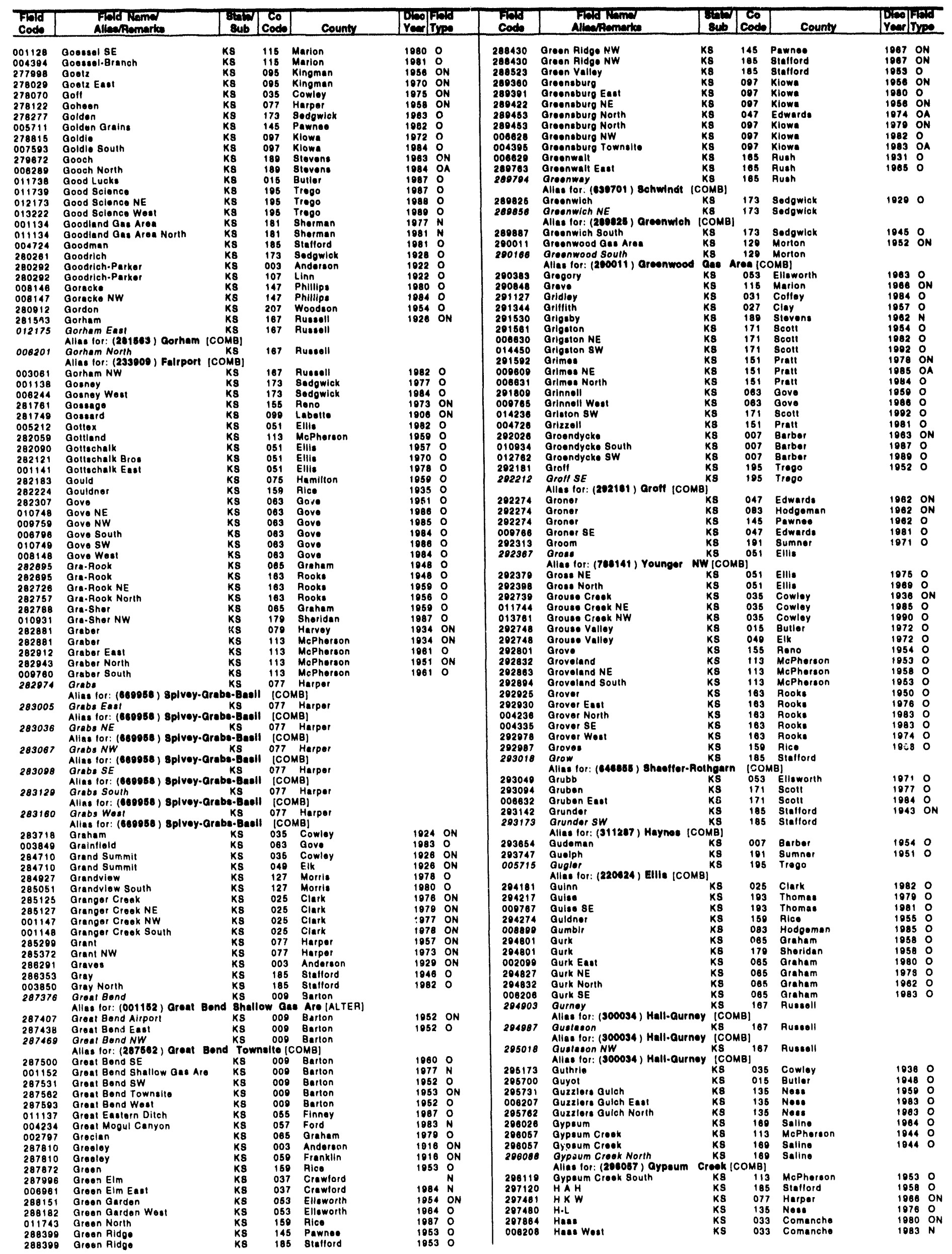


KANSAS

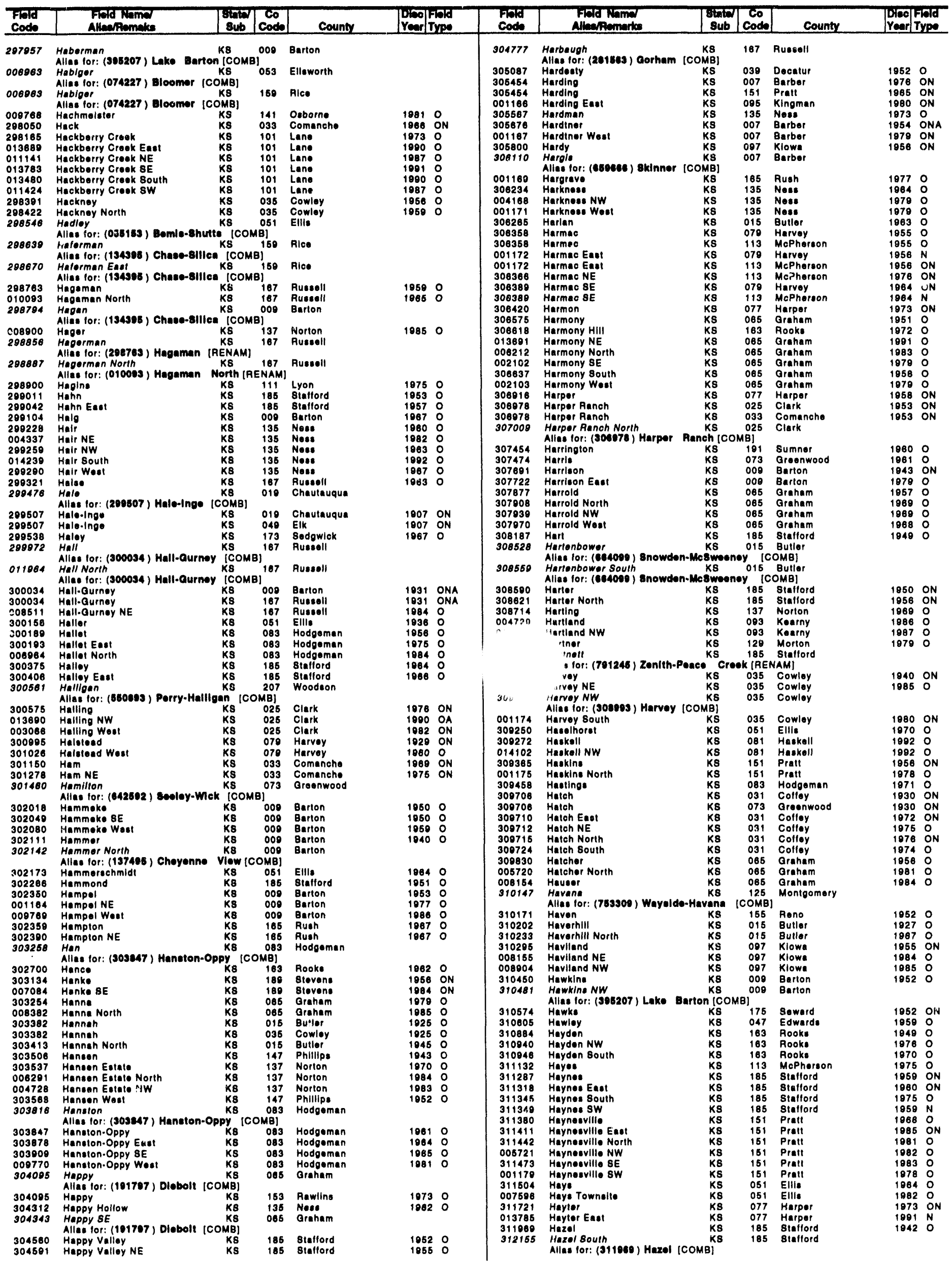


KANSAS

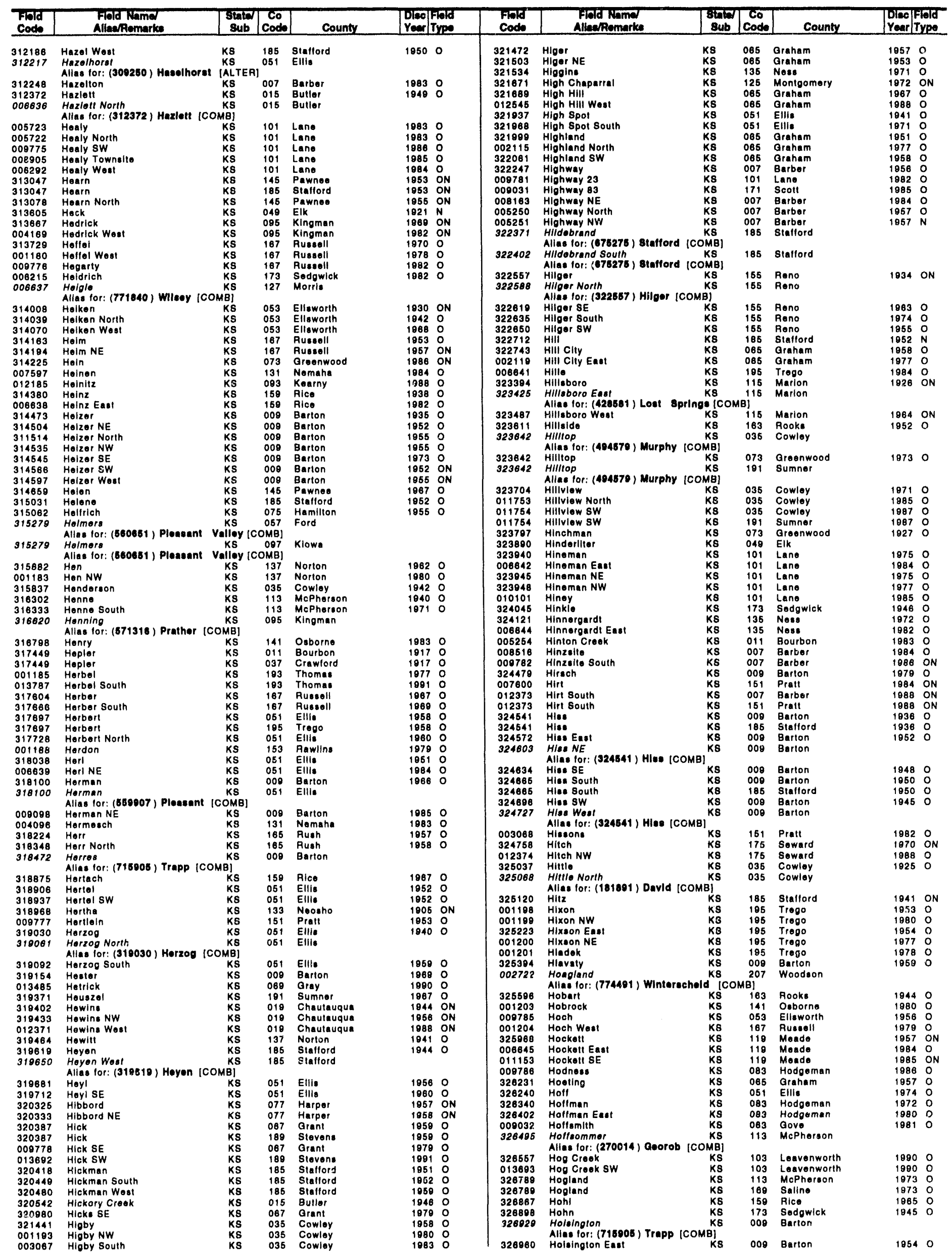


KANSAS

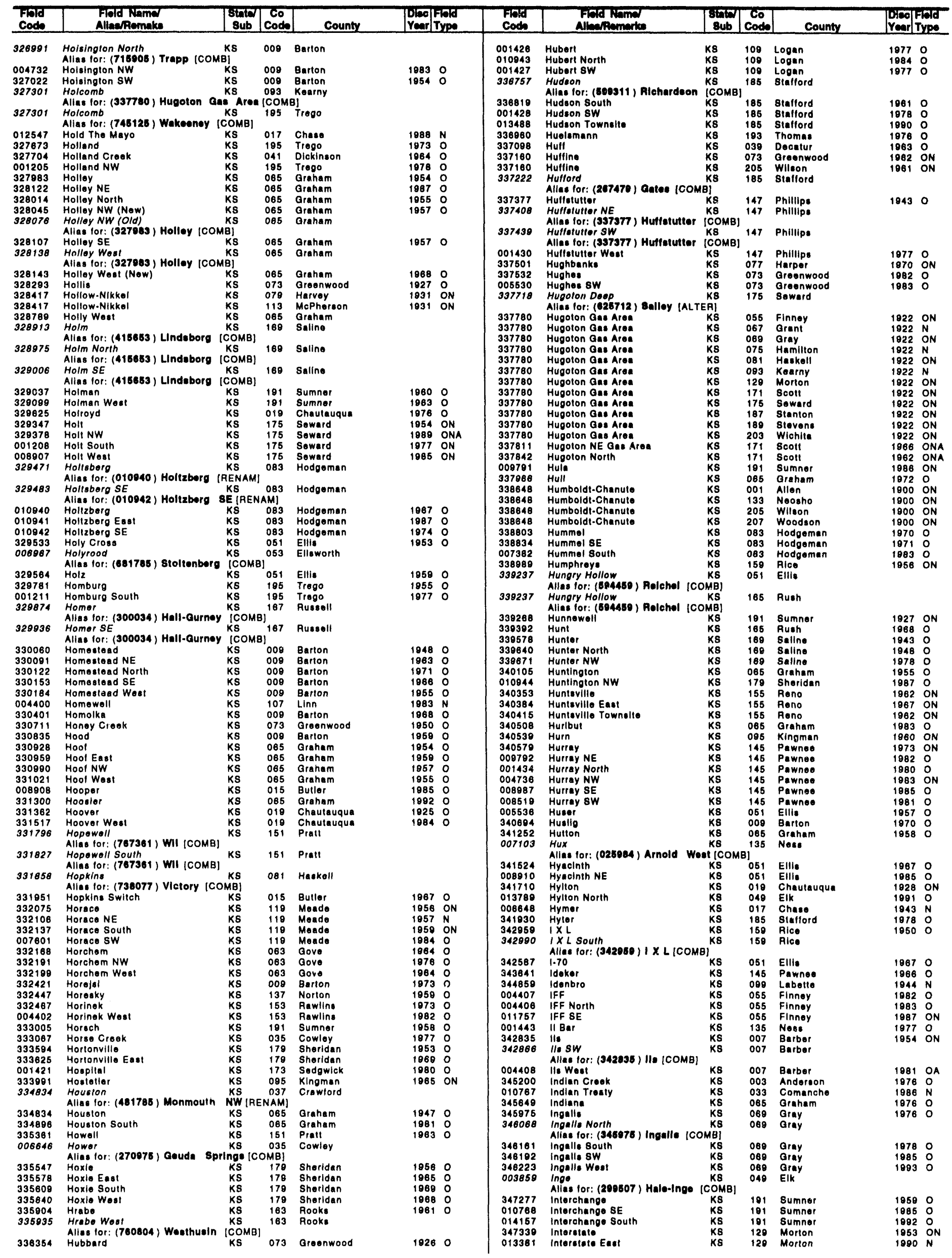


KANSAS

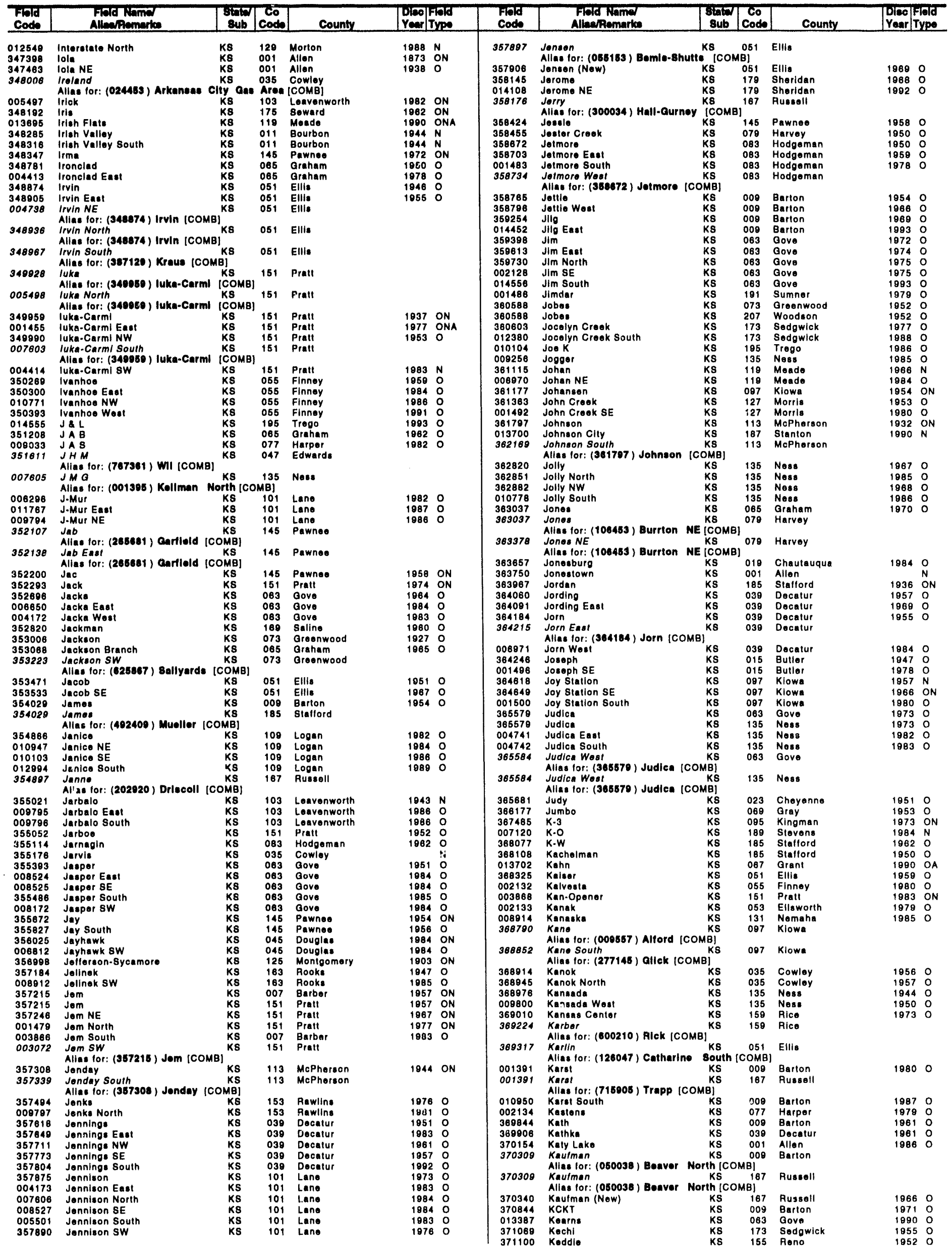


KANSAS

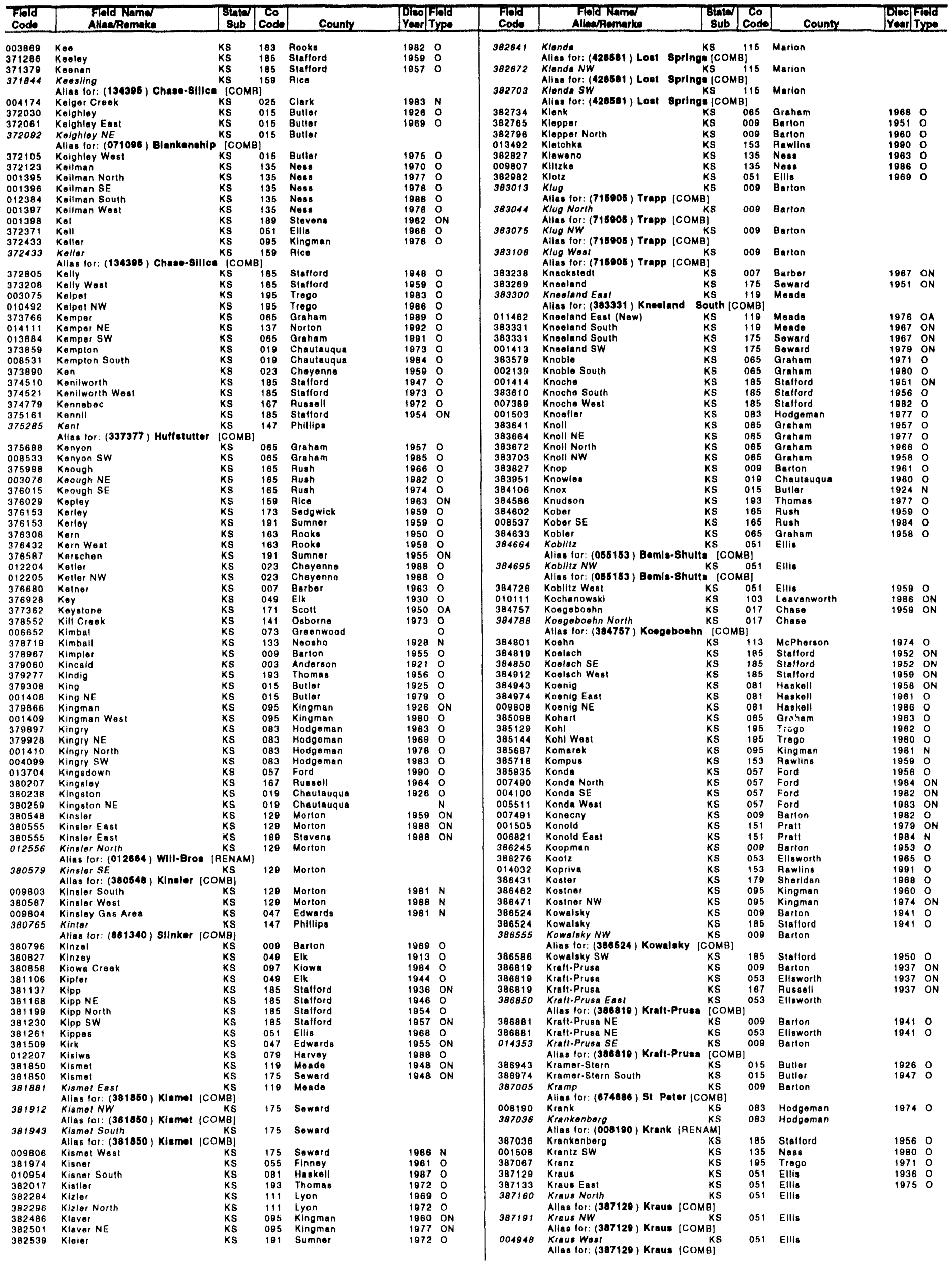


KANSAS

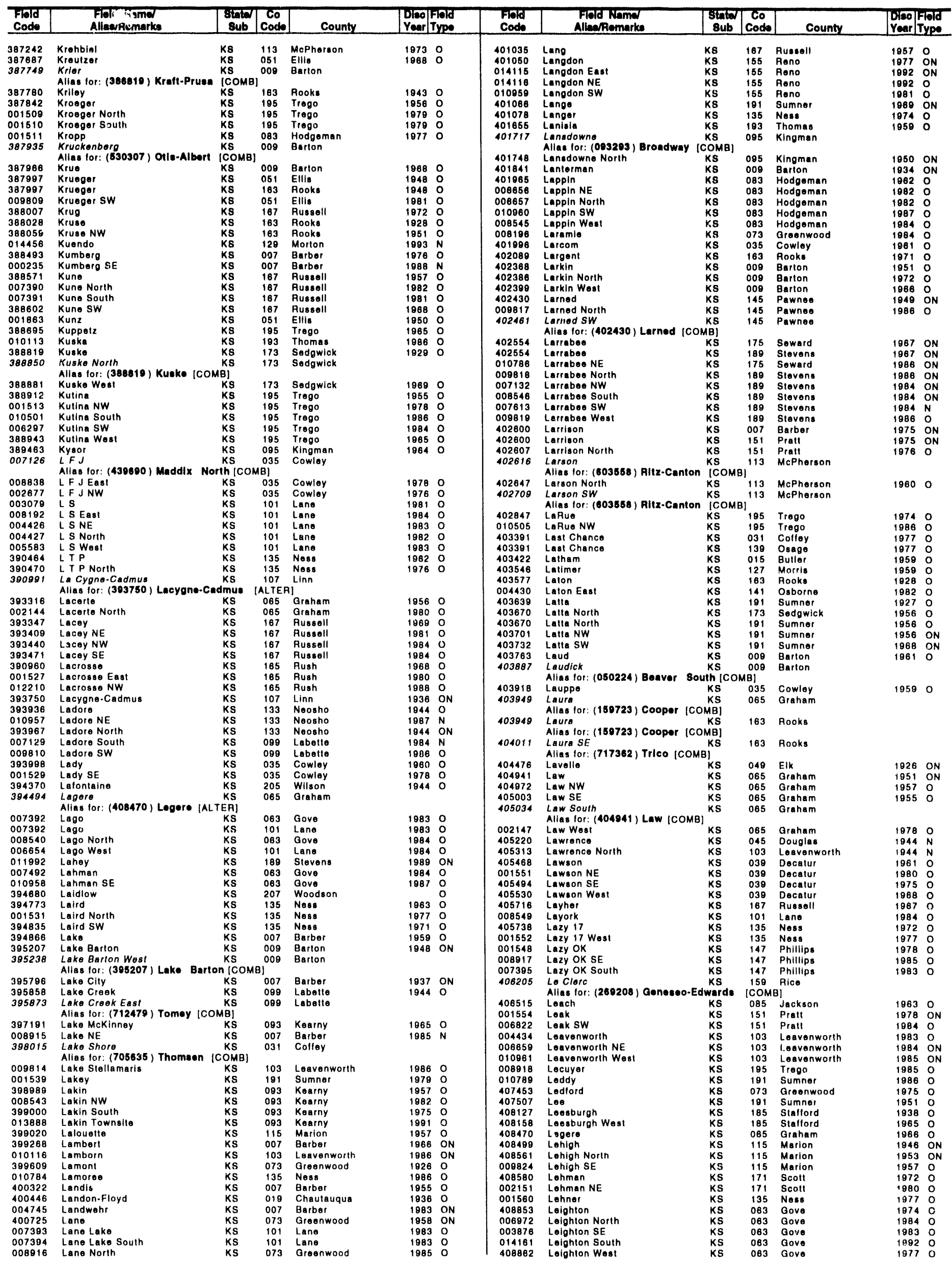




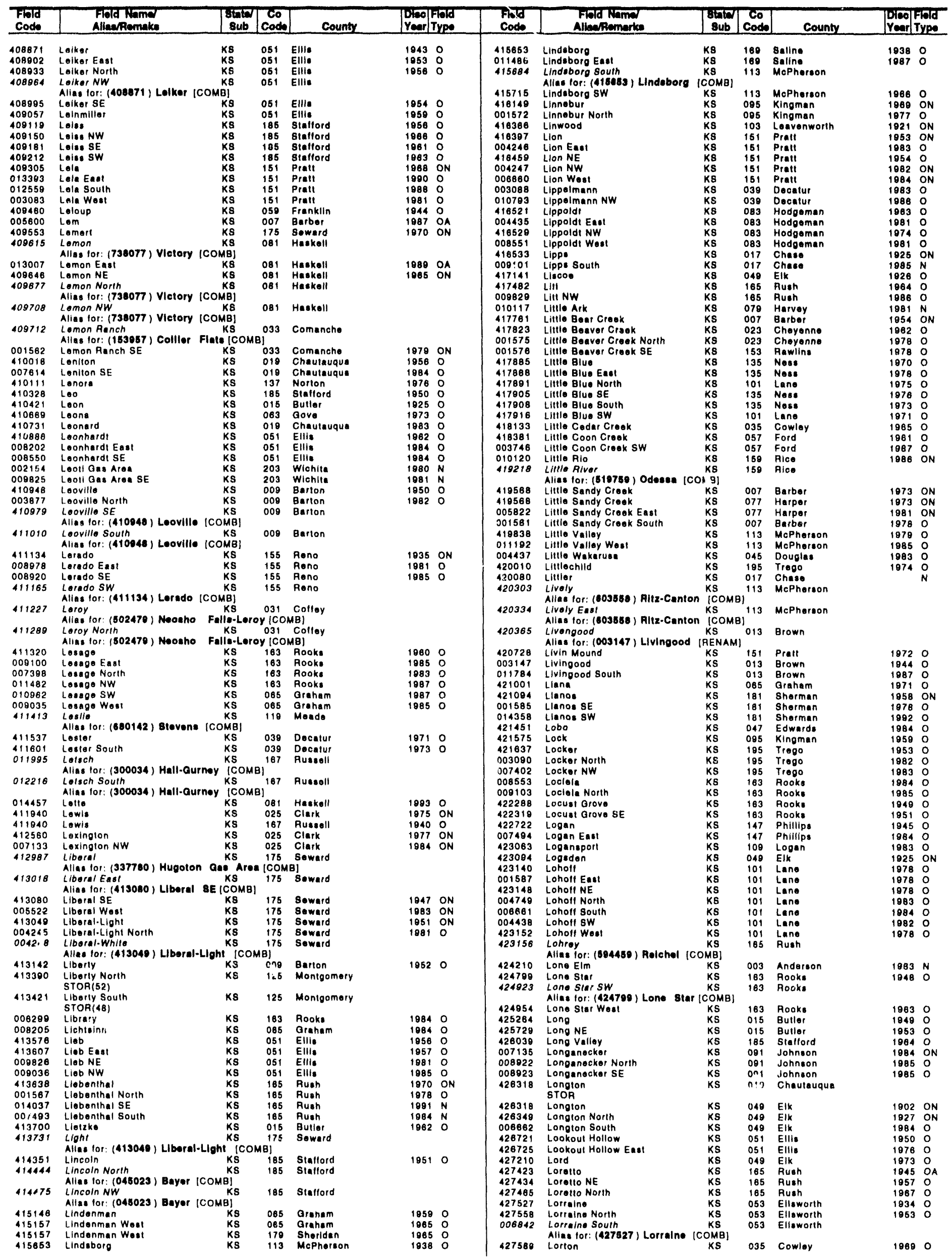


KANSAS

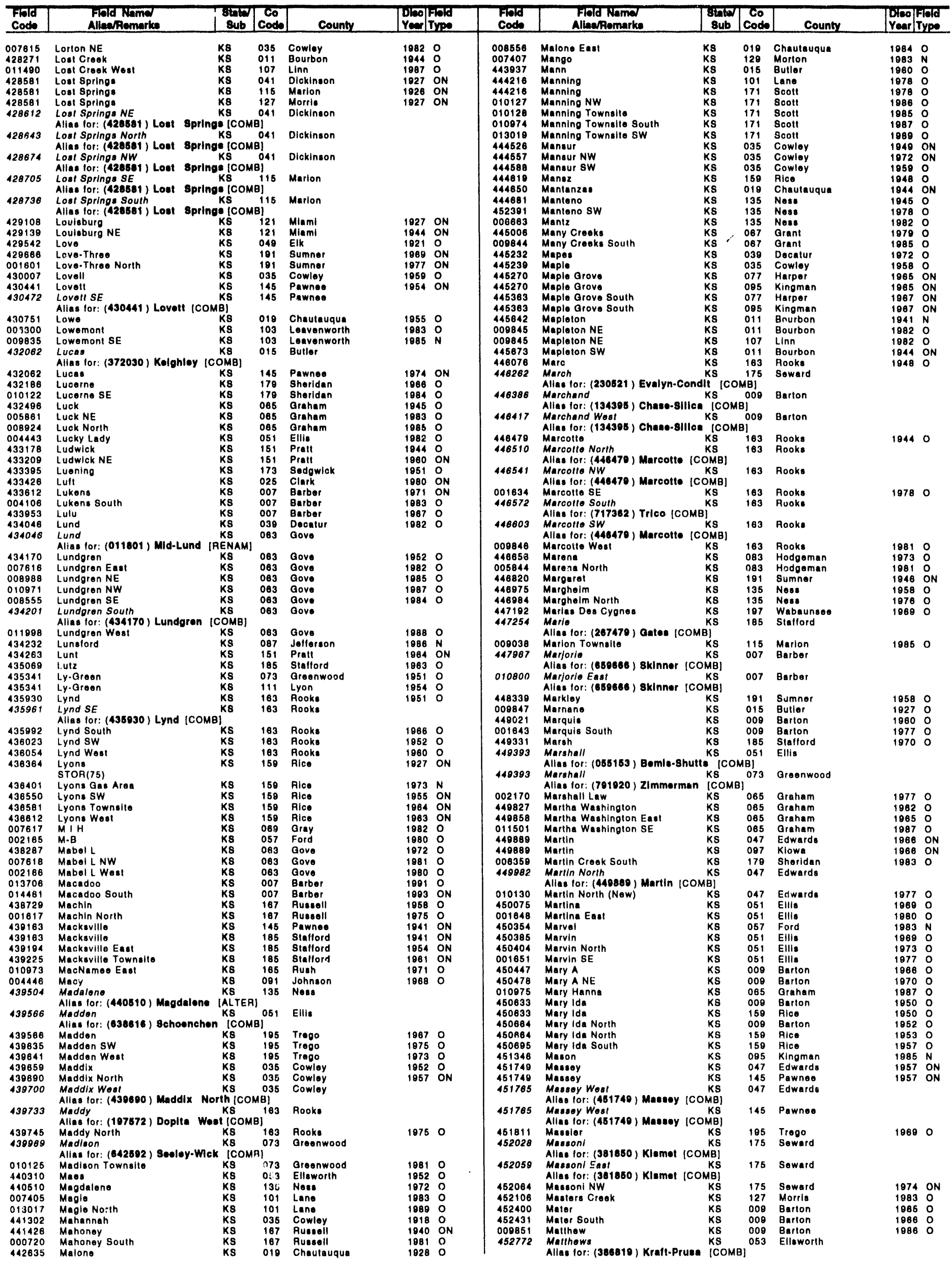


KANSAS

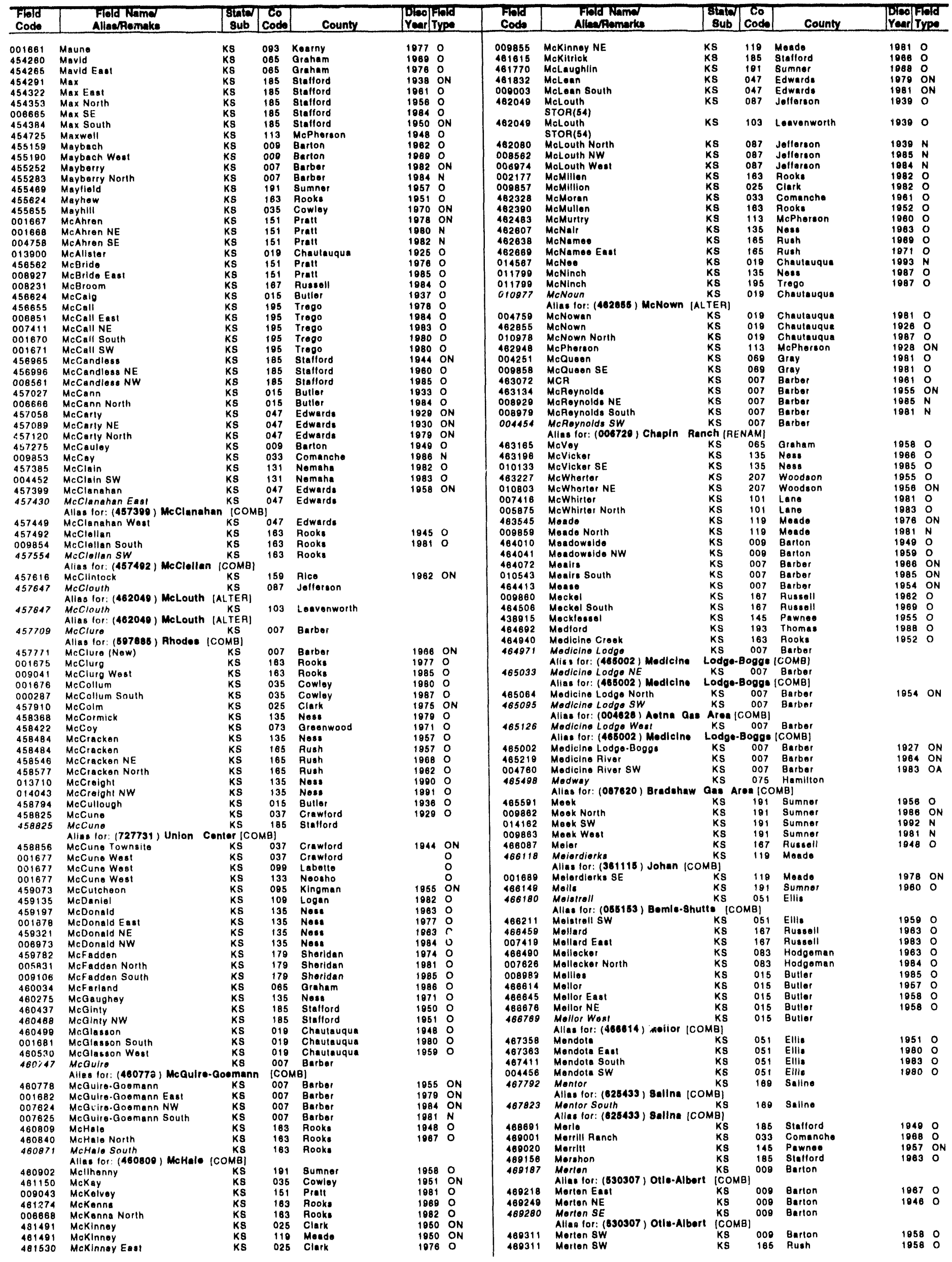


KANSAS

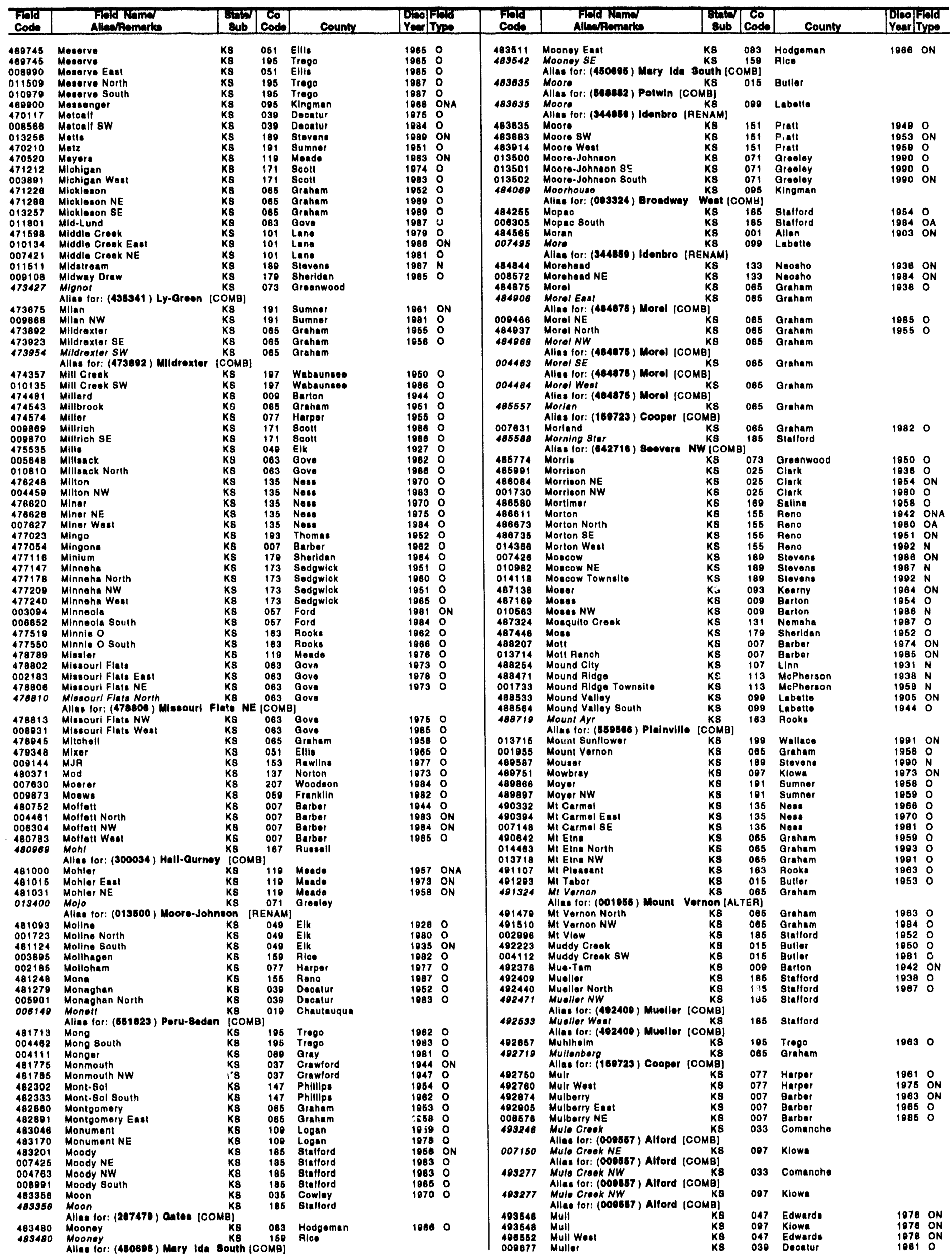


KANSAS

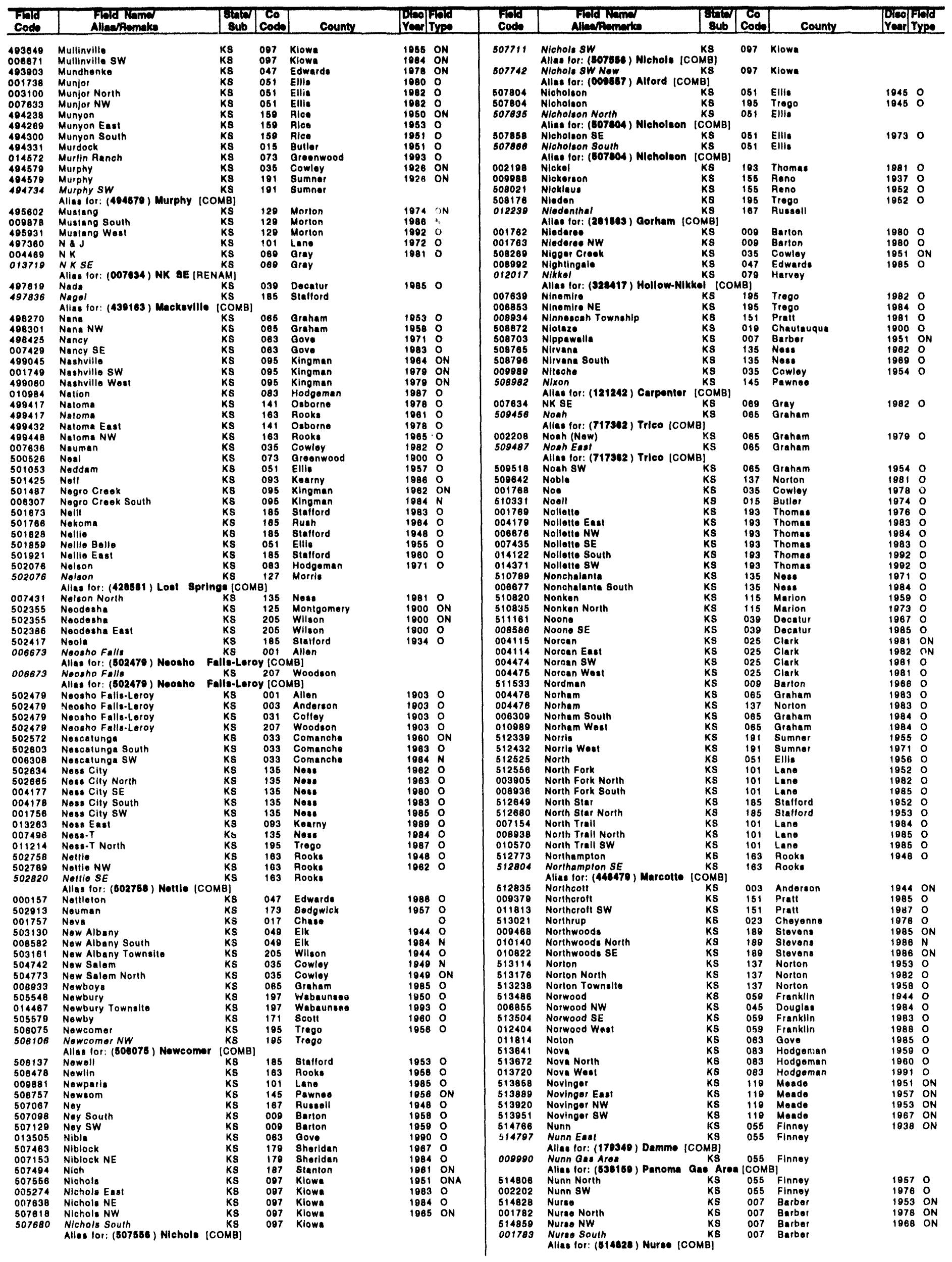


KANSAS

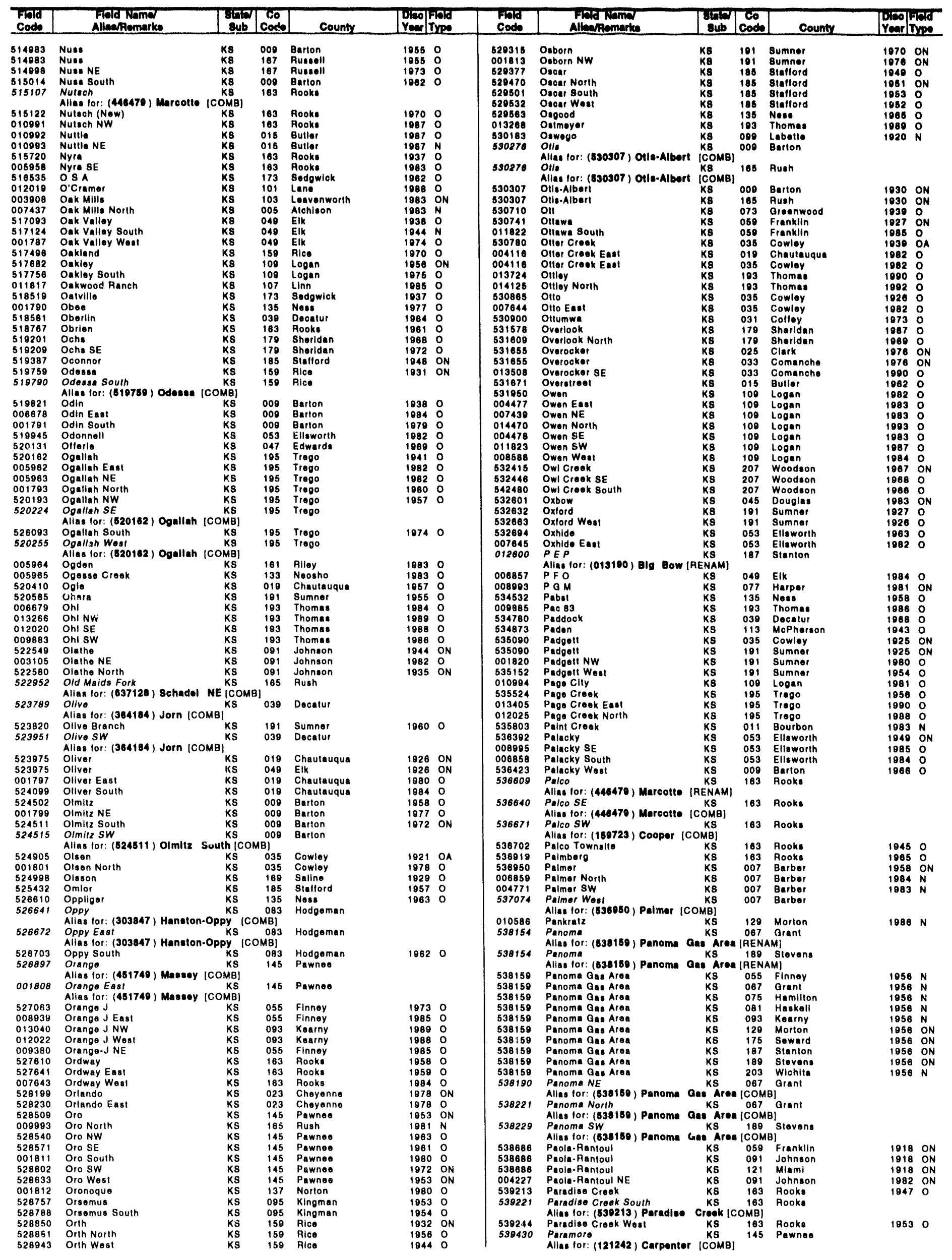


KANSAS

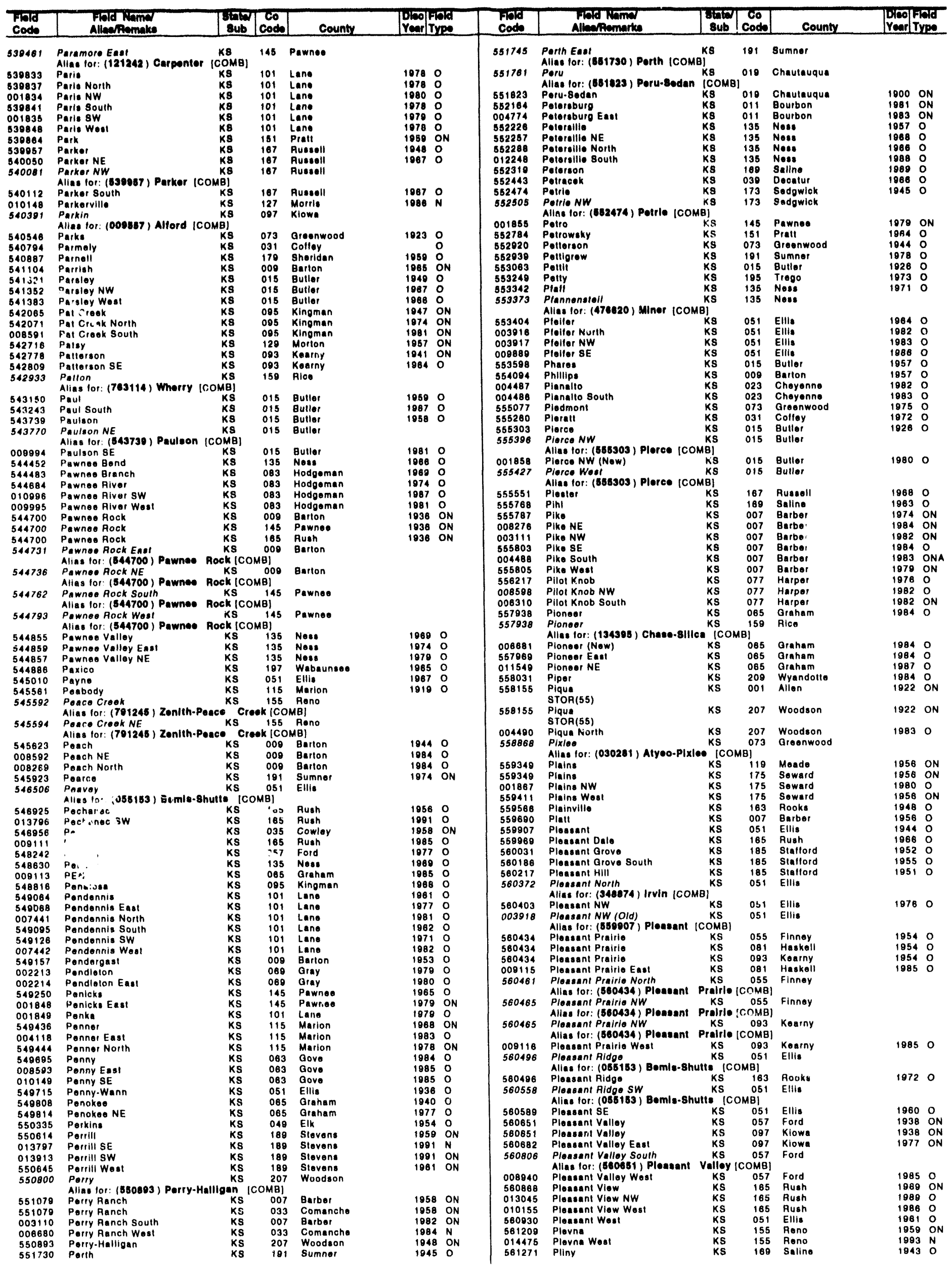


KANSAS

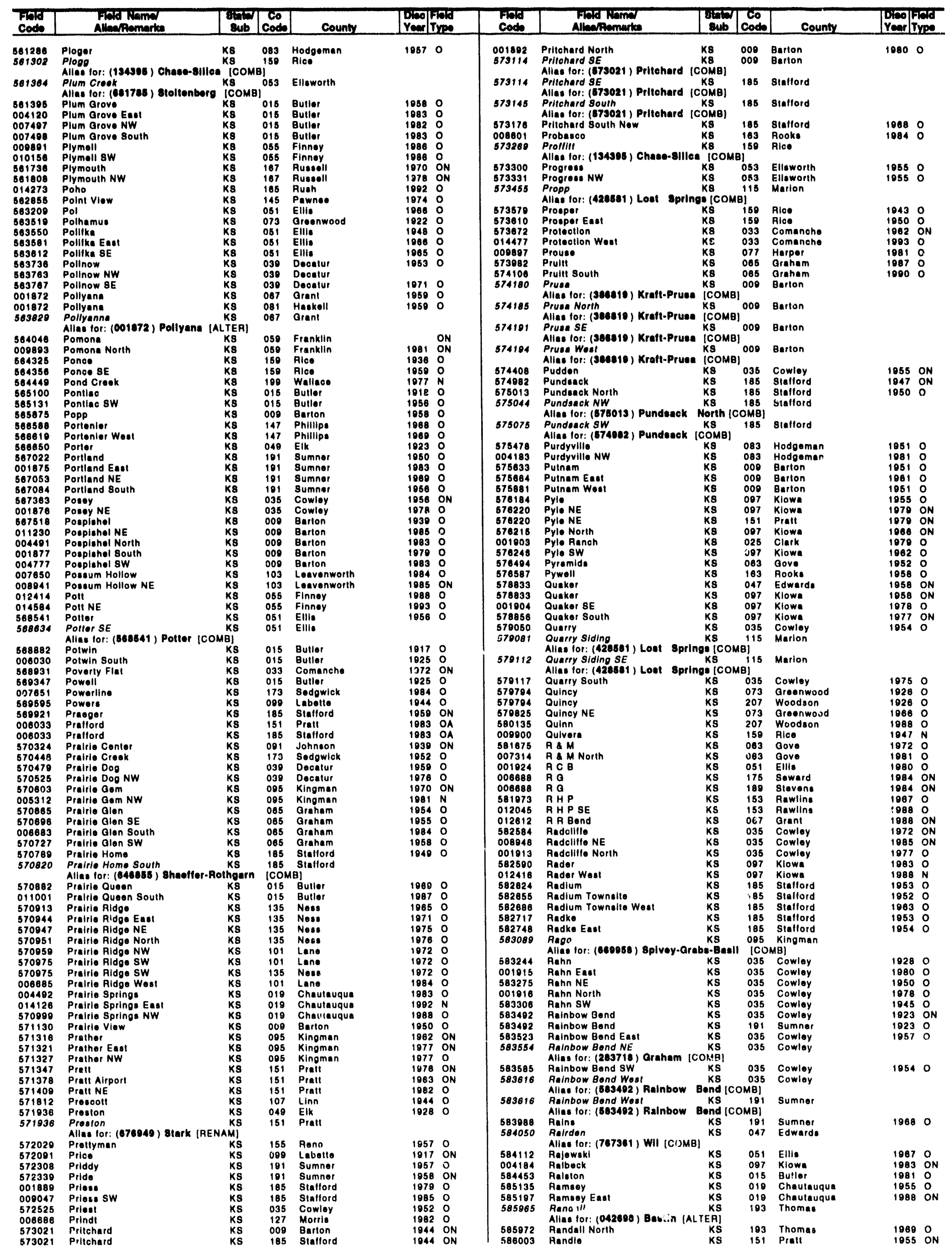




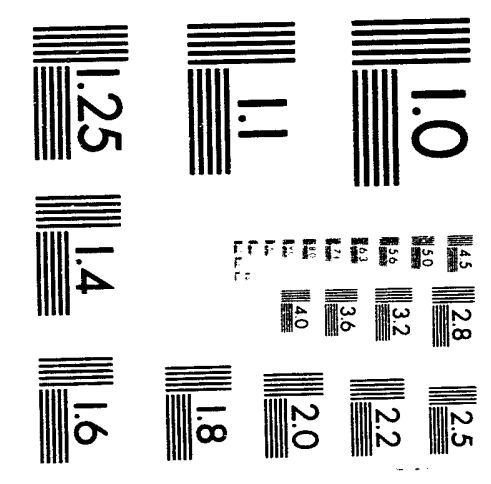



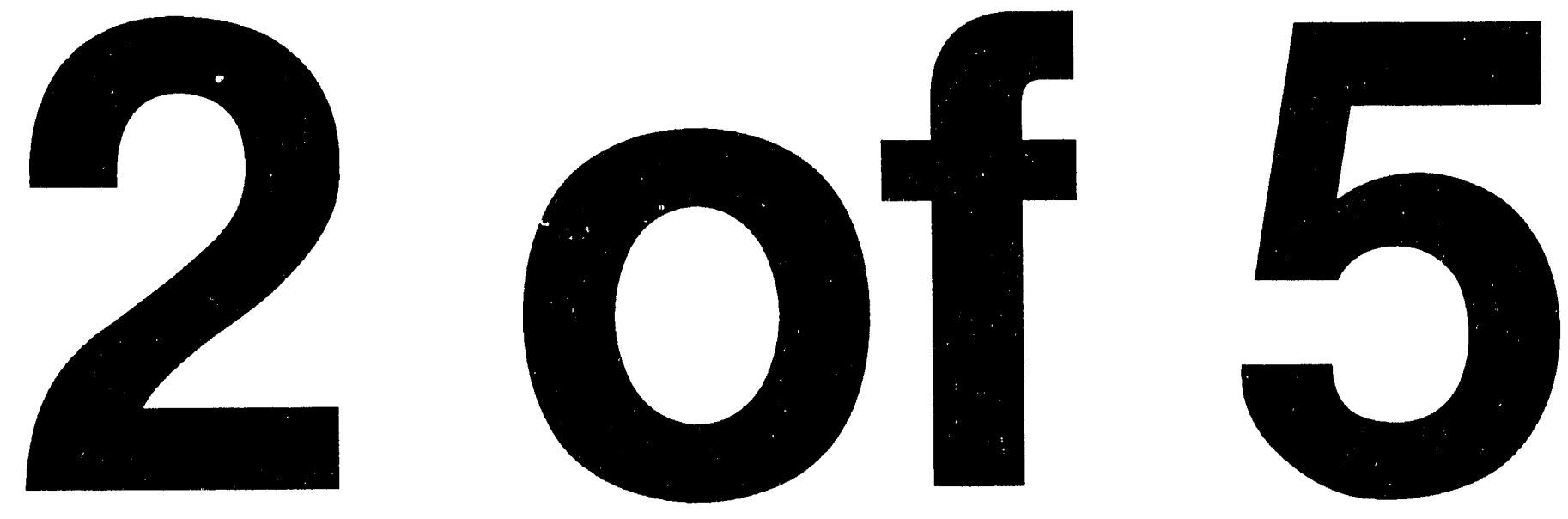
KANSAS

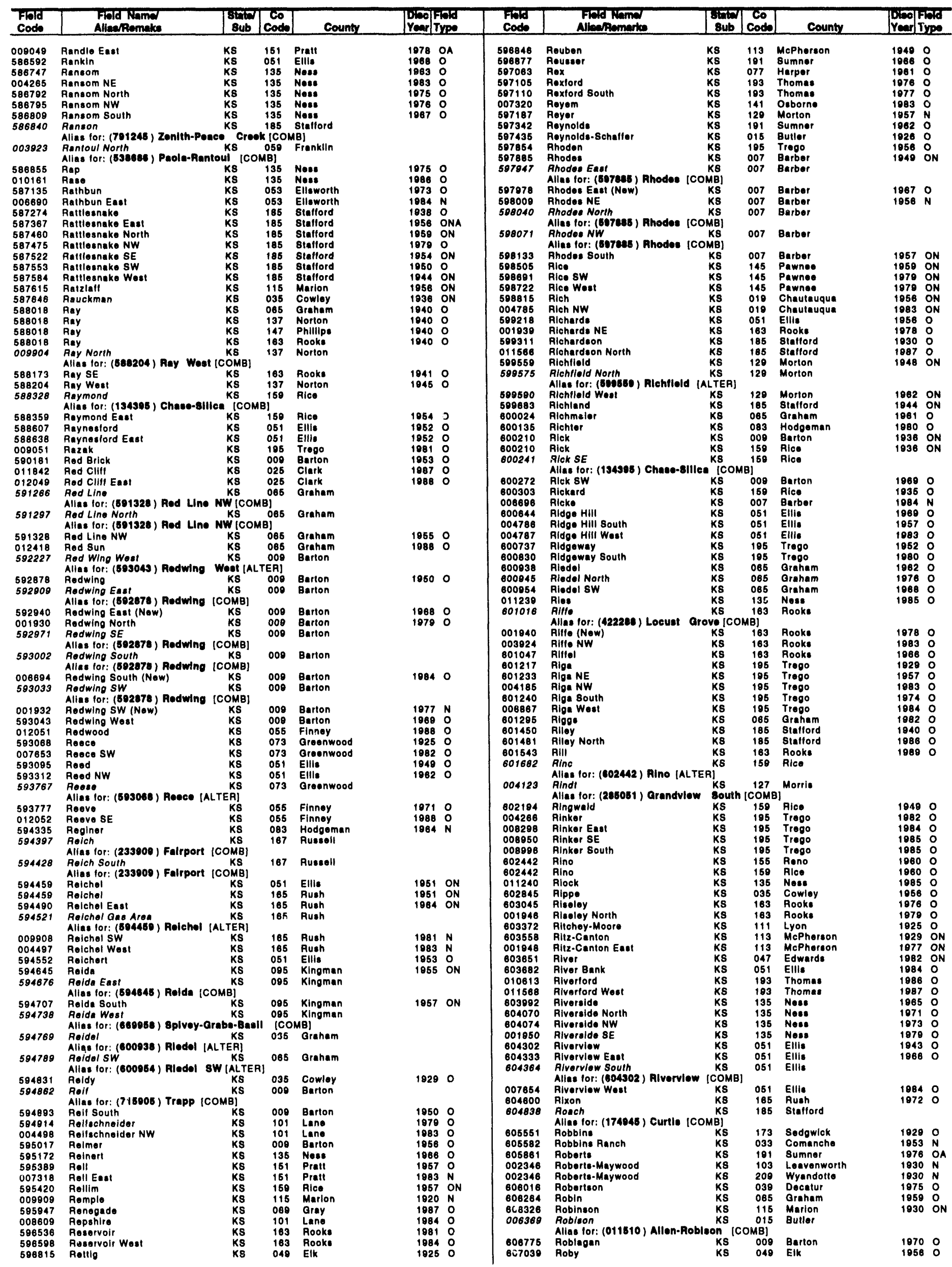


KANSAS

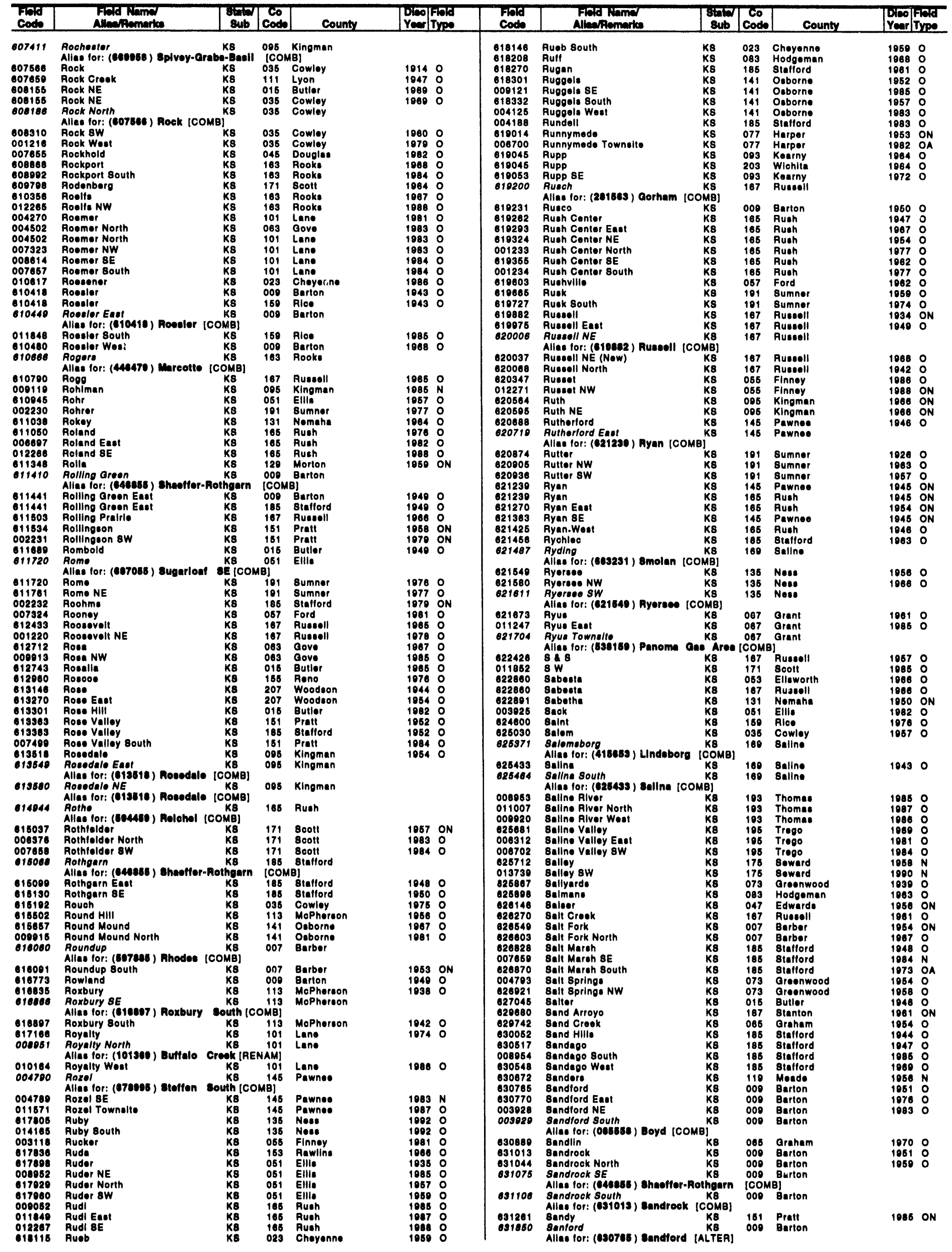


KANSAS

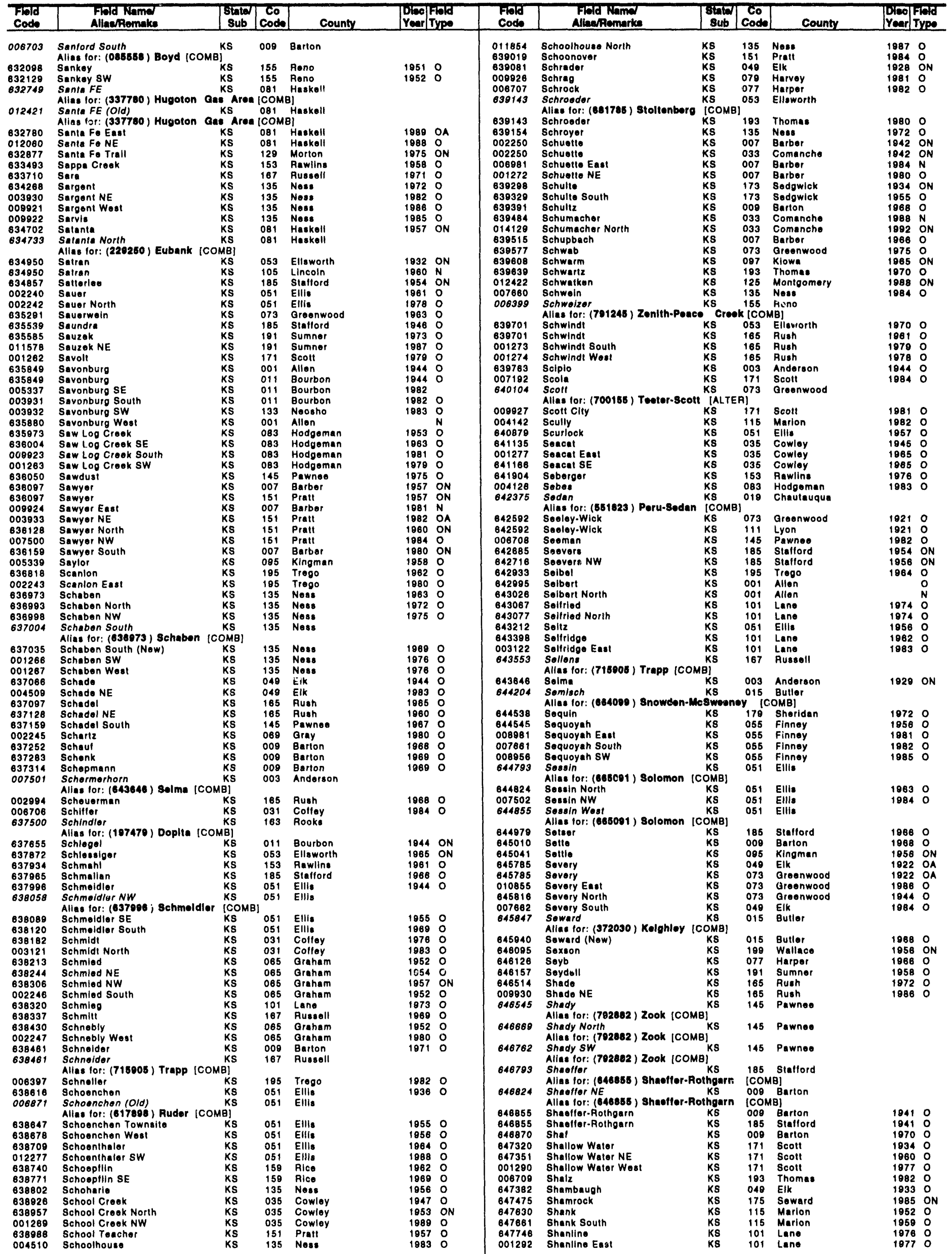


KANSAS

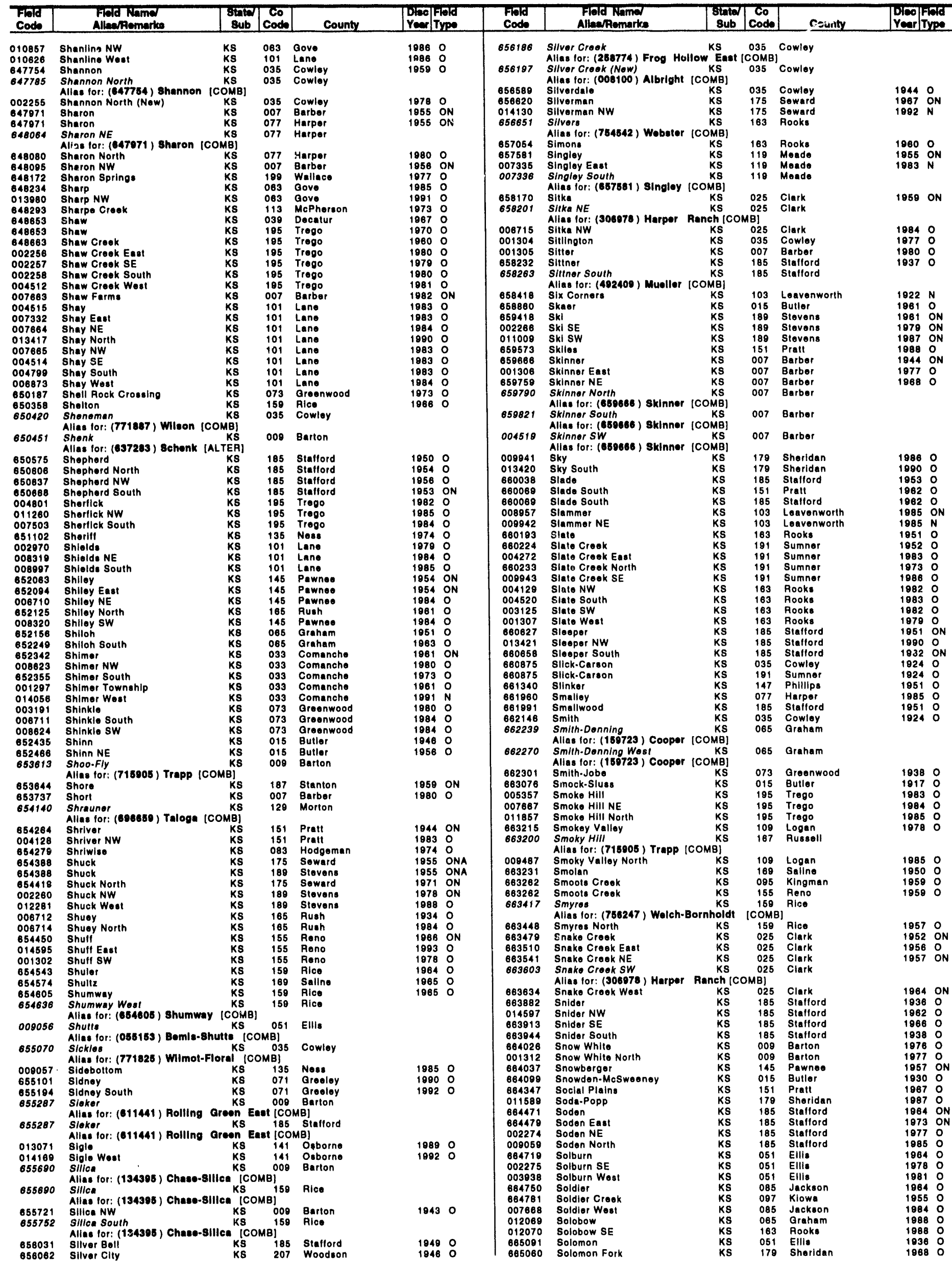




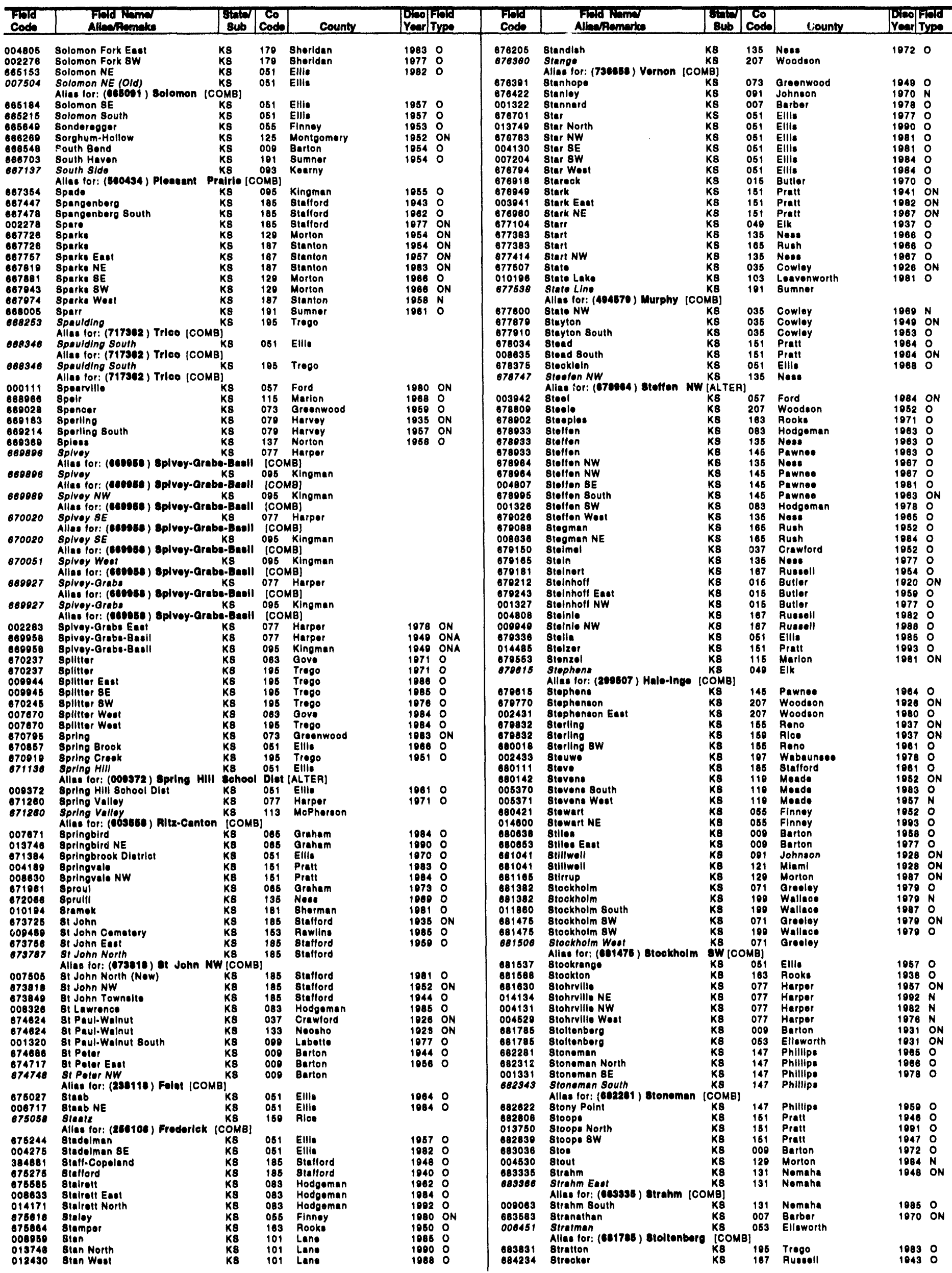


KANSAS

\begin{tabular}{|c|c|c|}
\hline $\begin{array}{l}\text { Fald } \\
\text { Code } \\
\end{array}$ & $\begin{array}{l}\text { Flo Noma } \\
\text { Allaerpemarks }\end{array}$ & $\begin{array}{c}\text { stad } \\
\text { sub }\end{array}$ \\
\hline $\begin{array}{l}684327 \\
014603 \\
001337\end{array}$ & $\begin{array}{l}\text { Stremel } \\
\text { Stromel East }\end{array}$ & $\begin{array}{l}\text { KS } \\
\text { KS }\end{array}$ \\
\hline $\begin{array}{l}001337 \\
684389\end{array}$ & $\begin{array}{l}\text { Stromol NW } \\
\text { Strick }\end{array}$ & KS \\
\hline $\begin{array}{l}684389 \\
684730\end{array}$ & $\begin{array}{l}\text { Strick } \\
\text { Strob }\end{array}$ & ks \\
\hline $\begin{array}{l}684730 \\
684761\end{array}$ & $\begin{array}{l}\text { Strob } \\
\text { Strobol }\end{array}$ & $\begin{array}{l}\text { KS } \\
\text { KS }\end{array}$ \\
\hline & $\begin{array}{l}\text { Strobof } \\
\text { Allas for: (6s20ss) Radlum }\end{array}$ & $\begin{array}{l}\text { KS } \\
\text { Townalte } 10\end{array}$ \\
\hline 684792 & $\begin{array}{l}\text { Strobol NW } \\
\text { Allas for: (582685) Radlum }\end{array}$ & Kownulte ic \\
\hline 685012 & $\begin{array}{l}\text { Altis for: (082.686) Radilum } \\
\text { Strong Clty }\end{array}$ & $\underset{\mathrm{KS}}{\text { Townsite }}$ \\
\hline 685093 & strother & Ks \\
\hline 685102 & Stroud & KS \\
\hline $\begin{array}{l}685257 \\
885310\end{array}$ & Stroud North & ks \\
\hline $\begin{array}{l}685318 \\
002442\end{array}$ & $\begin{array}{l}\text { Stroud South } \\
\text { Struse: }\end{array}$ & KS \\
\hline $\begin{array}{l}002442 \\
002442\end{array}$ & $\begin{array}{l}\text { Struss } \\
\text { Struss }\end{array}$ & KS \\
\hline 002443 & $\begin{array}{l}\text { Struss } \\
\text { Struss NE }\end{array}$ & $\begin{array}{l}\text { KS } \\
\text { KS }\end{array}$ \\
\hline 008998 & Struss West & KS \\
\hline 685722 & $\begin{array}{l}\text { Stuckey } \\
\text { Allas for: (108453) Burrton }\end{array}$ & $\begin{array}{l}\text { KS } \\
\text { ME [COMB] }\end{array}$ \\
\hline 002444 & Studor & kS \\
\hline $\begin{array}{l}685753 \\
005375\end{array}$ & $\begin{array}{l}\text { Studloy } \\
\text { Studloy North }\end{array}$ & $\begin{array}{l}\text { Ka } \\
\text { KS }\end{array}$ \\
\hline 685784 & Studioy SE & KS \\
\hline 685815 & Studloy South & ks \\
\hline 685846 & Studley sw & ks \\
\hline $\begin{array}{l}002445 \\
685877\end{array}$ & Stuhlaiz & Ks \\
\hline $\begin{array}{l}685877 \\
885939\end{array}$ & $\begin{array}{l}\text { Stukenbroker } \\
\text { Stumph }\end{array}$ & $\begin{array}{l}\text { KS } \\
\text { KS }\end{array}$ \\
\hline $\begin{array}{l}885939 \\
006454\end{array}$ & $\begin{array}{l}\text { Stumph } \\
\text { Stumps }\end{array}$ & $\begin{array}{l}\text { KS } \\
\text { KS }\end{array}$ \\
\hline & Alise for: (074227) Blocmer & [СОMB] \\
\hline 686001 & Sturgeon & KS \\
\hline 686218 & Stuttgart & K8 \\
\hline 686249 & Stuttgart South & KS \\
\hline 688280 & Stulz & ks \\
\hline 686288 & Stutz Enest & ks \\
\hline 688293 & Stutz NE & KS \\
\hline 001338 & Stutz North & KS \\
\hline 686342 & Subera & ks \\
\hline $\begin{array}{l}002450 \\
007508\end{array}$ & $\begin{array}{l}\text { Subera SW } \\
\text { Sublette }\end{array}$ & Ks \\
\hline $\begin{array}{l}007506 \\
686993\end{array}$ & $\begin{array}{l}\text { Sublette } \\
\text { Sugarloaf }\end{array}$ & KS \\
\hline $\begin{array}{l}686993 \\
687055\end{array}$ & $\begin{array}{l}\text { Sugarloaf } \\
\text { Sugarloal SE }\end{array}$ & KS \\
\hline $\begin{array}{l}687055 \\
687179\end{array}$ & $\begin{array}{l}\text { Sugarloal SE } \\
\text { Suiter }\end{array}$ & $\begin{array}{l}\text { KS } \\
\text { KS }\end{array}$ \\
\hline 002452 & Sukovaty & KS \\
\hline 687272 & Sullivan & ks \\
\hline 687272 & $\begin{array}{l}\text { Sullivan } \\
\text { Allas for: (281863) Gorham }\end{array}$ & $\begin{array}{c}\mathrm{Ks} \\
{[\mathrm{COMB}]}\end{array}$ \\
\hline 002453 & Sullivan Easi & Ks \\
\hline 687417 & Sullivan South & KS \\
\hline 004533 & Sum-Wick & KS \\
\hline 004531 & Sum-Wick East & ks \\
\hline 004532 & Sum-Wick South & Ks \\
\hline 008638 & Sumking & KS \\
\hline 688202 & Summors & ks \\
\hline $\begin{array}{l}688946 \\
001340\end{array}$ & $\begin{array}{l}\text { Sun Clly } \\
\text { Sun Clty sw }\end{array}$ & $\begin{array}{l}\text { KS } \\
\text { K8 }\end{array}$ \\
\hline 689225 & $\begin{array}{l}\text { Sun City Sw } \\
\text { Sunllower }\end{array}$ & KS \\
\hline 689473 & Sunny Slope & ks \\
\hline 008315 & Sunny Slope North & KS \\
\hline $\begin{array}{l}689535 \\
689597\end{array}$ & Sunny Slope NW & KS \\
\hline 689597 & $\begin{array}{l}\text { Sunny Valloy } \\
\text { Sunny Valloy East }\end{array}$ & KS \\
\hline 689628 & $\begin{array}{l}\text { Sunny Valloy East } \\
\text { Sunny Valloy NE }\end{array}$ & KS \\
\hline 689859 & $\begin{array}{l}\text { Sunny Valloy NE } \\
\text { Sunny Valloy SW }\end{array}$ & KS \\
\hline $\begin{array}{l}689690 \\
689752\end{array}$ & $\begin{array}{l}\text { Sunny Valloy SW } \\
\text { Sunnydalo }\end{array}$ & KS \\
\hline $\begin{array}{l}689752 \\
688783\end{array}$ & $\begin{array}{l}\text { Sunnydalo } \\
\text { Sunnyaido }\end{array}$ & $\begin{array}{l}\text { KS } \\
\text { KS }\end{array}$ \\
\hline 689876 & Sunnyviow & KS \\
\hline & Aline for: (669988) Splvey-G & irabe-Basll \\
\hline 689876 & Sunnyviow & Ks \\
\hline $\begin{array}{l}690000 \\
690279\end{array}$ & $\begin{array}{l}\text { Sunrise } \\
\text { Sunshine }\end{array}$ & ks \\
\hline 890341 & $\begin{array}{l}\text { Sunshine } \\
\text { Sunshine South }\end{array}$ & $\begin{array}{l}\text { KS } \\
\text { KS }\end{array}$ \\
\hline 012073 & $\begin{array}{l}\text { Sunshine South } \\
\text { Susank }\end{array}$ & $\begin{array}{l}\text { KS } \\
\text { KS }\end{array}$ \\
\hline & & OMB] \\
\hline 691116 & $\begin{array}{l}\text { Sutcliff } \\
\text { Alias for: }(691147 \text { ) Sutellffe }\end{array}$ & $\begin{array}{l}\text { KS } \\
\text { [ALTEA] }\end{array}$ \\
\hline 691147 & Sutclitfo & KS \\
\hline 691151 & Suteliffo Enst & KS \\
\hline 002454 & Sutcllfio SE & KS \\
\hline 681178 & Sutherland & KS \\
\hline 691364 & Sutton & ks \\
\hline 691527 & Swader & KS \\
\hline $\begin{array}{l}681868 \\
007673\end{array}$ & $\begin{array}{l}\text { Swank } \\
\text { Swayze }\end{array}$ & $\begin{array}{l}\text { KS } \\
\text { KS }\end{array}$ \\
\hline 692209 & Sweanoy & KS \\
\hline 692240 & Sweeney SW & KS \\
\hline 692271 & Swooney West & Ks \\
\hline 011888 & & $\underset{\mathrm{KS}}{[\mathrm{COMB}]}$ \\
\hline 692302 & $\begin{array}{l}\text { Swoeney Woat (Now) } \\
\text { Swoot }\end{array}$ & $\begin{array}{l}\text { KS } \\
\text { KS }\end{array}$ \\
\hline 004276 & $\begin{array}{l}\text { Swool } \\
\text { Sweot Adeline }\end{array}$ & $\begin{array}{l}\text { KS } \\
\text { KS }\end{array}$ \\
\hline 012432 & $\begin{array}{l}\text { Sweet Adellne } \\
\text { Swoet North }\end{array}$ & $\begin{array}{l}\text { KS } \\
\text { KS }\end{array}$ \\
\hline 013774 & $\begin{array}{l}\text { Swoet Norrh } \\
\text { Swoet SE }\end{array}$ & KS \\
\hline 692488 & Swoot William & KS \\
\hline 682519 & Sweat William NW & KS \\
\hline 692798 & Swonson & KS \\
\hline 693139 & Swishor & KS \\
\hline $\begin{array}{l}007344 \\
683333\end{array}$ & Swordfish & ks \\
\hline & Sycemore Creak & ks \\
\hline 006462 & Sycamore Valley & Ks \\
\hline 008963 & Sycamore Valloy East & KS \\
\hline 013751 & Sylvia & Ks \\
\hline 693922 & & $\begin{array}{l}\text { KS } \\
\text { MB\} }\end{array}$ \\
\hline & $\begin{array}{l}\text { Allas for: }(402400) \text { Mueller } \\
\text { Syms SE }\end{array}$ & $\begin{array}{l}\text { MB] } \\
\text { KS }\end{array}$ \\
\hline $\begin{array}{l}693984 \\
694415\end{array}$ & $\begin{array}{l}\text { Sym: SE } \\
T \& L\end{array}$ & $\begin{array}{l}\text { KS } \\
\text { KS }\end{array}$ \\
\hline $\begin{array}{l}694415 \\
694432\end{array}$ & $\begin{array}{l}T \& L \\
T \& L \text { Wost }\end{array}$ & KS \\
\hline $\begin{array}{l}685408 \\
007674\end{array}$ & $\begin{array}{l}\text { Tabing } \\
\text { Tabing Wost }\end{array}$ & $\begin{array}{l}\text { Ks } \\
\text { KS }\end{array}$ \\
\hline $\begin{array}{l}007674 \\
605563\end{array}$ & $\begin{array}{l}\text { Tabing Woat } \\
\text { Tacha }\end{array}$ & $\begin{array}{l}\text { KS } \\
\text { KS }\end{array}$ \\
\hline
\end{tabular}


KANSAS

\begin{tabular}{|c|c|c|c|c|c|c|}
\hline $\begin{array}{l}\text { Fiod } \\
\text { Code } \\
\end{array}$ & $\begin{array}{l}\text { Fiad Noma } \\
\text { Aliae/Remoke }\end{array}$ & sub & $\left.\begin{array}{l}c 0 \\
\text { code }\end{array}\right]$ & County & 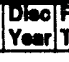 & $\begin{array}{l}\text { Frord } \\
\text { Type }\end{array}$ \\
\hline $\begin{array}{l}001369 \\
712479\end{array}$ & $\begin{array}{l}\text { Tolle } \\
\text { Tomey }\end{array}$ & KS & $\begin{array}{l}039 \\
099\end{array}$ & $\begin{array}{l}\text { Docatur } \\
\text { Labette }\end{array}$ & $\begin{array}{l}1980 \\
1944\end{array}$ & $\stackrel{0}{0}$ \\
\hline 712789 & Tonok & KS & 145 & Pawnee & 1971 & ON \\
\hline $\begin{array}{l}712820 \\
003848\end{array}$ & Toni-Mike & KS & 007 & Barber & 1967 & 0 \\
\hline $\begin{array}{l}003948 \\
713008\end{array}$ & $\begin{array}{l}\text { Tonl-Mike NE } \\
\text { Tonkin }\end{array}$ & ks & 007 & Barber & 1983 & $\mathbf{N}$ \\
\hline $\begin{array}{l}713006 \\
713037\end{array}$ & Tonkin & KS & 000 & Barton & 1863 & 0 \\
\hline $\begin{array}{l}713037 \\
713088\end{array}$ & Tonovay & ks & 073 & Groenwood & 1917 & ON \\
\hline $\begin{array}{l}713088 \\
713812\end{array}$ & Tonovay Weat & ks & 073 & Greenwood & 1950 & 0 \\
\hline $\begin{array}{l}713812 \\
713812\end{array}$ & Toronto & ks & 073 & Greonwood & 1813 & o \\
\hline $\begin{array}{l}713812 \\
002485\end{array}$ & Toronto & K8 & 207 & Woodson & 1913 & ON \\
\hline $\begin{array}{l}002485 \\
713843\end{array}$ & Toronto Woat & KS & 073 & Groenwood & 1078 & 0 \\
\hline 713843 & $\begin{array}{l}\text { Torrance } \\
\text { Alias for: }(692200) \text { swoonoy }\end{array}$ & $\begin{array}{c}\text { KS } \\
\text { [COMB] }\end{array}$ & 1451 & Pawnoe & & \\
\hline 714184 & Toulon & KS & 051 & Ellis: & 1035 & ON \\
\hline 004293 & $\begin{array}{l}\text { Toulon North } \\
\text { Alias for: (714184) Toulon } 10\end{array}$ & $\begin{array}{r}\text { Ks } \\
\text { (CоMB] }\end{array}$ & 051 & Elllo & & \\
\hline $\begin{array}{l}714215 \\
714240\end{array}$ & $\begin{array}{l}\text { Toulon SE } \\
\text { Toulon South }\end{array}$ & $\begin{array}{l}\text { KS } \\
\text { KS }\end{array}$ & $\begin{array}{l}051 \\
051\end{array}$ & $\begin{array}{l}\text { Ellis } \\
\text { Ellis }\end{array}$ & 1858 & 0 \\
\hline & Allas for: (7141e4) Toulon 10 & [COMB] & & & & \\
\hline 714277 & $\begin{array}{l}\text { Toulon SW } \\
\text { Alias for: (714184) Toulon }\end{array}$ & $\begin{aligned} \text { KS } \\
\text { [COMB] }\end{aligned}$ & 051 & Ellis & & \\
\hline 714354 & Toviea & KS & 023 & Cheyenne & 1978 & 0 \\
\hline 714354 & Tovros & Ks & 153 & Aawline & 1978 & 0 \\
\hline 714354 & Toviee & ks & 181 & Shermen & 1978 & 0 \\
\hline $\begin{array}{l}714401 \\
715115\end{array}$ & $\begin{array}{l}\text { Towenda } \\
\text { Truer }\end{array}$ & $\begin{array}{l}\text { KS } \\
\text { KS }\end{array}$ & $\begin{array}{l}015 \\
039\end{array}$ & Butlor & 1948 & $\begin{array}{l}0 \\
0\end{array}$ \\
\hline 001375 & Traer Traer SE & $\begin{array}{l}\text { KS } \\
\text { KS }\end{array}$ & $\begin{array}{l}039 \\
039\end{array}$ & Decatur & $\begin{array}{l}1974 \\
1880\end{array}$ & 0 \\
\hline 715130 & Trallas & KS & 007 & Barber & 1955 & ON \\
\hline 001376 & Tralfas East & KS & 007 & Barbor & & \\
\hline 715161 & $\begin{array}{l}\text { Traflas SE } \\
\text { Alles for: }(715130) \text { Traffes }\end{array}$ & $\begin{array}{r}\text { Ks } \\
{[\text { COMB] }}\end{array}$ & 007 & Barber & & \\
\hline $\begin{array}{l}006249 \\
000127\end{array}$ & Trallan south & $\begin{array}{l}\text { KS } \\
\text { KS }\end{array}$ & 007 & $\begin{array}{l}\text { Barbor } \\
\text { Ford }\end{array}$ & $\begin{array}{l}1984 \\
1985\end{array}$ & ON \\
\hline $\begin{array}{l}000127 \\
715905 \\
715905\end{array}$ & $\begin{array}{l}\text { Tragic } \\
\text { Trapp } \\
\text { Trapp }\end{array}$ & $\begin{array}{l}\text { KS } \\
\text { K8 } \\
\text { KS }\end{array}$ & $\begin{array}{l}057 \\
009 \\
167\end{array}$ & $\begin{array}{l}\text { Bartion } \\
\text { Ruesoll }\end{array}$ & $\begin{array}{l}1986 \\
1929 \\
1929\end{array}$ & ON \\
\hline 715936 & Trapp East & Ks & 000 & Berton & & \\
\hline 715936 & Allao for: (718008) Trapp (CC & 2OMB] & & & & \\
\hline 715936 & $\begin{array}{l}\text { Trapp East } \\
\text { Allas lor: (716005) Trapp ICC }\end{array}$ & $\begin{array}{l}\text { KS } \\
\text { COMB! }\end{array}$ & 187 & Russell & & \\
\hline 012078 & Trapp South & K8 & 000 & Barton & & \\
\hline 006250 & $\begin{array}{l}\text { Alias lor: (718008) Trapp ICC } \\
\text { Trapp West }\end{array}$ & LOMB] & 167 & Ruseoll & & \\
\hline 716029 & Allas lor: (718908) Trapp IC & SOMB] & 163 & & & \\
\hline & $\begin{array}{l}\text { Trarbech } \\
\text { Allas lor: (446470) Marcotte }\end{array}$ & $\begin{array}{c}\text { KS } \\
\text { (COMB) }\end{array}$ & & Rooks & & \\
\hline $\begin{array}{l}716432 \\
004545\end{array}$ & $\begin{array}{l}\text { Treos } \\
\text { Trogo Center }\end{array}$ & $\begin{array}{l}\text { KS } \\
K S\end{array}$ & $\begin{array}{l}036 \\
105\end{array}$ & $\begin{array}{l}\text { Cowley } \\
\text { Trogo }\end{array}$ & $\begin{array}{l}1935 \\
1983\end{array}$ & $\begin{array}{l}0 \\
0\end{array}$ \\
\hline 008877 & Tregeun & KS & 195 & Trogo & 1981 & 0 \\
\hline 716484 & $\begin{array}{l}\text { Trokell } \\
\text { Alles for: }(010840) \text { Anson } s\end{array}$ & $\begin{array}{c}\mathrm{KS} \\
\mathrm{SE}[\mathrm{COMB}]\end{array}$ & 101 & Sumner & & \\
\hline 008848 & Trekell Wost & KS & 101 & Sumner & 1984 & 0 \\
\hline 716509 & Trombley & K5 & 155 & Rono & 1978 & 0 \\
\hline 004135 & Trembley North & KS & 155 & Reno & 1082 & 0 \\
\hline 716587 & Trent & Ks & 133 & Noosho & 1951 & ON \\
\hline 716818 & Trent North & KS & 133 & Noosho & 1081 & 0 \\
\hline 716880 & Trenton & KS & 047 & Edwards & 1974 & 0 \\
\hline 716680 & $\begin{array}{l}\text { Trenton } \\
\text { Alias tor: (8600se) splvey-a }\end{array}$ & $\begin{array}{c}\text { KS } \\
\text { arabe-Baell }\end{array}$ & $\stackrel{095}{1 \mathrm{COM}}$ & $\begin{array}{l}\text { Kingman } \\
\text { MB] }\end{array}$ & & \\
\hline 718742 & $\begin{array}{l}\text { Trenton South } \\
\text { Allas tor: (860080) 8pivay-a }\end{array}$ & $\begin{array}{l}\text { KS } \\
\text { Oraba-Basil }\end{array}$ & $\begin{array}{l}095 \\
\text { lCOM }\end{array}$ & $\begin{array}{l}\text { Kingman } \\
\text { MB] }\end{array}$ & & \\
\hline 001379 & Trester & KS & 009 & Barton & 1880 & 0 \\
\hline $\begin{array}{l}009962 \\
004546\end{array}$ & $\begin{array}{l}\text { Tresler East } \\
\text { iriangle }\end{array}$ & $\begin{array}{l}\text { KS } \\
\text { KS }\end{array}$ & $\begin{array}{l}008 \\
103\end{array}$ & $\begin{array}{l}\text { Barton } \\
\text { Thomas }\end{array}$ & $\begin{array}{l}1986 \\
1983\end{array}$ & $\begin{array}{l}0 \\
0\end{array}$ \\
\hline 010869 & Triangle North & KS & 103 & Thomas & 1086 & 0 \\
\hline 013754 & Triengle NW & kS & 193 & Thomas & 1990 & 0 \\
\hline 008985 & Triangle South & Ks & 193 & Thomas & 1084 & 0 \\
\hline 717362 & Trico & Ks & 051 & Elllo & 1951 & 0 \\
\hline 717362 & Trlco & K8 & 065 & Graham & 1851 & 0 \\
\hline 717382 & Trico & K8 & 163 & Rooks & 1051 & 0 \\
\hline 717362 & Trico & K8 & 185 & Trego & 1851 & 0 \\
\hline 718202 & Tritech & KS & 009 & Barion & 1986 & 0 \\
\hline 718684 & Trousdale & ks & 047 & Edward: & 1956 & 0 \\
\hline 718685 & Troundale NE & $\mathbf{K S}$ & 047 & Edward: & 1058 & $O N$ \\
\hline 718726 & Trousdale North & KS & 047 & Edwarde & 1058 & ON \\
\hline 002490 & Trousdalo NW & KS & 047 & Edwarde & 1080 & 0 \\
\hline 718757 & Trousdale South & Ks & 047 & Edwards & 1056 & $B$ ON \\
\hline 718757 & Trousdalo South & ks & 097 & Klowa & 1856 & ON \\
\hline 008730 & Trove & KS & 063 & Gove & $\begin{array}{l}1984 \\
1988\end{array}$ & 0 \\
\hline 710036 & $\begin{array}{l}\text { Truax } \\
\text { Tucker }\end{array}$ & $\begin{array}{l}\text { KS } \\
\text { Ks }\end{array}$ & $\begin{array}{l}101 \\
070\end{array}$ & $\begin{array}{l}\text { Lane } \\
\text { Groenwood }\end{array}$ & $\begin{array}{l}1988 \\
1927\end{array}$ & $\begin{array}{ll} \\
7\end{array}$ \\
\hline $\begin{array}{l}718881 \\
007351\end{array}$ & $\begin{array}{l}\text { Tucker } \\
\text { Tucker SE }\end{array}$ & KS & 073 & Greenwood & 1883 & $\mathrm{~S}^{\mathrm{N}}$ \\
\hline $\begin{array}{l}007351 \\
005398\end{array}$ & Tucker Wost & KS & 073 & Groonwood & 1883 & 0 \\
\hline 010838 & Tullis & K8 & 103 & Leavenworth & 1986 & 8 ON \\
\hline 721307 & Turkey Croek & K8 & 007 & Barber & 1943 & 0 \\
\hline 721369 & Turkey Creek East & KS & 007 & Barber & 1857 & 7 ON \\
\hline 721400 & Turkey Creek North & K8 & 007 & Barber & 1851 & ON \\
\hline 721431 & Turkey Crook NW & KS & 007 & Berber & 1987 & 0 \\
\hline 721834 & Turkvillie & KS & 051 & Ellis: & 1053 & 0 \\
\hline 721865 & Turkville Upper & KS & 051 & Ellis & 1848 & 30 \\
\hline 002492 & Turkville Upper NW & KS & 051 & Elllo & 1979 & 0 \\
\hline 721896 & Turkvillo Wost & Ks & 051 & Ellis & 1959 & 0 \\
\hline 010227 & Turmorrow & ks & 129 & Morton & 1886 & ON \\
\hline 722175 & Turner. & ks & 035 & Cowley & 1837 & 0 \\
\hline 722330 & Turner North & KS & 035 & Cowley & 1040 & 0 \\
\hline 722392 & Turner Wost & Ks & 035 & Cowloy & 1953 & 30 \\
\hline 014611 & Turon & KS & 155 & $\begin{array}{l}\text { Aono } \\
\text { Reno }\end{array}$ & $\begin{array}{l}1983 \\
1993\end{array}$ & $\begin{array}{l}3 \\
0 \\
0\end{array}$ \\
\hline $\begin{array}{l}014612 \\
722981\end{array}$ & $\begin{array}{l}\text { Turon NE } \\
\text { Tuttle }\end{array}$ & $\begin{array}{l}\text { KS } \\
\text { KS }\end{array}$ & $\begin{array}{l}155 \\
033\end{array}$ & $\begin{array}{l}\text { Reno } \\
\text { Comanche }\end{array}$ & $\begin{array}{l}1993 \\
1957\end{array}$ & $\begin{array}{l}0 \\
\text { ON }\end{array}$ \\
\hline 723012 & Tuttle East & KS & 033 & Comanche & 1963 & 3 ON \\
\hline 723053 & Tuttlo NE & ks & 033 & Comenche & 1977 & $\mathrm{~N}$ \\
\hline 723065 & Tulllo SE & KS & 033 & Comanche & 1978 & $B$ ON \\
\hline 002288 & Tuttle South & KS & 025 & Clark & 1978 & ON \\
\hline 723074 & $\begin{array}{l}\text { Tuttle Wost } \\
\text { Allas for: (683470) Snake co }\end{array}$ & 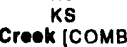 & B! & Clark & & \\
\hline & $\begin{array}{l}\text { Alias for: }(663470) \text { shake } \\
\text { Twenty }\end{array}$ & & 008 & & & \\
\hline 006731 & Twenty-Ono & Ks & 137 & Norton & 1882 & 20 \\
\hline 723733 & Twin Mound & KS & 163 & Pooks & 1085 & 50 \\
\hline 723784 & Twin Mounds & KS & 113 & McPherson & & \\
\hline & Allas for: (270014) Ceorob & [COMB] & & & & \\
\hline $\begin{array}{l}723888 \\
723919\end{array}$ & $\begin{array}{l}\text { Two-Elghty One } \\
\text { Two-Elghty Ono SE }\end{array}$ & $\begin{array}{l}\text { KS } \\
\text { KS }\end{array}$ & $\begin{array}{l}009 \\
009\end{array}$ & $\begin{array}{l}\text { Barton } \\
\text { Barton }\end{array}$ & $\begin{array}{l}1963 \\
1963\end{array}$ & $\begin{array}{ll}3 & 0 \\
3 & 0\end{array}$ \\
\hline 724826 & Tyro & ks & 125 & Montgomory & 1904 & 4 ONA \\
\hline
\end{tabular}


KANSAS

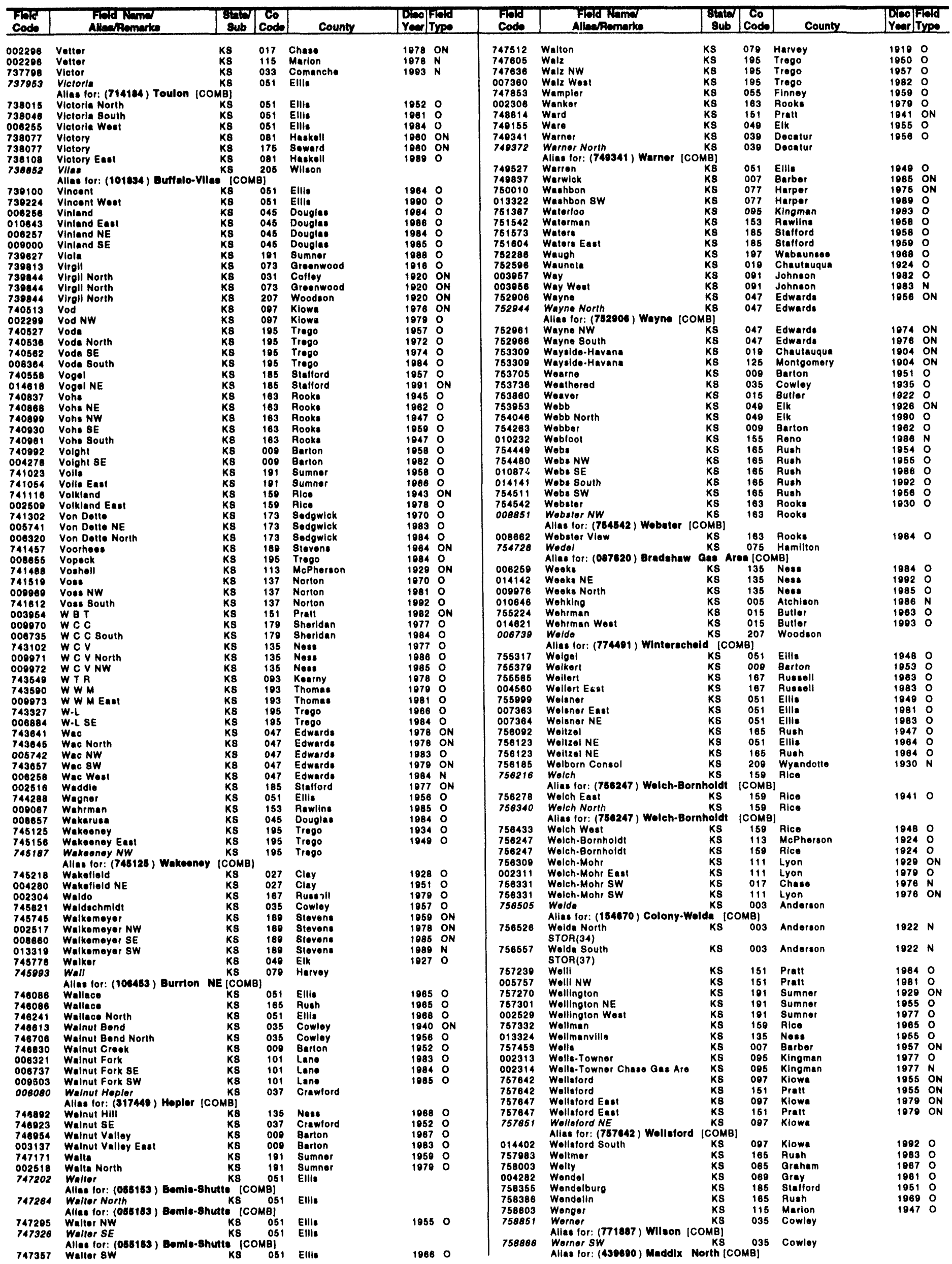


KANSAS

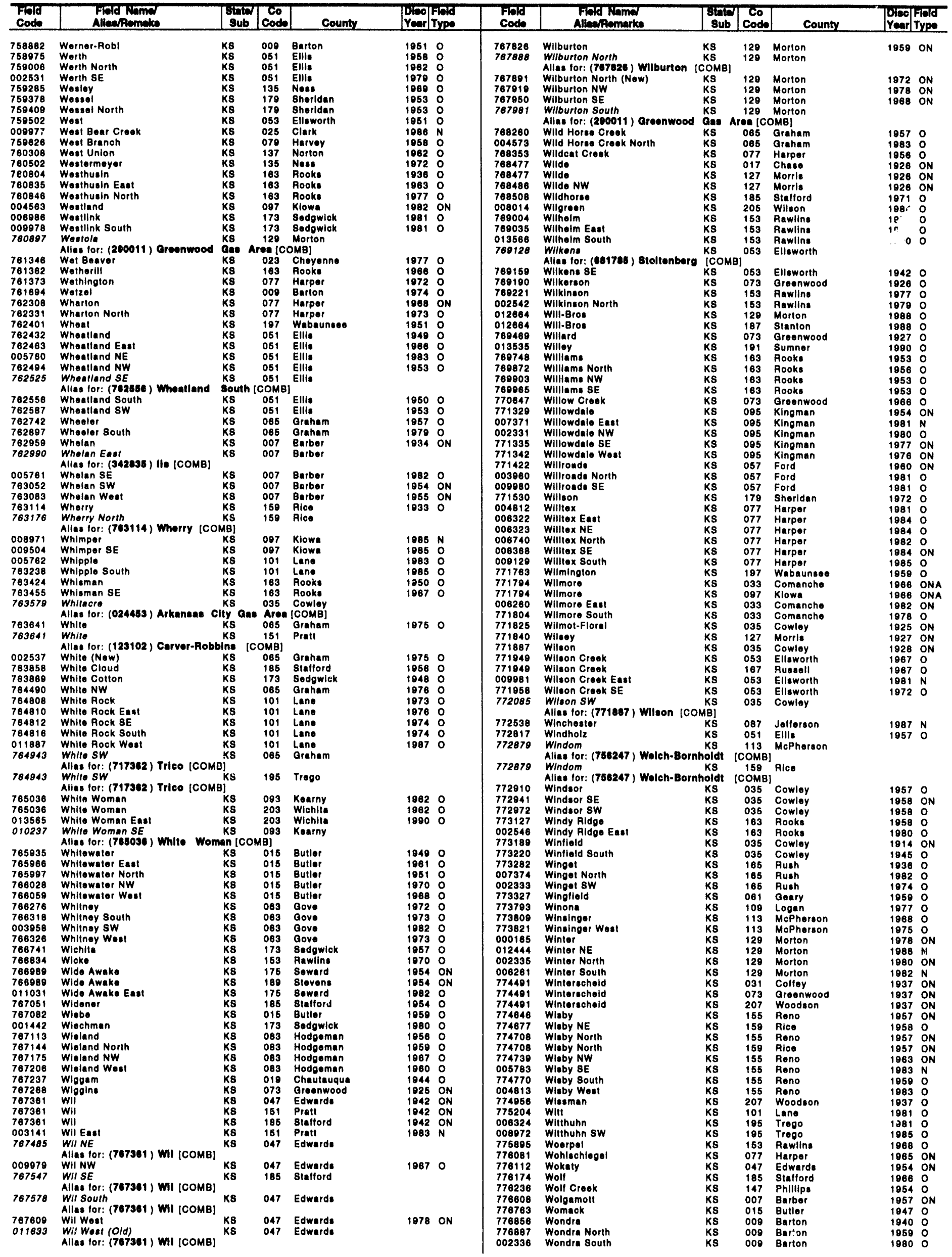


KENTUCKY

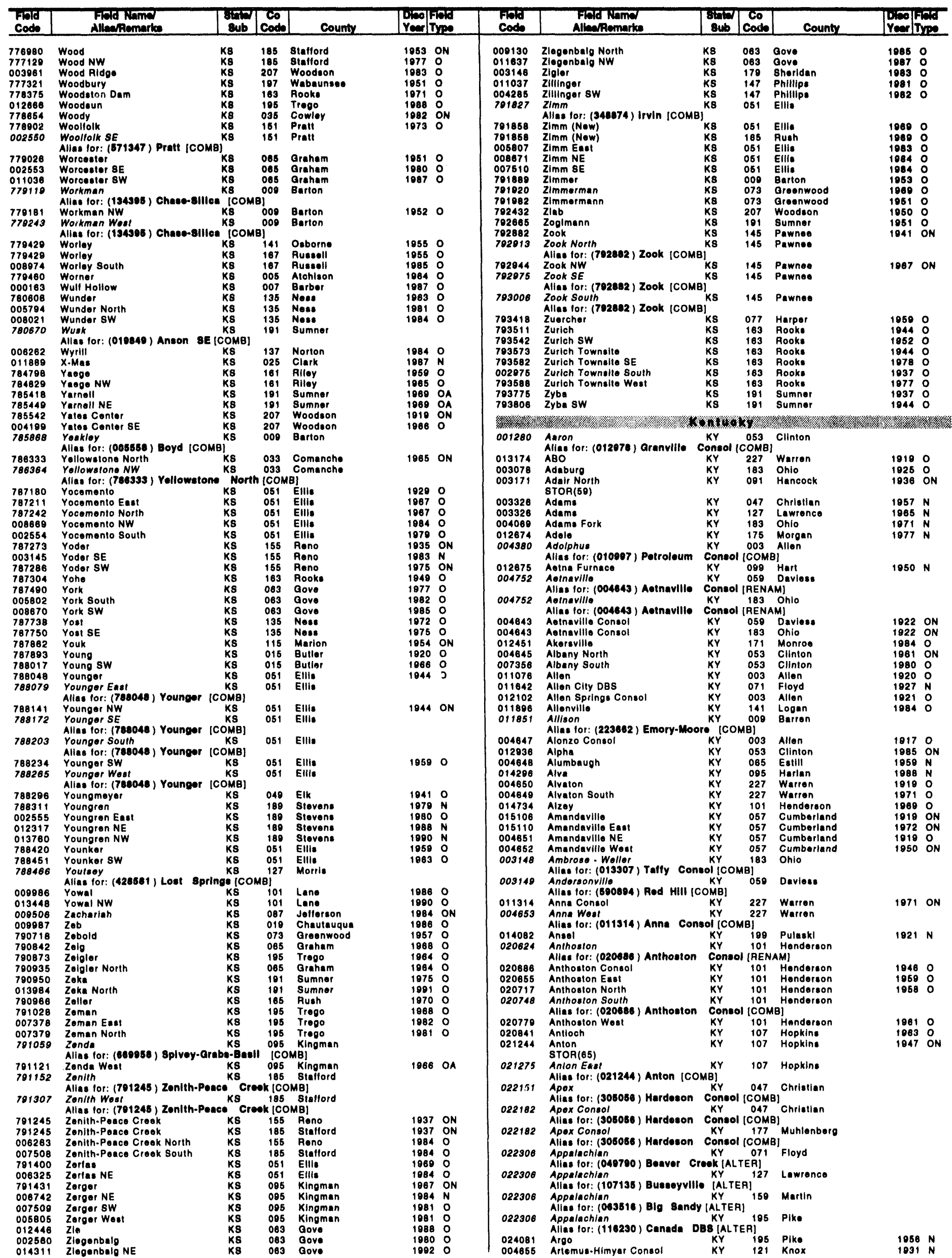




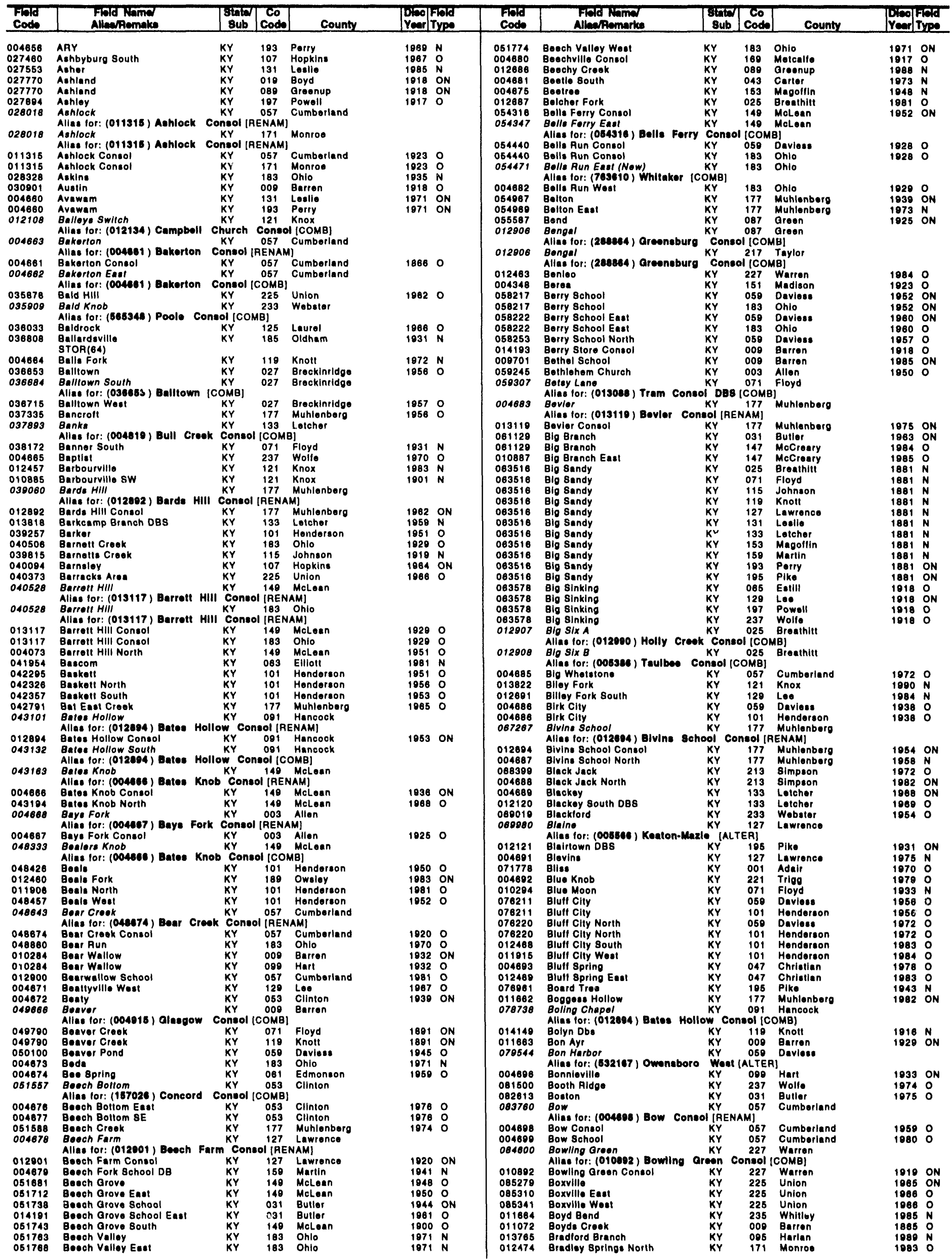


KENTUCKY

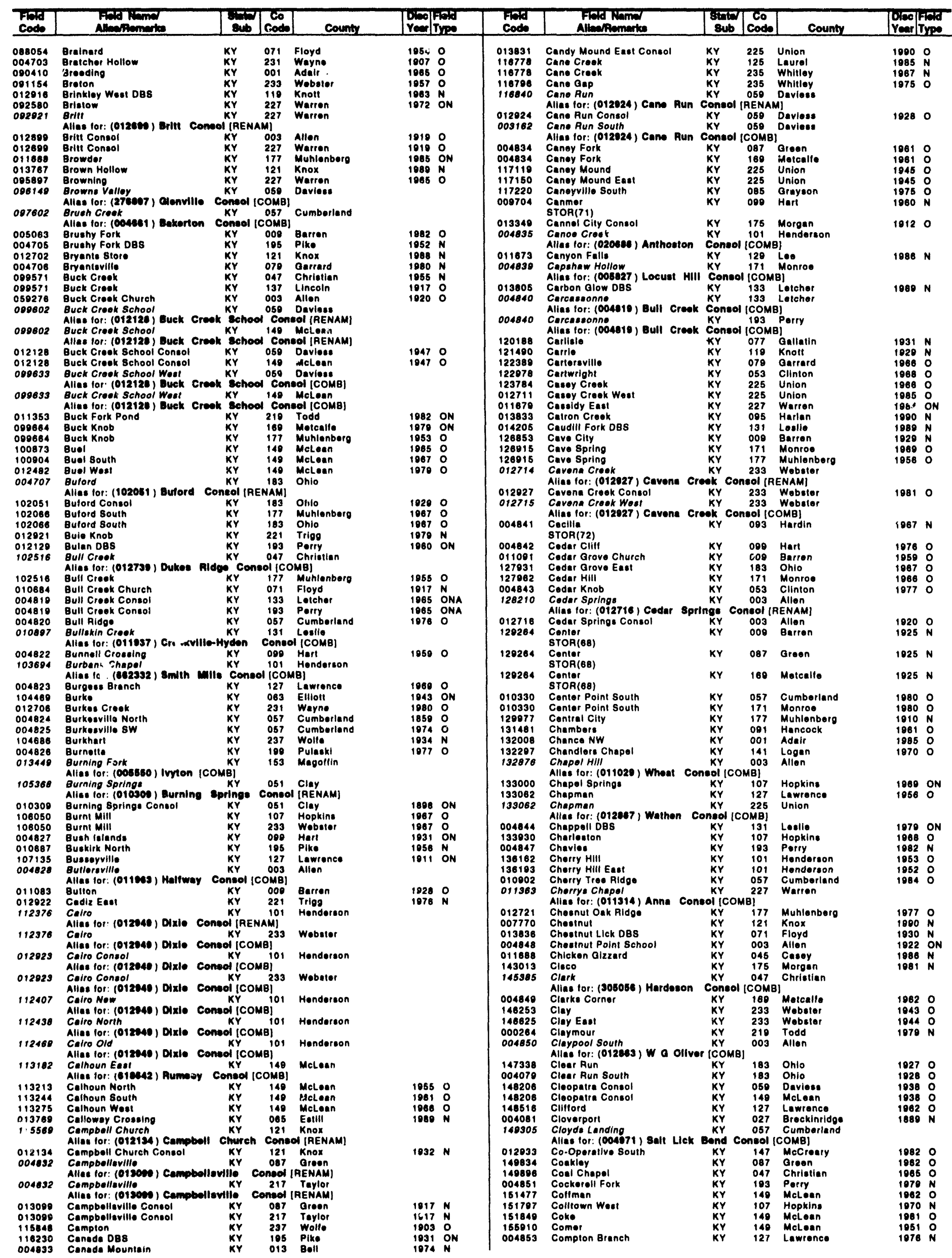




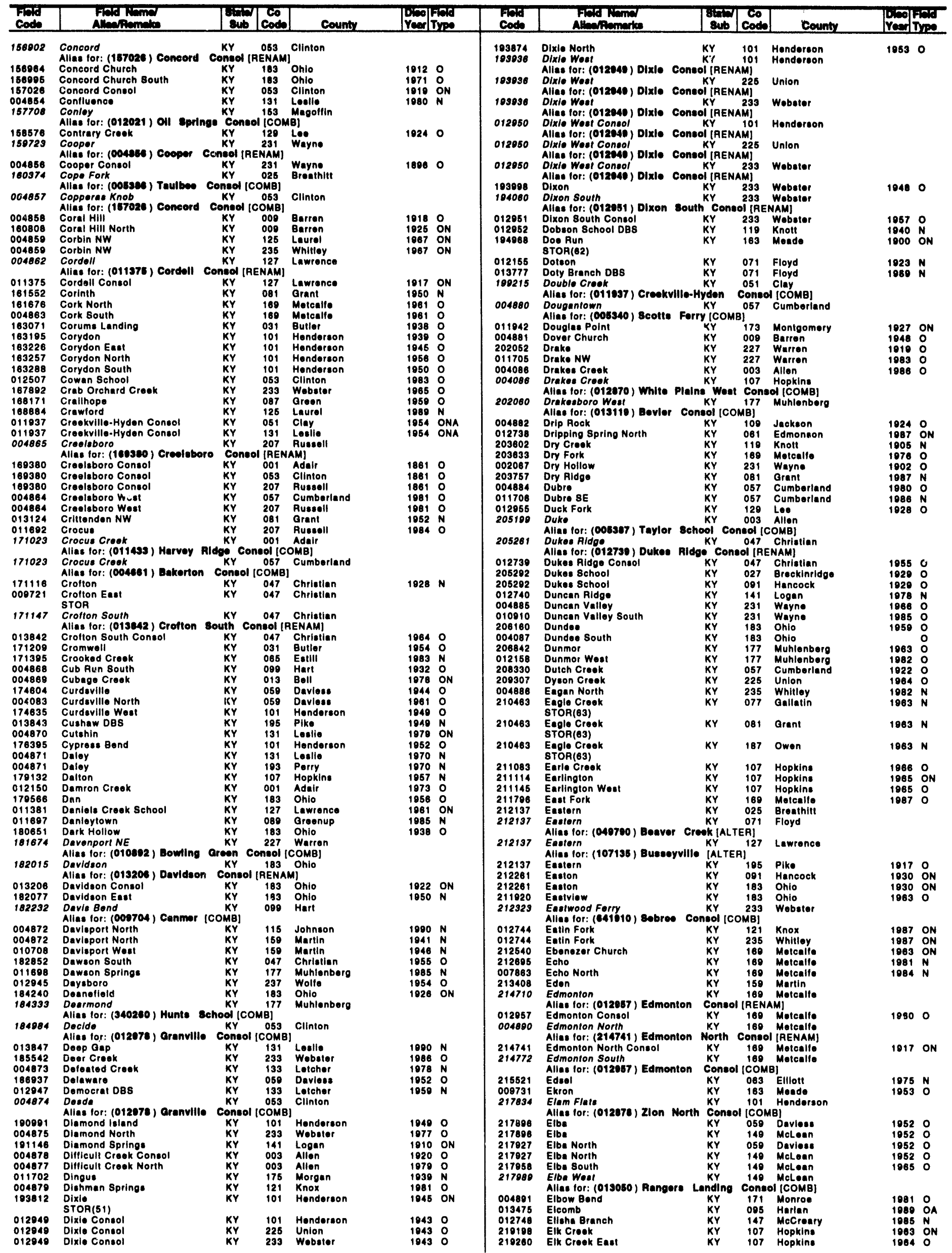


KENTUCKY

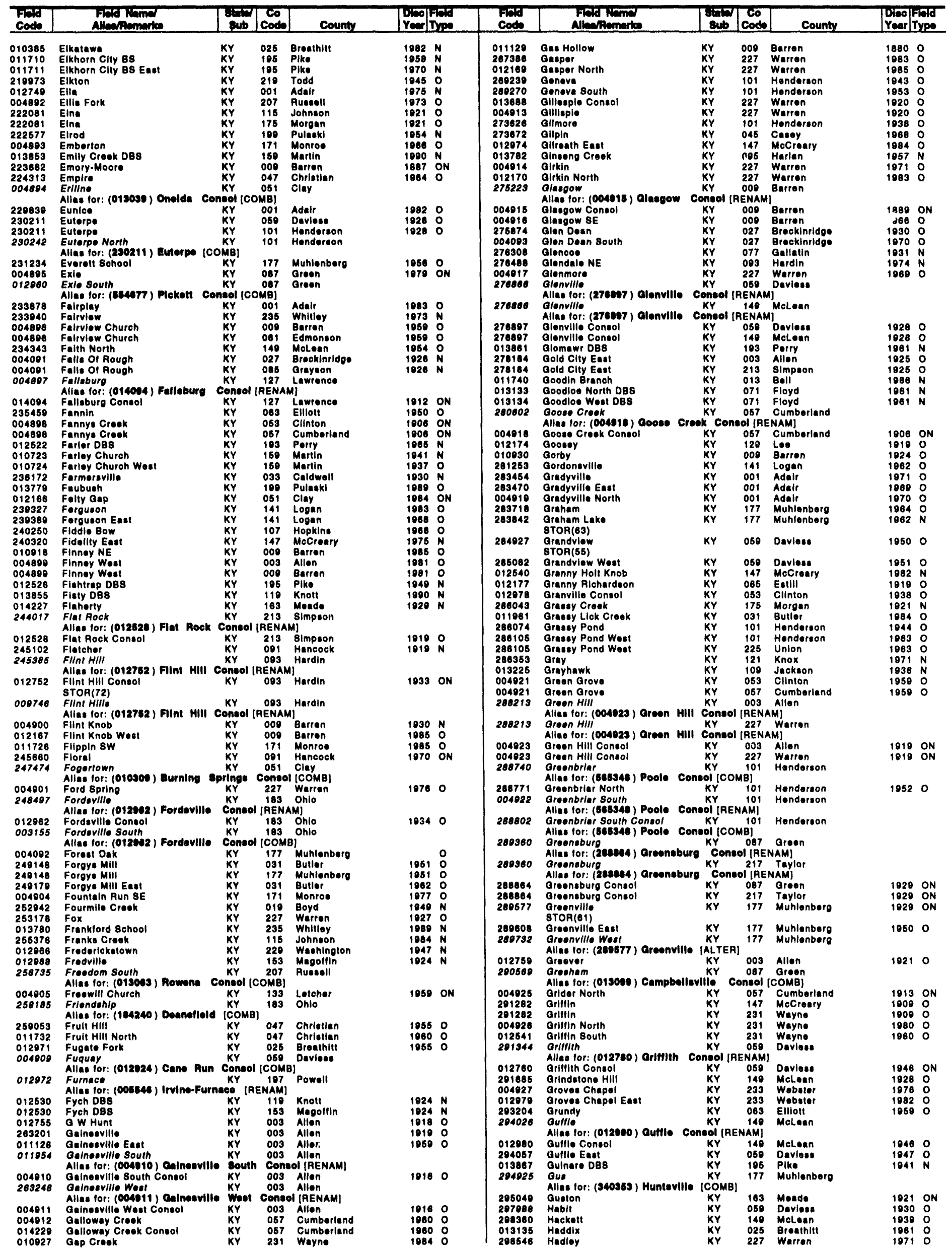




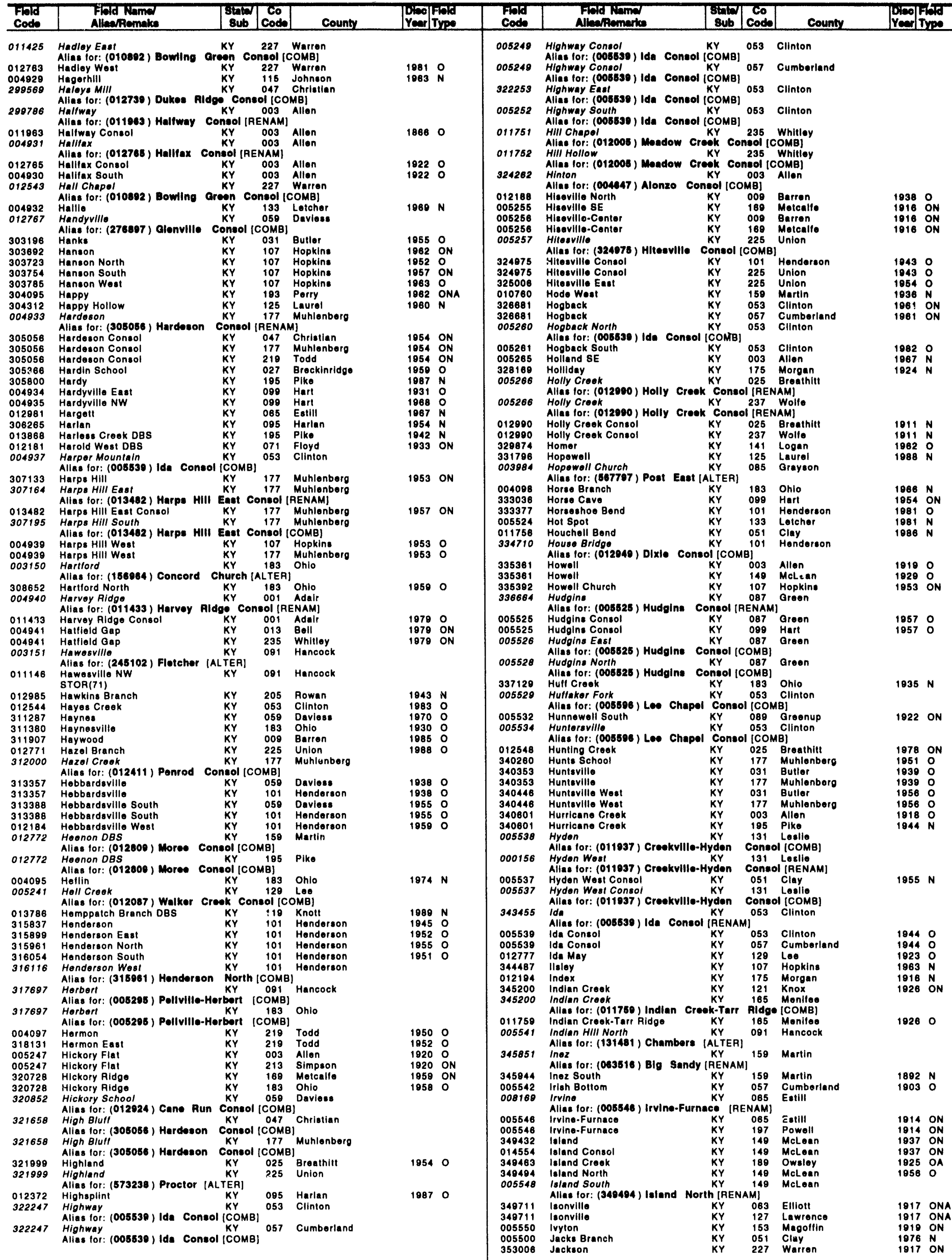


KENTUCKY

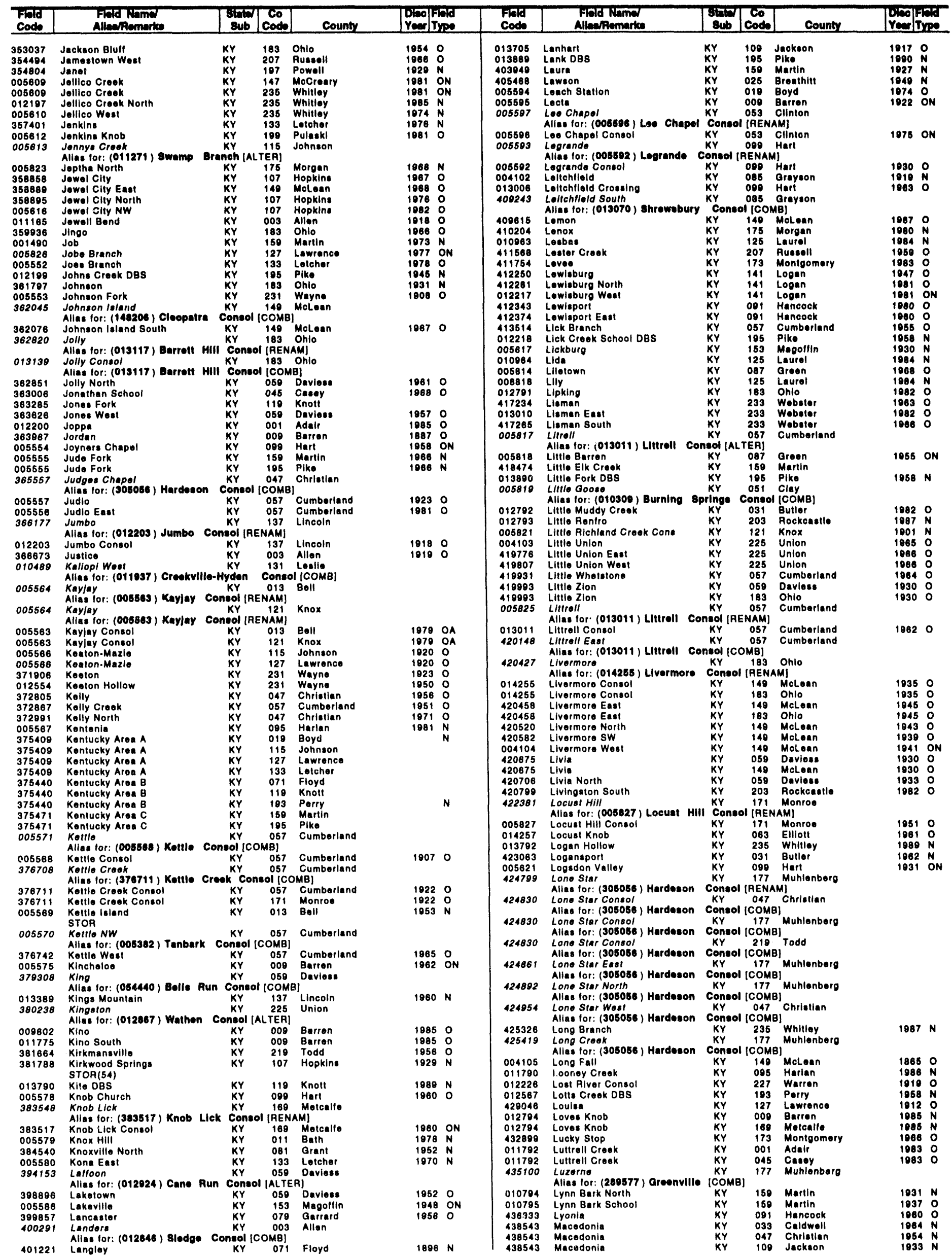




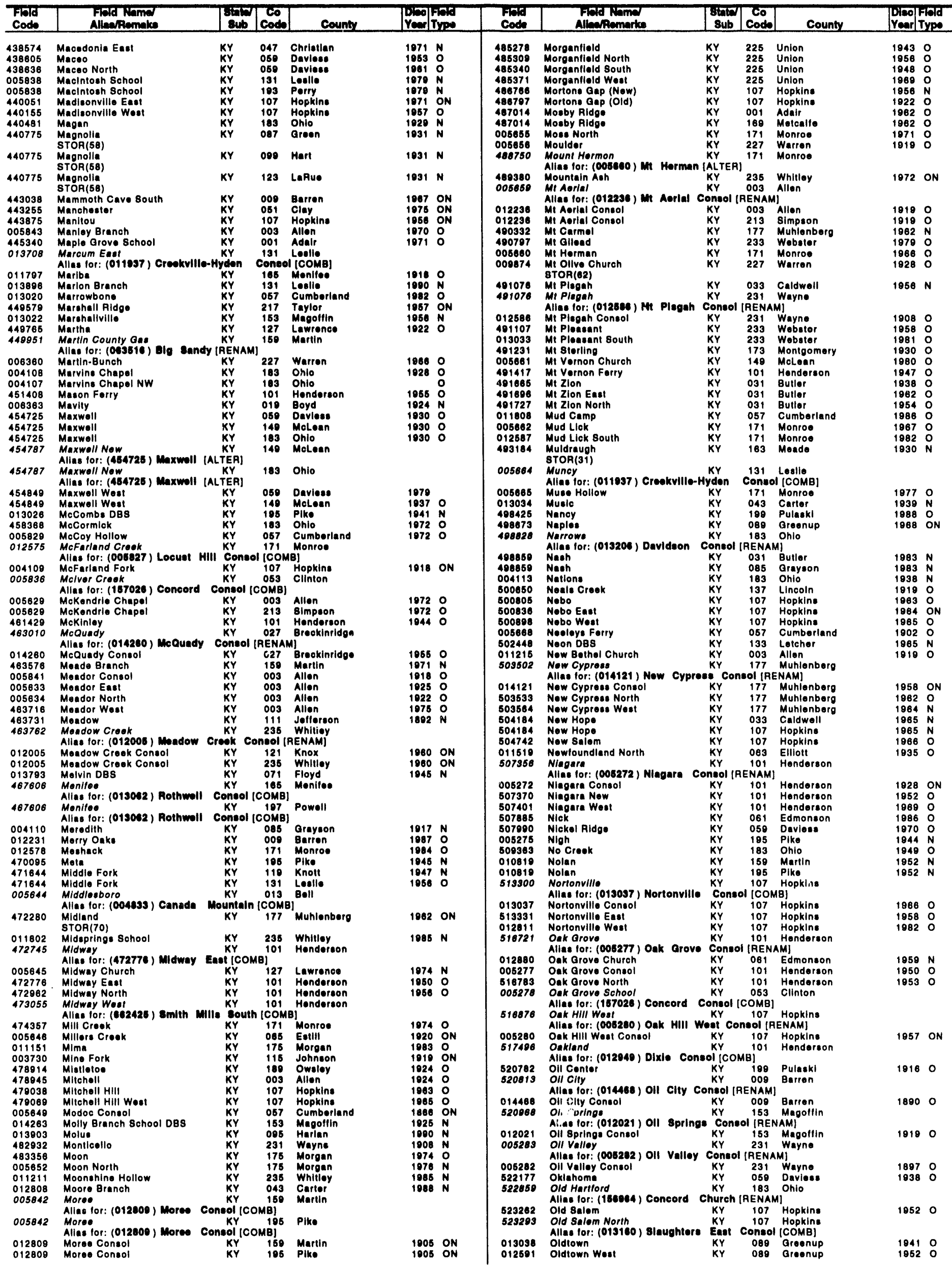


KENTUCKY

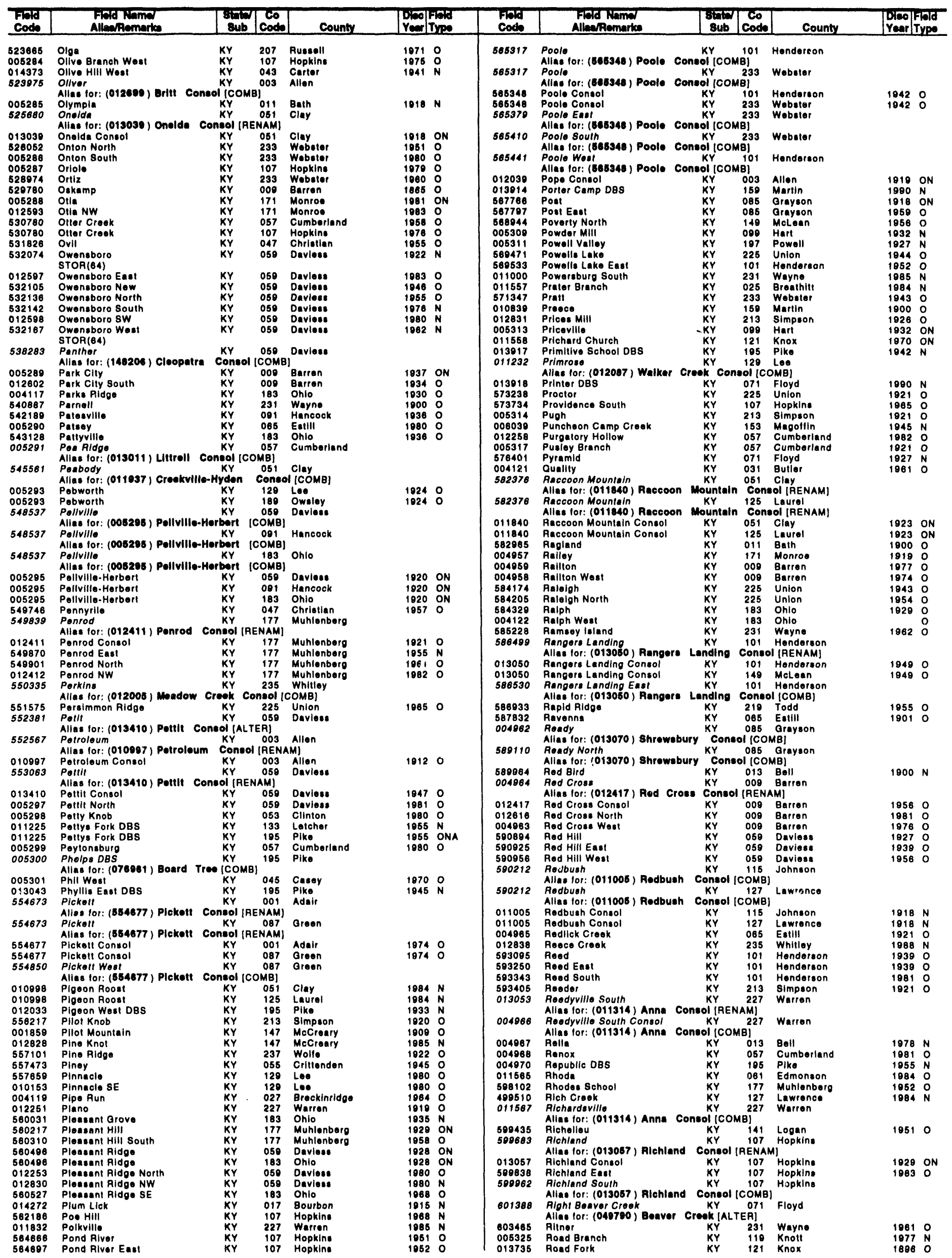




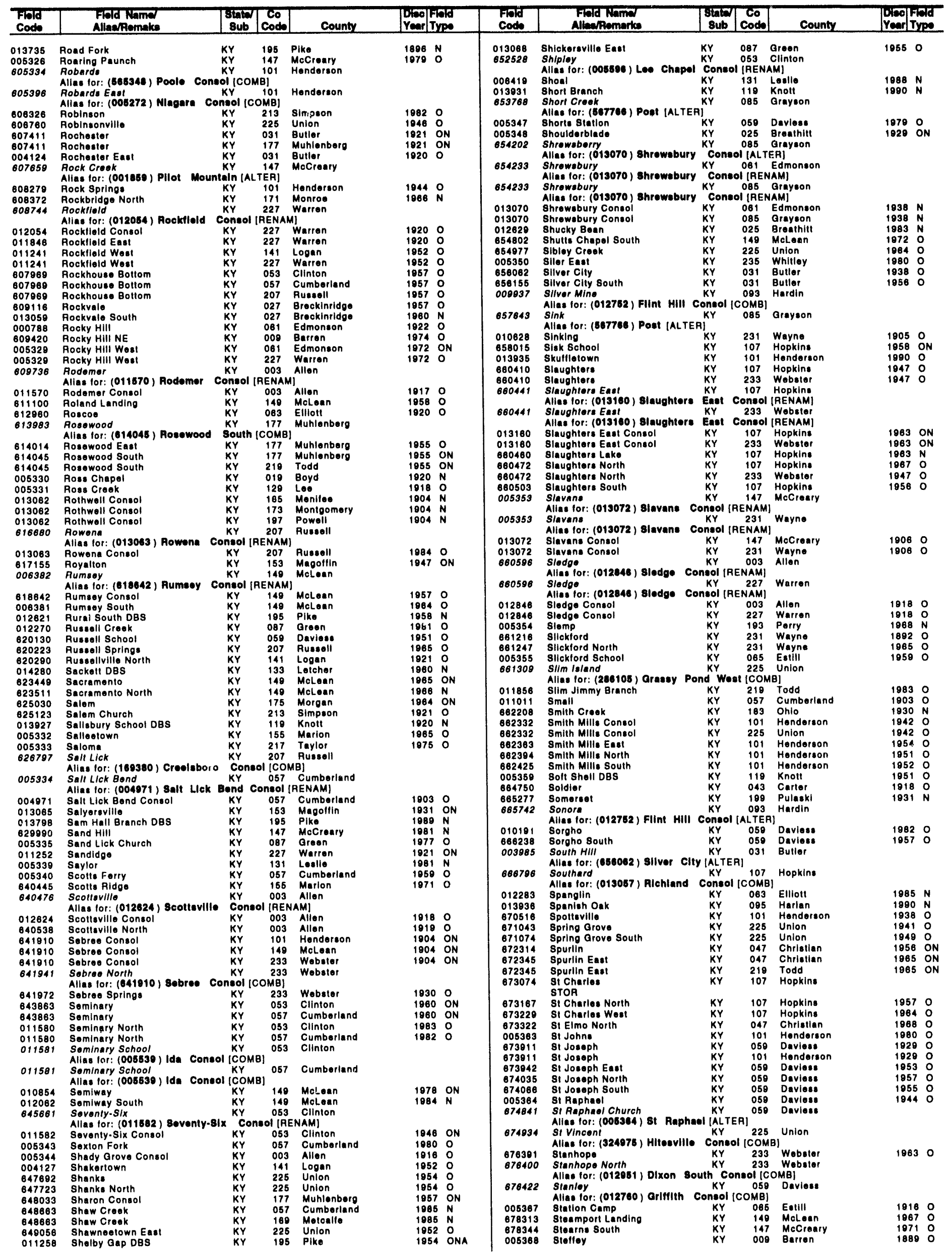


KENTUCKY

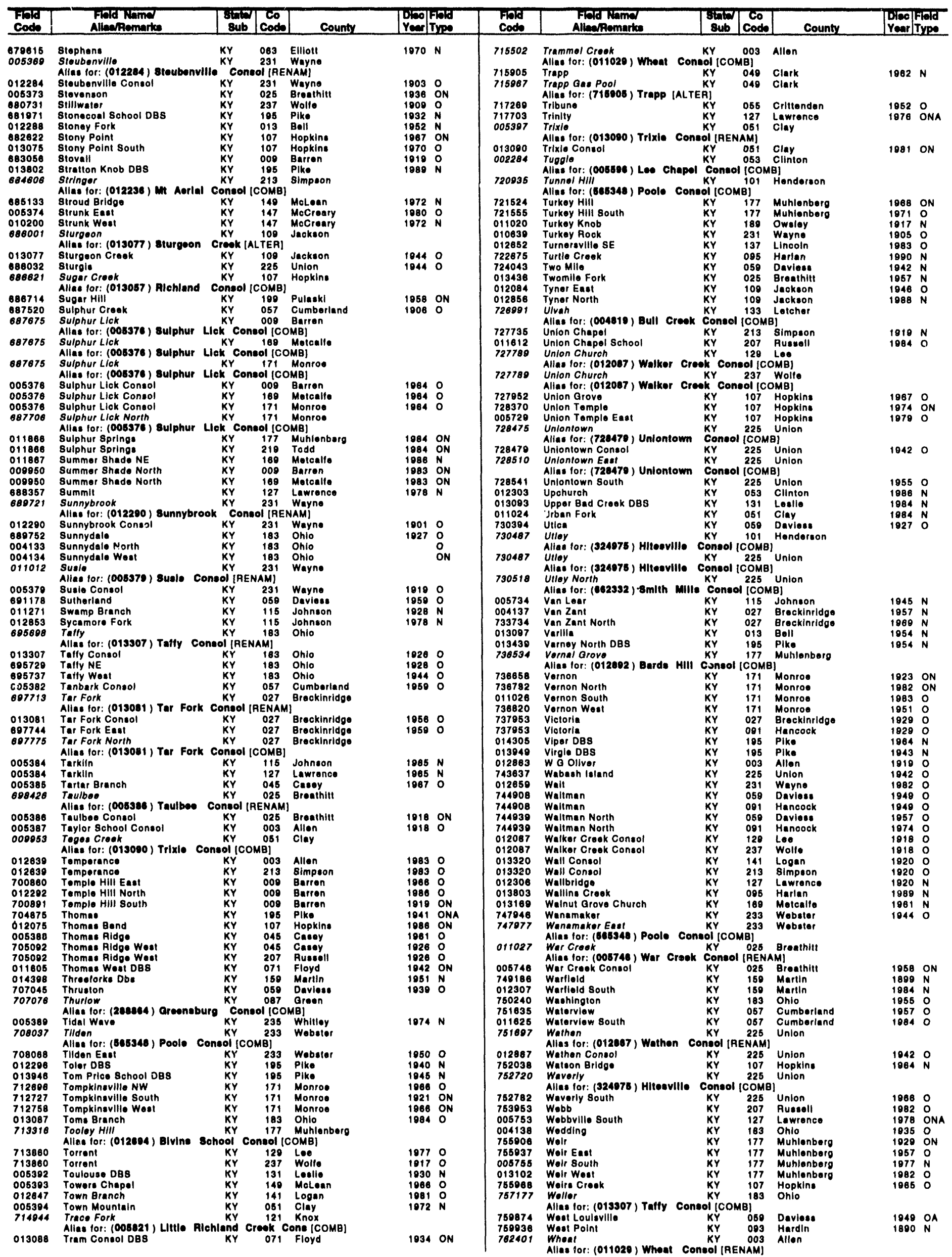




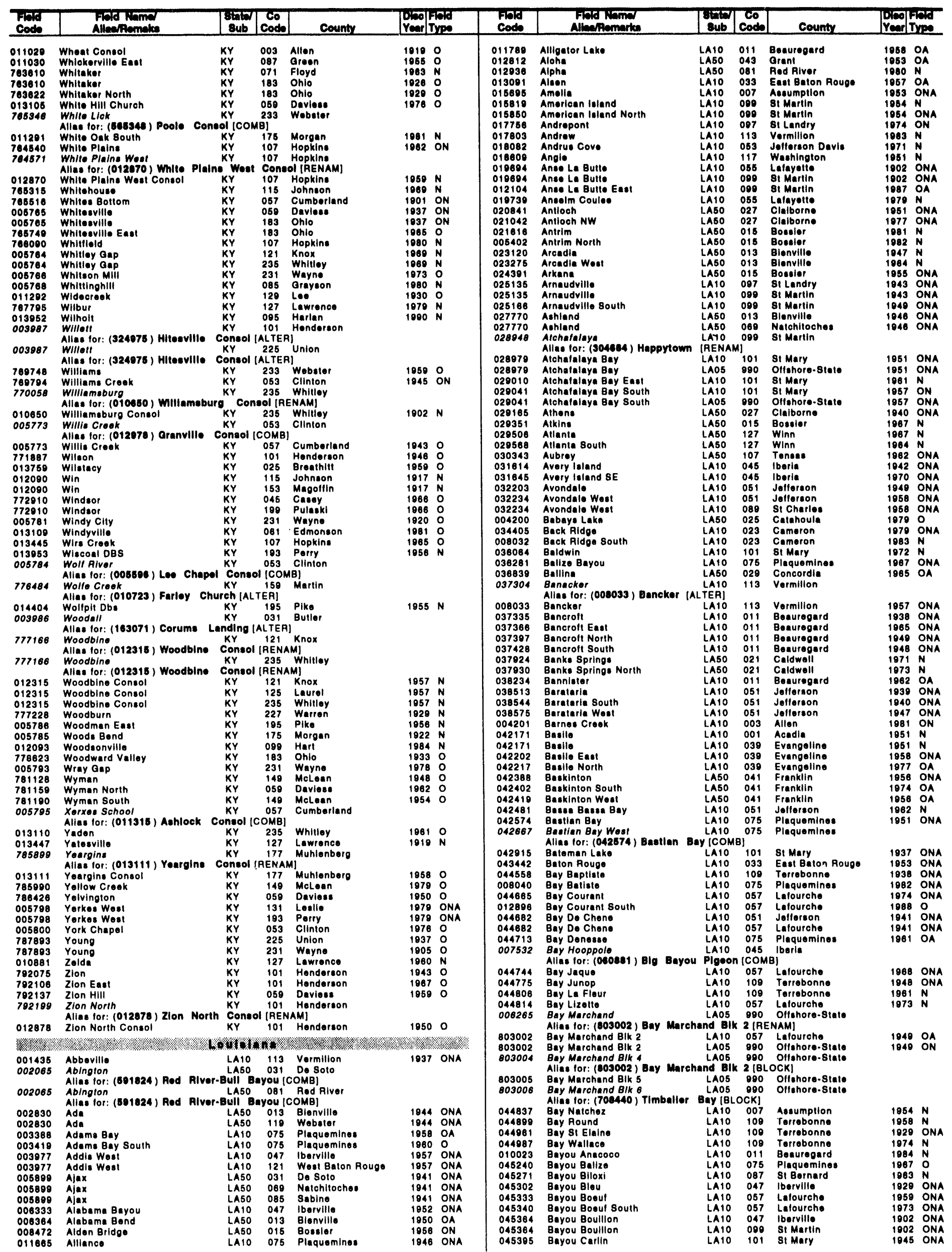


LOUISIANA

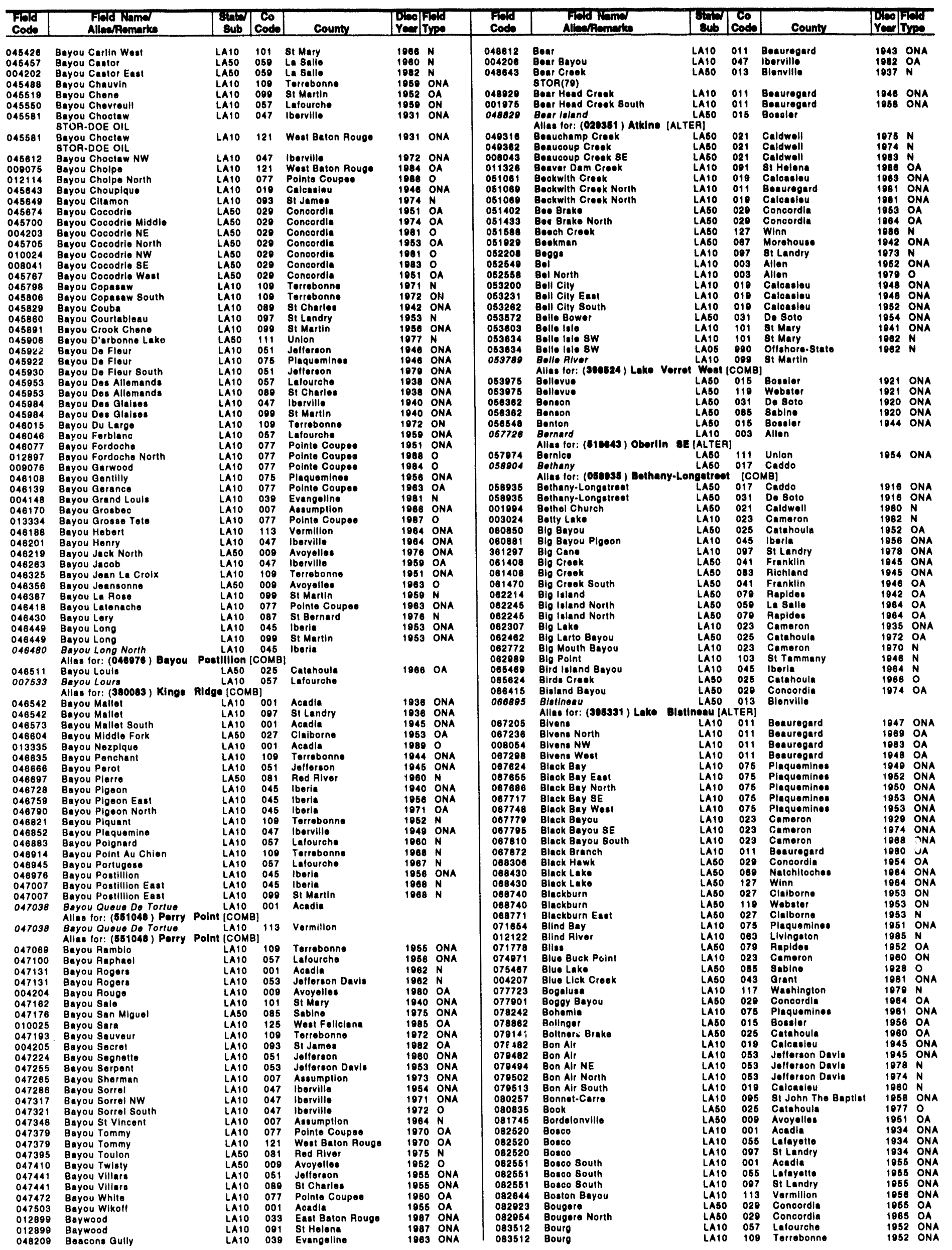


LOUISIANA

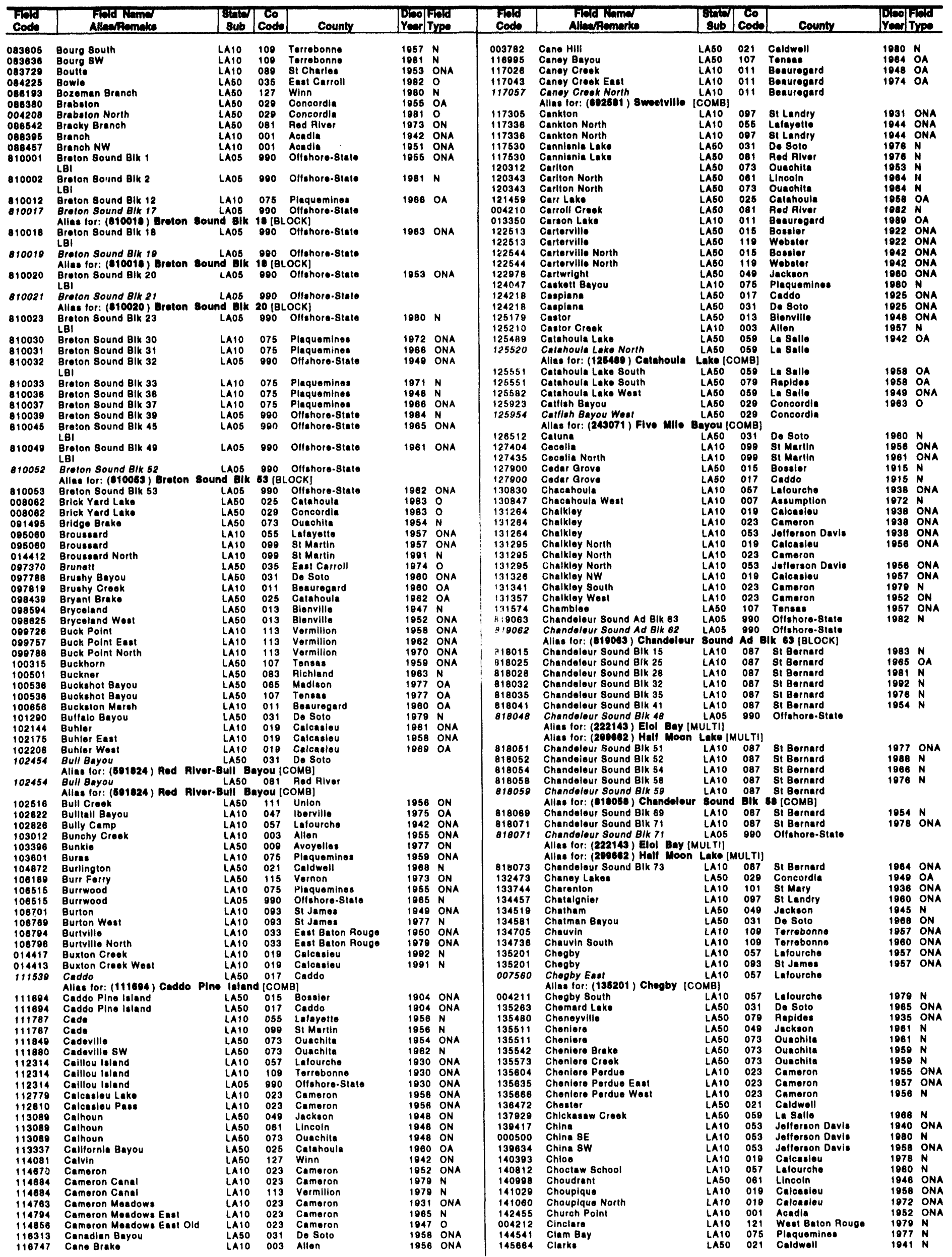


LOUISIANA

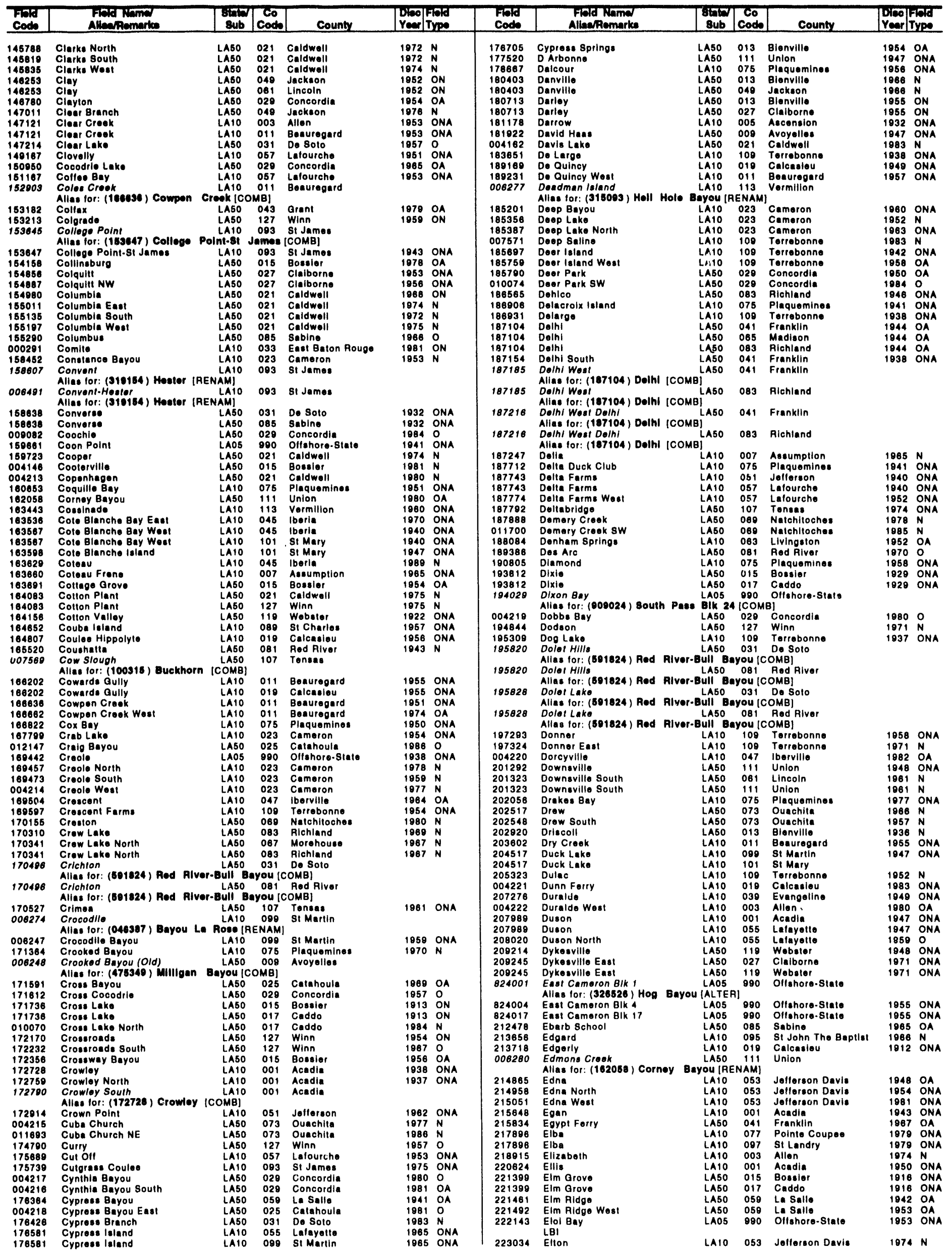




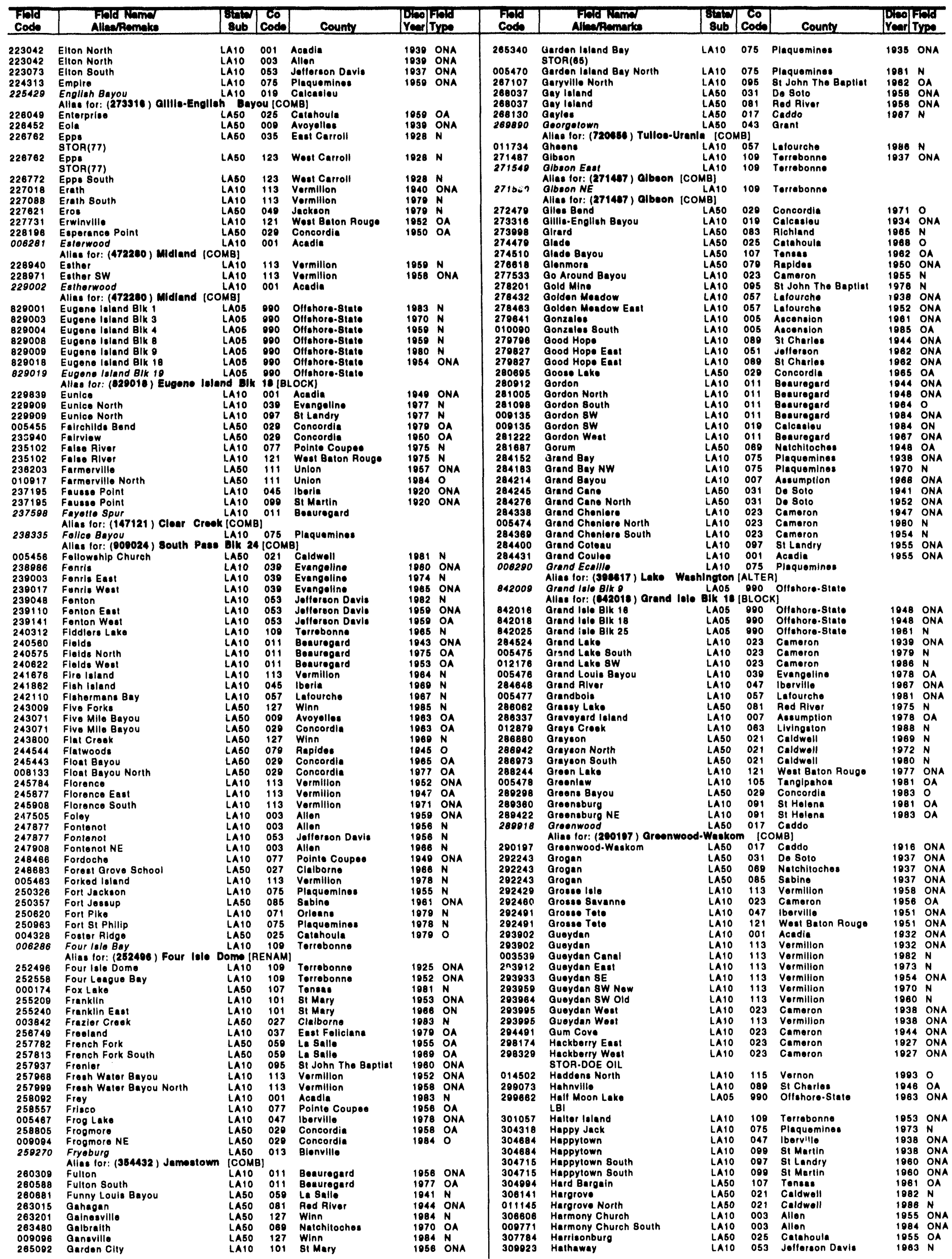


LOUISIANA

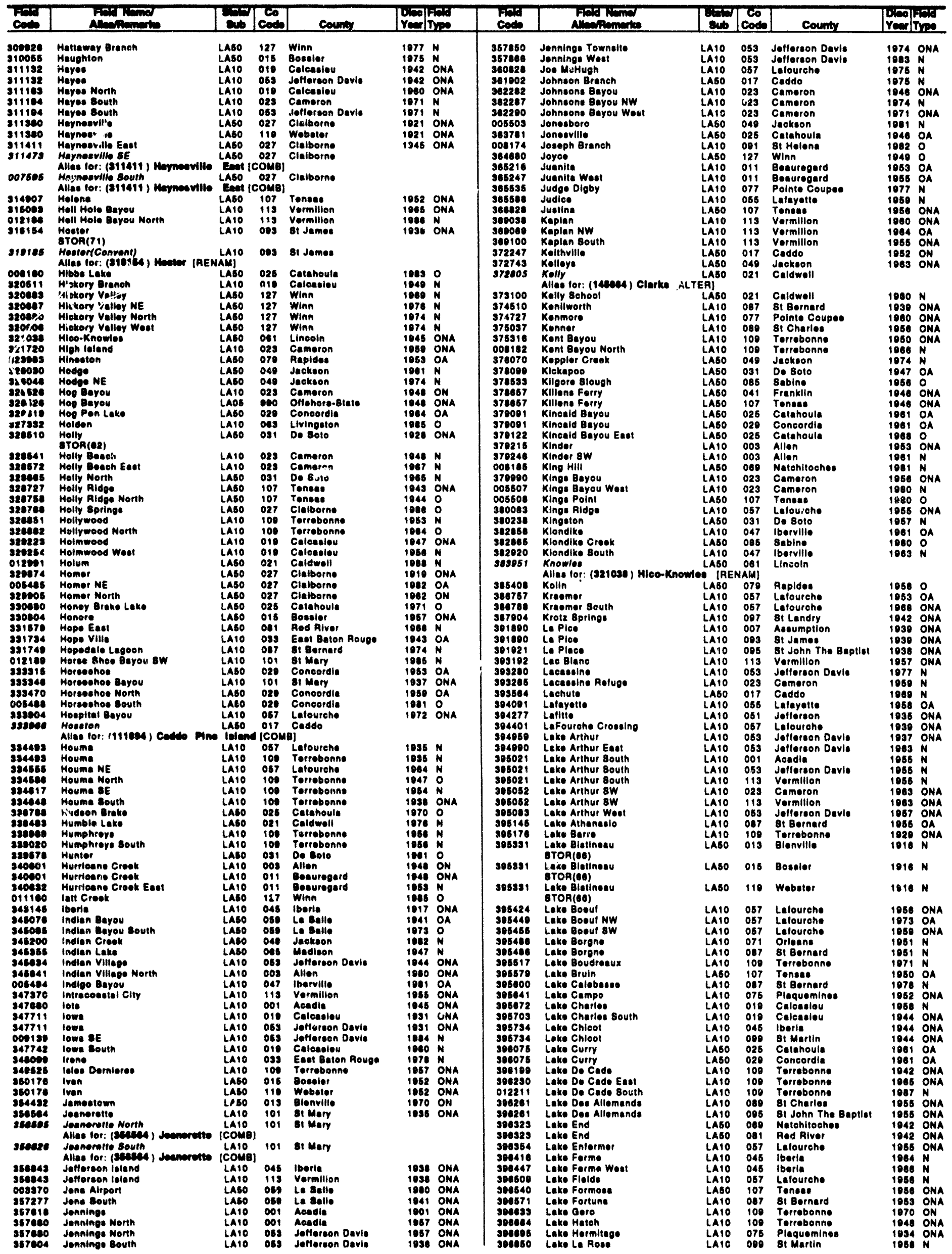




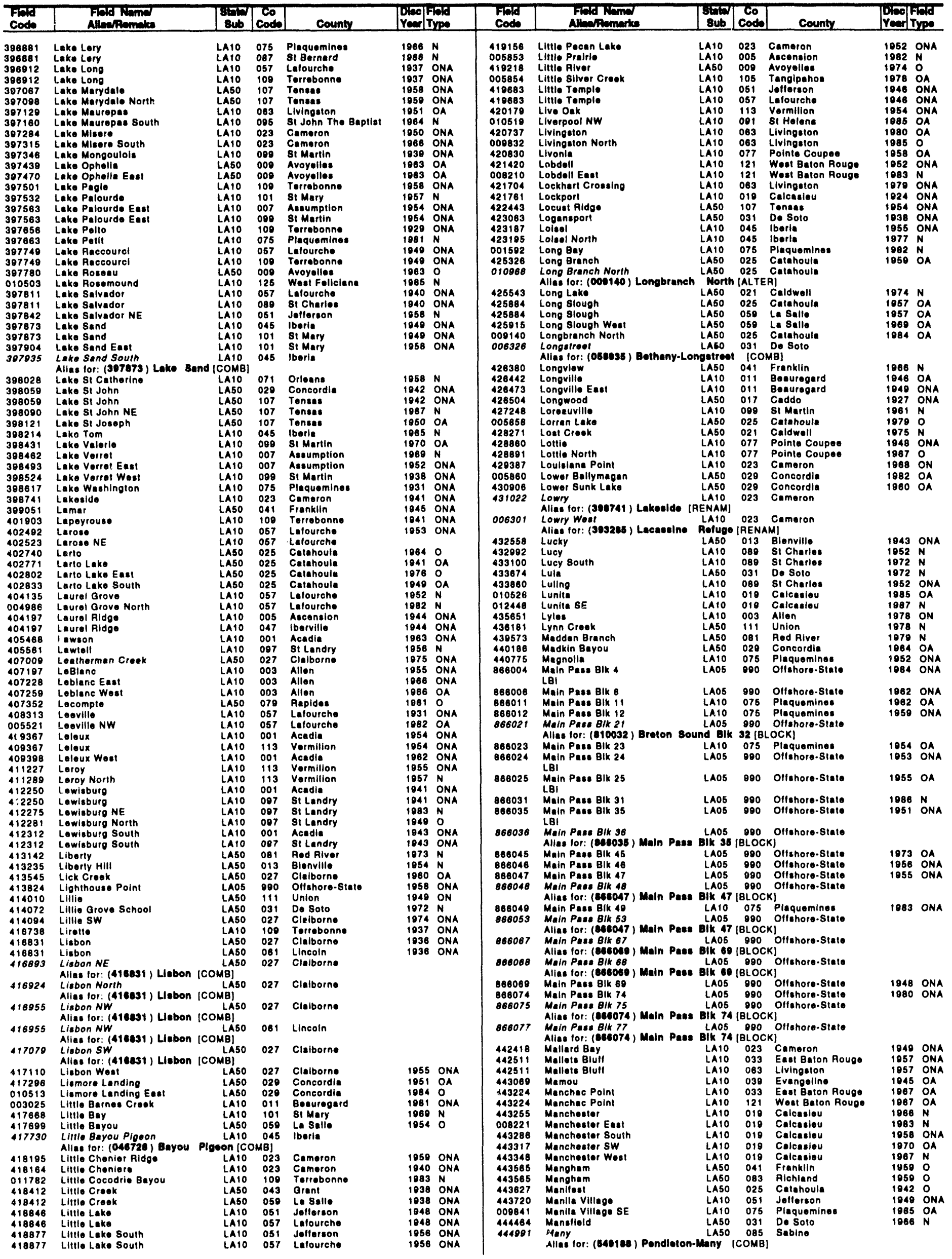


LOUISIANA

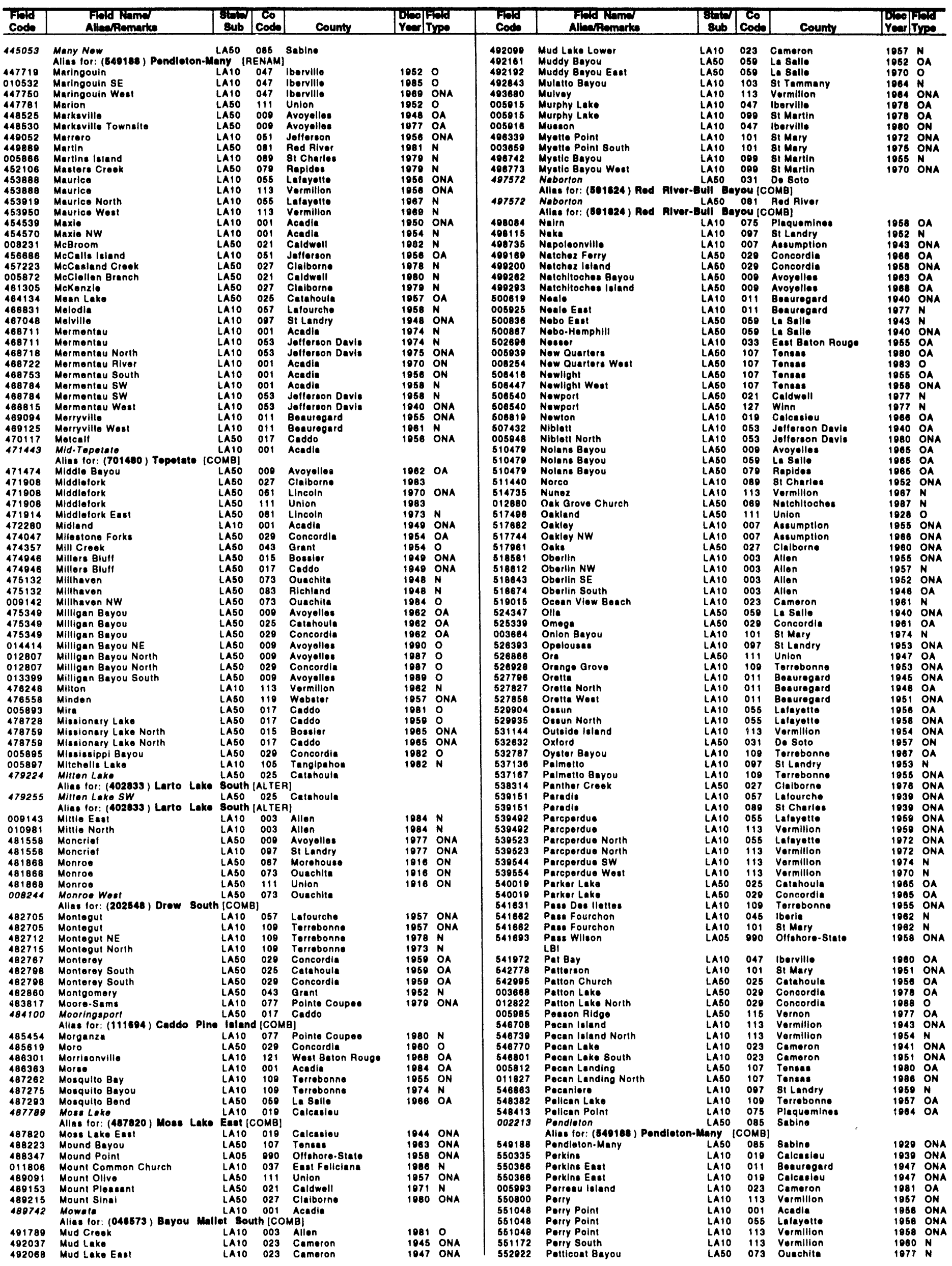




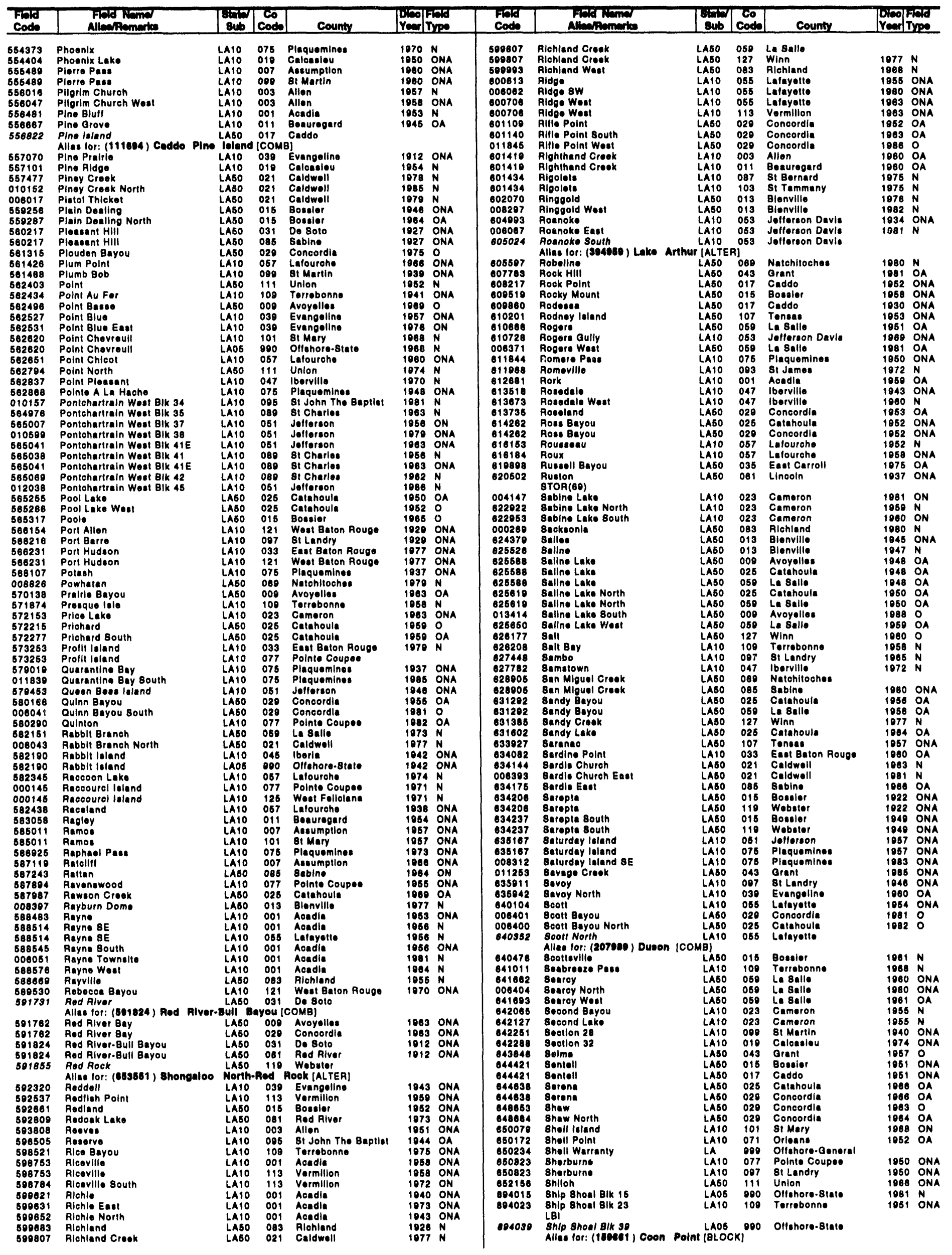


LOUISIANA

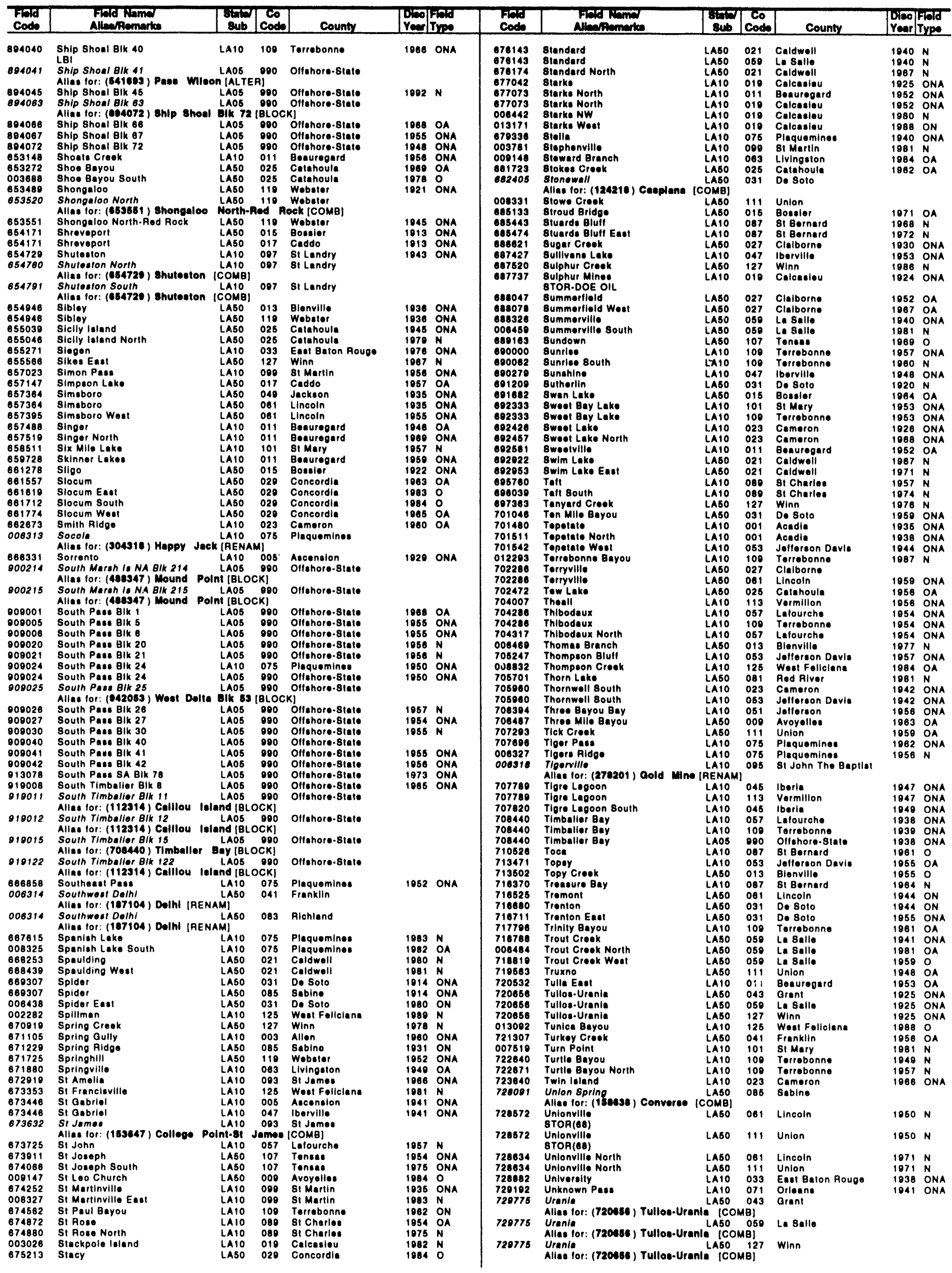




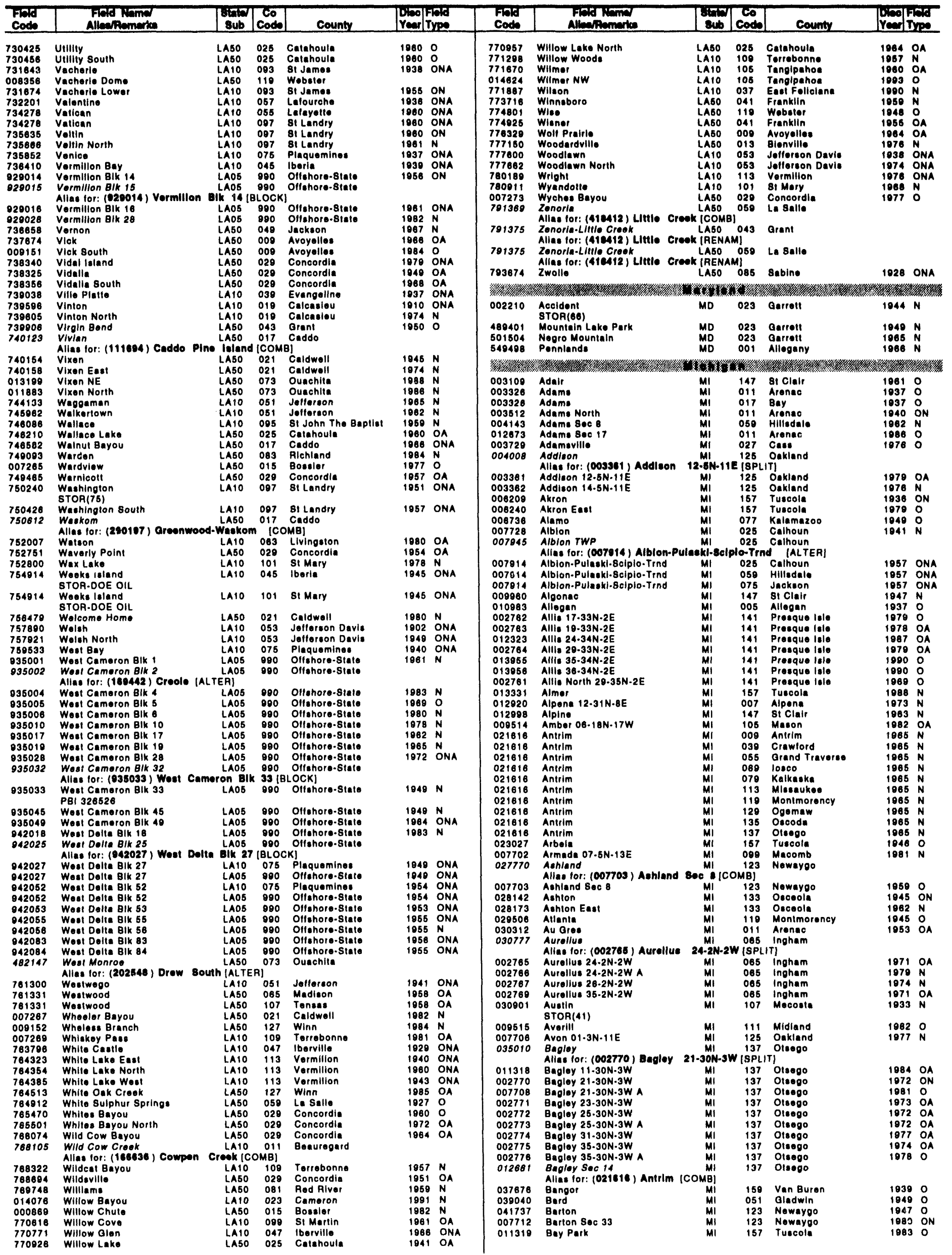


MICHIGAN

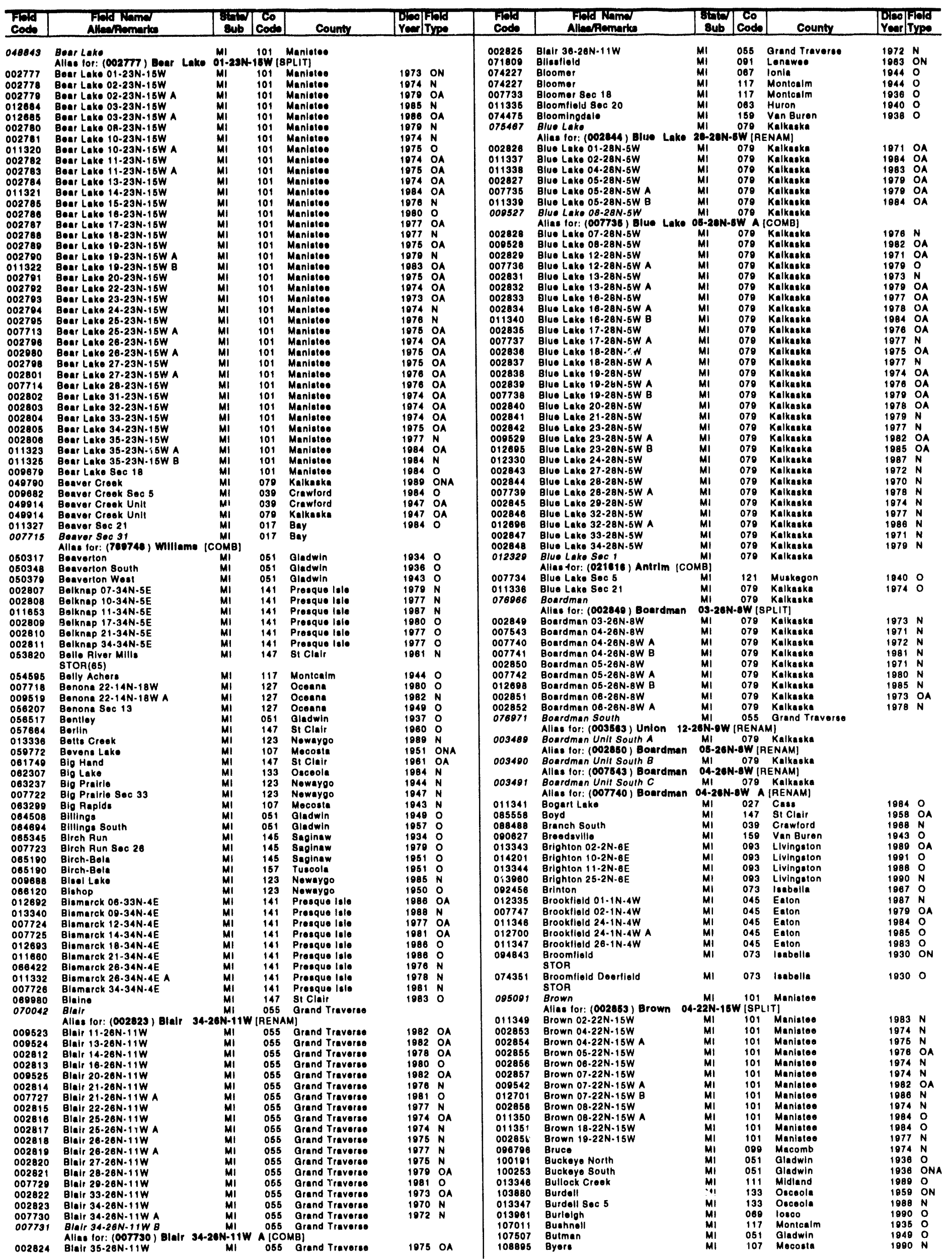




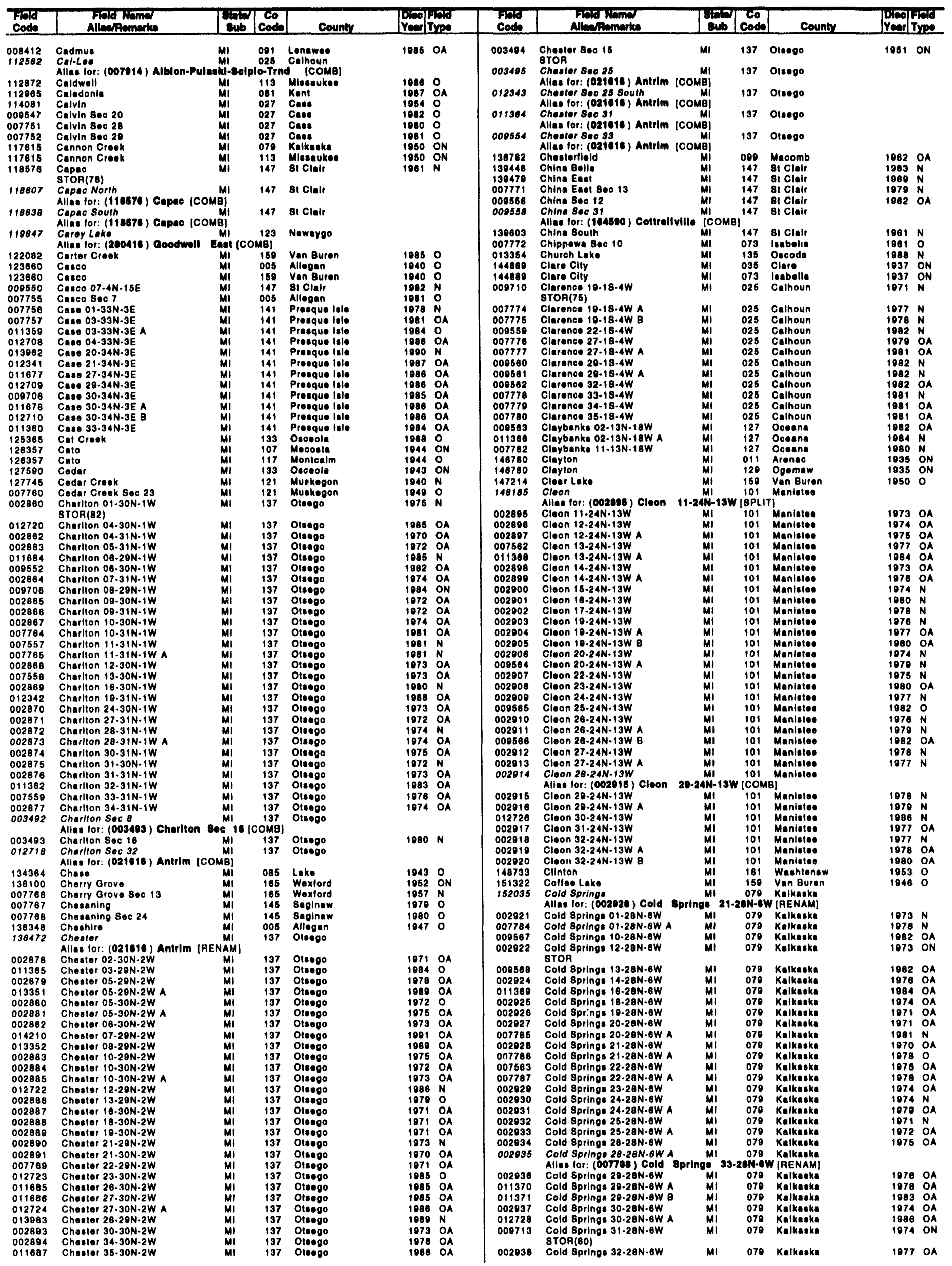


MICHIGAN

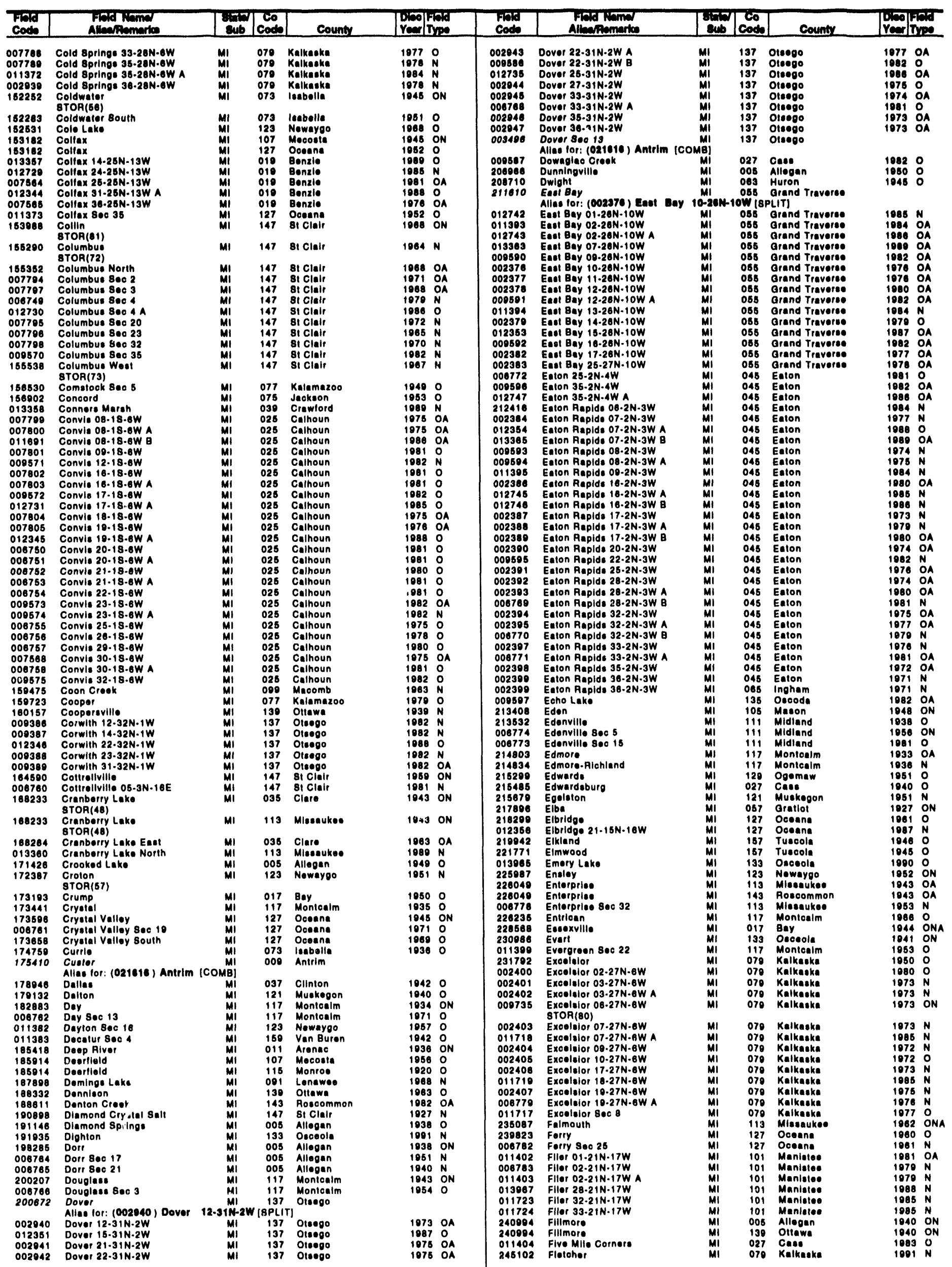




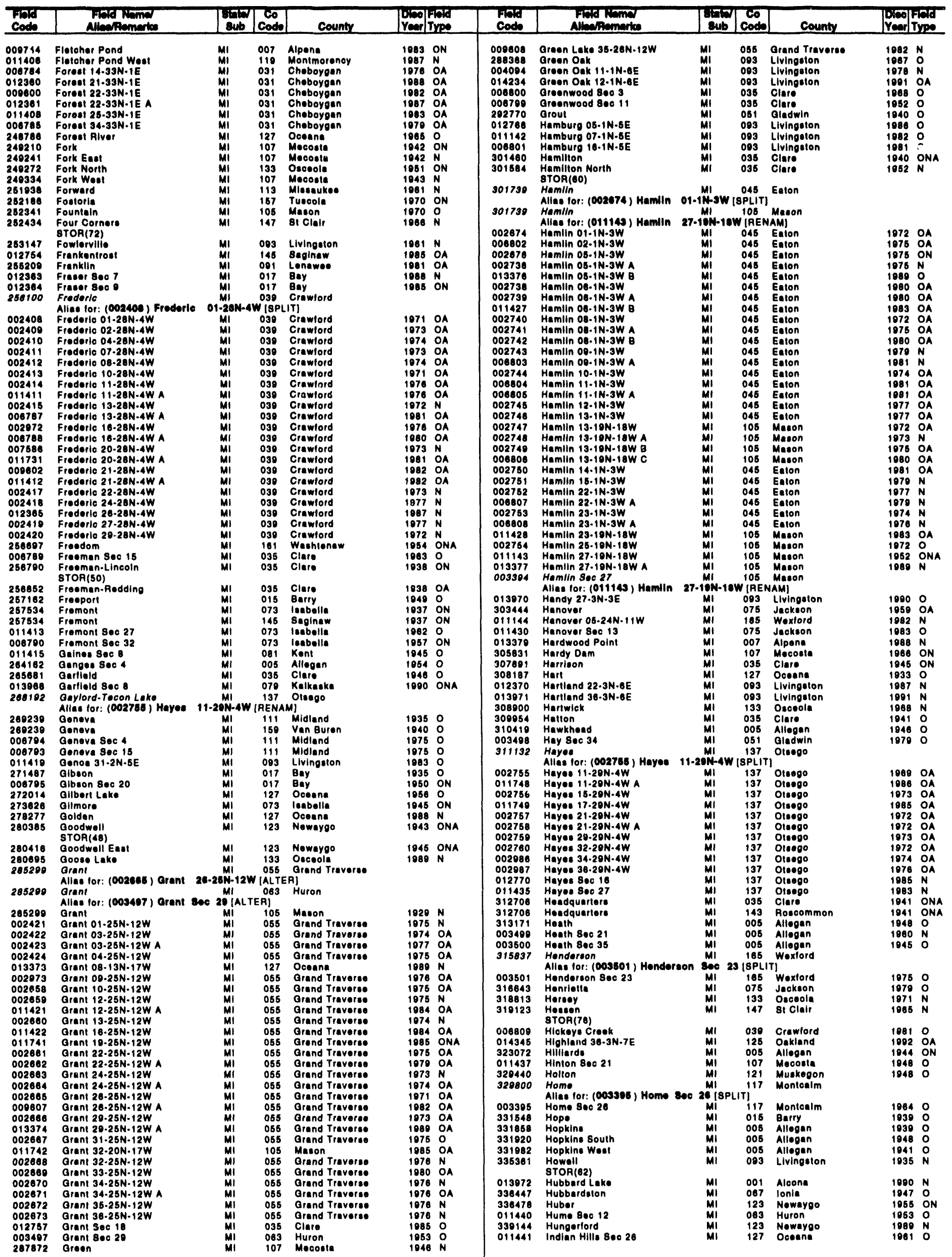


MICHIGAN

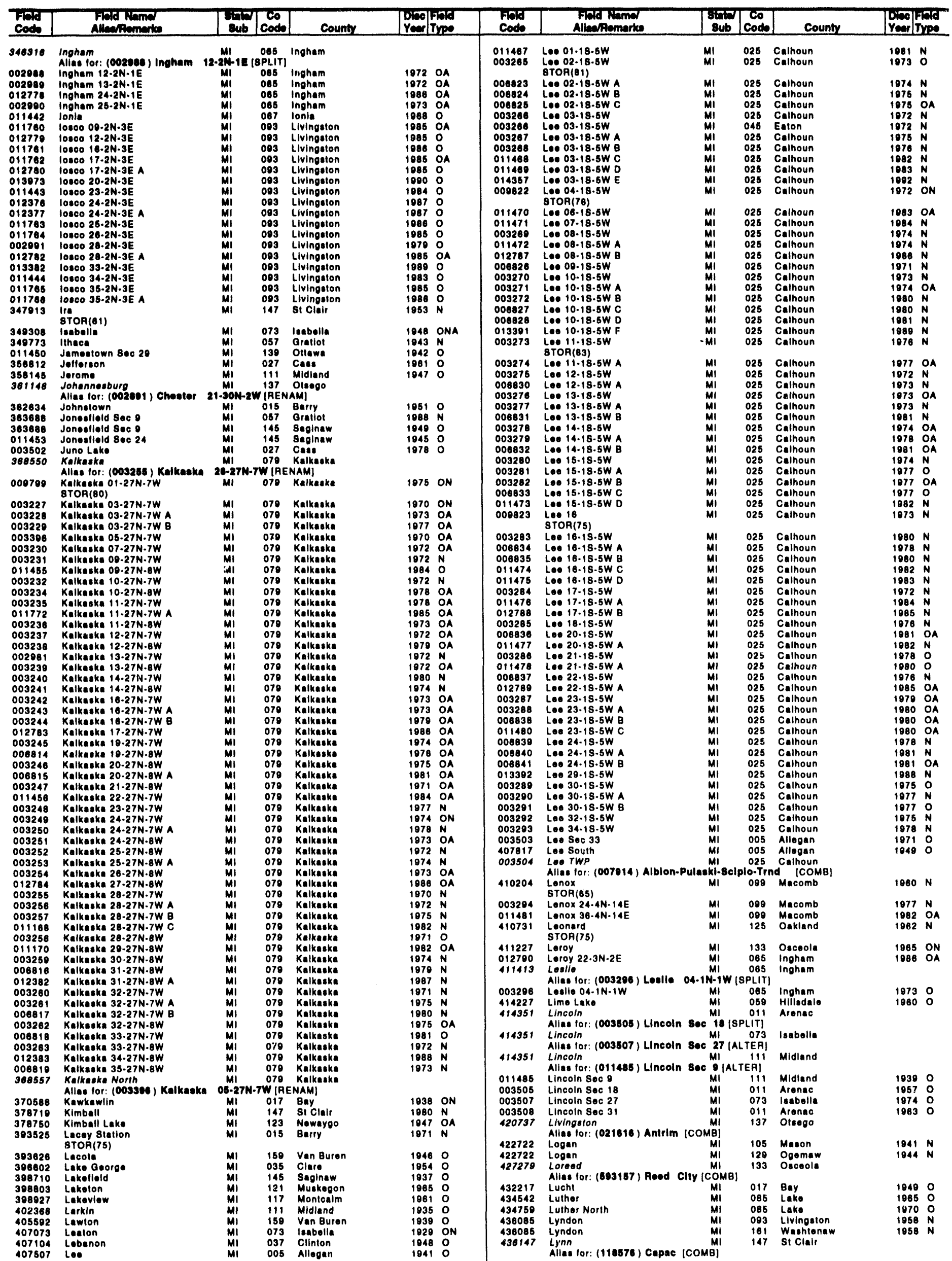




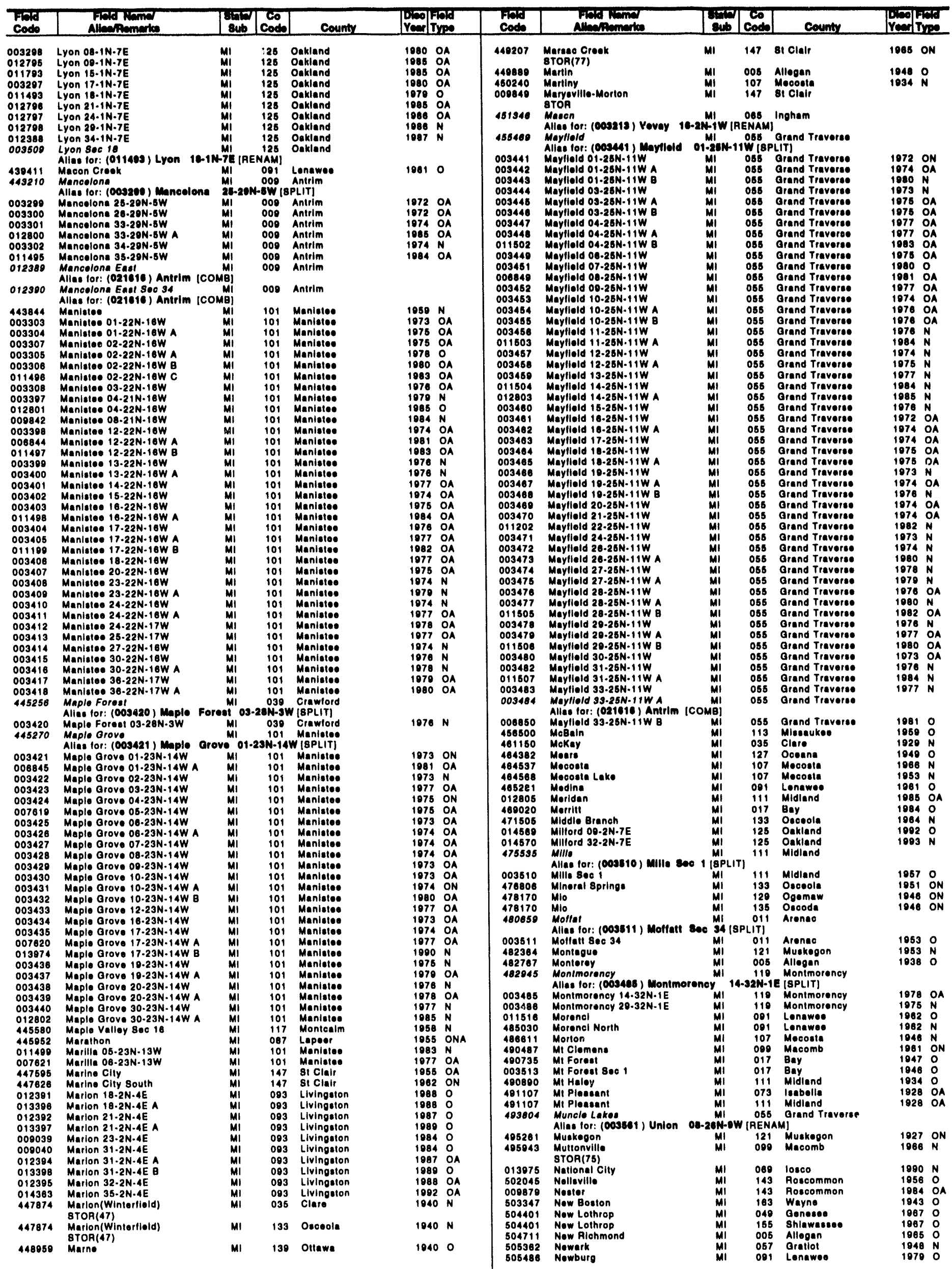


MICHIGAN

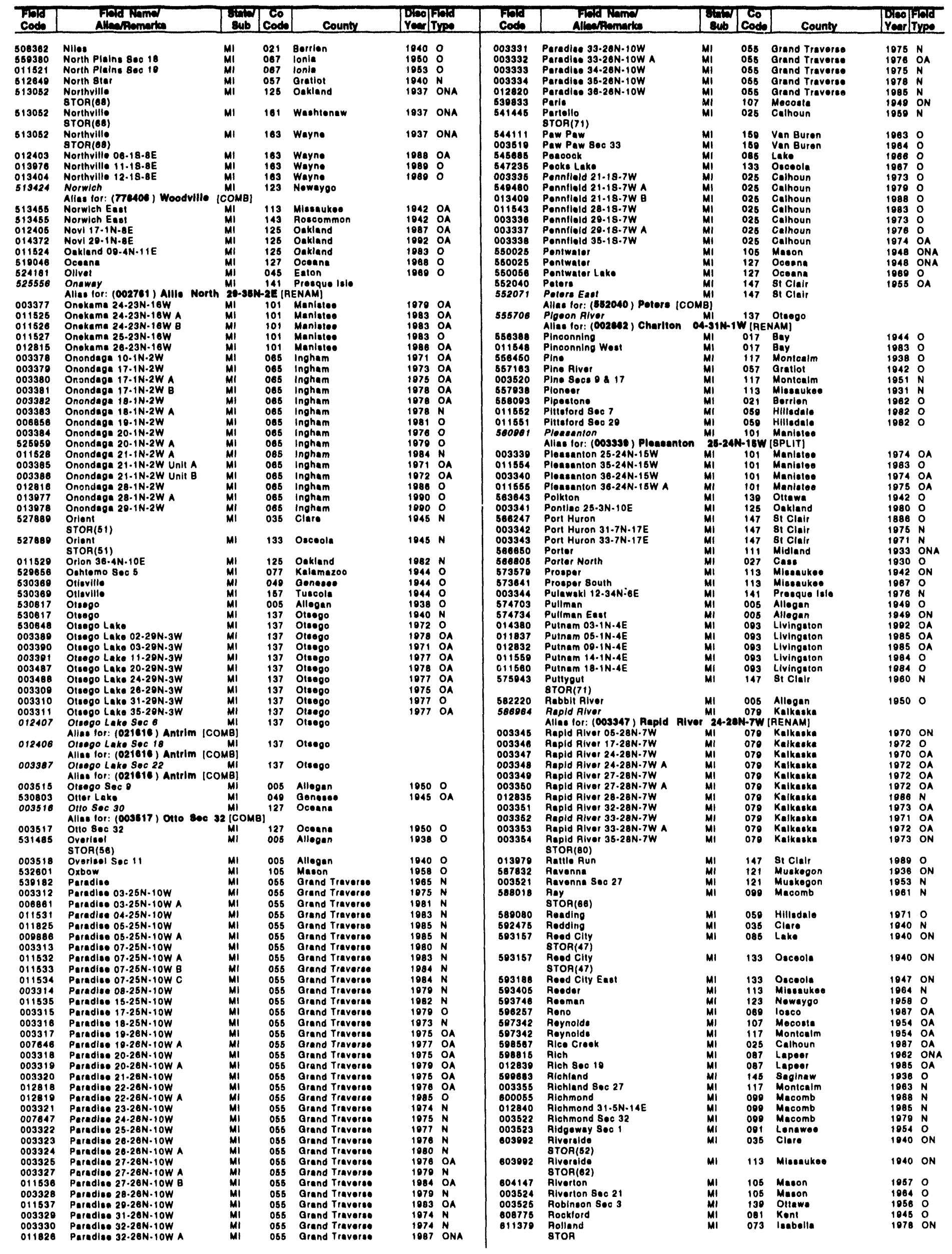




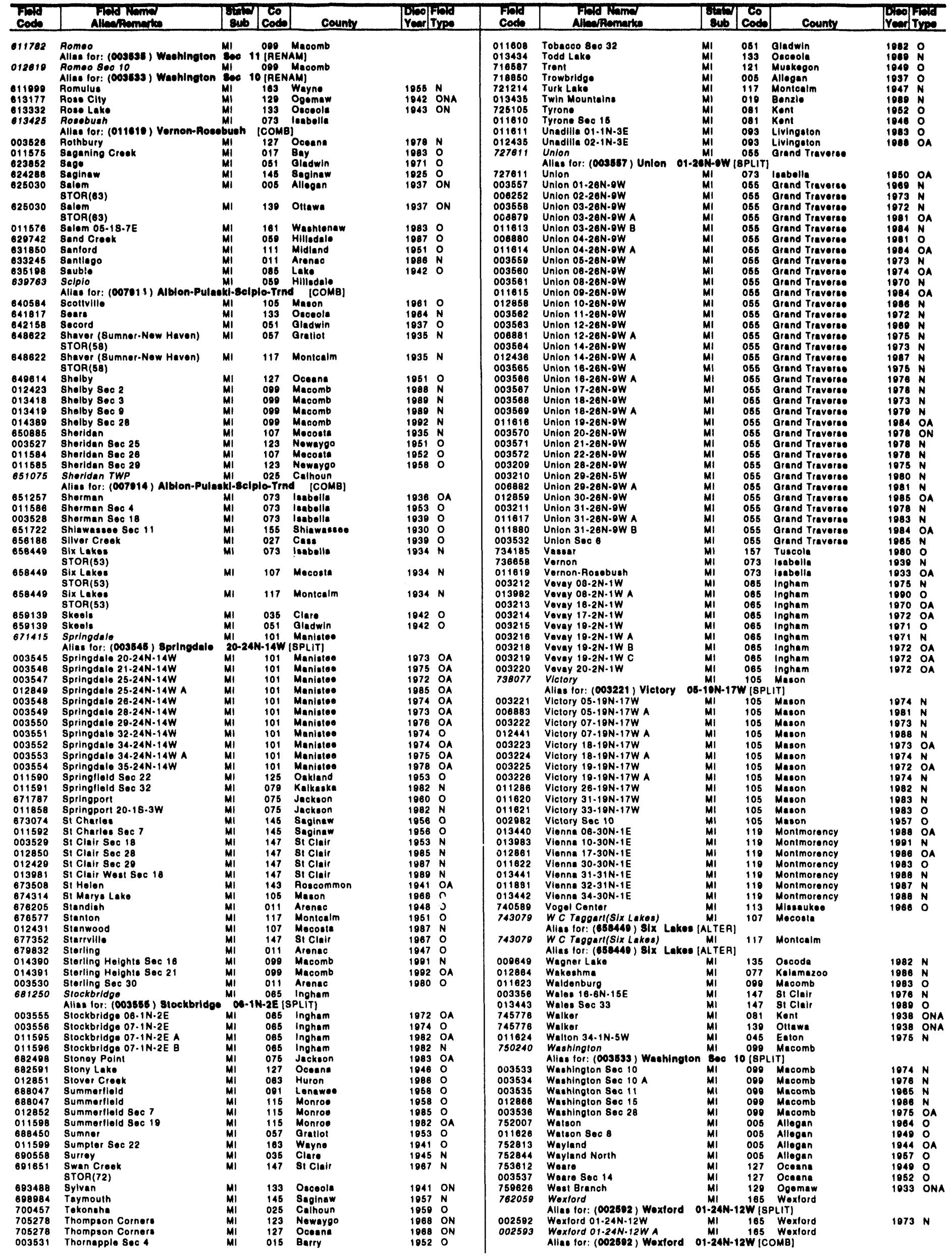


MISSISSIPPI

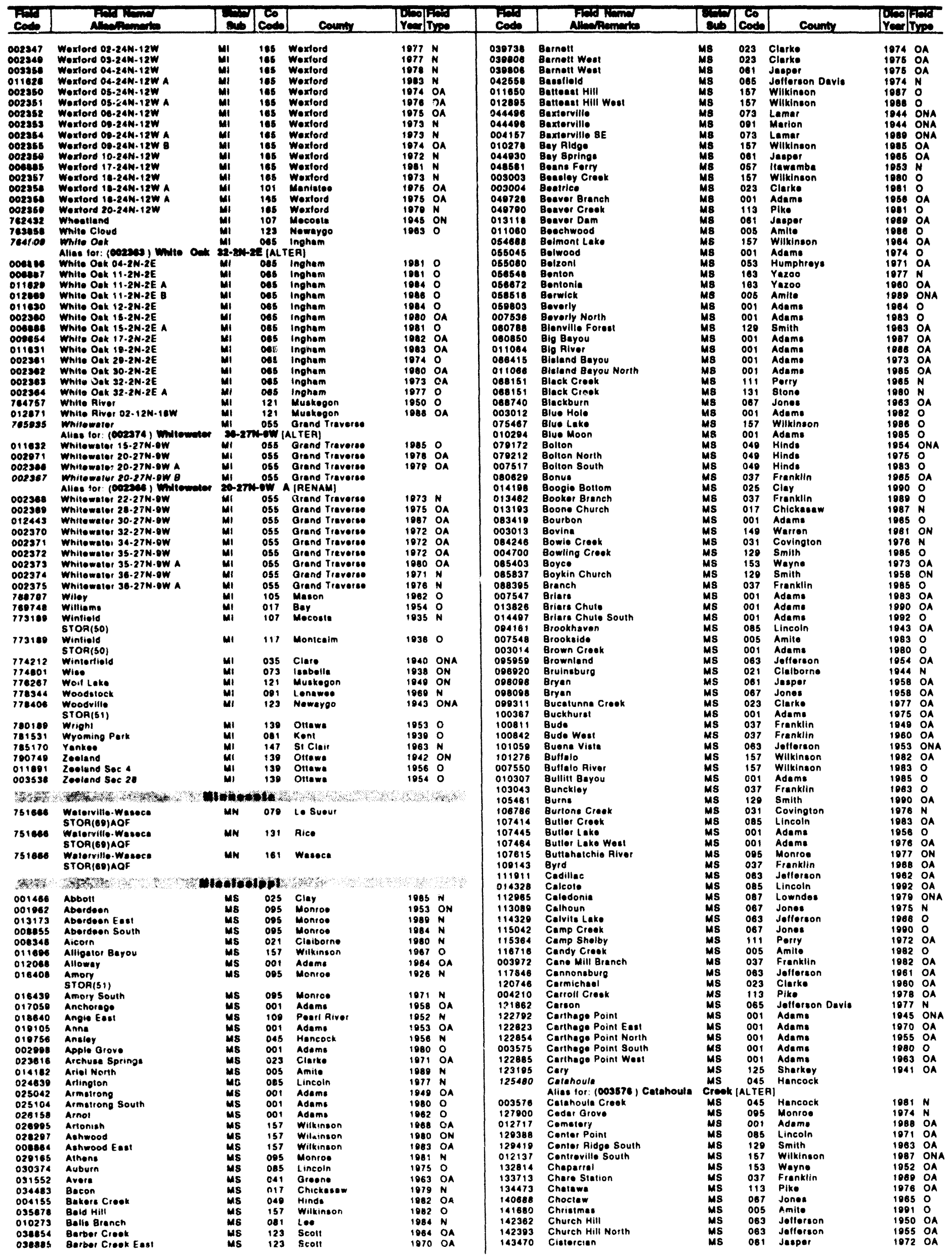




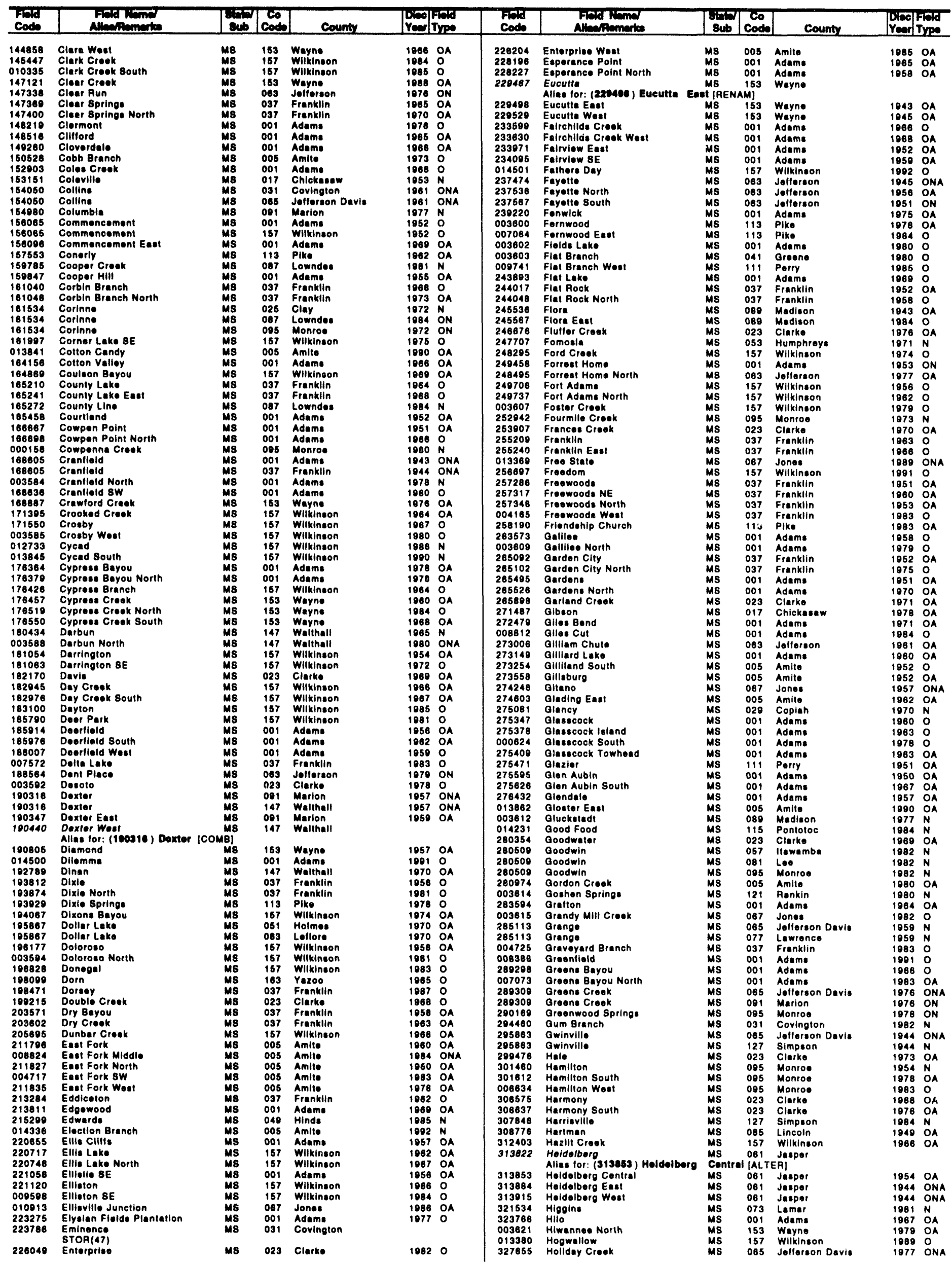


MISSISSIPPI

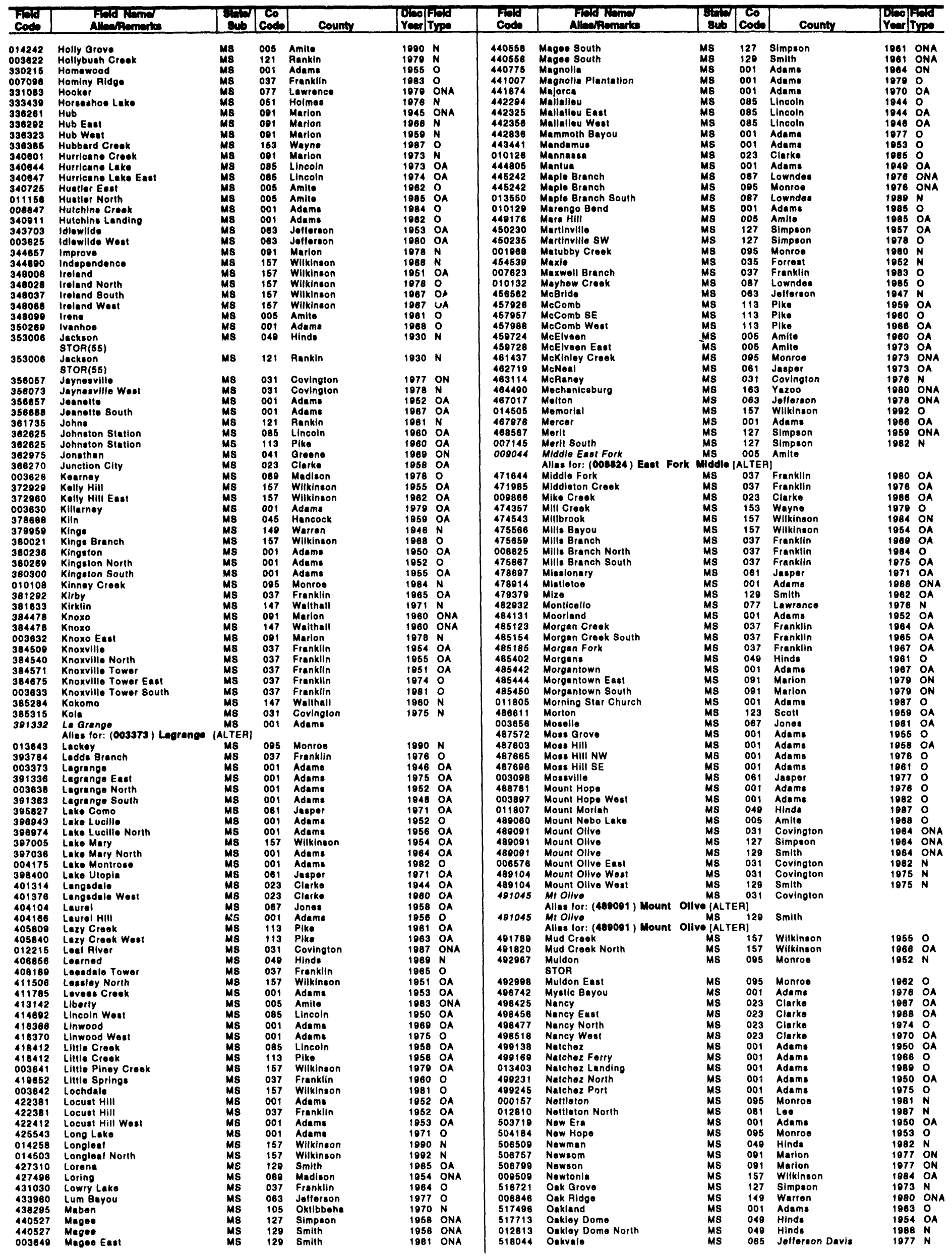




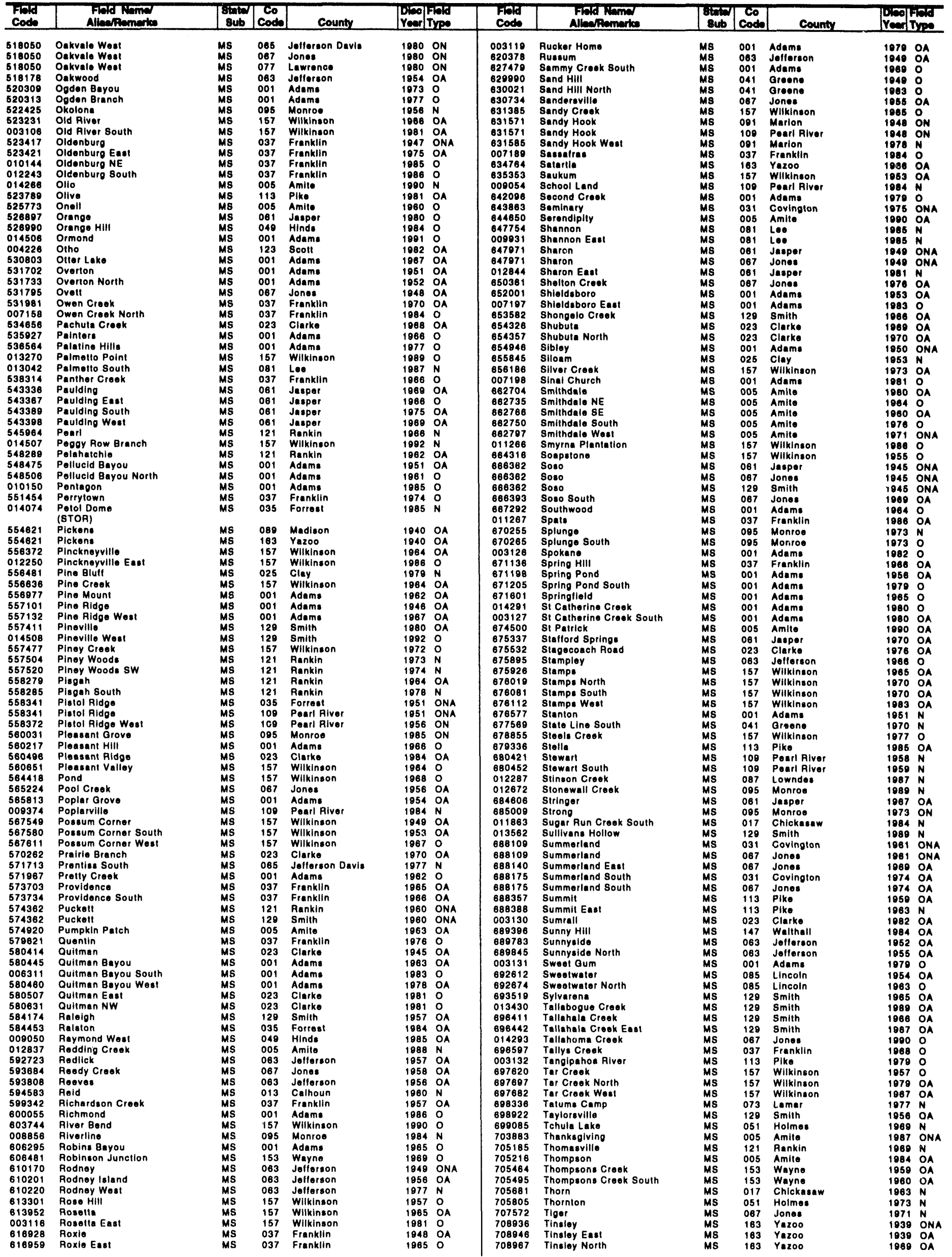


MONTANA

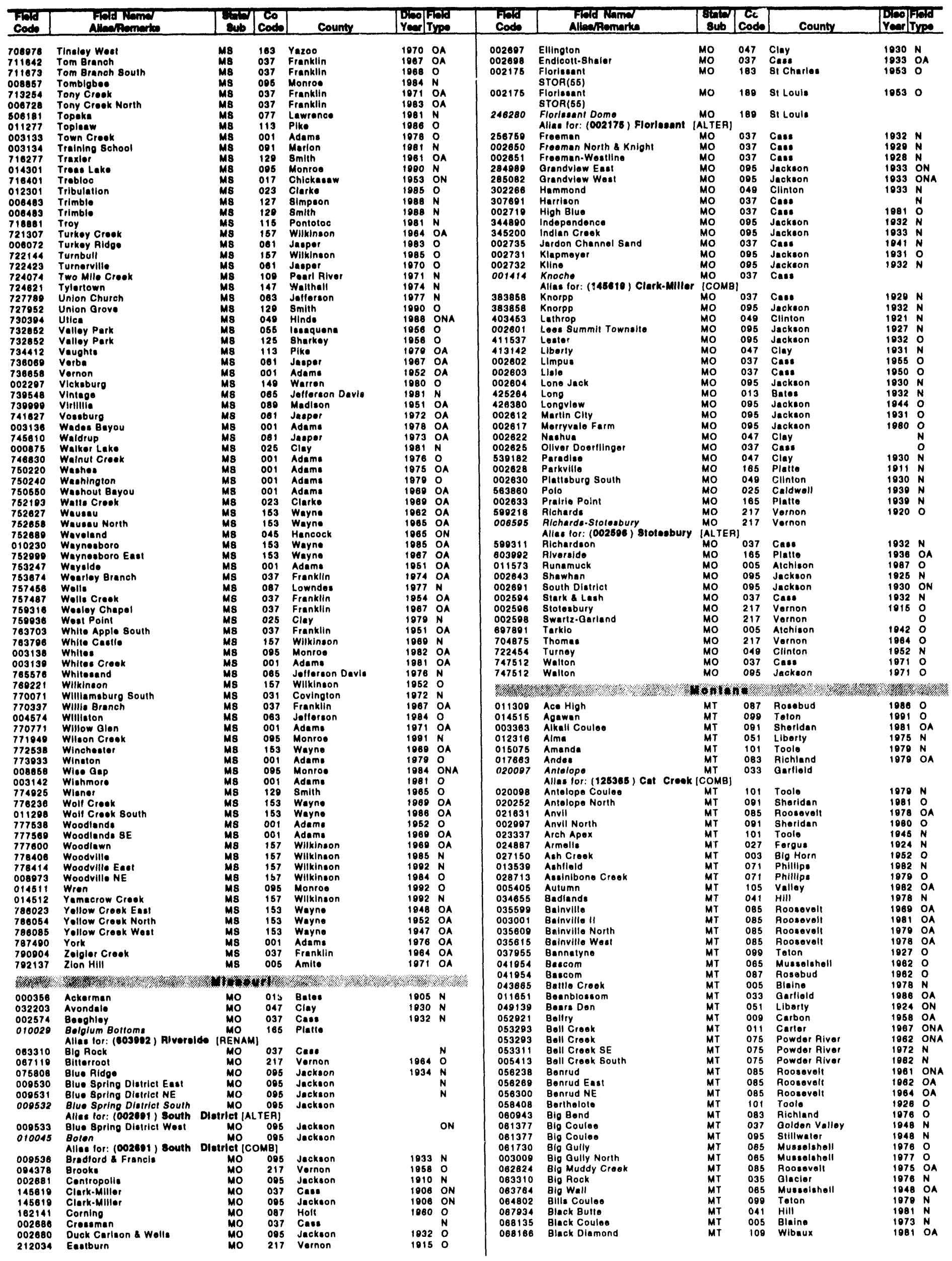


MONTANA

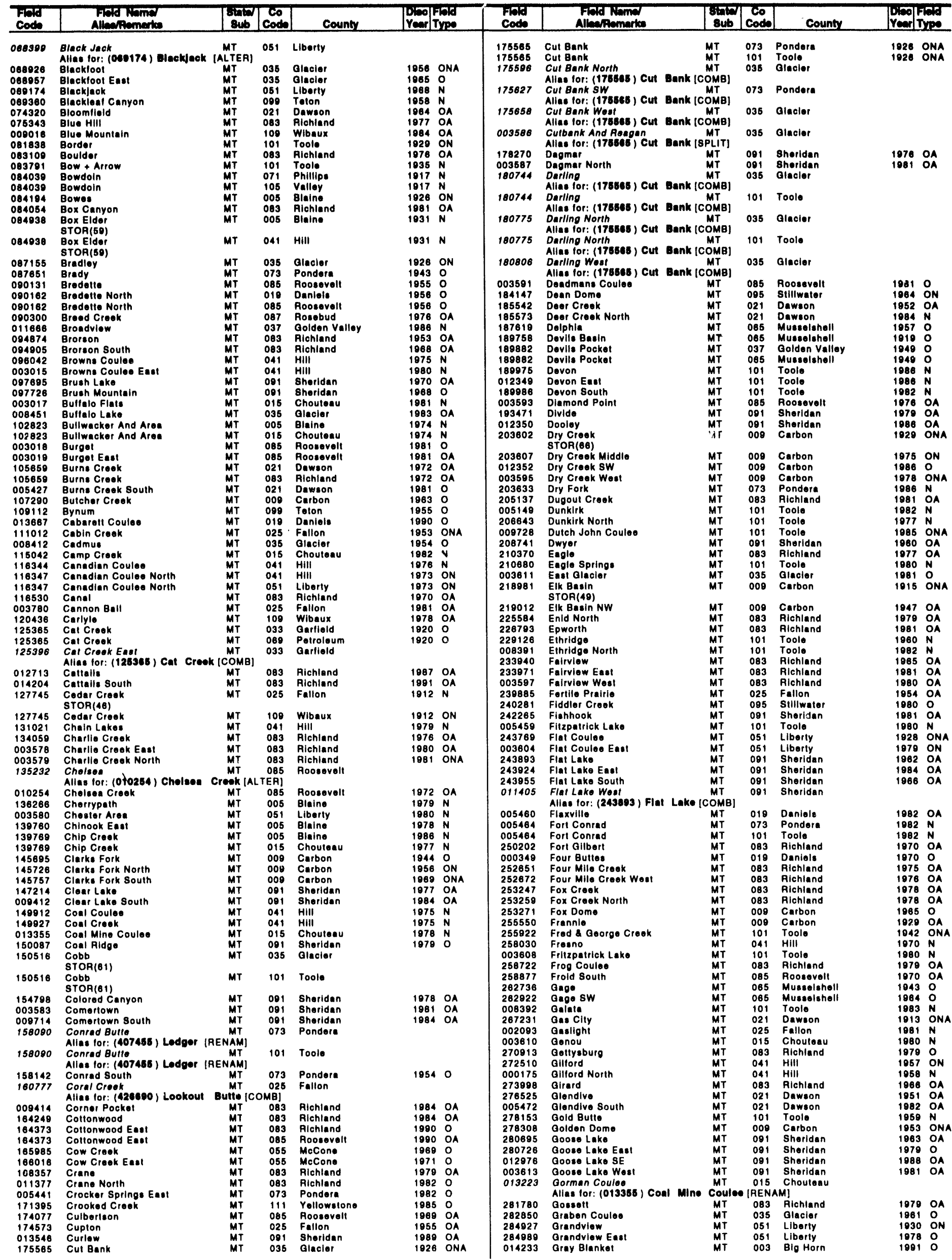


MONTANA

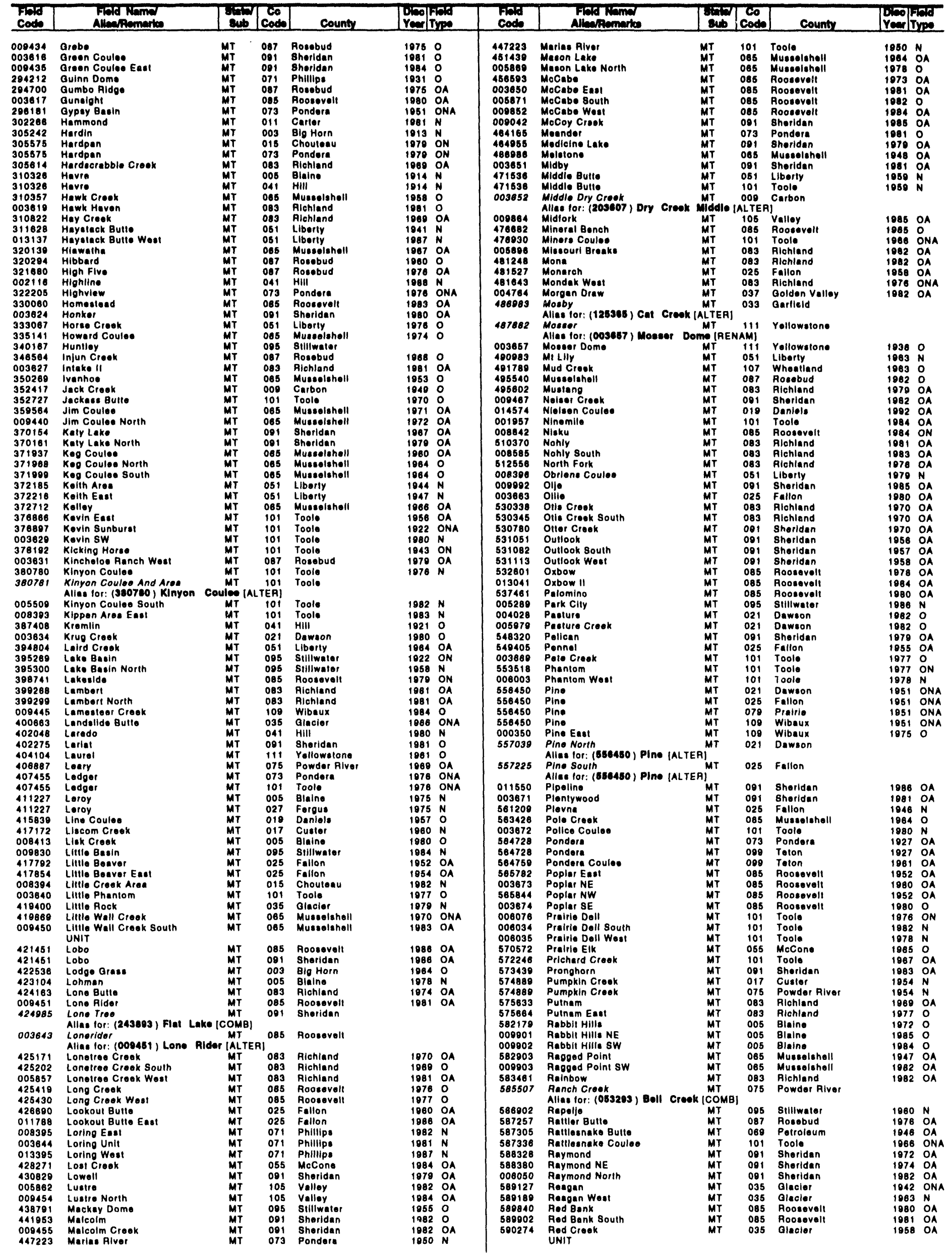


MONTANA

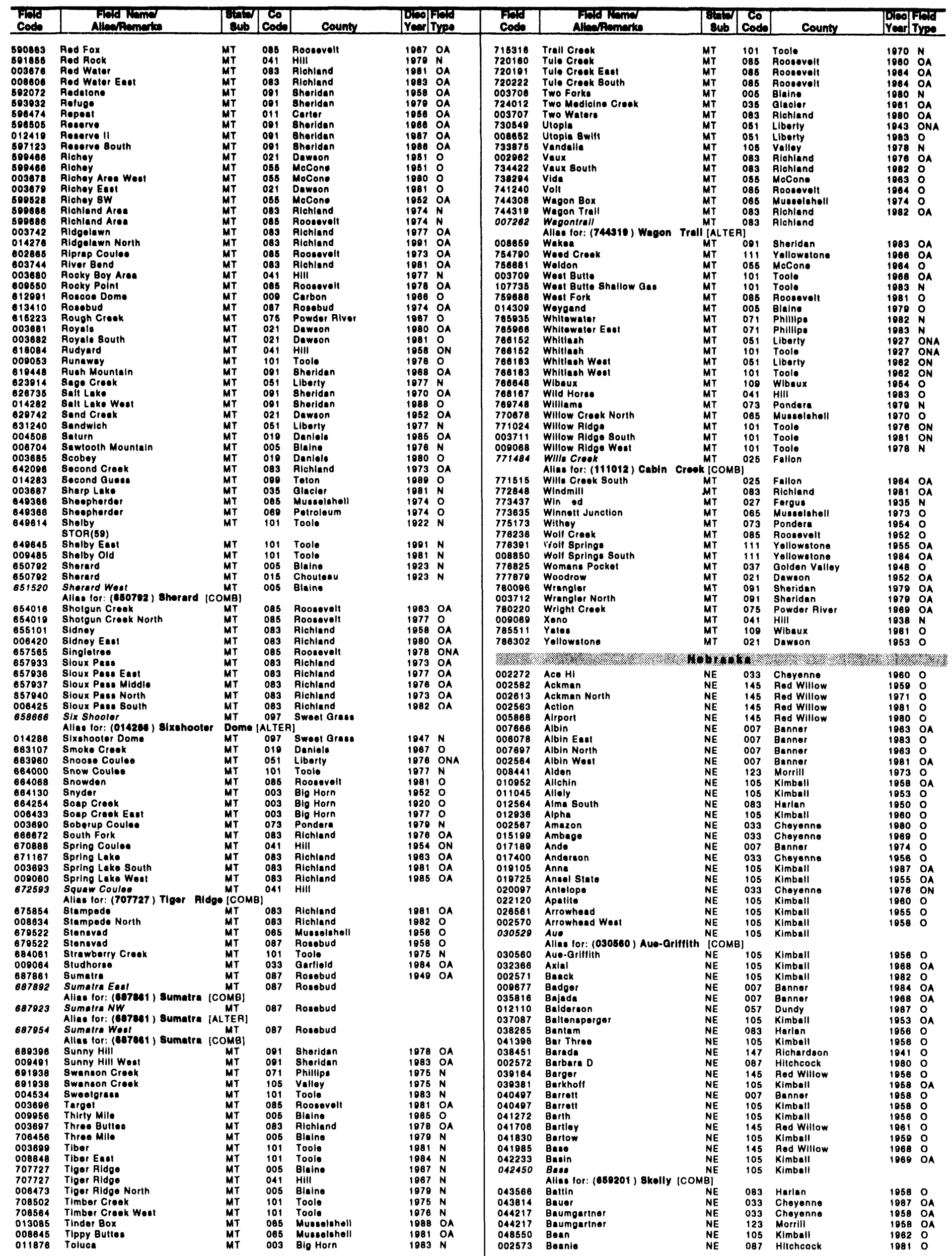


NEBRASKA

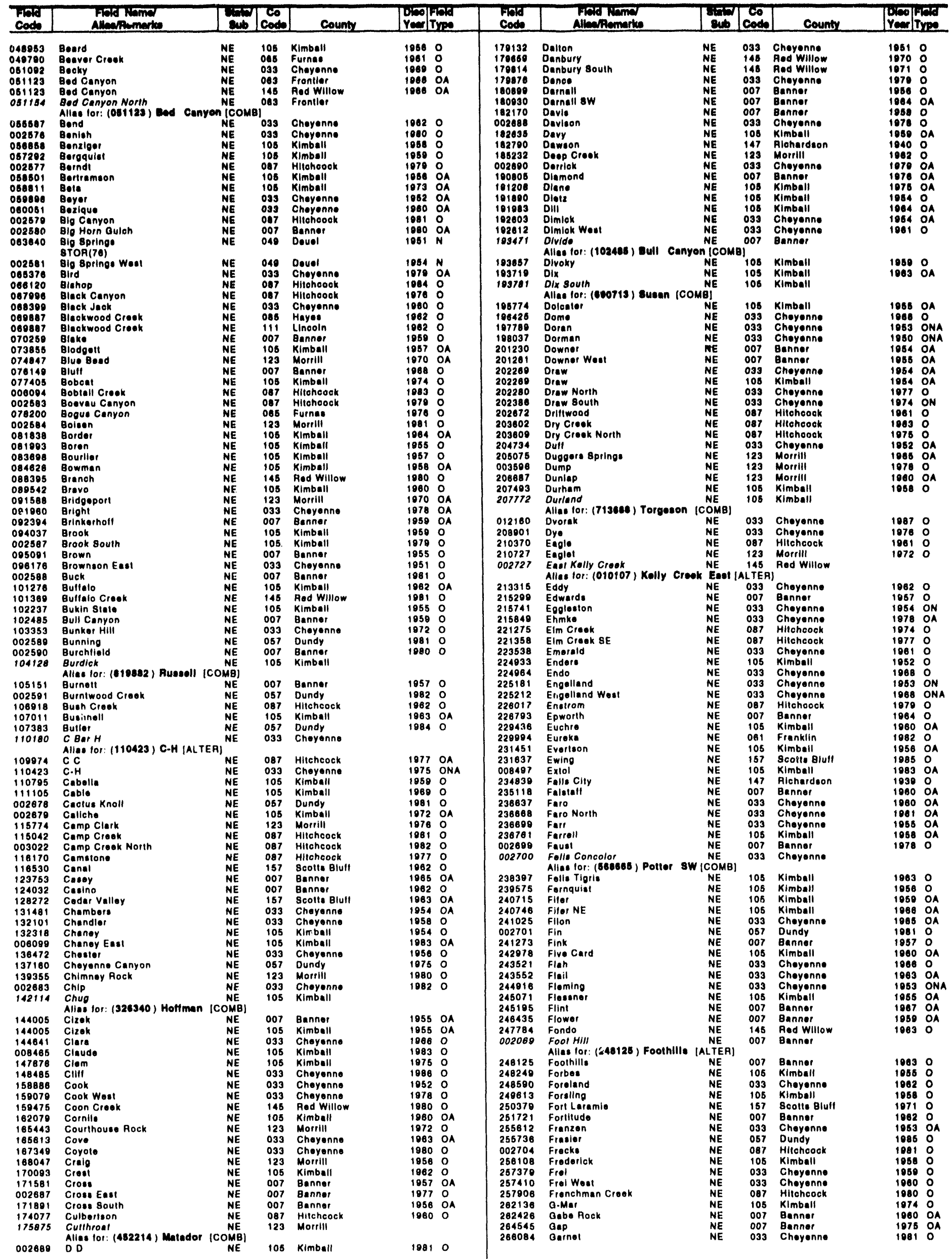


NEBRASKA

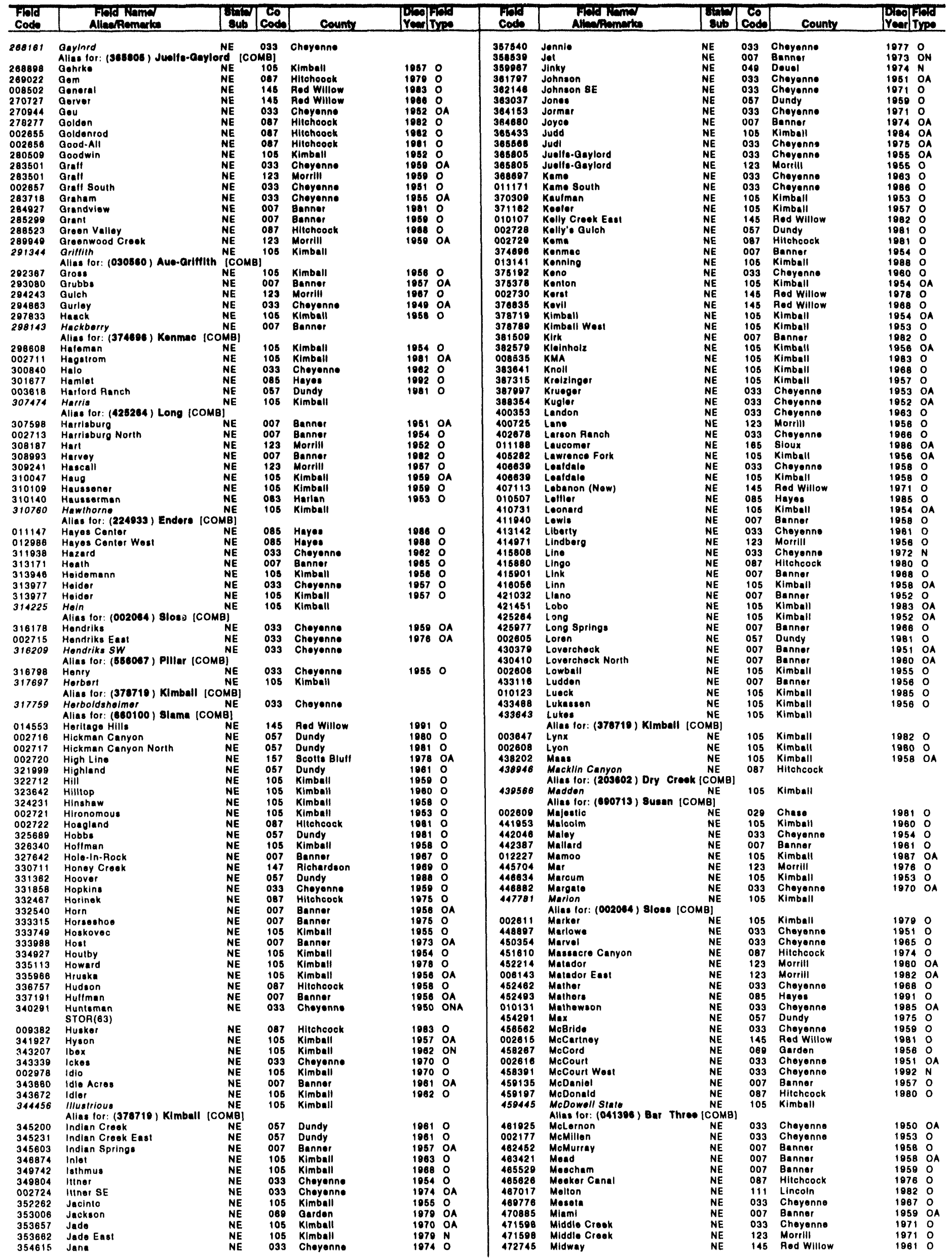


NEBRASKA

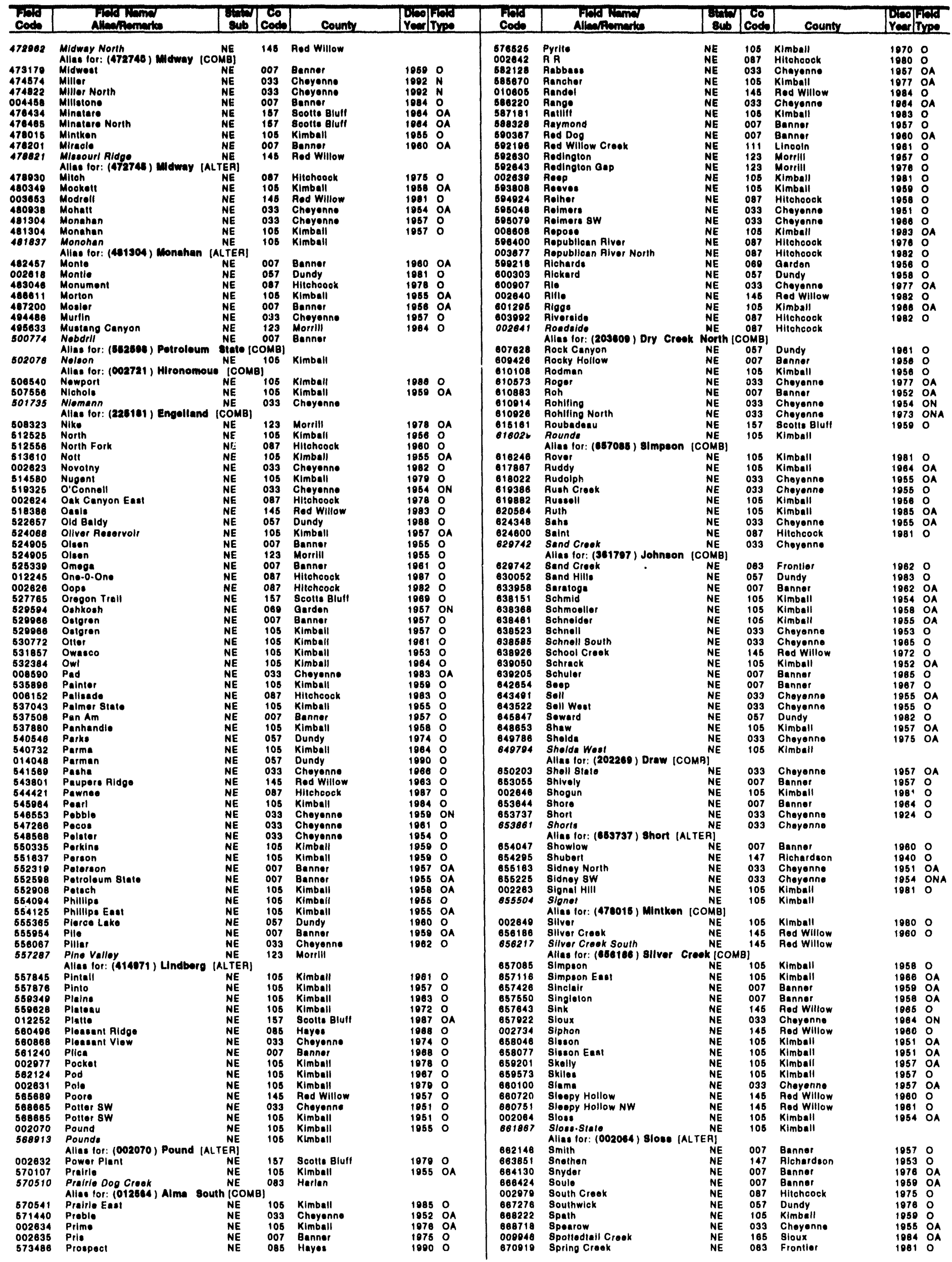


NEBRASKA

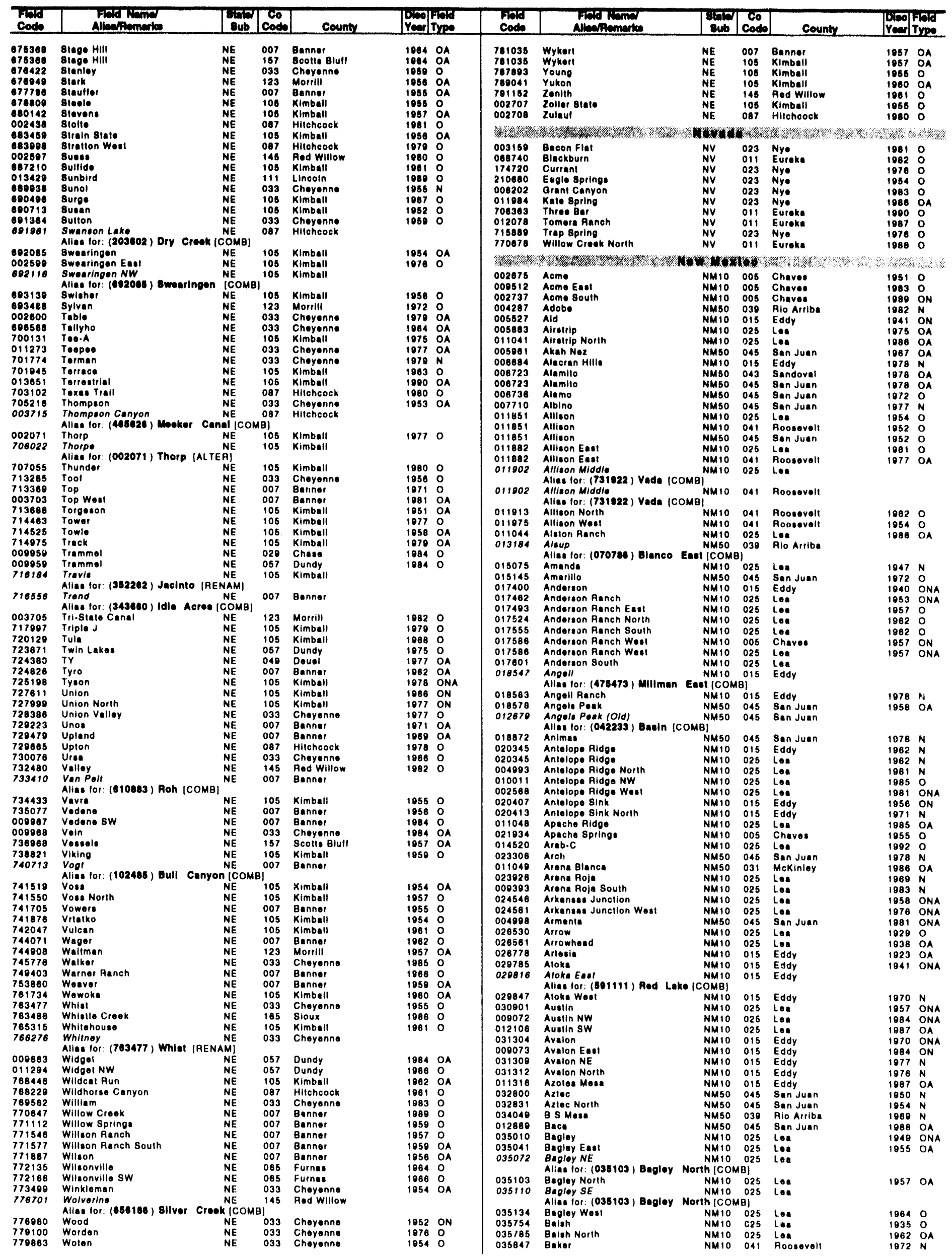


NEW MEXICO

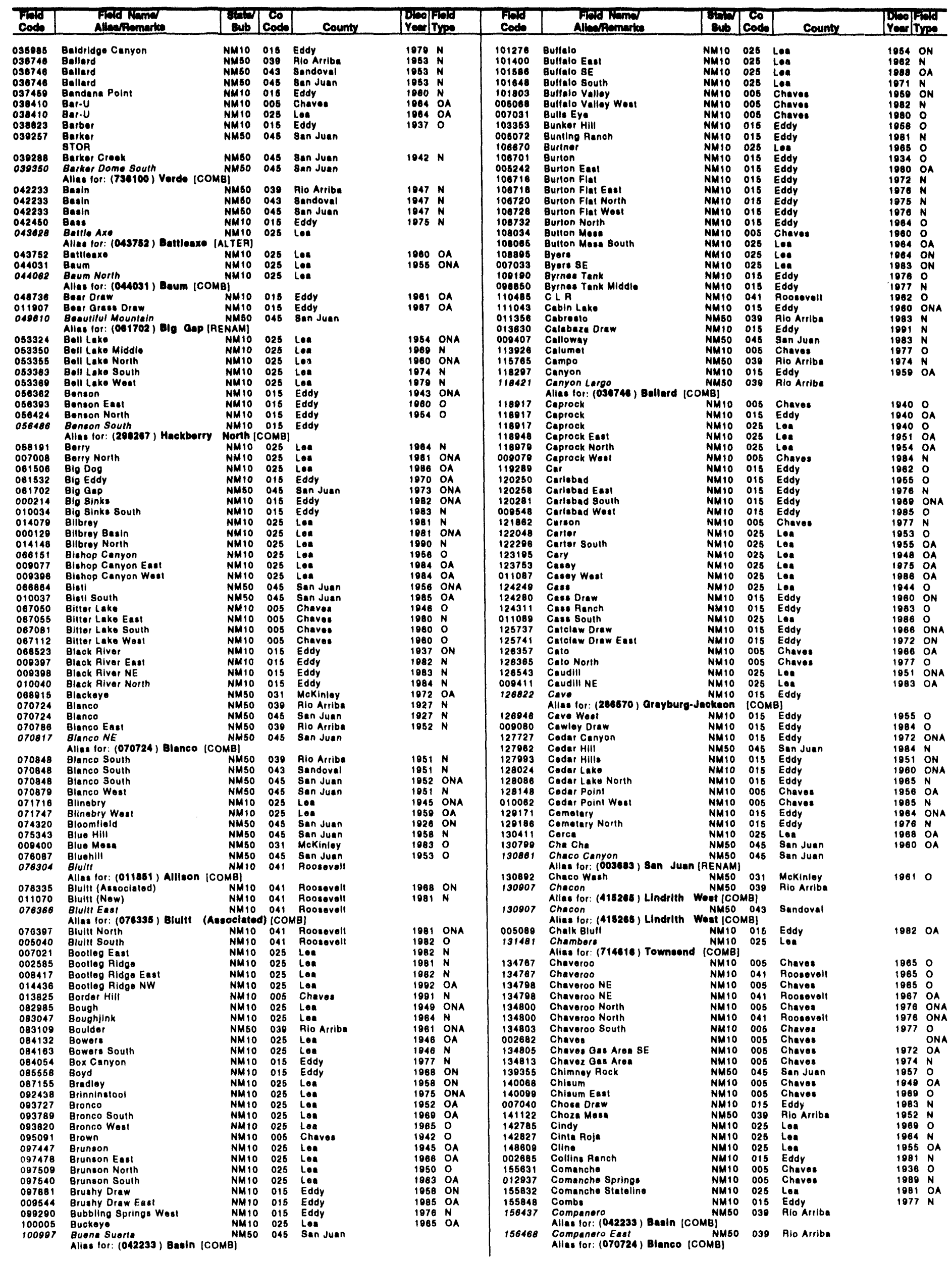




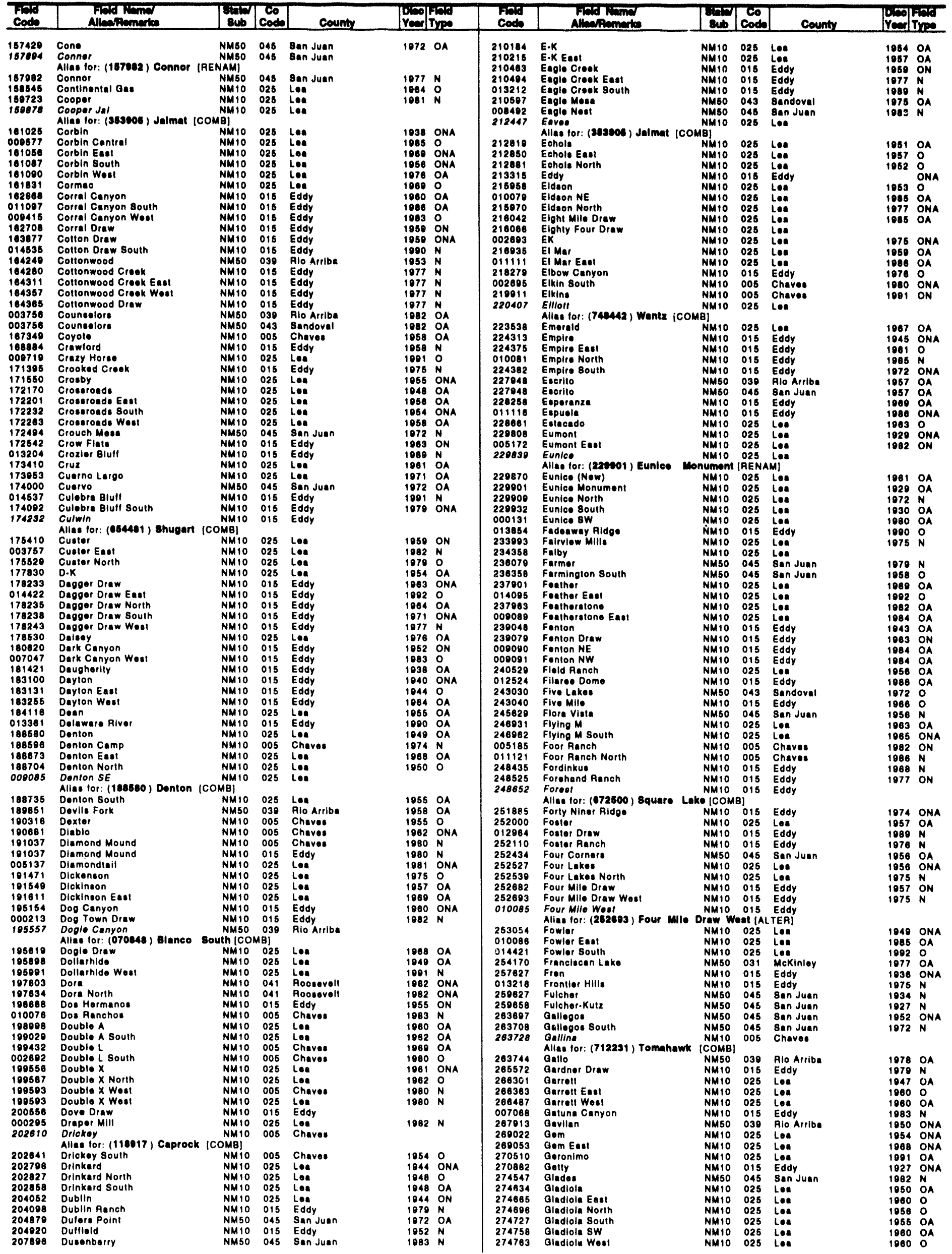


NEW MEXICO

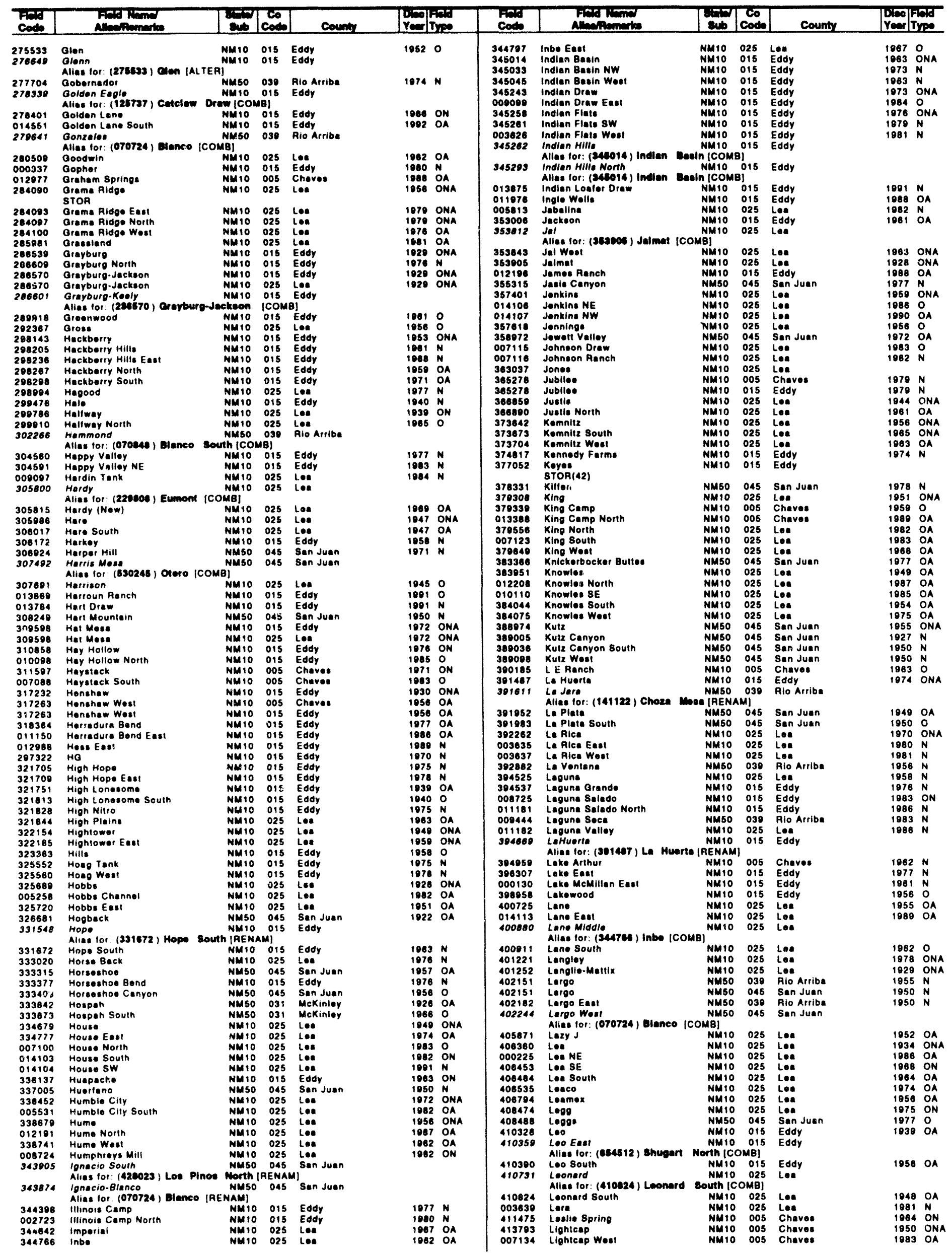


NEW MEXICO

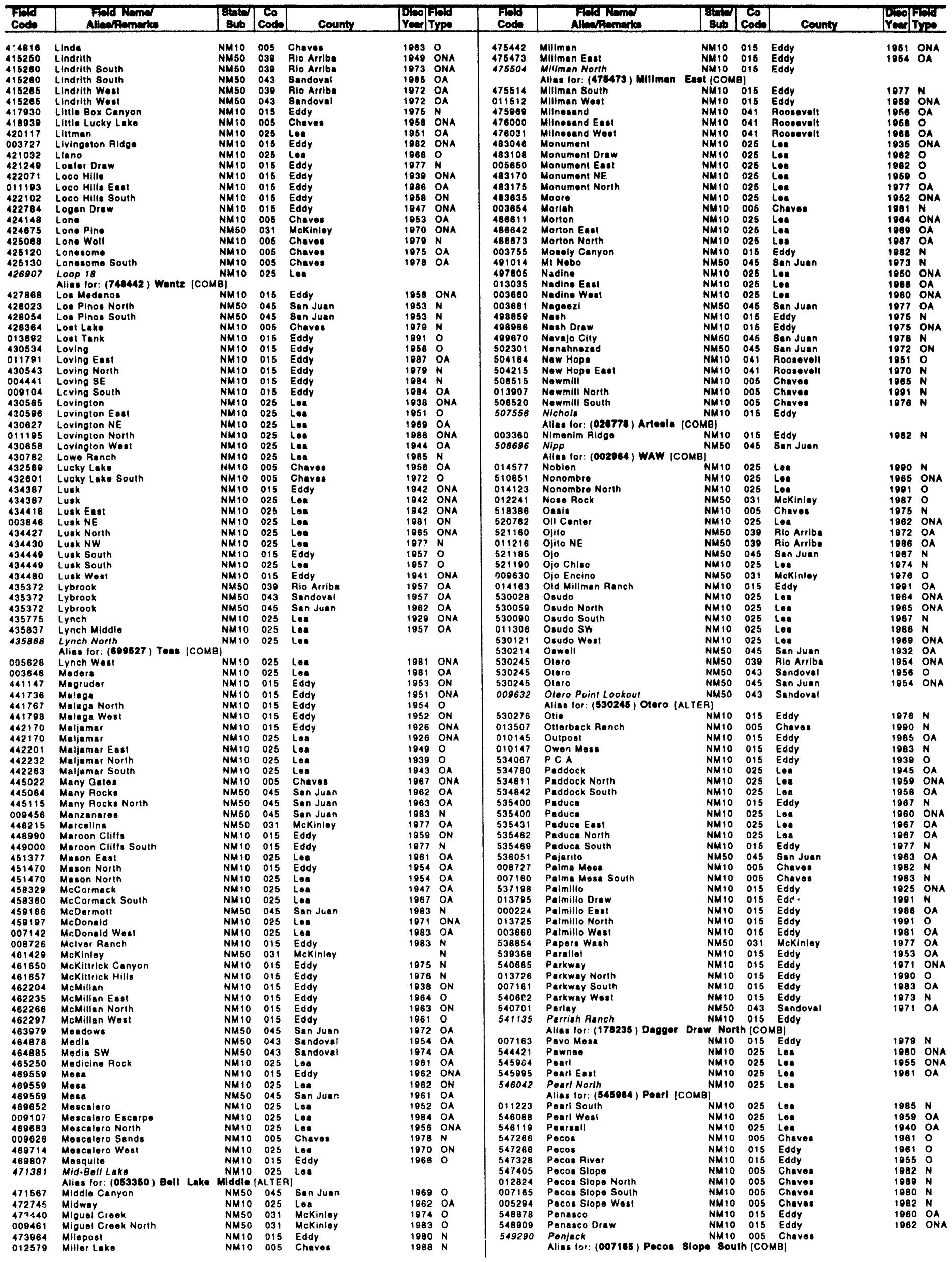


NEW MEXICO

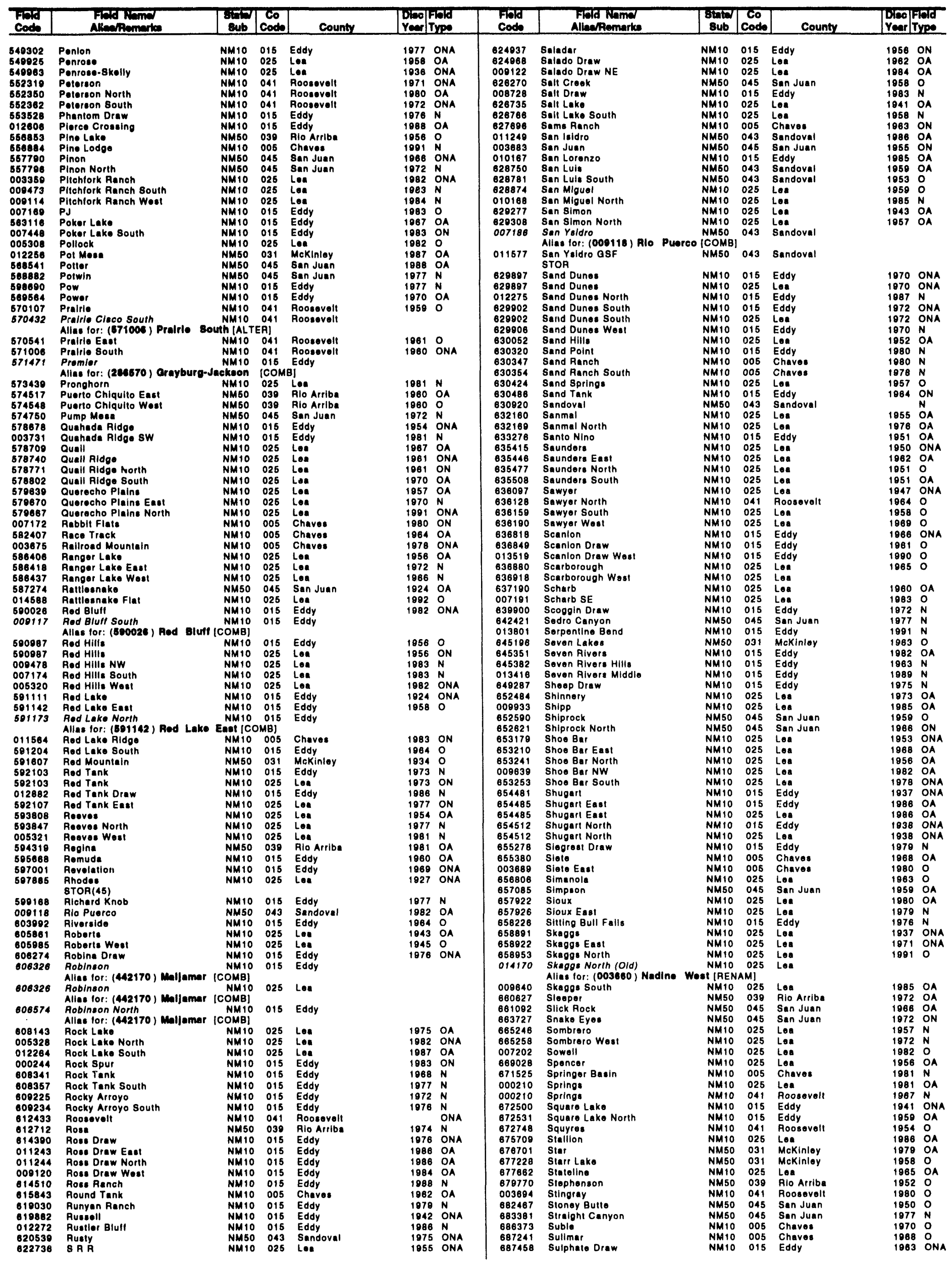




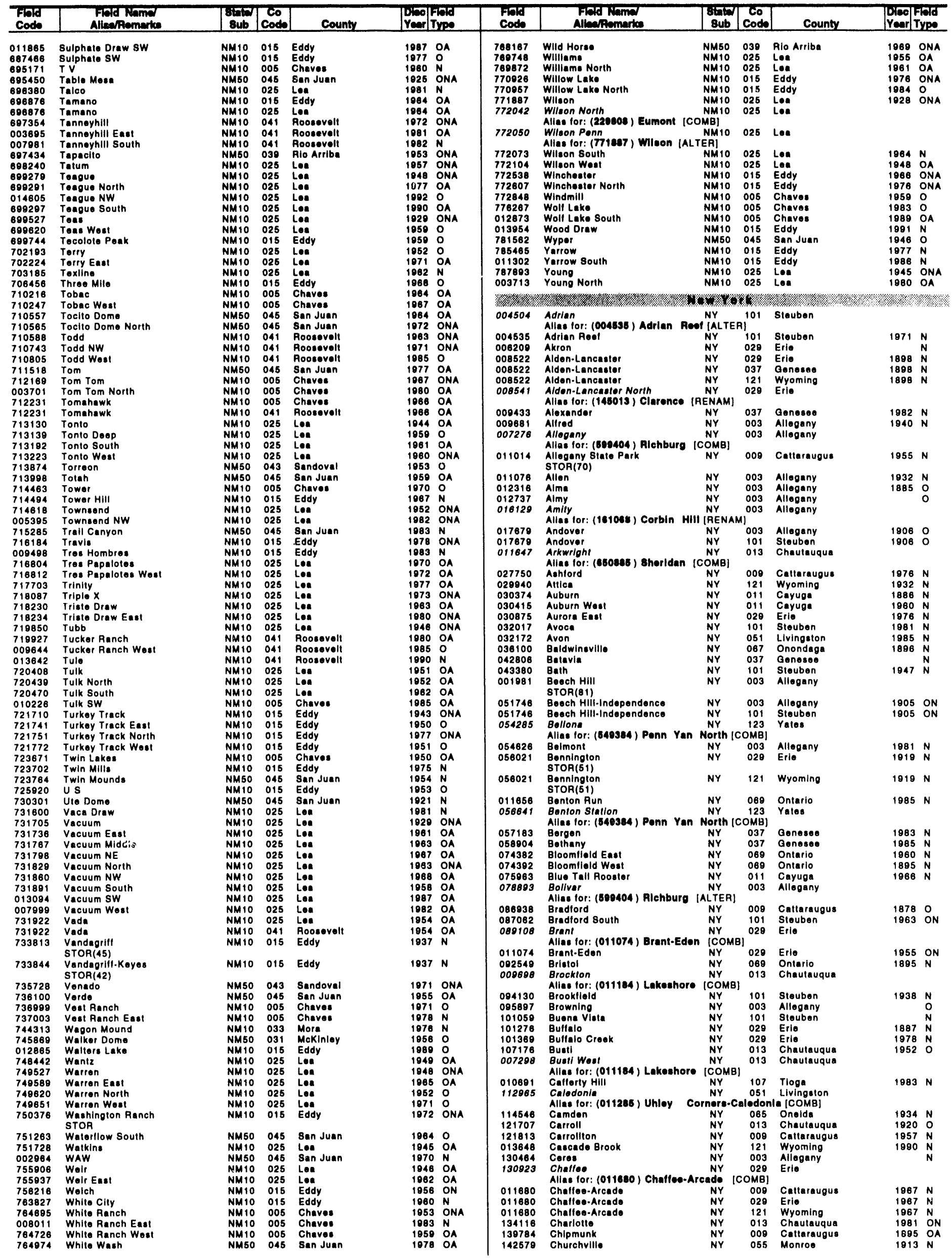


NEW YORK

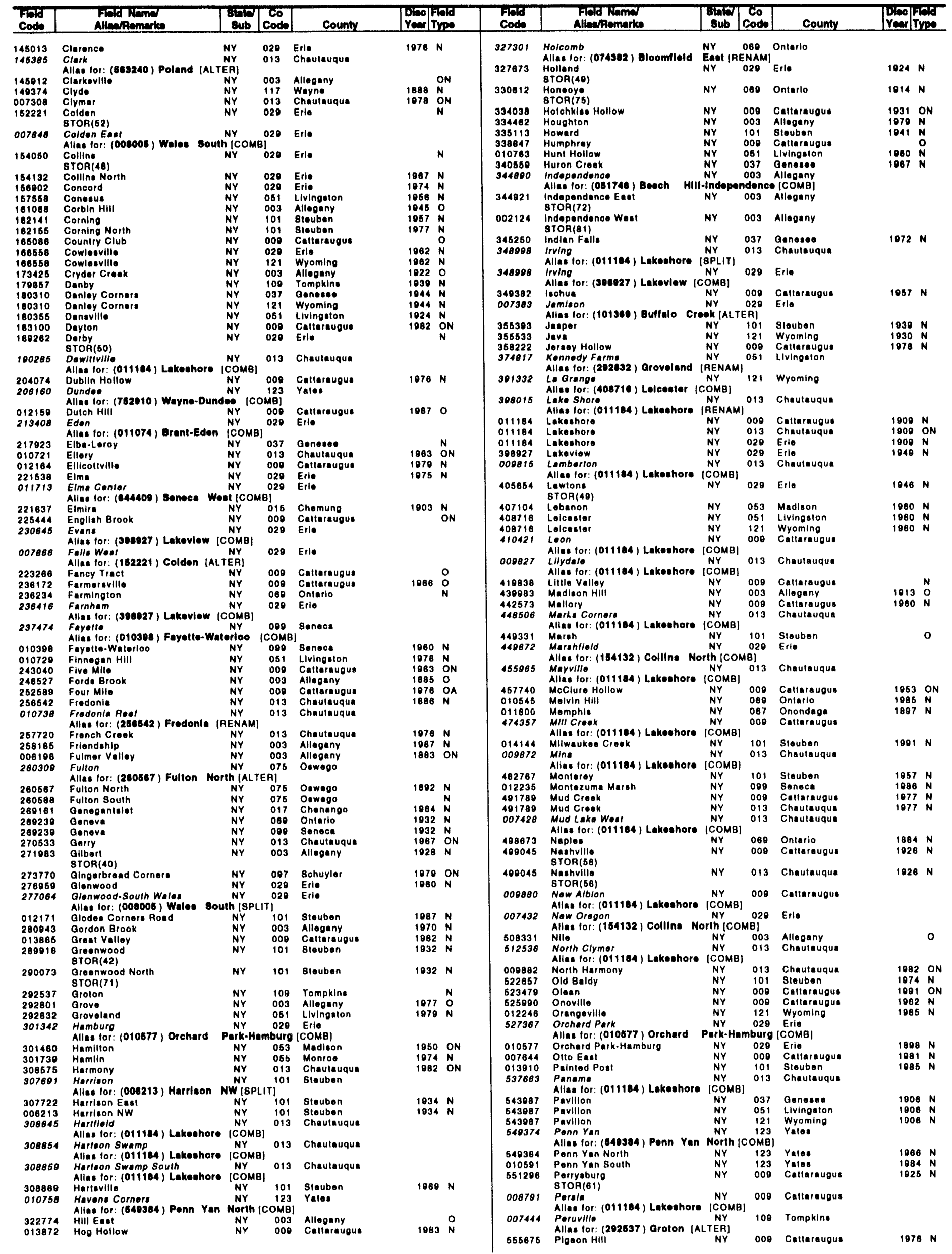




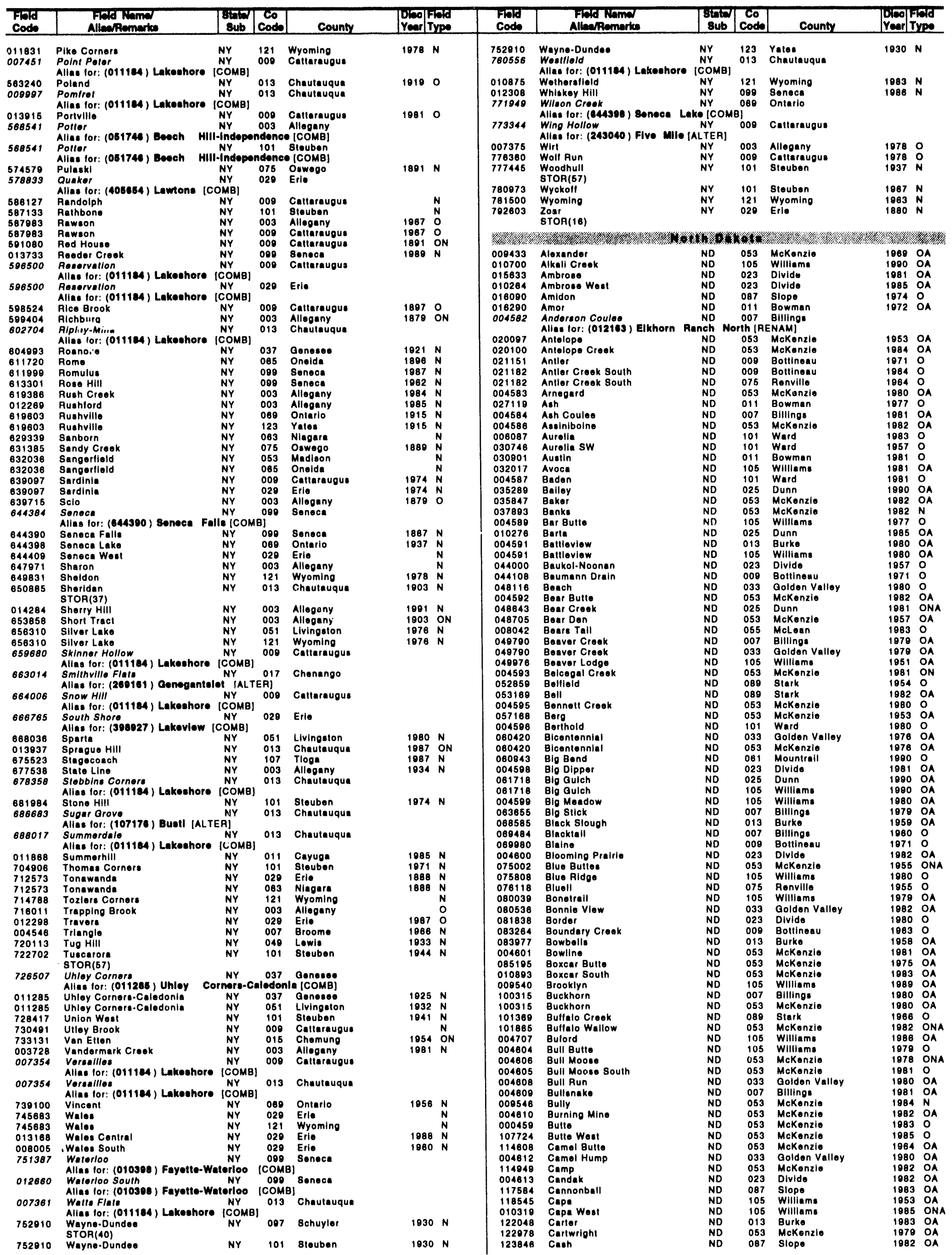


NORTH DAKOTA

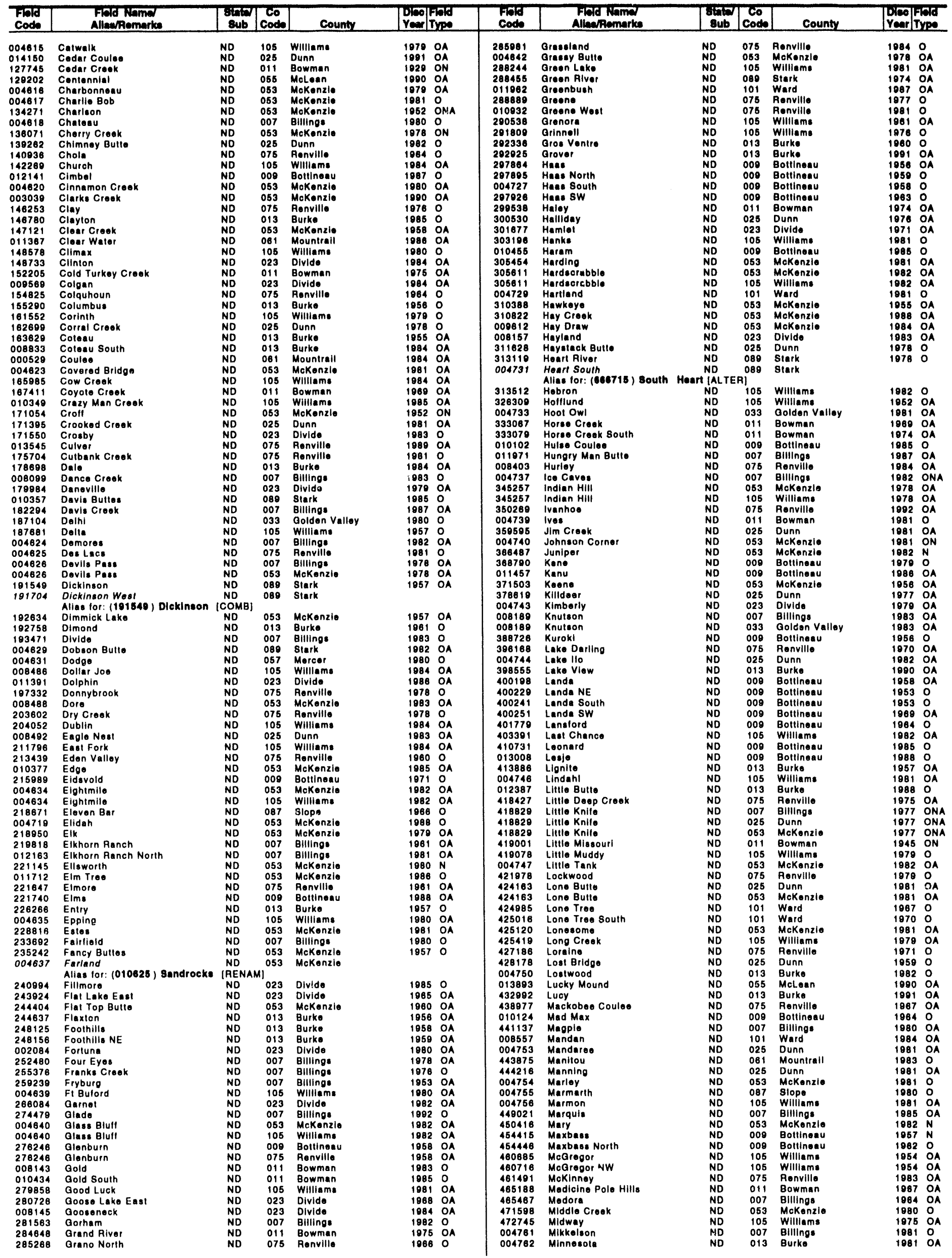


NORTH DAKOTA

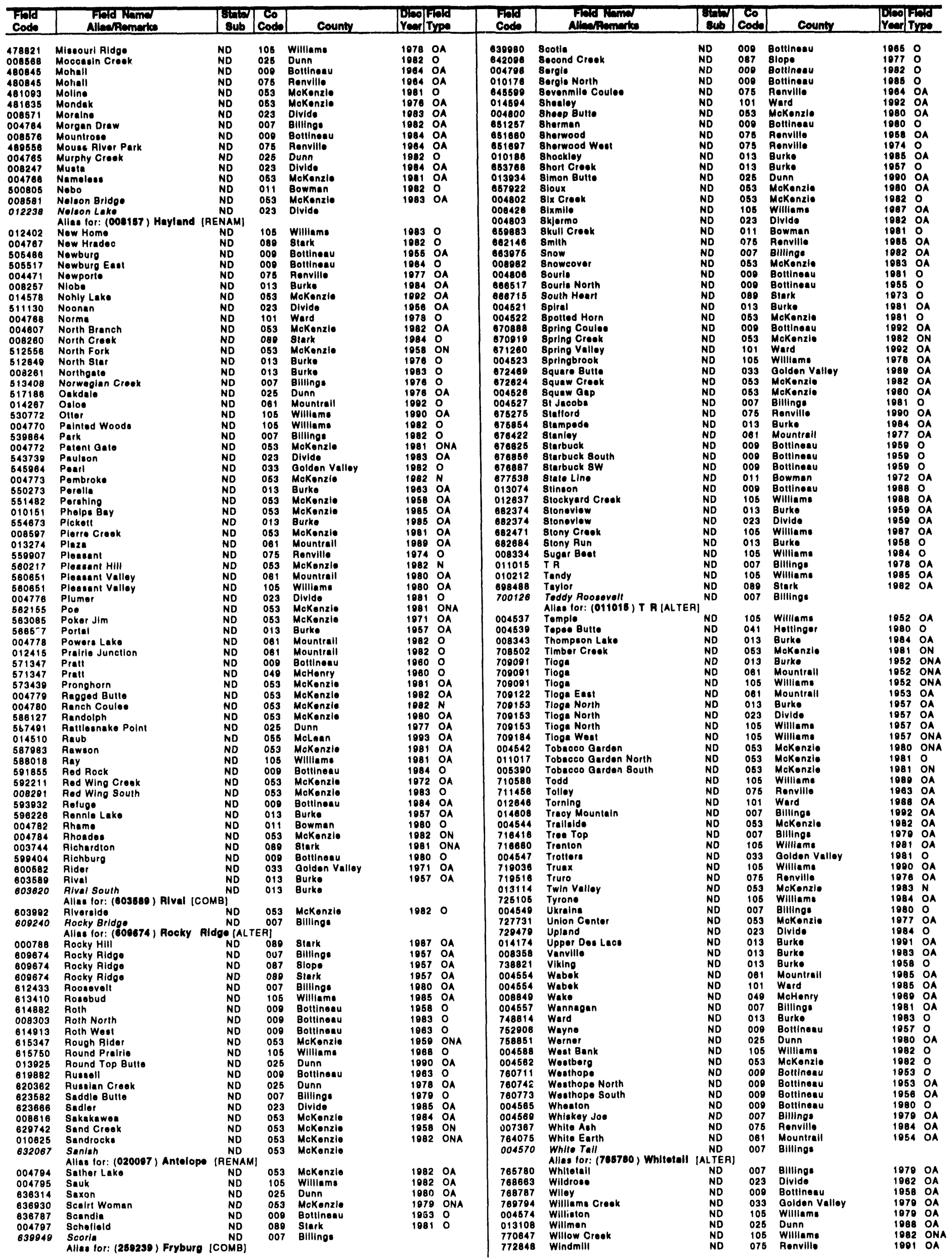


OHIO

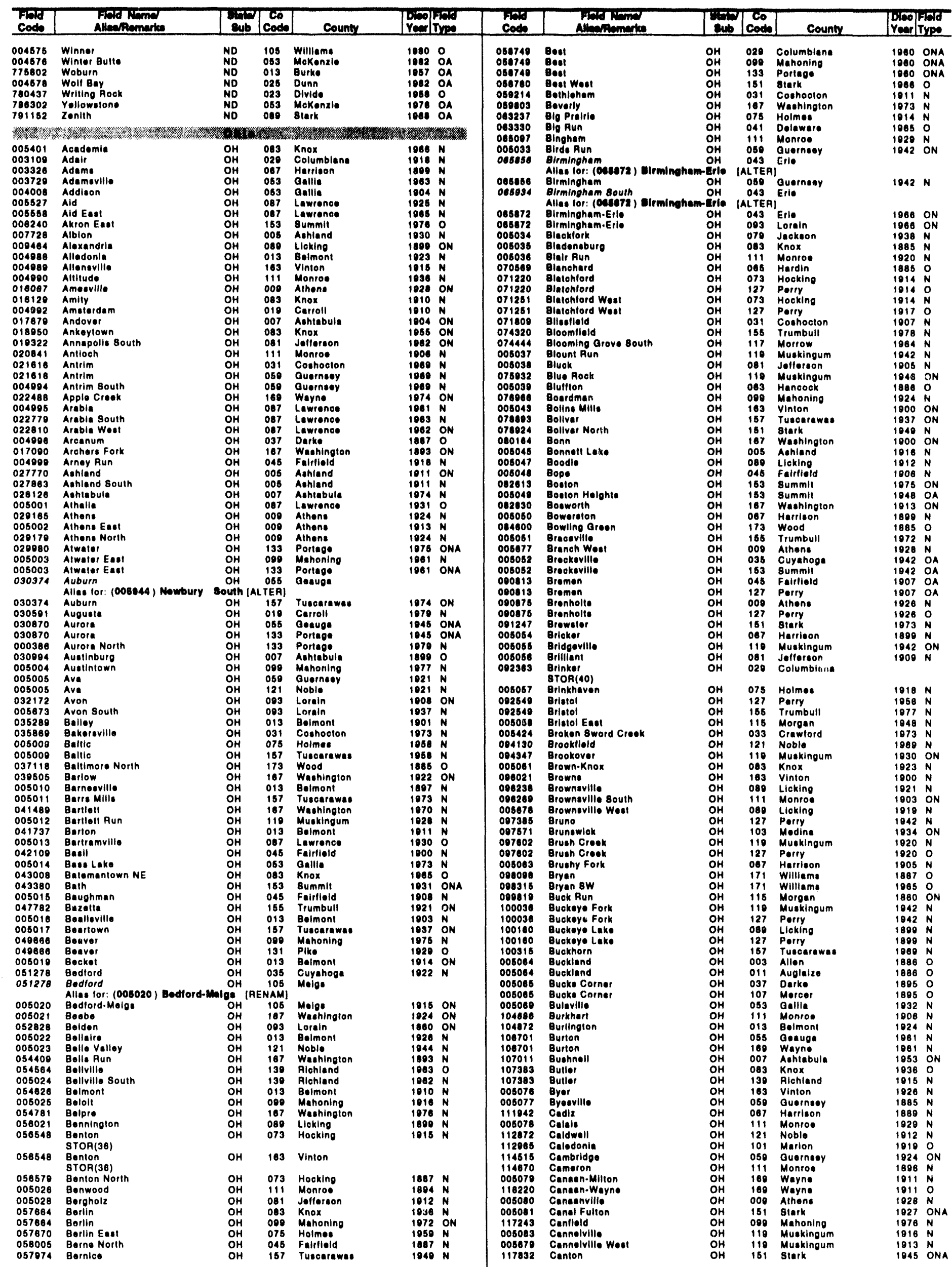




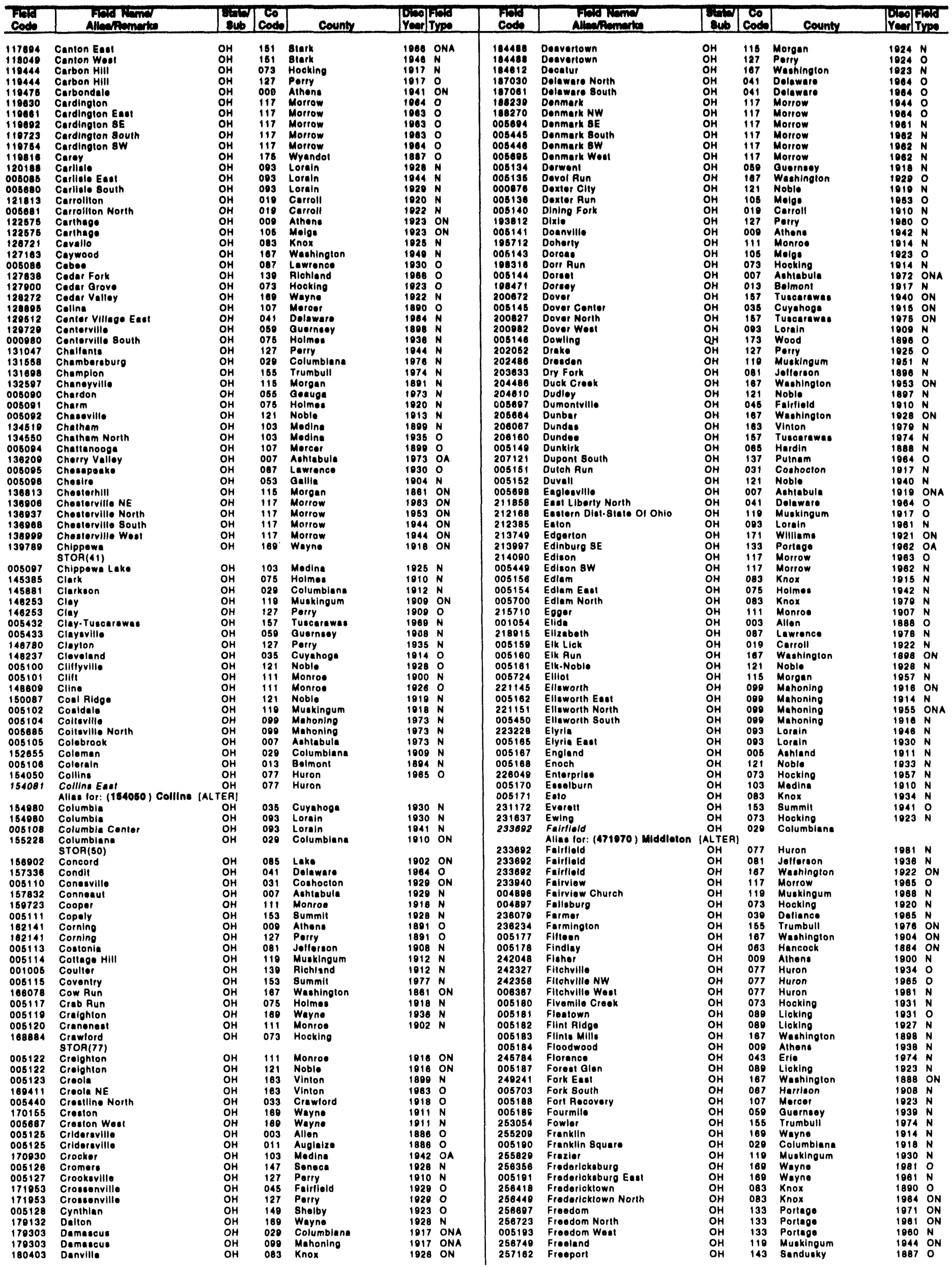




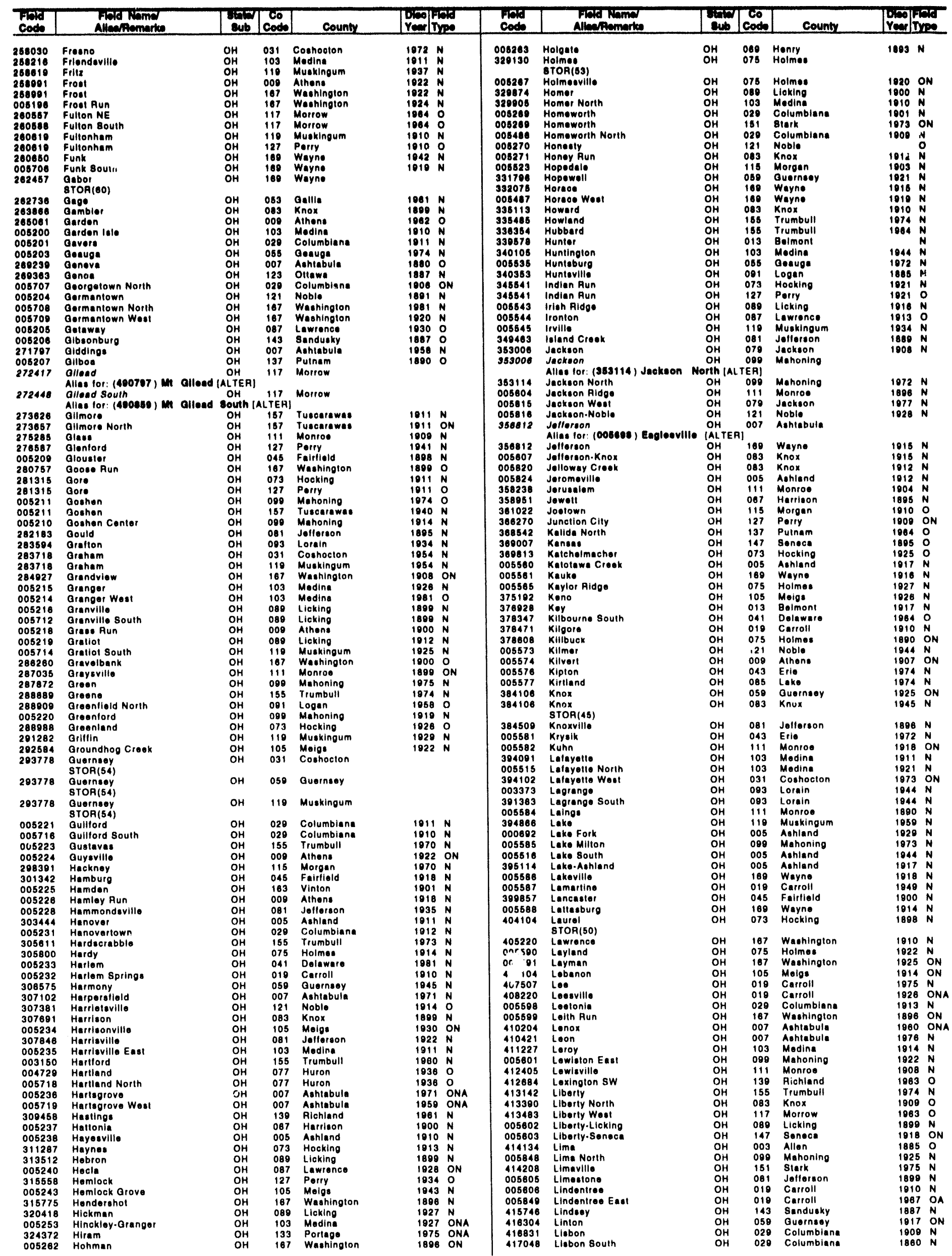




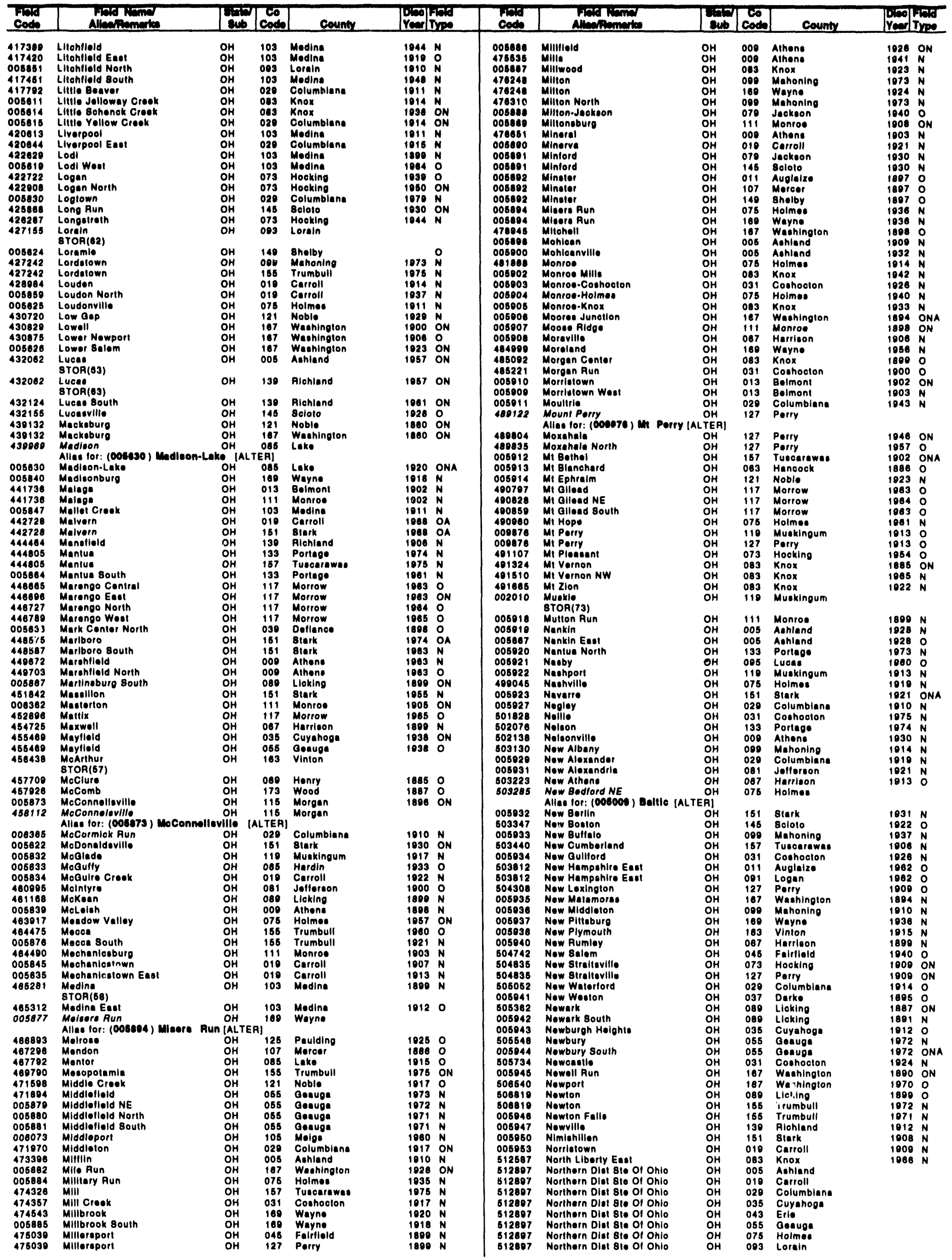




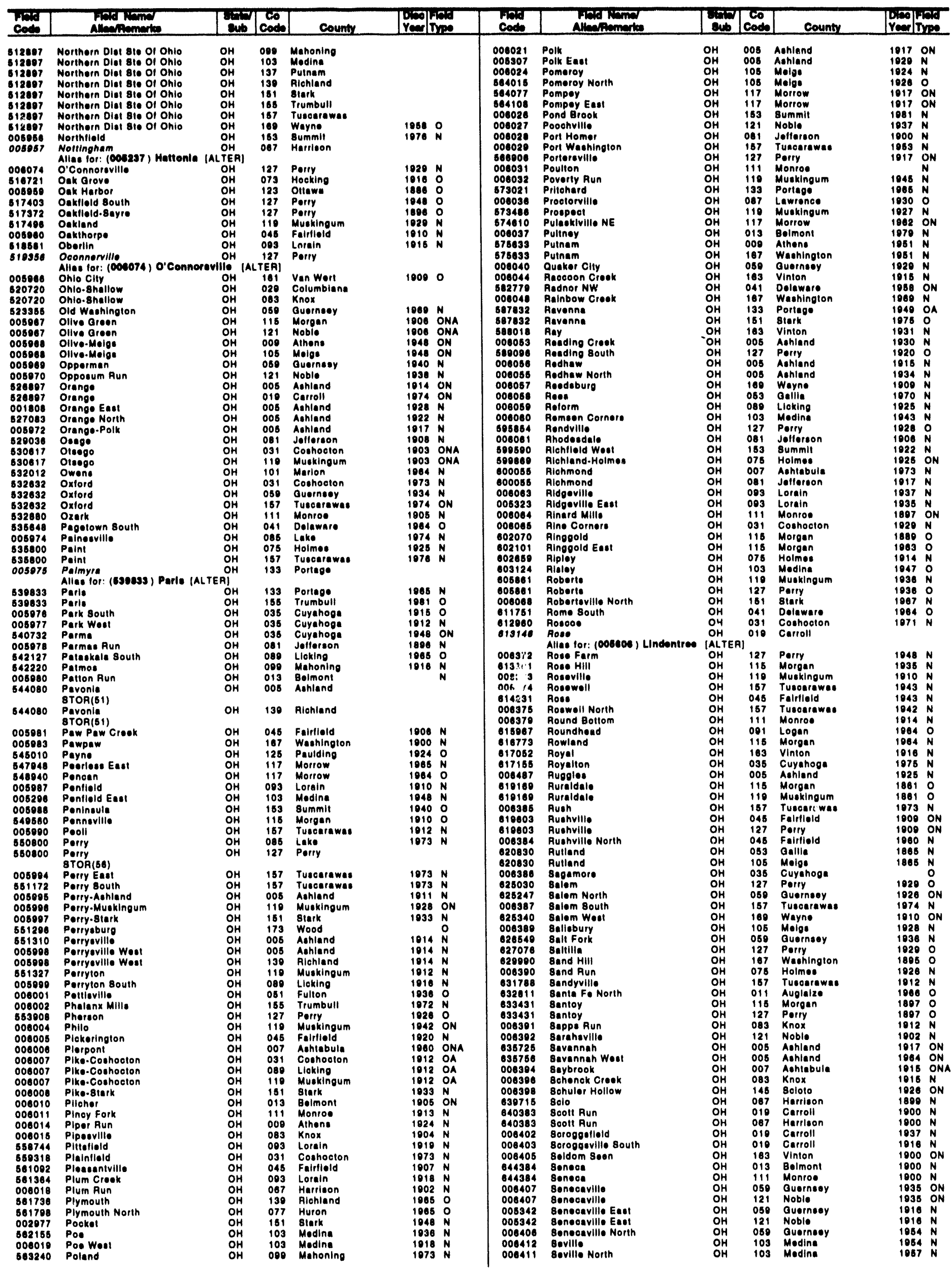




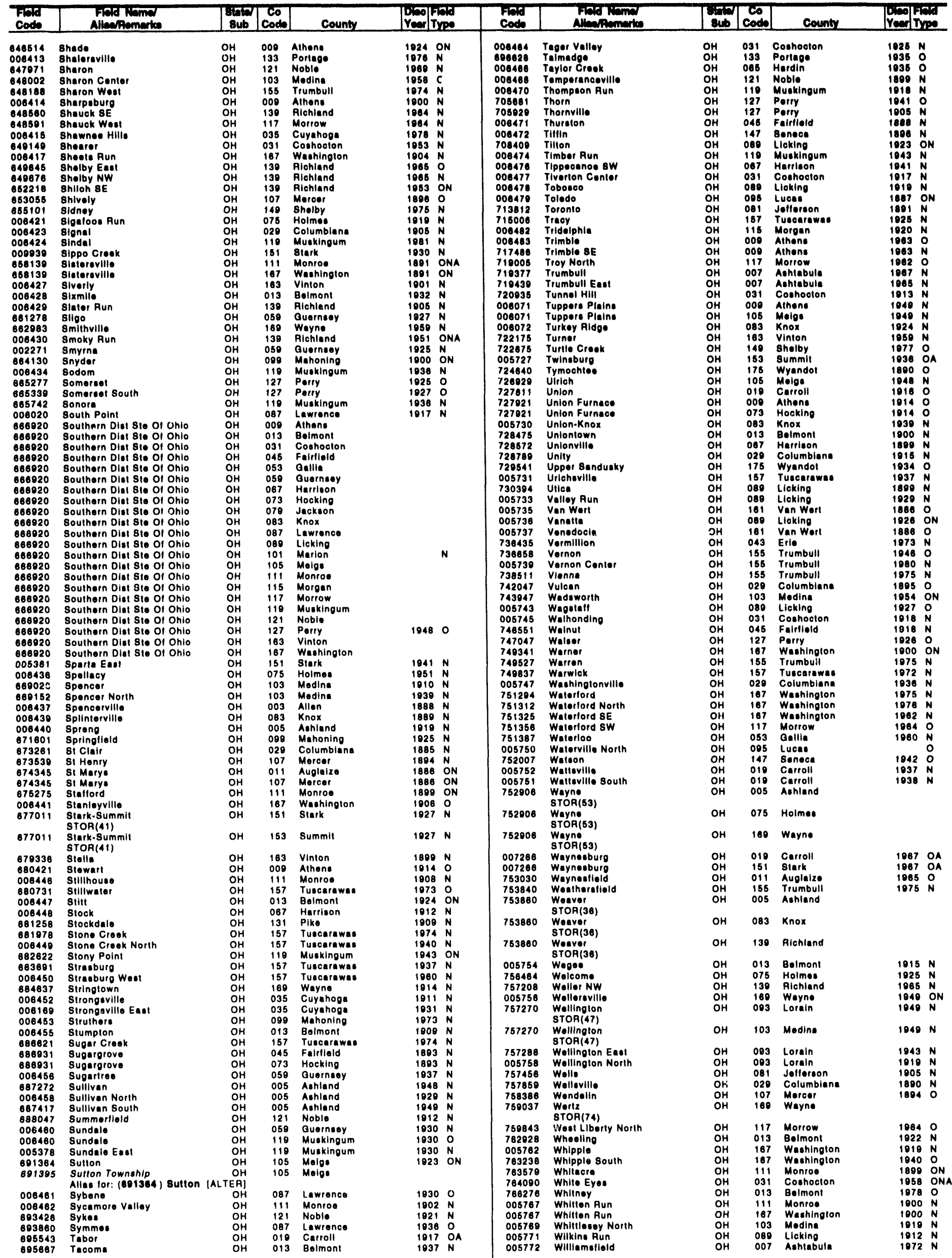


OKLAHOMA

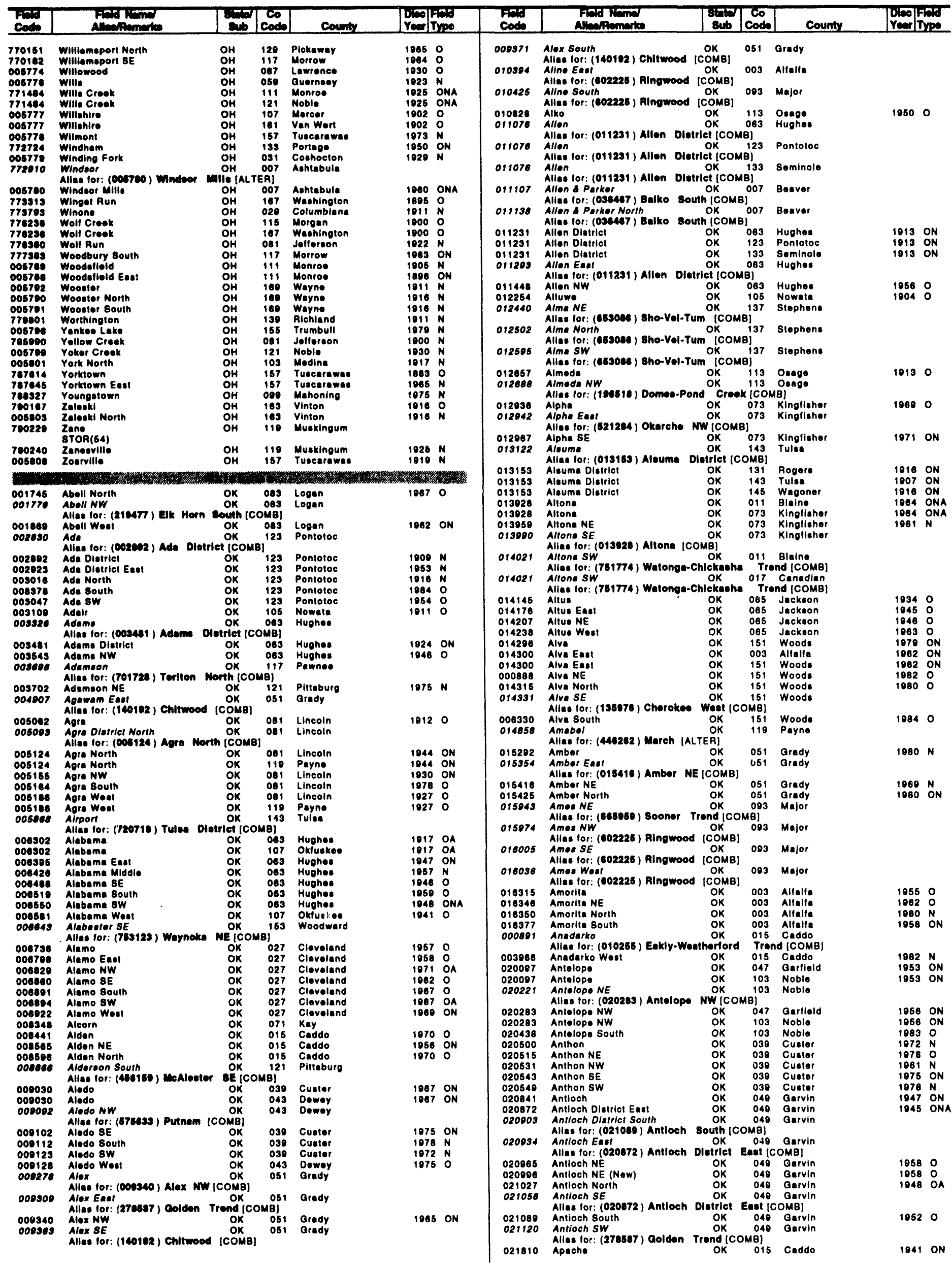




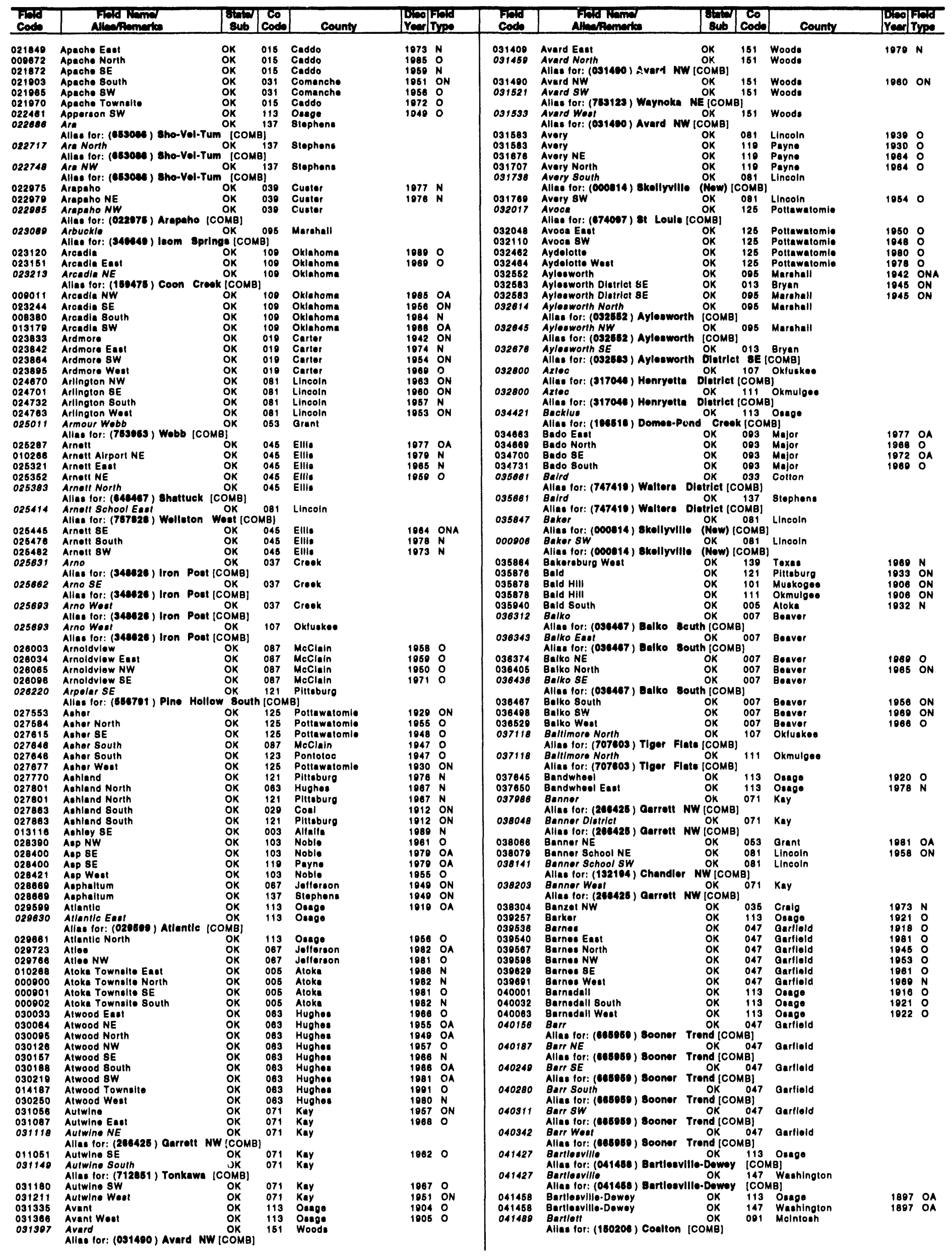


OKLAHOMA

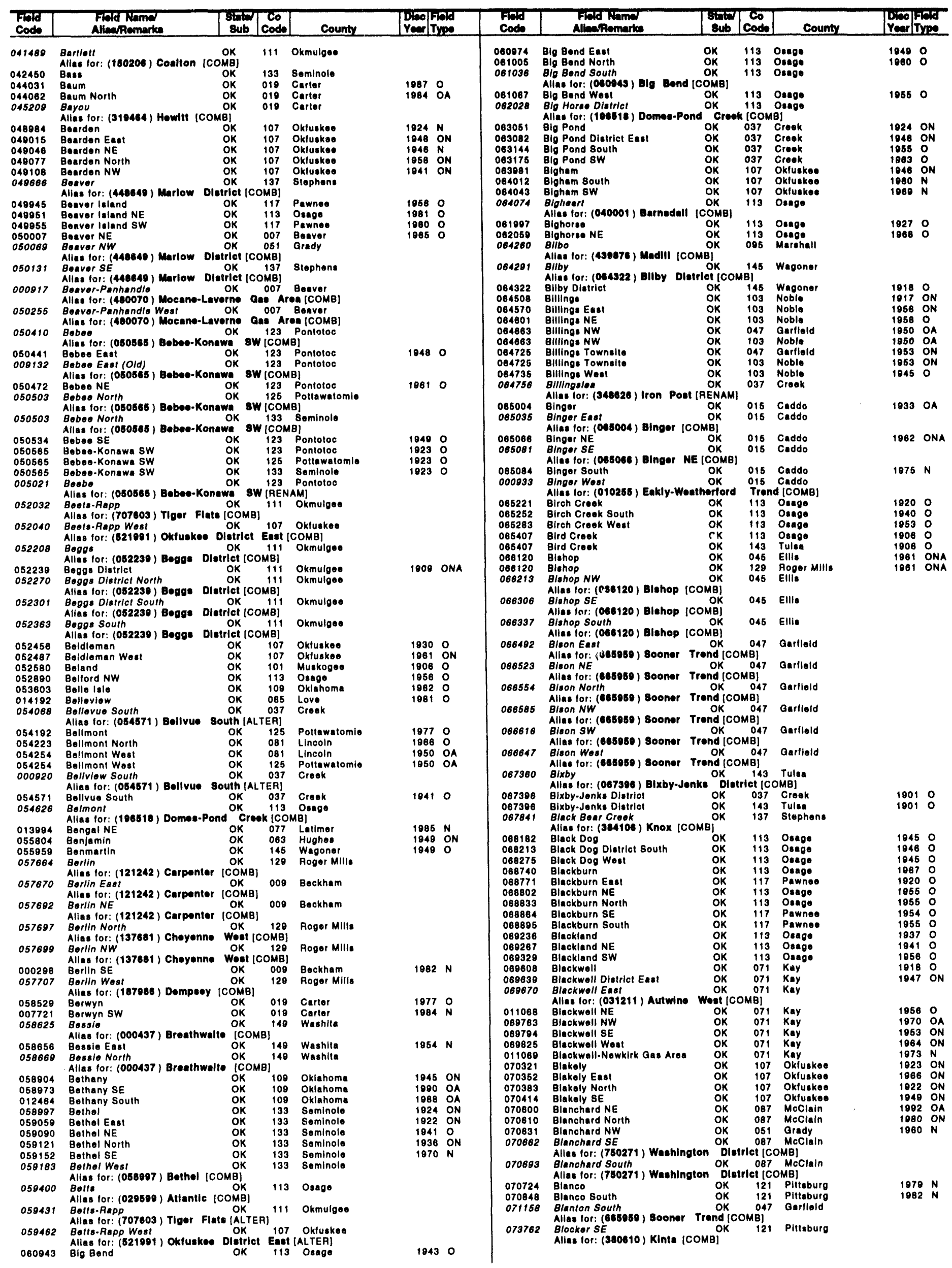




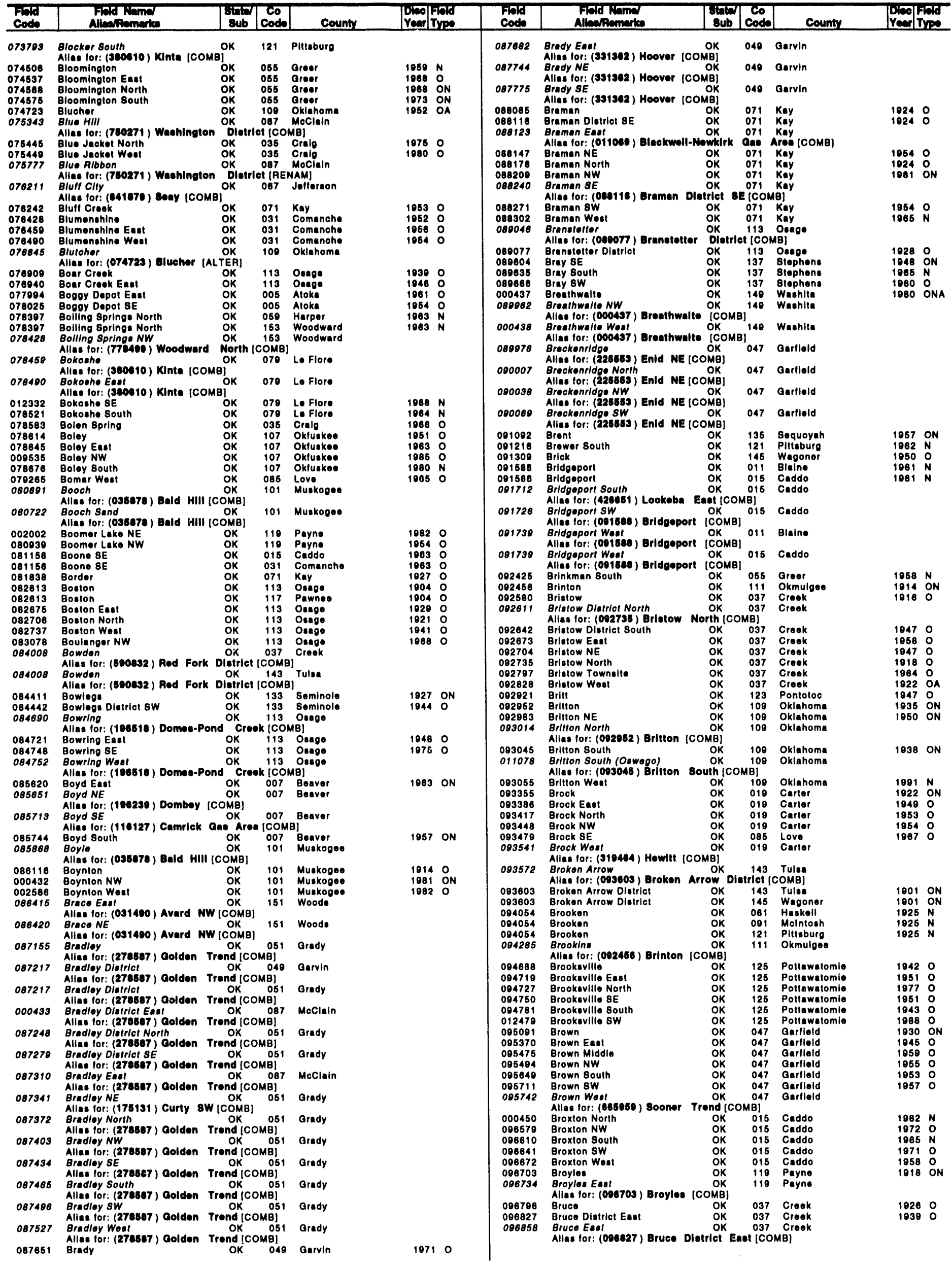


OKLAHOMA

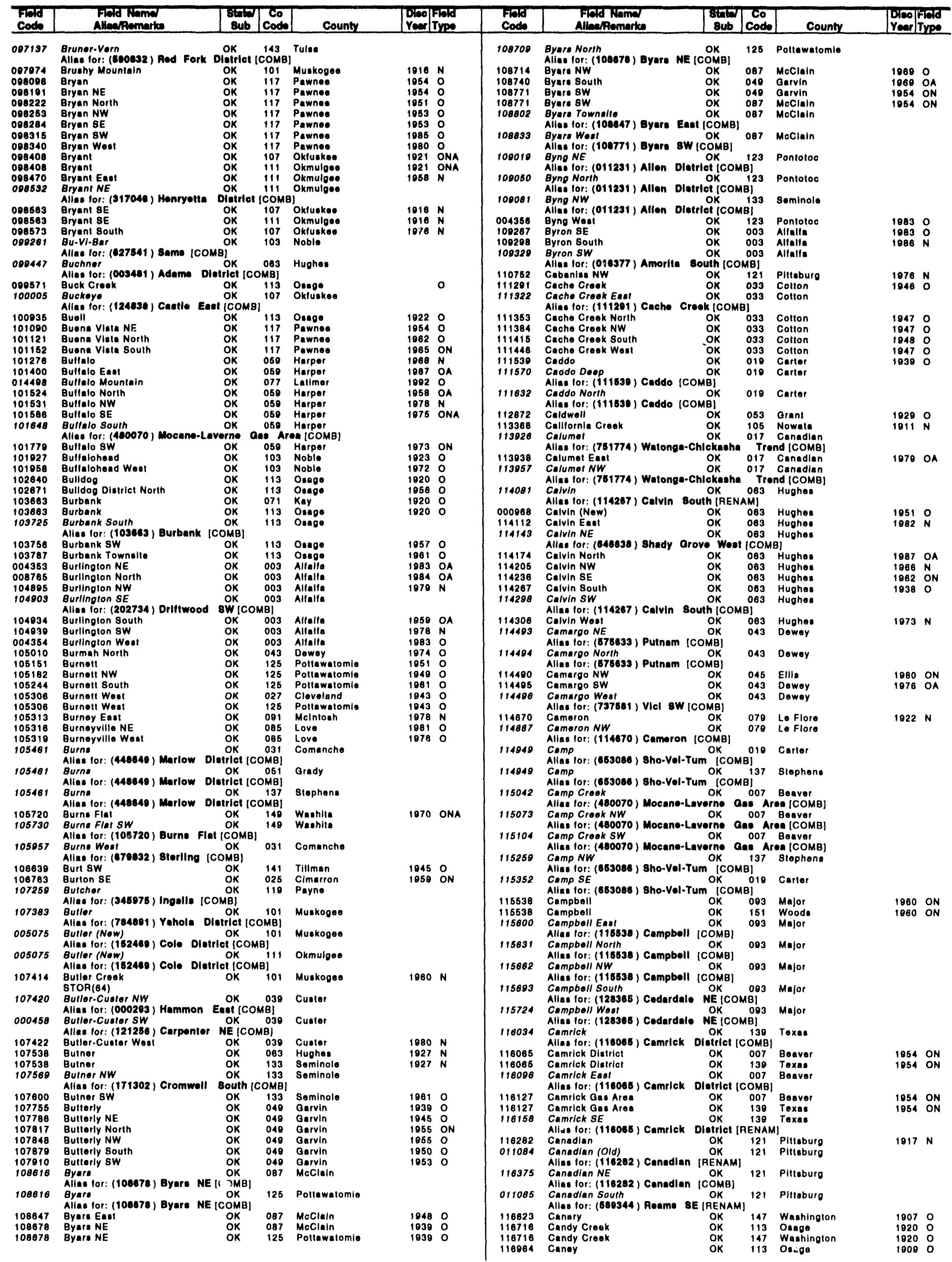


OKLAHOMA

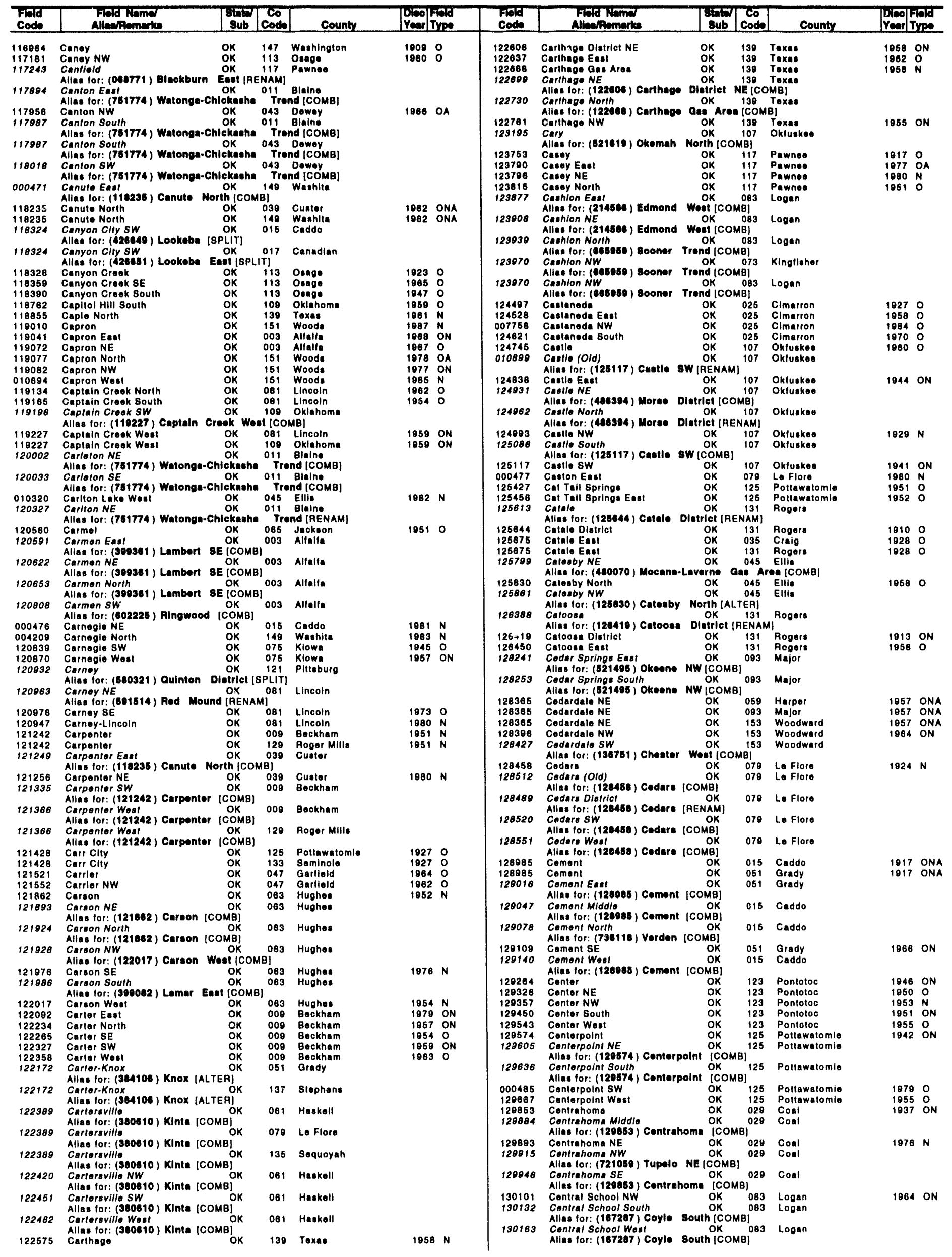


OKLAHOMA

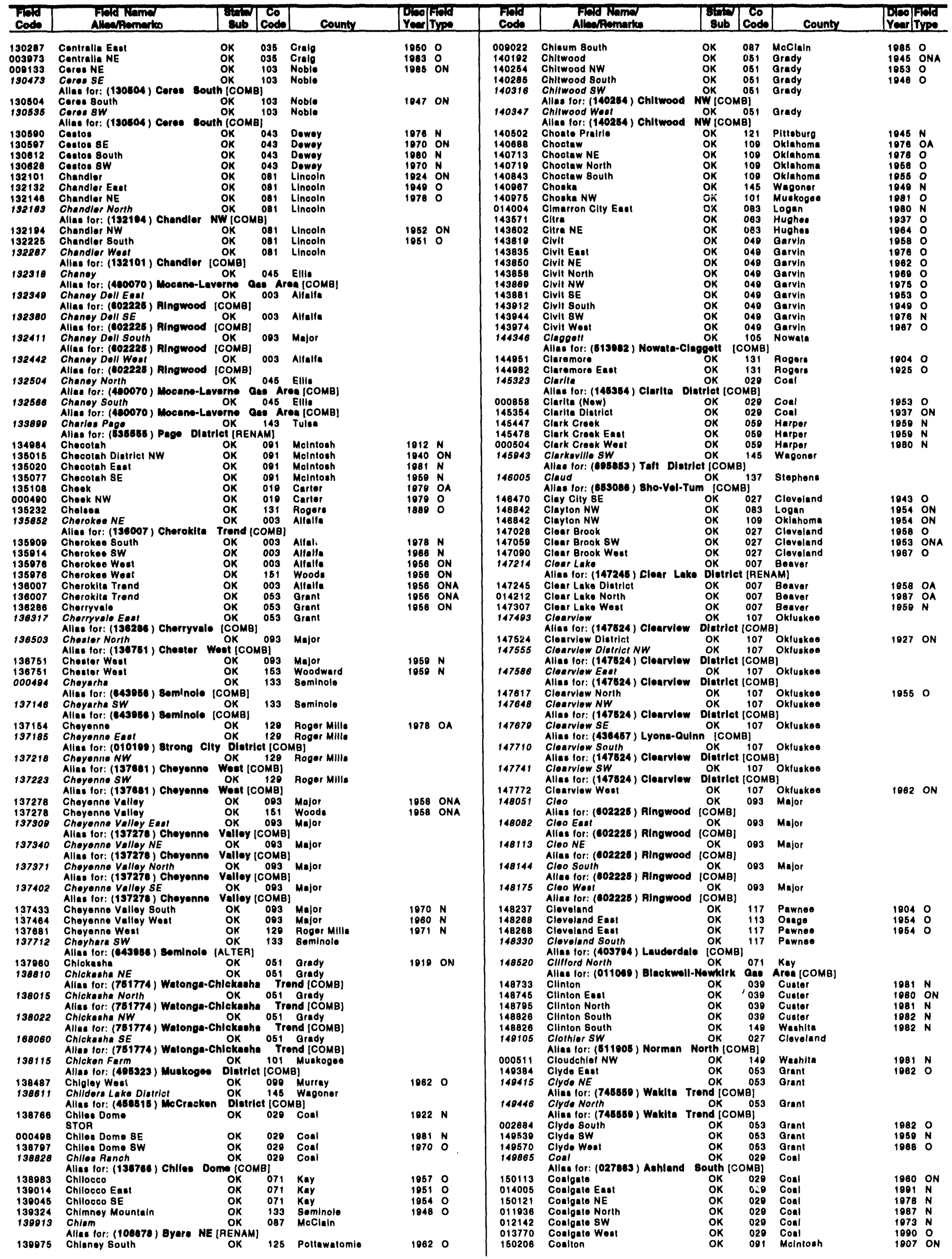




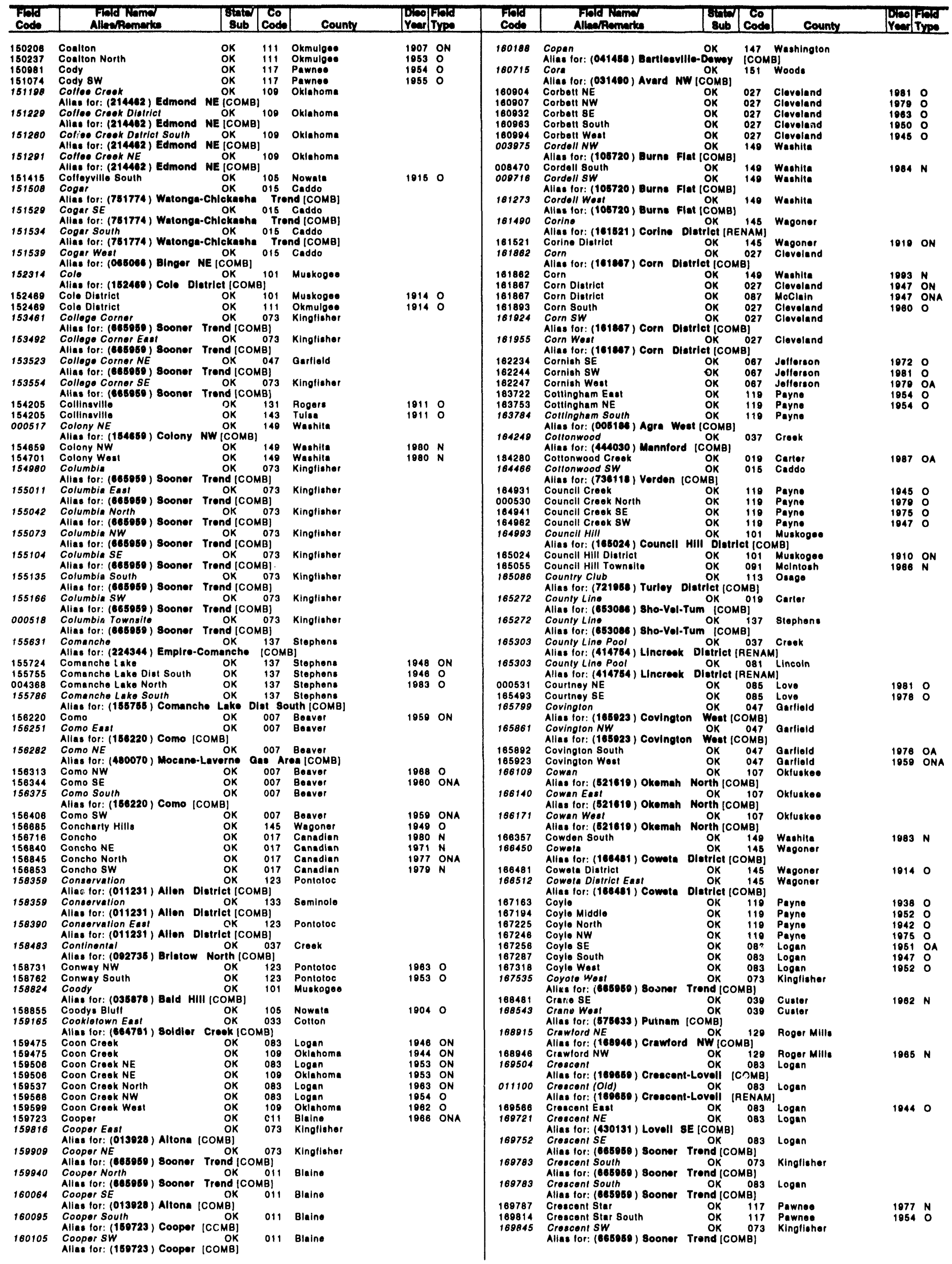


OKLAHOMA

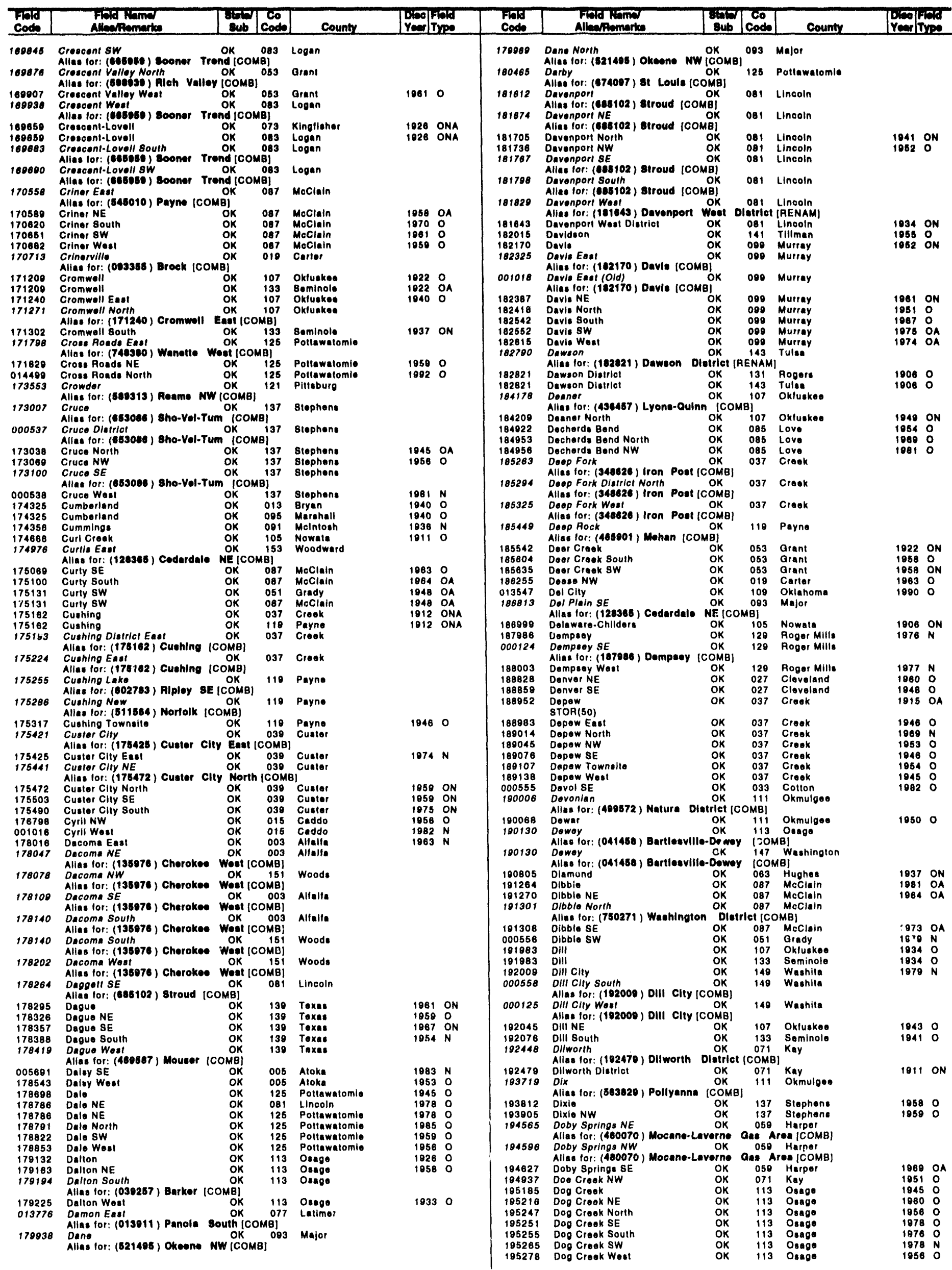




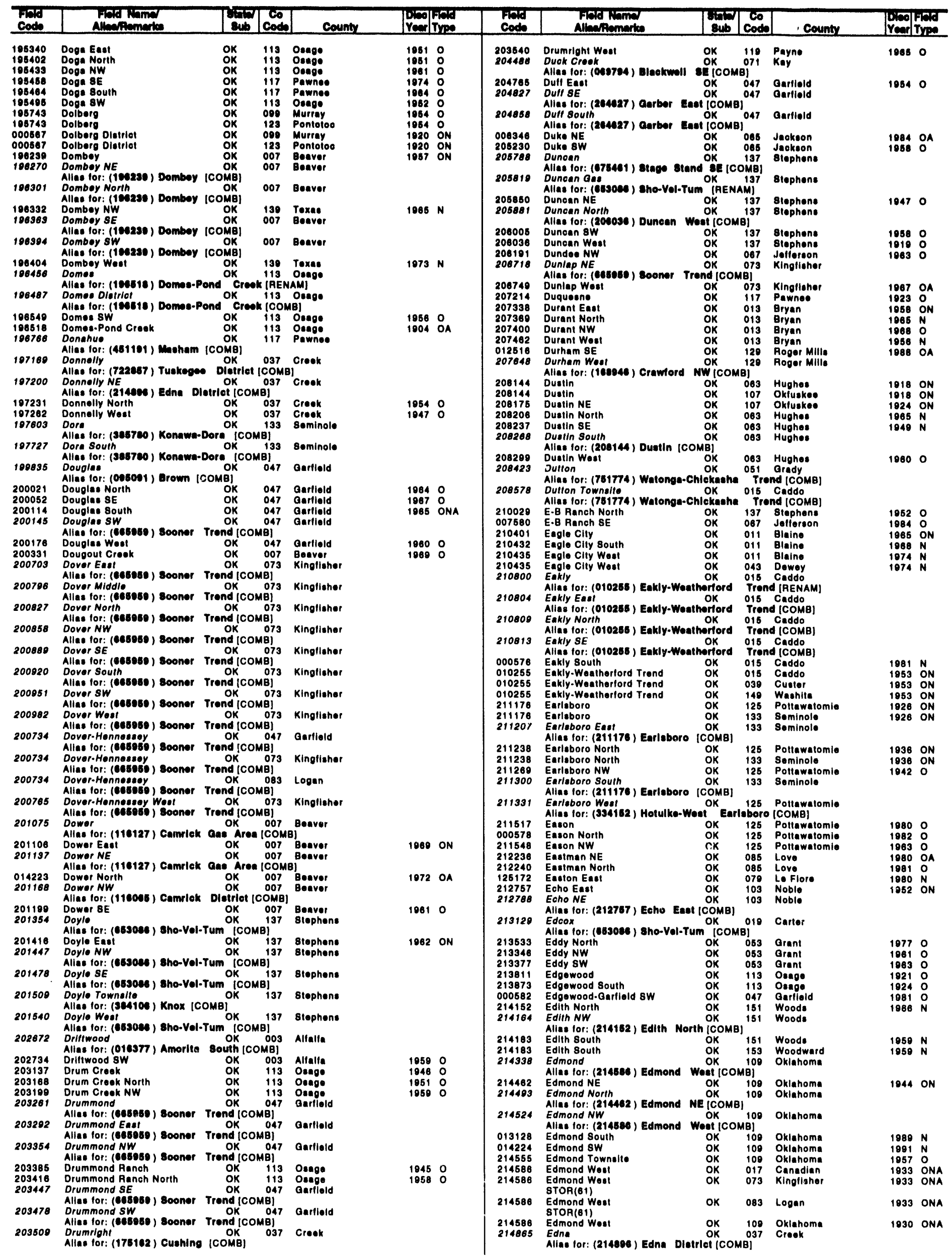


OKLAHOMA

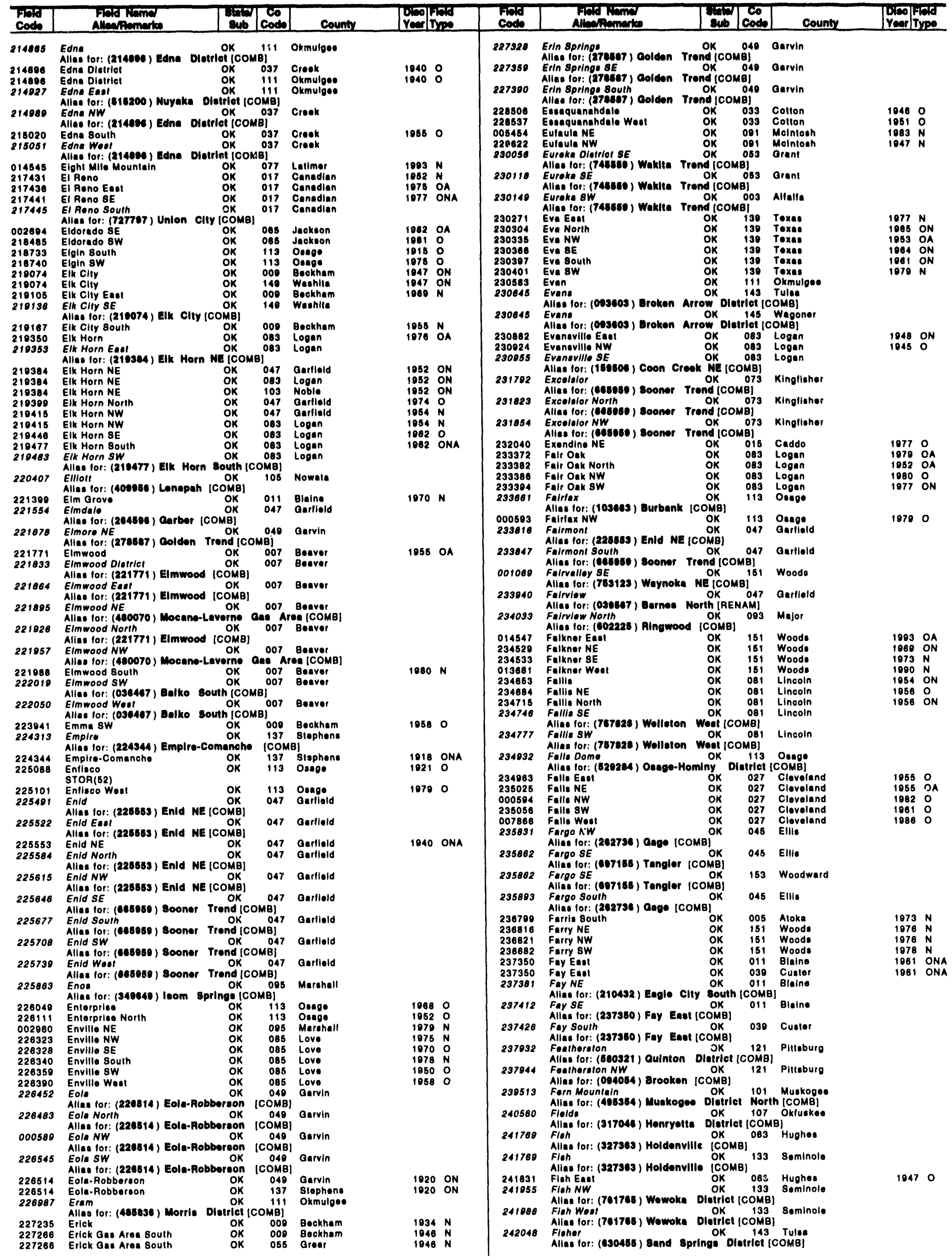


OKLAHOMA

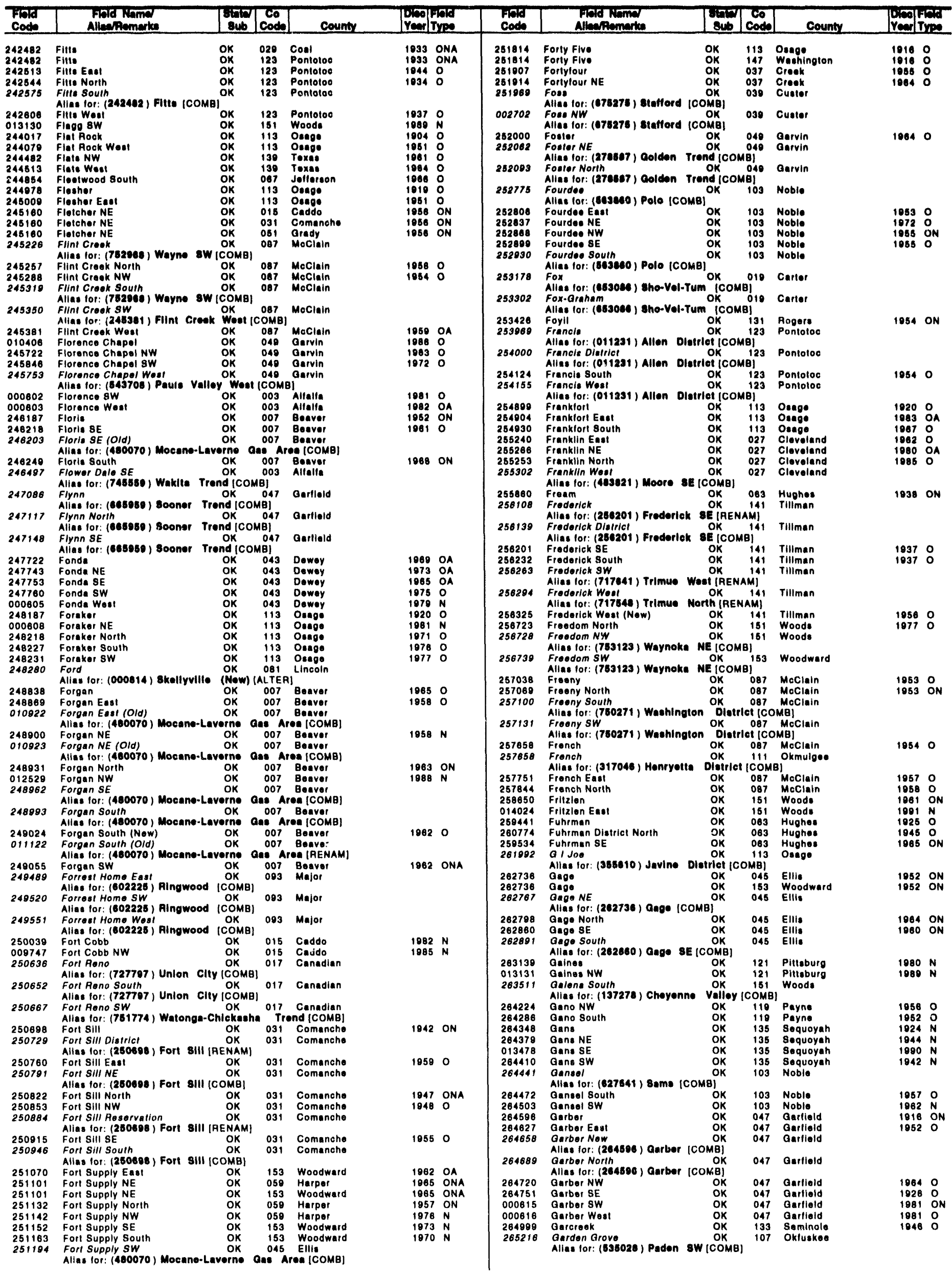


OKLAHOMA

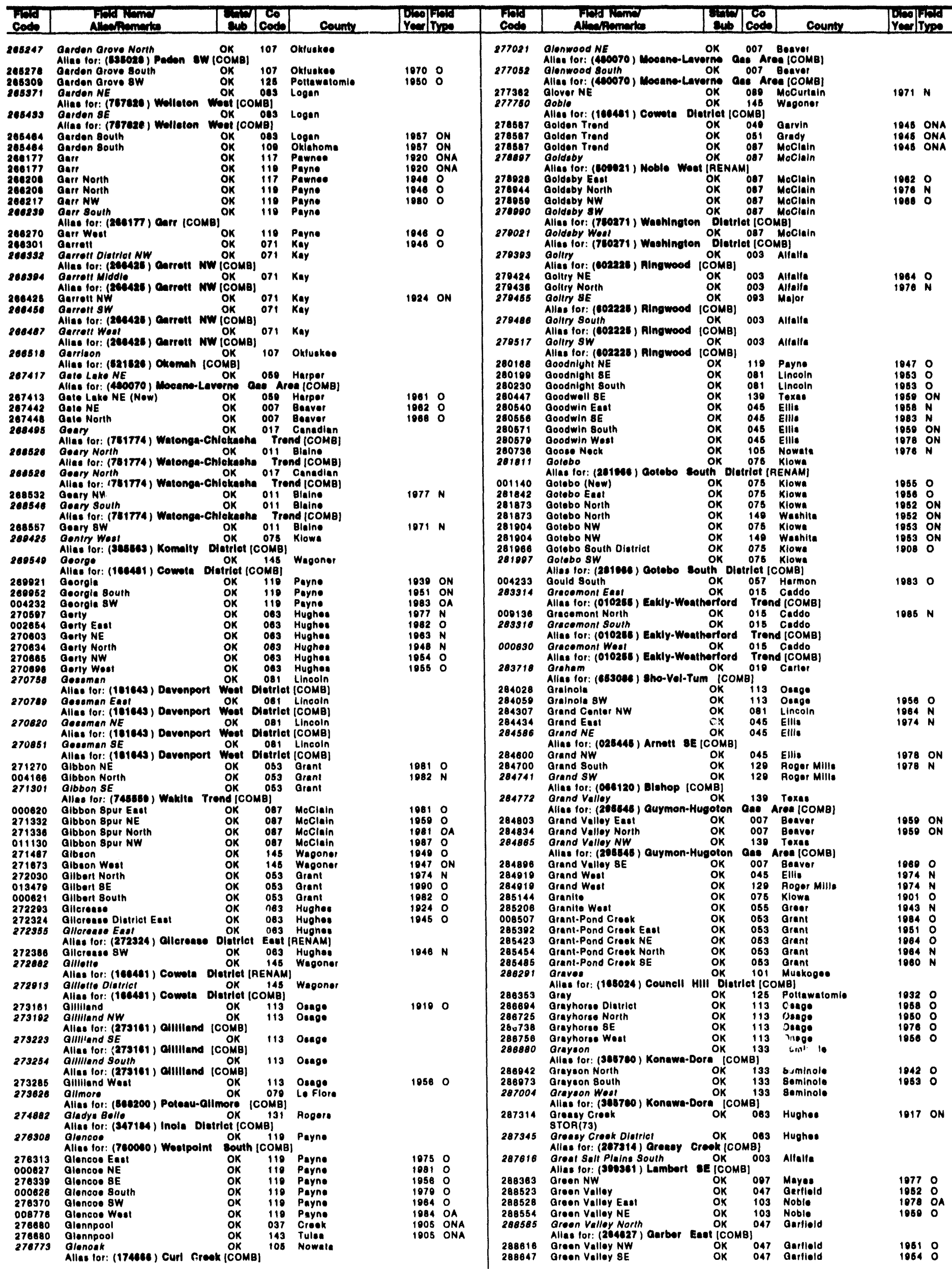




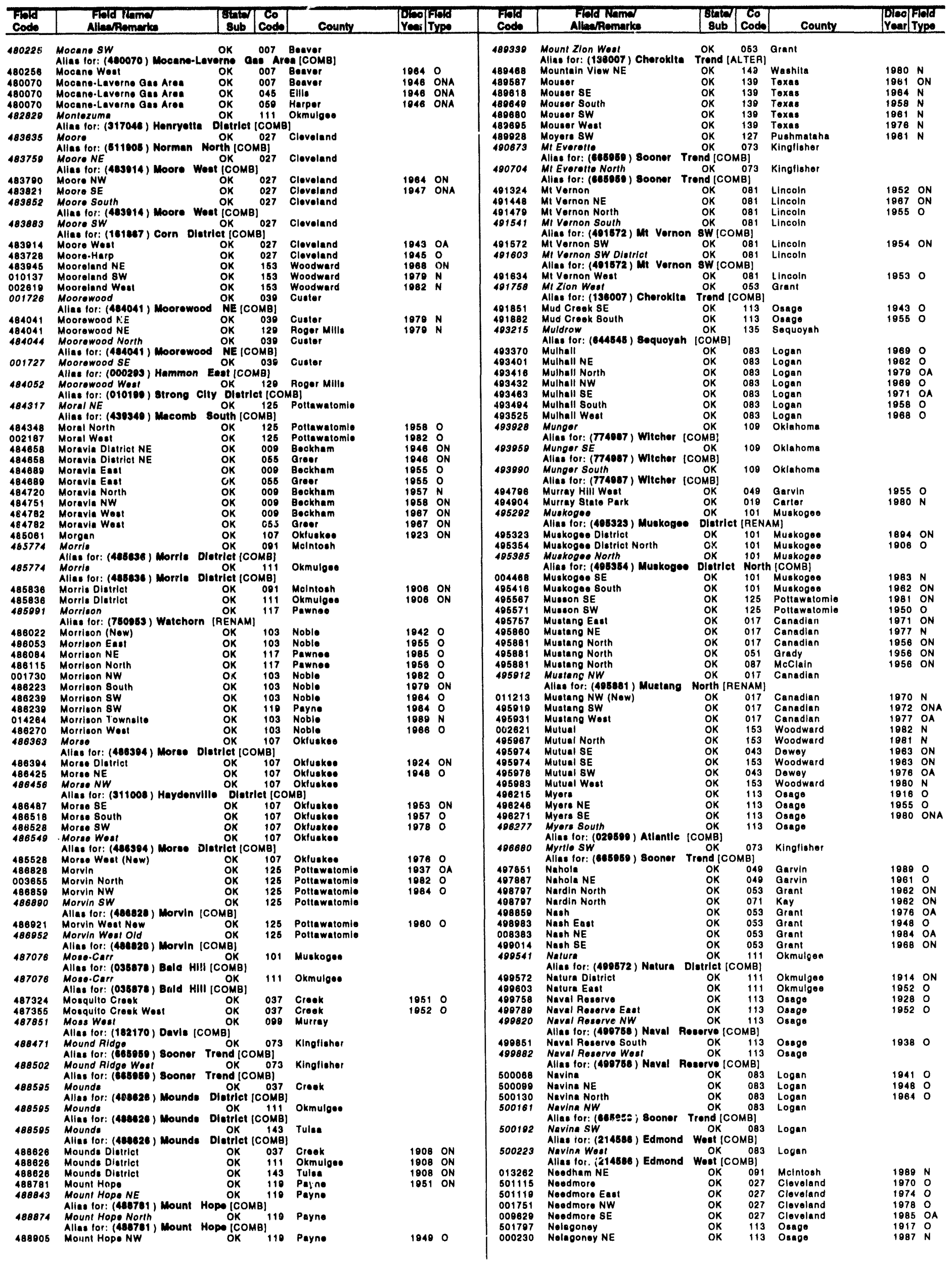




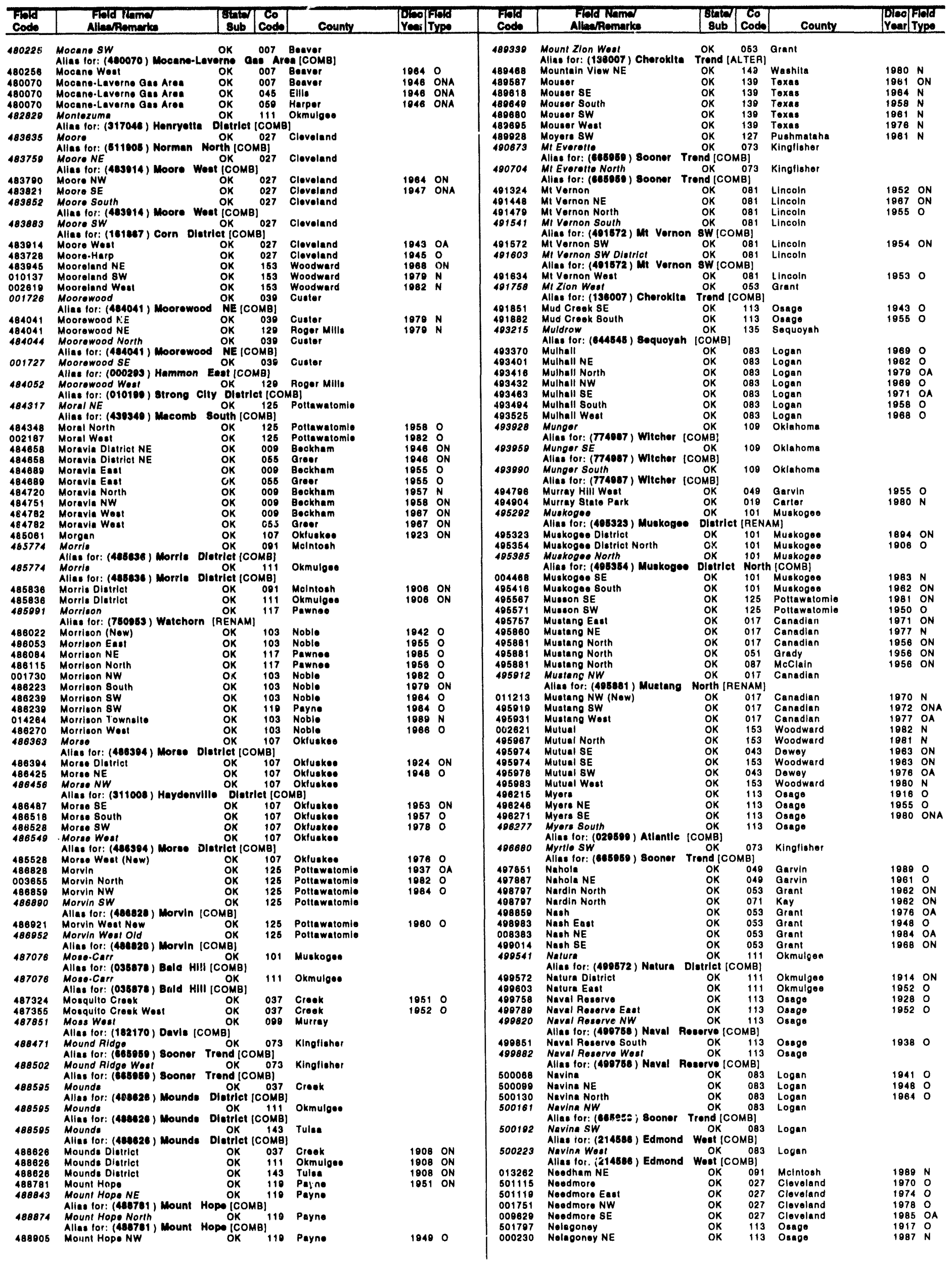


OKLAHOMA

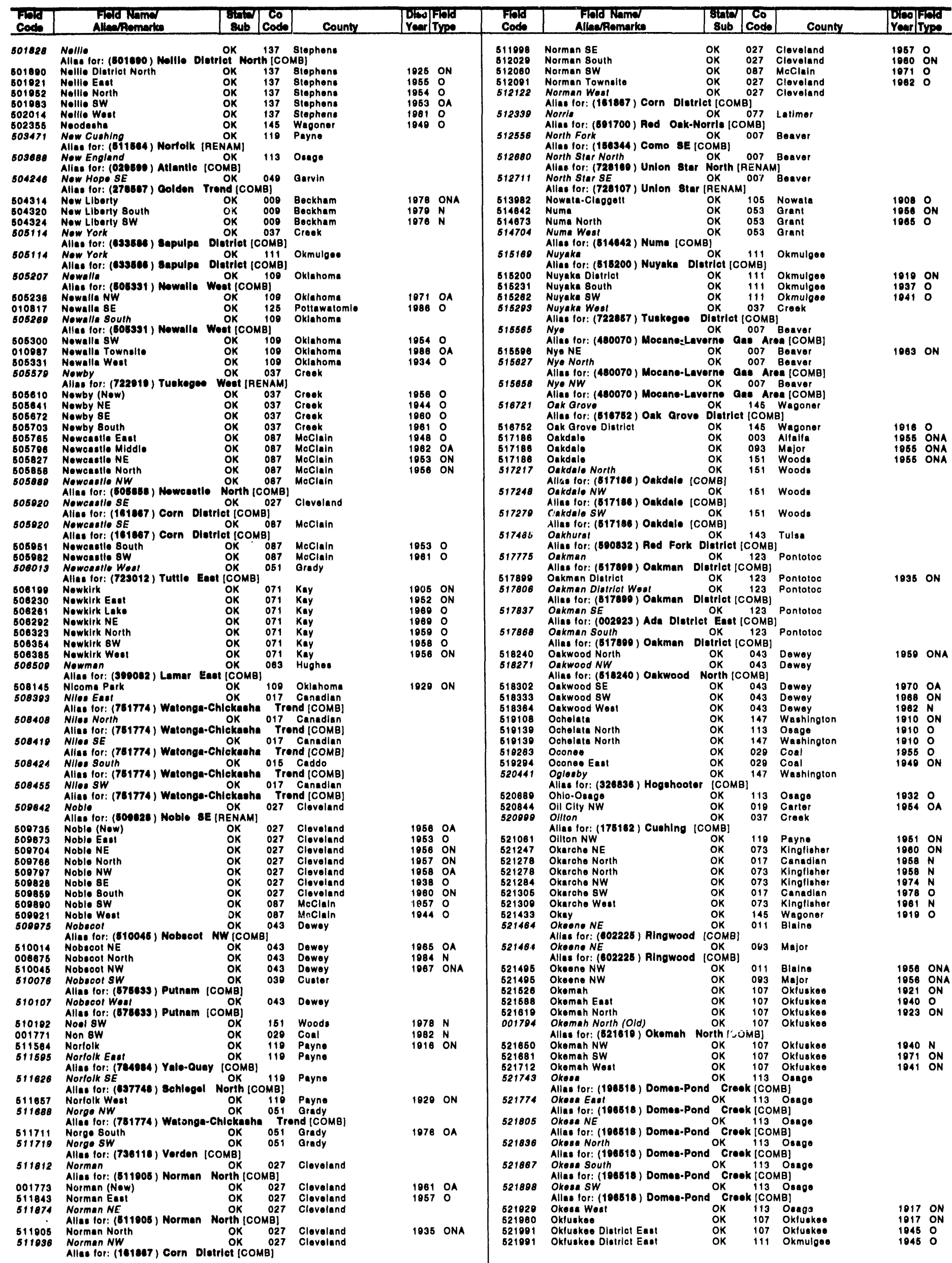




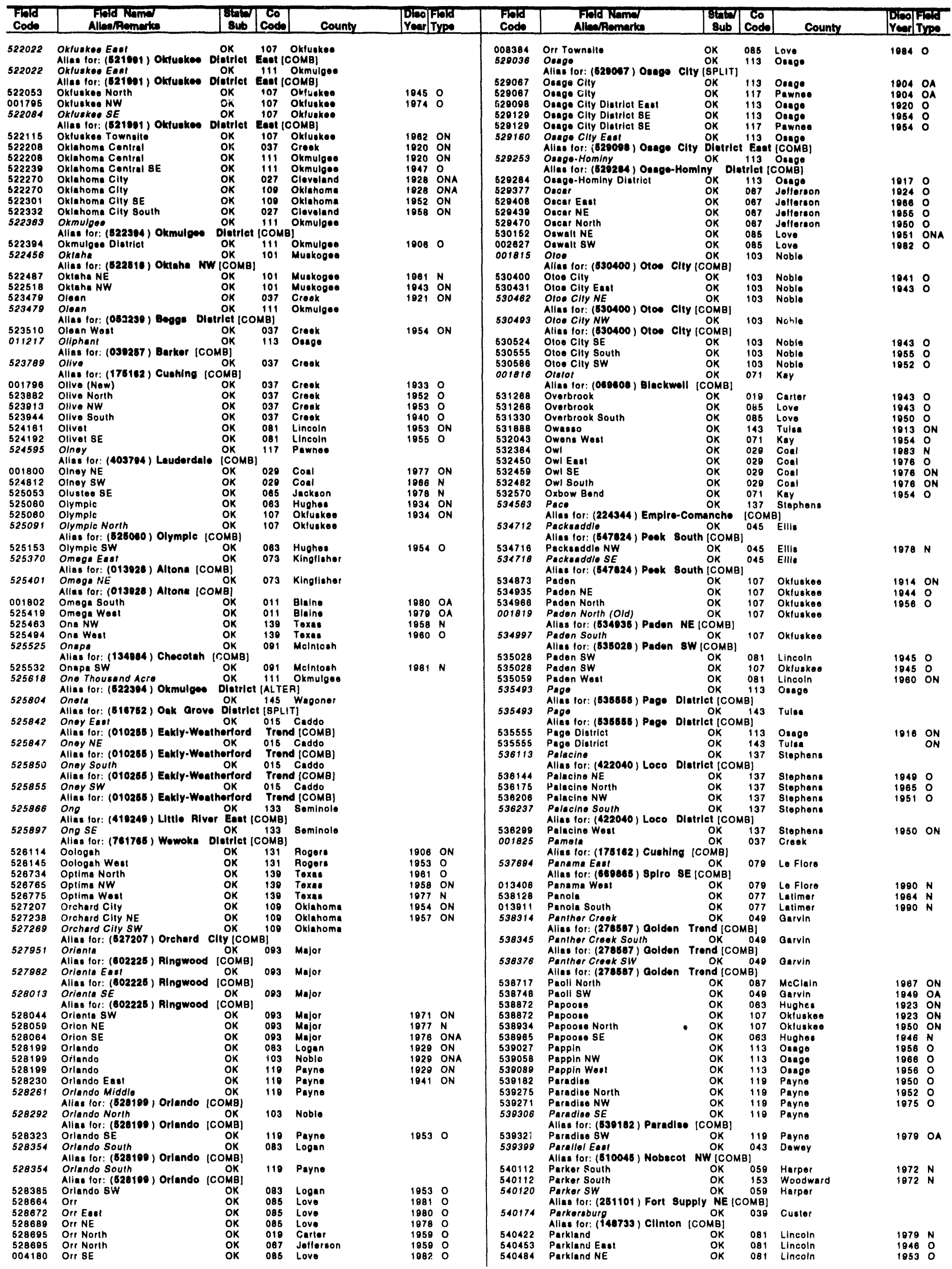


OKLAHOMA

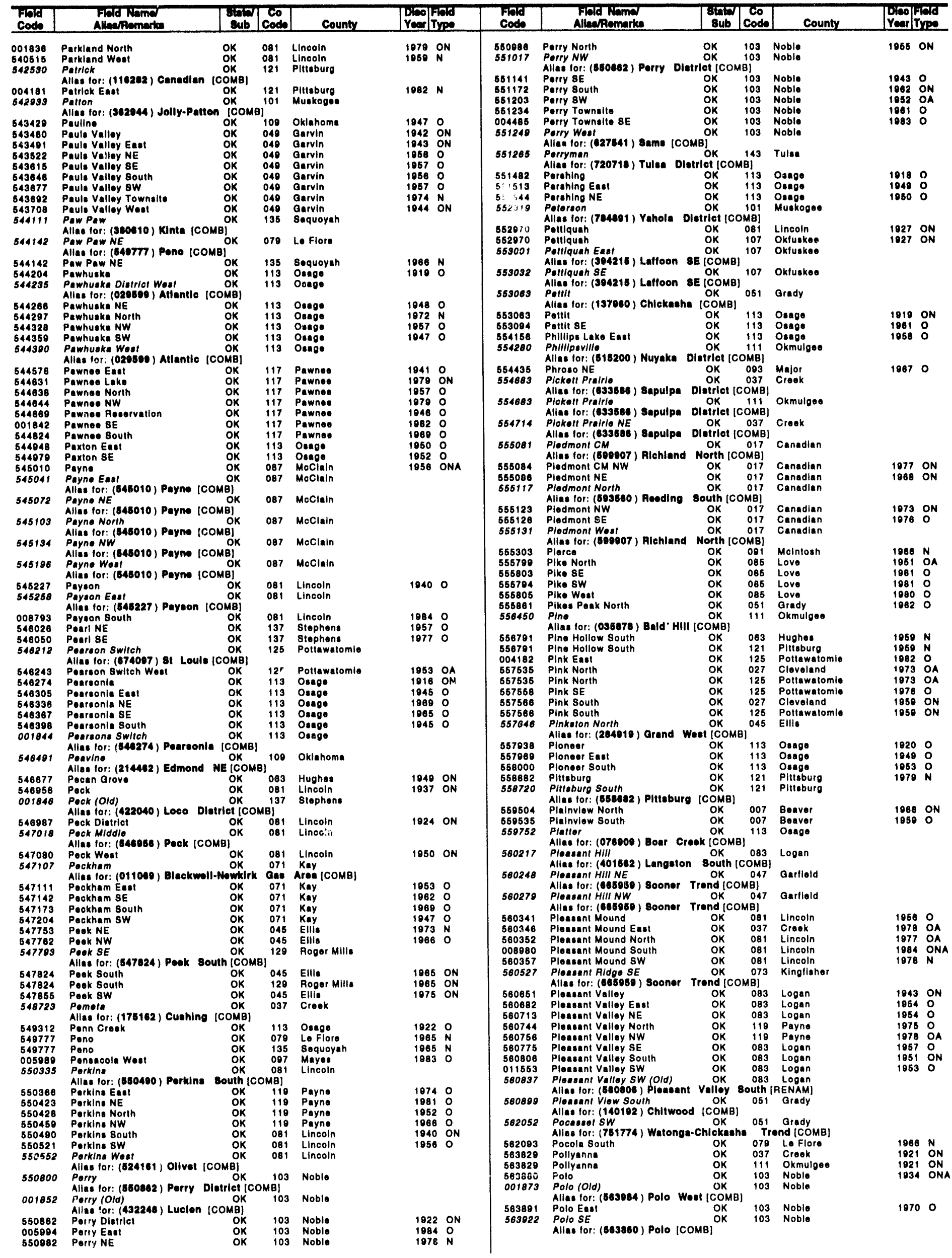




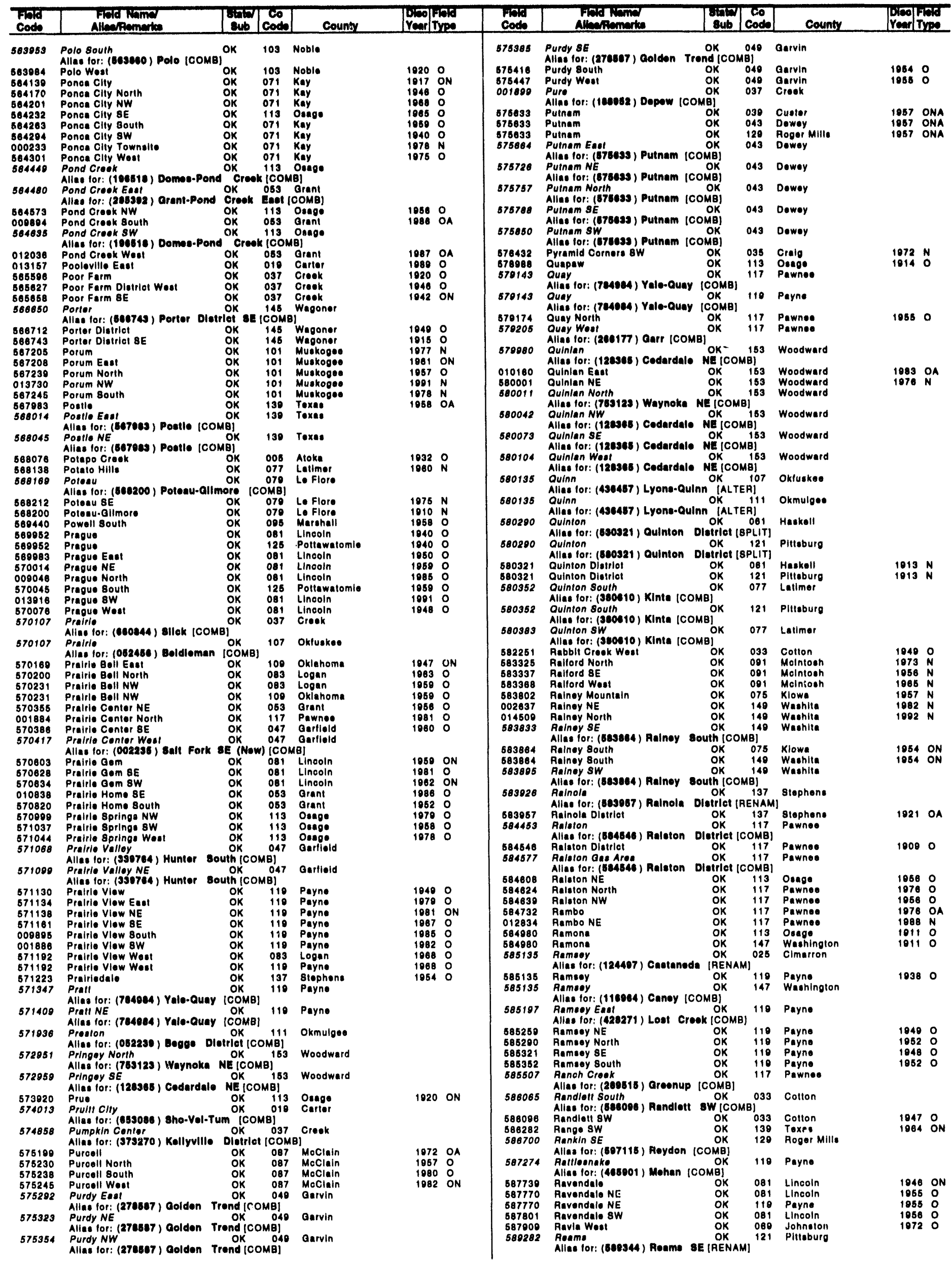


OKLAHOMA

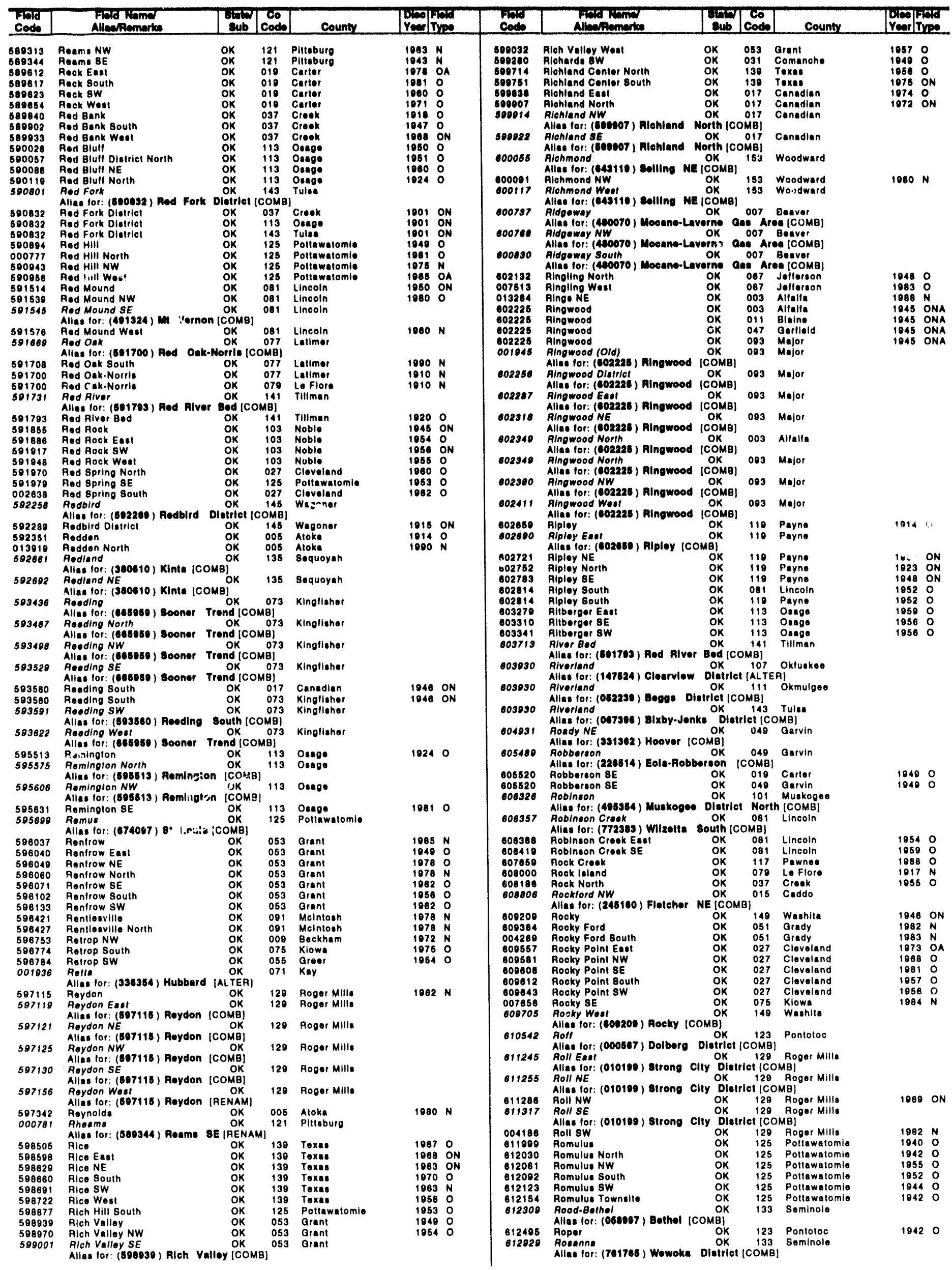




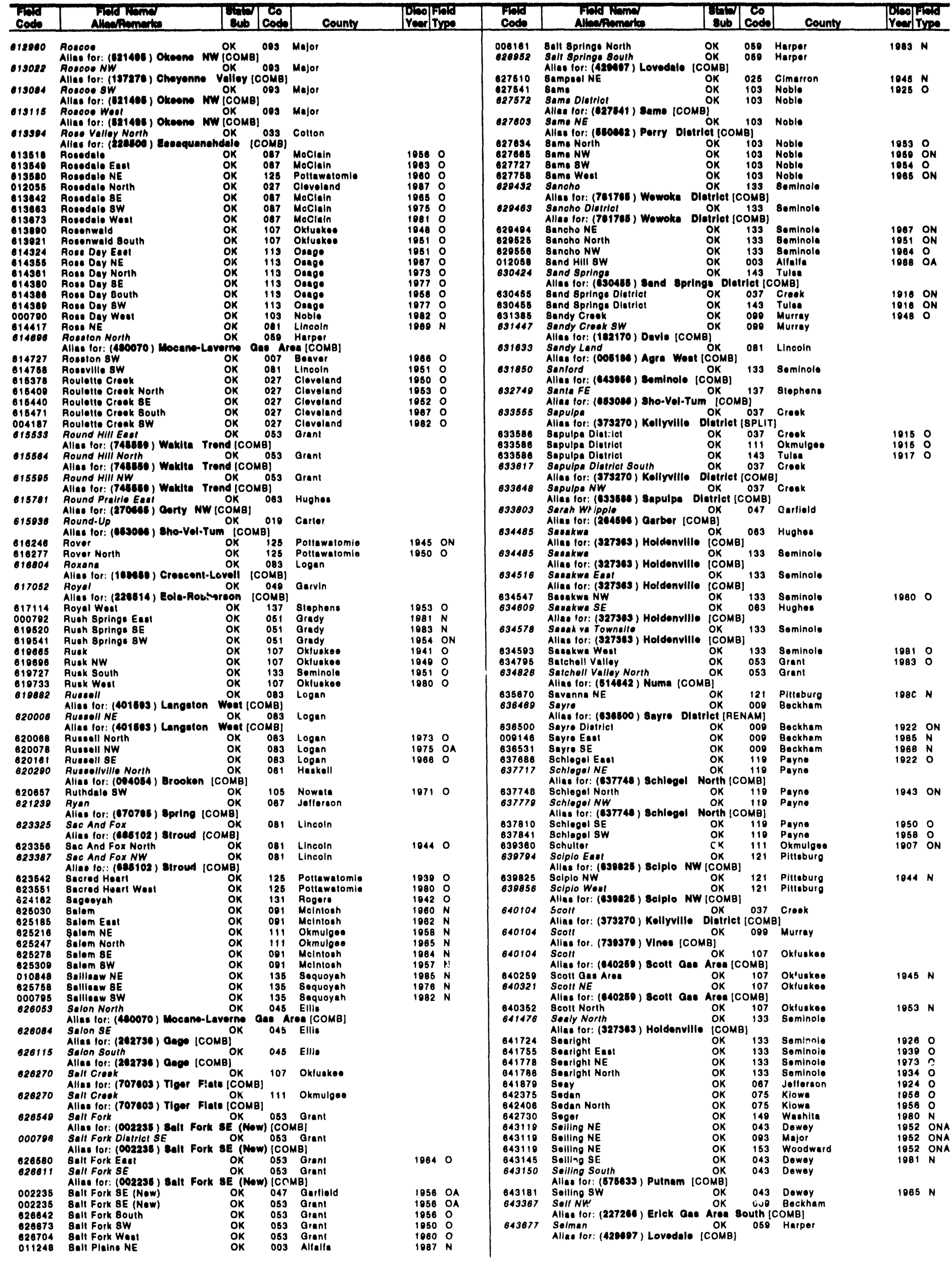


TEXAS

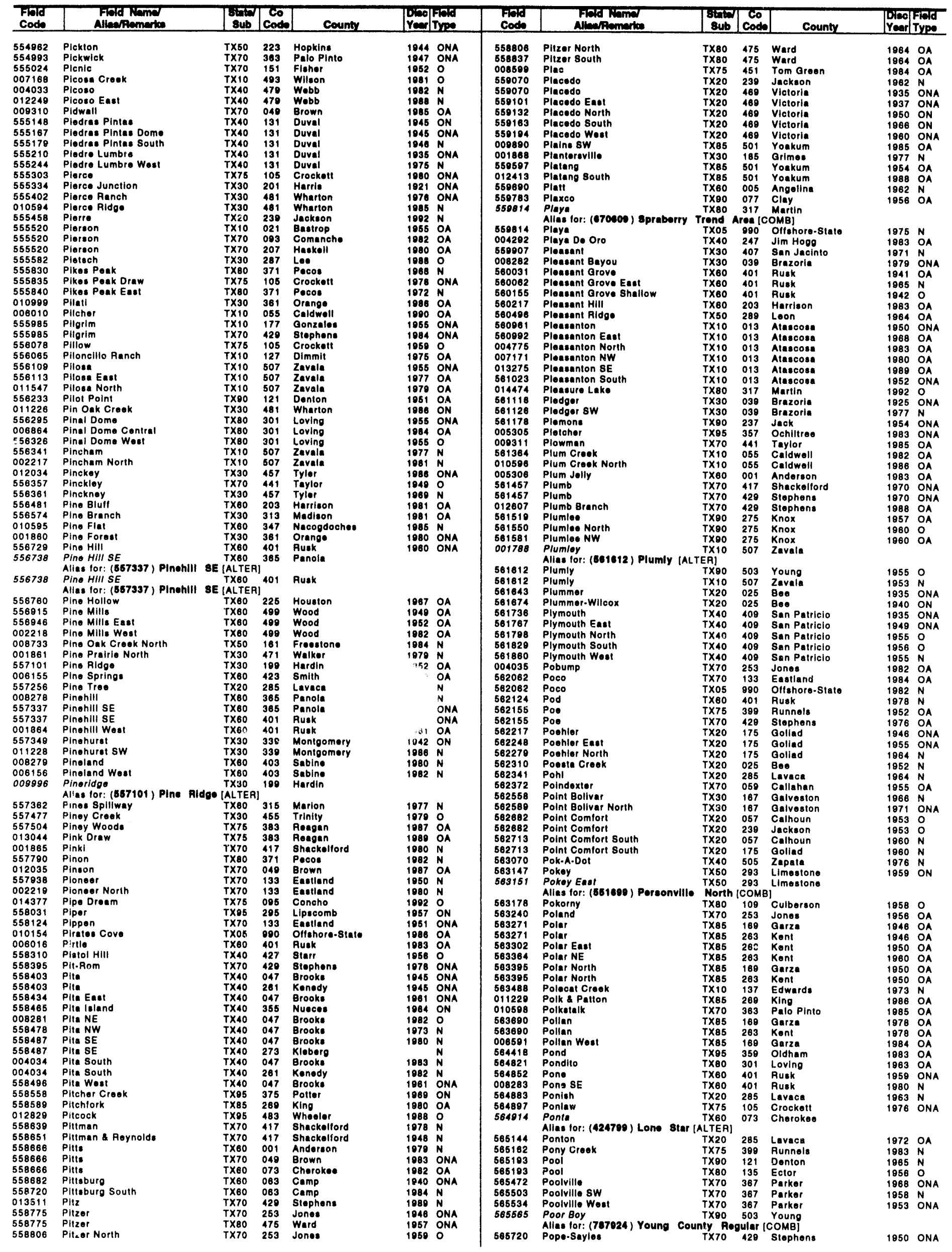


TEXAS

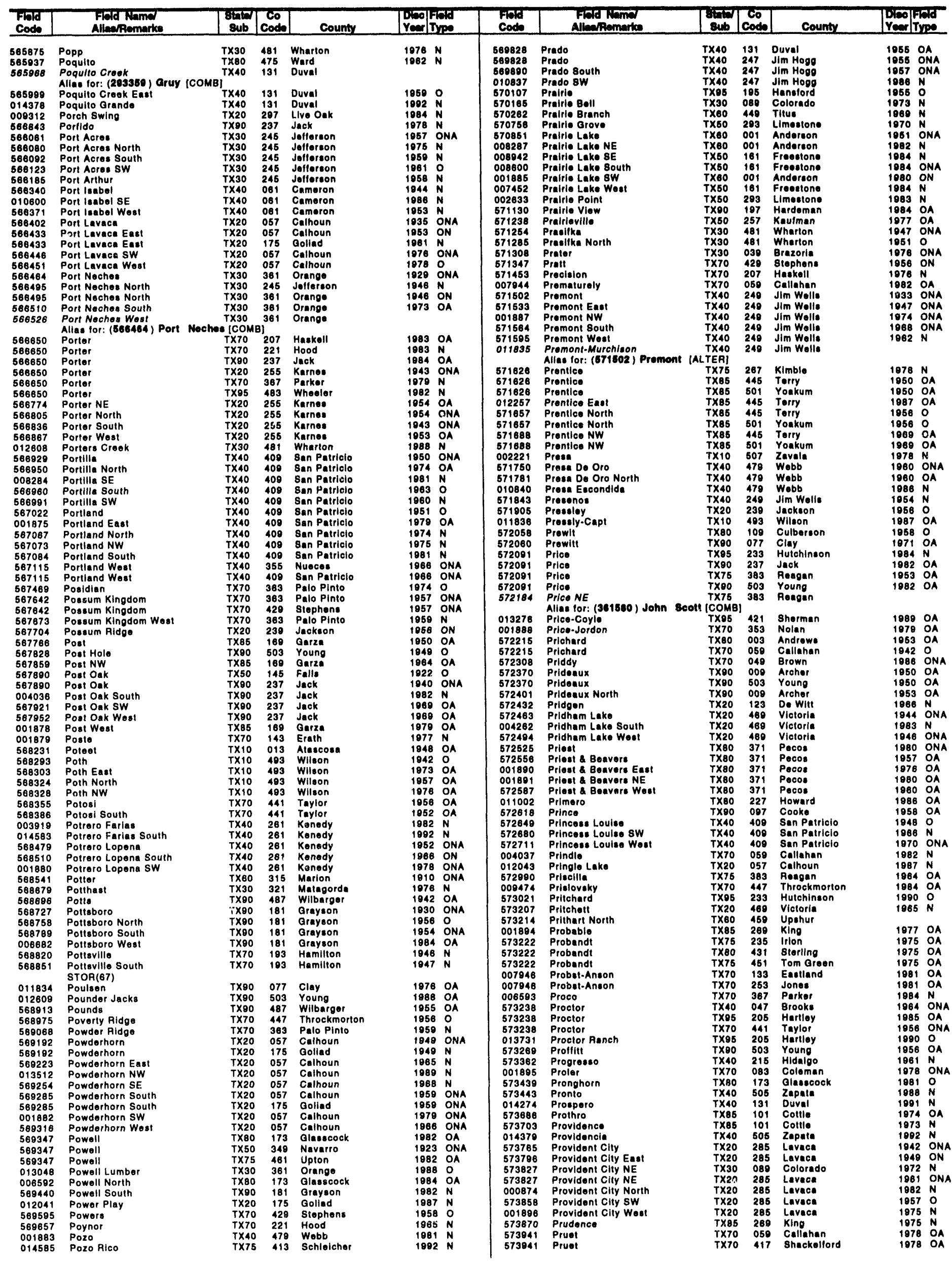


TEXAS

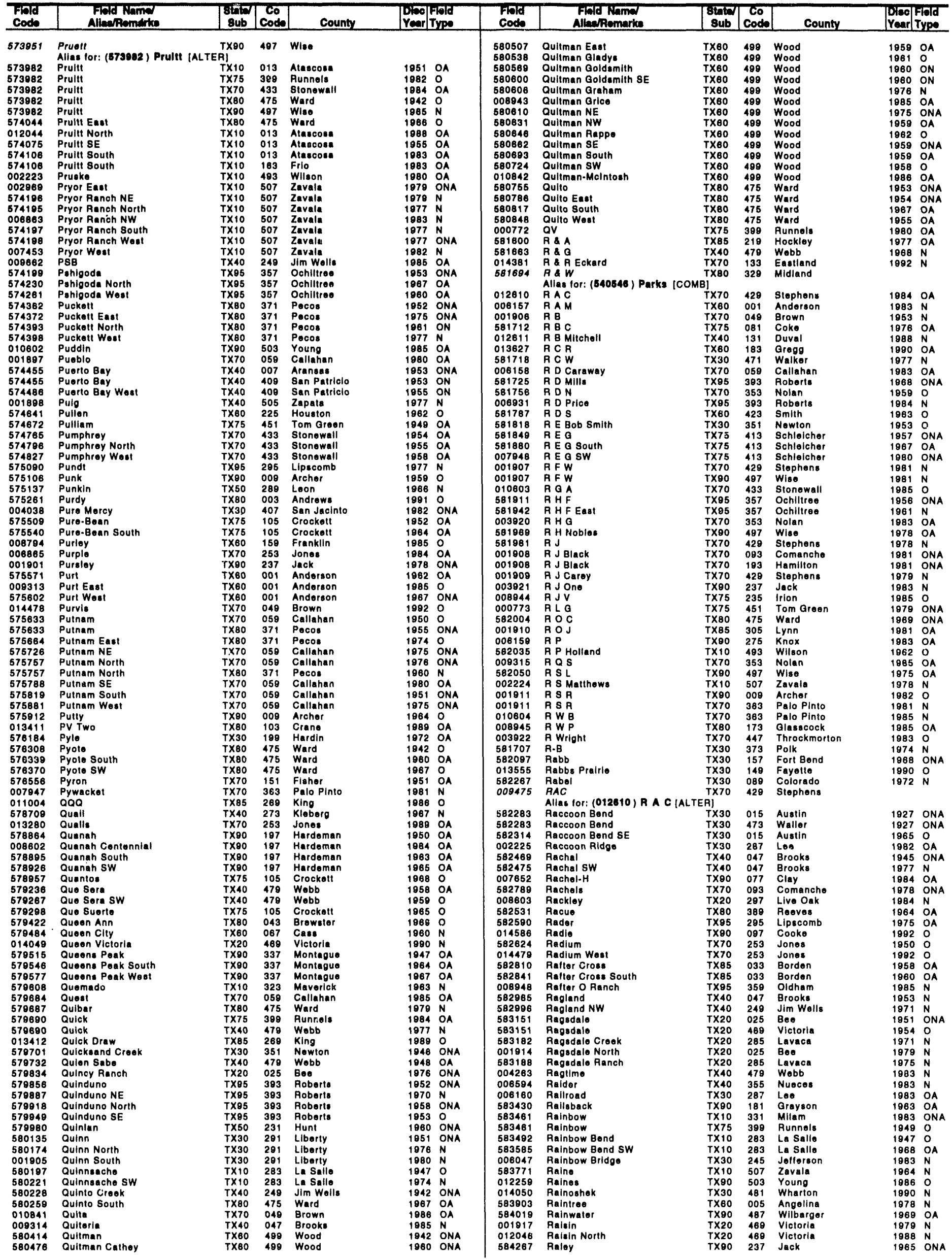


TEXAS

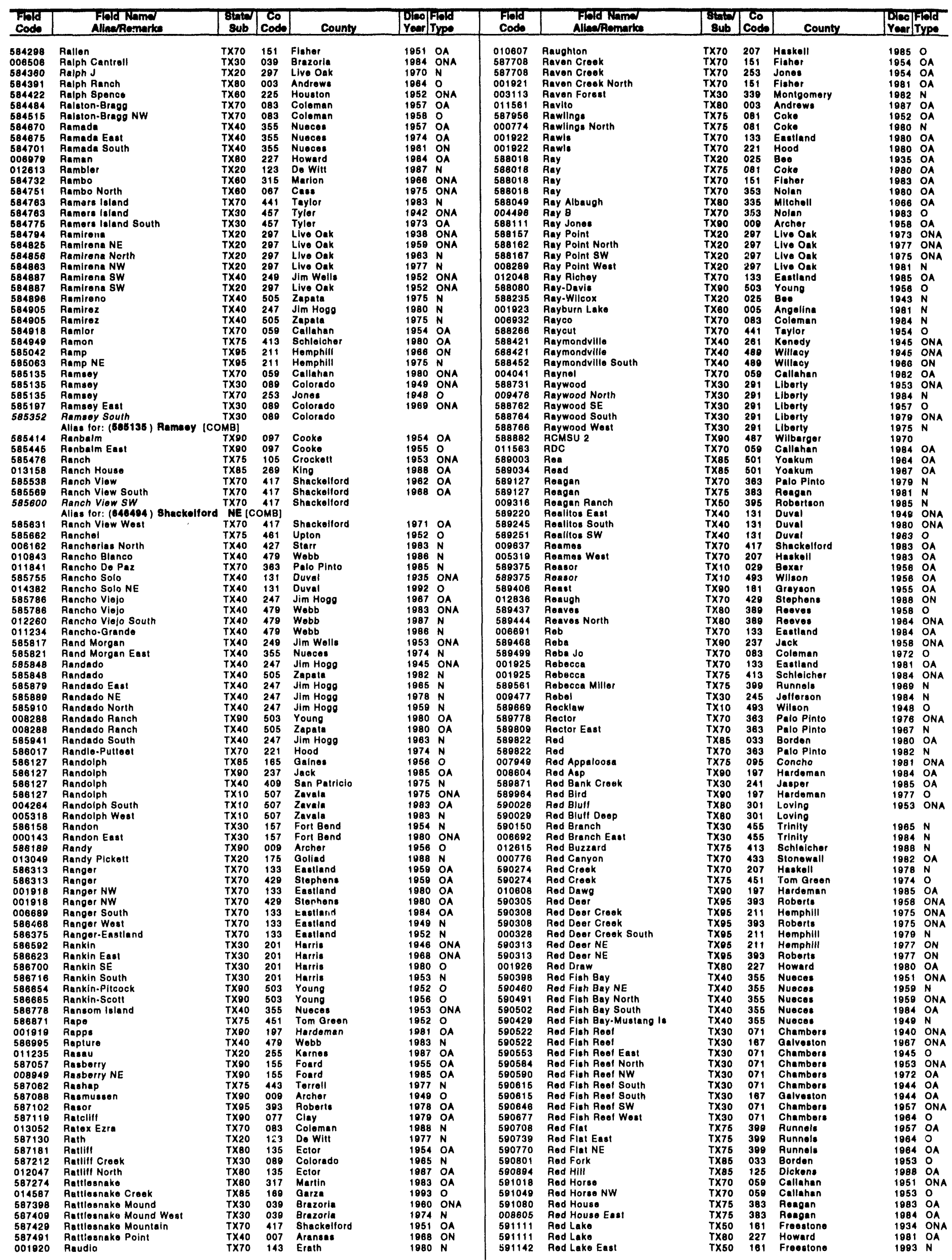


TEXAS

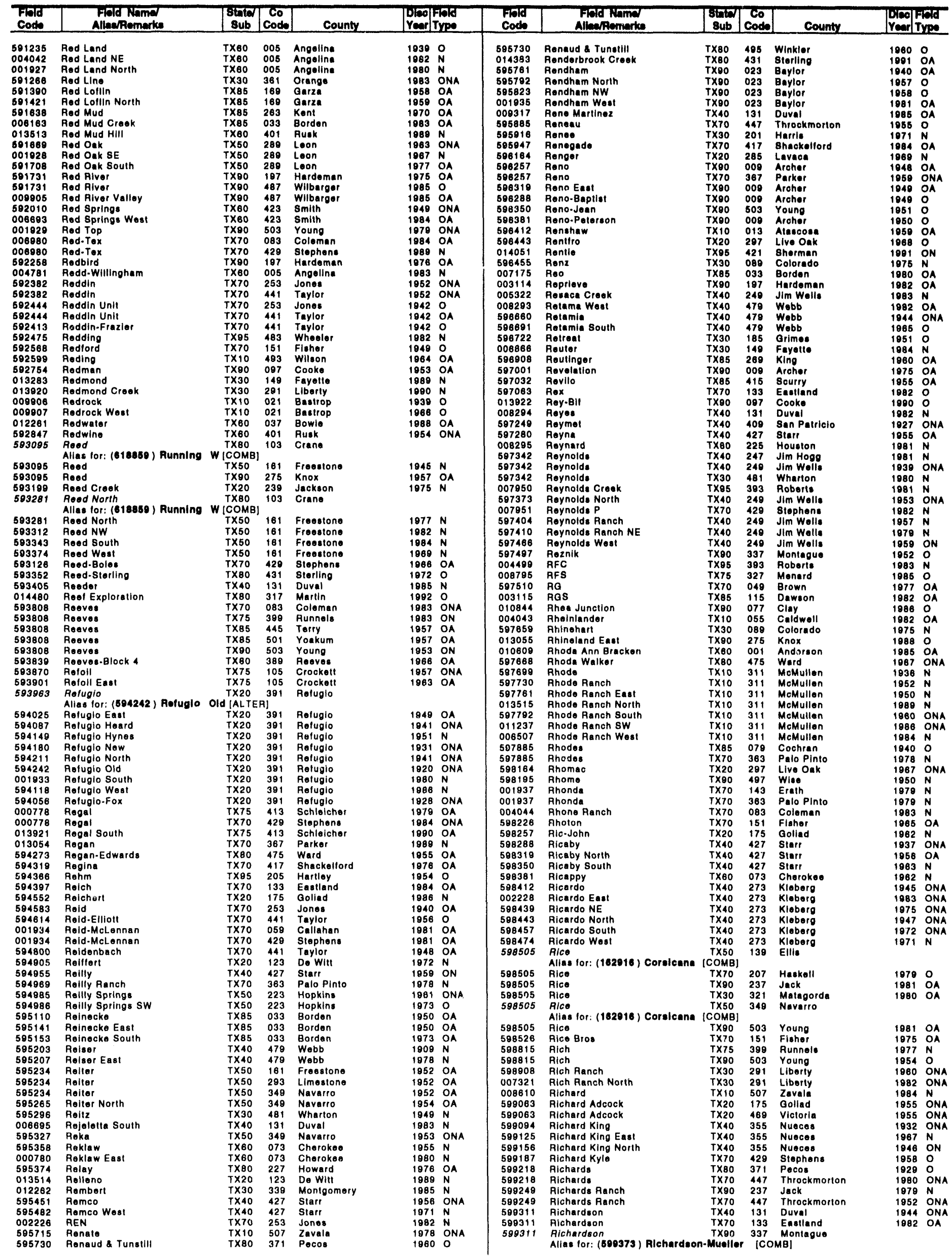


TEXAS

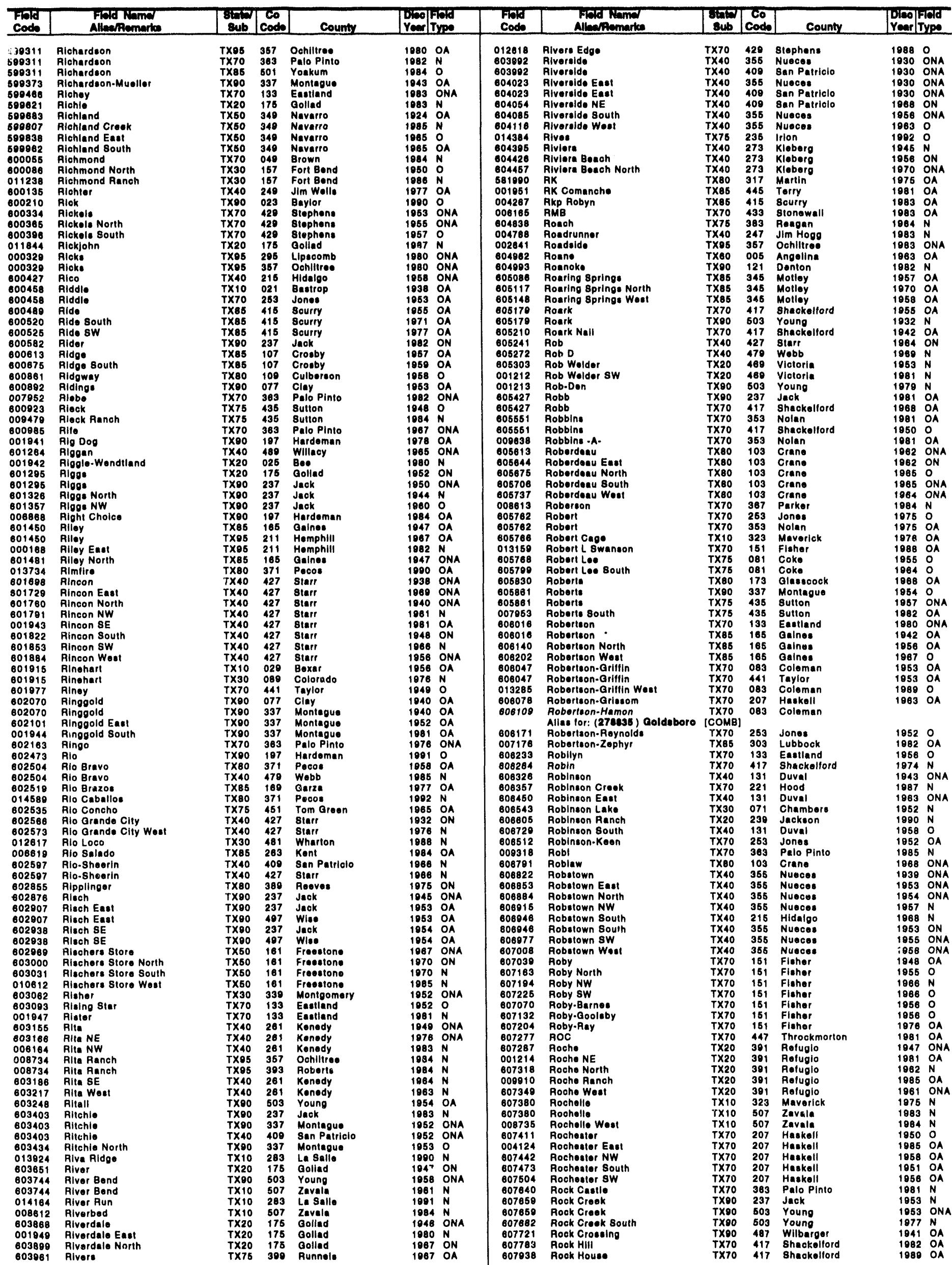


TEXAS

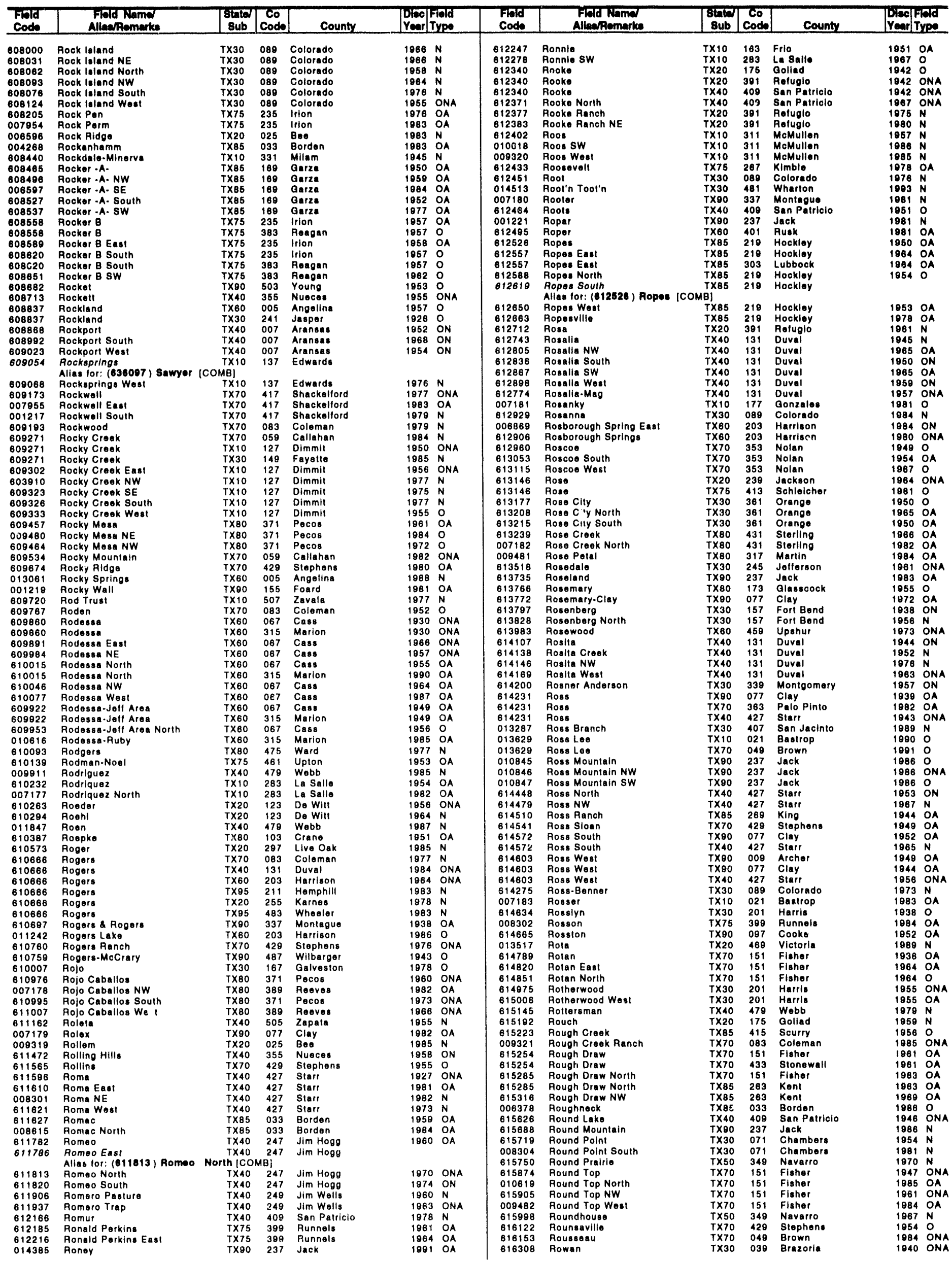


TEXAS

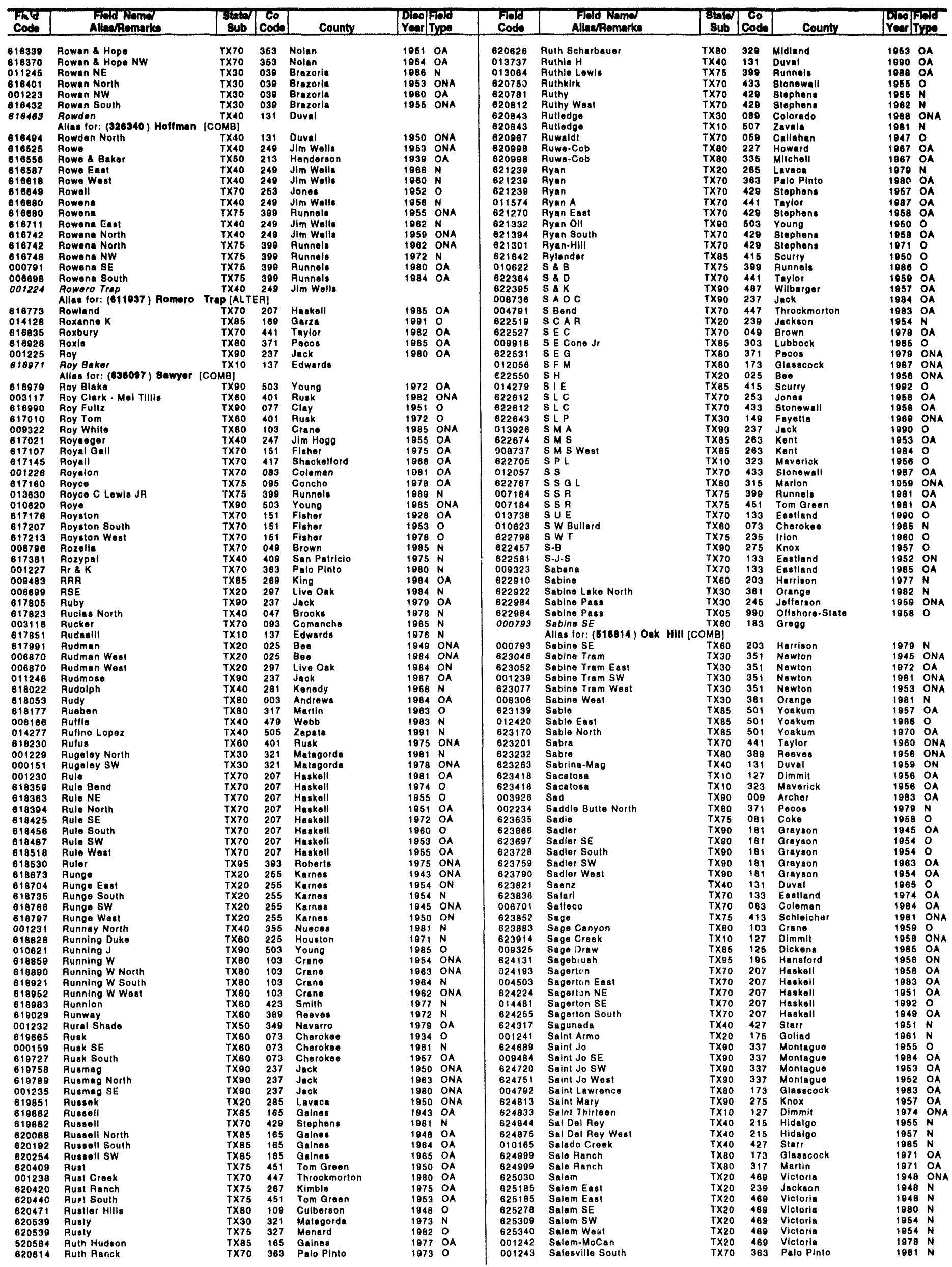


TEXAS

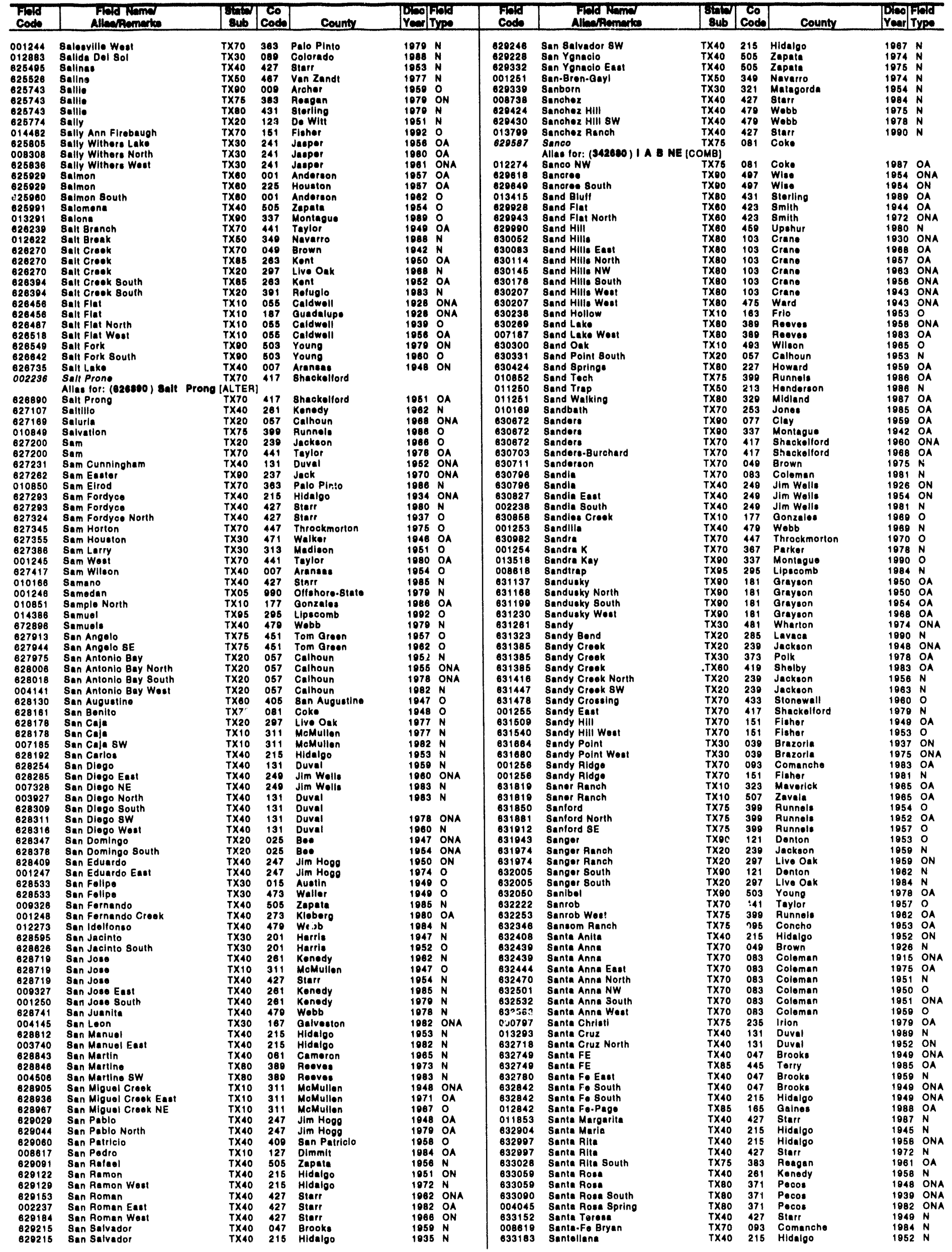


TEXAS

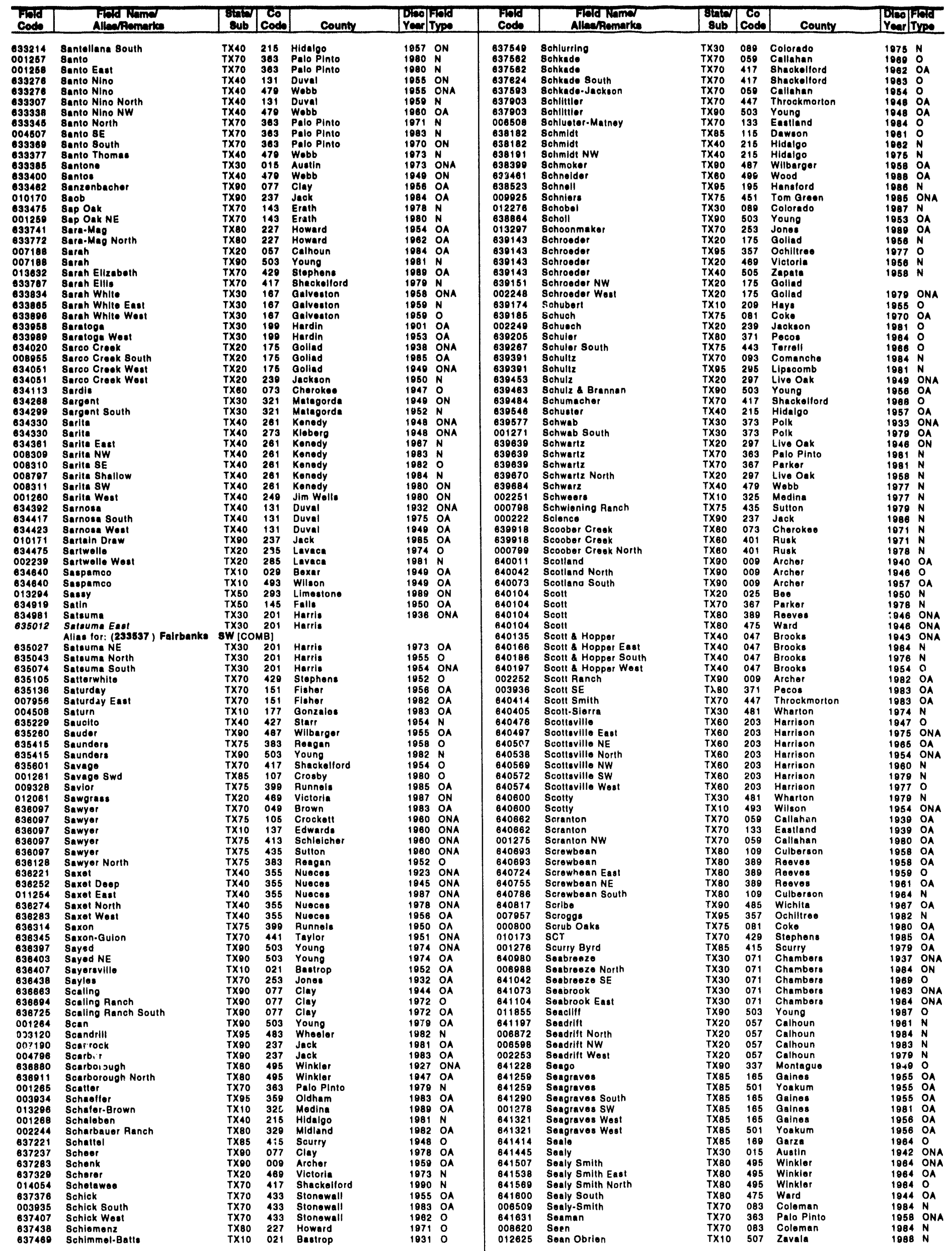


TEXAS

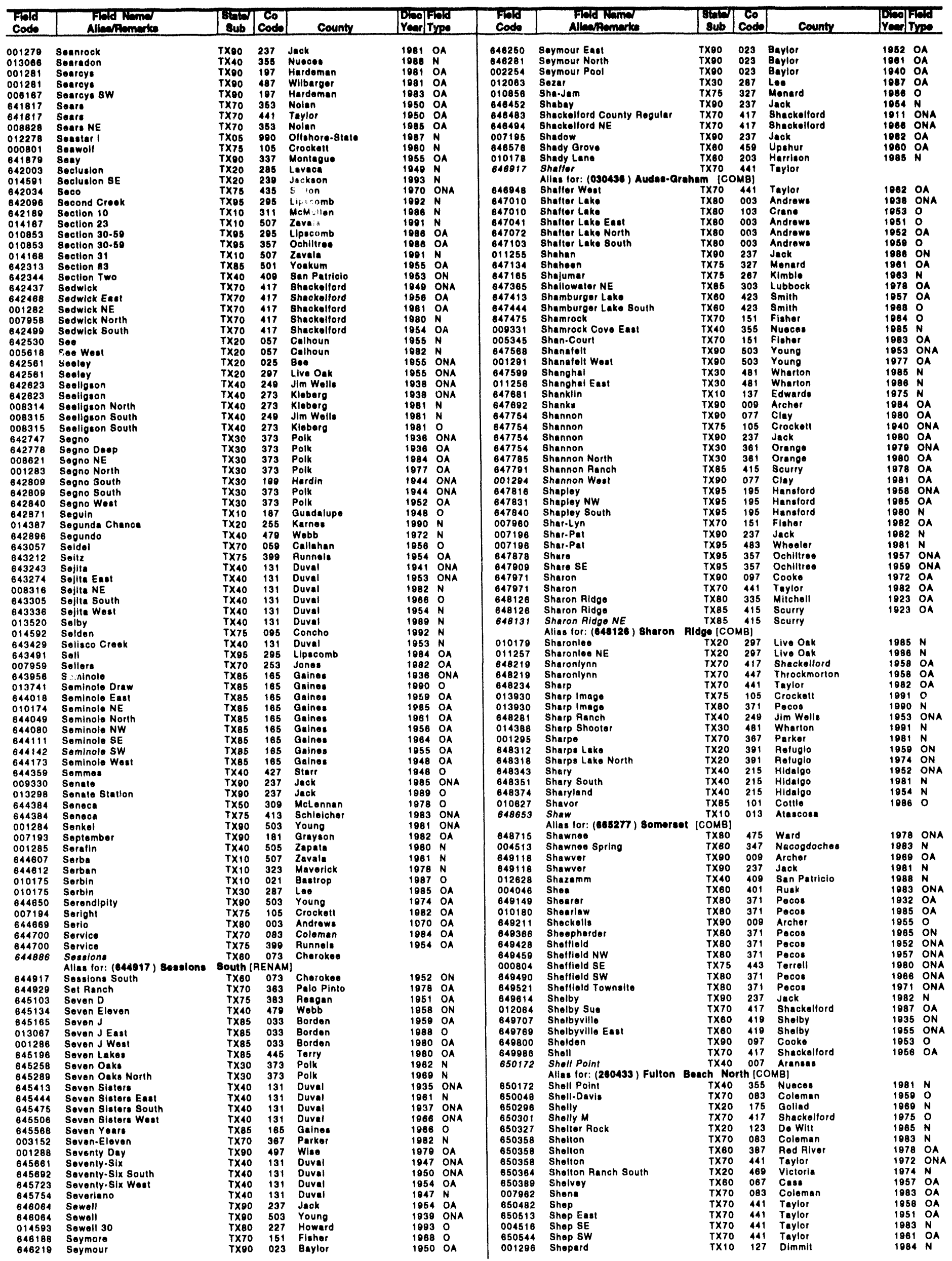


TEXAS

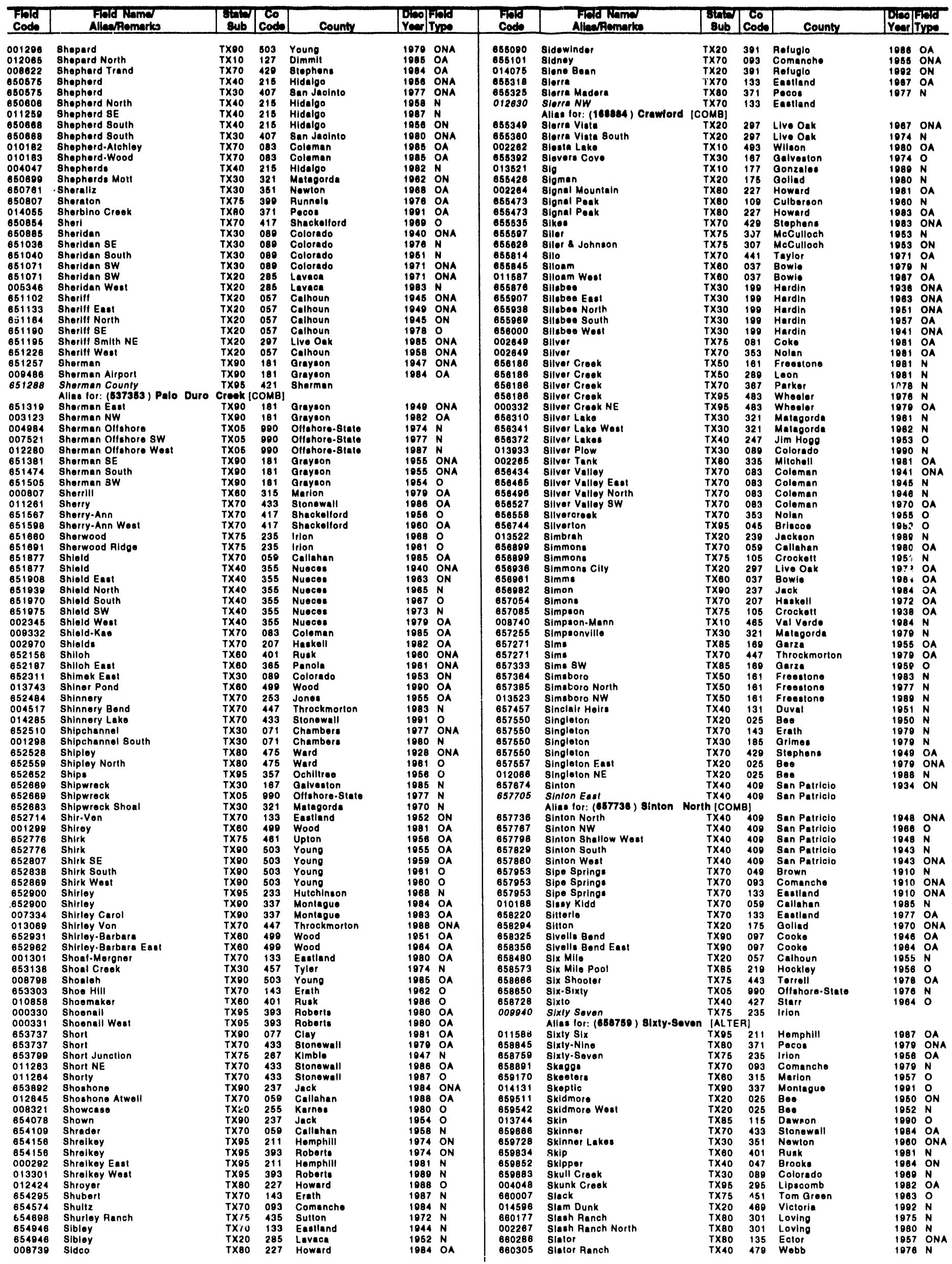


TEXAS

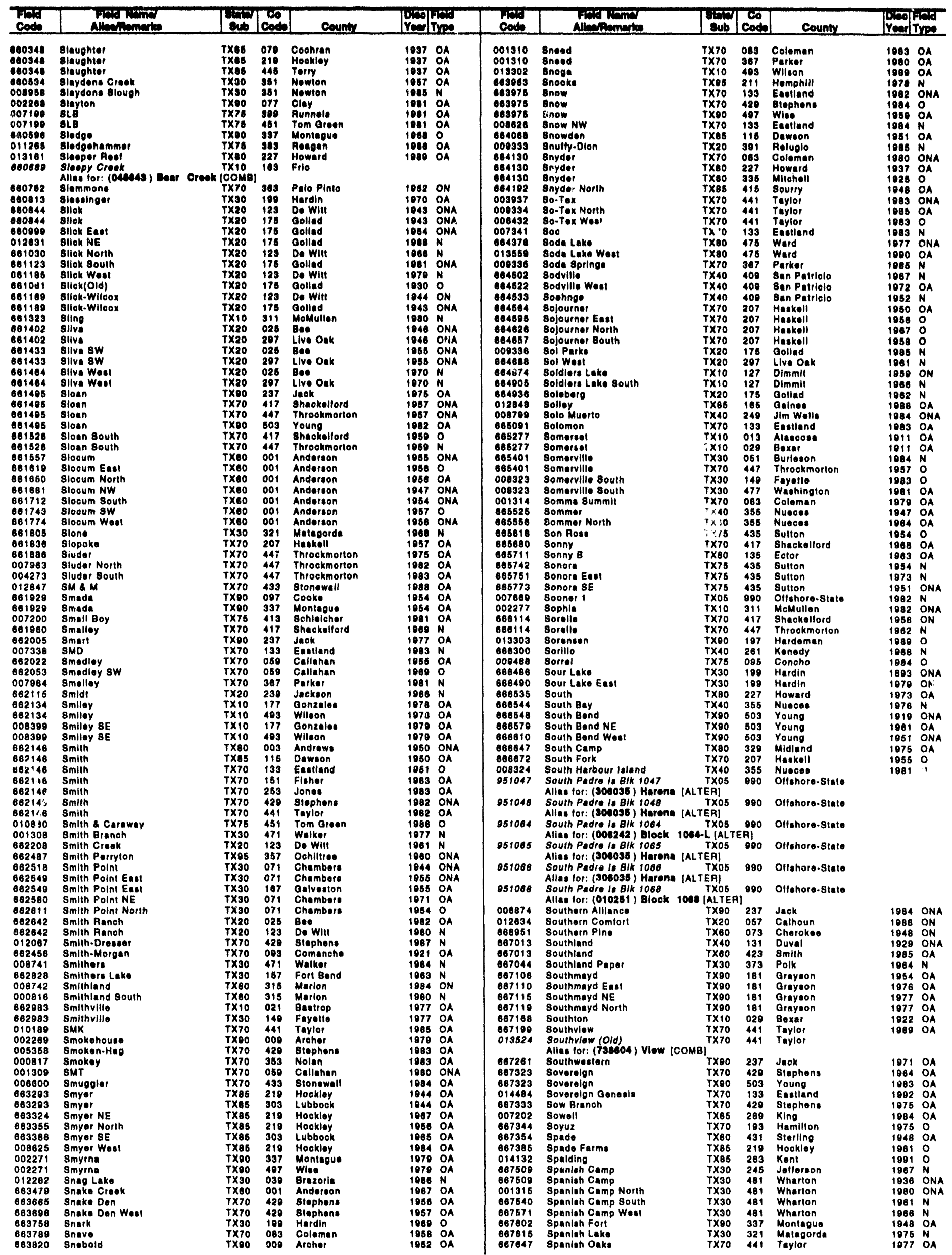


TEXAS

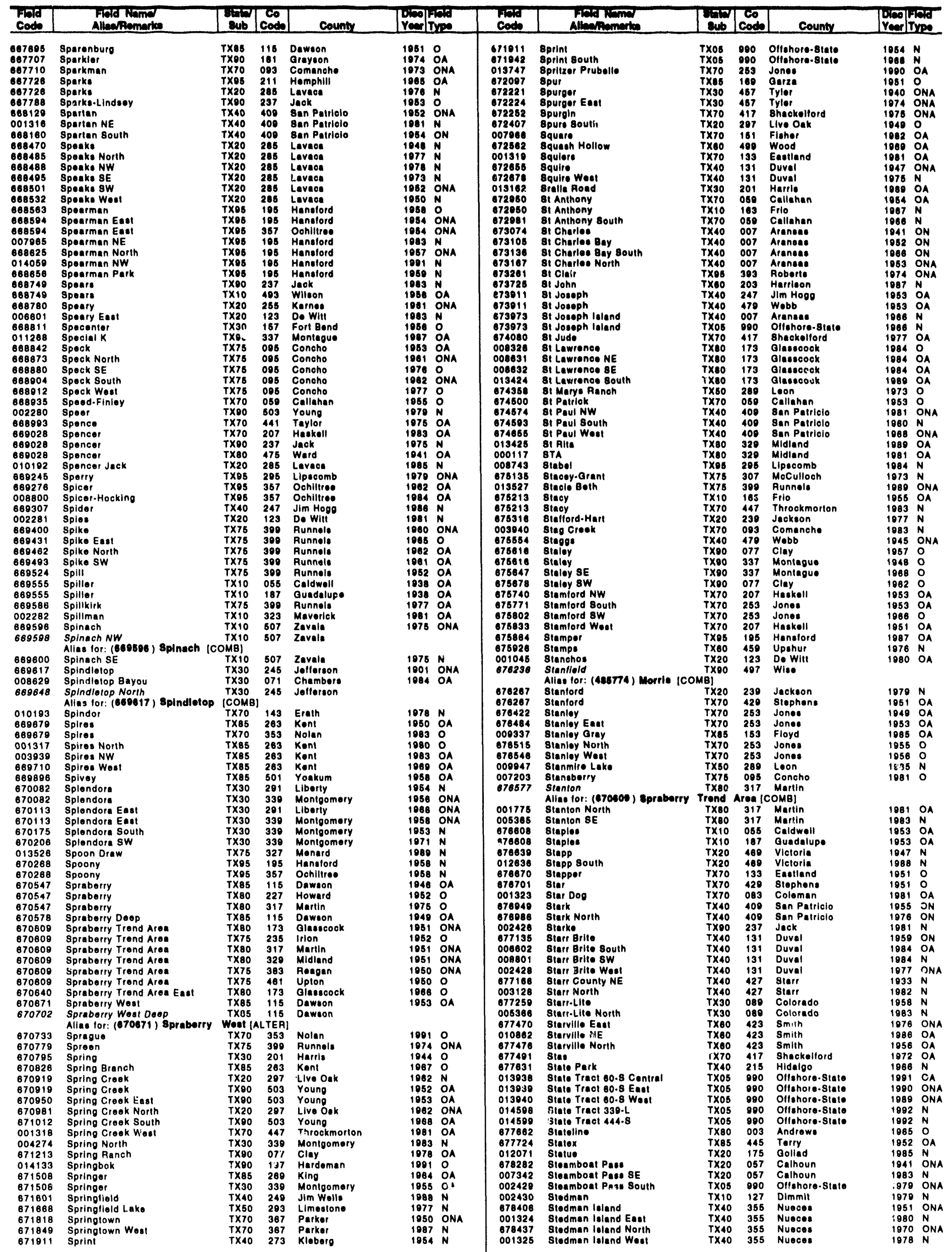




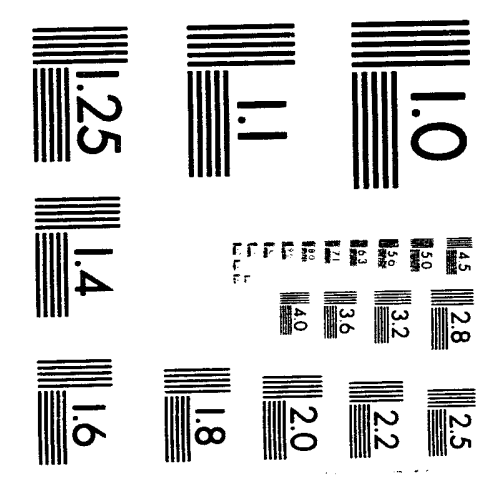



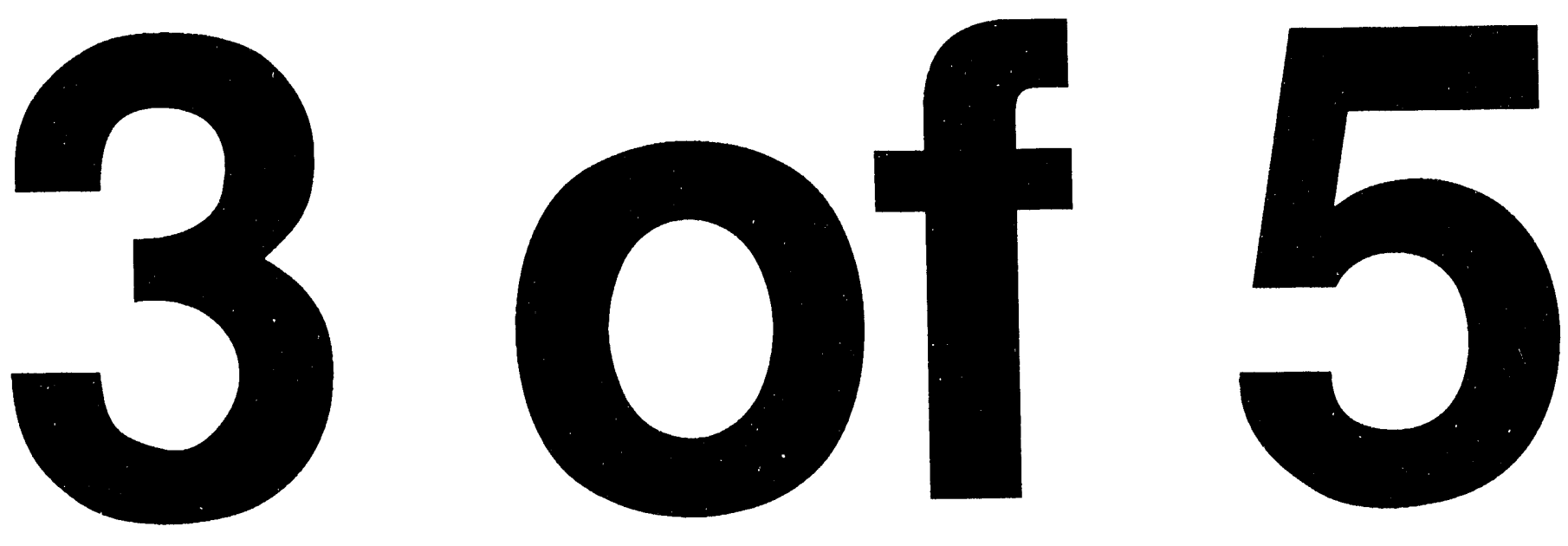
OKLAHOMA

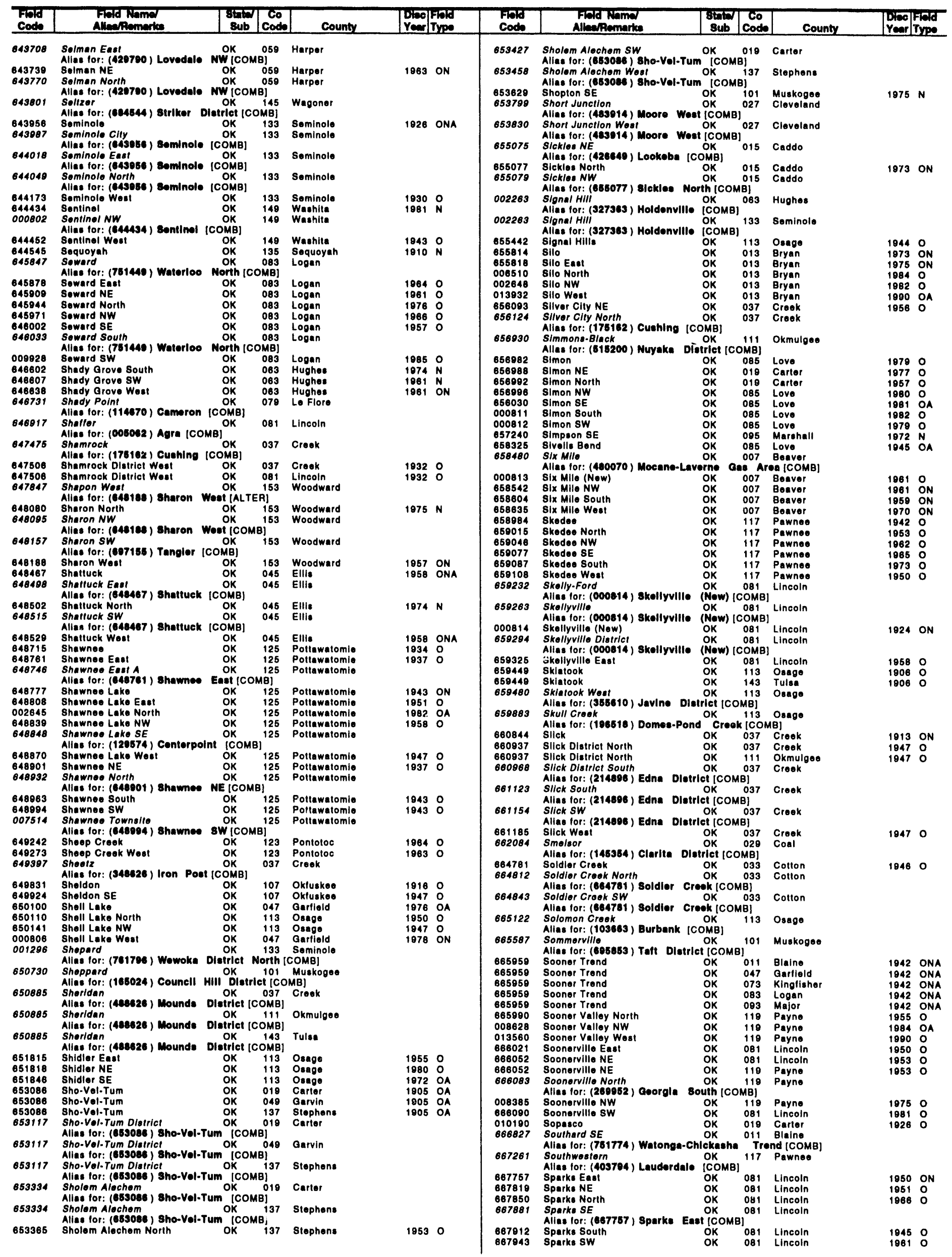




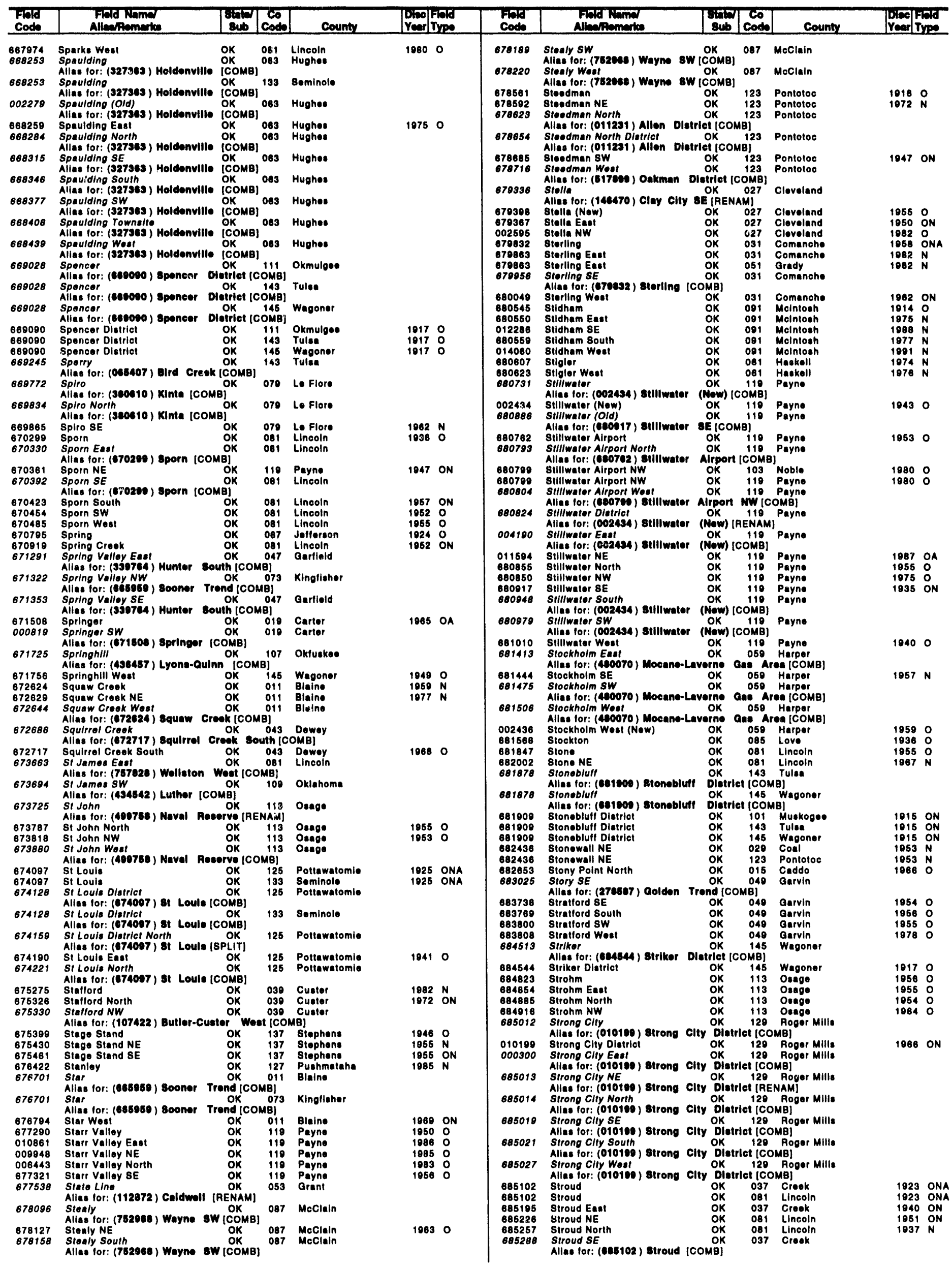


OKLAHOMA

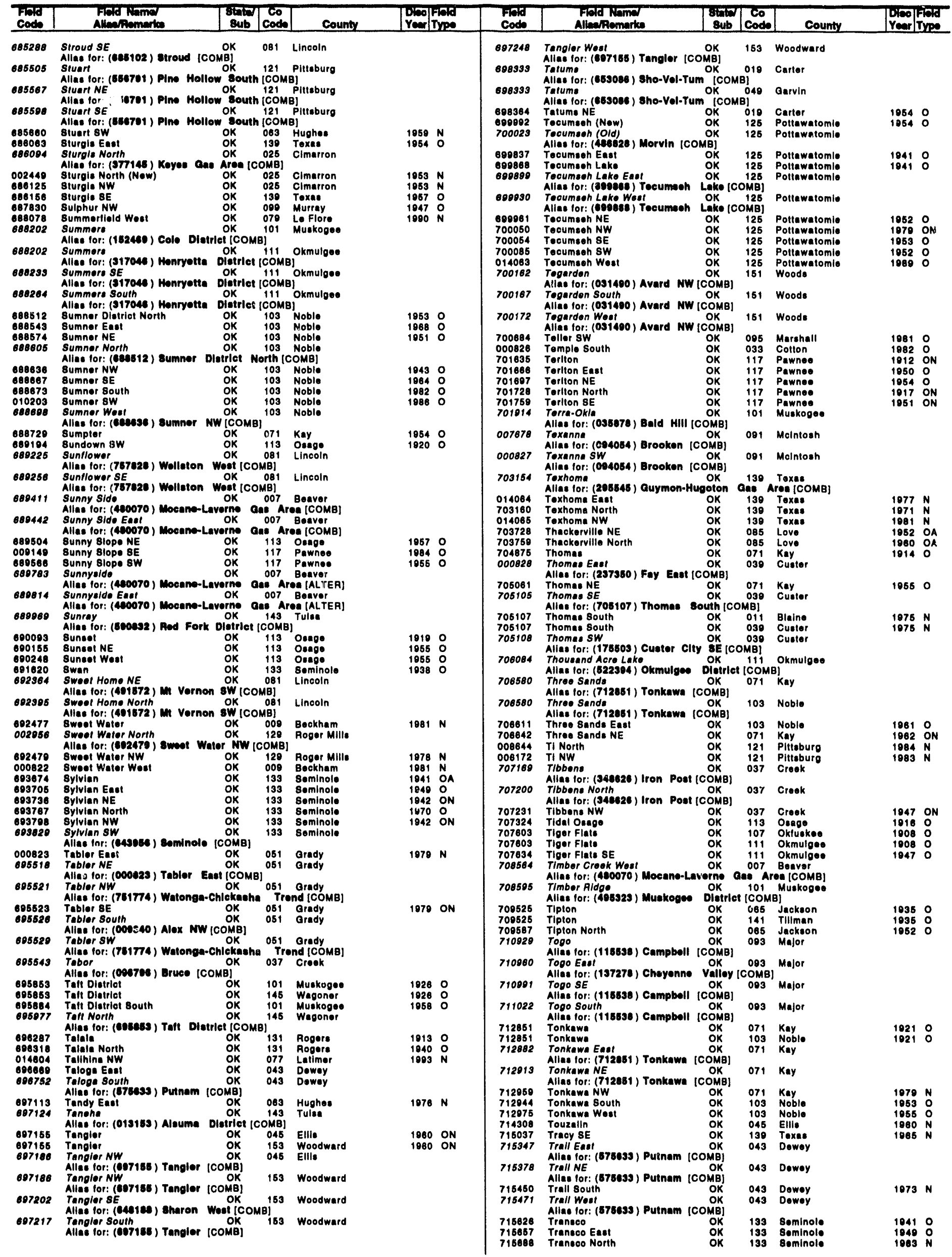




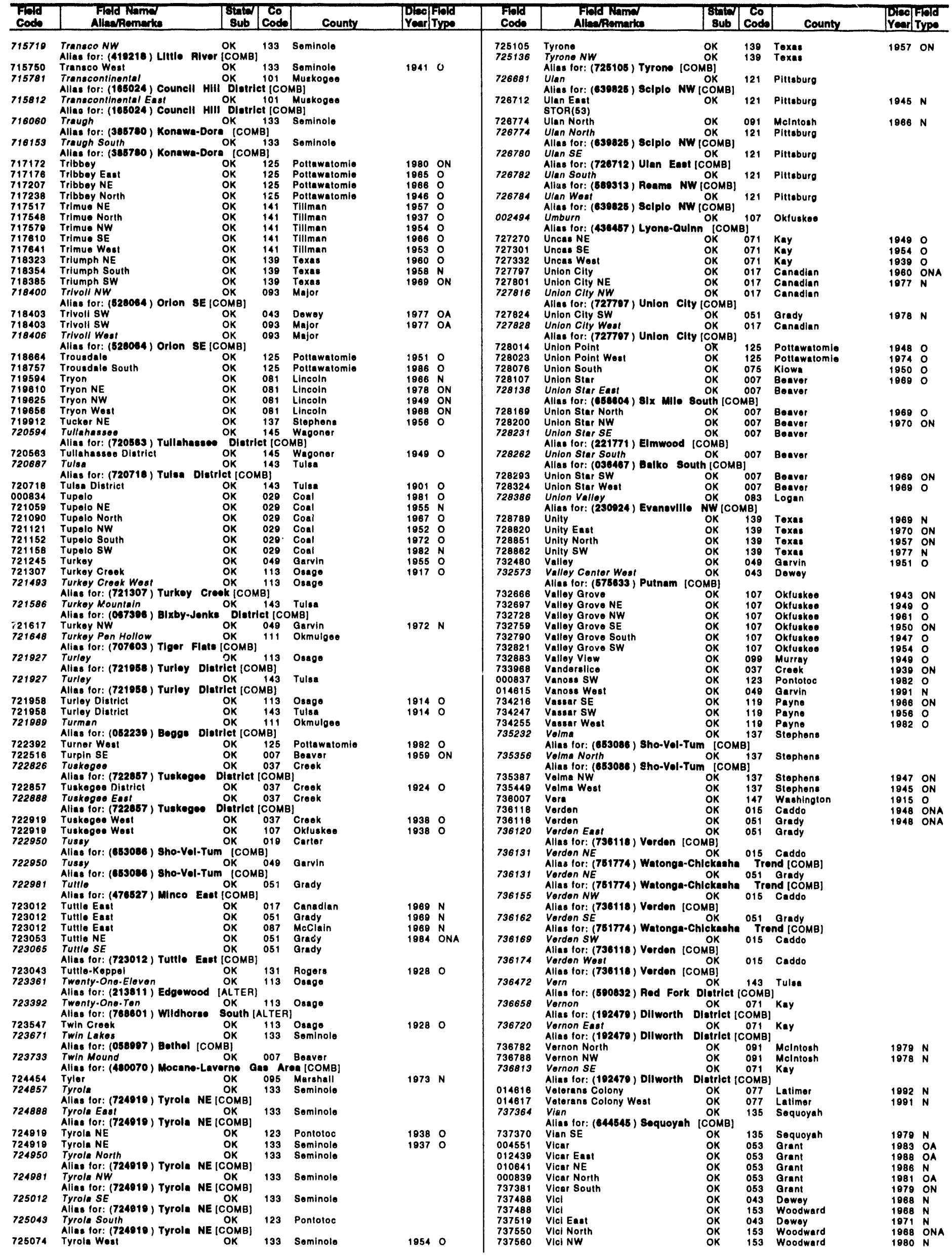


OKLAHOMA

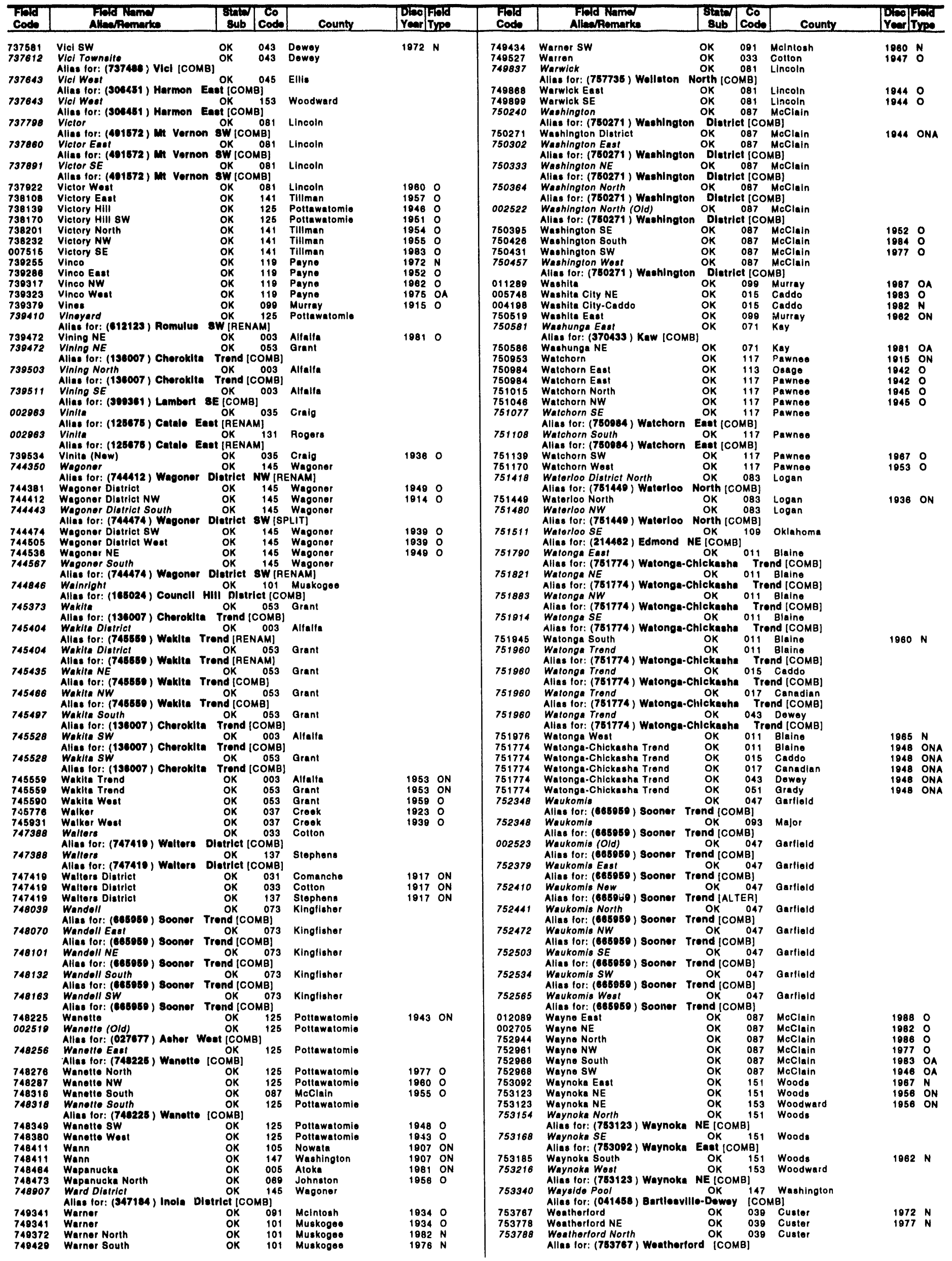




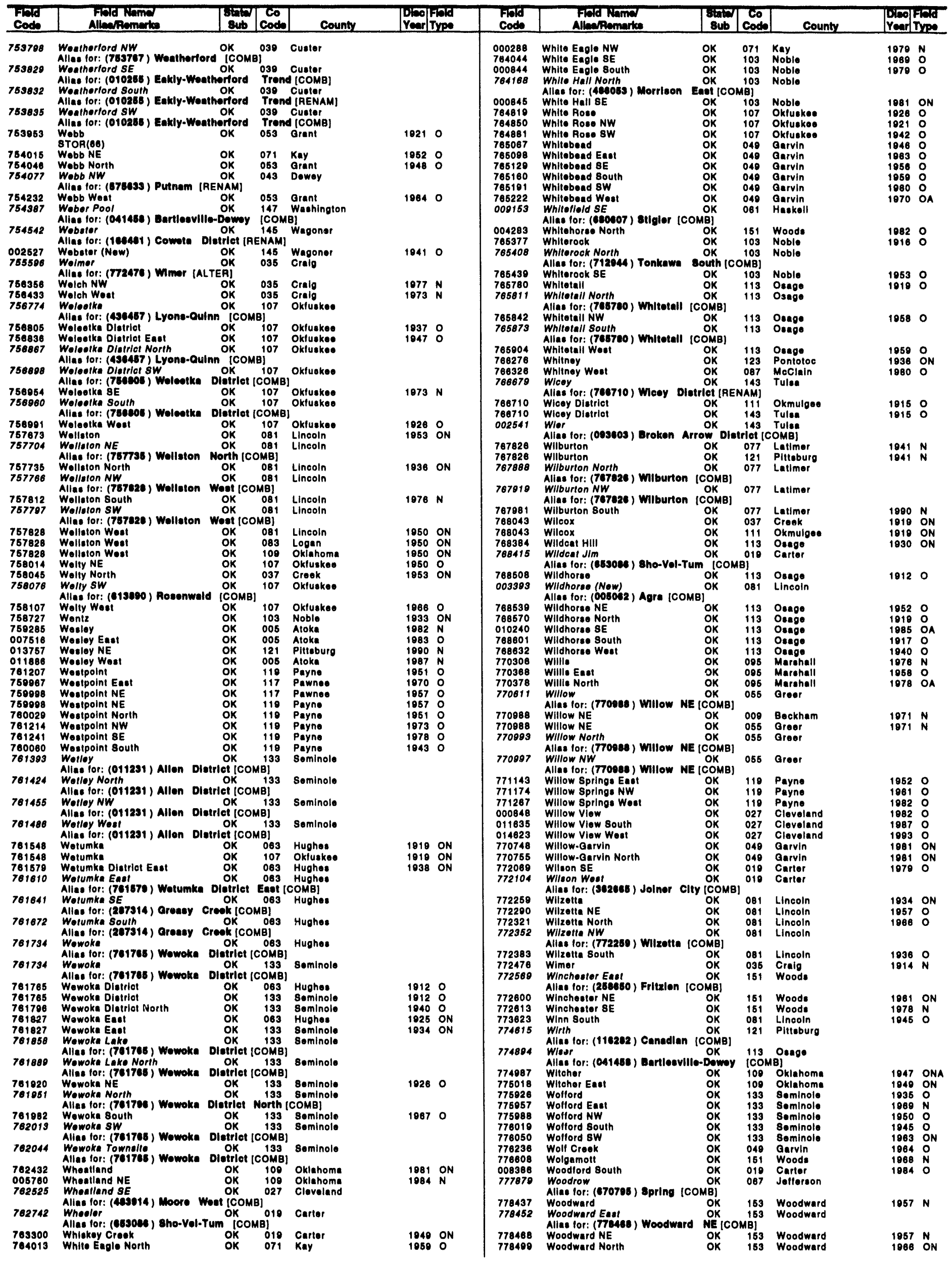


PENNSYLVANIA

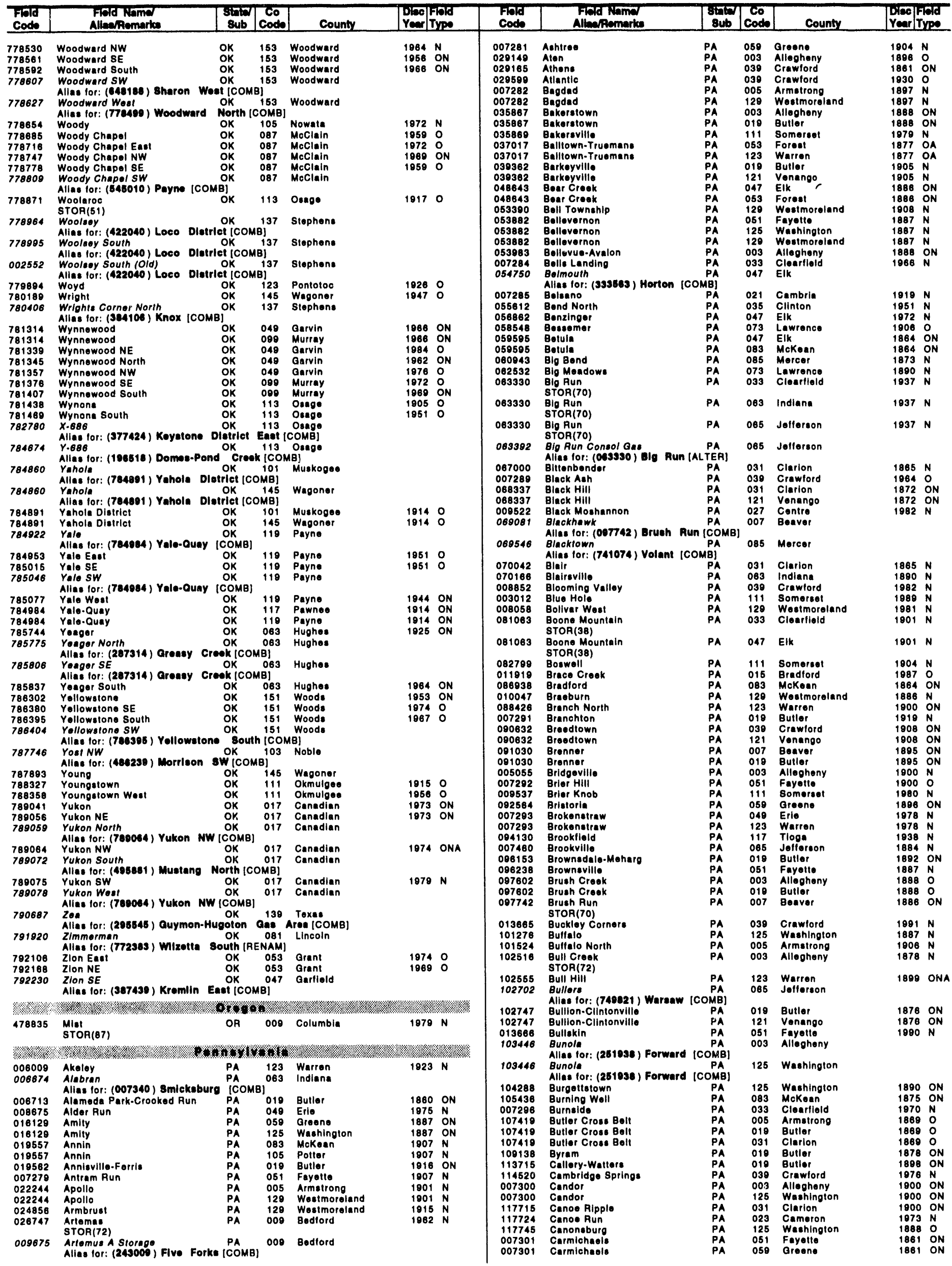




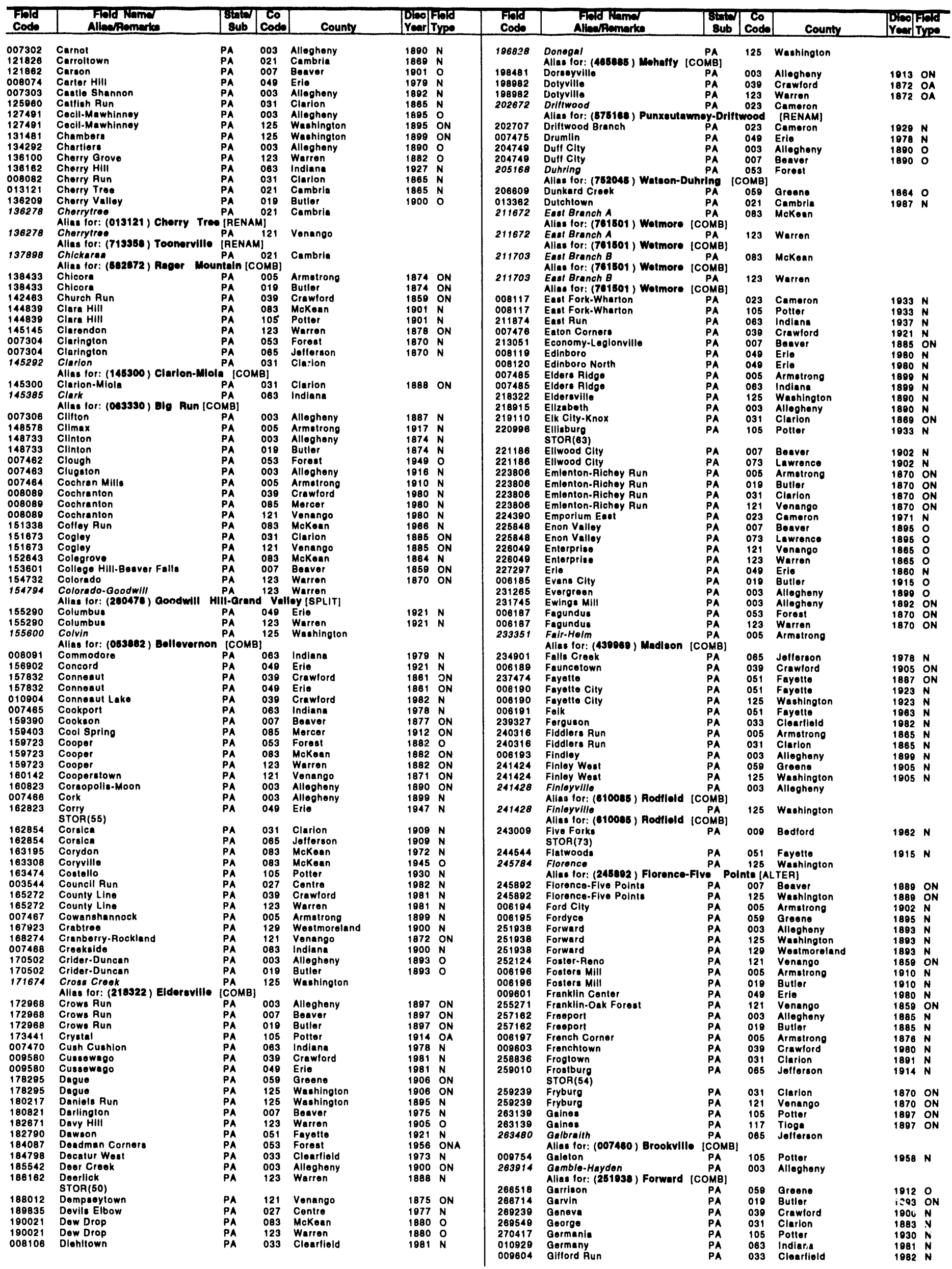


PENNSYLVANIA

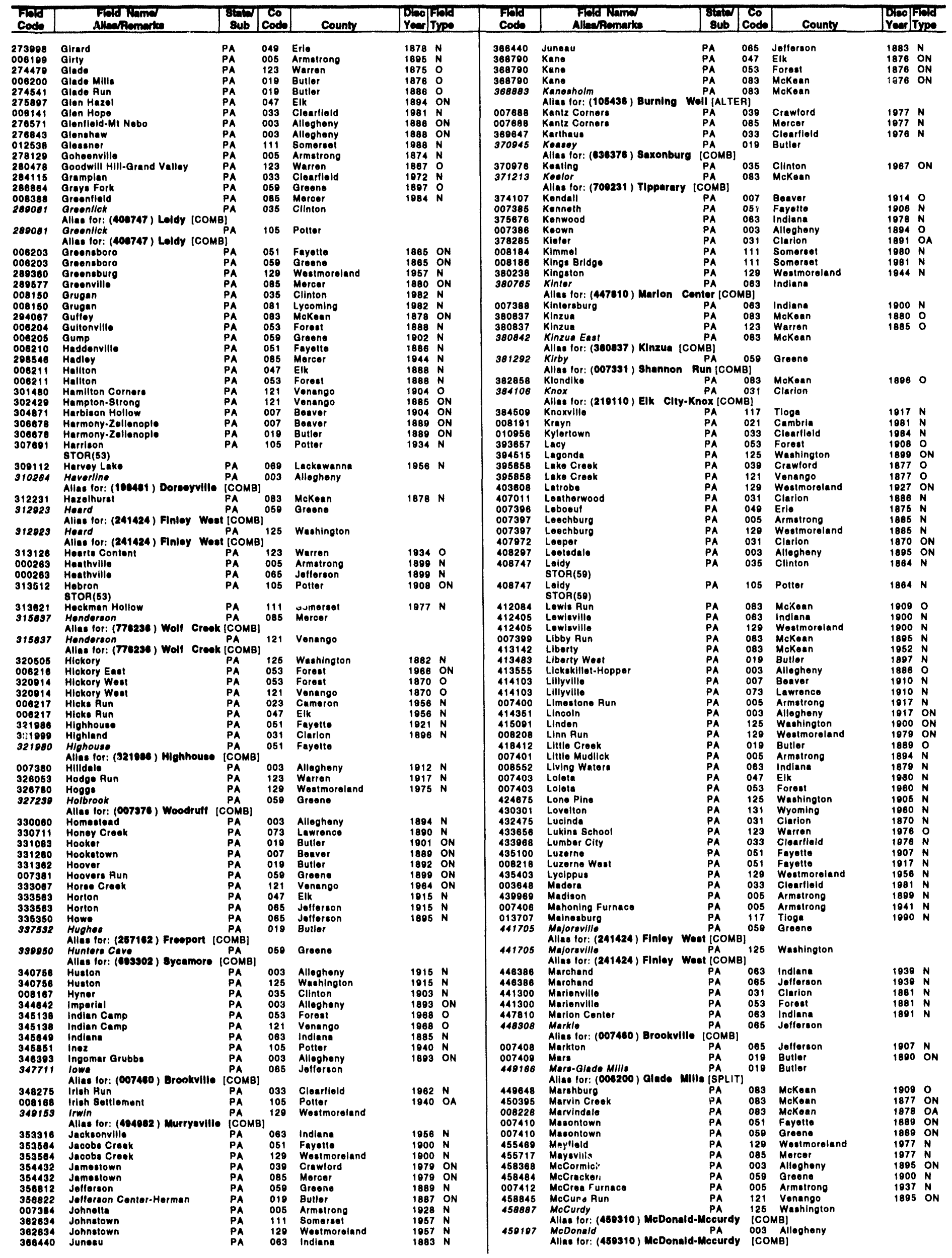


PENNSYLVANIA

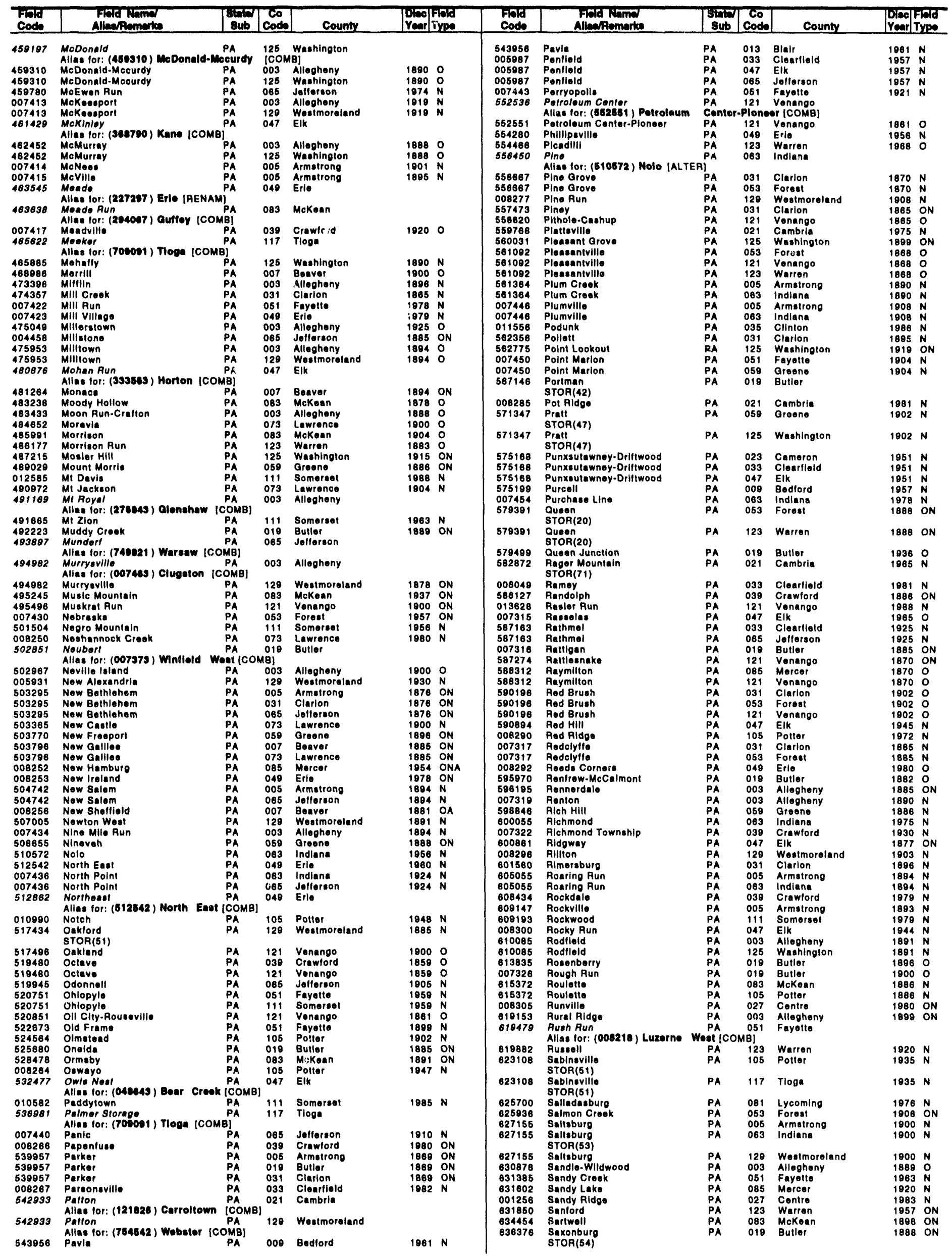


SOUTH DAKOTA

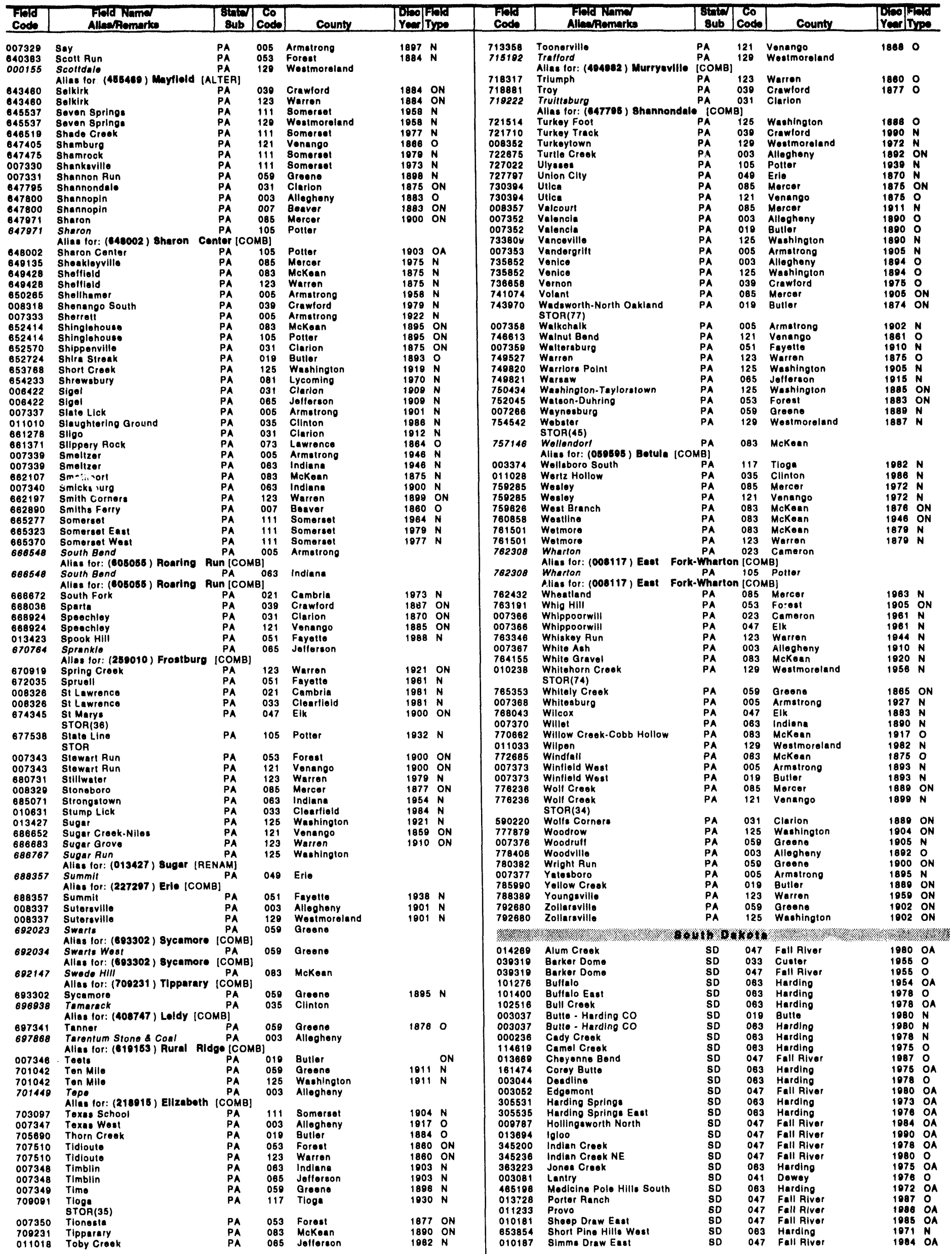




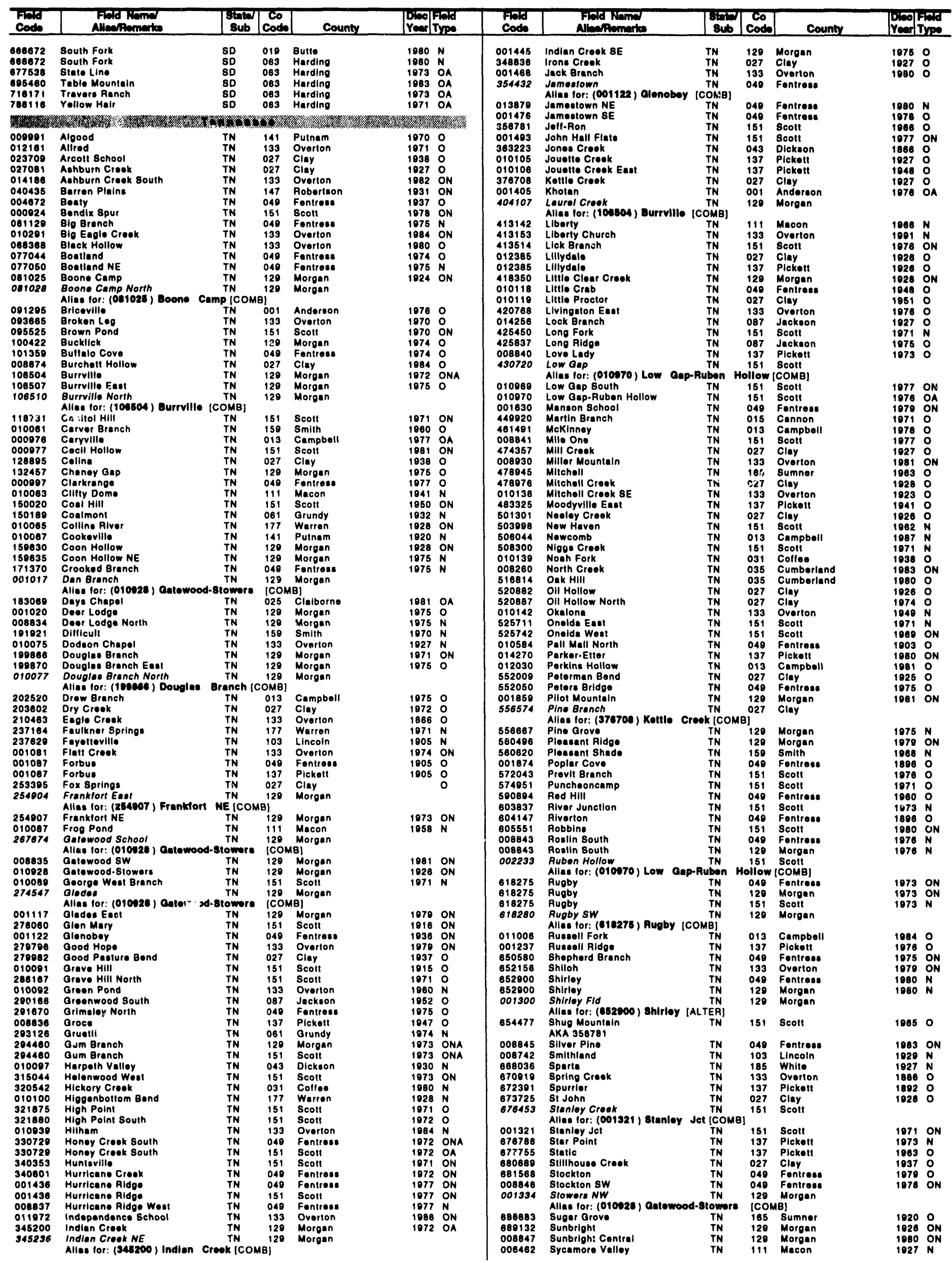


TEXAS

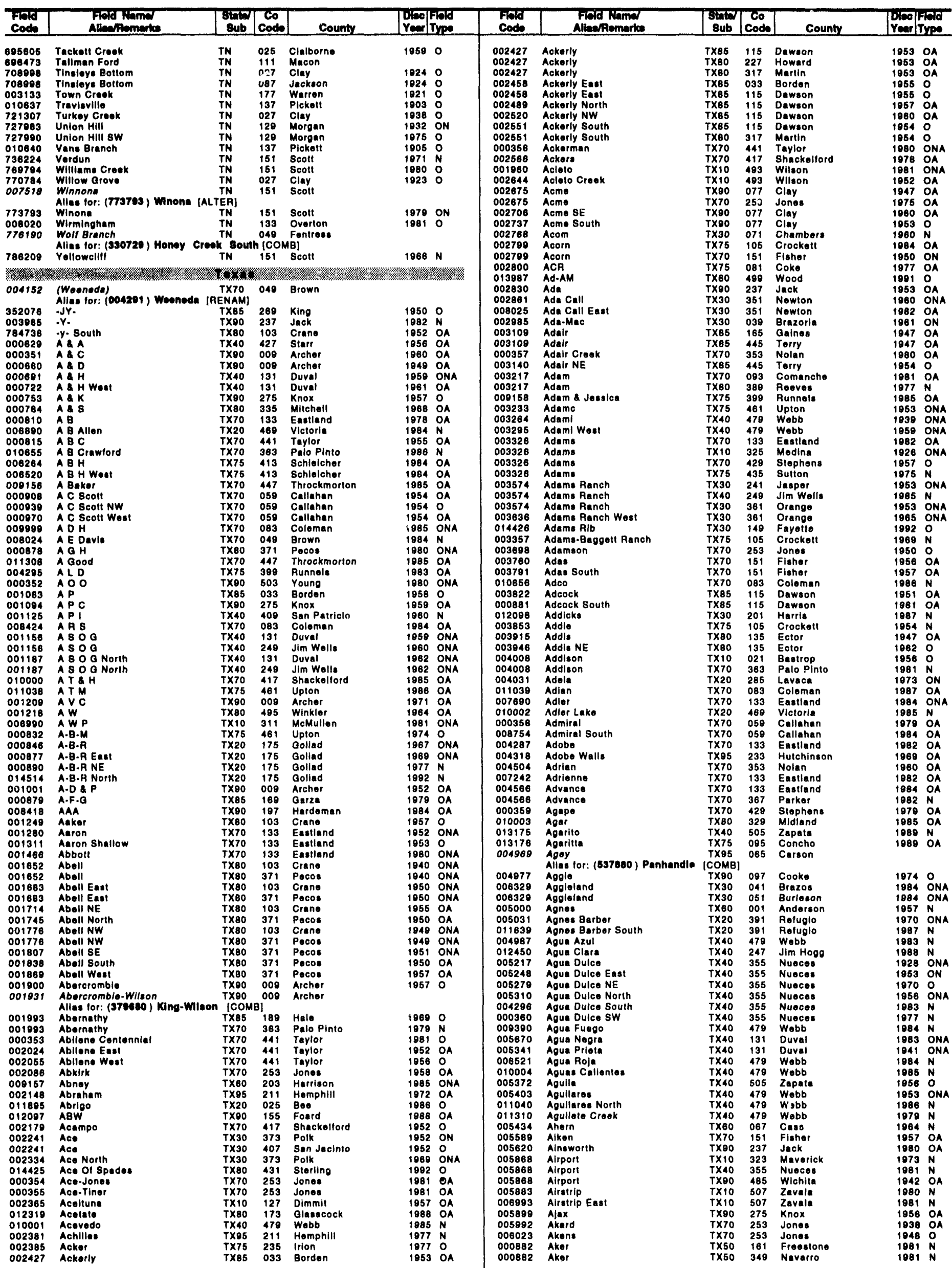


TEXAS

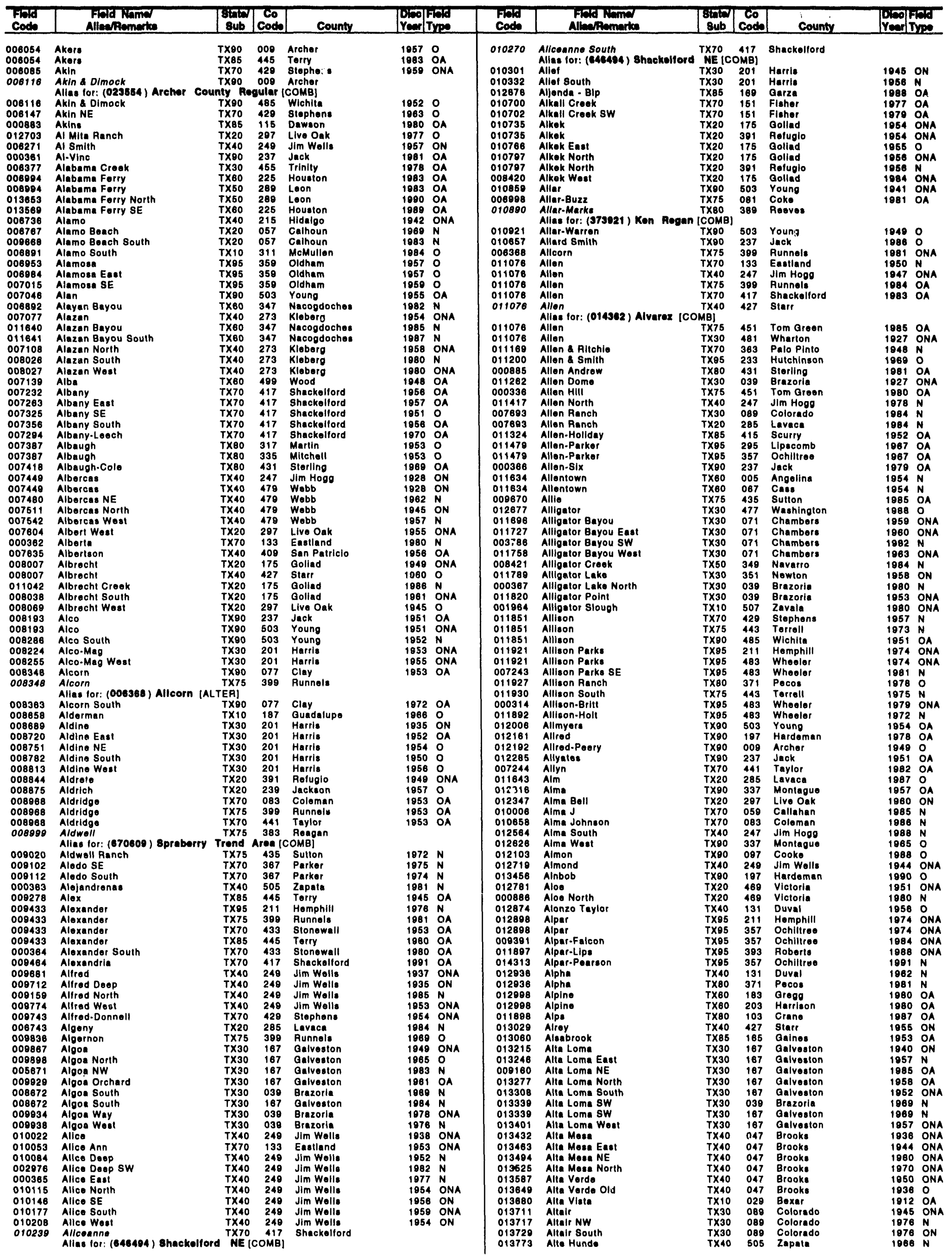


TEXAS

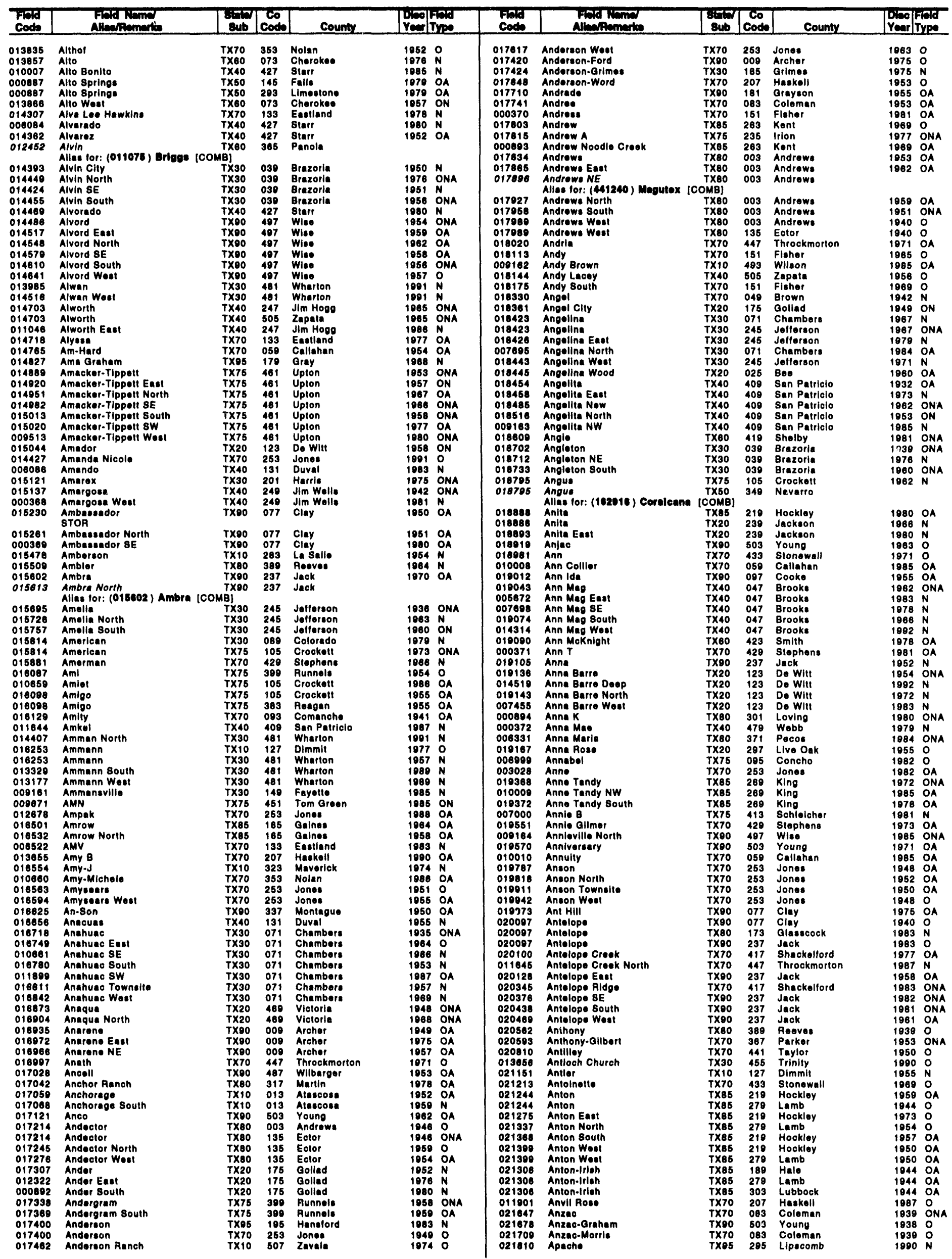


TEXAS

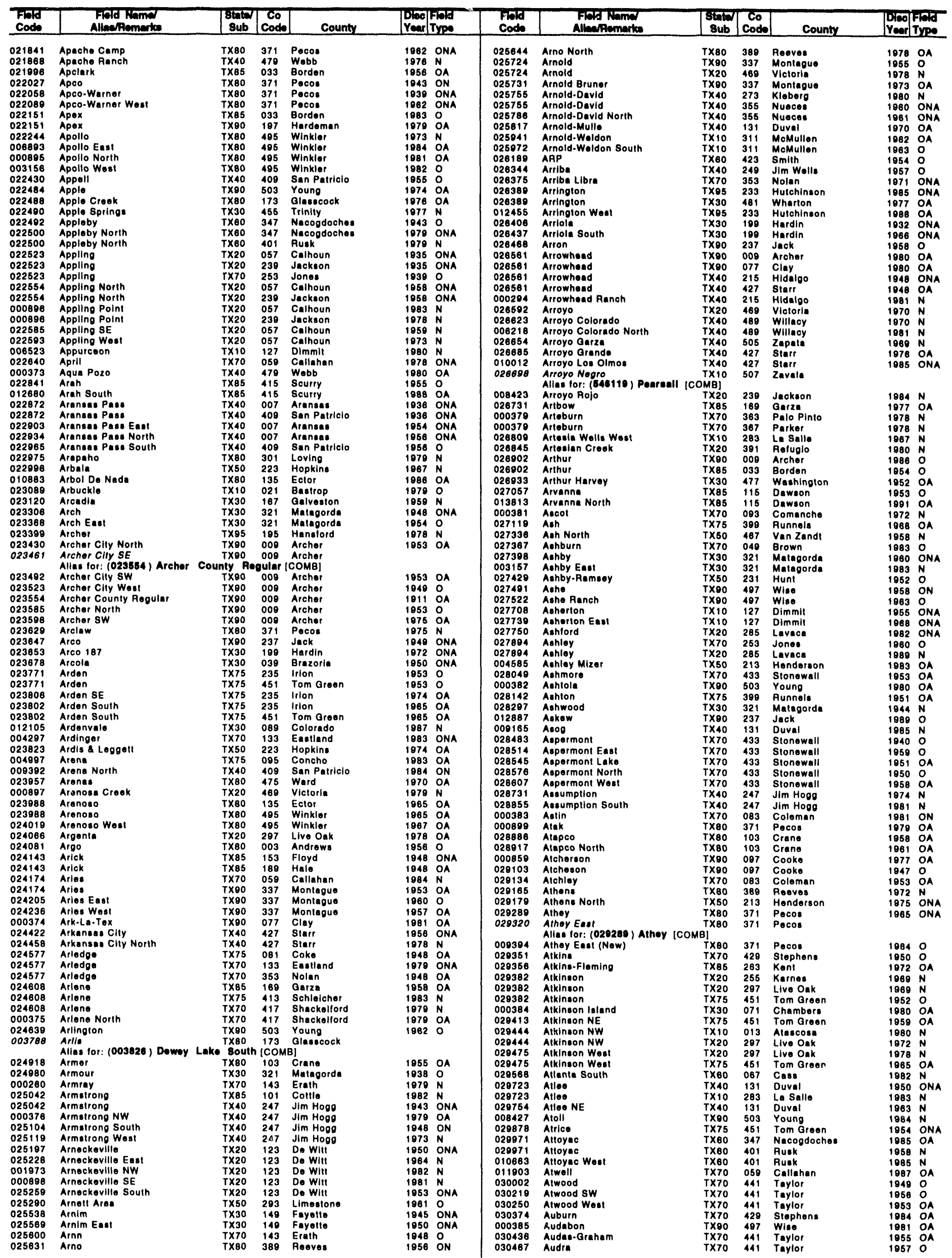


TEXAS

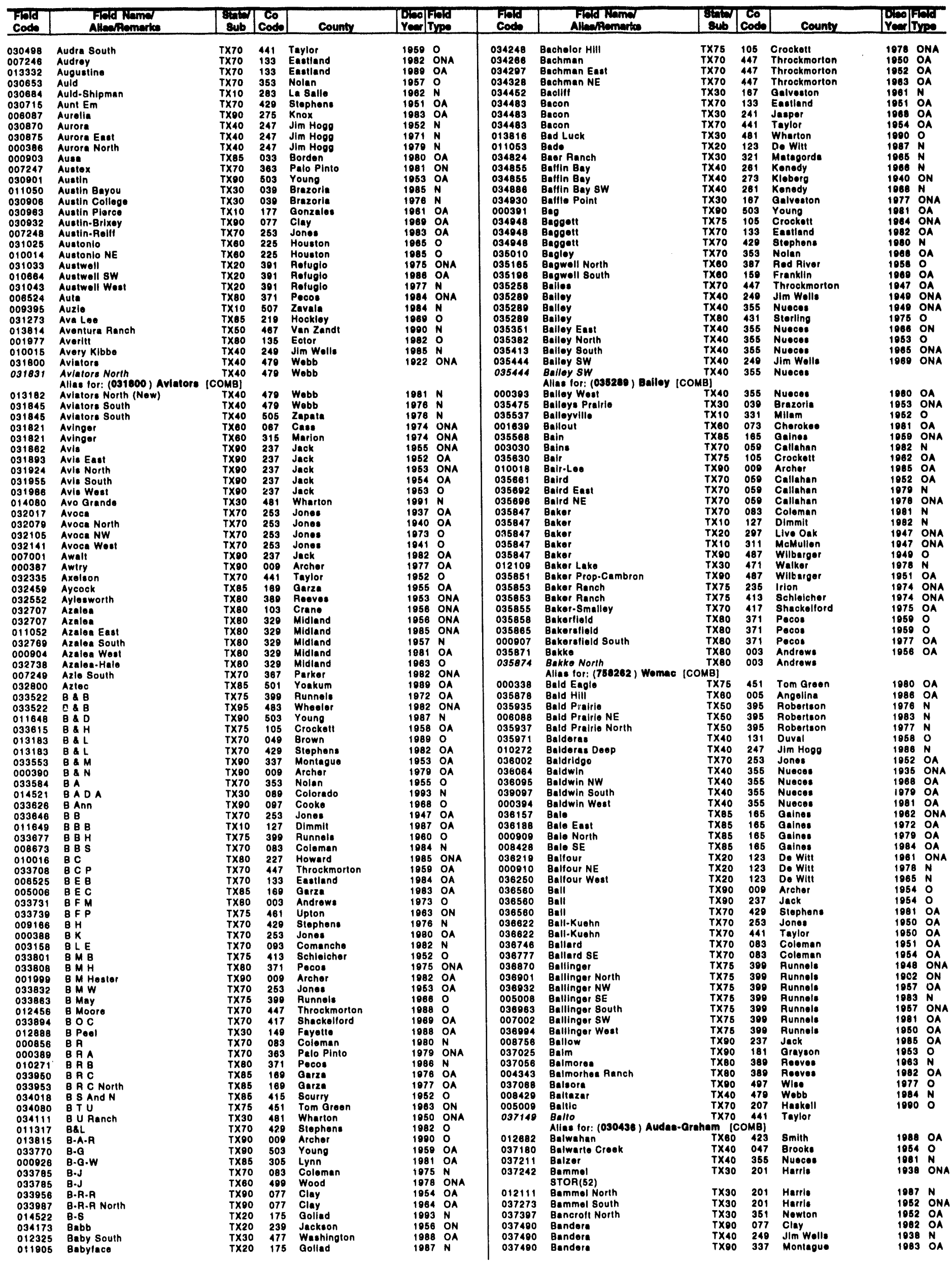




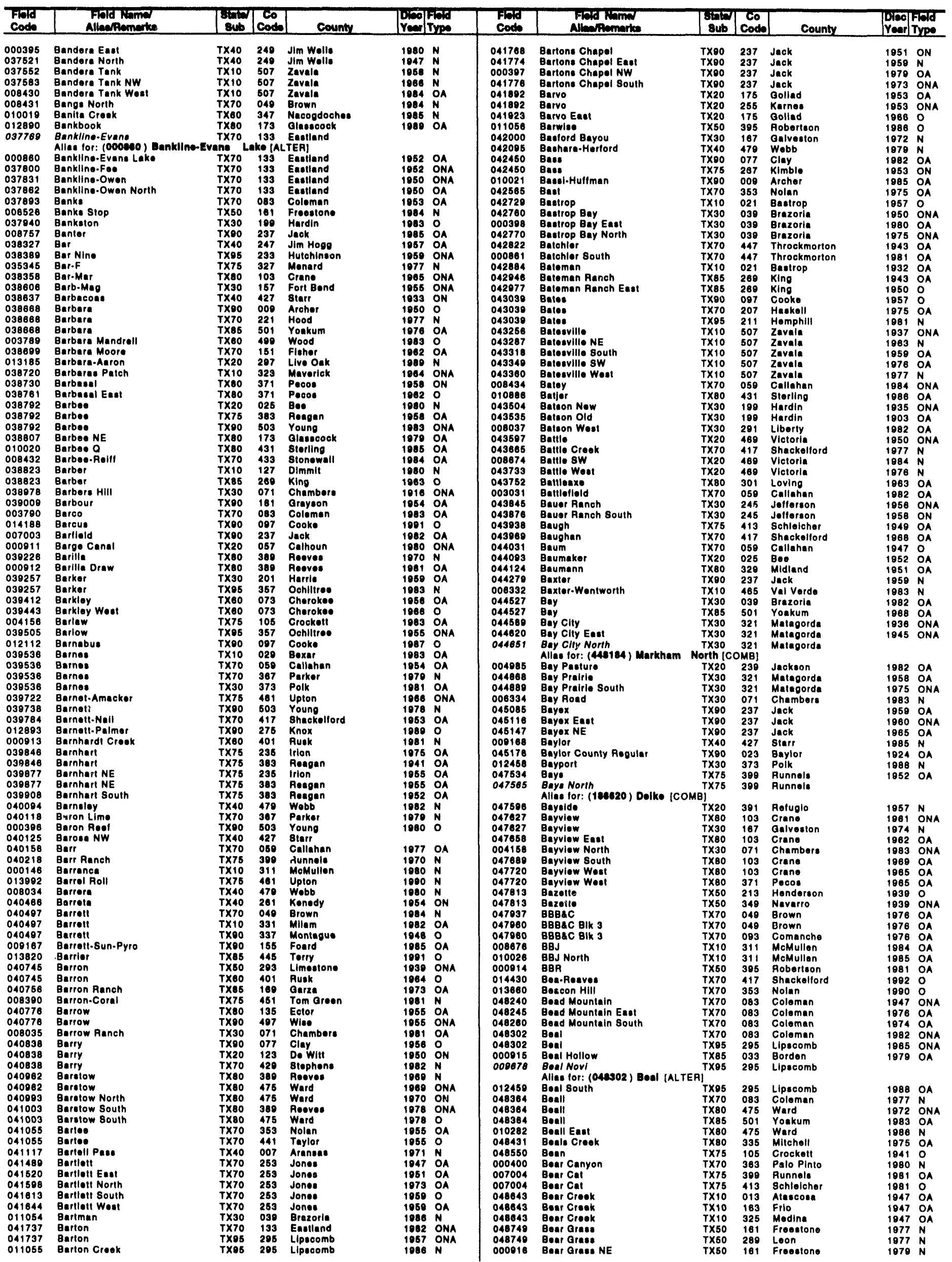


TEXAS

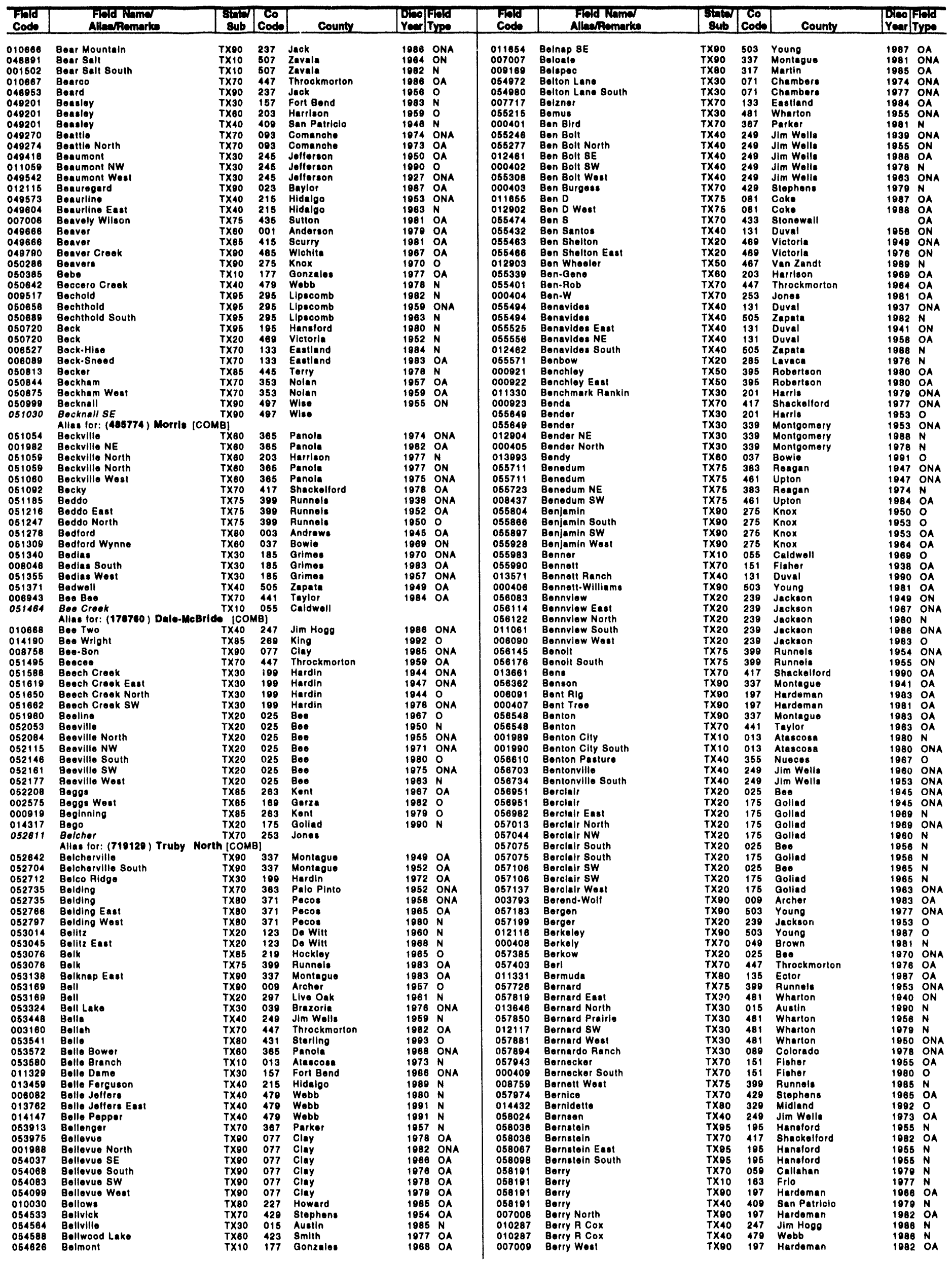


TEXAS

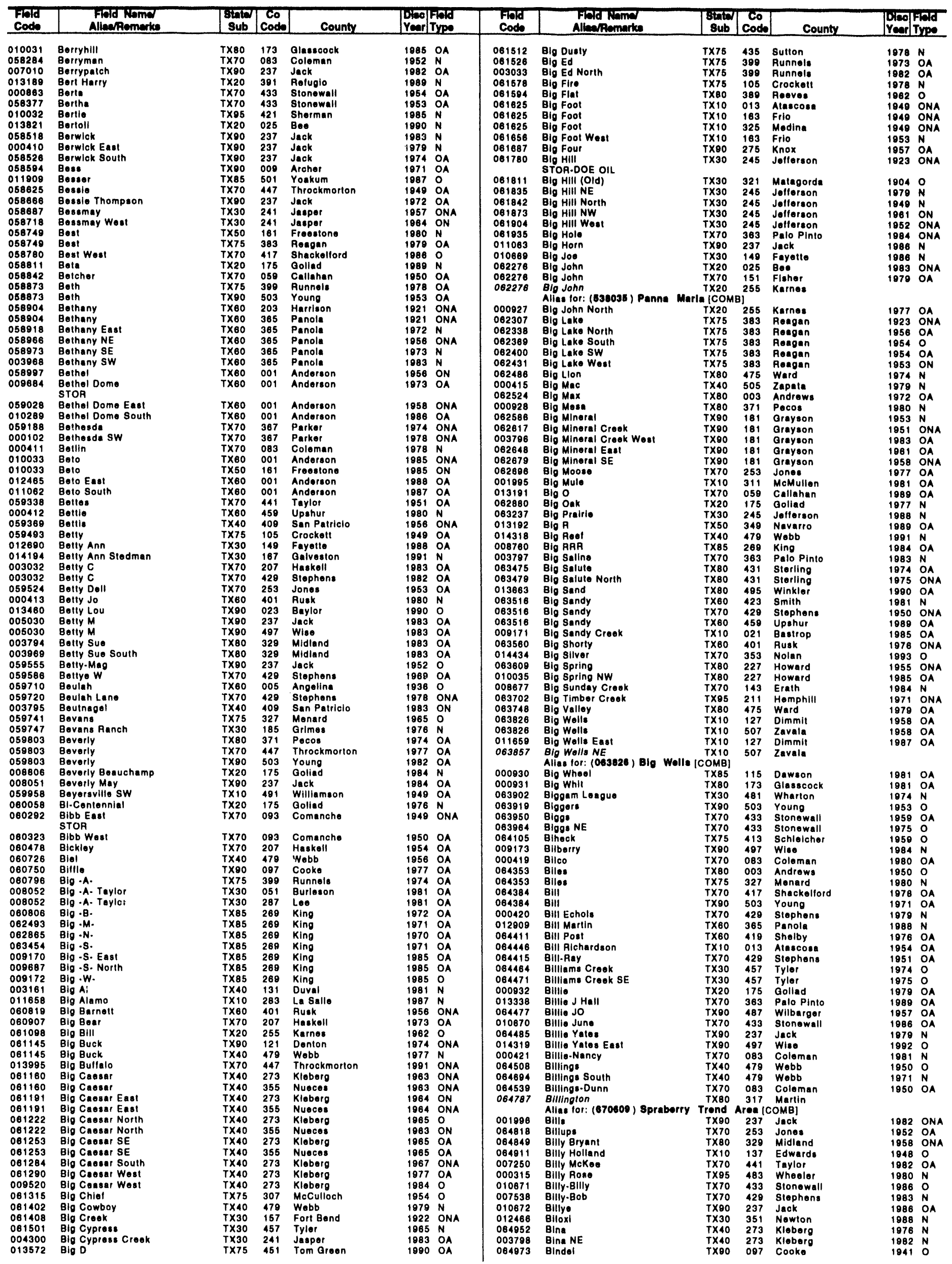


TEXAS

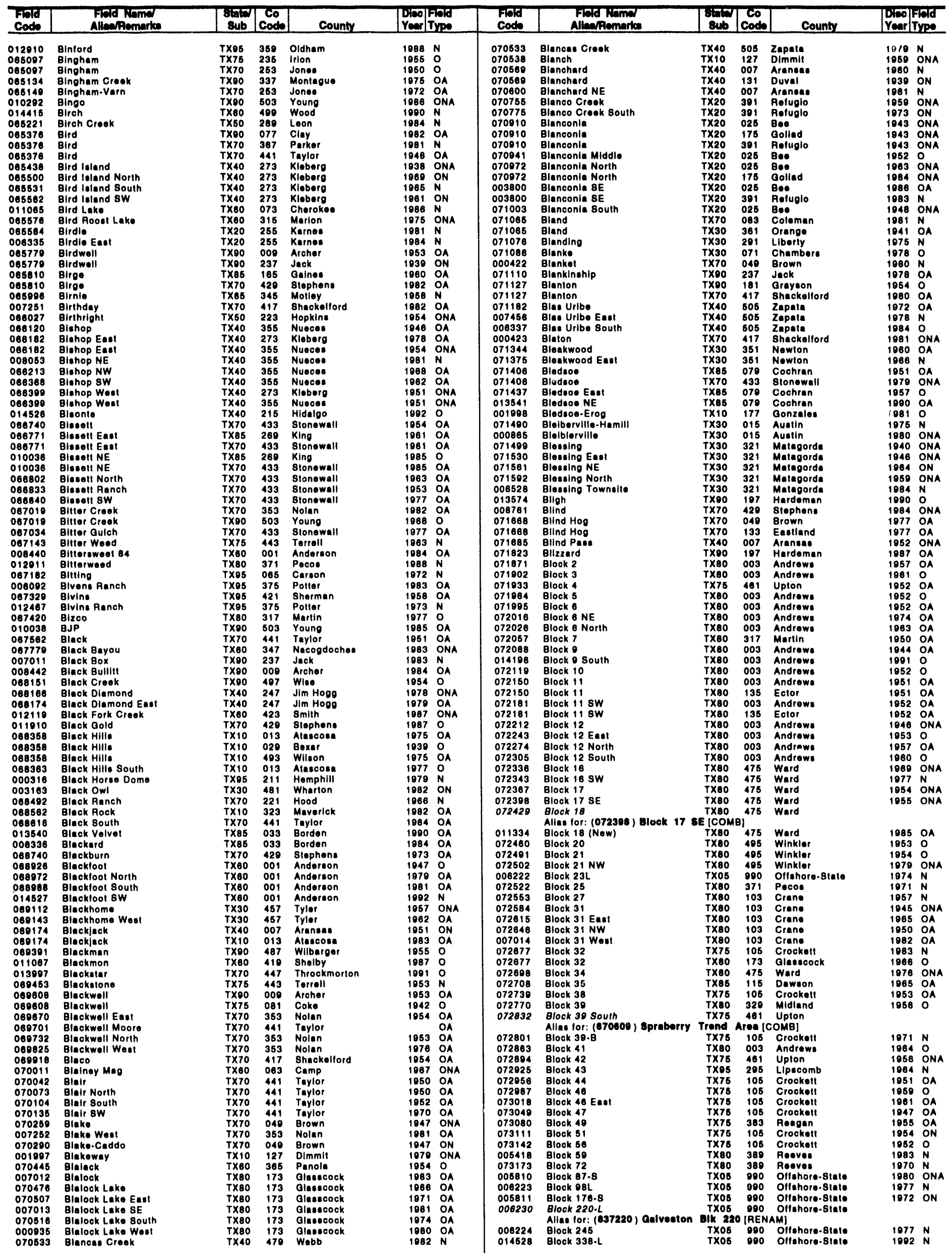


TEXAS

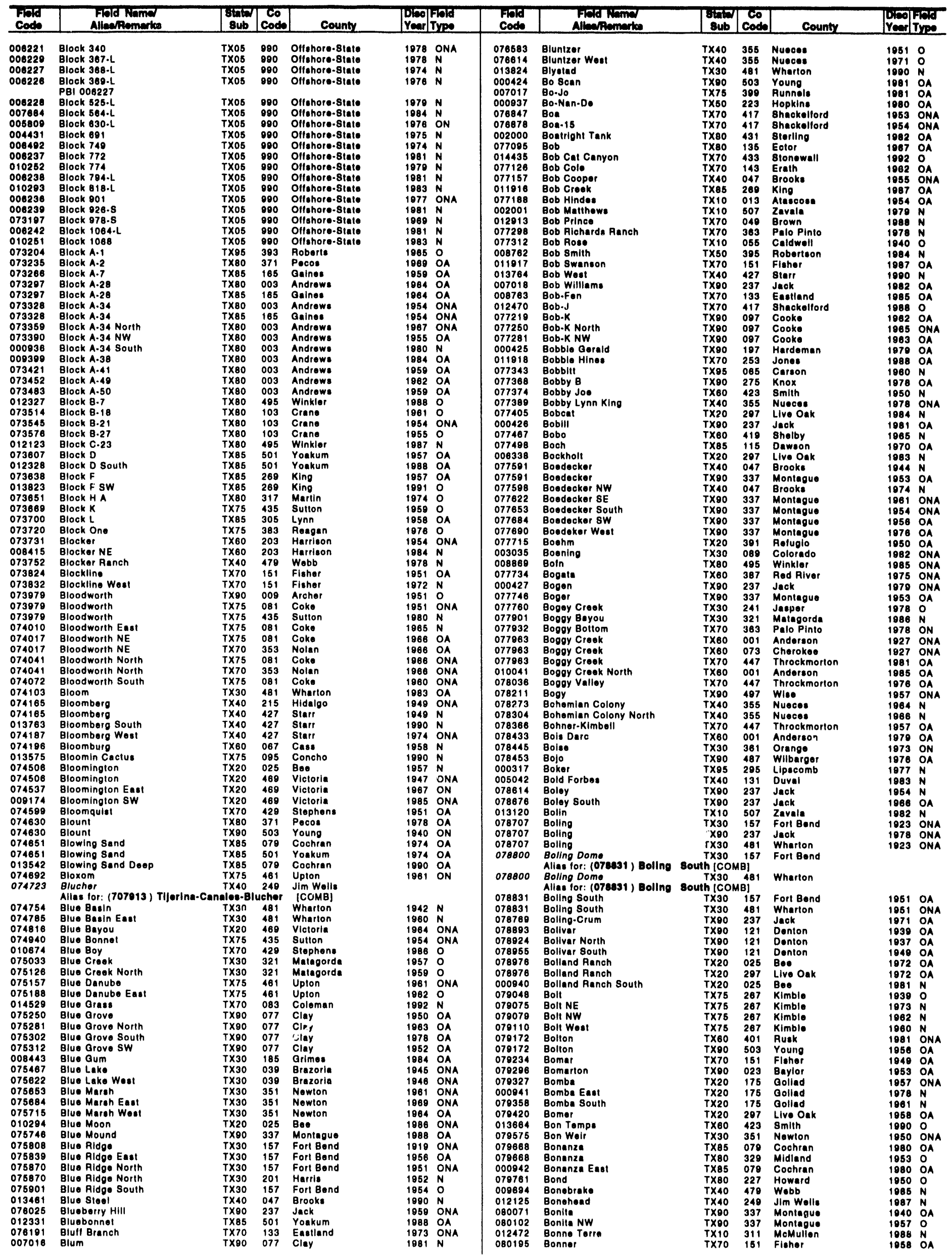


TEXAS

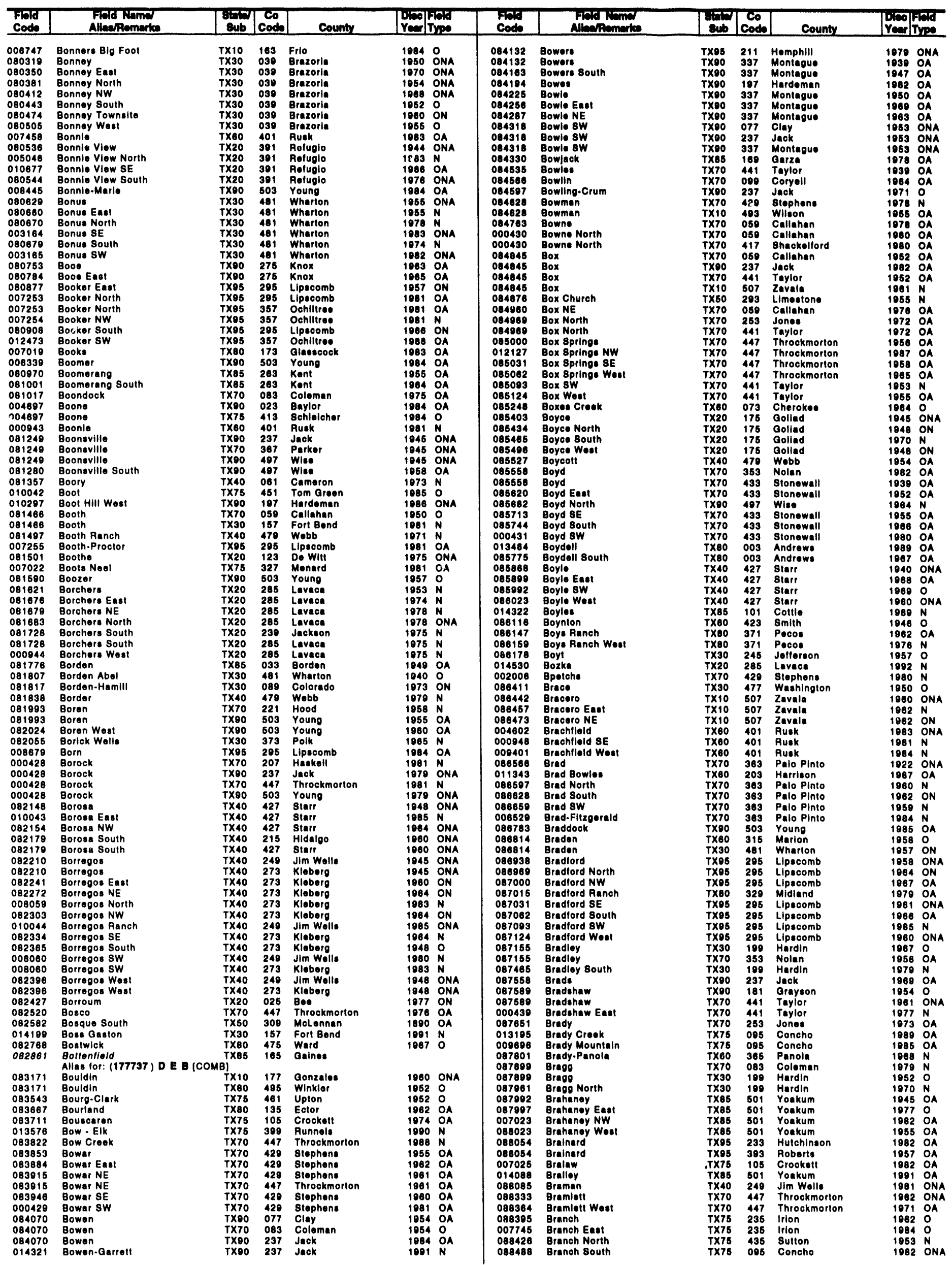


TEXAS

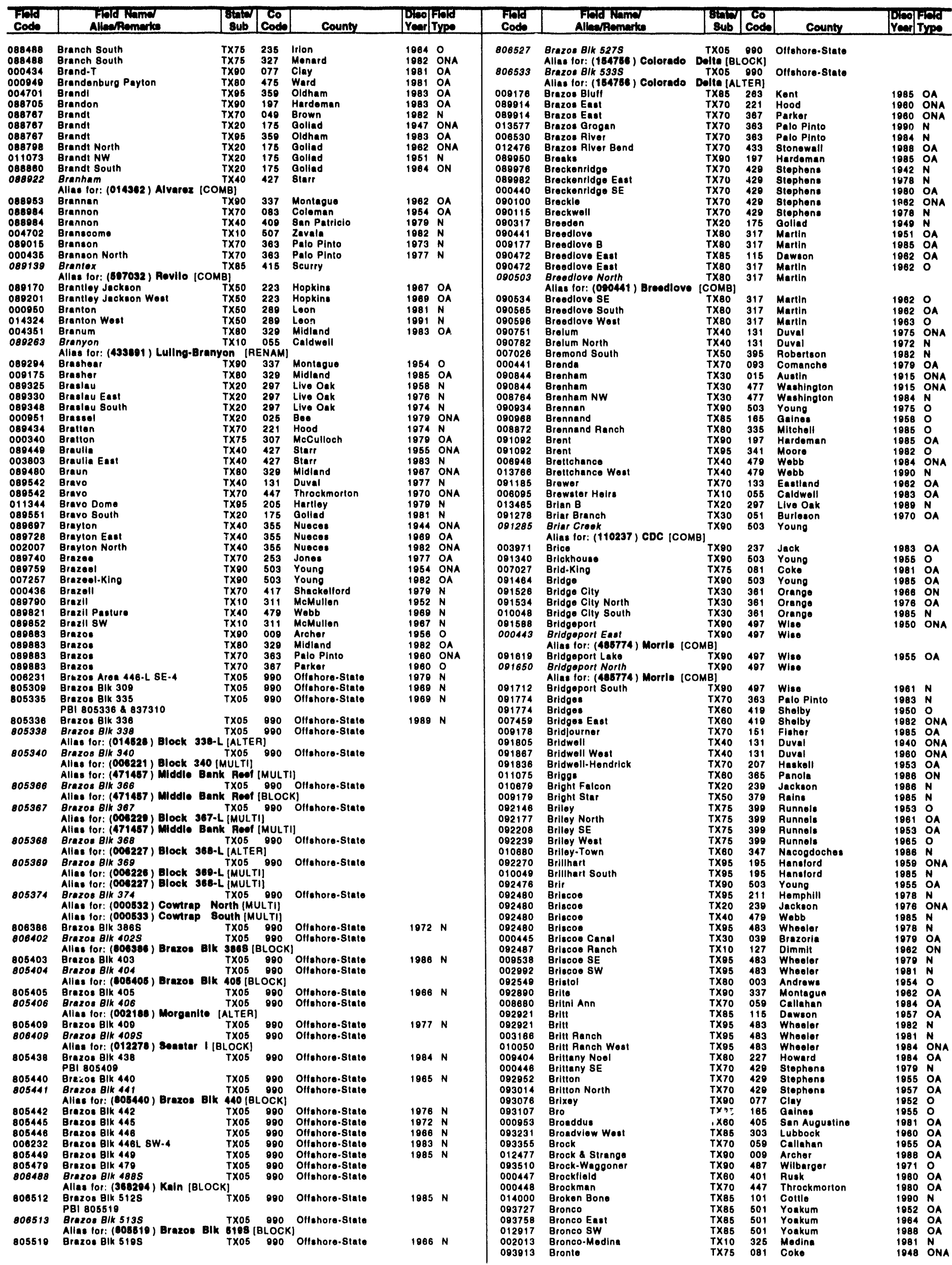


TEXAS

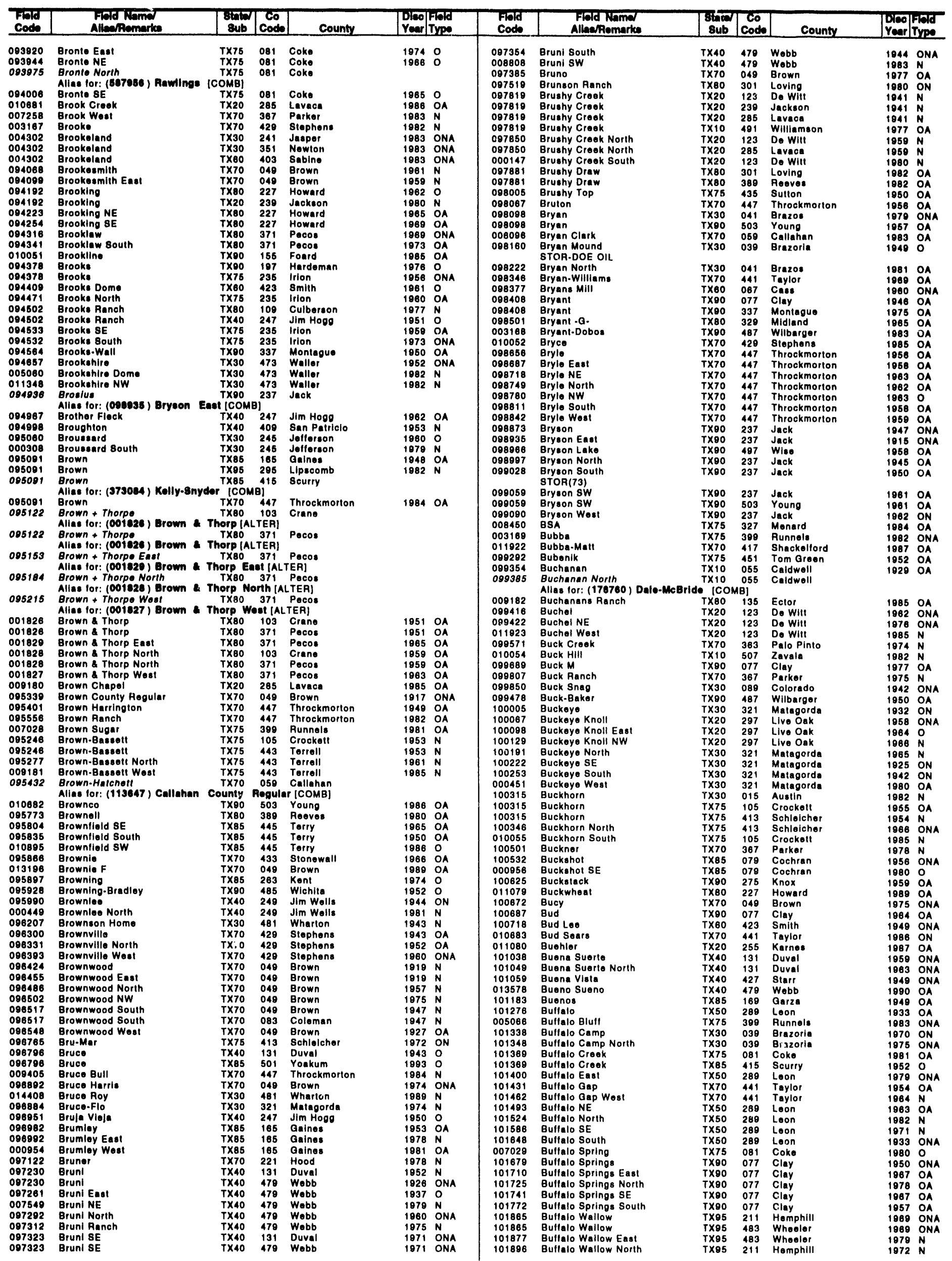


TEXAS

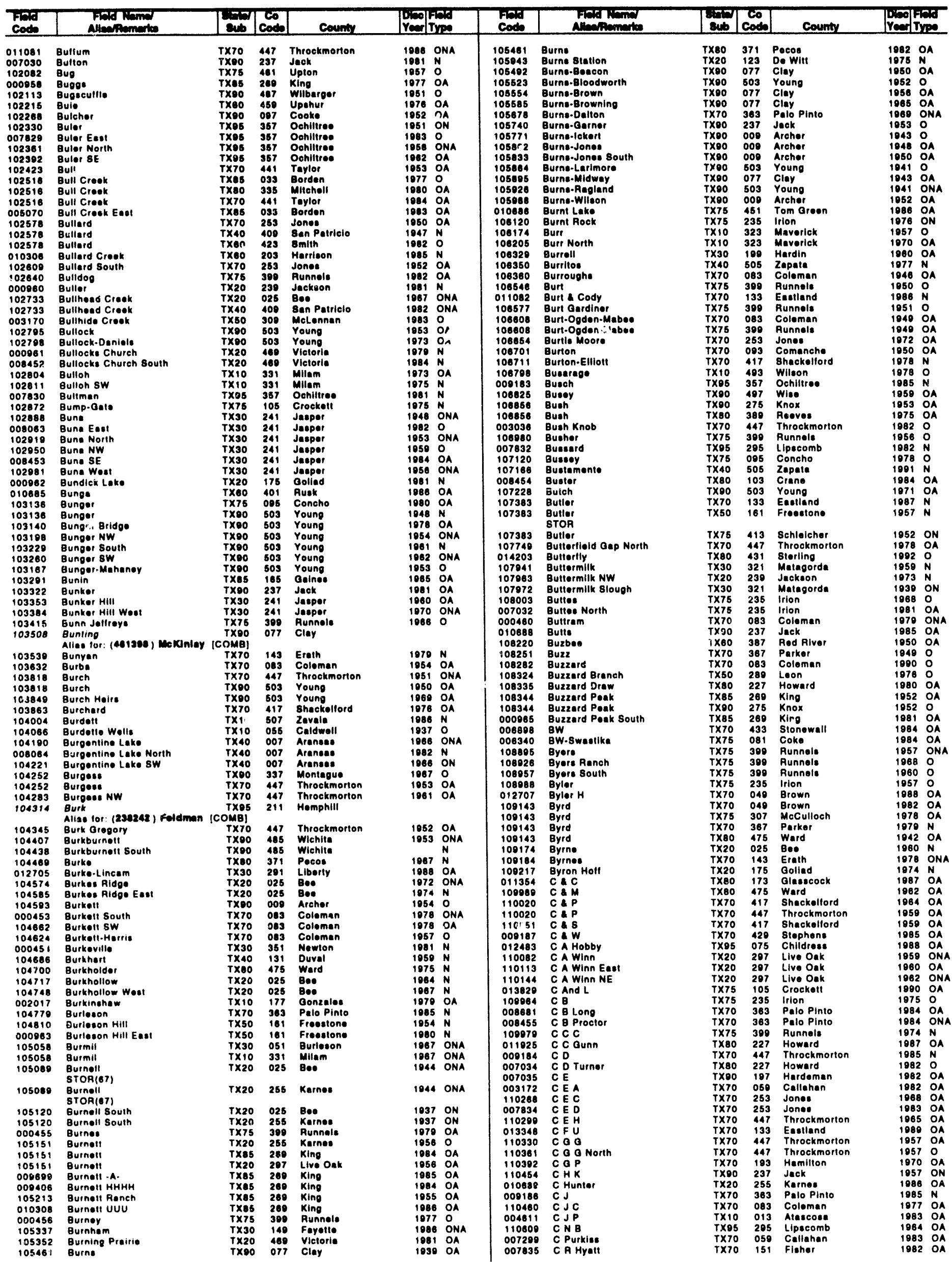


TEXAS

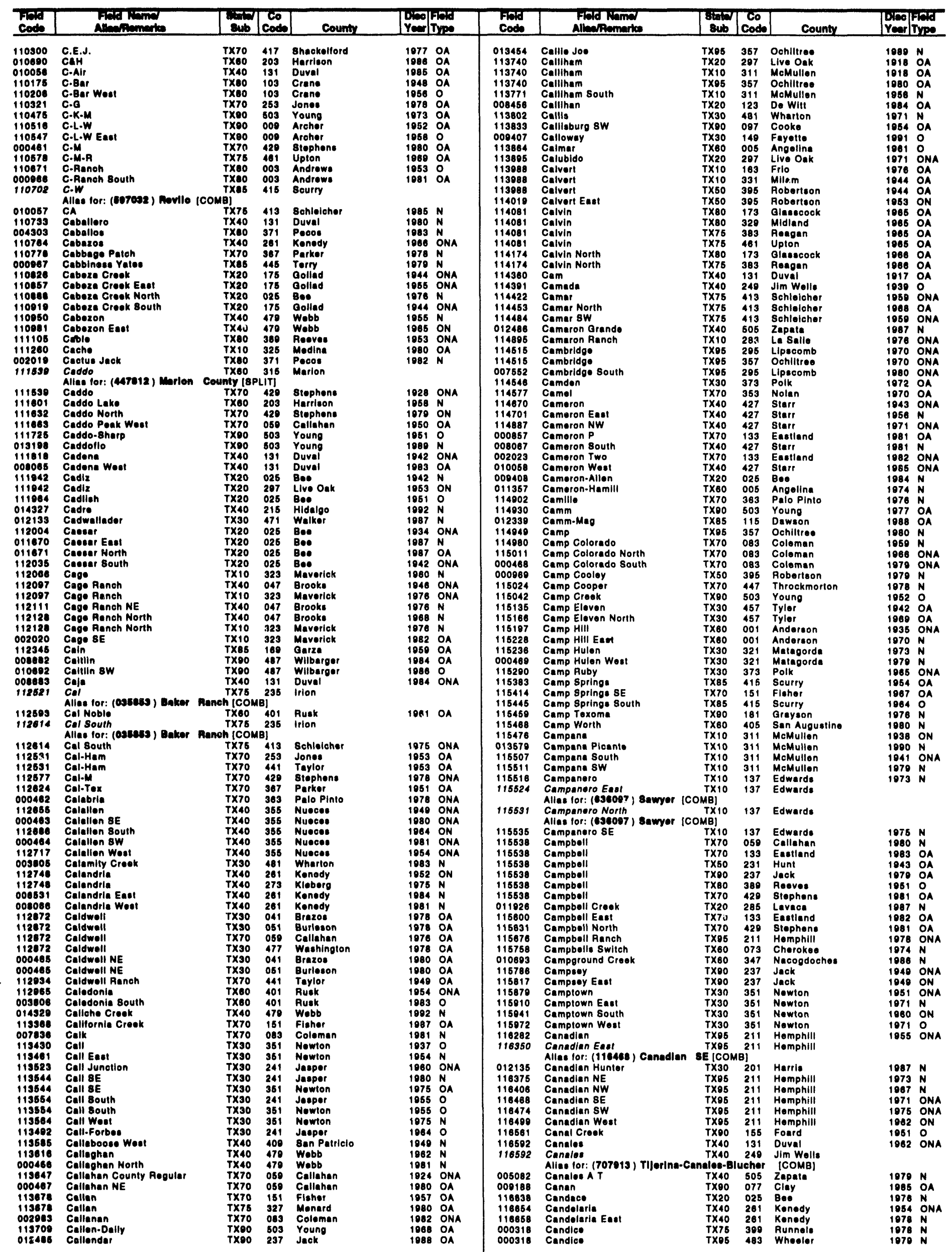


TEXAS

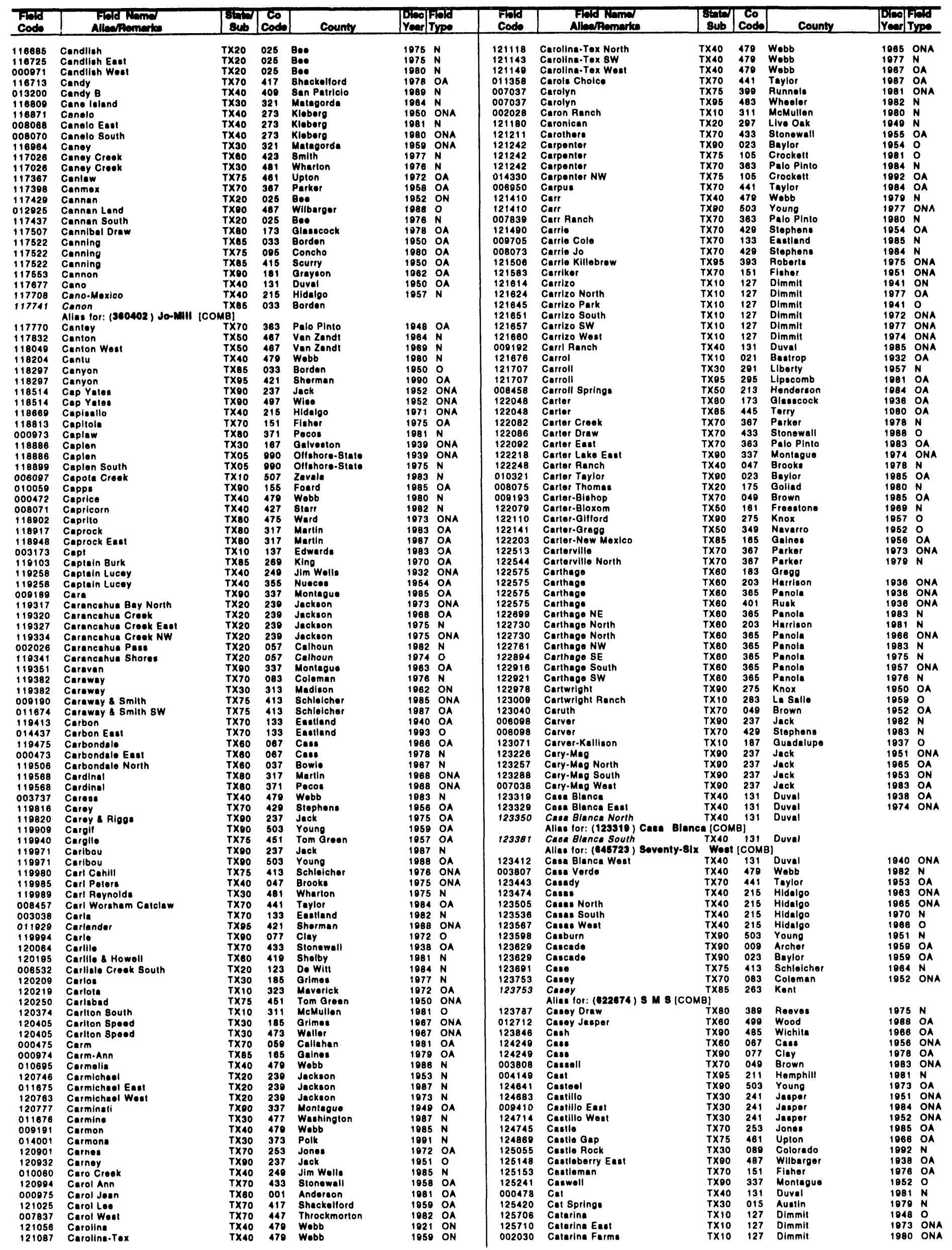


TEXAS

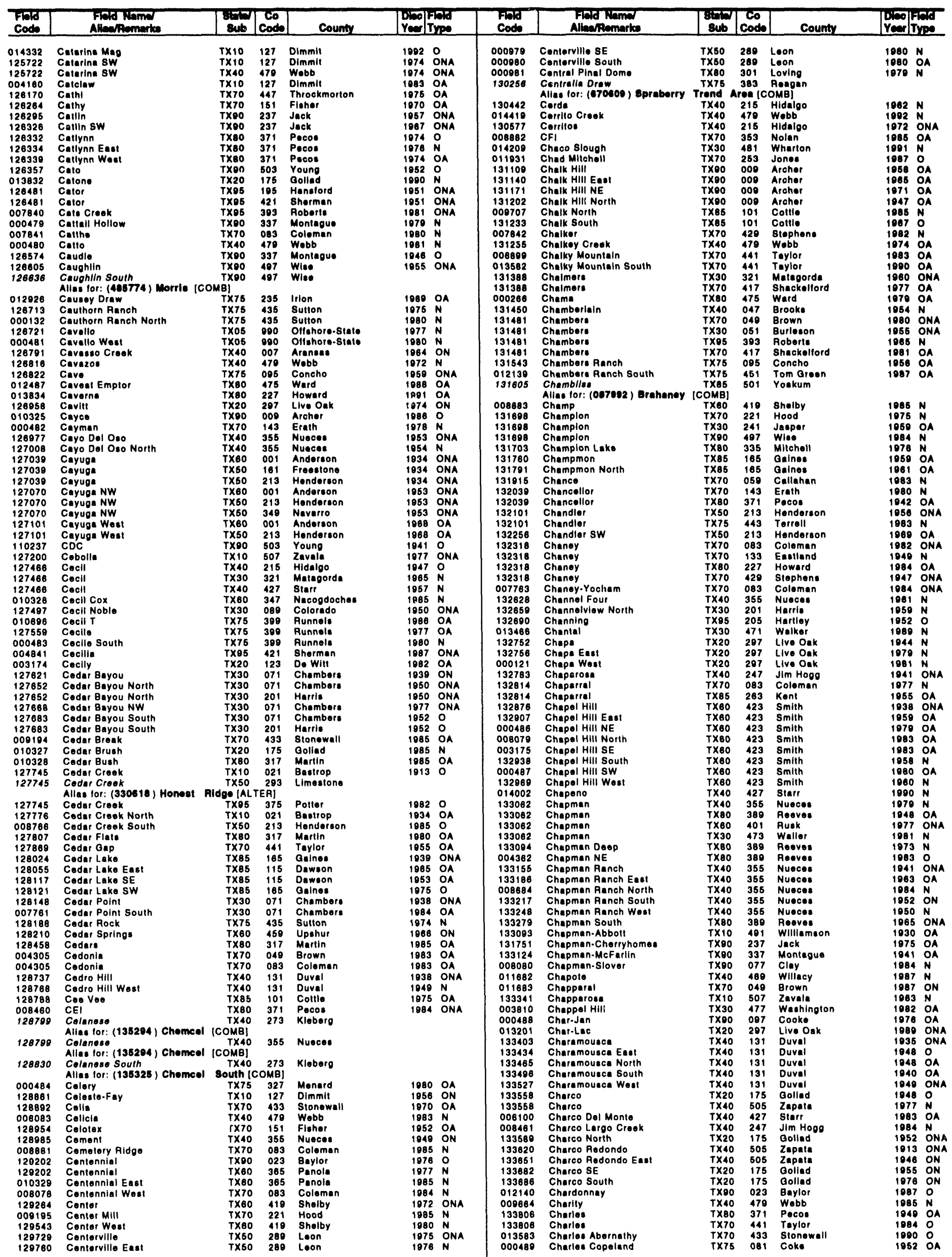


TEXAS

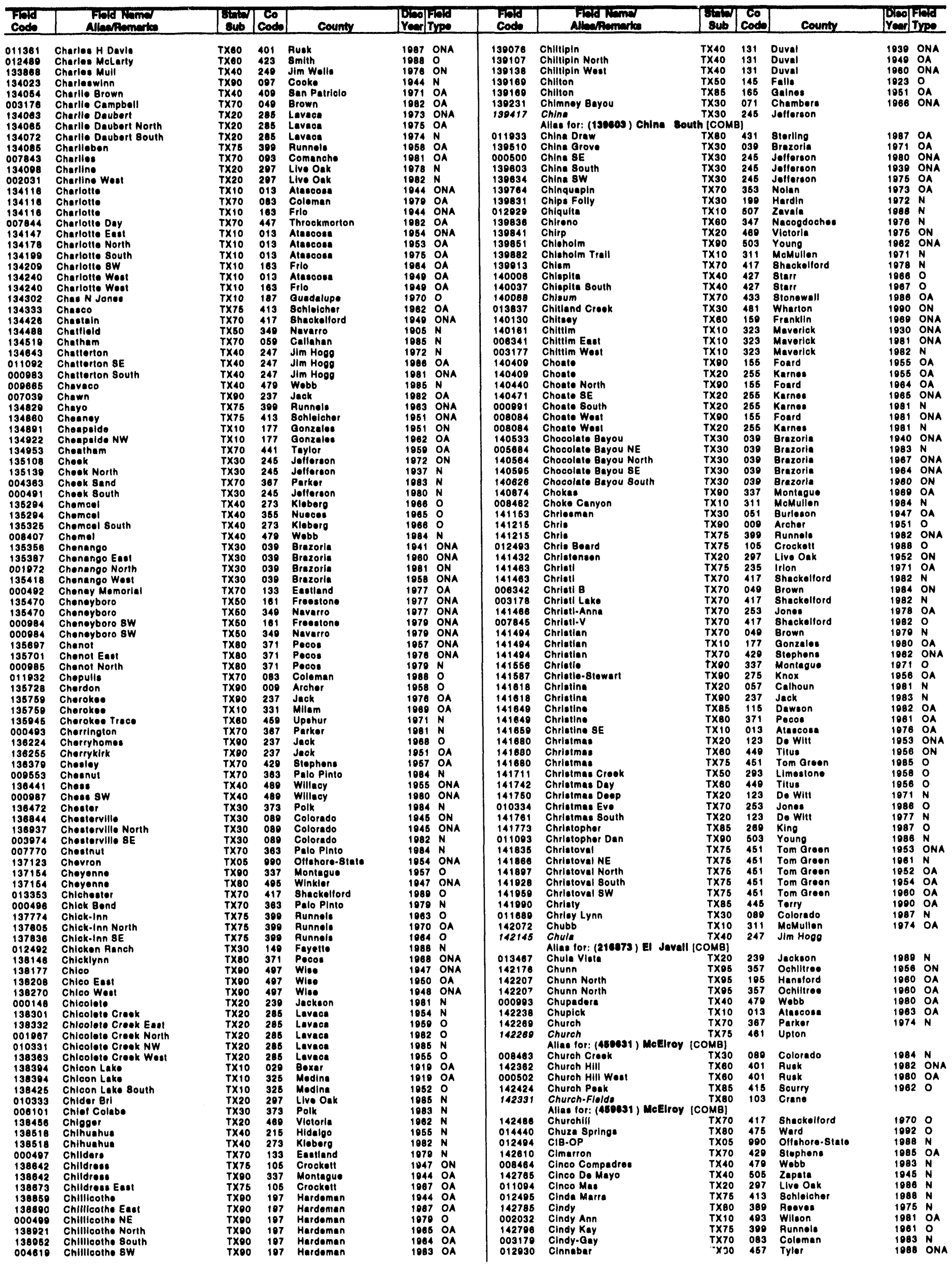


TEXAS

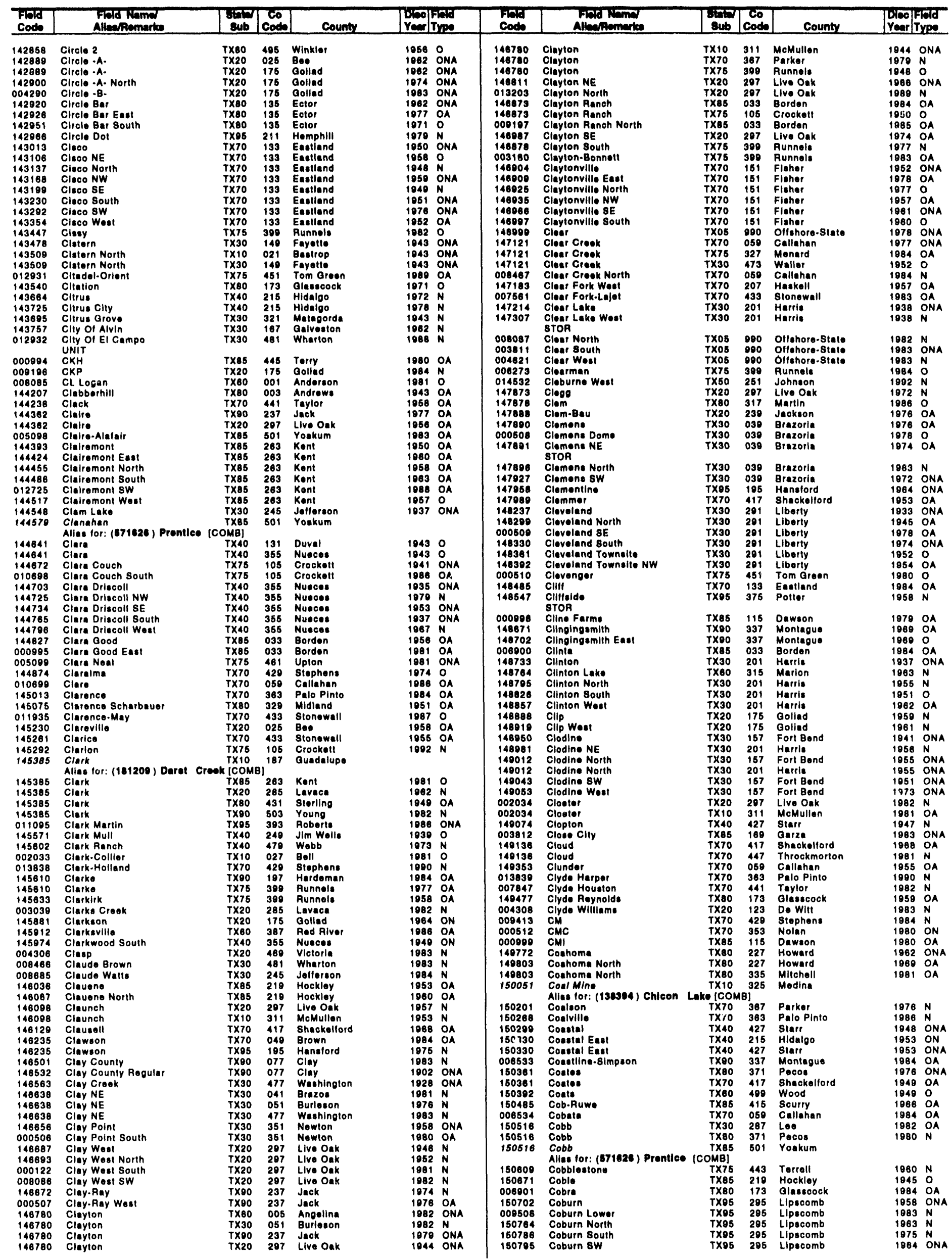


TEXAS

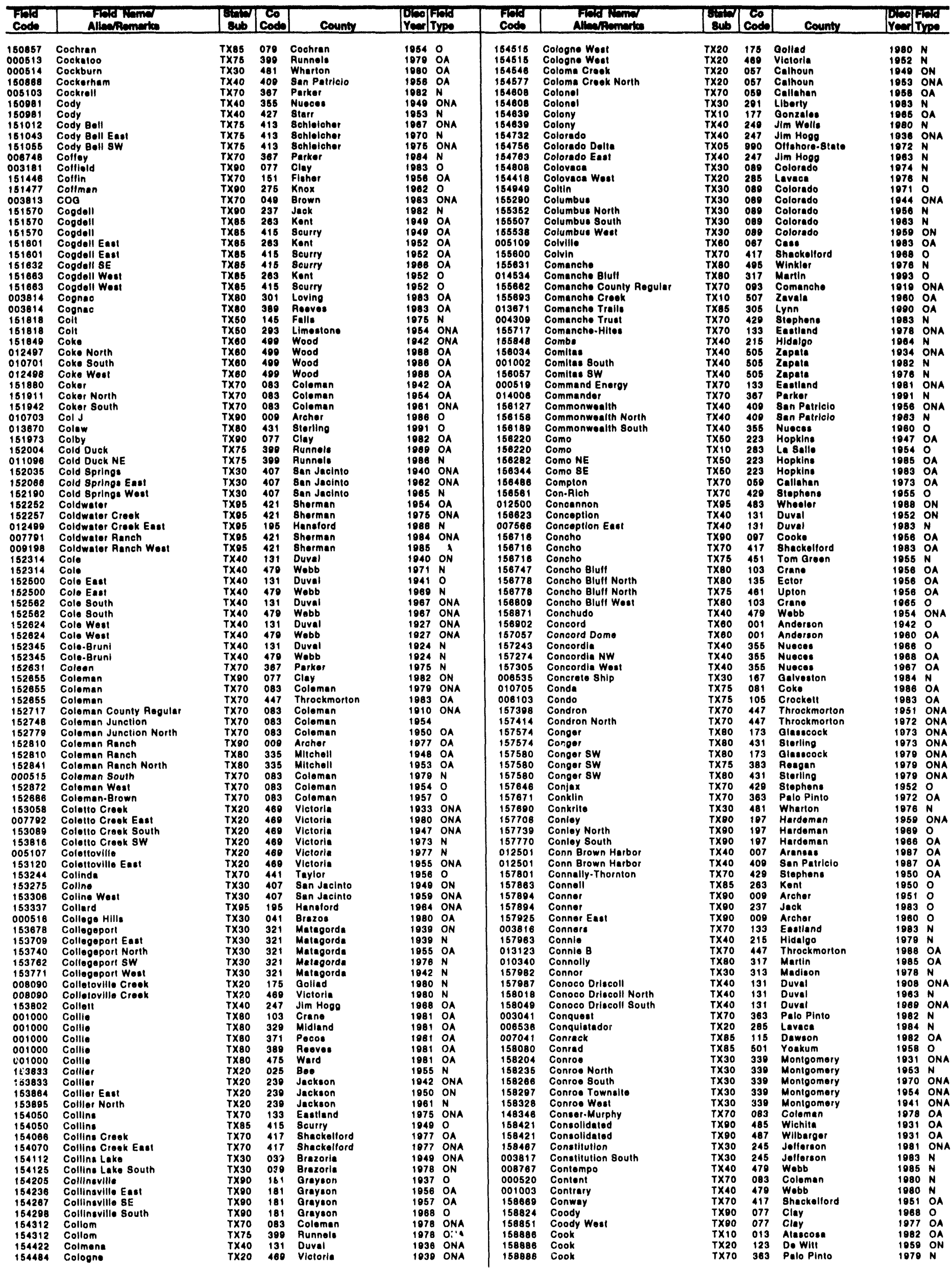


TEXAS

\begin{tabular}{|c|}
\hline $\begin{array}{l}\text { Finta } \\
\text { Code }\end{array}$ \\
\hline 008469 \\
\hline $\begin{array}{l}158917 \\
158010\end{array}$ \\
\hline 158041 \\
\hline 000621 \\
\hline $\begin{array}{l}160072 \\
150076\end{array}$ \\
\hline 150079 \\
\hline 158906 \\
\hline 150103 \\
\hline $\begin{array}{l}150134 \\
008760\end{array}$ \\
\hline 160140 \\
\hline $\begin{array}{l}180180 \\
000100\end{array}$ \\
\hline 004310 \\
\hline 159361 \\
\hline 160351 \\
\hline $\begin{array}{l}159382 \\
012503\end{array}$ \\
\hline 150413 \\
\hline 150413 \\
\hline 150444 \\
\hline 159444 \\
\hline 159476 \\
\hline $\begin{array}{l}150830 \\
150923\end{array}$ \\
\hline $\begin{array}{l}159723 \\
159723\end{array}$ \\
\hline 150723 \\
\hline 150785 \\
\hline 180002 \\
\hline 012145 \\
\hline $\begin{array}{l}000522 \\
160219\end{array}$ \\
\hline 160210 \\
\hline 160250 \\
\hline 160250 \\
\hline $\begin{array}{l}160281 \\
000092\end{array}$ \\
\hline 160312 \\
\hline 160343 \\
\hline 160343 \\
\hline 160343 \\
\hline 160343 \\
\hline $\begin{array}{l}160405 \\
160436\end{array}$ \\
\hline 160442 \\
\hline 160498 \\
\hline 160501 \\
\hline 000200 \\
\hline 160022 \\
\hline $\begin{array}{l}160746 \\
004713\end{array}$ \\
\hline 160870 \\
\hline 160001 \\
\hline 160901 \\
\hline 160001 \\
\hline 160001 \\
\hline 006343 \\
\hline 180003 \\
\hline 013468 \\
\hline 004622 \\
\hline 161118 \\
\hline 161149 \\
\hline 181180 \\
\hline $\begin{array}{l}161226 \\
161242\end{array}$ \\
\hline 161335 \\
\hline 101360 \\
\hline 161397 \\
\hline 101428 \\
\hline 161489 \\
\hline 012504 \\
\hline 012505 \\
\hline 161534 \\
\hline 161862 \\
\hline 161086 \\
\hline 011307 \\
\hline 000523 \\
\hline $\begin{array}{l}000524 \\
008094\end{array}$ \\
\hline 162017 \\
\hline 102048 \\
\hline 162048 \\
\hline 001004 \\
\hline 010706 \\
\hline 182200 \\
\hline 102327 \\
\hline 000526 \\
\hline 000526 \\
\hline 162358 \\
\hline 008003 \\
\hline 162389 \\
\hline $\begin{array}{l}003182 \\
162420\end{array}$ \\
\hline 162451 \\
\hline 102451 \\
\hline 162462 \\
\hline 162544 \\
\hline 162576 \\
\hline 162608 \\
\hline 162637 \\
\hline 162601 \\
\hline 003042 \\
\hline 162730 \\
\hline 162761 \\
\hline 102702 \\
\hline $\begin{array}{l}008886 \\
182823\end{array}$ \\
\hline 102854 \\
\hline
\end{tabular}


TEXAS

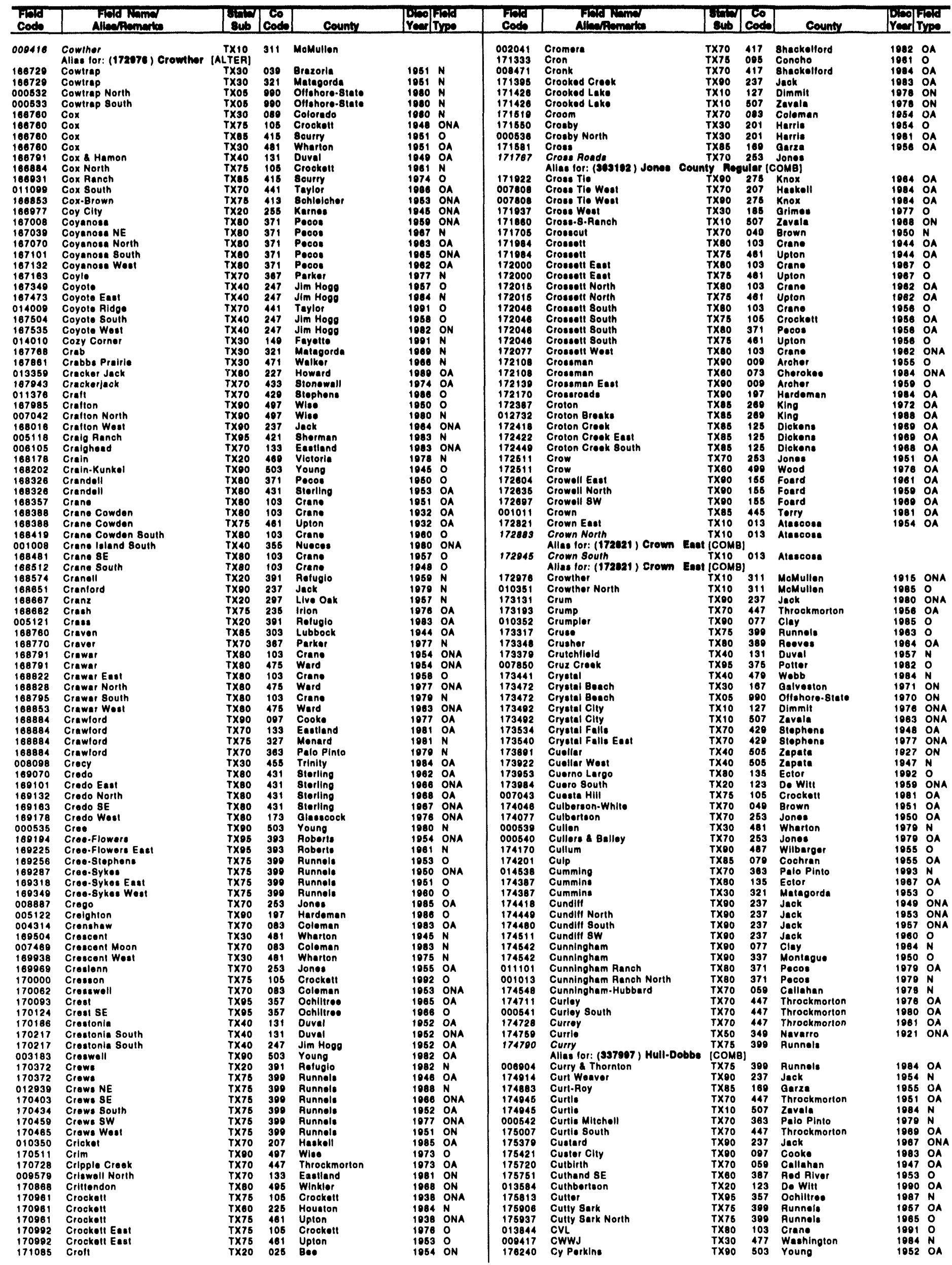


TEXAS

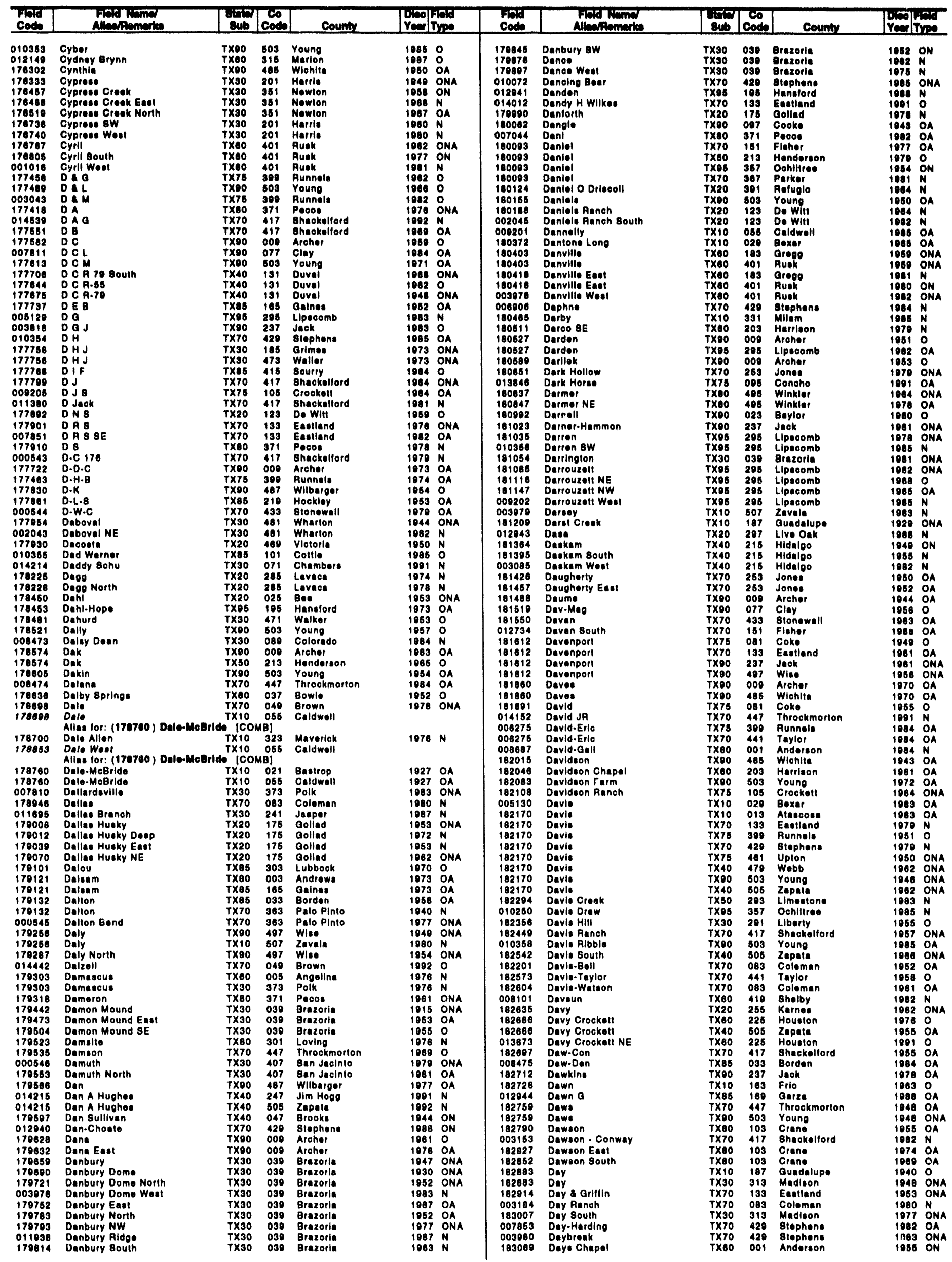


TEXAS

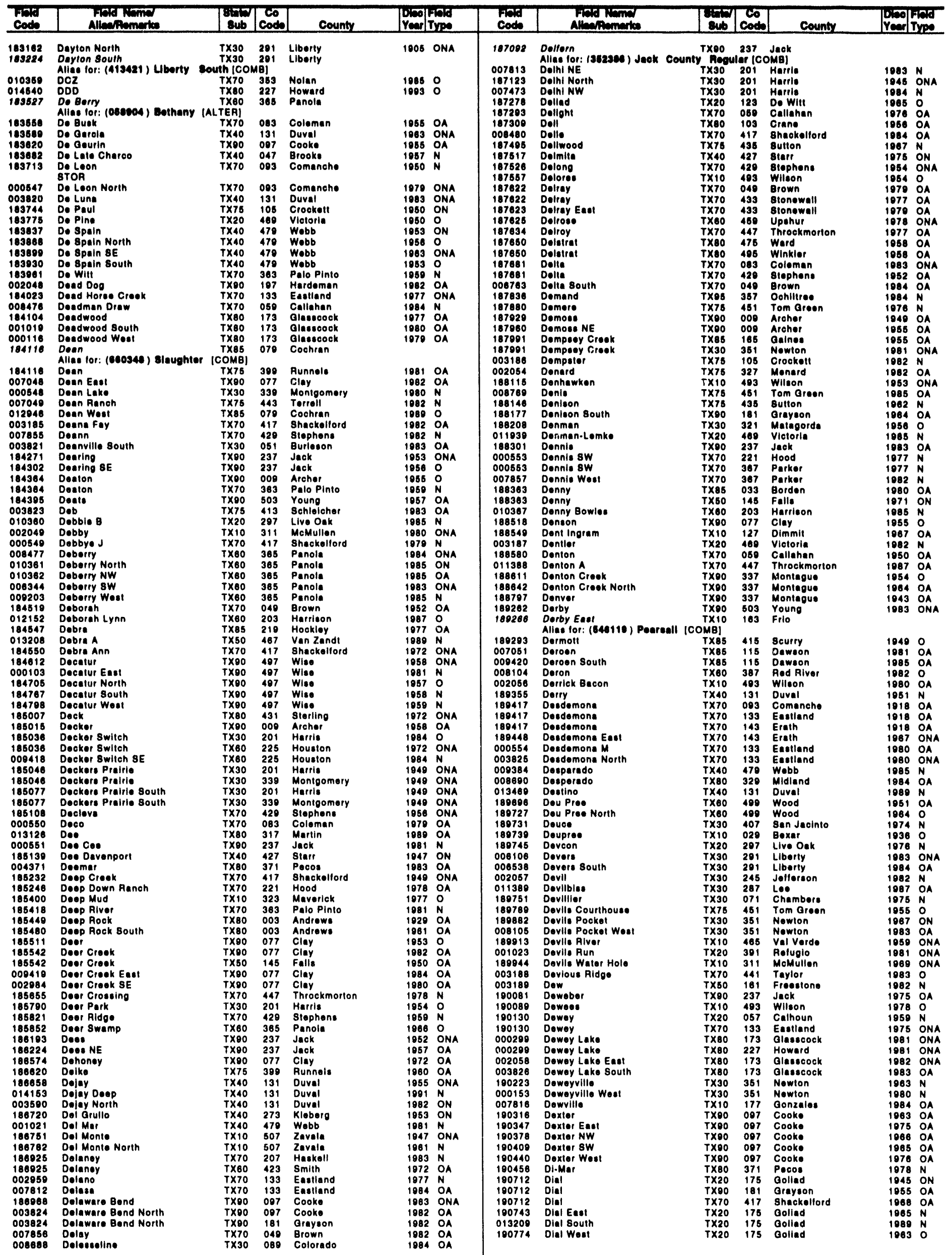


TEXAS

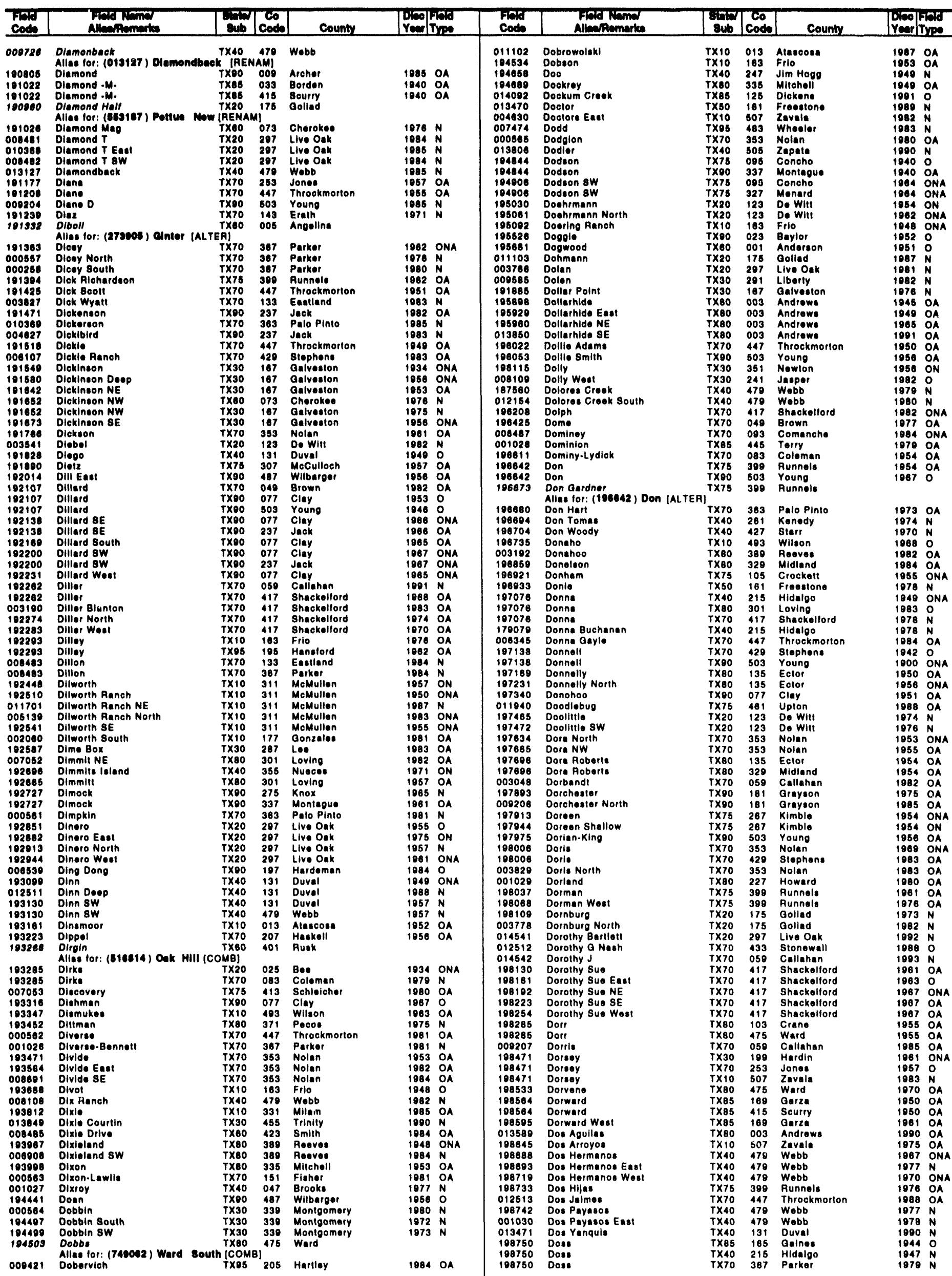


TEXAS

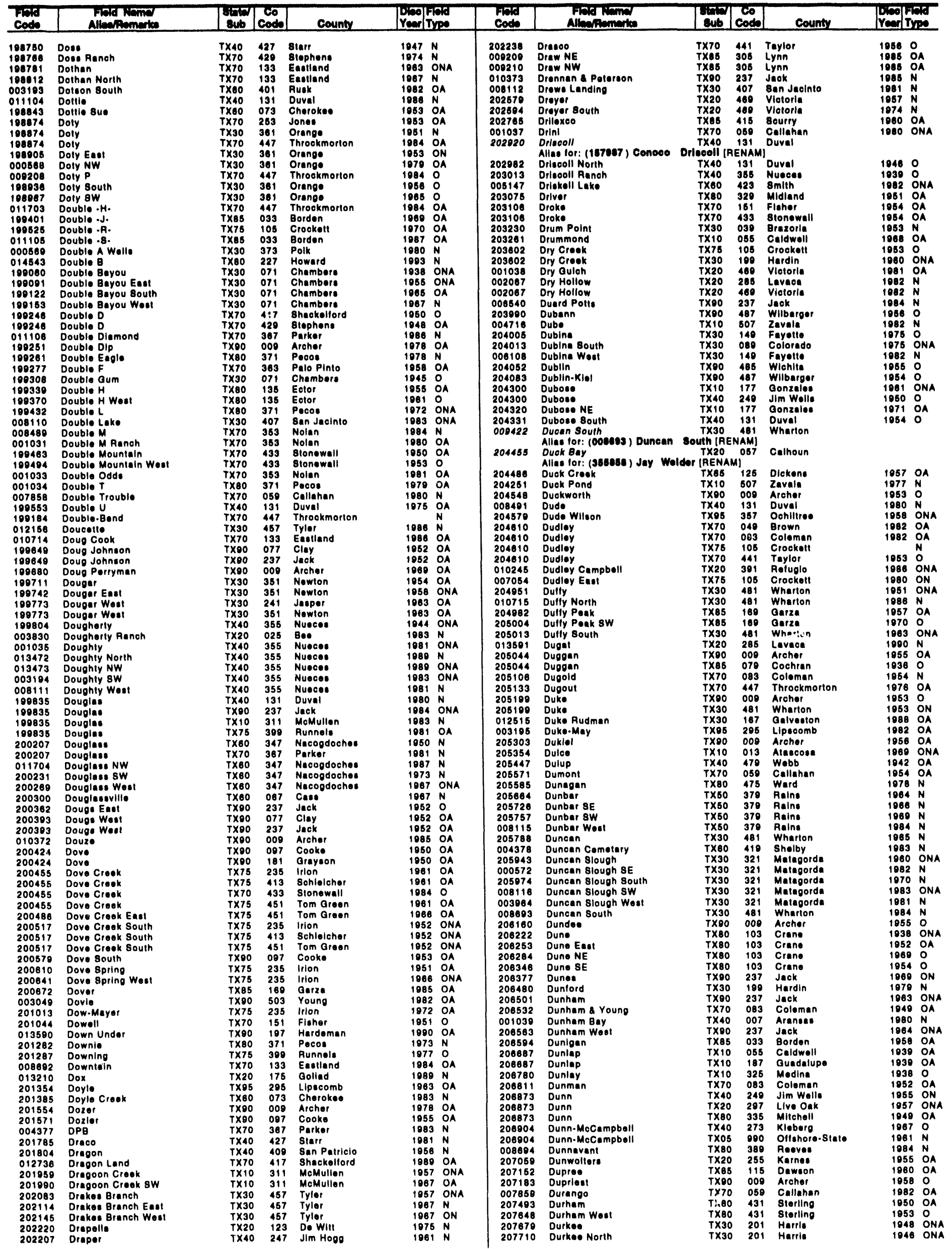


TEXAS

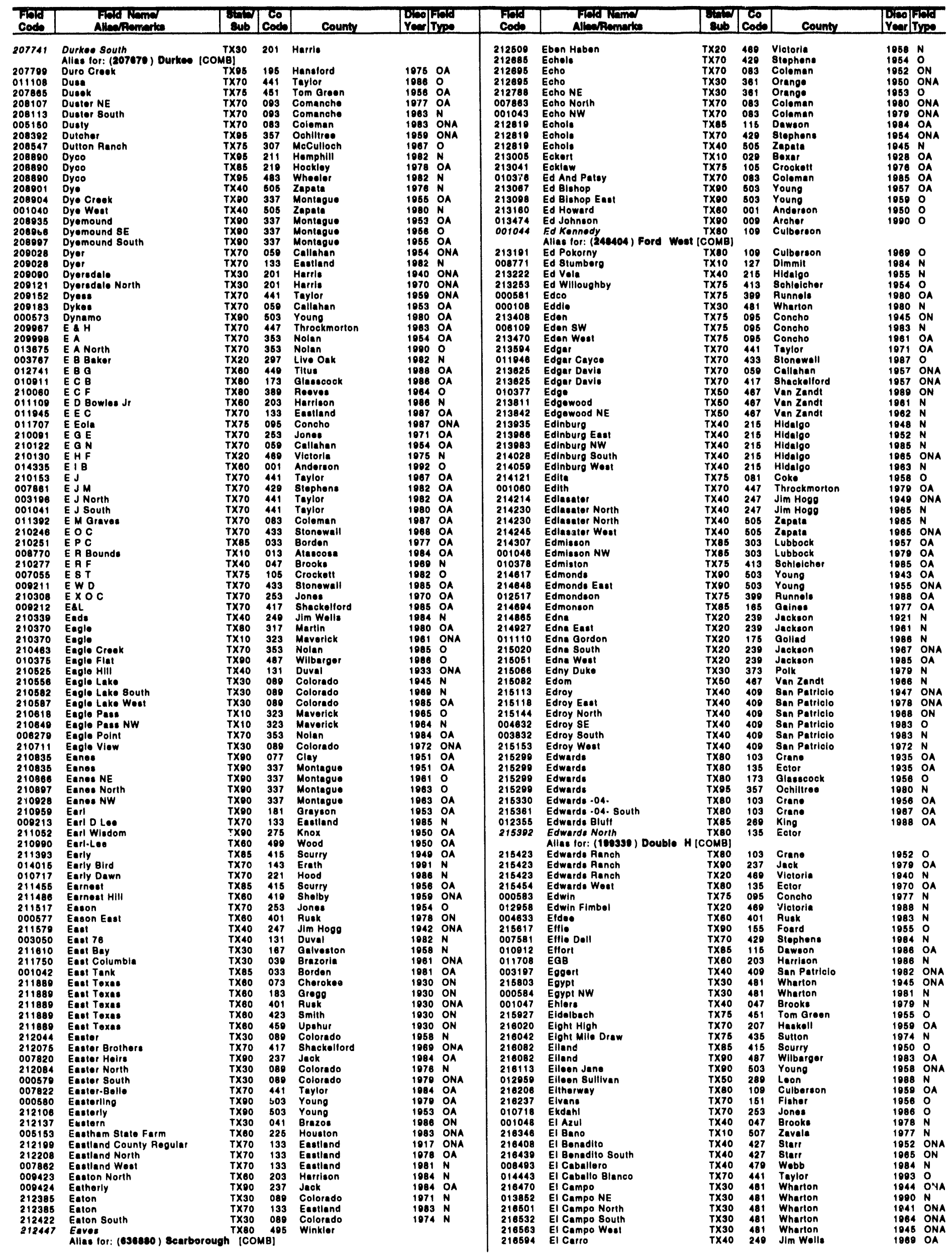


TEXAS

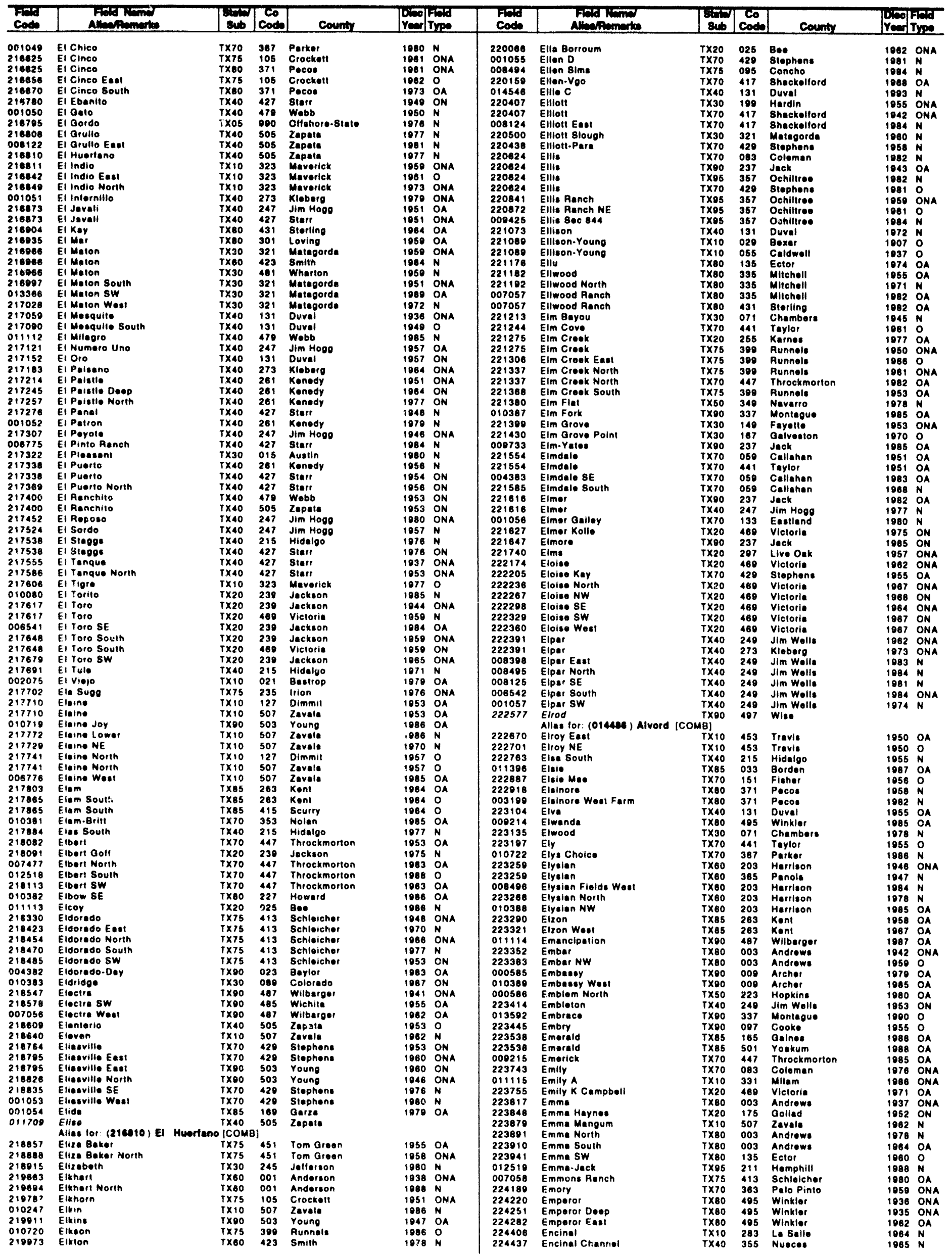


TEXAS

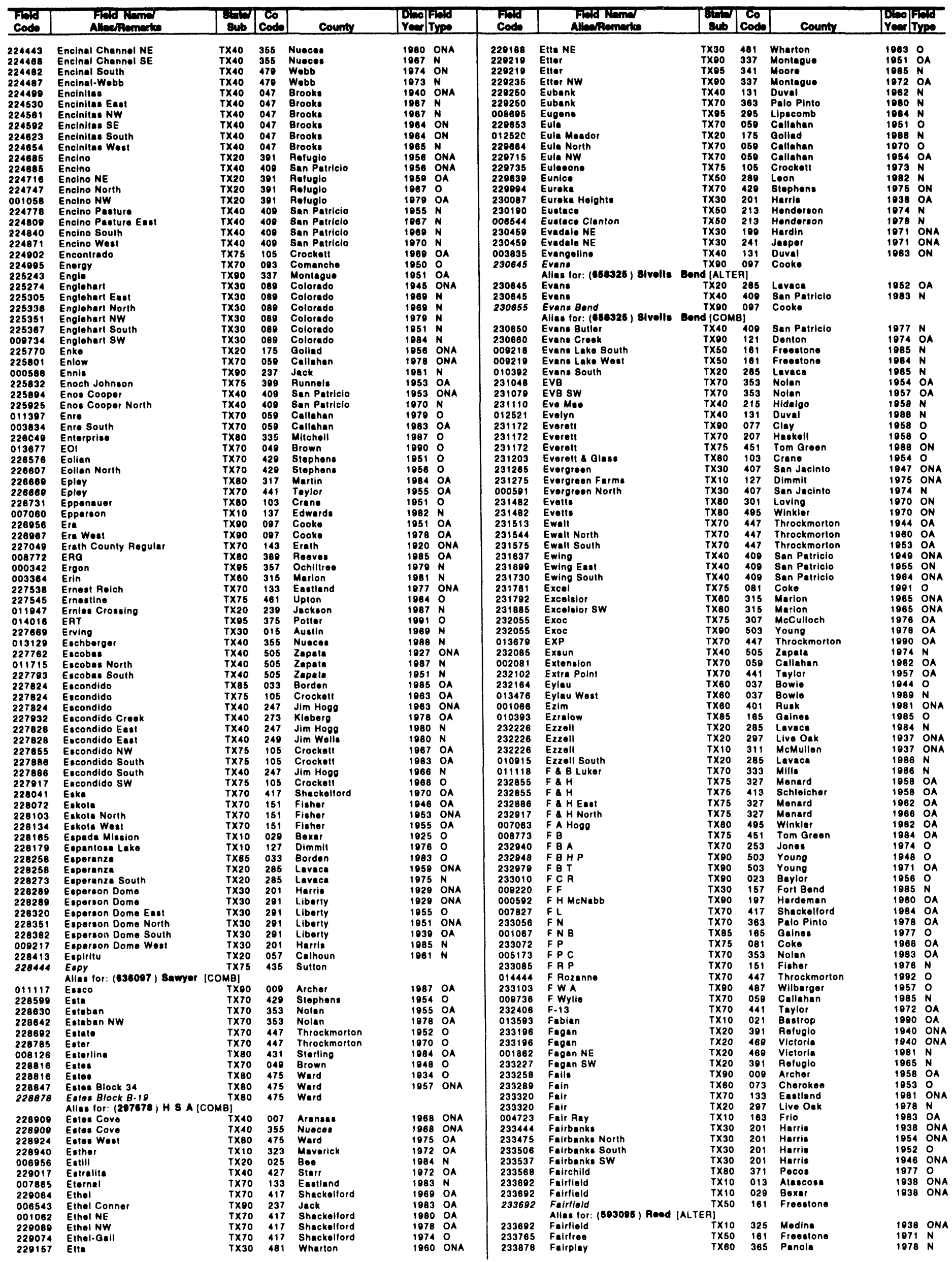


TEXAS

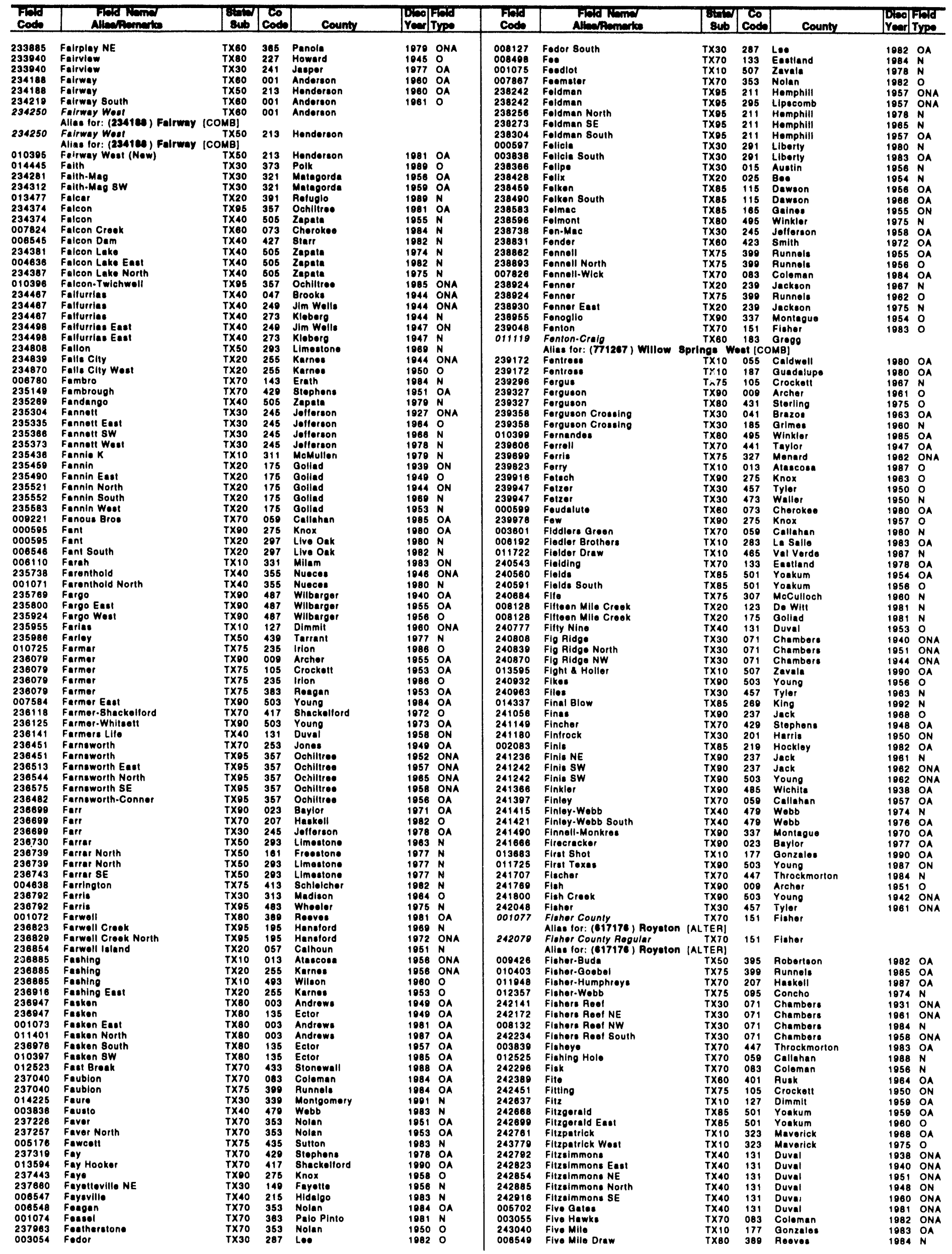


TEXAS

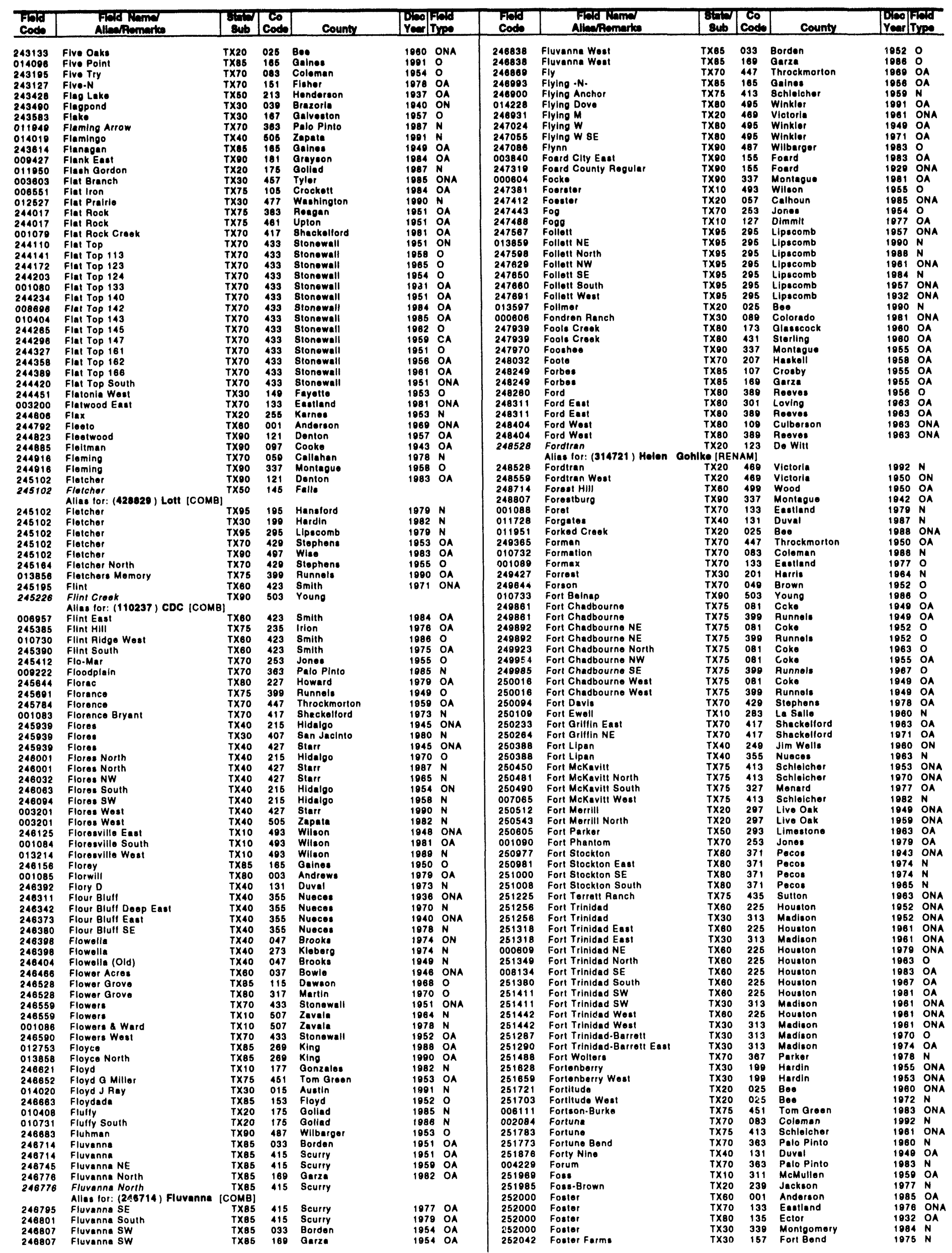


TEXAS

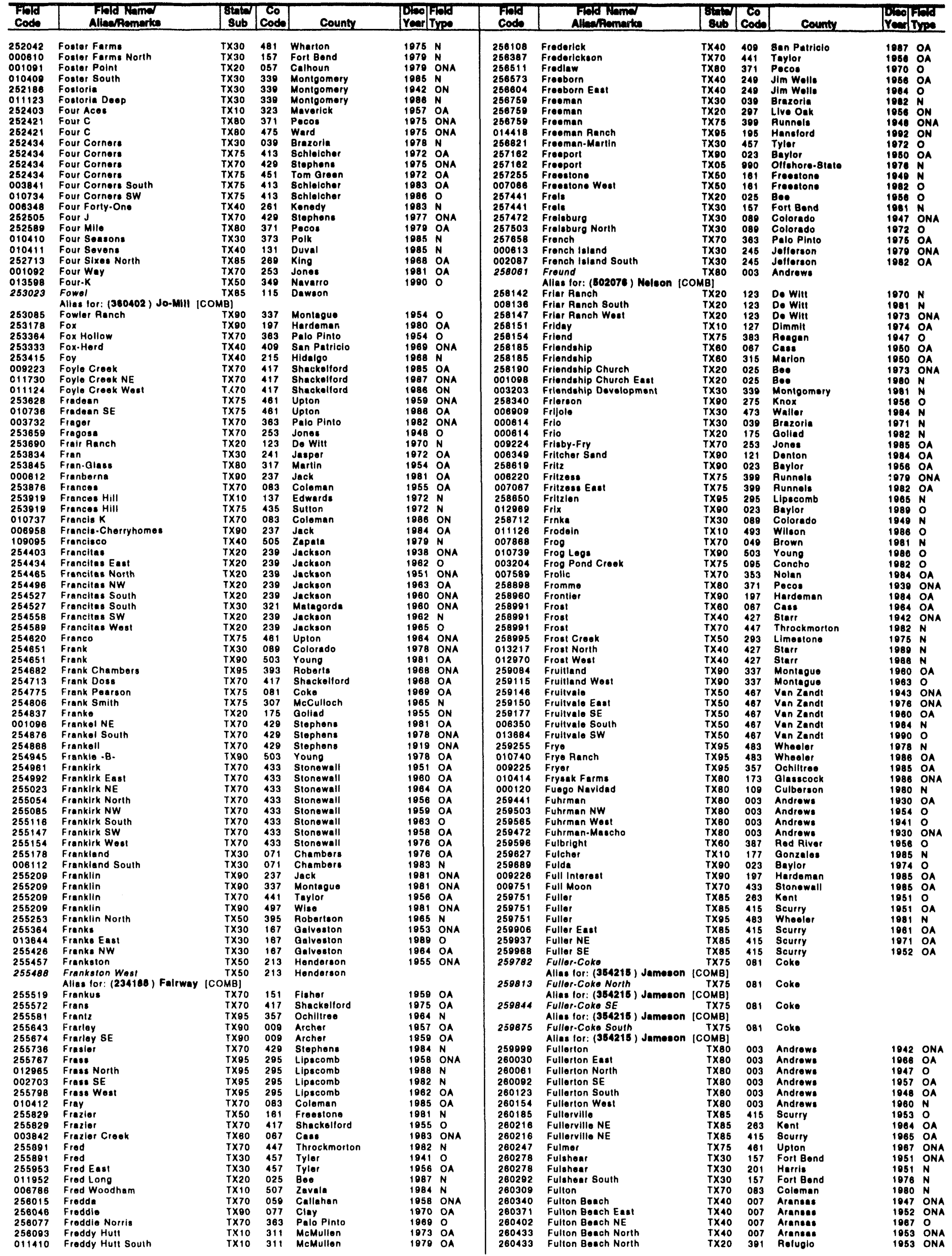


TEXAS

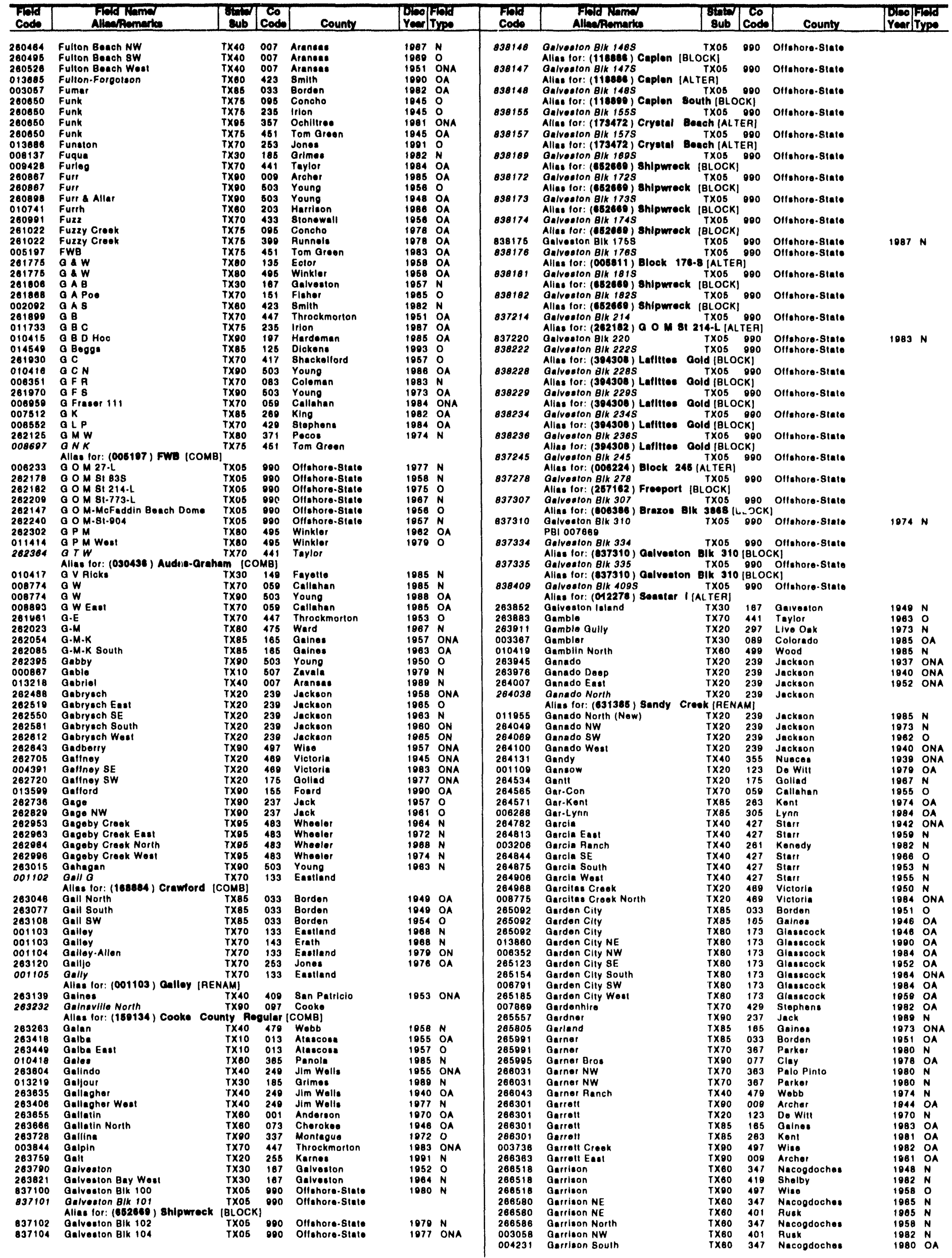


TEXAS

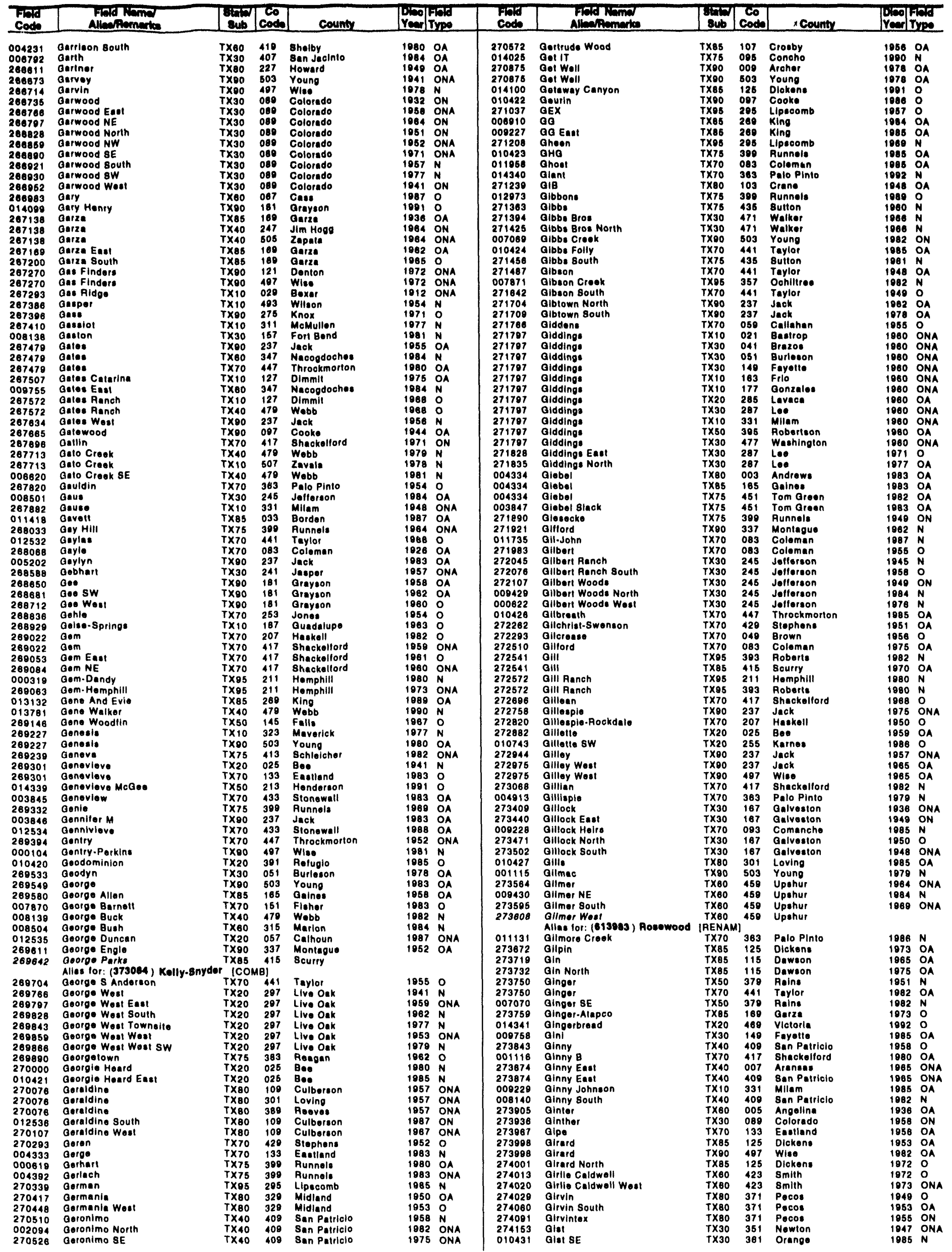


TEXAS

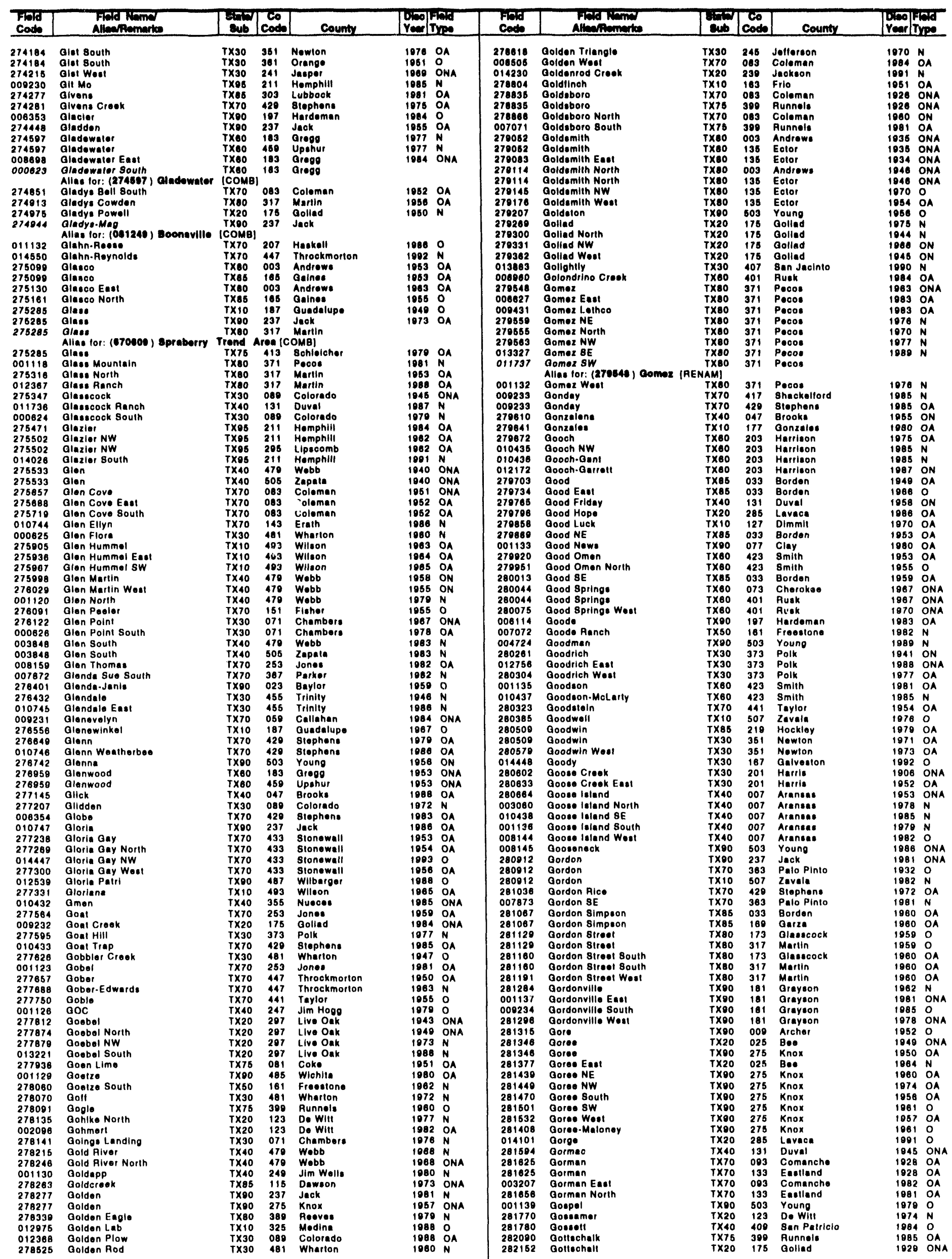




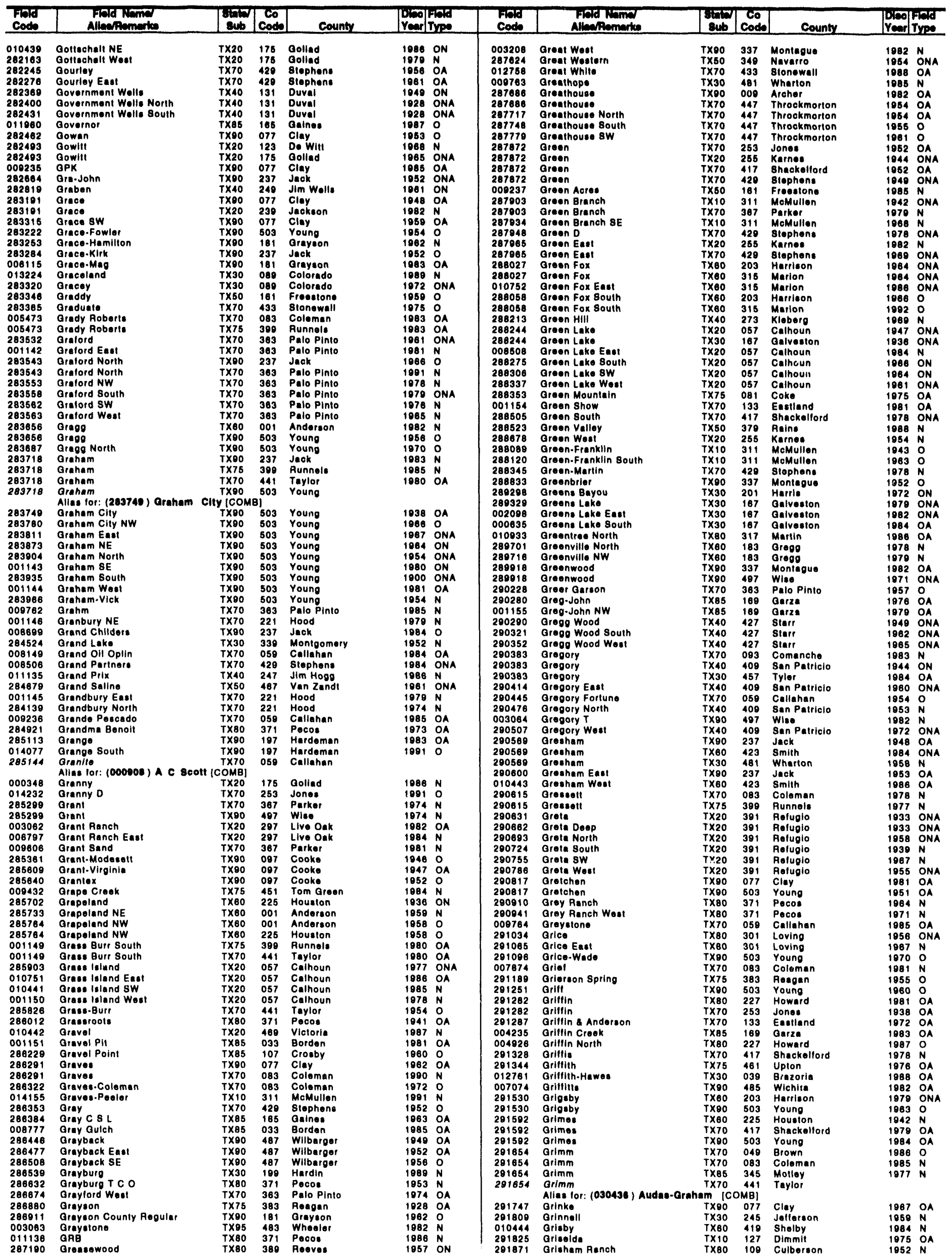


TEXAS

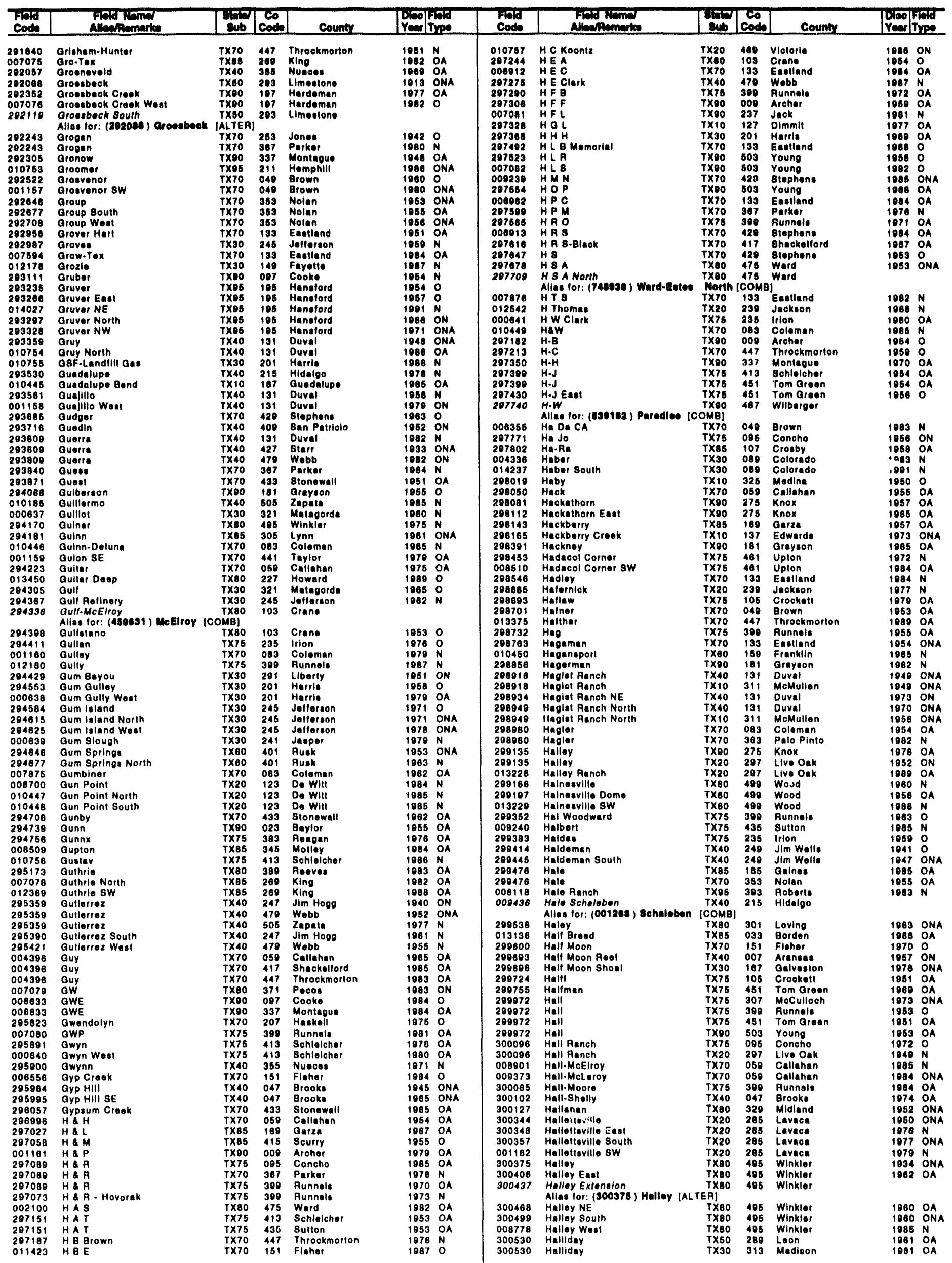


TEXAS

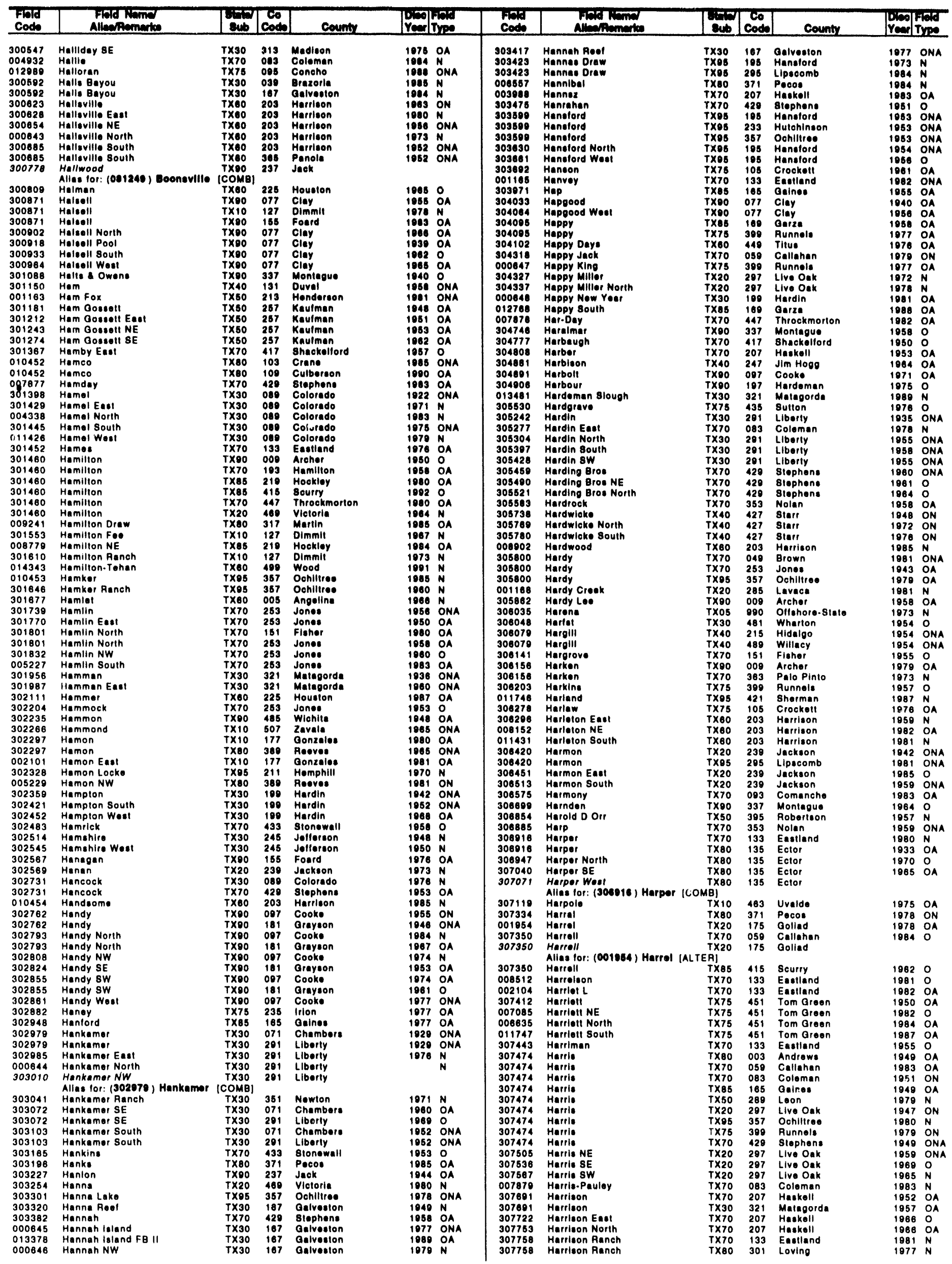


TEXAS

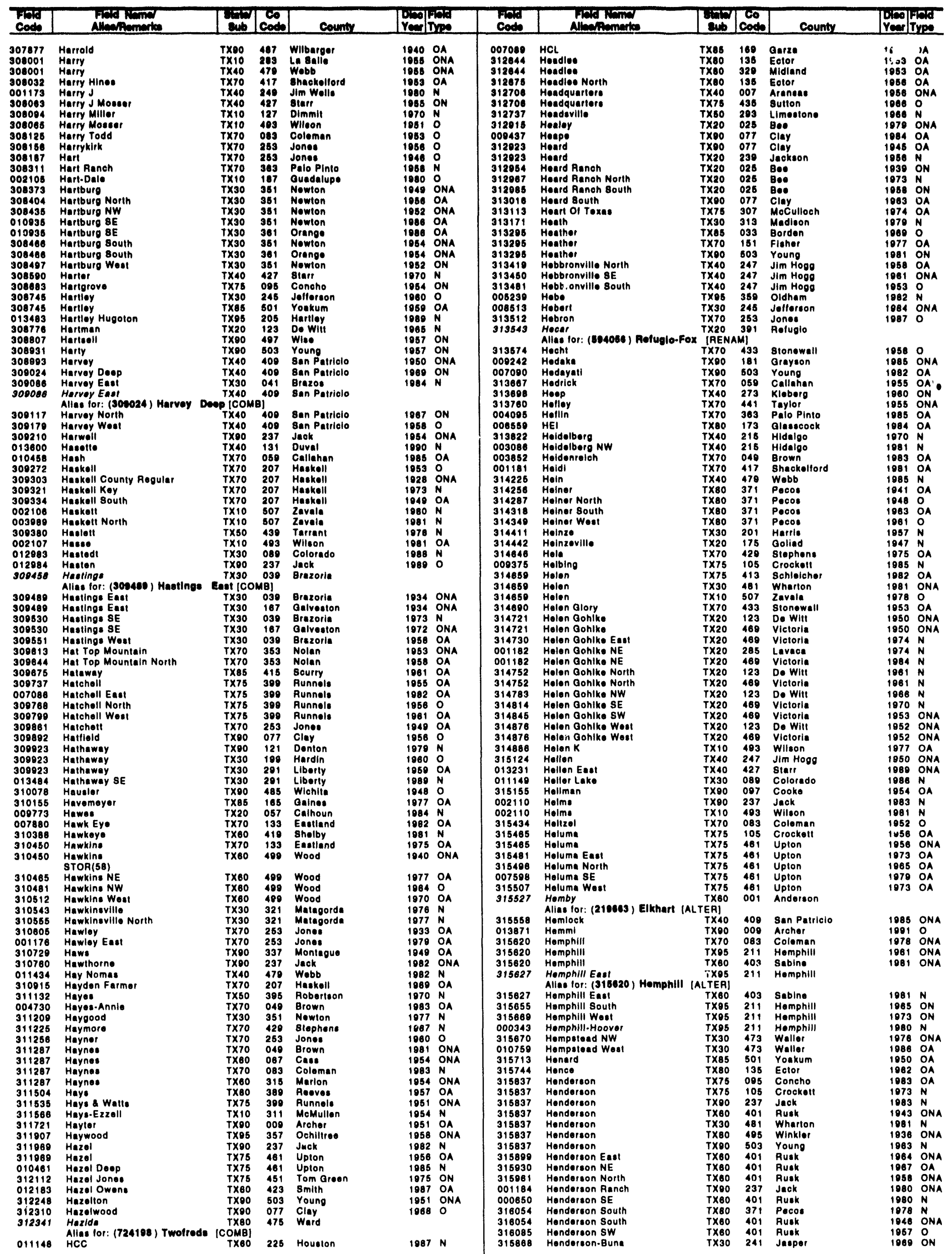




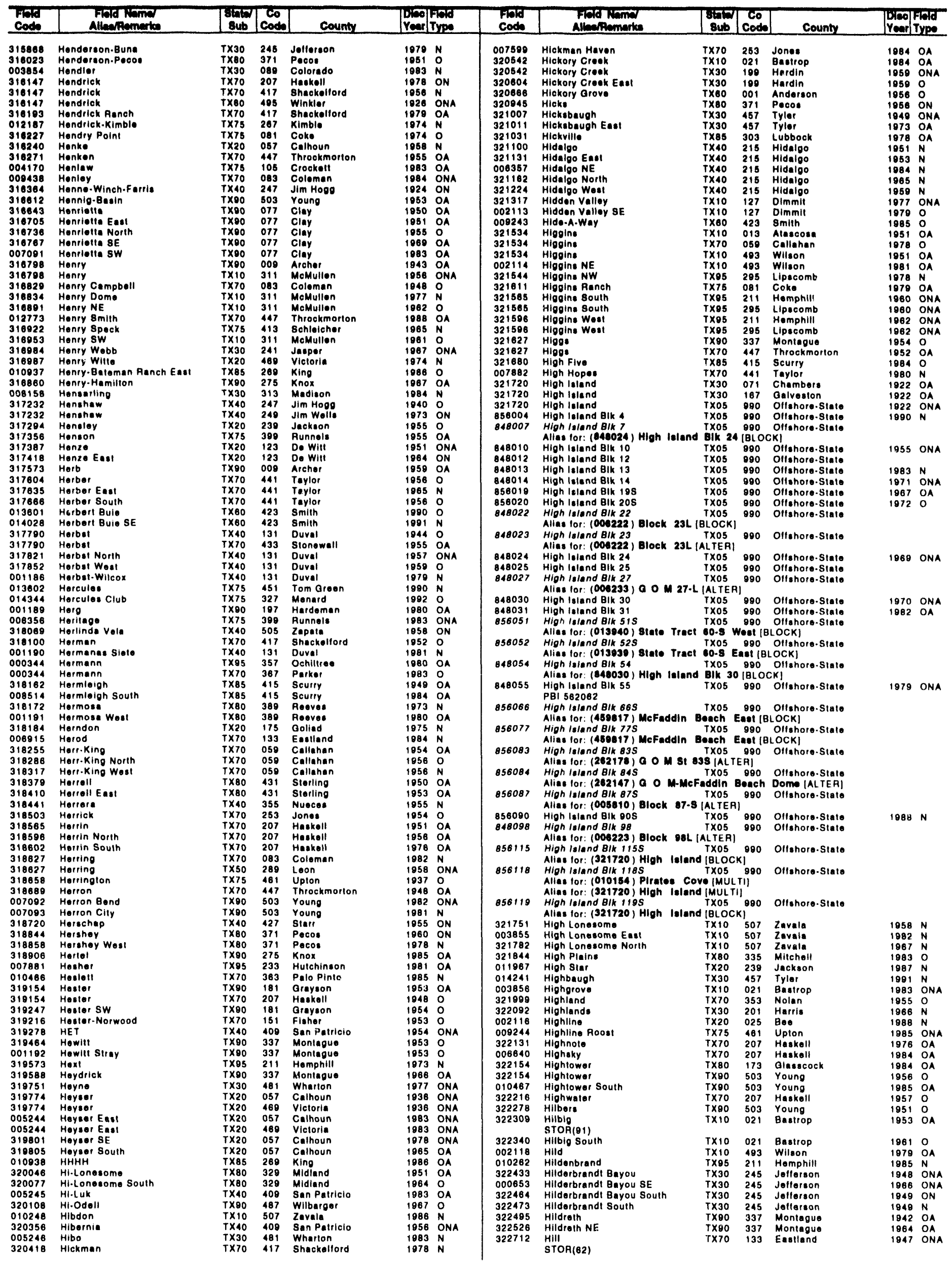


TEXAS

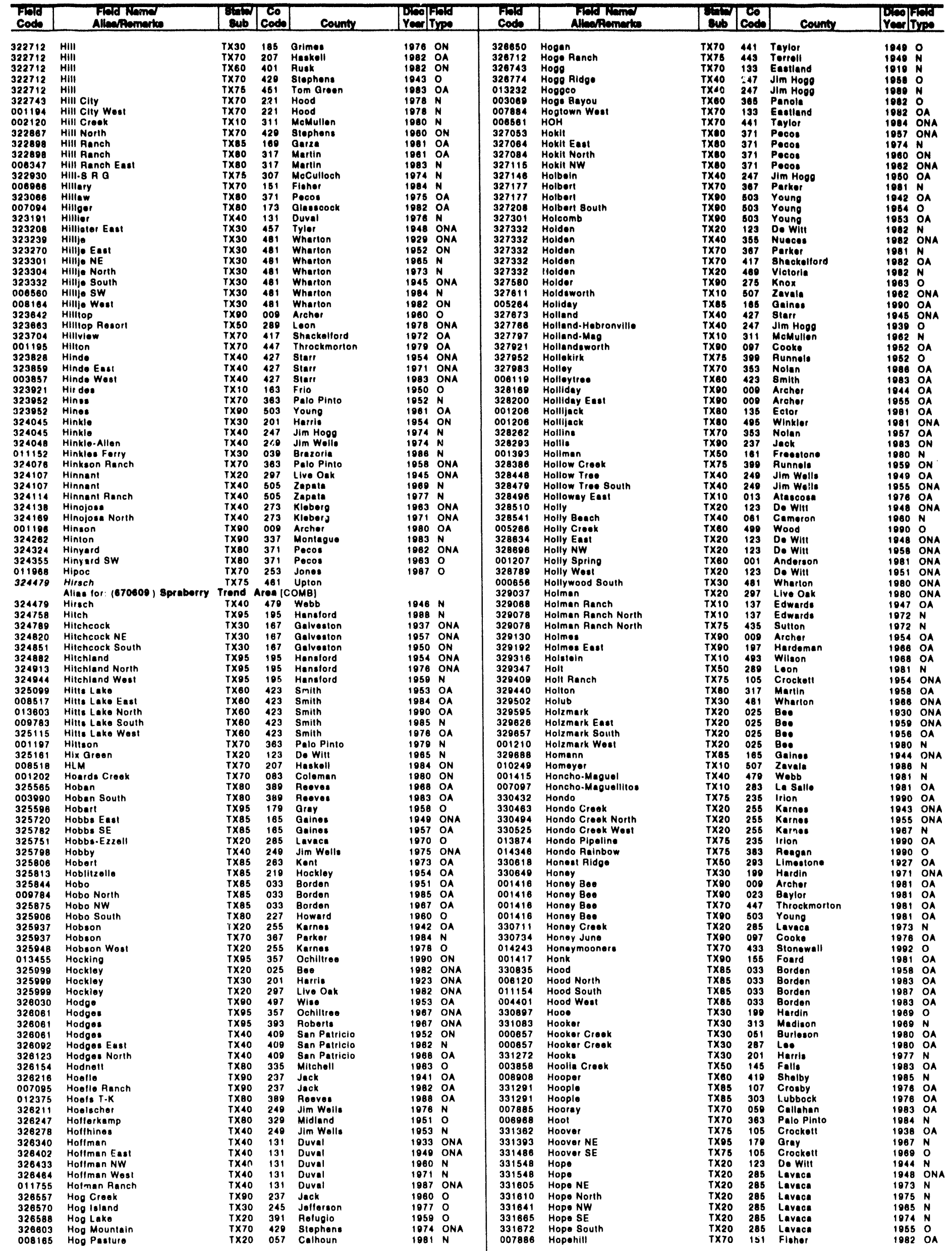


TEXAS

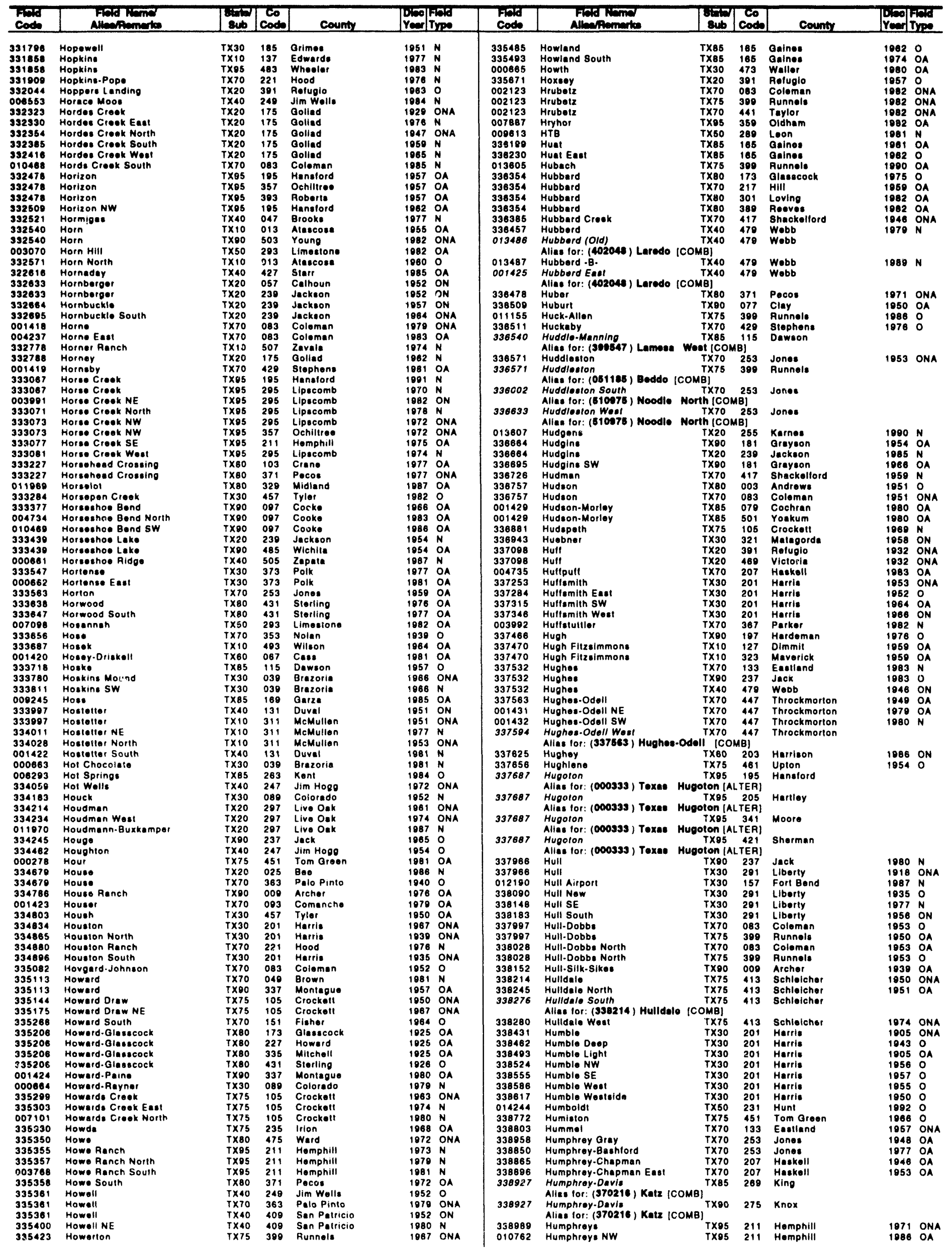


TEXAS

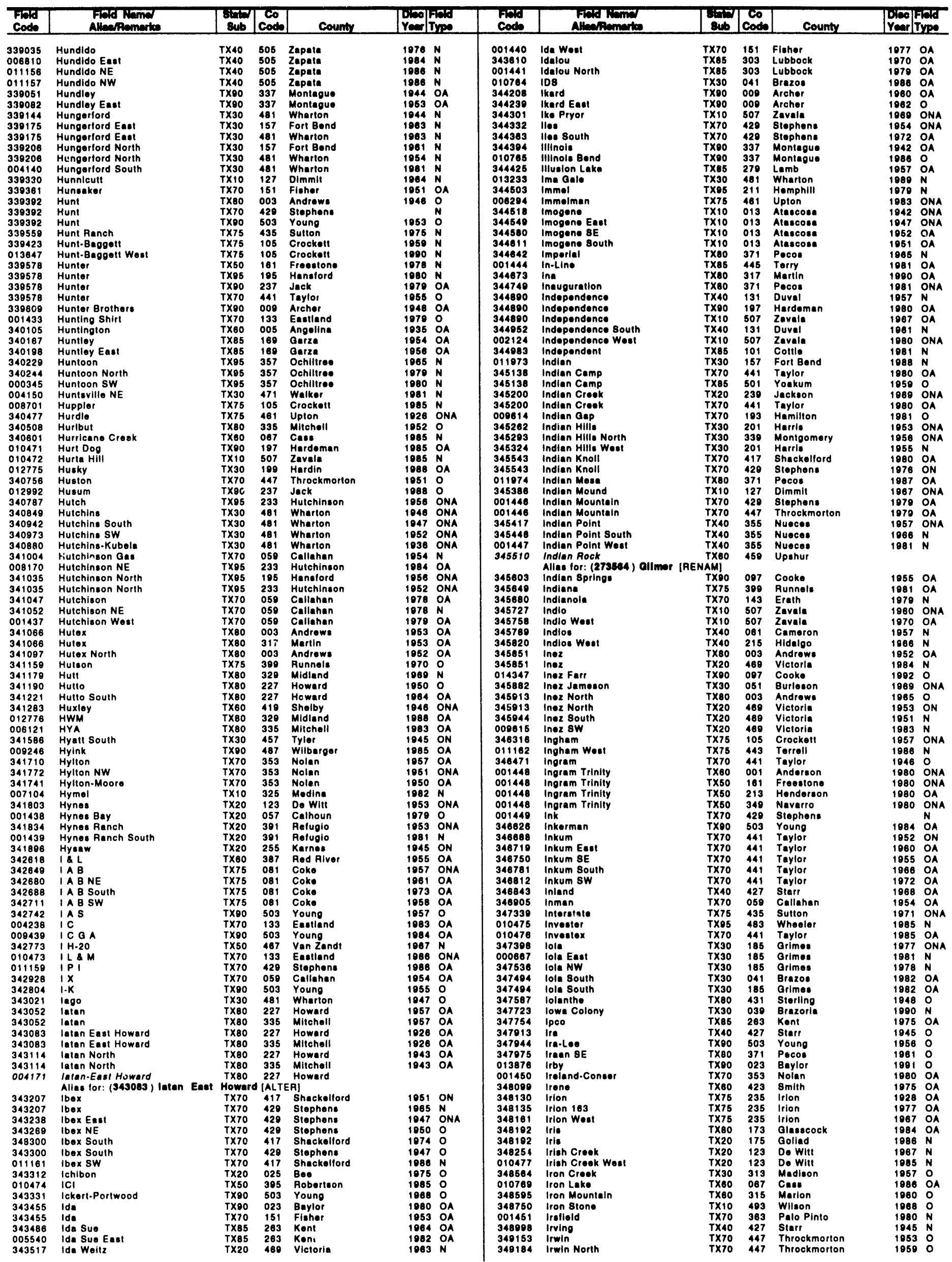


TEXAS

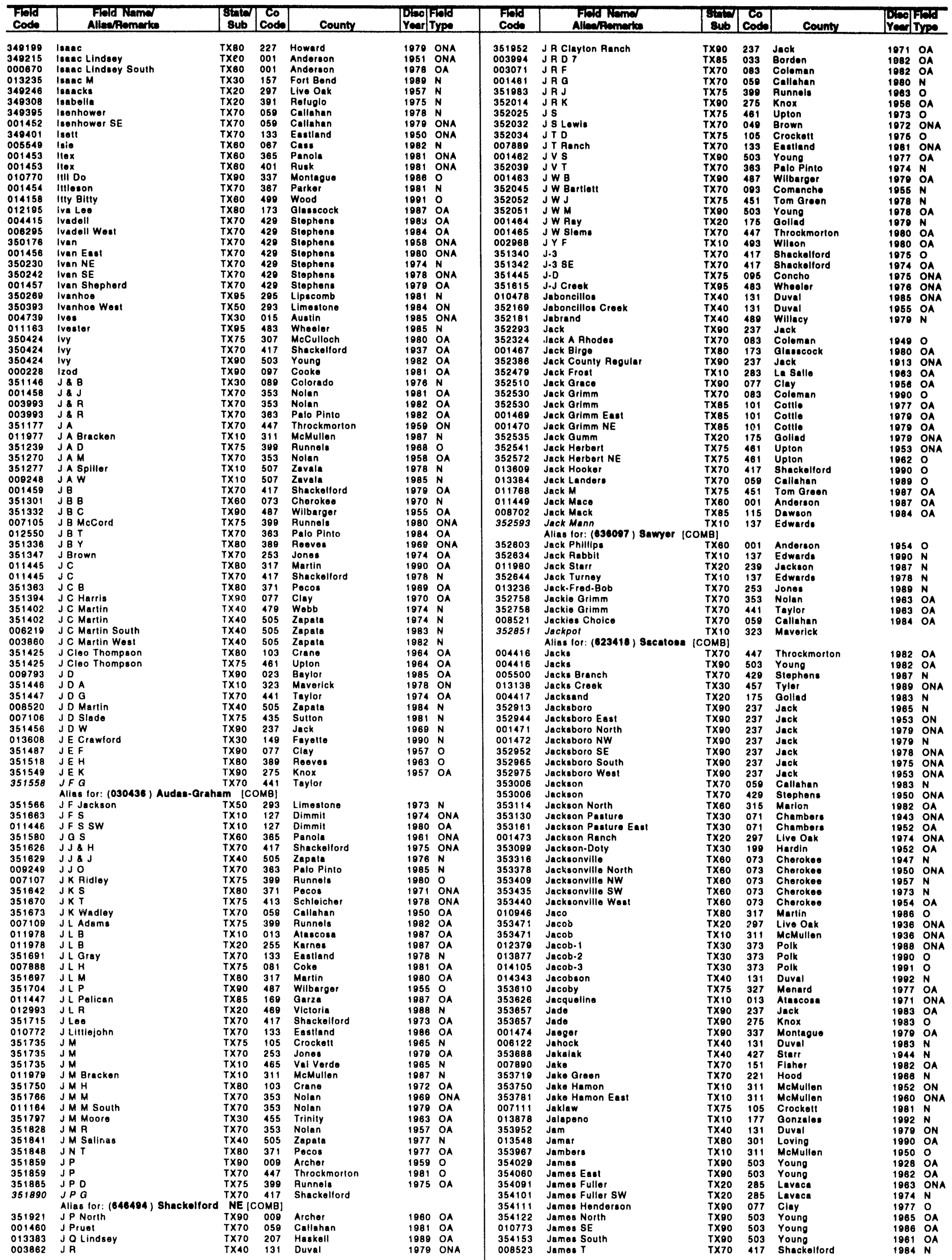


TEXAS

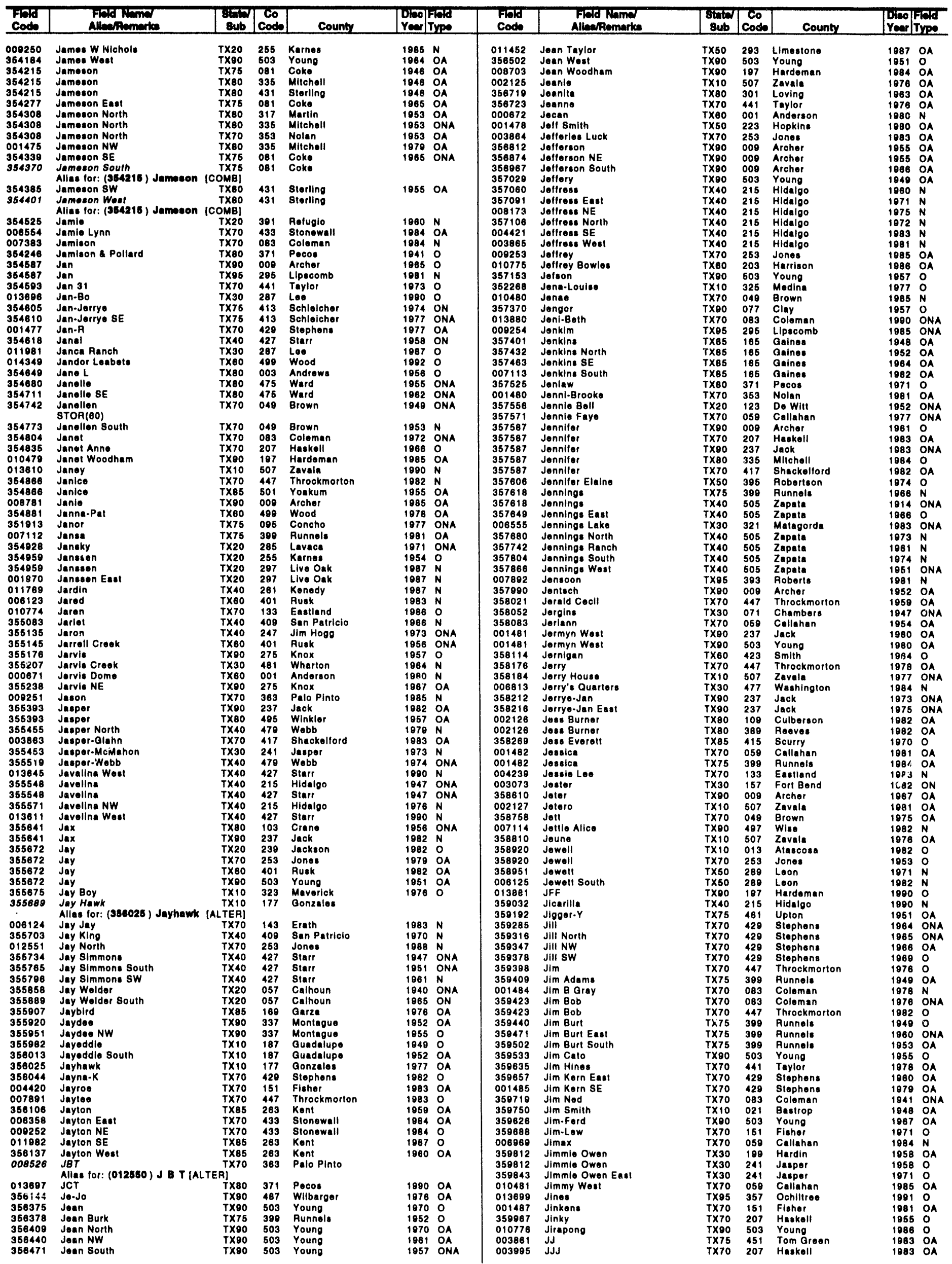


TEXAS

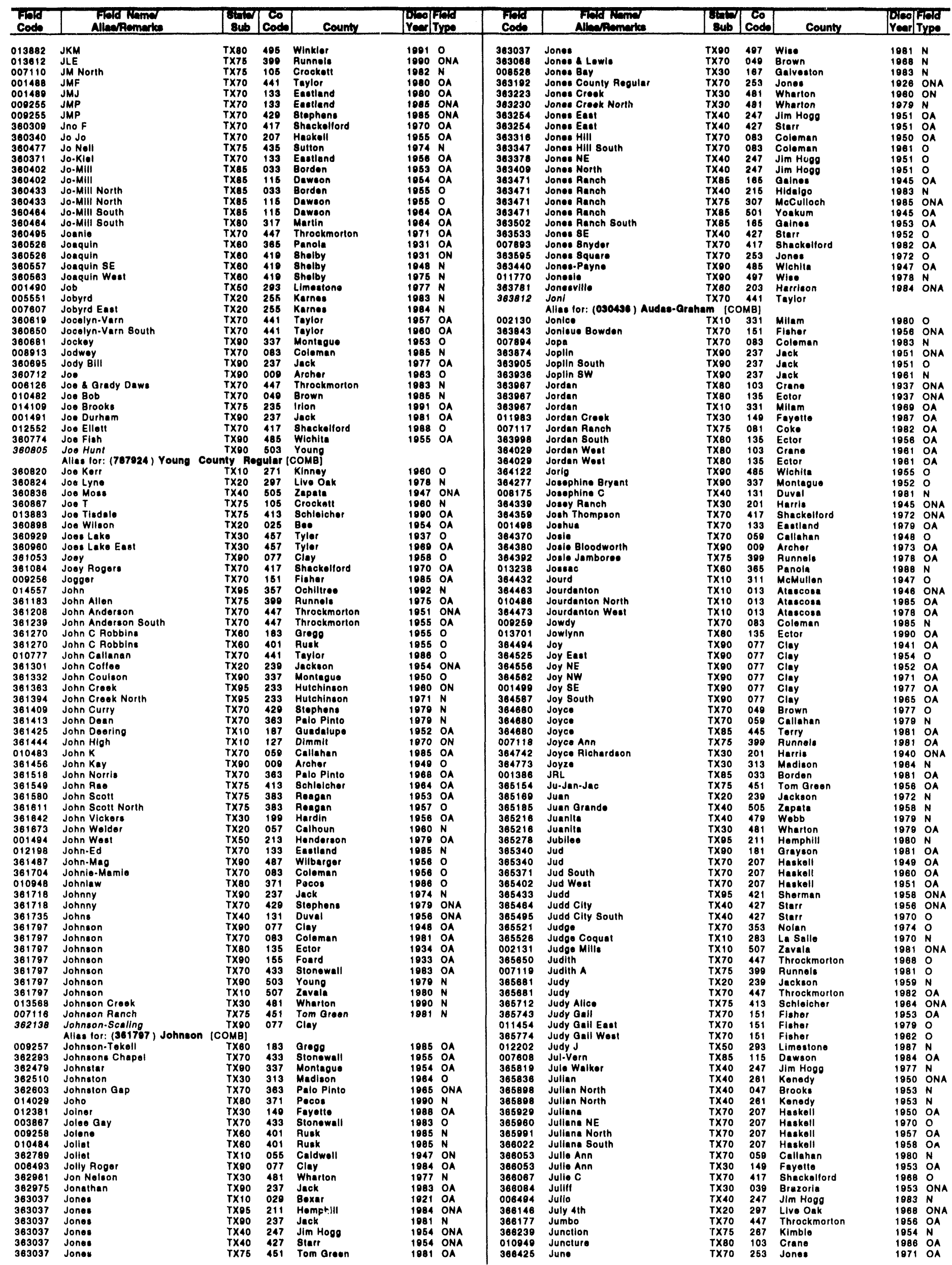


TEXAS

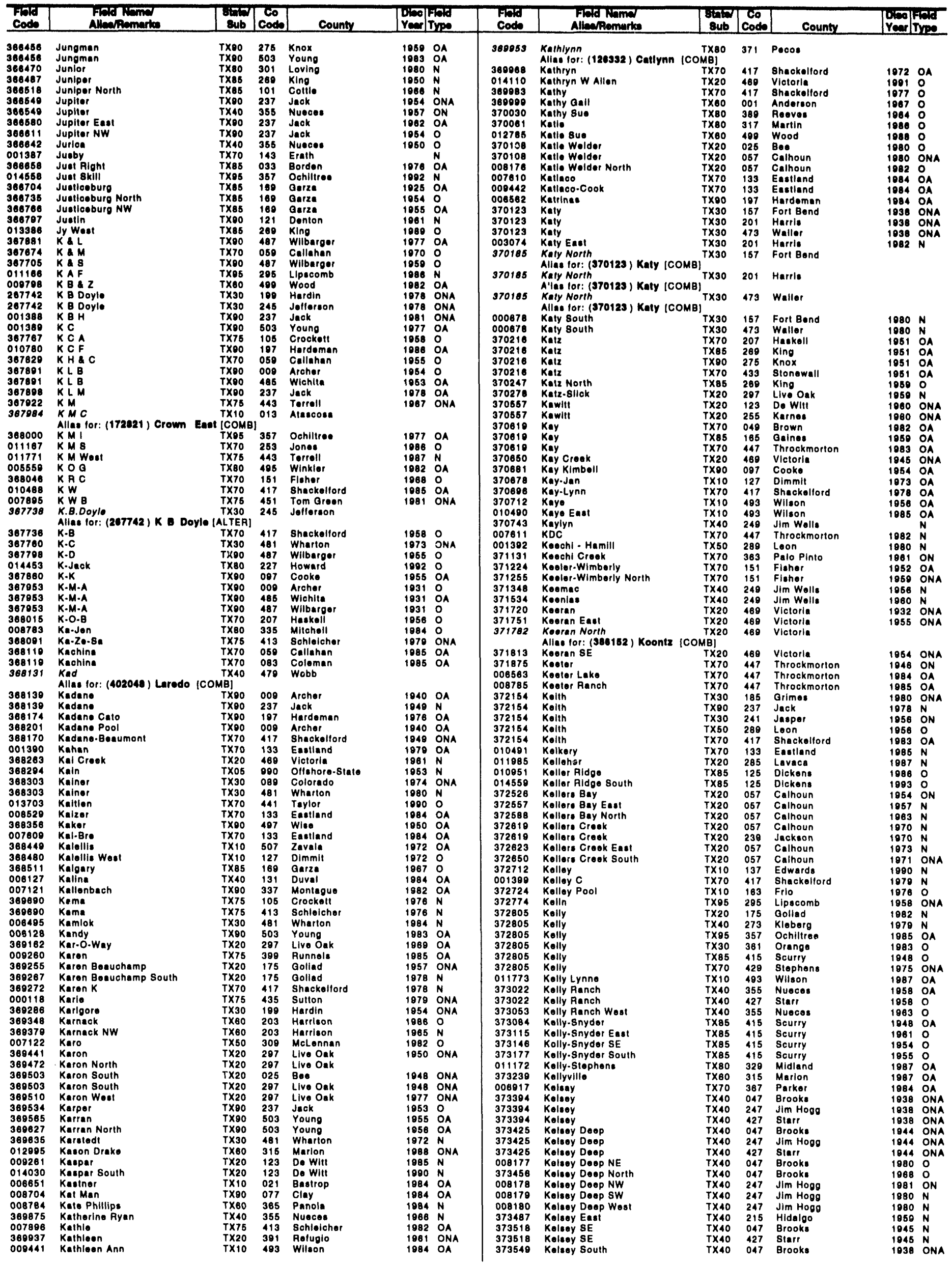


TEXAS

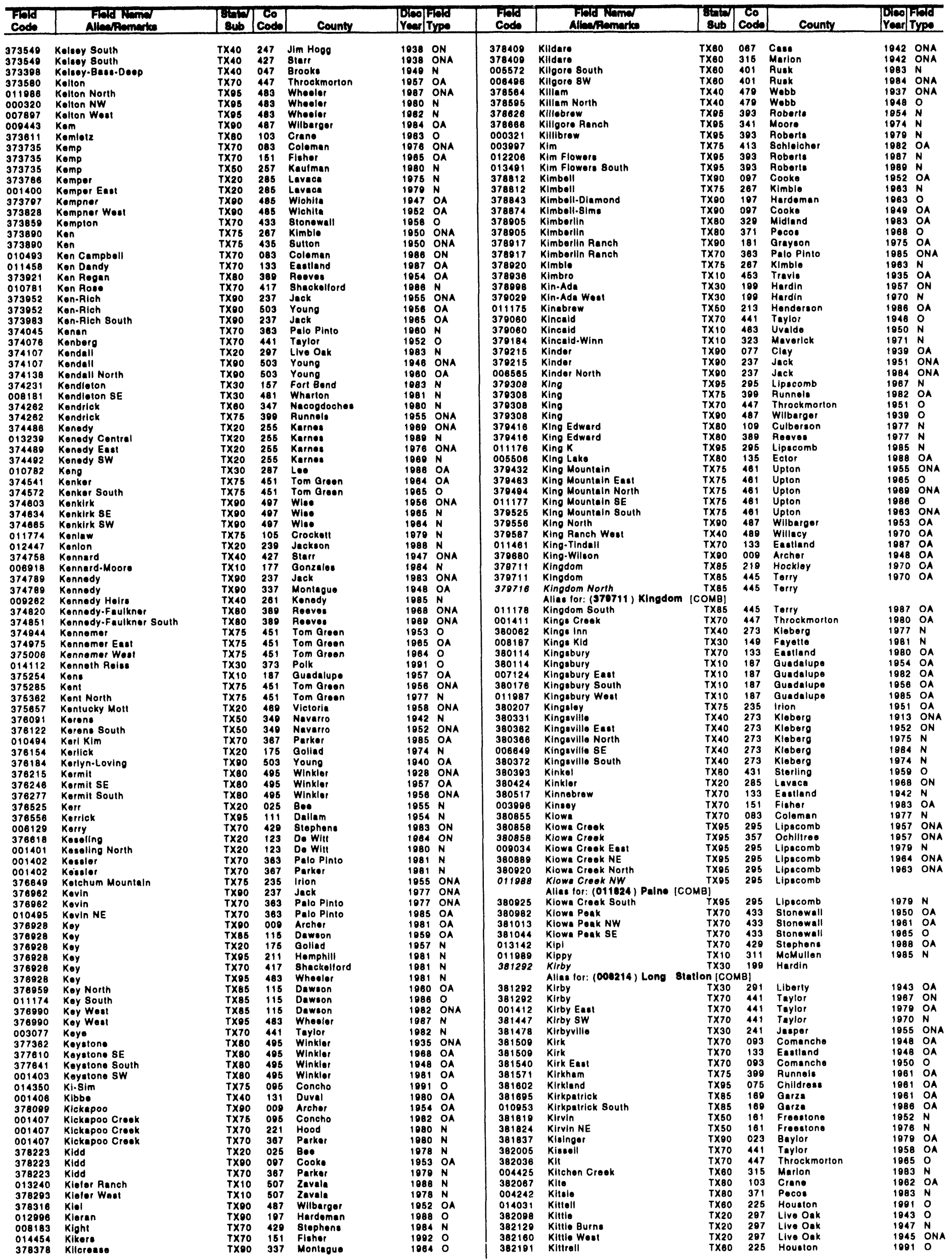


TEXAS

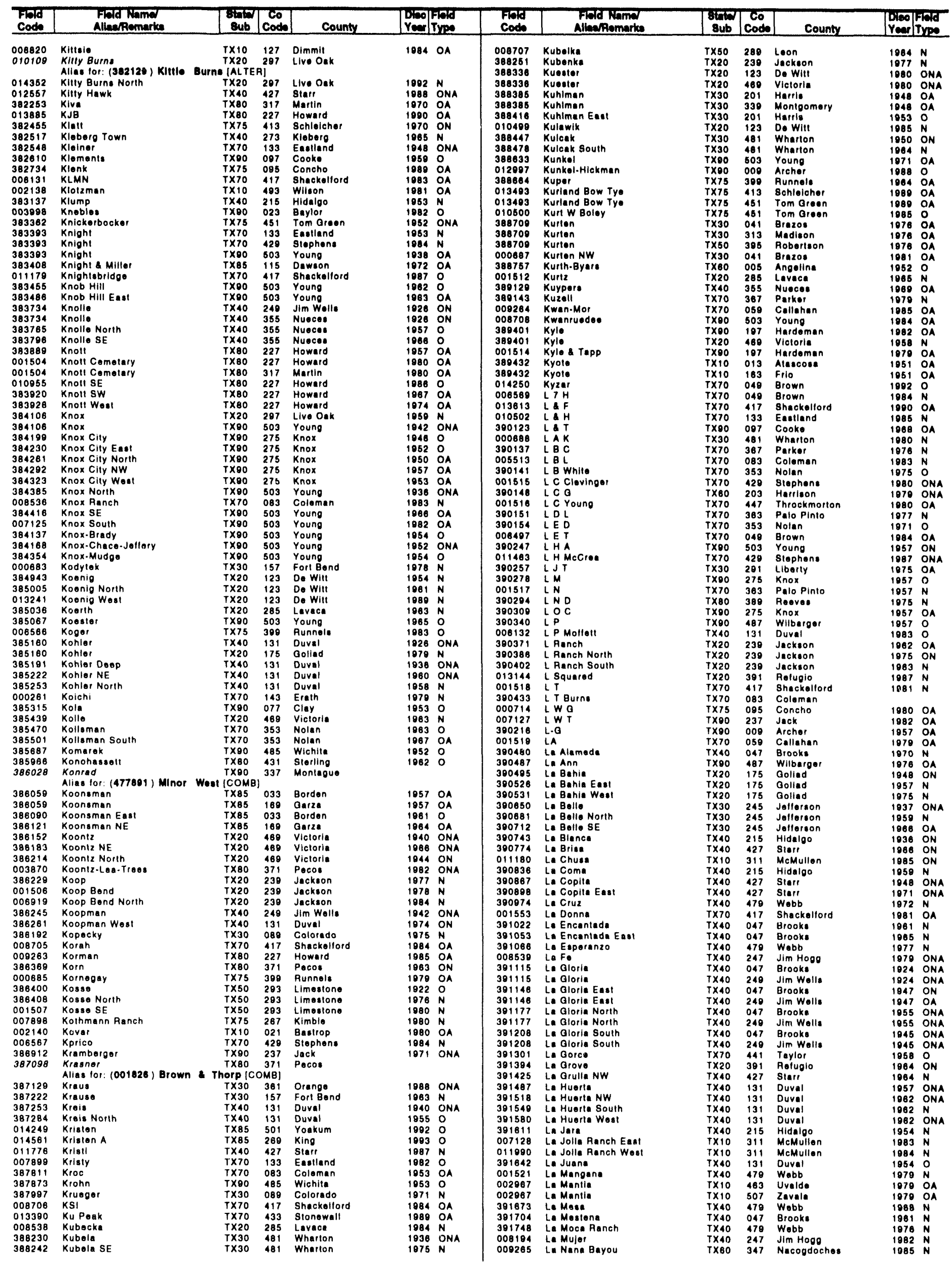


TEXAS

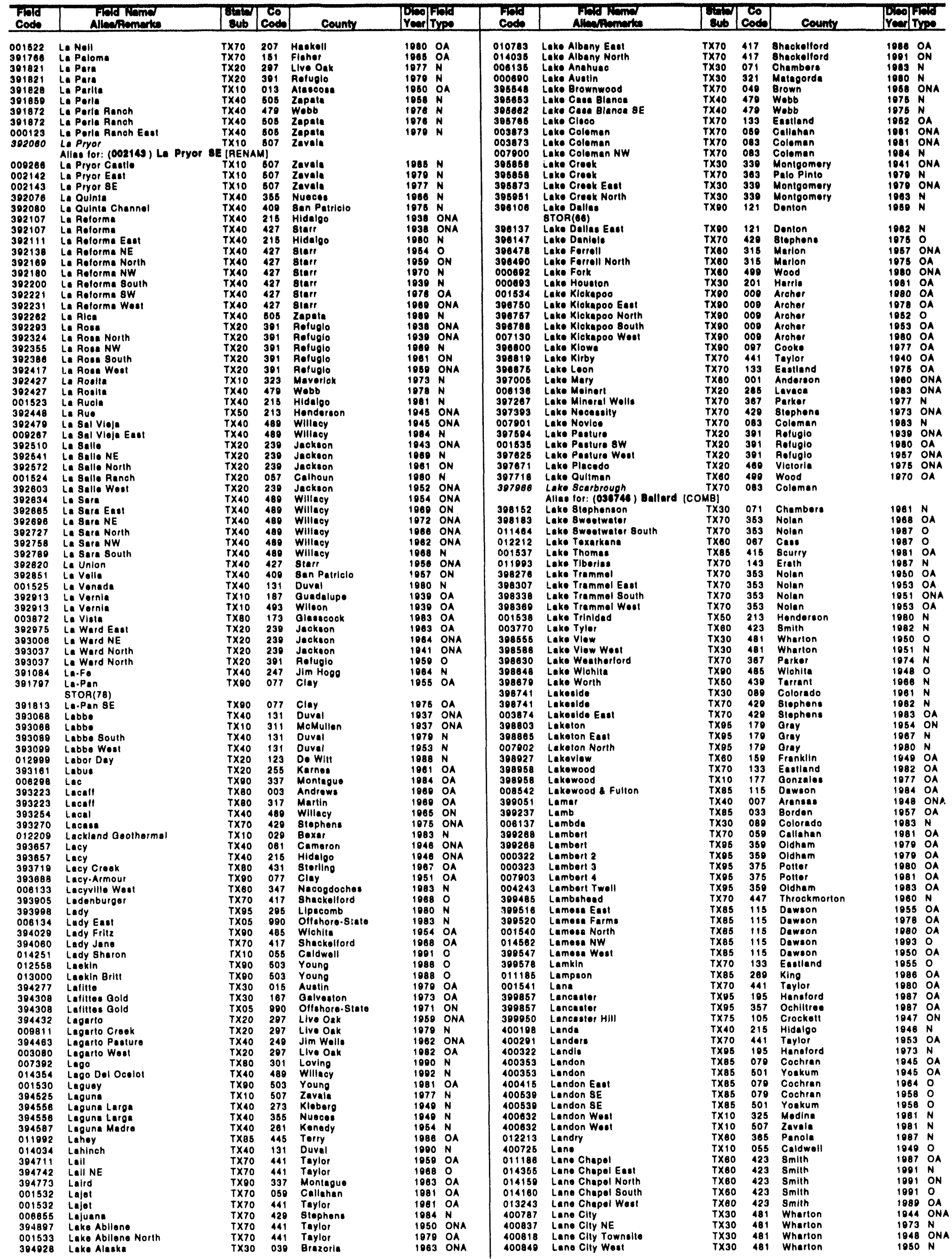


TEXAS

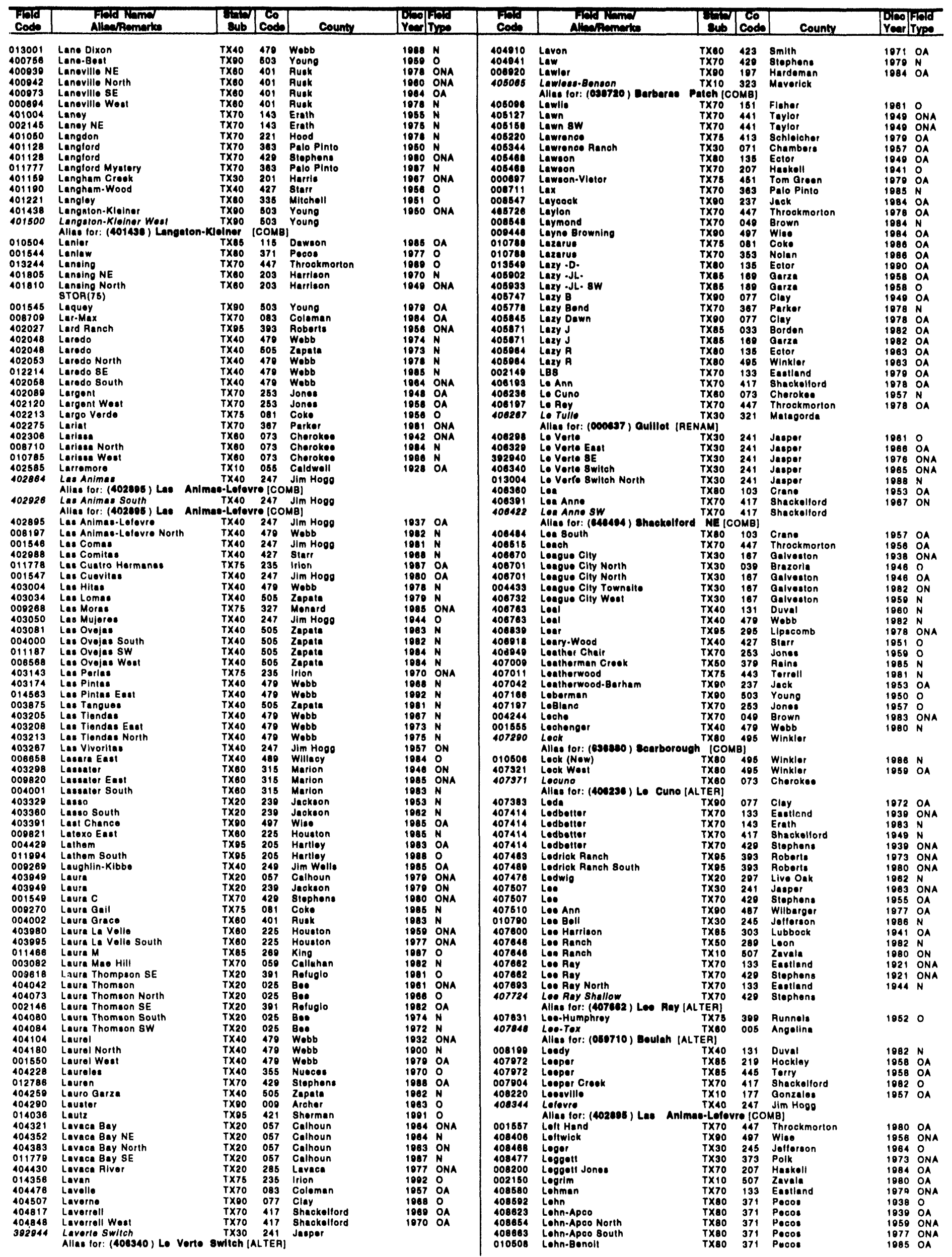


TEXAS

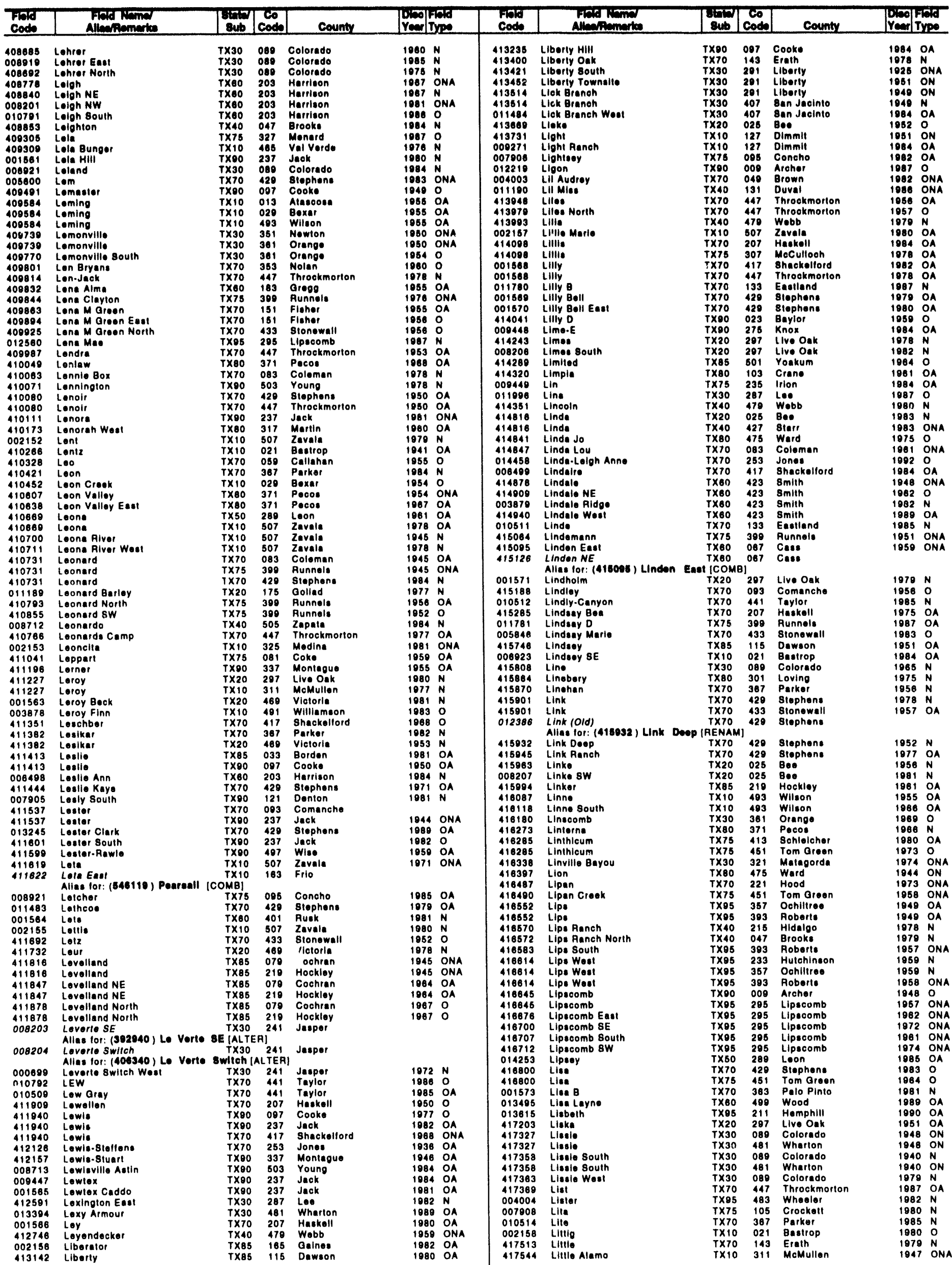


TEXAS

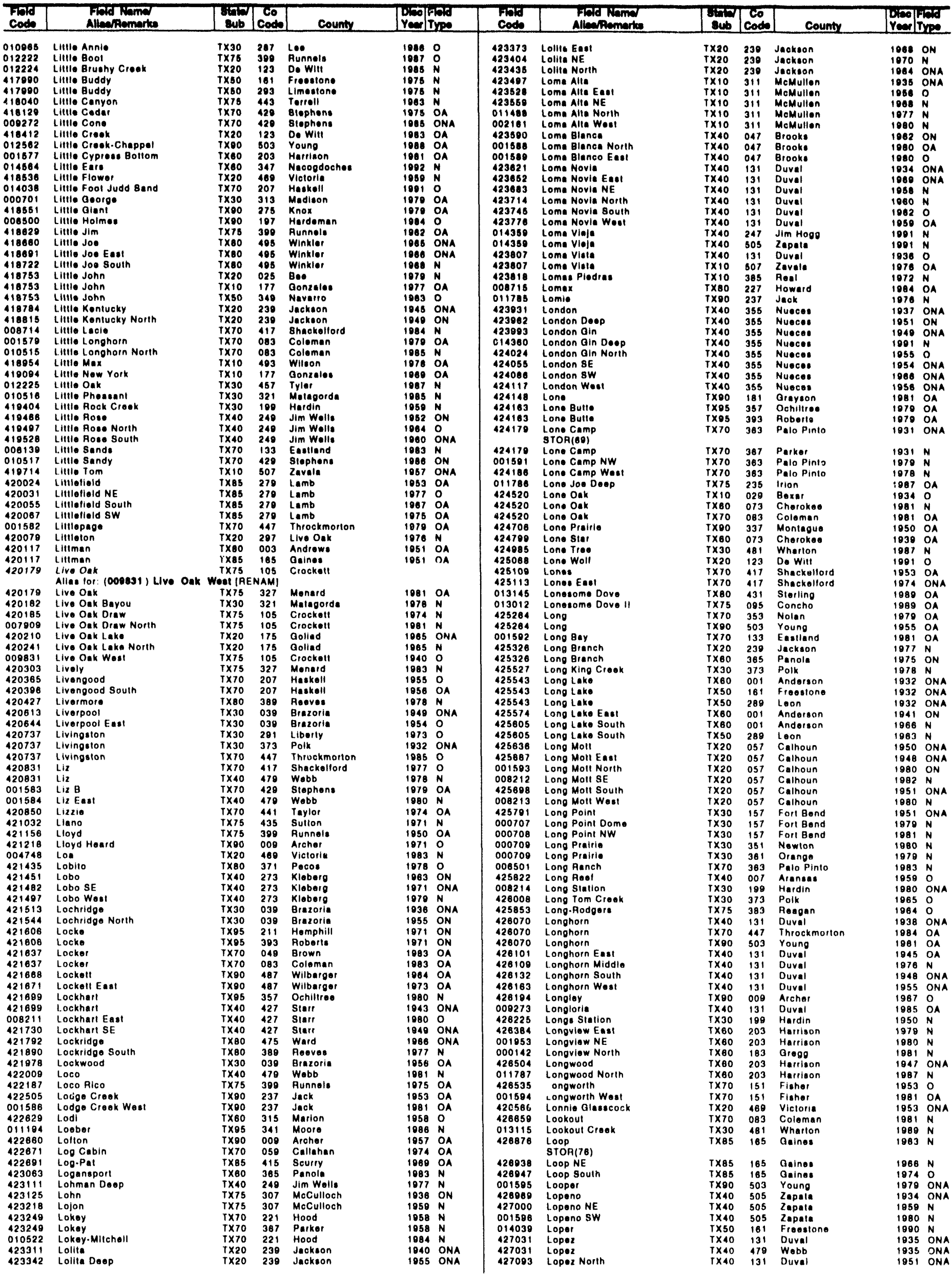


TEXAS

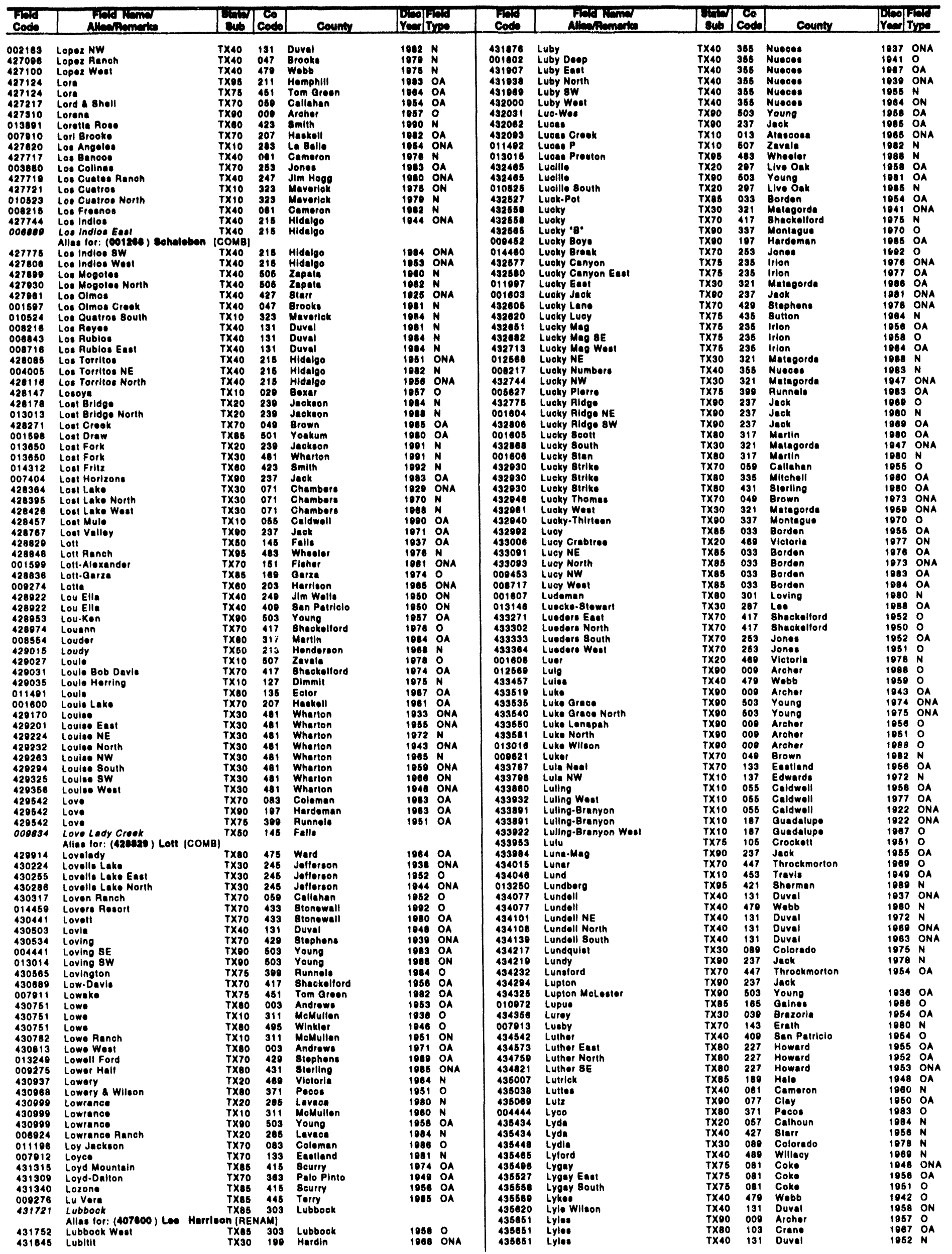


TEXAS

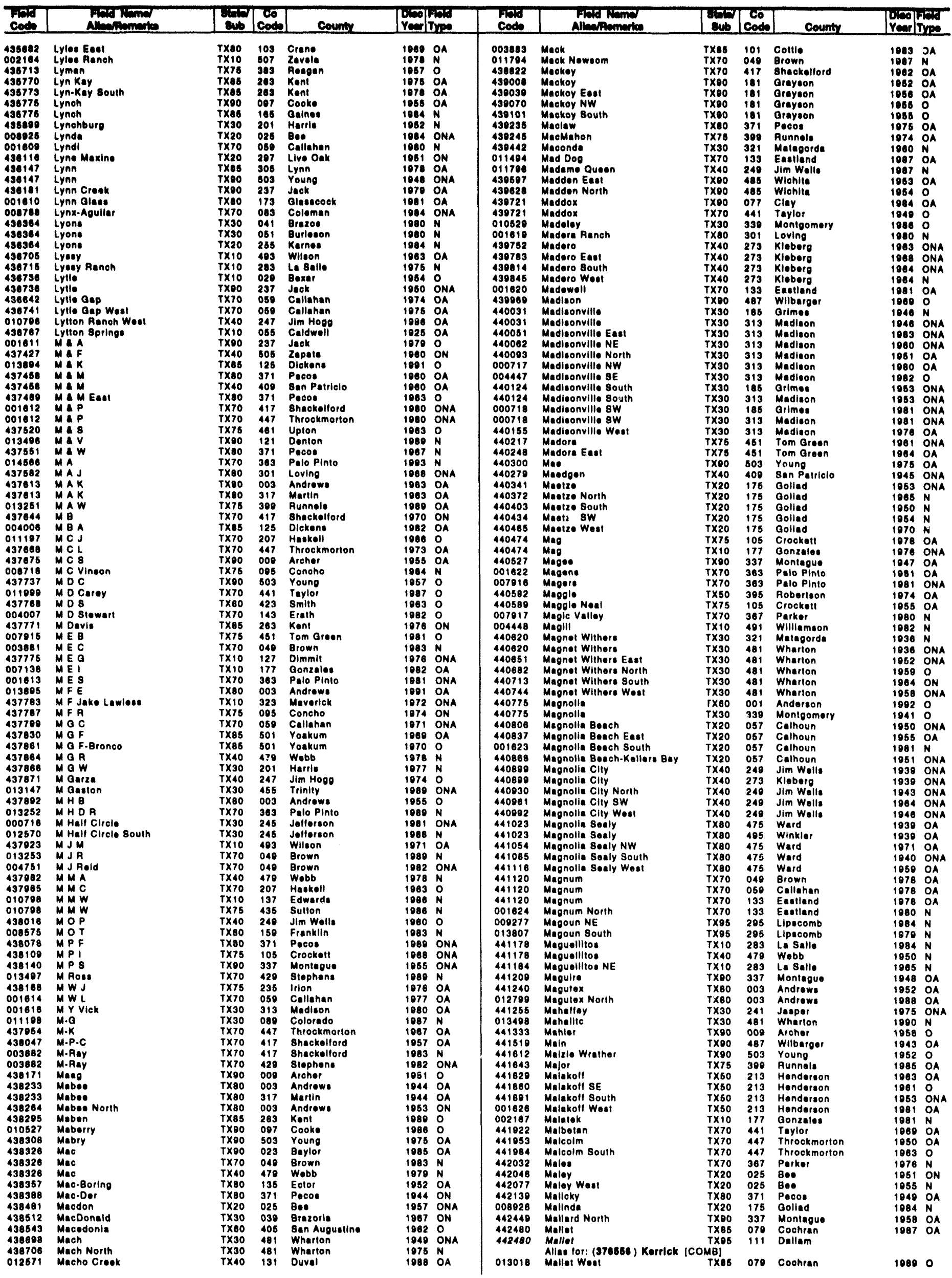


TEXAS

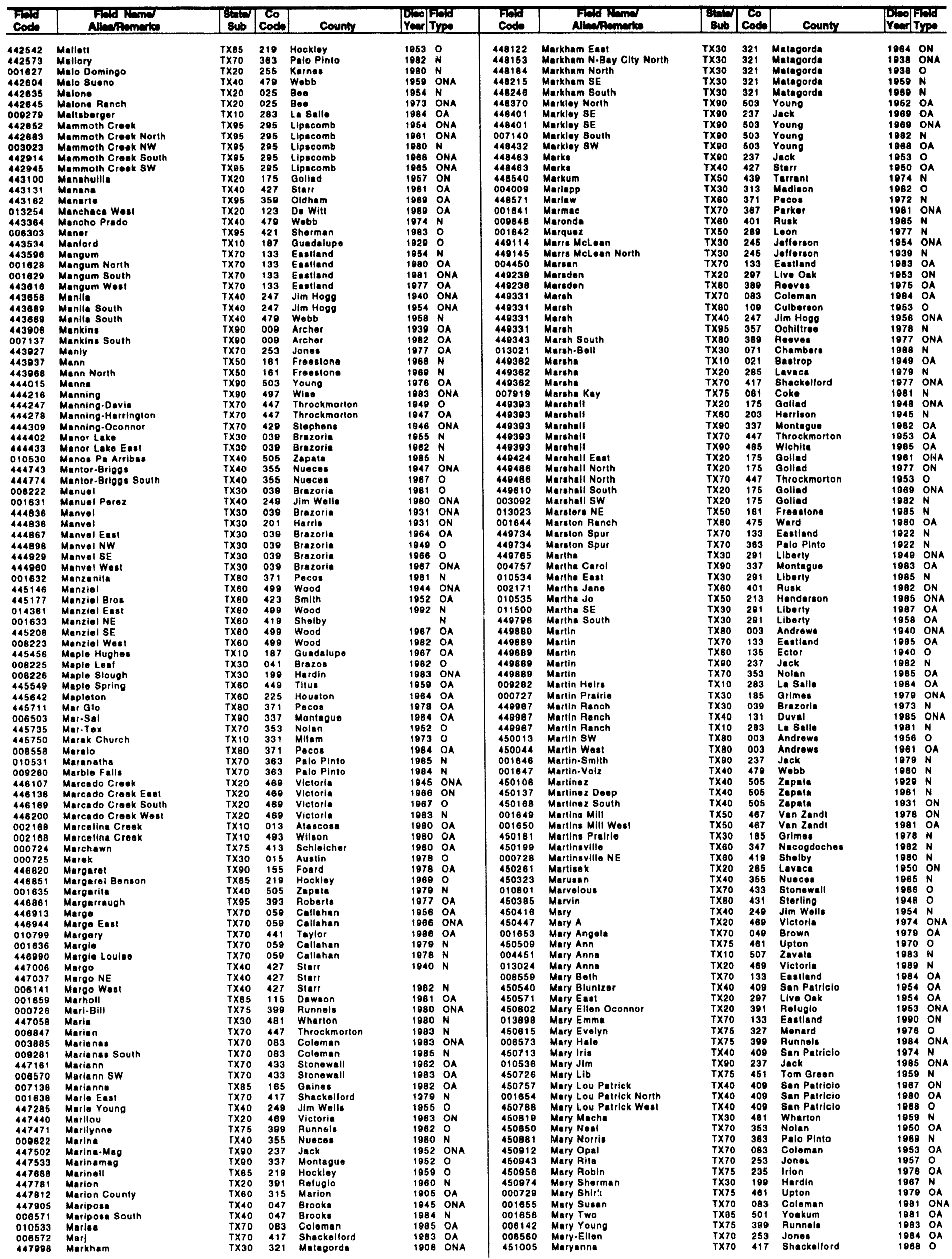


TEXAS

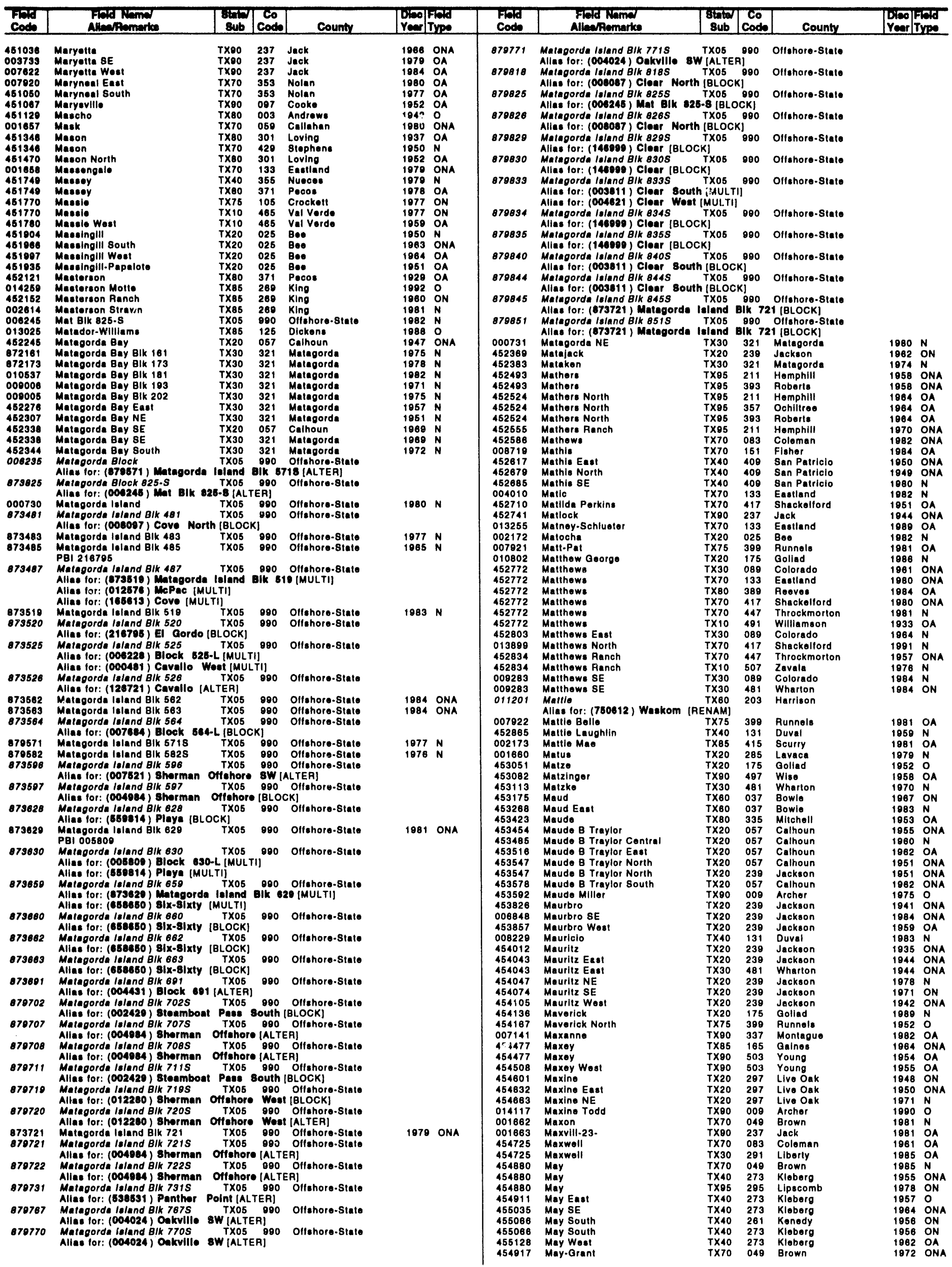


TEXAS

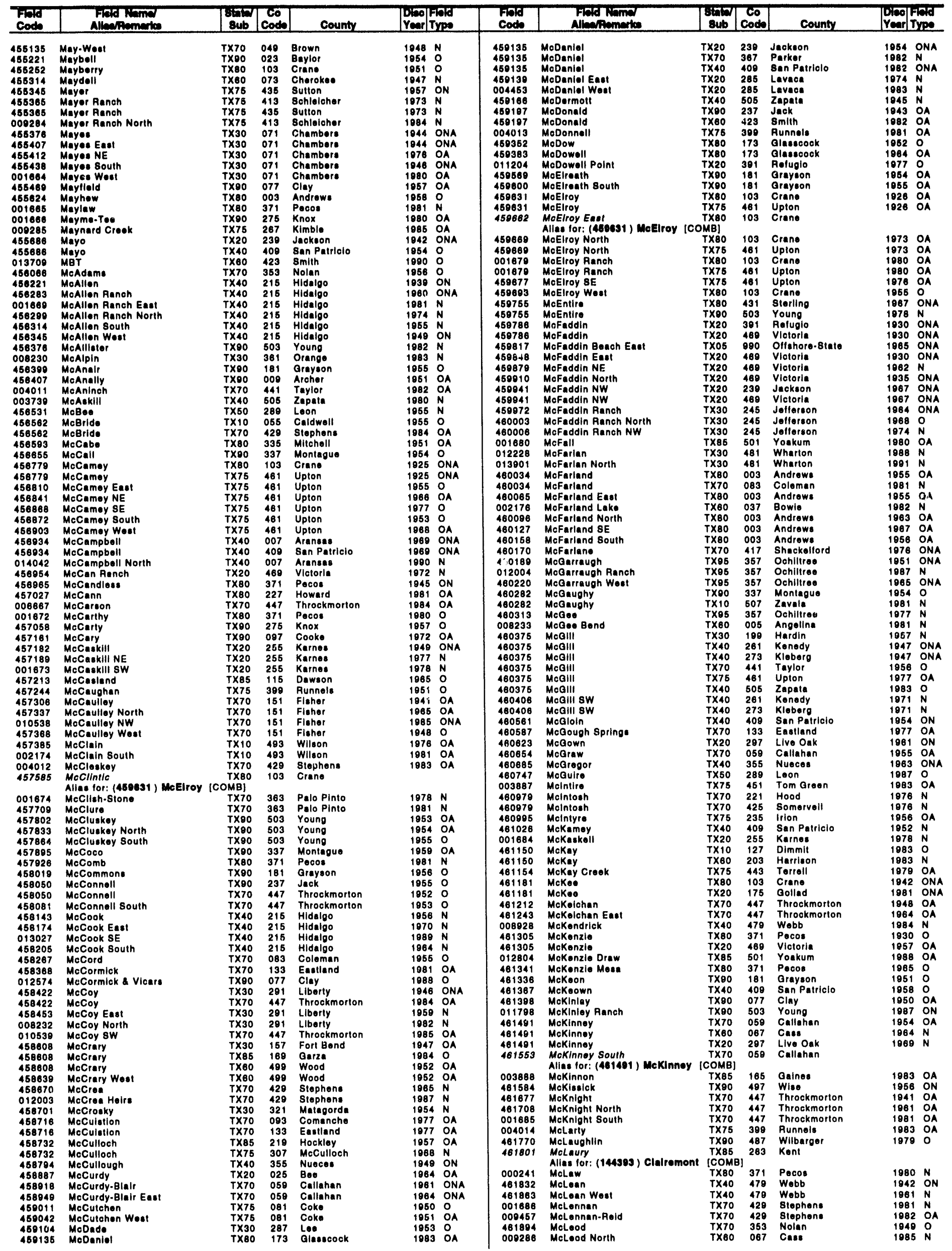


TEXAS

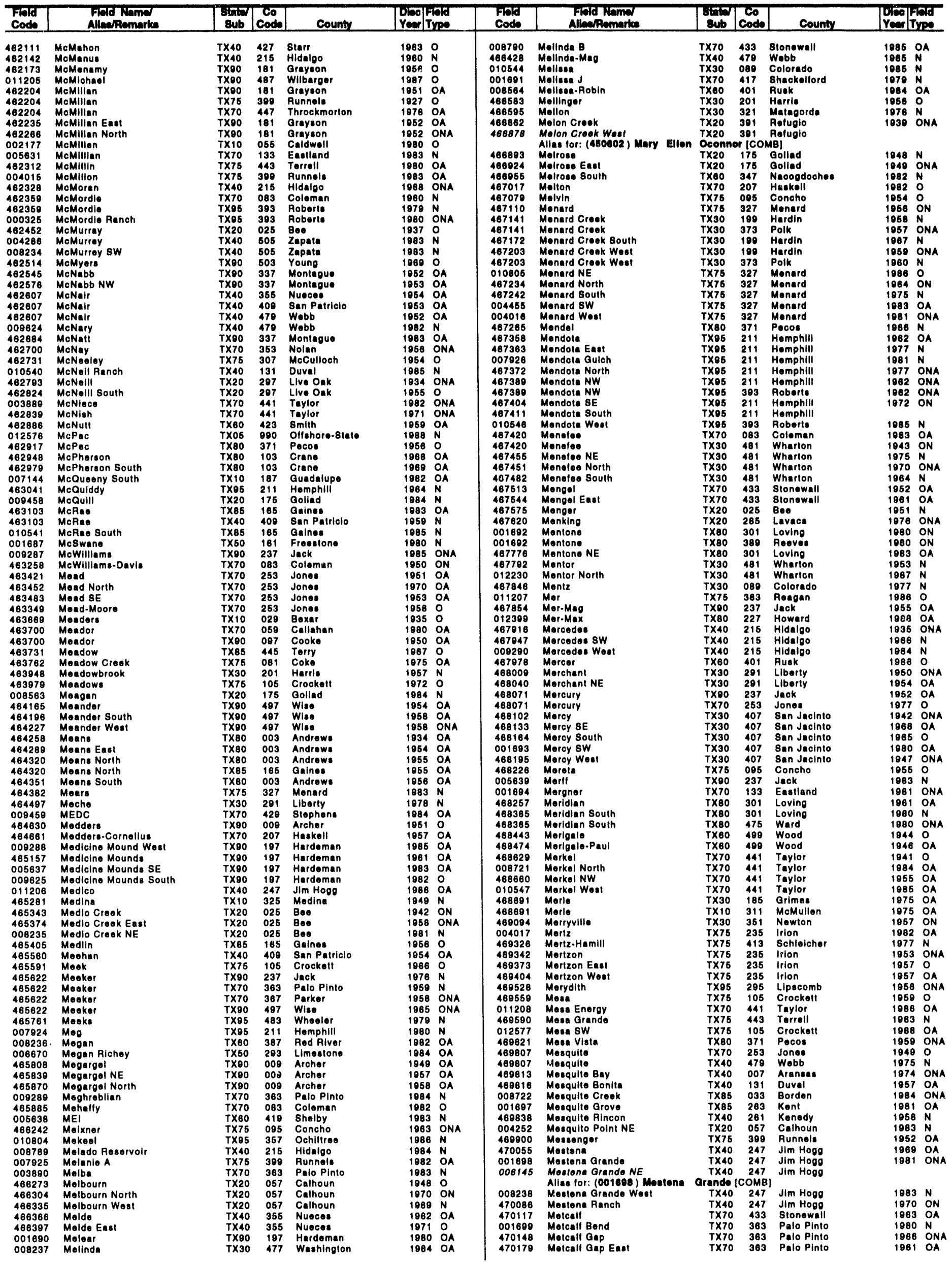


TEXAS

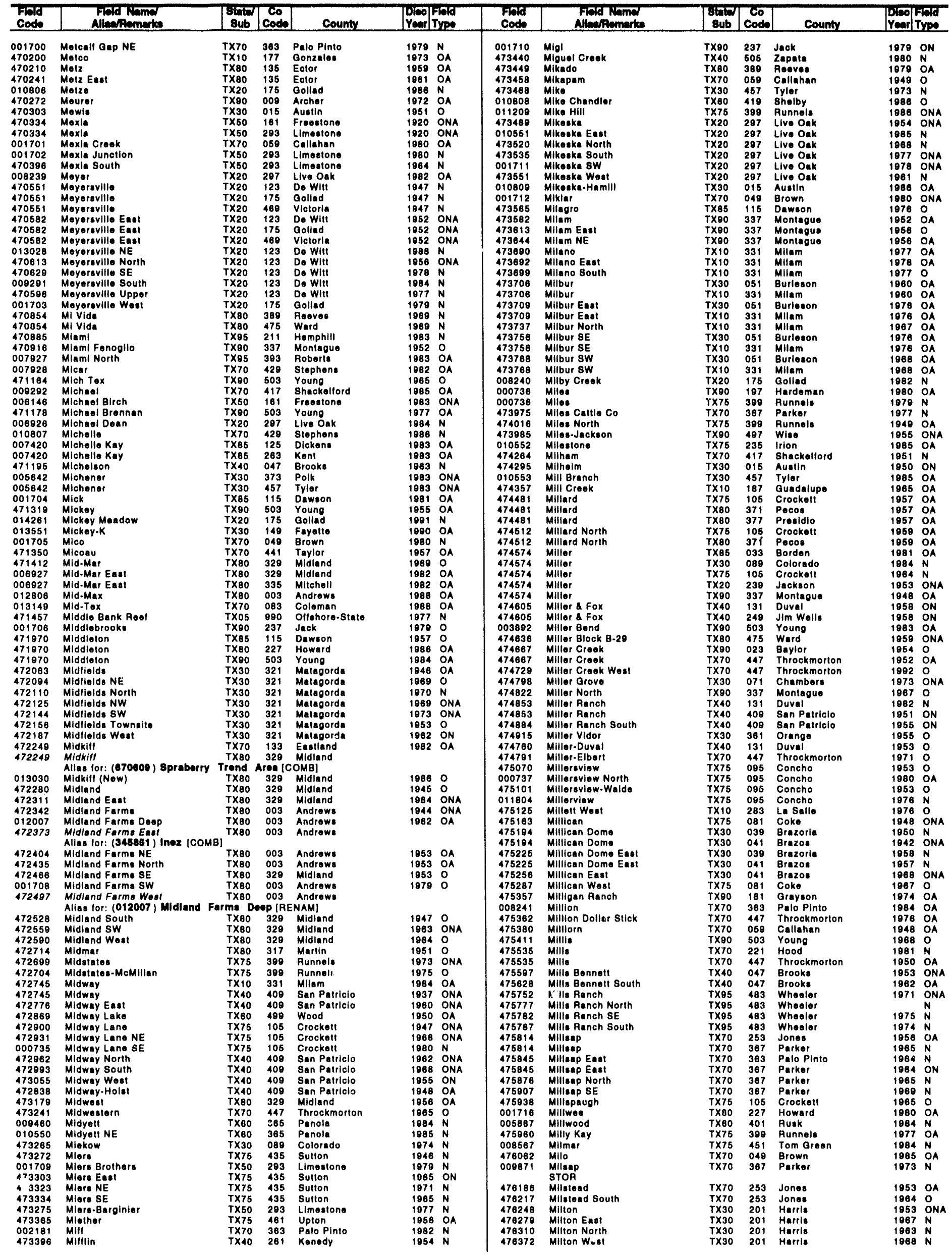


TEXAS

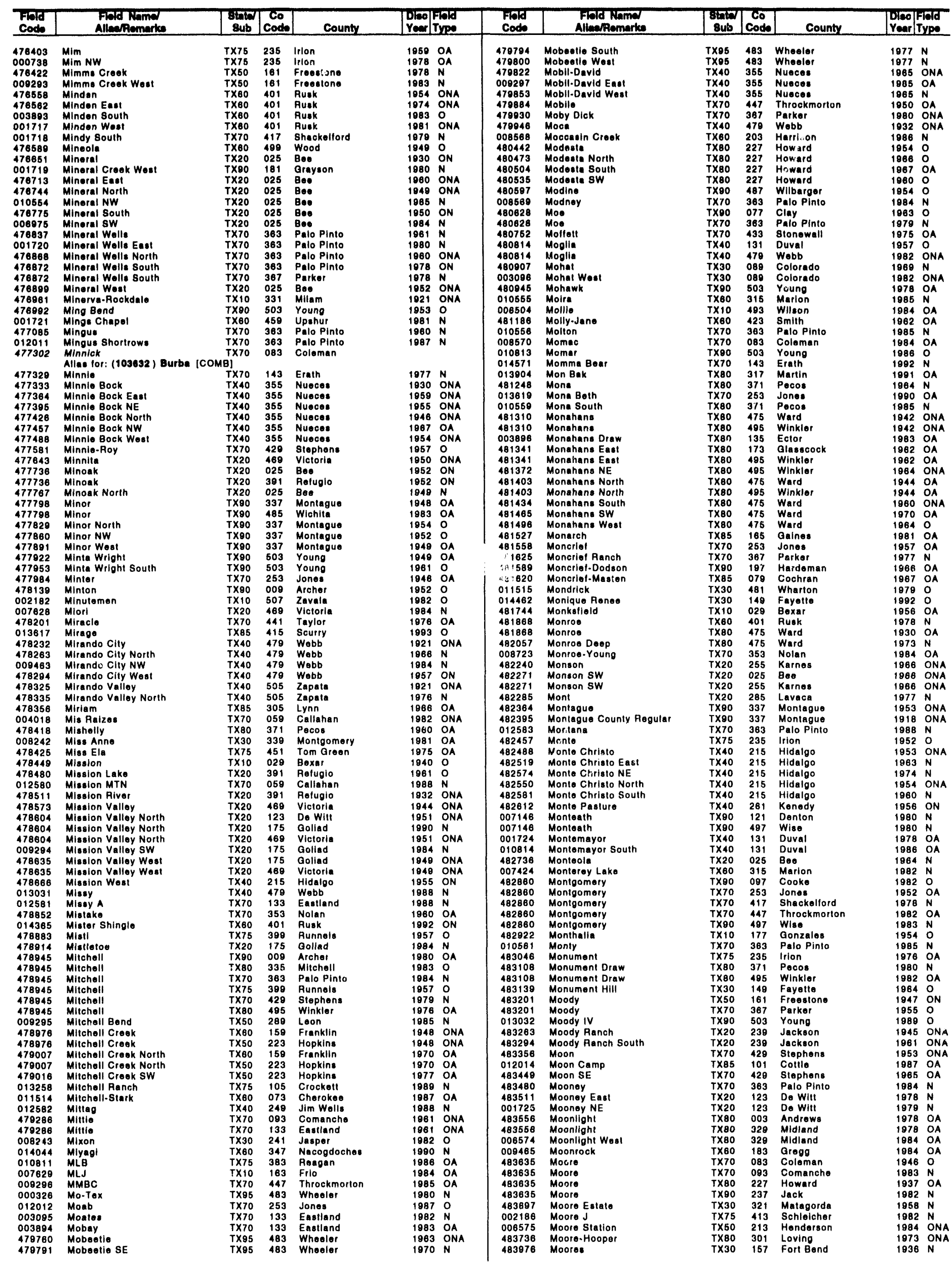


TEXAS

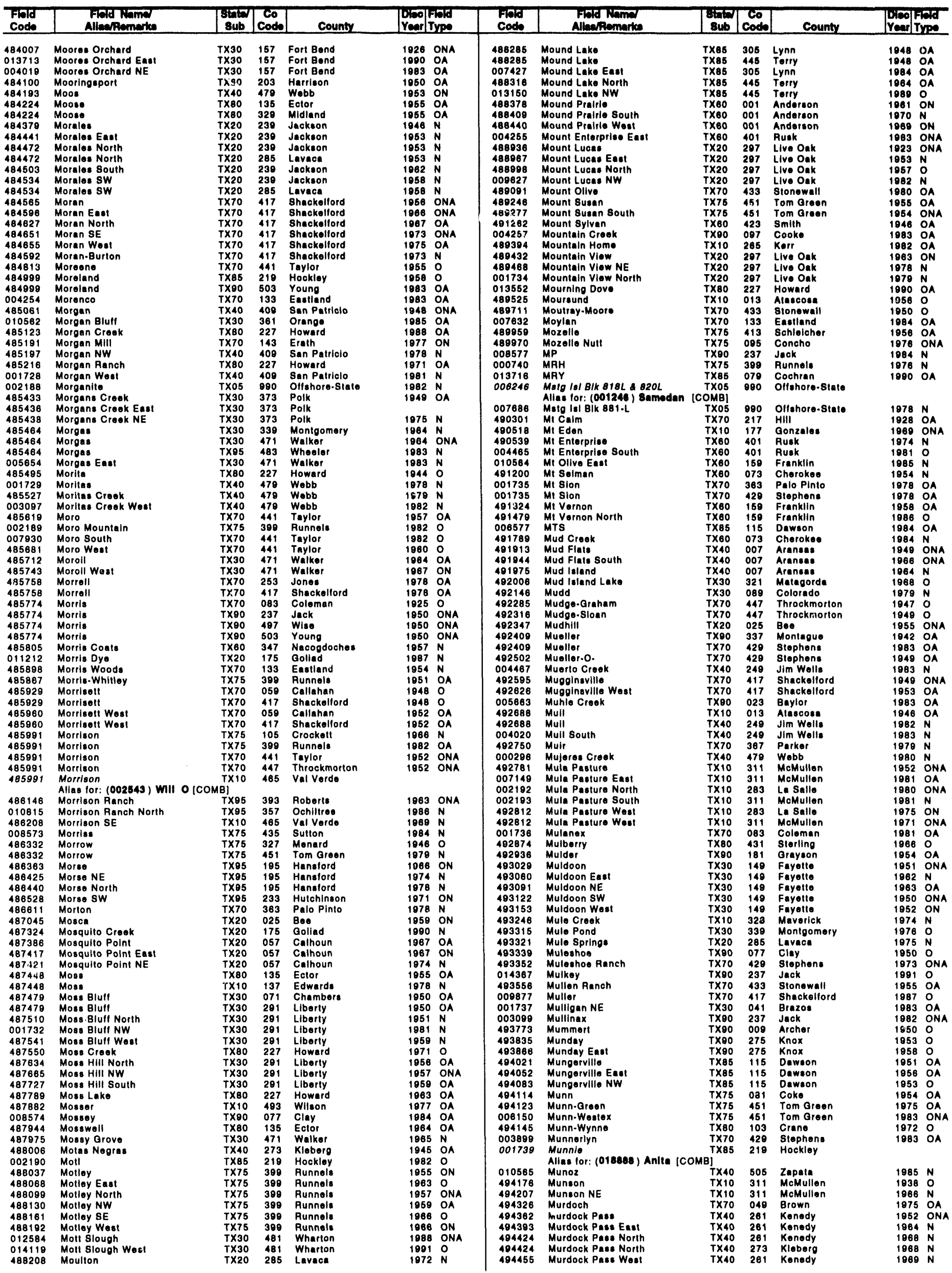


TEXAS

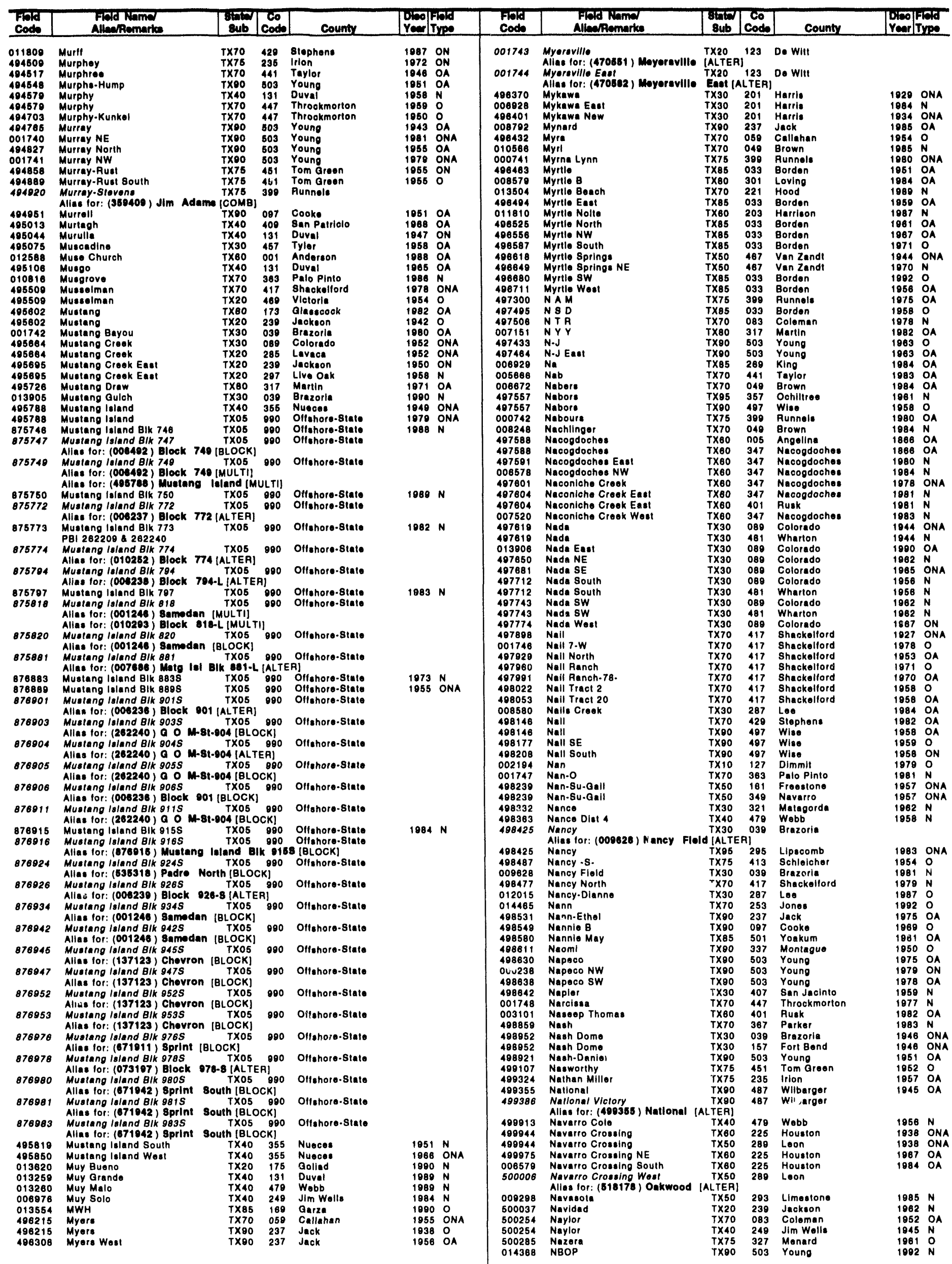


TEXAS

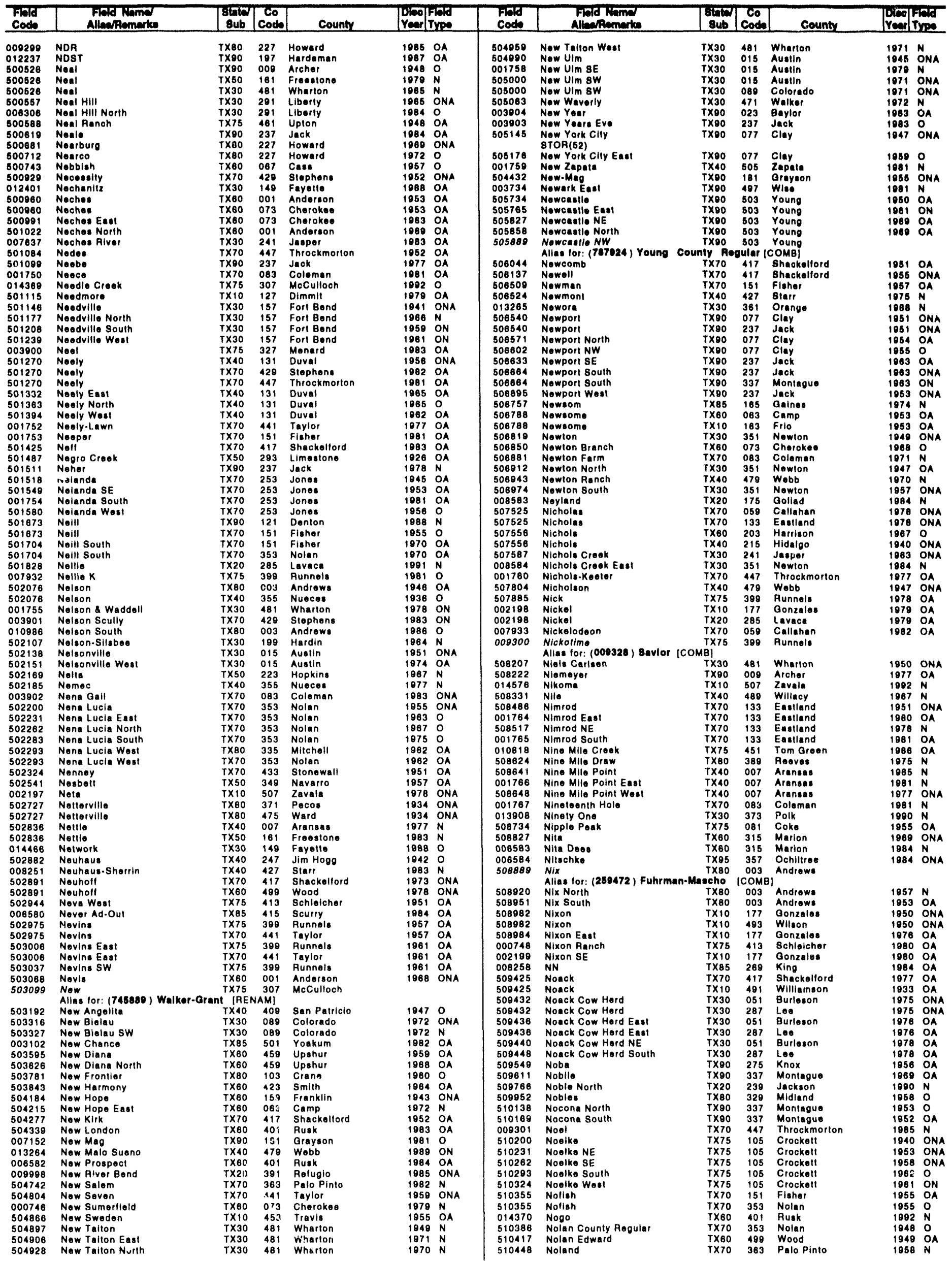


TEXAS

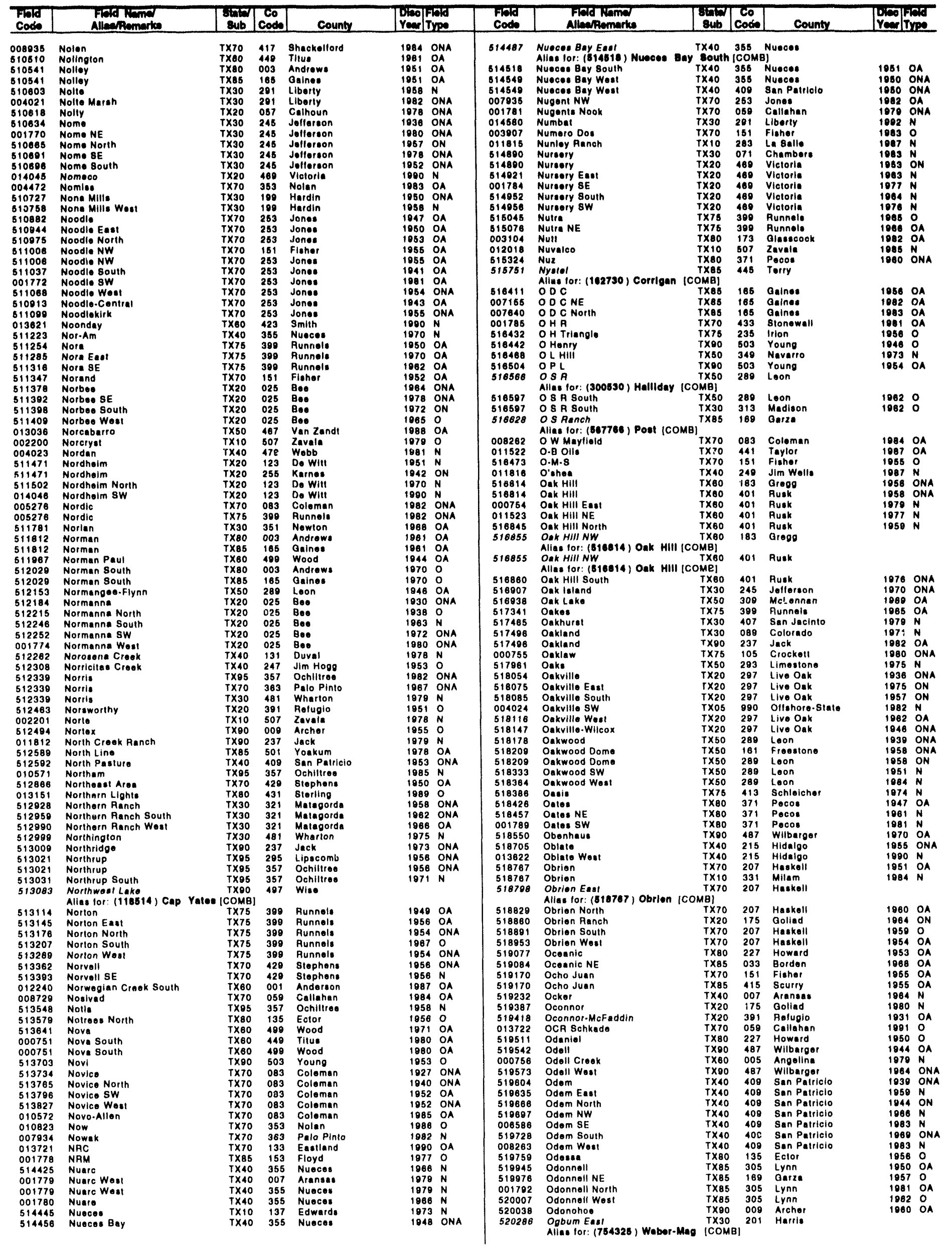


TEXAS

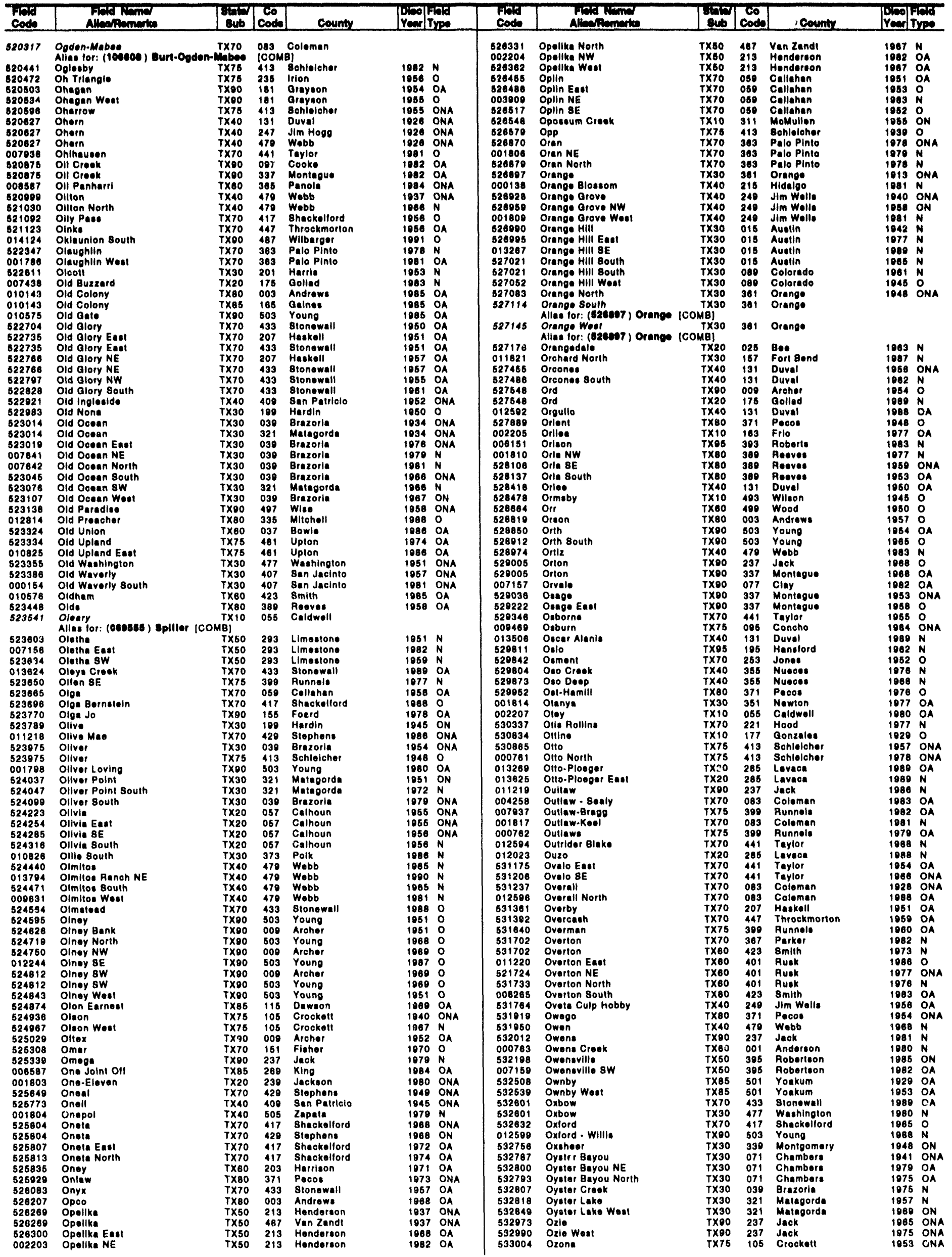


TEXAS

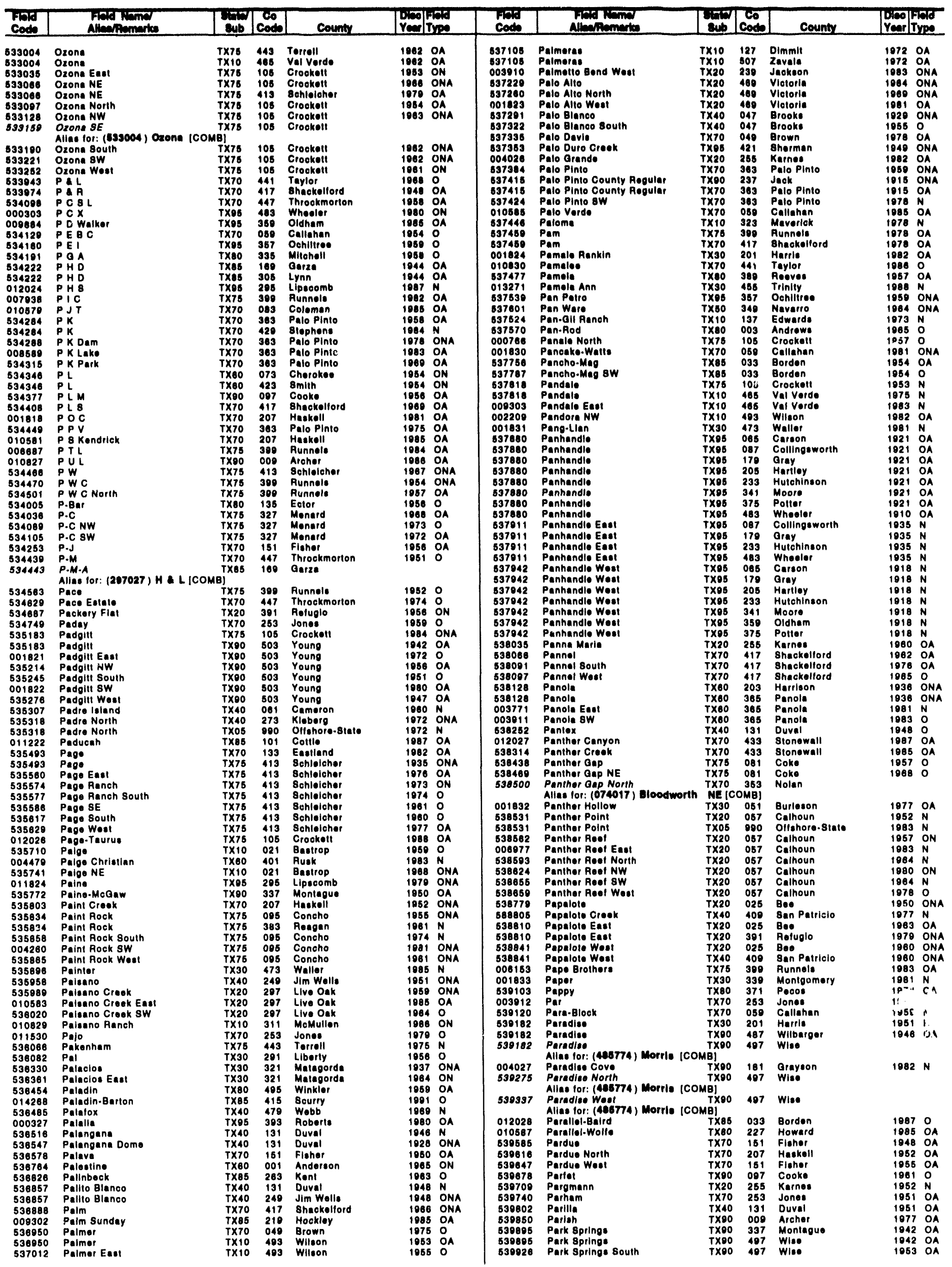


TEXAS

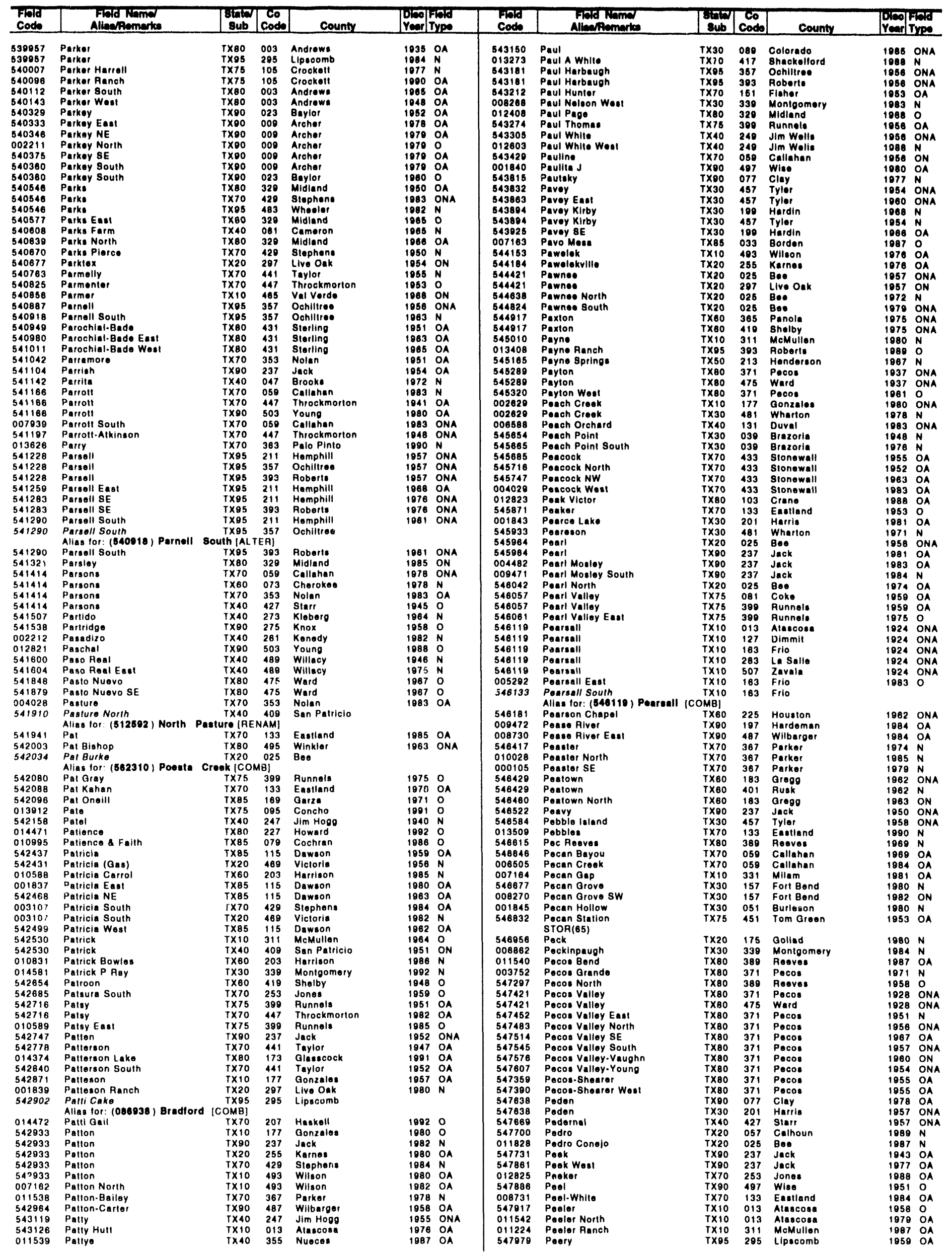


TEXAS

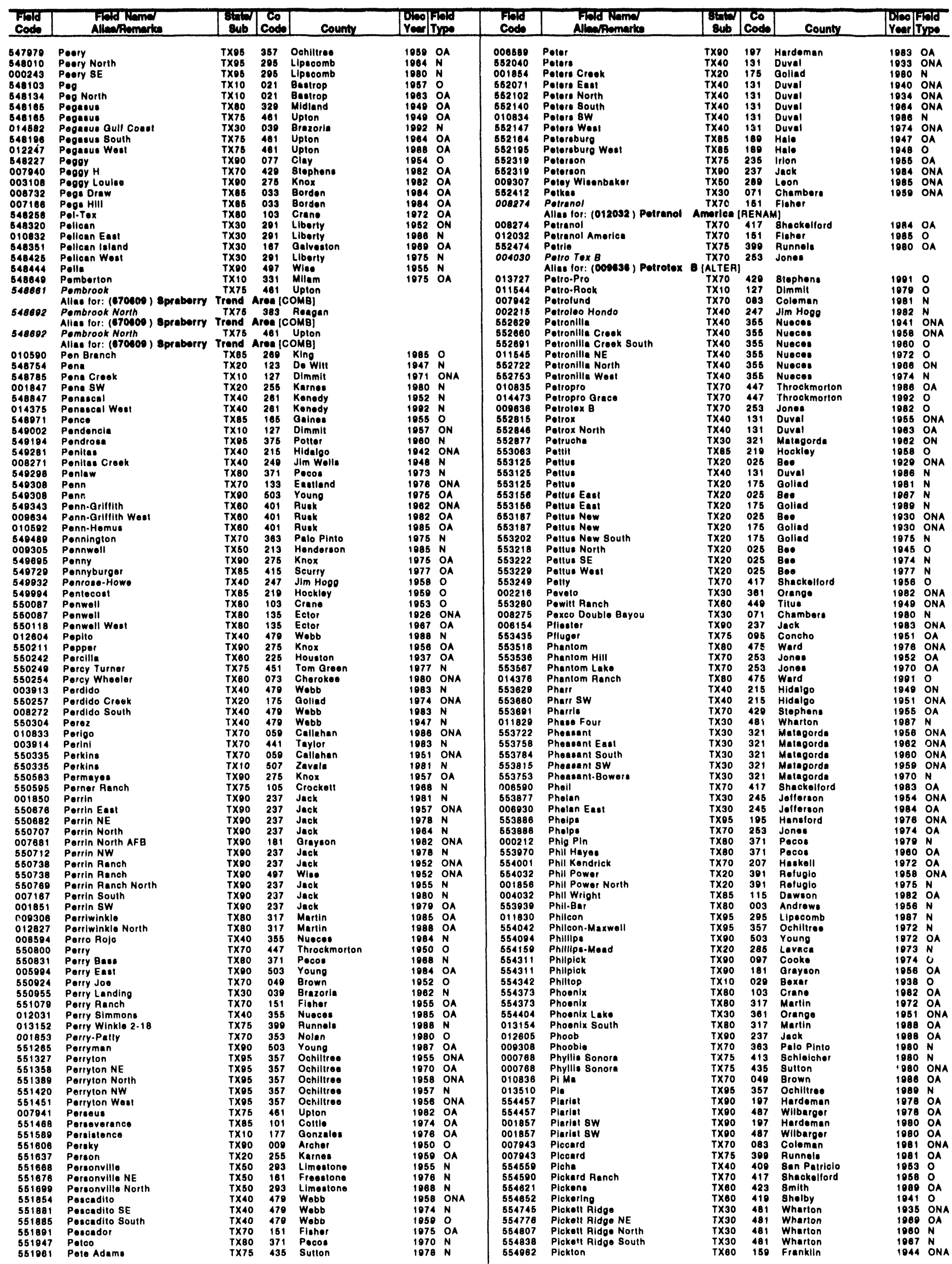




$$
\begin{aligned}
& \text { 震 }
\end{aligned}
$$

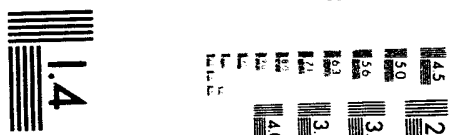

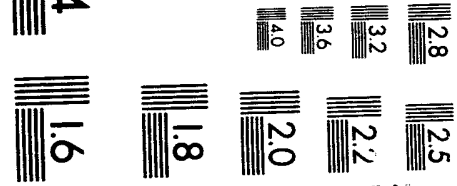



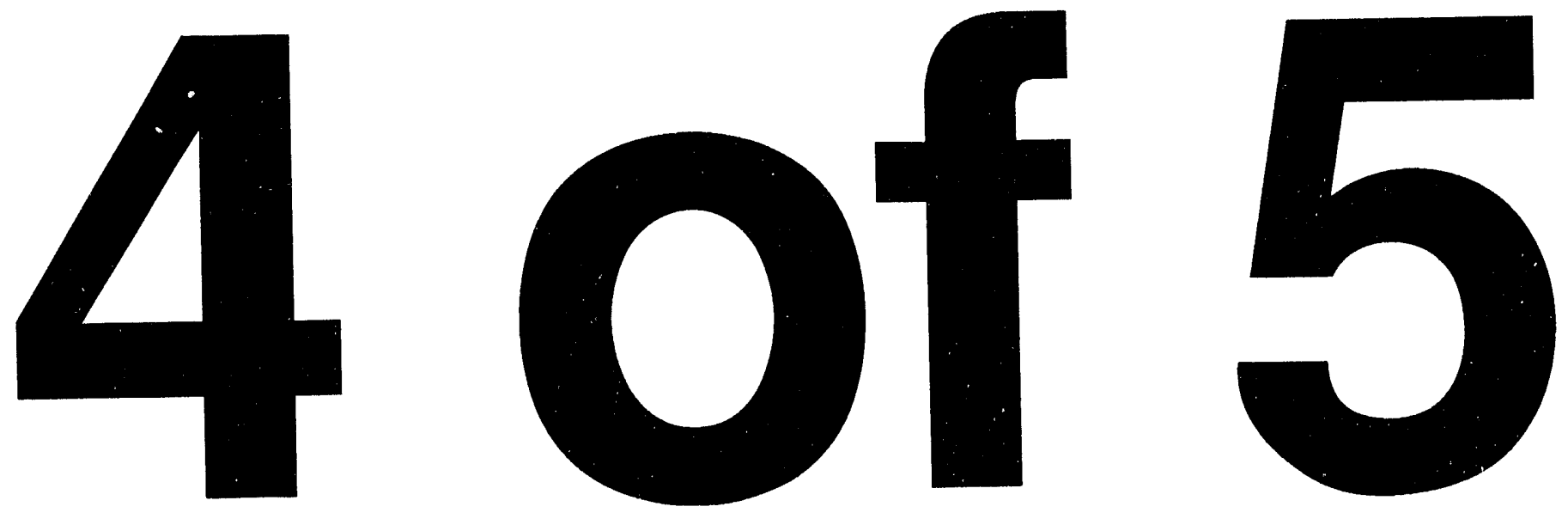
TEXAS

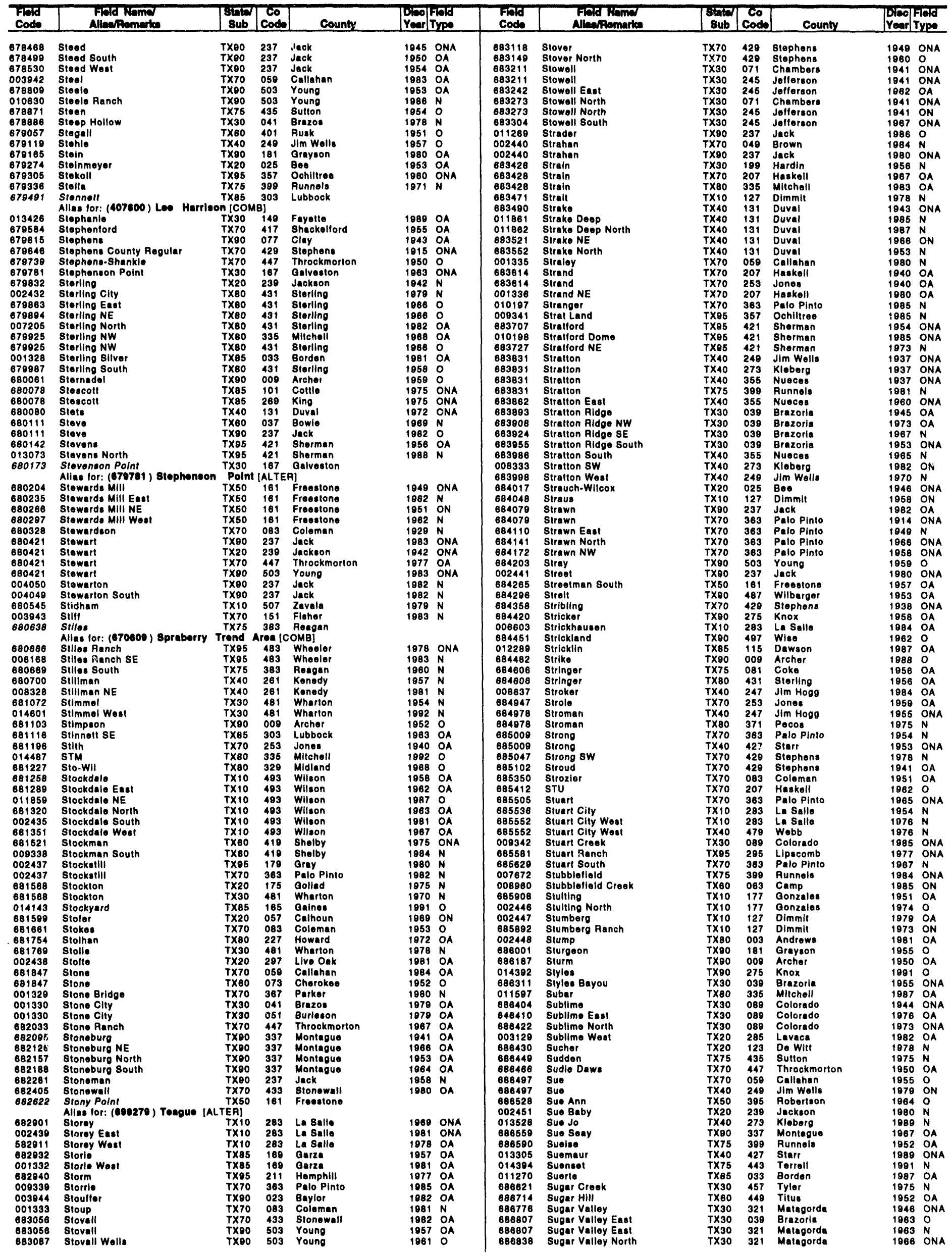


TEXAS

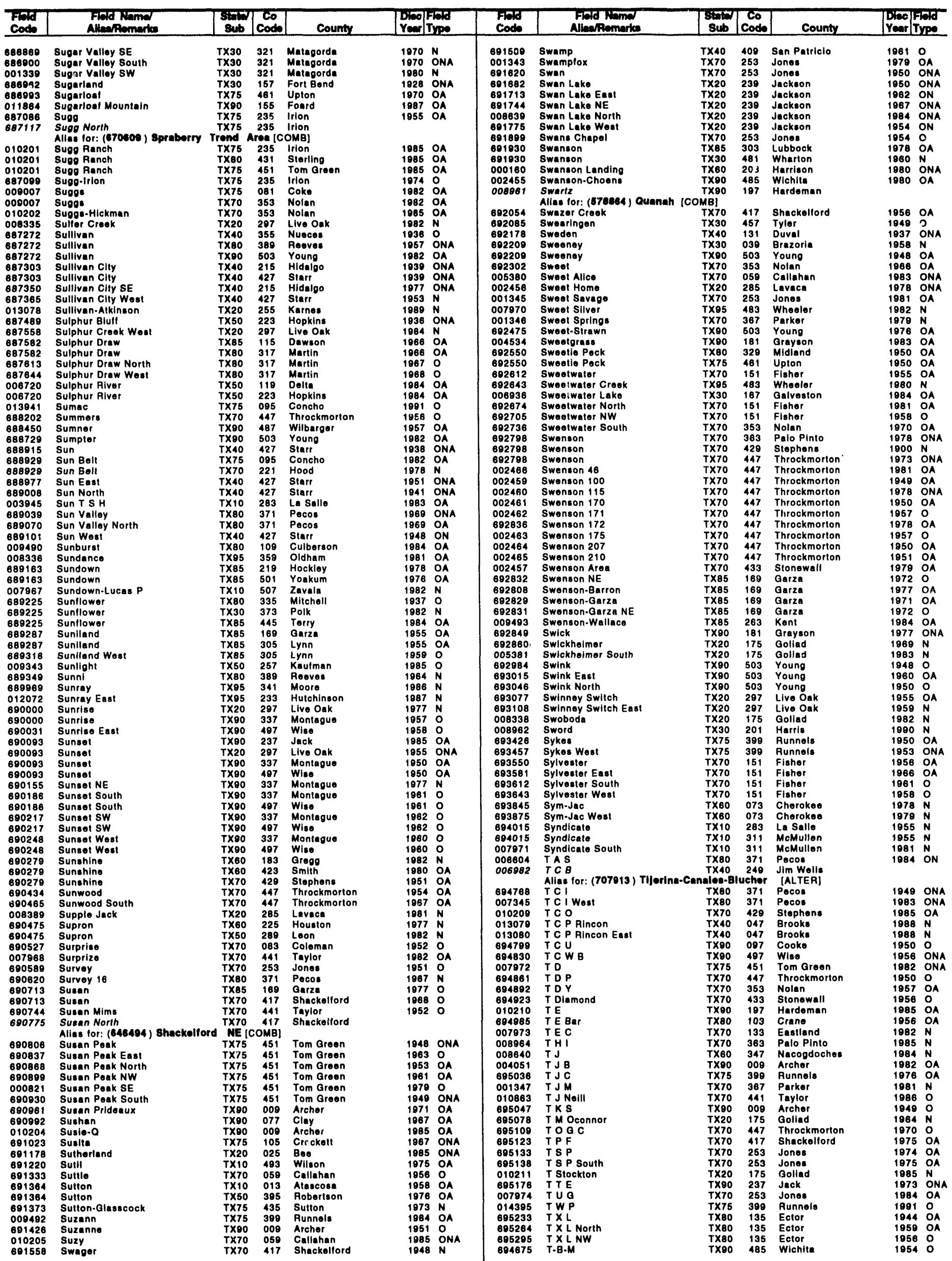


TEXAS

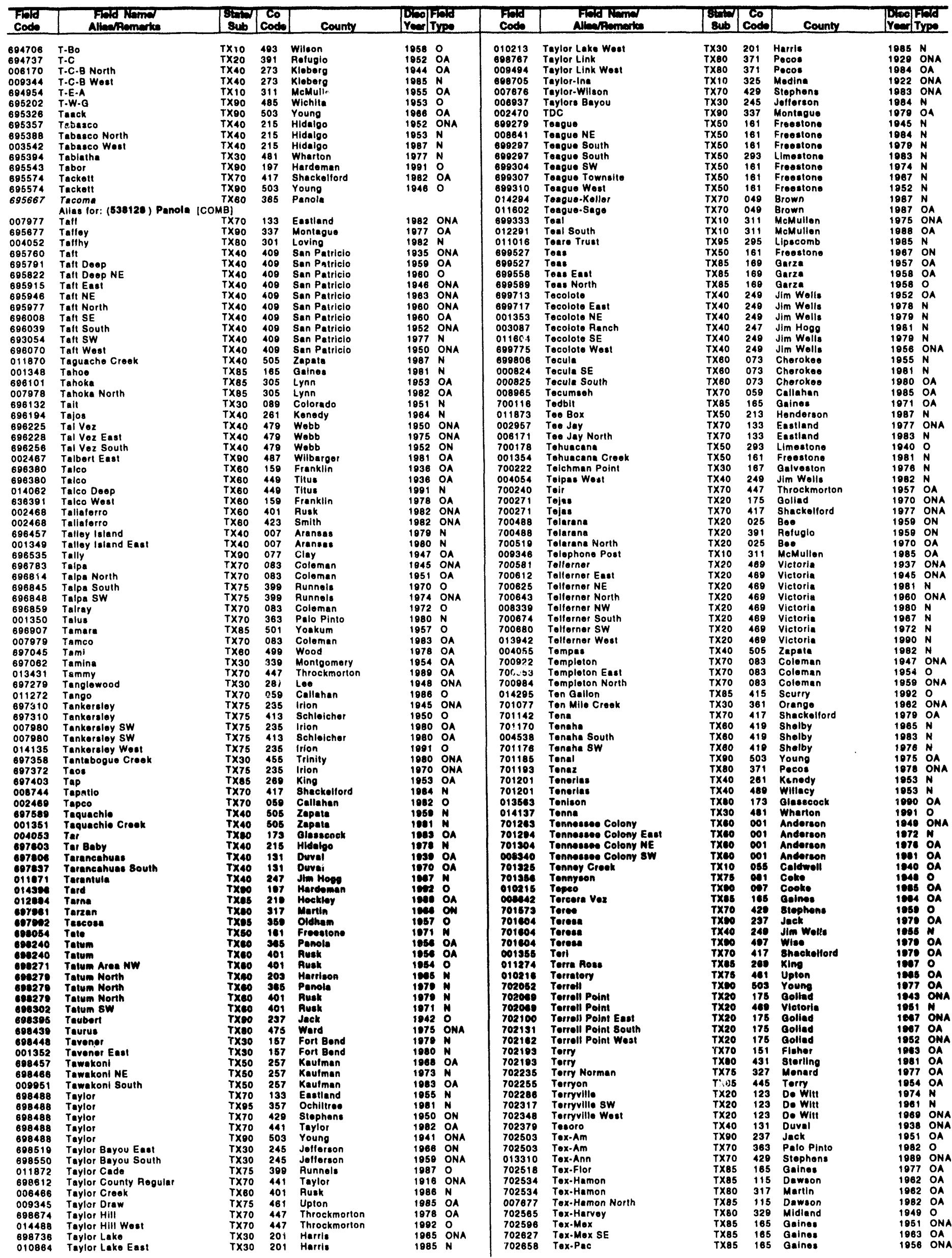


TEXAS

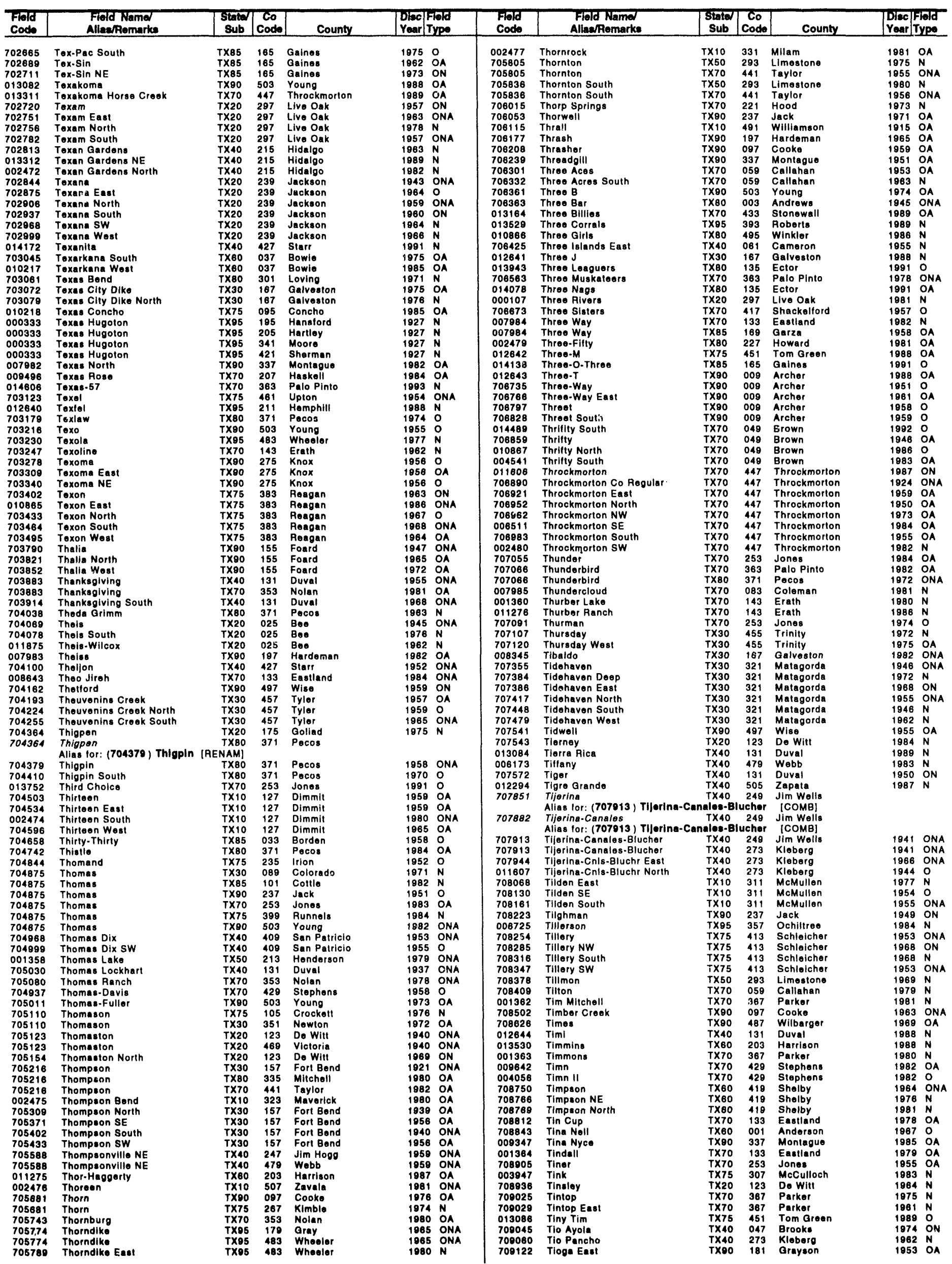


TEXAS

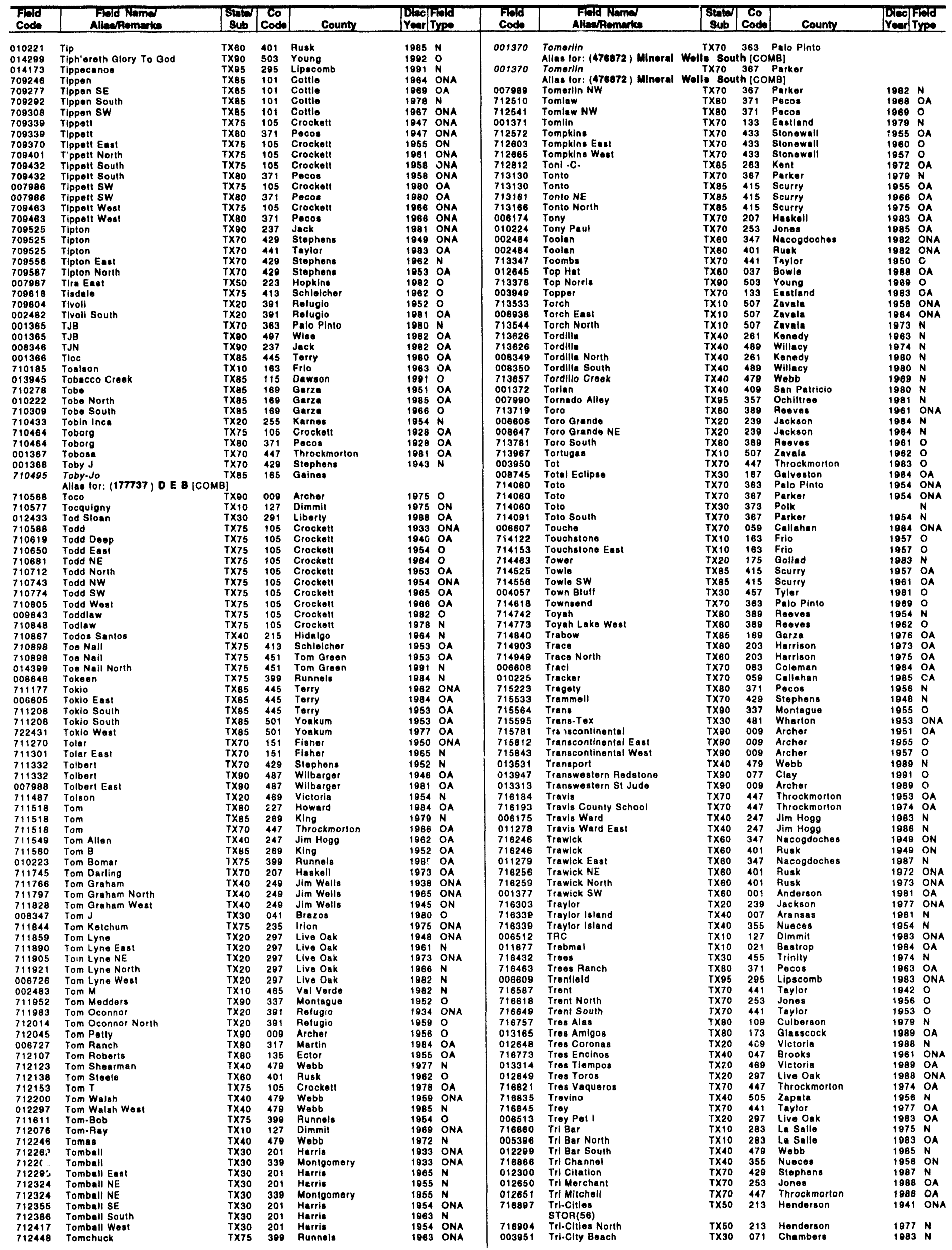


TEXAS

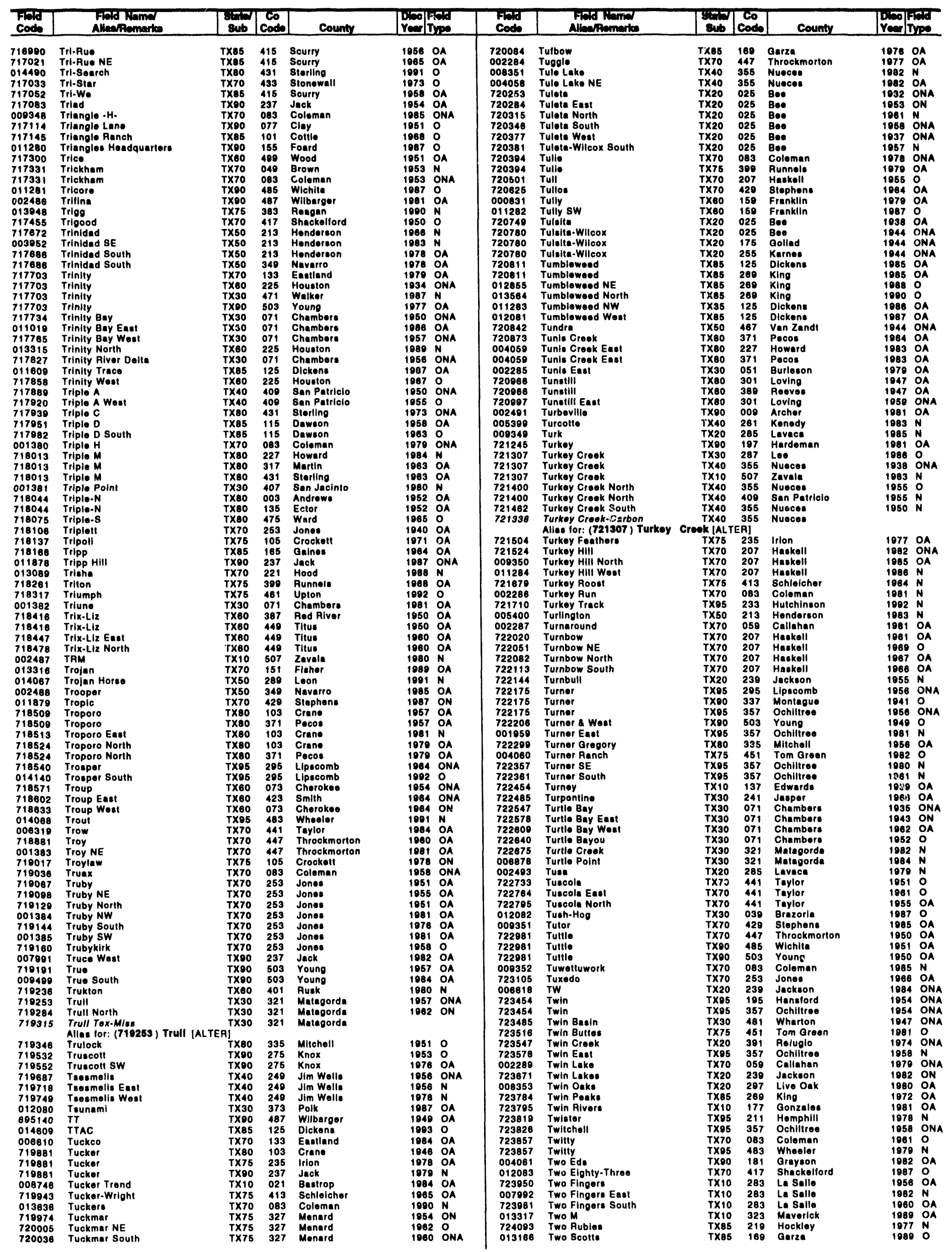


TEXAS

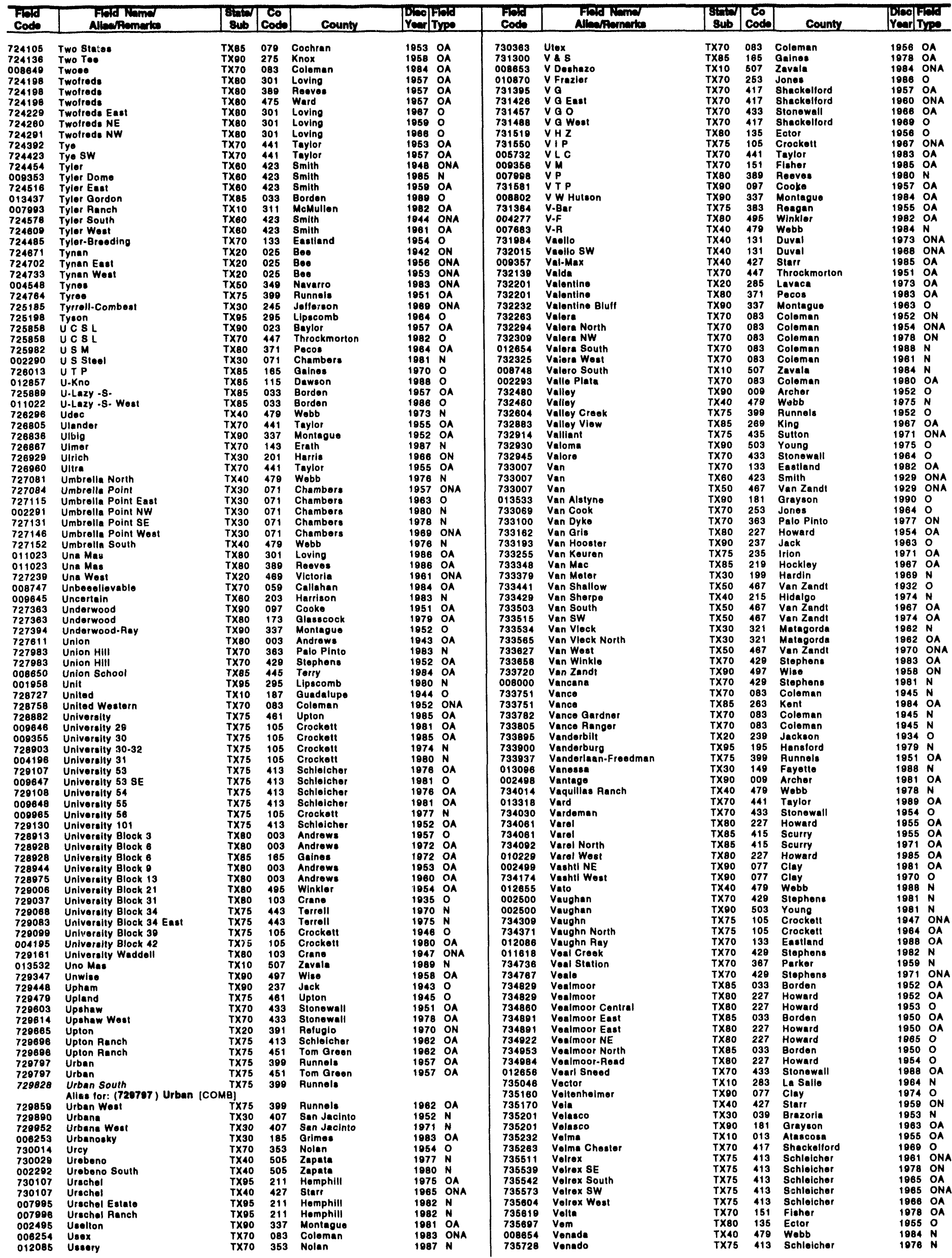


TEXAS

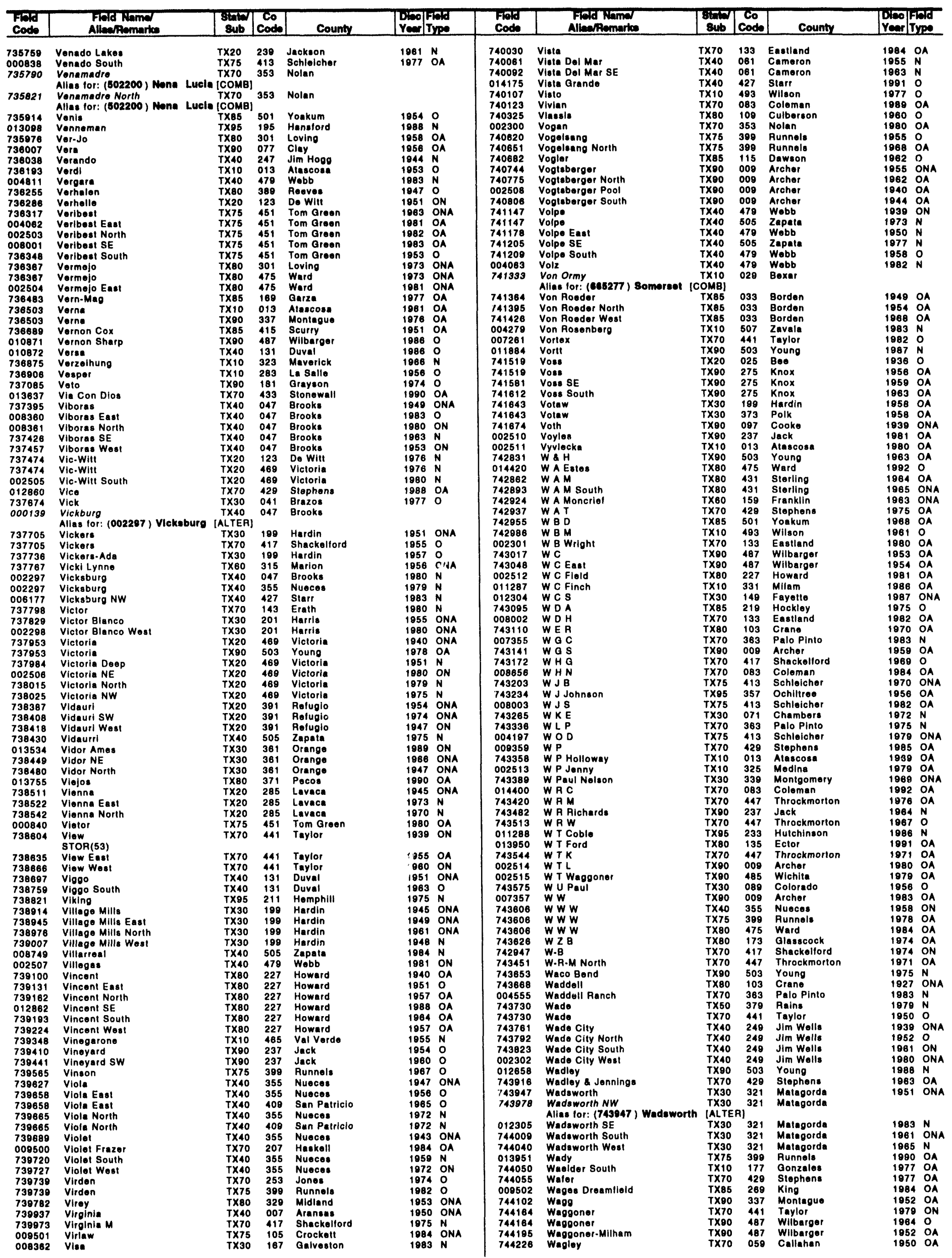


TEXAS

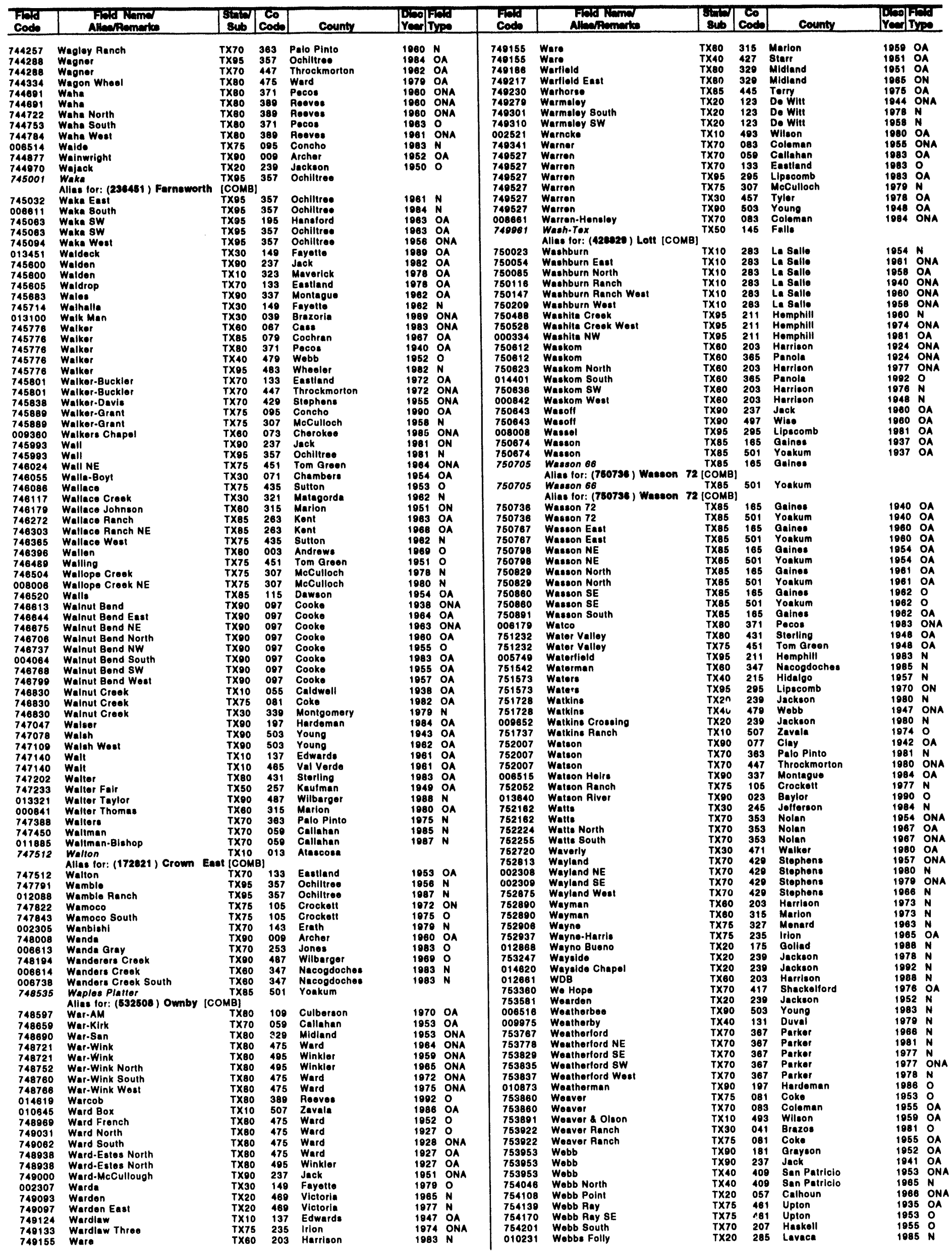


TEXAS

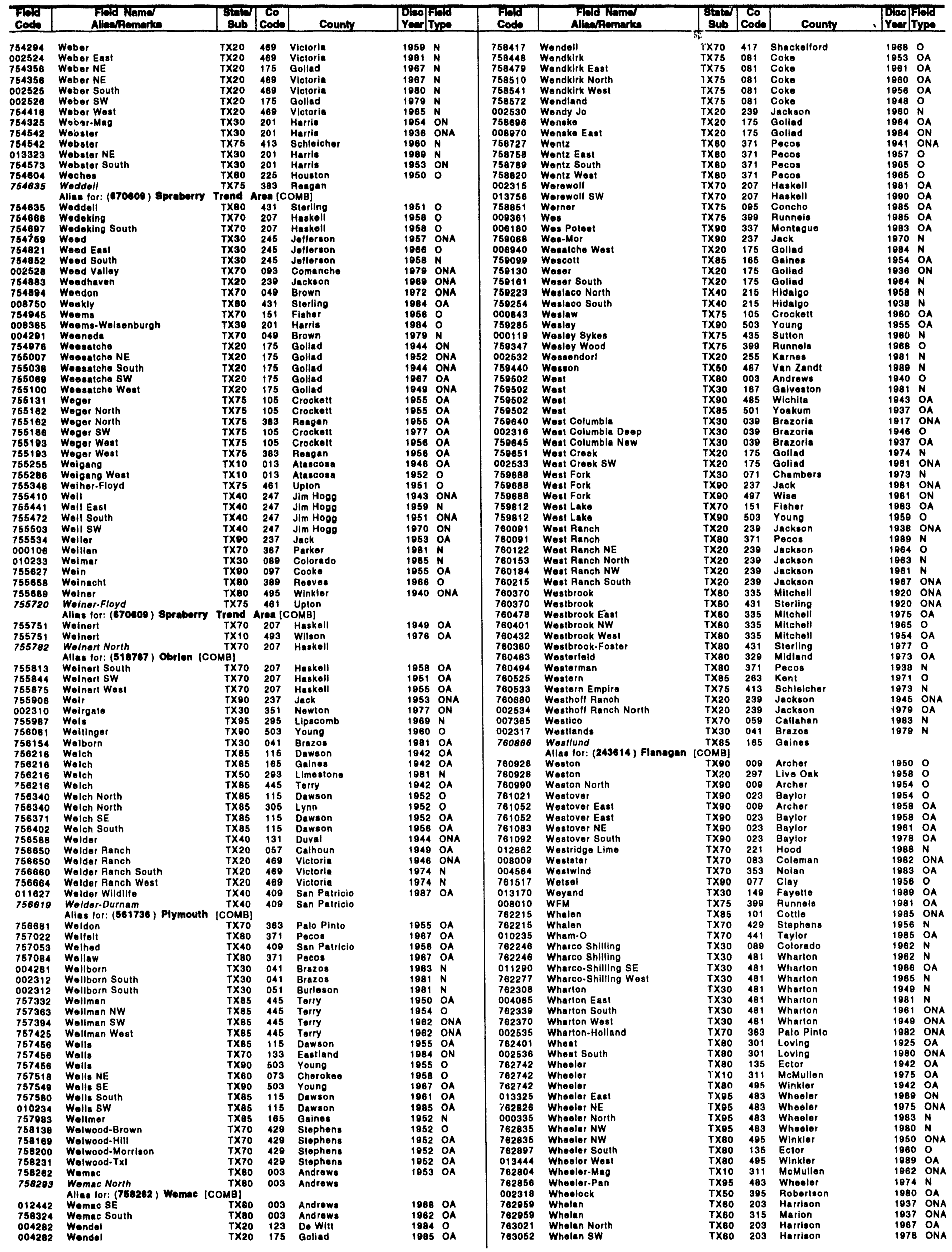


TEXAS

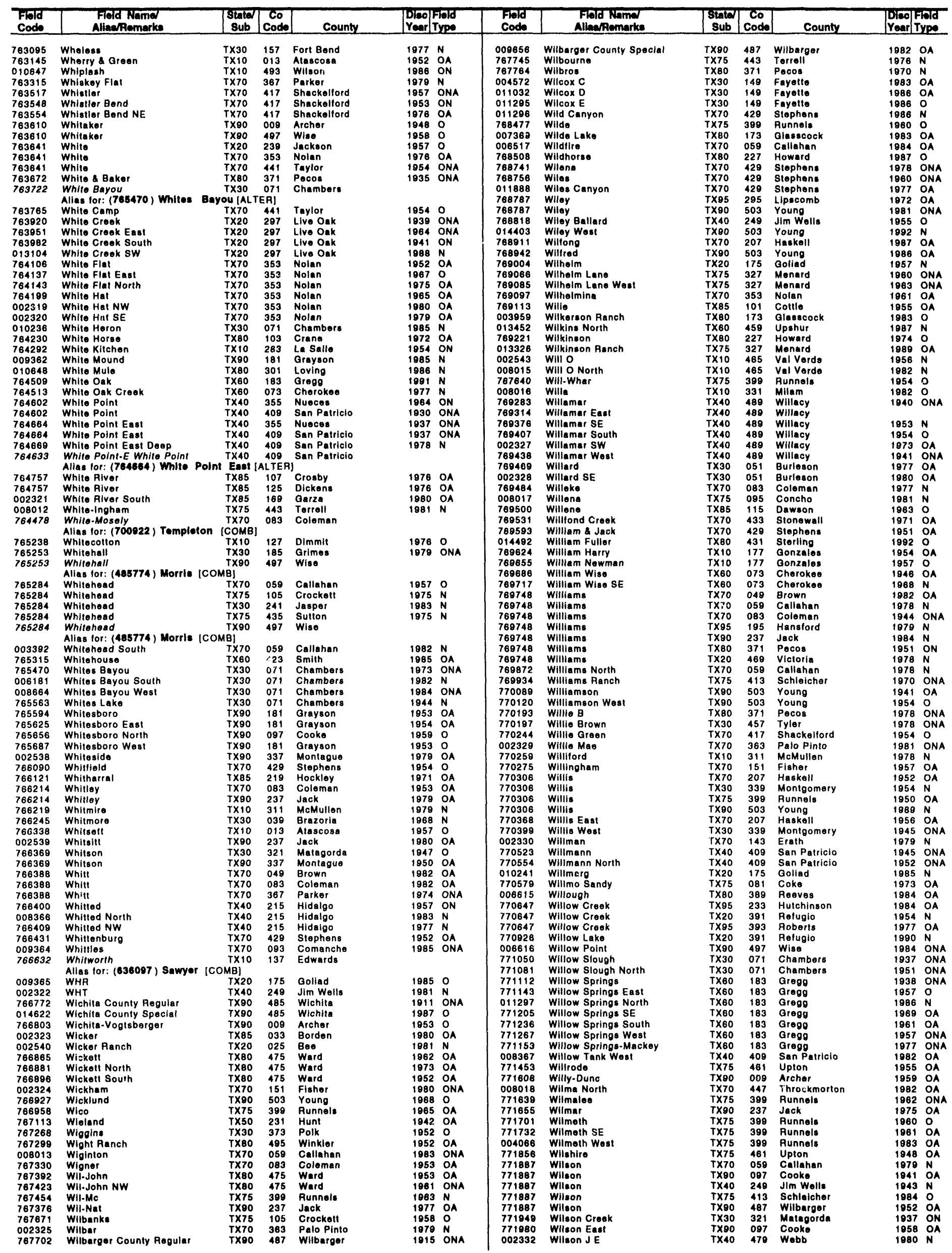


TEXAS

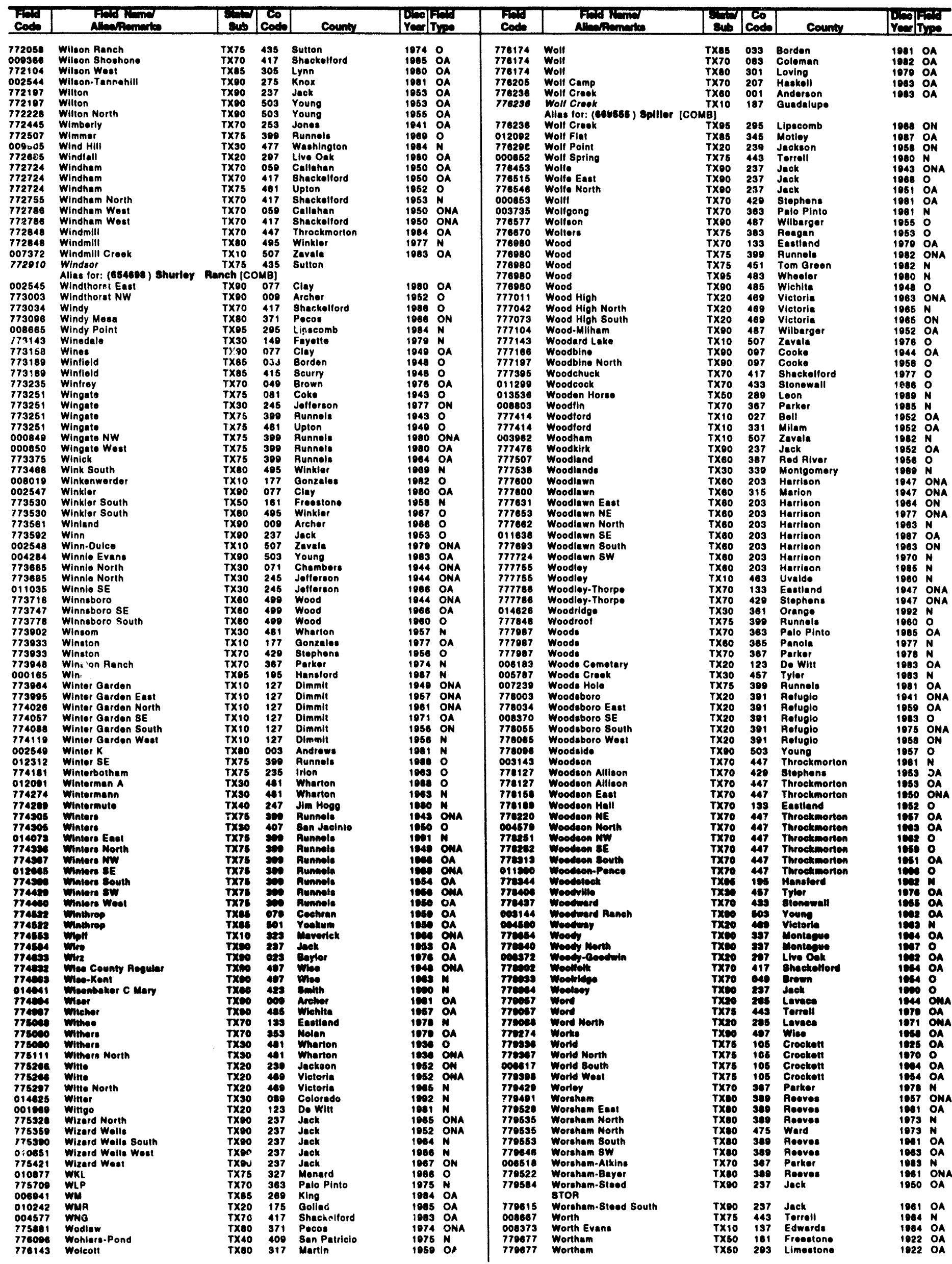


TEXAS

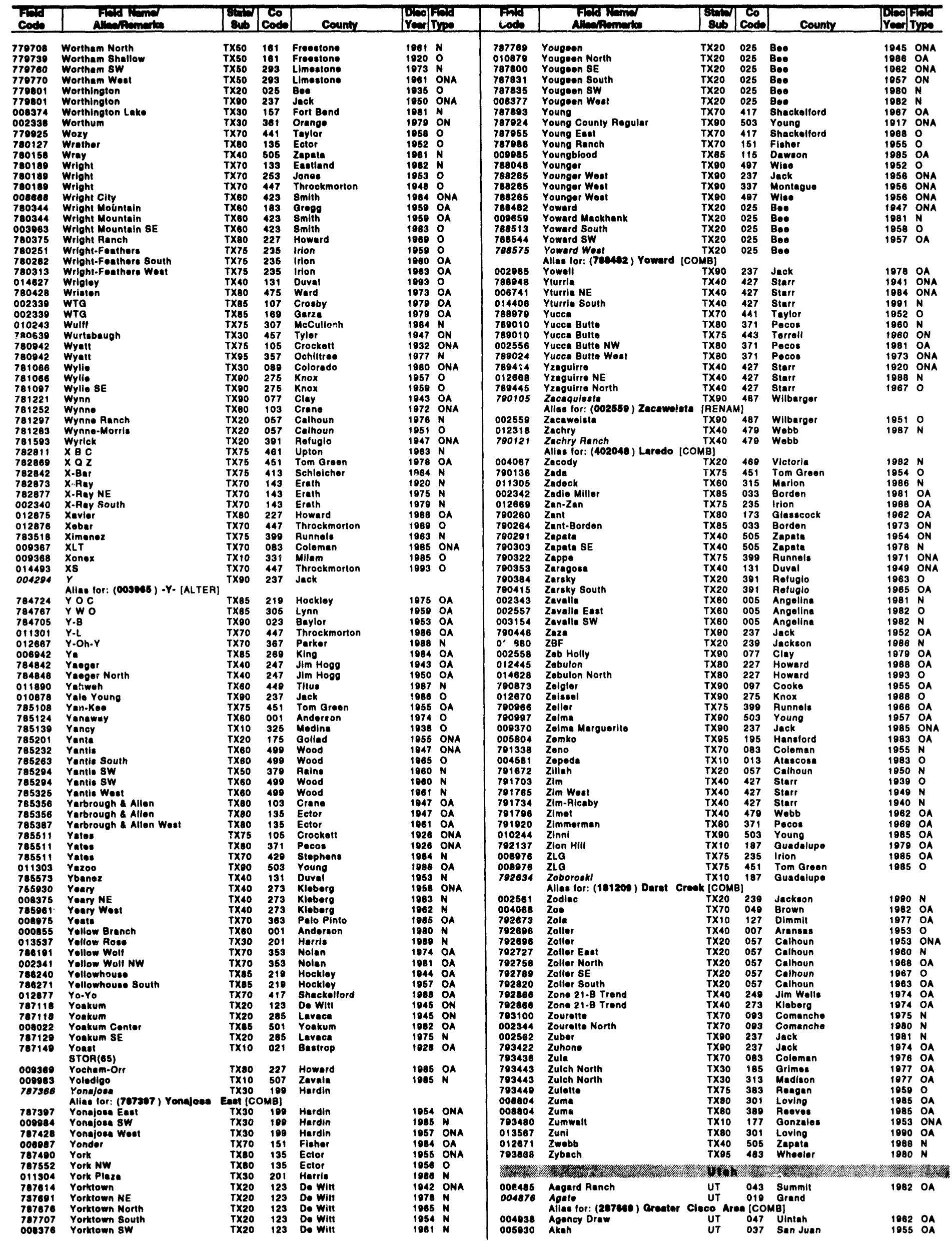


UTAH

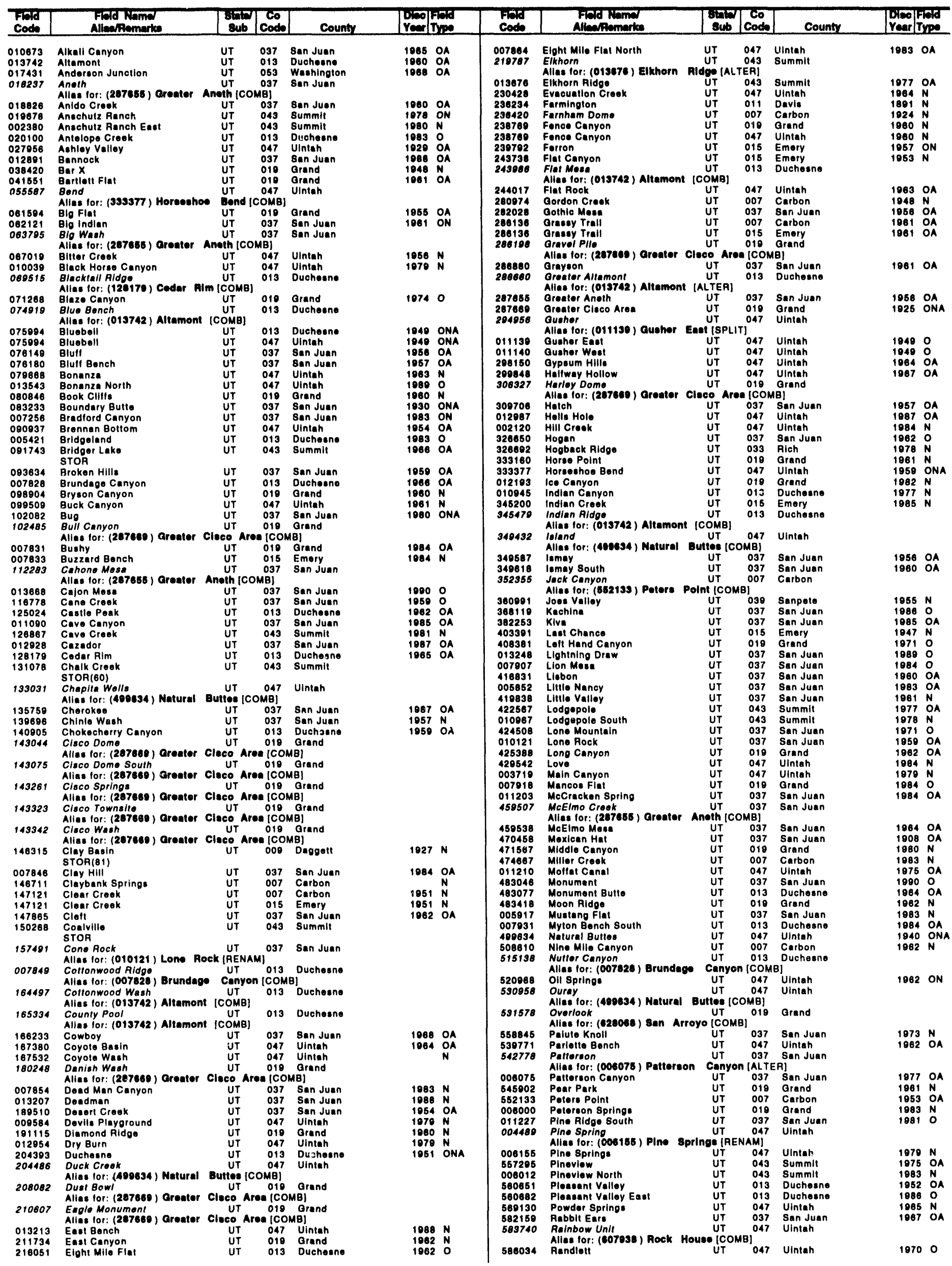




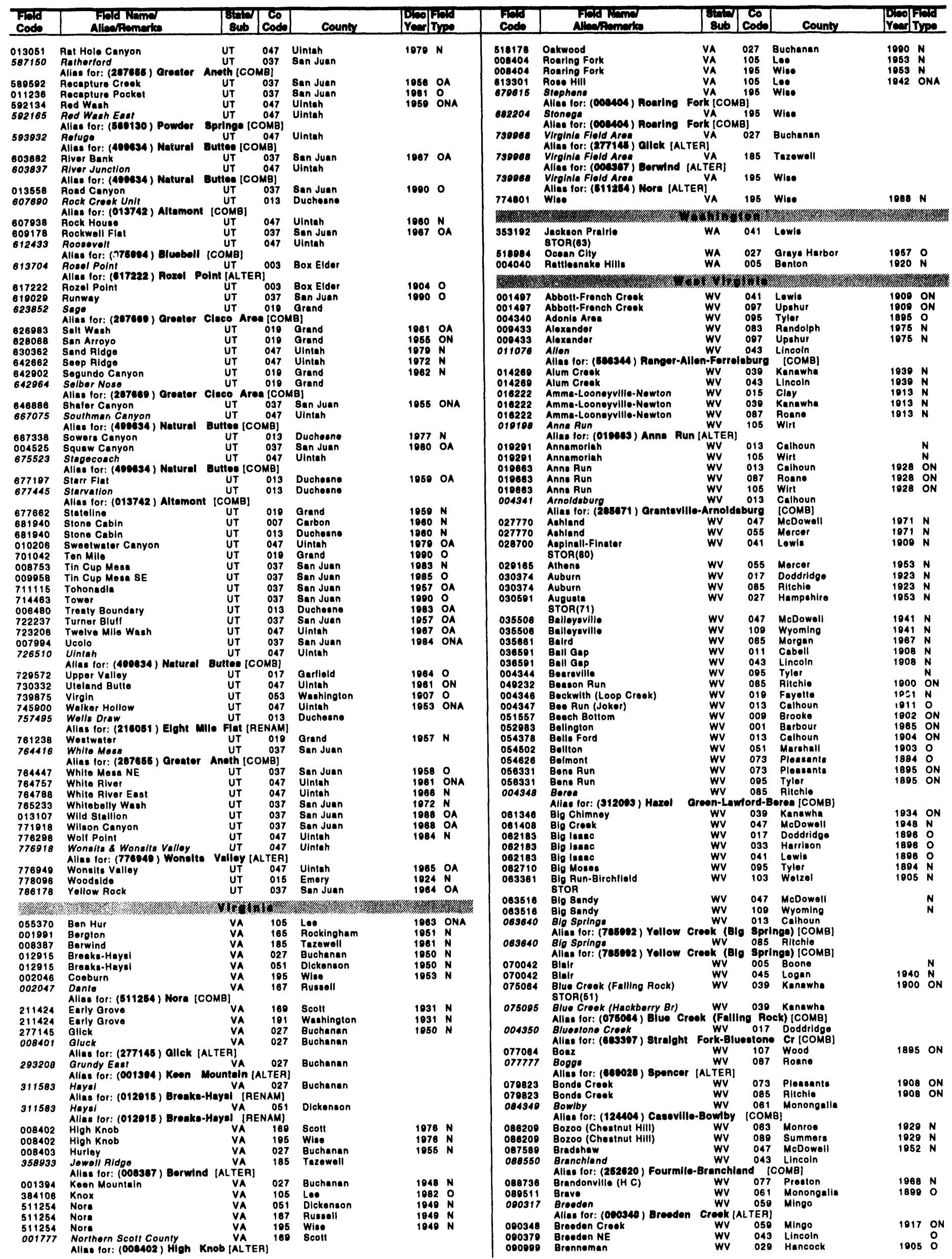


WEST VIRGINIA

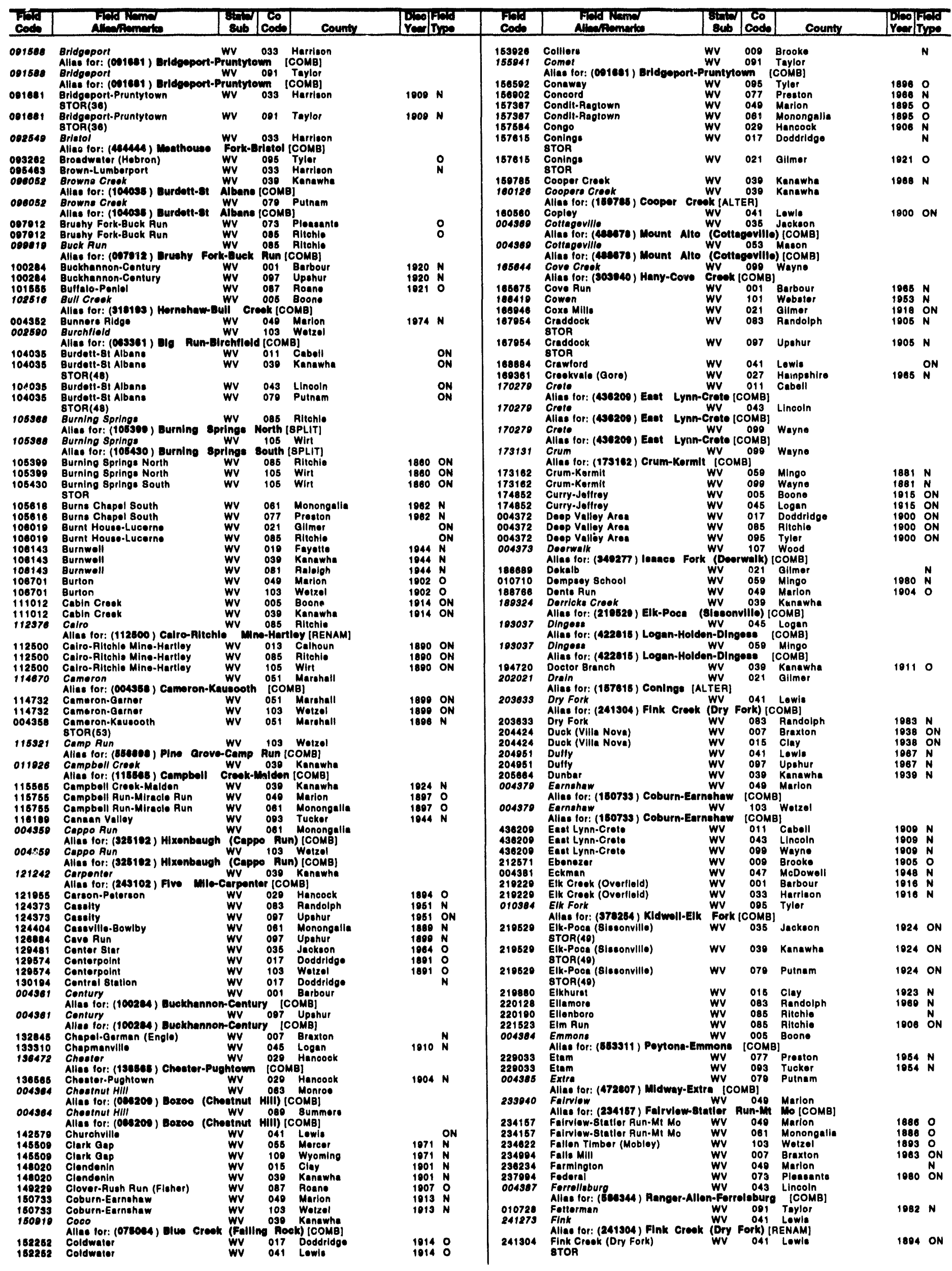




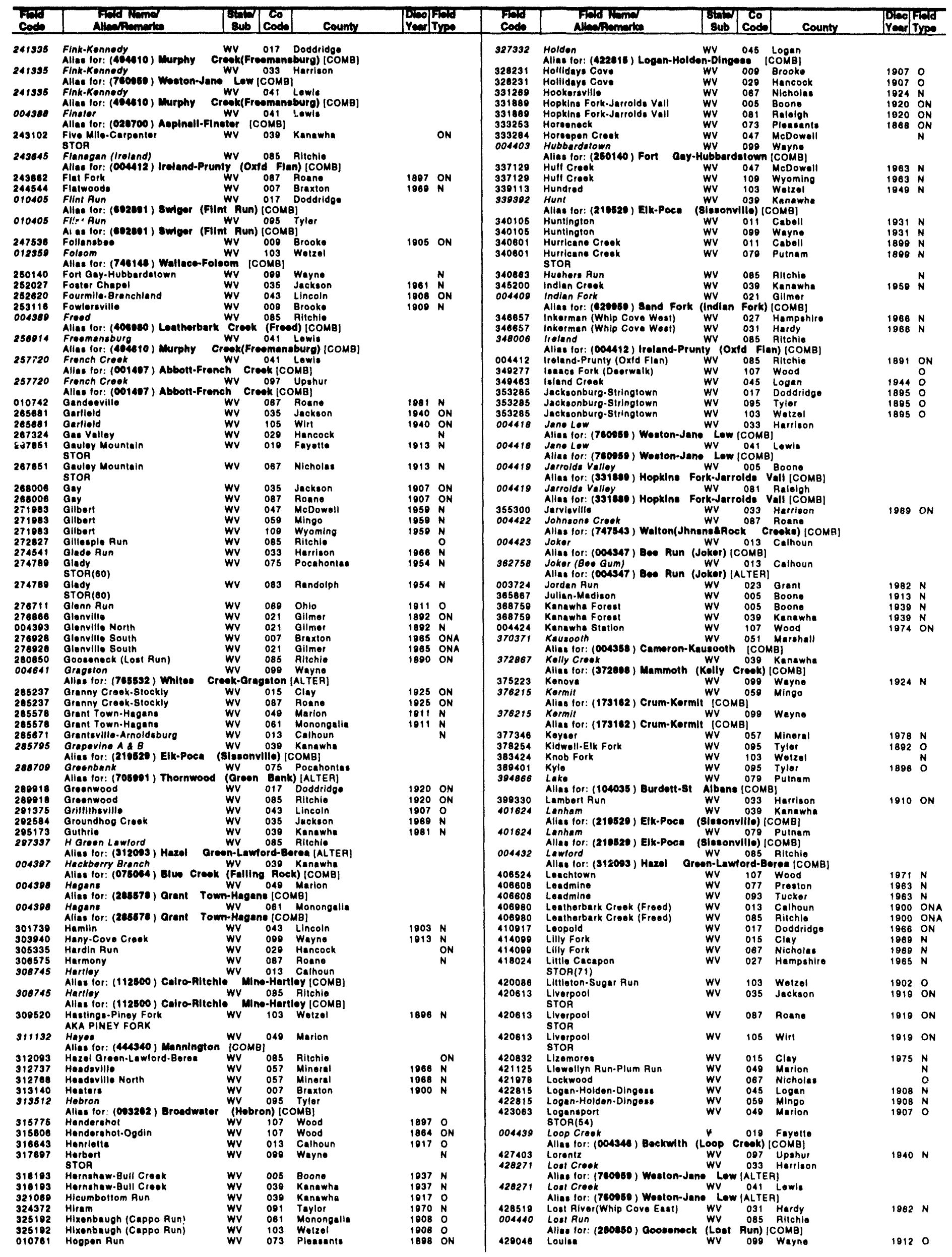


WEST VIRGINIA

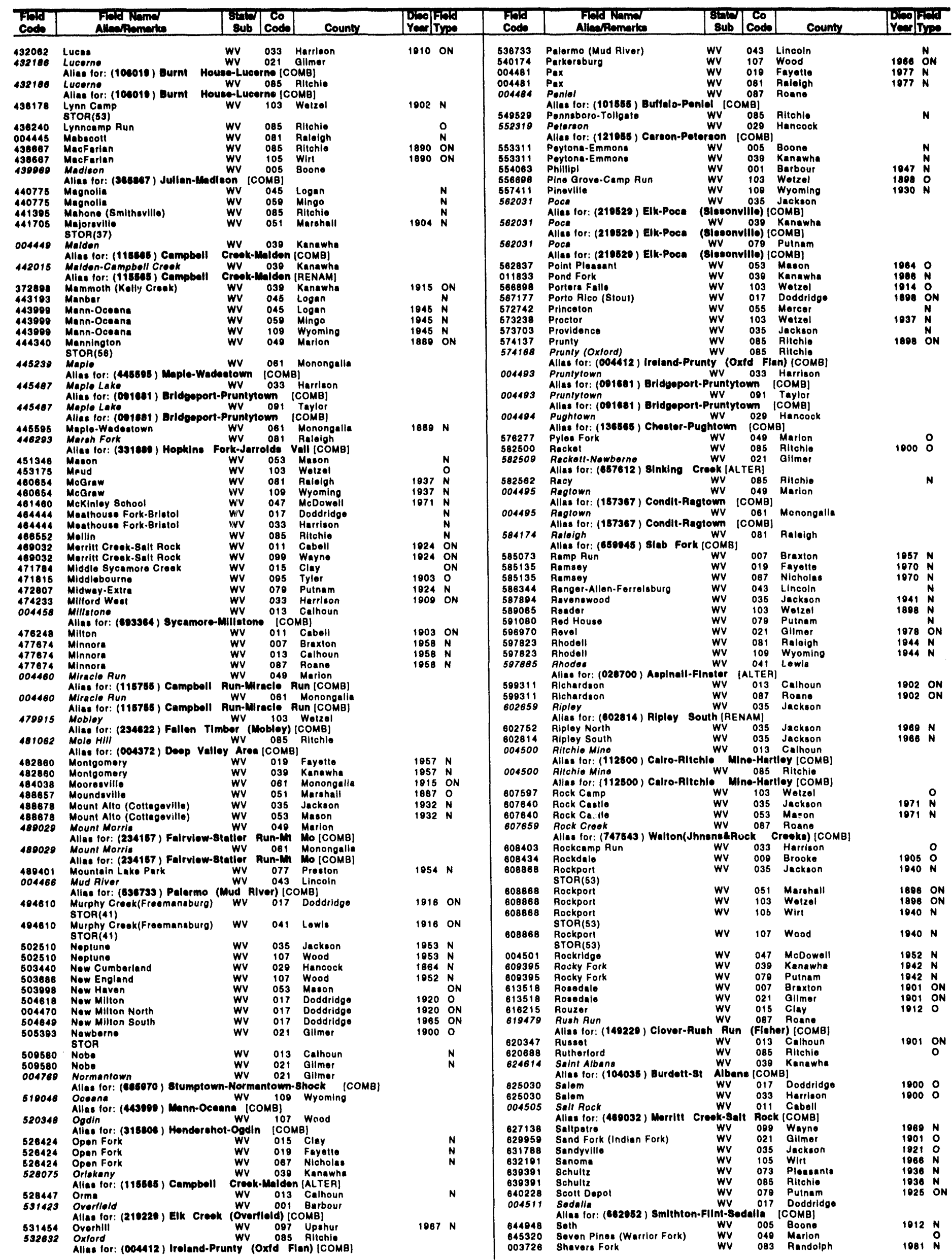




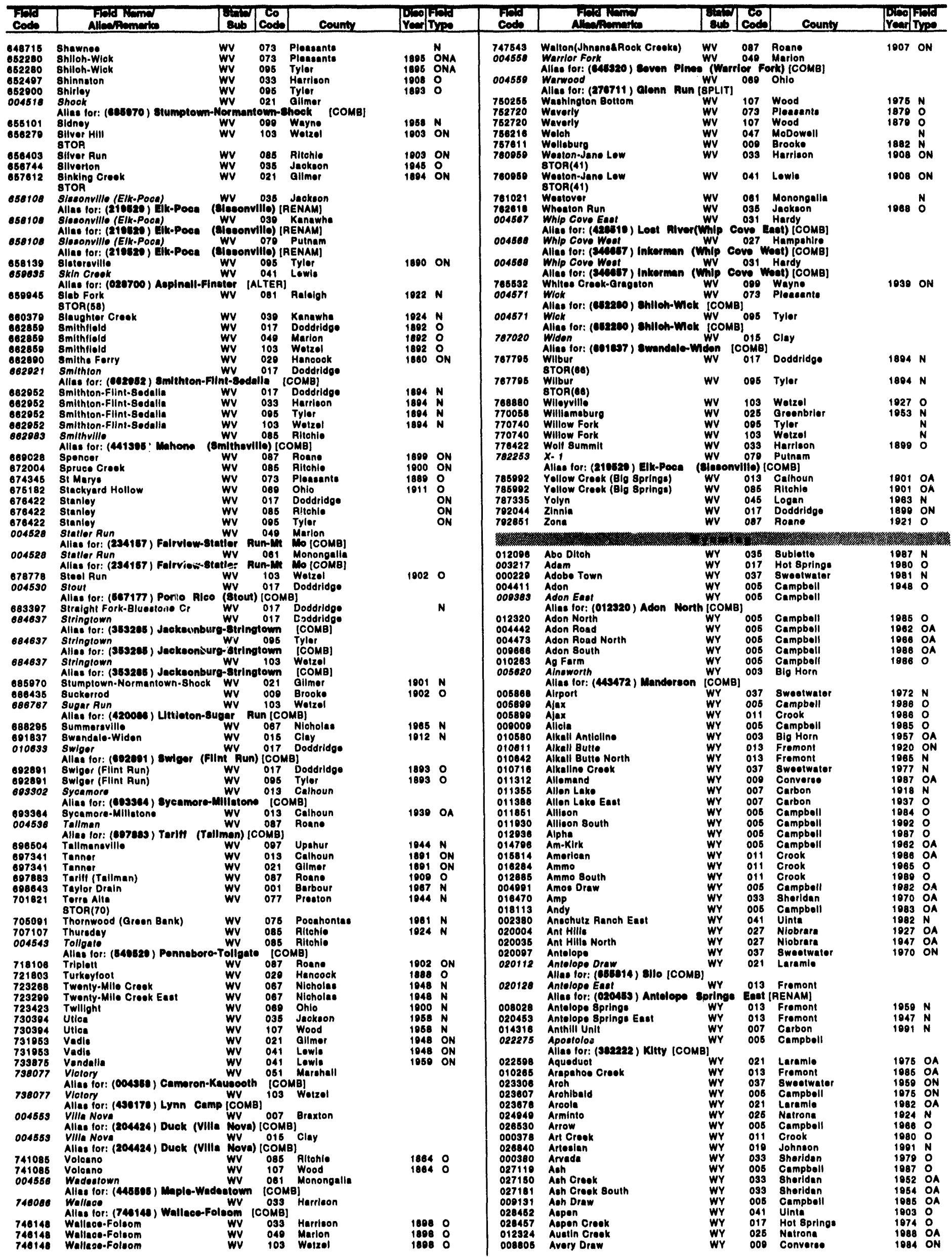


WYOMING

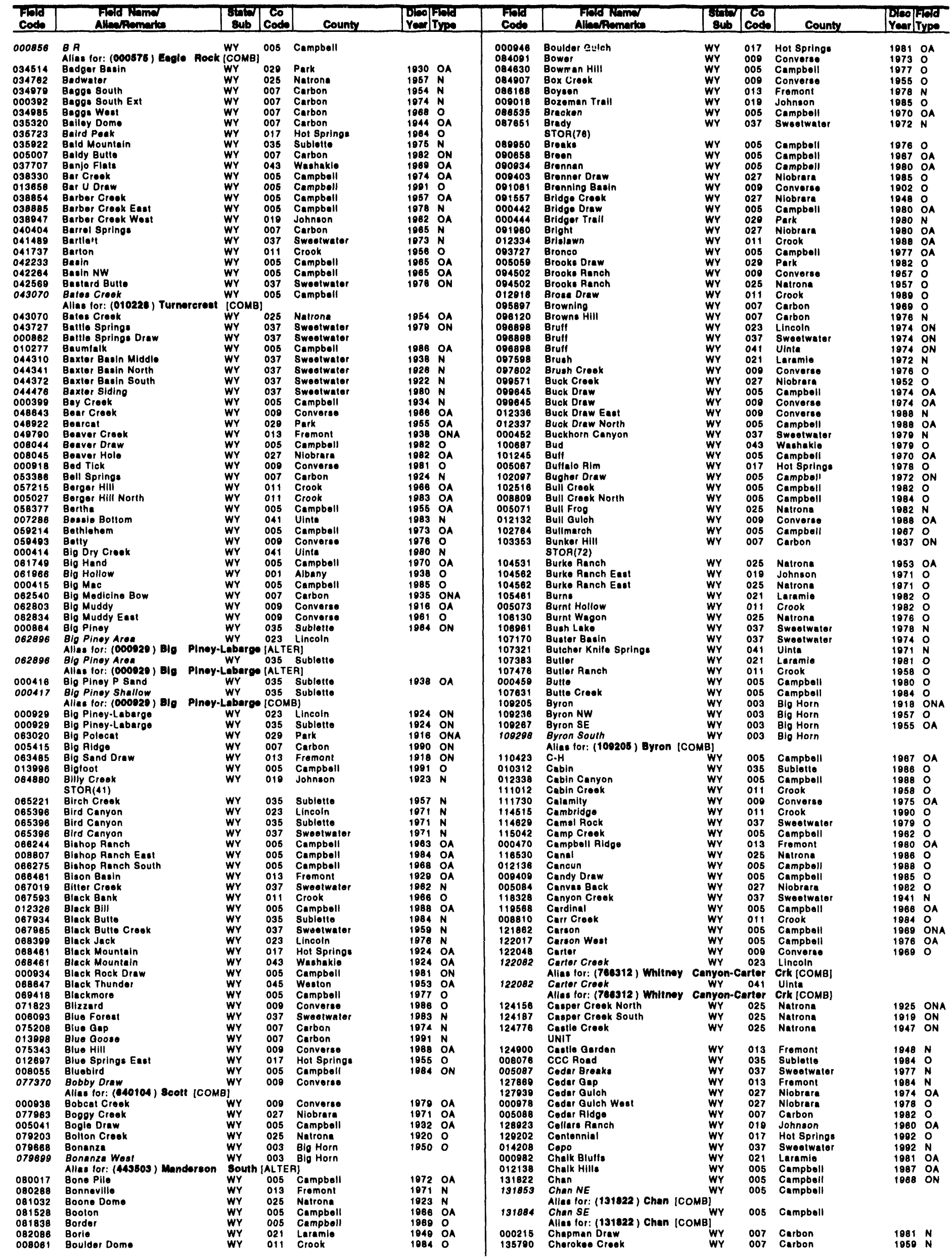




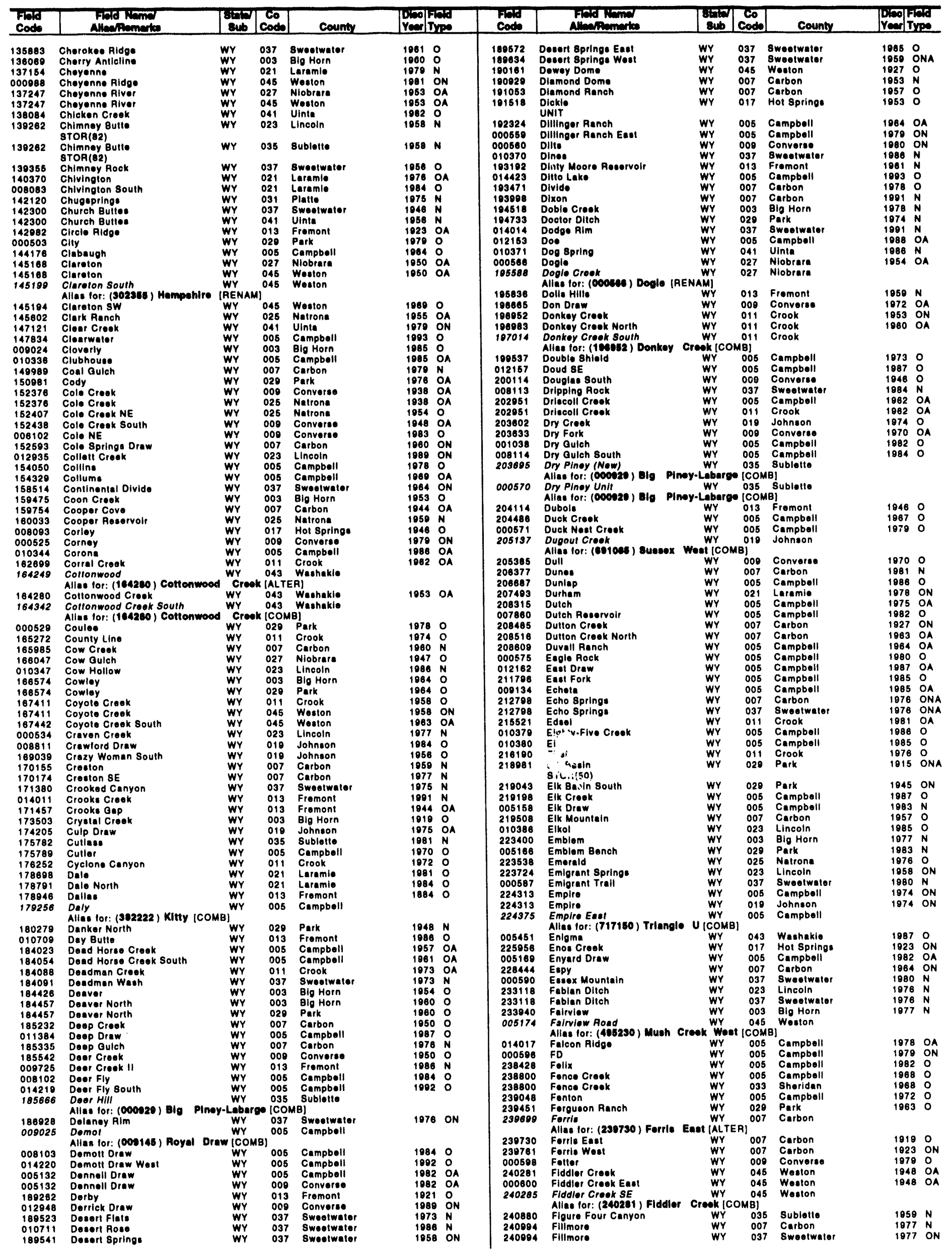


WYOMING

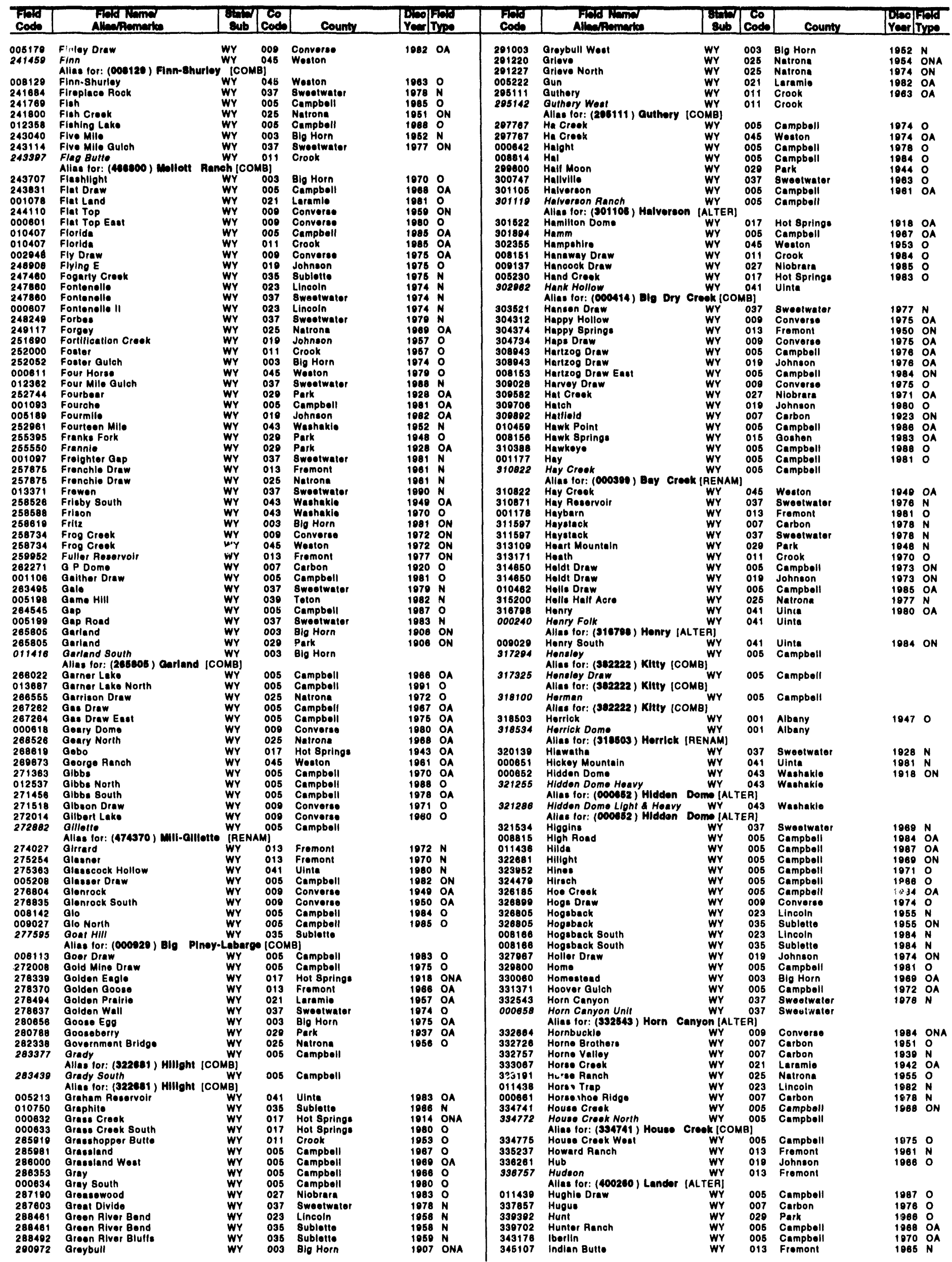




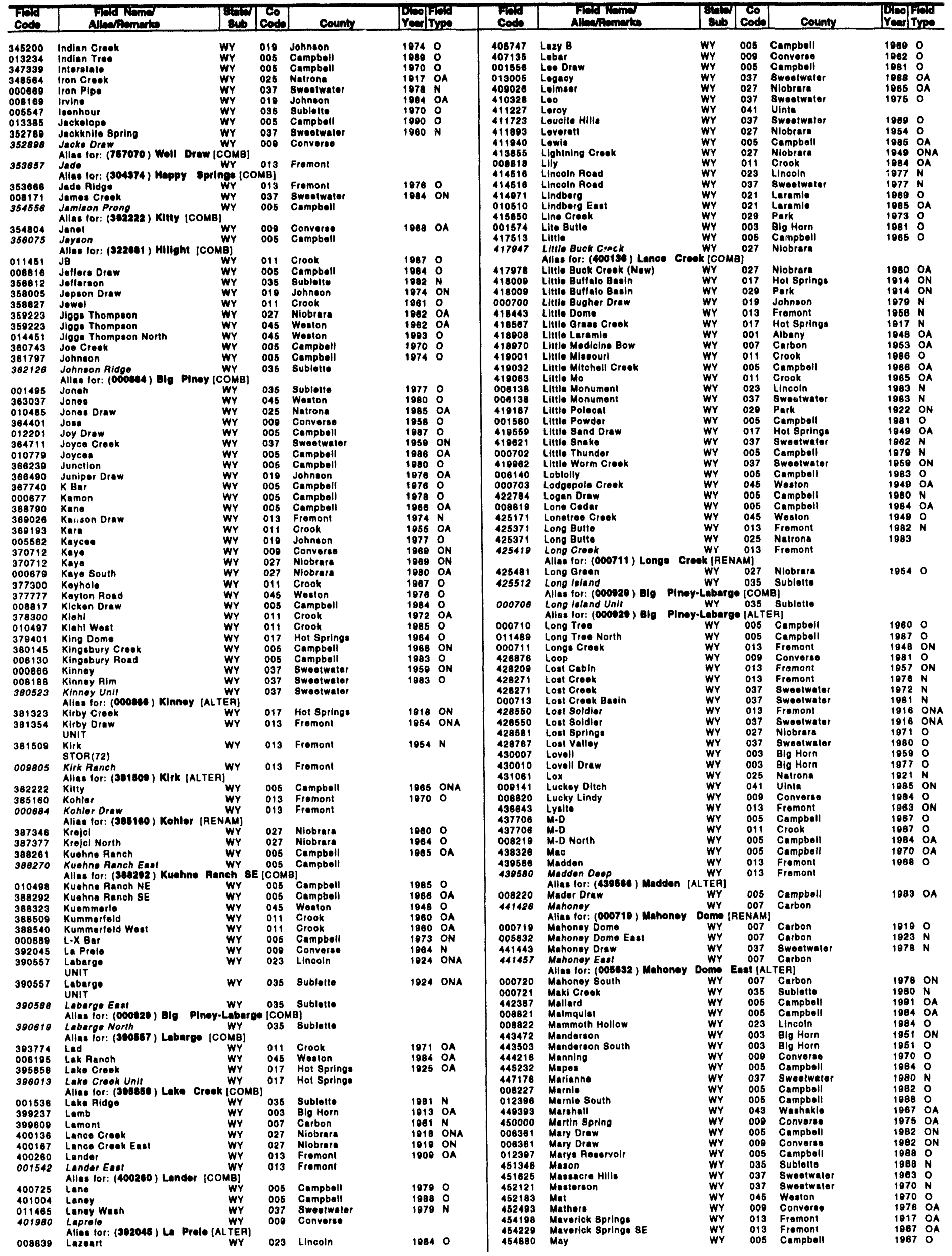


WYOMING

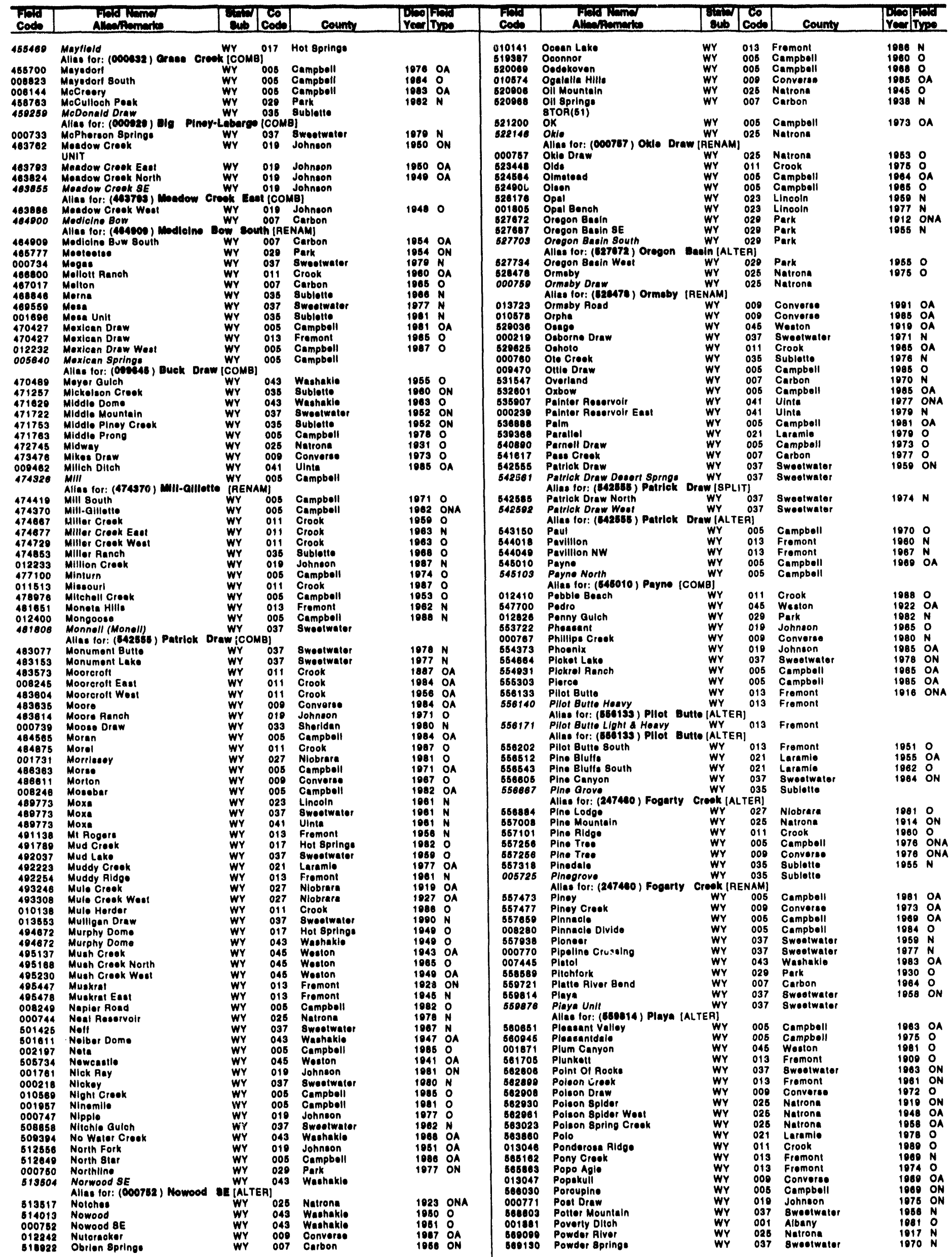




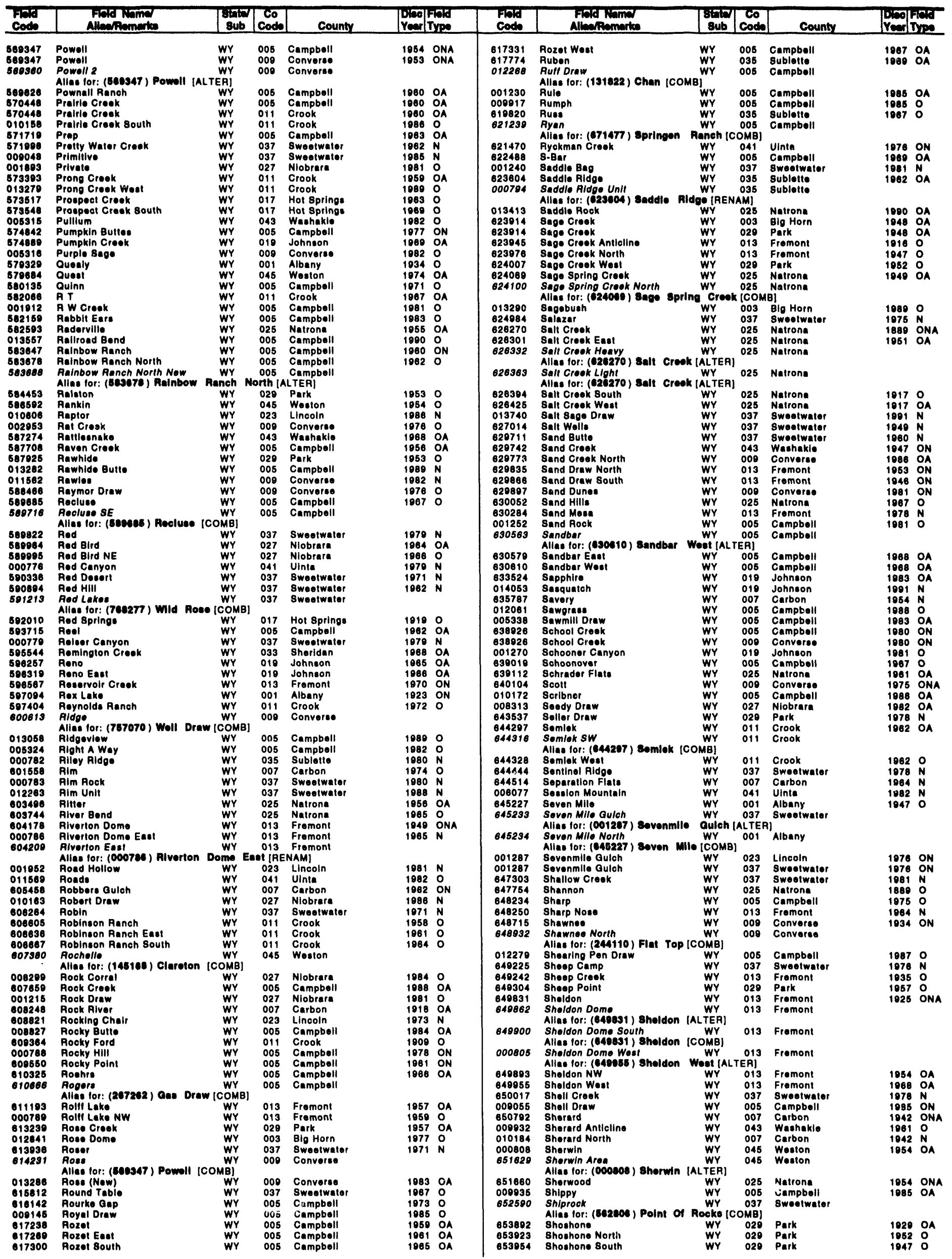


WYOMING

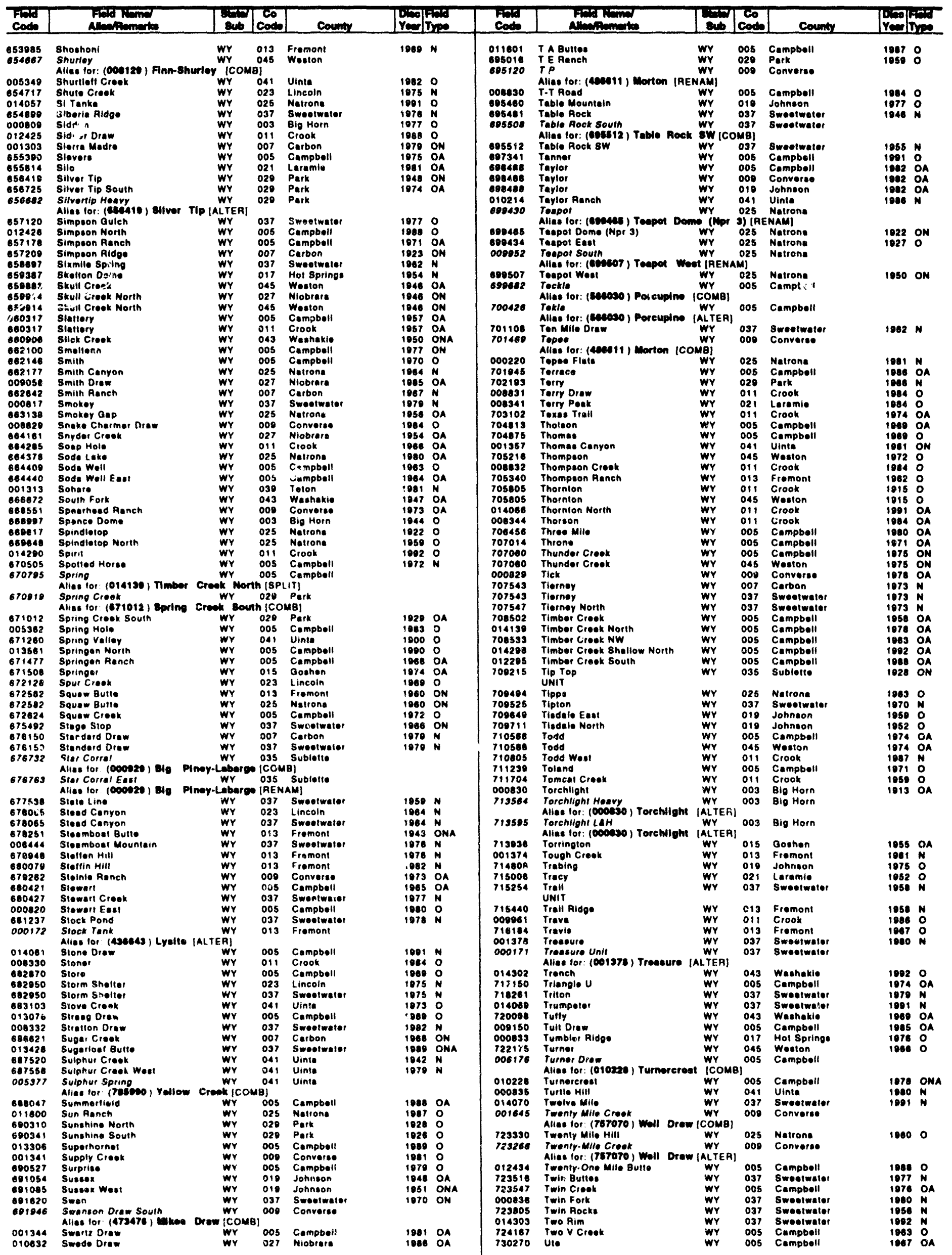




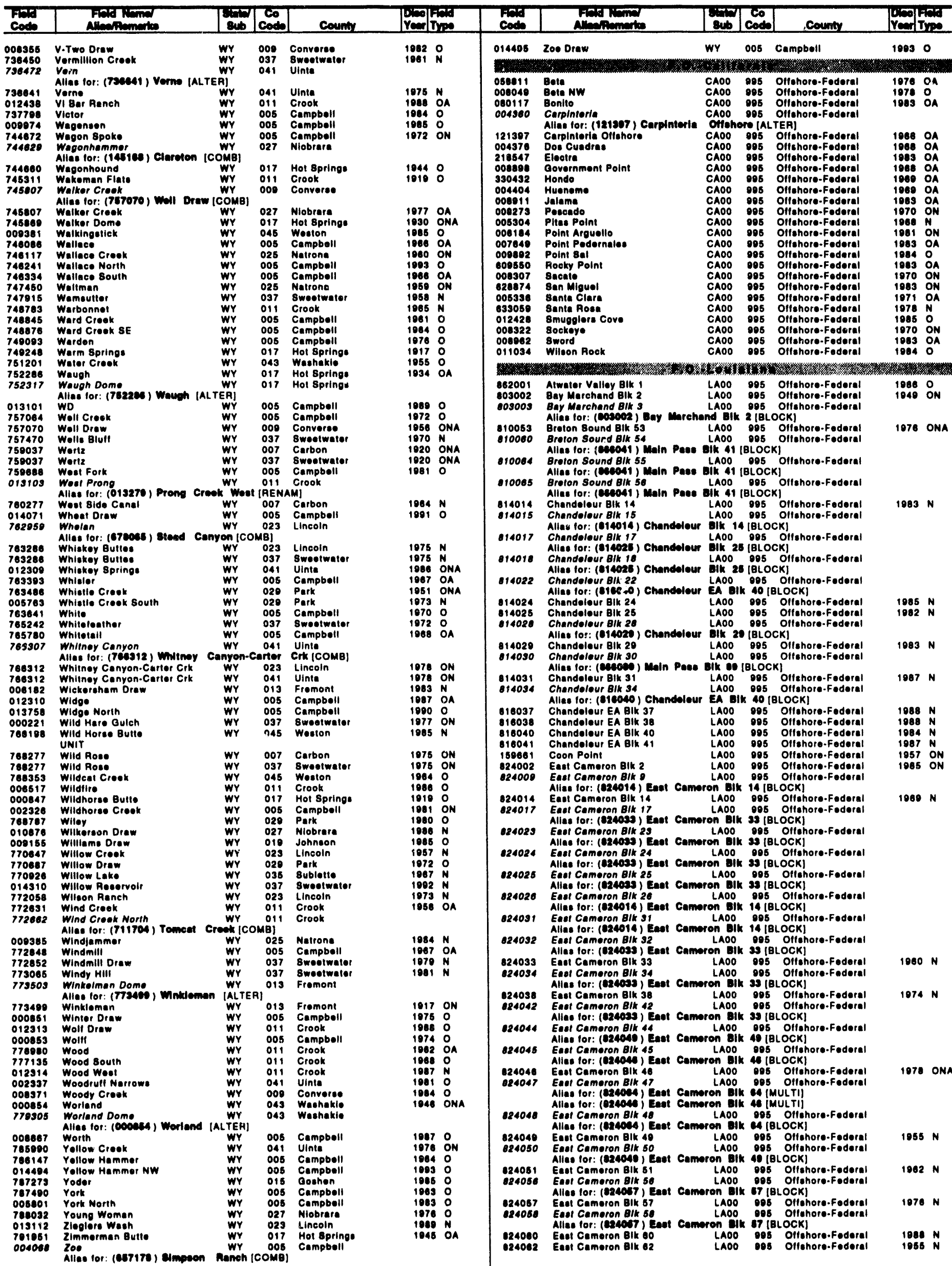


F.O.-LOUISIANA

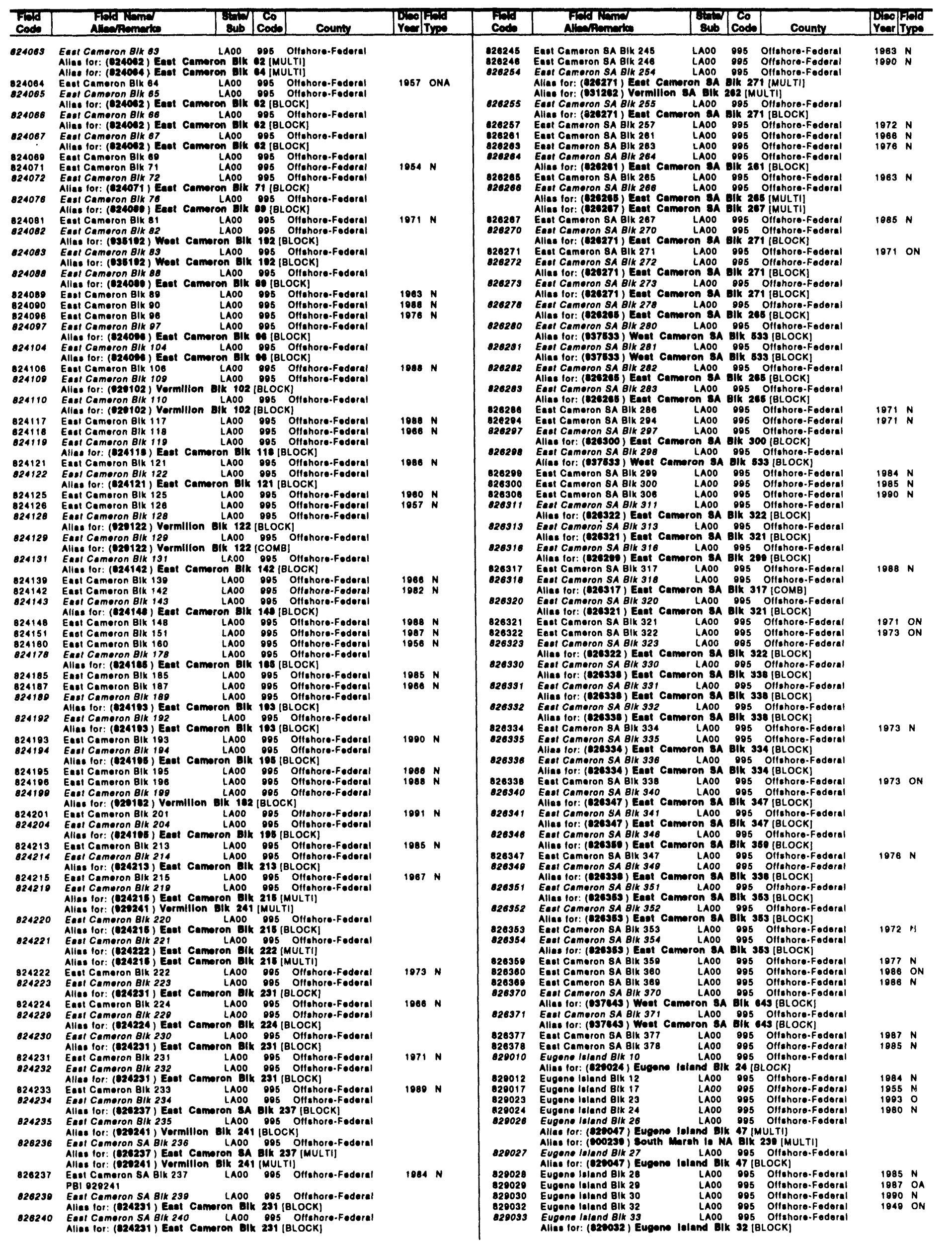




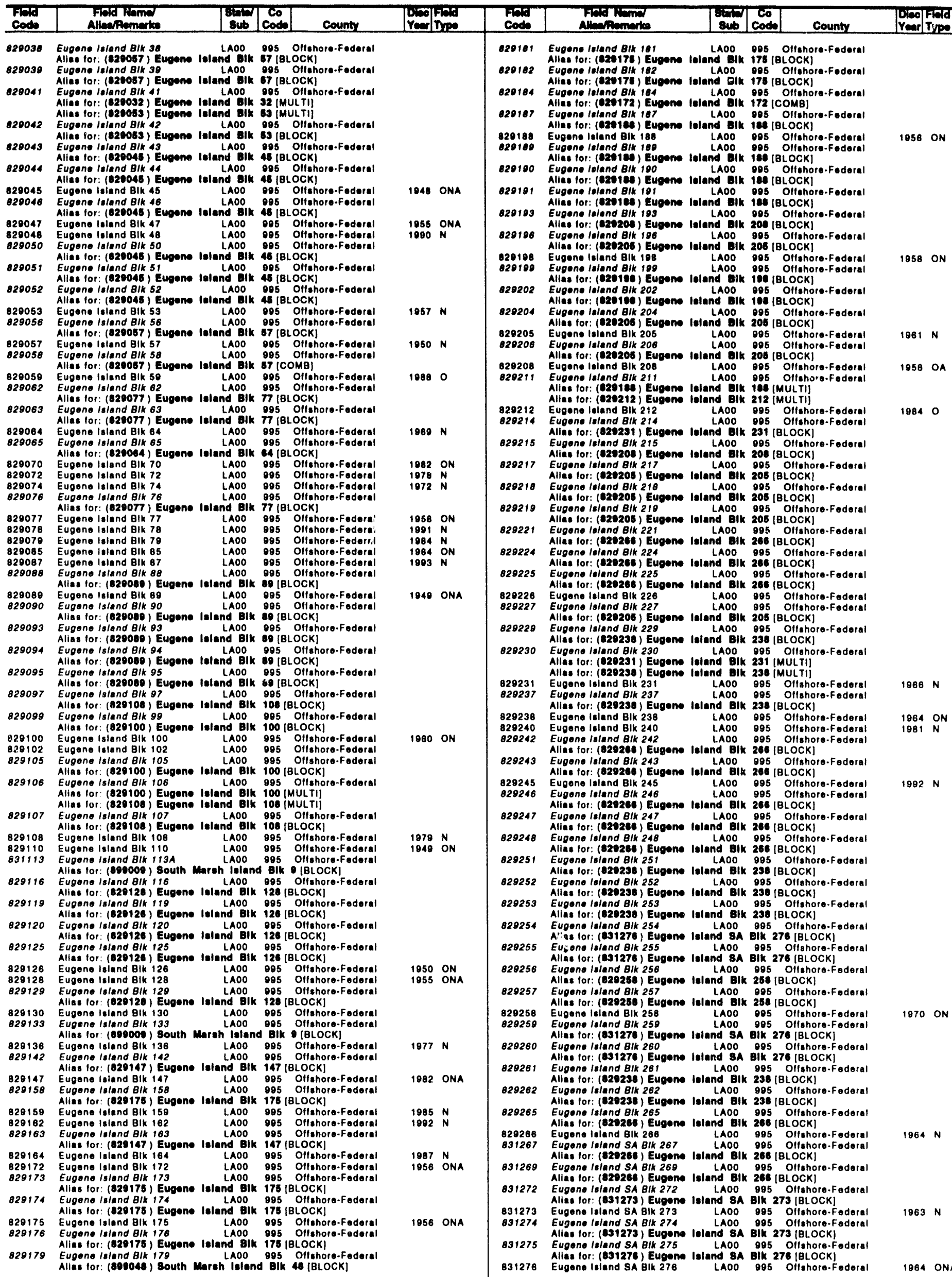




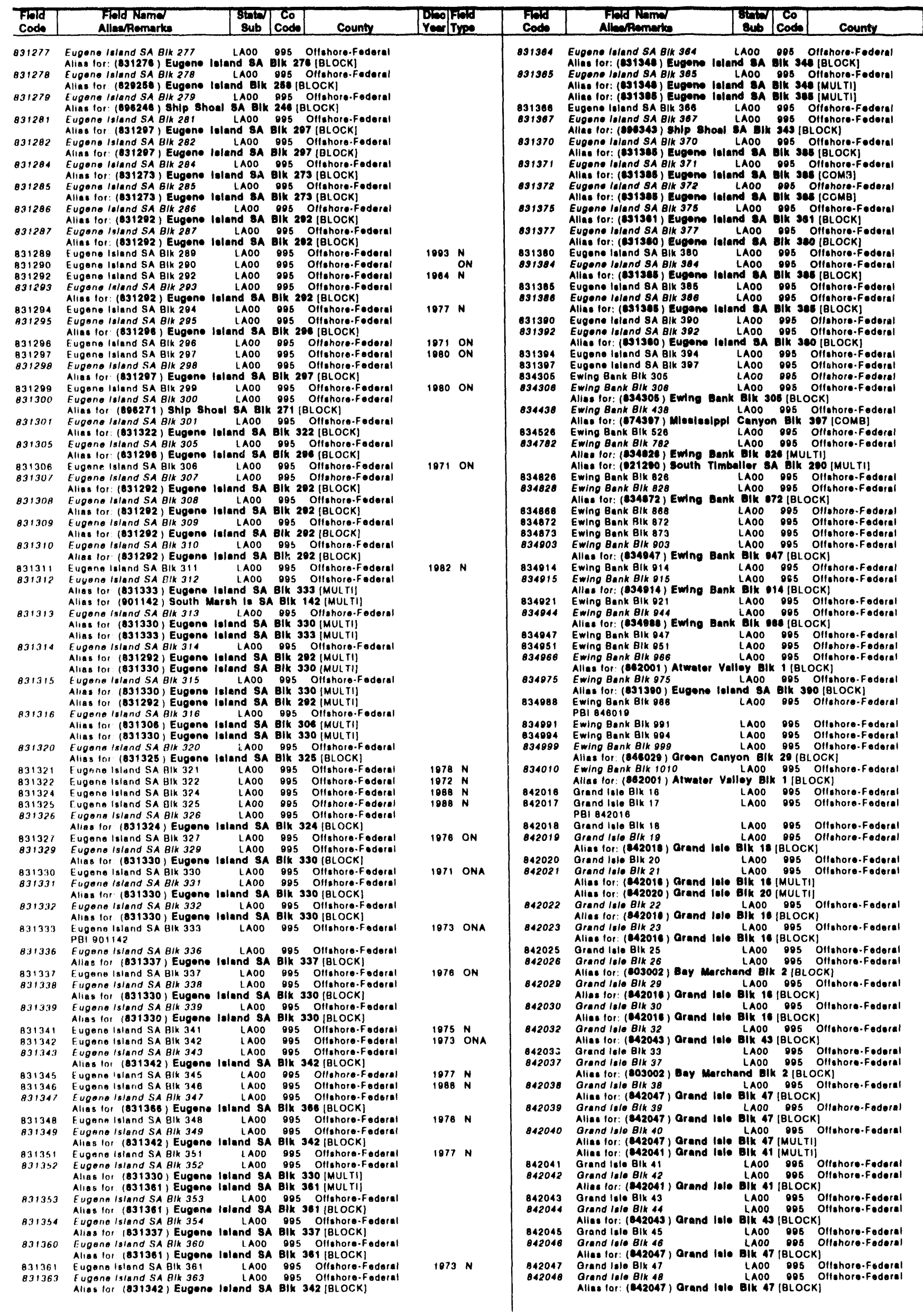

$1088 N$

1074 N

1076 N

$1088 N$

1084

1984 ON

$1082 \mathrm{~N}$

1085 ON

$1986 \mathrm{~N}$

$\begin{array}{ll}1086 & O \\ 1001 & N\end{array}$

1084 ON

10930

10840

19880

$\begin{array}{ll}1980 & 0 \\ 1086 & 0\end{array}$

1948 ON
1049 ON

1848 ON

$1078 N$

$1961 \mathrm{~N}$

$1068 \mathrm{~N}$

1004 ON

1958 ONA

$1072 \mathrm{~N}$ 


\begin{tabular}{|c|c|c|c|}
\hline $\begin{array}{l}\text { Fold } \\
\text { Cods }\end{array}$ & $\begin{array}{l}\text { Ficd Nama } \\
\text { Alleapominter }\end{array}$ & \begin{tabular}{|c|c|}
$\cos$ & $\cos$ \\
$\cos$ & cods
\end{tabular} & County \\
\hline
\end{tabular}

842040 Grand lolo B1k 49
Allas for: (842047) Grand lale Lik 47 [BLOCK]

042051 Grand lele Blk 51 LAOO 005 Oftehore.Fodera

o42052 Alias for: (\$42047) Grand lele Dik 47 (BLOCK)

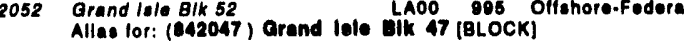

642053 Giand lolo BL 53 ) arand Talo dik 47 [BLOCK]

Alias lor: (842047) Orand lole Blk 47 [BLOCK]

642055 Allas lor: (84k2045) Crand lale bik 77 [BLOCK]

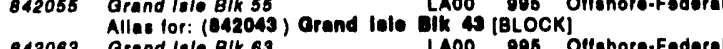

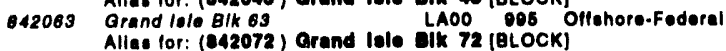

842072 Allas for: (842072) Grand lalo Lik 72 (BLOCK)

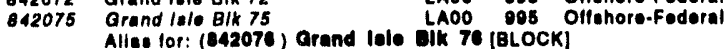

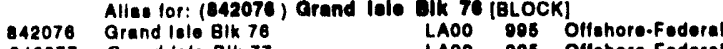

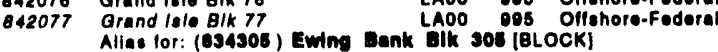

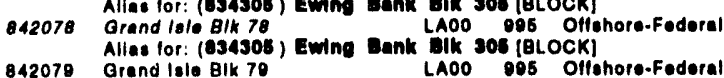

842079 Grand lale Bik 70
842081 Grand lalo B1k 81

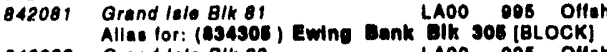

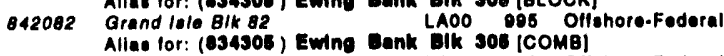

04208s Grand 1alo alk B3.

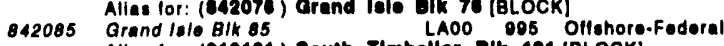

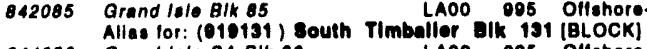

844086 Grand lolo SA BIK 86 LAOO 995 Olfehoro-Fodural

844086 Grand lolo SA Bik 86 Alias lor: (010131) South TImbellor BIK 131 [BLOCK]

844090 Grand lele SA BIK 90
LAOD 995 Otfshoro-Foderal

844001 Grand lele SA alk 01 LAOO 905 Olfohore.Fedoral

844093 Grand lalo SA 81k 93

844094 Grand 1810 SA B1k 94 G LAOO OOS OHthoro.Federal

844095 Grand lolo SA BIK O5 LADO OOS Ollshoro.Foderal

844096 Grand lalo SA Blk 86 LAOO 095 Oltahoro-Foderal

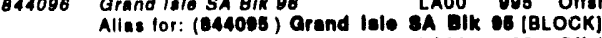

844101 Grand lole SA B1K 101 LAOO 095 Ollshore.Foderal

844102 Grand Isle SA BIK 102 LAOO 095 Otfehoro-Federal

844103 Grand lele SA Bik 103 LAOO 998 Ollehore-Foderal

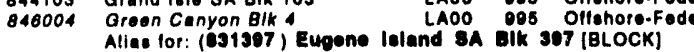

848000 Green Canyon Bik 6 LAOO 995 Olfehoro-Foderal

846018 Green Canyon Blk 18 LAOO 995 Olfeh

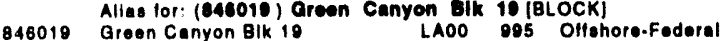

846021 Groon Canyon Blk 21 LAOO 995 Otthorr.Foderal

846027 Groen Cenyon BIk $27 \quad$ LAOO 995 Oftohore-Fodoral

846029 Green Canyon Blk 29
846031 Green Canyon Blk 31
LAOO 995 Olishoro-Foderal

Alial lor: (846075) Green Canyon BIk 78 (BLOCK)

846039 Groen Canyon Blk 39 LAOO 995 Oftuhore.Foderal

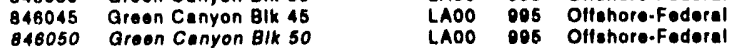

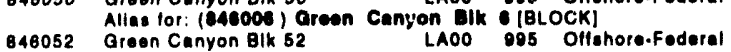

846053 Groen Canyon B1k 53 LAON 995 Offahoro-Foderal

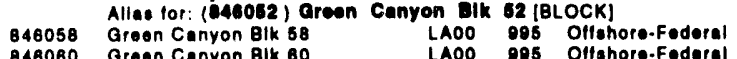

Q46060 Green Canyon Blk 60
846064 Green Canyon BIK 64 LAOO 905 Ollshore-Foderal

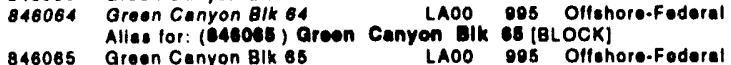

846085 Groen Canyon BlK 85
846066 Groen Canyon B1K 68 Aliae for: (BAc0ses) arcen Canyon Bik of [BLOCK]

846070 Green Canyon BIK 70 LAOO OOS Olfohore-Foderal

84607? Greon Canyon BlK 72 LAOO 095 Oftohoro.Federal

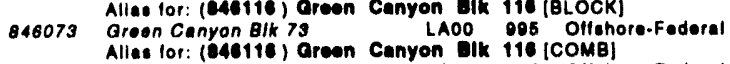

B46075 Groen Canyon Blk 75 LAOO 995 Olfehore.Foderal

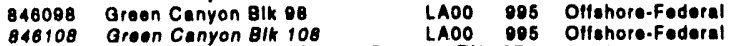

846108 Green Canyon BIK 108 LAOO 905 Olfah

846109 areen Cenyon Blk 100 LAOO 095 Oftehoro-Foderal Alies lor: (E400ses) Orean Canyon Blk 68 (BLOCK)

846110 Green Canyon Blk 110 LAOO 095 Oftohore-Foderal Allac tor: (B400s8) Green Canyon bik

B46118 Groen Canyon Bik 110 LAOO DOS Offahoro-Fodersl

846136 Green Canyon B1K 136
846147 Green Canyon BlK 147

846147 Groen Canyon Blk 147
846152 Green Canyon BIK 152

Allat lor: (C46183) Green Canyon BIK 163 [BLOCK]

846153 Green Canyon Blk 163 LAOO 995 Offehoro.Foderal

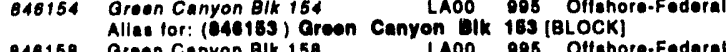

B46158 Groen Canyon BIk 158 LAOO 005 Offuhore-Foderal

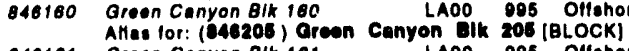

846181 Groen Canyon Bik 161 LAOO 095 Olfohoro.Foderal

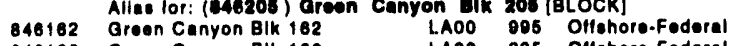

846186 LAOO 005 Oltehore-Fodera

846184 Groon Canyon Blk 184 LAOO DOS Oftehore-Foderal

846185 Green Canyon Blk 185 LAOO 095 Offshora-Federal

Allas tor: (e46104) Green Canyon BIk 104 [BLOCK]

846205 Gieen Canyon Blk 205 LAOO 095 Olfahore-Foderal

846228 Green Canyon Bik 228 LAOO 995 Othsioro-Fodora

$\begin{array}{llll}846235 & \text { Green Canyon B1k } 235 & \text { LAOO } 005 & \text { Offohore-Foderal } \\ 846472 & \text { Greon Canyon B1k 472 } & \text { LAOO } 095 & \text { Offehoro-Foderal }\end{array}$

846473 Green Canyon B1k 473 LAOO 995 OHtehoro-Foderal

Allas tor: (846472) Groen Canyon Bik 472 (BLOCK)

852330 High lolend EA 8X BIK A330 LAOO 095 Olfshore.Fodaral

852370 High loland EA SX BlK A370 LAOO 905 Olfohoro.Foderal

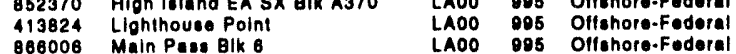

866007 Main Pase Bik Mallas lor: (866006) Main Pase Bik B [BLOCK]
$1080 \mathrm{~N}$

$1072 \mathrm{~N}$

$1080 \mathrm{~N}$

$1097 \mathrm{~N}$

$1072 \mathrm{~N}$

$1084 N$
1084

1985 ON

1980 OA

$1985 \mathrm{~N}$

$\begin{array}{ll}1089 & 0 \\ 1084 & 0\end{array}$

$\begin{array}{ll}1984 & 0 \\ 1088 & 0\end{array}$

1904 ON

$\begin{array}{ll}1085 & 0 \\ 1084 & 0\end{array}$

10840

10840

1988 OA
19840

1083 ONA

1004
$1008 \mathrm{~N}$

1984 ON

10020

$1088 \mathrm{~N}$

1081 ONA

$\begin{array}{ll}1988 & 0 \\ 1085 & 0\end{array}$

$\begin{array}{ll}1086 & O \\ 1084 & N\end{array}$

$1089 N$

$1073 \mathrm{~N}$

$1976 \mathrm{~N}$

1958 ON
Tina

Fad Nima
Alloperemente

-

Tinta co

County

Diso freld

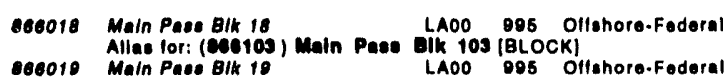

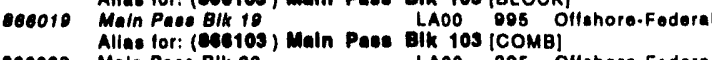

066029 Main Pase Blk 29 LAOO 995 Oflehoro-Foderal

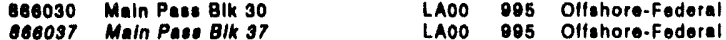

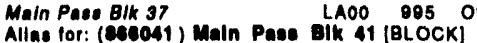

ceoose Main Pase Blk 98 . Aliae for: (Ceb127) Main Pase Bik 127 (BLOCK)

068030 Main Pase Blk 39 LAOO 096 Olfohoro-Foderal

$\$ 60040$ Main Pase Blk 40 LAOO 905 Ottehoro.Foderal Main Paso Blk 40
Allas for: (860041) main Pase Dik 41 [BLOCK]

968041 Main Pass Blk 41 LAOO 805 Olfehoro-Foderal

086042 Maln Paos Blk 42 LAOO 995 Ollshoro-Foderal

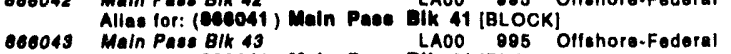

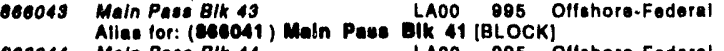

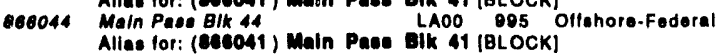

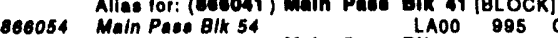

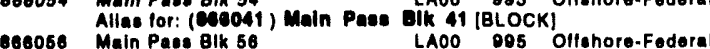

860056 Main Pass Bik 56
860057 Main Paes Blk 57 Malins for: (Booses) Main Pase DIK of [MULTI]

Alins for: (e00044) Main Pase bik sa (MULTI]

oesoss Main Pase B/k 58 LAOO 995 Ollshore.Foderal

006058 Main Pass Blk 58 ) Main Pags Blk 41 (BLOCK)

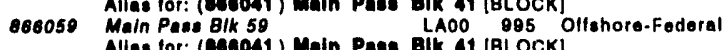

668064 Main Pase BIK 64 LAOO 995 Offshoro.Foderal

\$60065 Main Paee Blk 65 LAOO 995 Olfohoro-Foderal

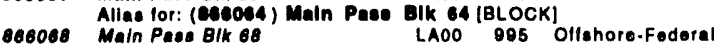

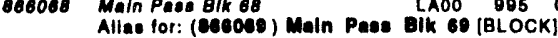

860069 Main Pass Bik 60 LAOO 995 Olishore-Foderal

868072 Main Pass Blk 72 La LAO 995 Olfshoro-Foderal

868073 Main Pase BIk 73
806074 Main Pase BIk 74 LAOO 995 Ollshore-Foderal

Alias for: (e60075) Main Pase Bik 73 (MULTI)

868077 Main Pase B/k 77 LAOO 995 Offehoro-Foderal

Aline for: (Eeb1B1) Main Pase Bik 181 [BLOCK]

808078 Main Page Blk 78 Lain Page LAOO 995 Oftehore-Foderal

868080 Alias for: (\$66151) Main Pase Bik 151 (BLOCK)

LAOO 985 Olrshore-Faderal Main Pass Blk 90 LAOO 995 Ollshoro.Foderal Allea for: (Eseoos) Maln Pase Blk B (BLOCK)

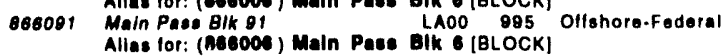

806002 Mein Pase Blk 82 L LAOO 995 Olishoro-Foderal Allas for: (868006) Maln Pase Bik \& [BLOCK]

868093 Main Pass BIk 93 LAOO 995 Olfshore-Foderal

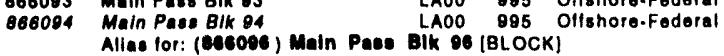
868095 Main Pass Bik 95
Alies for: (60006) Main Pase Bik 90 [COMB]

868098 Main Pass Blk 96 LAOO 995 Offehore-Foderal

886008 Main Pase Blk OB LAOO 995 Oltshoro.Foderal

866099 Main Paes B/k 99 LAOO 995 Olfohoro-Fodoral Allas tor: (Eesto7) Wain Pass Bik 107 [BLOCCK]

060100 Main Pase Blk 100 LAOO 995 O" shoro-Foderal

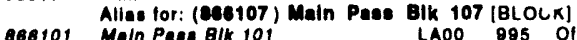

068101 Maln Pase Blk 101 L LAOO 995 Offer. O.Foderal

Alias for: (866107) Main Paes Blk 107 [BLOCK)

806102 Maln Paen Blk 102 LAOO 995 Ollehoro.Fodoral

PBI 886008

LAOO 905 OllshorO-Foderal

Alias lor: (868006) Main Pase Bik 6 [BLOCK]

866100 Main Pase Blk 106 LAOO 095 Oftenore-Fodoral

Allas for: (666107) Main Pase Bik 107 (BLOCK)

886107 Main Pass Blk 107 LAOO 995 Ottshore-Foderal

868108 Main Pass Blk LAOO 895 Olfshoro-Foderal

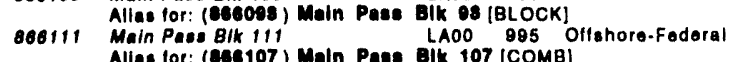

o66112 Main Pase Blk 112 LAOO PO5 Olishoro.Foderal

Alles for: (E66107) Maln Pase Bik 107 [BLOCK]

806113 Main Pase Blk 113 LAOO 895 Olfshoro.Foderal

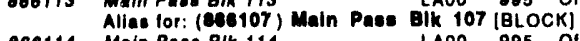

866114 Main Pase Bik 114 LAOO 995 Offshoro-Foderal

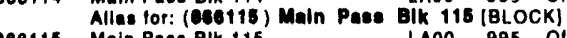

868115 Main Pass elk 115 LAOO 995 Olfshoro-Foderal

Besite Main Pase B1k 116 LAOO 995 Offehore-Foderal

Alius for: (806127) Main Paee Bik 127 [BLOCK]

Allas Por: (Batk 117 maln Page Blk 127 (BLOCK) Mallas for: (E66127) Maln Pase Bik 127 (BLOCK]

868120 Main Pass Blk 120 LAOO 995 Ollshore-Federal

808121 Main Paso BIK 121 LAOO 995 Oltshoro-Foderal

808121

Allas for: (868133) Maln Pase Bik 133 (BLOCK)

Main Pase BIK 124 Main Pase B Main Pase Blk 124 , Main Paes Blk 128 (COMB)

B66125 Main Pase BIK 125 LAOO 985 Oltshoro.Foderal

B86126 Maln Pass Blk 126 LAOO 895 Ollshoro-Fodoral

868127 Maln Pase Blk 127 LAOO 995 Offahoro.Federal

866128 Maln Pass Blk 128 LAOO 995 Olishoro-Foderal

806120 Main Pass Blk 129 LAOO 995 Ollshore.Fedora

Allas for: (866133) Main Pase Blk 133 (BLOCK

860133 Main Paes Blk 133 LAOO 995 Ollshore.Federa

868138 Mein Pase Blk 138 LAOO 905 Oflsholo-Fodoral

$68 B 130$ Main Pass BIk 139 LAOO 995 Offehoro-Foderal

806140 Main Pass BIK 140 LAOO 985 Olfshoro-Foderal

868141 Main Pass Blk 141 LAOO 995 Olfshore-Foderal

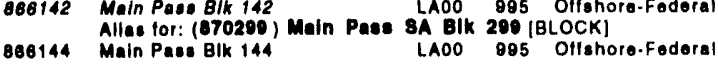

$\begin{array}{ll}1982 & N \\ 1084 & \text { OA }\end{array}$

$1984 \mathrm{~N}$

1056 ONA

$1988 \mathrm{~N}$

19820

1848 ONA

1075 ONA

$1986 \mathrm{~N}$

$1885 \mathrm{~N}$

1869
$1984 \mathrm{~N}$

$\begin{array}{ll}1983 & \text { ON } \\ 1968 & \text { ON }\end{array}$

1982 ON
$1985 \mathrm{~N}$

$1978 \mathrm{~N}$

$1877 \mathrm{~N}$

$1981 N$

$1986 \mathrm{~N}$

$1985 N$

$1985 N$

$1961 \mathrm{~N}$

1970 ONA

$1984 \mathrm{OA}$

$1988 \mathrm{~N}$

1893 O

1967 ONA 
F.O.- LOUISIANA

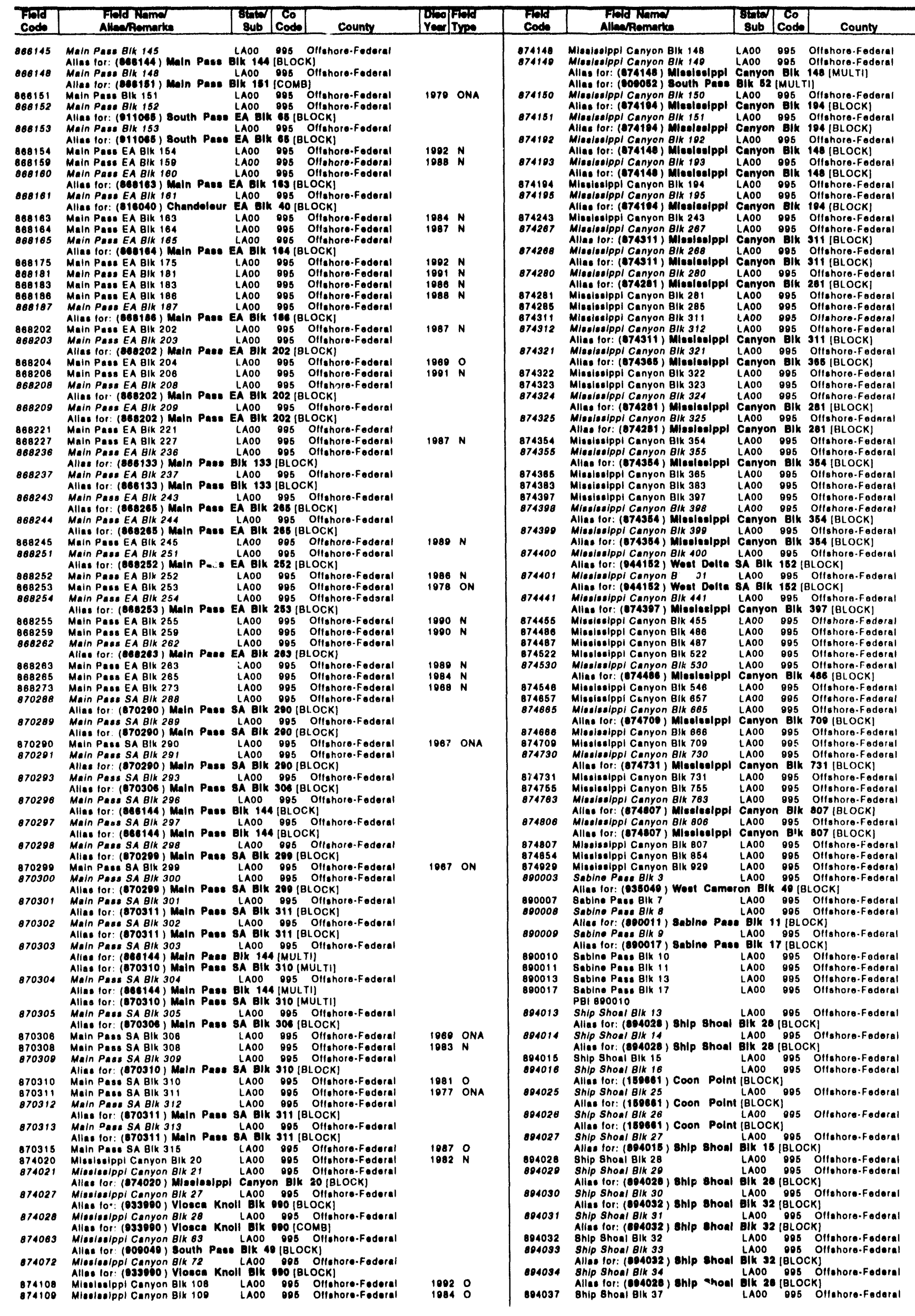

$1875 \mathrm{~N}$

$1993 \mathrm{~N}$

976 ONA

987
$O$

$1087 \mathrm{~N}$
$1884 \mathrm{~N}$

9770

$1985 \mathrm{~N}$

$1887 \mathrm{O}$
$1984 \mathrm{~N}$

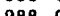

$\begin{array}{ll}1988 & \mathrm{O} \\ 1978 \mathrm{~N}\end{array}$

$1892 \mathrm{~N}$

$1880 \quad 0$

18880

$1988 N$

$1987 \mathrm{~N}$

$1886 N$

19820

$1988 \mathrm{~N}$

$1884 \mathrm{~N}$

$1978 \mathrm{~N}$

$1980 \mathrm{~N}$

$1879 \mathrm{~N}$
$1880 \mathrm{~N}$

$1880 \mathrm{~N}$

$1940 \mathrm{ON}$

$1047 \mathrm{ON}$

$1085 \mathrm{~N}$ 


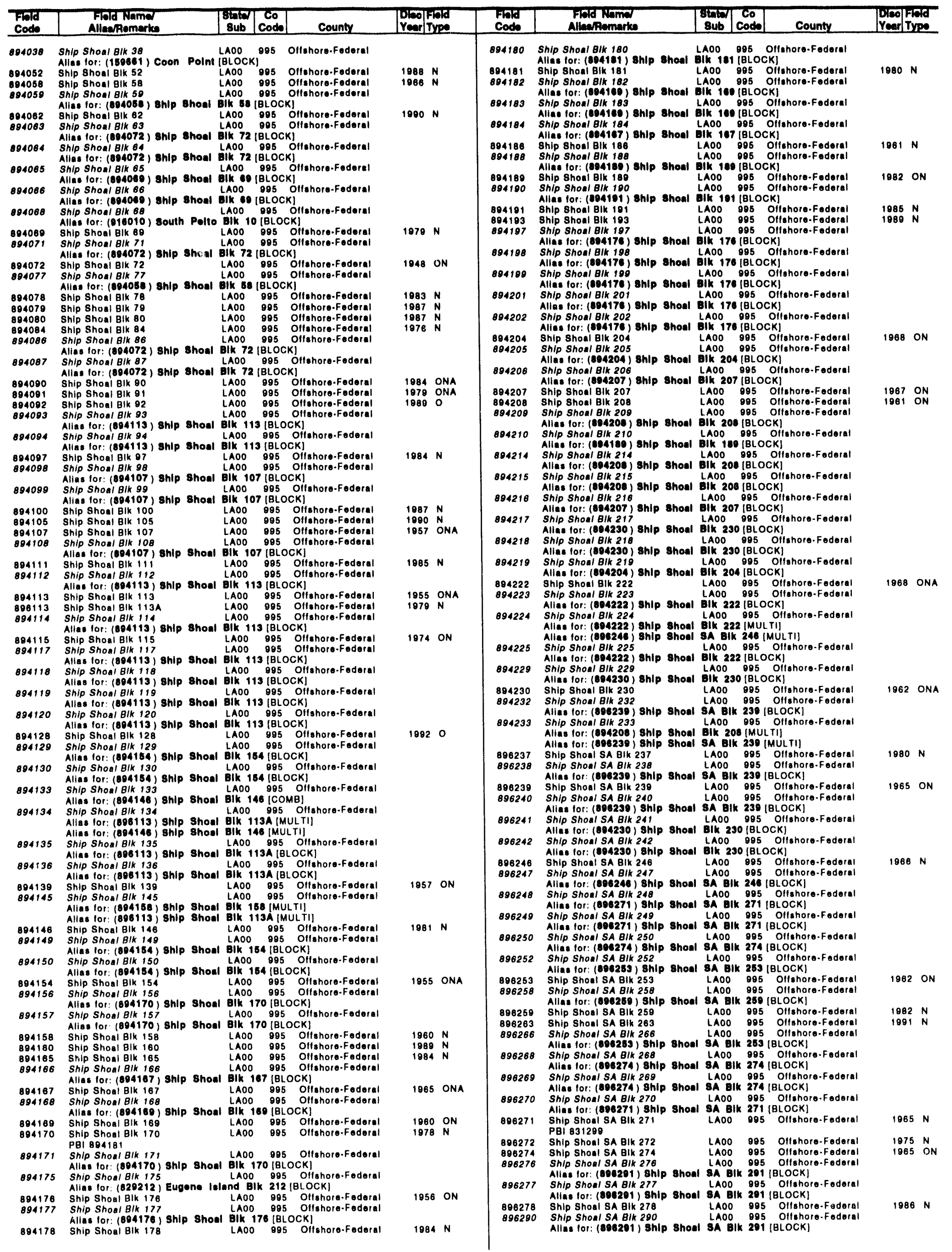


F. O.-LOUISIANA

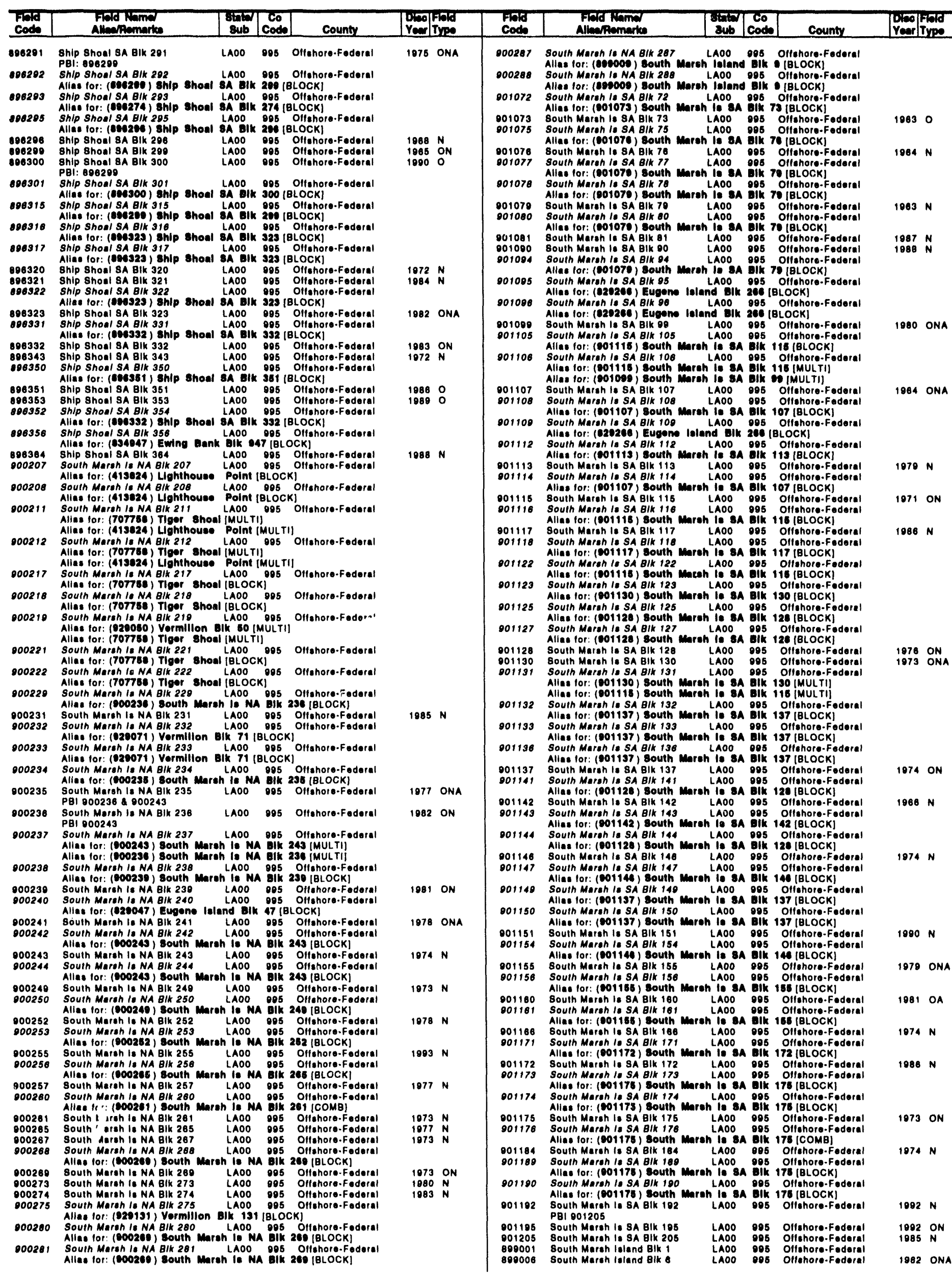




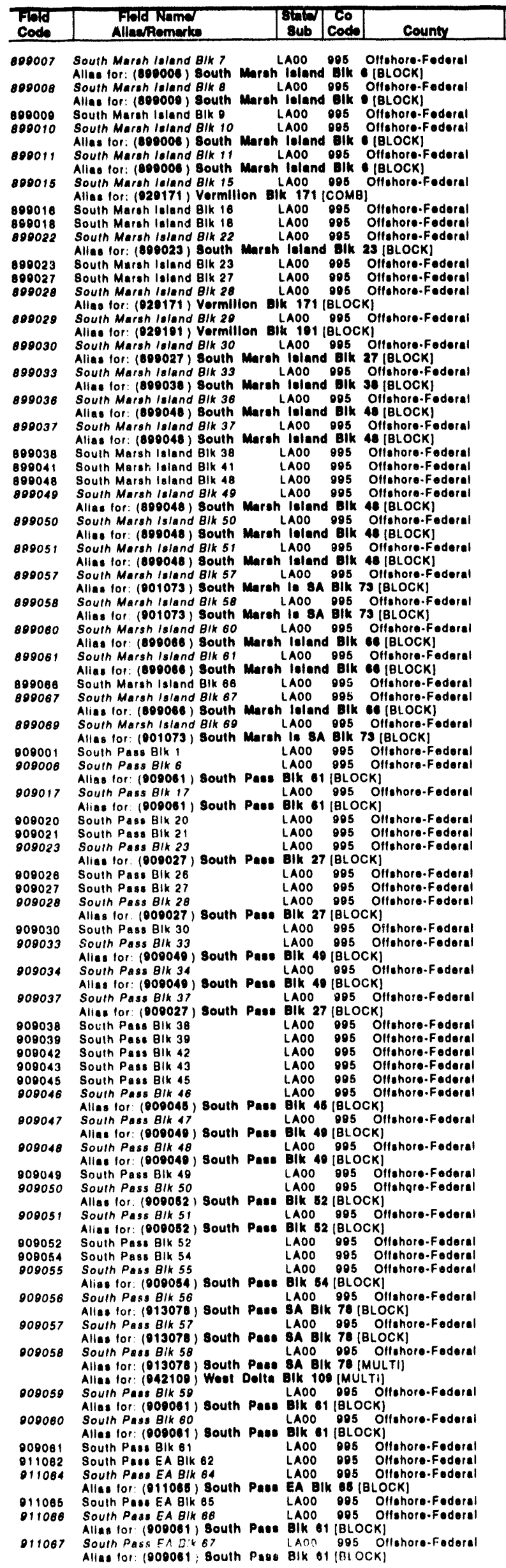

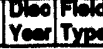

1000 O

1963 ON

1063 ON
1081 ON

1088 OA

$1050 \mathrm{~N}$

1058 ON

1957 N

$1955 \mathrm{~N}$

(

1962 ON

$\begin{array}{ll}1056 & N \\ 1080 & \text { ONA }\end{array}$

1081 N

(

1074 ONA

1988 ONA

1989 ON

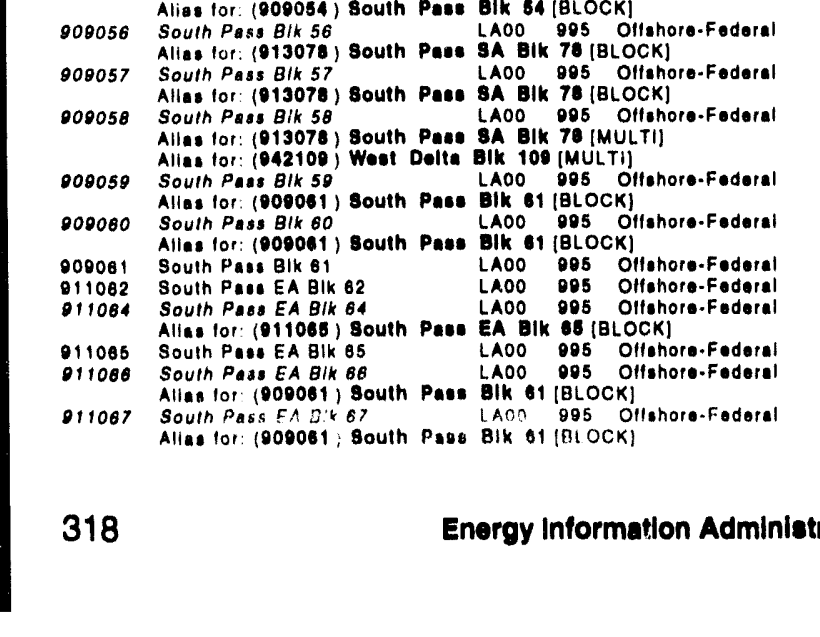

1060 OA

Cods

Find Noma

con

County

limentirat

O11070 South Pase EA BIK $70 \quad$ LAOO OOS Olfshore-Foderal LAOK o1 [BLOCK)

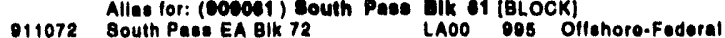

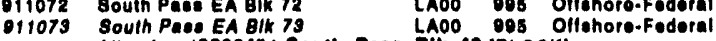

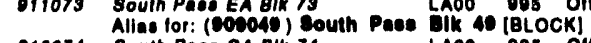

013074 South Pane SA BIK 74 LAOO 095 Ollohore-Foderal

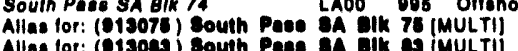

013078 South Pace 8A Bik 75 LAOO OOS Olth

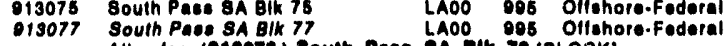

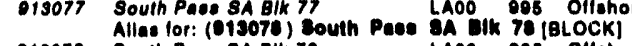

013078 Bouth Paise $8 A$ Blk 78

LAOO 085 Ollehore-Foderal PBI 042100

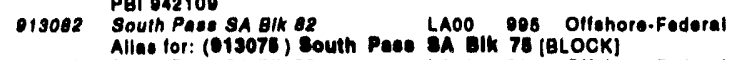

O13083 8outh Paes 8A BIK BS LAOO OOS Oltohore-Federal

O13008 South Paed SA Blk 86 LAOO 995 Olfohore-Fodoral

o1s087 South Pags SA Bik Br

Alies for: (013000) Bouth Pase OA BIK 10 [BLOCK]

913088 South Pase SA BIK 88 A LOO 905 Oltahore.Foderal

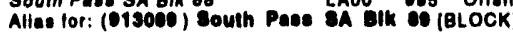

913089 South Pase 8A B1k 89 LAOO 995 Otishore.Foderal

Allas for: (O13000) South Pase BA BIK 00 [BLOCK]

013094 South Paes SA BlK 94 . LAOO 905 Olfshore.Faderal

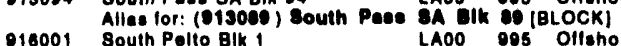

LAOO 095 Offehore.Federal

016002 South Pollo Blk 2 LAOO 995 Offohore-Foderal

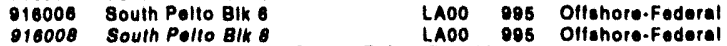

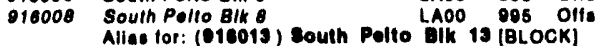

010000 South Pelto Blk 9 .

016010 Allat for: (\$16010) Bouth Polto Bik 10 (BL.OCK)
LAOO 095 Ollehore-Federal

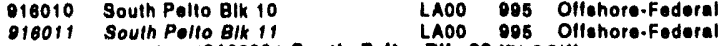

South Polto Blk '1
Alias tor: (E16020) south Pelto LAOK 20 (BLOCK)

016012 Soulh Polto Blk 12 LAOO 995 Oftuhore.Foderal

010013 Slias for: $(010020)$ south Pelto BlK 20 (BLOCK)
LAOO 005 Ollshore.Foderal

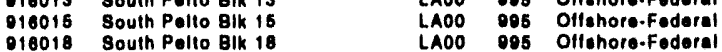

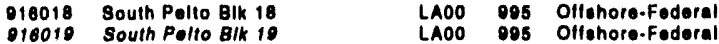

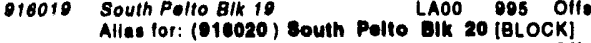

016020 South Pollo Blk 20 LANO 905 Ollshore-Foderal

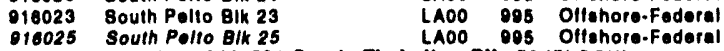

016025 South Pollo Blk 25
Allas for: (010082) south TImballor OIK 82 (BLOCK)

010021 South Timballer BIK 21 LAOD 096 Olfehoro-Federal

019022 South Timballor Blk 22 LAN TImbo 095 Ollehore.Federal

010023 Allas tor: (918021) Bouth Timbelior Blk 21 (BLOCK)

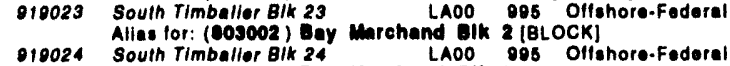

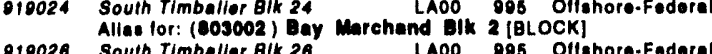

010028 South Timballer Blk 20

Alles for: (003002) Bay Marehand Bik 2 (BLOCKI

010027 South Timbellor Bik 27 LAOO 905 Ollehore-Federal

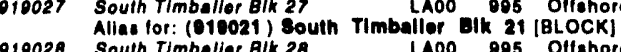

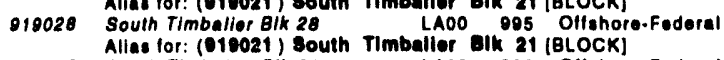

919029 South Timballor Bik 29 LA00 905 Ollshoro-Foderal

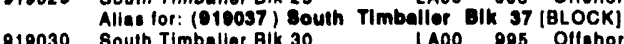

South Timballer Bik 30
910031
South Timballor Bik 31

Allas lor: (O19030) Bouth TImballor Bik 30 [BLOCK]
[BLE

910034 South Timbalier BIK 34 LAOO DOS Otfehoro.Fodoral

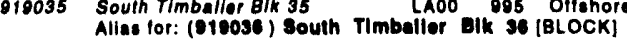

910036 South Timballor Blk $3 B$ LAOO 995 Ollohoro.Foderal

010037 Bouth Timbalior BIK 37 LAOO 095 Offehore. Fodoral

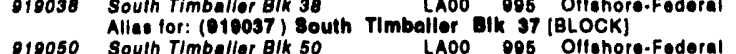

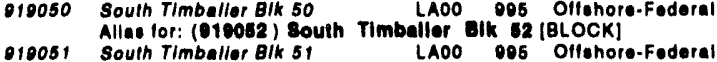

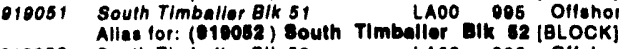

919052
0 South Timballer Bik 52

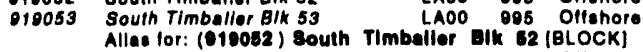

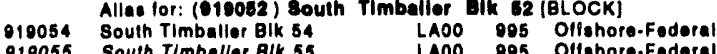

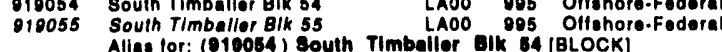

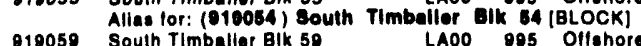

018059 South Timbalier BIK 50 LAOO 995 Oltshoro.Foderal

919003 South TImballer BIK B3 LAOD 995 Ollahoro.Fodoral

018000 Allas for: (018008) Bouth TImballer BIK 20 [BLOCK]

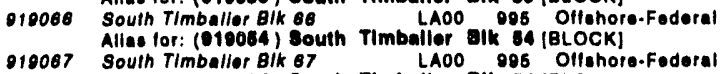

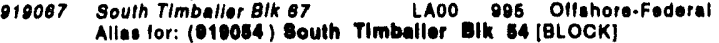

918088 South Timballor Bik 88 LA LAOO 905 Oltohore.Foderal

Allas for: (019084) South Timballer BIK BA (BLOCK)

919071 South Timbeller BIK 71 LADO 905 Offehoro.Foderal

010072 South Timbalior B1k 72 LAOO 095 Offohoro.Foderal

Alla for: (010023) Bouth Polto BAk 23 [BLOCK]

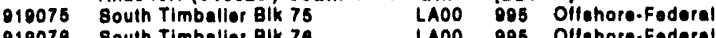

0

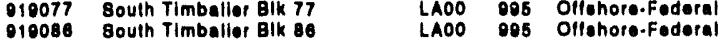

019087 South TImballor Blk 87 LADO 006 Olfehoro.Foderal

Allas for: (O18000) South TImballor BIK es [BLOCK]

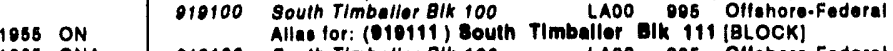

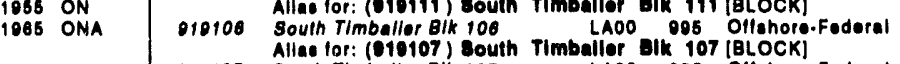

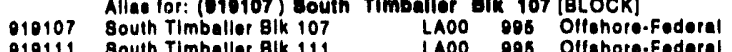

019111 Bouth Timballor Blk $111 \quad$ LAOO OOS Offohoro-Foderal

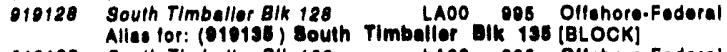

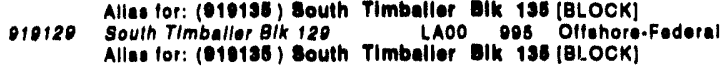

$1900 \mathrm{~N}$

1984 ONA

1073 ON

$1008 \mathrm{~N}$

1000 ONA

$1081 \mathrm{OA}$

$1082 N$
$1003 N$

1975 ON

1078 ON

$\begin{array}{ll}1082 & N \\ 1070 & 0\end{array}$

1951 ONA

1962 ONA

10870

1970 N

1940 ON

1974 ONA

10500

$1955 O N$

$1079 \mathrm{~N}$

$1088 \mathrm{~N}$

1086 ONA

$1080 N$
$1986 \mathrm{~N}$ 


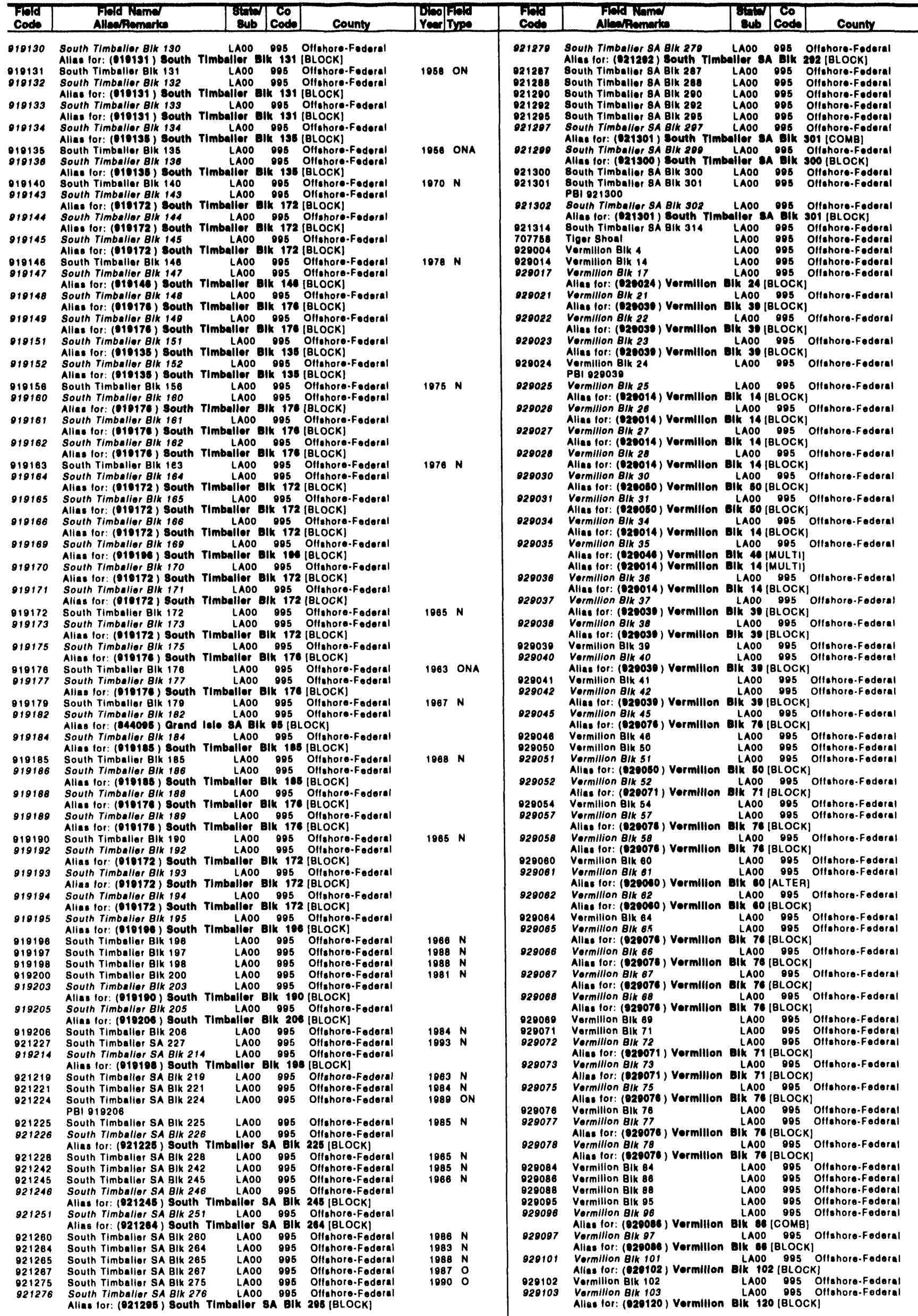

$1086 \mathrm{~N}$

1905 O
1087 O

$\begin{array}{ll}1087 & \mathrm{O} \\ 1082 & \mathrm{~N}\end{array}$

$1002 N$
$1084 N$

1081 ON

$1987 \mathrm{~N}$

$1058 \mathrm{ON}$

$1063 \mathrm{~N}$

$1902 \mathrm{~N}$

1949 ON

$1093 \mathrm{~N}$

1956 ON

$1983 \mathrm{~N}$

$1975 \mathrm{~N}$

$1875 \mathrm{~N}$

$1984 \mathrm{~N}$
1848 ON

$1840 N$

$1977 \mathrm{~N}$

$1057 \mathrm{~N}$

$1088 \mathrm{~N}$

$1056 \mathrm{~N}$ 


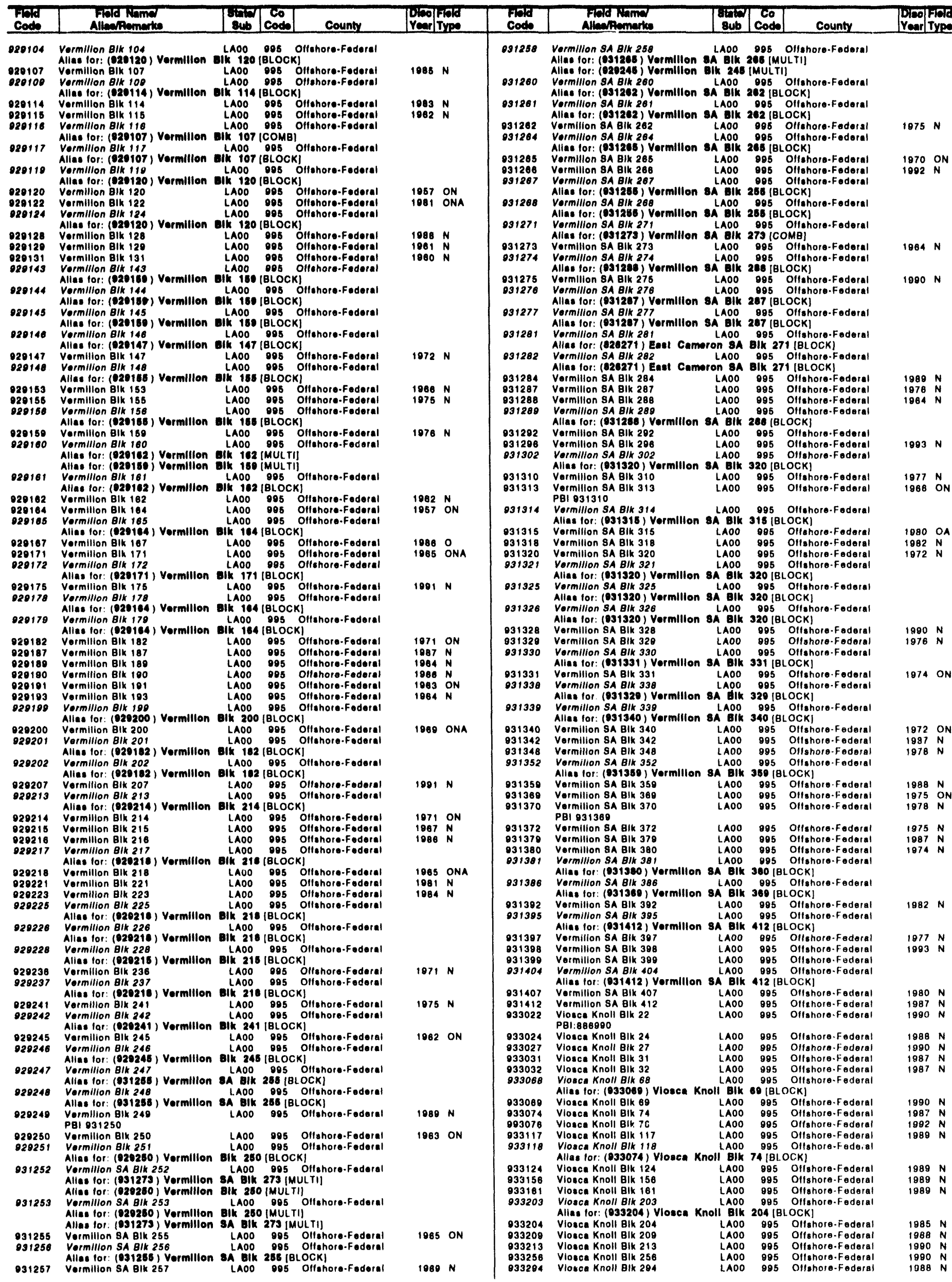




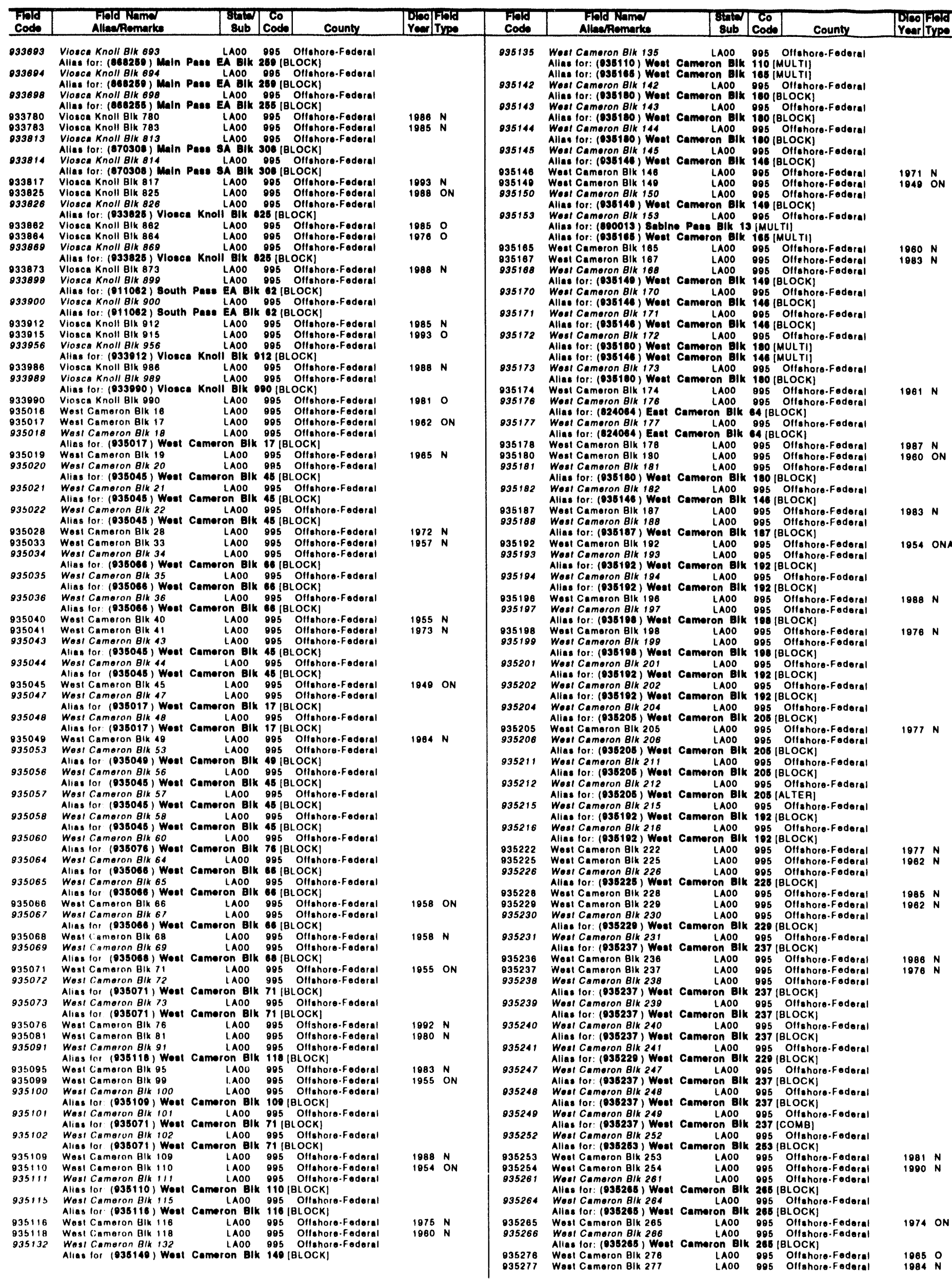




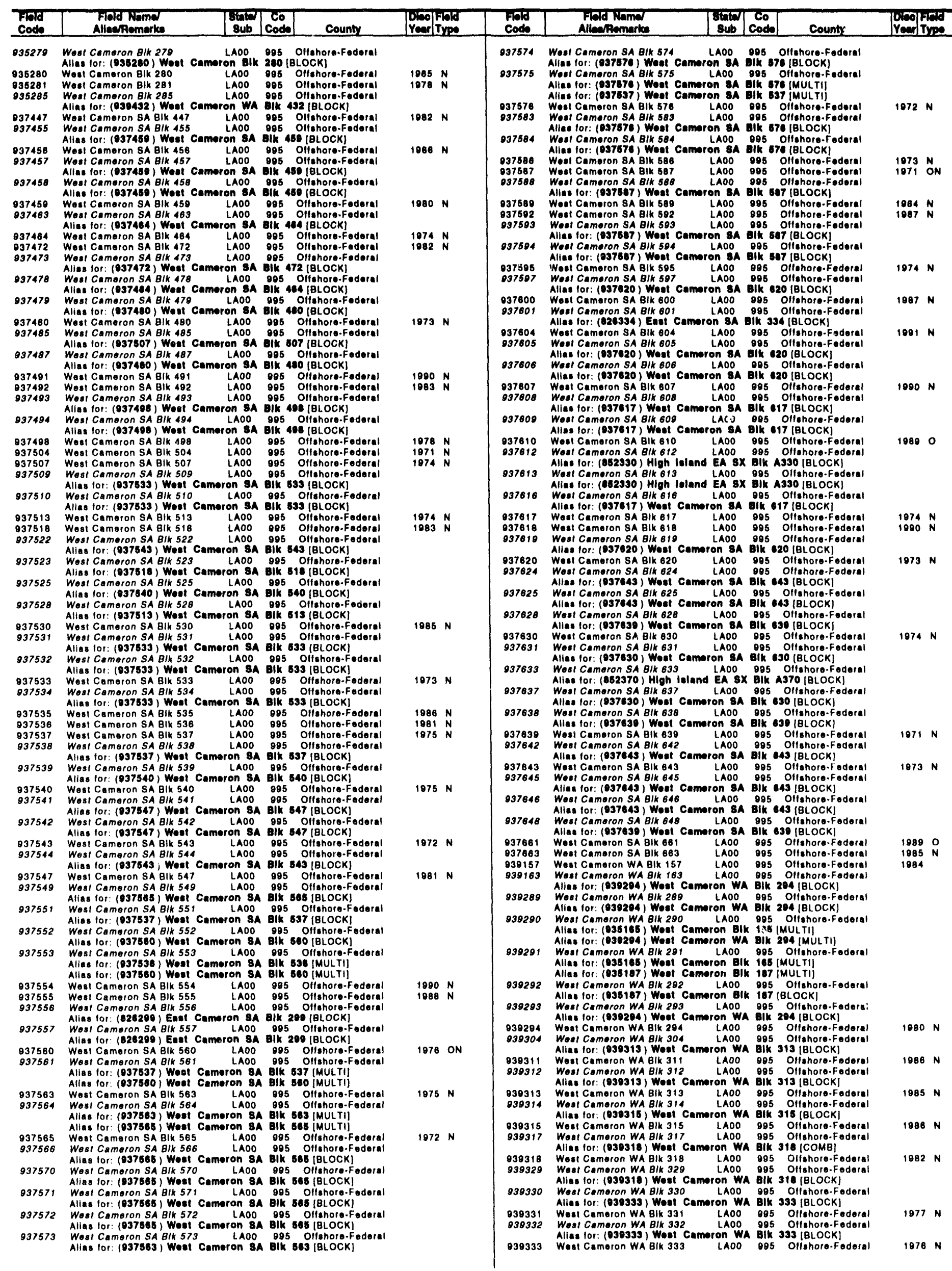




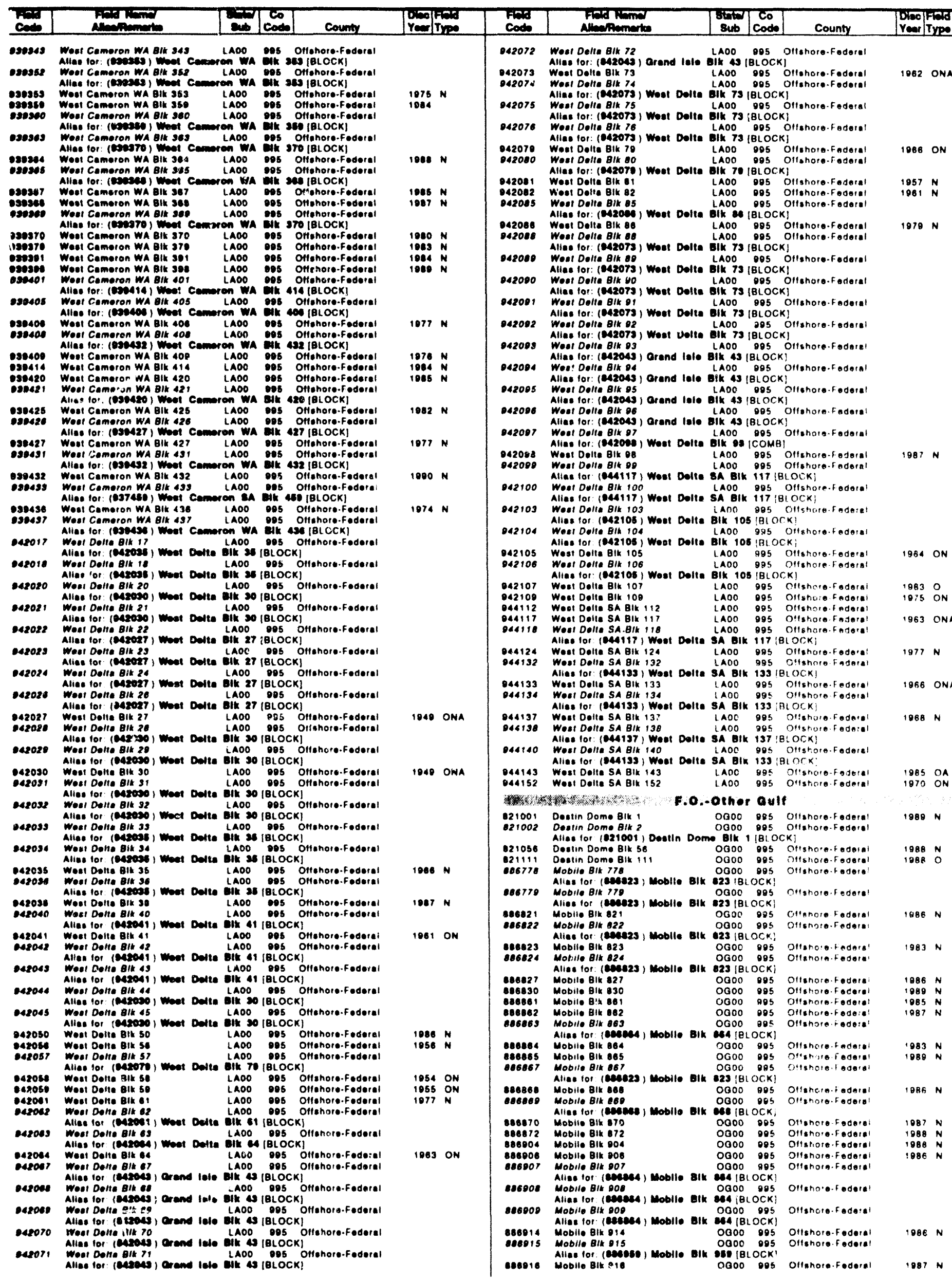




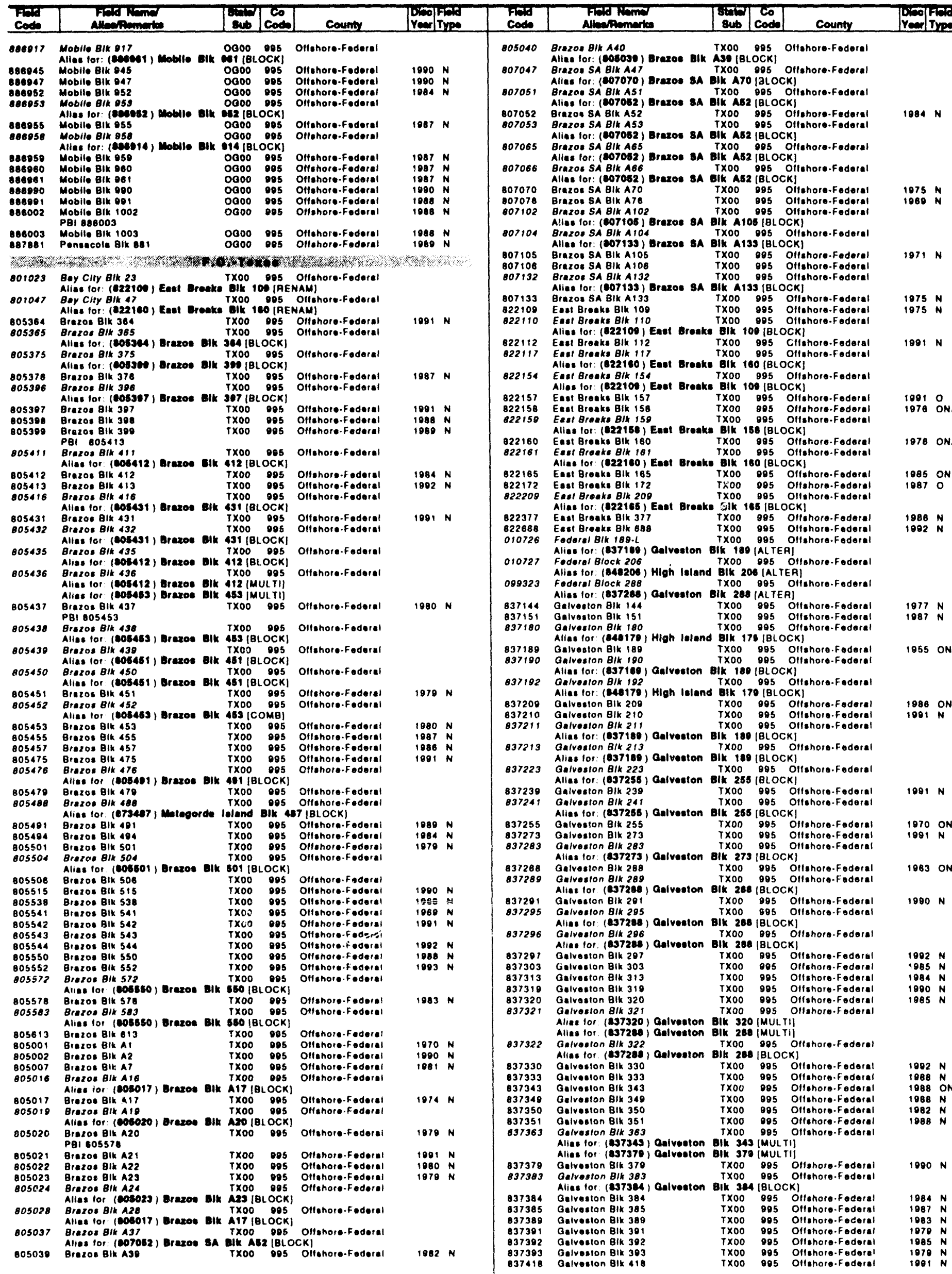




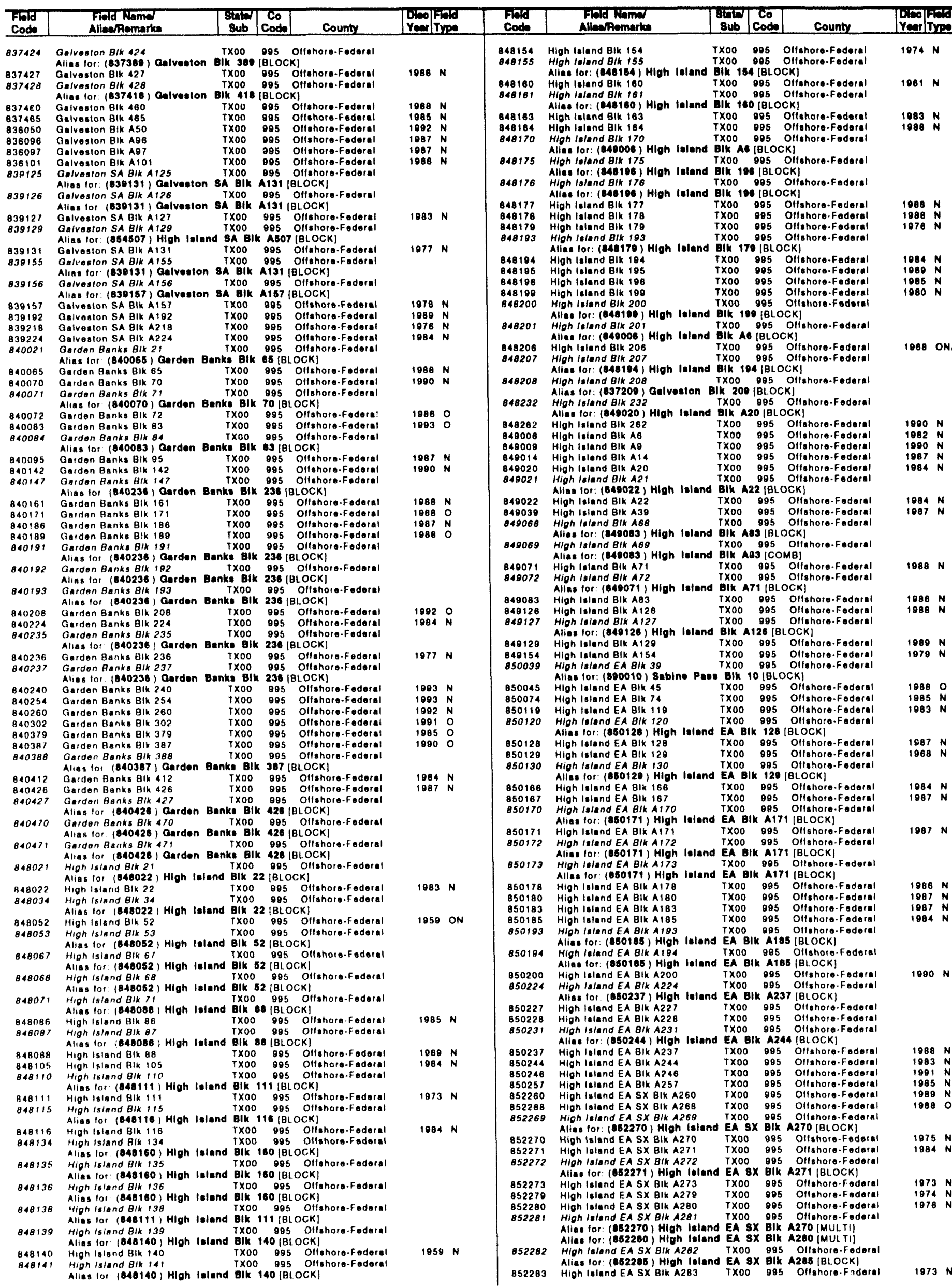




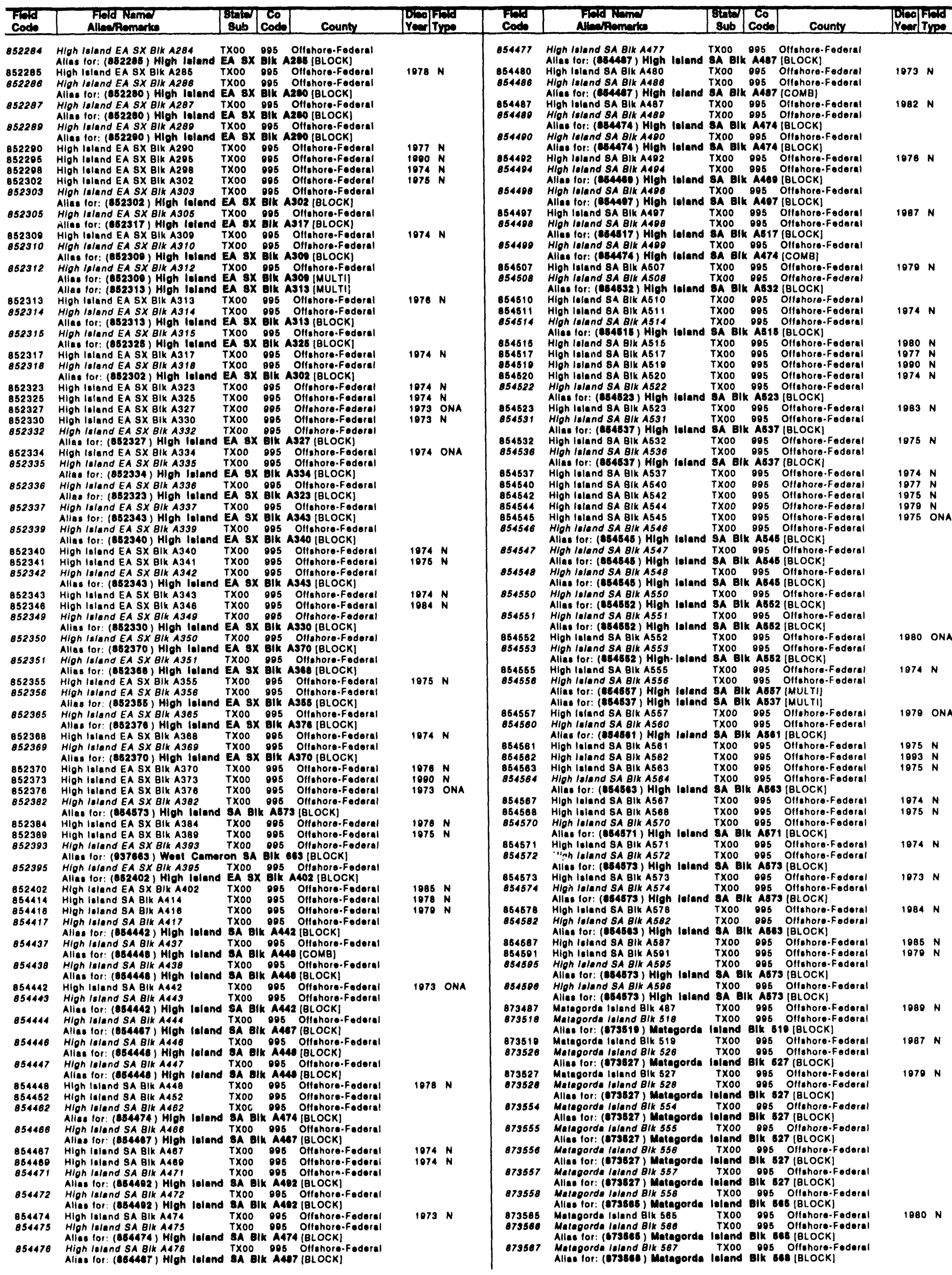


F.O.-TEXAS

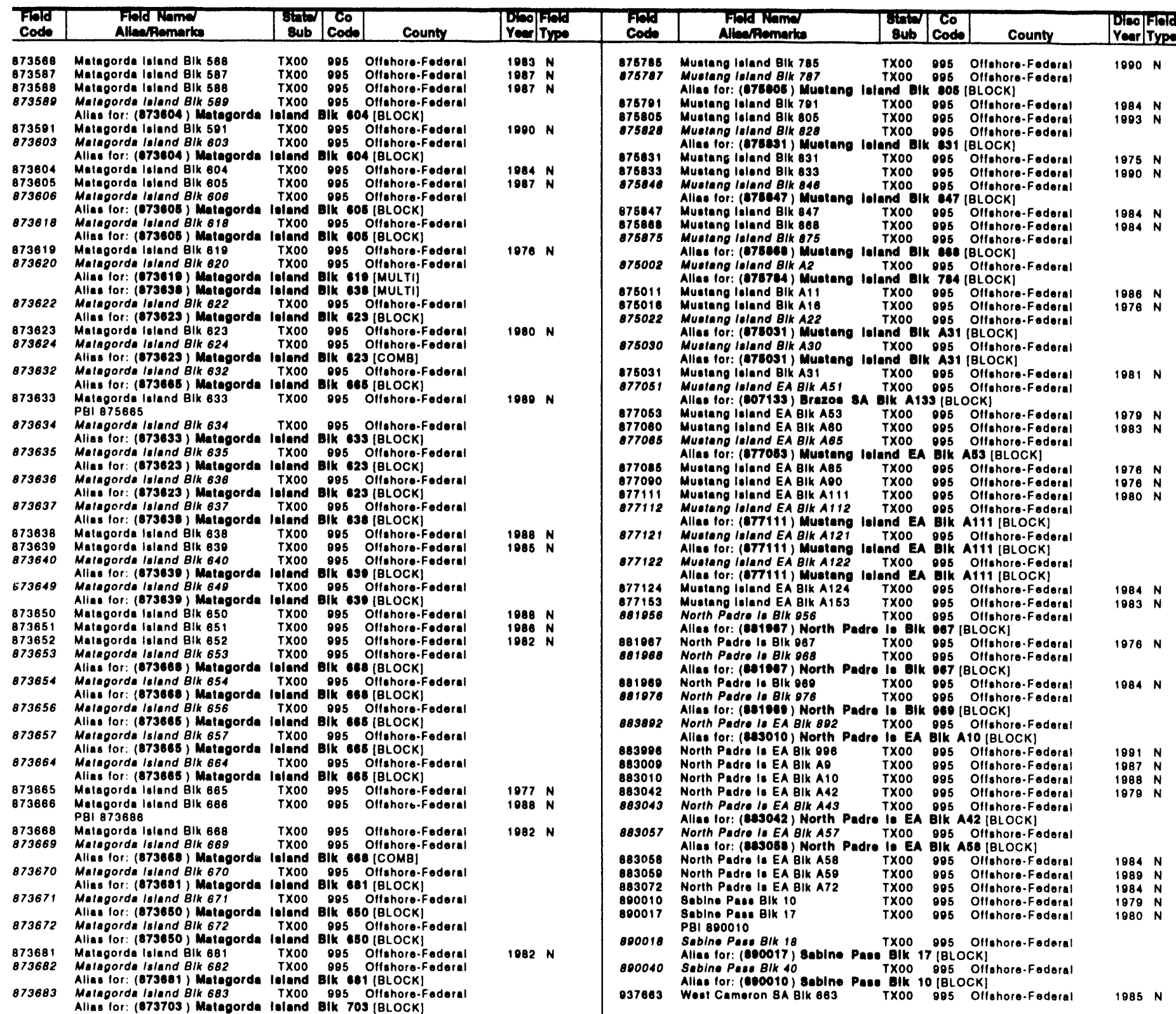

$\begin{array}{ll}1978 & N \\ 1988 & N\end{array}$

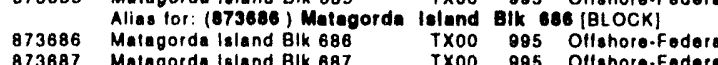

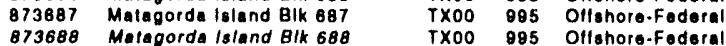
Alias lor: (873687) Matagorda leland bik 887 [BLOCK]

873698 Mategorda laland Blk 696 TX00 995 Otfohorn.Fodoral

Alles lor: (873696) Matagorda laland Bik 696 (BLOCK)

Metegorde Island Blk 699 TXOO 995 Oltshore-Foderal Allas for: (873700) Mategorda Ialand Bik 700 [BLOCK]

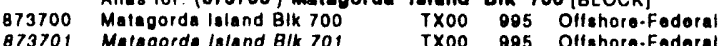
Matagorda laland Blk 701 TX00 995 Olfahora.Foda
Alias lor: (873700) Matagorda laland Bik 700 [BLOCK]

873703 Masagorda tsland Bik 703 TX00 985 Olfshore.Foderal

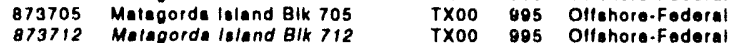
Malagorda lsland Blk 712 TX00 895 Olfshora-Foderal
Allas lor: (873700) Matagorda leland Bik 700 [BLOCK] Allas lor: (873700) Matagorda leland Bik 700 [BLOCK]
Malegorda ls/and Blk 713 TX00 995 Otfehoro.Fodorat Alias for: (073700) Matagorda leland Bik 700 (BLOCK)

873004 Malagorda lsland Blk A4
873007 TXOO 985 Oltehoro-Foderal

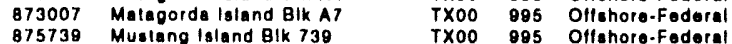

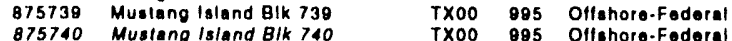

875742 Mustang island B/K 742 TX00 995 Olfohore.Fodoral Alins for: (875754) Muetang Island BIK 754 (BL.OCK)

875752 Musteng lsland Blk $752 \quad$ TX00 995 Ollshore-Foderal

875754 Muslang laland Bik 754 TX00 895 Olfehoro-Foderal

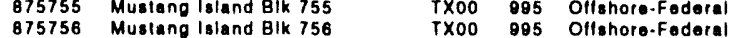

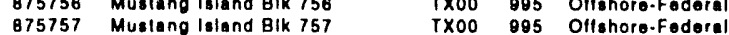

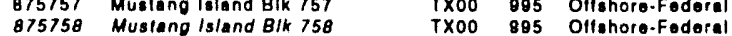

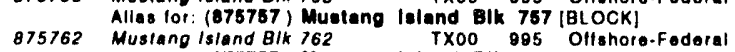

875762 Mustang lsland Blk 762 TX00 985 Olfshora-Fodoral
Allas lor: (878757) Mustang Island B1K 757 (COMB)

875763 Mustang island Blk 763 TX00 995 Otishoro-Foderal

875778 Musteng laland Blk 778 TX00 895 Oltshore-Foderal

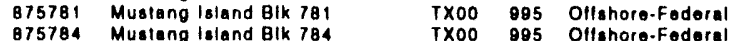

$1982 N$

$1975 \mathrm{~N}$

$\begin{array}{ll}1982 & N \\ 1088 & N\end{array}$

$1988 \mathrm{~N}$

$1084 \mathrm{~N}$

$1977 N$

$1988 \mathrm{~N}$

1985 ON

$1977 \mathrm{~N}$

$\begin{array}{ll}1088 & N \\ 1078 & N\end{array}$

$1988 \mathrm{~N}$

$1987 N$
1084 


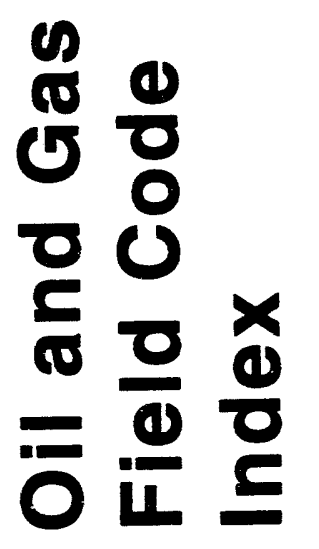




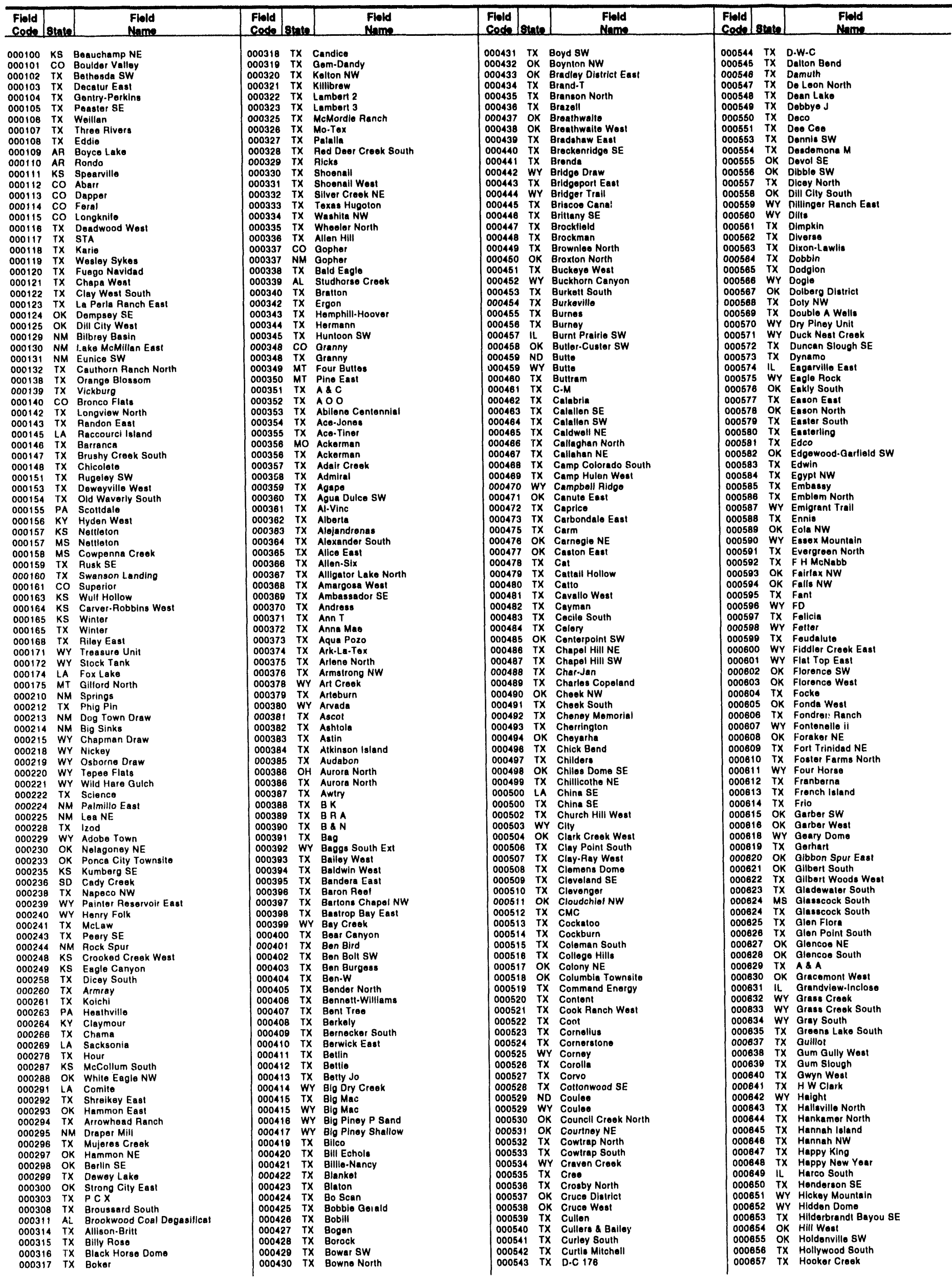


001110

\begin{tabular}{|c|c|c|c|c|c|c|c|c|c|c|c|}
\hline $\begin{array}{l}\text { Fleld } \\
\text { Code }\end{array}$ & 8tats & $\begin{array}{l}\text { Fild } \\
\text { Name }\end{array}$ & $\begin{array}{l}\text { Fiold } \\
\text { Code }\end{array}$ & State & $\begin{array}{l}\text { Flold } \\
\text { Name }\end{array}$ & \begin{tabular}{l|l|l} 
Fleld & \\
Codp & 8 \\
\end{tabular} & State & $\begin{array}{l}\text { Flold } \\
\text { Name } \\
\end{array}$ & $\begin{array}{l}\text { Field } \\
\text { Code }\end{array}$ & Btate & $\begin{array}{l}\text { Fiold } \\
\text { Name }\end{array}$ \\
\hline 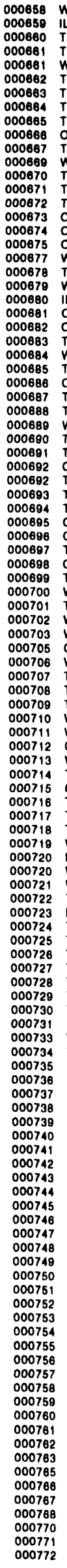 & 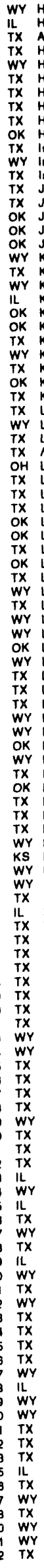 & 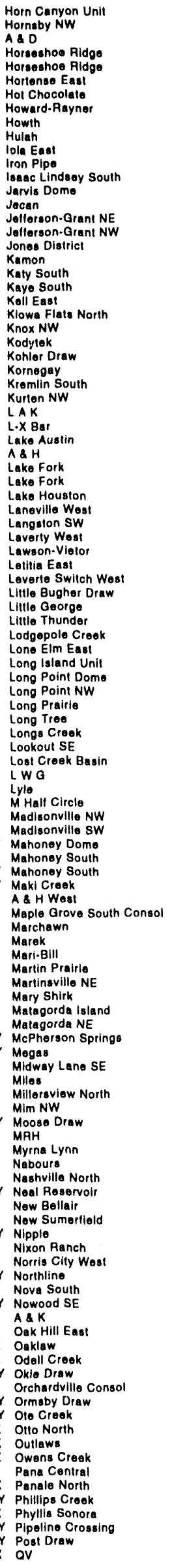 & 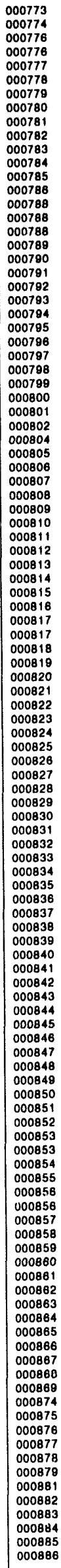 & 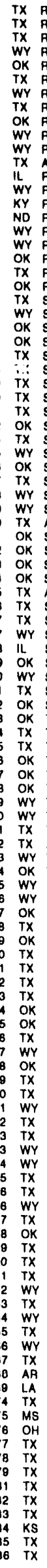 & 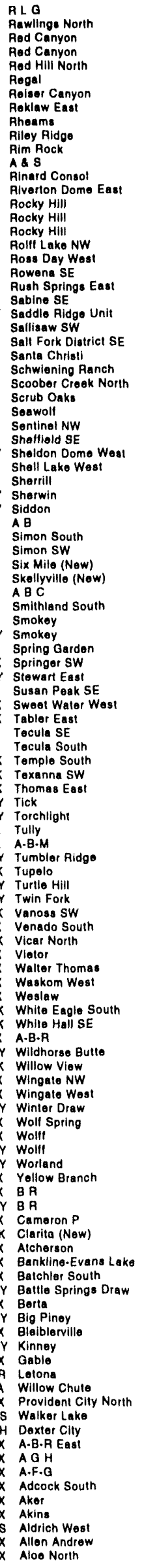 & 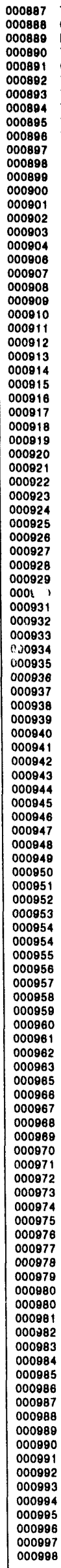 & 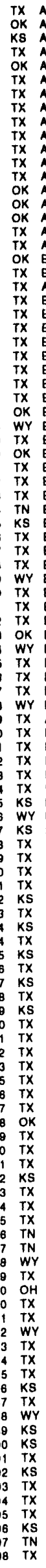 & 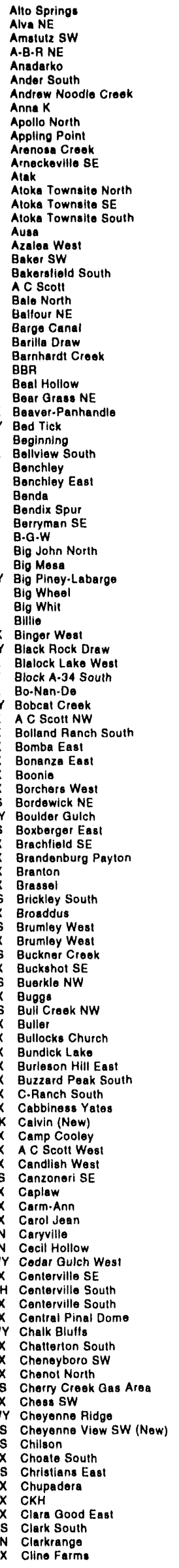 & 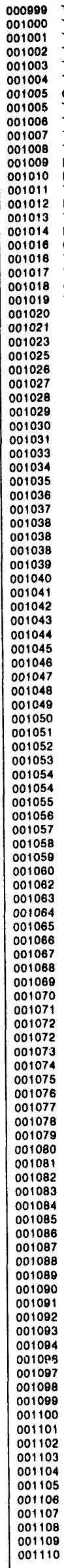 & 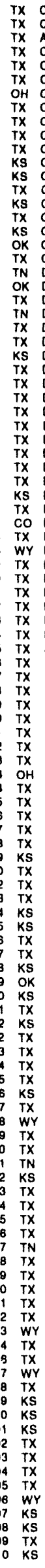 & 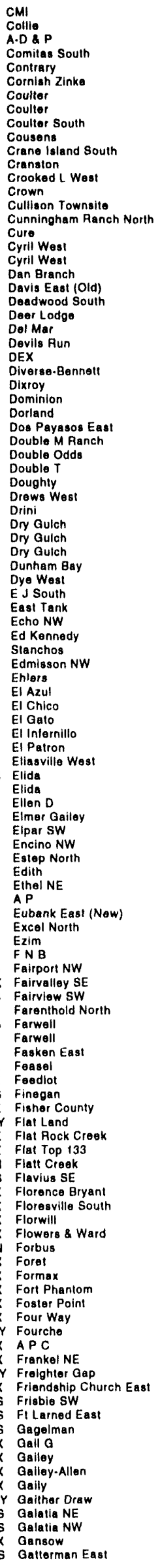 \\
\hline
\end{tabular}




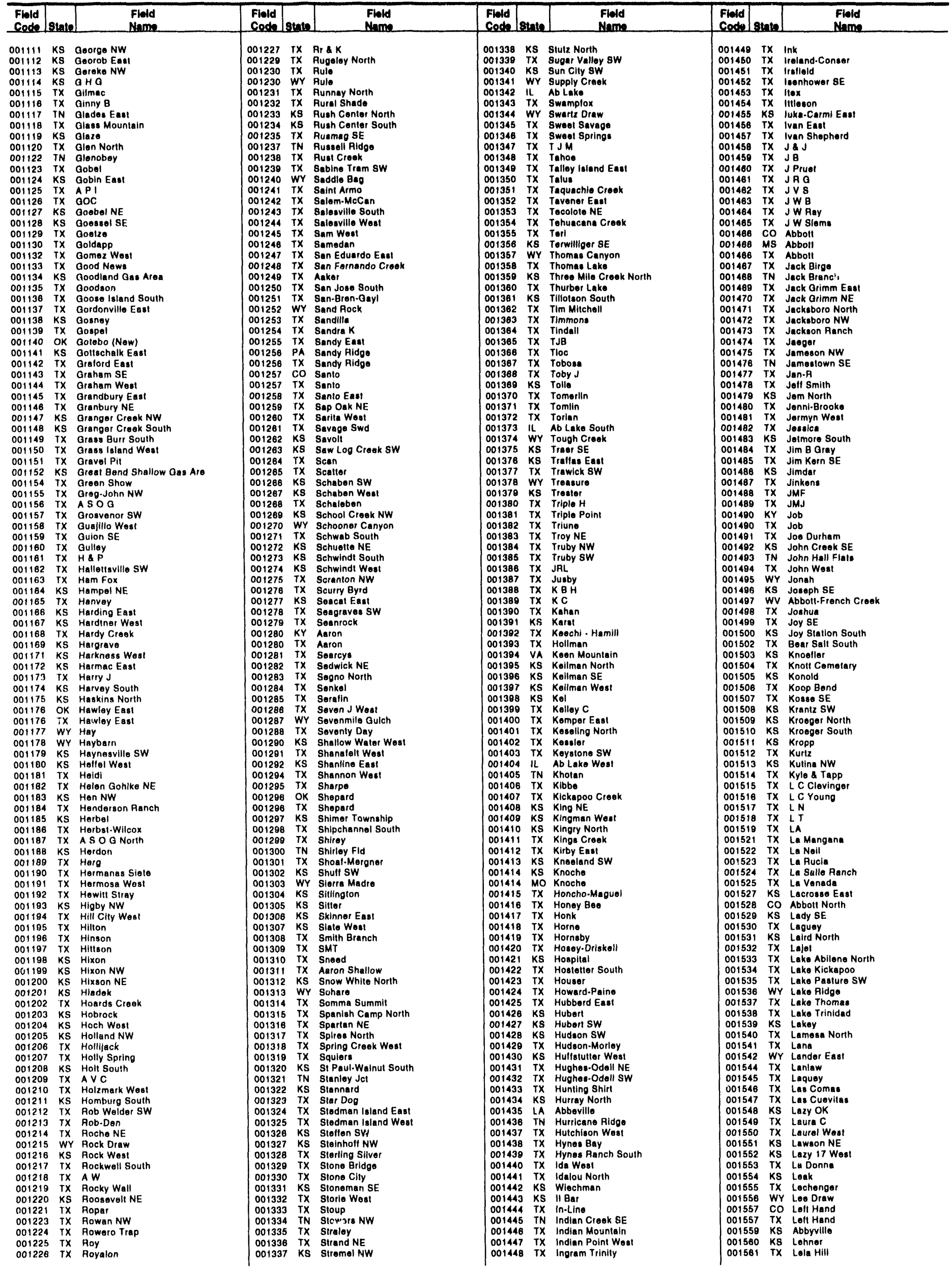




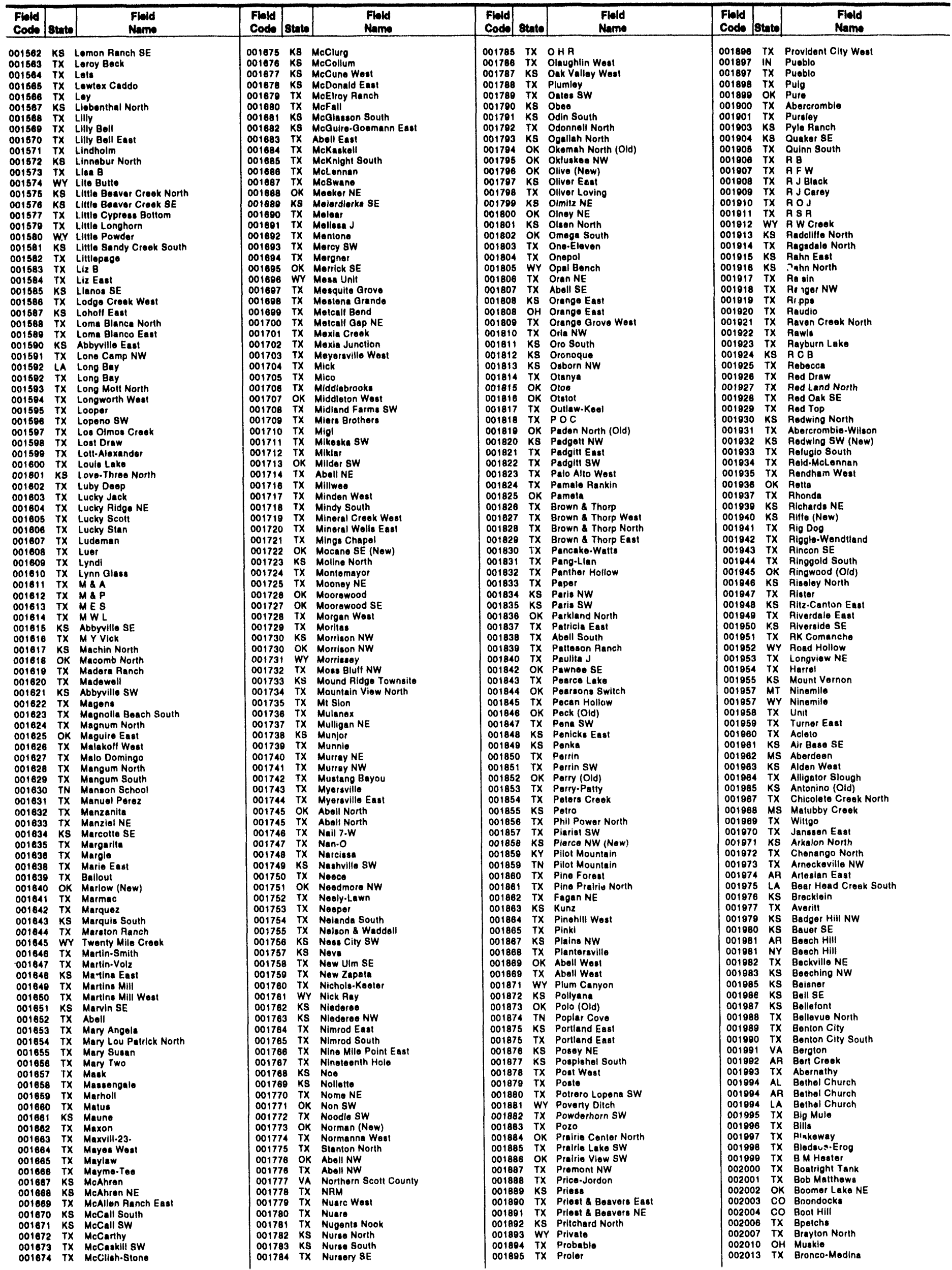




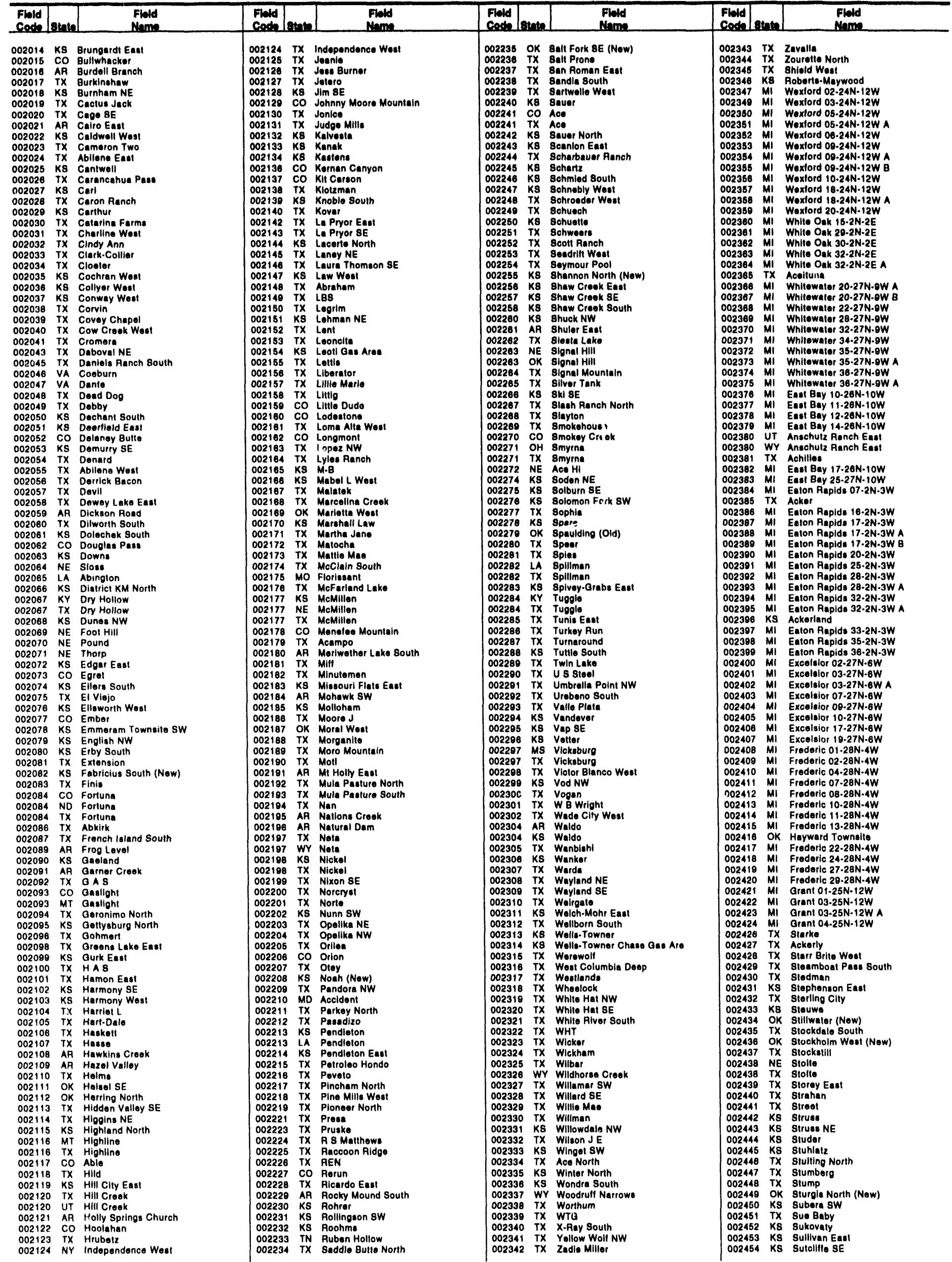




\begin{tabular}{|c|c|c|c|c|c|c|c|c|c|c|c|}
\hline $\begin{array}{l}\text { Fibld } \\
\text { Cods }\end{array}$ & stoto & $\begin{array}{l}\text { Flold } \\
\text { Name }\end{array}$ & $\begin{array}{l}\text { Flald } \\
\text { Cods }\end{array}$ & Btate & $\begin{array}{l}\text { Fiold } \\
\text { Neme }\end{array}$ & \begin{tabular}{l|l} 
Find & Cods
\end{tabular} & Stulo & $\begin{array}{l}\text { Fleld } \\
\text { Name }\end{array}$ & $\begin{array}{l}\text { Field } \\
\text { Code }\end{array}$ & 8tate & $\begin{array}{l}\text { Fiold } \\
\text { Name }\end{array}$ \\
\hline 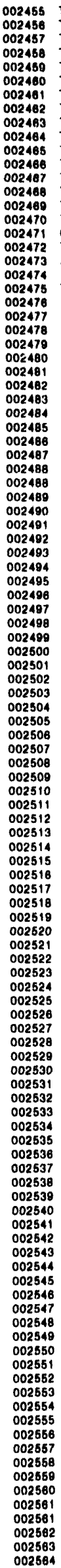 & 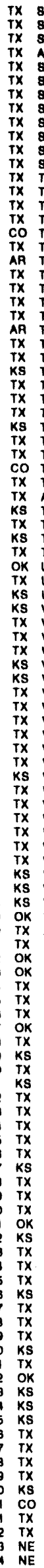 & 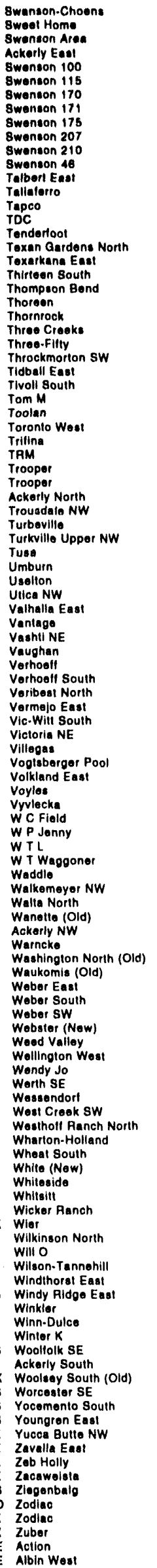 & 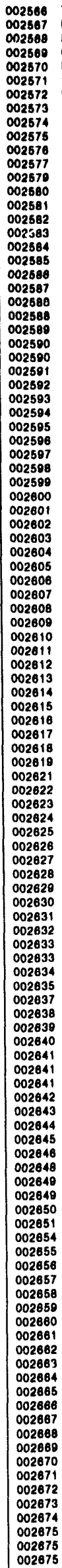 & 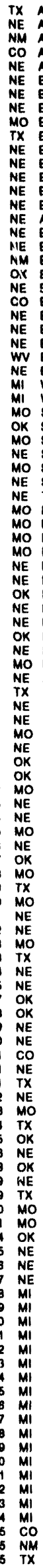 & 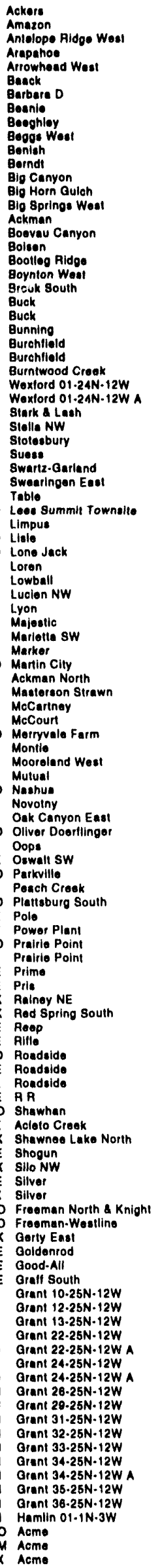 & 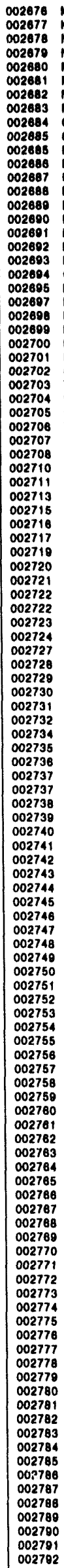 & 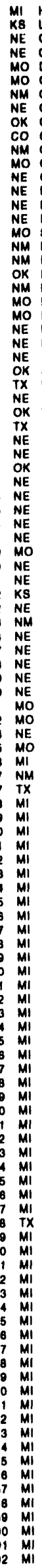 & 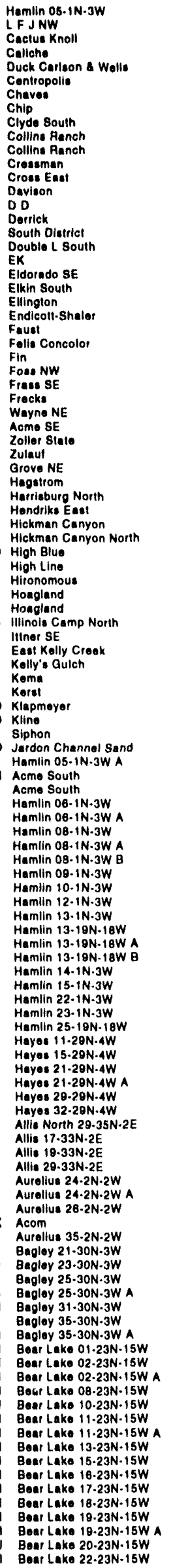 & 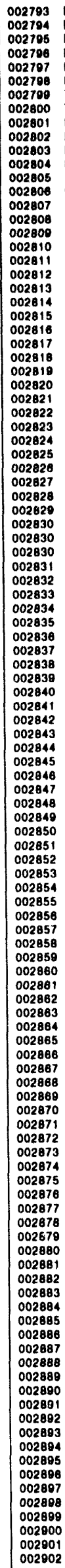 & 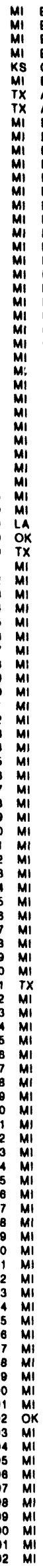 & 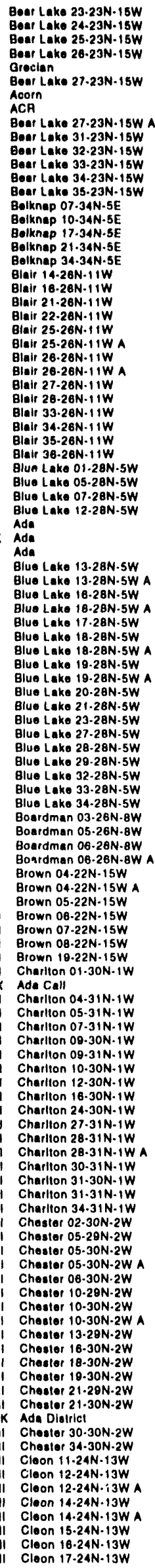 \\
\hline
\end{tabular}




\begin{tabular}{|c|c|c|c|c|c|c|c|c|c|c|c|}
\hline $\begin{array}{l}\text { Fiold } \\
\text { Code }\end{array}$ & $\mathrm{at}$ & $\begin{array}{l}\text { Fleid } \\
\text { Nem. }\end{array}$ & $\begin{array}{l}\text { Field } \\
\text { Coed? }\end{array}$ & & $\begin{array}{l}\text { Fild } \\
\text { Name }\end{array}$ & \begin{tabular}{|l|} 
Field \\
Cost
\end{tabular} & & $\begin{array}{l}\text { Fleld } \\
\text { Nom }\end{array}$ & $\begin{array}{l}\text { Fiold } \\
\text { cosels }\end{array}$ & & $\begin{array}{l}\text { Fiald } \\
\text { Mame }\end{array}$ \\
\hline 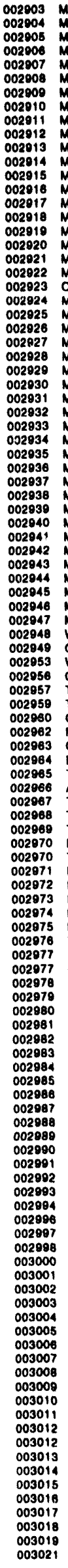 & 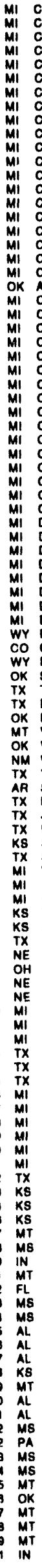 & 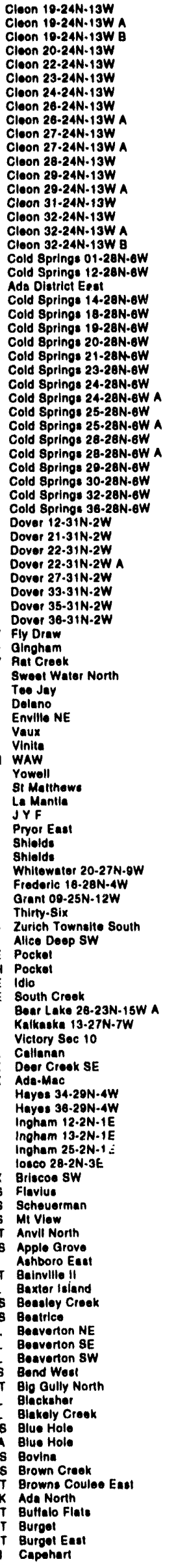 & 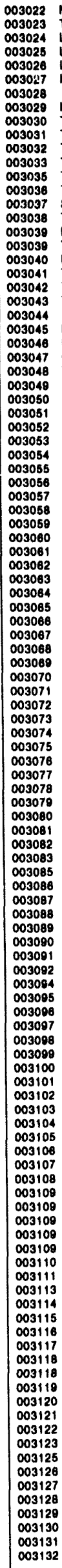 & 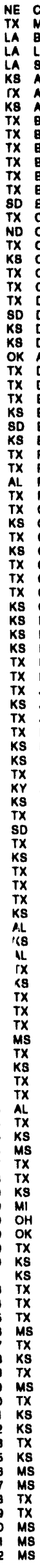 & 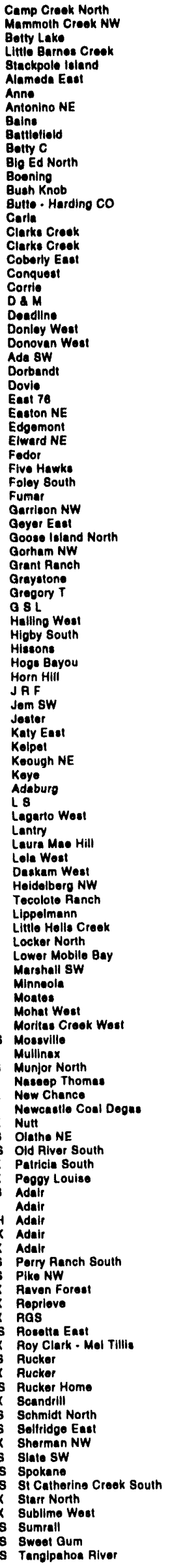 & 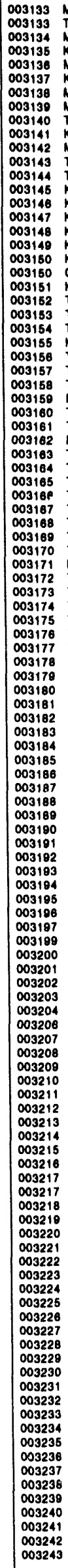 & 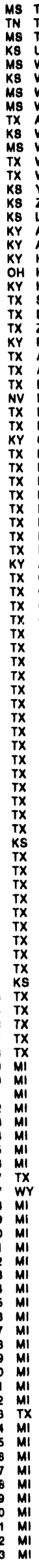 & 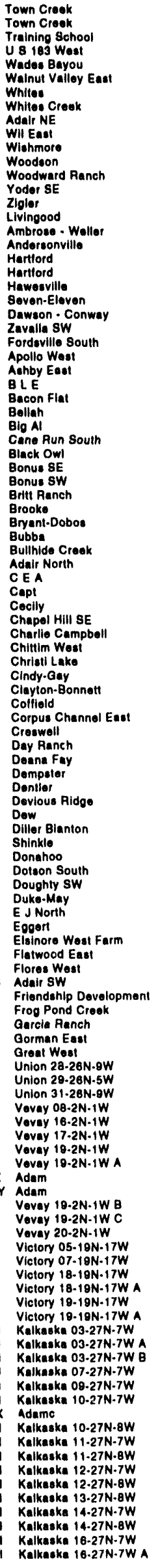 & 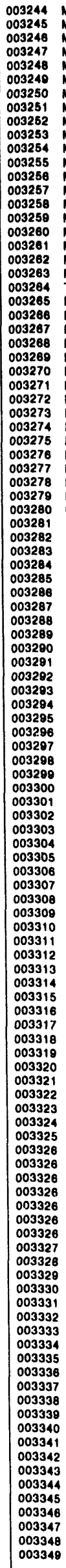 & 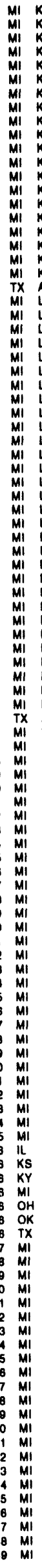 & 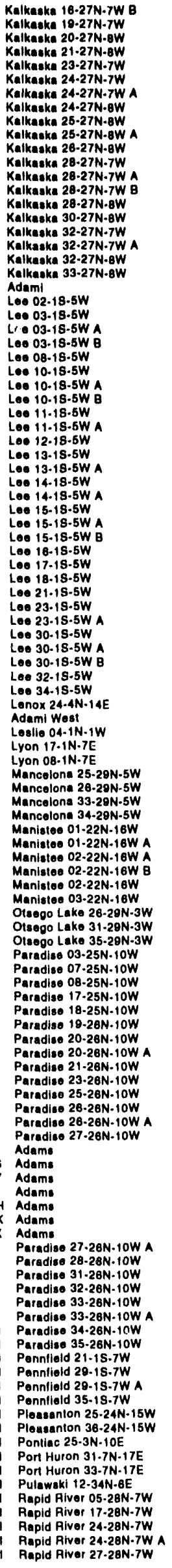 \\
\hline
\end{tabular}




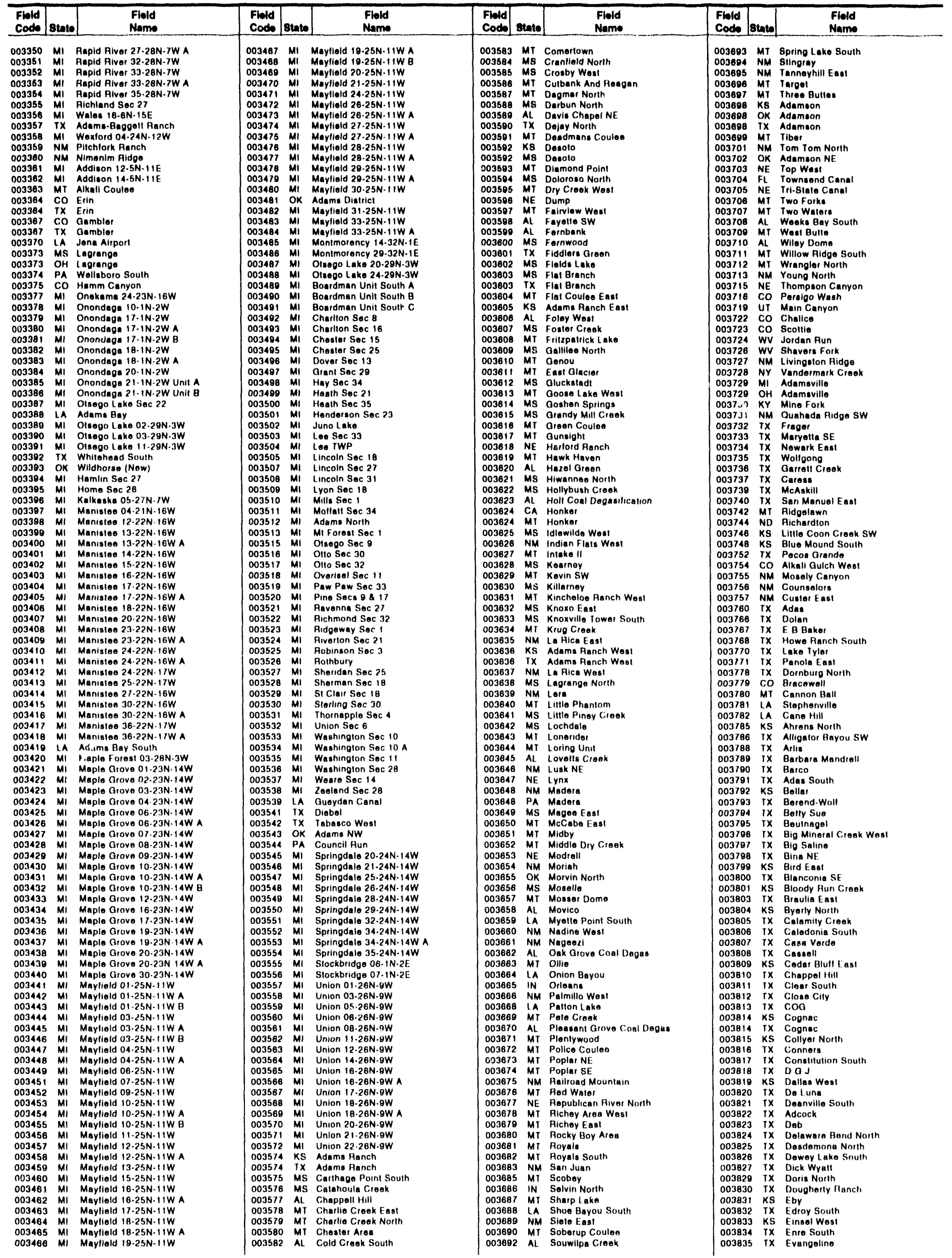




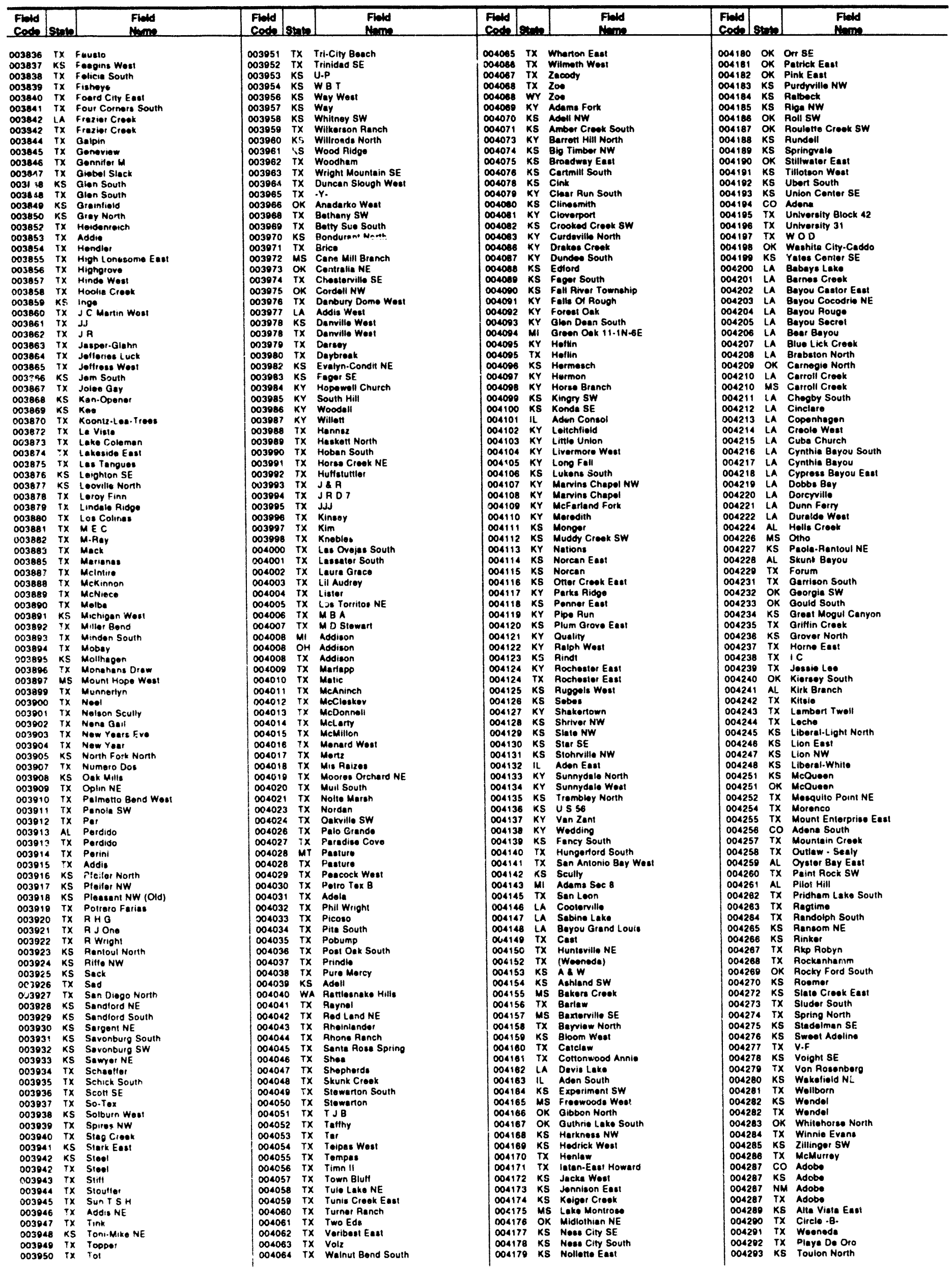




\begin{tabular}{|c|c|c|c|c|c|c|c|c|c|c|c|}
\hline $\begin{array}{l}\text { Fisid } \\
\text { Cods }\end{array}$ & stanto & $\begin{array}{l}\text { Findd } \\
\text { Name }\end{array}$ & $\begin{array}{l}\text { Findd } \\
\text { Code }\end{array}$ & state & $\begin{array}{l}\text { Finld } \\
\text { Name }\end{array}$ & $\begin{array}{l}\text { Fiold } \\
\text { Code }\end{array}$ & Stete & $\begin{array}{l}\text { Fild } \\
\text { Name }\end{array}$ & $\begin{array}{l}\text { Fiold } \\
\text { Code }\end{array}$ & State & $\begin{array}{l}\text { Fild } \\
\text { Narme }\end{array}$ \\
\hline 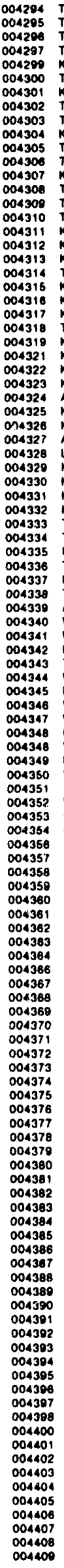 & 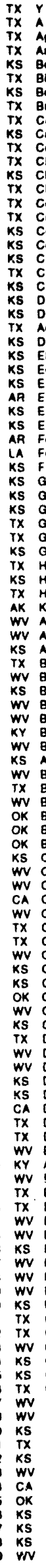 & 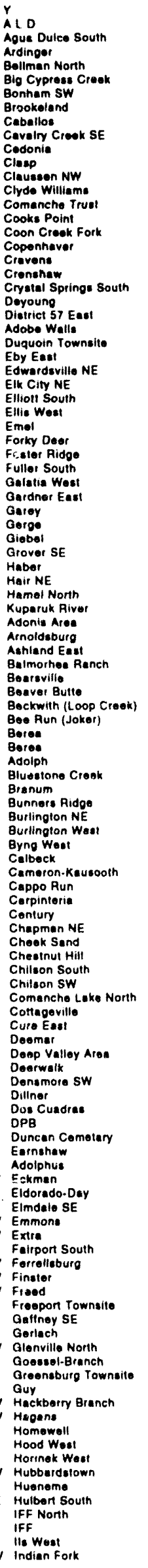 & 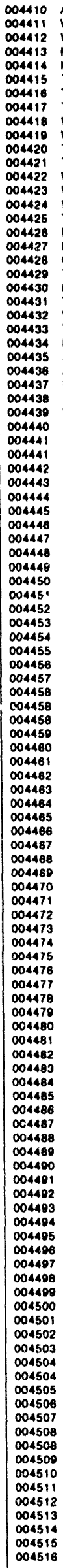 & 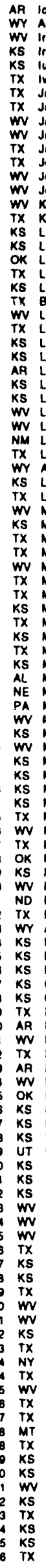 & 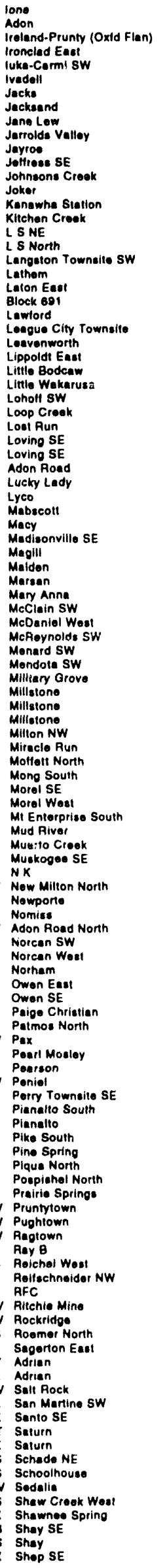 & 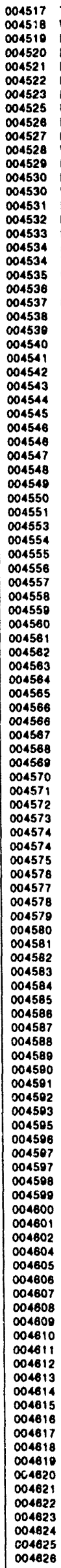 & 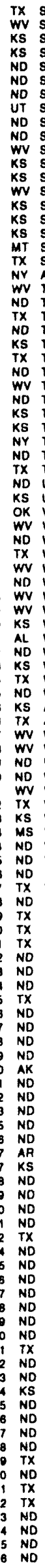 & 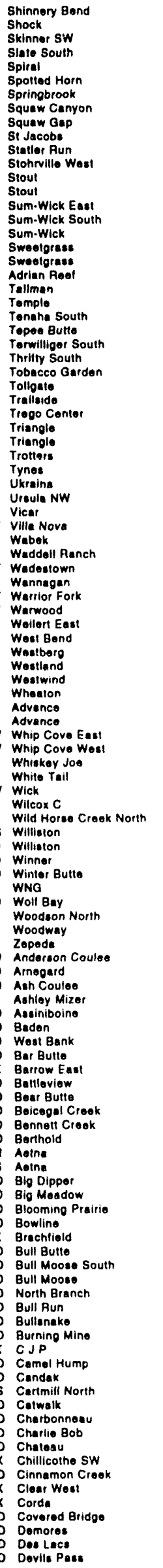 & 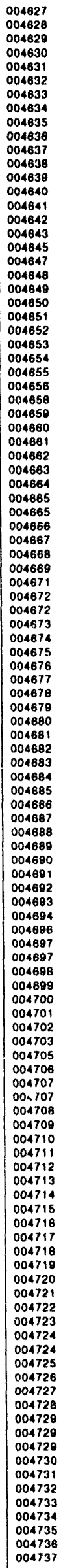 & 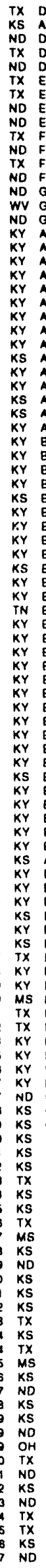 & 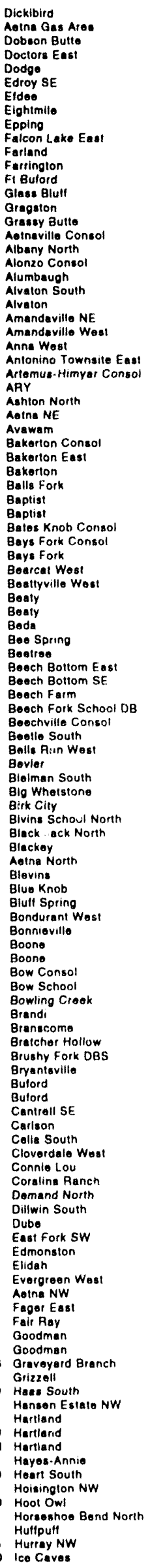 \\
\hline
\end{tabular}




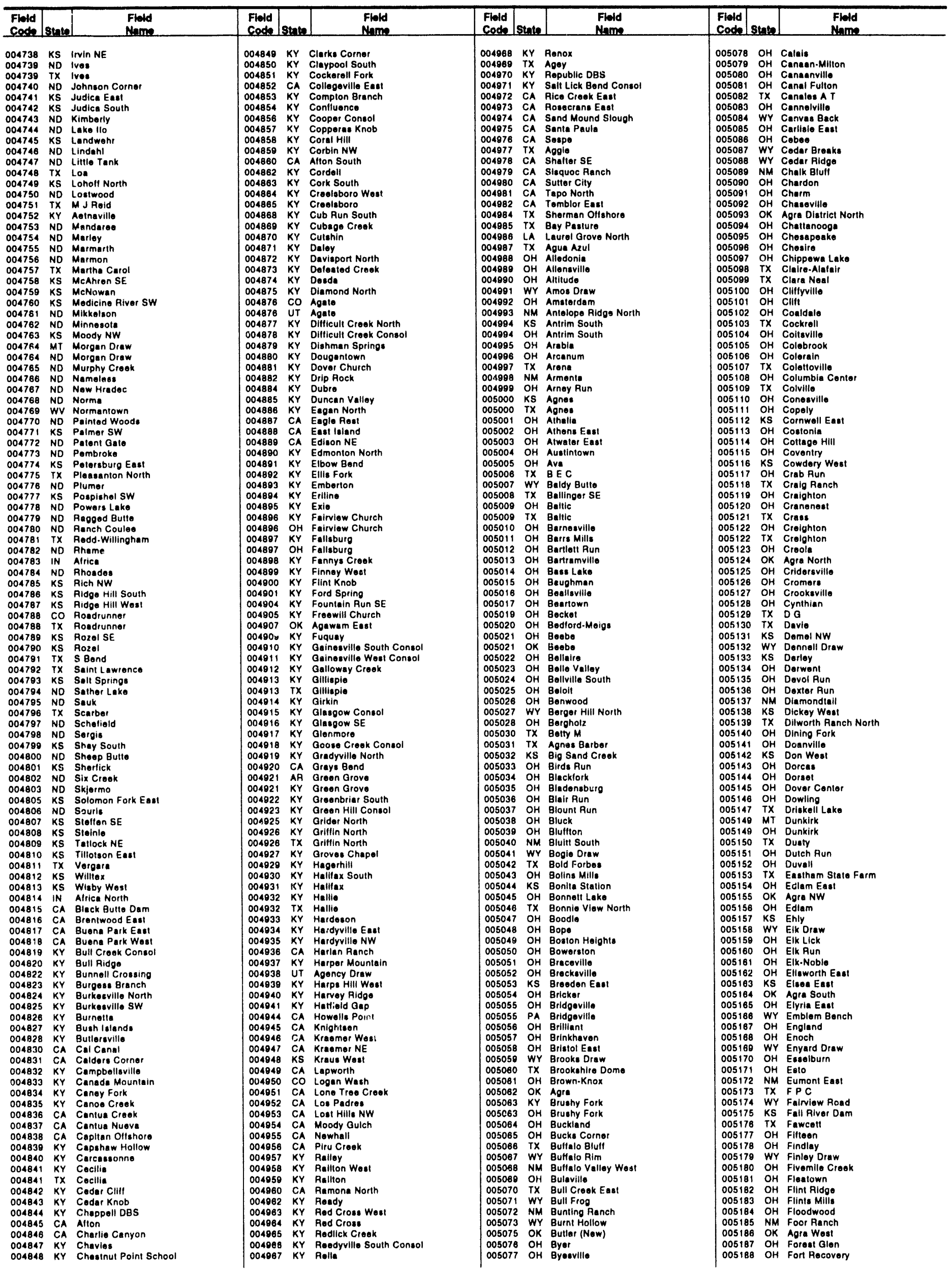




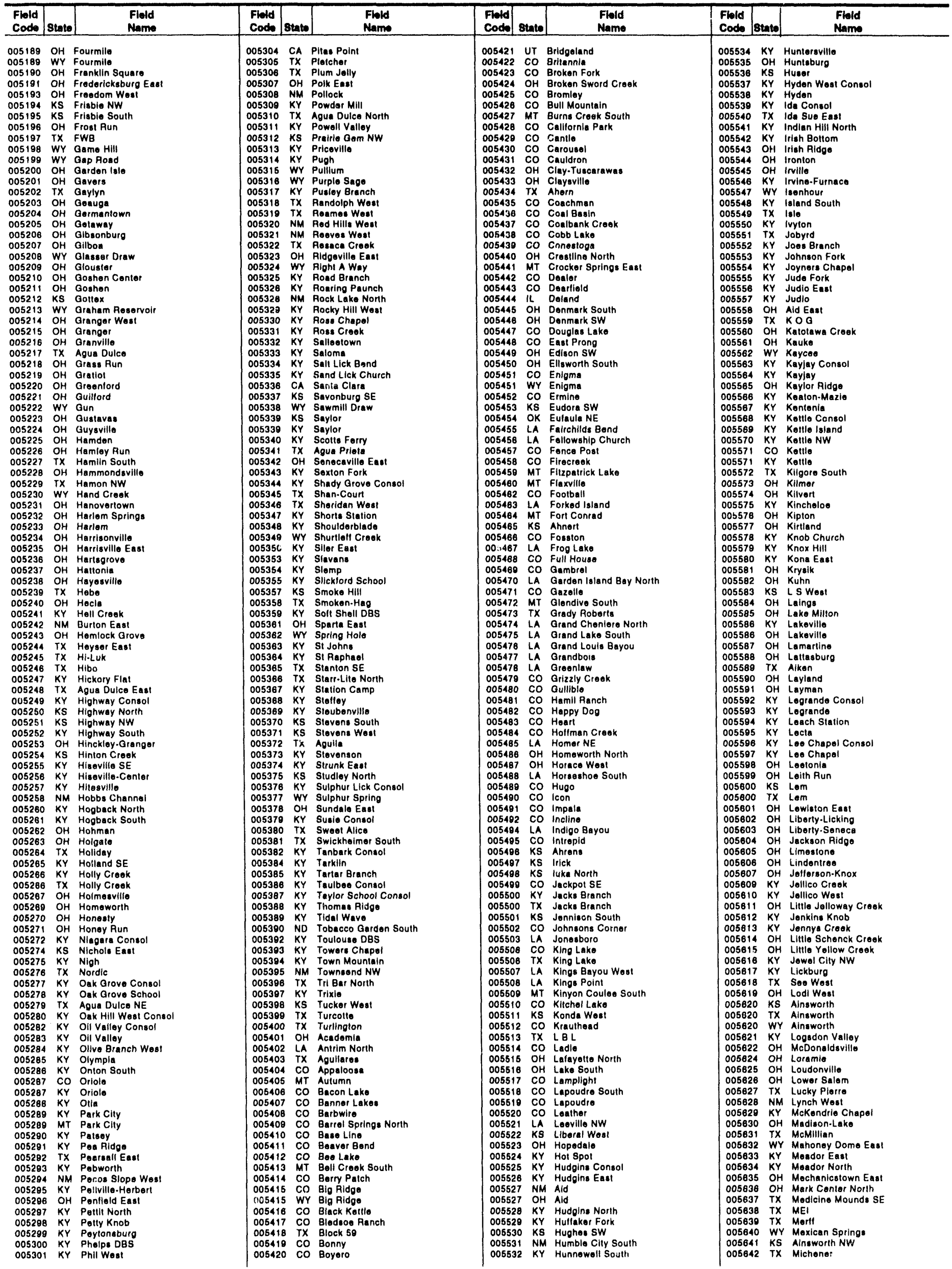




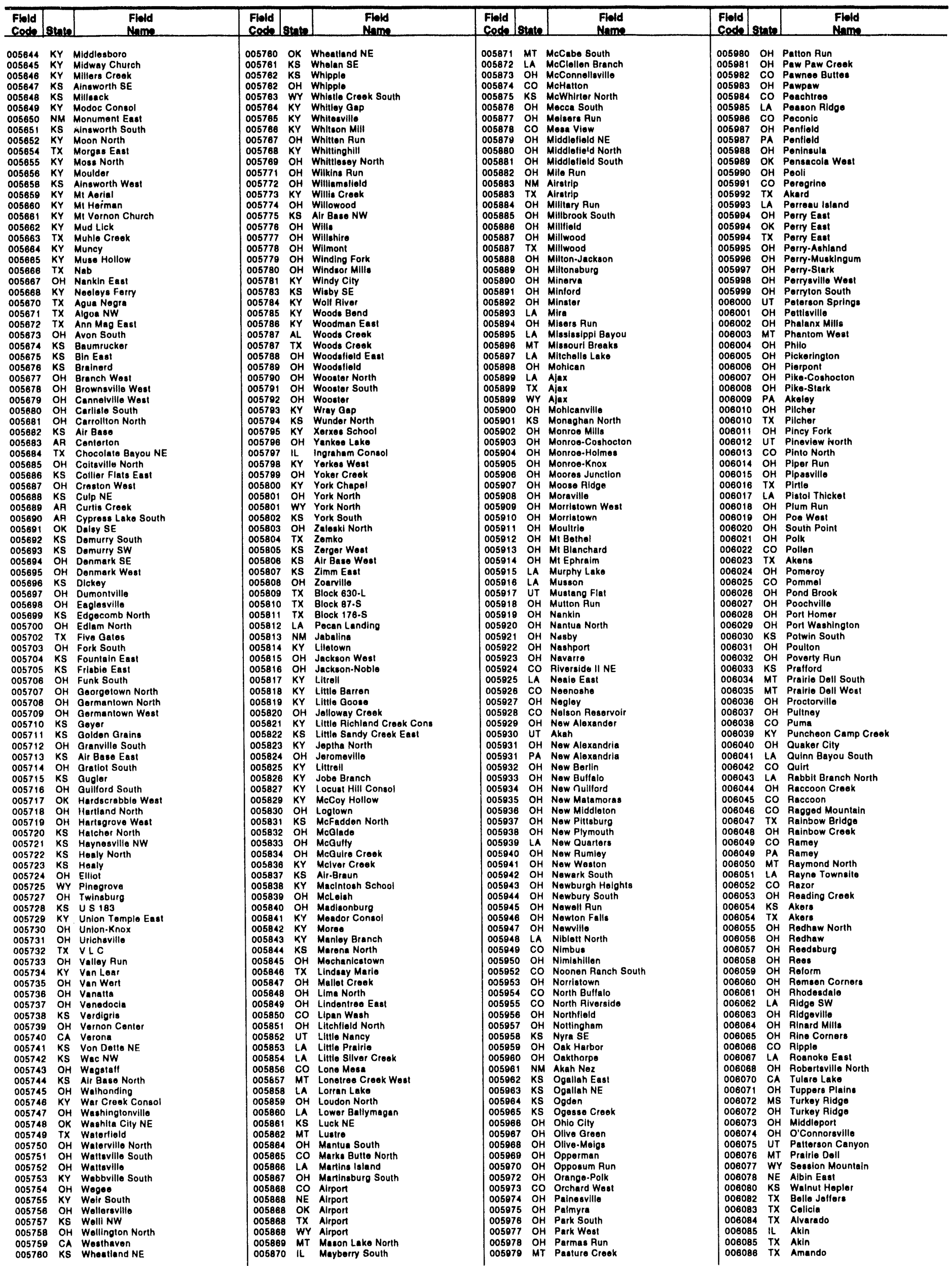




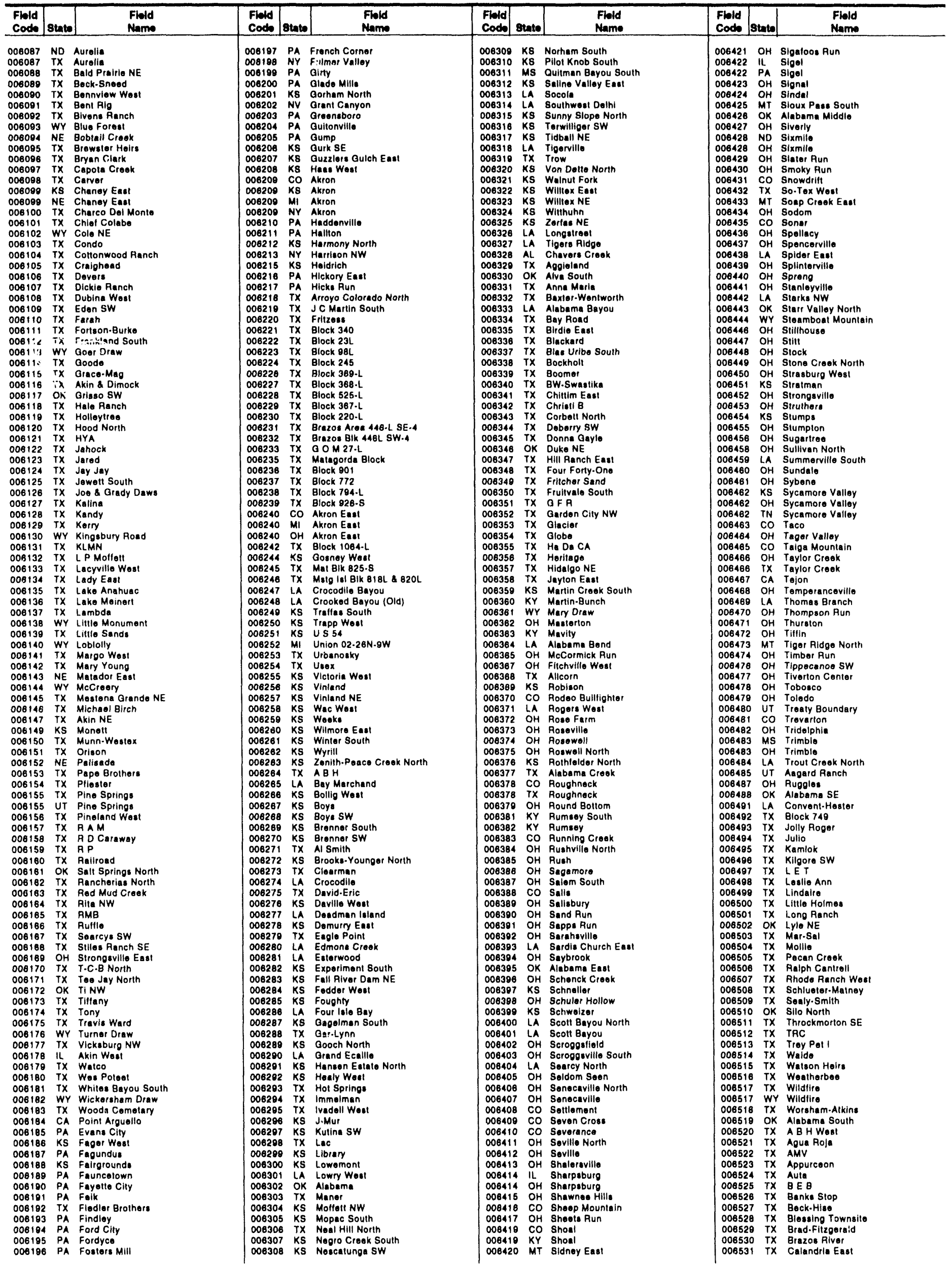




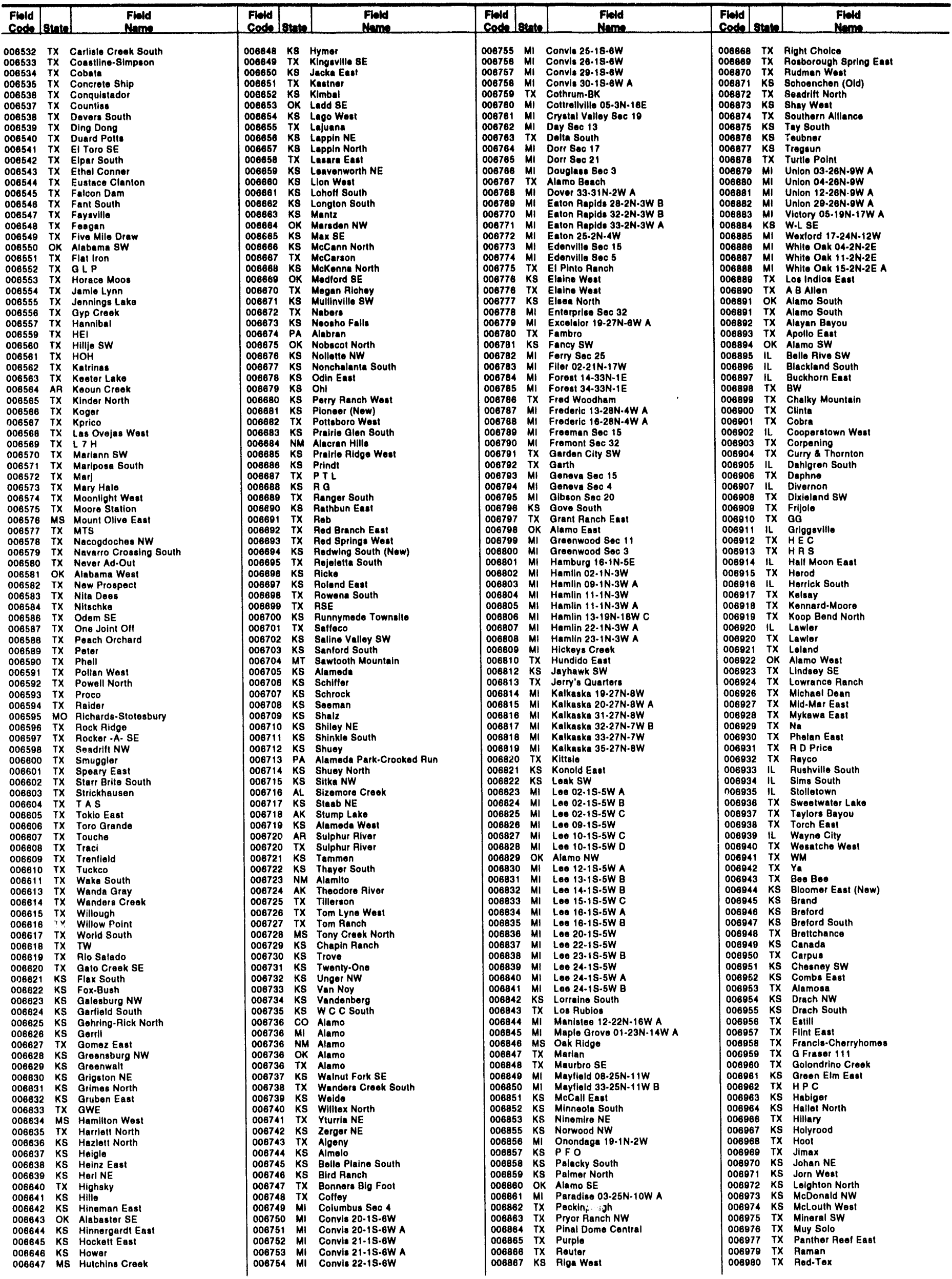




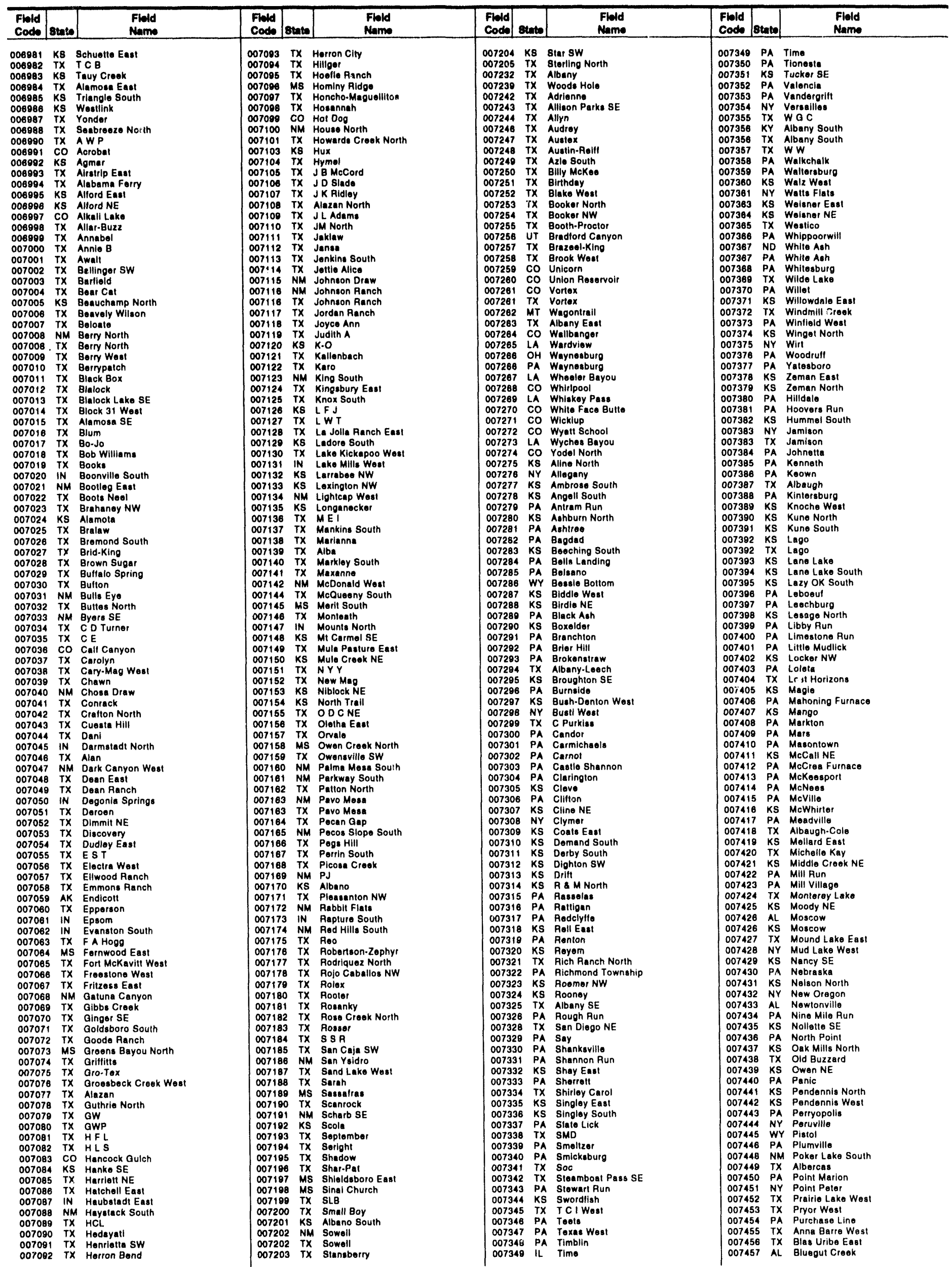




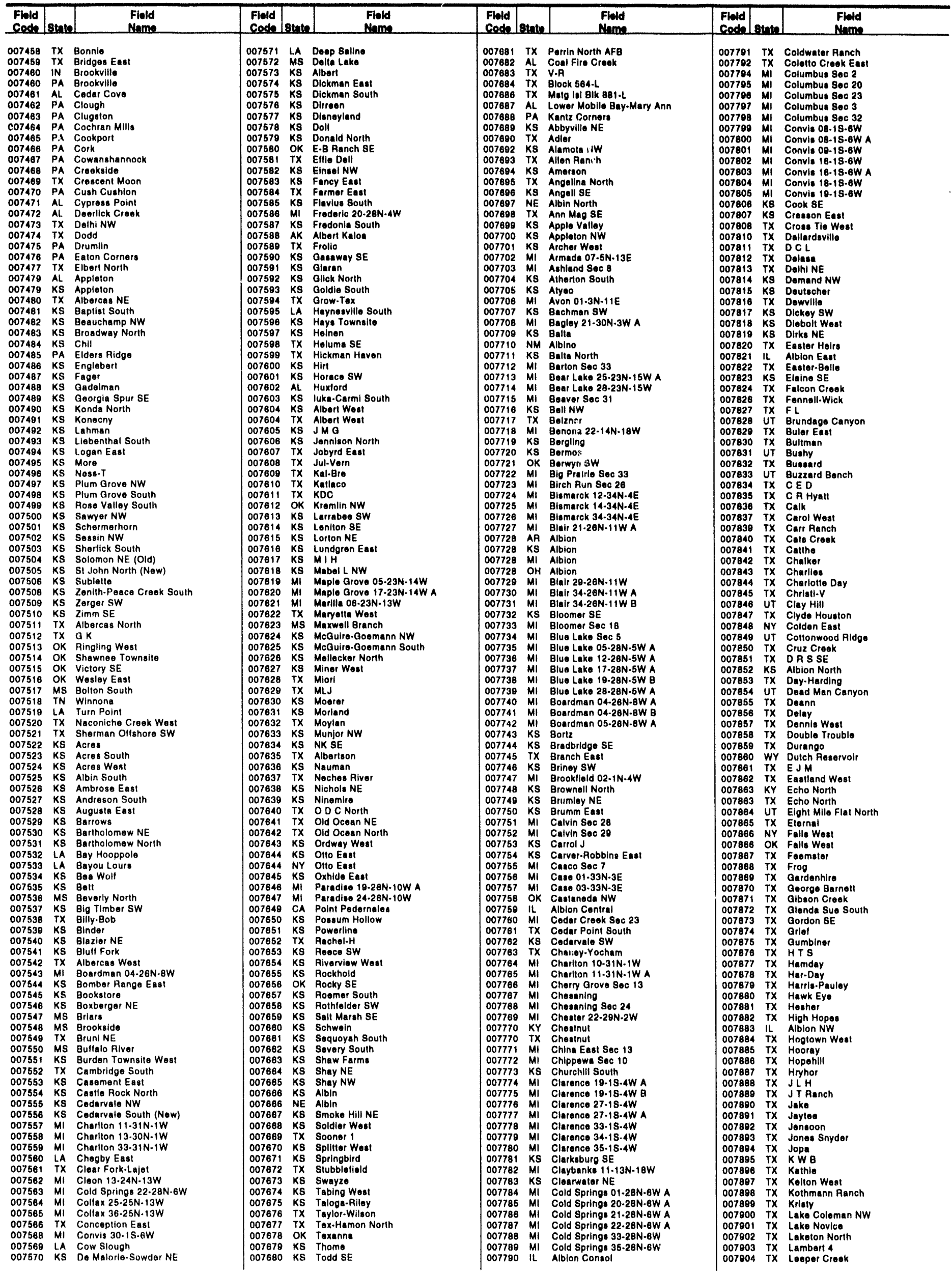




\begin{tabular}{|c|c|c|c|c|c|c|c|c|c|c|c|}
\hline $\begin{array}{l}\text { Fild } \\
\text { Code }\end{array}$ & state & $\begin{array}{l}\text { Fibld } \\
\text { Name }\end{array}$ & $\begin{array}{l}\text { Fleld } \\
\text { Code }\end{array}$ & state & $\begin{array}{l}\text { Fiold } \\
\text { Name }\end{array}$ & $\begin{array}{l}\text { Filld } \\
\text { Code }\end{array}$ & sime & $\begin{array}{l}\text { Fleld } \\
\text { Name }\end{array}$ & $\begin{array}{l}\text { Fiold } \\
\text { Cods }\end{array}$ & state & $\begin{array}{l}\text { Fibld } \\
\text { Name }\end{array}$ \\
\hline 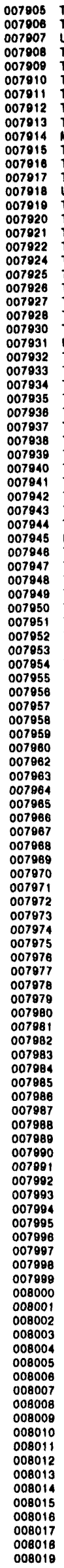 & 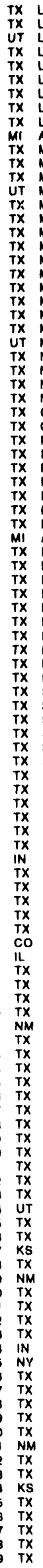 & 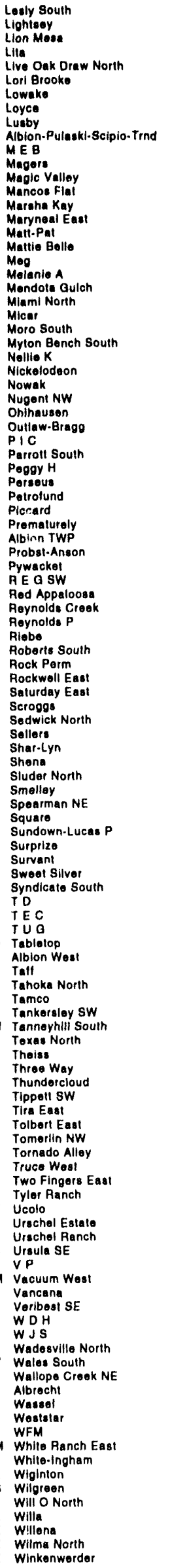 & 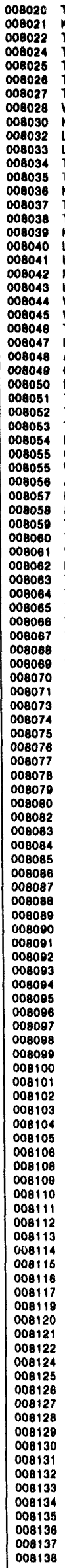 & 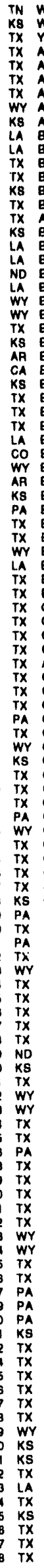 & 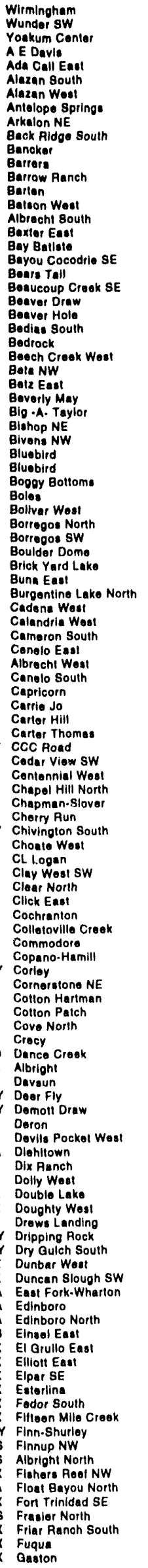 & 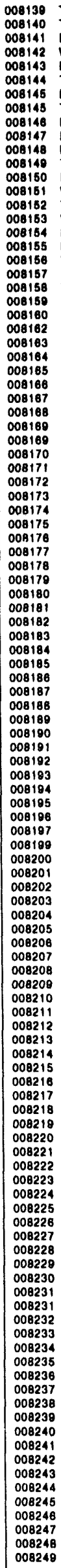 & 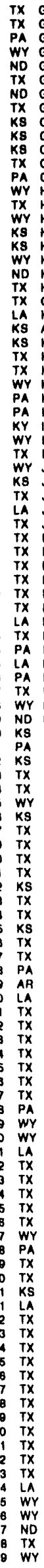 & 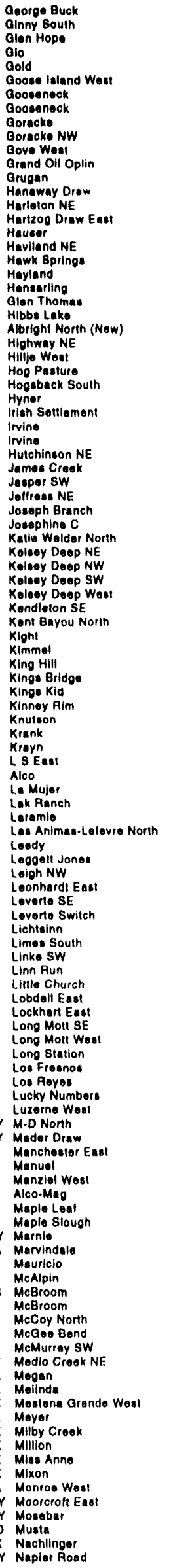 & 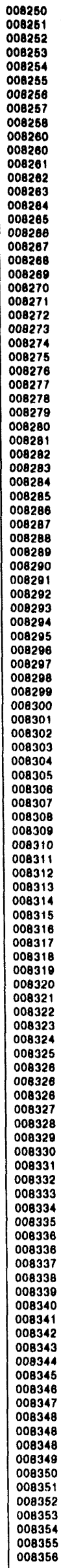 & 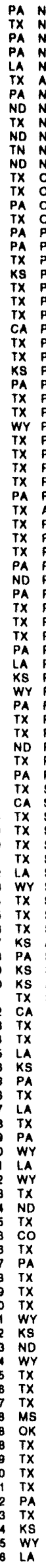 & 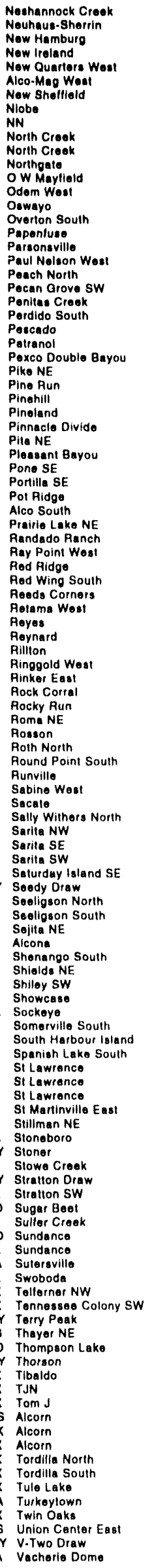 \\
\hline
\end{tabular}




\begin{tabular}{|c|c|c|c|c|c|c|c|c|c|c|c|}
\hline $\begin{array}{l}\text { Flold } \\
\text { Cod? }\end{array}$ & sinte & $\begin{array}{l}\text { Field } \\
\text { Nem: }\end{array}$ & $\begin{array}{l}\text { Field } \\
\text { Code }\end{array}$ & stant & $\begin{array}{l}\text { Fiold } \\
\text { Name }\end{array}$ & $\begin{array}{l}\text { Flold } \\
\text { Code } \\
\end{array}$ & Sinte & $\begin{array}{l}\text { Finld } \\
\text { Ninme }\end{array}$ & $\begin{array}{l}\text { Fleld } \\
\text { Coet. }\end{array}$ & sinte & $\begin{array}{l}\text { Fiold } \\
\text { Nam, }\end{array}$ \\
\hline 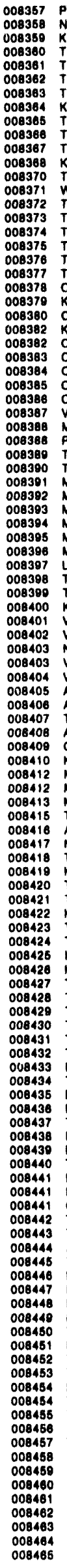 & 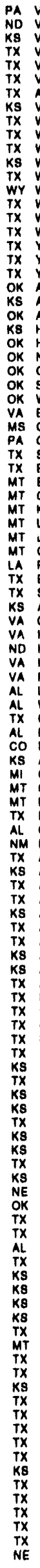 & 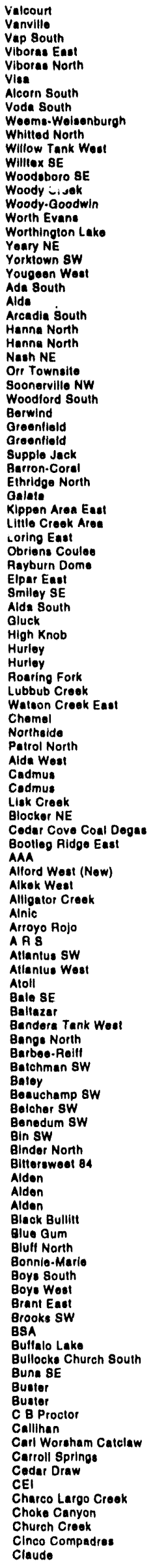 & 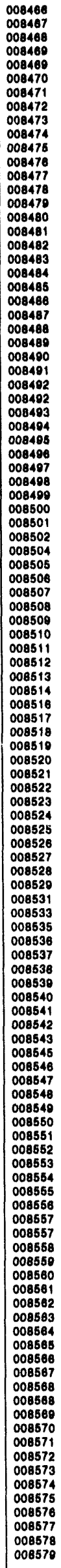 & 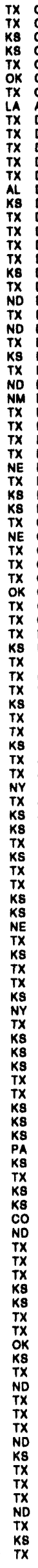 & 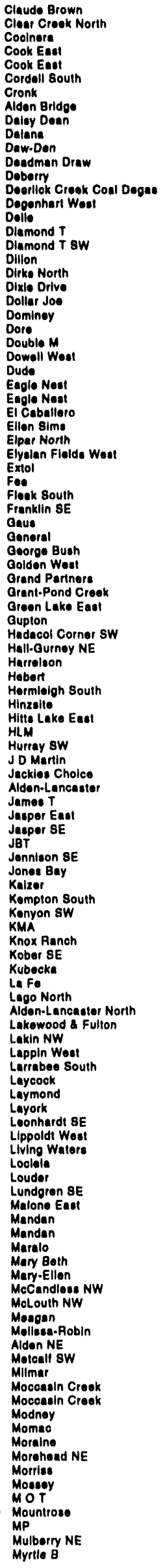 & 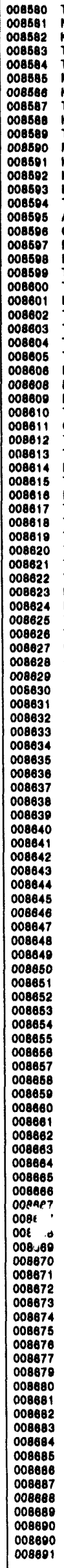 & 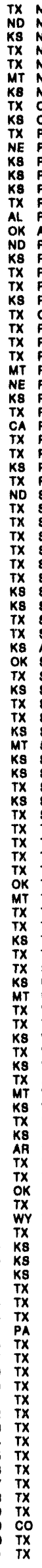 & 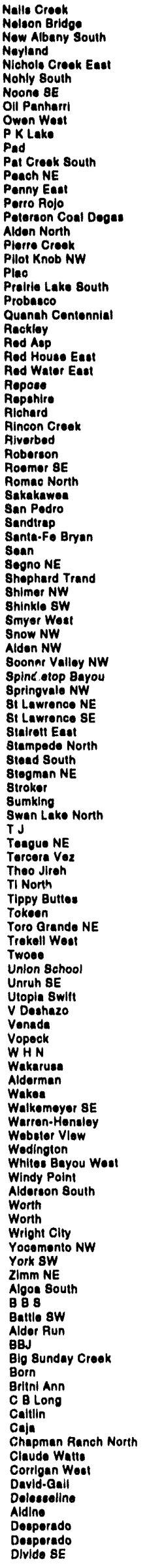 & 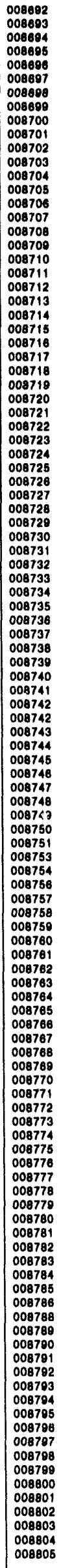 & 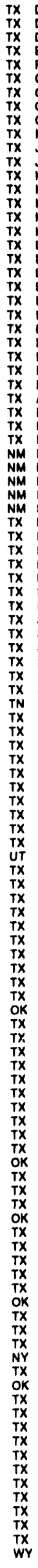 & 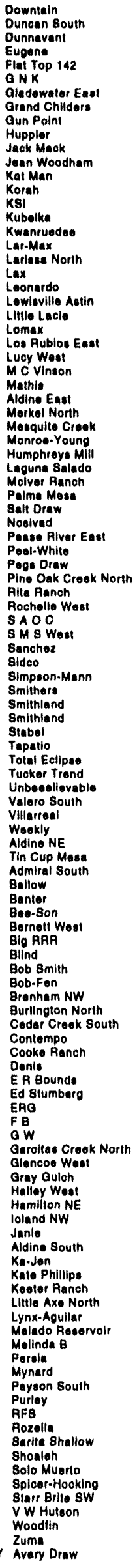 \\
\hline
\end{tabular}




\begin{tabular}{|c|c|c|c|c|c|c|c|c|c|c|c|}
\hline \begin{tabular}{l|l} 
Fibld \\
Code
\end{tabular} & 8tato & $\begin{array}{l}\text { Fibld } \\
\text { Name }\end{array}$ & $\begin{array}{l}\text { Flold } \\
\text { Code }\end{array}$ & state & $\begin{array}{l}\text { Fiold } \\
\text { Name }\end{array}$ & $\begin{array}{l}\text { Field } \\
\text { Cods }\end{array}$ & 8tate & $\begin{array}{l}\text { Fleld } \\
\text { Name }\end{array}$ & $\begin{array}{l}\text { Fisld } \\
\text { Cods }\end{array}$ & State & $\begin{array}{l}\text { Fleld } \\
\text { Name }\end{array}$ \\
\hline 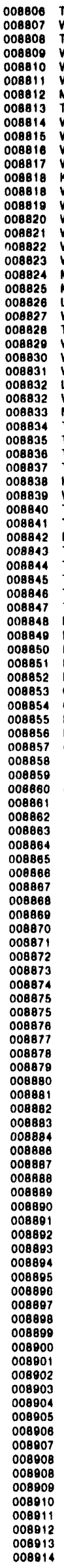 & 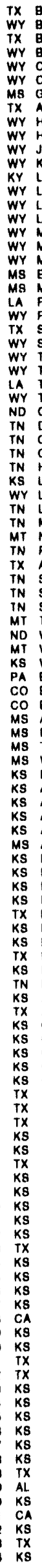 & 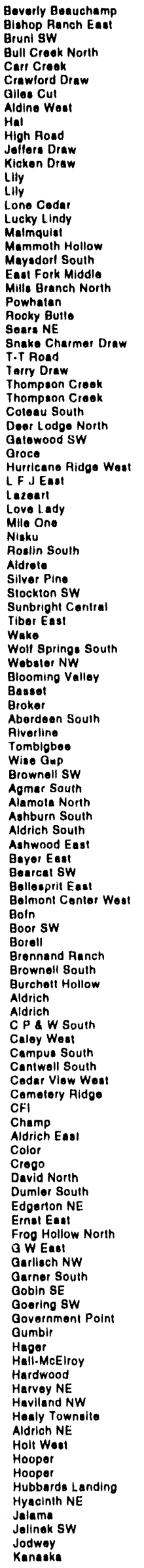 & 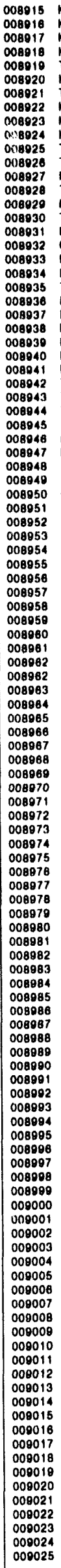 & 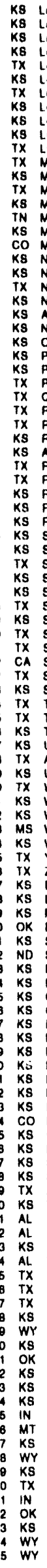 & 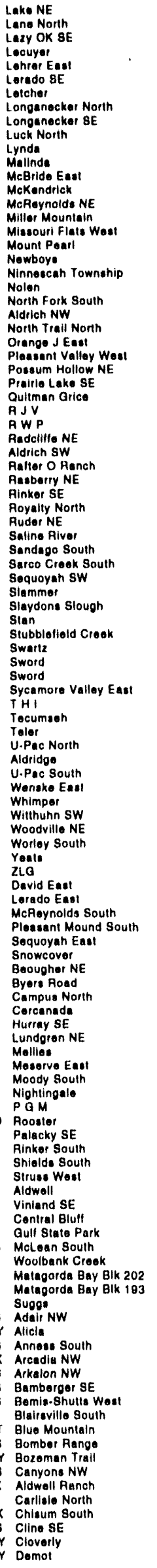 & 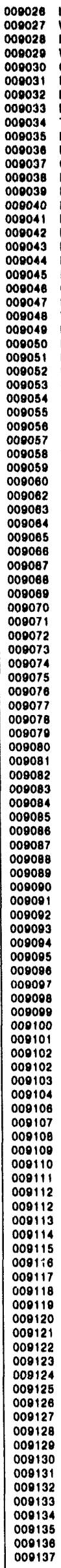 & 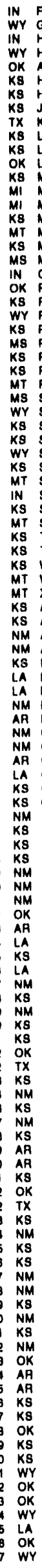 & 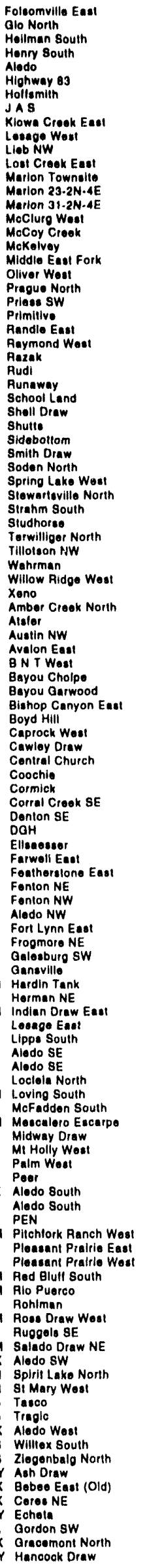 & 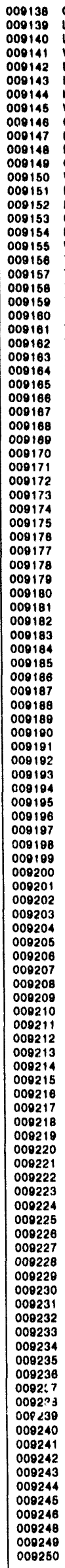 & 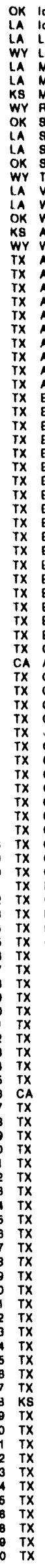 & 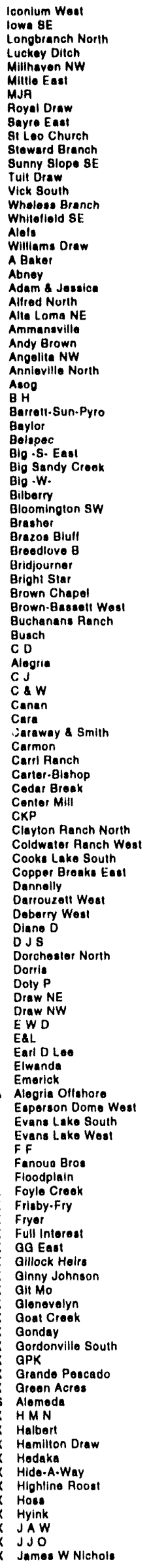 \\
\hline
\end{tabular}




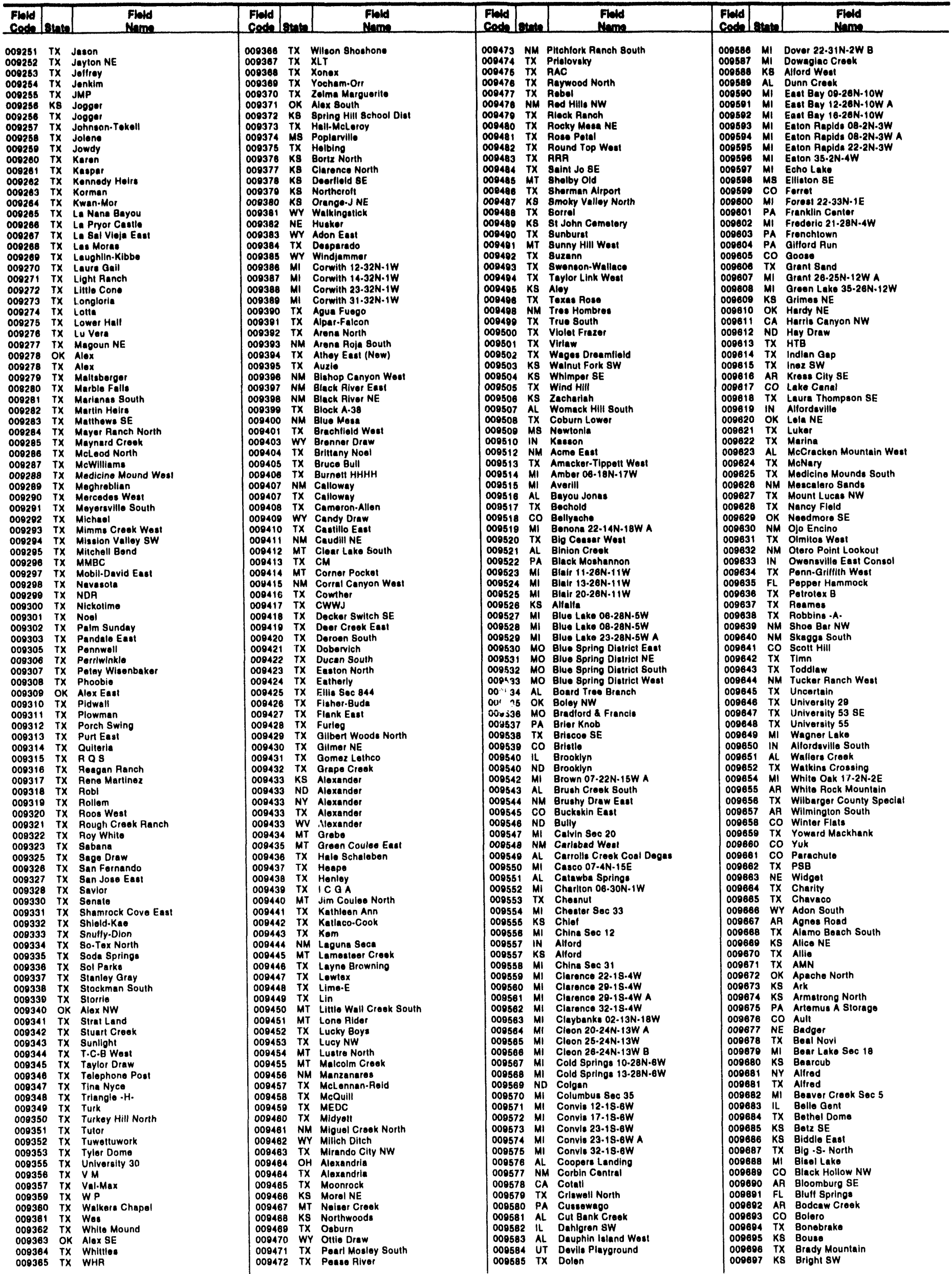




\begin{tabular}{|c|c|c|c|c|c|c|c|c|c|c|c|}
\hline \begin{tabular}{l|} 
Fiold \\
Code
\end{tabular} & etan & $\begin{array}{l}\text { Fild } \\
\text { Name }\end{array}$ & $\begin{array}{l}\text { Fildd } \\
\text { Code }\end{array}$ & state & $\begin{array}{l}\text { Fbld } \\
\text { Name } \\
\end{array}$ & \begin{tabular}{l|l} 
Flatd \\
Code
\end{tabular} & state & $\begin{array}{l}\text { Fleld } \\
\text { Neme }\end{array}$ & $\begin{array}{l}\text { Finld } \\
\text { Code }\end{array}$ & 8tate & $\begin{array}{l}\text { Fleld } \\
\text { Name } \\
\end{array}$ \\
\hline 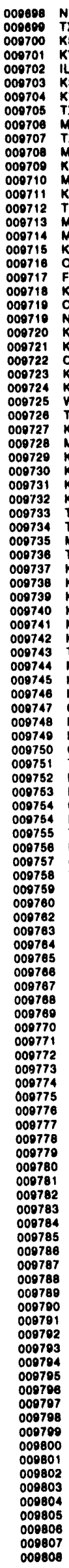 & 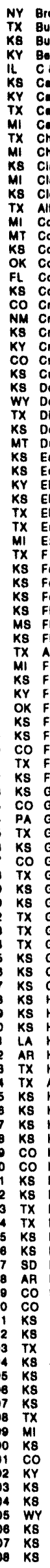 & 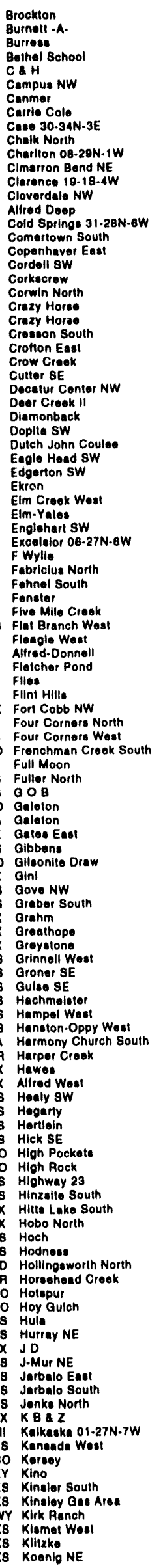 & 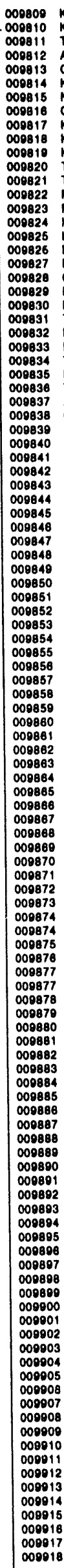 & 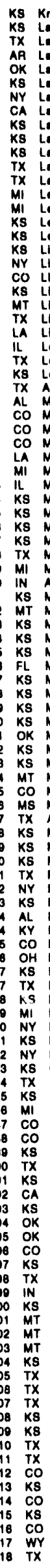 & 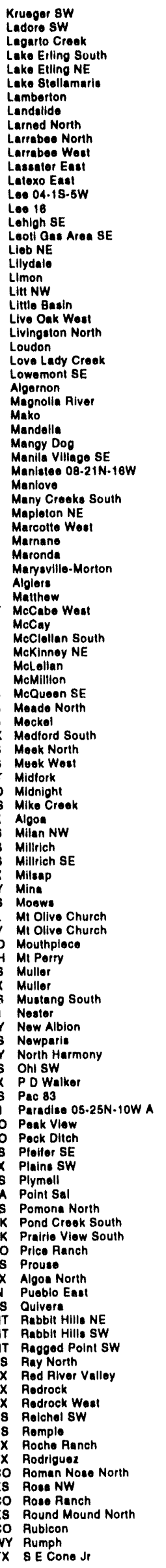 & 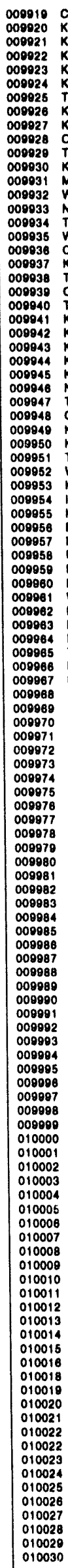 & 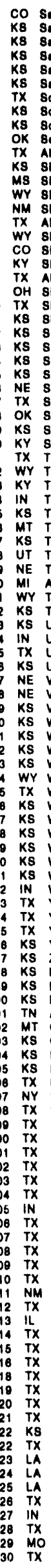 & 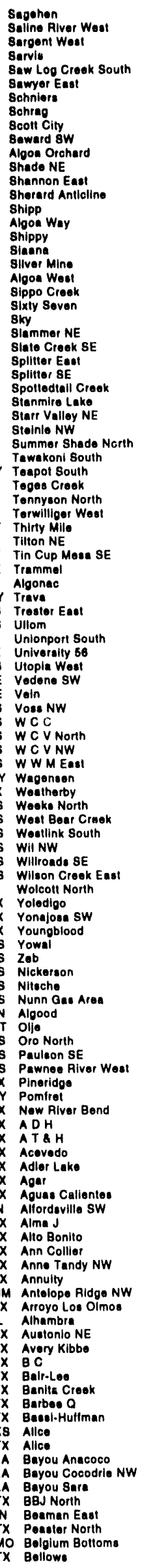 & 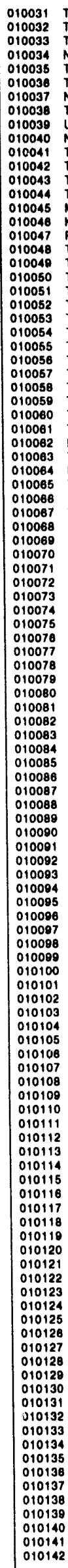 & 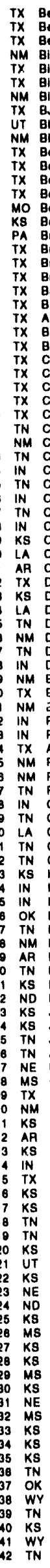 & 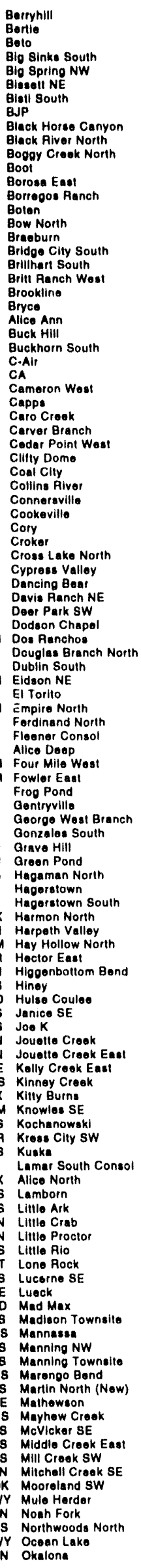 \\
\hline
\end{tabular}




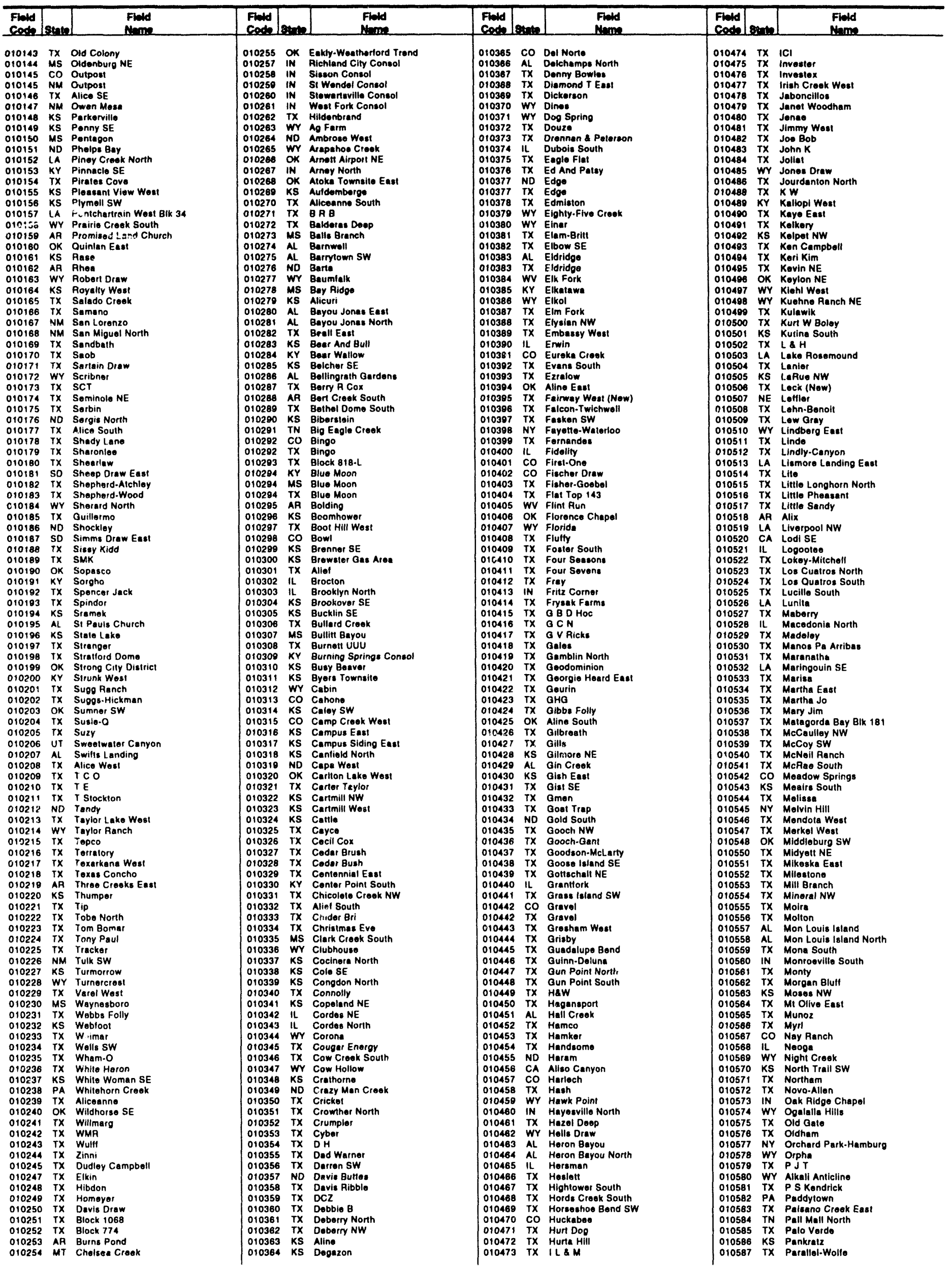




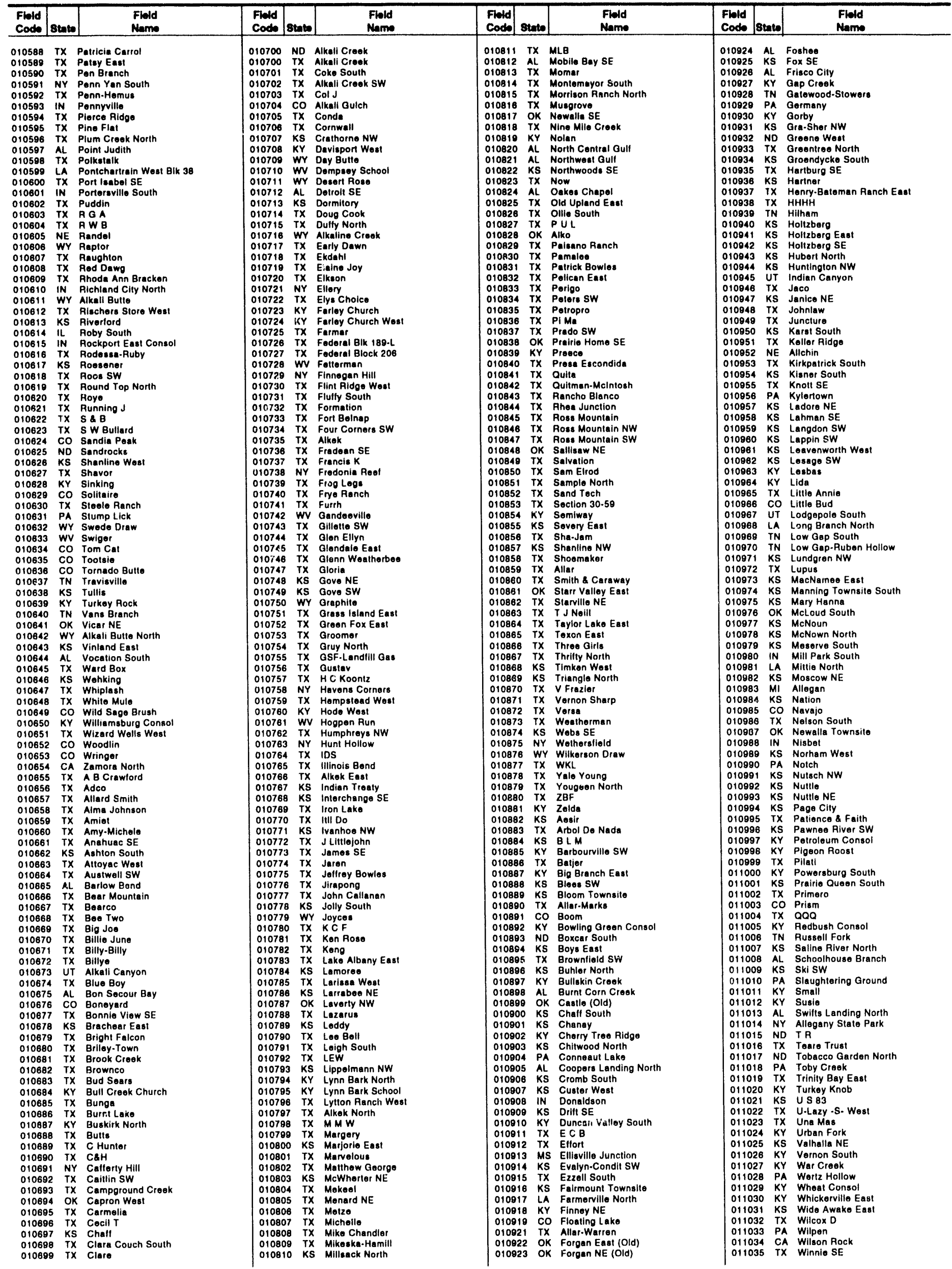




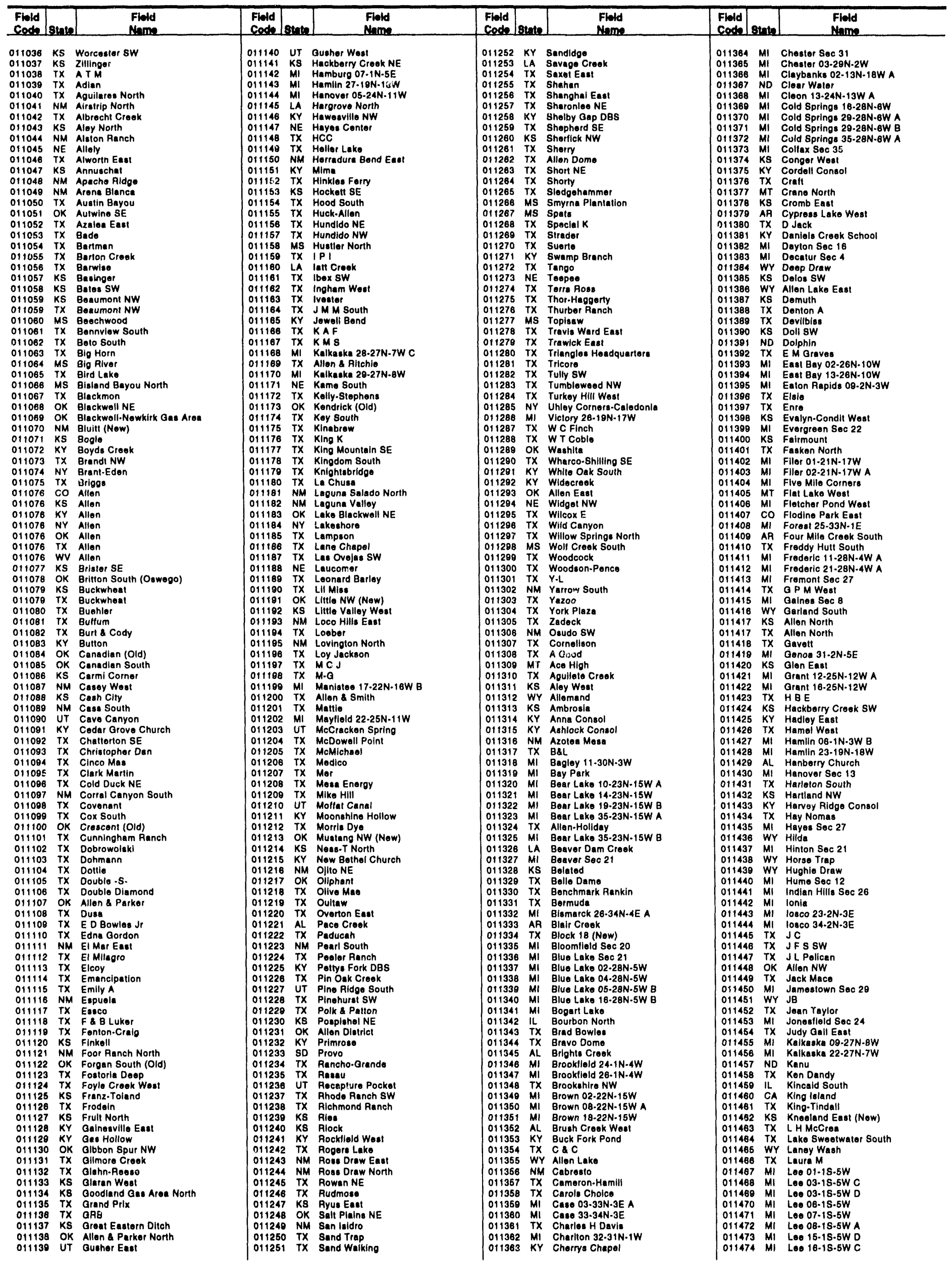




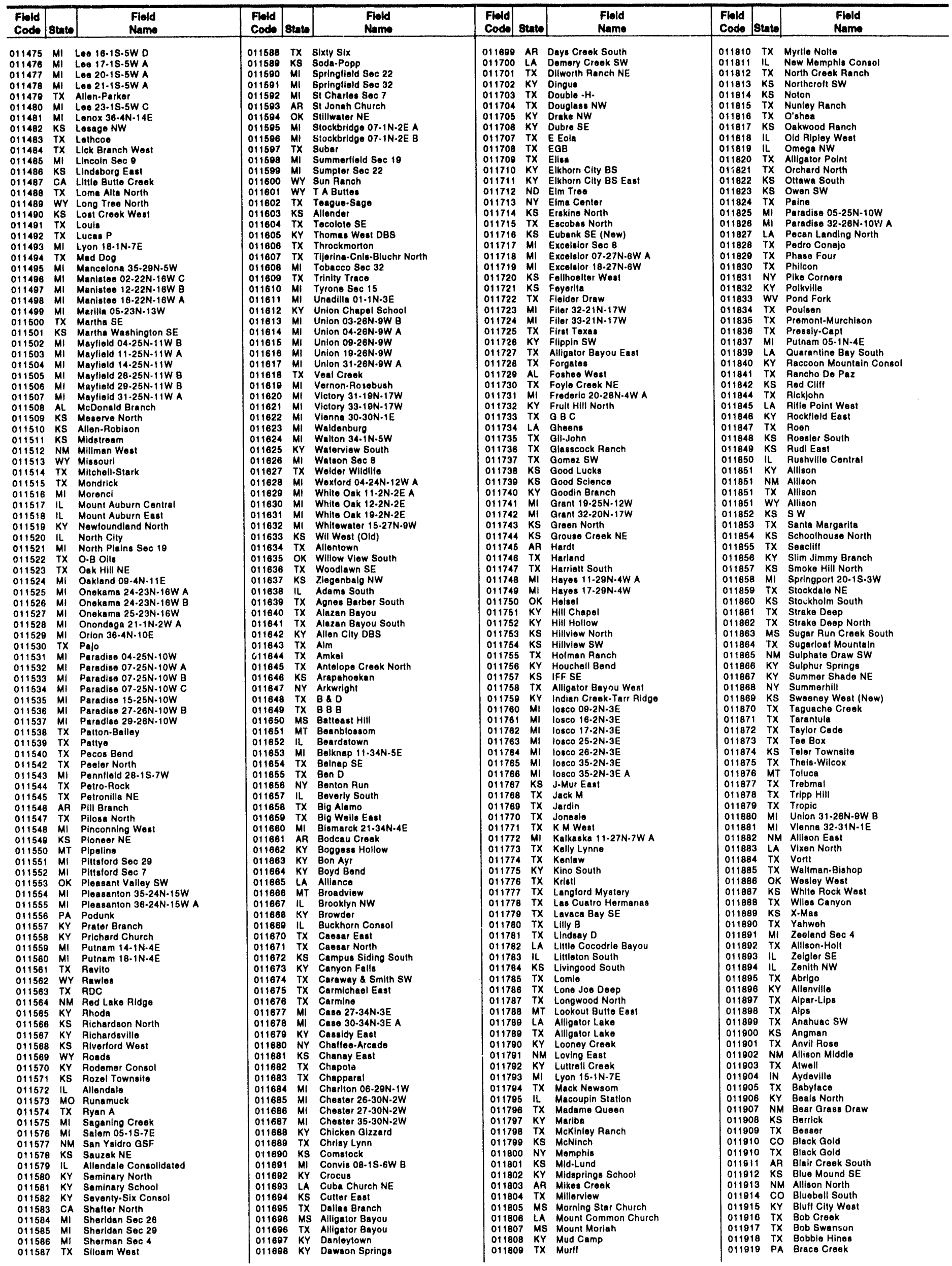




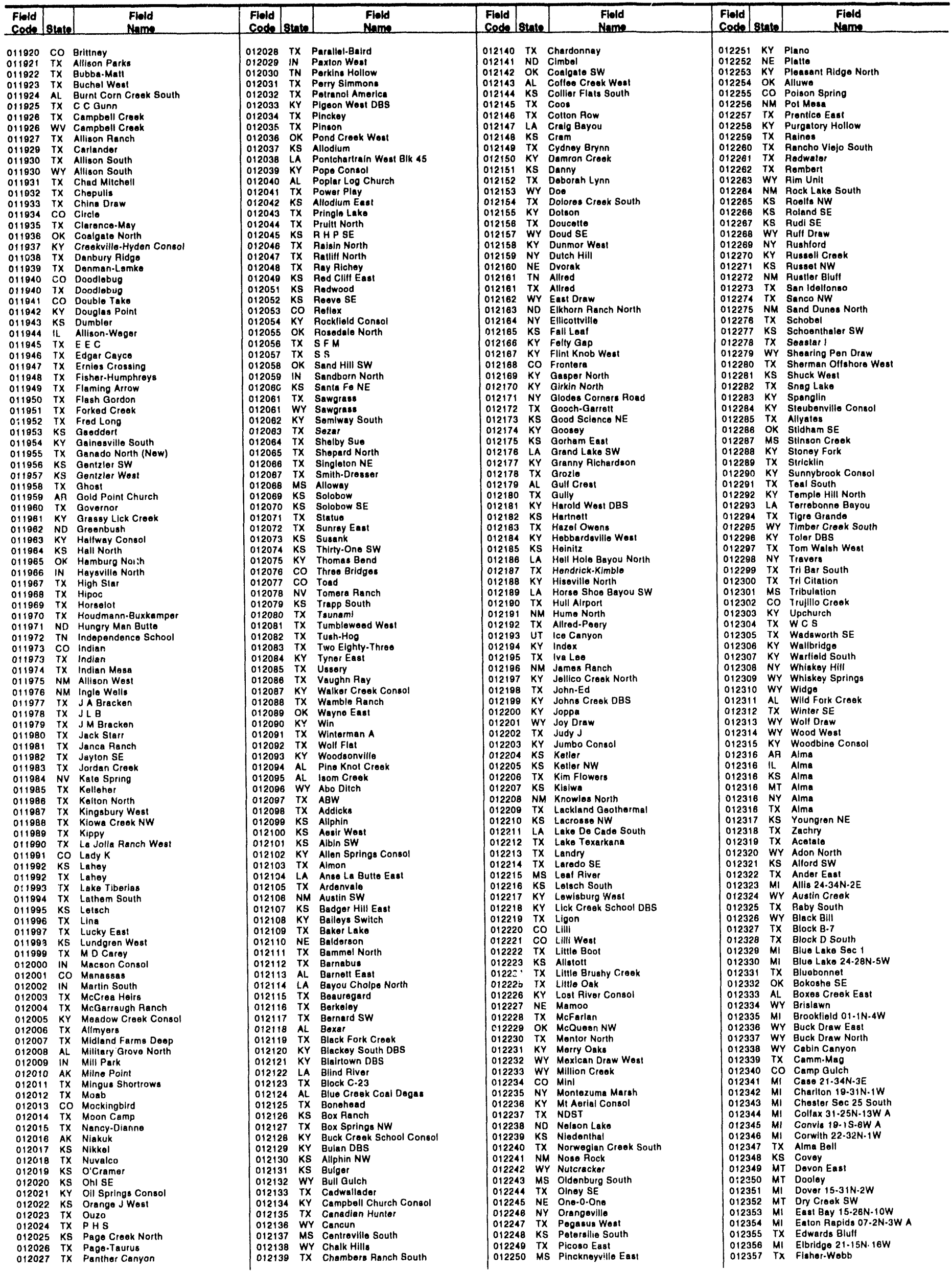




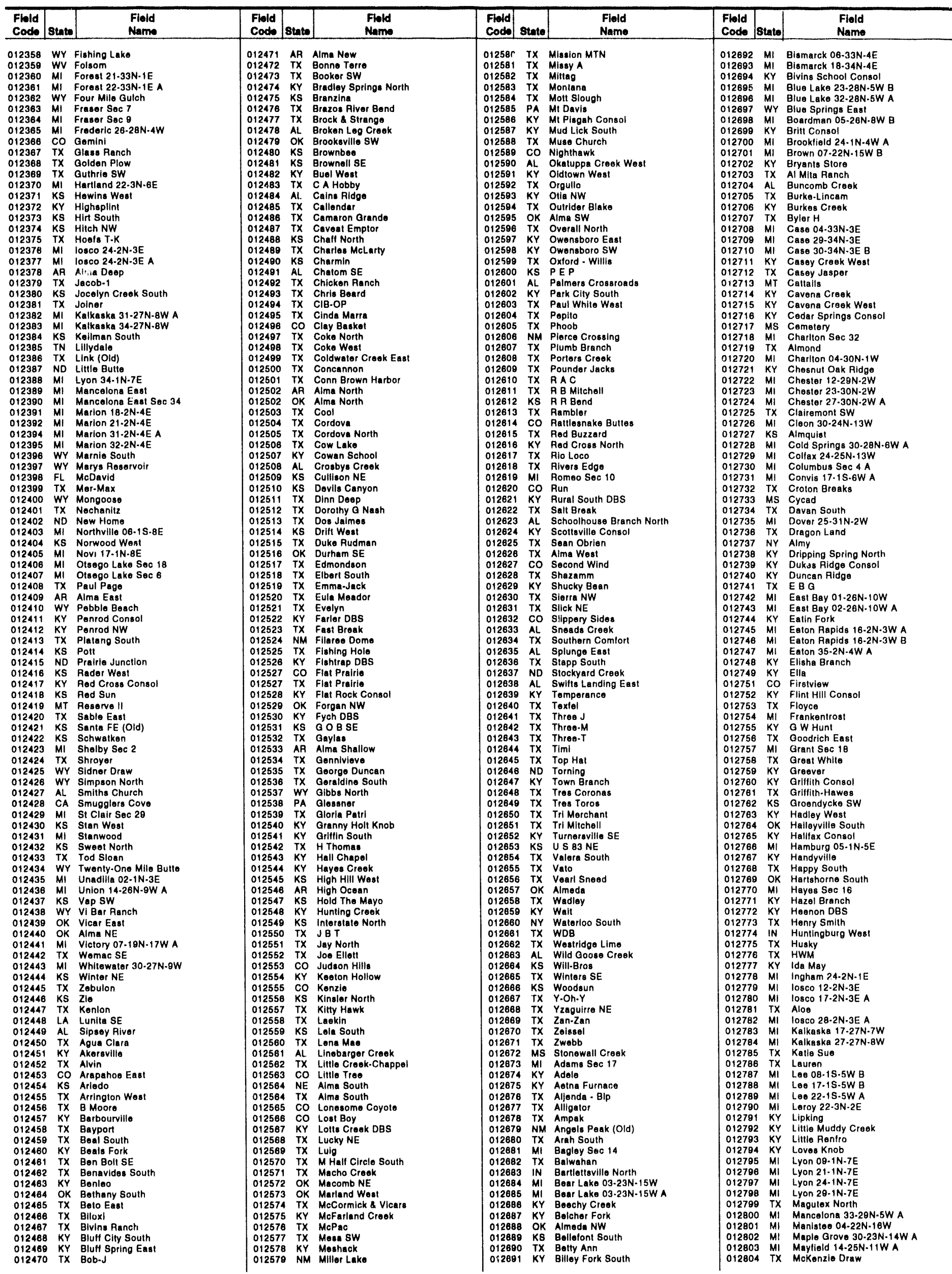




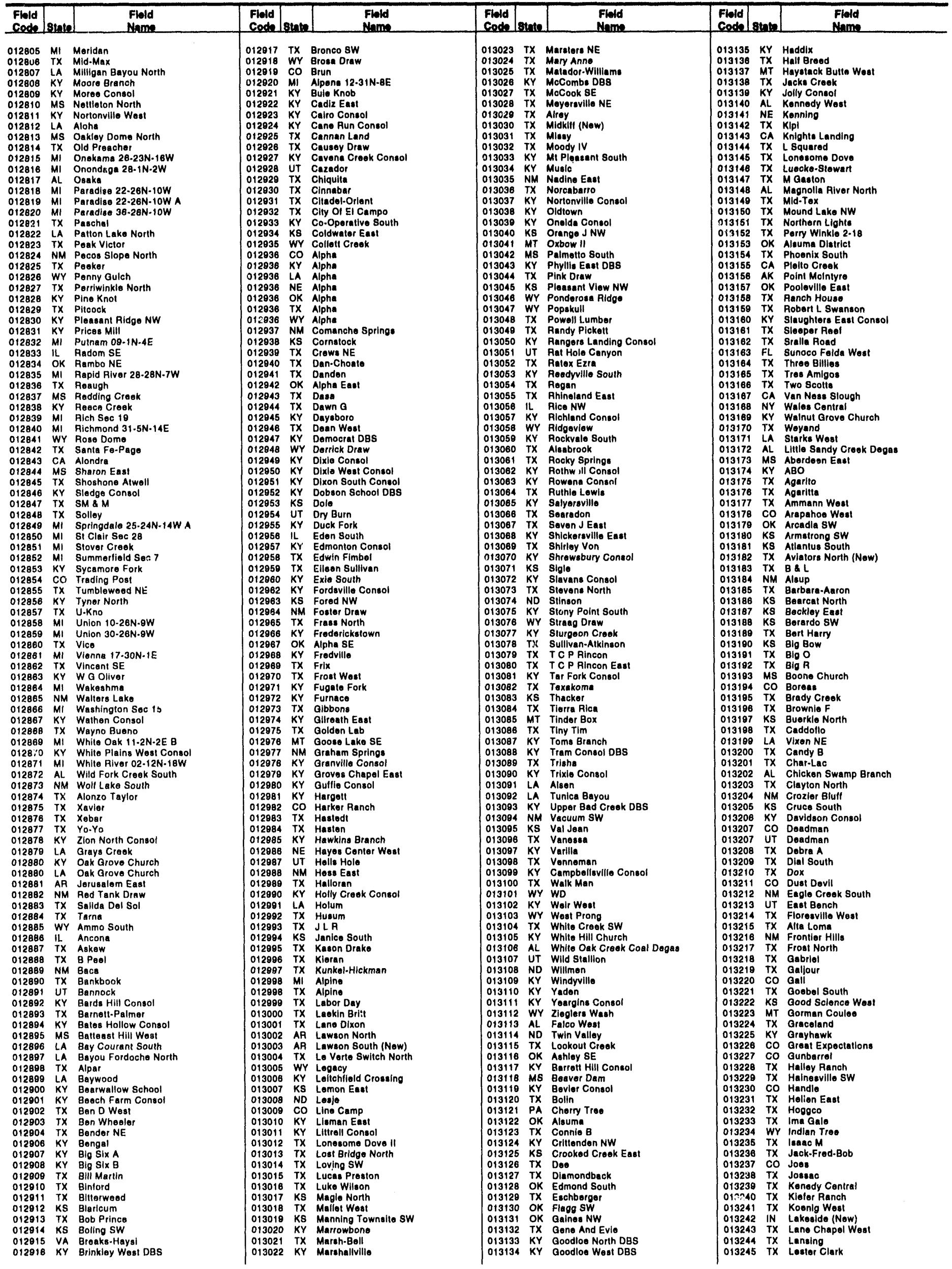




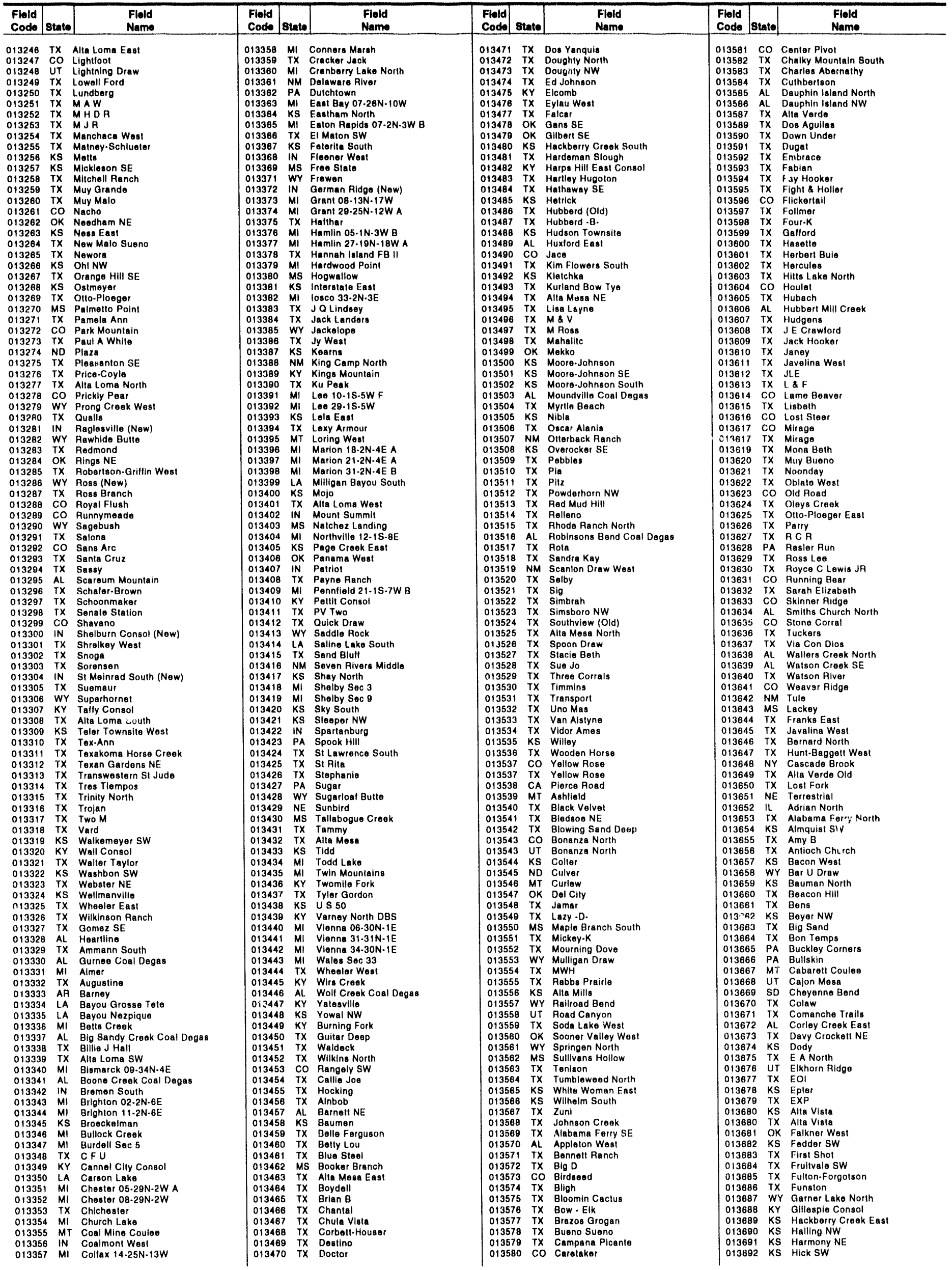




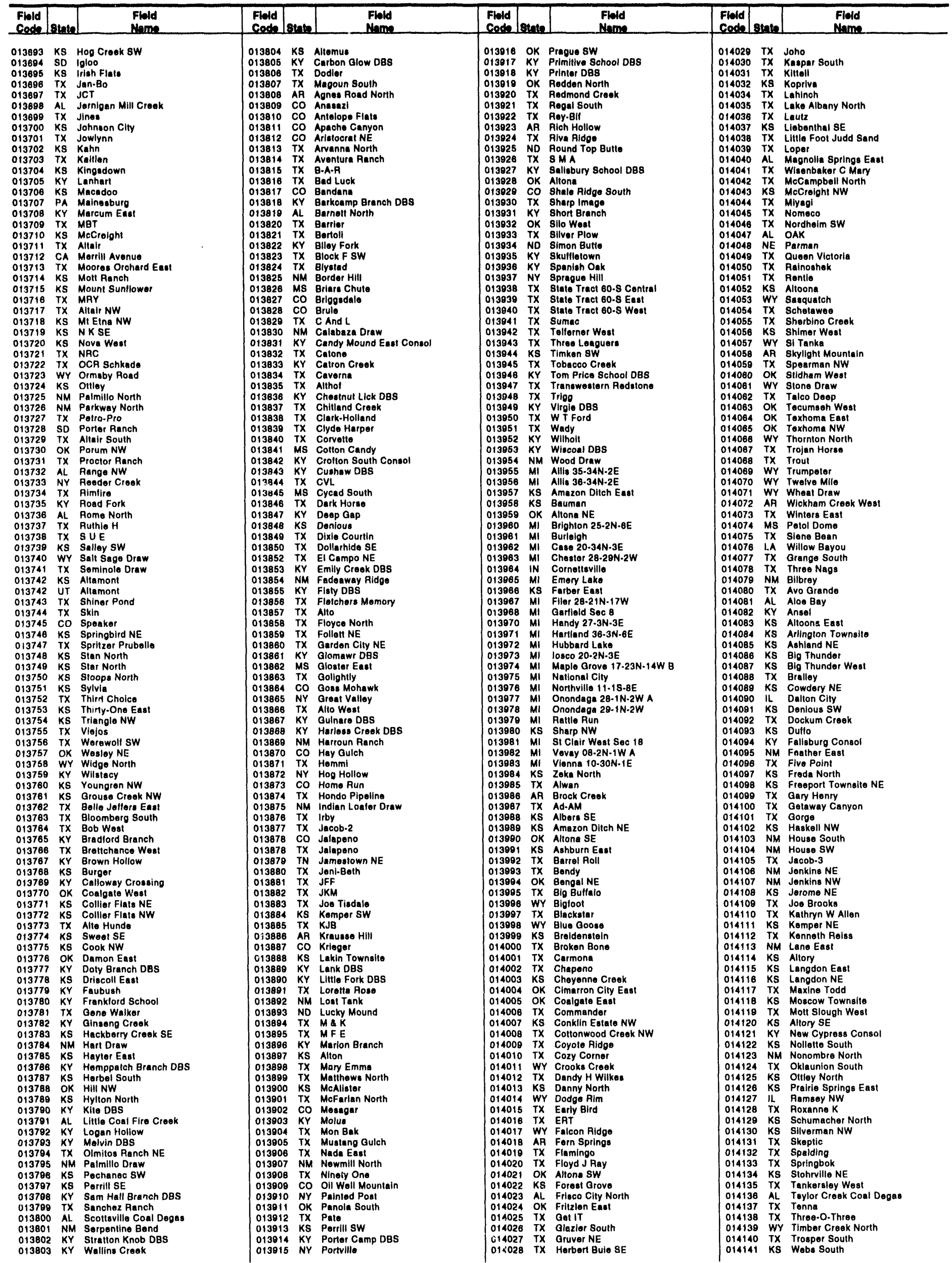




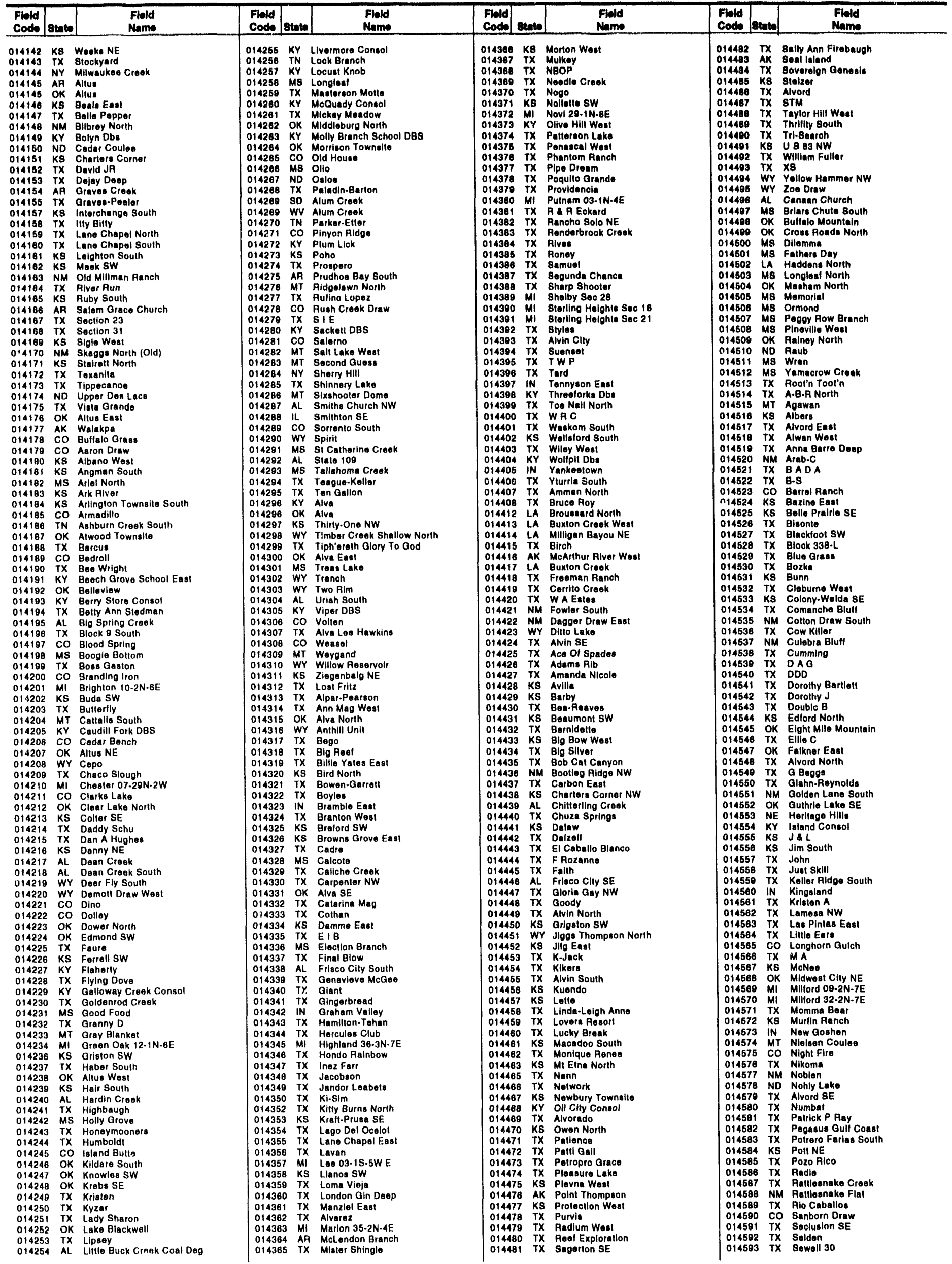




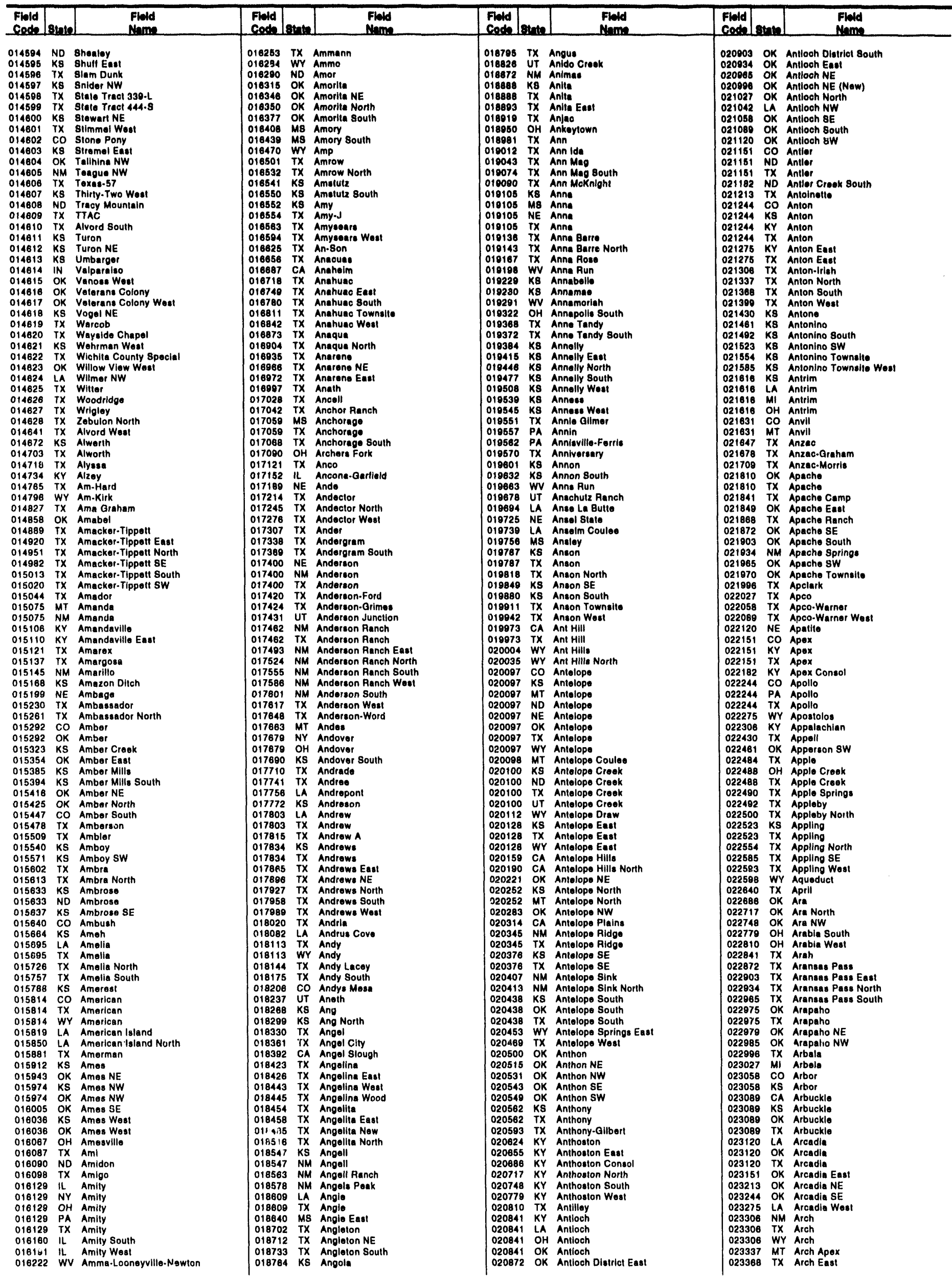




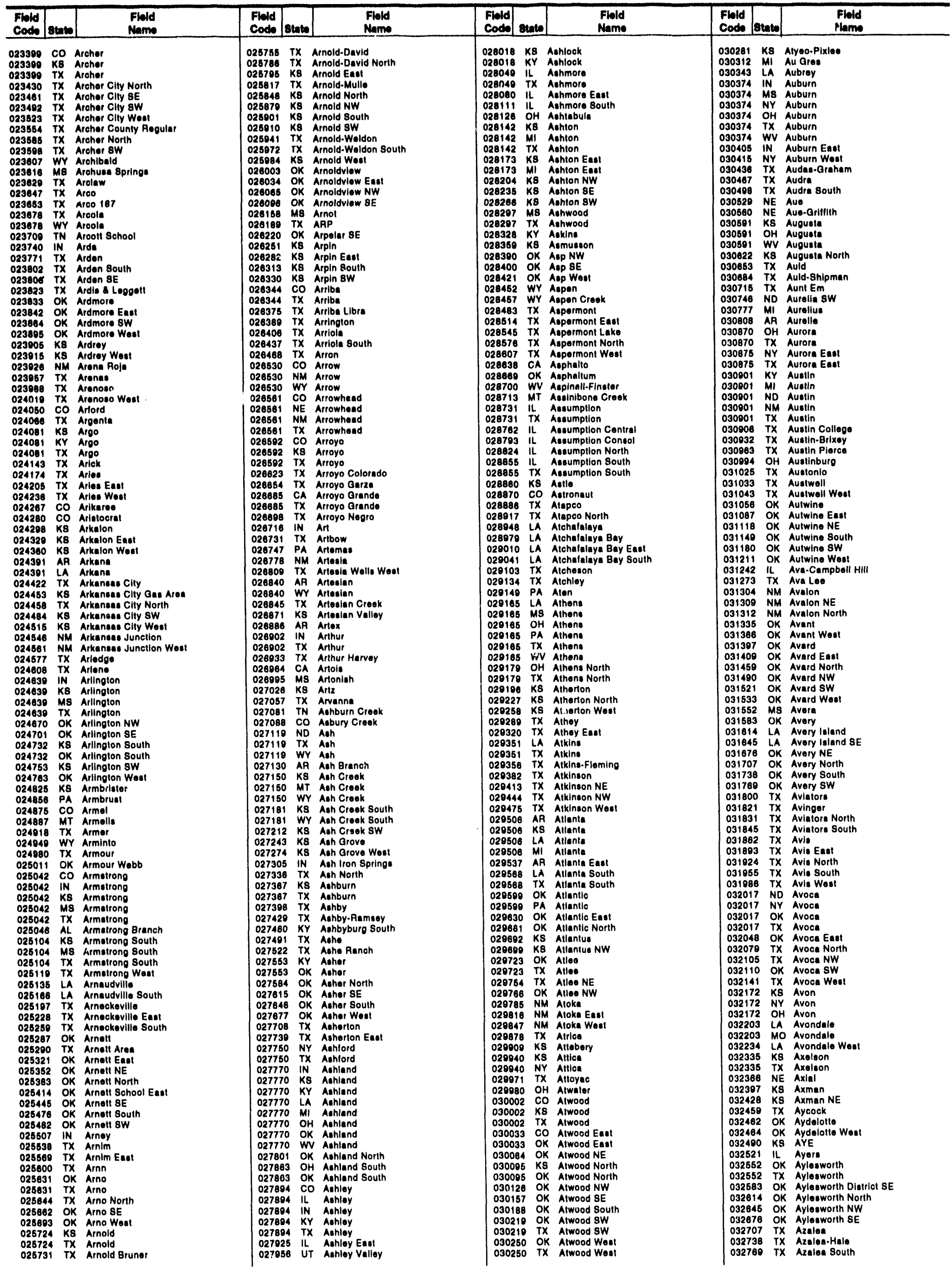




\begin{tabular}{|c|c|c|c|c|c|c|c|c|c|c|c|}
\hline $\begin{array}{l}\text { Fibld } \\
\text { Codt }\end{array}$ & Sinte & $\begin{array}{l}\text { Field } \\
\text { Nem }\end{array}$ & $\begin{array}{l}\text { Froid } \\
\text { Codt }\end{array}$ & stintel & $\begin{array}{l}\text { Fiold } \\
\text { Nime }\end{array}$ & $\begin{array}{l}\text { Flold } \\
\text { Code }\end{array}$ & & $\begin{array}{l}\text { Fild } \\
\text { Nume. }\end{array}$ & $\begin{array}{l}\text { Fildd } \\
\text { Codt. }\end{array}$ & sentel & $\begin{array}{l}\text { Flold } \\
\text { Ninme }\end{array}$ \\
\hline 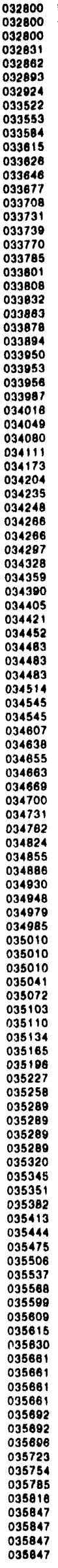 & 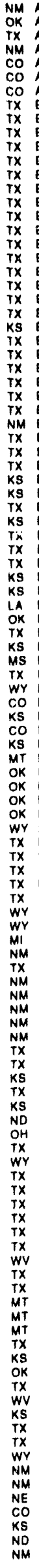 & 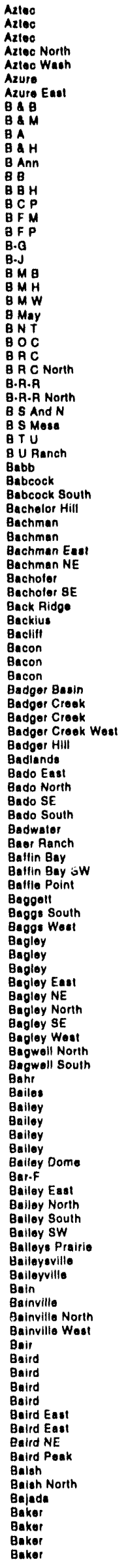 & 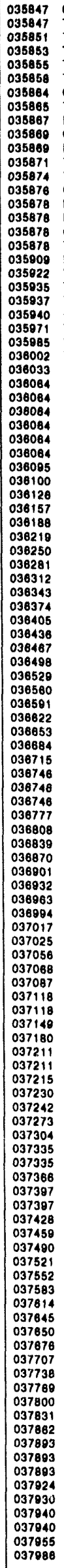 & 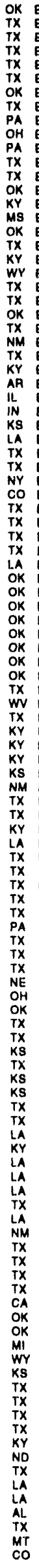 & 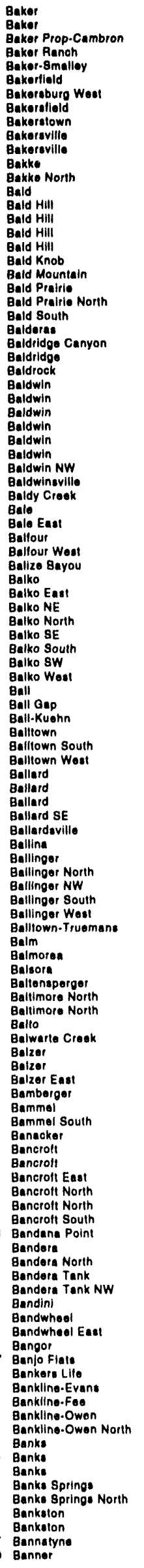 & 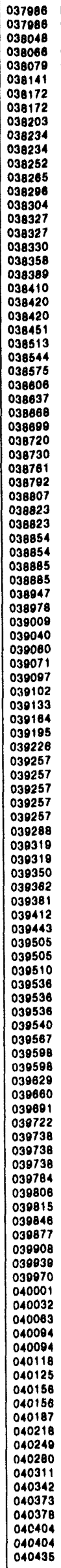 & 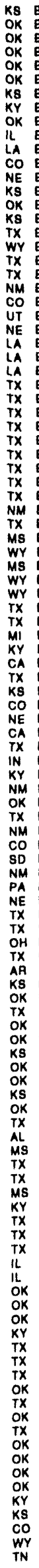 & 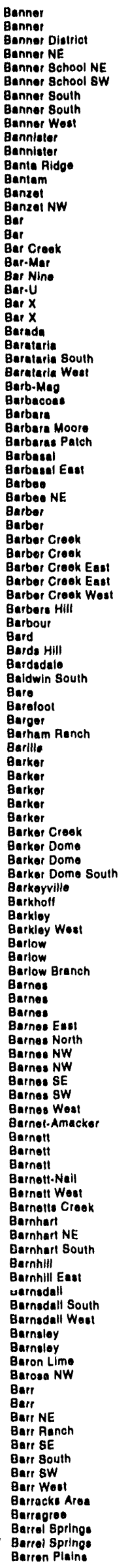 & 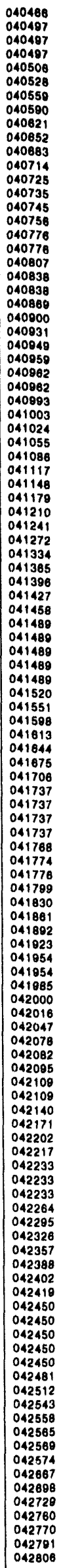 & 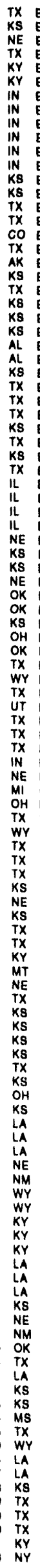 & 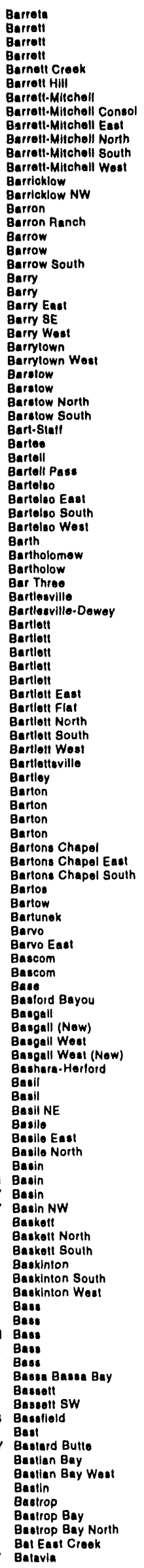 \\
\hline
\end{tabular}




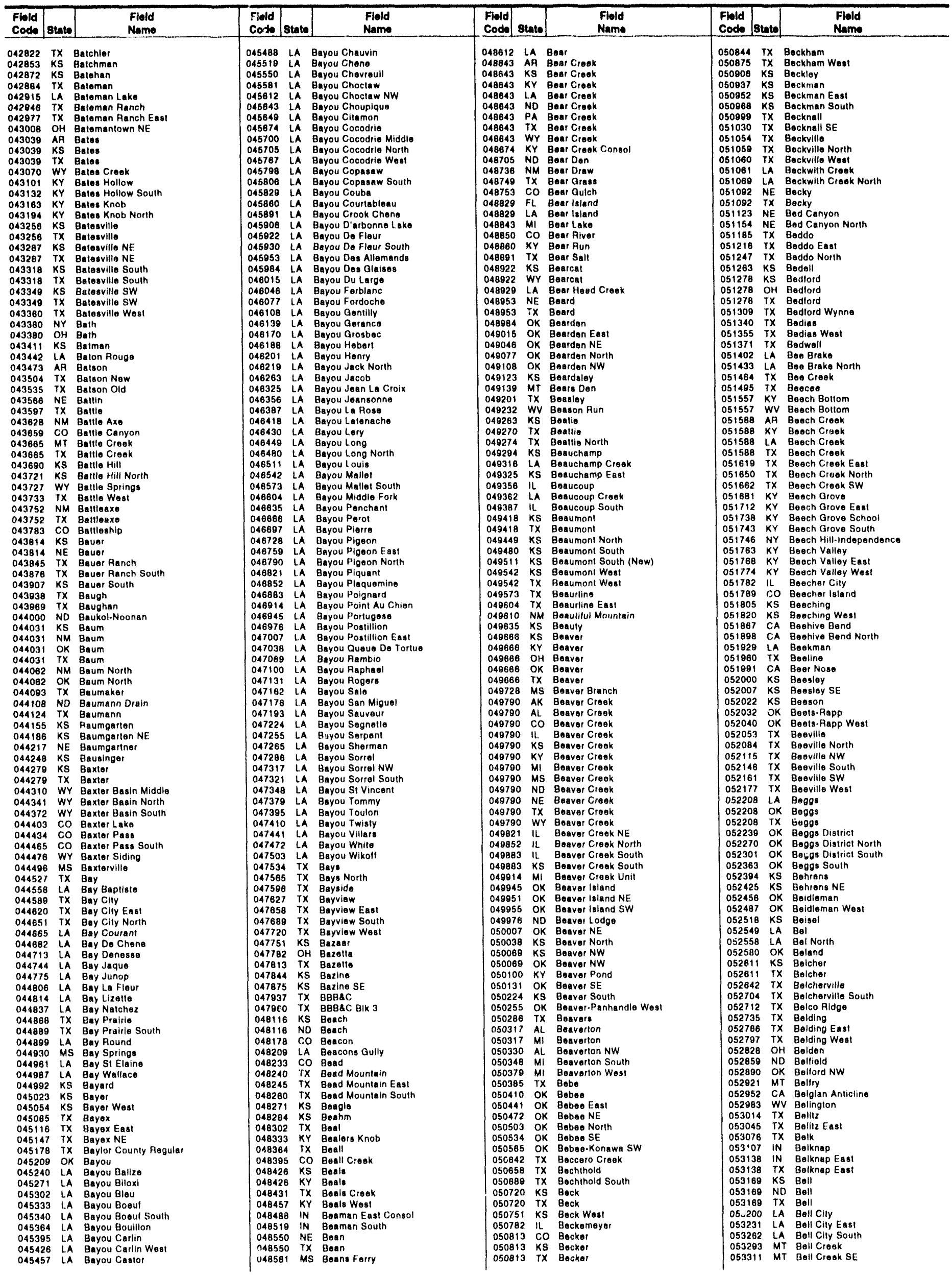




\begin{tabular}{|c|c|c|c|c|c|c|c|c|c|c|c|}
\hline $\begin{array}{l}\text { Fiold } \\
\text { Code }\end{array}$ & State & $\begin{array}{l}\text { Fildd } \\
\text { Ninge }\end{array}$ & $\begin{array}{l}\text { Field } \\
\text { Coots }\end{array}$ & stat & $\begin{array}{l}\text { Findd } \\
\text { Nimen. }\end{array}$ & \begin{tabular}{|l|} 
Ficid \\
Code
\end{tabular} & Sint & $\begin{array}{l}\text { Findd } \\
\text { Nems }\end{array}$ & \begin{tabular}{l|l} 
Finid \\
Coded
\end{tabular} & sinte & $\begin{array}{l}\text { Fleld } \\
\text { Neme }\end{array}$ \\
\hline 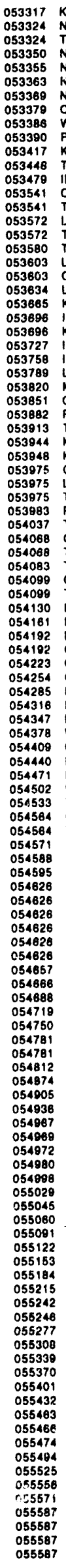 & 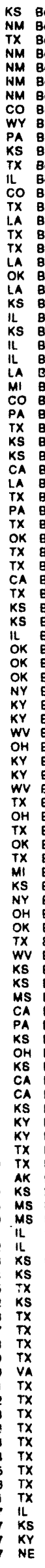 & 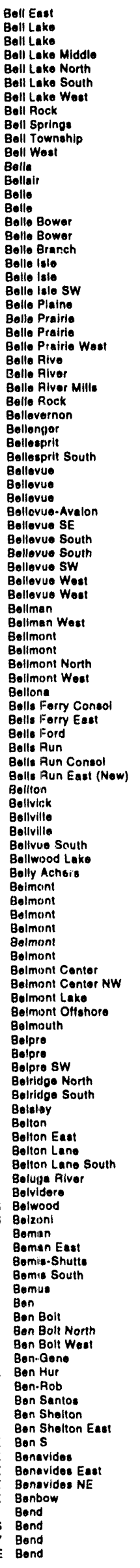 & 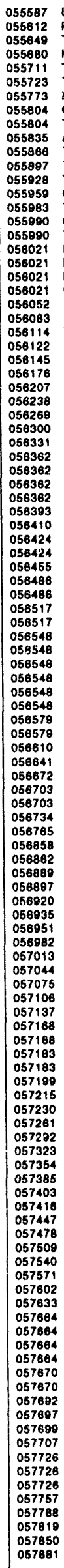 & 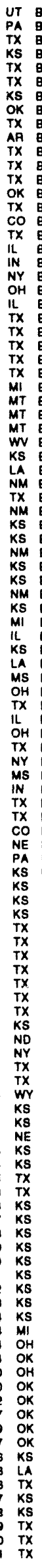 & 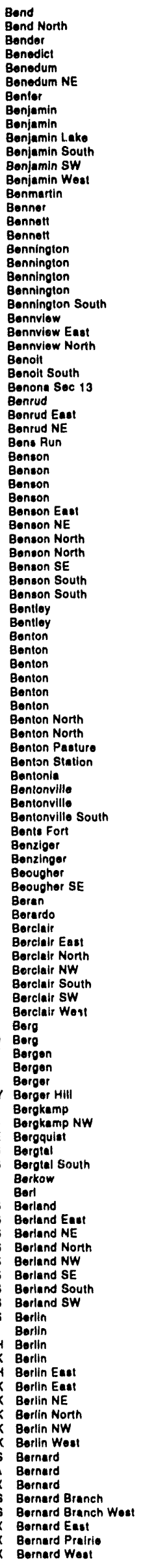 & 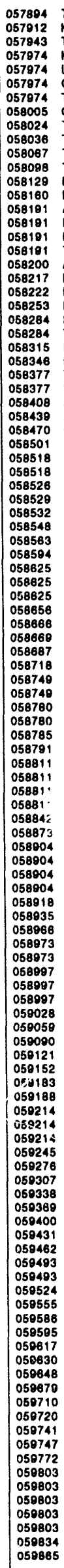 & 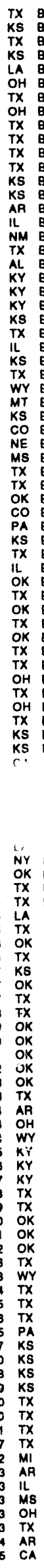 & 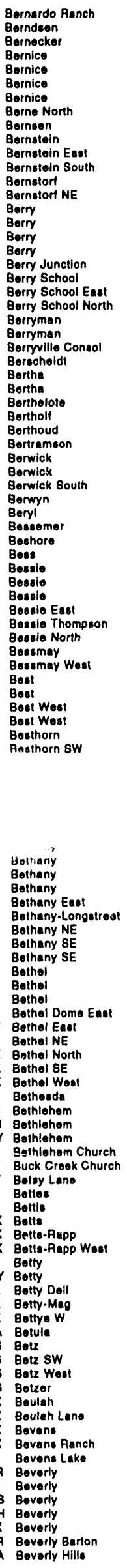 & 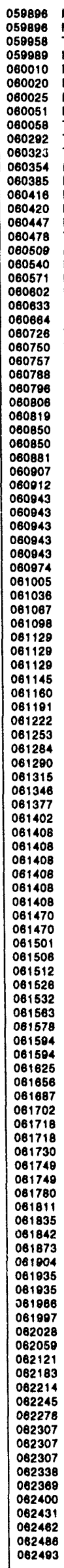 & 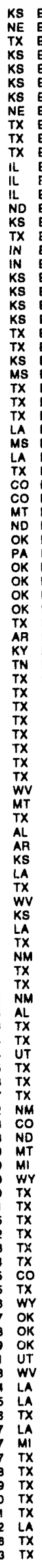 & 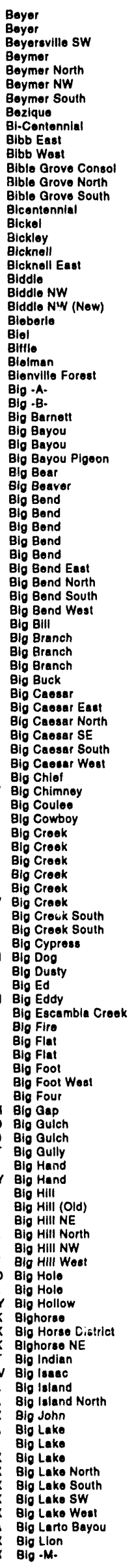 \\
\hline
\end{tabular}




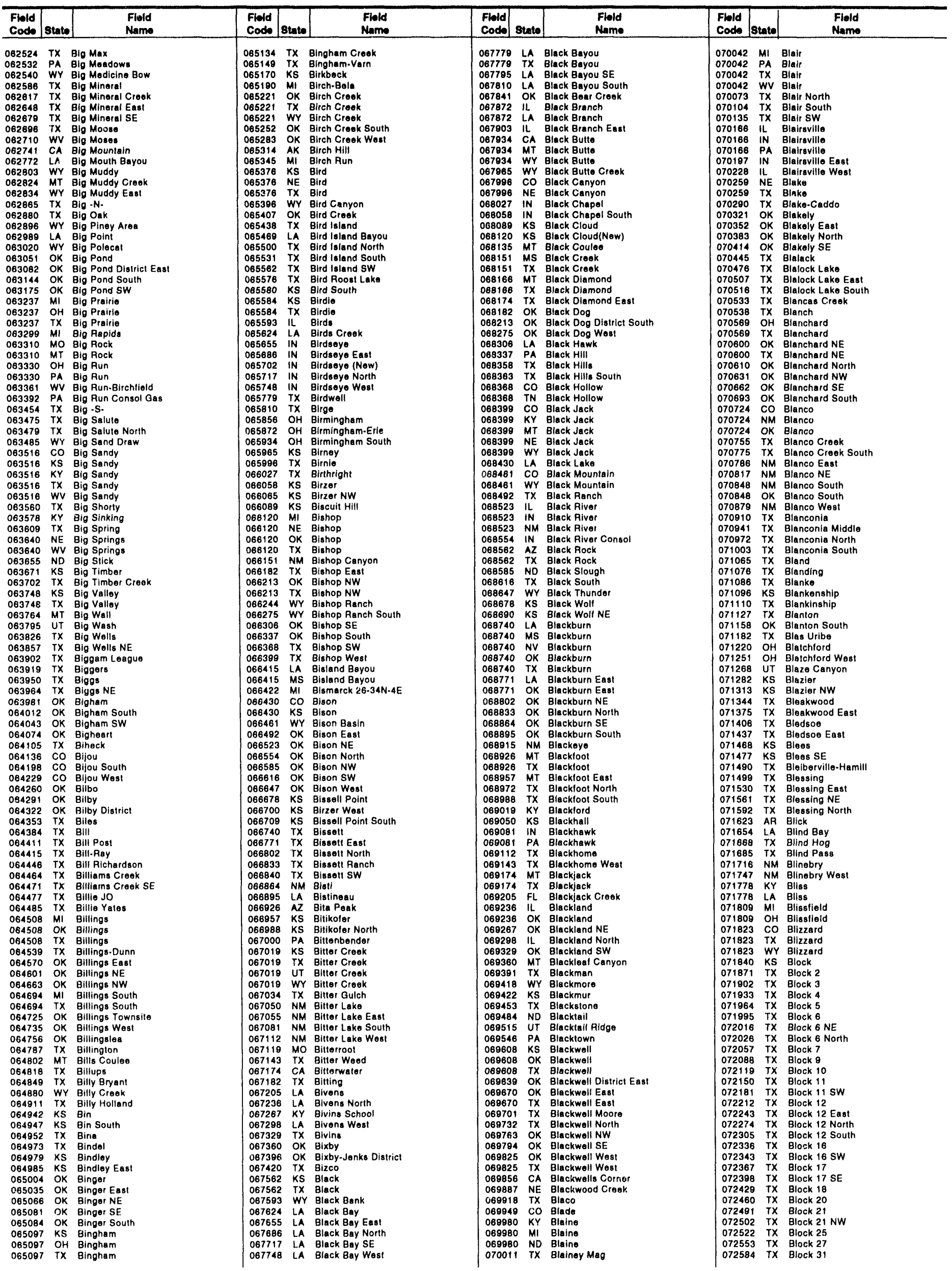




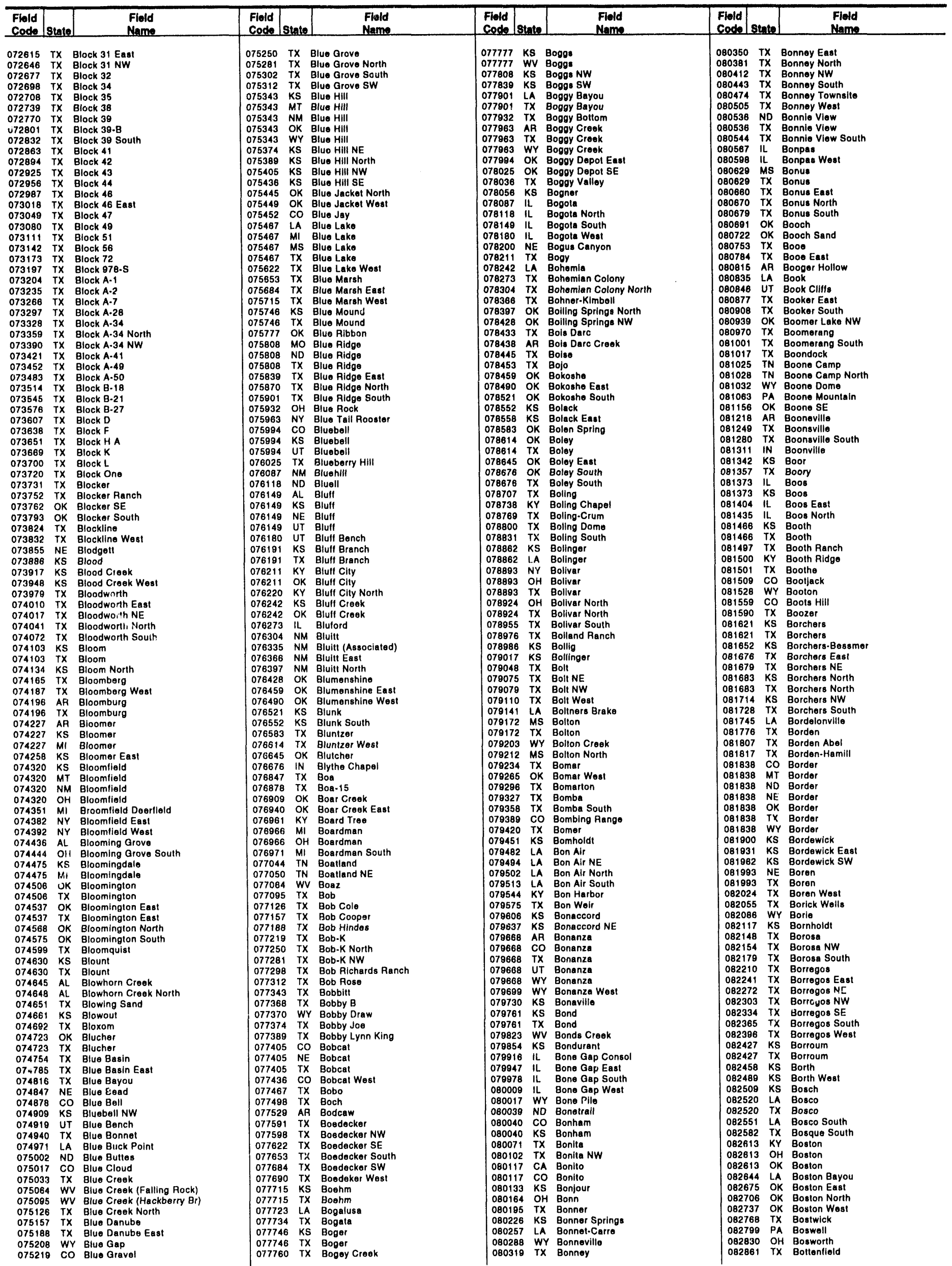




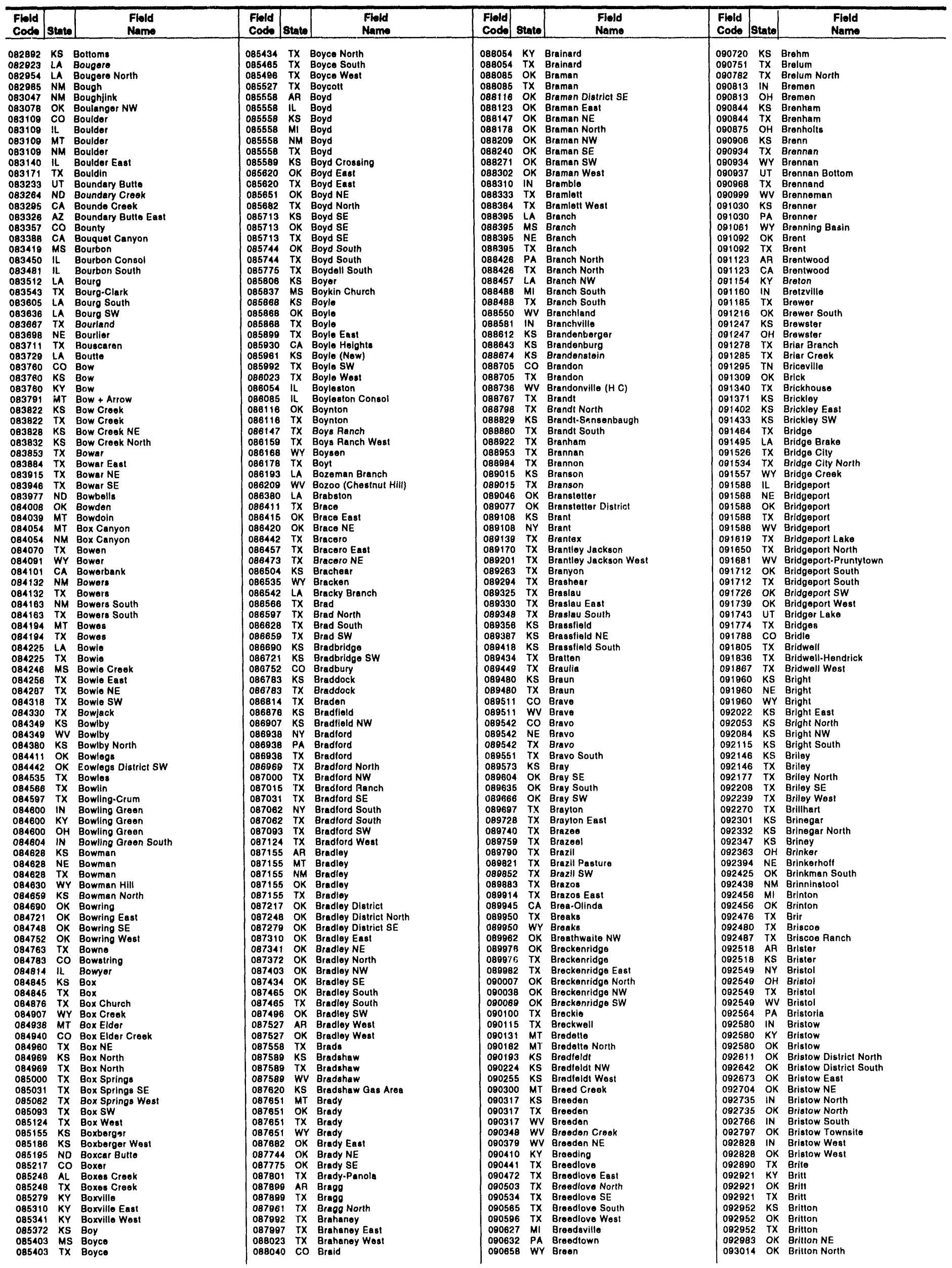




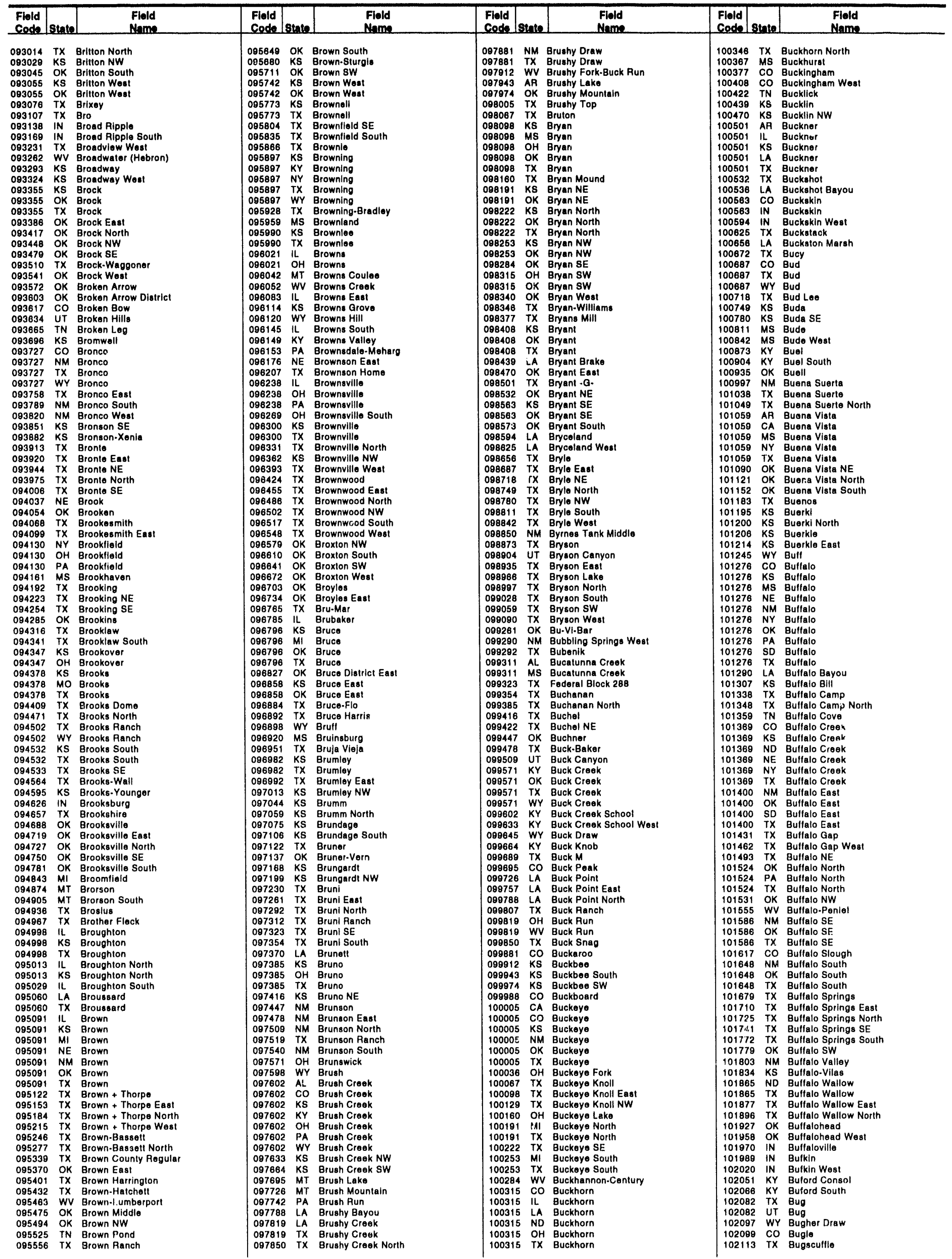




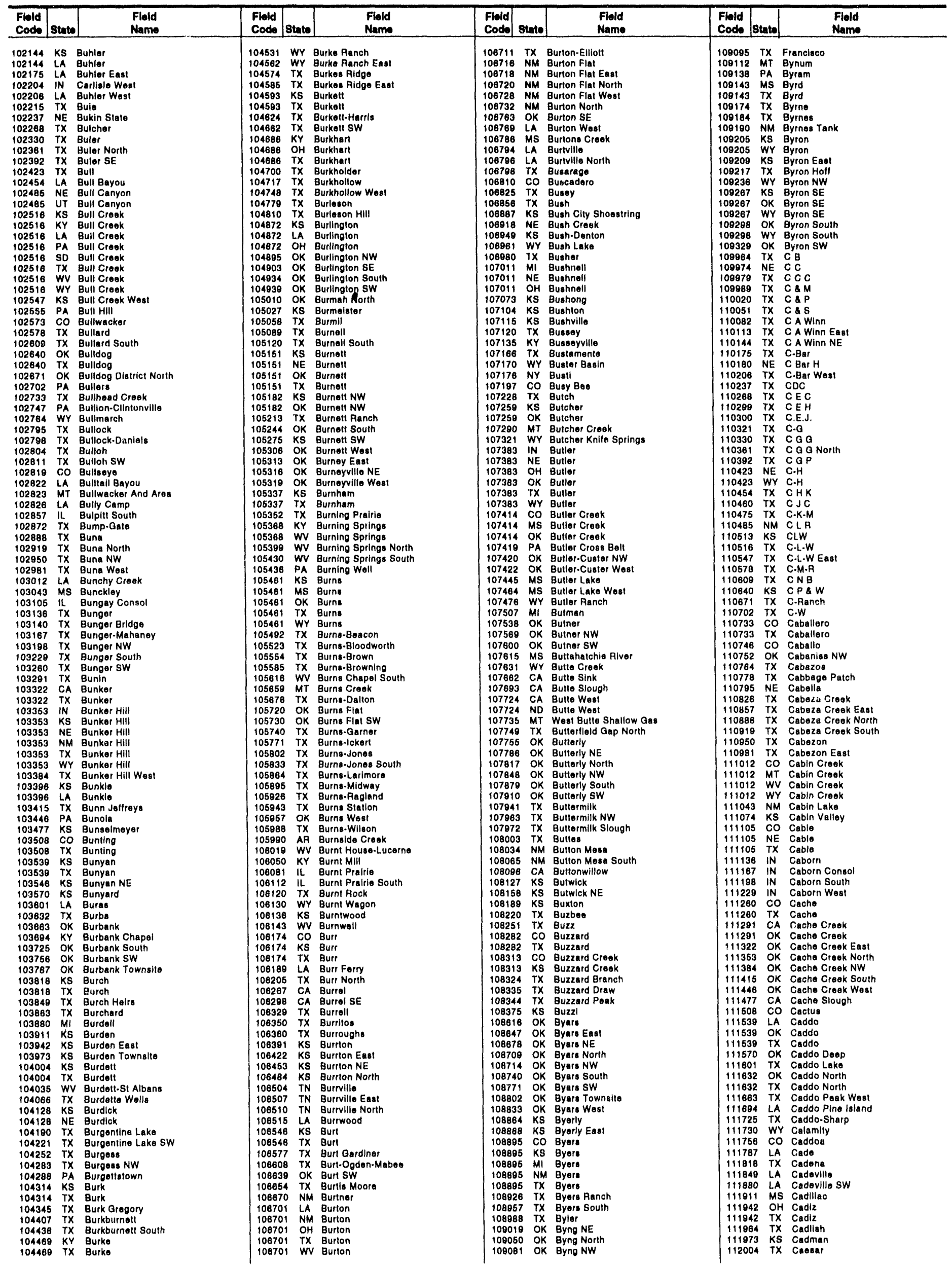




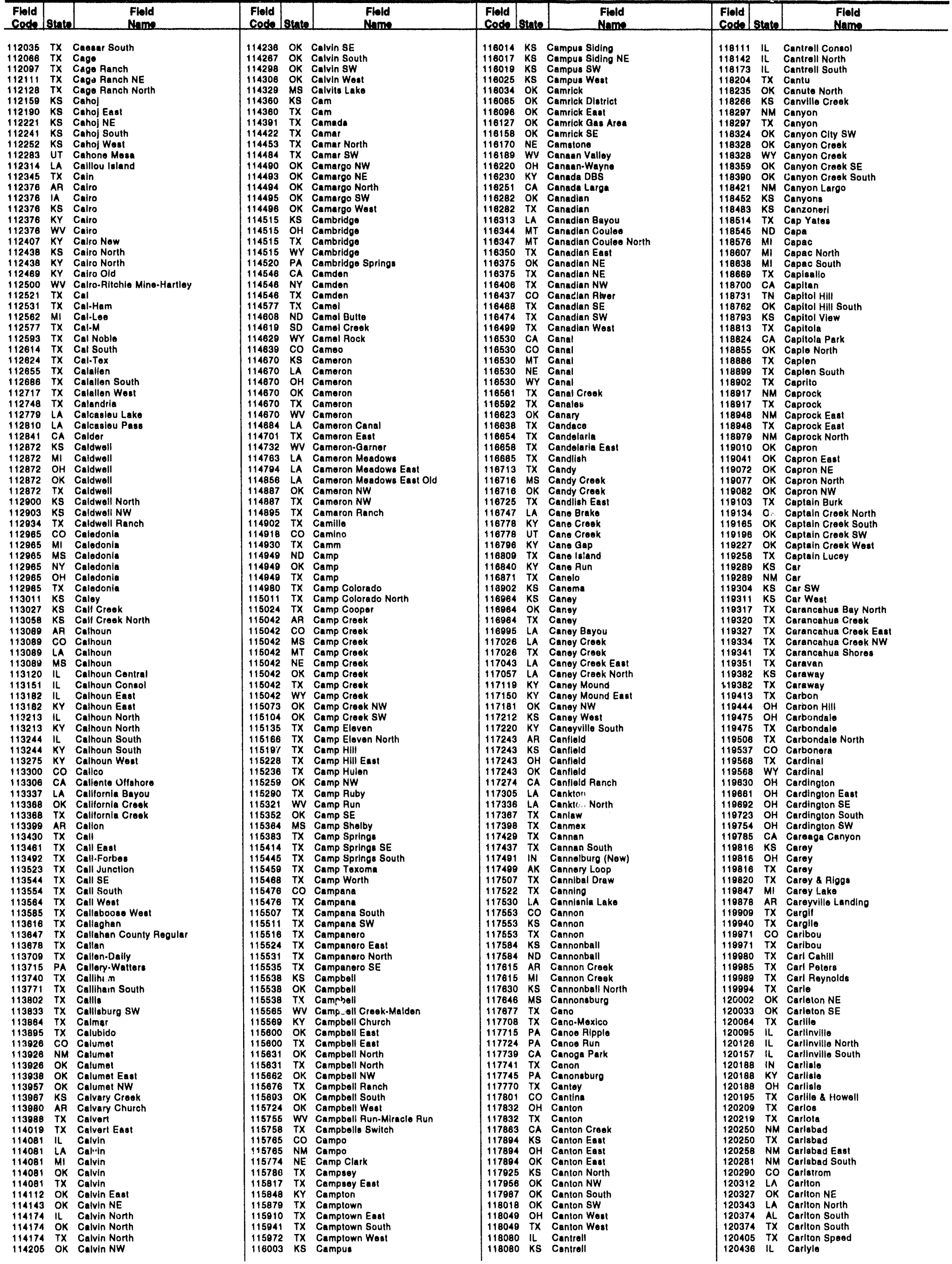




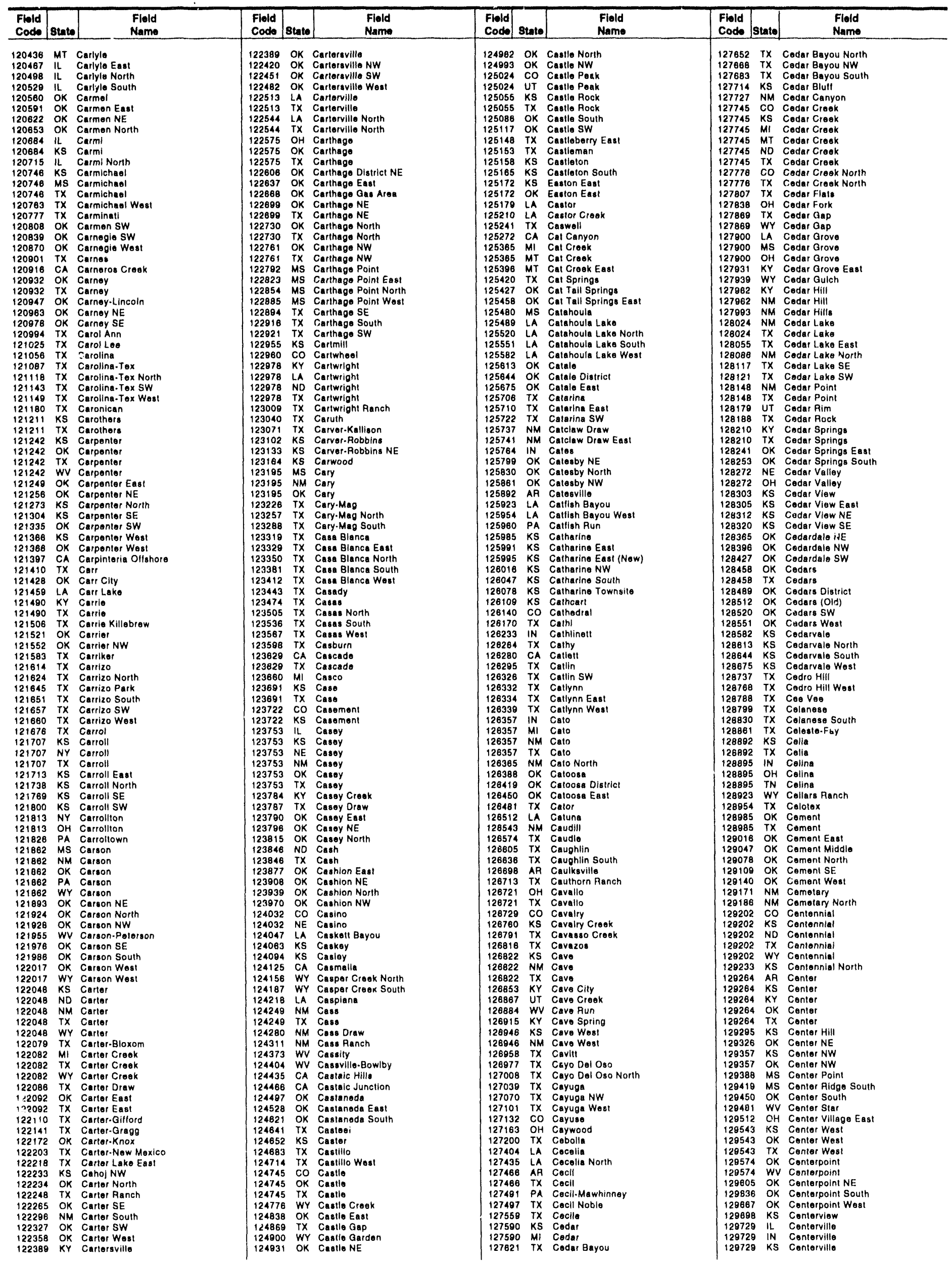




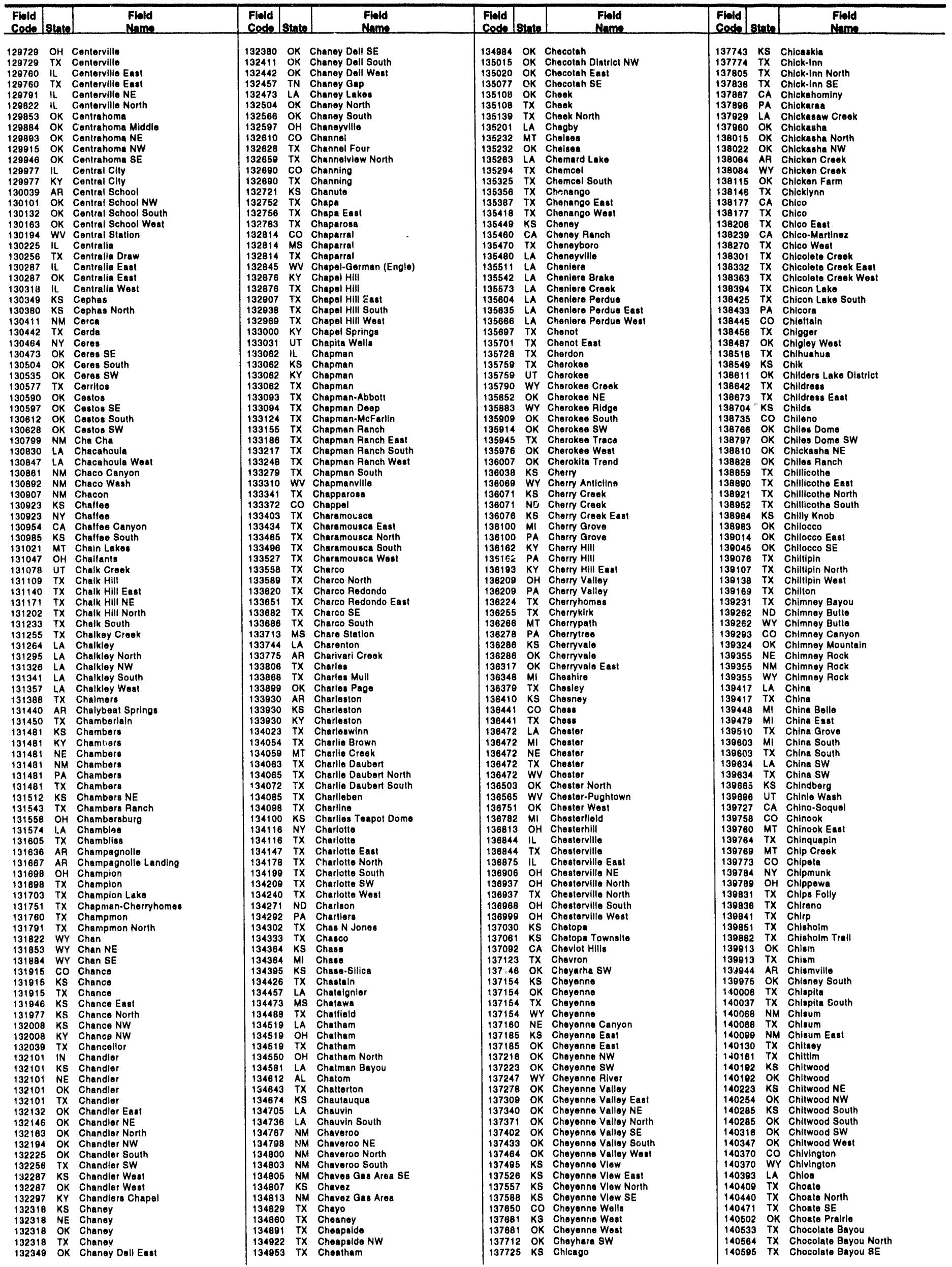




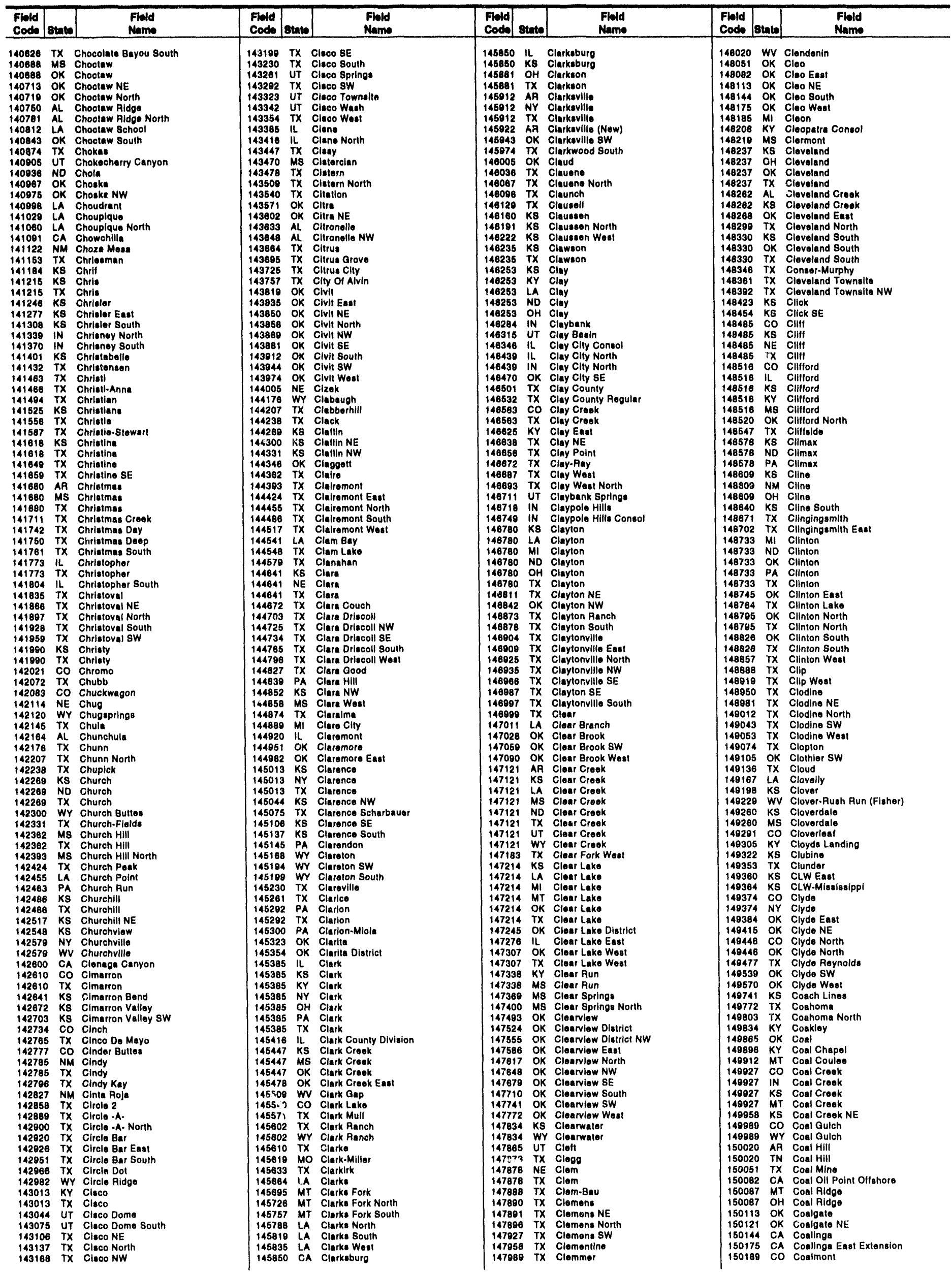




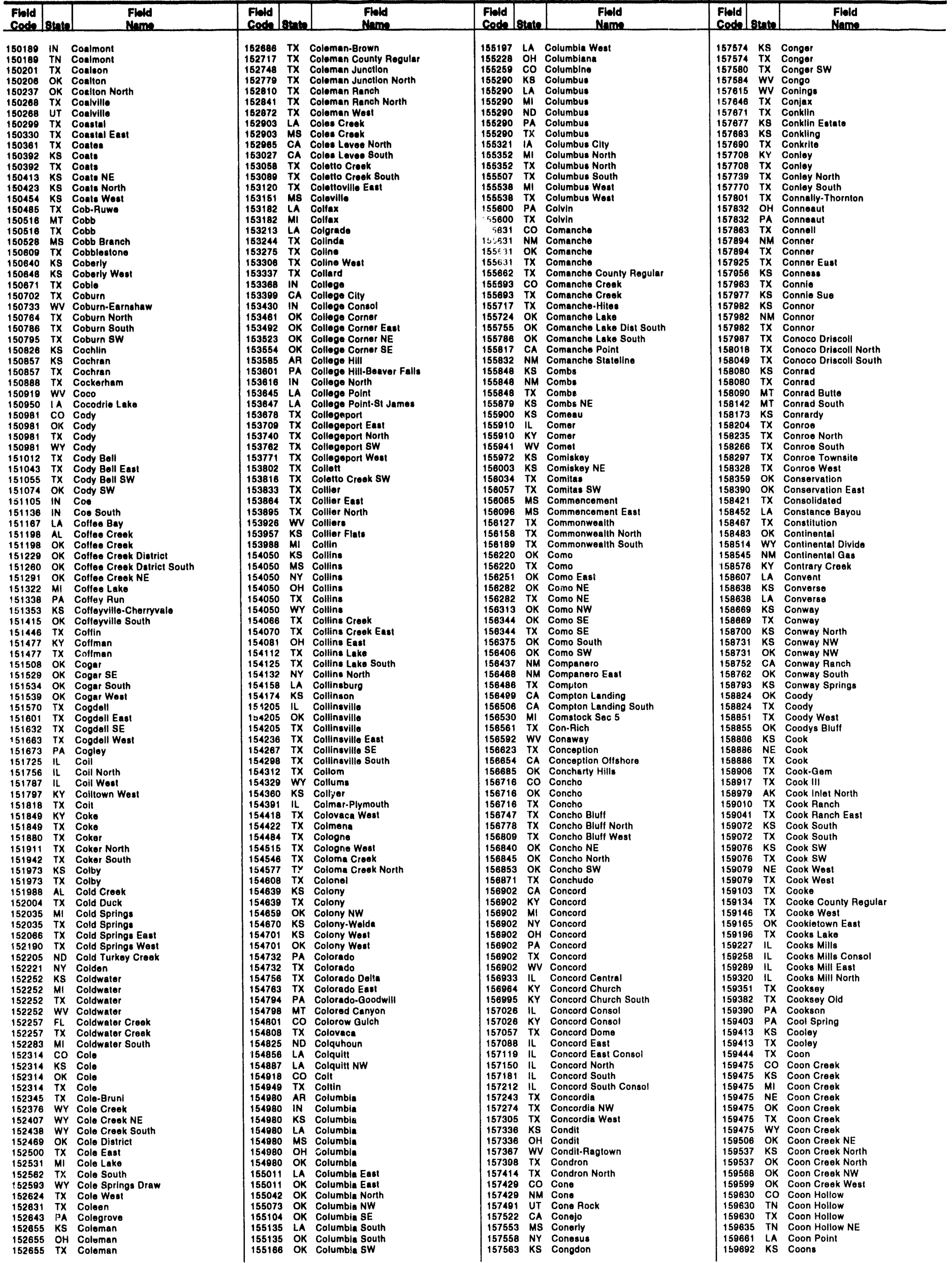




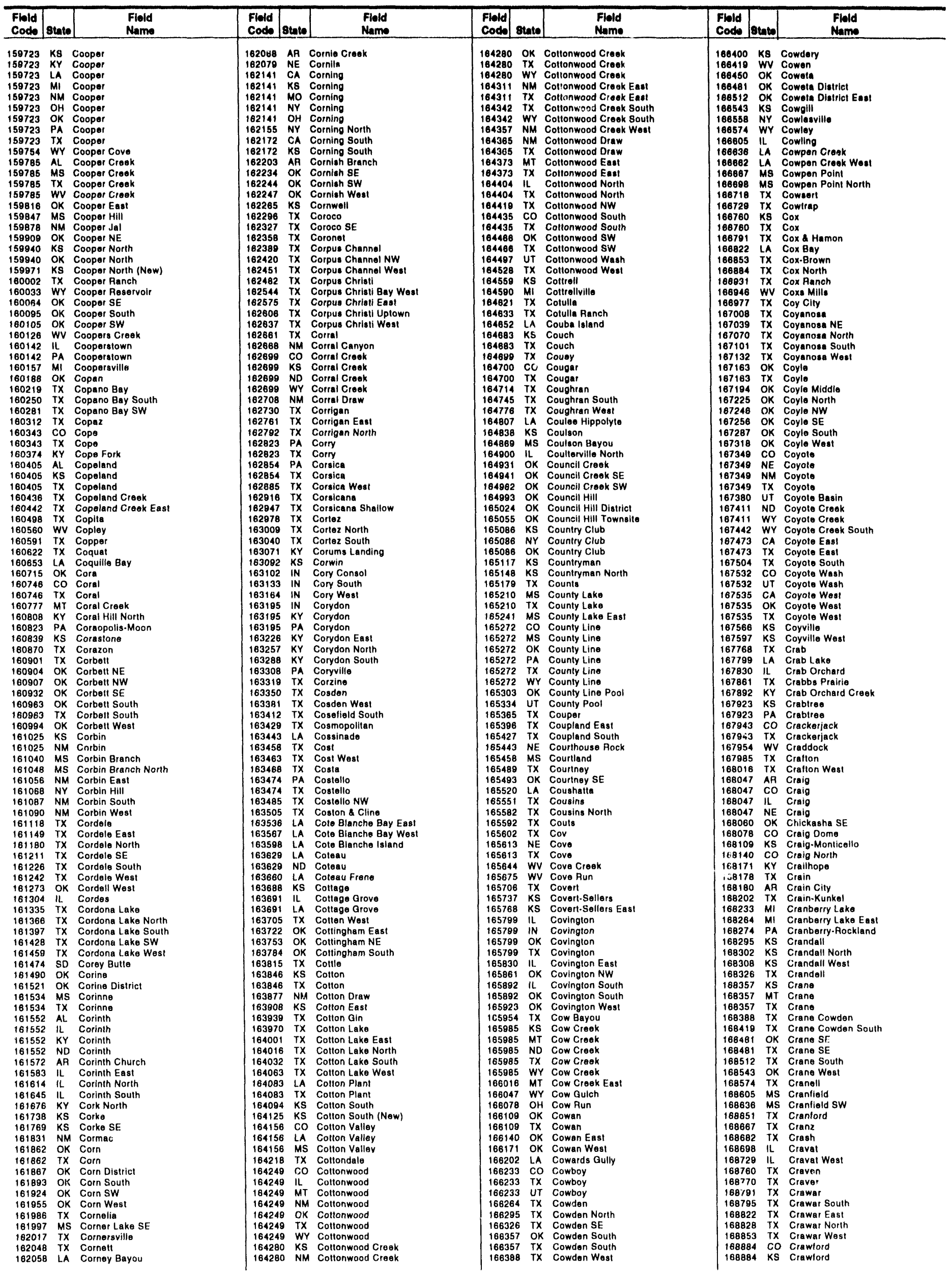




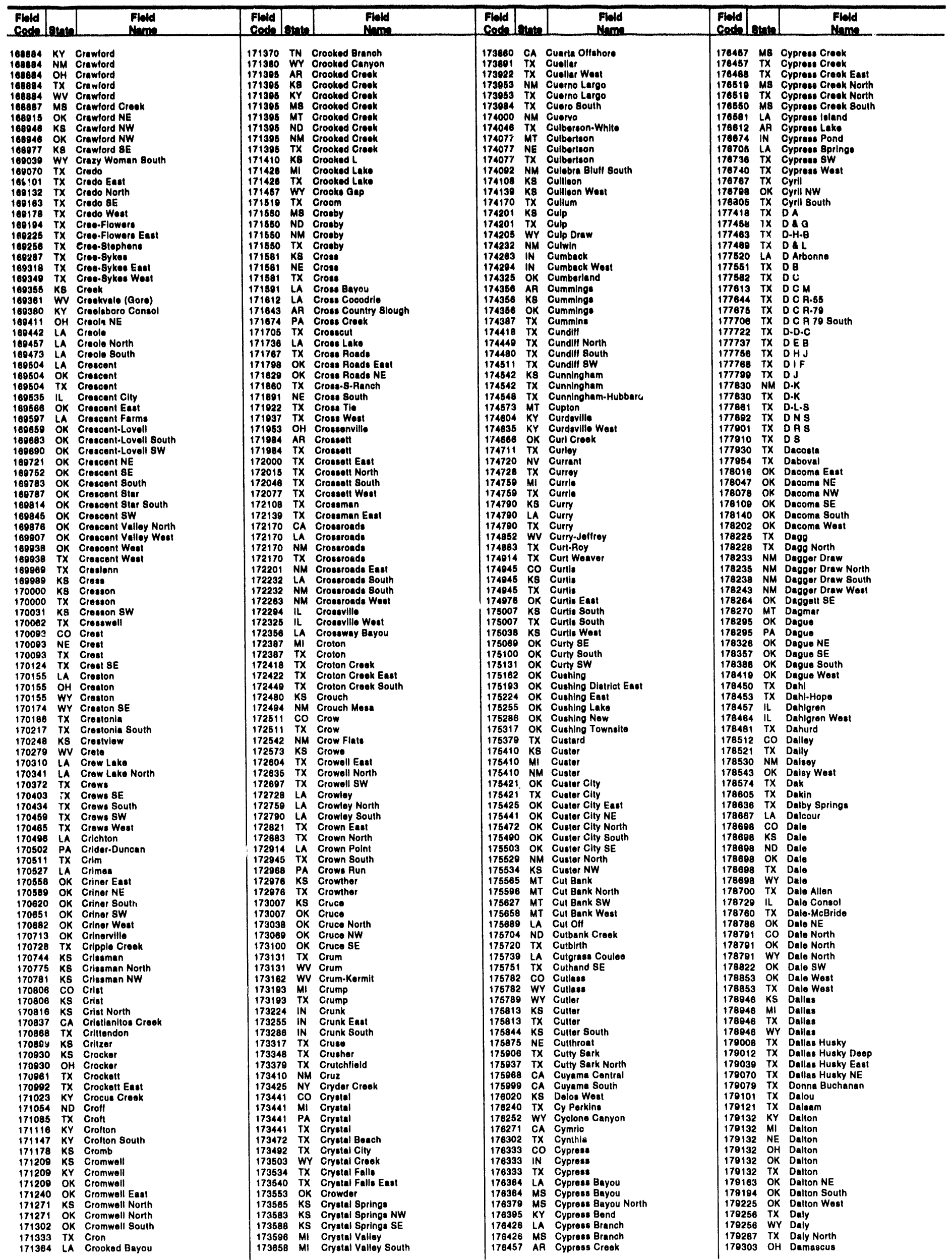




\begin{tabular}{|c|c|c|c|c|c|c|c|c|c|c|c|}
\hline $\begin{array}{l}\text { Fild } \\
\text { Code } \\
\end{array}$ & Etatio & $\begin{array}{l}\text { Fild } \\
\text { Nome }\end{array}$ & $\begin{array}{l}\text { Fileld } \\
\text { Code }\end{array}$ & Etate & $\begin{array}{l}\text { Fild } \\
\text { Name }\end{array}$ & \begin{tabular}{l|l} 
Fild & \\
Code & 8 \\
\end{tabular} & State & $\begin{array}{l}\text { Flold } \\
\text { Name }\end{array}$ & \begin{tabular}{l|l} 
Fiold \\
Code 8 \\
\end{tabular} & 8tate & $\begin{array}{l}\text { Fiold } \\
\text { Namo }\end{array}$ \\
\hline 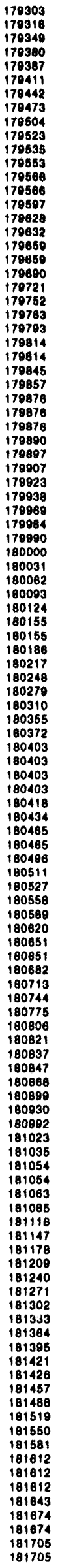 & 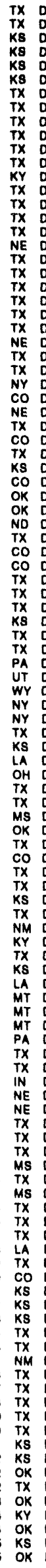 & 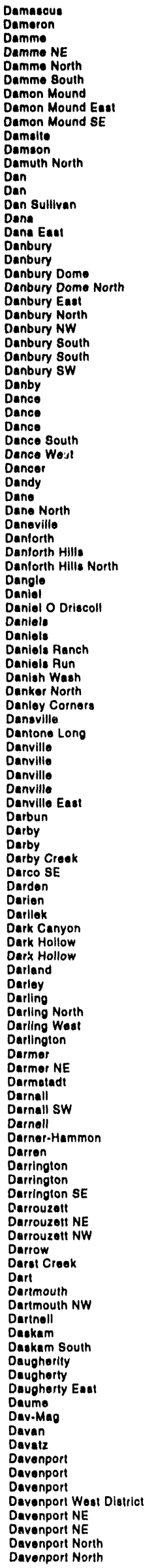 & 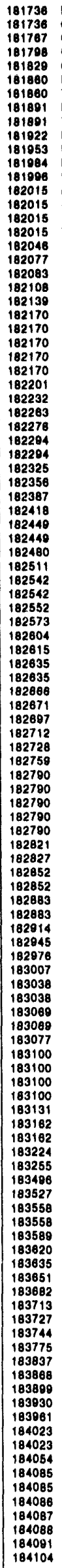 & 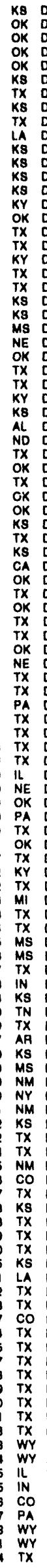 & 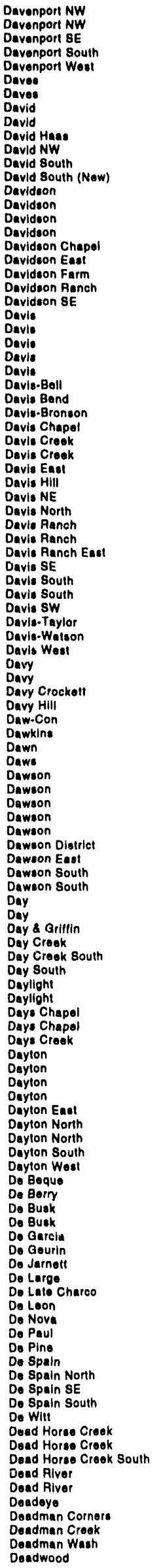 & 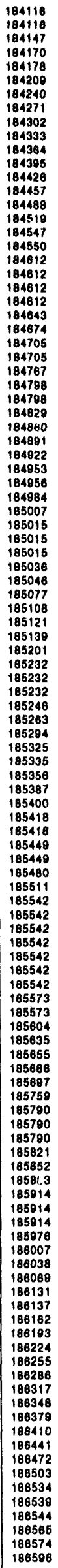 & 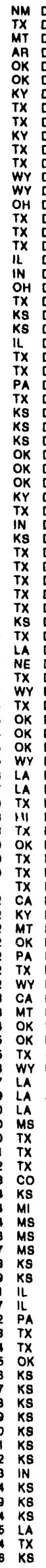 & 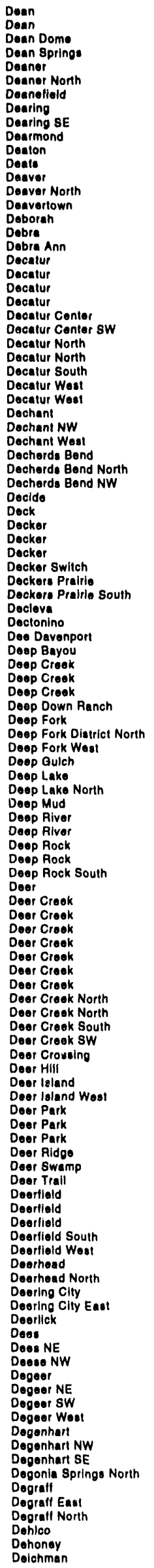 & 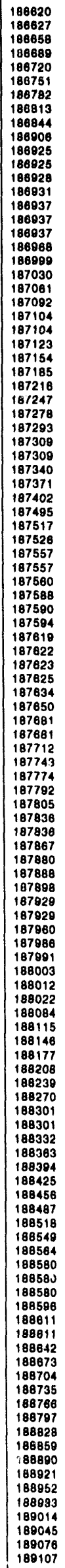 & 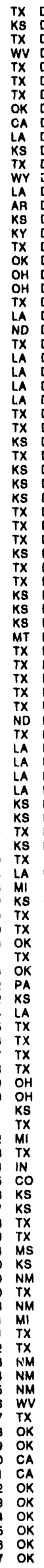 & 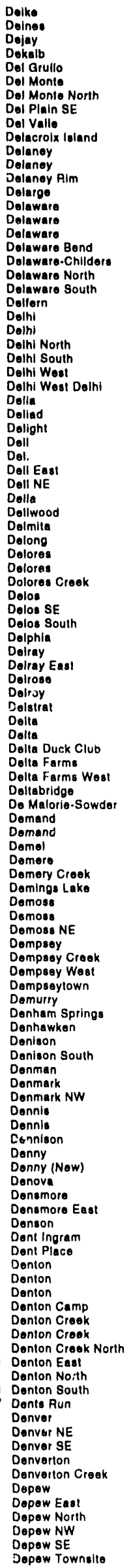 \\
\hline
\end{tabular}




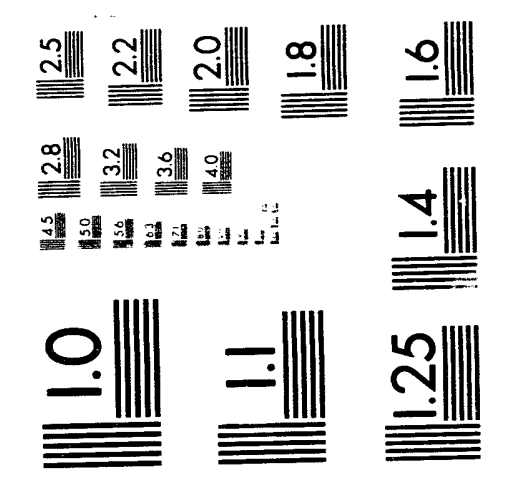




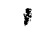
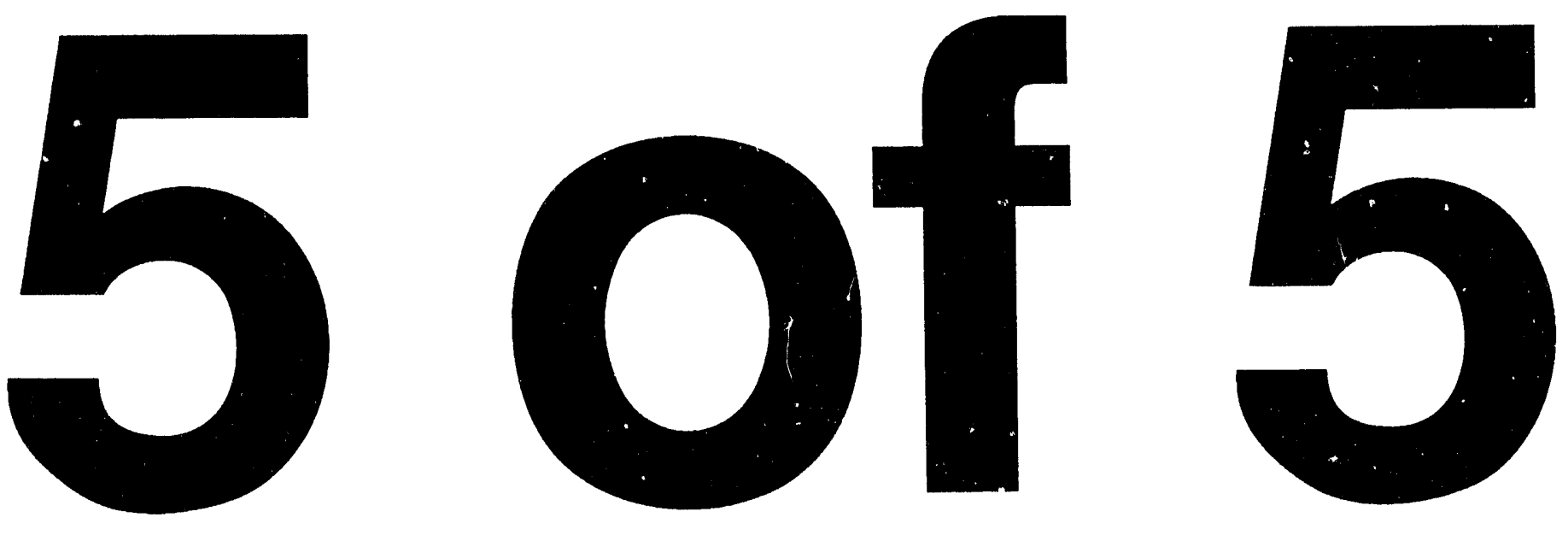


\begin{tabular}{|c|c|c|c|c|c|c|c|c|c|c|c|}
\hline $\begin{array}{l}\text { Fiold } \\
\text { Code }\end{array}$ & Statel & $\begin{array}{l}\text { Fiold } \\
\text { Name }\end{array}$ & $\begin{array}{l}\text { Field } \\
\text { Cook }\end{array}$ & Stat & $\begin{array}{l}\text { Fiold } \\
\text { Name }\end{array}$ & $\left.\begin{array}{l}\text { Field } \\
\text { Cood. }\end{array}\right]$ e & Bint & $\begin{array}{l}\text { Findd } \\
\text { Ninm: }\end{array}$ & $\begin{array}{l}\text { Filld } \\
\text { codels }\end{array}$ & State & $\begin{array}{l}\text { Fild } \\
\text { Nam. }\end{array}$ \\
\hline 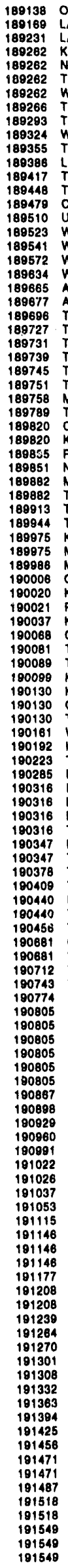 & 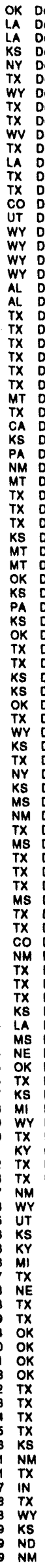 & 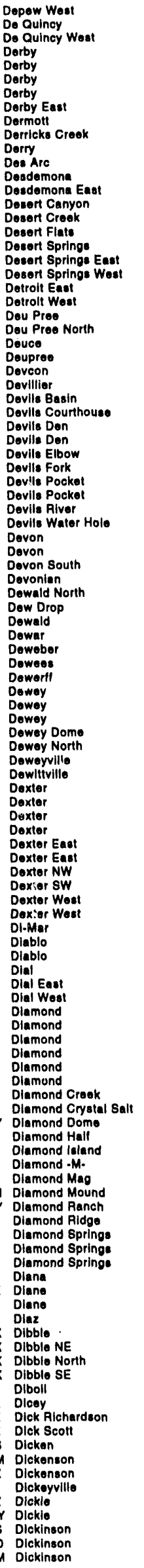 & 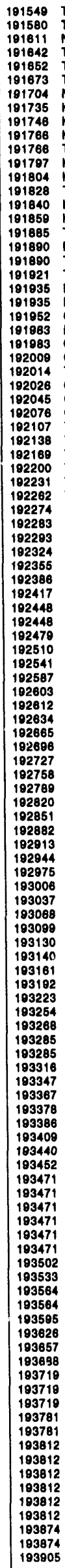 & 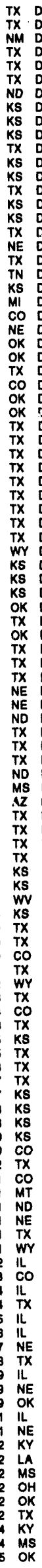 & 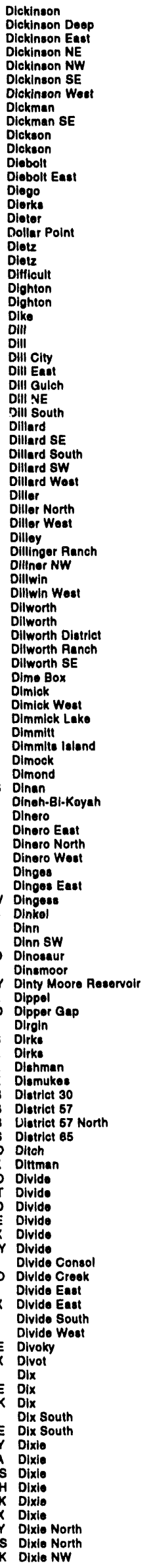 & 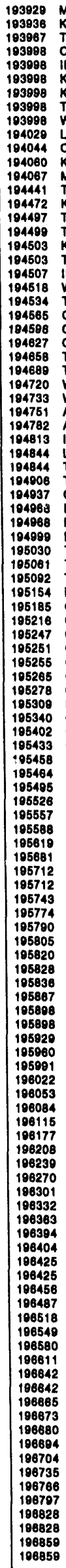 & 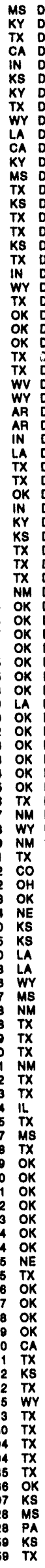 & 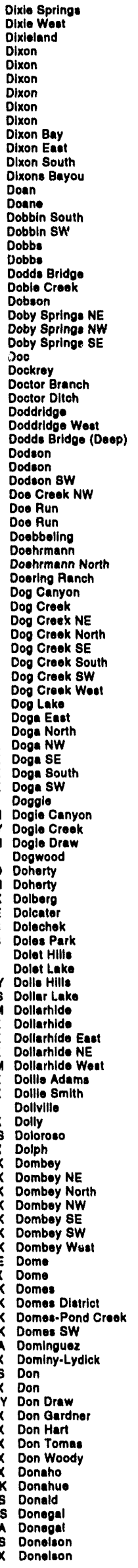 & 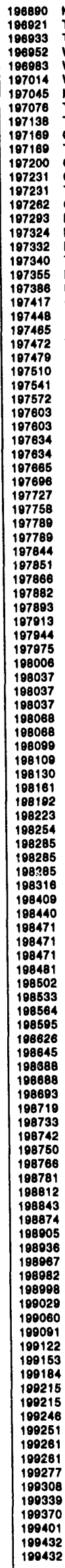 & 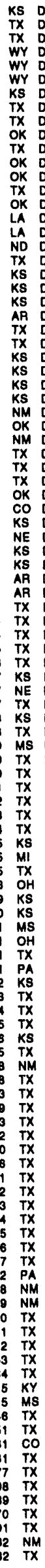 & 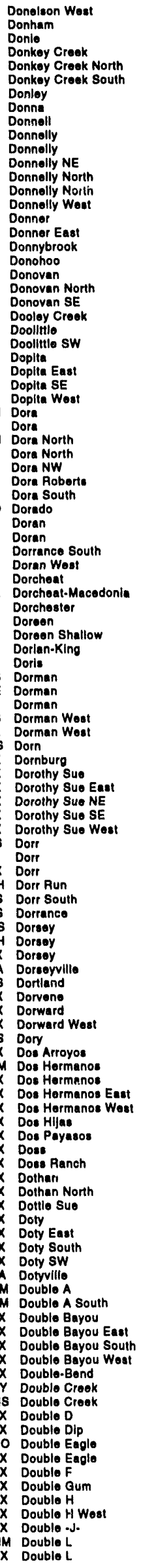 \\
\hline
\end{tabular}




\begin{tabular}{|c|c|c|c|c|c|c|c|c|c|c|c|}
\hline $\begin{array}{c}\text { Fild } \\
\text { Coda }\end{array}$ & state & $\begin{array}{l}\text { Fiold } \\
\text { Nams }\end{array}$ & $\begin{array}{l}\text { Fild } \\
\text { Code }\end{array}$ & State & $\begin{array}{l}\text { Fiold } \\
\text { Name }\end{array}$ & \begin{tabular}{l|l} 
Fild & \\
Code & 8 \\
\end{tabular} & State & $\begin{array}{l}\text { Field } \\
\text { Namo }\end{array}$ & $\begin{array}{l}\text { Flold } \\
\text { Codd }\end{array}$ & State & $\begin{array}{l}\text { Fleld } \\
\text { Name } \\
\end{array}$ \\
\hline 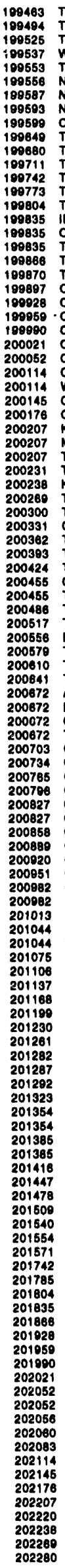 & 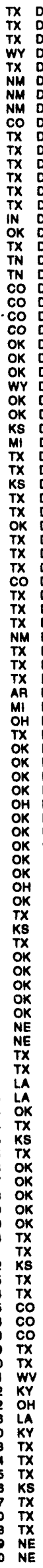 & 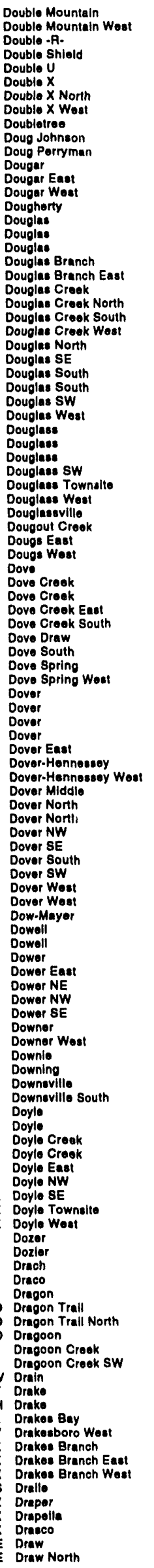 & 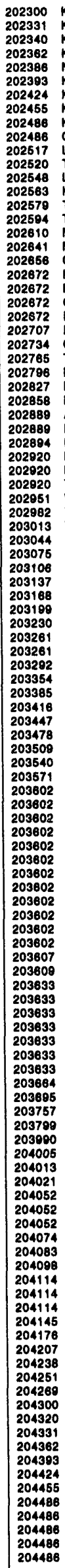 & 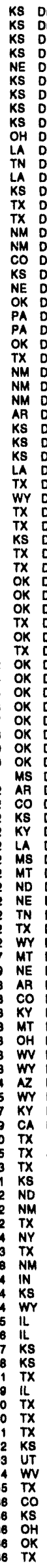 & 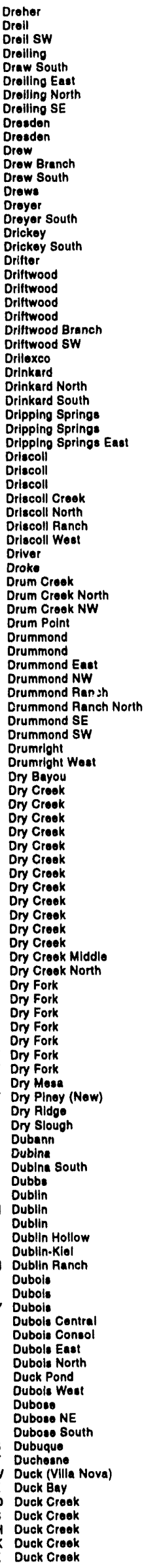 & 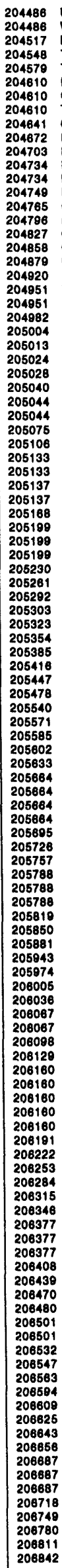 & 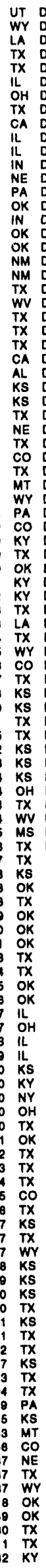 & 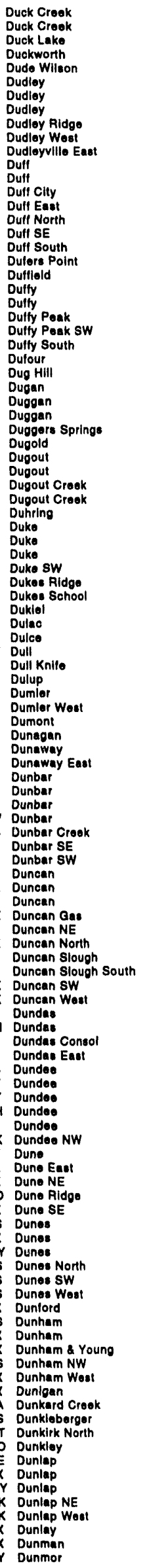 & 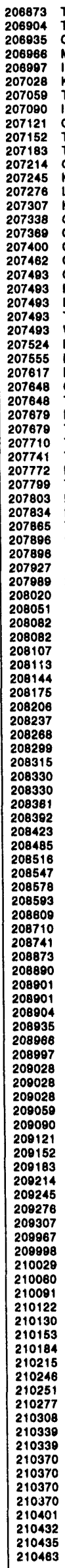 & 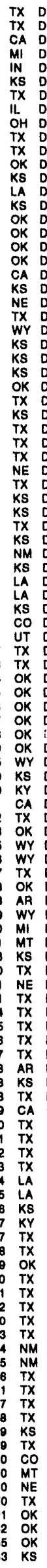 & 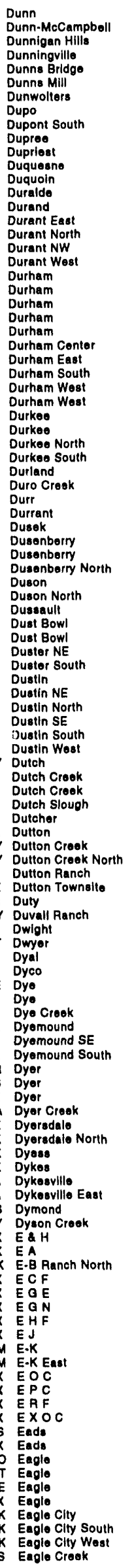 \\
\hline
\end{tabular}




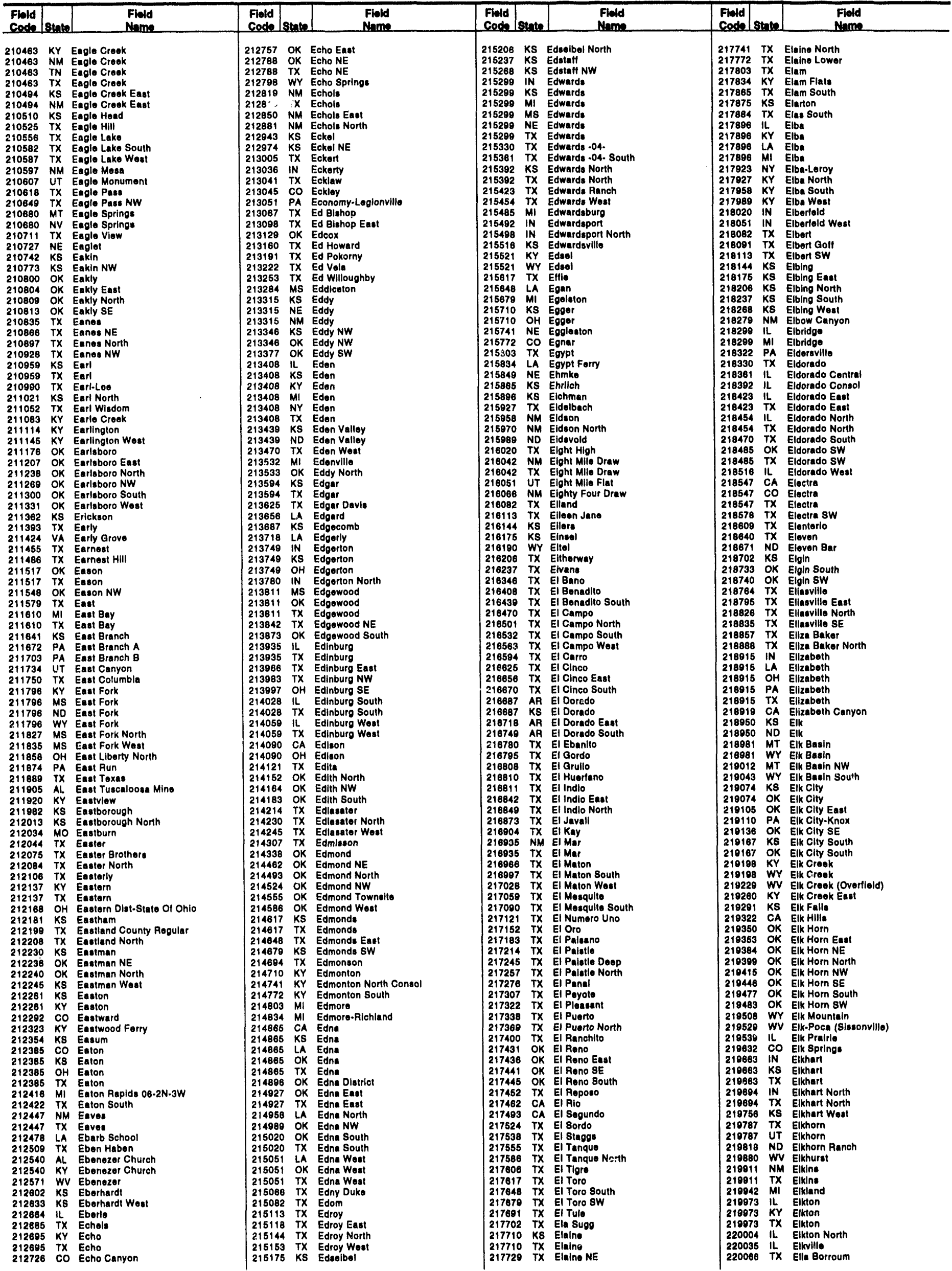




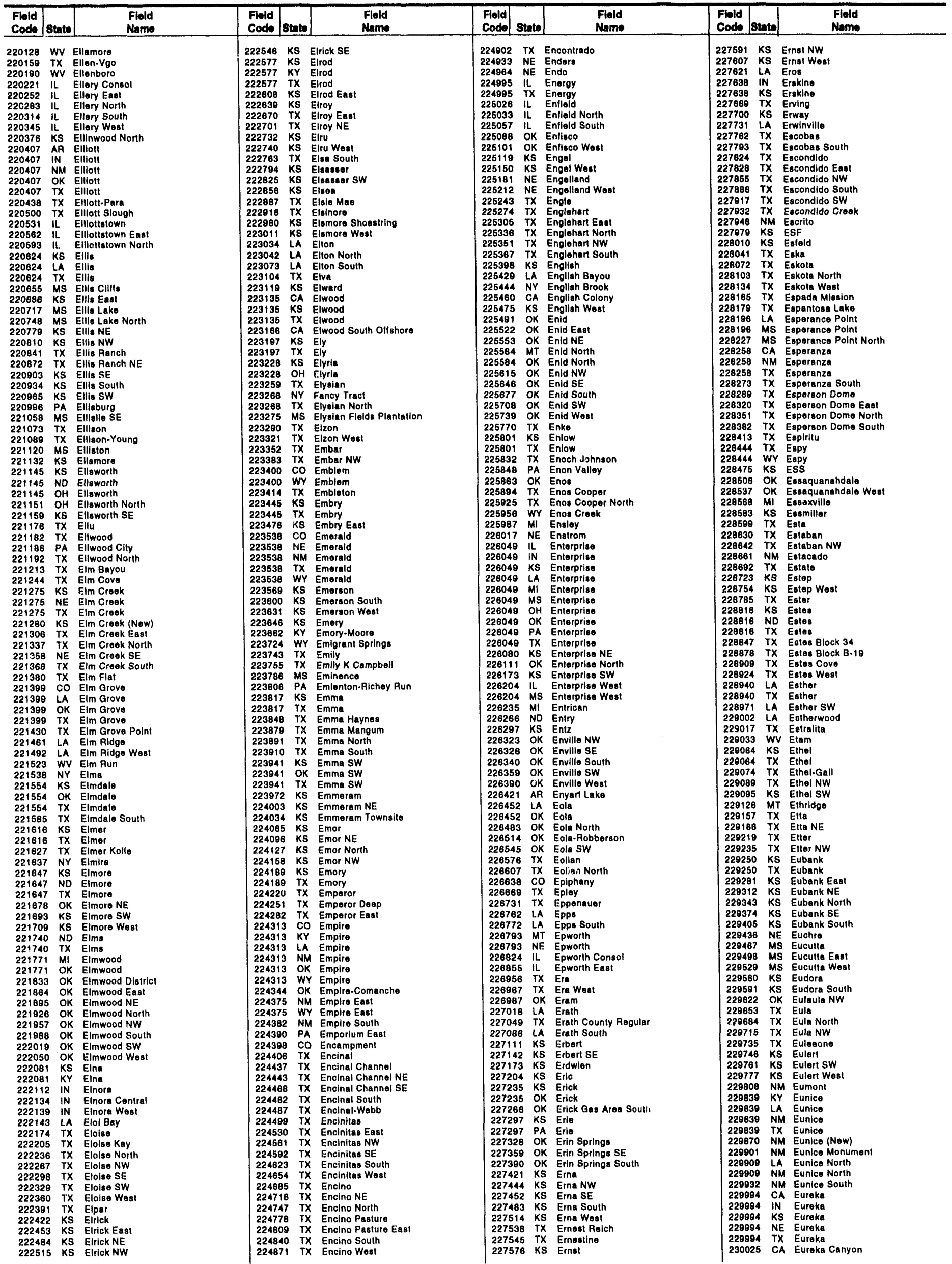




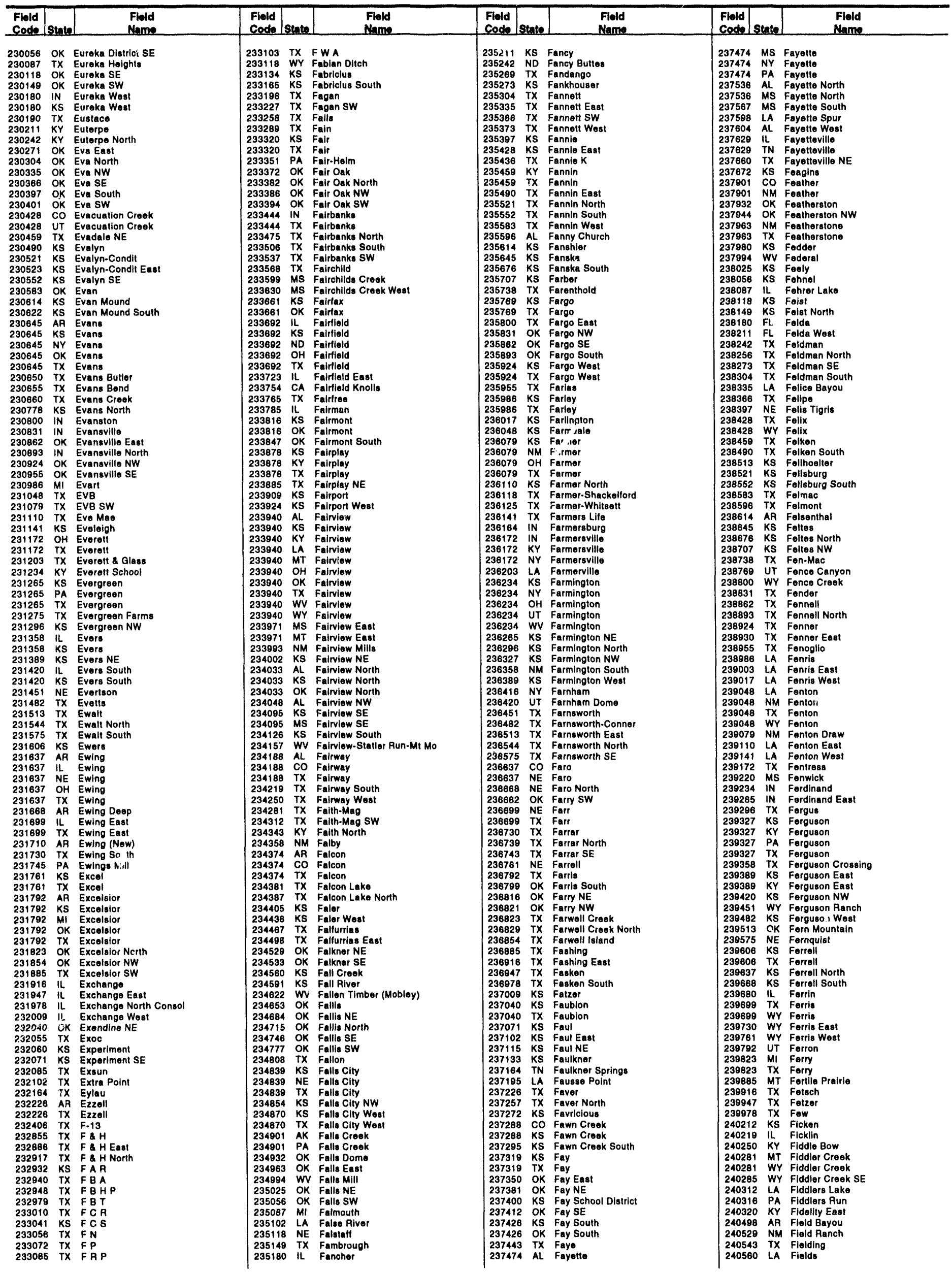




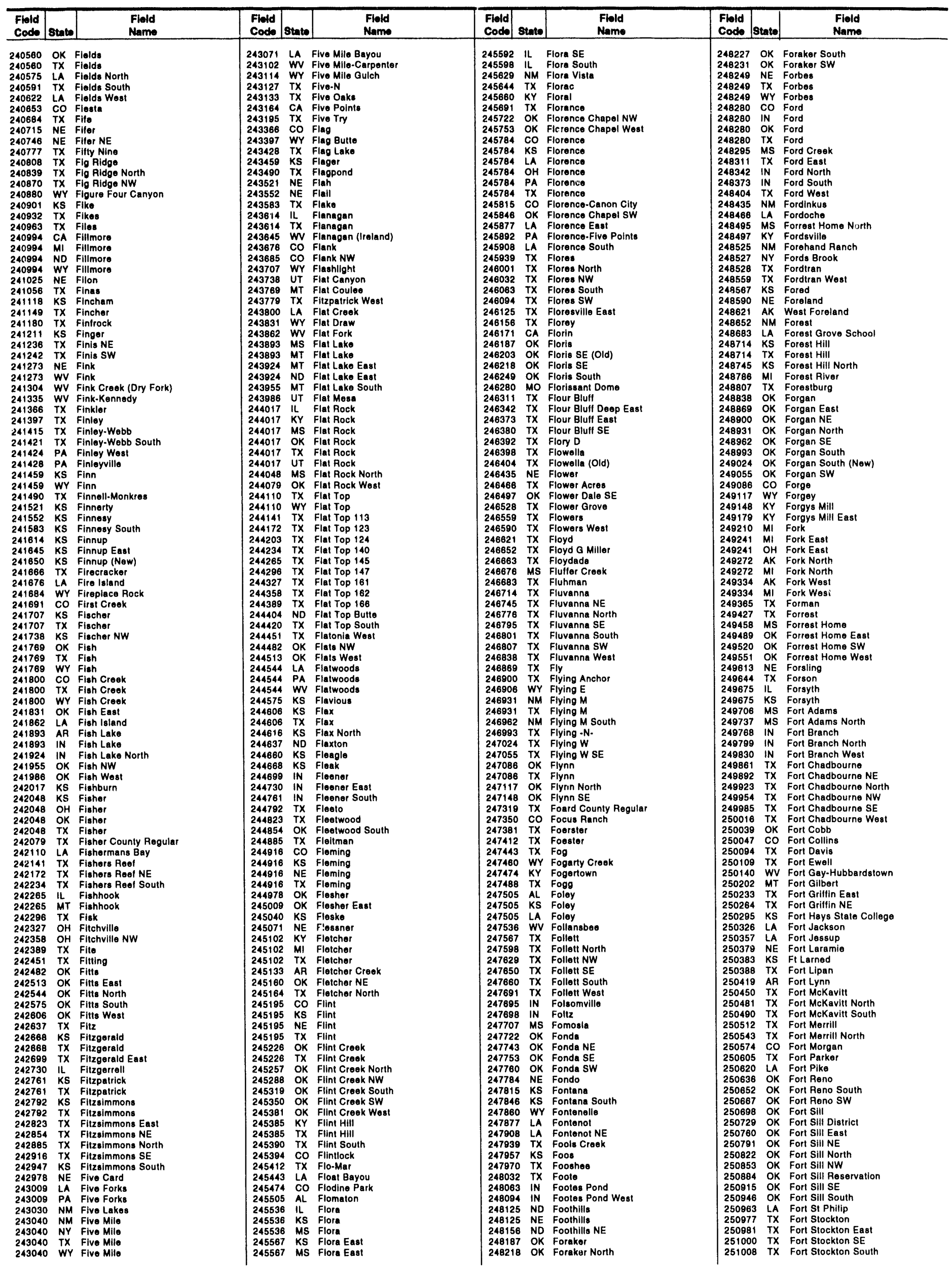




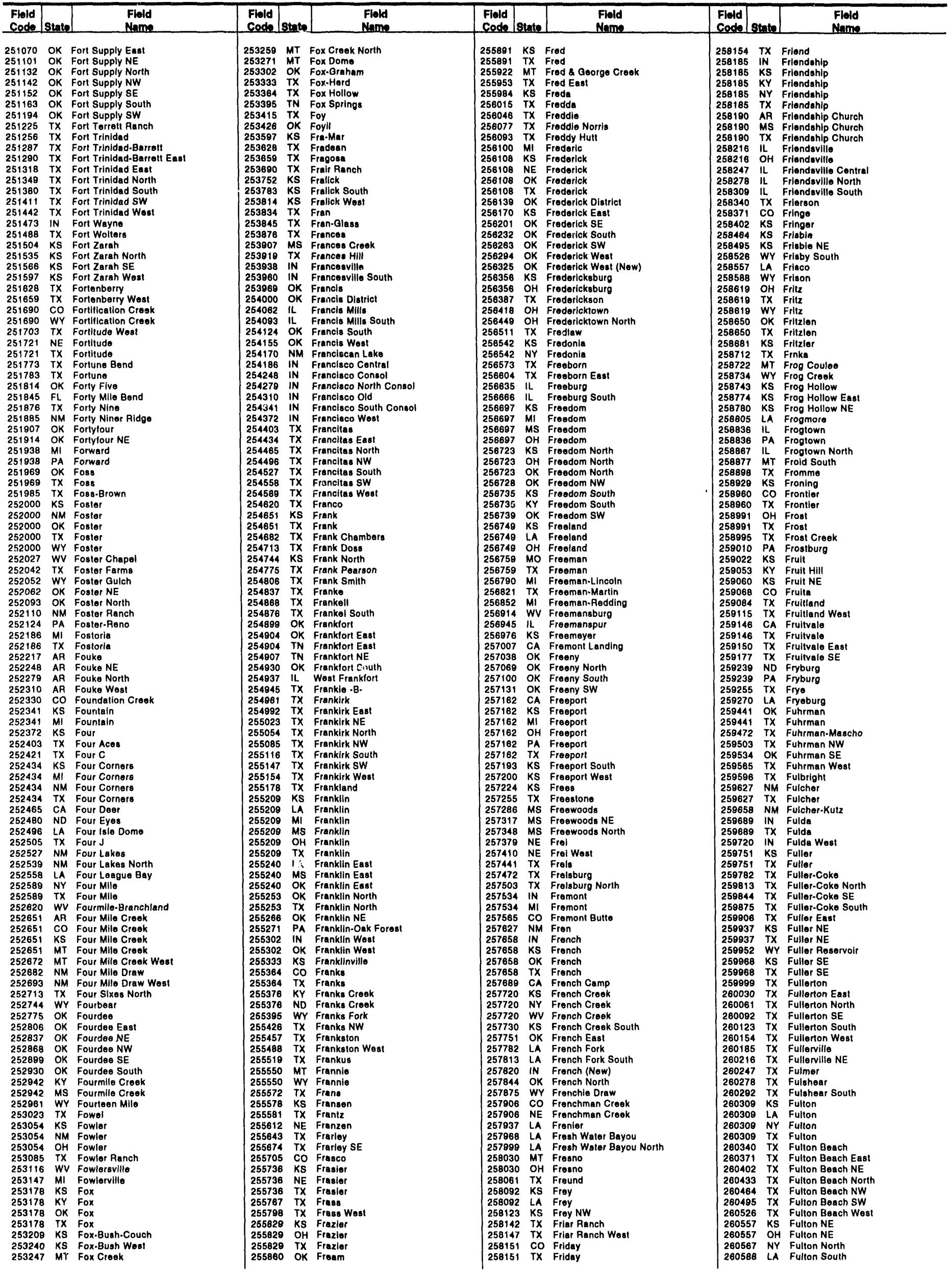


271673

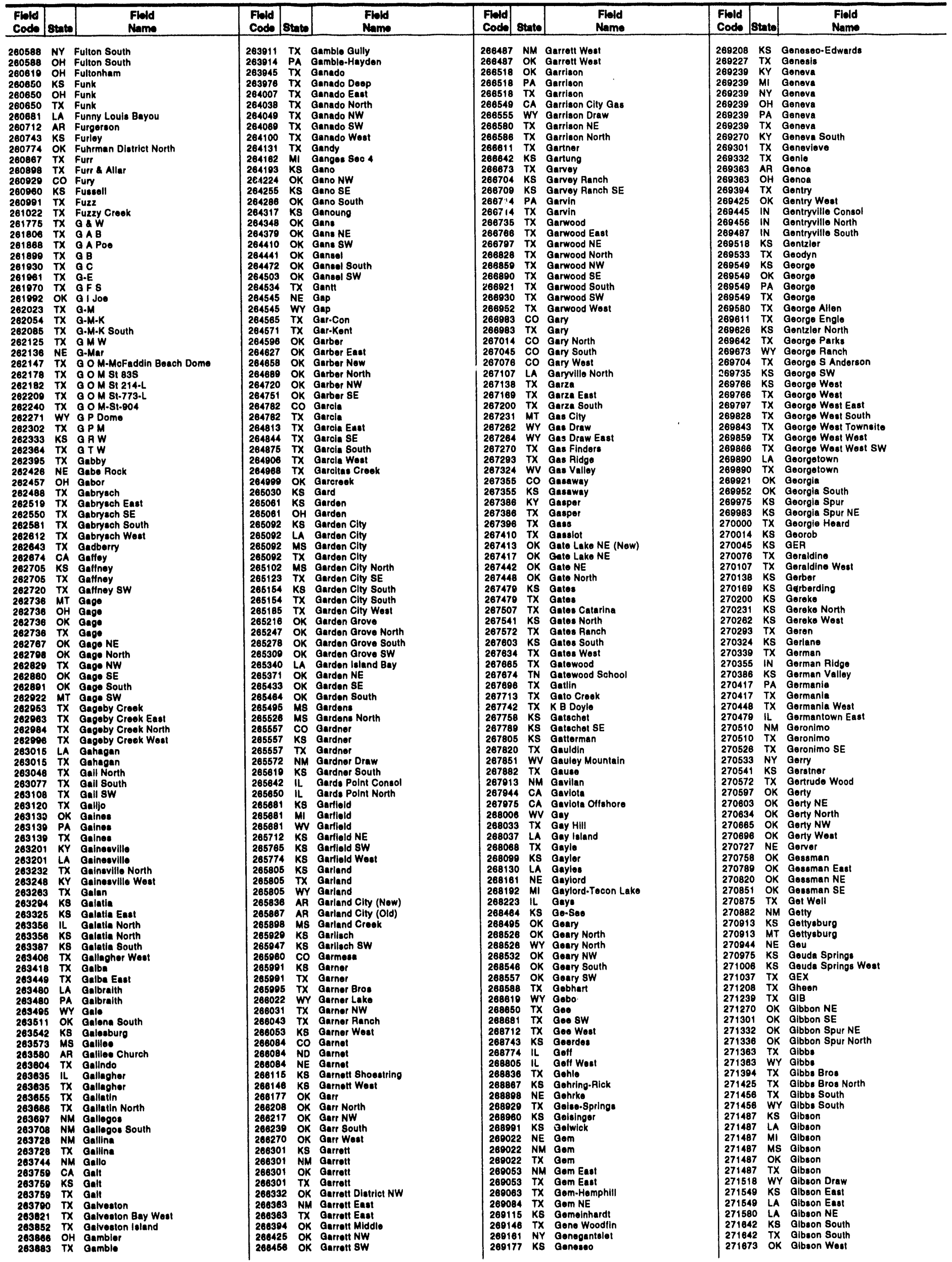




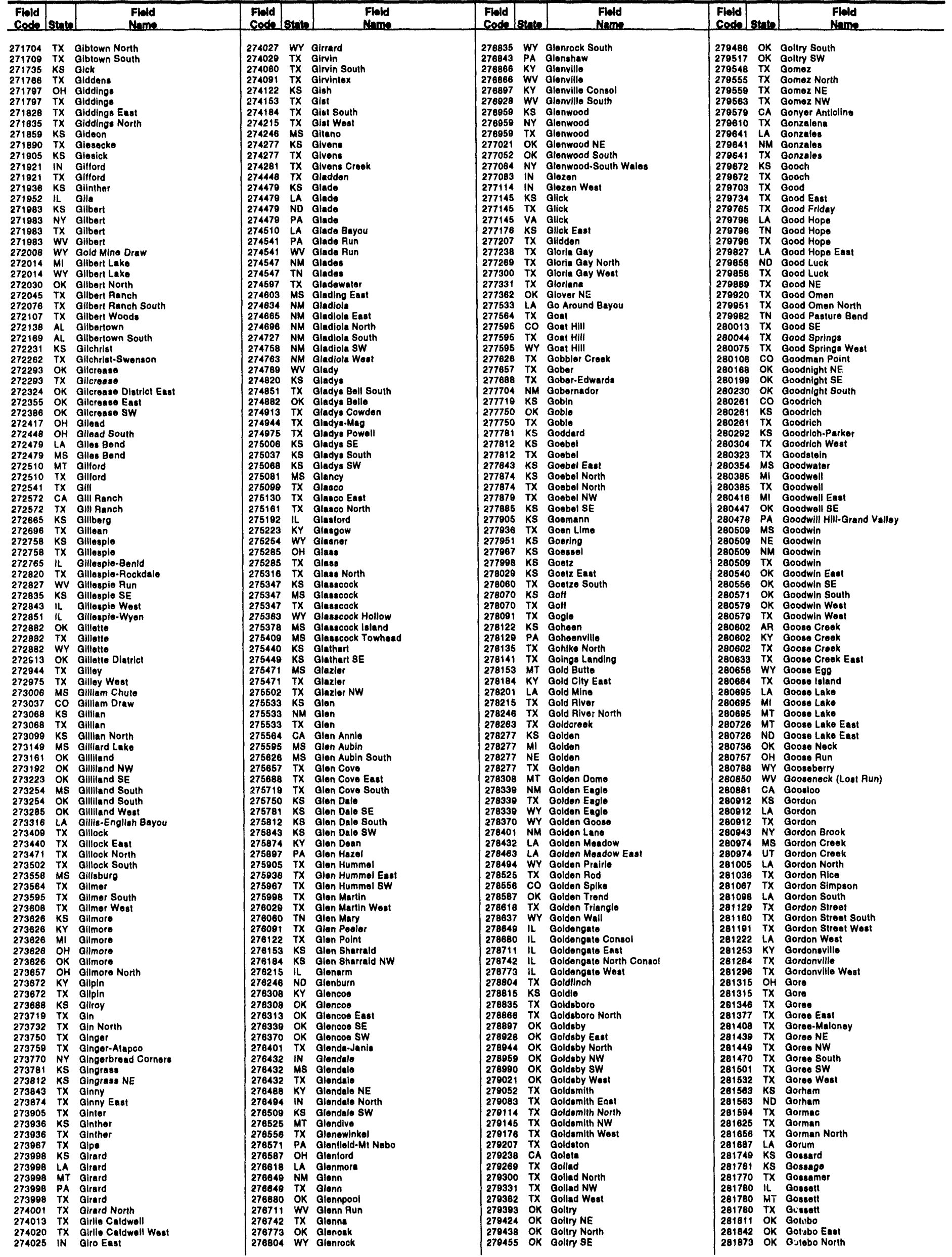


291592

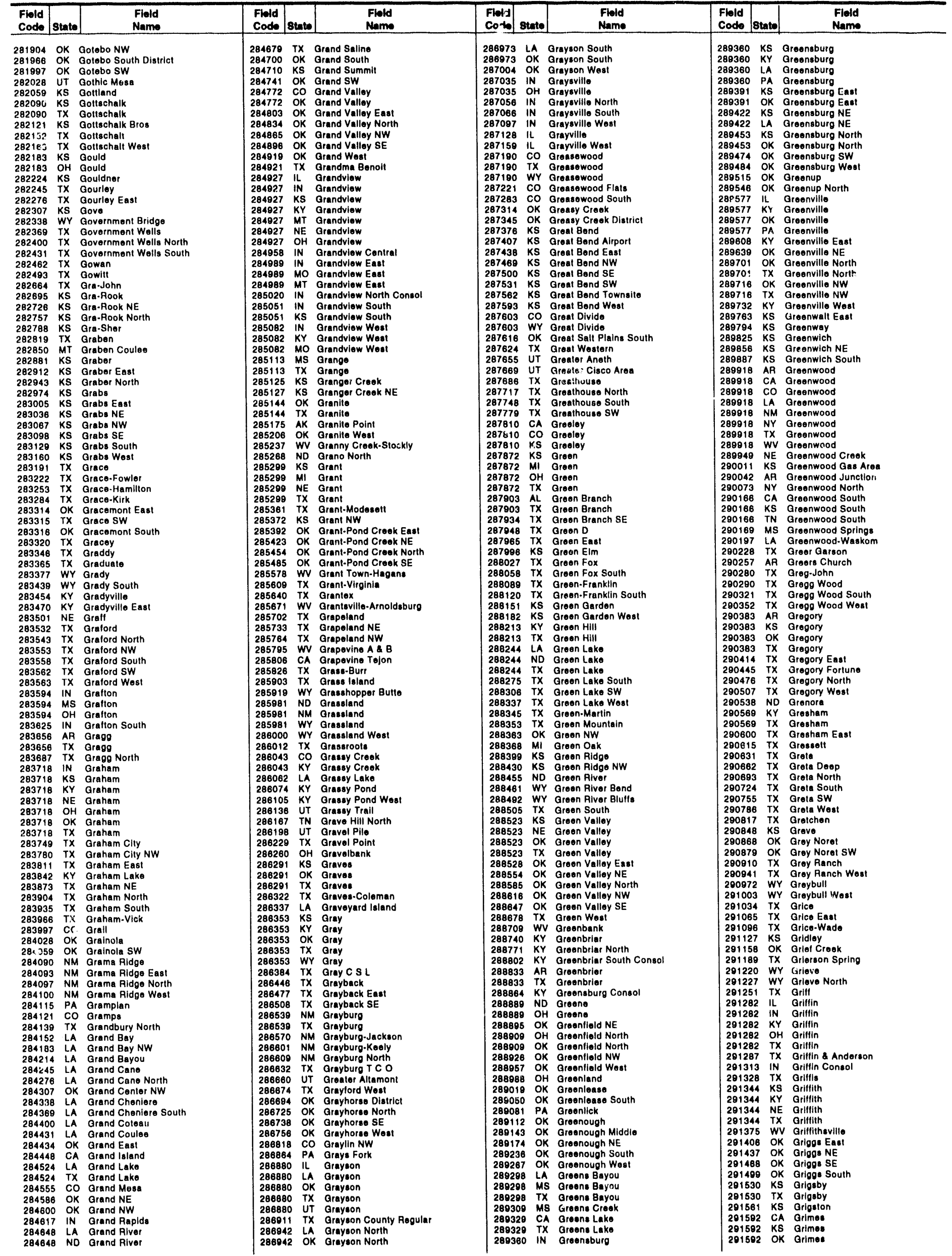




\begin{tabular}{|c|c|c|c|c|c|c|c|c|c|c|c|}
\hline $\begin{array}{l}\text { Fiold } \\
\text { Coded }\end{array}$ & Sinte & $\begin{array}{l}\text { Field } \\
\text { Name }\end{array}$ & $\begin{array}{l}\text { Fiold } \\
\text { Code }\end{array}$ & sinte & $\begin{array}{l}\text { Fiald } \\
\text { Name }\end{array}$ & $\begin{array}{l}\text { Fleld } \\
\text { Codh } 1 \text { s }\end{array}$ & Stath & $\begin{array}{l}\text { Fiold } \\
\text { Neme }\end{array}$ & $\begin{array}{l}\text { Fild } \\
\text { coothls }\end{array}$ & Sthets & $\begin{array}{l}\text { Fileld } \\
\text { Name }\end{array}$ \\
\hline 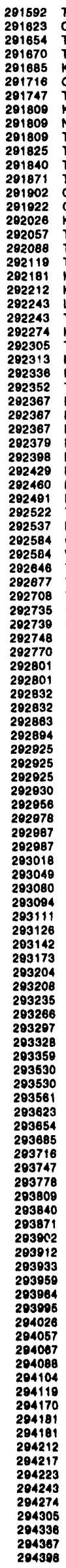 & 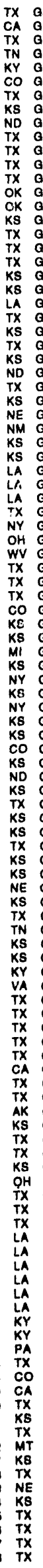 & 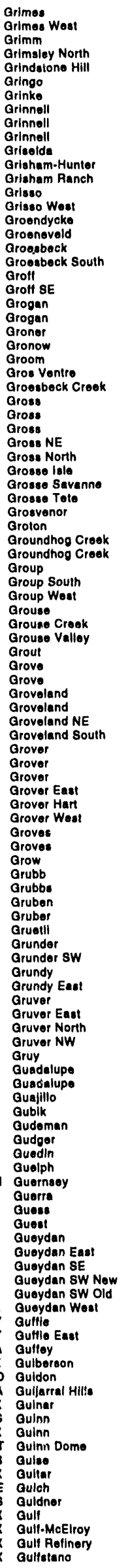 & 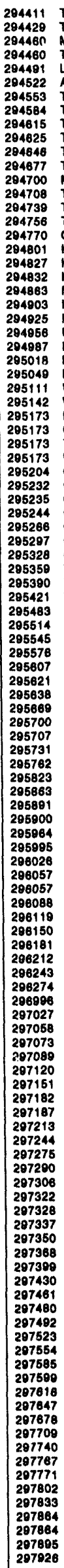 & 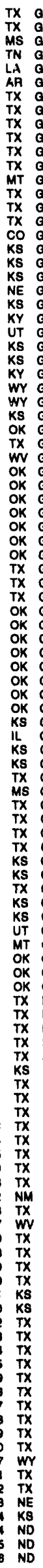 & 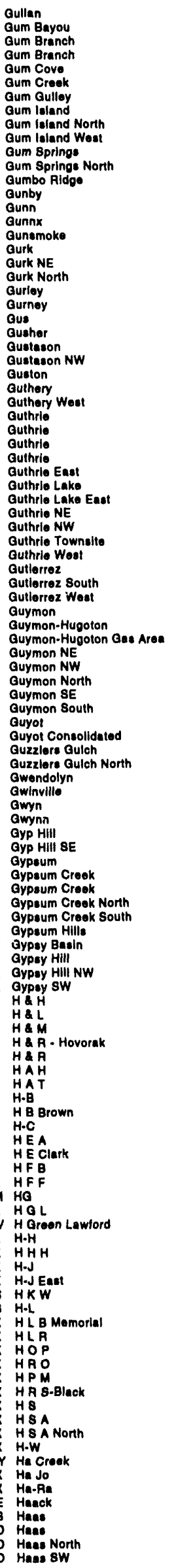 & 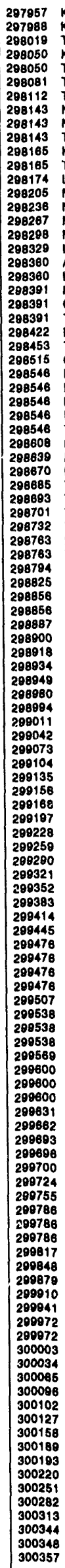 & 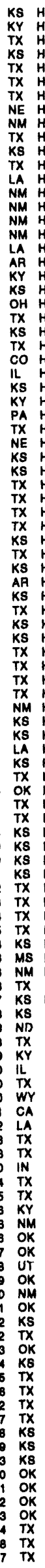 & 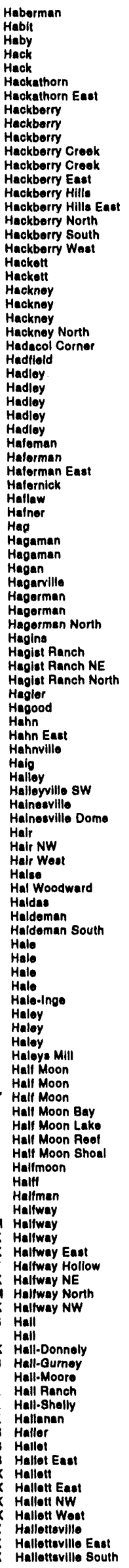 & 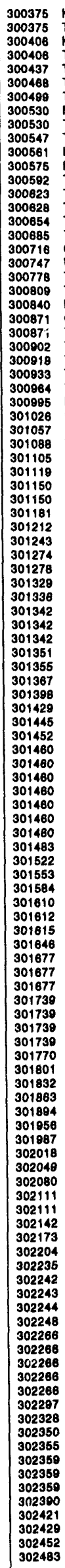 & 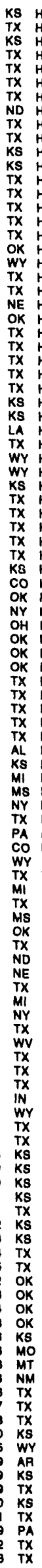 & 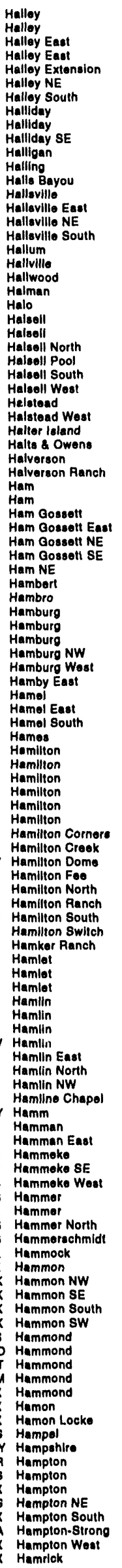 \\
\hline
\end{tabular}




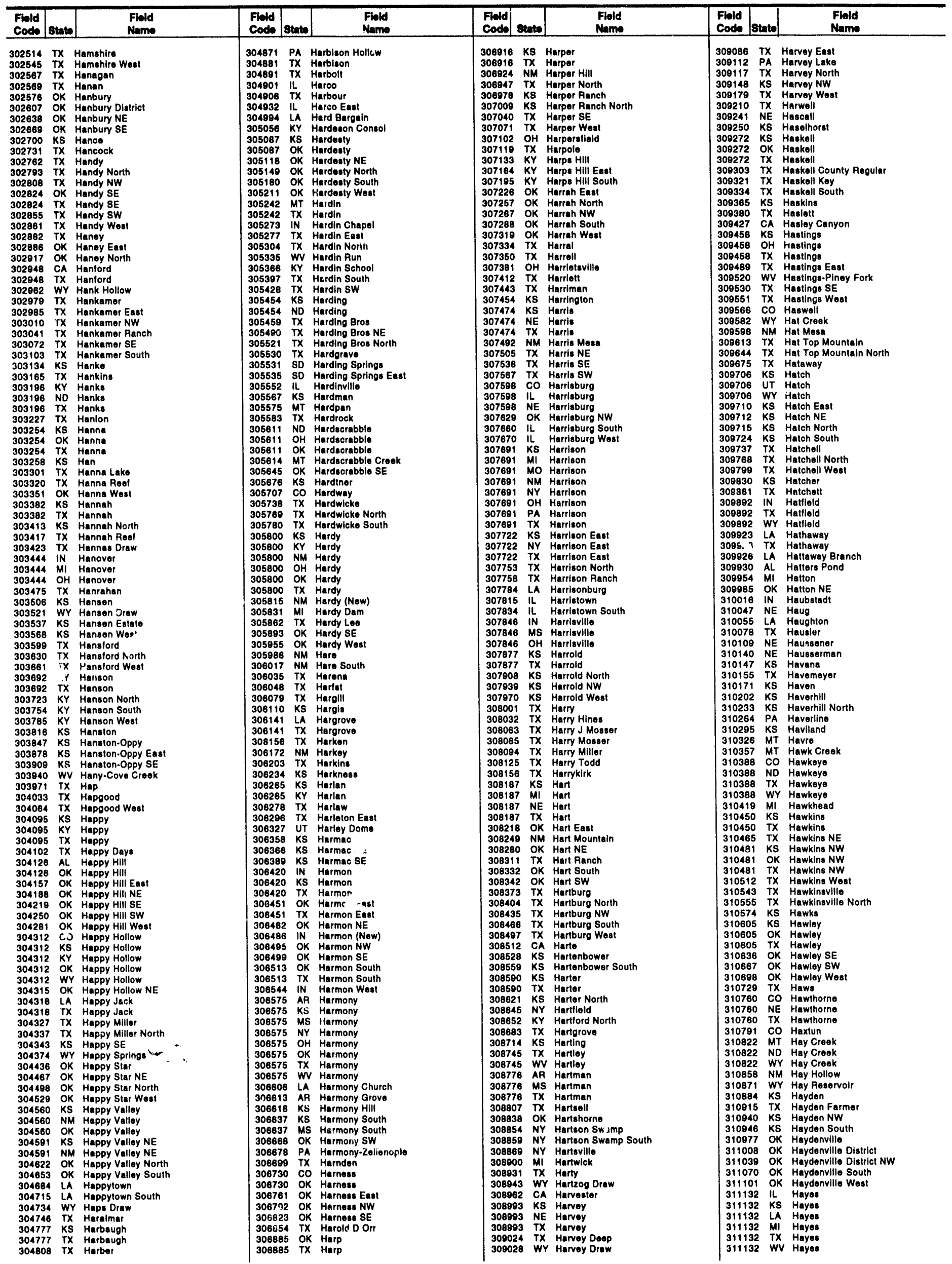




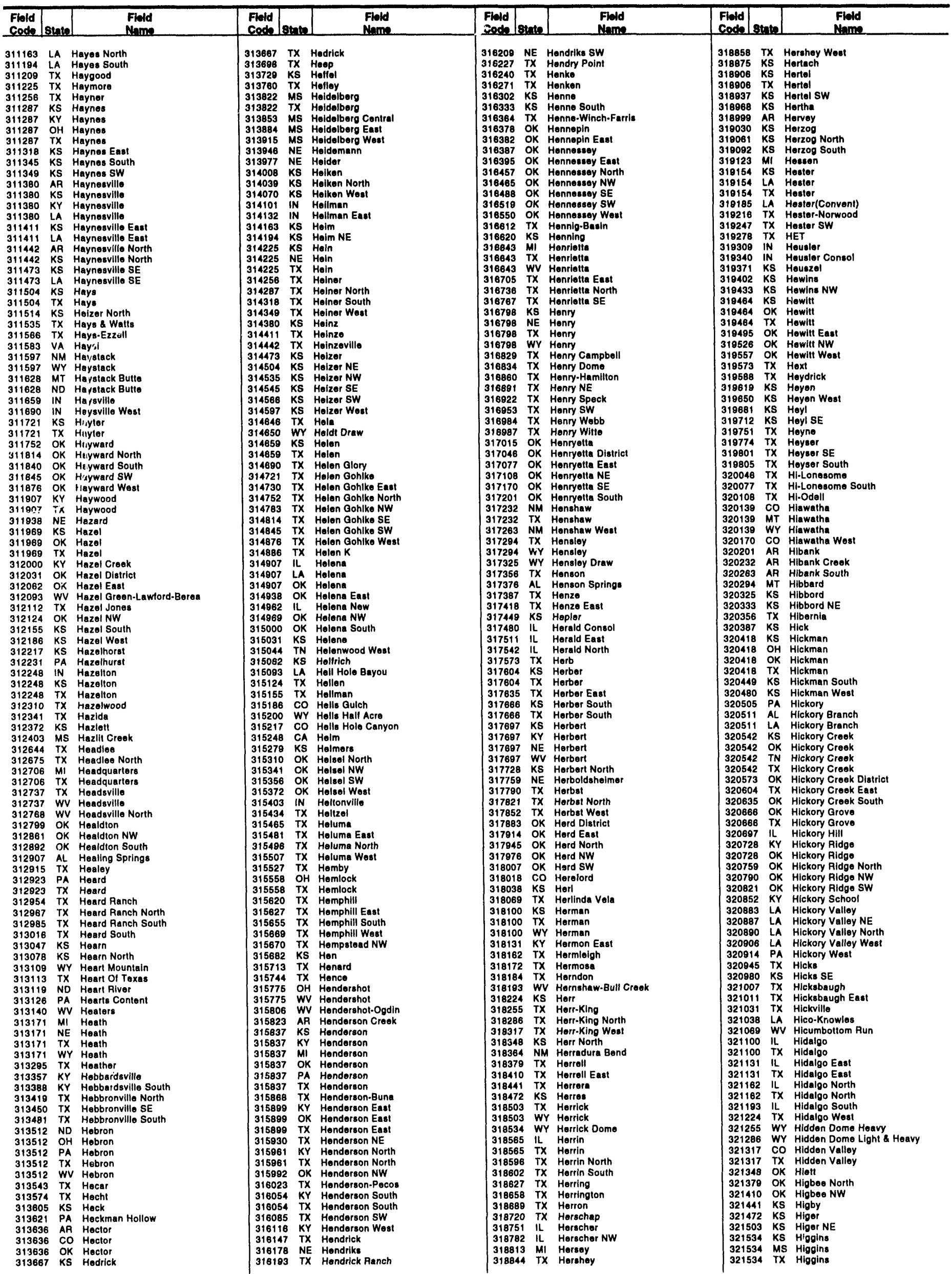




\begin{tabular}{|c|c|c|c|c|c|c|c|c|c|c|c|}
\hline \begin{tabular}{l|l} 
Fiold \\
Code
\end{tabular} & 8tate & $\begin{array}{l}\text { Fileld } \\
\text { Nanno }\end{array}$ & $\begin{array}{l}\text { Field } \\
\text { Code }\end{array}$ & 8tan & $\begin{array}{l}\text { Fiold } \\
\text { Name }\end{array}$ & \begin{tabular}{l|l} 
Fild & \\
Code & 8 \\
\end{tabular} & $8 \tan \theta$ & $\begin{array}{l}\text { Field } \\
\text { Neme }\end{array}$ & $\begin{array}{l}\text { Fiold } \\
\text { Code } 8 \\
\end{array}$ & State & $\begin{array}{l}\text { Fleld } \\
\text { Neme }\end{array}$ \\
\hline 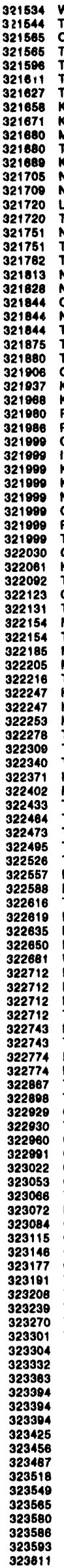 & 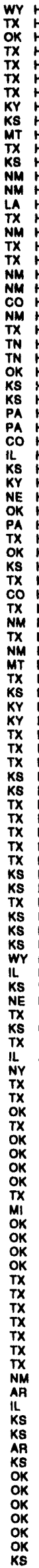 & 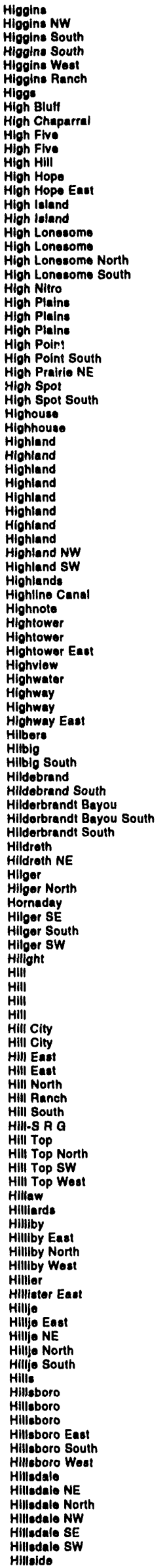 & 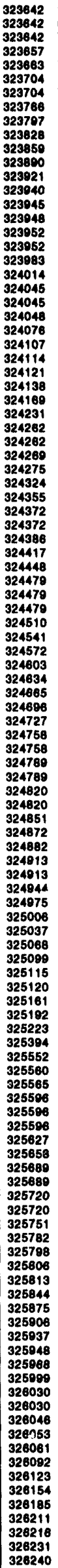 & 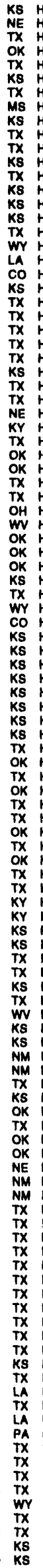 & 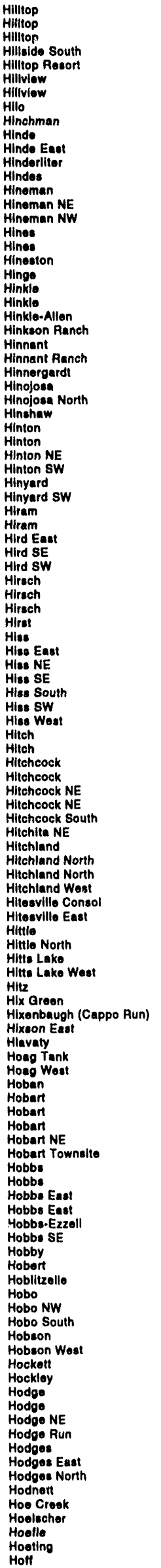 & 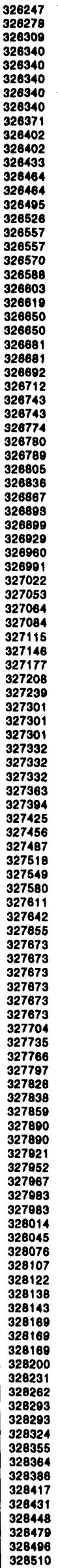 & 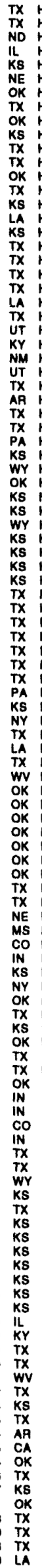 & 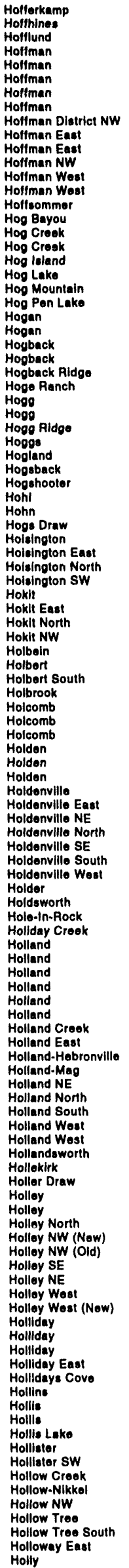 & 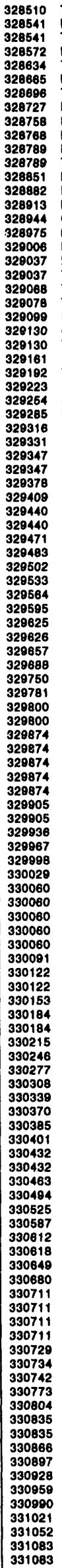 & 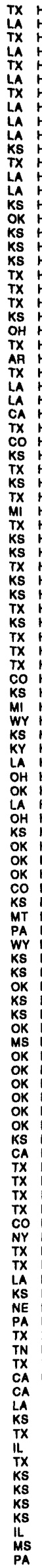 & 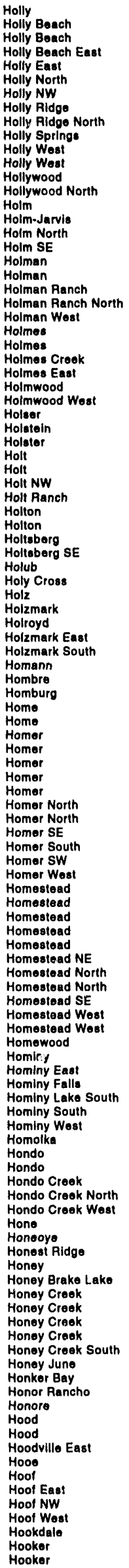 \\
\hline
\end{tabular}




\begin{tabular}{|c|c|c|c|c|c|c|c|c|c|c|c|}
\hline $\begin{array}{l}\text { Finld } \\
\text { Coed? }\end{array}$ & & $\begin{array}{l}\text { Fild } \\
\text { Neme }\end{array}$ & $\begin{array}{l}\text { Fland } \\
\text { Coods }\end{array}$ & & $\begin{array}{l}\text { Find } \\
\text { N.mm. }\end{array}$ & $\begin{array}{l}\text { Findd } \\
\text { Cond. }\end{array}$ & & $\begin{array}{l}\text { Find } \\
\text { Nem. }\end{array}$ & $\begin{array}{l}\text { Flaid } \\
\text { Codt }\end{array}$ & & $\begin{array}{l}\text { Fiveld } \\
\text { N-It: }\end{array}$ \\
\hline 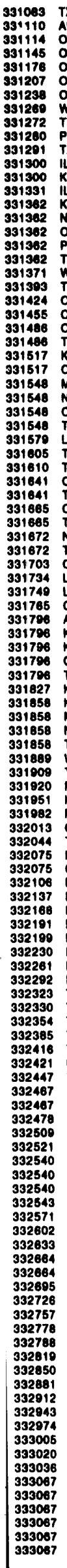 & 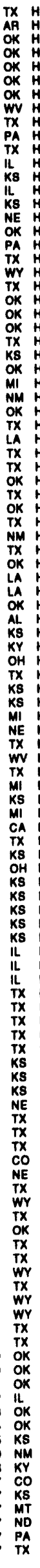 & 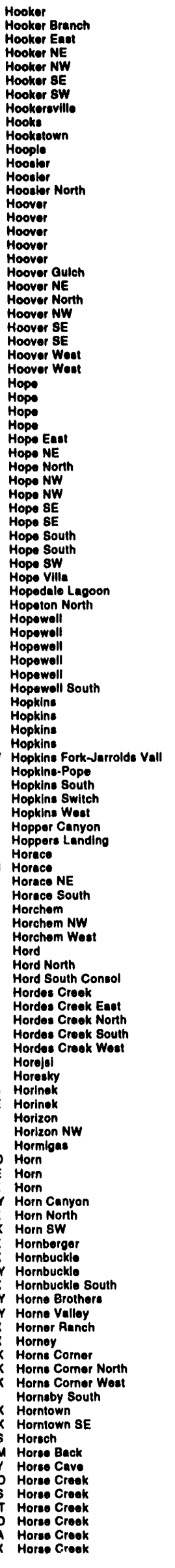 & 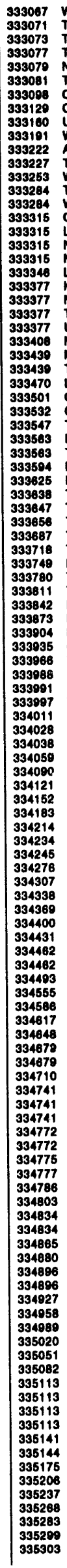 & 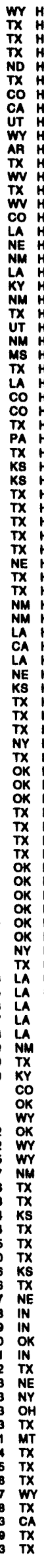 & 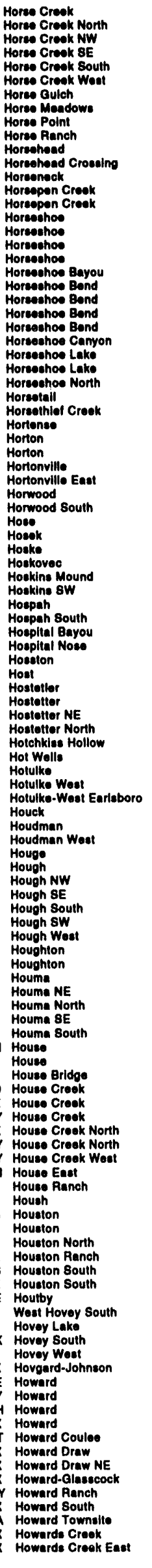 & 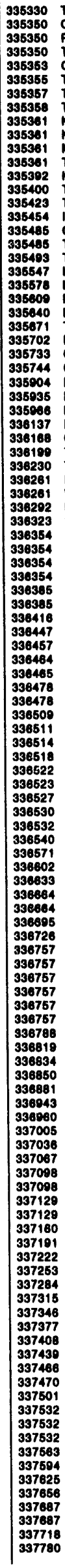 & 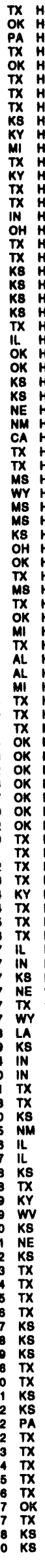 & 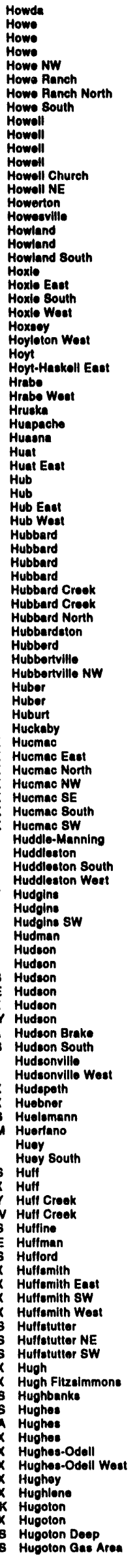 & 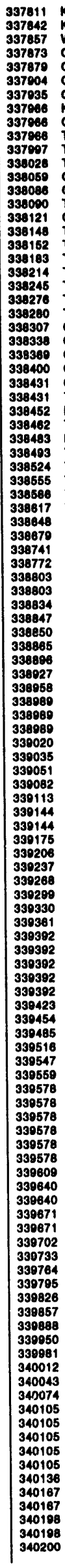 & 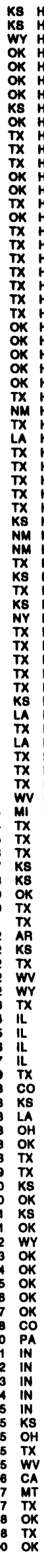 & 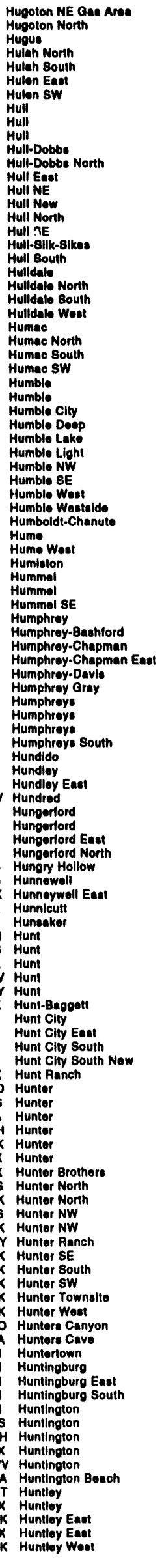 \\
\hline
\end{tabular}




\begin{tabular}{|c|c|c|c|c|c|c|c|c|c|c|c|}
\hline $\begin{array}{l}\text { Finld } \\
\text { Code }\end{array}$ & 8tas & $\begin{array}{l}\text { Fisld } \\
\text { Namo }\end{array}$ & $\begin{array}{l}\text { Fildd } \\
\text { Code }\end{array}$ & 840 & $\begin{array}{l}\text { Fildd } \\
\text { Name }\end{array}$ & $\begin{array}{l}\text { Finld } \\
\text { Codo }\end{array}$ & | مشتيك & $\begin{array}{l}\text { Field } \\
\text { Neme }\end{array}$ & $\begin{array}{l}\text { Fleld } \\
\text { Code }\end{array}$ & 8tat & $\begin{array}{l}\text { Fuld } \\
\text { Nome } \\
\end{array}$ \\
\hline 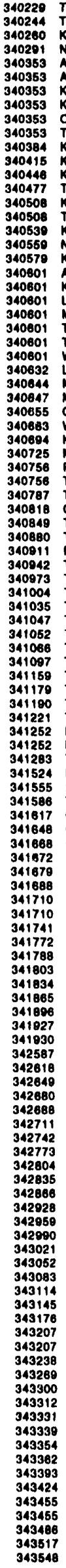 & 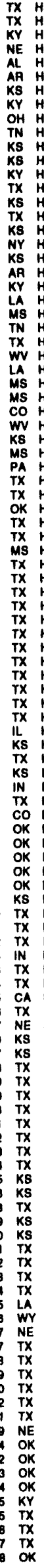 & 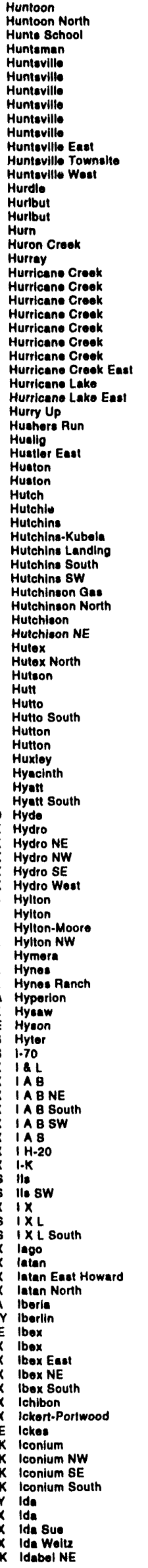 & 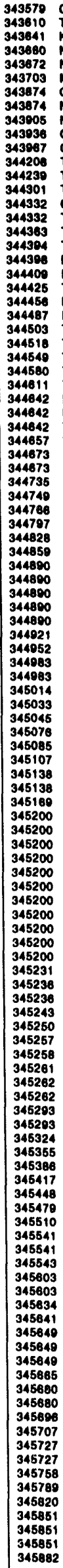 & 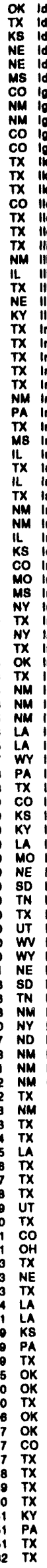 & 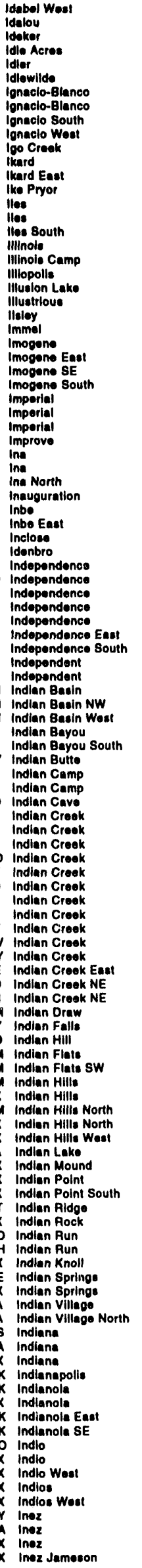 & 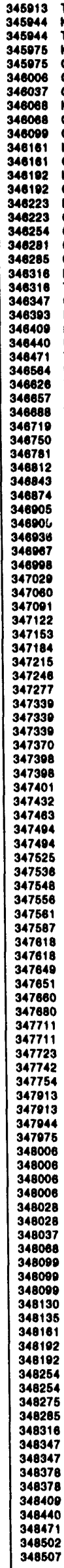 & 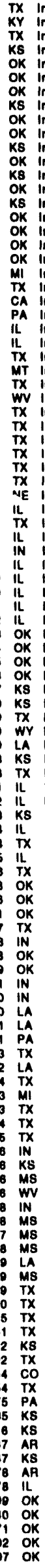 & 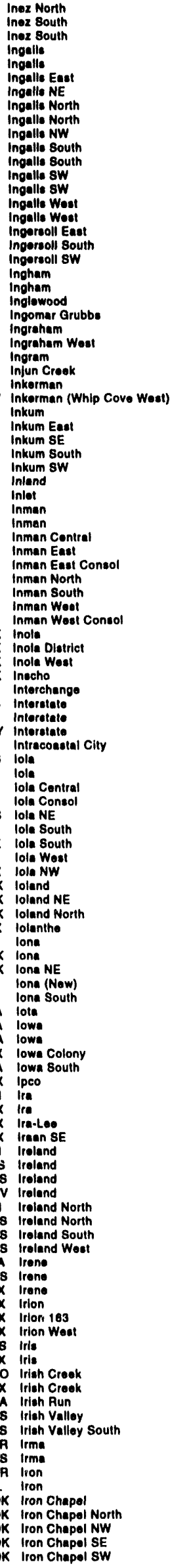 & 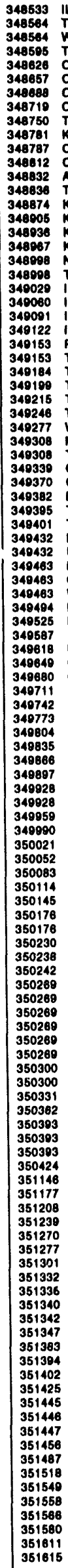 & 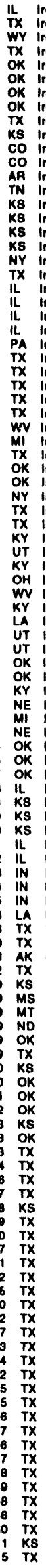 & 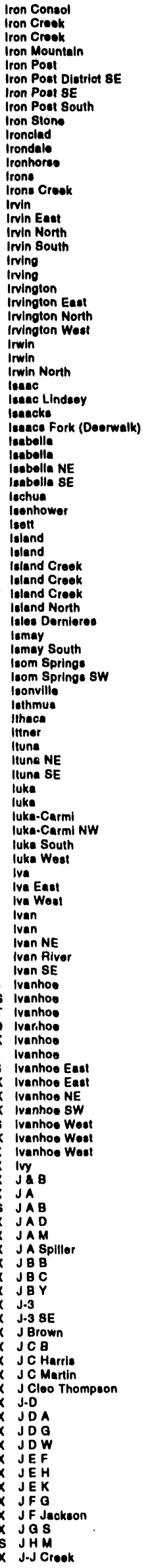 \\
\hline
\end{tabular}




\begin{tabular}{|c|c|c|c|c|c|c|c|c|c|c|c|}
\hline $\begin{array}{l}\text { Fileld } \\
\text { Codte }\end{array}$ & sintel & $\begin{array}{l}\text { Field } \\
\text { Neme }\end{array}$ & $\begin{array}{l}\text { Field } \\
\text { Coote }\end{array}$ & sthel & $\begin{array}{l}\text { Fild } \\
\text { N.me }\end{array}$ & $\begin{array}{l}\text { Field } \\
\text { Code }\end{array}$ & Sinte & $\begin{array}{l}\text { Field } \\
\text { Name }\end{array}$ & $\begin{array}{l}\text { Fleld } \\
\text { Code }\end{array}$ & sinte & $\begin{array}{l}\text { Fild } \\
\text { Name }\end{array}$ \\
\hline 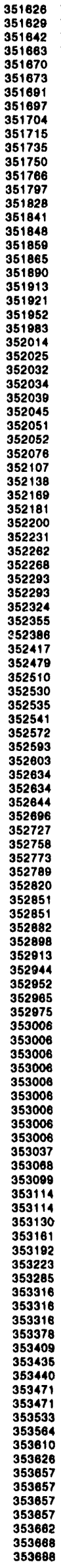 & 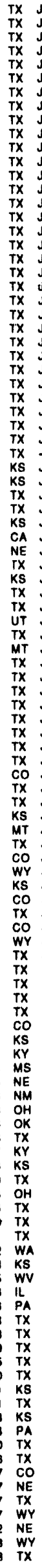 & 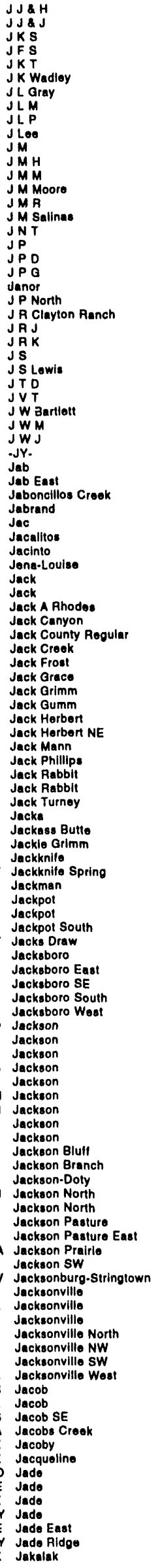 & 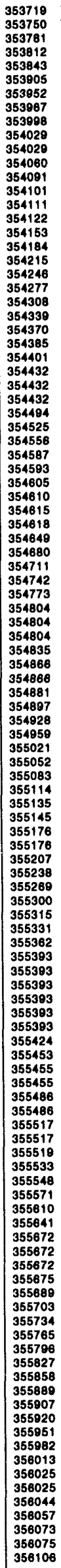 & 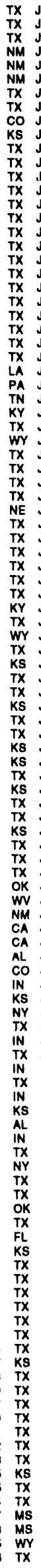 & 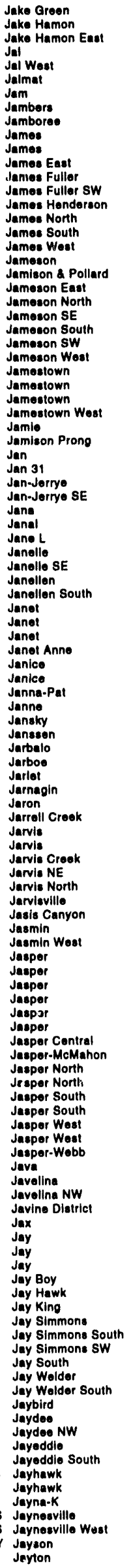 & 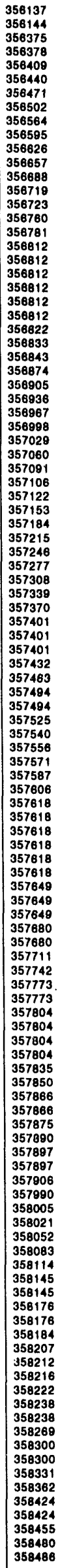 & 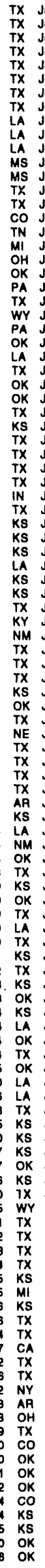 & 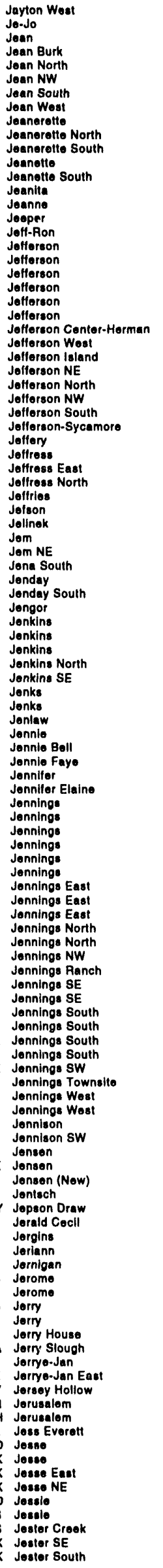 & 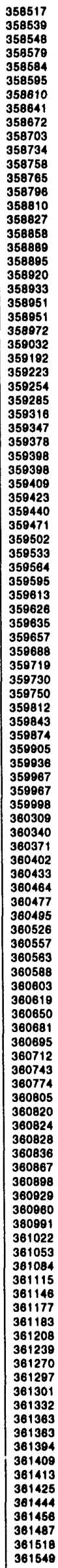 & 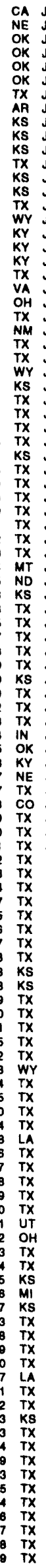 & 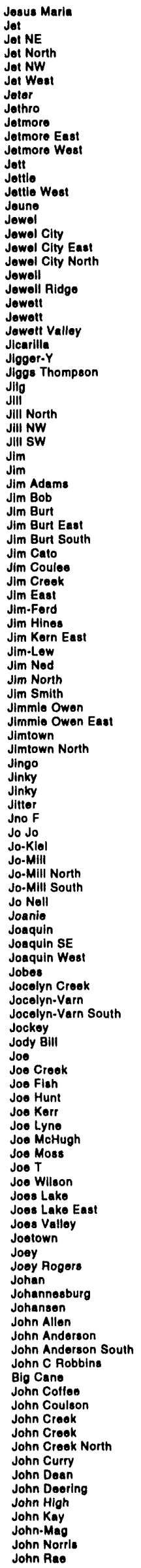 \\
\hline
\end{tabular}




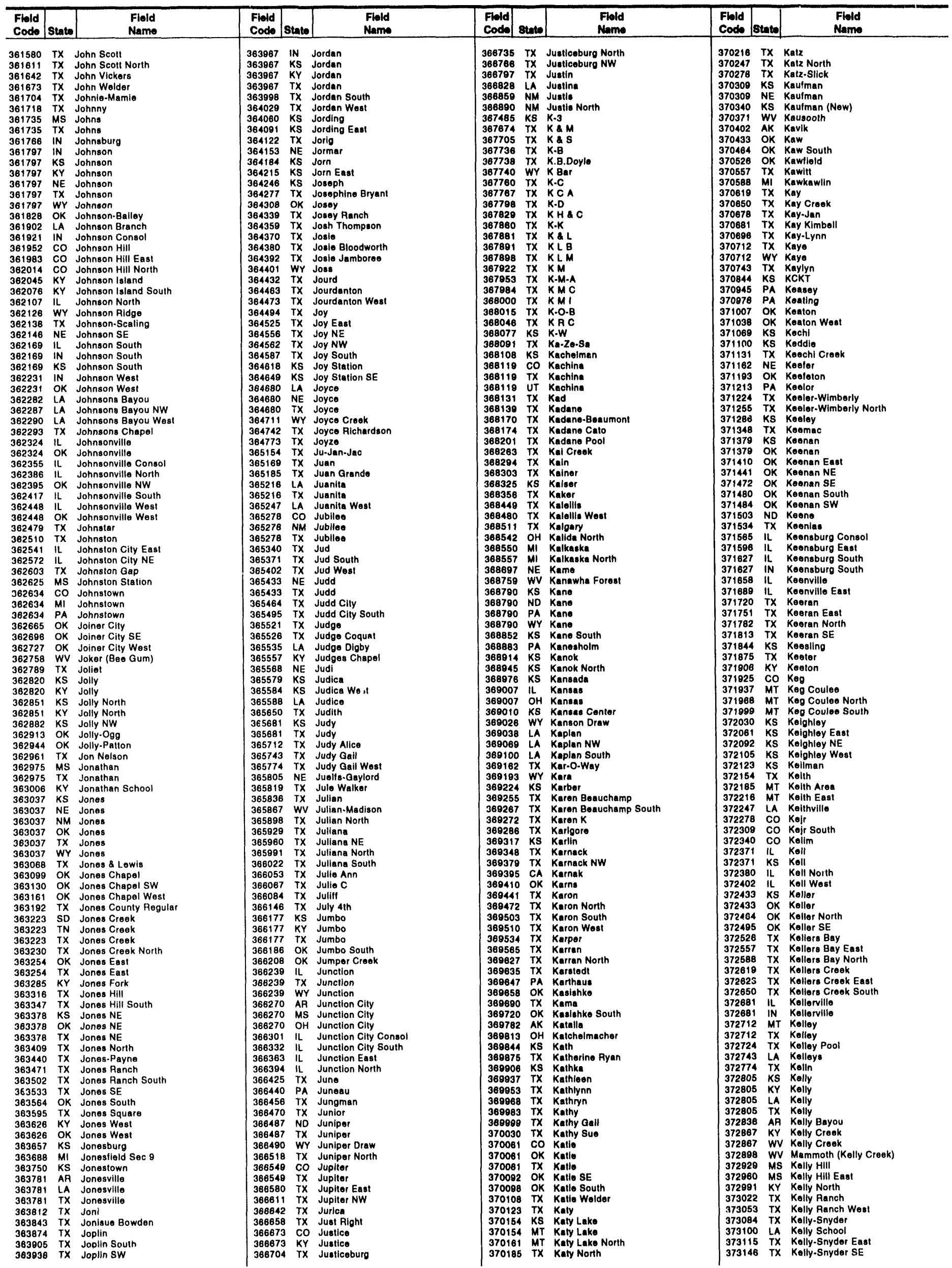




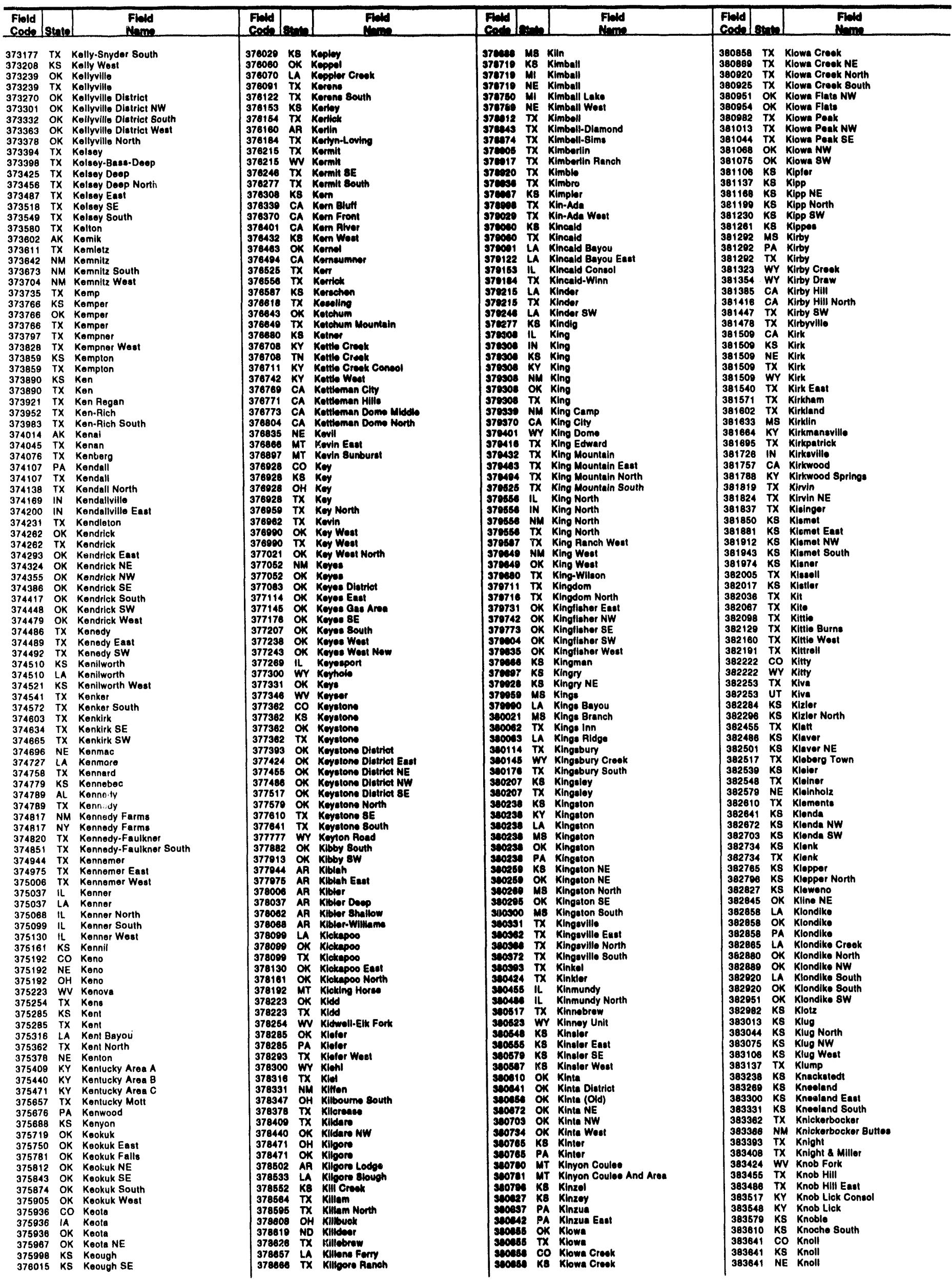


395703

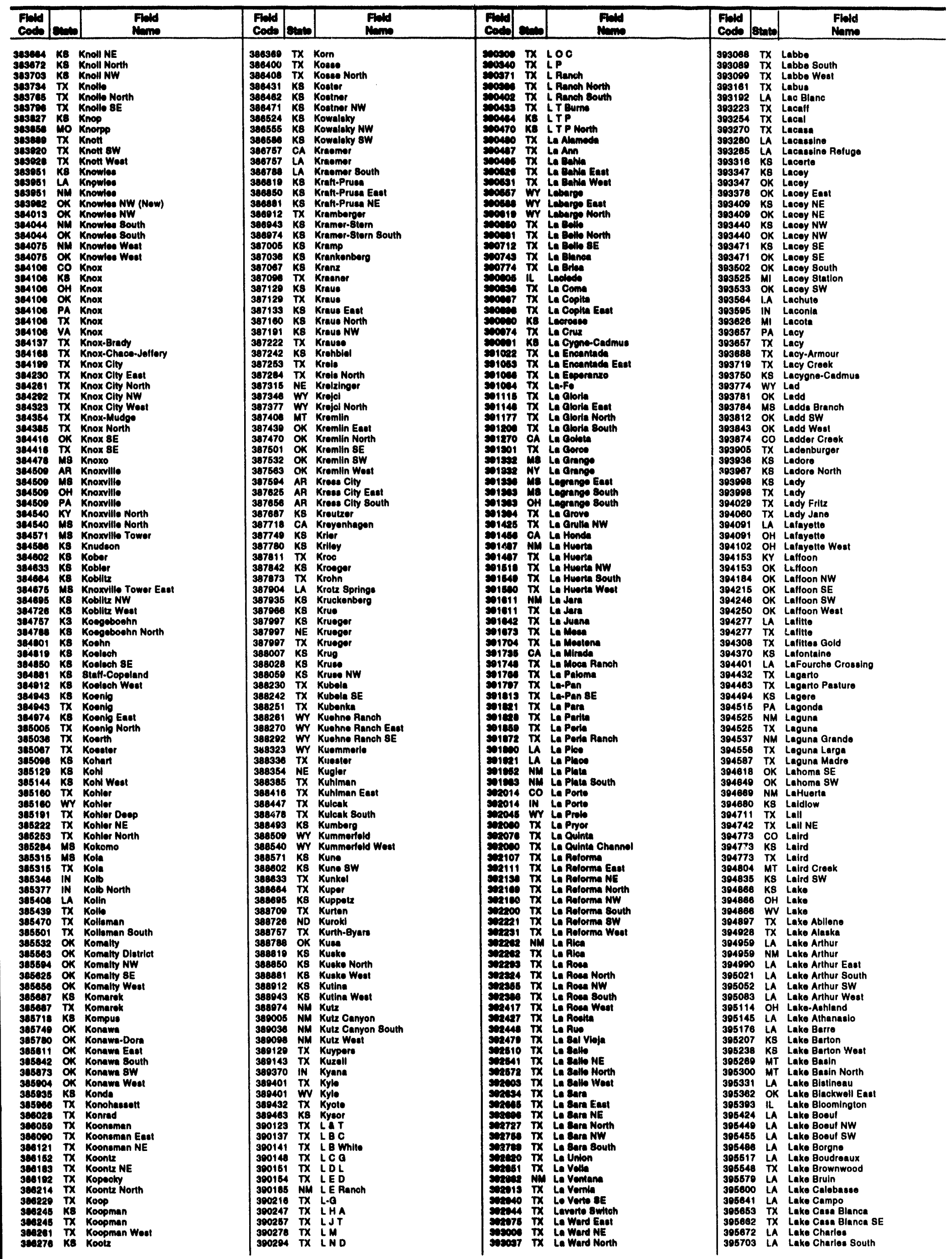




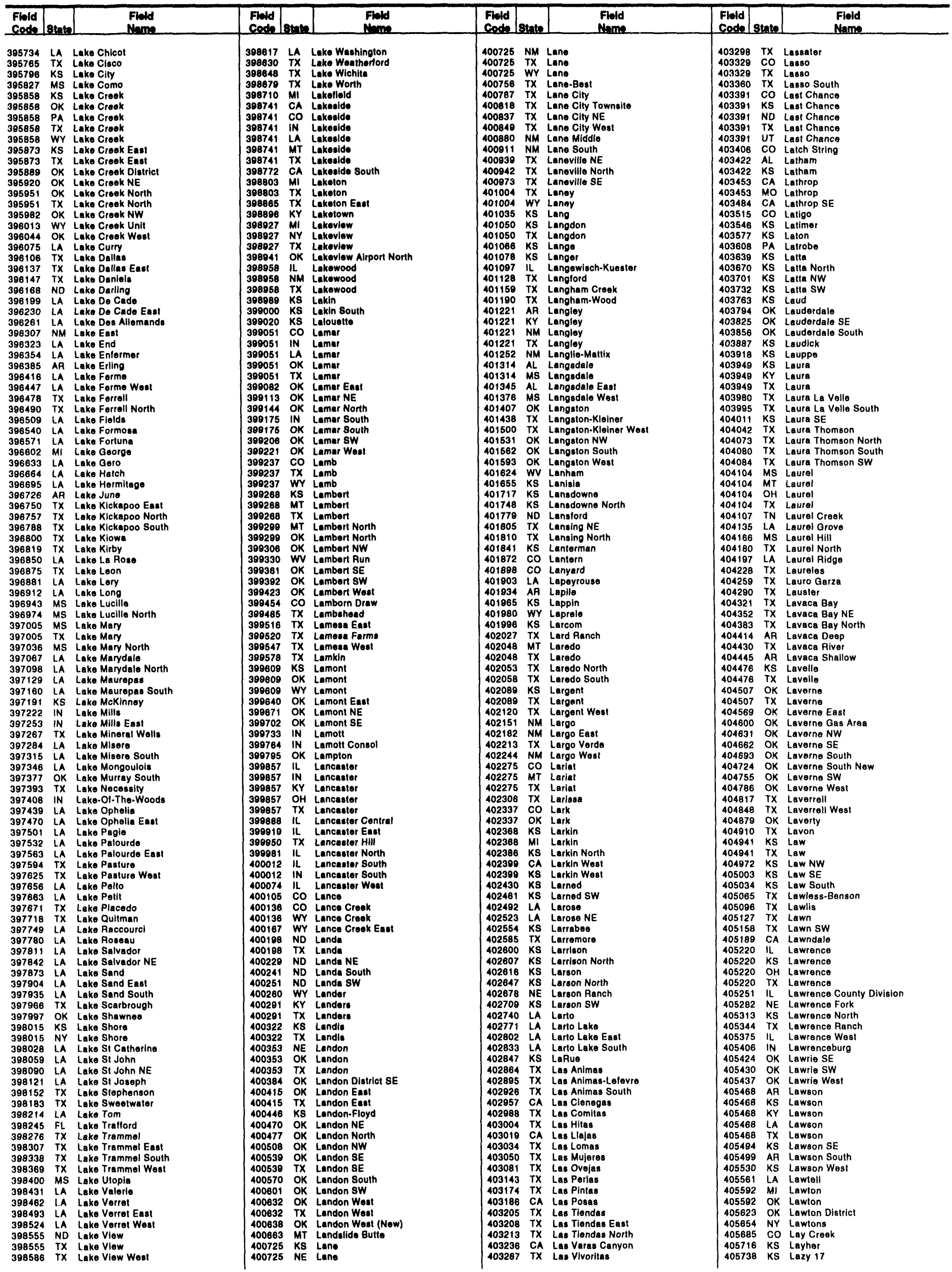




\begin{tabular}{|c|c|c|c|c|c|c|c|c|c|c|c|}
\hline $\begin{array}{l}\text { Flold } \\
\text { Codo }\end{array}$ & State & $\begin{array}{l}\text { Field } \\
\text { Name } \\
\end{array}$ & $\begin{array}{l}\text { Findd } \\
\text { Cods }\end{array}$ & Sento & $\begin{array}{l}\text { Flold } \\
\text { Name }\end{array}$ & $\begin{array}{l}\text { Fleld } \\
\text { Code }\end{array}$ & state & $\begin{array}{l}\text { Fidd } \\
\text { Name }\end{array}$ & $\begin{array}{l}\text { Fiold } \\
\text { Cods }\end{array}$ & Btale & $\begin{array}{l}\text { Field } \\
\text { Nemp }\end{array}$ \\
\hline
\end{tabular}




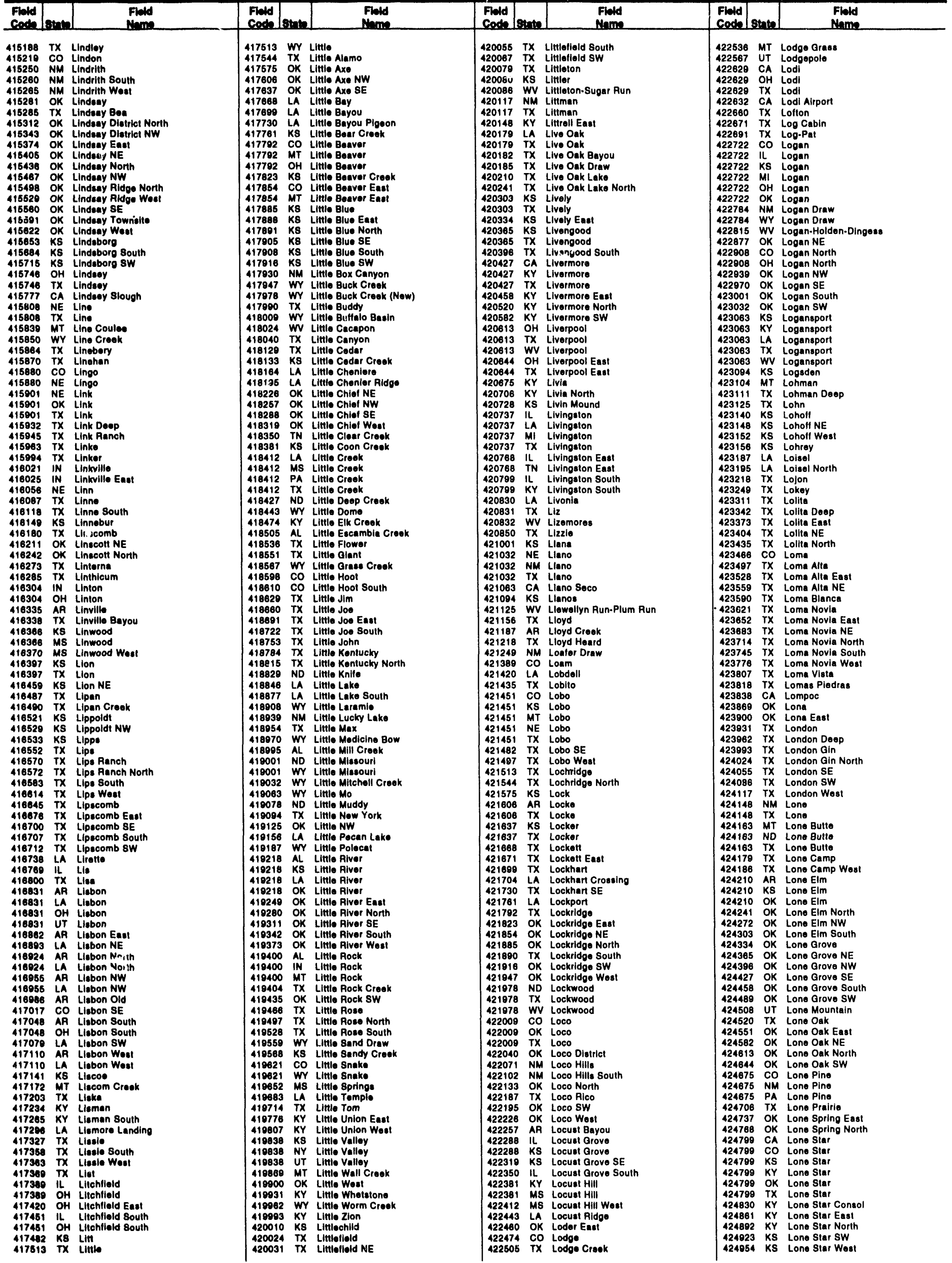




\begin{tabular}{|c|c|c|c|c|c|c|c|c|c|c|c|}
\hline $\begin{array}{l}\text { Fildd } \\
\text { Code }\end{array}$ & stan & $\begin{array}{l}\text { Field } \\
\text { Name }\end{array}$ & $\begin{array}{l}\text { Finld } \\
\text { Cods }\end{array}$ & 8tan & $\begin{array}{l}\text { Flold } \\
\text { Neme }\end{array}$ & \begin{tabular}{l|l|} 
Fluid \\
Code
\end{tabular} & $80+1$ & $\begin{array}{l}\text { Field } \\
\text { Neme } \\
\end{array}$ & \begin{tabular}{l|l} 
Fiskd \\
Code
\end{tabular} & stanl & $\begin{array}{l}\text { Field } \\
\text { Neme } \\
\end{array}$ \\
\hline 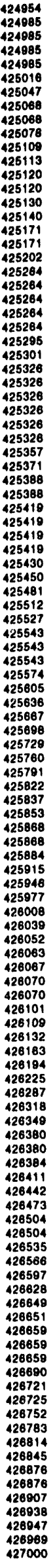 & 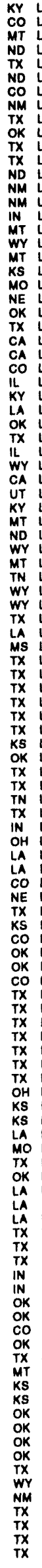 & 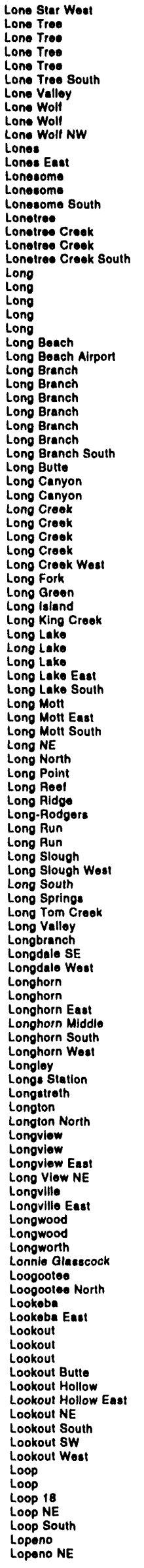 & 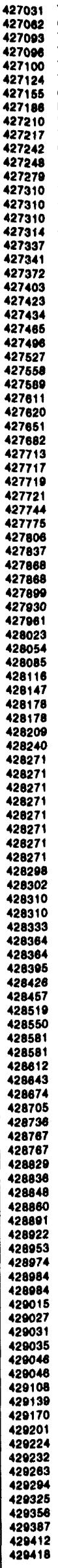 & 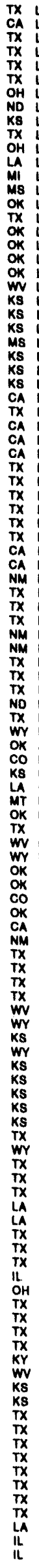 & 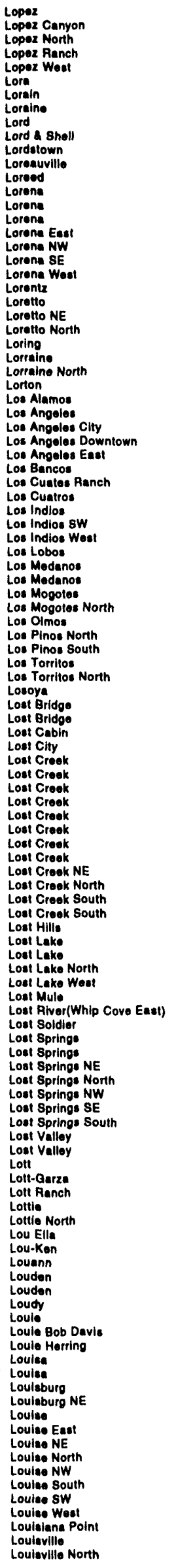 & 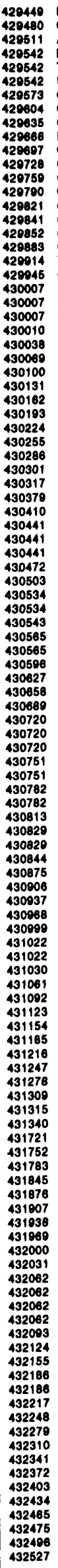 & 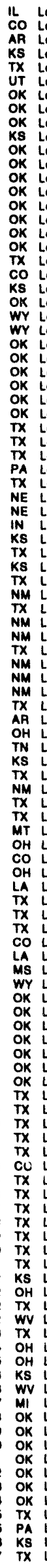 & 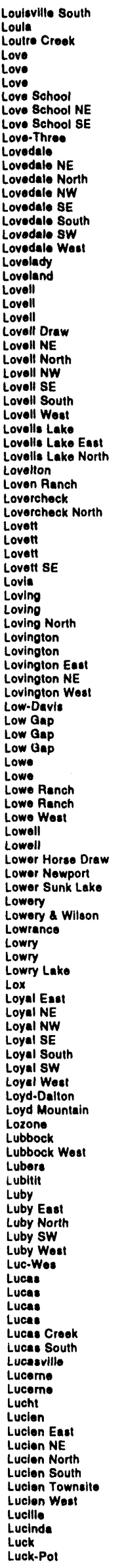 & 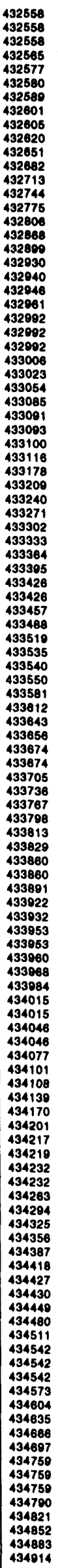 & 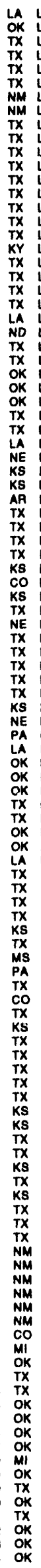 & 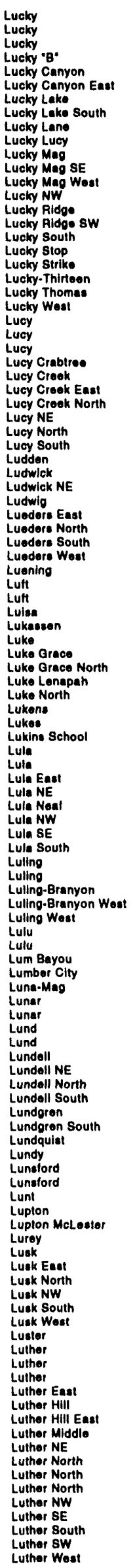 \\
\hline
\end{tabular}




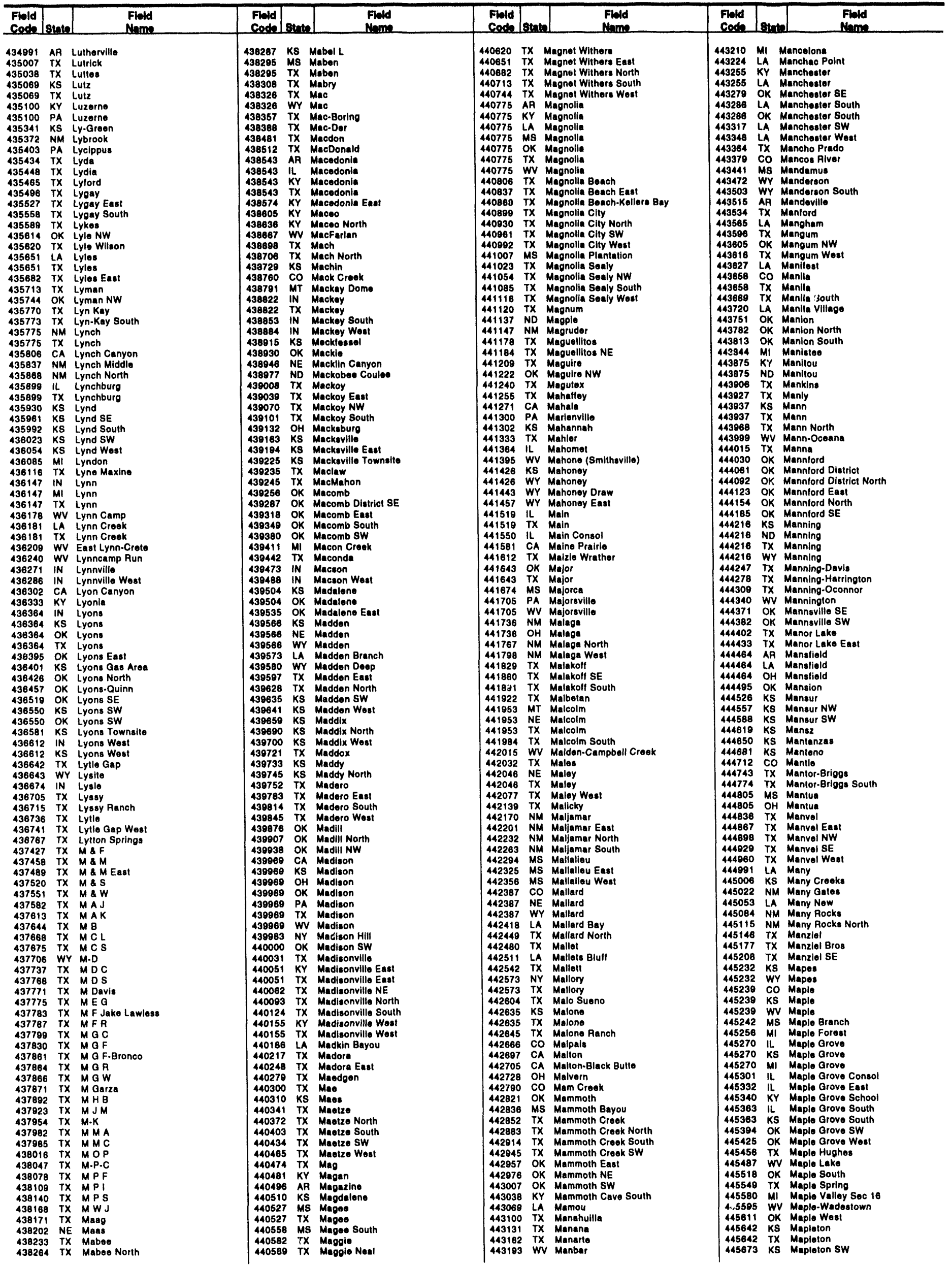




\begin{tabular}{|c|c|c|c|c|c|c|c|c|c|c|c|}
\hline $\begin{array}{l}\text { Fiold } \\
\text { Code }\end{array}$ & Stato & $\begin{array}{l}\text { Fild } \\
\text { Neme }\end{array}$ & $\begin{array}{l}\text { Finkd } \\
\text { Code }\end{array}$ & Stote & $\begin{array}{l}\text { Fild } \\
\text { Name }\end{array}$ & $\begin{array}{l}\text { Fiold } \\
\text { Code }\end{array}$ & 8tow & $\begin{array}{l}\text { Fild } \\
\text { Name }\end{array}$ & $\begin{array}{l}\text { Filald } \\
\text { Code }\end{array}$ & gina & $\begin{array}{l}\text { Field } \\
\text { Name }\end{array}$ \\
\hline 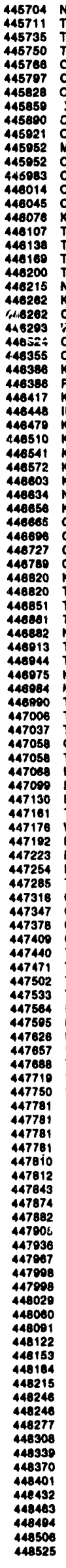 & 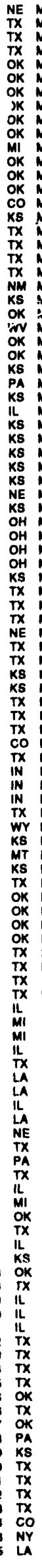 & 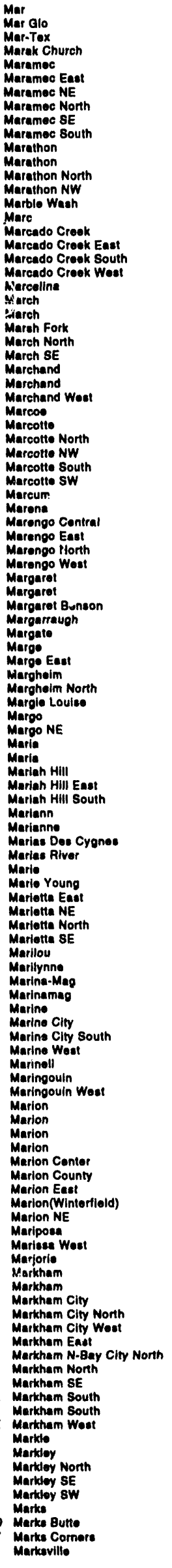 & 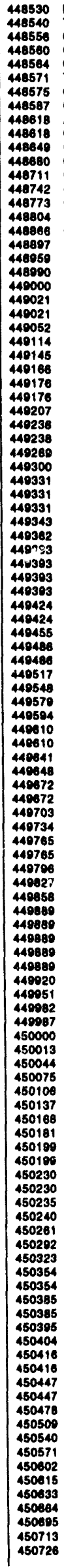 & 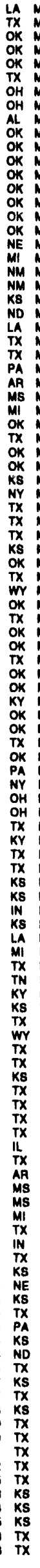 & 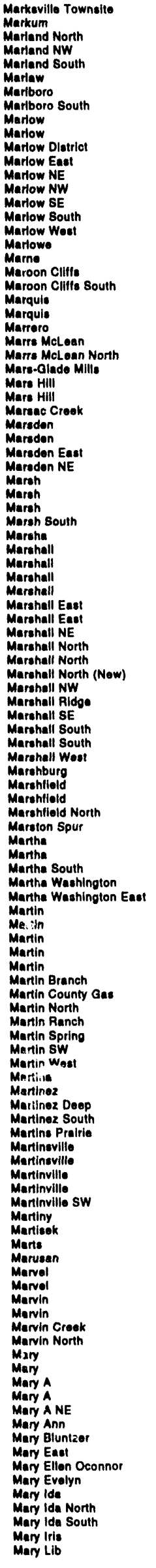 & 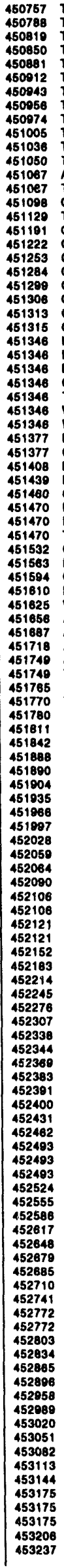 & 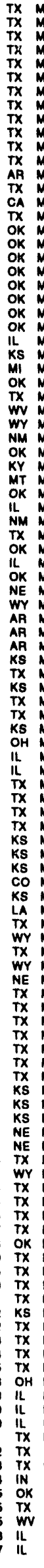 & 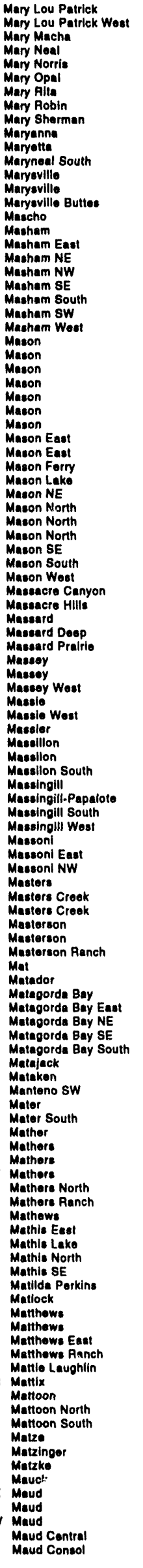 & 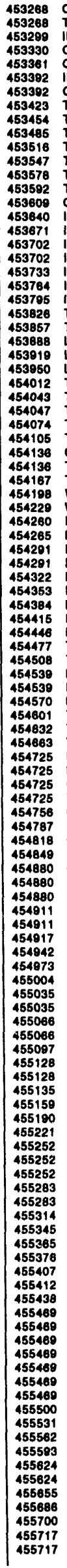 & 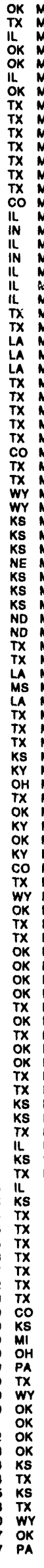 & 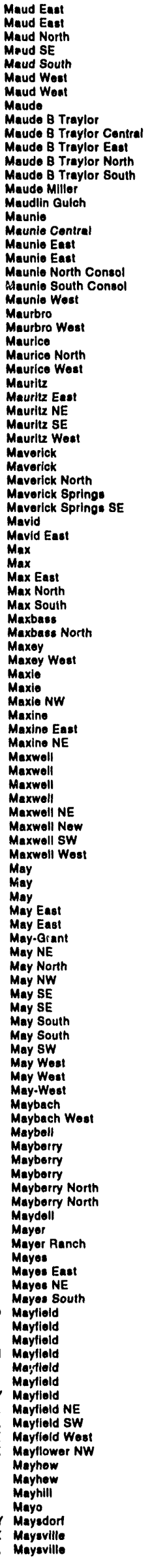 \\
\hline
\end{tabular}




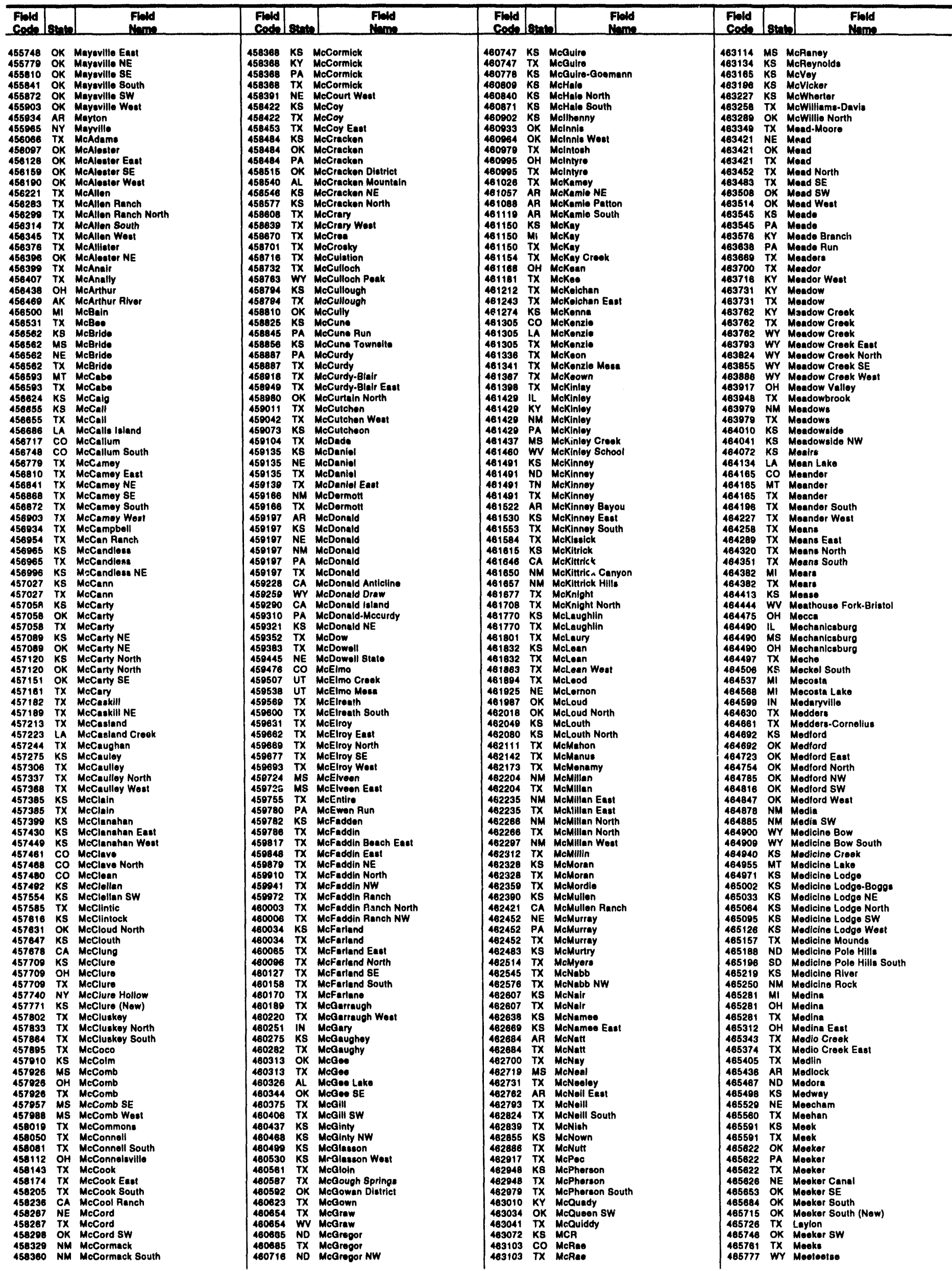




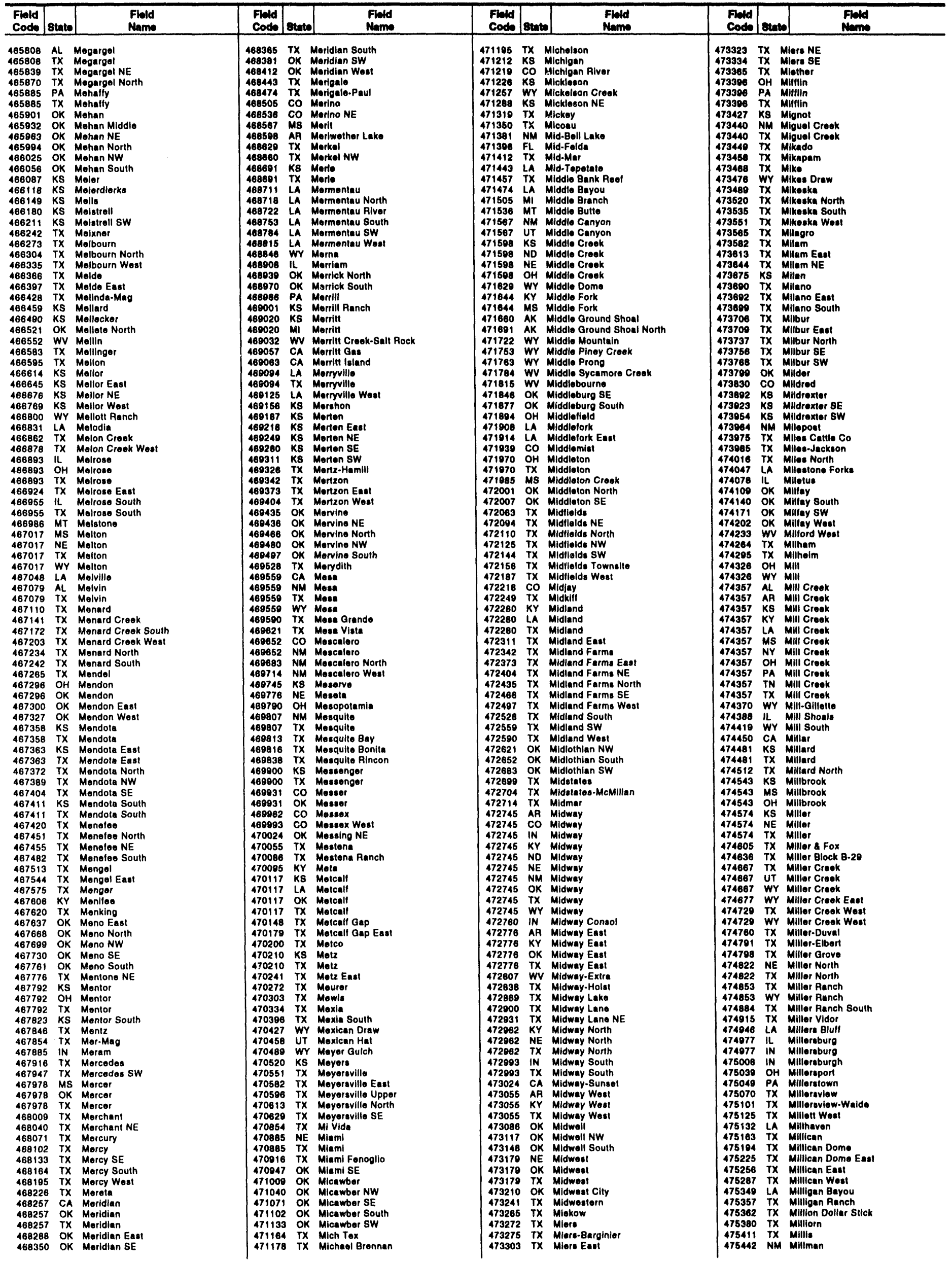




\begin{tabular}{|c|c|c|c|c|c|c|c|c|c|c|c|}
\hline $\begin{array}{l}\text { Flold } \\
\text { Cod. }\end{array}$ & & $\begin{array}{l}\text { Finld } \\
\text { then. }\end{array}$ & $\left.\begin{array}{l}\text { Field } \\
\text { Code. }\end{array}\right]$ & & $\begin{array}{l}\text { Findd } \\
\text { Nonde }\end{array}$ & $\begin{array}{l}\text { Finld } \\
\text { conde }\end{array}$ & & $\begin{array}{l}\text { Field } \\
\text { Nem: }\end{array}$ & $\begin{array}{l}\text { Fildd } \\
\text { Codte }\end{array}$ & & $\begin{array}{l}\text { Fiold } \\
\text { Neme }\end{array}$ \\
\hline 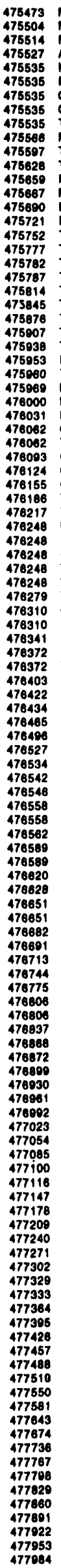 & 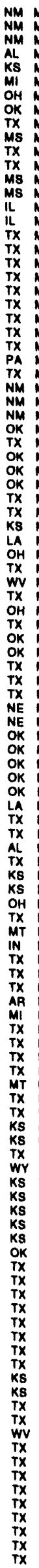 & 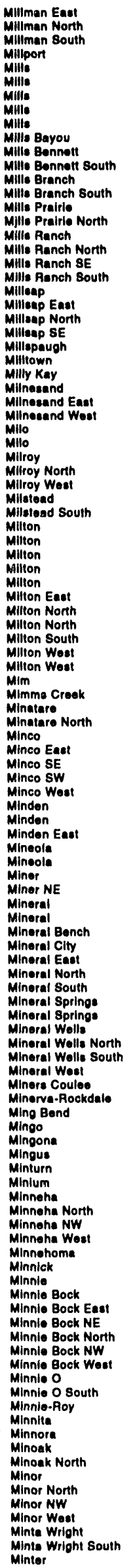 & 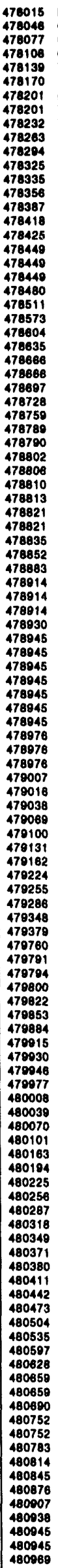 & 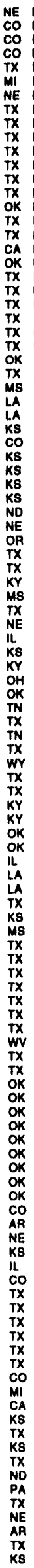 & 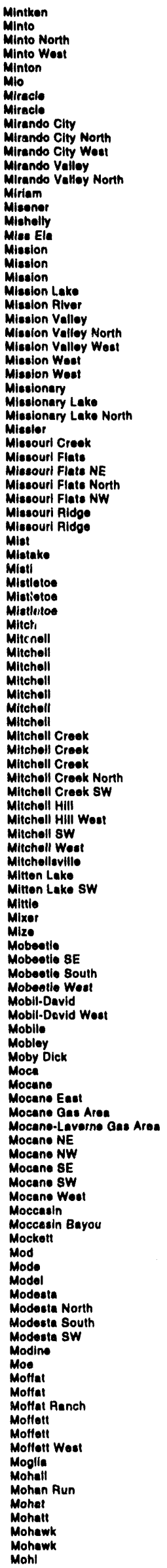 & 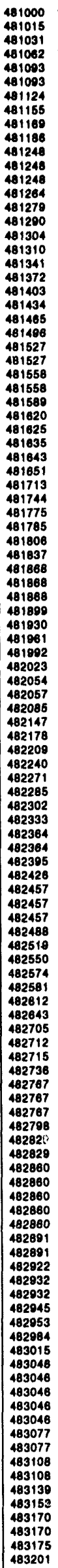 & 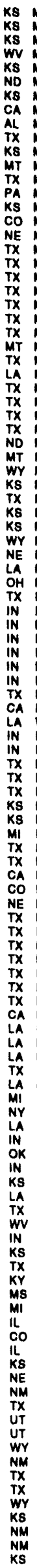 & 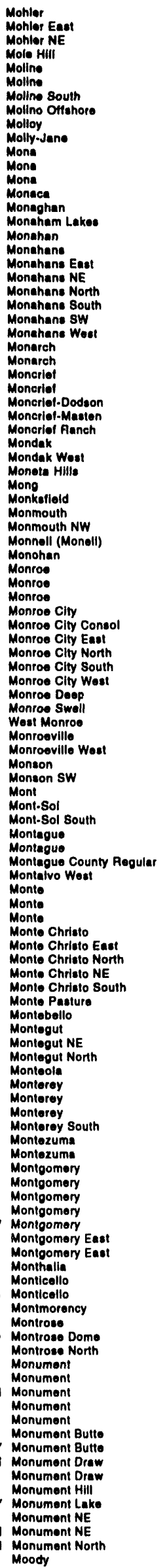 & 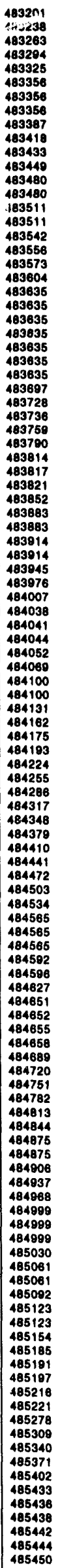 & 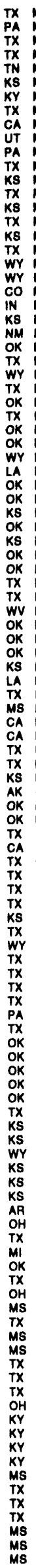 & 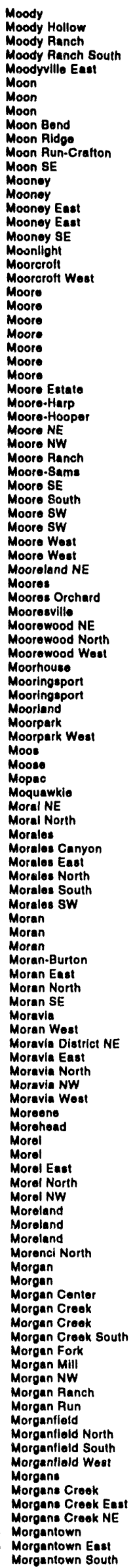 \\
\hline
\end{tabular}




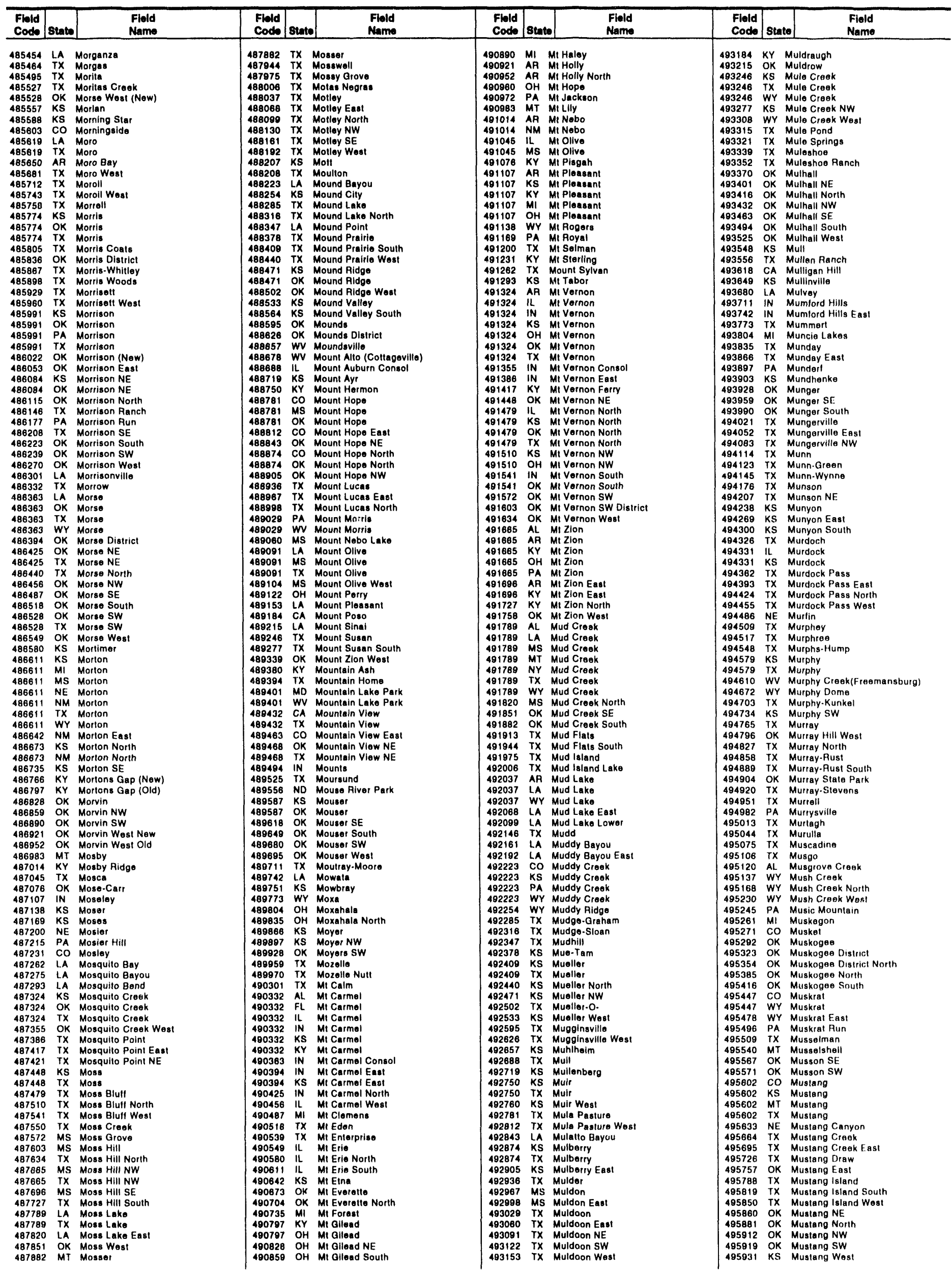




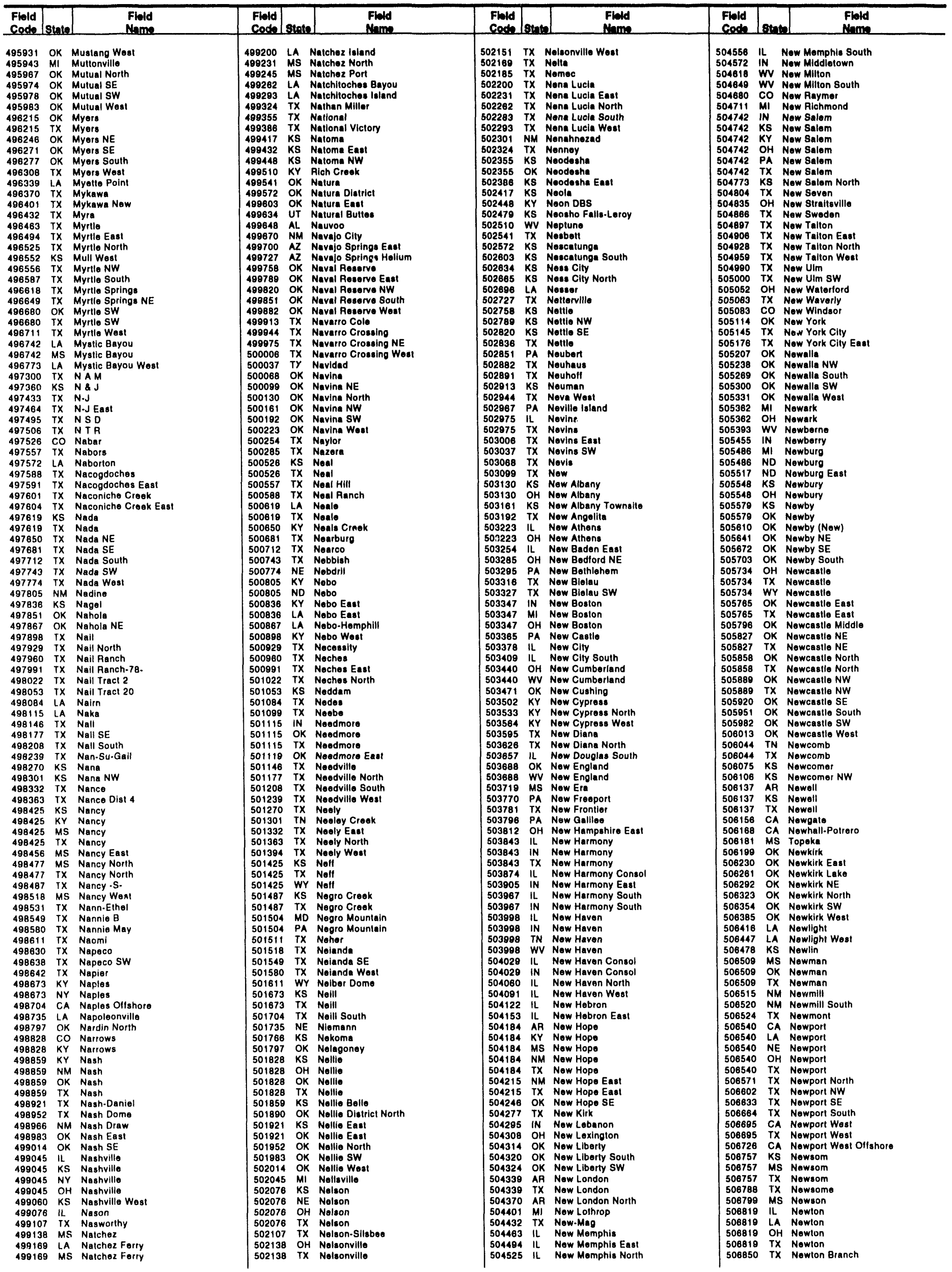


518829

\begin{tabular}{|c|c|c|c|c|c|c|c|c|c|c|c|}
\hline $\begin{array}{l}\text { Fild } \\
\text { Code }\end{array}$ & Sorate & $\begin{array}{l}\text { Fileld } \\
\text { Nams }\end{array}$ & $\begin{array}{l}\text { Flold } \\
\text { Codo }\end{array}$ & 8tint: & $\begin{array}{l}\text { Fild } \\
\text { Mams }\end{array}$ & $\begin{array}{l}\text { Fiald } \\
\text { code }\end{array}$ & enene & $\begin{array}{l}\text { Fleld } \\
\text { Neme }\end{array}$ & $\begin{array}{l}\text { Fiold } \\
\text { Code }\end{array}$ & State & $\begin{array}{l}\text { Fiold } \\
\text { Name }\end{array}$ \\
\hline 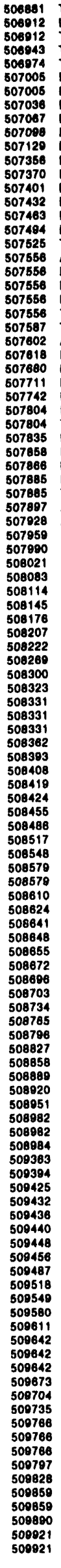 & 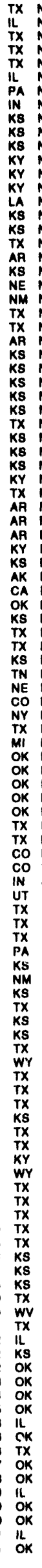 & 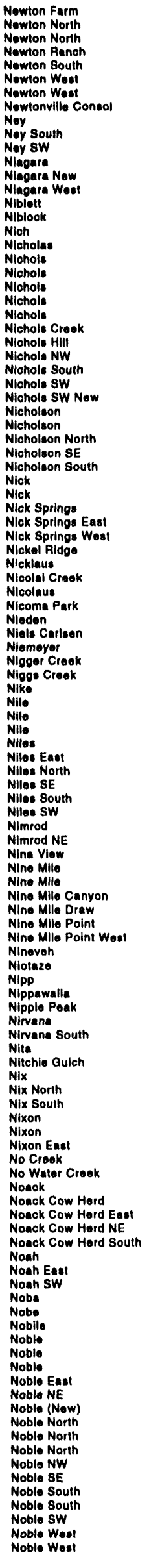 & 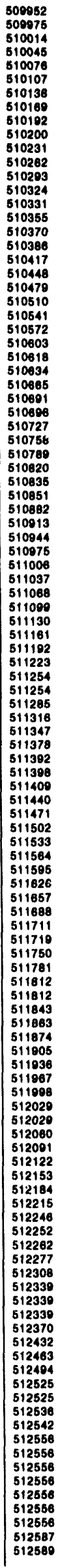 & 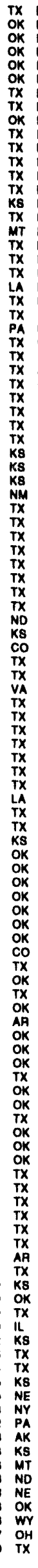 & 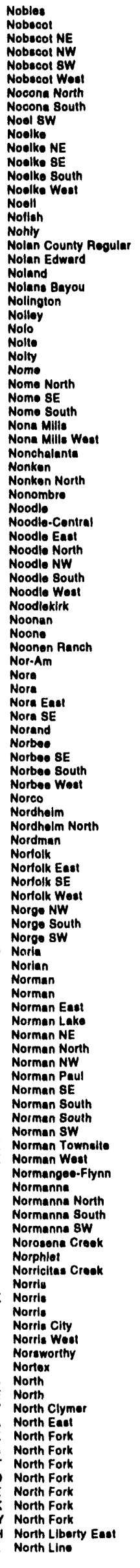 & 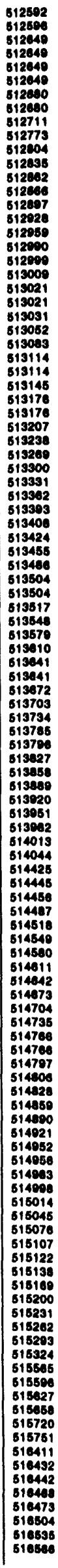 & 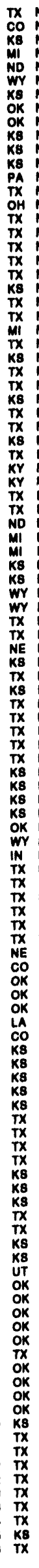 & 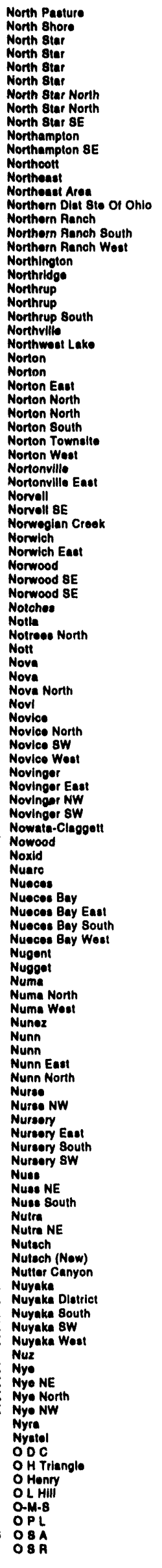 & 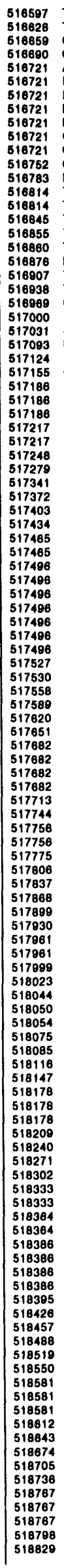 & 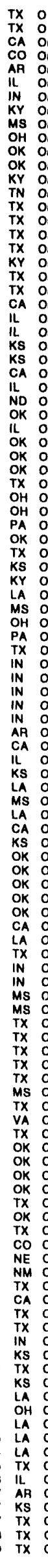 & 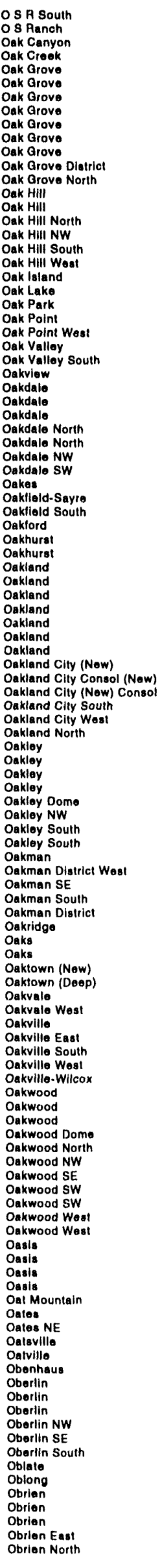 \\
\hline
\end{tabular}




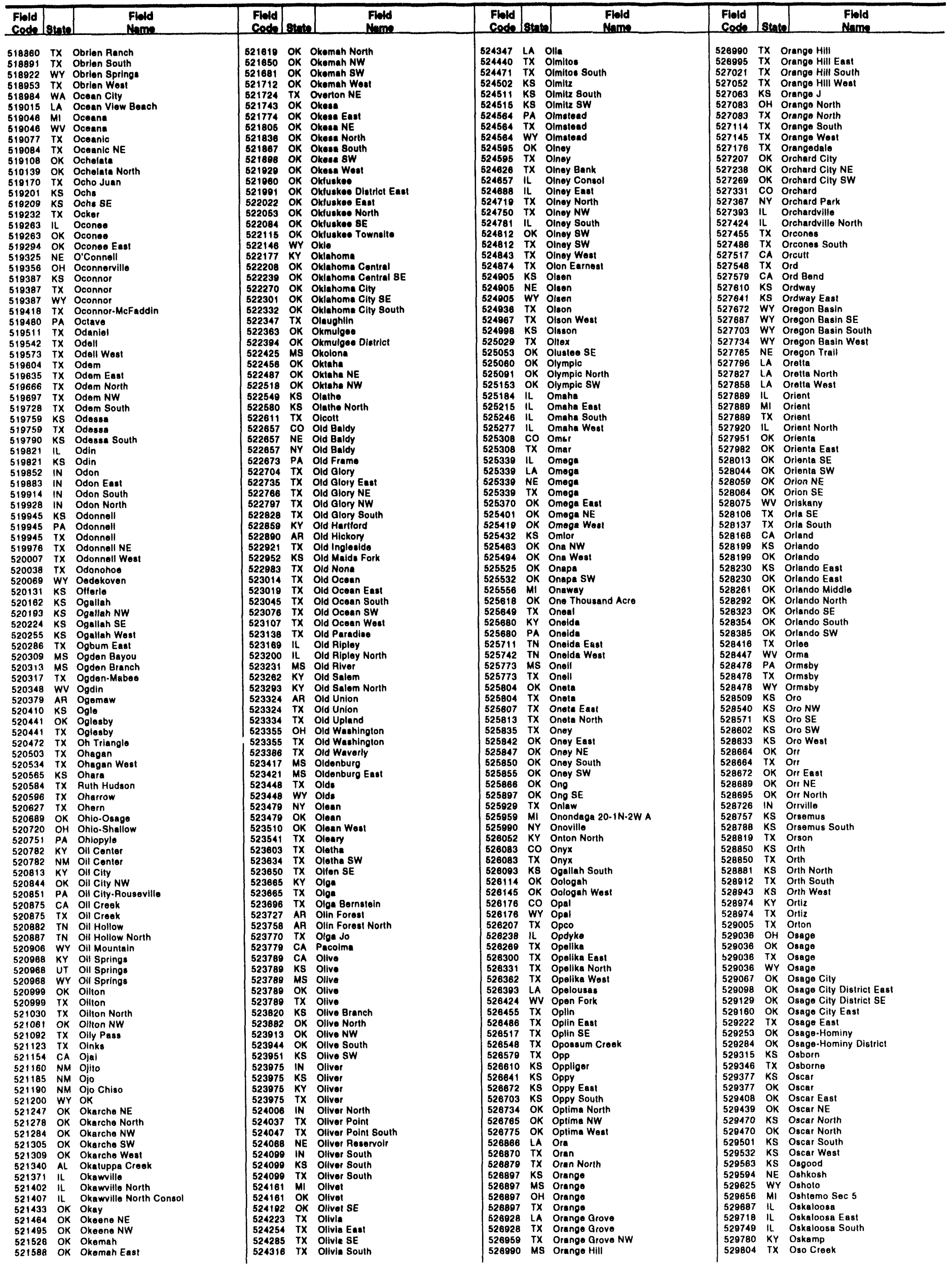




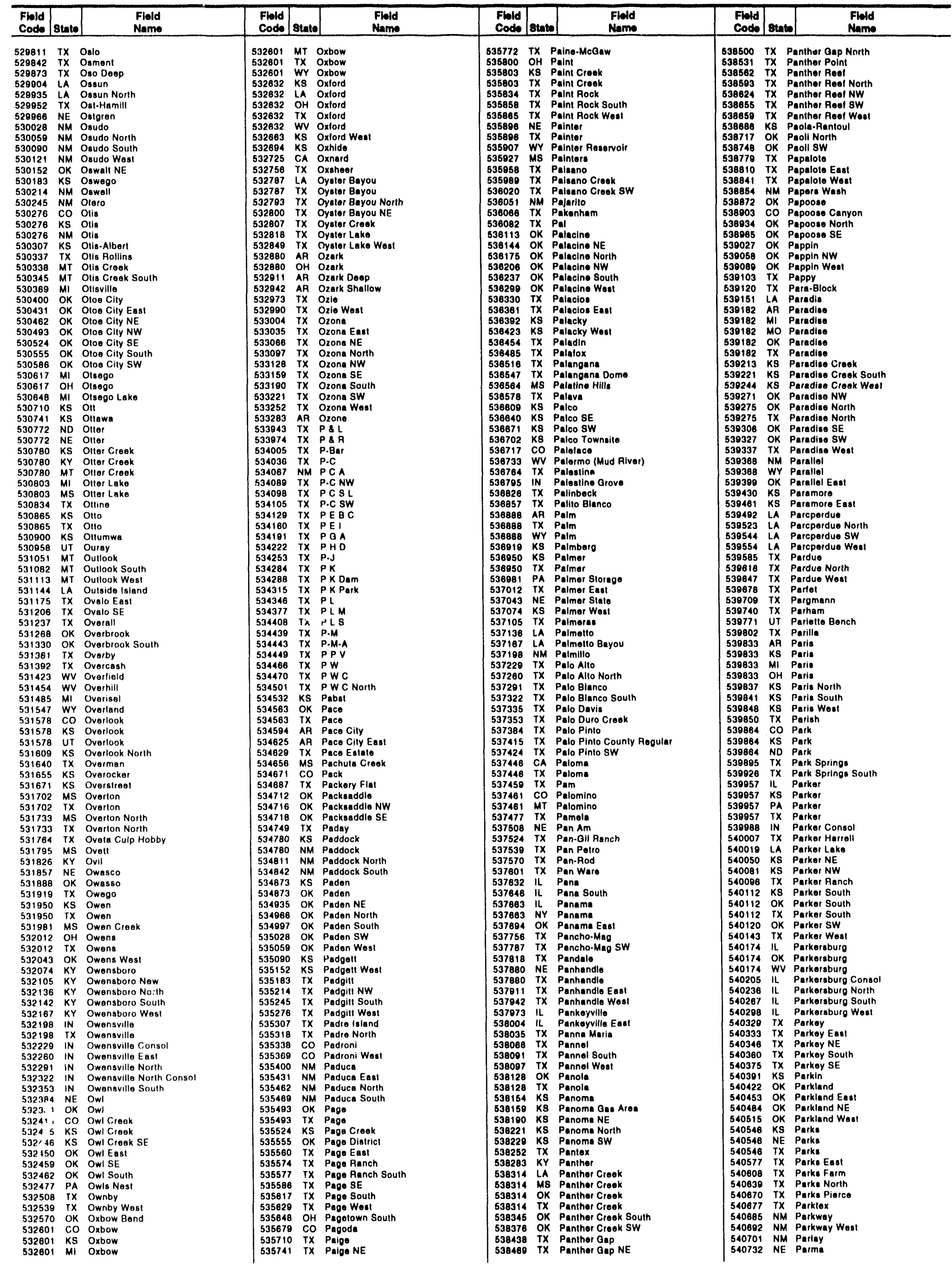




\begin{tabular}{|c|c|c|c|c|c|c|c|c|c|c|c|}
\hline $\begin{array}{l}\text { Flold } \\
\text { Code }\end{array}$ & Stat & $\begin{array}{l}\text { Field } \\
\text { Neme }\end{array}$ & $\begin{array}{l}\text { Fild } \\
\text { Coede }\end{array}$ & & $\begin{array}{l}\text { Findd } \\
\text { N'min: }\end{array}$ & $\begin{array}{l}\text { Fileld } \\
\text { Codt2 }\end{array}$ & & $\begin{array}{l}\text { Filold } \\
\text { Name }\end{array}$ & $\begin{array}{l}\text { Flold } \\
\text { Coode }\end{array}$ & Stat: & $\begin{array}{l}\text { Fibld } \\
\text { Nhm }\end{array}$ \\
\hline 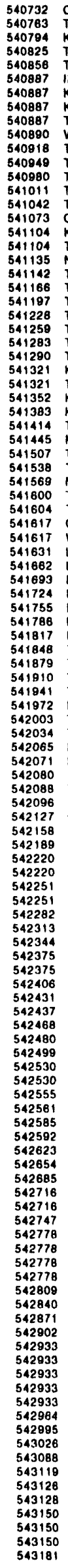 & 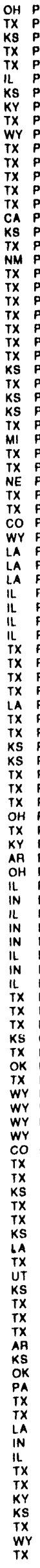 & 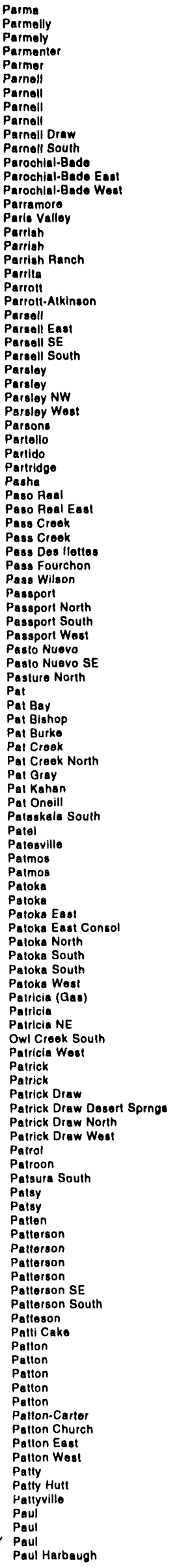 & 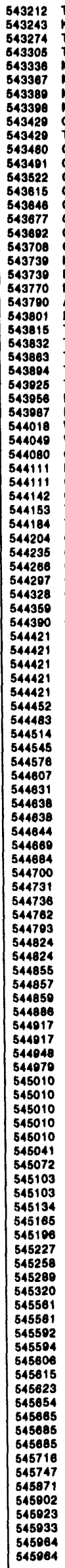 & 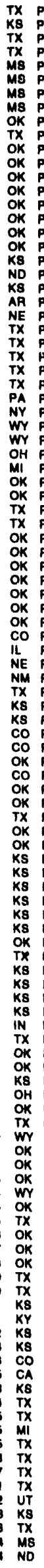 & 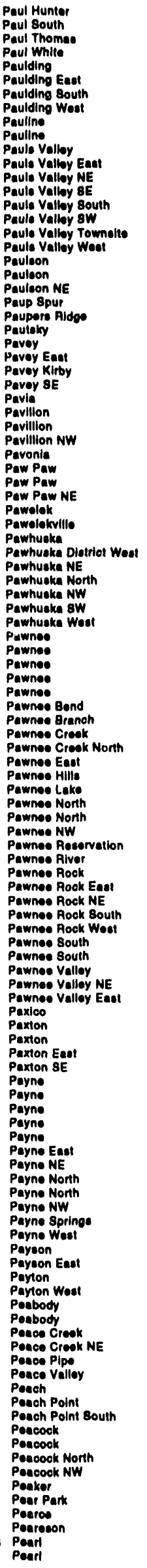 & 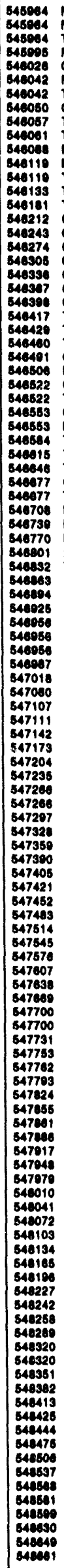 & 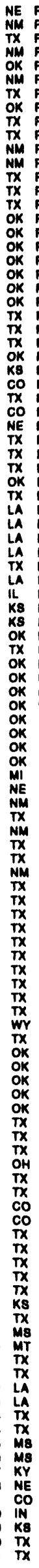 & 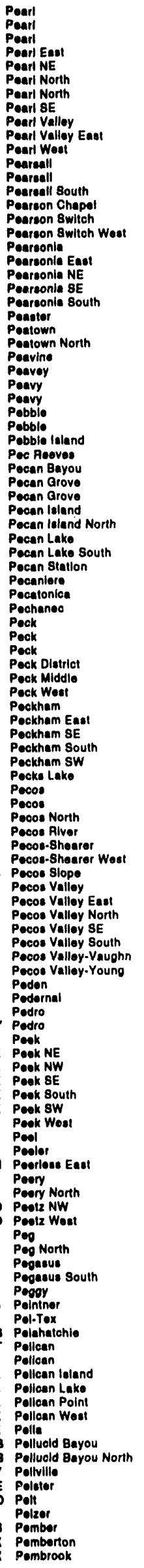 & 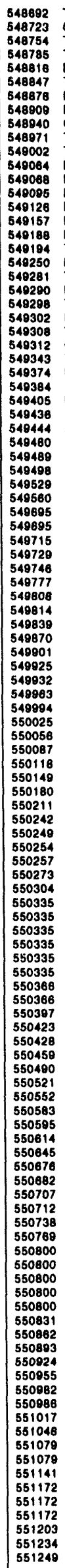 & 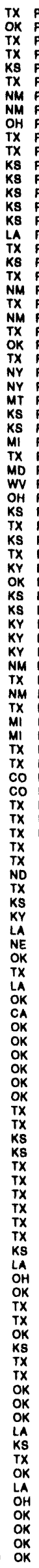 & 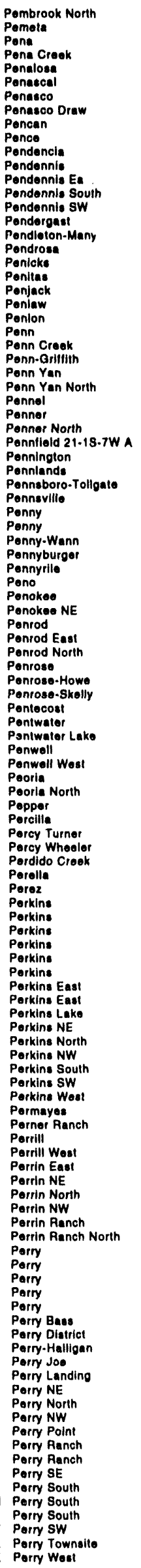 \\
\hline
\end{tabular}


560217

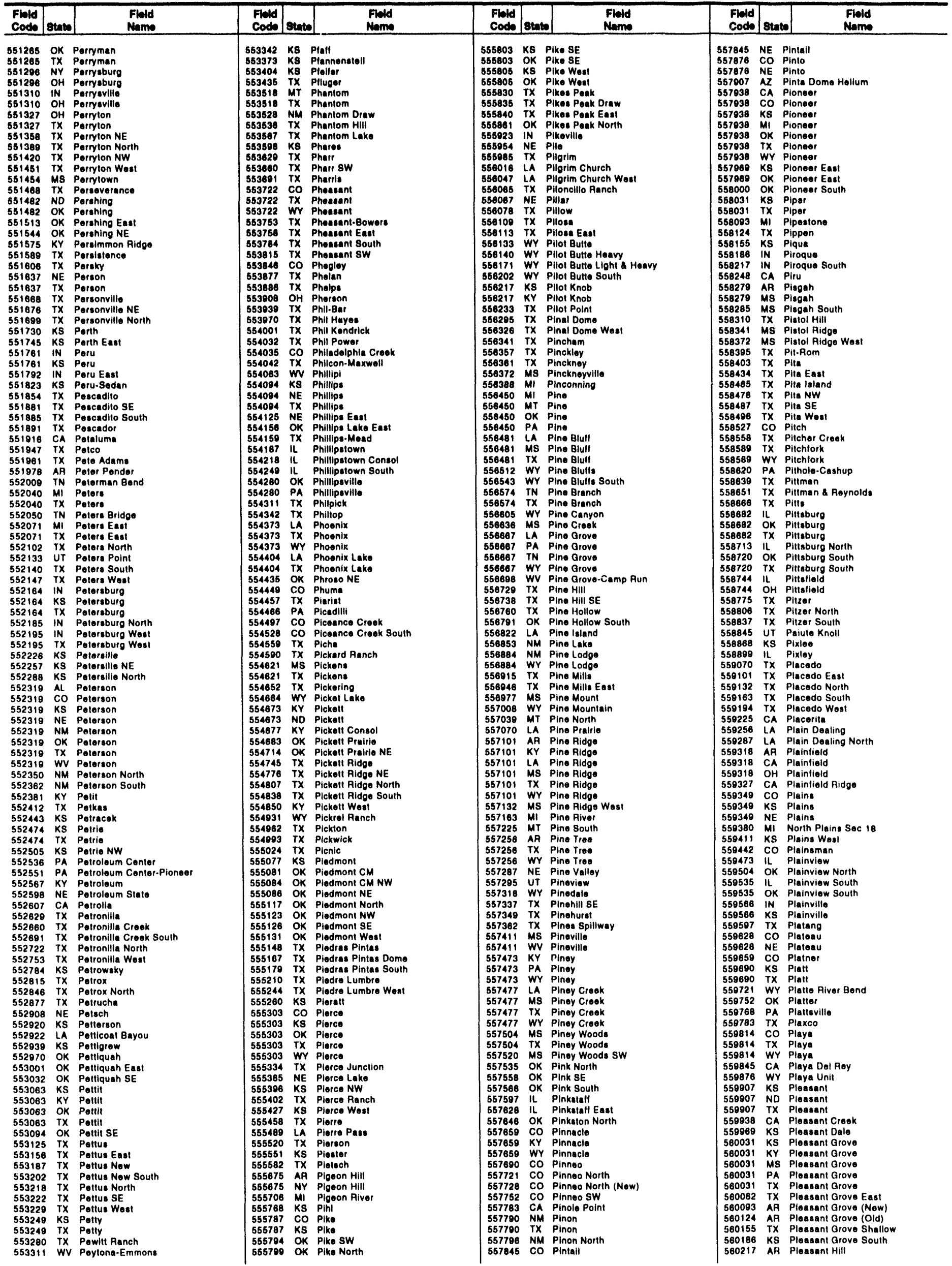




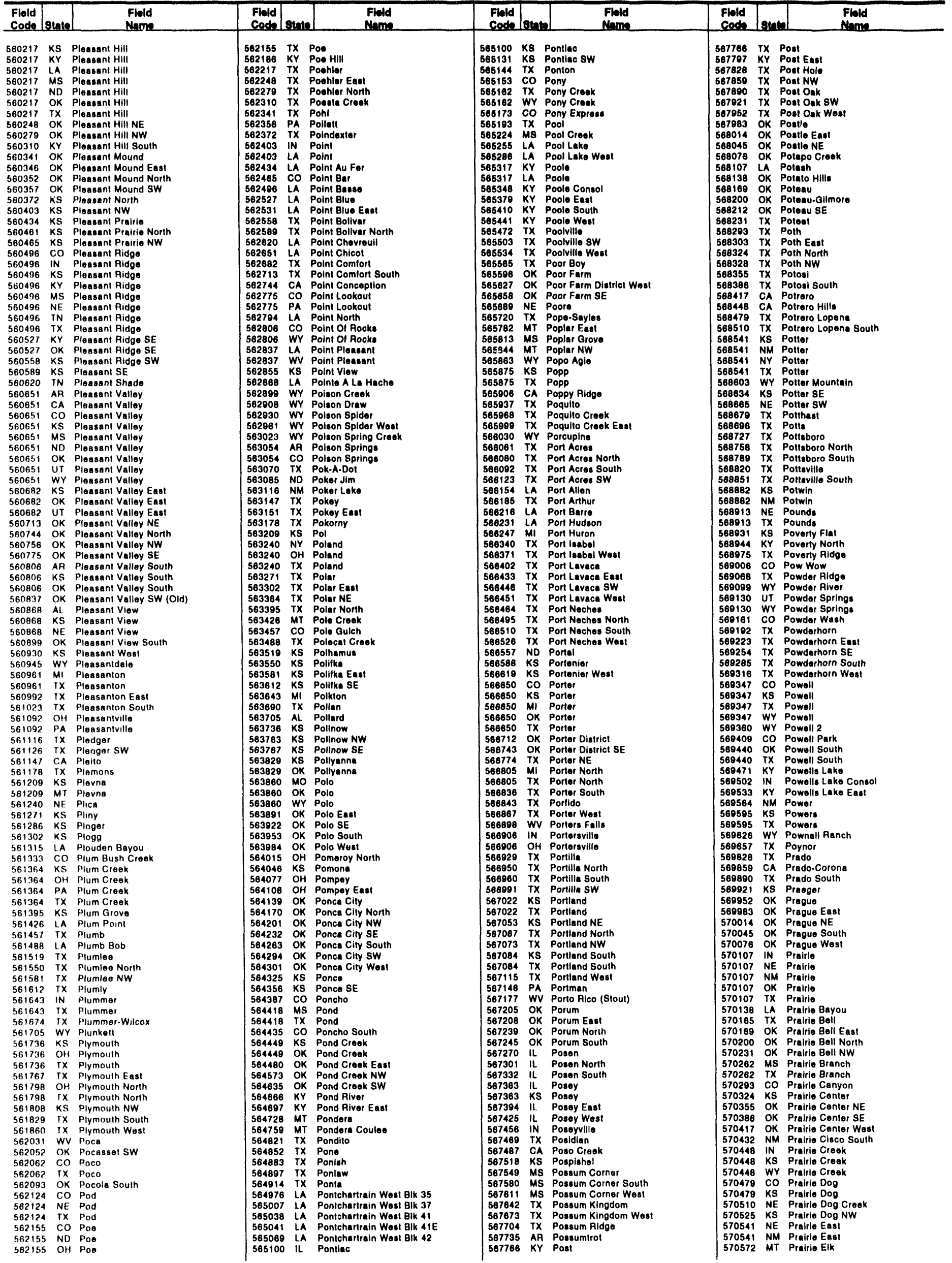




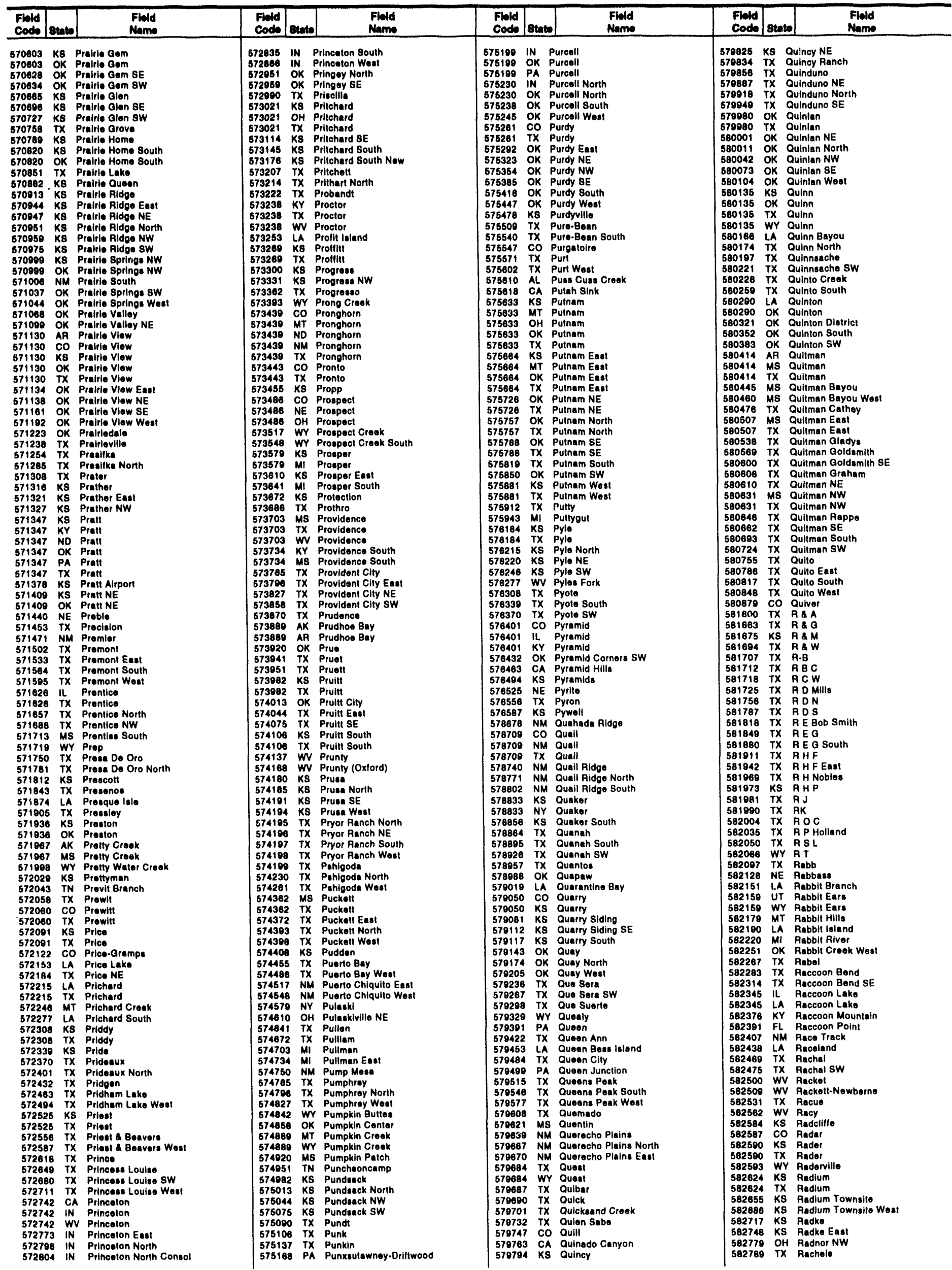




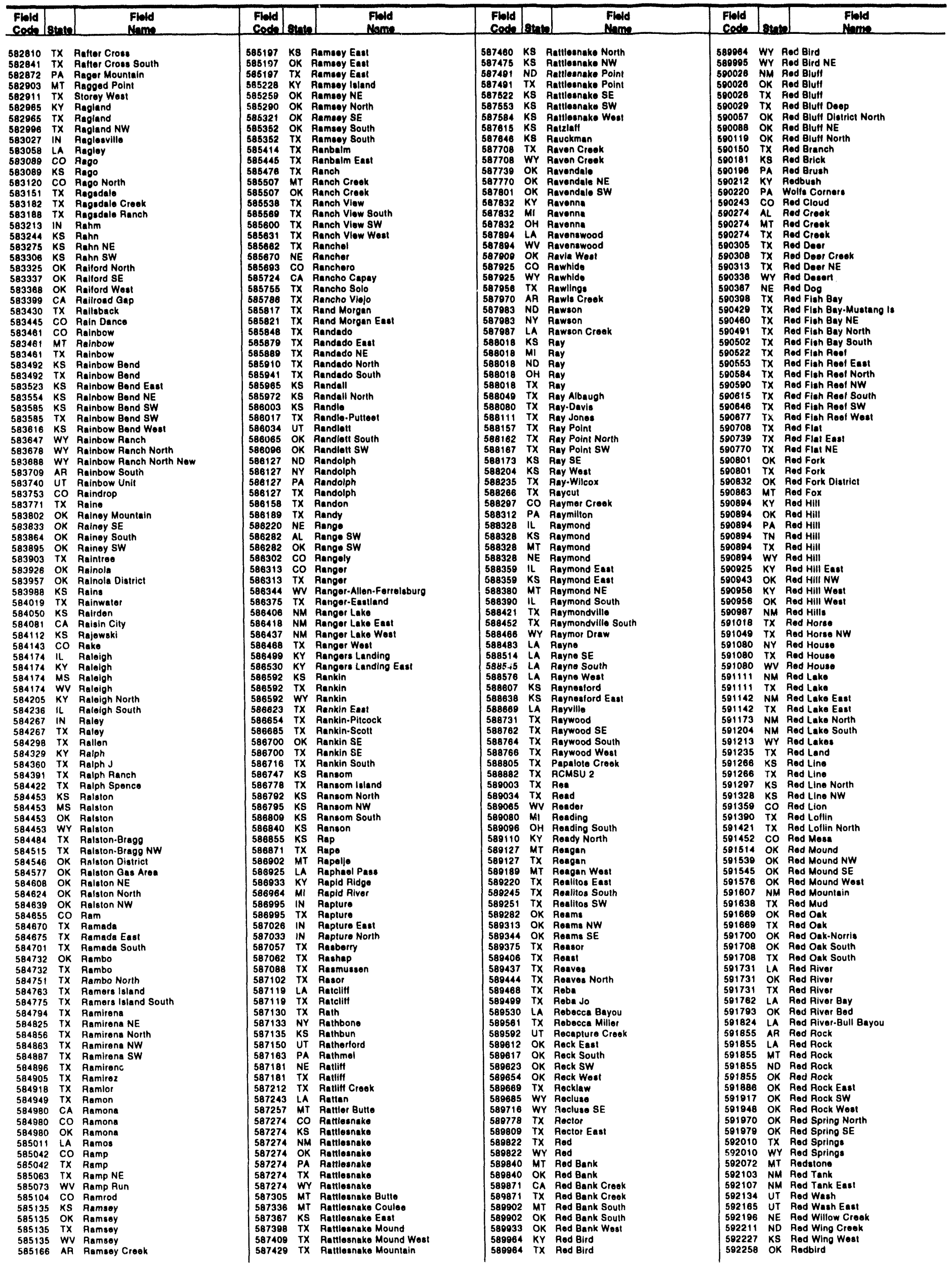




\begin{tabular}{|c|c|c|c|c|c|c|c|c|c|c|c|}
\hline $\begin{array}{l}\text { Finld } \\
\text { Code }\end{array}$ & State & $\begin{array}{l}\text { Fileid } \\
\text { Nemo }\end{array}$ & $\begin{array}{l}\text { Find } \\
\text { Code }\end{array}$ & Sinte & $\begin{array}{l}\text { Field } \\
\text { Name } \\
\end{array}$ & $\begin{array}{l}\text { Fiold } \\
\text { Code }\end{array}$ & State & $\begin{array}{l}\text { Field } \\
\text { Name }\end{array}$ & $\begin{array}{l}\text { Fild } \\
\text { Codo }\end{array}$ & state & $\begin{array}{l}\text { Ficid } \\
\text { Name } \\
\end{array}$ \\
\hline 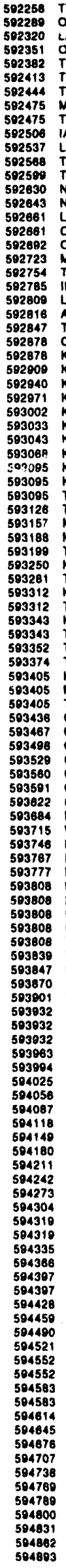 & 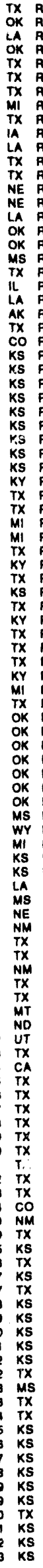 & 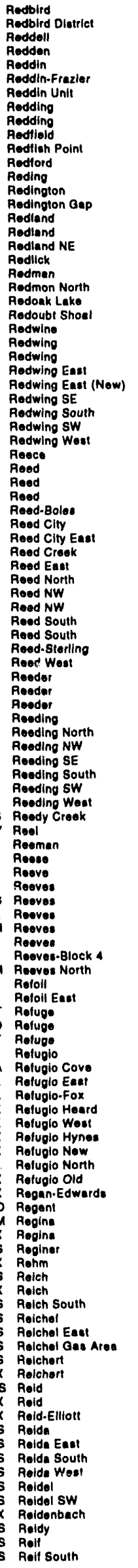 & 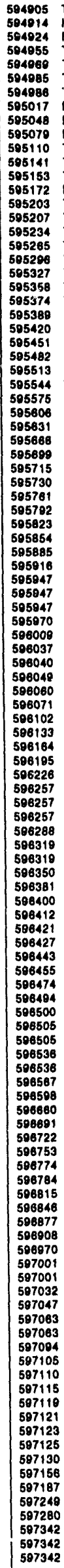 & 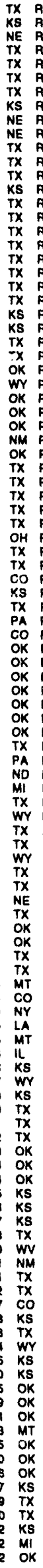 & 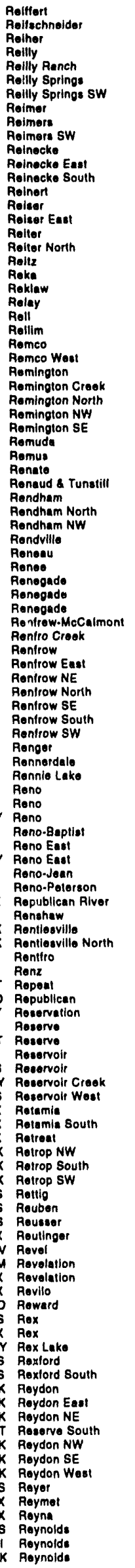 & 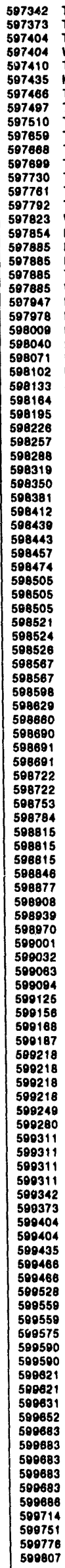 & 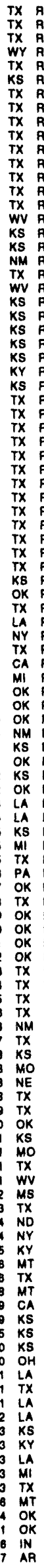 & 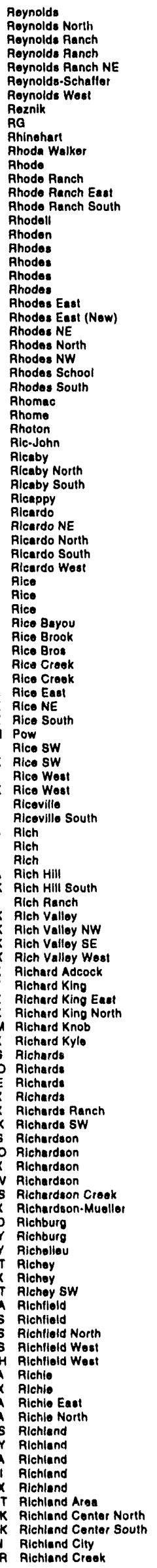 & 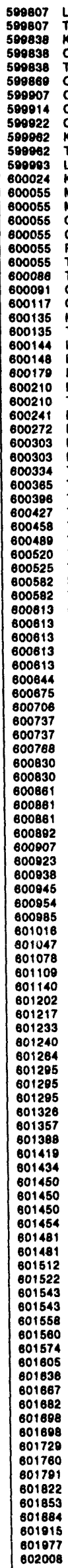 & 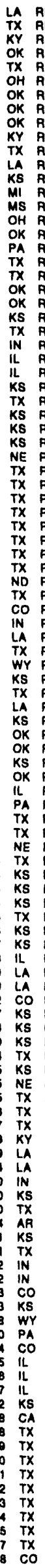 & 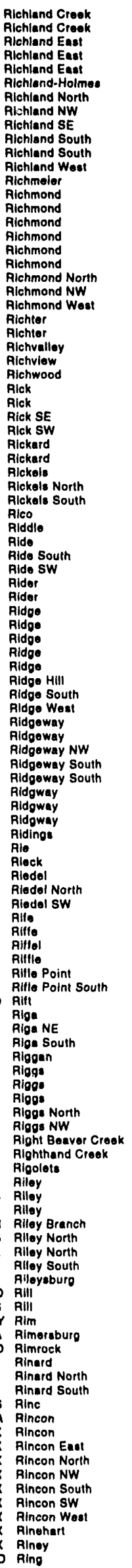 \\
\hline
\end{tabular}




\begin{tabular}{|c|c|c|c|c|c|c|c|c|c|c|c|}
\hline $\begin{array}{l}\text { Fiold } \\
\text { Codh }\end{array}$ & stata & $\begin{array}{l}\text { Field } \\
\text { Name }\end{array}$ & $\begin{array}{l}\text { Field } \\
\text { Coded }\end{array}$ & Ethe & $\begin{array}{l}\text { Fiold } \\
\text { Name }\end{array}$ & $\begin{array}{l}\text { Fiold } \\
\text { Codt. }\end{array}$ & Sintel & $\begin{array}{l}\text { Fild } \\
\text { Name }\end{array}$ & $\begin{array}{l}\text { Fileld } \\
\text { Code }\end{array}$ & Stint: & $\begin{array}{l}\text { Fibld } \\
\text { Name }\end{array}$ \\
\hline 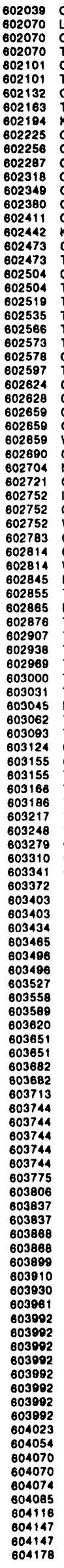 & 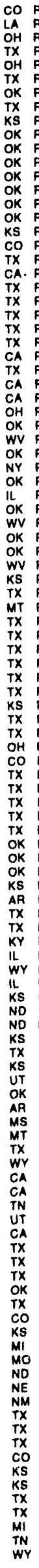 & 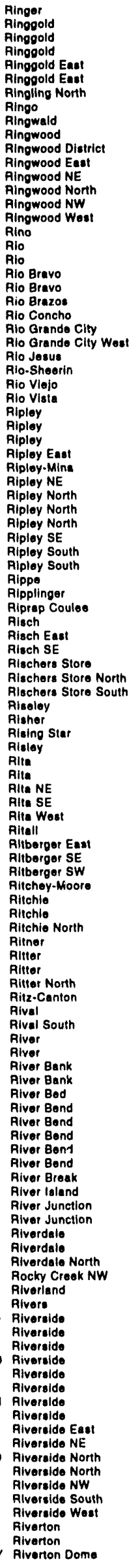 & 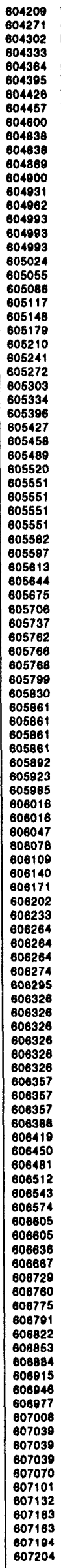 & 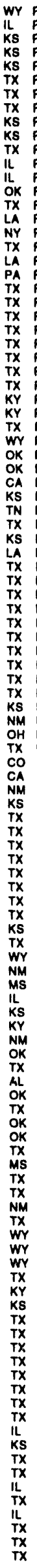 & 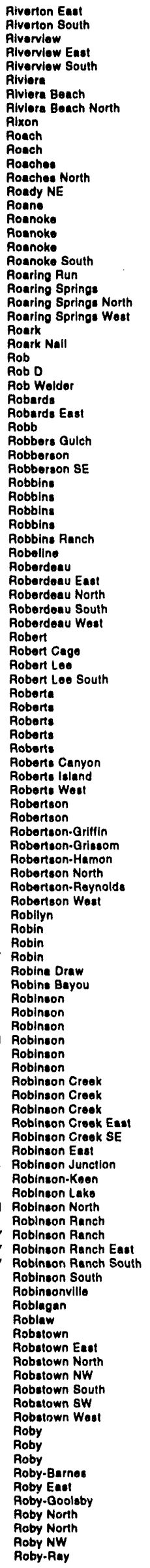 & 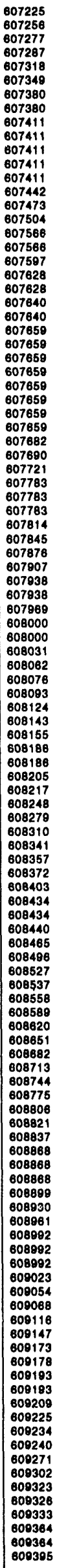 & 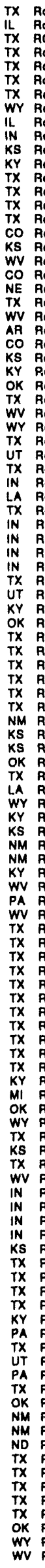 & 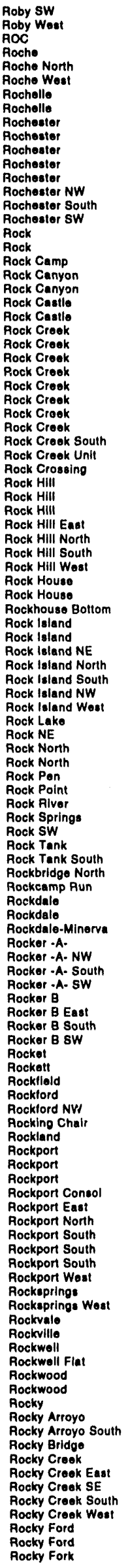 & 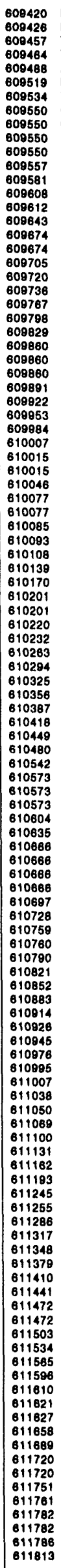 & 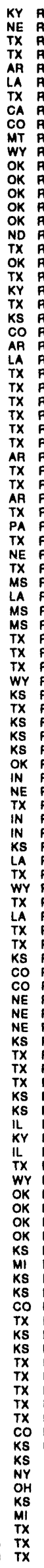 & 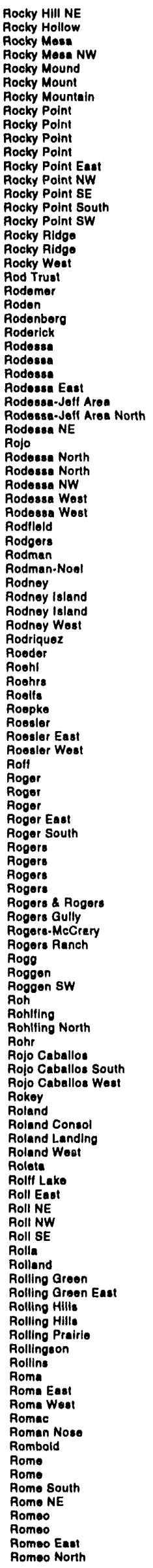 \\
\hline
\end{tabular}




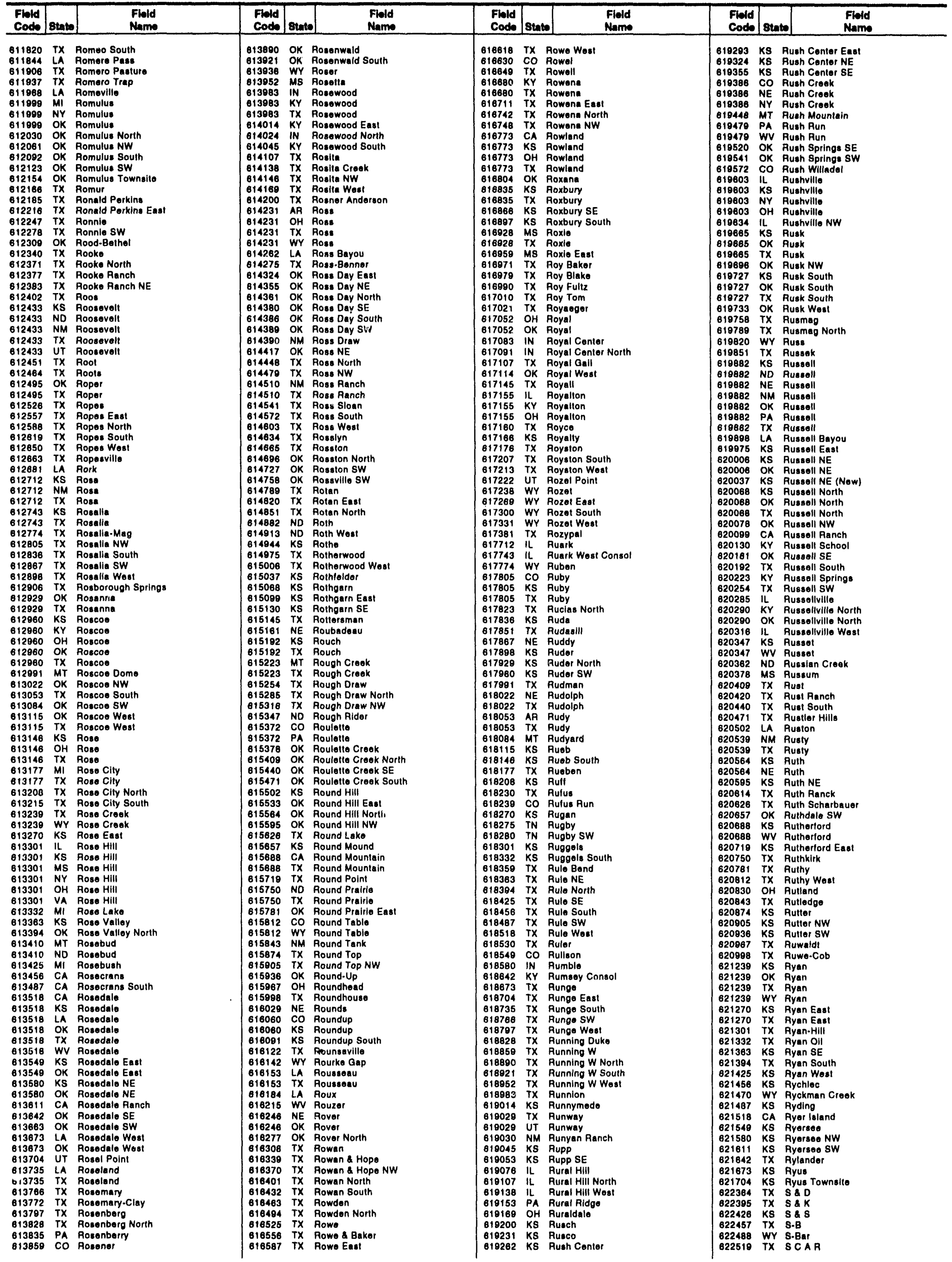




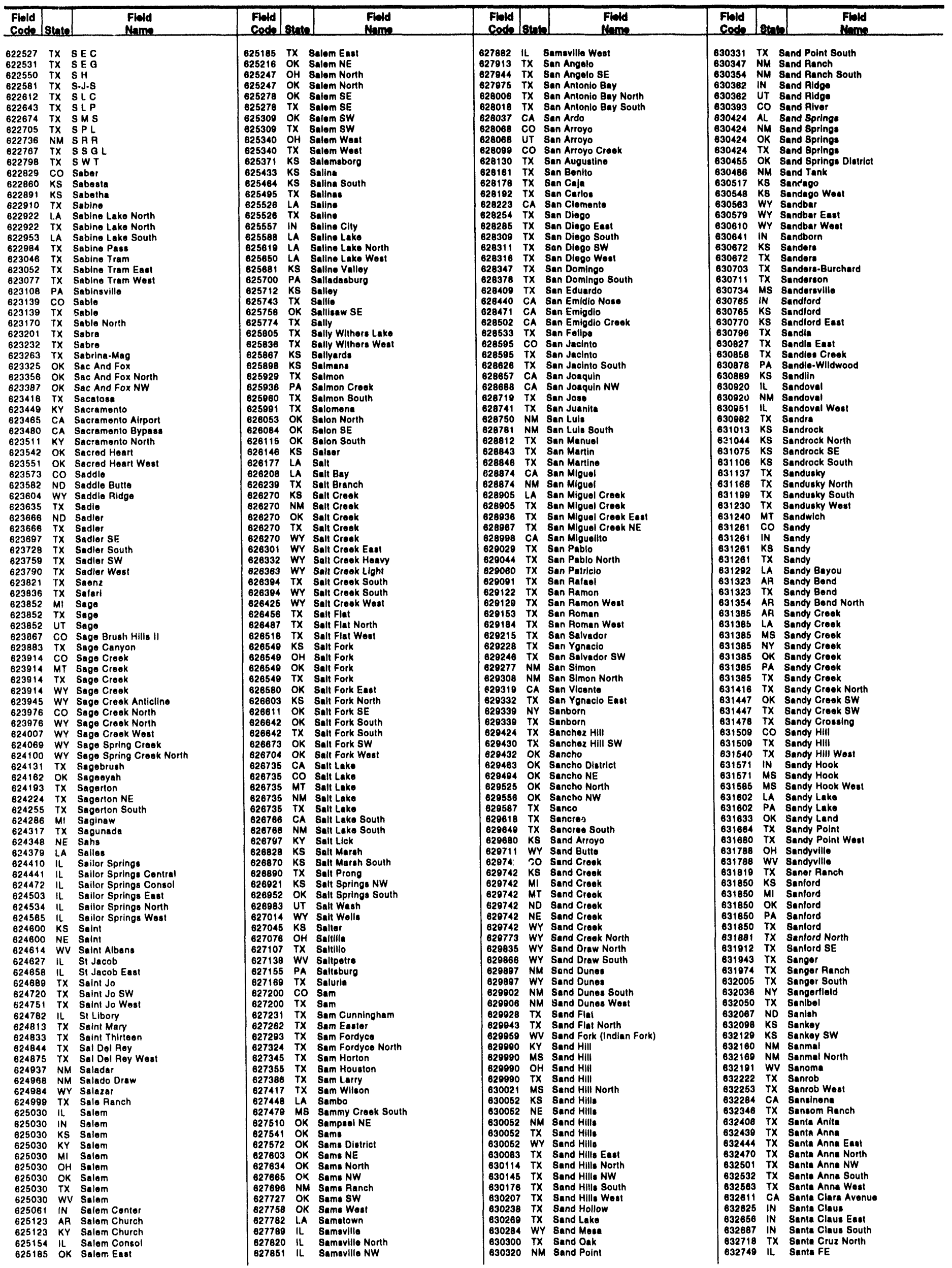




\begin{tabular}{|c|c|c|c|c|c|c|c|c|c|c|c|}
\hline $\begin{array}{l}\text { Flold } \\
\text { Code }\end{array}$ & 8tate & $\begin{array}{l}\text { Flold } \\
\text { Name } \\
\end{array}$ & $\begin{array}{l}\text { Fildd } \\
\text { Code } \\
\end{array}$ & Eute & $\begin{array}{l}\text { Flold } \\
\text { Name }\end{array}$ & $\begin{array}{l}\text { Fold } \\
\text { Code } \\
\end{array}$ & Stane & $\begin{array}{l}\text { Fild } \\
\text { Name } \\
\end{array}$ & $\begin{array}{l}\text { Flold } \\
\text { Code }\end{array}$ & State & $\begin{array}{l}\text { Fiold } \\
\text { Name } \\
\end{array}$ \\
\hline 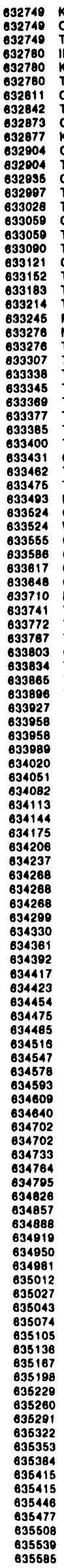 & 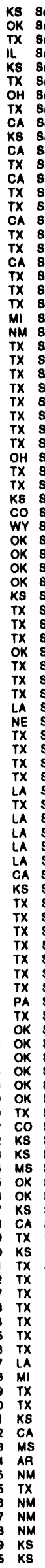 & 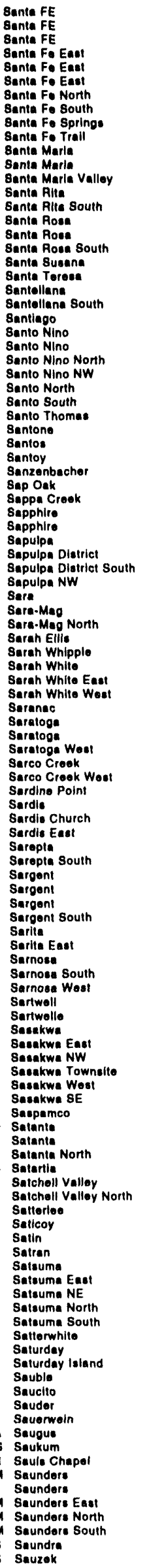 & 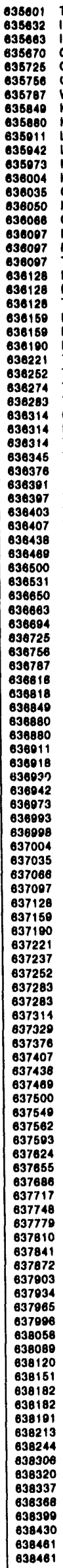 & 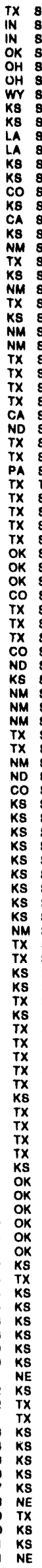 & 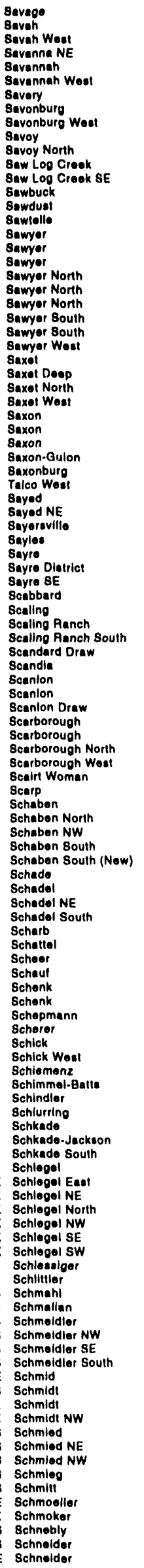 & 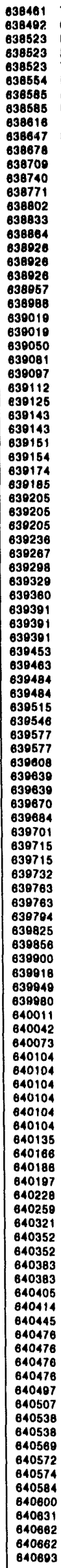 & 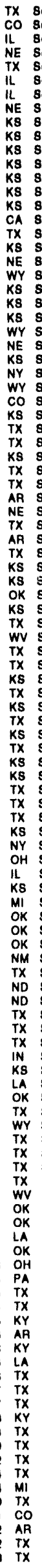 & 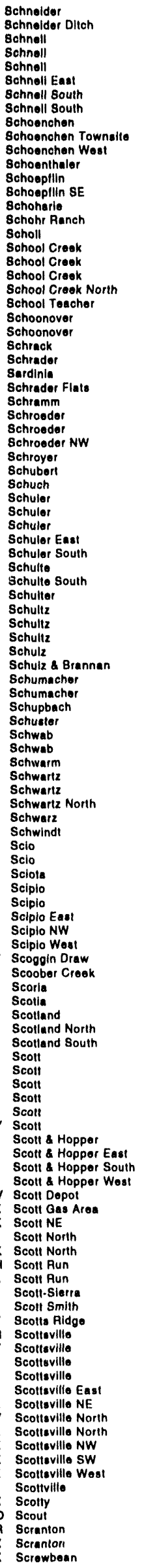 & 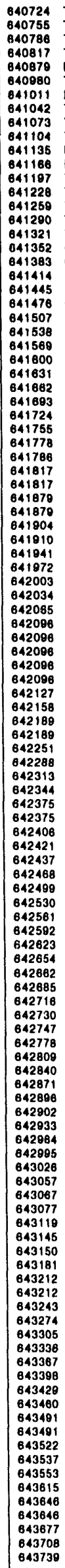 & 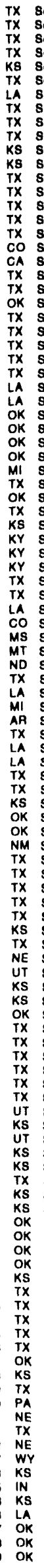 & 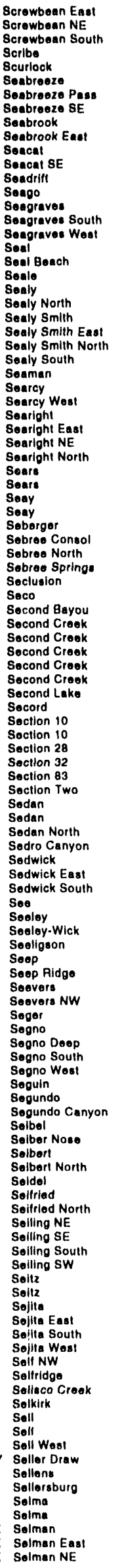 \\
\hline
\end{tabular}




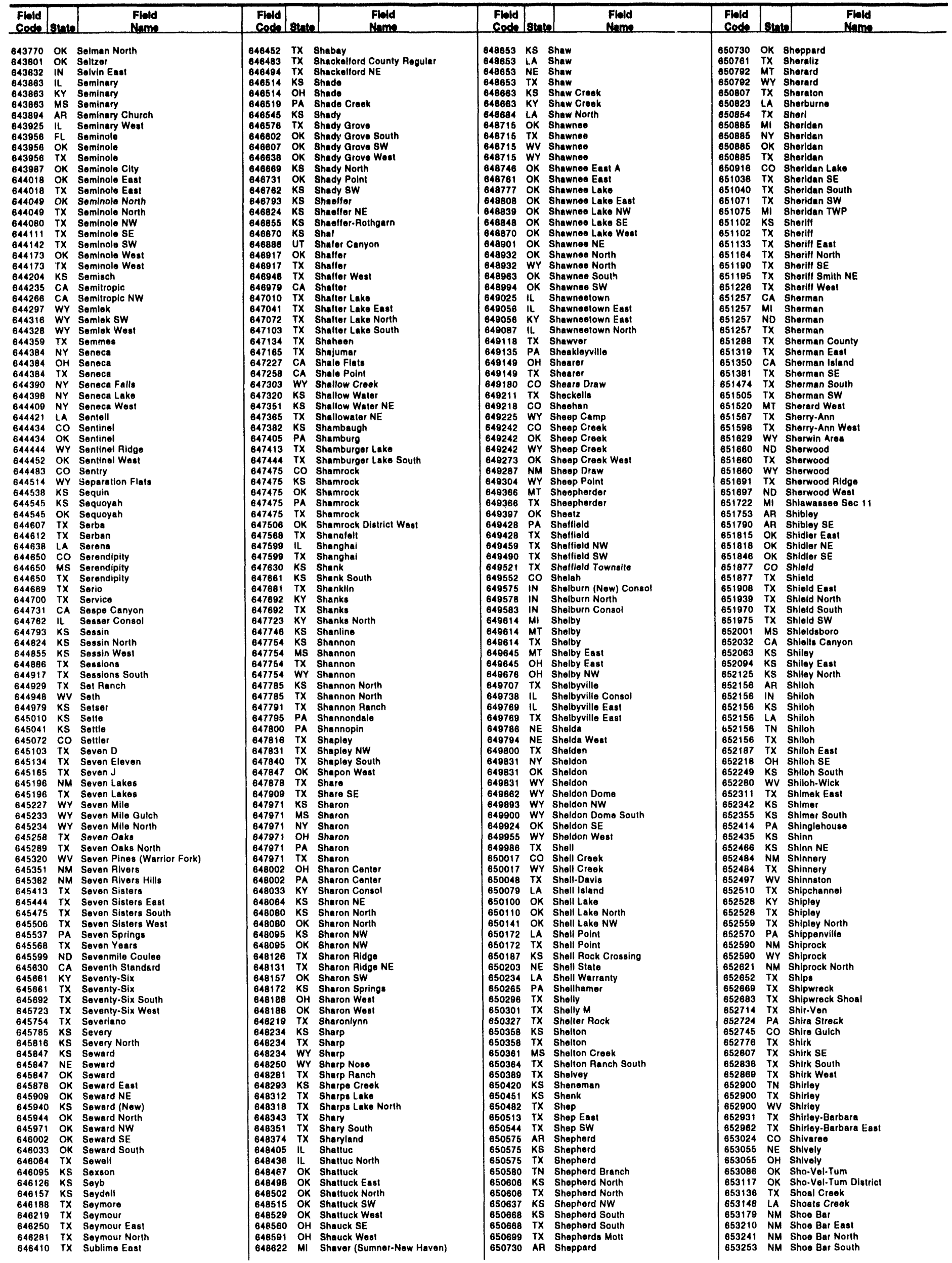




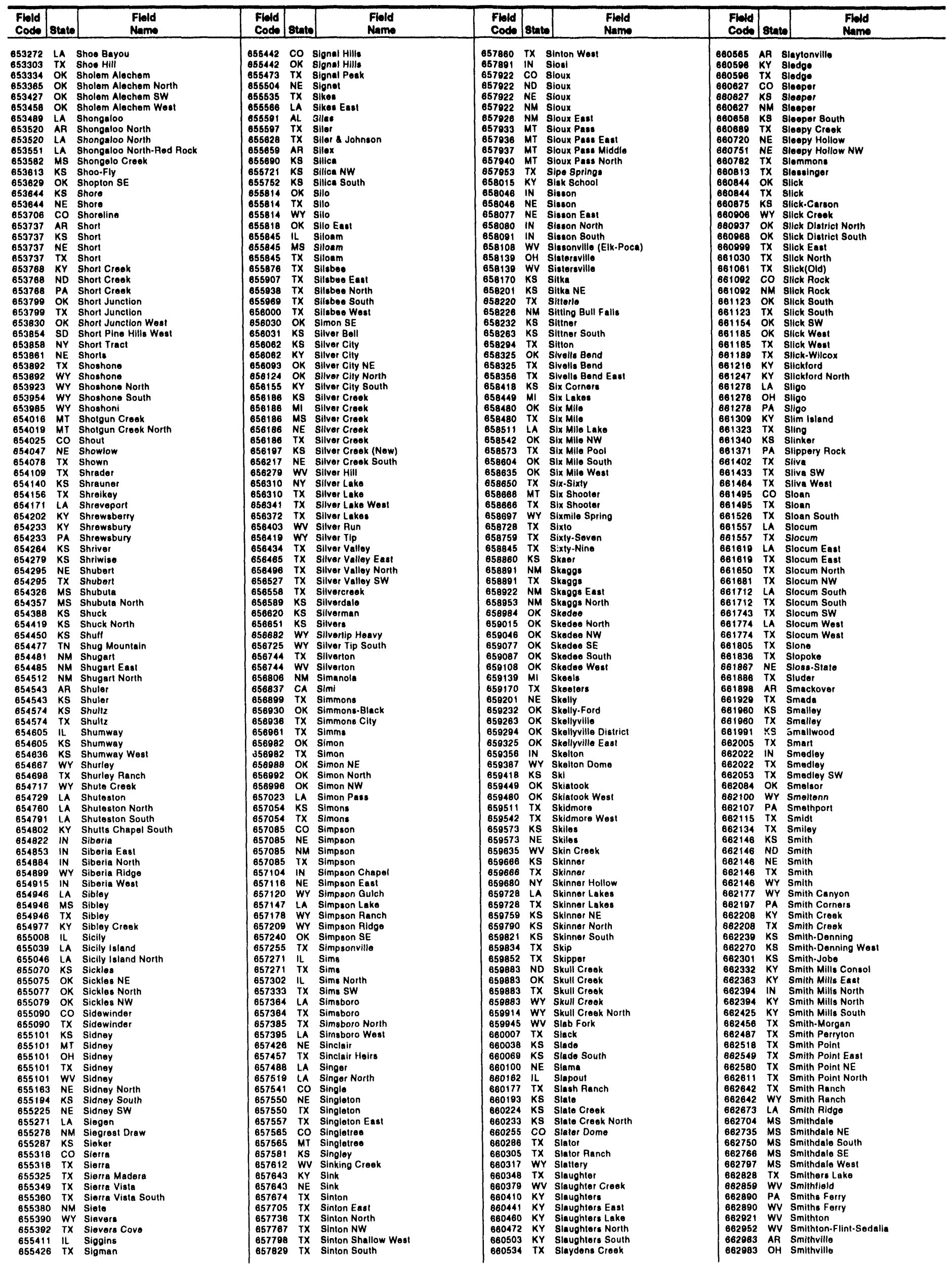




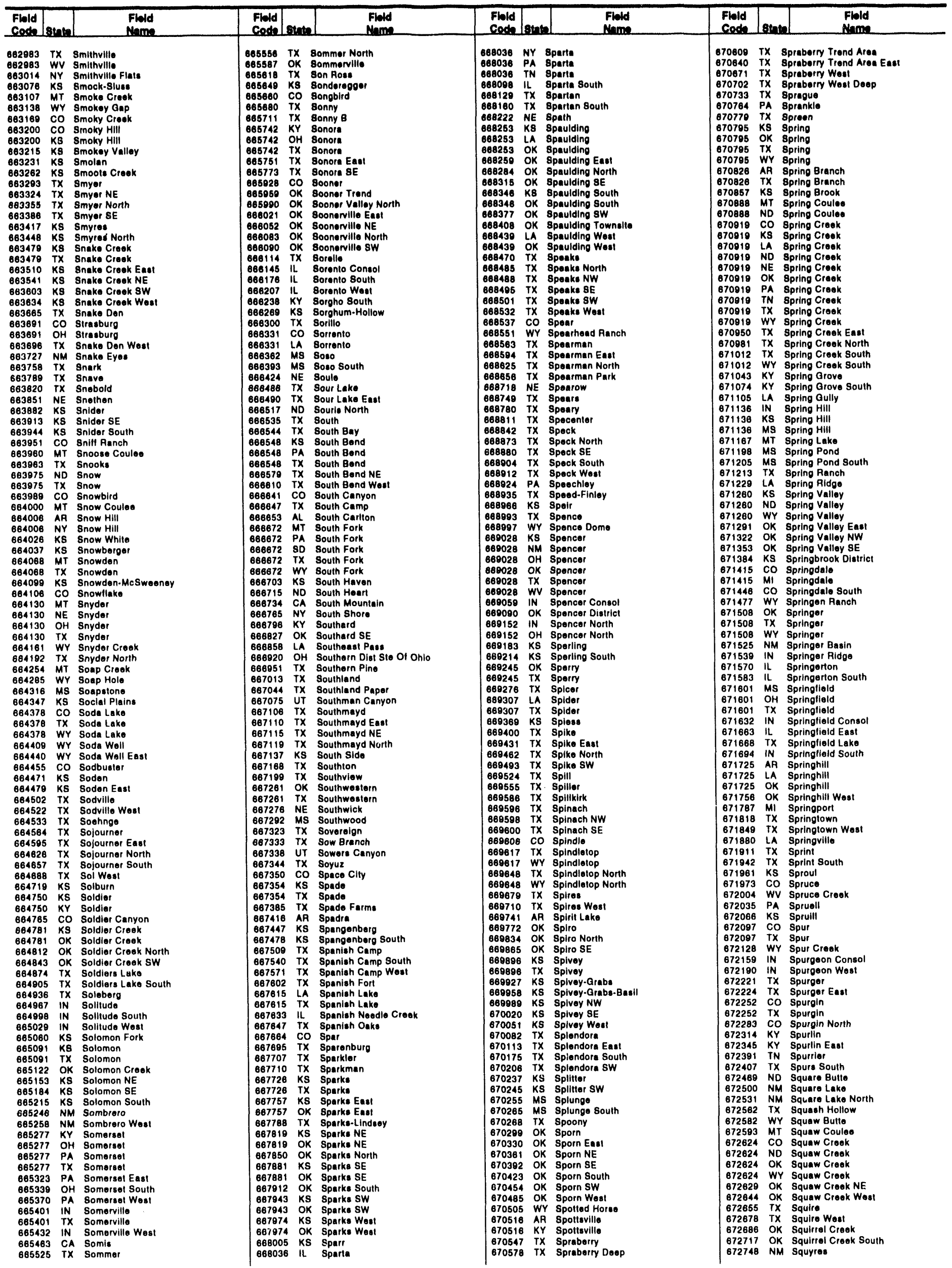




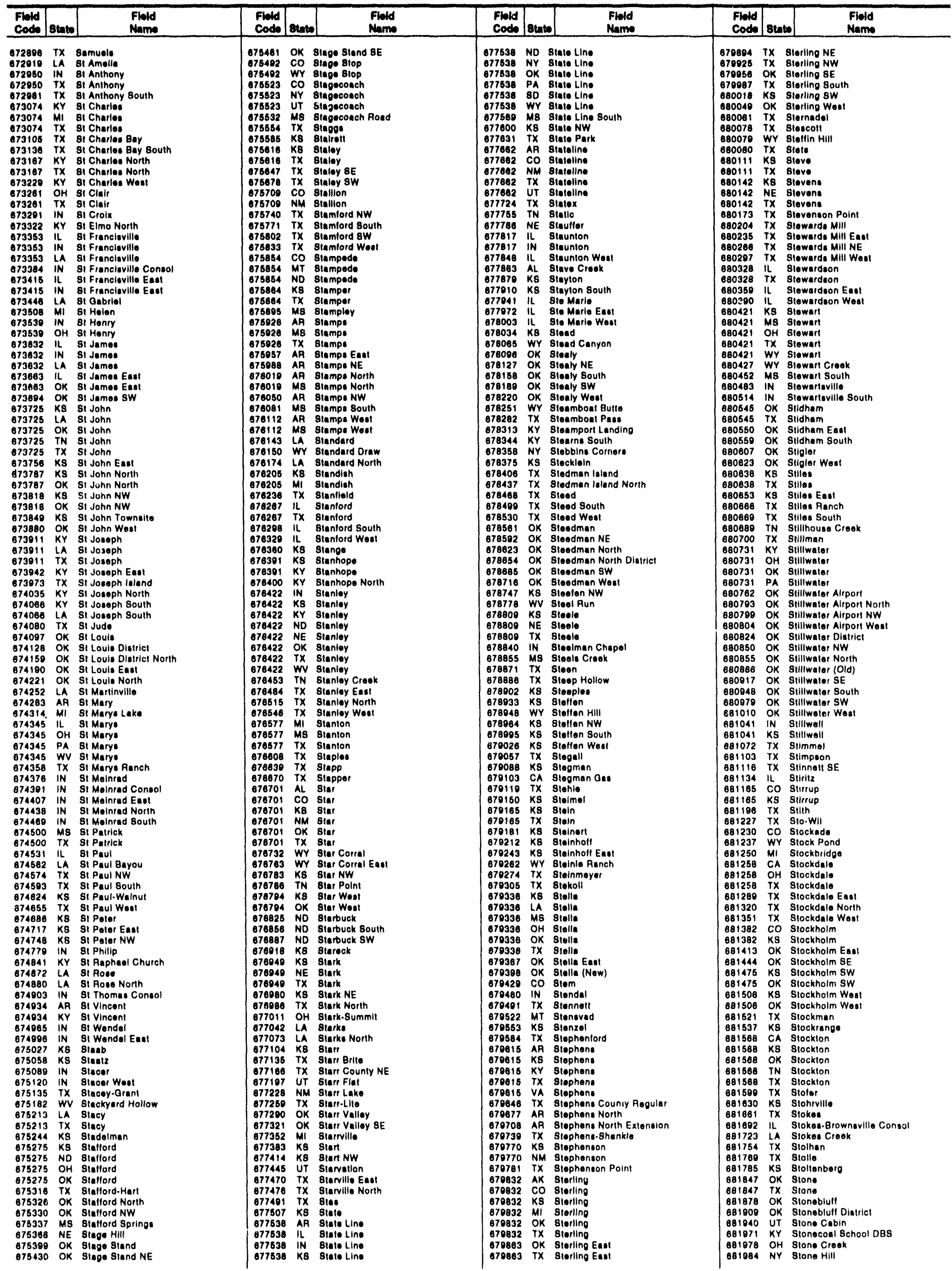




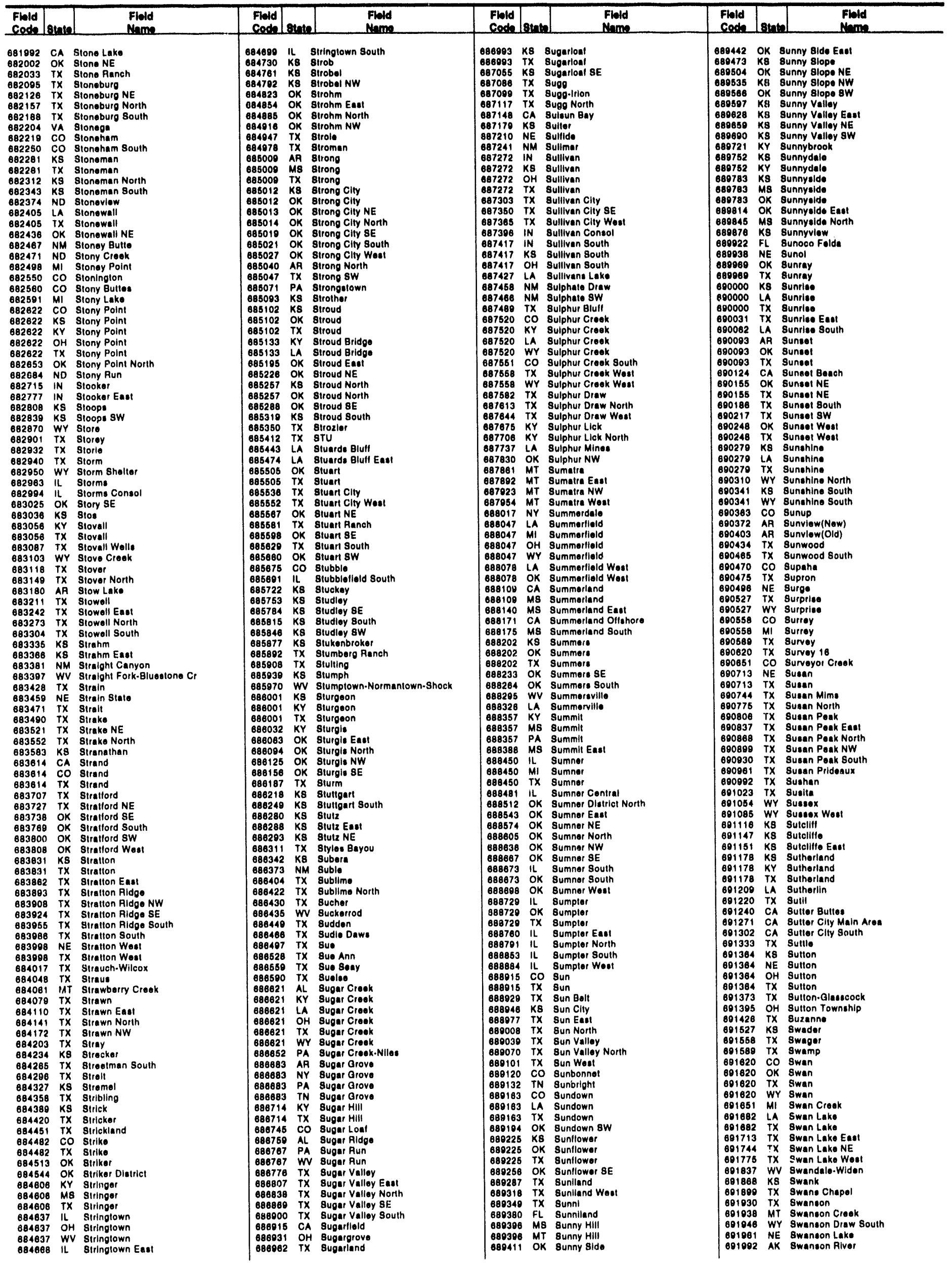




\begin{tabular}{|c|c|c|c|c|c|c|c|c|c|c|c|}
\hline $\begin{array}{l}\text { Flovid } \\
\text { Code }\end{array}$ & State & $\begin{array}{l}\text { Fild } \\
\text { Name }\end{array}$ & $\begin{array}{l}\text { Findd } \\
\text { code }\end{array}$ & Einel & $\begin{array}{l}\text { Field } \\
\text { Nome }\end{array}$ & $\begin{array}{l}\text { Fild } \\
\text { Code }\end{array}$ & onele & $\begin{array}{l}\text { Flold } \\
\text { Name }\end{array}$ & $\begin{array}{l}\text { Filid } \\
\text { Coded } \\
\end{array}$ & Sowe & $\begin{array}{l}\text { Fibld } \\
\text { Name } \\
\end{array}$ \\
\hline 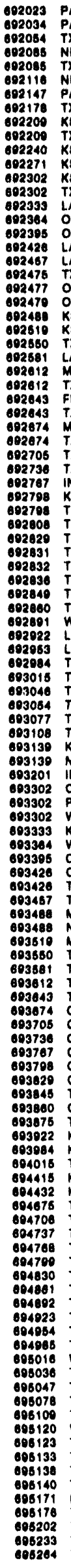 & 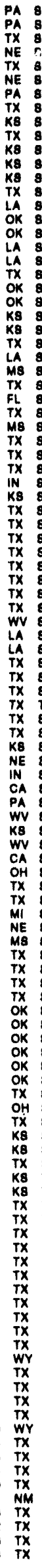 & 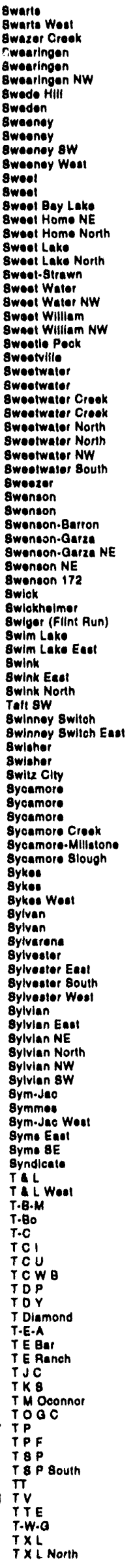 & 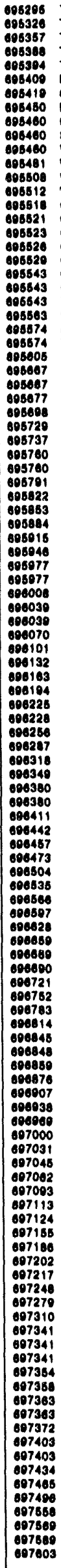 & 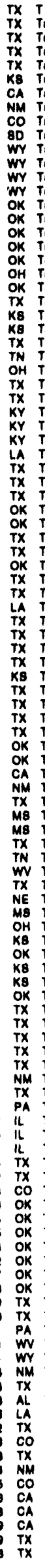 & 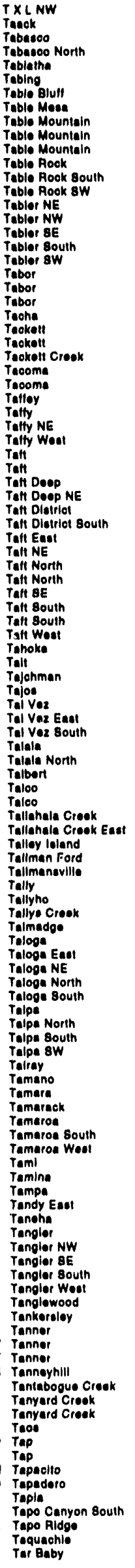 & 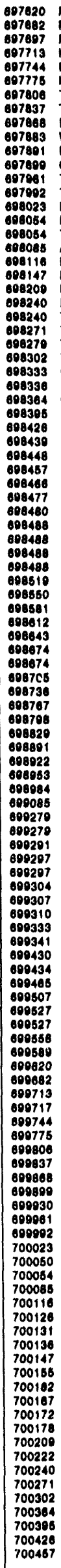 & 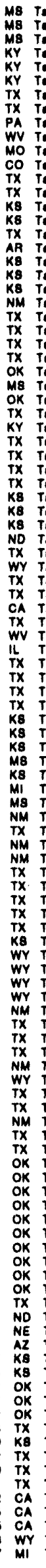 & 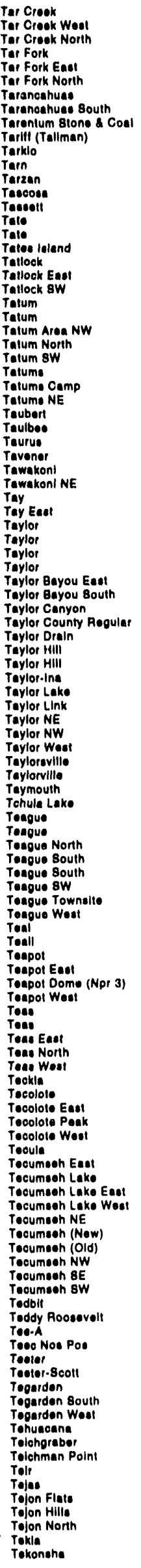 & 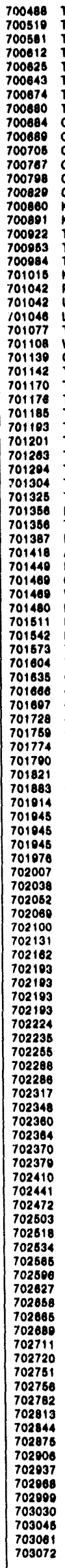 & 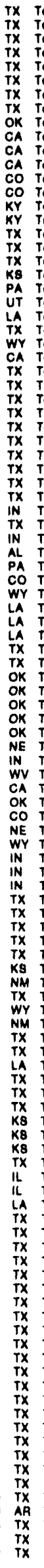 & 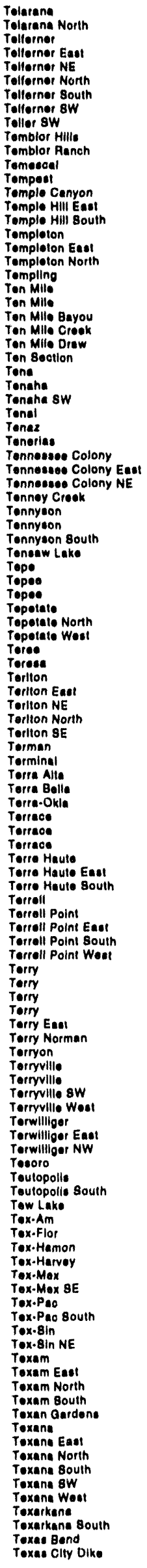 \\
\hline
\end{tabular}




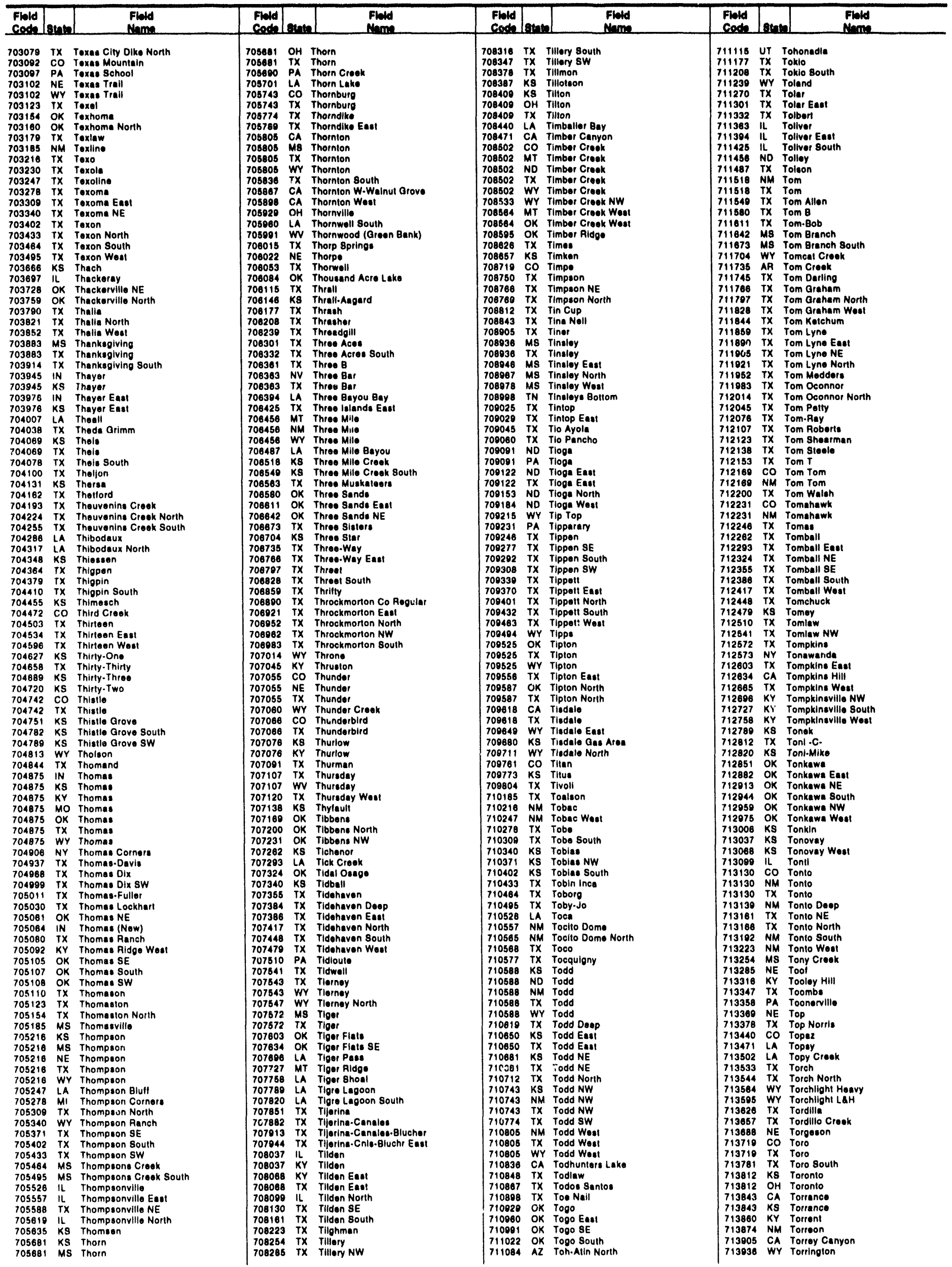




\begin{tabular}{|c|c|c|c|c|c|c|c|c|c|c|c|}
\hline $\begin{array}{l}\text { Fild } \\
\text { Coode }\end{array}$ & state & $\begin{array}{l}\text { Fileld } \\
\text { Hamo }\end{array}$ & $\begin{array}{l}\text { Field } \\
\text { Code }\end{array}$ & Etatol & $\begin{array}{l}\text { Fild } \\
\text { Name }\end{array}$ & $\begin{array}{l}\text { Finld } \\
\text { Code }\end{array}$ & Etato & $\begin{array}{l}\text { Fild } \\
\text { Name }\end{array}$ & $\begin{array}{l}\text { Fild } \\
\text { Code }\end{array}$ & etine & $\begin{array}{l}\text { Field } \\
\text { Name } \\
\end{array}$ \\
\hline
\end{tabular}




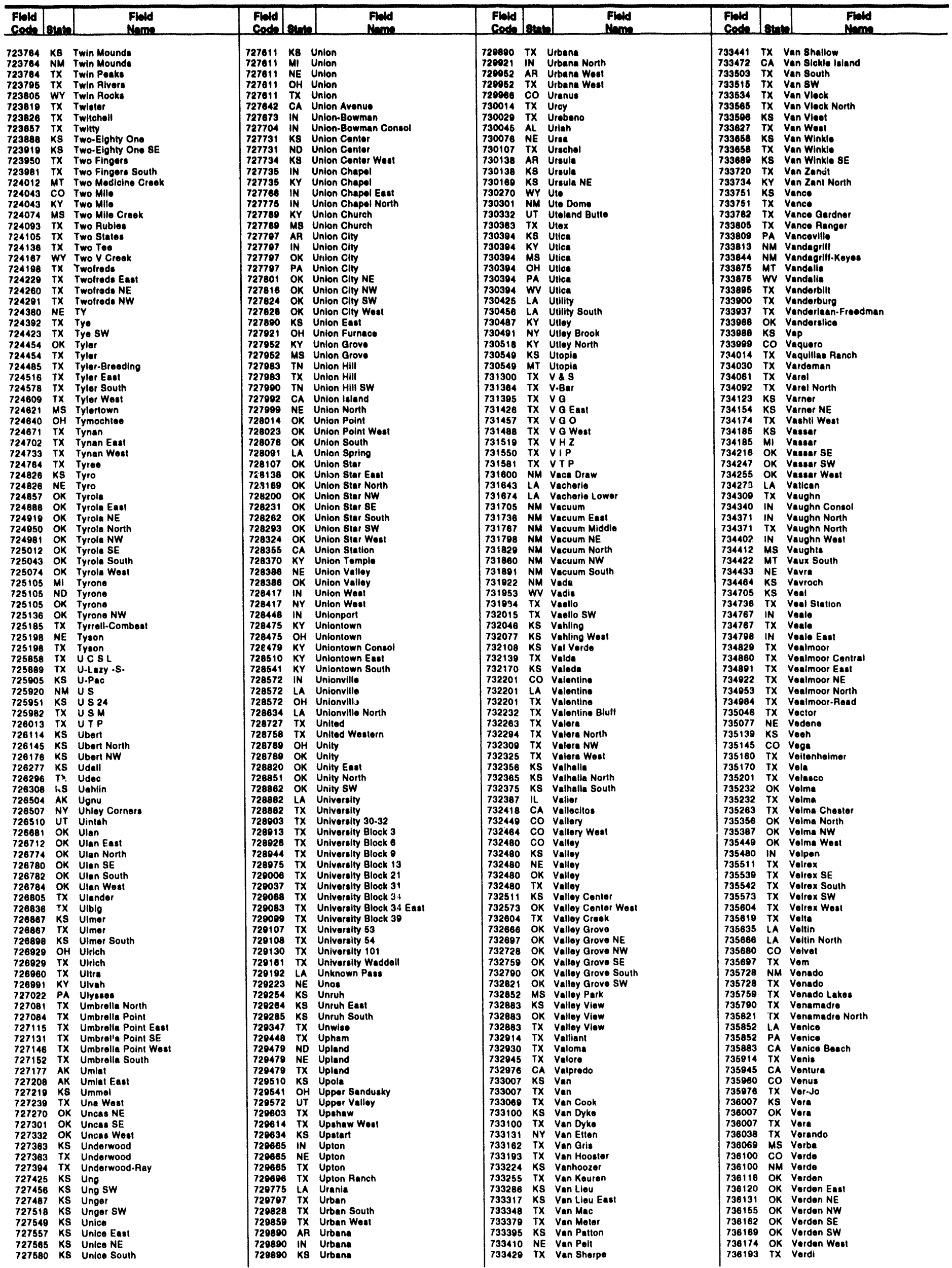




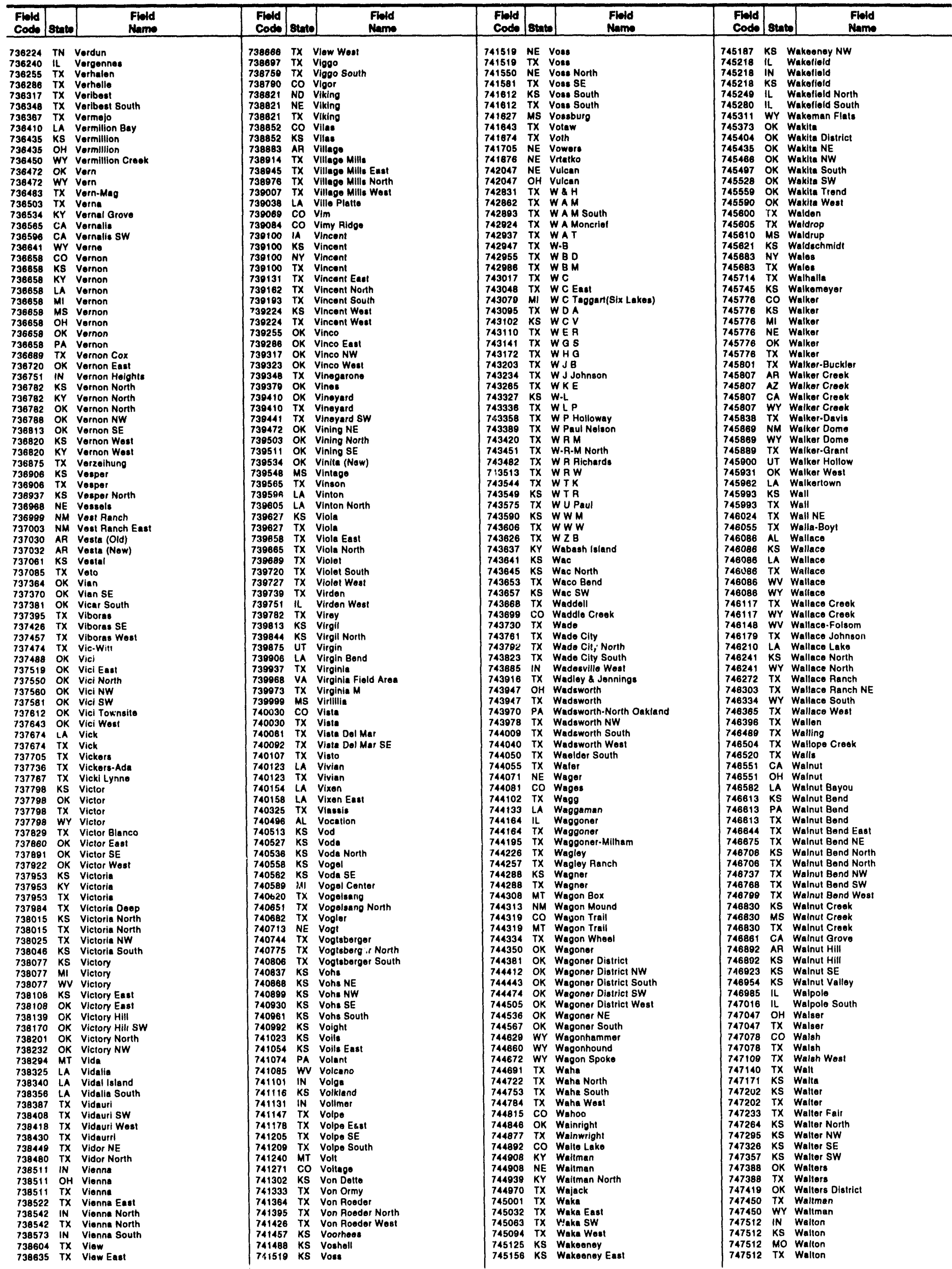




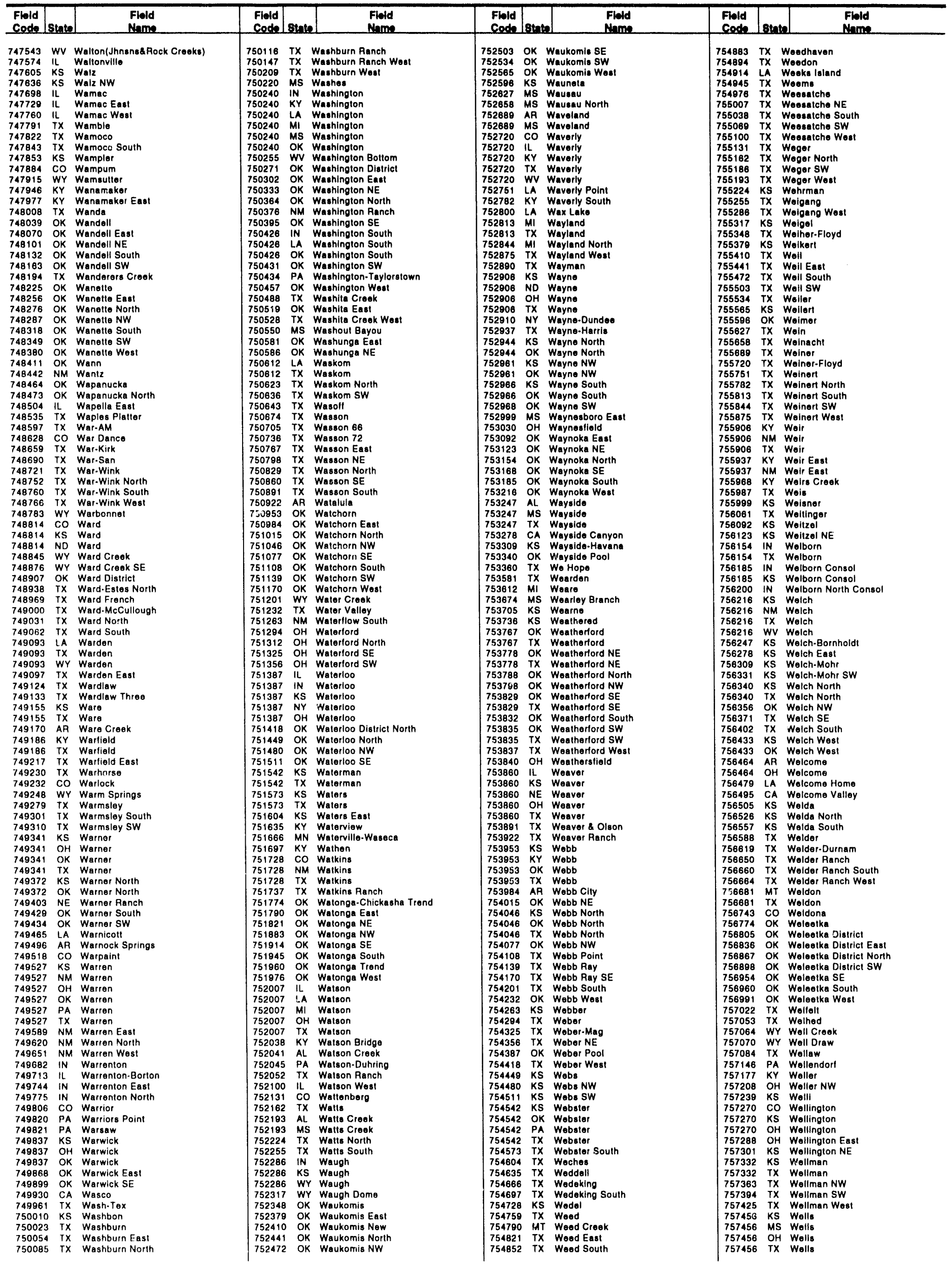




\begin{tabular}{|c|c|c|c|c|c|c|c|c|c|c|c|}
\hline $\begin{array}{l}\text { Filld } \\
\text { Coode }\end{array}$ & 8tate & $\begin{array}{l}\text { Field } \\
\text { Namo }\end{array}$ & $\begin{array}{l}\text { Field } \\
\text { Code }\end{array}$ & 8tate & $\begin{array}{l}\text { Ficld } \\
\text { Name } \\
\end{array}$ & $\begin{array}{l}\text { Fiold } \\
\text { Codte }\end{array}$ & State & $\begin{array}{l}\text { Fild } \\
\text { Namo } \\
\end{array}$ & $\begin{array}{l}\text { Findd } \\
\text { Code }\end{array}$ & Stato & $\begin{array}{l}\text { Field } \\
\text { Name } \\
\end{array}$ \\
\hline 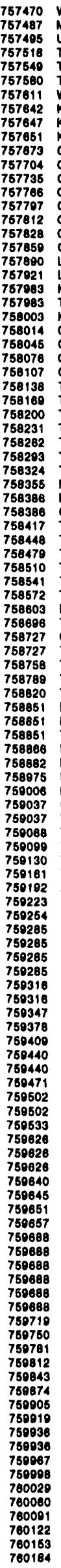 & 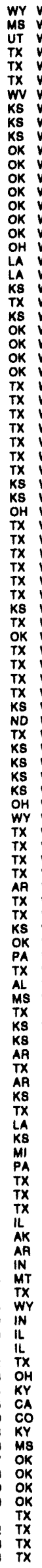 & 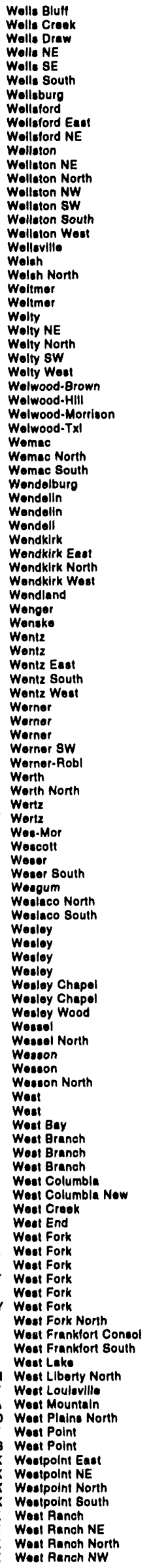 & 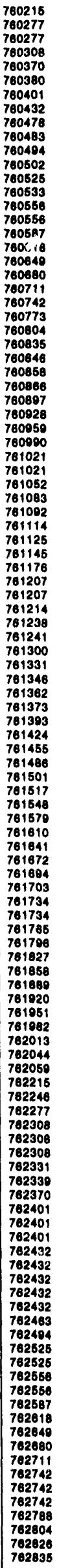 & 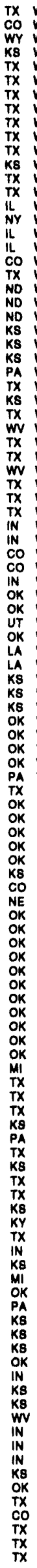 & 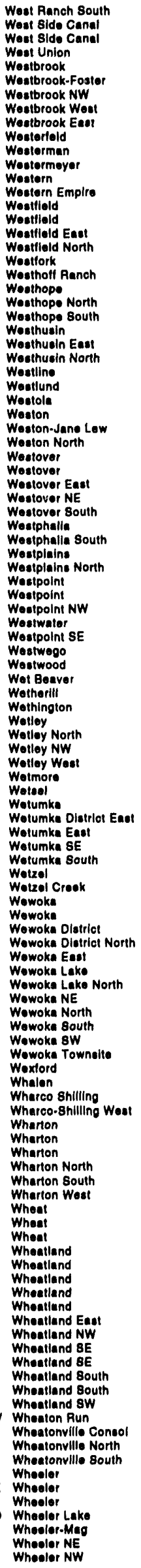 & 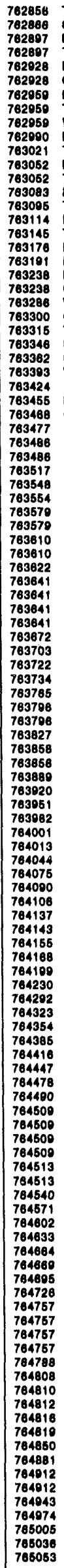 & 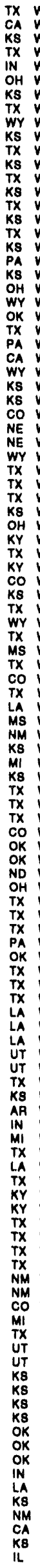 & 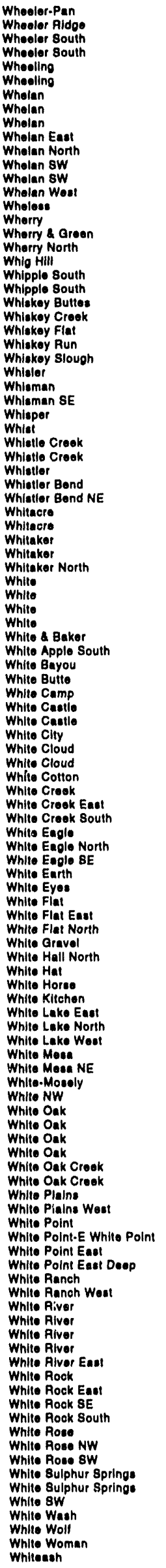 & 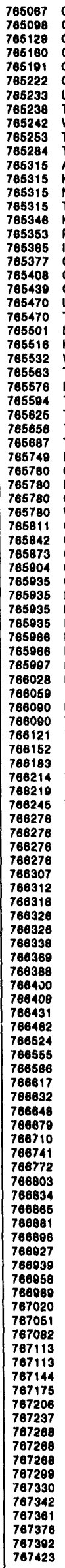 & 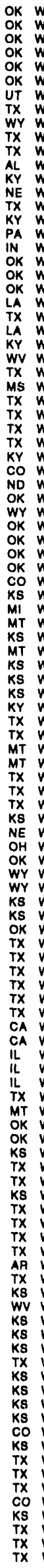 & 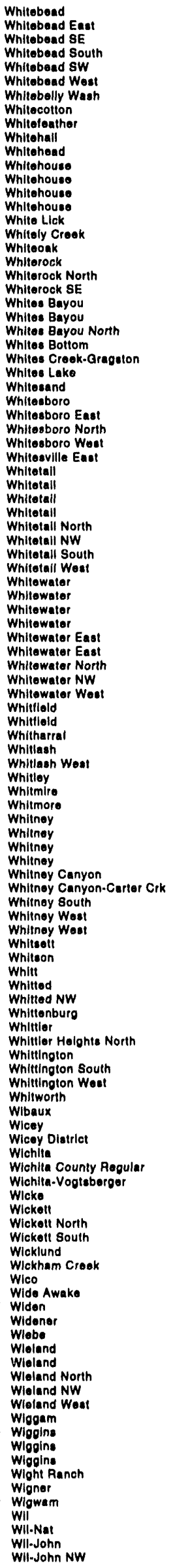 \\
\hline
\end{tabular}




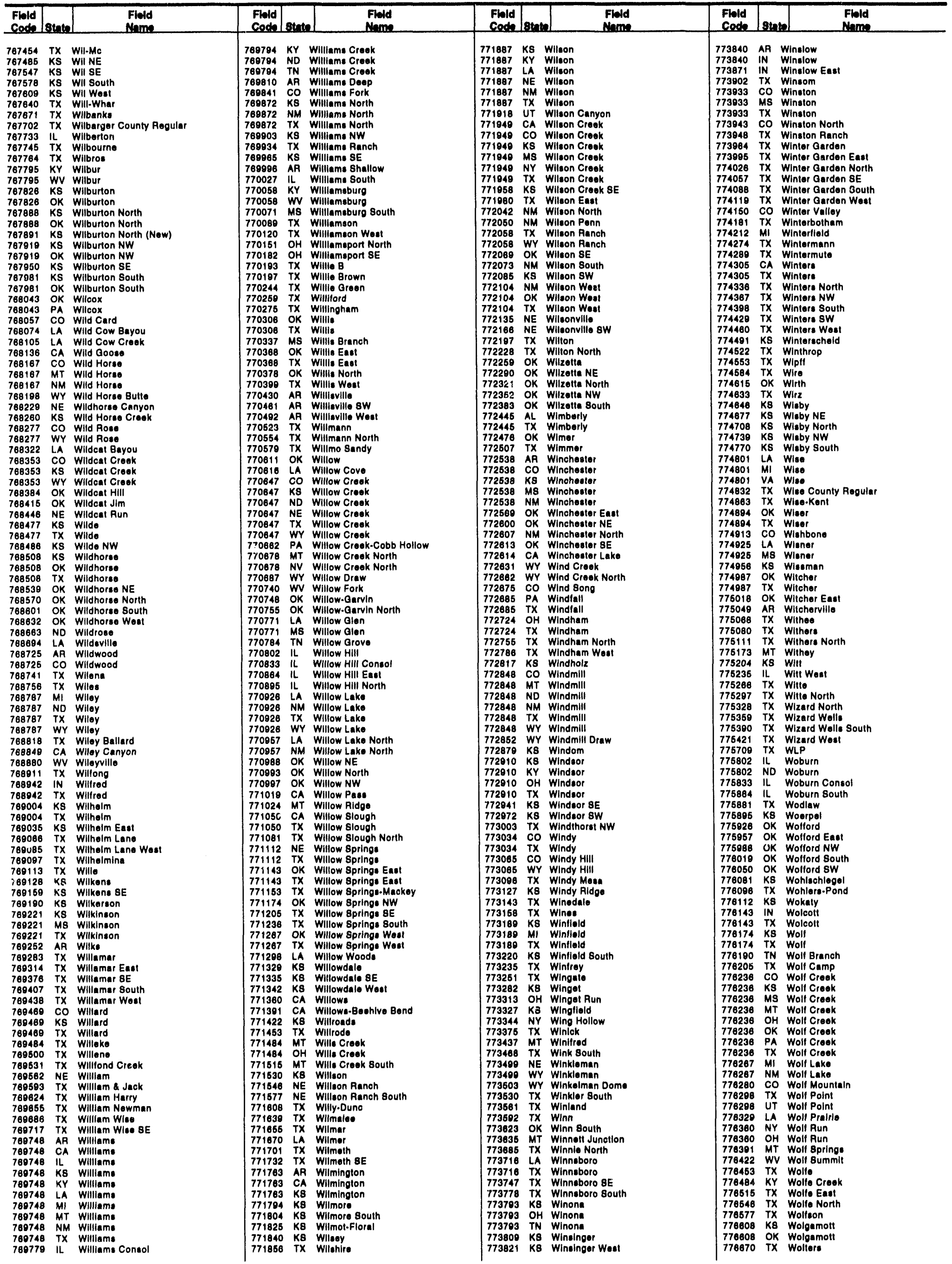




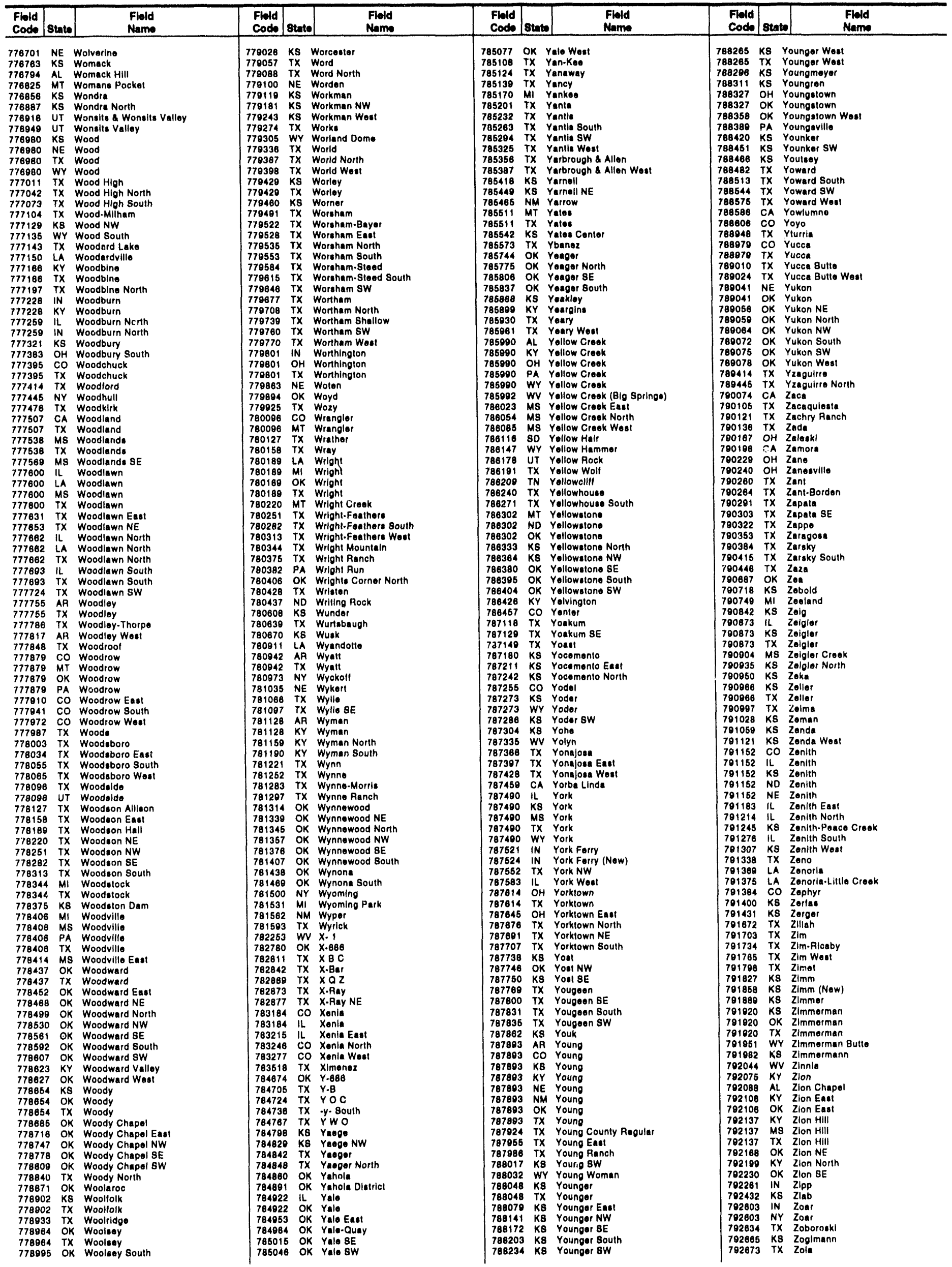




\begin{tabular}{|c|c|c|c|c|}
\hline $\begin{array}{l}\text { Fiold } \\
\text { Ceode }\end{array}$ & Sintel & $\begin{array}{l}\text { Fleld } \\
\text { Neme }\end{array}$ & $\begin{array}{l}\text { Fild } \\
\text { Coded }\end{array}$ & in \\
\hline $\begin{array}{l}792686 \\
792727\end{array}$ & $\begin{array}{l}\text { TX } \\
\text { TX }\end{array}$ & $\begin{array}{l}\text { Zoller } \\
\text { Zoller East }\end{array}$ & $\begin{array}{l}805544 \\
805550\end{array}$ & $\begin{array}{l}T X \\
T X\end{array}$ \\
\hline 792758 & $T \hat{x}$ & Zoller North & 805552 & $T X$ \\
\hline $\begin{array}{l}792789 \\
702820\end{array}$ & $T X$ & Zollor SE & 805572 & $T x$ \\
\hline $\begin{array}{l}792851 \\
792866\end{array}$ & $\begin{array}{l}\text { WV } \\
T X\end{array}$ & $\begin{array}{l}\text { Zona } \\
\text { Zone } 21.8 \text { Trend }\end{array}$ & $\begin{array}{l}805583 \\
805613\end{array}$ & $\begin{array}{l}\text { TX } \\
\text { TX }\end{array}$ \\
\hline $\begin{array}{l}792882 \\
792913\end{array}$ & $\hat{\mathrm{KS}}$ & Zook & $\begin{array}{l}805613 \\
806386\end{array}$ & $\begin{array}{l}T X \\
T x\end{array}$ \\
\hline $\begin{array}{l}792913 \\
792944\end{array}$ & $\begin{array}{l}\text { KS } \\
\text { KS }\end{array}$ & $\begin{array}{l}\text { Zook North } \\
\text { Zook NW }\end{array}$ & 806402 & $\underline{T x}$ \\
\hline $\begin{array}{l}792944 \\
792975 \\
7930006\end{array}$ & $\begin{array}{l}\text { KS } \\
\text { KS }\end{array}$ & $\begin{array}{l}\text { Zook NW } \\
\text { Zook SE }\end{array}$ & $\begin{array}{l}806409 \\
806488\end{array}$ & $\begin{array}{l}T X \\
T X\end{array}$ \\
\hline $\begin{array}{l}793000 \\
703037\end{array}$ & KS & Zook South & $\begin{array}{l}808488 \\
808512\end{array}$ & $\begin{array}{l}\text { TX } \\
T X\end{array}$ \\
\hline $\begin{array}{l}793037 \\
793100\end{array}$ & co & Zorichak & 806513 & $T x$ \\
\hline $\begin{array}{l}793100 \\
793418\end{array}$ & $\begin{array}{l}\text { TX } \\
\text { KS }\end{array}$ & $\begin{array}{l}\text { Zourette } \\
\text { Zuerchei }\end{array}$ & & TX \\
\hline $\begin{array}{l}793418 \\
793422\end{array}$ & $\begin{array}{l}\text { KS } \\
\text { TX }\end{array}$ & $\begin{array}{l}\text { Zuercher } \\
\text { Zuhona }\end{array}$ & $\begin{array}{l}808533 \\
807047\end{array}$ & $\begin{array}{l}\text { TX } \\
\text { TX }\end{array}$ \\
\hline 703436 & $T x$ & Zula & 807051 & $T x$ \\
\hline $\begin{array}{l}793443 \\
793440\end{array}$ & $T X$ & Zulch North & 007052 & $T X$ \\
\hline $\begin{array}{l}793448 \\
793480\end{array}$ & $T X$ & $\begin{array}{l}\text { Zulotte } \\
\text { Zumwalt }\end{array}$ & $\begin{array}{l}807053 \\
807065\end{array}$ & $\begin{aligned} T X \\
T X\end{aligned}$ \\
\hline $\begin{array}{l}793480 \\
793511\end{array}$ & $\begin{array}{l}T X \\
\mathrm{KS}\end{array}$ & $\begin{array}{l}\text { Zumwalt } \\
\text { Zurich }\end{array}$ & $\begin{array}{l}807065 \\
807088\end{array}$ & $\begin{array}{l}T X \\
T X\end{array}$ \\
\hline 793542 & KS & Zurich SW & 807070 & Tx \\
\hline 783573 & KS & Zurlch Townalte & 807076 & $T X$ \\
\hline $\begin{array}{l}793582 \\
793588\end{array}$ & KS & $\begin{array}{l}\text { Zurich Townsite SE } \\
\text { Zurich Townsite Weat }\end{array}$ & 807102 & $T x$ \\
\hline $\begin{array}{l}793588 \\
793674\end{array}$ & $\begin{array}{l}\text { KS } \\
\text { LA }\end{array}$ & $\begin{array}{l}\text { Zurich Townsite West } \\
\text { Zwollo }\end{array}$ & $\begin{array}{l}807104 \\
807105\end{array}$ & $\begin{array}{l}T X \\
T X\end{array}$ \\
\hline 793775 & KS & Zyba & 807106 & $T x$ \\
\hline Pyjouso & ks & Zybs sW & 807132 & $T X$ \\
\hline $\begin{array}{l}793868 \\
901023\end{array}$ & $T X$ & Zybach & 807133 & $T x$ \\
\hline $\begin{array}{l}901023 \\
81: 047\end{array}$ & $\begin{array}{l}T X \\
T X\end{array}$ & $\begin{array}{l}\text { Bay City Blk } 23 \\
\text { Bay City Blk } 47\end{array}$ & $\begin{array}{l}810001 \\
810002\end{array}$ & LA \\
\hline $\begin{array}{l}81: 047 \\
80 ; 012\end{array}$ & $\begin{array}{l}T X \\
\text { LA }\end{array}$ & $\begin{array}{l}\text { Bay City Blk } 47 \\
\text { Bay Marchand Blk } 2\end{array}$ & $\begin{array}{l}810002 \\
810012\end{array}$ & LA \\
\hline 803003 & LA & Bay Marchand BIK 3 & 810017 & LA \\
\hline $\begin{array}{l}803004 \\
803005\end{array}$ & LA & $\begin{array}{l}\text { Bay Marchand Blk } 4 \\
\text { Bay Marchand Blk } 5\end{array}$ & $\begin{array}{l}810018 \\
810018\end{array}$ & LA \\
\hline $\begin{array}{l}803005 \\
803006\end{array}$ & LA & $\begin{array}{l}\text { Bay Marchand Blk } 5 \\
\text { Bey Marchand Blk } 6\end{array}$ & $\begin{array}{l}810019 \\
810020\end{array}$ & LA \\
\hline $\begin{array}{l}805001 \\
805002\end{array}$ & Tx & Brazoe BIK A1 & 810021 & LA \\
\hline $\begin{array}{l}805002 \\
805007\end{array}$ & $T x$ & $\begin{array}{l}\text { Brazos Blk } A 2 \\
\text { Brazos Blk } A 7\end{array}$ & $\begin{array}{l}810023 \\
810030\end{array}$ & LA \\
\hline $\begin{array}{l}805007 \\
805018\end{array}$ & $\begin{array}{l}T X \\
T X\end{array}$ & $\begin{array}{l}\text { Brazos B1k A7 } \\
\text { Brazos B1k A16 }\end{array}$ & $\begin{array}{l}810030 \\
810031\end{array}$ & LA \\
\hline $\begin{array}{l}805018 \\
805017\end{array}$ & $\begin{array}{l}\text { TX } \\
T x\end{array}$ & $\begin{array}{l}\text { Brazos Bik A16 } \\
\text { Brazos Bik A17 }\end{array}$ & $\begin{array}{l}810031 \\
810032\end{array}$ & LA \\
\hline $\begin{array}{l}805019 \\
805020\end{array}$ & $T x$ & Brazo Blk A19 & 810033 & LA \\
\hline $\begin{array}{l}805020 \\
805021\end{array}$ & $T X$ & Brazos B1k A2O & 810036 & LA \\
\hline 805021 & $T X$ & Brazos BIK A21 & 810037 & LA \\
\hline $\begin{array}{l}805022 \\
805023\end{array}$ & $T X$ & Brazos B1k A22 & 810039 & LA \\
\hline $\begin{array}{l}805023 \\
805024\end{array}$ & $T_{-x}^{x}$ & $\begin{array}{l}\text { Brazos Bik } 123 \\
\text { Brazos Bik } A 24\end{array}$ & 810045 & LA \\
\hline $\begin{array}{l}805024 \\
805028\end{array}$ & $T X$ & $\begin{array}{l}\text { Brazos Bik A24 } \\
\text { Brazos B1k A28 }\end{array}$ & $\begin{array}{l}810049 \\
810052\end{array}$ & LA \\
\hline $\begin{array}{l}805028 \\
805037\end{array}$ & $\begin{array}{l}T X \\
T X\end{array}$ & & $\begin{array}{l}810052 \\
810053\end{array}$ & LA \\
\hline 805039 & Tx & $\begin{array}{l}\text { Brazos Bik A37 } \\
\text { Brazos Blk A39 }\end{array}$ & $\begin{array}{l}810053 \\
810060\end{array}$ & $L A$ \\
\hline 805040 & ix & Brazos Blk A40 & 810064 & $L A$ \\
\hline 605309 & $T X$ & Brazos Bik 309 & 810065 & LA \\
\hline $\begin{array}{l}805335 \\
805336\end{array}$ & $\tau x$ & Brazos Blk 335 & 814014 & LA \\
\hline $\begin{array}{l}805338 \\
805338\end{array}$ & $T X$ & Brazos Blk 338 & 814015 & LA \\
\hline $\begin{array}{l}805338 \\
805340\end{array}$ & $\begin{array}{l}T X \\
T X\end{array}$ & $\begin{array}{l}\text { Brazos Blk } 338 \\
\text { Brazos Blk } 340\end{array}$ & 814017 & LA \\
\hline $\begin{array}{l}805340 \\
805364\end{array}$ & $\begin{array}{l}T X \\
T X\end{array}$ & $\begin{array}{l}\text { Brazos Blk } 340 \\
\text { Brazos Blk } 364\end{array}$ & $\begin{array}{l}814018 \\
814022\end{array}$ & $\stackrel{L A}{L A}$ \\
\hline $\begin{array}{l}805365 \\
805386\end{array}$ & Tx & $\begin{array}{l}\text { Brazos Bik } 364 \\
\text { Brazos Blk } 365\end{array}$ & 814024 & LA \\
\hline $\begin{array}{l}805366 \\
805367\end{array}$ & $T X$ & Brazos Blk 386 & 814025 & LA \\
\hline $\begin{array}{l}805367 \\
805368\end{array}$ & IX & Brazo: B1k 387 & 814028 & LA \\
\hline $\begin{array}{l}805368 \\
805368\end{array}$ & $T X$ & $\begin{array}{l}\text { Brazos Blk } 388 \\
\text { Brazos Bik } 360\end{array}$ & 814029 & $L A$ \\
\hline $\begin{array}{l}805389 \\
805374\end{array}$ & $\begin{array}{l}\text { TX } \\
T X\end{array}$ & $\begin{array}{l}\text { Brazos 日ik } 369 \\
\text { Brazoos dik } 374\end{array}$ & $\begin{array}{l}814030 \\
814031\end{array}$ & $L_{A}$ \\
\hline 805375 & TX & Biazoo Blk 375 & $\begin{array}{l}814031 \\
814034\end{array}$ & LA \\
\hline 805376 & $T x$ & Brazos Bik 376 & 816037 & LA \\
\hline $\begin{array}{l}805398 \\
805307\end{array}$ & $T X$ & Brazos Blk 396 & 816038 & LA \\
\hline $\begin{array}{l}805307 \\
805308\end{array}$ & & Brazos Blk 397 & 816040 & $L A$ \\
\hline $\begin{array}{l}805308 \\
805399\end{array}$ & $\begin{array}{l}T X \\
T X\end{array}$ & $\begin{array}{l}\text { Brazo Blk } 398 \\
\text { Brazos Blk } 399\end{array}$ & $\begin{array}{l}818041 \\
818015\end{array}$ & $L_{A}$ \\
\hline 805403 & & $\begin{array}{l}\text { Brazos Blk } 399 \\
\text { Brazos Blk } 403\end{array}$ & $\begin{array}{l}818015 \\
818025\end{array}$ & $L A$ \\
\hline $\begin{array}{l}805404 \\
805405\end{array}$ & $T X$ & Biazos Blk 404 & 818028 & LA \\
\hline $\begin{array}{l}805405 \\
805406\end{array}$ & $\begin{array}{l}T X \\
T X\end{array}$ & $\begin{array}{l}\text { Brazos Blk } 405 \\
\text { Brazos 81k } 406\end{array}$ & $\begin{array}{l}818032 \\
818035\end{array}$ & LA \\
\hline $\begin{array}{l}805406 \\
805409\end{array}$ & $T x$ & $\begin{array}{l}\text { Brazos 81k } 406 \\
\text { Brazos Blk } 109\end{array}$ & $\begin{array}{l}818035 \\
818041\end{array}$ & LA \\
\hline 805411 & $\hat{T x}$ & Brazos Bik 411 & 818048 & LA \\
\hline $\begin{array}{l}805412 \\
805413\end{array}$ & $T X$ & $\begin{array}{l}\text { Brazoo Blk } 412 \\
\text { Brazos Blk } 413\end{array}$ & 818051 & LA \\
\hline $\begin{array}{l}805413 \\
805418\end{array}$ & $\begin{array}{l}T X \\
T X\end{array}$ & $\begin{array}{l}\text { Brazos Blk } 413 \\
\text { Brazos Blk } 418\end{array}$ & $\begin{array}{l}818052 \\
818054\end{array}$ & LA \\
\hline 805431 & $\mathrm{Tx}$ & $\begin{array}{l}\text { Brazos Blk } 418 \\
\text { Brazos Blk } 431\end{array}$ & $\begin{array}{l}818054 \\
818058\end{array}$ & LA \\
\hline 805432 & $T X$ & Brazol Blk 432 & 818050 & LA \\
\hline 805435 & $T X$ & Brazos Blk 435 & 818068 & LA \\
\hline 805436 & $I x$ & $\begin{array}{l}\text { Brazos Blk } 436 \\
\text { Brazon Blk } 437\end{array}$ & 818071 & LA \\
\hline $\begin{array}{l}805437 \\
806438\end{array}$ & $\begin{array}{l}T X \\
T X\end{array}$ & $\begin{array}{l}\text { Brazon Blk } 437 \\
\text { Brazo Blk } 438\end{array}$ & \begin{tabular}{|l}
818073 \\
810062
\end{tabular} & LA \\
\hline $\begin{array}{l}805438 \\
805438\end{array}$ & $\begin{array}{l}1 x \\
T x\end{array}$ & $\begin{array}{l}\text { Brazos Blk } 438 \\
\text { Brazos Blk } 439\end{array}$ & $\begin{array}{l}810082 \\
810083\end{array}$ & LA \\
\hline 805440 & $T x$ & Brazos 日1k 440 & 821001 & oa \\
\hline $\begin{array}{l}805441 \\
805442\end{array}$ & $T X$ & Brazos Blk 441 & 821002 & $O Q$ \\
\hline $\begin{array}{l}805442 \\
805445\end{array}$ & $\begin{array}{l}T X \\
T X\end{array}$ & $\begin{array}{l}\text { Brazos Blk } 442 \\
\text { Brazos Blk } 445\end{array}$ & $\begin{array}{l}821056 \\
821111\end{array}$ & $\begin{array}{l}\text { OG } \\
\text { OG }\end{array}$ \\
\hline $\begin{array}{l}805445 \\
805446\end{array}$ & $T \hat{x}$ & $\begin{array}{l}\text { Brazos Blk } 445 \\
\text { Brazos Bik } 446\end{array}$ & 822100 & \\
\hline 805448 & $T X$ & Brazos BIK 448 & 822110 & $T x$ \\
\hline 805450 & $T X$ & Brazos Blk 450 & 822112 & $T X$ \\
\hline $\begin{array}{l}805481 \\
805462\end{array}$ & $\begin{array}{l}T X \\
T X\end{array}$ & $\begin{array}{l}\text { Brazos Blk } 451 \\
\text { Brazos Blk } 452\end{array}$ & $\begin{array}{l}822117 \\
822154\end{array}$ & $\begin{array}{l}T X \\
T X\end{array}$ \\
\hline $\begin{array}{l}805452 \\
805453\end{array}$ & $T x$ & $\begin{array}{l}\text { Brazos Blk } 452 \\
\text { Brazos Blk } 453\end{array}$ & 822157 & $T X$ \\
\hline 805455 & $T \hat{x}$ & Brazos Blk 456 & 822158 & $T X$ \\
\hline 805457 & $T X$ & Brazos Blk 457 & 822159 & $T X$ \\
\hline 805475 & $T x$ & Brazos Blk 475 & 822180 & $T X$ \\
\hline 805476 & $T x$ & Brazos 81k 478 & 822161 & $T X$ \\
\hline 806470 & $T x$ & Brazos Bik 478 & 822165 & TX \\
\hline 805488 & $T X$ & $\begin{array}{l}\text { Brazos Blk } 488 \\
\text { Brazos Blk } 401\end{array}$ & \begin{tabular}{|l|}
822172 \\
822209
\end{tabular} & $\begin{array}{l}T X \\
T X\end{array}$ \\
\hline 805491 & $\begin{array}{l}T x \\
T X\end{array}$ & $\begin{array}{l}\text { Brazos Bik } 401 \\
\text { Brazos Blk } 494\end{array}$ & $\begin{array}{l}822209 \\
822377\end{array}$ & $\begin{array}{l}T X \\
T X\end{array}$ \\
\hline $\begin{array}{l}808494 \\
805501\end{array}$ & $\begin{array}{l}T X \\
T X\end{array}$ & $\begin{array}{l}\text { Brazos Blk } 494 \\
\text { Brazos Blk } 501\end{array}$ & 822688 & \\
\hline $\begin{array}{l}805501 \\
805804\end{array}$ & $\begin{array}{l}1 x \\
T x\end{array}$ & $\begin{array}{l}\text { Brazos Blk } 501 \\
\text { Brazos Blk } 504\end{array}$ & 824001 & LA \\
\hline $\begin{array}{l}805604 \\
805500\end{array}$ & $T \hat{x}$ & $\begin{array}{l}\text { Brazos Blk } 504 \\
\text { Brazos Blk } 506\end{array}$ & 824002 & LA \\
\hline $\begin{array}{l}805500 \\
805515\end{array}$ & $T \hat{X}$ & & 824004 & LA \\
\hline 805515 & $T X$ & $\begin{array}{l}\text { Brazoe B1k } 515 \\
\text { Brazos Blk 519S }\end{array}$ & 824000 & $\hat{\Lambda A}$ \\
\hline $\begin{array}{l}805510 \\
805538\end{array}$ & $\begin{array}{l}T X \\
T X\end{array}$ & $\begin{array}{l}\text { Brazos Blk 519S } \\
\text { Brazos Blk } 538\end{array}$ & $\begin{array}{l}824008 \\
824014\end{array}$ & LA \\
\hline $\begin{array}{l}805638 \\
805841\end{array}$ & $7 x$ & $\begin{array}{l}\text { Brazos Bik } 538 \\
\text { Brazos B1k } 541\end{array}$ & 824017 & LA \\
\hline 805542 & $T x$ & Brazos Blk 542 & 824023 & LA \\
\hline
\end{tabular}




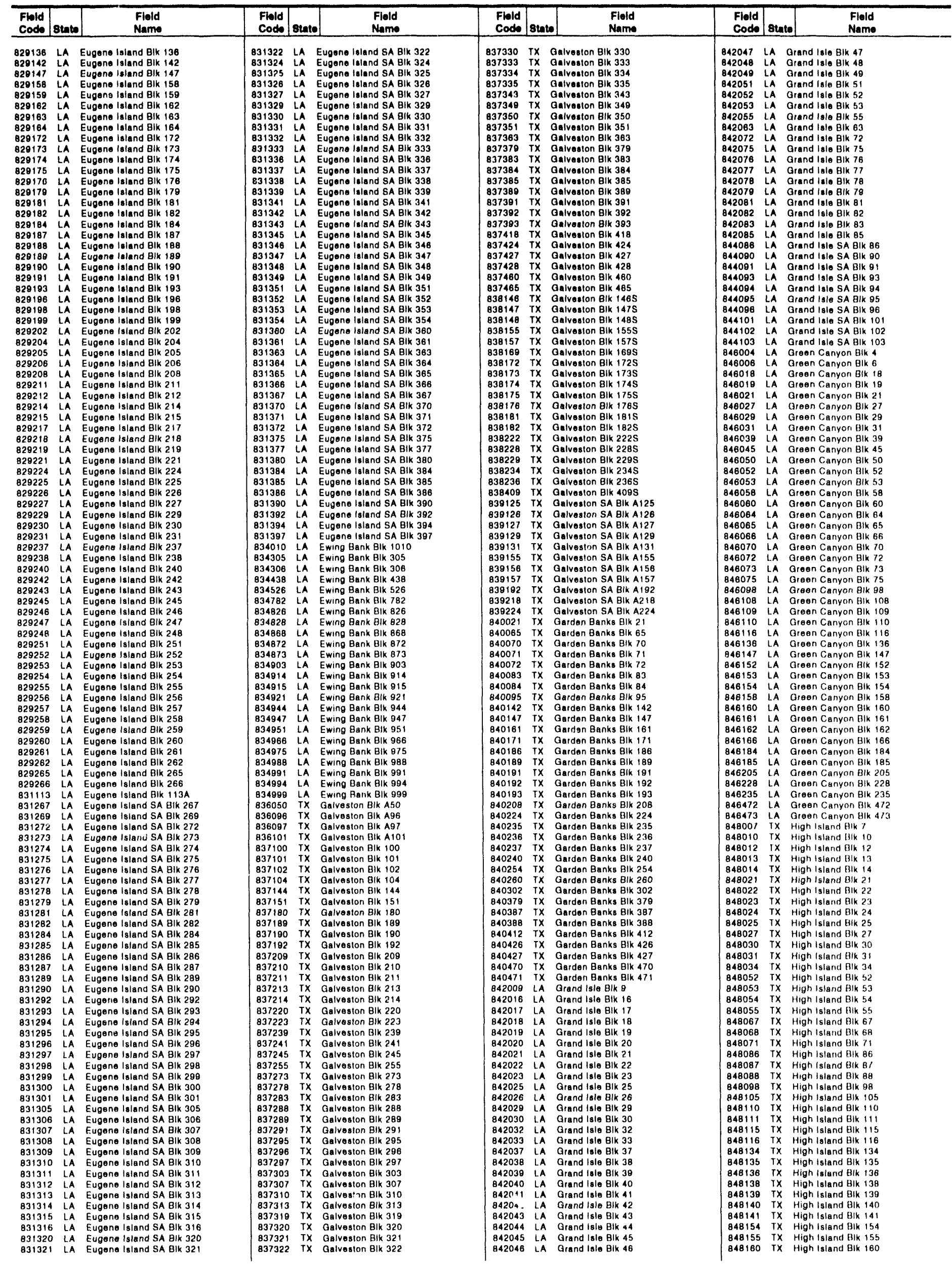




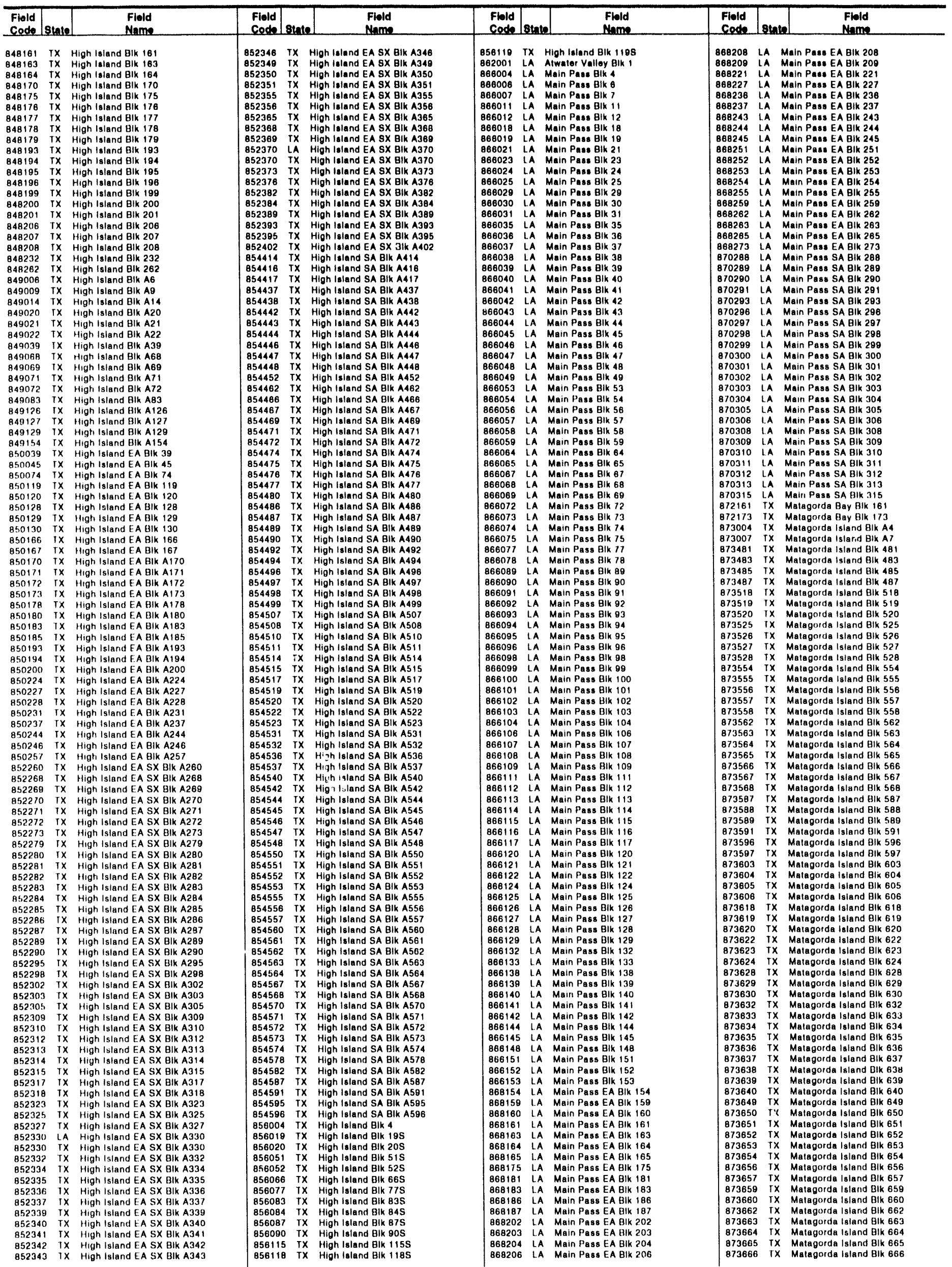




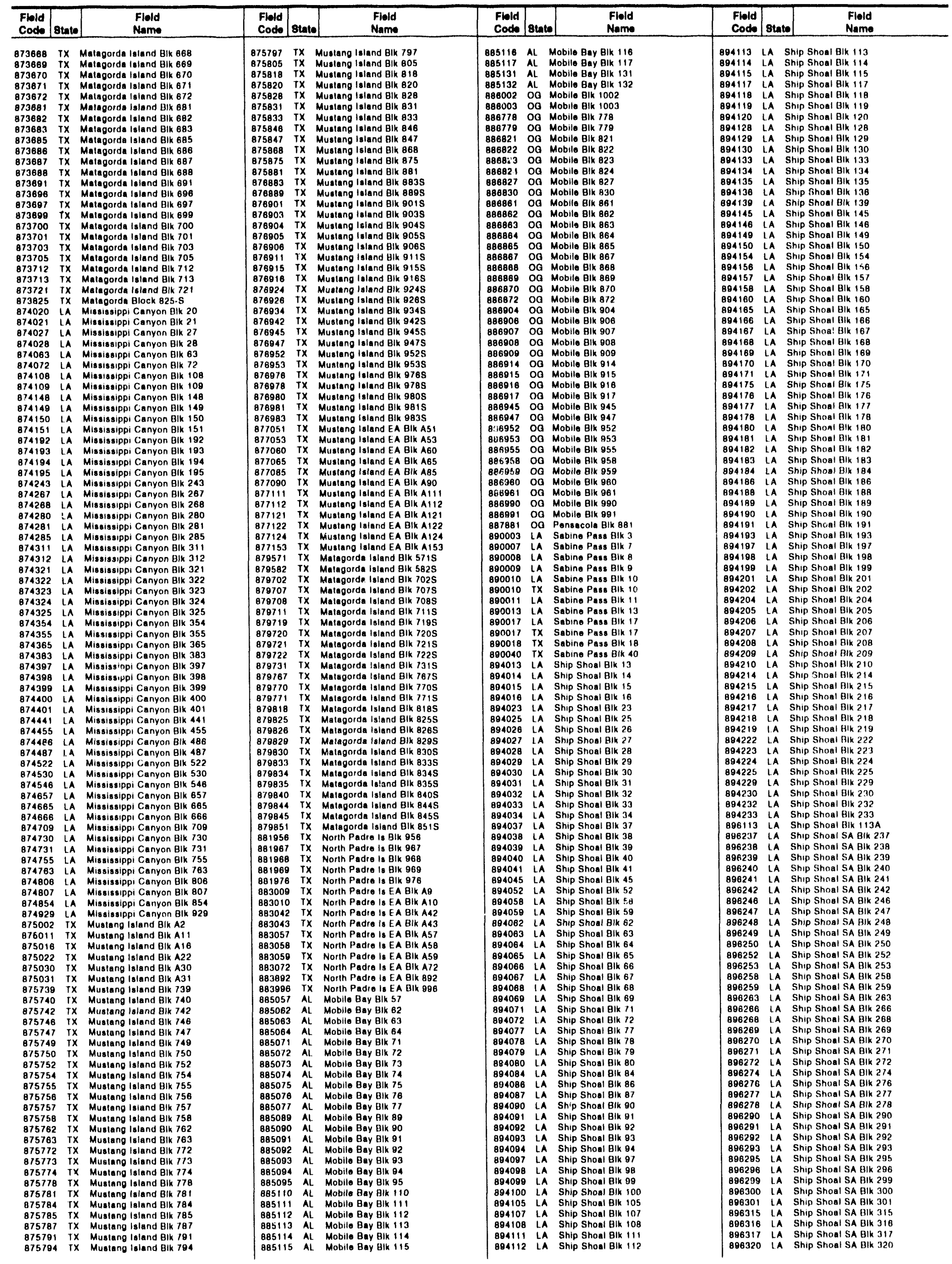




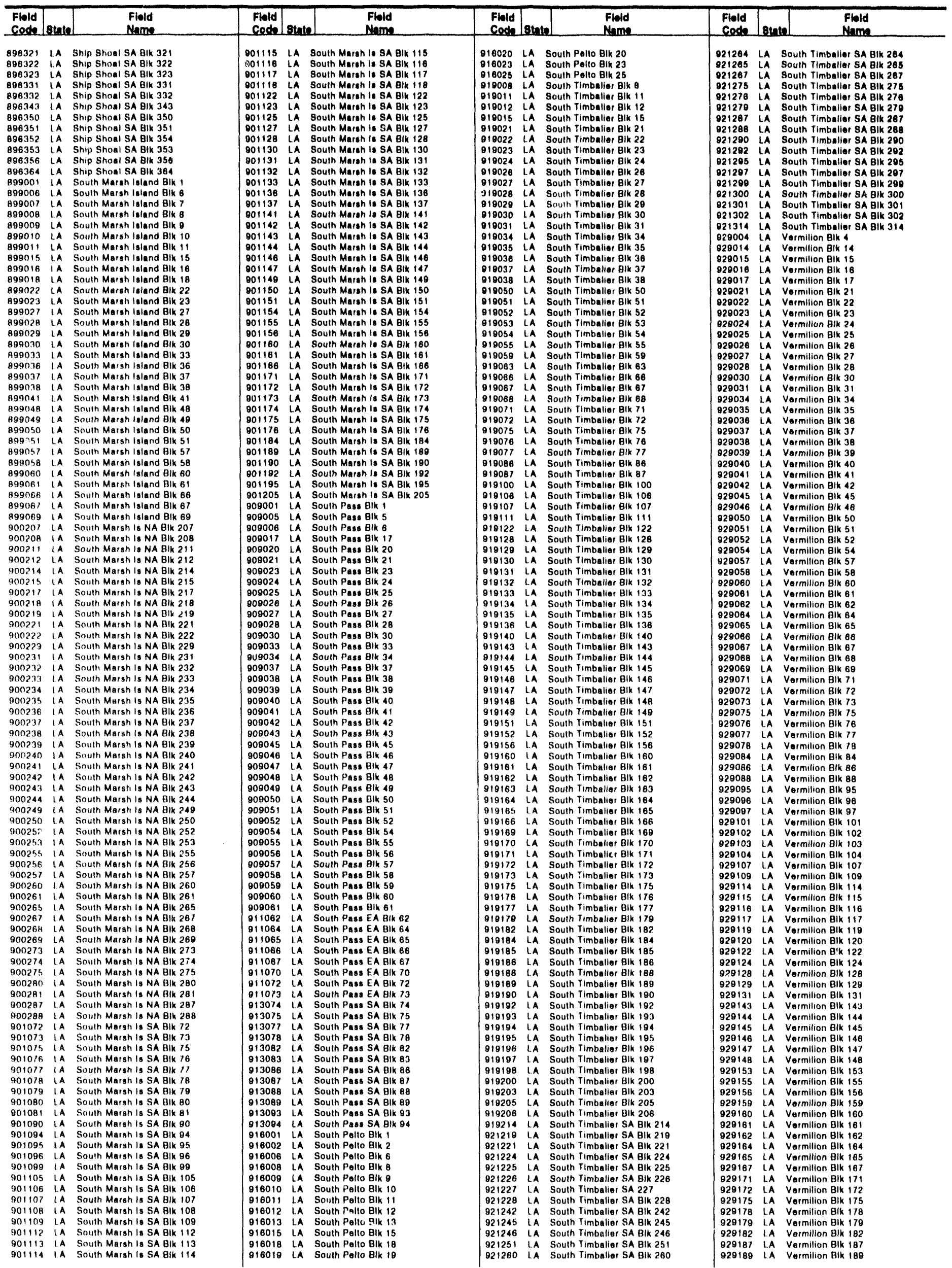




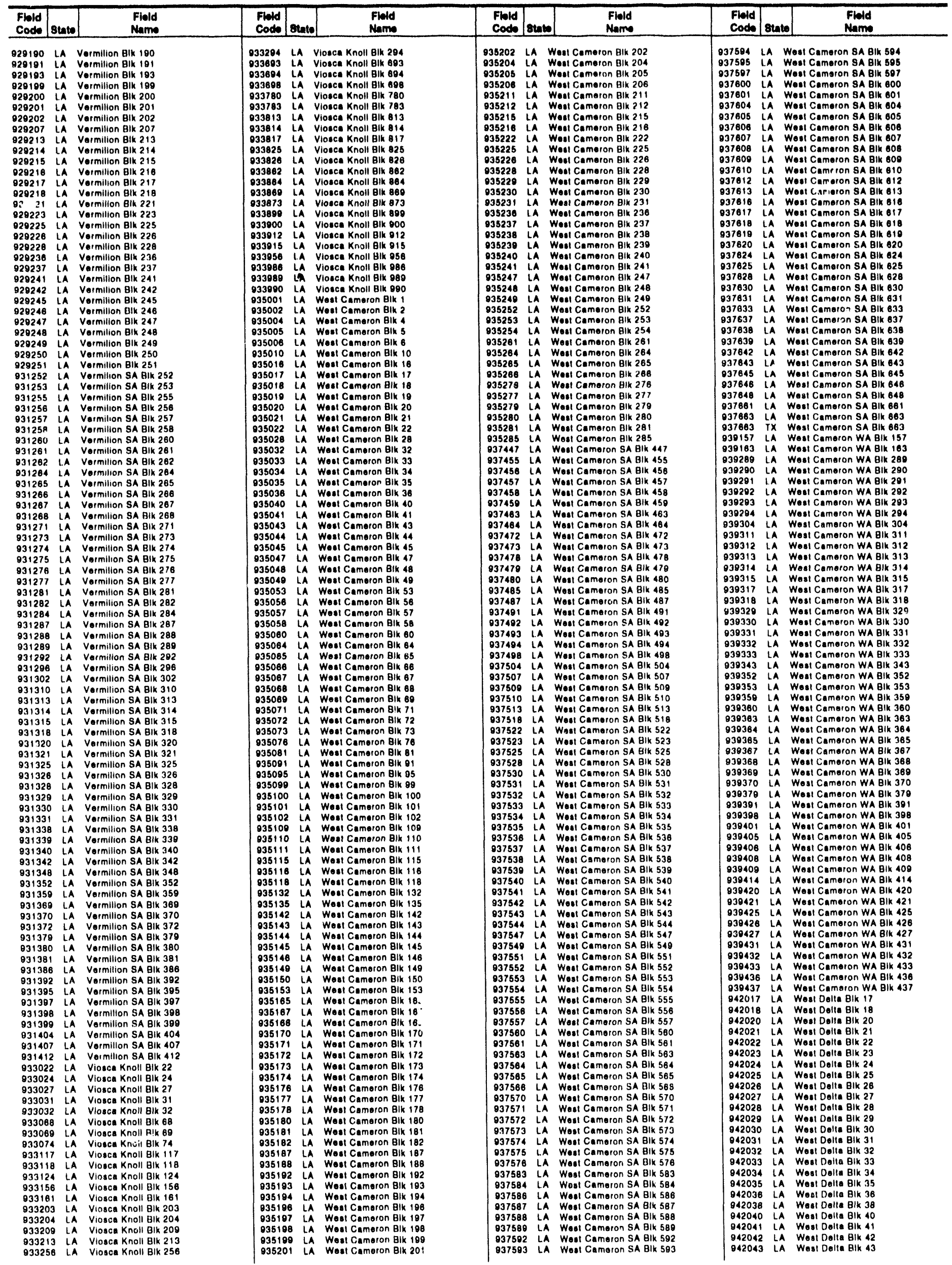




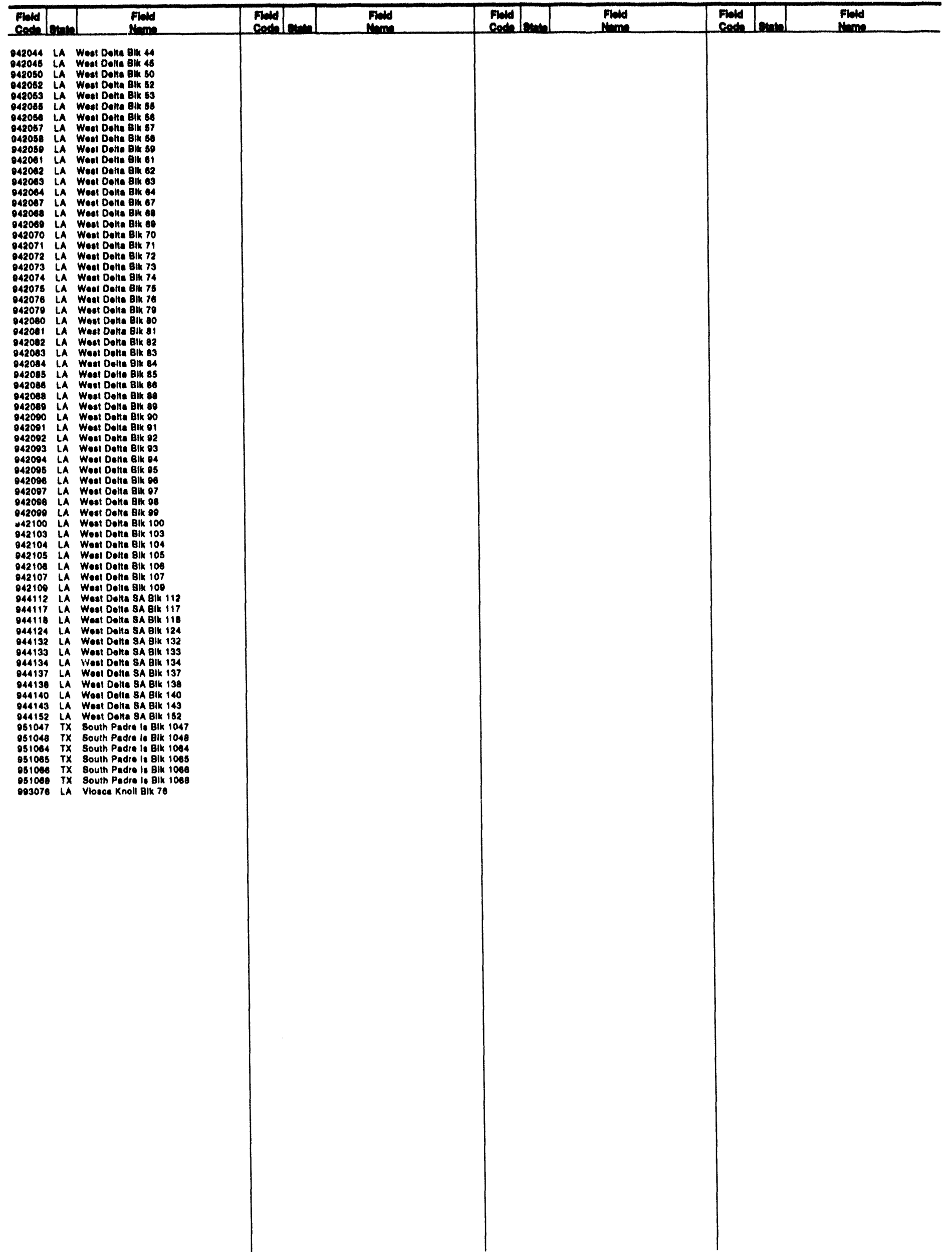




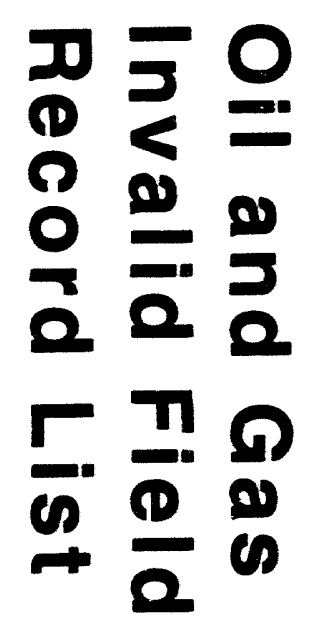




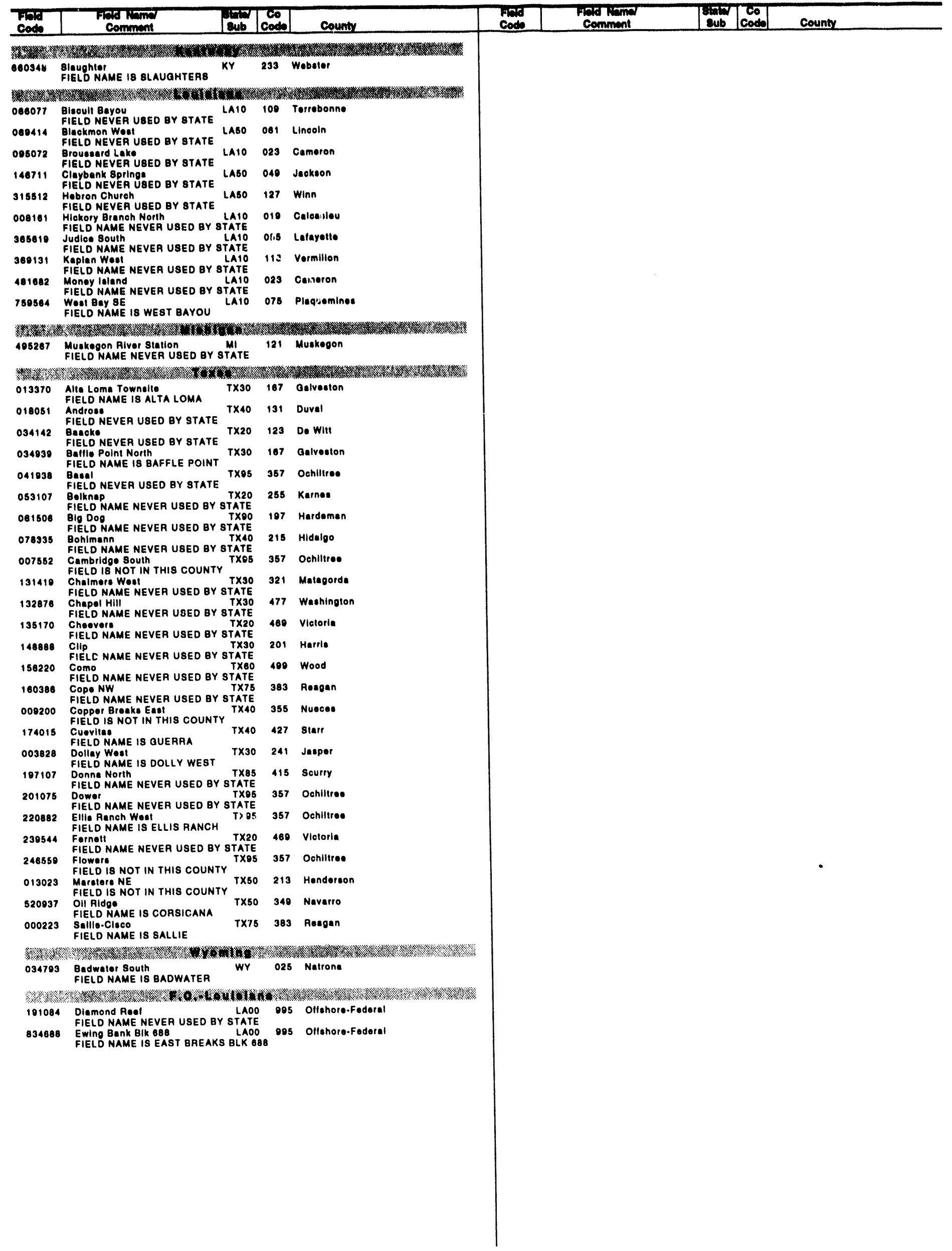



